\title{
MASTER
}

\section{Bibliography of Atomic and Molecular Processes}

March 1980

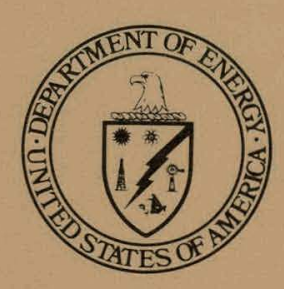

U.S. Department of Energy

Office of Energy Research

Office of Basic Energy Sciences

Division of Chemical Sciences 


\section{DISCLAIMER}

This report was prepared as an account of work sponsored by an agency of the United States Government. Neither the United States Government nor any agency Thereof, nor any of their employees, makes any warranty, express or implied, or assumes any legal liability or responsibility for the accuracy, completeness, or usefulness of any information, apparatus, product, or process disclosed, or represents that its use would not infringe privately owned rights. Reference herein to any specific commercial product, process, or service by trade name, trademark, manufacturer, or otherwise does not necessarily constitute or imply its endorsement, recommendation, or favoring by the United States Government or any agency thereof. The views and opinions of authors expressed herein do not necessarily state or reflect those of the United States Government or any agency thereof. 


\section{DISCLAIMER}

Portions of this document may be illegible in electronic image products. Images are produced from the best available original document. 


\section{Bibliography of Atomic and Molecular Processes}

March 1980
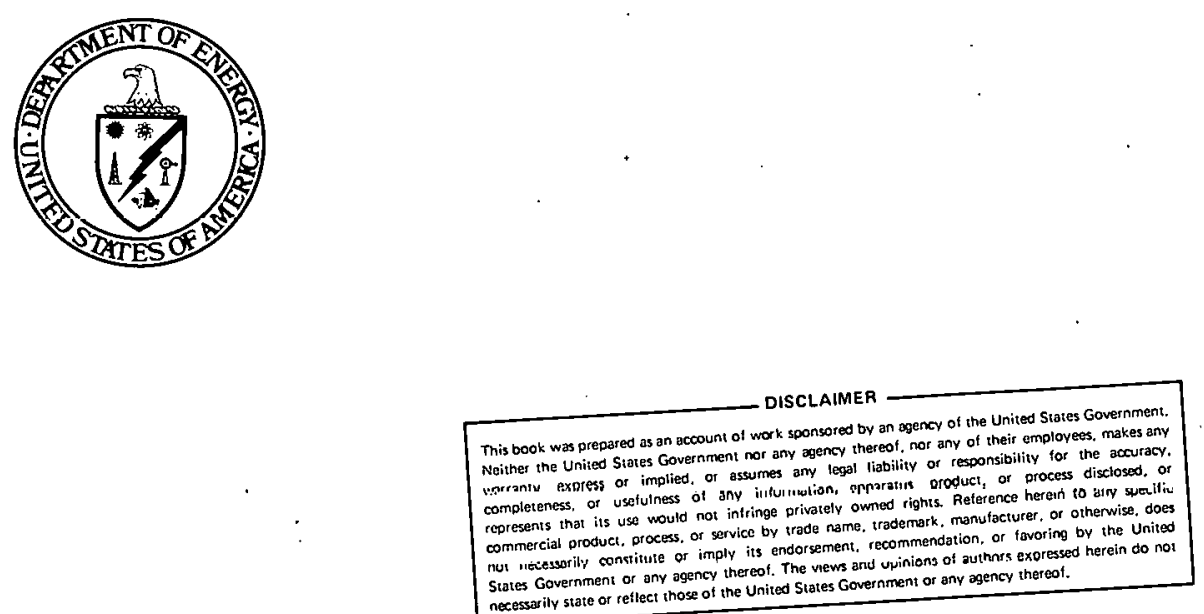

\section{U.S. Department of Energy}

Office of Energy Research

Office of Basic Energy Sciences

Division of Chemical Sciences

Washington, D.C. 20545 
Avallable from:

Nationa1 Technical Information Service (NTIS)

U.S. Department of Comnerce

5285 Port Royal Road

Springfleld, Virginie 22161

Price: $\quad$ Printed Copy: $\$ 13.25$

Microfiche: $\quad \$ 3.00$ 


\section{FOREWARD}

IT IS A PLEASURE FOR THE DEPARTMENT OF ENERGY, OFFICE OF BASIC ENERGY SCIENCES, TO MAKE AVAILABLE THIS 1978 BIBLIOGRAPHY OF ATOMIC AND MOLECULAR PROCESSES.

THE WORK WAS COMPILED BY A GROUP OF ACTIVE AND OUTSTANDING SCIENTISTS IN THE PHYSICS DIVISION OF THE OAK RIDGE NATIONAL LABORATORY UNDER THE ABLE LEADERSHIP OF C. F. BARNETT. ON BEHALF OF THE DEPARTMENT, I WISH TO EXPRESS OUR GRATITUDE TO THESE RESEARCHERS FOR SHARING THIS WORK WITH THE SCIENTIFIC COMMUNITY.

THE DEPARTMENT APPRECIATES THE VALUE OF THIS WORK TO RESEARCHERS IN THE ENERGY. AREA. IT ALSO UNDERSTANDS THAT BIBL IOGRAPHIC WORK PROVIDES SCIENTISTS AN INDISPENSABLE TOOL TO MAINTAIN A QUALITY RESEARCH EFFORT.

J. V. MARTINEZ, MANAGER

ATOMIC PHYSICS PROGRAM

MARCH, 1980 


\section{BIBLIOGRAPHY OF ATOMIC AND MOLECULAR PROCESSES}

1978

compiled by

C. F. Barnett

D. H. Crandall

B. J. Farmer

H. B. Gilbody
S. W. Hawthorne

M. I. Kirkpatrick

E. W. McDaniel

R. H. Mcknight
F. W. Meyer

T. J. Morgan

R. A. Phaneuf

E. W. Thomas

Oak Ridge National Laboratory

Physics Division

March 1980 
Table of Contents

Abstract ......................... v

Introduction ........................... vii

Categorization List ................... . . . .

Bibliography ................... 3

Reactant Index ................ 183

Author Index .. . . . . . . . . . . . . . 285 
THIS PAGE

\section{WAS INTENTIONALLY LEFT BLANK}




\section{Abstract}

This annotated bibliography lists 2,557 works on atomic and molecular processes reported in publications dated 1978. Sources include scientific journals, conference proceedings, and books. Each entry is designated by one or more of the 114 categories of atomic and molecular processes used by the Controlled Fusion Atomic Data Center to classify data. Also indicated is whether the work was experimental or theoretical, what energy range was covered, what reactants were investigated, and the country of origin of the first author. Following the bibliographical listing are indexes of reactants and authors. 
THIS PAGE

\section{WAS INTENTIONALLY LEFT BLANK}




\section{Introduction}

This annotated bibliography lists 2,557 works on atomic and molecular processes reported in publications dated 1978. It is the first such bibliography to be formatted under an open-field computer code recently developed for the Controlled Fusion Atomic Data Center. Unlike previous bibliographies in which entries were arranged alphabetically by first author, there is no significance to the ordering of entries in this publication. The benefits of such alphabetical arrangement by author are delegated to the author index. Also unlike bibliographies formatted using our earlier computer code, the entries in this bibliography are not grouped according to a particular atomic or molecular process (i.e., there is no independent section on electron transfer, ionization, etc.). Instead each entry is labeled by one or more of the 114 categories of atomic and molecular processes used by the Controlled Fusion Atomic Data Center to classify data. (A list of these categories follows this Introduction.) Grouping according to specific categories may be found in the reactants index. The author index is likewise arranged by assigned category. The absence of an author and reactants index for any one of these 114 categories indicates that there were no entries designated t. n that category in 1978.

Each entry indicates whether the work was experimental (E) or theoretica? ( $T$ ), what energy range was covered, and what reactants were investigated.

The following remarks are offered to facilitate the use of the bibliography.

1. The entries have been edited for incorrect categorization. Misplaced reactants were removed from their original reactants index category. 
and added to the correct index. Authors jisted for these entries were similarly removed to the appropriate index. The addition of a reactant to the reactants index is indicated by an asterisk beside the entry's alphabetically correct position; the reactant is given at the end of the appropriate column. The same method is used to add authors to the author index. When necessary, double asterisks, triple asterisks, and daggers are used to show second, third, or fourth additions to a column.

2. Due to computer manipulation, any differences in symbolic representation or formatting resulted in different entries in the index. For example, a superscript ++ is handled differently from a superscript $\underline{2+}$. All attempts have been made to minimize these discrepancies. However, the user should keep these possible differences in mind when performing searches.

3. Sequencing of reactants in the index follows the order $N, N^{*}$ (excited state), $\mathrm{N}^{+}, \mathrm{iN}^{2+}, \mathrm{N}_{2}, \mathrm{~N}^{-}, \mathrm{NO}, \mathrm{Na}$, Ne, etc.

4. Many papers do not refer to a particular collision system. The reactants in these cases are listed as undefined, denoted as Undef. Review papers are labeled Keview rather than list all reactants discussed in the paper. The abbreviation Seq preceded by an atom indicates all members of the isoelectronic sequence for that particular atom. PERT symbolizes "periodic table"; this notation is used when reactions involving a large number of the elements are covered by a publication. All of these codes are used in a more or less general sense to avoid handling thousands of additional reactants at every stage in the production of these bfbllographies.

5. The country listed at the end of each bibliographic entry is derived from the address of the first author given in the original publication. 
1. hEAYY PARTICLE - MEAYY PARTICLE IMTERACTIONS

2. General Elastic Scattering Collistons

3. Excitation

- Olssectacton

(photon emtsston by unspecified processes)

6. Electron Capture

. Recombination or Mutual Meutrallzation Leading to Neutral

o. Eroducts (Ton-Ion)

1. Collis (onal De-Excitation (total)

12. Collis lonal Line Broadening

3. Heavy Particie interchange. Rearrangement. aad Association

(one or more lonic reactants)
4eavy Particle Interchange, Rearrangement, and Association (only neutral reactants)

15. Epln Exchonge Detachent from Negative Ions Into Cont inum

17. Interaction Potentiais

18. Angular Scattering (specifled process or othenwise)

19. Inner-Shell Interactions (not covered under other

20. Attenuation (unspecified process)

8. IMTERACTIONS WITH STATIC OR TIME-VARYING ELECTRIC AMO MAGNETIC FIELDS

2. General Oissociation

3. Ionization

5. Detachment

7. Colitsions in Presence of Intense Electronagnetic Fields

C. PARTICLE PENETRATION IN maCrosCopic MATter (IONS, heUtrals, aNo
1. General
2. Energy Loss
3. Energy to Crecte an Ion Palr
4. Particle Range.
6. Charge State Fopulation
Chelted State population
8. Channeling

D. particle interactions With SOLIOS

3. General

(totol removal coertelents) Charge and Quantum (Excited) State Oistribution

4. Secondary Electron Ejectíon by Heary Parthele and Electrons

5. Photoelectric Ejection of Electrons (coefflefints)

6. Reflection of Electrons from Surfaces (coef

- Reflectlon of heavy Particles rron

8. Charge and Quantum State O1stributions of Reflected Heavy Particles

at Macroscopic Distances from Surfaces

9. De-Excltation. Neutralization, Ionization, or Dissociaction of Particles interacting with Surfaces
10. Interaction Potentials Between Surfaces and Free Particles Located External to the Surface (electrons and heavy particles)

11. Sticking Coefficients (thermal energies). Impact on Surfaces

13. Desorption of Gases from Surfuces

14. Blistering, Volds, and Surface Strain in Metals

17. Electron-. Ion-, and Photon-Induced Chemical Changes to Surfaces

E. ELECTRON-PARTICLE INTERACTION
1. Generai
2. Elastic Collitsions
3. Excitation
4. Dissociation
6. Recombination (electron-ion)
. Collisional De-Excitation
8. Colltsion Line Broadening
10. Spin Exchange
11. Free-Free Transitions (Bremsstrahlung)
12. Positron Collistons
14. Binary Electron-Electron
16. Fluorescence and Luminescence
17. Angular Scattering (specifled process)
18. Attenuation (unspecified process)

G. TRAMSPORT PHENOMENA AND AVERAGE PROPERTIES IN GASES

1. General

2. Diffusion of Neutrals

3. Diffusion of Electrons

5. Drift Velocity of Electrons

6. Drift Velocity of Ions

Scattering and Energy Loss Parameters of Electrons.

Keutrals, and lons in Gases

8. Energy Distribution (energy distribution of lons and

9. Momention Transfer

10. First and Second Townsend coeffictents

11. Electron Attachment Coefficients

. PHOTON COLLISIONS WITH MEAYY PARTICLES, ELECTRONS, ANO

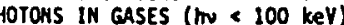
1. General
Elastic Scattering
. Excitation
5. Dissociation
7. Photodetachent
8. Fluorescence ond Luminescence Yielos
0. Photochentstry
1. Free-Free Absorotion or Inverse Brensstrahluna
12. Photon-Electron Seatterina
14. Photon-photon collistions
4. Photo-De-Excitation. Quenchina. and Stimulated
Emission (cross sections)

J. DATA COMPILATION

1. Heavy Particle

2. Electrons

3. Photons

5. Transport

K. REVIEUS AND BOOKS

1. Heary Particle

Electrons

. Particles on Surfaces and Sollds

5. Transpor

6. Structure

L. BIBLIOGRAPHIES

1. Heavy Particlo

2. Electrons

Photons

On Surfaces and Solids.

Transport

ABBREVIATIONS:
L1 Seq - sequence (LI)
PERT - perlodic table
Undef - undefined 
BIBLIOGRAPHY OF ATOMIC AND MOLECULAR PROCESSES

1978 
BIBLIOGRAPHY 
REF . REACTANT S

NO.

1

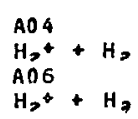

2

$\mathrm{HO} 1$

Undef

3

A06

$\mathrm{H}^{+}+\mathrm{Kr}: \mathrm{H}^{+}+\mathrm{Xe}^{\circ}$

A 03

$\mathrm{H}^{+}+\mathrm{Kr}_{\mathrm{r}} \mathrm{H}^{+}+\mathrm{Xe}$

4 A06

A0 6
$H e^{2+}+H$

5

A03

$H+N_{2}: H+C O: H+\mathrm{O}_{2}: \mathrm{He}+\mathrm{N}_{2}: \mathrm{He}$

$+\mathrm{CO}$ : $\mathrm{He}+\mathrm{D}$,

$\mathrm{H}^{+}+\mathrm{Al}: \mathrm{H}^{+}+\mathrm{SI}: \mathrm{H}^{+}+\mathrm{B}: \mathrm{H}^{+}+\mathrm{CO}^{+}$

$\mathrm{H}_{2}^{+}+\mathrm{Al}: \mathrm{H}_{2}^{+}+\mathrm{SI}: \mathrm{H}_{2}^{+}+\mathrm{B}: \mathrm{H}_{2}^{+}+$

Co

7

EO 3

e+ He

8

A19
Br+ + PERT: $\mathrm{I}+$ + PERT: $\mathrm{N} 1+$ + PERT

9

A11

E

$300 \mathrm{~K}$

REFERENCE

Melerjohann. B.: Vogler.. M. Vitrationally resolved

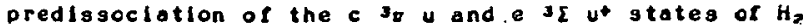
by flight-time-difrerence. spectroscopy. Phys. Rev. A 17. 47 (1978)

West Germany

I Under

Le Gouet, J.-L.: Berman, P. R. Elfect of

velocity-chongling colligl cro.upon .cptical coherences. In

a three-level sygtew. Fhys. Rev. ... 17. 52 (157e)

France

$10-150 \mathrm{keV}$

Doughty。 B. H.: Hrandon, F. L.: Bray. Co Wo: Cernosek, R. W.: Goad. H. L. Electron capture Into the $3 g$ state of atomic hydrogen by $H^{*}$ on $K r$. and $X e$. Phys. Rev..A 17. 59 (15.78)

United states

I $0.1-20 \mathrm{keV}$

Winter. T. G.: Lone. N. F. Electrcn transfer in collisicns of Hett ions with.H. aters at Hett impoct enerales of $0.1-20.0$ keV. Physa.. Bey. A. 17.66 (1978) United States

$0 \cdot 15-2 \cdot 4$

Bearman, G. H.: Leventhal, J. J. Excited-szate production In collisions of H. and He with $N_{2}, \mathrm{CO}$, and $O_{>}$over the eneray range $150-2400$.eV. Fhyse. fiev. A 17. 80 (1978)

United states

E $\quad 300-2400$

Lurlo, A.: Andergen. H, H.: Feldsan, L. C. Seerch lor cluster effects in $x$-ray prcduction by lost hydrogen wolecules. Fhys. Fev. A, 17..90,(1978.)

United states

E $\quad 80 \mathrm{eV}$

Sutcilere, V. C.: Haddad, G. N.: Steph," N. C.: Golden, D. E. Electron-photon angular correlation gessurements for the 2ip, atate of hel lum.. Phus. Rev. A 17. 100 (1578)

United states

E T 45-110 M.eV

Meyerhol. W. E.: Anholt, R.: Elchler, J.: Solop, A. $K$-vacancy production In heavy-ion collisiong. IV. $K-L$ level Eatchlng. Phys. Rev. A. 17, 108 (1978) Unlted States

Gallagher. T. F.: Cooke. W. E.: Edelstein. S. A. Collisicnal deactivation of the 58 . and 40 .states of Na by N,. Fhys. Rev. A 17, 125. (15.78)

United Staties 
REF.
NO.

REACTANTS

10

$\mathrm{CO} 2$

$\mathrm{H}+\mathrm{Hi} \mathrm{H}+\mathrm{He}: \mathrm{H}+\mathrm{LI:H}+\mathrm{Be:} \mathrm{H}+$ $\mathrm{B}: \mathrm{H}+\mathrm{C}: H+\mathrm{N}: \mathrm{H}+\mathrm{O}: \mathrm{H}+\mathrm{F}: \mathrm{H}+$ $\mathrm{Ne}: \mathrm{He}+\mathrm{H}: \mathrm{He}+\mathrm{He}$ : $\mathrm{He}+\mathrm{LI}: \mathrm{He}+$ $\mathrm{Be}$ : He $+\mathrm{B}$; $\mathrm{He}+\mathrm{C}$ : $\mathrm{He}+\mathrm{N}$ : He $+\mathrm{O}$ : $\mathrm{He}+\mathrm{Fa} \mathrm{He}+\mathrm{Ne}=\mathrm{LI}+\mathrm{Hz} \mathrm{LI}+\mathrm{He}:$

$\mathrm{LI}+\mathrm{LI}=\mathrm{LI}+\mathrm{Be}_{\mathrm{L}} \mathrm{LI}+\mathrm{B}_{\mathrm{O}} \mathrm{LI}+\mathrm{C}:$

$\mathrm{LI}+\mathrm{N}: \mathrm{LI}+\mathrm{O}: \mathrm{LI}+\mathrm{F}: \mathrm{LI}+\mathrm{Ne}: \mathrm{Be}$

$+\mathrm{HZ} \mathrm{Be}+\mathrm{He:Be}+\mathrm{Cl}: \mathrm{Be}+\mathrm{Be}: \mathrm{Be}$

$+\mathrm{B}: \mathrm{Be}+\mathrm{C}: \mathrm{Be}+\mathrm{N}: \mathrm{Be}+\mathrm{O}: \mathrm{Be}+$ $\mathrm{B}+\mathrm{Be}: \mathrm{B}+\mathrm{B}$ : B $+\mathrm{C}: \mathrm{B}+\mathrm{N}: \mathrm{B}+\mathrm{O}$ :

$\mathrm{B}+\mathrm{F}: \mathrm{B}+\mathrm{Ne}$ : $\mathrm{C}+\mathrm{H}: \mathrm{C}+\mathrm{He}$ : $\mathrm{C}+$

LI: $C+B e: C+E: C+C: C+N: C$ $+\mathrm{D}: \mathrm{C}+\mathrm{F}: \mathrm{C}+\mathrm{Ne}: \mathrm{N}+\mathrm{H}: \mathrm{N}+\mathrm{He}:$ $N+L 1: N+B_{0}: N+B: N+C: N+$ $N: N+0: N+F: N+N e: O+H: O+$ $\mathrm{He}: \mathrm{O}+\mathrm{LI}: \mathrm{O}+\mathrm{Be} ; \mathrm{O}+\mathrm{B} ; \mathrm{O}+\mathrm{C}: \mathrm{O}$ $+\mathrm{N}: \mathrm{O}+\mathrm{O}: \mathrm{O}+\mathrm{F}: \mathrm{O}+\mathrm{Ne}: \mathrm{F}+\mathrm{H}: \mathrm{F}$ + Ho: F+ L.1:F + Re:F + B: F + C: $F+\mathrm{N}: F+0: F+\mathrm{F}_{\mathrm{F}}+\mathrm{Ne}: \mathrm{Ne}+$ $\mathrm{H}: \mathrm{Ne}+\mathrm{He}: \mathrm{Ne}+\mathrm{LI}$ : Ne + Be: $\mathrm{Ne}+$ $\mathrm{B} ; \mathrm{Ne}+\mathrm{C}: \mathrm{Ne}+\mathrm{N}: \mathrm{Ne}+\mathrm{O}: \mathrm{Ne}+\mathrm{F}:$

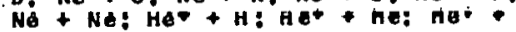
LI: net De: He' $\rightarrow$ B: Hel + C. Ho' $+\mathrm{N}: \mathrm{He}^{+}+\mathrm{O}: \mathrm{He}^{\circ}+\mathrm{F}: \mathrm{He}^{+}+\mathrm{Ne}$ :

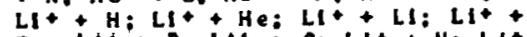

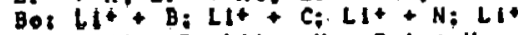
$+\mathrm{O}: \mathrm{Ll}_{+}+\mathrm{F}_{\mathrm{Z}} \mathrm{LI}_{1}+\mathrm{Ne} \mathrm{Be}_{+}^{+}+\mathrm{HI}$

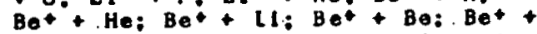
$\mathrm{BF} \mathrm{Be}^{+}+\mathrm{C}_{\mathrm{i}} \mathrm{Be}^{+}+\mathrm{N}: \mathrm{Be}^{+}+\mathrm{O}$ : $\mathrm{Be}^{+}+$ $\mathrm{F}: \mathrm{Be}^{*}+\mathrm{Ne} \mathrm{B}^{*}+\mathrm{H}: \mathrm{B}^{*}+\mathrm{He}^{\mathrm{O}} \mathrm{Bt}^{+}+$ $\mathrm{LI}: \mathrm{B}^{+}+\mathrm{Be}: \mathrm{B}^{+}+\mathrm{B}: \mathrm{B}^{+}+\mathrm{C}^{+} \mathrm{B}^{+}+$ $\mathrm{Ni} \mathrm{Bt}_{+}+\mathrm{O}=\mathrm{Bt}+\mathrm{Fi}+\mathrm{B}+\mathrm{NaO} \mathrm{C}+\mathrm{H}$ $\mathrm{C}^{+}+\mathrm{He} ; \mathrm{C}^{+}+\mathrm{H}: \mathrm{C}^{+}+\mathrm{Be} \mathrm{C}^{+}+\mathrm{C}^{+}$ $\mathrm{C}^{+}+\mathrm{C}_{\mathrm{F}^{+}} \mathrm{C}^{+}+\mathrm{N}_{\mathrm{H}^{+}} \mathrm{C}^{+}+\mathrm{O}^{+} \mathrm{C}^{+}+\mathrm{F}^{+} \mathrm{C}^{+}$ $+\mathrm{Ne}^{+} \mathrm{N}^{+}+\mathrm{H}: \mathrm{N}^{*}+\mathrm{He}^{+} \mathrm{N}^{+}+\mathrm{Ll}^{+} \mathrm{N}^{+}$ $+\mathrm{Be}^{+} \mathrm{N}^{+}+\mathrm{B}: \mathrm{N}^{+}+\mathrm{C}_{2} \mathrm{~N}^{+}+\mathrm{N}^{+} \mathrm{N}^{+}+$ $\mathrm{O}: \mathrm{N}^{+}+\mathrm{F}: \mathrm{N}^{+}+\mathrm{Ne}: \mathrm{O}^{+}+\mathrm{H}^{+} \mathrm{O}^{+}+$ He: $\mathrm{O}^{+}+\mathrm{Cl}: \mathrm{O}^{+}+\mathrm{Be}: \mathrm{O}_{+}+\mathrm{B}=\mathrm{O}^{+}+$

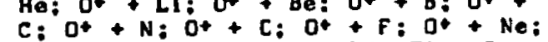
$\mathrm{F}^{+}+\mathrm{H}^{+} \mathrm{F}^{+}+\mathrm{He}=\mathrm{Ft}^{+}+\mathrm{Ll}^{+} \mathrm{Ft}^{+} \mathrm{Be}^{+}$ $\mathrm{F}^{+}+\mathrm{B}: \mathrm{F}^{+}+\mathrm{C}_{\mathrm{O}} \mathrm{F}^{+}+\mathrm{N}: \mathrm{Ft}^{+} \mathrm{O}:$ $+\mathrm{F}: \mathrm{Ft}+\mathrm{Ne}$ : $\mathrm{Ne}+\mathrm{H}_{\mathrm{O}} \mathrm{Ne}^{+}+\mathrm{He}$ $\mathrm{Ne}^{+}+\mathrm{Ll}$ : $\mathrm{Ne}^{+}+\mathrm{Ne}: \mathrm{No} \mathrm{Ne}^{+}+\mathrm{F}: \mathrm{Ne}+$ $\mathrm{Na}: \mathrm{Na}+\mathrm{H}_{\mathrm{i}} \mathrm{Na}^{+}+\mathrm{He} \mathrm{Na}^{+}+\mathrm{Li}$ : $\mathrm{Na}^{+}+\mathrm{Be}: \mathrm{Na}^{+}+\mathrm{B}$ : $\mathrm{Na}^{+}+\mathrm{C}^{2} \mathrm{Na}^{+}+$ $\mathrm{Nz} \mathrm{Na}+\mathrm{N}_{+}+\mathrm{O}$; $\mathrm{Na}+\mathrm{F} ; \mathrm{Na}++\mathrm{Ne}$

11

A19

Al0 + Al: $\mathrm{Al}^{+}+\mathrm{CU}: \mathrm{Al}^{+}+\mathrm{S}: \mathrm{Al}^{+}$

Ar: $\mathrm{SI}^{+}+\mathrm{Al}: \mathrm{SI}+$ + $\mathrm{Cu}$ : $\mathrm{SI}_{+}^{+}+\mathrm{S}$;

$\mathrm{SI}+$ + $\mathrm{Ar}: \mathrm{Cl}_{+}+\mathrm{Al:Cl}+\mathrm{Cl}+\mathrm{Cl}+$

S: Cl+ + Ar: Art+Al: Art+Cu:

Art + B: $\mathrm{Ar}^{+}+\mathrm{Ar}$

12

$A D ?$

$\mathrm{H}^{+}+\mathrm{He}^{+} \mathrm{H}^{+}+\mathrm{He}: \mathrm{H}^{+}+\mathrm{Ar}$

13

$$
\begin{aligned}
& \mathrm{EO2} \\
& \mathrm{E}+\mathrm{H}, \\
& \mathrm{Enz} \\
& \mathrm{E}+\mathrm{N}, \\
& \mathrm{E} 17 \mathrm{~N}, \\
& \mathrm{O}+\mathrm{N}, \\
& \text { SOg }
\end{aligned}
$$

$0+\mathrm{N}$.

E02

E He

O+ He
MIddleṣngth, F. M.e dr.; Banahue. D. J.: McInture. L. C.. Jr.: Bornatoln. E. M. K-oholi. Nacanoy production in.collisions of aluolinum. Sllicon, chlorine, ono argon lons ith ool id and as targets. Phys. Rev. A. 17. 141 (1578)

United Statea

$0.1-1.5$ MeV

Toburen, L. H.: Harson, S. T.: Kla, X.-K. Energy distributions of secondary electrons. III. Projectlle eneray dependence lor lonizaticn of He. Ne. ond At by protons. Phys. Gev. A 17.148 (1578) Uilted Btetes

$0.5-10 \in V$

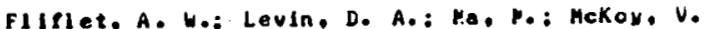

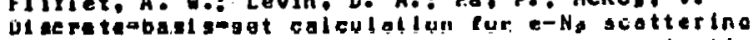
cross sections in the static-exchange ecproximetion. Phya. Rev. A 17. 160 (1578) United states

$100-700$ eV

Byron. W. Jr. Totol cross sections In electronand positrontheliua seattering. Phys. Fev. A 17,170 (1578)

Unlted states

$20-40$ eV
Gustafsson, T.: Pl uamer, E. W. : Eagtman, D. E.: Gudat, W. Partial photalonlaclloll cruss. stet lons of $\mathrm{CO}$ between 20 and 40 oV studled with synchrotron radiation. Phys. Aev. A 17, 176 (1578) radiation. Phited States 
REF.
NO.

REACTANT S

16

HO?

he + H-

17

HOP

ho $+\mathrm{Cs}^{-}: \mathrm{hv}_{\mathrm{N}}+\mathrm{K}-$

18

HOP

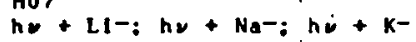

19

$\stackrel{\mathrm{AOS}}{\mathrm{C} 1+}+\mathrm{He}$

20

HOS

Under

21

H12

hut

H14

ho + e

22

HOS

Under

23

008

$\mathrm{H}^{+}+\mathrm{HO}_{\mathrm{O}} \mathrm{H}^{+}+\mathrm{CU}_{2} \mathrm{H}^{+}+\mathrm{SS}: \mathrm{H}^{+}+$

Ho: $\mathrm{H}_{+}++\mathrm{Cu}_{3} \mathrm{H}_{2}++\mathrm{SS}$ : $\mathrm{H}_{3}++\mathrm{Cu}$;

$\mathrm{H}_{3}+\mathrm{Mo:} \mathrm{H}^{+}+\mathrm{SS}^{2}$

E03

- He*

25

A29

SI2t+ He: SI3t+ He: SIt+ $+\mathrm{Ha}$

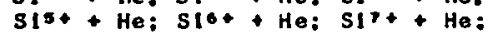

$\mathrm{SI}+4 \mathrm{He}$ : Slot He: Sl10+ $+\mathrm{He}$ Silit He

EO2

C. H*

27

A 06

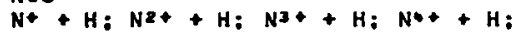

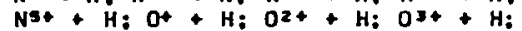

$\mathrm{Os+}+\mathrm{H}: \mathrm{C}^{+}+\mathrm{H}_{\mathrm{Z}} \mathrm{C}^{2+}+\mathrm{H}$ : $\mathrm{C}^{3+}+\mathrm{H}$ :

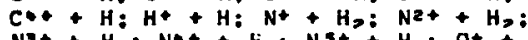

$\mathrm{N3}+\mathrm{H}_{3}: \mathrm{N}+4+\mathrm{H}_{3}: \mathrm{N}^{3}+\mathrm{H}_{2}: \mathrm{O}_{+}+$

$\mathrm{H}_{2}=\mathrm{O}_{2}+\mathrm{H}_{2} \mathrm{O} \mathrm{O}_{3+}+\mathrm{H}_{2} \mathrm{O} \mathrm{O}+4+\mathrm{H}_{2}=$

$\mathrm{H}_{3}: \mathrm{COH}_{4}+\mathrm{H}_{3}: \mathrm{N}^{2}+\mathrm{H}_{3}+\mathrm{H}_{3}: \mathrm{N}^{3}:+\mathrm{H}_{3}:$

$\mathrm{NOS}+\mathrm{H}$,

28

B07

$f *+x_{e}$

$29^{-}$

$\operatorname{Eo3}$

$e+\mathrm{K} ; e+\mathrm{Rb} ; e+\mathrm{Cs}$
EXP

ENERGY

RANGE

THEOR

T $\quad 10 \cdot 9-11 \cdot 1$

iv

E $\quad 1-2$ ev

$0-24000$ AO

E

$2-200 \mathrm{keV}$

$3-1000$ eV

15-60 MeV

9-1650 keV

$20-500 \mathrm{eV}$

the

Wendolosk 1 . J. J.: Reinhardt, W. P. Elfects of an external electric fleld on 1 fo rescnances of $\mathrm{H}^{-}$. Physo Rev. A.17. 195 (1978)

United stotes

Sl ater, J.: Read, F. H.: Novlck, S. E,: Lineberger, b. C. Alkoli neqative lons. III. Vultichennel photodetachment study of $\mathrm{Cs}^{-}$and $\mathrm{K}-$. Fhys. Rev. A 1 ? 201 (.1S7B)

United státes

Lama, G.: Szabo, A.: Adelaan, S. A. Extended asymptotic model for the alkali-anion photodetachment cross sectlcns and multipcle cclarizatilities. Fhys. Rev. A 17,238 (1978)

United stotes

KIng, D. B.: Head, C. E. Experimental litetimes of some laser levels of $\mathrm{Cl}$. Fhys. Rev. A 17, 243 (1578)

Unlted stotes

Ackerhalt, J. R. Resonant pulse excltation leading to Ionizatlon. Phys. Rev. A 17, 293 (1578)

Unlted States

Kroll, N. M.: McMullin. W. A. Stlaulated ealssicn frca relativistlc eloctrons passing thrcugh spatially perlodic tranguerse wagnet ic field. Phys. Rev. A 17. $300(2 \leq 78)$

United States

Ackerhalt, J. R. Cooment on theory of resonance fluoroscence. Phys. Rev. A 17.471 (1578) Untied states

Rausch, E. O.: Inouye, H.: Senol, A. J.: Thcoas, E. W. Exclted $H$ Coreaticn by 2-200-keV $H_{1}+h_{3}+$, and $H_{3}+$ ion Impact on metal surfaces. Fhys. Rev. A 17,473 ( 1578 )

Unlted states

Khoyrallah, G. A.: Chen, S. T.: Rugte, J.K..Jr. Inelastic scattering of electrong by metastable helium: FIrst Bcrn and Glauber crosa gecticns tor 2JS-3JS excitation. Fhys. Rev. A 17. 513 (1578) United states

Dovle. B. L.: Schlebel, U.: Macdonald, J. R.: Ellgworth, L. De Charge-stote dependence of the mean K-shell livorescence vields of Sil aper $a^{+}$) ions. Phys. Rev. A 17, 523 (IS7E) United States

Ho, T. S.: Chan, F. T. Elostic scottering of electrons by metastable 23 atonic hydrogen. Phys. fiev. A 17. 529 (1978) United States

Phaneut, R. A.: Meyer, F. W.: Makniaht, R. h. Single-electron capture by aultiply charged icns of carbon. nitrogen, and oxygen In atcmic ond wolecular hydrogen. Phys. Rev. A 17,534 (1s7e) United states

Devrlea, P. L.: Mahlab, M. S.: George, T. F. Computational study of a molecular ccillalicn process in the presence of an intense radiaticn tield: Enchanced quenching of $F$ by $X e$ in the 240-ng light of the $K r F$ laser. Phys. Rev. A 17, 546 (1578) United States

E $\quad 1.5-1500 \mathrm{eV}$
Chen, S. T.: Gallogher. A. C. Electron excltation of the resonance 1 ines of the alkall-aetal ators. Fhys. Rev. A 17.551 (1978)

United states 
REF.

REACTANTS

ADG

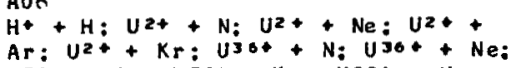
$\mathrm{U}_{36+}+\mathrm{Ar}$ : U36t + $\mathrm{Kr}$ : U92+ + N: $\mathrm{UQ2+}+\mathrm{Ne}$ : U924 + Ar: U92+ + Kr

E05

e+Ne: e+Xe

34

E05

$e+H e ; e+A r: e+N e$

35

A 12

$\mathrm{He}+\mathrm{Ne}^{*}: \mathrm{Ne}+\mathrm{Ne}$ *

HO7

37

HO3

$h_{v}+H_{2}: h_{v}+H_{e}$

38

Hns

hu $+H$

39

603

e+Be+

40

$\varepsilon 05$

e + He

41

A 19
$X e^{*}+U 1$

42

$0^{004}+(\mathrm{Cu}+\mathrm{BO}+7)$

43

EO5

$e+N e ; e+A r: e+H_{2}: e+N$,

\section{EXP
OR}

ENE KGY

RANGE

THEOR

$T$$$
\begin{aligned}
& 1-7 \\
& M e V
\end{aligned}
$$

E $\quad 1-4, \mathrm{eV}$

$T$

E T $0.2-1.2 \mathrm{keV}$

E

$\mathbf{T}$

E

$j-12$ keV

$0.1-10.6$

ov

$26-500 \mathrm{eV}$

Undef

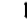

E T $460 \mathrm{MeV}$
E $\quad 0.05-3 \mathrm{eV}$

E

Lee; C. M. Radlative charae-exchonge frocess in high-energy ion-atom collisions. Phys. Rev. A 17, 566 (1578)

United States

Wong, S. F.: Dube, L. Fo tat lonal excitation of $\mathrm{N}_{2}$ by electrcn incact: 1-4 eV. Fhys. Rev. A 17, 370 ( 1578 )

United stotes

Trioathy. D. N.; Roo. B. K. Charge trangfer In prcton-hydrogen collisicns. Yhys. Rev. A 17,587 (1978) ( 1978 )

DIxon, A. J.: McCarthy. I. E.: Noble: C. J.: Helgcld. E. Factorized distorted-wove ofprcxidotion for the (e.2e) reaction on atoms: Noncoplanar symmetric. Phys. Rev. A 17, 5S7 (is?E) Australla

Fuss, I,: HçCarthy, I. E, : Noble. C, J.: Weloold. E. Factorized distorted-wave approxiarion for the (e,2e) roacticn on atoos: Coolanar syauctilc. Phys. Fev. A 17. 604 (1578) Augtralia

Salour, M. M. Isotopic shift, pressure shift. and pressure brcadening of the $7 d$ states ct neon using Doppler-pree two-photon absorption spectroscopy. Phys. Rev. A 17, 614 (15?A)

Uiiltod atoles

Regelanc, T. N.: Eender. C. F.: Mckov, B. U. Study of the photodetachment cross section of $F$-. Phys. Rev. A 17.645 (1978)

United States

Ice, G. E.: Chen, H. H.: Crosemann, B.

Photon-gcattering cross sections of $H$, ond he measured with synchrotion radiation. Phys. Rev. A 17,650 (1578)

Unlted States

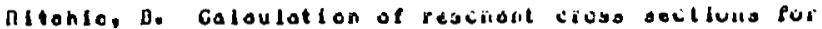
multiphoton ionization using very-narrou-bandwidth sources. Phys. Rev. A 17.659 (1978) unirea siares

Henry, F. J. H.: van Hyngaarden, H.-L.: Matese, J.J. Excltation of Bet by electron lopact. Fhys. Fev. A 17,798 (1678) United States

Oda. N.: Tahira, S.: NIshimura, F.: Kolke, F. Erratud Energy ond angular distribution of electrons ejected rrom autolonization states in hellum by electron impact [Phya. Paw o 15, S?A (167)il. Fhyo. Foy. A 17.401 ( 1578 )

Javan

Anholt, R. Analyofs of Depoler-brcadened BI K-a d-rav lines observed in $460-\mathrm{MeV} x e+$ Ei collistono. Fhyse Rev. A 17, 834 (1578) United states

Borst, b. L.: Nowak, G.: Fricke, J. KInetlc-energy deuendence of llik suranilary-ulartern yioln tor low-eneray $C(5 S)$ metastables on a Cu-be-U surface. Phys. Rev. A 17, 838 (LS7E) West Germany

DuBols. R. D.: Fudd. M. E. Absolute dcubly differential cross sectlons for ejecticn of secondary electrcns trom aases by electron lupact. II. 100-500-eV electrcna on neon. Arnon, aclacil! ar hydrogen, and molecular nitrogen. Phyo. Kev. A 17 , 843 ( 1578 )

United states

Boun. W. L. Ion scattering scectrol teotures in oxides caused by inelastic eneray logas. Phys. Rev. A 17. 849 (1578)

United states 
REF.

REACTANTS

NO.

45

A06

$\mathrm{SI}^{2+}+\mathrm{H}: \mathrm{Sl}_{2+}+\mathrm{H}_{2}: \mathrm{SI}^{3+}+\mathrm{H}^{\mathrm{S}} \mathrm{SI}^{3+}$

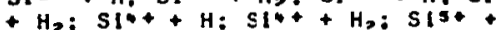

$\mathrm{Hi}$ Sist $+\mathrm{H}_{3}$ : $\mathrm{SlOH}_{+}+\mathrm{H}_{\mathrm{Z}} \mathrm{SlO}_{\mathrm{O}}+\mathrm{H}_{3}$ :

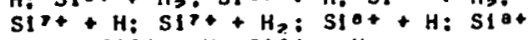

$+\mathrm{H}_{3} \mathrm{OSI}+\mathrm{H}$ : SlOt $+\mathrm{H}_{2}$

46

$\mathrm{CO} 2$
$\mathrm{O}^{+}+\mathrm{Al}$
$\mathrm{COS}$
$\mathrm{O}^{+}+\mathrm{Al}$
$\mathrm{AOS}$
$\mathrm{OO+}+\mathrm{Al}$
$\mathrm{AO}+\mathrm{Al}$
$\mathrm{O}^{2}+\mathrm{Al}$

47

E06

et $A r_{r}+$

EOA Ars+

EO3

$\mathrm{C}+\mathrm{He}$

A06

$\mathrm{H}^{+} \mathrm{Seq}+\mathrm{H}: \mathrm{H}^{+} \mathrm{Seq}+\mathrm{Cs}: \mathrm{H}^{+} \mathrm{Seq}+$

$\mathrm{Rb}: \mathrm{H}^{+} \mathrm{Seq}+\mathrm{K} ; \mathrm{H}+\mathrm{Sea}+\mathrm{Na}^{+} \mathrm{H}^{+} \mathrm{Sea}$

+ Ll: $\mathrm{H}^{*} \mathrm{Seq}+\mathrm{He}^{+} \mathrm{H}^{+} \mathrm{Seq}+\mathrm{Ne}^{+} \mathrm{H}^{+}$

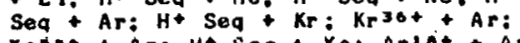

$\mathrm{H} 17+\mathrm{L}^{+}+\mathrm{H}+\mathrm{Na}+\mathrm{H}+\mathrm{K}^{+}: \mathrm{H}+\mathrm{Rb}^{+}:$

$\mathrm{H}+\mathrm{Cs}^{+}$

A06

$\mathrm{H}^{-}+\mathrm{Na}^{+}: \mathrm{H}^{-}+\mathrm{Cs}^{+}: \mathrm{H}^{-}+\mathrm{Cl}^{+}: \mathrm{H}^{+}+$

$\mathrm{K}+\mathrm{H}+\mathrm{H}+\mathrm{Rb}+$

$\mathrm{HOO}^{-}+\mathrm{Na}^{+} \mathrm{OH}-+\mathrm{Cs}^{+}: \mathrm{H}^{-}+\mathrm{CH}^{+}: \mathrm{H}_{-}+$

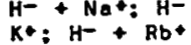

A07 + L : D + Na: D + K: D +Ro: D+

51

A19.

$\mathrm{Kr}^{2}+\mathrm{Ge}+\mathrm{Ge}$

E

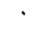

52

$\mathrm{AO3}$

A10

$\mathrm{Na*}+\mathrm{He}$; $\mathrm{Na}+\mathrm{Ne} ; \mathrm{Na}+\mathrm{Ar}$

53

EO2 $+H_{2}: \mathrm{e}+\mathrm{N}_{2}$

609

$++N_{2}$

54

$\mathrm{EO3}$

e+CsF: e+KI: Revlew

55

E

E

\section{$T$}

$\mathbf{T}$
Na* + He: Na* + Ne: Na* + Ar

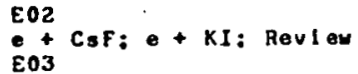

$\begin{array}{ll}\text { EXP } & \text { ENERGY } \\ \text { CR } & \text { RANGE } \\ \text { THEOR } & \end{array}$

5-50 MeV

$300-8500 k$

$20-1000 \mathrm{eV}$

$0.004-50000$
$k \in V$

$0.01-10 \mathrm{keV}$

$33.6 \mathrm{MeV}$

$300 K$

$0.01-14.0$

kev

Under

e+CaF: + LIf: Revlew

E.03

+ corte I LIT: Revieu

$0.01-10000$
HEFERENCE

KI G, H. J.: Phaneul, R. A.: Meyer, F. D.: Stelson, P. H. Single electron capture by aultiply charged 2 osi lons In atoric and molecular hyorosen. Phys. Fev. A 17. 854 (1978)

United states

Sofield, C. J.: Cowern, N.E. 8.: Fetty, K. J.:

Freeman, J. Mo: Mason, d. P. Chorce-exchange eftects in the energy-loss gtragoling of 100 long in $A l$. Phys. Rev. $A$ 17. 859 (1578)

United Kingdow

Shlu, Y.-J.: Blondl, M. A. Dissoclative recciblination In argan: Dependence ct the total rote coefficlent and exclted-state production on electron temperature.

Phys. ReV. A l?. $t 68$ (1S7E)

United States

Oh. S. [.: Macek, J.: Kelsex, E. Electron excltation of hydrogenlike ions in the Coulomb Born apfroxlation. Phys. Rev. A 17. E73 (1S7E)

United States

Grozdancv. T. P.: Jonev. R. K. Charpe-exchonge cclligicns of multiply charged tons with atcos. Phys. Reve A 17.880 (1578)

Yugoslavía

Janev, R, K.: Radulovic, 2. M. Ior-icr recambination and lon-palr foriation crocesses in alkall-hydrogen diotomic systems. Phys. Rev. A 17. BEg (1978) Yugoslavia

Llesen, D.: Macdenald, J. F.: Mckler, P.: Warczak, A. Mechanisos of K-vacancy production deterifined frod the lapact-paradeter dependence of $K$-roy froduction in 1.4-Mevjamu Kr Ge collisicns. Phys. Rev. A 17, es7 (1578)

We gt Gergany

Gallagter, T. F.: Cooke, W. E.: Edelsteln. S. A. Collisicnal angular momentum mixing of states cr Na. Collisicnal angular momentum
Phys. Rev. A 17, 904 . (1s7e)

United States

Morrtsen M. A.: Collins..L. A. Exchonge in low-energy electrcn-molecule scater Ing: Free-electron-gas wodel exchange cotentiols and actications te e-H, and $e-N$,

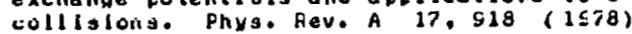
United states

Mukherjee, D.: Snlth, F. T. Semlclagsical certurbation theory cf electren-folar-mclecule ccllisicns: Total excitation and scattering cross sectlong. Hhys. Rev. A 17. $954(1 \leq 78)$

United states

Hlekman. A.CF.: Solth, H. T. Mcaeriua eransier in electron-colar-molecule collisions: Results or sealciosical perturbation gcattering theory. Fhys. Rev..A 17, 96a (1978)

United Stotes 
REF .

REACTANTS

NO.

56

$\mathrm{H}^{+}+\mathrm{Ca}: \mathrm{H}^{+}+\mathrm{Zn}: \mathrm{H}^{+}+\mathrm{Zr}: \mathrm{H}^{+}+\mathrm{Sn}:$

$\mathrm{H}^{+}+\mathrm{NO}^{+} \mathrm{H}^{+}+\mathrm{Yb}^{+} \mathrm{H}^{+}+\mathrm{AU}^{+} \mathrm{H}^{+}+\mathrm{U}:$

$\mathrm{He}^{2+}+\mathrm{Cn}_{\mathrm{H}} \mathrm{He}^{2+}+\mathrm{Zn}: \mathrm{He}^{2+}+\mathrm{Zr}$ :

$\mathrm{He}^{2+}+\mathrm{Sn}: \mathrm{He}^{2+}+\mathrm{Nd} ; \mathrm{He}^{2+}+\mathrm{Yb}^{2 t}$

$\mathrm{He}^{2+}+\mathrm{Au}: \mathrm{He}^{2+}+\mathrm{U}$

$\mathrm{AO3}$

$\mathrm{H}^{+}+\mathrm{Ca}_{\mathrm{a}} \mathrm{H}^{+}+\mathrm{Zn}: \mathrm{H}^{+}+\mathrm{Zr}: \mathrm{H}^{+}+\mathrm{Sn}:$

$\mathrm{He}^{2+}+\mathrm{Ca}: \mathrm{He}^{2+}+\mathrm{Zn}: \mathrm{He}^{2+}+\mathrm{Zr}:$

$\mathrm{He}^{2+}+\mathrm{Sn}: \mathrm{He}^{2+}+\mathrm{Nd:He} \mathrm{He}^{2+}+\mathrm{Yb}^{2}$

$\mathrm{HO}^{2+}+\mathrm{AUI} \mathrm{He}^{2+}+\mathrm{U}$

57

A 19

$\mathrm{H}^{+}+\mathrm{Y}_{\mathrm{O}} \mathrm{H}^{+}+\mathrm{MO}_{\mathrm{O}} \mathrm{H}^{+}+\mathrm{Ag}_{\mathrm{O}} \mathrm{H}^{+}+\mathrm{Sn}^{+}$

$\mathrm{H}^{+} \mathrm{Pr}^{+} \mathrm{H}^{+}+\mathrm{Sm}: \mathrm{H}^{+}+\mathrm{HO}^{+} \mathrm{H}^{+}+\mathrm{HC}^{+}$

$\mathrm{H}^{+}+\mathrm{Ta}: \mathrm{H}^{+}+\mathrm{Re}: \mathrm{H}^{+}+\mathrm{Pt}^{+} \mathrm{H}^{+}+\mathrm{Au}:$

$\mathrm{H}^{+}+\mathrm{Ta}^{+} \mathrm{H}^{+}+\mathrm{Re}: \mathrm{H}^{+}+\mathrm{Pt}: \mathrm{H}^{+}+\mathrm{AU}:$

$\mathrm{He}^{2+}+\mathrm{Y}_{\mathrm{Z}} \mathrm{He}^{2+}+\mathrm{HO}_{\mathrm{O}} \mathrm{He}^{2+}+\mathrm{Ag}_{\mathrm{a}}$

$\mathrm{He}^{2+}+\mathrm{Sn}: \mathrm{He}^{2+}+\mathrm{Pr}: \mathrm{He}^{2+}+\mathrm{Sn}$;

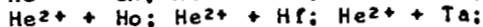

$\mathrm{He}^{2 t}+\mathrm{Re}: \mathrm{He}^{2+}+\mathrm{Pt}_{\mathrm{H}} \mathrm{He}^{2+}+\mathrm{Au}_{\mathrm{i}}$

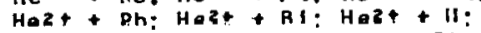

$\mathrm{He}^{+}+\mathrm{Th}_{\mathrm{O}} \mathrm{O}^{+}+\mathrm{Y}_{\mathrm{O}} \mathrm{O}^{+}+\mathrm{MO}: \mathrm{O}^{+}+$

Aa: $0^{+}+S_{n}: 0^{+}+P r: n^{+}+S_{m}: D^{+}+$

llu: $0 *$ I llfi $0^{*}$ I Ta: $0^{*}$ i Roi $0^{*}$ ।

$\mathrm{Pt}_{\mathrm{Z}} \mathrm{O}_{+}+\mathrm{Au}: \mathrm{O}^{+}+\mathrm{Pb}_{\mathrm{O}} \mathrm{O}_{+}+\mathrm{Bl}: \mathrm{O}_{+}+$

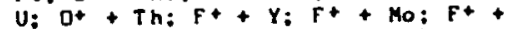

Aa: $F^{+}+\mathrm{Sn}_{\mathrm{F}} \mathrm{F}^{+}+\mathrm{Pr} \mathrm{F}^{+}+\mathrm{S}^{+} \mathrm{F}^{+}+$

Ho: $F^{*}+H^{+}: F^{+}+T^{+}: F^{+}+R e: F^{+}+$

$\mathrm{Pt}: \mathrm{F}^{+}+\mathrm{Au}: \mathrm{F}+\mathrm{Pb}_{+} \mathrm{F}_{+}^{+} \mathrm{BI}: \mathrm{F}^{+}$

$\mathrm{U}: \mathrm{F}^{*}+\mathrm{Th}: \mathrm{Cl}^{+}+\mathrm{Y}_{\mathrm{Z}} \mathrm{Cl}^{+}+\mathrm{HO}_{\mathrm{O}} \mathrm{Cl}^{+}$

$+\mathrm{Ag}: \mathrm{Cl}^{+}+\mathrm{Sn}: \mathrm{Cl}^{+}+\mathrm{Pr}: \mathrm{Cl}^{+}+\mathrm{Sm}$

$\mathrm{Re}=\mathrm{Cl}^{+}+\mathrm{Pt}: \mathrm{Cl}^{+}+\mathrm{Au}: \mathrm{Cl}^{+}+\mathrm{Pb}$ :

$\mathrm{Cl}+\mathrm{Bl}_{\mathrm{Cl}} \mathrm{Cl}_{+} \mathrm{U}: \mathrm{Cl}+$ + Th

\section{E06}

etArtro: e+Ar10+: e $+A+13+: e$

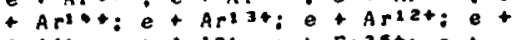

Ar11t: $e+A r^{10+}:$ e $+\mathrm{Fe}^{25+}: \mathrm{e}+$

$\mathrm{Fe}^{2+:} \mathrm{C}+\mathrm{Fe}^{23+} ; \mathrm{e}+\mathrm{Fe}^{22+} \mathrm{Fe}$

$\mathrm{Fe}^{21+}: \mathrm{e}+\mathrm{Fe}^{20+} ; \mathrm{e}+\mathrm{Fe}^{10+} ; \mathrm{e}+$

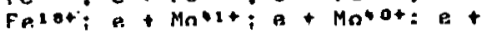

Mo394: e+Mo38+: e + Mo37+: e+

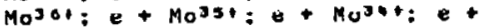

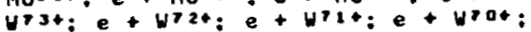

etwo

59

$\mathrm{EO}^{+}+\mathrm{H}$

60

$\mathrm{H}^{+} \mathrm{Seg}+\mathrm{H} \operatorname{Sed}$

91

E16 $\operatorname{Sn}: e+\mathrm{Te}: e+1$

62

HO4

hiv. 0

63

$\mathrm{HOS}$

$h v+Y: h v+2 r: h w+M O: h N+A_{G}:$

$h v+C d: h v+S n$

1106

$h \nu+C ; h \nu+S n: h \nu+U: h \nu+H g:$

$\mathrm{hv}+\mathrm{Al}: \mathrm{h \nu}+\mathrm{Ne}: \mathrm{hv}^{+} \mathrm{Au}: \mathrm{h \nu}+\mathrm{Fb}$

$h_{\nu}+\mathrm{Nd}: h_{\nu}+\mathrm{BI}: h_{\nu}+\mathrm{Ag}: h_{\nu}+\mathrm{Kr}:$

$h \nu+G e: h \nu+A r: h \nu+X e$
EXP ENERGY

THEOR

I Under

E

$0.5-70 \mathrm{MeV}$

Undef

$1-10$ ev

(1)

$\tau$

Unde $\mathrm{f}$

E

$662 \mathrm{keV}$

$0.5-1400$
$k \in V$
FEFEFENCE

Anholt. R. Theoretical investigation of electionic ralativistic eflects on $K-v a c a n c y$ froduction by charged portlcles. Phys. Feu. A 17.976 (1578)

UnIted stateg

Ainalt. Fiectronic relativistic and Coulcab deflection effecta cn la sigmo-vacency production. Phys. Fev. A 17.983 (197e)

United Stotes

Retter, J. A.: Gau, J. N.: Hahn, Y. Scaling properties of the olelectronlc recombination amulltudey. Phys. Rev. A. 17, SST8 (1578)

United states

Klar. H.: Klar. M. Elast ic Dhase hitt of $-H(13)$ and arcund-state energy of $H$ calculated in the post-odiabotic ecproxleotion. Fhys. lieve A 17, 1007 (1578)

West Germany

Shakeshaft. R. Aayoptotic tor of the third Born

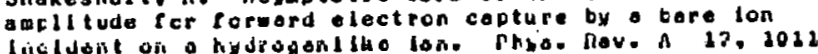
(15?R)

United states

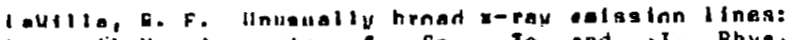

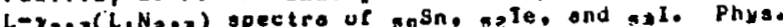
Rev. A 17. 1018 (is7e)

Unted states

Pindzolo. M. S. Two-photon excitation ol atomic

oxygon. Phyo. Hov. A d\%, 1021 (167t)

Unlted States

Al lamadhl, K. L.: Veroa, S. L.: Ghumar, B. S.: Sood,

A. S. ceterination of Integral Ccoptcr-scattering

cross sections of $662-k e V$ rrays fros $k$-shell electrons in intereediate 2 elements. Phys. Rev. A 17. 1058 $(1 \leqslant 78)$

India

Taeng, H. K.: Pratt, R. H. Y Yu, S.I Rcr. A.

Photoelectron angular olstributions. Fhys. Rev. A 17. 1061 (1978)

China 
REF.

REACTANTS

NO.

65

$\mathrm{BO} 3$
$\mathrm{Na*}$

66

$\cos$

H Ar: He + Ar: Ll + Ar: Be + Ar:

$B+A r: C+A r: N+A r: D+A r: F+$ Ar: $\mathrm{Ne}+\mathrm{Ar}: \mathrm{Na}+\mathrm{Ar}: \mathrm{Ma}+\mathrm{Ar}_{\mathrm{A}} \mathrm{Al}+$ Ar: Si + Ar: P + Ar: S + Ar: Cl +

Ar: Ar $+A r$

$\mathrm{CO} 2$

H+Ar: $\mathrm{He}+\mathrm{Ar:} L \mathrm{Ll}+\mathrm{Ar:} \mathrm{Be}+\mathrm{Ar}$ :

$B+A r: C+A r: N+A r: D+A r: F+$ Ar: $\mathrm{Ne}+\mathrm{Ar}: \mathrm{Na}+\mathrm{Ar}: \mathrm{Mg}+\mathrm{Ar}: \mathrm{Al}+$ Ar: SI + Ar: P + Ar: $S+A r: C_{1}$.

Ar: Ar + Ar

$\varepsilon 02$

$-\mathrm{N}_{2}$

$\mathrm{EO} 3$

$+\mathrm{N}$,

$68 \quad \mathrm{CO2}$

Roulew

69

HO4

he + H

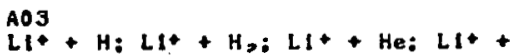
$\mathrm{C}: \mathrm{LI}^{+}+\mathrm{Na} \mathrm{LI}_{1+}+\mathrm{O}: \mathrm{Cs}^{+}+\mathrm{H}=\mathrm{Cs}_{3}+$ $\mathrm{H}_{3}: \mathrm{C}_{3}+\mathrm{He}^{+} \mathrm{C}_{3}+\mathrm{C}_{2} \mathrm{Cs}_{3}^{+}+\mathrm{N}: \mathrm{Cs}_{3}+$ $+\mathrm{D}_{-} \mathrm{Au}^{+}+\mathrm{H}: \mathrm{Au}^{+}+\mathrm{H}=\mathrm{Au}^{+}+\mathrm{He}$ : Aut $+\mathrm{C}: \mathrm{Au}^{+}+\mathrm{N}: \mathrm{Au}^{+}+\mathrm{O}$

Aut

A07

$\mathrm{C:} \mathrm{LI}^{+}+\mathrm{N}: \mathrm{Ll}^{+}+\mathrm{O}: \mathrm{Cs}^{+}+\mathrm{H}_{3} \mathrm{Cg}^{+}+$

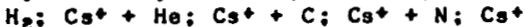
$+\mathrm{O}: \mathrm{Au}^{+}+\mathrm{H}: \mathrm{Au}^{+}+\mathrm{H}: \mathrm{Bu}+\mathrm{He}$

$+\mathrm{O}: \mathrm{Au}^{+}+\mathrm{C}: \mathrm{H}: \mathrm{Au}+\mathrm{N}+\mathrm{Hu}+\mathrm{H}+\mathrm{O}$

Als

$\mathrm{Al}_{+}+\mathrm{H}: \mathrm{Ll}_{+}+\mathrm{H}_{2}: \mathrm{Ll}+\mathrm{He}_{+} \mathrm{LI}_{+}+$ $\mathrm{C}: \mathrm{Ll}_{1}+\mathrm{N}: \mathrm{Cl}_{1}+\mathrm{O}_{\mathrm{O}} \mathrm{Cs}_{3}+\mathrm{H}_{3} \mathrm{Cs}_{3}+$

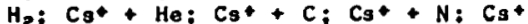
$+\mathrm{O} ; \mathrm{Au}^{+}+\mathrm{H}: \mathrm{Au}^{+}+\mathrm{H}_{2} \mathrm{O} \mathrm{Au}^{+}+\mathrm{He}$

$\mathrm{Au}^{+}+\mathrm{C}: \mathrm{Au}^{+}+\mathrm{N}: \mathrm{Au}^{+}+\mathrm{O}$

71

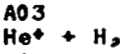

$\mathrm{He}+\mathrm{H}_{2}$

72

A0 3

$\mathrm{O}^{2+}+\mathrm{SI}_{\mathrm{O}} \mathrm{O}^{2+}+\mathrm{S}_{\mathrm{O}} \mathrm{O}^{2+}+\mathrm{Cl}^{2} \mathrm{O}_{2+}+$

Ar: $\mathrm{O}_{2+}+\mathrm{SIH}_{a}: \mathrm{C}^{2+}+\mathrm{SIF}_{0}: \mathrm{O}_{2+}+$

$\mathrm{H}_{3} \mathrm{3} ; \mathrm{O}^{2+}+\mathrm{SU}_{>}: \mathrm{O}^{2+}+\mathrm{SF}_{n}: \mathrm{O}^{2+}+$

$\mathrm{HCl}: \mathrm{O}_{2+}+\mathrm{Cl}_{2} \mathrm{C}^{2+}+\mathrm{CCl}_{4} \mathrm{O}^{2+}+$

$\mathrm{NaCl}: \mathrm{O}_{2+}+\mathrm{KCl}: \mathrm{O}_{2+}+\mathrm{P}_{4}: \mathrm{O}_{2+}+\mathrm{S}_{2}$

73

E06

a $+\mathrm{NH}_{0} \cdot$

74

$\mathrm{AO} 2$

$\mathrm{He}^{+}+\mathrm{He}$

AnG

Het+ He

75

A12

$\mathrm{Kr}+\mathrm{Na*}: \mathrm{Xe}_{\mathrm{e}}+\mathrm{Na}$

$\mathrm{HOB}$

$h_{v}+\mathrm{Na}_{0}+\mathrm{Kr}_{r}: \mathrm{hv}_{v}+\mathrm{Na}+\mathrm{Xe}_{e}$

76

H06
$\mathrm{Ll}+\mathrm{H}: \mathrm{Ll}_{+}+\mathrm{HOH}_{2} \mathrm{Ll}_{+}+\mathrm{He}_{0} \mathrm{Cl}_{+}+$

$\begin{array}{ll}\text { EXP } & \text { ENERGY } \\ \text { CR } & \text { RANGE }\end{array}$

T

10-105 eV

$0.05-30 \mathrm{MeV}$

$0.4-30 e V$

$0.065-2.0$ eV

A12

$300 \mathrm{~K}$

$H 08$
$2 \mathrm{eV}$

E
GEF EFENCE

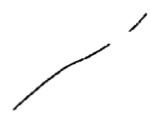

Cooke, ti. E.: Gollagher T. Fo Cecendence of Ry dbero-state cleld-tonization thresholos on [*1. Phys. Rev. A 17.1226 (1578)

United Stotes

Inokut l. M.: Baer. T.: [ehmer.J.L. Aodendum: Systematlcs of moments of dipole oscillator-gtrength di otributions for otos in the first ino second rcw. Phys. Fev. A 17, 1229 (1578) United States

Teak in. A. Internuclear dependence of the polarizobility. of N,. Phys. Fev. A 17, 1232 (1578) United States

Ahlen. S. P. $Z_{1}$ stopolng-cover formula for fost heavy lons. Fhys. Rev. A 17, 1236 (1978)

Untied states

Salzan, W. R. Erratum Semiclassical calculations on one- two-, three- and four-photon absorption in truncated wcdels of the hyorogen otca f fhys. hev. Al6, 1552 (1577)) . Fhys. Rev. A 17. 1240 (157E) Unlted states

Glliesple, G. H.: Kla, Y, $-K_{0}$ : Cheng, K. Born crcss sections for lon-atoo collisicns. Hnys. Fev. A 17. $12 \varepsilon 4$ ( 1578$)$

United States

1.5-3.0 keV ErIksen, F. J.: Jaecks. D. H. Ha( 23f) excltotion in 1.5- anc 3.0-kev Het+ H, ccillsions. Fhys. Rev. A 17. 1256 (1578)

United States

Demarest, J. A.: Wotson, R. L. Ior-excited $k$ a x-roy

satellite spectro of $S i, S, C l$, and $A r$ in the gas

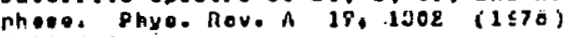

Unlted States

DuBols. R. D.: Jeffrles, J. B.: Durn, G. H

Dissociotive recoablnation cross secticns er NHat Ions and electrons. Phys. Rev. P 17, 1314 (2578) Unlted States

Vestal, M. L.: Blakley, C. F.: Futrell, J. H. Crossed-bear measurements cf diflerentiol cross sectlong lor eldatic gcottering and charge exchange in low-energy Het-He cclilisions. Fhys. Fev. A 17,1321 (1578)

United stoter

Hest. H. P.: Gollagher. A. Fressure dependence of Na resonance it ine broadening ty $K r$ and $x e$. Fhys. Fev. A 17.1431 (1978) United Etotes

Nygaard, K. J.: Corbln, R. J.: Jones, J. D. Two-step photolonizetion of potassiug otoms. Phys. Rev. A 17, 1543 (2578) Unl ted states 


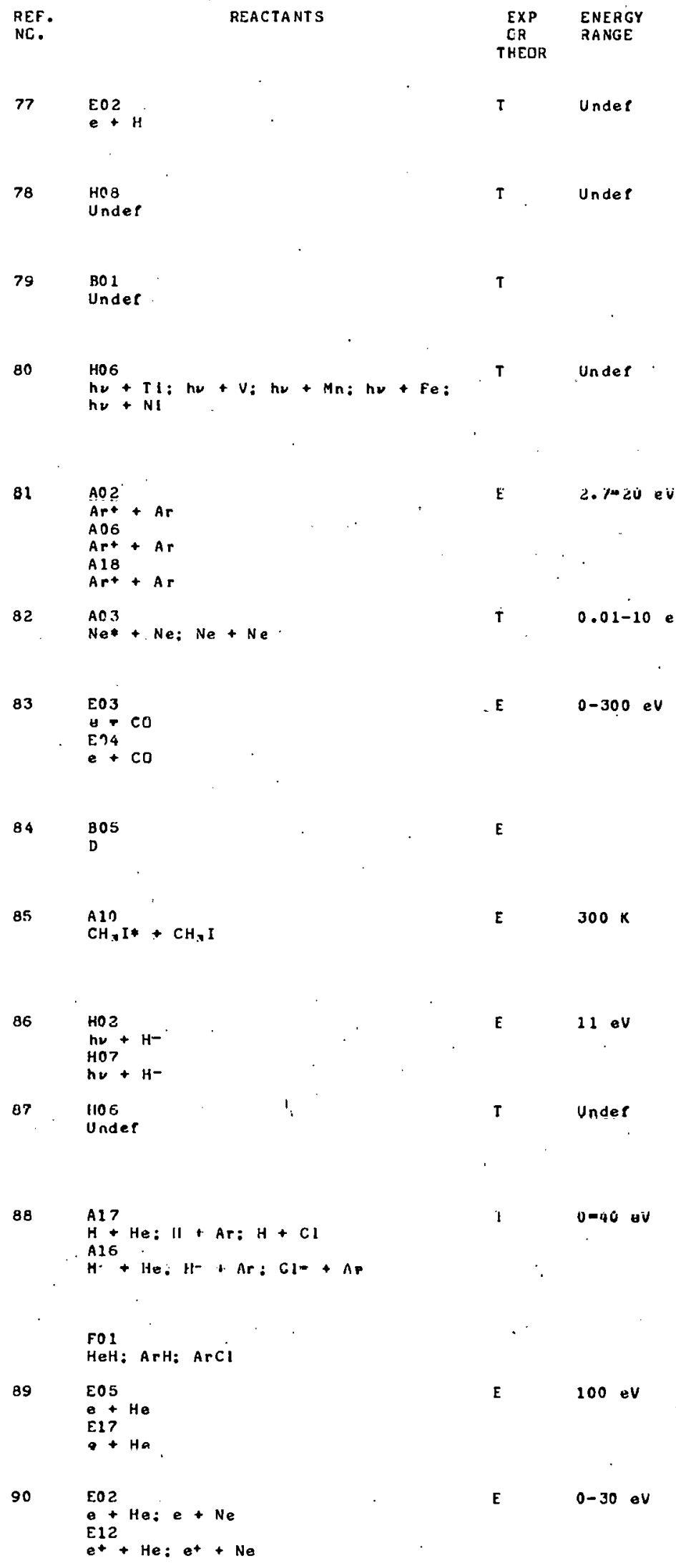

Byron, F. W.. Jr. Comment on dispersicn relationg for the static-exchonge asclltude. Fhys. Fev. A 17, 1545 (1578)

Unlted Etates

Knlght, P. L.: Molonder, H. A.: Stroud, C. F., Jr. Asymmetric resonance lluorescence spectra in portiolly coherent llelds. Fhys. Rev. A 17, $1 \leqslant 47$ (1978) United Kingdom

Molonex, J. V.: Meath, W. J. Induceo trangiticn probablilties and energles for the strongly coupled two-level gystem. Phys. Fev. A 27,1550 (1578) Canada

YIn, L. I.: Adler, I.: Tsong, T.: Chen, M. h.: RIngers, D. A.: Crosemonn. B. Errotum Wioths of atomlc $M-s h e l l$ vocancy stotes and auastatcalic aspects ct radlaticnless translitions in sclids ifhus. Fev. As, 1070 (1574)\}. Fhys. Rev. A $1 \%$. 1E56 (157e) United states

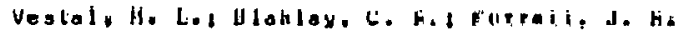

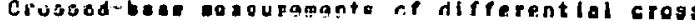
gectiono for elagtle gcattering and charge exchange in loy-energy Art-Ar collisicrs. Fhyse feve A 17, 1337 (2578)

Unlted stotes

Cohen, Je So: Colling, L. A,: Lane, N. F. Theory in Inelastic cclllglens between low-lying exclted- and ground-state $\mathrm{Ne}$ atoms. Phyg. Fev. A 17,2343 (1578) Unitad stateg

Wells, H. C.; Borst, W. L.; 21 pl, E. C. Translatlonal soeftrogccpy of metostatle progrents crcduced ty dissociotive excitation of atmosphéric oaseg oy electrcn lapact: 1I. Carbcr monoxide. Phys. Fev. A electrcn (ar ract: United stotes

van Wijngoorden. A.: Drake, G. W. F. Leuteriua Lanb shift via quenching-radiaticn onisictrocy messurements. Fhys. Fev. A 17,1366 (1578)

Conodo

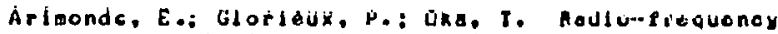
spectrcscopy Inside a laser coulty: cure nuclear guadrupcele regononce of gasecus Chis. Fhys. Fev. A 17.1375 (1.978)

Callada

Behrlnger, K.: Thowa. P. Coservetion of the 'H- shape resonanoe in. the hydrogentarc talsilen spectrum. Fhys. Rev. A 17, 140E (IS7E)

West Gerwany

de Meljere, J.L. F.: Eberly, J. H. Fate of resonant two-photon lonlzation in ine preserce ci a jortially coherent roclaticn lield. Phys. Rev. A 17,1416 (1570)

United states

Ol sou, F. E.t Liu. P. Selpecenalstent-tieló notentlal energles for the pround negative-icn and neutral atates or HeH. ArH. and ArCl. PhNs. Hev.A 17. 1568 (1578) Unlted States

Beoty. E. C.: Heanel bacher, K. Fe: Hiono, B. P.I Meore, J. H. Measurementg of the triple-differential crcas section for low-energy elestrcn-iagact lonization of hellum. Fhys. Fev. A 17. 15s2 (157e) United states

Steln, 1. S.: Kaufrlla, U. E.: Pcl, V.: Saert, J. H.: Jeslon. G. Measurements. cl totol scottering cross sections for low-eneray positrong and electrons colliding ith hellum and neon atoas. Fhys. Rev. A 17. 1600 (1578)

United states 
REF.

REACTANTS

91

H02

$h_{\nu}+K_{r} *+H_{e}: h_{\nu}+K_{r} *+A r$

A12

$\mathrm{Kr}^{*}+\mathrm{He}: \mathrm{Kr}^{*}+\mathrm{Ar}$

92

A06

Under

93

E03

a He Sea

E05

a He Seq

94

E05

e $+\mathrm{He}$

95

$\mathrm{HL}^{+}+\mathrm{Al}: \mathrm{LI}^{+}+\mathrm{Al}: \mathrm{O}^{+}+\mathrm{Al}$

96

$\mathrm{H}^{+}+\mathrm{C}: \mathrm{H}^{+}+\mathrm{N}: \mathrm{H}^{+}+\mathrm{O}: \mathrm{H}^{+}+\mathrm{Ne}: \mathrm{H}^{+}$

$+\mathrm{Ar:} \mathrm{H}^{+}+\mathrm{H}$

A19

$\mathrm{H}^{+}+\mathrm{C}: \mathrm{H}^{+}+\mathrm{N}: \mathrm{H}^{+}+\mathrm{O}: \mathrm{H}^{+}+\mathrm{Ne}: \mathrm{H}^{+}$

+ Ar: $\mathrm{H}^{+}+\mathrm{H}$

97

A07

$\mathrm{H}^{+}+\mathrm{Al}: \mathrm{H}^{+}+\mathrm{Ni}: \mathrm{D}++\mathrm{Al}: \mathrm{D}^{+}+\mathrm{Ni}:$

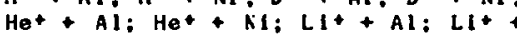

$\mathrm{NI}$ : $\mathrm{C}^{+}+\mathrm{NI}: \mathrm{O}^{+}+\mathrm{NI}: \mathrm{F}^{*}+\mathrm{NI}$

AO 6

$\mathrm{H}^{+}+\mathrm{Al}: \mathrm{H}^{+}+\mathrm{NI}: \mathrm{D}+4 \mathrm{Al}: \mathrm{D}^{+}+\mathrm{NI}:$

$\mathrm{He}^{+}+\mathrm{Al}: \mathrm{He}^{+}+\mathrm{NI}: \mathrm{LI}_{+}+\mathrm{Al}: \mathrm{LI}^{+}$

$\mathrm{NI:} \mathrm{C}^{+}+\mathrm{NI}: \mathrm{O}^{+}+\mathrm{NI:F+}+\mathrm{NI}$.

98

E11

99

$\stackrel{B O 1}{H}^{\text {Bo1 }}$

100

B07

Undet

HO6

Under

101

B07
Undef

Unde

Under

102

A12

$\mathrm{CF}_{4}+\mathrm{CF}_{4}$

$\mathrm{HO4}$

$\mathrm{hy}+\mathrm{CF}_{4}+\mathrm{CF}$.

$\operatorname{lng}$

011

$\mathrm{He}+\mathrm{Pd:} \mathrm{CO}+\mathrm{Pd:} \mathrm{O},+\mathrm{Pd}$

D07

$\mathrm{Ho}+\mathrm{Pd}: \mathrm{Co}+\mathrm{Pd}: \mathrm{O}$, $+\mathrm{Pd}$

$104 \quad$ FOI
LIH
E

\section{EXP ENERGY
CR \\ THEOR}

E

$300 \mathrm{~K}$

T

Under

$40-400$ eV

$\varepsilon$

$400-800 \mathrm{eV}$

E

4-26 MeV

E. T

2-90 Mel

$300 \mathrm{keV}$

西

Under

Under

$300 \mathrm{~K}$

$300-1020 \mathrm{~K}$

Brechlonac, C.: Vetter, R.: Heran, F. F. Study ol velocity-changlng collisions In exclted Kr using saturation spectroscopy. Fhys. Fev. A 17,1605

(1578)

France

Arora, I.: Turner, J.E. Theoretical oethed for solving two-level collisicn systes. Fhys. hev. A. 17. 1614 (1978)

Indla

Sagpson, D. H.: Parks, A. D.: Clark. F. E. H. Intermediate-coupling cclilsicn strengths for fine-structure transitions tetieen $S$ and $p$ levels and $S$ and D levels In highly chorged He-like lons. Fhys. Rev. A 17,1619 (1978)

Unlted stotes

Casllicnl, R.: Guiconi, A. G.: MeCarthy, I. E.:

Stetani. G. Mechaniso cf the $(e, 2 \in)$ reacticn with atcos. Fhys. Rev. A 17, 1634 (257d)

Italy

Jaolson, K. A.: Hilchard, P.: Hookins, F.: Matthews, D. L. Nonstatistical copulaticn of deanetic gubstotes of

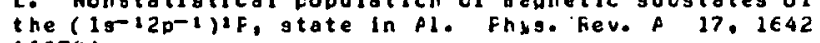
(1576)

Unlted States

LIn, C. D.: Sccng. S. C.: Tunnell, L. N. Two-state atomlc exponston methods for electron capture frca multielecticn atorg by fast crctcrs. Fhys. Fev. A 17. 1646 (1978)

Unlted States

Basbas, G.: Brandt, W.: Loutert, R. Univergal cress sectiong lor K-shell icnization by heabycharaed particles. II. Intermediate farticle welocities.

Phys. Fev. A 17, 1655 (1578)

Unlted States

Behncke, H.-H.: Nokel, t. Angular dependence of the photon linear polarlzation in the eleaentary process of atomic-lielc bremsgtrahlung. Phys. Rev. A 17, 1675 ( 1578 )

west Gerang

Ehrlch. H.: Kelleher, L. E. hudrcsen Iline-structure ellects at low electron densitles. Phys. Rev. A 17, $16 \varepsilon 6$ (1578)

United states

Nayfeh. H. H.; Payne, M. G. Fadiative

collisicn-induced thotcionizaticn. Frys. Fev. A 17. 1655 (1578)

Unltad Statoo

Kruper, H.: Jung, C. Lou-frequency apcroach to wultiphoton free-tree trangiticns inouced ty raliatic laser Fulses. Fhys. Rev. P 17, 1706 (1S7e) west Gerpany

Le Dutf, Y.: Gharbl. A. Collision-induced scattering at a vibrational haman frecuency. Fhys. Hev. A 17. $1729(1 \leqslant 78)$

United states

Engol, 1. A molecular ceas investigation of He, CC, and $C$, scattering trow Fd(111). J. Cher. Phys. ES, $373(1 \leqslant 78)$

West tọerangy

Zeske, H. I.: Won. K. F.: Stralley, . C. Fodlotive

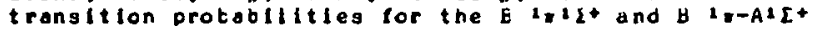
bands of 'LiH. Je Chem. Phys. 65, 40z (1578) Unlted states

Zeake, b. T.: Stwalley. W. C. Fadiotive lifetimes for vitraticnal ievels of the E is atate ci NiH.J. Chea. Phys. ES, 40S. (1578)

Unlted States 


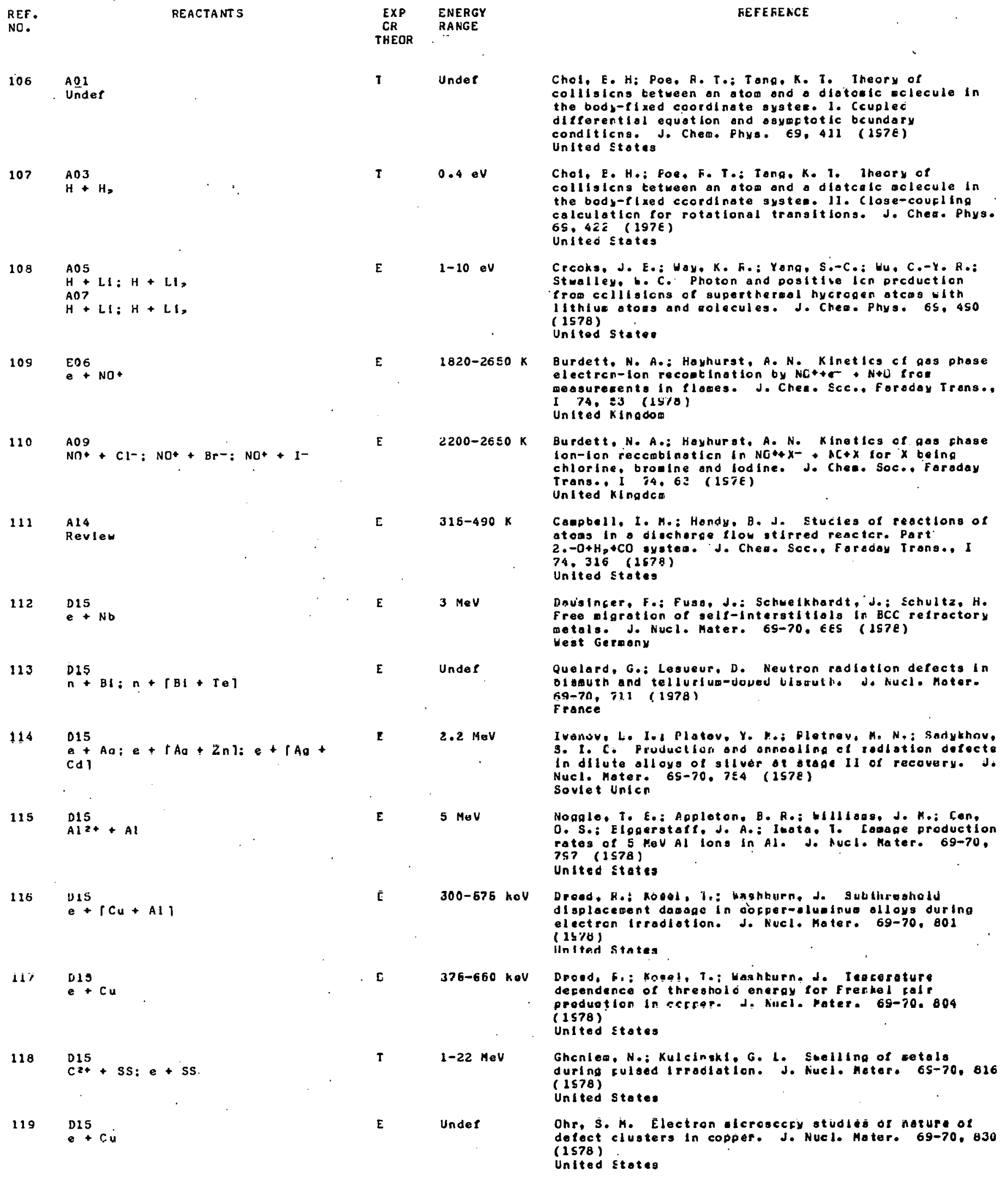


REF.

REACTANT 3

NO.

120

$\mathrm{AO2}$

NO $+A r: H_{2}+A r: H_{2}+K_{r}: H_{2}+X_{e}$

121

Ar $+N_{2}$

122

$\mathrm{LI}^{\mathrm{AO3}}+\mathrm{CO}$

A17

$\mathrm{LI}+\mathrm{CO}$

123

A 03

$\mathrm{He}+\mathrm{HD}$

124

A 14

Review

125

$\mathrm{All}$
$\mathrm{HO}$ + $\mathrm{He}: \mathrm{H}_{2}+\mathrm{Ar}$

$\mathrm{H}_{2}+\mathrm{He}: \mathrm{H}_{2}+\mathrm{Ar}$

$126 \quad A 01$

Under

127

$\mathrm{A} 17$
$\mathrm{CO}+\mathrm{H}$

128

$\stackrel{A 05}{N O}+0$.

129

$\mathrm{HO} I$ + $\mathrm{He}: \mathrm{HCl}$ + $\mathrm{Ar}$

$\mathrm{HCl}+\mathrm{He}: \mathrm{HCl}+\mathrm{Ar}$

130

HO5

hy + $\mathrm{H}_{3}$ *

131

$\mathrm{AO2}$
$\mathrm{LO}$
$\mathrm{AO}$

$\mathrm{LI}+\mathrm{Na}: \mathrm{Na}++\mathrm{H}$

132

A17

$\mathrm{Ne}+\mathrm{He}_{*}+$

133

Al1

$H_{2}+H_{2}: N_{*}+N_{i} N_{2} *+N_{2}$

134

$\mathrm{HO}_{\mathrm{H}}+\mathrm{H}=\mathrm{O}$

HOS

hV $+H=0$

HOG.

hv $+\mathrm{H}, \mathrm{O}$

135

$\begin{array}{ll}\text { EXP } & \text { ENERGY } \\ \text { GR } & \text { RANGE } \\ \text { THEOR } & \end{array}$

$\tau$

T $0.26-1 . \varepsilon$

kcal toole

T $300 \mathrm{~K}$

Under

1 Under

E $\quad 0-1$ eV

T $5-30$

keal/nole

T $\quad 12-20$ eV

Under

$300 \mathrm{~K}$

E $\quad 10-60$ eV

Alo

REF EFENCE

Klaasen. D.: Thuls, H.: Stclte, S.: Reuss, J. Colculaticn of the glory ocottering ith anlsotroplc molecule-aton interactions in cudden acproxisation. Chem. Fhys. 27. 107 (1978)

The Netherlands

Alexander. H. H. Polorization in elatic scattering: close-ccurling otudies cn Ar-N, Cher. Phys. 27, 229 (1578)

United stotes

Thcmas, L. D.: Kraemer, W. F.: DIerckgen, G. H. F.: MeGuire. F. Coaparison of classical wechanics anc the coupled states approximation for Li*-CO scattering on an ab initlc calculated CI cotenticl eneray gurtace. Chem. Frys. 27. 237 (1978) West Germony

Ge1b. A. Clasical trajectory atudy cf rotaticnal excltation cf HD by collisicns with He. Chem. Fhys. 27. 245 (1578) Hexico

Flscher, S.: Venzl. G.: Rotin, J.: Fatrer. M. A. Product eneray digtributicn fcrexcthesalic reoctive collisicns. Chem. Phys. 27. 251 (2578)

West Gerouny

Liv. Ho-K.: MCCcurt. F. H. DUBA colculations et relaxation and kinetic cress secticns. II. Application to orthc-H,-He ond ortho-H,-Ar. Ched. Physe 27, 281 (1578)

The Netherlands

Eu. B. C. P stachastlc thecry ct collision phenomeno, distritution or otgervables and Inforwation entrciy. Char. Phys. 27. 301 (1STE)

Conada

Prissett. J.: KochongkI, E,: Flcuer, [, R. Ireoretical

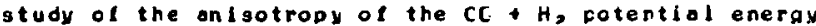
surface in the non-reactive region. Chem. Fhys. 27. surface in

France

Redpath, A. E.; Menzinger, M.: Carringtcn, T. Molecular beas chediluininescence $X 1$ : 'kinetic ono internal energy dependence of the $N O+0_{0}, N_{2}, *$ NO, reacticn. Cheo. Fhys. 27, 4CS (1978) Canada

Folanyl. J. C.: Sothyaeurthy. N. Fctotional eneray tronster (theory). II. HCl + He, Ar. Cham. Physe 29; g (1978)

canada

Kulander. K. C.: Eottcter, C. Fhotofregsentation of Ho. Chem. Phys. 29, 141 (1S78) United kingdom

Wi inaendts von Resondt, $R$, H.: de vreuod, $C_{\text {: }}$ : Chaopion R. L.: Lca, J. Differentid scaterinf cross sections for collisicns of alkall icns ond etons. III. Lit 4 Na and $\mathrm{Not}+\mathrm{Ll}$. Chea. Prys. 2S, 151 (1S78) The Netherlands

Scholdt, H. M.: ven Hirschhaugen, H.: telfrich, K. Ab Initio fotential gurfaces for Nebe, in the frozen orbital apcroxidation. Chem. Fhys. ZS. zig (ISTE) West Geriany

Euing. G. The role of van der tools aclecules in vitraticnal relaxation processes. Ches. Phys. 25, 253 (1578)

France

Tan, K. H.: Brion, C. E.: van der Leeuw. Ph. E.: van der Wiel, M. J. Absolute osclllatcr strenaths (10-60 eV) for the photoobsorfticr. chctoicnisation and fragmentation of H,C. Chell. Phys. 2S. 29S (ISTE) Canada

Murty. F. S. Electronlc tronsitions ol aludinum monoxlde. Chem. Prys. 25. 3SI (1578) Ind 19 


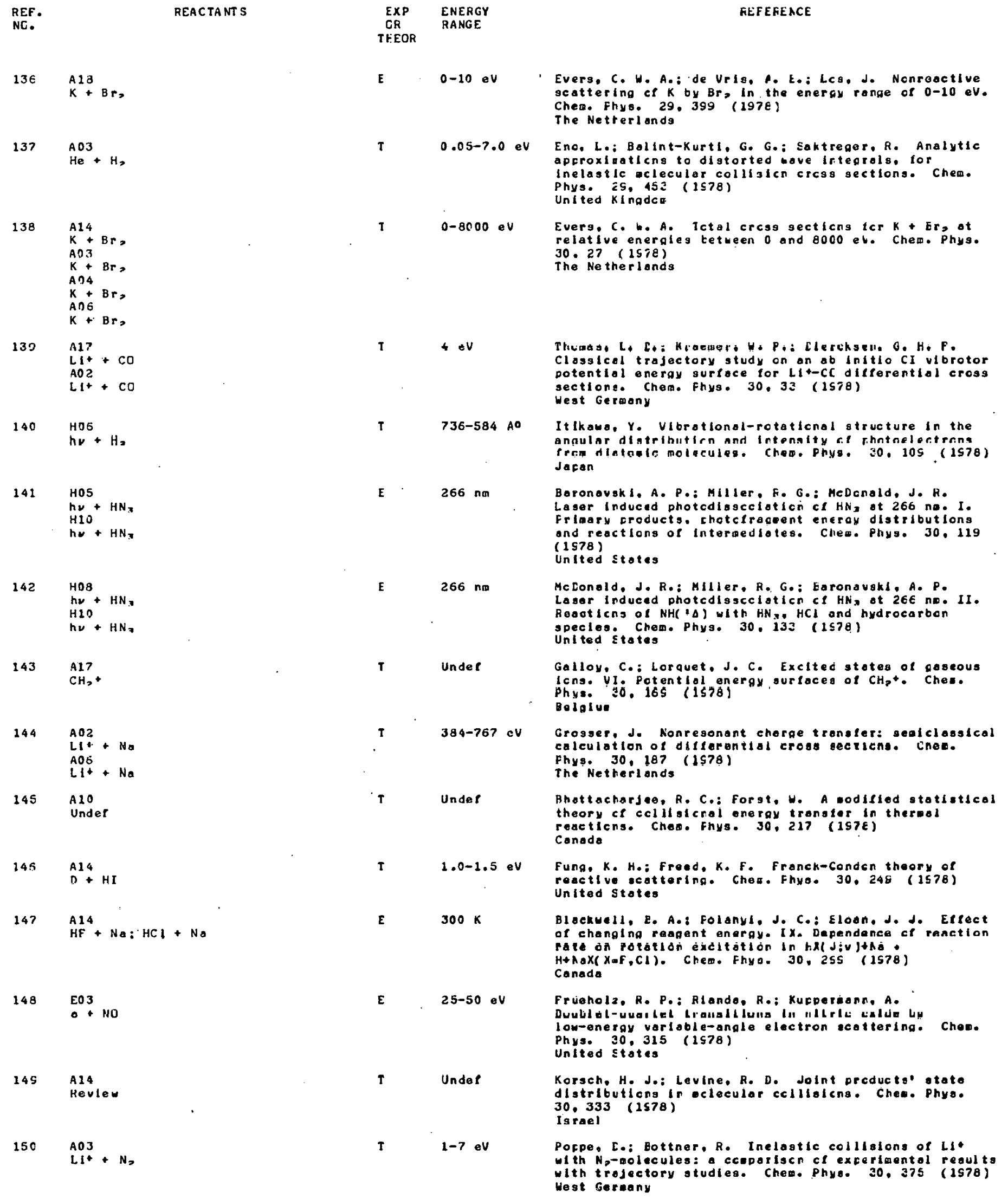


REF.

REACTANT $S$

NO.

$\mathrm{H}_{3} *+\mathrm{He}: \mathrm{D}_{2} *+\mathrm{He}$

A11

$H_{2}+$ He: $D_{2} *+H_{e}$

152

$\mathrm{N}_{0 *}+\mathrm{N}_{2} \mathrm{Cl}_{2}+\mathrm{Cl}_{2}$

153

154

155

604

Revlew

G06

Review

156

A 17 F

A06

Under

EOS

$\mathrm{e}+\mathrm{H}_{3}: \mathrm{e}+\mathrm{H}_{3}+$

H06

$h_{\nu}+H_{3}: h_{\nu}+H_{3}+$

159

HO2

Under

A03

$\mathrm{He}+\mathrm{H}_{3} \mathrm{OHe}+\mathrm{Cl}$,

161

AOS 3

Undor

Under

$162 \quad$ An3

$\mathrm{He}^{\mathrm{n}}+\mathrm{H}$,

163

H08

hv + I,

164

H10

hv + I,

HO6

$h_{2}+510$

166

AO2

$\mathrm{Ar} *+\mathrm{CO}_{*}$

Al1 $+\mathrm{CO}^{\circ}$

167
A02

$\mathrm{H}^{+}+\mathrm{Ar}_{\mathrm{O}} \mathrm{CH}_{+}+\mathrm{Ar}_{\mathrm{H}} \mathrm{He}+\mathrm{Ne}$

\section{EXP . ENERGY \\ RANGE}

T $\quad 0.3-1.5 \mathrm{eV}$

$100-600 x$

$568-444 \mathrm{~nm}$

Under

$0-20 \mathrm{eV}$

Un def

Unde?

Unde

Undef

$0.4-1.2 \mathrm{eV}$

Unde?

$0.5-7.0 \mathrm{eV}$

$5145 A^{\circ}$

$5145 A^{\circ}$

$10-18$ eV

$300 k$

$58-126$ a
REFEFENCE

B $111 \mathrm{ing}$ G. D. Cress secticns and rote ccnstants lor rotaticnal and vibraticnal excltoticn $C l H_{2}$ and $D$, colliding with He. Chem. Fhys. 20.3e? (1578) Deneart

Niveland, C.: Blliling. G. C. Fictationol reloxation of homonuclear diatoolc nolecules by claesical trajectcry cooputoticn. Chem. Phys. 30, 401 (1578) Denoerk

Petersen, A. E.: Salth, I. W. Me relc of Er*(42P,lo) as a functicn of wavelength in the fhctodissoclotion of $8 \mathrm{r}$, and IBr. Chew. Phys. 30,407 ( $1 \leqslant 78$ ) United KIngdom

Bengurlo, R.; Lieb. E. H. Many-body otcalc potentials In Thoas-Ferel theory. Anr. Fhys. (Ni) 110, 34 (1578) United stotes

Vlehland, L. W.: Moson. E. A: Gaseous lon cobllity and diffusicn in electric lields ce ortitrory strengths. Ann. Fhys. (NY) 110, 2E? (1S7E)

United states

Wilson. S. Diagrogeatic certurtation theory.

Potentiol curves fcr the hydrcoen tiucride oolecule. Mol. Plys: 35. 1 (1S7E)

United states

Shipsey. E. J. Accroxleaticn wetheds tor certoln charge-trongfer collisions. Mol. Fhys. 35,El (1s78) United stotes

Llu, J. H.: Salth, V. H.. Jr. Thecretical Coscton proflles ond mooentue distributions $\mathrm{CCI} \mathrm{H}_{2}+\left({ }^{2} \Sigma a^{+}\right)$ond $H \rightarrow(2 E Q+)$. Mol. Fhys. 35,145 (157E) West Gereany

Brickmann, J. Vibronlc coupling ir wolecular exclted states. Model occroach to ealsglor. anc atscrption spectro. Mcl. Phys. 3E, ISS (157e)

West Germony

Sehatz, G. C. A direct method lor determining acuents or linal stote distributions in goleculor cclilisions. Mel. Phys. 3E. $47 \overline{7}$ ( $L S 7 E$ )

United states

Case, D. A.: Mcclelland, G. M.: Hersertoch, D. F. Angular wodentuo cclarizaticn in aclecular collisions: Classical and quentum theory for measurements ising resonance fluorescence. Mcl. Fhys. 3E. 541 (157e) United Stotes

Eno, L.: Soktreger. R. The staticnary-colnt structure and colculaticn of distorteo wave inteprals in atomd lotoo seattering. Mcl. Fhys. JE. EOl (1S7E) United lingdom

Clark. F.; McCalfery. A. J. Loser Ilucrescence studles of solecular lodine. I. Scectrol asslgraento of the 5145 Ao tlucrescence. Rol. Phys. $3 E$. $\operatorname{LOS}(1 \leq 7 \varepsilon)$ Unlted Kingdco

Clark. F.: HeCattery. A. J. Laser flucrescence studies of moleculas lodine. II. Felaxation ct oriented ground and exclted molecules. Mol. Phys. sE. EIT (ISTE) Unt ted KIngcoor

Colbourn, E. A.: Dyke, J. M.: Lee,E.F.F.: Mcrils, A.: Trickle. I. F. The vocuum ultro-viclet

photoelectrcn spectrum of the sio(xil+) molecule. Mol. Phys. 35, 872 (1578)

Unlted kinodeo

Froites, J. L.: Wiricur, C. H. Cifferential elastic and querching crosa sectiong for Ar*( $3 F)$ and $\mathrm{CO}_{2}\left(\mathrm{XI}^{2}\right.$. $\mathrm{E}$ $a+1$. Hcl. Fhys. 35, 927 (1578) United States

Ccnnor. J. A. L.: Jakubet 2, H. Rainbcu scattering in atoolc collistong: A Regge pole analyals. Pol. Fhyso 35.945 (1578) United Kingdom 
REF.

REACTANTS

No.

\section{8}

e+ He

E07

e $+\mathrm{He}^{*}$

165

$\mathrm{Hl}_{\mathrm{He}}+\mathrm{H}_{7}: \mathrm{He}+\mathrm{D}_{9}: \mathrm{He}+\mathrm{T}_{2}$

170

A11 $1+N$,

171

F $+\mathrm{H}$,

T

$300 \mathrm{~K}$

172

A13

Revien

173

$\mathrm{NOB}_{+}^{+}+\mathrm{He}: \mathrm{O}_{+}^{+}+\mathrm{He}$

$174 \quad \mathrm{AOA}_{4}-+\mathrm{He}$

175

Ho6

$h_{\nu}+\operatorname{css}_{2:} h \nu+\operatorname{ccs}$

176

$\underset{\mathrm{H}>\mathrm{O}^{+}+\mathrm{H}_{2}}{\mathrm{A13}}$

177

DO?

Review

E05

$O+\mathrm{H}_{3}: e+\mathrm{N}_{2} \mathrm{O}+\mathrm{O}, \mathrm{O} e+\mathrm{CO}: \mathrm{E}$

E NO

คอส

$\mathrm{He}^{+}+\mathrm{CH}_{4}: \mathrm{He}^{+}+\mathrm{H}_{2} \mathrm{O}: \mathrm{Ne}++\mathrm{H}_{2} \mathrm{O}$ : $\mathrm{Ar}^{+}+\mathrm{H}_{\infty} \mathrm{O}$

1 A0 $\stackrel{\text { A L L }}{0^{-}}+\mathrm{N}$,

$181 \quad$ A13

Reviow

$300 \mathrm{~K}$

Intap $\mathrm{KeV}$

$\operatorname{sinn} x$
GEFEEENCE
Herrick, D. F. 1-changing electron faroct cross secticns for high Fyoberg atows ith afflicoticn to hellur exferiments. Mol. Fhys. 3E, 1211 (1578) United States

$0-100$ eV

$0-10 \mathrm{eV}$

$50-75$ oc

$0-50$ eV

$0-2$ ov

$1230-620 A^{0}$

$0.1-2.0 \mathrm{eV}$

$0.04-1.0$

$0.03-0.6$ OV

Spe

atudies between $\mathrm{C}^{-}$lons and nitrcoen. Int. J. Mass Spectrce. ICn Phyo. 26, 321 (1S7E) Unlted Kingdom potentiol acproach to the calculation cl vib-rctaticnal relaxation rates. Mol. Phys. 35, 1248 (1 1578 )

Israel

Zorltsks, N. C.: Minglegrlr, U.: Gerber, H. B.

Vit-rotational reloxation in $L i+A$, ccillisicns. caloulatiens bith the oftleal-fetertial method. Mol. Phys. $25,12 \in S \quad(1 \leq 78)$

Is rae I

Connor, J. K. L.: Jakutetz, H.: Morz, J. The f + ho $(v=c)$ FH ( $v$ < 3 ; H reaction: Quantua collinear reacticn probablilties on tree different pctential energy urfeces. Mat. Phye. 3E, 1301 (isba) United KIngdom

Polley, C. W.: Munson. E. The proton offinities cf the halngen arids. Int. I. Ma... Spartmom. Ign chyel 36. 49 (1578)

Unlted states

Noveed-cllah, K.: Mothur. D.: Hooted, Jo B. Energy diotributicns of diatodic acleculor cositive icns in a drift tube. Int. J. Mass Epectron. Ier Phys. $2 \epsilon$, $s 1$ (1578)

United Kingdod

Refoey, K. M. A.: Frarklln, J. L. Callisicnal

decompcsittlen of SF-.. Int. J. Mazs spectrem. ICn Phys. $z 6,125$ (1578)

United States

Frey, F.: Gctchev, E.: Festuan, H. E.: Follok, H.: Schlag. E. H. Fhotolonization rescnance study of the $x$ tilde $(2 x)$. A tilde $\left(2 x^{2}\right)$, B tilde $(2 \Sigma+)$ and $C$ tilde $\left(2 L^{+}\right)$otates cl $C S_{,+}$and $C C S^{+}$. Int. J. Maso Spectrom. Ion Phys. 26, 137 (1578)

West Germany

Yeneha, A. Jo: Pacok, Vo: Herman, z. Kinesatics of the

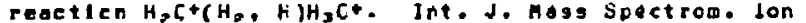
Phys. $z \epsilon, 20 E$ (.1578)

Czechosicuaklo

Pluln, J. C. Roguea-Gorooe, G.1 Slooeion, C. Varlation des rendements deenissicr icnlque seconcalre des alliages NI-Cr. Fe-Cr. Fe-Nl or-lcreticn de 10 tanaur en sclute. Int. J. poss Sotctico. Ion thyo. 26,219 (1578)

Fronce

Breha, F.: de frenes, $G$. Investlgation of lon palrs from tast decay frccesses cf ocutly-cherged molecular icns. Int. J. Mass Spectror. Ion Fhys. 26, 251 (3678) hest Germanx

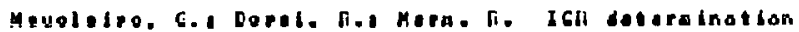
of kinetically excited ion produced in water and aethane by charge transfer fice therad rare gos ions. Int. J. Hass Spectrow. Ion Fhys. zE, zes (1S78) Fronce

Adams, N. G.: Salth, D.; Grier, D. Racetlena ef $H(n) C C$ + ions ifth colecules of $300 \mathrm{~K}$. Int. J. Mags Spectrox. Ion Phys. 26, 405 (157E) United Kingdus.

Banks, C.: Leopold, J. G. Closglcel stork icnisation threshold electric rield ond energy for hydrogenic lons. J. Phys. B 11. L5 (1S7E)

United Kingdo: 
REF.

REACTANTS

No.

183

E02

e+ He

E03

C He

184

$\mathrm{E} 03$

$e+\mathrm{K}: \mathrm{e}+\mathrm{Rb} ; \mathrm{e}+\mathrm{Cs}_{3}$

E.05

$e+K: e+R b: e+C s$

185

$B a+X e$

186

B0 3

H Seq

187

hy $+\mathrm{H}=\mathrm{C}$

HOS

he $+\mathrm{H}>\mathrm{O}$

188

A10

$\mathrm{LI}+\mathrm{He}$

189

$A 10$
$C+$

$\mathrm{No}+\mathrm{Ne}$

191

$\mathrm{Ha}^{\mathrm{AO}}+\mathrm{H}: \mathrm{He}+4 \mathrm{H}$

A.06

$\mathrm{He}^{2+}+\mathrm{H}: \mathrm{He}^{+}+\mathrm{H}$

192

A0 6

$\mathrm{He}^{+}+\mathrm{He}: \mathrm{Ar}^{+}+\mathrm{Ar}$

193

EO3

$++H$

E05

e + Mg

195

$\mathrm{EO} 2$

e+No:e+CO

$\varepsilon 03$

$e+N_{2}: e+C O$

804

$+N_{D}:-\leftarrow C O$

E05

$e+N_{2}: e+C O$

196

A0 3

H* $+H$

$19 ?$

AO 3

$\mathrm{Ne}+\mathrm{Ne}$

198

A06

H* $+\mathrm{He}^{*}$

A07

$\mathrm{H} *+\mathrm{He}^{+}$
T

\begin{tabular}{ll}
$\begin{array}{l}\text { EXP } \\
\text { OR } \\
\text { THEOR }\end{array}$ & $\begin{array}{l}\text { ENERGY } \\
\text { RANGE }\end{array}$ \\
\hline & $22-23 \mathrm{eV}$ \\
\hline & $30-600 \mathrm{eV}$ \\
\hline & \\
\hline & \\
\hline
\end{tabular}

E

$2800-3300$

A 0

T

$0.005-0.01$

50-1000K

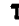

$T$

E

$4-343 k \in V$

E T 1-10 kaV

$100-300 \mathrm{eV}$

$30-280$ ov

E $\quad 6-16$ ○V

$T$

$10-700 \mathrm{keV}$

$40-386 k e V$

Nesbet, F. K. Fesonances, cugp effects and a vitual state in e--He scottering neor the $n=3$ thresholds. J. Fhys. E 11, L21 (1978)

United Stotes

Peterkof, R.; Liepingh. A. Electrcn lacact excltation of autolonising levels in heovy olkell otoos. J. Fhys. B 11, $127(2978)$

soviet linion

Kielkoft, J. The interactlon of excited states ch barium ith xencn atoss deterelinec ty sectral-line. contour meaguredentg. J. Fryg. E 11,25 (1578) Unlted states

Banks, D.: Leopold, J. G. Ionlaaticn ct highly-exclted atoos bs electric lields I. Closelcal theory of the critical electric lield for hydrogenic lons. J. Phys. B 11. 37 (157E)

Unlted Kingdco

Lee, L. C.: Oren. L.: Phlliles. E.: Juoge, E. L. Cross sections for production of the OH( $z_{2} L^{+} X Z_{n}$ ) tluorescence by fhetodiasccloblen ct H,C vafour. J. Phys, E 11, 47 (1578)

Untied States

Reld. F. H. G.: Rankin.R.F. The elqenohose formulaticn of fine-structure tionsiticn crcss sections. J. Phys. B 11 . EE (1SiE)

Unlted Kingdom

Harel, C.: Lopez, L.; HeCarroll, F.: Elera, A.: Wahnon, F. 'Collisicn models of Intromulticlet transitions at thermol eneraies. J. Fhys. E 11.71 (1978) France

Gaunaca, J. P. Thecreticol study cl he-Ne collitions. J. Phys. 11. as (1578) France

Shah, H. B.: Giltody, H. B. Electren capture and Het(2s) fcreaticn in tost tezt-t ond het-h collisions. J. Phy o. B 11. 121 (1578)

United kingdoo

Hegerberg. R.: Stelansson. T.: Elfcrd, M. T. Measuretent of the syosetric charge-exchange cross section in telitun and orgon in the impoct energy range 1-10 kev. J. Phys. P 11. 133 (1578) Norway

Winters, K. H. Cn distorted-wave apprcximaticrs lor excltation. d. Fhyo. B 12. 14S (1S7E) Belgiur

Karstensen. F.: Schnelder. H. Absclute cross oectiong for single and doutle ton leotion of $\mathrm{kg}$ atcas by electrcn infact. J. Phys. B 11. 167 (ISTE) Weat Germony

Brunt, d. N. H.: King, G. C.: Read, F, H. Excltation of carton monoxlde and nitrogen oolecules by electrcn lopect anerales belcu $l \in$ eV : studies of resononces in the excitation functiong or metostatle and in the excitation functiong or detostate and 173 ultravicit (1578)

Unlted KIngdom

Demangan. D. P. A aodel ter len-atod ccllisions at interceciate energies. J.Fhys. B 11. L37 (1S7E) India

Fitsch, inilie, U. Approxicate clcsed-tcro solutlen of a twc-state medel fcr rotationolly Incuced excitation in atomic collibiong. J. Fhys. E 11. L 43 (1578) Weat Germany

Angel. G. Co: Dunn, K. F.: Eemell, E. C.: Glltody, H. B. Ioniaction ond charge trangler in last $\mathrm{H}^{*-\mathrm{He}}$ colllalcns: further wesureants ol isfroveo accuracy. J. Phys.B 11, L4S (1578)

UnI ted KI ngdor 


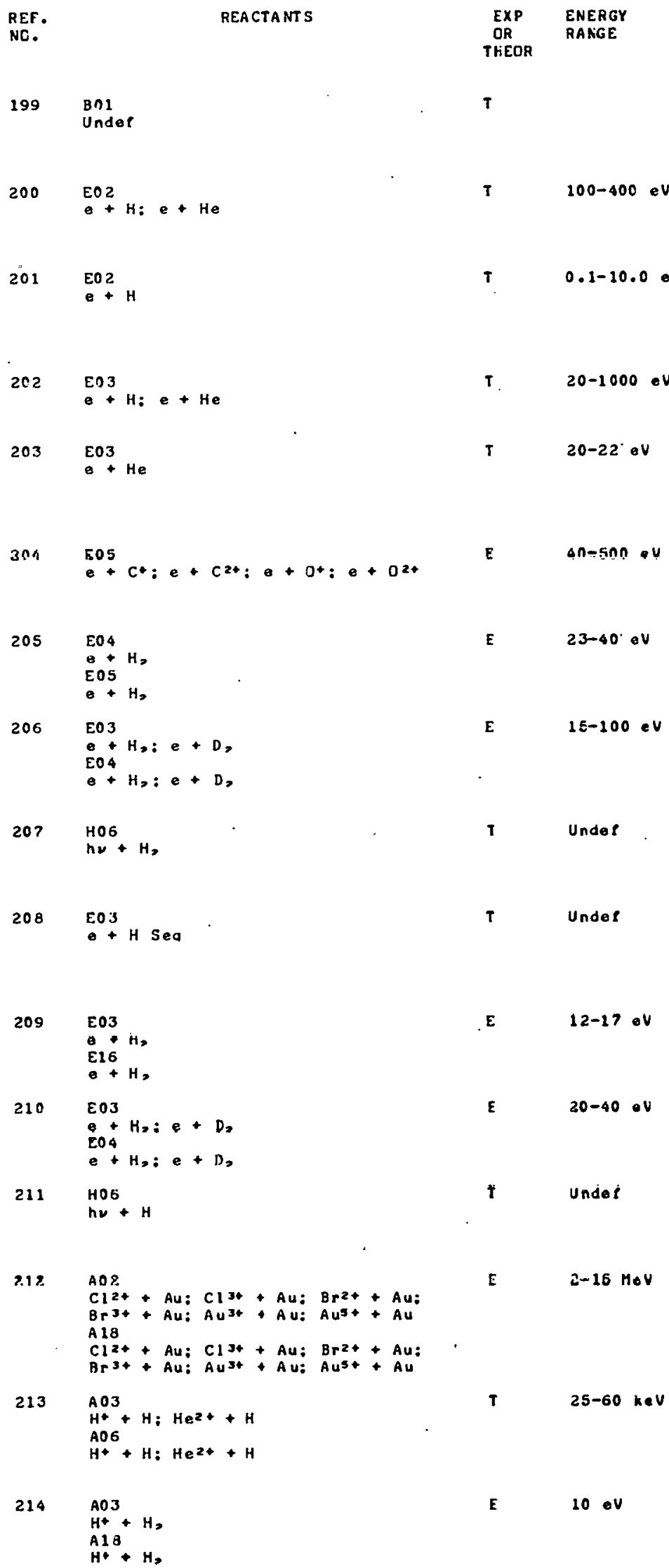

Propln. R. Stark effect for the rctational levels of a dlatoole celar oolecule in. strenctield. J. Fhys. B 11. 257 (1578)

Soviet lnion

Vanderscorten, R.: Hinters, K. H. A ccoparison of local cetical potentials ler elostic electron-otom scattering. J. Fhys. E 11, 2E1 (1S7E) Belgive

Seaton. H. J.: Steenman-Clork. L. Ettectlue potentlals ror electron-otod ocatterirg telcu Inelostic thresholdo II. The linite-ronge probles. Je Fhys. B 11, 293 (1578) Unlted kingdoe

Srivastovo, fio: fal, C. K. Electron-lafact excitation of H onc He. J. Physe B 11,305 (1578)

India

Fon, H. C.: Berrington, K. A.: Eurke, F. G.: Kingston, A. E. Electron excitotion tunctiors for the $2^{3} \leq, 21 \mathrm{~s}$, $2^{3}$ f and $21 \mathrm{p}$ states of hellug between the $n=2$ ono $n=3$ excitation thresholds. J. Fhys. 11 , J2E (1S7E) United Kingcioo

Huasian, M.: H!rk!nehan, K.: Hasted, Je fio Icniantion of positlue licns by elections in the hallow-beos trop. J. Phys. B 11, 331 (1978) United Kingdom

Kollmanr. K. Energetic protong irco outolonising gtates cr Hz. J. Fhys. B 11. 339 (1678) West Germany

Karolis, C.: Hartling. E. Electron locact dissoclation crcos secticne in hydrogen ond deutoriur. leoding to Balear alphe and bete ewielion. Je Fhys. E 11,35? (1578)

Austrollo

Chang, E. S. Angular distributiona of phctcelectrons with analyals on the rctaticnel otetes of Ho. J. Fhys. B $11.169(1978)$

United Kingdoo

Hayes, M. A.: Seoton. M. J. Revonances In $18-2$ and $18-2 p$ cclllelch strengths lor electron lapoct excliasion or nyarogente ions. J. Pnyse B 11. L7s (1578) Unted Mingoci.

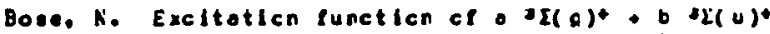
lluht ealoglun lil $\mathrm{H}$, meosured by cincenterwotlo electrens. J. Phys. B 11. Lez (J97E) Hest Gerwony

Glese-Heujean. M. Electron impect discelation of $H$. and $D_{2}$ etudied sice the anticroseing ignals and by the

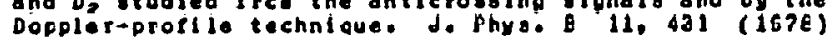
France

Karul e. E, Tuo-photon lonlaction ct otoric hydrogan silultaneously ulth ona-pheten icnisaticn. J. Fhys. B 11. 441 (1578)

goviet intion

Knudsen. Ilo: Foteroen. Fo Mo Meosurosents ar differertial of nole-scottering crobs octions of heavy lons of HeV energits on solid targets. J. Phys. B 11. 4ES (IS7E)

Deneark

Morriecn. H. Go: CoIk, U. An lepact-porameter wethod for heoverarticle collision involving one electrcn I. theory ond eaple results cn he-h ond text-h collislcns. J. Fhyse E 11. 473 (1S7E) United KIngdoe

Herean, V.: Scheldt, H.: LInder, F. Fctatlenel and vibraticnal excltation in ICw-energy $H_{0-H}$ ocottering experiaente. J. Fhya. E 11. 493 (1578) Weat German 


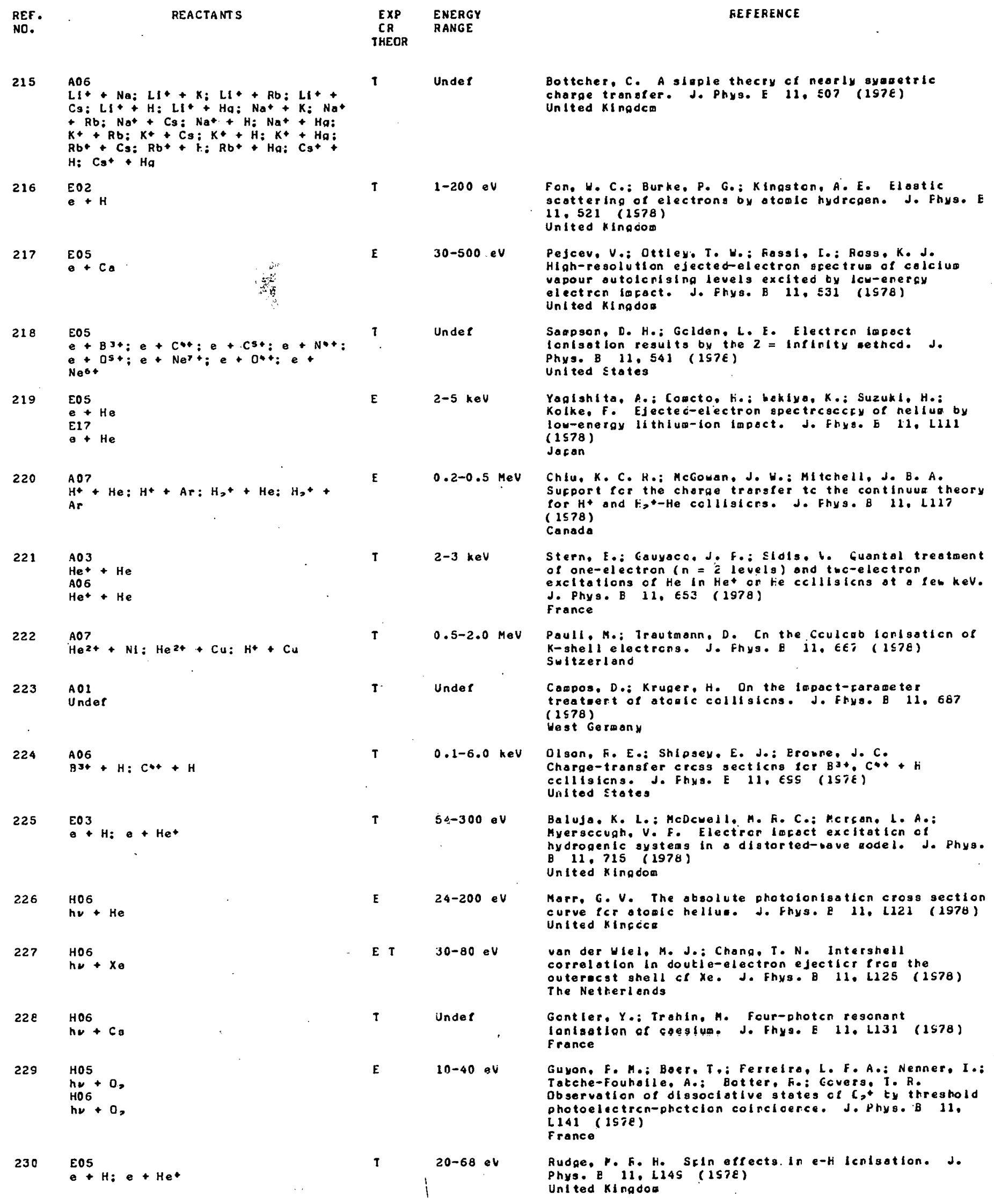




\begin{tabular}{|c|c|c|c|c|}
\hline $\begin{array}{l}\text { REF. } \\
\text { NO. }\end{array}$ & REACTANT S & $\begin{array}{l}\text { EXP } \\
\text { ER } \\
\text { THEOR }\end{array}$ & $\begin{array}{l}\text { ENERGY } \\
\text { RANGE }\end{array}$ & FEFEFENCE \\
\hline 231 & $\begin{array}{l}\mathrm{A}^{10}+\mathrm{N} \\
\mathrm{A18} \\
\mathrm{K}+\mathrm{N}\end{array}$ & $\mathbf{T}$ & $0.1-1.0 \mathrm{k} \in \mathrm{V}$ & 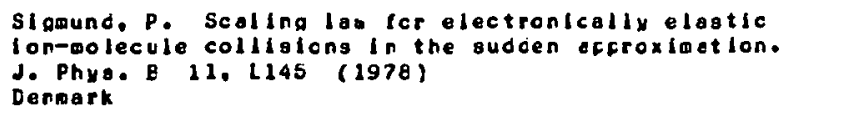 \\
\hline 232 & $\begin{array}{l}\mathrm{HOG} \\
\mathrm{ho}+\mathrm{Na}\end{array}$ & $\mathbf{E}$ & $\begin{array}{l}24000-29000 \\
c m-1\end{array}$ & 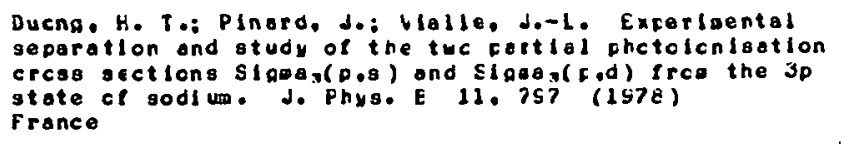 \\
\hline 233 & $\begin{array}{c}\mathrm{AOT} \\
\mathrm{H*}+\mathrm{Al}: \mathrm{H}+\mathrm{PE} \mathrm{KT}\end{array}$ & $\mathbf{T}$ & $\begin{array}{l}0.16-4.6 \\
G e V\end{array}$ & 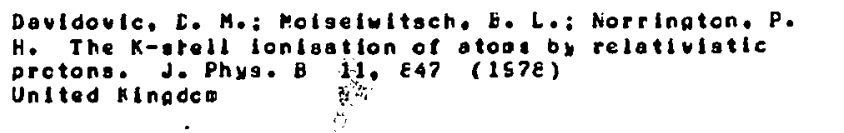 \\
\hline 234 & $\begin{array}{l}\mathrm{AO3} \\
\mathrm{N} *\end{array} \mathrm{~N}_{2}$ & . & $35-200 \mathrm{keV}$ & 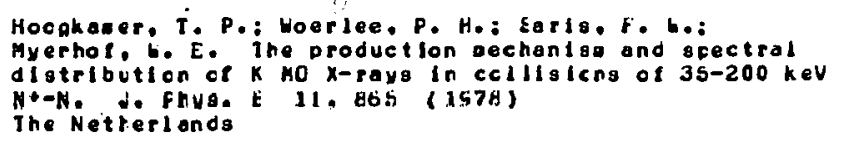 \\
\hline 235 & 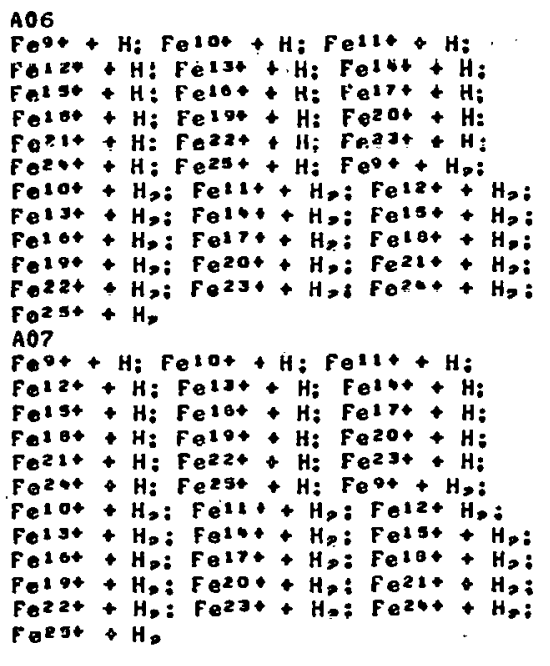 & E $\mathbf{T}$ & $\begin{array}{l}15.5-62.8 \\
\operatorname{MeV}\end{array}$ & 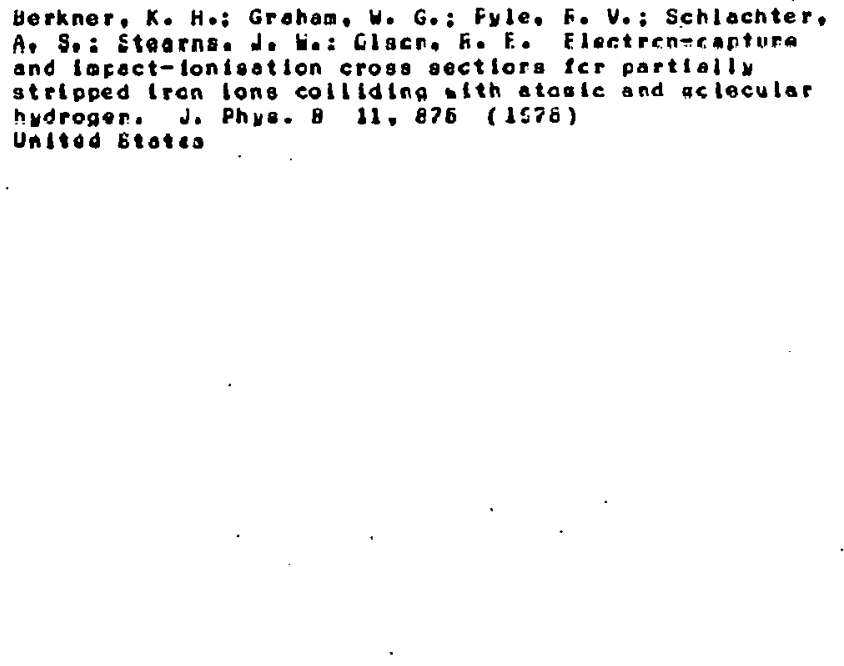 \\
\hline 236 & $\begin{array}{ll}\mathrm{EO} 2 \\
\mathrm{H}\end{array}$ & $\mathbf{T}$ & $100-10 \mathrm{Co} \mathrm{aV}$ & 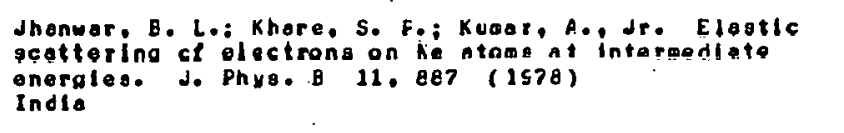 \\
\hline 237 & $\begin{array}{l}\text { E03 } \\
\text { EOS He } \\
\text { E.He } \\
\text { E17 He }\end{array}$ & $\mathbf{E}$ & $57-61 \mathrm{eV}$ & 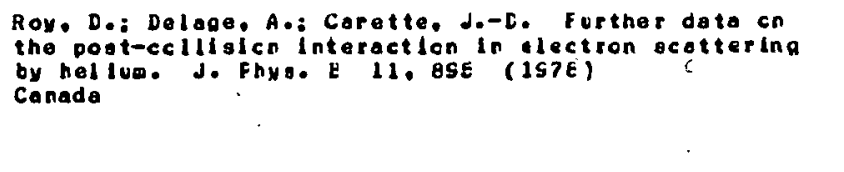 \\
\hline 2.39 & $\begin{array}{l}\mathrm{AOS}^{\circ} \\
\mathrm{Cl}_{2}+\mathrm{H}: \mathrm{Tl}_{2}+\mathrm{H}: \mathrm{C}_{2}+\mathrm{H}_{3}: \mathrm{TI}^{2+}+\end{array}$ & $\mathbf{E}$ & $\begin{array}{l}0.0-1+60 \\
k \in V\end{array}$ & 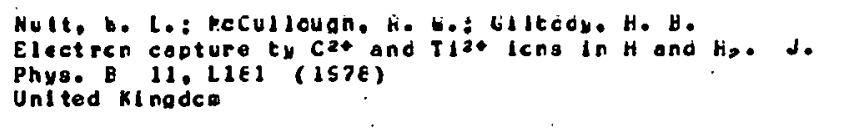 \\
\hline 239 & 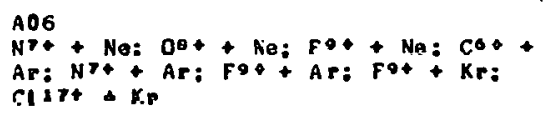 & $\mathrm{T}$ & $14-160 \mathrm{rev}$ & 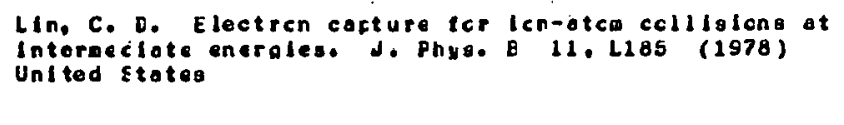 \\
\hline 240 & $\begin{array}{l}\mathrm{AOG} \\
\mathrm{CO}+\mathrm{H}\end{array}$ & $\mathbf{T}$ & $\begin{array}{l}0.1-1 \cdot 0 \\
a \cdot 4 .\end{array}$ & 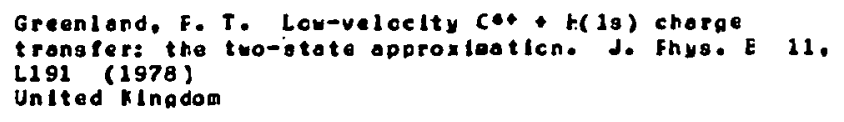 \\
\hline 241 & 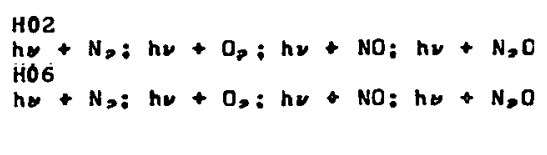 & $\mathbf{E}$ & $50-340 A^{\circ}$ & 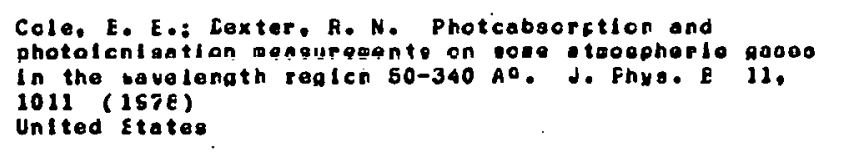 \\
\hline 242 & $\begin{array}{l}\text { A03 } \\
\text { Under } \\
\text { A06 } \\
\text { Under }\end{array}$ & $\mathbf{T}$ & Unde $t$ & 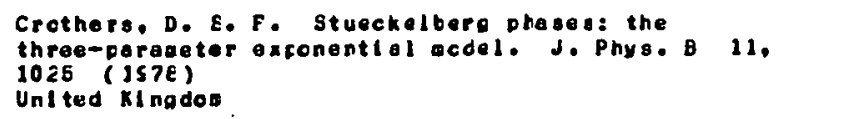 \\
\hline
\end{tabular}




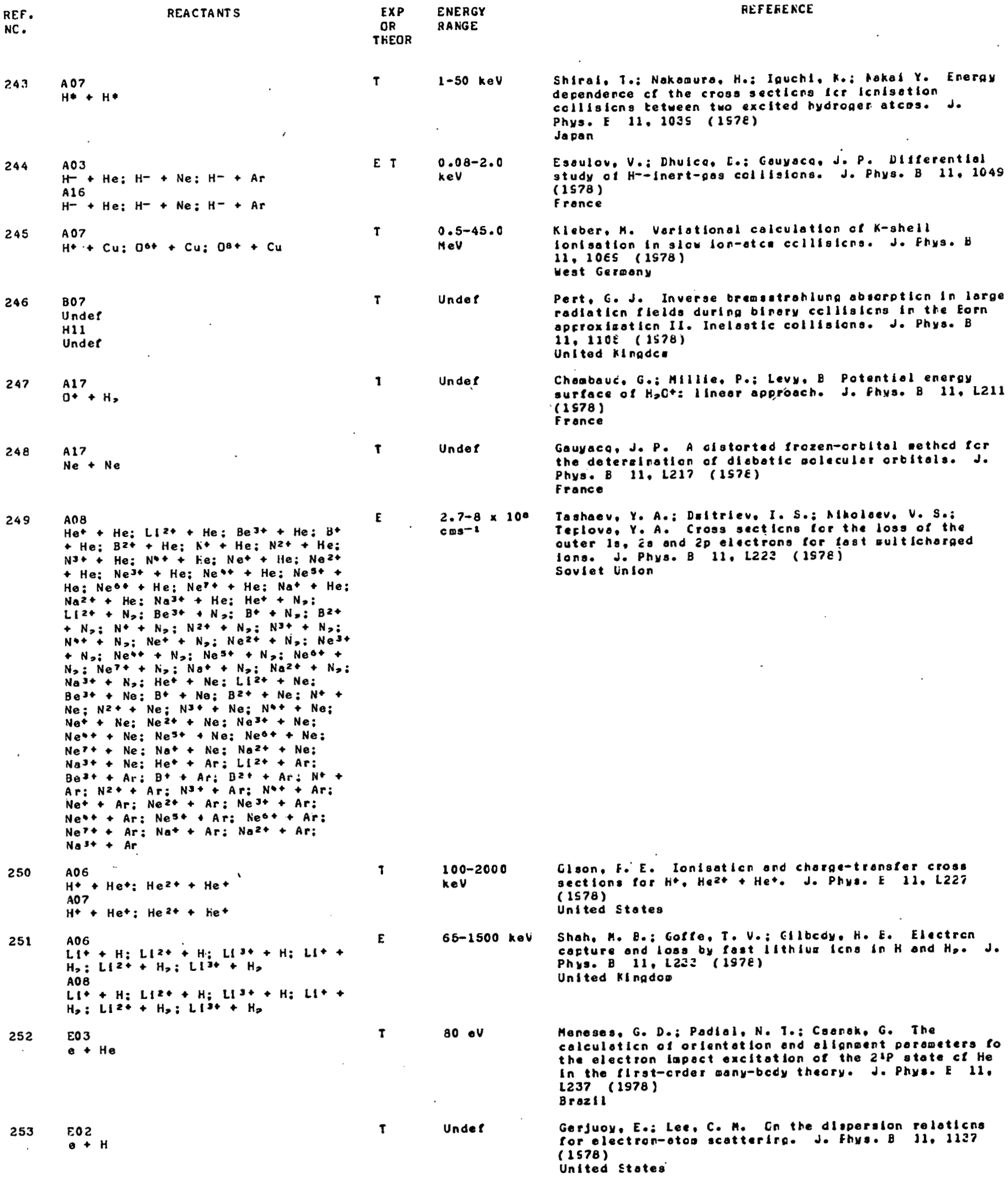

Shiral. T.: Nakomuro, Ho: I I uchl, Ko: Nokal Y. Eneray

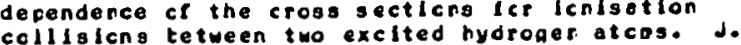
Phys. F 11. 2035 (157e)

Japan

Esoulor, V.: Dhulca, [.: Gouyaca, j. P. Differentiol study of H--inert-gos col lisions. J. Phys. B 11,1049 ( 1578 )

Fronce

Kleber. M. Varlotional colculation of K-ahell lontsation in slou ion-atca cclilisicna. J. Fhys. 11. $20 \in S$ (1978)

west Germony

Pert. 6. J. Inverge bremsetrohlung absorpticn in large radiaticn flelds during binery cellisicns in the Forn apcroxicoticn II. Inelogtic collisiono. J. Physe. B $11,1208(1578)$

Unitod kinadea

Choabauc, G.: Mlllie, P.: Levy, D Potentiol eneroy surface of $\mathrm{H}_{0} \mathrm{C}^{+}$: 1 ineor approach. J. Fhys, B 11, L211 ( 1578$)$

fronce

Gauyaca, J. P. A alstorted frazen-orbltal wethed fer the deterelnation of diabotic aolecular orbitals. J. Phys. B 11, L217 (1S7E)

France

Tashaev, Y. A.: Daltriev. I. S.: Alholeev, V. S.: Teflovo, Y.A. Cross sectiens lor the loss of the outer 1s, as ond $2 p$ electrong for fost aulticharged lona. J. Phys. B 11. L223 (1978)

soviet union

Clson. Fo: E. Lonlsaticn ond charge-transfer crose sections for Ho. Hezt+Het. J. Physe E 11. 1227 (1578)

United States

Shah, H. B.: Golfe, T. V.: Cllbcoy. H. E. Electron carture and 1080 by fast lithiug icns in $H$ and $H, \ldots$ Phys. B 11, l.2zz (197e)

United Kingdoo

Meneses, G. D.: Padial, N. T.: Coenok, G. The calculoticn ol orlentotion ond allanoent porometers to the election impoct oxcitstion of the 2 ip atate ct He In the flrot-crder wany-bcdy thecry. J. Phys. E 11. 1237 ( 1978$)$ Brazll

Gerjuoy, E.; Lee, C. M. Cn the dispersion relaticns lor electron-otos acateriro. J. Fhyseg j1. 1237 (1578)

United Stotes 
H06

$2 h \nu+L I: 2 h \nu+N a: 2 h v+K: 2 h v+$ Cs: $2 h y+R b$

25

A10

$\mathrm{Na}+\mathrm{N}$,

256

$\mathrm{AO3}_{\mathrm{He}+\mathrm{H}}$

$\mathrm{He*}+\mathrm{H}$

$\mathrm{He}^{+}+\mathrm{H}$

257

$\mathrm{Cl}^{-}+\mathrm{Ne}: \mathrm{Cl}^{-}+\mathrm{Ar}_{\mathrm{Cl}}+\mathrm{Kr}: \mathrm{Cl}^{-}$

258

$\mathrm{CO}+\mathrm{C}: \mathrm{N}+\mathrm{C}: \mathrm{O}+\mathrm{C}: \mathrm{F}+\mathrm{C}: \mathrm{Ne}+\mathrm{C}$

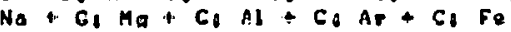

$+\mathrm{C}$

2ss

$\mathrm{EOJ}_{+\mathrm{Be}} \mathrm{Be}+\mathrm{Mg} \mathrm{CO}^{+}+\mathrm{Ca}^{*}$

260

$\mathrm{EO} 2$

a $H$ *

E17

e+H*

261

05

e $+K$

262

H06

hv + Na*: 2 hv + Na*

263

HO6

hv + Na

A07

Under

265

107

$\mathrm{H}^{+}+\mathrm{C}: \mathrm{H}^{+}+\mathrm{O}: \mathrm{H}^{+}+\mathrm{Mg}: \mathrm{H}^{+}+\mathrm{Al}$

$\mathrm{H}^{+}+\mathrm{Fe}: \mathrm{H}^{+}+\mathrm{C}_{0}: \mathrm{H}^{+}+\mathrm{Cu}_{4} \mathrm{H}^{+}+\mathrm{Zn}$

$\mathrm{H}^{+}+\mathrm{Ga}^{\circ} \mathrm{H}^{+}+\mathrm{Ge}: \mathrm{H}^{+}+\mathrm{As}_{3}$

266

$\mathrm{Ne}+\mathrm{Ne}: \mathrm{Ne}++\mathrm{Ne}: \mathrm{Ne}^{2+}+\mathrm{Ne}$

267

A06

$\mathrm{He}^{2+}+\mathrm{H}_{2} ; \mathrm{He}^{2+}+\mathrm{H}$

268

A06

$\mathrm{Ne}^{2+}+\mathrm{He}: \mathrm{Ne}^{2+}+\mathrm{Ne} \mathrm{Ne}^{2+}+\mathrm{Ar}^{2+}$

$\mathrm{Ne}^{2 t}+\mathrm{Kr}: \mathrm{Ne}^{2 t}+\mathrm{Xe}$

269
$\mathrm{H}^{*}+\mathrm{He}^{*}$

A 07

$\mathrm{H}^{*}+\mathrm{He}$
ENERGY

RANGE

FEF EFENCE
Undef

$0.004-0.05$

ev

$4-4000 \mathrm{keV}$

$80-2000$ eV

$10-300$

kev/amu

T J-100 eV

$0.1-30.0 \mathrm{eV}$

E $6-80$ eV

2400-1100

A 0

nder

T. $\quad 10-10 \cdot k e v$

$0.3-400 \mathrm{keV}$

E $\quad 0.4-10.0$

keV

$60-200 \mathrm{keV}$
$60-180 \mathrm{keV}$
Mlehule, Go: Groncecon. E, H. A.: von cer Wlel, M. J. Formaliom tor two-photon ionisotion of alkall otces vio - resoront Interaeolate stete. J. Fhys. B 11.1203 (1578)

The Netrerlonds

Amaee, E.: Botteher, C. Collitalong between sodlus atong and nitrager aolecules: rctotionel excitotion ano ilne-structure-chonging collisions. J. Phye. B 11. 1249 (1578)

United Kingdoe

Bell. K. L.: Kingaton. A. E. Excitoticn ond Ionisotión oroaesoes in Hot(10) H(le) cellisions. J. Phys. H $11.1255(1928)$

United Kingoo:

Gyetcn, J.: Chulca, D.: Eorat, M. Stuoy ot electrcn-detacheent and inelastic crocesges in Cl-prare-gos collisions. J. Phys. E 11, i267 (1578) Frence

Baudinet-fotinet, Y.: Cumont, P. C.: Carnil P. H. P. Analyolo of charge-state distritutions of heovy Icns in

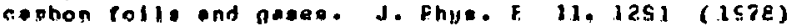
Belgiue

Kentedy, J. Vo: Hyerscough, V. I.: Rotcrell, H. H. C. Electer locaci excltation ef the cescrailce lines uf Bet. Mot onc Cat. J. Fnyse E 11. $130 \mathrm{a}$ (1\$7E) United kingdom

Gallitia. H. The ongular dependence cl electrct ocottering by awclted hydrcgon oteos. d. Fhyo. 11, L 279 (157e) soviet $\ln i e n$

HIls, D.: Kleinpoppen, H. The lonisaticn Cl pelaplised potasolue atces by colarisec electicna. J. Fhys. E 11. 1283 (1578) United Kingdom

Laughlin. C. One- and two-photen lonisaticn of the 39 and $3 \mathrm{p}$ tates of Nie I. J. Phys. E 11. 139s (2578) Unlted stotes

Aymar. M. Intluence of core-polarloatlon etlects on the phetcicnisaticn crcss secticns ct he arouni leve ono exclted ns levels ct neutral scdiua. J. Phys. B 11. 1412 (1578)

France.

Langenterg. A.: von Eck, J. A tlnery-encounter calculotion ith realitic velocity distributicn ci the target lectrcn. J. Phys. B 11. 142a (1s78) The Netterlands

Kuar. A.: Foy, E. N. Modilled blnary encounter model for proton impact k-shell lonlaticn cl atces. J. intis 1 . $11,1435(1570)$

Anderson, T.: Boving, E.: hedegaard, P.: Ulaen. J. O. Producticn of 23 vaconcles in collitsicns of $0.3-400 \mathrm{keV}$ NoO, Ne' and hea. ith He. J. Fhys. I 11. 1445 ( 1578 ) Denturk

Nutt, W. L.: HeCullough. F. H.: Erody, K.: Ehoh, A. B.: Gllbody. H. B. Electrcn ceoture by Hezt lors in culllsers olth lI and Il, at lecoet encreles tolow 10 keV. J. Fnys. E 11. 1457 (1578) United Kingdoo

Suk, H. C.: Gullbaud, A.: Hird, B. Crasa gectlons for electren carture ty Ne ${ }^{2}$ Ir. He. Ae, Ar, Kr and $X e$ between 60 and $200 \mathrm{keV}$. J. Fhys. F. 11. 1463 (1578) Canada

Angel . C. C.: Sewell, E, C.: Dunn, K. F.: Gllbcdy. H. B. Charge transfer and icnisotion in lost $h^{+-H e *}$ collisicns: Iurther meosurements using o colncidence technlave. J.Phys. E 11. L257 (157e) United kingdom 


\section{$\begin{array}{ll}\text { EXP } & \text { ENERGY } \\ \text { CR } & \text { RANGE }\end{array}$ \\ THEOR}

$$
270 \stackrel{E 05}{a+A r} \text {. }
$$

271

E03

C $+\mathrm{D}$,

$C+D$.

272

A09

$\mathrm{H}^{*}+\mathrm{H}^{-}$

273

502

$6+4$

$0+\mathrm{H}$,

274

A24
$H$ .

275

$\mathrm{A} 17$ $+\mathrm{Na}: \mathrm{H}+\mathrm{K}$

$\mathrm{HO2}$

hv + $\mathrm{NO}_{3}: \mathrm{hv}+\mathrm{NO}_{3}: \mathrm{h}_{\nu}+\mathrm{HNO}_{3}: h_{\nu}+$

$\mathrm{N}, \mathrm{O}$,

A 24

Revtev

276

$\mathrm{Ha}_{\mathrm{a}}^{\mathrm{AOG}}+\mathrm{H}_{2} \mathrm{O}^{+}: \mathrm{Mg}+\mathrm{NH}_{3}+: \mathrm{Mg}+\mathrm{H}_{9}+$

A13

$\mathrm{Mq}+\mathrm{H}_{3} \mathrm{O}^{+}: \mathrm{HQ}_{\mathrm{Q}}+\mathrm{H}_{3} \mathrm{~S}^{+}$

277

A23

$\mathrm{Zn}_{\mathrm{n}}+\mathrm{H}_{3}+\mathrm{Zn}+\mathrm{D}_{\mathrm{x}}+\mathrm{Zn}+\mathrm{CH}_{4} \mathrm{D}+$

A06

$\mathrm{Zn}_{\mathrm{n}}+\mathrm{H}_{\mathbf{9}} * \mathrm{Zn}+\mathrm{D}_{\mathbf{3}}+$

278

Al4
Under

279

$\mathrm{Cl}^{\mathrm{AL}}+\mathrm{H}=\mathrm{OCl}+\mathrm{D}$,

280

A 14

Revien

$\mathrm{EO} 4$

Revien

EOS

Reviou

281

Reviou

282

AL1

Revien

283

${ }_{C d *}^{A L}+H_{2}: C d *+H D_{0} C d *+D_{2}$

Cd* + $H_{2}: C d *+H D: C d *+D_{*}$

284
E

$25-1000$ eV

$200 \mathrm{eV}$

$5-2000 \mathrm{keV}$

$\mathbf{T}$

$\varepsilon$

$\mathbf{T}$

$\mathrm{HO} 2$

$1-15$ eV

A 14

$198-1000 \mathrm{~K}$

A17

Undef

E $\quad 1-20 \mathrm{eV}$

E

$0.04-20 \mathrm{eV}$

Under

1

E

$0.16-10 \cdot 0$

ov

A 14

$785-825$ o C

E04: EO5

$10-60$ eV

$E$

$300 \mathrm{~K}$

$2300 \mathrm{C}$

$190-2800^{\circ} \mathrm{C}$

1 Under

Under

FEFEFENCE

Wocdruff, F. F.: Hublet. M. C.: harrlscr, M. F. A. A weosureaent of the crosa section ler electrcn irfoct lonisation ct Art. J. Fhyse b 11, L3C5 (1578) UnIted lingdo.

Bose, N. Direct cclncldent detecticn ct twc D( Z p) dissociation products iroo a doubly excited state of D,. J. Phya. B 11. L3OS (1S7E)

Hest Gerany

Moore. J. C.; Bonyard, K. E. Ccntinuvo-distortec-eave calculaticna tor electron copture lro hydrcgen negative lcr.s by fost crotcres J. Fhys.b 11.1613 (1578)

United Kinociom

Klonover, Ao: Kolcor, U. At Initic electron-aclecule seattering theory including celorisaticn: elastlc scattering and rotational excitaticn cl $H_{2}$ J. Phys. B 11. 1623 (1578) Israel

Hashlda, N.: Akiacto, Hi.: Ckuda, M. Farmaticn of singlet stote molecular oxygen in the reaction of $H$. 0 .. J. Phya. Cheo. 82, 18 (ISTE) Jacan

Nuarich, R. W.: Truhlar. D. G. Detelled study ct the Interaction ci covelent anc icnic ototes in ccliligions of No ond $K$ with h. J. Phys. Cheo. Ea, I6E (ISTE) United States

Radus, 1. F.: Pcrter. F. F. An energy dependent kinetic study of the reactions of Mg(a) atces with a gerles cl protonated lons. J. Fhys. Cher. 82,513 (1578) United stotes

Po. P. L.: Fadus. T. P.: Forter. F. F. An energy dependent hinetic gtudy of the reactlcrs of $2 n(p)$ atoms uth $\mathrm{H}_{4}+\left(\mathrm{D}_{3}{ }^{+}\right)$and $\mathrm{CH}_{4} \mathrm{D}^{+}$. J. Fhys. Chea. Eá, E2O (1578)

United states

Thakur. A. K.: Fegclanc. A.: Lellgl. C. Stcchastic theory of seccnd-order cheolicel reacticns. J. Phys. Chea. E2, ESE (1578)

United stetes

Stevens, D. J.: Sclcer. L. L. Charocterlzetion of hot chlorine otcm reaction uith hydrogen. J. Phys. Chem. 82. 627 (1578)

United States

Grialey, R. T.: Forsmon, J. A.: Grinostati, O. G. A mass sfectrcmetric atudy of the fragaertaticn co the lithlus flucride vocor systes. J. Fhys. Chem. 82, 632 1 I thi us (1578)

United stotes

Hasegato, K.: Neta, P. Rate ccnotanto ond cechantsoms of reaction of Cl- rodicals. J. Fhys. Ches. Éc, 854 (1578) United states

Breckenrldge. Wo he: Rerlund, A. H. Guenchlng of exclted cadolum( IF, )atoms by several aclecules. Crosg sections and chewical and phyalcal exit chonnels. J. Phys. Chew. 82,1474 (1578) United States

Breckenzldge. W. Hei Renl und. A. M. Feactlon ct excl ted codalum (JFI JI) anc cadolun (IF, otoms with $H, H D$, and $D, 0$ Cuenching cross secticns ond CoH(CdD) fieldo. J. Phys. Chea. 8z. 1484 (1578) United stotes

Bonhas. F. A.: Keroka. S. The secend Ecrn opfroxleaticn for electron cattering. I. The high energy listt for seall ongle elastlc acottering troa atcise. J. inem. Fhys. o5, 525 (1578) Hest Geroony 


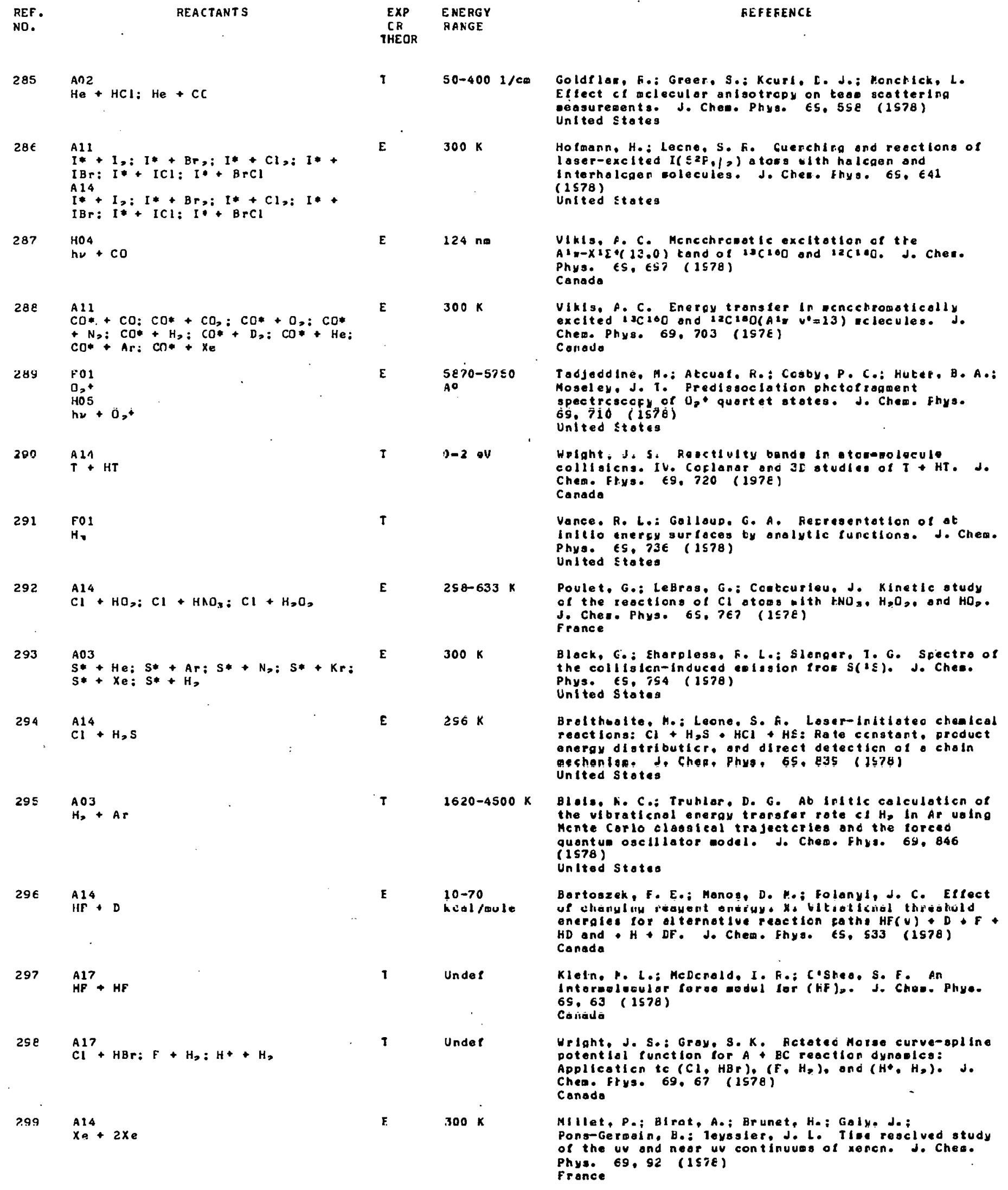


REF.

REACTANTS

NO.

300

$\mathrm{Al7}$.
$\mathrm{HOL}$
$\mathrm{FO}$

$\mathrm{HeH} *$

301

H06

hv + Ars: hr + Krs

A17

302

A17

$\stackrel{\mathrm{Ne}}{\mathrm{Ne}}+\mathrm{F}: \mathrm{Ar}+\mathrm{F}: \mathrm{Kr}+\mathrm{F}: \mathrm{Xe}+\mathrm{F}$

303

A14: $::$
Under :

304

$A 17$
$C+0$

Fol 1

co

305

$\mathrm{Cl}^{\mathrm{Cl}}+\mathrm{CH}_{\text {。 }}$

306

AO2

$\mathrm{Ar}+\mathrm{CH}_{\text {. }}$

A 03

$\mathrm{Ar}+\mathrm{CH}_{\text {。 }}$

307

$\mathrm{LOS}_{\mathrm{C}}+\mathrm{O}_{2}: \mathrm{Y}+\mathrm{O}_{2}: \mathrm{Sc}+\mathrm{O}_{2}$

308

A 14
$I$ $\begin{array}{ll}\underset{\text { EXP }}{\text { CR }} & \text { ENERGY } \\ \text { THEOR } & \end{array}$

T

Undef

E

I

Under

T

Under

'

Undef

E

$220-423 K$

$300 K$

E

Under

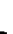

$300-46$ CO K

GEFEAEACE

Grean, T. A.: HIchels. H. H.: Brosne, J.C. Configuraticn Interacticn gtudies ct the Hetit oolecular lon. IV. The triplet sigoe. pl. ond delto otates. J. Chem. Phys. 69, 101 (197E)

United states

Dehmer. F. M.: Cehwer. J. L. Fhctcelectren spectro of $A r$, and $\mathrm{Kr}$, and $d \mathrm{lgsoclation} \mathrm{energles} \mathrm{ct} \mathrm{the} \mathrm{rate} \mathrm{gas}$ dloer Icns. J. Chem. Fhys. ES. 125 (1978) United states

Dunnlng. T. H.. Jr.: Hoy. F. J. The ccuolent and lonic stateg ce the rore aas oonofluorides. J. Chea. Phys. 69.134 (157E)

United Stateg

Case. D. A.: Herschbach, D. R. Intorastion theory anolysis of anpular mosentuo dispoeal in cheaical peacticns. J. Chea. Phys. 6S, 150 (1578)

United Stoteg

KIrby, K.: LIu, E. Theoretical gtudy ct molecular difole wowent functions. II. The dus and aj $a^{3}$ states of CO. J. Chem. Phys. 6S, 200 (ISTE) United stotes

Keyser. $L$. F. Atsolute rate and teaperature decendence of the reacticn between chlcrine ( $2 F$ ) etcas ono gethane. J. Cheq. Fhys. ES. 214 (ISTE) Untied stotes:

Hell, 1. G.: Secreat. D. A comparison of clcse coupling ond coupled states fer lcw energy apherical topatco collisions: Ar-CH. J. Cheo. Physe ES, zis $(1578)$

United states

Hanos. [. P.: Parsen. J. M. Cheallualrescent reactions of aroup IIIb atom uith $G_{0}$ : Spectiol ilouloticns and extended eneray decenderce. J. Ches. Fhys. 6S, 231 ( $1 \leqslant 78$ )

United Etotes

Groy, J. C.: Iruhlor, D. G.: Clewers, Lo: Dufl, Jobie; Chapman. F. M.. Jro: Herrell, C. C.: Heyes, E. F. Quasiclossical trojectory colculoticns ccmpared to quantum mechantcol rosction probabilities. rate

constants. ond octivaticn energles tor tuc cilierent potentiol surlaces lor the collinear reacticn ti, I. H HI. Including dependence on Inltial vibrational state. J. Chem. Phys. 6S, 240 (IS7E)

United atotes 
REF.

REACTANT S

309

$\mathrm{N}^{+}+\mathrm{NH}_{3}: \mathrm{N*}+\mathrm{H}>\mathrm{S}: \mathrm{N}^{*}+\mathrm{H}_{2} \mathrm{CO}: \mathrm{N}^{*}+$

COS: $\mathrm{N}^{*}+\mathrm{O}, \mathrm{N}^{+}+\mathrm{H}_{2} \mathrm{O}: \mathrm{N}^{+}+\mathrm{CH}_{*}:$

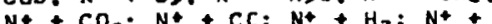

$\mathrm{N}_{0}: \mathrm{N}_{2}+\mathrm{NH}_{3}: \mathrm{N}_{2}+\mathrm{H}, \mathrm{S}: \mathrm{N}_{2}+\mathrm{H}_{>} \mathrm{CO}:$

$\mathrm{N}_{2}++\cos : \mathrm{N}_{2}+\mathrm{O}_{2}: \mathrm{N}_{2}+\mathrm{H}_{2} \mathrm{O}: \mathrm{N}_{2}$

$+\mathrm{CH}_{4}: \mathrm{N}_{2}+\mathrm{CO}_{2}: \mathrm{N}_{+}^{+}+\mathrm{CC}^{+} \mathrm{N}_{2}+$

$\mathrm{H}_{0}: \mathrm{N}=+\mathrm{N}_{2}: \mathrm{N}_{3}:+\mathrm{NH}_{3}: \mathrm{N}_{0}+\mathrm{H}_{2} \mathrm{~S}:$

$\mathrm{N}_{3}+\mathrm{H}_{2} \mathrm{CO}: \mathrm{N}_{3}{ }^{*}+\cos : \mathrm{N}^{*}+\mathrm{O}_{3}:$

$\mathrm{N}_{3}^{*}+\mathrm{H}_{7} \mathrm{O}: \mathrm{N}_{3}+{ }^{*} \mathrm{CH}_{4} \mathrm{ON}_{3} \mathrm{~N}^{+}+\mathrm{CO}_{3}:$

$\mathrm{N}_{4}^{+}+\mathrm{CO}: \mathrm{N}_{3}^{+}+\mathrm{H}_{2}: \mathrm{N}_{2}^{+}+\mathrm{N}_{2}: \mathrm{N}_{4}^{+}+$

$\mathrm{NH}_{3}: \mathrm{N}_{0}^{+}+\mathrm{H}_{3} \mathrm{~S}: \mathrm{N}_{4}^{+}+\mathrm{H}_{2} \mathrm{CO}: \mathrm{N}_{+}^{+}+$

COS: $\mathrm{N}_{4}^{+}+\mathrm{O}_{7}: \mathrm{N}_{4}^{+}+\mathrm{H}_{3} \mathrm{O}: \mathrm{N}_{4}^{+}+$

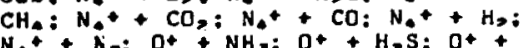

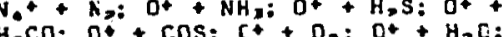

$\mathrm{O}^{*}+\mathrm{CH}^{\circ} \mathrm{O}^{+} \mathrm{CO}^{\circ} \mathrm{O}^{+}+\mathrm{CO} \mathrm{O}^{+}+\mathrm{CO}^{+}$

$\mathrm{H}_{0}: \mathrm{O}^{+}+\mathrm{N}=\mathrm{O} \mathrm{O}_{2}+\mathrm{NH}_{3}: \mathrm{O}_{2}^{+}+\mathrm{H}_{2} \mathrm{~S}:$

$\mathrm{O}++\mathrm{H}_{2} \mathrm{CO}: \mathrm{O}_{2}+\cos : \mathrm{O}_{2}+\mathrm{O}_{2}:$

$\mathrm{O}_{+}^{+}+\mathrm{H}_{3} \mathrm{O}: \mathrm{O}_{2}+\mathrm{CH}_{4}: \mathrm{O}_{2}^{+}+\mathrm{CO}_{3}:$

$\mathrm{O}_{*}^{+}+\mathrm{CO}: \mathrm{O}_{+}^{+}+\mathrm{H}_{2}: \mathrm{O}_{2}+\mathrm{N}_{2} \mathrm{NO}_{+}+$

CoS: $\mathrm{NOt}+\mathrm{O}_{2} \mathrm{NO}^{+}+\mathrm{H}_{7} \mathrm{O}$ : $\mathrm{NO}+$

$\mathrm{CH}_{4}: \mathrm{NO}+\mathrm{CO}_{2}: \mathrm{NO}_{+}^{+}+\mathrm{CO}_{\mathrm{O}} \mathrm{NO}_{+}+\mathrm{H}_{2}:$

$\mathrm{NO*}+\mathrm{H}_{2}$

A13

$\mathrm{N}^{*}+\mathrm{NH}_{3}: \mathrm{N}^{+}+\mathrm{H}_{>} \mathrm{S}: \mathrm{N}++\mathrm{H}_{2} \mathrm{CO}: \mathrm{N}^{+}+$

cos: $\mathrm{N}^{+}+\mathrm{O}_{2}: \mathrm{N}^{+}+\mathrm{H}_{2} \mathrm{O}: \mathrm{N}^{+}+\mathrm{CH}_{*}:$

$\mathrm{N}^{*}+\mathrm{CO}_{2}: \mathrm{N}^{+}+\mathrm{CO}: \mathrm{N}^{+}+\mathrm{H}_{2}: \mathrm{N}^{+}+$

$\mathrm{N}_{-}: \mathrm{N}_{2}+\mathrm{NH}_{3}: \mathrm{N}_{2}+\mathrm{H}_{2} \mathrm{~S}: \mathrm{N}_{2}+$

$\mathrm{H}_{0} \mathrm{CO}: \mathrm{N}_{2}+\cos : \mathrm{N}_{2}+\mathrm{O}_{2}: \mathrm{N}_{2}+$

$\mathrm{H}_{3} \mathrm{O}: \mathrm{N}_{2}^{+}+\mathrm{CH}_{4}: \mathrm{N}_{2}+\mathrm{CO}_{2}: \mathrm{N}_{2}+$

$\mathrm{CO}: \mathrm{N}_{2}+\mathrm{H}_{3}: \mathrm{N}+\mathrm{N}_{3}: \mathrm{N}_{3}+\mathrm{NH}_{3}:$

$\mathrm{N}_{3}+\mathrm{H}_{2} \mathrm{~S}: \mathrm{N}_{3}^{+}+\mathrm{H}_{2} \mathrm{CO}: \mathrm{N}_{3}+\mathrm{COS}:$

$\mathrm{N}_{3}++\mathrm{O}_{30} \mathrm{~N}_{3}++\mathrm{H}_{2} \mathrm{O}: \mathrm{N}_{3}+\mathrm{CH}_{3}: \mathrm{N}_{3}+$

$+\mathrm{CO}_{3}: \mathrm{N}^{+}+\mathrm{CO}^{*} \mathrm{~N}_{3}^{+}+\mathrm{H}_{3}: \mathrm{N}^{+}+$

$\mathrm{N}_{0}: \mathrm{N}_{+}^{+}+\mathrm{NH}_{2}: \mathrm{N}_{0}^{+}+\mathrm{H}_{3} \mathrm{~S}: \mathrm{N}_{+}^{+}+$

$\mathrm{H}_{\mathrm{P}} \mathrm{CU}: \mathrm{N}_{+}+\operatorname{COS}: \mathrm{N}_{4}+t \mathrm{O}_{2}: \mathrm{N}_{4}++$

$\mathrm{H}_{7} \mathrm{O}: \mathrm{N}_{*}^{+}+\mathrm{CH}_{4}: \mathrm{H}_{4}^{+}+\mathrm{CO}_{2}: \mathrm{N}_{+}^{+}+$

$\mathrm{CO}: \mathrm{N}_{+}^{+}+\mathrm{H}_{\rightarrow}: \mathrm{N}_{+}^{+}+\mathrm{N}_{3}: \mathrm{O}^{+}+\mathrm{NH}_{4}:$

$\mathrm{O}^{+}+\mathrm{H}_{2} \mathrm{~S}: \mathrm{O}^{+}+\mathrm{H}_{2} \mathrm{CO}: \mathrm{O}^{+}+\mathrm{COS}: \mathrm{O}^{+}+$

$\mathrm{O}: \mathrm{O}^{+}+\mathrm{H}_{2} \mathrm{O} ; \mathrm{O}^{+}+\mathrm{CH}_{4}: \mathrm{O}^{+}+\mathrm{CO}_{2}$

$\mathrm{O}_{+}^{+}+\mathrm{CO}^{+} \mathrm{C}^{+}+\mathrm{H}_{3}: \mathrm{O}+\mathrm{N}^{+}: \mathrm{O}_{2}+$

$\mathrm{NH}: \mathrm{O}_{3}+\mathrm{H}_{3} \mathrm{~S}^{-} \mathrm{C}_{2}^{+}+\mathrm{H}_{2} \mathrm{CO}: \mathrm{O}_{2}^{+}+$

$\operatorname{COS}: \mathrm{O}_{2}+\mathrm{O}_{2}: \mathrm{O}_{2}+\mathrm{H}_{2} \mathrm{O}_{3} \mathrm{O}_{2}++$

$\mathrm{CH}_{4}: \mathrm{O}_{3}+\mathrm{CO}_{7:} \mathrm{O}_{2}+\mathrm{CO}^{+} \mathrm{O}_{2}^{+}+\mathrm{H}_{2}:$

$\mathrm{O}_{+}^{+}+\mathrm{N}>\mathrm{NO}+\mathrm{NH}_{3}: \mathrm{NO}+\mathrm{H}_{2} \mathrm{~S}: \mathrm{NO}+$

$+\mathrm{H}_{3} \mathrm{CO}: \mathrm{NO}^{+}+\mathrm{COS}: \mathrm{NO}^{+}+\mathrm{C}_{3}: \mathrm{NO}^{+}+$

$\mathrm{H}_{2} \mathrm{NO}^{+}+\mathrm{Cll}_{4} \mathrm{NO}^{+}+\mathrm{CO}_{3}: \mathrm{NO}^{+}+$

$\mathrm{CO}: \mathrm{NO}++\mathrm{H}_{2}: \mathrm{ND}++\mathrm{N}$,

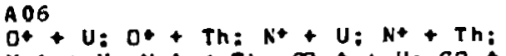

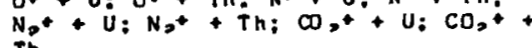

311

A

$\mathrm{NOF}+\mathrm{N}_{2}$

AI1

NO* + NO: NO* + He: NO* + $\mathrm{H}_{2}$ : NO*

$\mathrm{CO}: \mathrm{NO} *+\mathrm{CF}_{*}: \mathrm{NO}+\mathrm{N}_{2}: \mathrm{NO} *+\mathrm{CO}_{2}$

312

A14

$\mathrm{H}+\mathrm{O}_{\mathbf{3}}$

313

$\mathrm{K17}^{+}+\mathrm{Na}: \mathrm{Rb}++\mathrm{Na}: \mathrm{Cs}_{+}+\mathrm{Na}: \mathrm{Na}++$

$\mathrm{Na}: \mathrm{Rb}^{+}+\mathrm{K}: \mathrm{Cs}^{+}+\mathrm{K}: \mathrm{Cs}^{+}+\mathrm{Hb} ; \mathrm{K}+$

$+\mathrm{K}: \mathrm{Rb}^{+}+\mathrm{Rb}: \mathrm{Cs}^{+}+\mathrm{Cs}$

314

$\mathrm{GO2}$

$\mathrm{He}_{+}+\mathrm{Xe}_{\mathrm{H}} \mathrm{Ne}+\mathrm{Xe}: \mathrm{Ar}+\mathrm{Xe}: \mathrm{Kr}+\mathrm{Xe}_{\mathrm{H}}$

E

25-505 oc

$\mathrm{GO2}$

$K r+K r$
EXP
OR

ENERGY

RANGE

THEOR

E

$300 K$

E

$1-500$ ev

$300 K$

$219-360 \mathrm{~K}$

Unde t

\section{1}

E

$293 \mathrm{~K}$
FEFEFEACE

Solth, C.: Adams, N. G.: Mlller, T. H. A lobcratcry

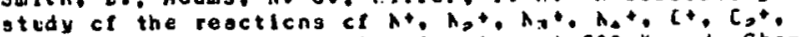
anc NCt ion. Ith several oclecules at $200 \mathrm{~K}$. J. Chea. Phys. ES. $20 E$ ( $157 \mathrm{e})$

United Kingdon
Rutherfero. J. A.: Uroos. D. A. Production of uranivo and therlus lens ty cherge tronster fem $U^{+}, N^{+}, A^{*}$, and CC.*. J. Chea. Phys. GS. 33z (1578) United states

Hiklda, T.: Morl, t. Induced diseclation cS NOLE2. $v \times 9$ ) by $N$, J. Chea. Fhys. ES, 346 (1578) Jatain

Lee. J. H.: Michael, J. V.: Fayne, ti, A.: Stiel, L. J." Absolute rate of the reaction of hyorogen atoms ith

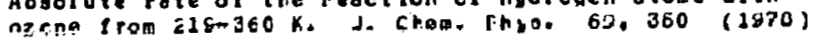
United states

Valance, A. Adlatatic cotentiol ererales for Mekt.

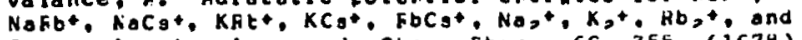
Cs, eclecular lons. J. Chea. Fhys. 6S, 355 (is78) france

Keatin. Jo: Khalite, h. E.: Wokehol, b. A. The viscosity ond diflusion coefflelents of the binary al atures of xencn ith the cther nctio oosea. Fhysica A 90 , 215 (1978)

United states

Codastefano, P.: Ficcl. M. A.: Zanza. W. Eehavicur of the soli-difusicn coeftictent ct kr at low densities. Physico A S2, 315 (1578) 


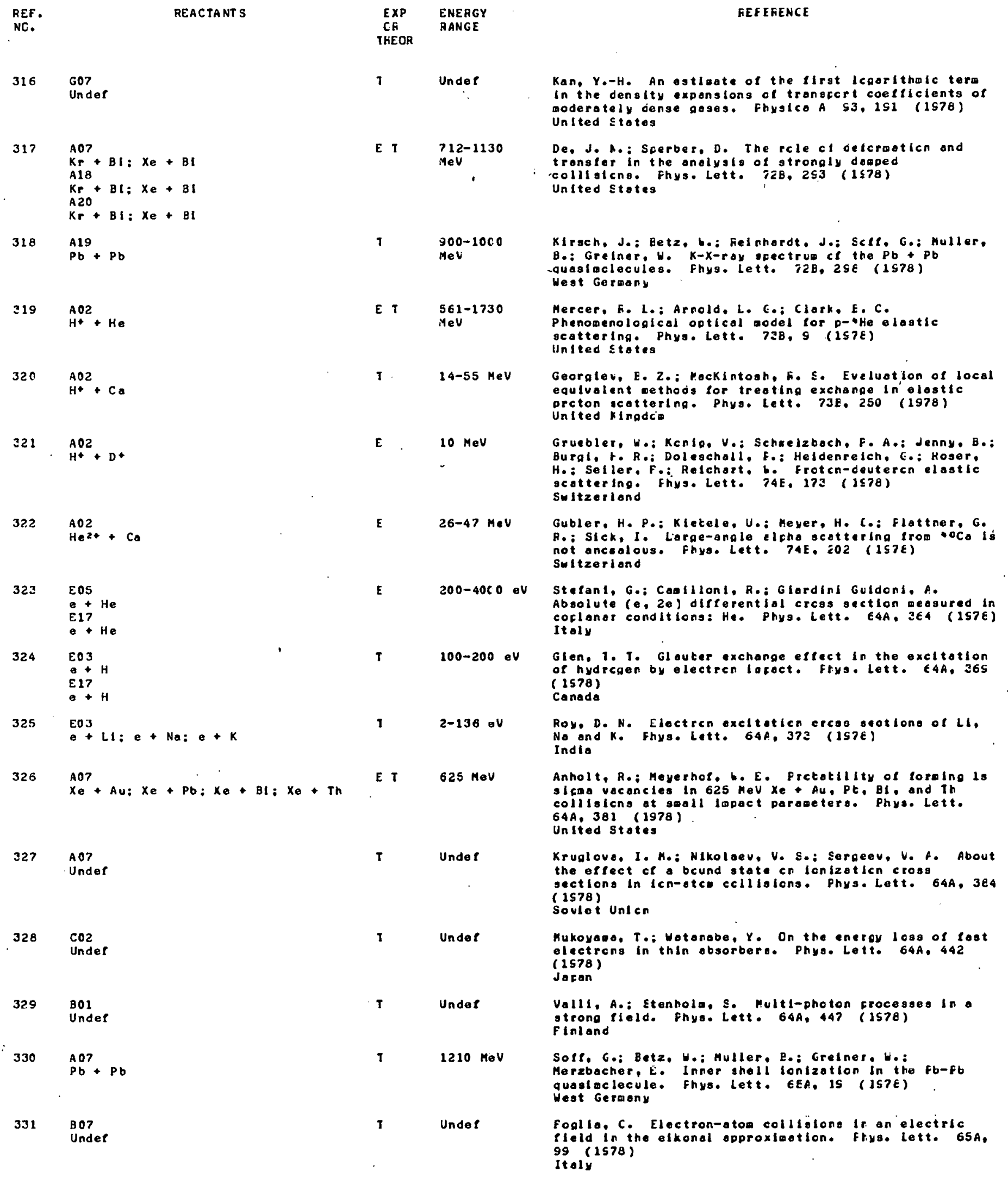




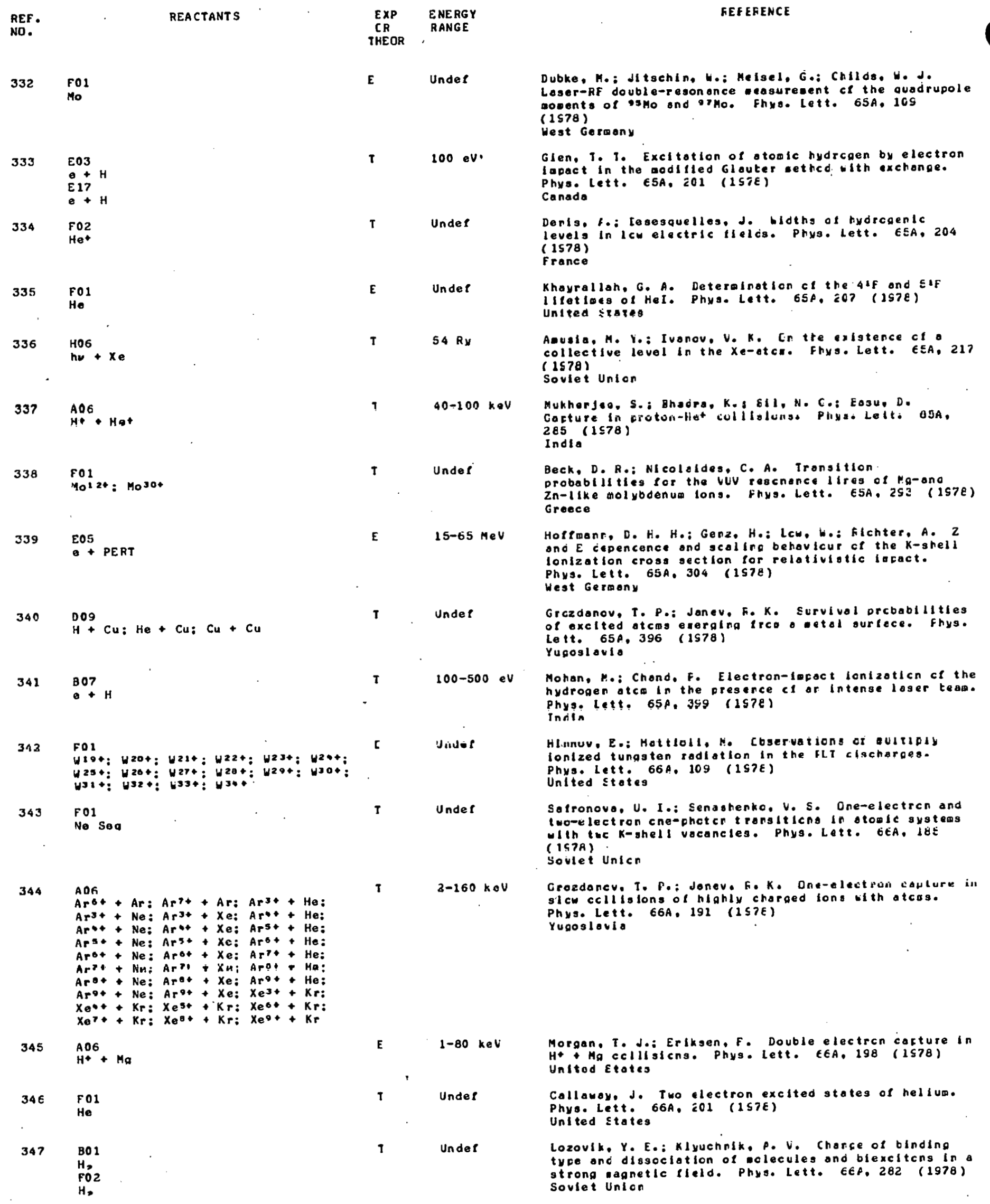


tEF.

REACTANTS

NO.

348

$\mathrm{AO} 07$
$\mathrm{H}+\mathrm{Xe}$

$349 H 06$

he + C 1

350

$\stackrel{\mathrm{AO7}}{\mathrm{H}+\mathrm{C}: \mathrm{He}^{2+}+\mathrm{C}}$

351

E05

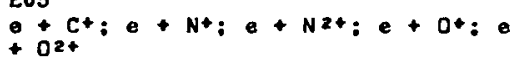

$352 \quad F 02$

$\mathrm{H}$

352

354

$\mathrm{E} 02$

e $+\mathrm{He}$

355

$\mathrm{H}^{\mathrm{AO}}+\mathrm{Cu}: \mathrm{D}^{*}+\mathrm{Cu}: \mathrm{He}^{+}+\mathrm{Cu}_{4}$

A18

$\mathrm{H}^{+}+\mathrm{Cu}: \mathrm{D}^{+}+\mathrm{Cu}: \mathrm{He}^{+}+\mathrm{Cu}$

$356 \quad$ FO1

LI Sea

357

A 05

$\mathrm{Cl}+4+\mathrm{Cu}$

D12

$\mathrm{Cl}++\mathrm{Cu}$

358

$\mathrm{DO}^{*}+\mathrm{Cu}$
$\mathrm{A}$

359

D04

$\mathrm{H}^{+}+\mathrm{Al}: \mathrm{H}++\mathrm{CH}: \mathrm{H}^{+}+\mathrm{Ag}: \mathrm{D}++\mathrm{Al}$ :

$\mathrm{D}^{+}+\mathrm{CU}: \mathrm{D}^{+}+\mathrm{Ag}_{\mathrm{O}} \mathrm{H}_{2}+\mathrm{Al}^{+} \mathrm{H}_{2}+$

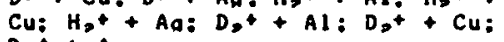

$D_{0}+A$ a

360

$\mathrm{CO} 1$

S* + PERT: S24 + PERT: S3* + PERT:

S*+ + PERT: S5* + PERT: So+ + PERT: ST+ + PERT: So + + PERT: SO + PERT: S10+ + PERT: S11+ + PERT: S12+ +

PERT: SIOH + PERT: SIO + PERT:

SI5 + + PERT: S10 + + PERT

361

A07

H* + Au

362

$\mathrm{H}^{\circ}+\mathrm{Na} \mathrm{H}^{+}+\mathrm{Ha}: \mathrm{H}^{+}+\mathrm{Al}: \mathrm{H}^{+}+\mathrm{SI}:$

$\mathrm{H}+\mathrm{Na}:$
$\mathrm{H}+\mathrm{Tl}$
EXP ENERGY

THEOR

T

T

100 kevjamu

$0.1-10 \mathrm{MeV}$

13-50 eV

$50-50 C_{0} \mathrm{eV}$

Undef

1-2 MeV

$200 \mathrm{eV}$

$0.5-2.0 \mathrm{MeV}$

Undef

20-80 MeV

$0.1-10 \mathrm{keV}$

$2-50 \mathrm{keV}$

E T $64 \mathrm{MeV}$

$0.4-1.0 \mathrm{MeV}$

$E$

15-65 keV
Komarov, F. F.; Novikov, A. P. Lonlzotion cross section of $n-s u b s h e l l$ electrons in the tinary-enccunter apfroxisaticn. Fhys. Lett. GEP, iBf (ISTE) Soviet inton

Brewn, E. R.: Carter, S. L.: Kelly, H. F.

Phctoicnizaticn crcss secticn cf cticitine 1 . Fhys.

Lett. E6A, ESO (157E)

United states

Jank, H.: Bell, F.: Heter, K, -H. The Inpoct ceroceter dependence ct corbcn Kaghil lcnizeticr by croton and hellus impoct. Fhys. Lett. eEA. ZSJ (1S7E) West Geroony

Kugar, f.: Roy, B. N. Electrcn leroct lonlzotion of posttive icns. Fiys. Lett. 66P. 262 (1S78)

Ind i a

VIrtamc, J. T.: Slool a, J. T. A. The spreading ci the prcton of a hydrocen otco in strene oonetic tielca.

Phys. Lett. E6A, 371 (ISTE)

Finland

Paul 1. P.: Fosel. F.: Trautsann. [. Screening ettects in the coulcot Ionlzation ot inner shell electrcise Phys. Lett. 67A, 28 (1978)

Sultzerland

Singh, S. A.: Tripathi, A. N. Application cr the modified Gleuber opproximation to the gtudy of elastic scattering of electrons by hellue etors. Fhys. Lett. $67 A, 31(1 \leq 78)$

Ind la

Cheeln. J. F.: Andrtaoonje. S.: Fcturler, J.: Soboyo B.: Goyet, F.: Sollin. A. Frojectlle charge ond woss dependence cl lorge ongle k-shell lonlzation

prcbabliltles cn ccpper. Fhys. Lett. 67A. LIE (157E) france

Tunnell, T. Wh: Ehalla, C. F. Thecretical llfetlaes and line flucrescence yielos of $192 p^{2}$ f levels. Fhys. Lett. 67A, i19 (1578)

United Stotes

Tanis, J. A.: Sholroth, S. M. Projectlle tlucrescence ylelds In reavy icn collilalcng. Frys. Lett. 67A, 124 (1578)

United stotes

Falcone, G.: Piperno, $f$. In the vie ct the Erinkaan cross secticn for sputterirg ylelo's ct argon icns on pclycrystalline ccper target. Phys. Lett. 67A, 138 (1578)

Italy

Baragiclo, F. A.: Alcnsc. E. V.: Auclillo, C:E Ferron. J.: Lontgchner. G.: Oliva Florlc. A. Molecular effects In lon-electron ealsicicice cleor detel surteces. Phys. Iett. 67A. 211 (157E) Argont ino

Watson, R. L.: White, J. F.: Jenson. F, E, Yield ratios tor $k$ atelilte ord hypersotellite $x$-ray ealsicn trcm $64 \mathrm{MeV}$ sulfur lons penetrating thich solld targets. Phys. Lett. ETP. 265 (ISTE) United States

Aashamar, O.: Adundsen. P. A.: Koctach. L. Prcton-induced L-shell lorizoticn ot lierge scattering anoles. Fhys. Lett. ETA. 34S (1578) Normay
Shlaa. K. Na. Ma, Al, El and $11 k$-shell ionizotion crcss sections for 15-es kev protcns. Phys. Lett. $67 A, 35)$ (1578)

Japan 


\begin{tabular}{|c|c|c|c|c|}
\hline $\begin{array}{l}\text { REF. } \\
\text { NC. }\end{array}$ & REACTANTS & $\begin{array}{l}\text { EXP } \\
\text { CR } \\
\text { THEOR }\end{array}$ & $\begin{array}{l}\text { ENERGY } \\
\text { RANGE }\end{array}$ & FEFEFENCE \\
\hline $3 \in 3$ & $\stackrel{\mathrm{AO3}}{\mathrm{Ne}+\mathrm{Ne}}$ & $\mathbf{E}$ & $292 \mathrm{~K}$ & 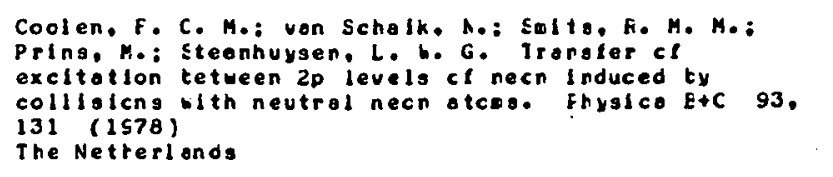 \\
\hline 364 & 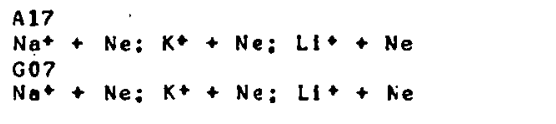 & $\mathbf{T}$ & $200-900 \mathrm{~K}$ & 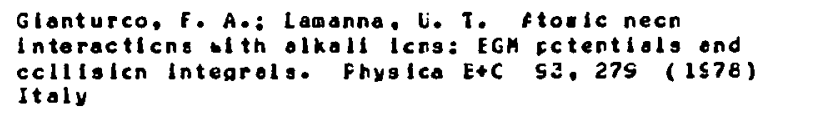 \\
\hline 365 & $\mathrm{H}^{+}+\mathrm{He}: \mathrm{H}^{+}+\mathrm{Ne}: \mathrm{H}^{+}+\mathrm{Ar}: \mathrm{H}^{+}+\mathrm{Kr}$ & $T$ & $\begin{array}{l}100-1200 \\
k \in V\end{array}$ & 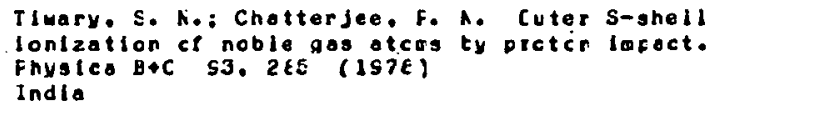 \\
\hline 366 & 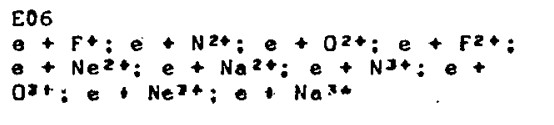 & $T$ & $200-4000 \mathrm{~K}$ & 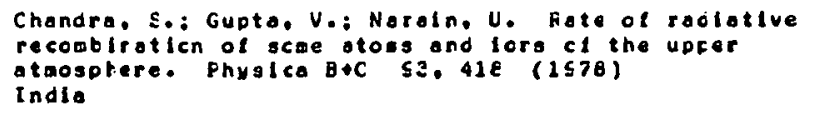 \\
\hline 367 & $\mathrm{hN}_{\mathrm{hN}}+\mathrm{NI}$ & $\mathbf{E}$ & $\operatorname{lov}^{16.8-21.2}$ & 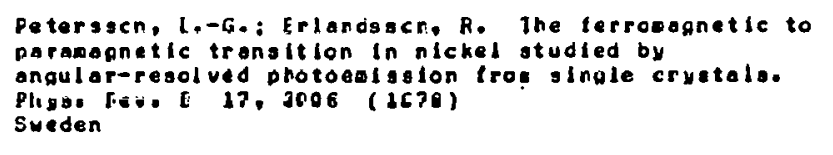 \\
\hline 360 & $\begin{array}{l}E 02 \\
e+H_{2}: e+N_{2}: e+C O \\
E 03 . \\
e+H_{2}: e+N_{2}: e+C O \\
E 17+H_{2}: e+N_{2}: e+C O\end{array}$ & $i$ & $0.5-10 \mathrm{eV}$ & 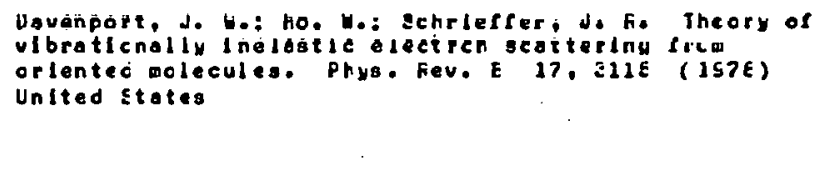 \\
\hline 369 & $\mathrm{DOS}_{\mathrm{H}}+\mathrm{O}+\mathrm{NI}: \mathrm{hv}+\mathrm{S}+\mathrm{NI}$ & $\mathbf{T}$ & $21.2 \mathrm{eV}$ & 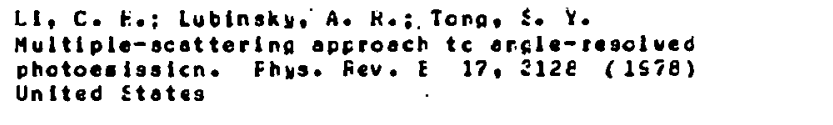 \\
\hline 370 & $\begin{array}{l}\text { COZ } \\
\text { PERT+ + PERT }\end{array}$ & $\mathbf{T}$ & $0-90 \mathrm{MeV}$ & 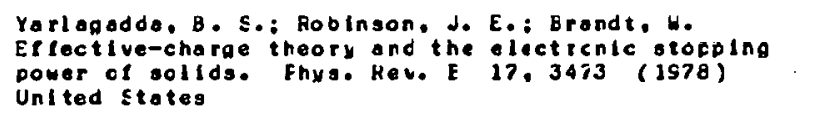 \\
\hline 371 & $\begin{array}{l}\mathrm{CO}^{+} \\
\mathrm{HO}^{+}+\mathrm{SI} \\
\mathrm{COB}^{+}+\mathrm{SI}\end{array}$ & E T & $30-100.0 \mathrm{keV}$ & 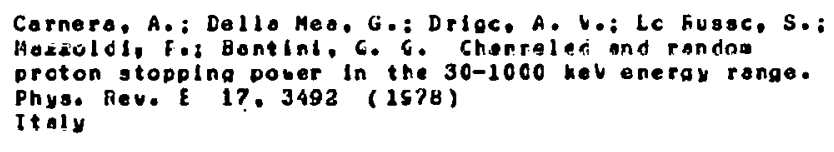 \\
\hline 372 & 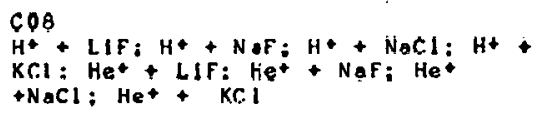 & $\mathbf{E}$ & $0.7-1.3 \mathrm{MeV}$ & 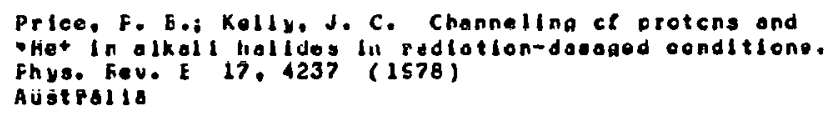 \\
\hline 373 & $\begin{array}{l}\text { DOS } \\
h v+P b: h v+P b C l=: h_{\nu}+P O S: h_{v}+ \\
\text { PbSe } \\
\text { HOG } \\
h v+P b\end{array}$ & $\mathbf{E}$ & $32-40$ eV & 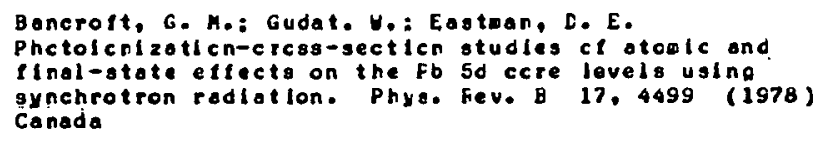 \\
\hline 374 & $\begin{array}{l}\text { BOS } \\
h_{\nu}+\mathrm{CO}_{0}+\mathrm{NI} \\
\mathrm{HOG} \\
h_{\nu}+\mathrm{CO}_{\mathrm{H}} \mathrm{HL}_{2}+\mathrm{C}_{a} \mathrm{H}_{0}\end{array}$ & 1 & $10 \div 100 \mathrm{eV}$ & 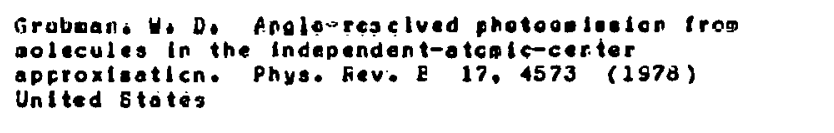 \\
\hline 375 & $\begin{array}{l}\text { DOZ } \\
\text { Ar' }+\mathrm{CCU}+\mathrm{N} 1 \mathrm{I}\end{array}$ & $E$ & $500 \bullet V$ & 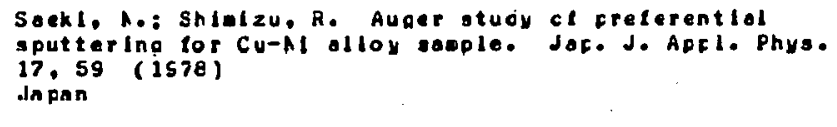 \\
\hline $37 \epsilon$ & 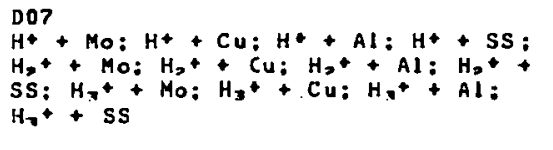 & $\boldsymbol{E}$ & $10-30 \mathrm{keV}$ & 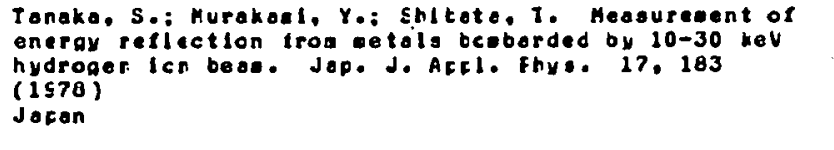 \\
\hline 377 & $\begin{array}{l}\text { Dos } \\
\text { hv }+ \text { GaAs }\end{array}$ & E & $1.2 \mathrm{keV}$ & 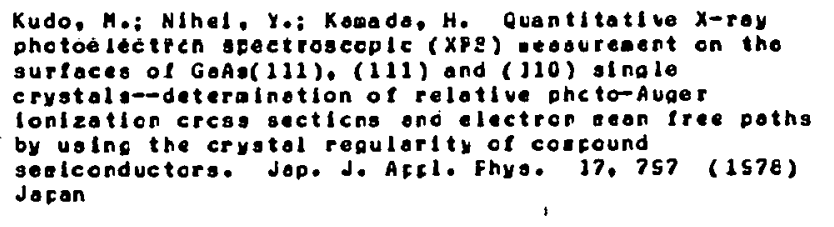 \\
\hline
\end{tabular}


REF
NO.

378

A13

$\mathrm{He}^{+}+2 \mathrm{He}$

REACTANTS

37

G08

$+N_{\text {, }}$

380

D04

$\mathrm{Ar}^{+}+[\mathrm{MgO}+\mathrm{Ag}]$

381

D 02

$A \mathrm{rt}^{+}+\mathrm{PtSi}$

D14

Art+Ptst

382

All $F *$ Ar: KrF* + Kr: KrF* +F,

383

hy + Au

384

A13

$\mathrm{HaCl} *+\mathrm{He}: \mathrm{HaCl}^{*}+\mathrm{Ar}: \mathrm{HaCl} *+\mathrm{Xe}$

$\mathrm{HaCl}^{*}+\mathrm{N}: \mathrm{HaCl} *+\mathrm{Cl}$ : $\mathrm{HaCl} *+$

$\mathrm{HCl}: \mathrm{HoCl} *+\mathrm{CCl}$.

HOS

$\mathrm{h \nu}+\mathrm{HgCl}$,

38

E + Ar

A14

Ar* $+A r+A r$

386

$\mathrm{He}^{+}+\mathrm{C}: \mathrm{H}^{+}+\mathrm{C}$

D14

$\mathrm{He}^{+}+\mathrm{C}: \mathrm{H}^{+}+\mathrm{C}$

387

Cl5 $+\mathrm{Cu}^{+} \mathrm{NI}^{+}+\mathrm{Cu}_{\mathrm{O}} \mathrm{He}^{+}+\mathrm{Cu}$

388

$\mathrm{Ne}^{+}+\mathrm{C}$

369

$\mathrm{H}^{+}+\mathrm{HO}^{2}$

390

D14 P $^{+}+$Mo

$\mathrm{DO} 2$

$\mathrm{Ar}^{+}+\mathrm{Mo}: \mathrm{He}^{+}+\mathrm{Mo}^{+}$

391

$\mathrm{DO} 2$

e+ SS

D14

392

$\mathrm{N14}+\mathrm{Nb}: \mathrm{NI}+\mathrm{NI}+\mathrm{Nb}$

393
D14

$n+A 1,0$,

$\begin{array}{cl}\text { EXP } & \text { ENERGY } \\ \text { CF } & \text { RANGE } \\ \text { THEOR } & \end{array}$

$3000 \mathrm{~K}$

$0-80$ eV

E

20-160 heV

$1,058-1.062$

Him

A13

$300 \mathrm{~K}$

$\mathrm{HOB}$

$1 \& 10 A^{\circ}$

E16

$600 \mathrm{keV}$

A13

$300 \mathrm{~K}$

$100 \mathrm{keV}$

4-58 $\mathrm{HeV}$

350-850 keV

C $0.5 \mathrm{MoV}$

$1 \mathrm{MeV}$

$3.2 \mathrm{MeV}$

E $\quad 100 \mathrm{k} \in \mathrm{V}$
FEFEFENCE

Matsuura, Y.: Fukuca. K. Fcreatcr of He, golicules in the early ofterglcti of fe culsec discherge. Jap. J. APDI. Phys. 17. ST7 (197E)

Jacan

Govinda Faju, G. F.: Guruaurthy. G. F. Electron energy distritutions and transport coeficients in $N$, In $E \times B$ rields. Int. J.Electron. 44, 3ES (1578) India

Koshida, N.: Yoshlde, S. High-efliciency secondary electrcr ea ission produced by lons incident cn a rorous MgO-Ag layer. Affl. Phys. Lett. 32,708 (1578) Japan

Llau, Z. L.: Sheng. T. T. Aracr tuttle toreaticn in the sputtering of fisi. Appl. Fhye. Lett. 32.716 (2578)

Unitod Statea

Eden, J. G.: bounont. F. W.: Seorles, E. K.: Eurnhoa, R. New quenching rotes afrilcable tc the Krf loser. Aprl. Frys. Lett. 32,732 (157e) United stotes

Lcopre, L. A.; Mainfray, G.: Manus, C.: Thebault, di: Farkas, G.: Horuath. 2. A new eftect in wultiphoton photoeftect of a gold gurface induced ty plcoseccnd laser Fulses. Appl. Fhys. Lett. 23 . 124 (1578) France

Mandl. A.: Farks, J. H. Ccllisicrel quenching kinetics for the $\mathrm{HgCl}$ (B $1 / 2$ ) state. ApDl. Phys. Lett. 33 , $458(1578)$ United states

Diegeloonn. M.: hrcbel. W. C.: tichlo. H. Tioe-resolved spectrcacopy of the Ar*,-exciwer edisich. Accl. Phys. Lett. $33,52 E$ ( 1578$)$

West Gerang

Sone, K.: Ate, T.: Cbara, K.: Yanace, F.: Ortguka, H. Graphite surfoce erosion by $100 \mathrm{kel}$ hellud ond hyorchen boubardaent. J. Aucl. Mater. 71,82 (1578) Jafan

Narayan, J.: Cen, C. S.: Ncgale. T. S. Icn roolation canoge in ccpoer. J. Nucl. Mater. 71,160 (1S7E) Unlted Stotes

Kazumata, $Y$. Surfoce erosicn cf curclytic srafhlte and glassy carbcns by len tomtordment. J. Nucl. Mater. $71,17 E$ (ISTE)

Japan

Mootop: D, H.: Ferd, A. G. Vaice frepotion in prcton-irrocioted bolybdenua. J. Nucl. Mater. 71, Le? $(1 \leq 78)$

United stotes

Kaaada, K.: Haranatc, H.: Kazusato, Y. Rocm temperature aging effects of blisterirg and surface roughering after Art icn tcotordiert cn ho single crystale. J. Nuel. Mater. 71. 245 (J578) Jacan

Makin. M. J. Vold shrinkoge and dlsocfearance in stainiess ateel duringelectren irrodietion. J. Nucl. Mater. 71, 300 (157e)

United KIngdor

Local a. B. A.: Gerter. S. E.: Eusct, C. E. Hecuction of vold number censity ond size in icn-irracloted II-coated Nt. J. Nucl. Mater. 73 . EE (1978) United States

Bunch, J. H.: Heteson, J. C.: Zeltaonn, A. H. Cn the noture cl leatures seen by TEM In fast neutron irradiaticn Al, 0,. J. Aucl. Moter. 73,65 (1578) United States 


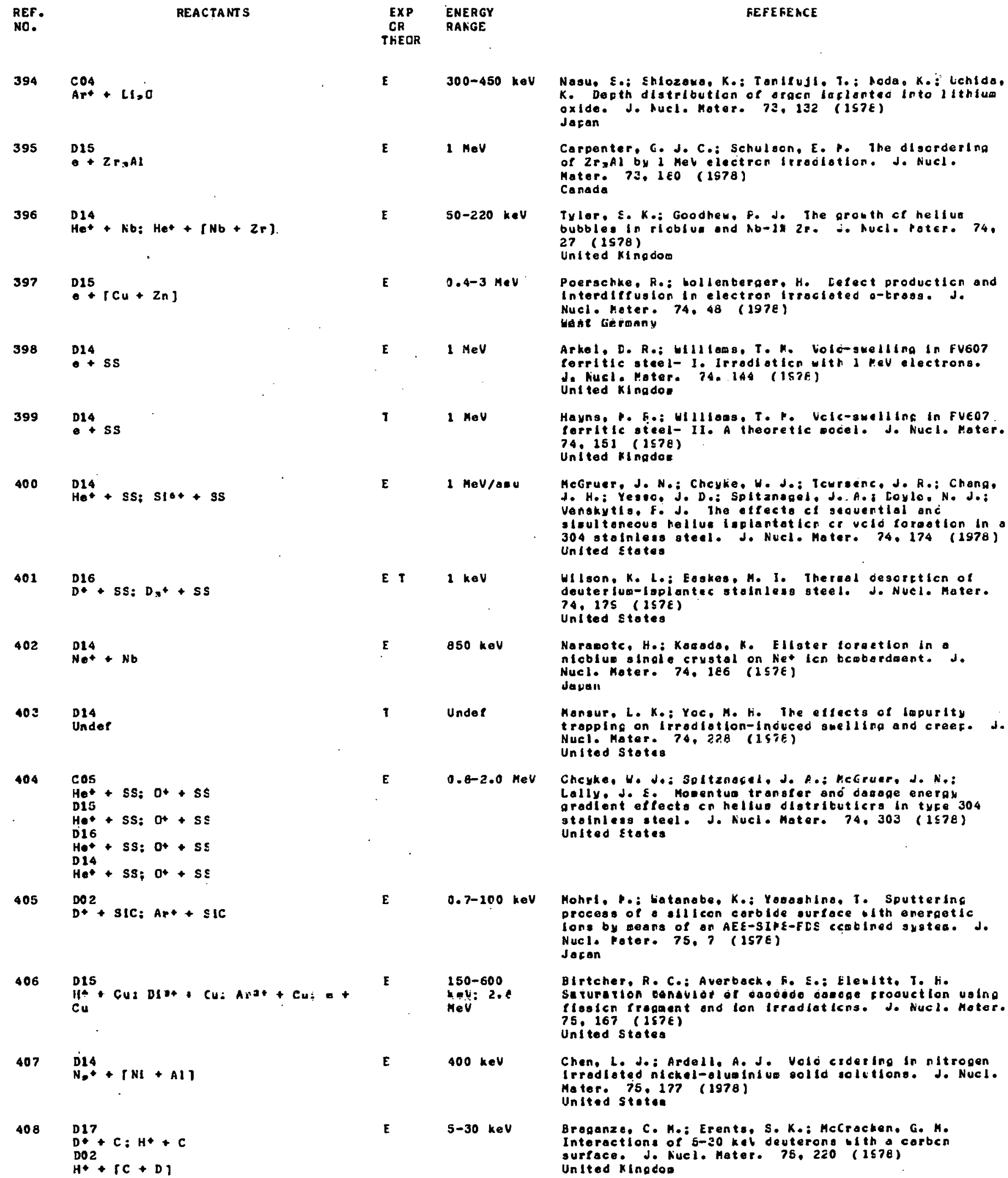

Cos

Hot + SS: $0+$ + SS

D15

$\mathrm{He}+$ SS: $\mathrm{O}^{+}+\mathrm{SS}$

D16

Hot + SS: O* + SS

$\mathrm{Het}^{+}+\mathrm{SS}: \mathrm{O}^{+}+\mathrm{SE}$

Under

$0.8-2.0 \mathrm{HeV}$

Nasu, E.: Shlozewa. K.: Tanlfujl. T.: Ncda, K. : lehida, $K$. Deoth distribution of orgen iaclanted into lithium oxide. J. Nucl. Moter. 73,132 (ISTE) Jaran

Carpenter, C. J. C.: Schulaon, E. P. The disorderlng of Zral by 1 Mel electron irrodition. J. Nucl. Mater. 73, $1 \in 0$ (1578)

Canada

Tyler. S. K.: Goodhew. F. de The grouth ct hellue

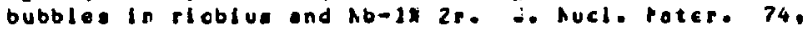
27 (1528) United kingoom

Poerschke. R.: hollenterger. H. Cefect productlicn and interdiffusion in electron irrocioted o-trosa. J. Nucl. Noter. 74, 48 (197e) Habt Germany

Arkel. D. R.: Wlilamg. T. M. Volo-swelling in fv607 territic steel- I. Irrodioticn with l peV alectrons. d. Nucl. Hoter. 74. IAS (ISTE) Unt ted KIngdoe

Hayns, H. H.: Wllloas, T. P. Velc-swelling in FVea? lerritic ated- II. A theoretic pocel. J. Nucl. Mater. $74,251$ ( 1578$)$ United IIngdoe

HeGruer, J. No: Chcyke. W. J.: Icurence J. R.: Chang. H. H.: Yeovo, J. D.: Spitanogel, J..A. Loylo. N. J.: Venskytio. F. Je ine effecto cl sequential anc sieviteneous heliua iaplantaticr cr velo topootion in a 304 stoiniets steel. J. Nucl. Mater. 74.174 (1978) United ctates

Wl lson, K. L.: Eoskes. H. I. Therdal desorticn of douteriun-iapiantec stainieas ateel. J. Nuel. Mater. 74,175 ( $257 E$ ) United States

Naranotc, He: Kacada, N. Elloter foraetion in a nicblue alnole crystal on Net icn tcobardment. J. Nucl. Moter. 74. 186 (IS7E) Javeil

Mansur. L. K.: Yoc, M. H. The eltects of lopurlty tropping on lirodiotion-inouced swelling and creef. J. Nuel. Mater. $74,22 B$ (15TE)

Unitod stoteo

Chcyke, H. Jo: Soltznapel, J. A.: HeGruer, d. N,: Lally. J. E. Mogentud transer onó dagoge energy aradient effects cn helluo distritutiers in tyce 304 stoinleas teel. J. Nucl. Mater. 74.303 (1578) Unlted Etates

0.7-100 heV Mohrl. P.: Latanabe, K.: Yobaahino. T. Sputtering process of allicen corblde uriace ith energetic lons by means of on AEE-SIPE-FCS ccobined syatea. Jo Nuc1. Pater. 75, 7 (1576) Jacon

150-600 Birtcher, R. C.: Averback, F. E.: Elewitt. T. H.

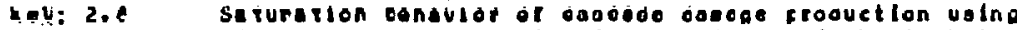
MeV liselcn froment and lon Irradiaticns. J. Nucl. Moter. $75,167$ ( $257 \varepsilon)$ Unitod states

$400 \mathrm{keV}$

Chen, L. d.: Ardell, A. J. Volo crdering in nltrogen Irradleted nickel-olueintuo solld soltiliong. J. Nucl. Mater. 75,177 (1978)

United steten

Broganze. C. M.: Erents, S. K.: MeCrachen, G. H. Interactions of $5-30 \mathrm{k}$ i devterong bith a carben surface. J. Nucl. Mater. 75, 220 (1578) Unlted Mingdoo 
REF.

REACTANTS

$\mathrm{DO}^{+}+\mathrm{SIC}: \mathrm{D}^{+}+\mathrm{SIC} ; \mathrm{Ar}^{+}+\mathrm{SIC}$

410

A14

$\mathrm{OH}^{\mathrm{H}}+\mathrm{COS}: \mathrm{OH}+\mathrm{CS}$,

A14

$\mathrm{CO}+\mathrm{OH}$

412

HOB

$h \nu+K r: h v+X_{e}$

413

D09

HD + W

414

A17

$\mathrm{He}+\mathrm{He}+\mathrm{He}$

$415 \quad A 14$

$U+0$,

$\mathrm{AO}+\mathrm{O}$

$416 \quad$ A11

$\mathrm{I} * \mathrm{HBr}$

417

$\mathrm{Ne}^{\mathrm{N} 7}+\mathrm{Ar}: \mathrm{Ar}+\mathrm{Kr}$

$\mathrm{GO2}$

$\mathrm{Ne}+\mathrm{Ar}: \mathrm{Ar}+\mathrm{Kr}$

418

A14

$X e^{*}+B r$,

$419 \quad 1106$

Review

420

A05

$\mathrm{Cl}_{2}+\mathrm{Ha}_{\mathrm{Q}}: \mathrm{Cl}_{2}+\mathrm{Sr}_{\mathrm{r}}$

$421 \quad$ A14

Al4
Under

$422 \quad$ A14

$N+N$

A 11

$\mathrm{Br}+\mathrm{I}_{2} \mathrm{Br}+\mathrm{Br}_{2}: \mathrm{Br} *+\mathrm{Cl}_{2}: \mathrm{Br}$

$20-120$

kJ/ncl

$11-21$ ov

$900-100.0 \mathrm{~K}$

Undef

$10-300 k$
MohrI, M.: Watanabe, K.: Yarahina, I.: Dol, hi.: Hayakawo. K. Measurement ct erogicn yielda for a sic surface on $H^{+}, D^{*}$ and $A^{+}$. J. Nucl. Pater. 75, 309 (1578)

Japan

Atkinson. R.: Perru. R. A.: Fitts. J. A.. Jr. Fate constants $\mathrm{l}$ cr the reaction cf Ct rodicels with CCS, CS, onc $\mathrm{CH}_{3} \mathrm{SCH}$, over the temperature ronge $295-430 \mathrm{~K}$.

Chew. Phys. Lett. 54, 14 (157E)

United States

Butler, F.: Sclemcn, 1. J.: Snelscn, A: Pressure decendence of the $\mathrm{CC}+\mathrm{CH}$ rate conatart in $\mathrm{O},+\mathrm{N}$, mixtures. Chem. Phys. Lett. E4. is (1978)

United states

Matthias. E.: White, M. G.: Pollohcte. E. E.:

Rosenberg, F. A.; Lee, S.-1.: Shirley, L. A.

TIae-resolved VUV acectroscopy ualing anchrcticn

radiaticn. Zeecan beats in rescnance lluoreacence of the Jp, stotes in krypton ond xenor. Chem. Phys. Lett. 54. 30 (157e)

United stotes

Wolken, G.. Jro: Mccreery, J. h. Gos-gallo energy transfer: etfect ol internal vibrational eneray. Chem. Phys. Lett. 54. 35 (1578)

Unted States

Llcyd. J.: Fugh. D. Sowe ctservaticns on the electron gas codel scr three-body interato ic torces. Chea. Fhys. Lett. E4, ES (1978)

United kingdom

Vasu, P.: Lo, H. Hi.: Fite, H. L. Energy distributicn of olectrons frco $b+C$, asciotive icnizotion. Chem. Phys. Lett. 54, es (1578)

United stotes

Fotaklo. C.: Donoven. F. J. Teoperoture decendence for

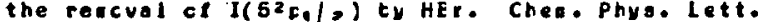

$54.91(1578)$

Arcra. F. S.: Carson, F. Je: Dunlof, P. J. Determinaticn of cctential farameters lor the systems Nie-Ar ond Ar-Kr $l \mathrm{rcm}$ the teacerature decencence ot thelr tinory difluolion coefliciente. Cheo. Phyo. Lett. 54. $117(1578)$ Austral to

Levy, H. F.: Fettner, C. I.: Steons, J.P. Moleculor beom inetics: the excitation function of the resction

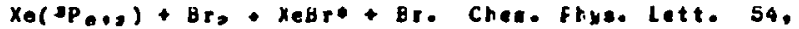
$120(1578)$

United Kinodon

de Leeub. D. M.: Mooyaon. F.: de Longe. C. A. He(I) photoelectren ocectroscopy ce holecen atose. Ches. Phys. Lett. 54, 231 (1978)

The Netherlands

Kowalskl. A.: Heldt. J. Chenllualnescent studles of exclted $\mathrm{Hg}$ and $\mathrm{Sr}$ otoug with Cl, Ir. the bedo-gog arrangesent. Chem. Phya. Lett. 54, 240 (157e) Poland

Northruf, S, hi.: Hynes. J. I. Cn reoction tote constonts and rote kernels. Ches. Phyo. Lett. E4, 248 (1578) United Etates

Gordan. E. H.: Hezhcu-Dealin. L. F.: Pugachev, O. F.: Khmelenko, $U$. V. Gas-phase apectro of the radiative recoobinaticn of nitrogen atcos ot hellue temperotures. Chea. Fhys. Lett. 54,282 (1578) Soutat Union
Holeann, Hi: Leone, S. F. Collibicnal deactivoticn of laeer-exclted Br*(2p,le) atces with holcgen ono interhaloger dolecules. Chep. Fhys. Lett. 54, 314 (1978)

United Etotes 


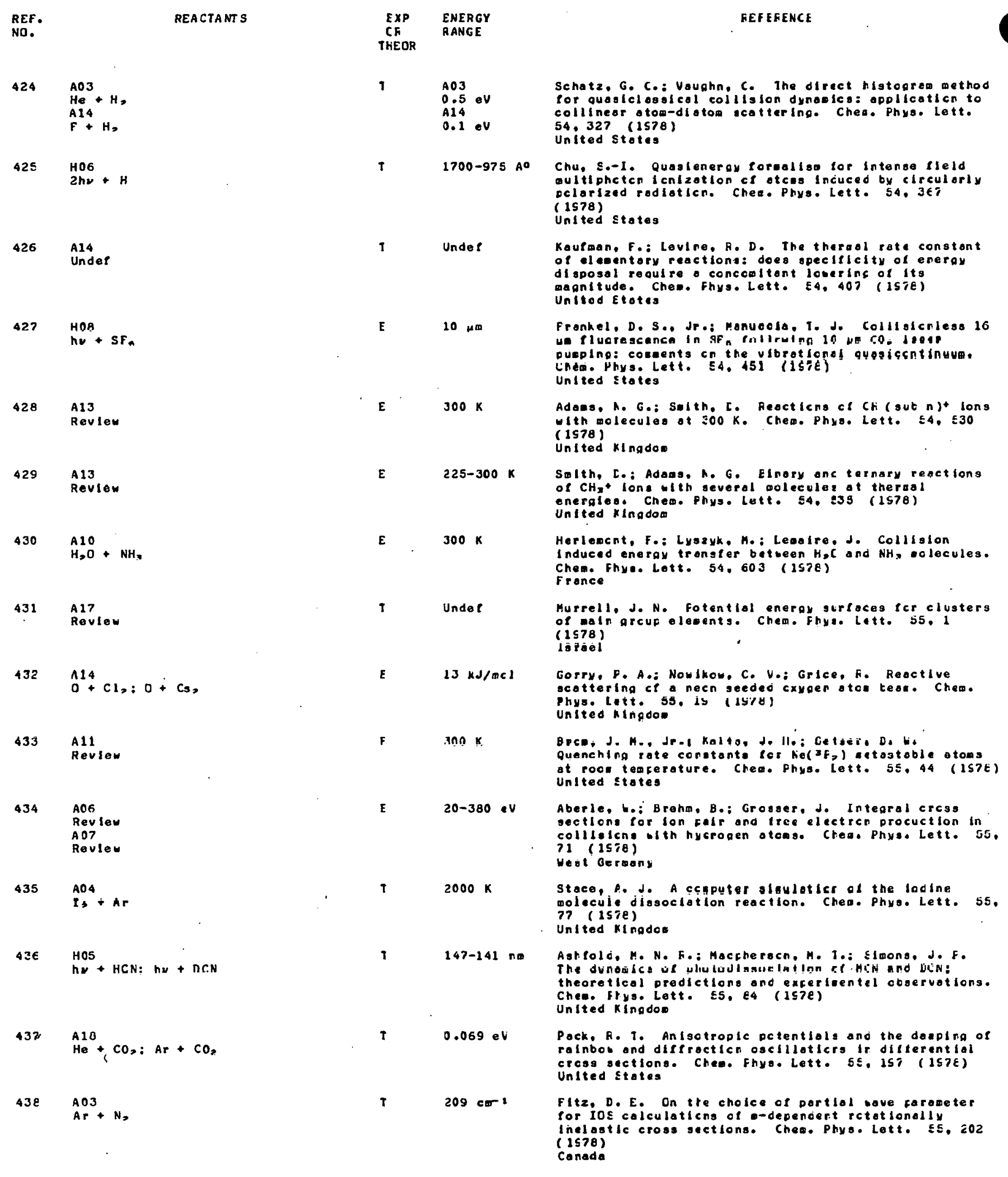




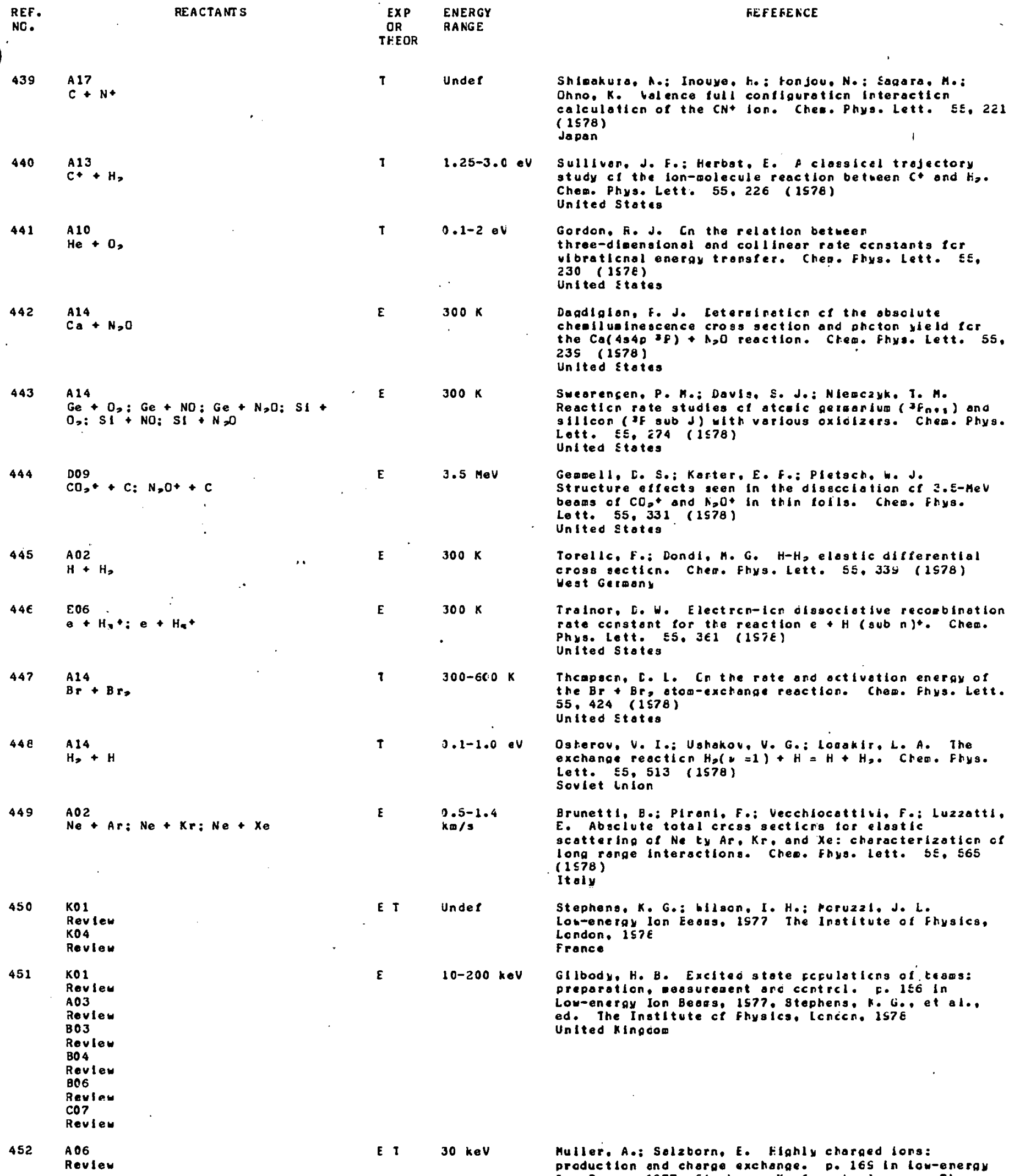
$\mathrm{O}$ : $\mathrm{SI}+\mathrm{NO}: \mathrm{SI}+\mathrm{N} \infty$

$\mathrm{AO3}$

Revlew

BO3

Revlew

$\mathrm{BO}_{4}$

Revile

Reviou

COF

Revieu

Shleakura, A.: Inouye, he: tonjou, No: Sagara, M. Ohno. $K$. bolence full contlguraticn interacticn calculaticn of the CN Ion. Chea. Phys. Lett. Es. 221 (1578)

Japan

Sulliven, J. Fo: Herbot, E. A classicel trojectory study ct the lon-wolecule reaction between $C^{*}$ ond tig. Ches. Phys. Lett. 55, 226 (1578) United Stotes

Gordon. F. J. Cn the relation between

three-diaenalional and collinear rate censtants $f c r$ vibratlicnal energy tronsfer. Chea. Fhys. Lett. se, 230 (157E)

United states

Dagdiglon. F. J. Leterinoticn cl the obsolute chealluelnescence cross section and phcton yield $\mathrm{scr}$ the $C a(4340, f)+A>0$ reaction. Chem. Fhys. Lett. 55 , 23s (1578)

Unlted stotes

Sweorengen, P. M.: Davls, S. J.: Nlemczyk. T. H. Reacticn rote studies cl atcelc geisarlum ( $\mathrm{F}_{\mathrm{n}}$...) and sillcon (if sub J) ith varlous oxlolzerse Ched. Phys. Lott. $\leqslant \leq . \leq 74$ ( $1 \leqslant 78)$

United stotes

Gemell, C. S.: Karter, E. F.: Pletgch, W. J. Structure elfects seen in the disgcelotion ct a.E-MeV beams of $\mathrm{CO}_{3}+$ and $\mathrm{H}_{3} \mathrm{O}^{+}$in thin tolls. Chese Fhys. Lett. 55,331 . (1578)

United stotes

Torelle, F.: Dondi. H. G. H-H, elastlc differential cross secticn. Chea. Fhys. Lett. 55, 33y (2578) West Gerouny

Tralnor, $[$. W. Electrcn-icn olssociotive recosbinotion rate ccnstent lor the reaction + $H(\text { sub n })^{+}$. Chem. Phys. Lett. ES. 3E1 (1STE)

United Stotes

Thcapecn, c. L. Cn the rote and octivation energy of the Br+Br, atom-exchange reaction. Chem. Fhys. Lett. $55,424(1578)$

United states

Ogtierov, V. I.: Ushakov, U. G.: Looakir, L. A. The exchange reacticn $H_{0}(v=1)+H=H+H_{2}$. Chear. Fhys. lett. 55,513 (1578)

Soviet inion

Brunett1, B.: Pironl, F.: Vecchlocottlit, F.: Luzzattl, E. Absclute total crcss secticris tor elastic scattering of Ne ty Ar. Kr. and Xe: characterizaticn of long ronge interactiong. Chea. Fhyo. lett. 55.565 (1578)

It aly

Stephens, K. G.: hllson, I. H.: Moruzzl, J. Lo Lon-energy Ion Eeors, isp? the Inatitute of fhysics, Lendon, Is?E France

Gllbody, H. B. Exclteo stote fCrulaticns of teass: Preporation, wosureaent orc ccrtrcl. F. 156 In Low-energy Ion Bears, is77. Stephens, k. 6. et al., ed. The Instltute ce fhysica, Lcncen. is7e United kinpiom 


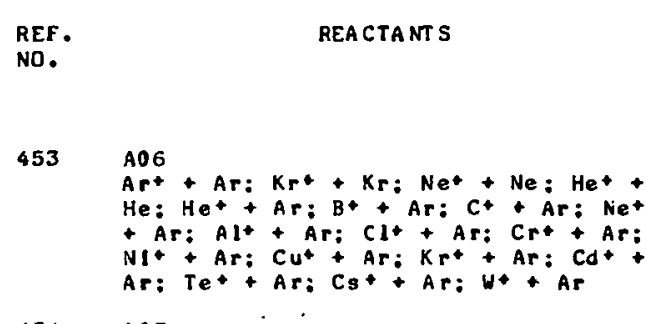

454

$\mathrm{I}+\mathrm{CH}$

455

007

$\mathrm{Ne}^{+}+\mathrm{NL}: \mathrm{He}^{+}+\mathrm{NI}: \mathrm{No}^{+}+\mathrm{Ag}$

D13

$\mathrm{Art}_{+}+\mathrm{CO}+\mathrm{NI}: \mathrm{Ar}_{+}^{+}+\mathrm{O}+\mathrm{NI}: \mathrm{Ne}_{+}^{+}$

$S+\mathrm{NI}: \mathrm{Ne}++\mathrm{CO}+\mathrm{NI}$

$\mathrm{DO}_{2}$

$\mathrm{He}^{+}+\mathrm{AP}_{\mathrm{A}} \mathrm{He}^{+}+\mathrm{Tn}: \mathrm{Ne}^{+}+\mathrm{Ta}^{\mathrm{N}} \mathrm{Ne}^{+}+$

$\mathrm{W:} \mathrm{Ne}^{+}+\mathrm{HI}: \mathrm{He}^{+}+\mathrm{W}: \mathrm{He}^{+}+\mathrm{Hl}^{+} \mathrm{He}^{+}$ $+40$

\section{A05}

$\mathrm{Ha}+\mathrm{TI}$

$A 03$

All + T

$\mathrm{HOZ}^{+}+\mathrm{H}: \mathrm{H}^{+}+\mathrm{D}$

$\mathrm{H}^{+}+$

$\mathrm{A06}$

$\mathrm{H}^{+}+\mathrm{H}^{-\mathrm{H}^{+}+\mathrm{D}}$

$\mathrm{H}^{+}+\mathrm{H}: \mathrm{H}^{+}+\mathrm{D}$

$46 \varepsilon$

\section{EXP ENERGY \\ THEOR}

E

E

$$
\begin{aligned}
& 200-600 \text { ov } \\
& 013 \\
& 200-2000 \text { eV }
\end{aligned}
$$

102

$100-2010$ ov

(n)

5-35 keV

HO2

$\mathrm{h}_{2}+\mathrm{N}_{2}: \mathrm{h}_{2}+\mathrm{CO}_{2}$

$\mathrm{N}_{2}+\mathrm{N}_{2}: \mathrm{CO},+\mathrm{CO}_{0}$

207-778 K

E $\quad 4.6 \mathrm{ctr}^{-1}$
$\mathrm{Ho}+\mathrm{Tl}_{1}$
GEFEFENCE

Leyland, K.: Arwour, D. G.: Corter, G.: Freemen, J. H. Cherge trongler cross aectlons in tilgh-llux. lou-energy eccelerotors. p. 175, in lormenergy Icn Bease, 1977. London, 1978

United Kingdow

Becker, M.: Hellgelst, Mo: Holl, G. K. An offaratus for the Inveatigation of the interecticn between Ion beams ond gases. c. les in Low-eneray Ion Eeases. 1577. stephona. K. G.. I al.. ed. The Inatitute of Phyalcs. London, 1978

Weot Gereany

Helland, W.: Taglover, E. Seattering Ct lon claos from surfaces. D. 2E? in Low-eneray Ion beoss. 1977, Stephens, K. G.. et ol.. ec. The Institute of Fhysics, London, 1978

weat Gerang

van den Eerg. J. A.: Armour, L. G.: Verheld. L. K. A 2-100 keV. UHV ion inpoct ipectroveter for lon-solld Interaction studies. $c .258$ in Lon-ereray Ion Beses, 1977. Stophens. K. G.. at al.. ed. Tre Instlitute of Physics, London, 1578 Uni ted Kingdo:

Kaolingky, M.: Das, S. K.: Ekern. F.: Hess, C. C, An accelerator systeo for producing twc-cceconent beas lor studies of Interoctive surlace elfoets. D. 305 in Low-energy Ion Eeare, 1977, Stephers. K. G.. et al.. ed. The Institute of Fhyoloo, London, is7e United stotes

Mashkove. E. E.l Molchenov. V. A. Surface anolysis using the dcuble-scottering eflect. F. 313 in Low-energy Ion Eeaes, 1577, Stephens, K. G.. et al.. ad. The Inatitute of Fhysics, London, 1977 Soviet inicn

PInningson. E. H.: Donnelly, K. E.: Kernation. J. A.: Irwin. I. J. G. Eqoo-loll apectricicopy of civaen in the wavelength region troa 270 to 450 po. Can.J. Phys. E6, 508 (1578)

Canada

$26-136$ e V

Kulhar, V. E, shoetry, C. E. Fuclted atales of J. Phys. Ee. 565 (1578)

Ind ia

Fulls, A. M.: Salth. M. M.: Scoles, G. Interoclecular rorces in hydrogen-noble gos ixtureo. Con. J. Fhys. $56.753(1578)$

Canada

chien, $i$. T.: Delly, fo ke van dor linde, te Radiative lifeties and hycerfine ccnatonts tos the 3 s ooplex of solecular hydropen. Can. J. Physe E6, 82: (1578)

Canada

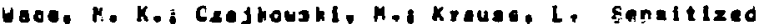
tluorescence in tholltue Incuceo in celllsich olth

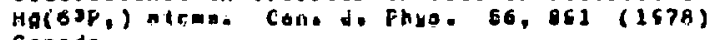
Conede

Davis, d. P.: Therscn, th. A. Very lou eneray scattering in HH* and HD*. Can.J. Phye. 56, 996 (1578)

canade stephens. K. G.. et ol... The Institute of Fhysica, pooltrentide In castrcn-nydicgen chorpe exclonge. Can
Dogg, 1. R.: Reesor, G. E.: tong, P. A alcrowave $\mathrm{N}$, and $\mathrm{CC}_{2}$ ot $4.6 \mathrm{cor} 1$. Can. J. Phys. 56. 1037 (1578) cavity ueastrament cl cclilsicn-incucec obsorption in 
REF.

REACTANT $S$

466

467

EO2

Eo3 Cd

$\mathrm{EO3}$

Bos

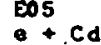

608

cot Ar

$0+c d$

$\cos 5$

DOB

$\mathrm{B}+\mathrm{C}_{4}: \mathrm{B}+$ + $\mathrm{HO}$

DO3

$B *+C u: B++$ Ho

A06

$\mathrm{H}+\mathrm{Ca}^{+} \mathrm{H}+\mathrm{Cs}$

A20

$\mathrm{H}+\mathrm{C} \& \mathrm{H}+\mathrm{Ce}$

477

$$
\text { All }+ \text { COS He* + SP }
$$$$
\mathrm{Xe}^{+}+\mathrm{Ca} \mathrm{Xe}_{+}+\mathrm{Sr}
$$

$478 \quad$ DO4

Undef

$479 \quad 006$

C 51 $\begin{array}{ll}\text { EXP } & \text { ENERGY } \\ \text { CR } & \text { RANGE } \\ \text { THEOR } & \end{array}$

E $4.4 \mathrm{car}^{-1}$

$20-10000$
$k \in V$

E

$20-60^{\circ} \mathrm{k} \in \mathrm{V}$

$320 k$

E

t

EO2: EO3:

E05: $G 0 E$

$0-15$ eV

607: 608

$0.1-100$ Id
Degg. I. R.: Reesor, G. E.: Wong. P. Colllolicn-linduced alcroveve otsorpticn in Ne-xe ond fr-xe gageova

witures. Can.J. Fhys. 56, 1046 (1578)

Conodo

Ojha, E. P.: Ilwarl, F.: Erivastavo, K. F. Crcas aection for the excltation cl hellua ty Het. Con. J. Phys. 56. 1232 (1978)

Ind I a

Kunar. A.: Rey. B: N. BInery encounter calculoticns on electrcr iefact double lonlabticn cr retle gas otoms. Can. J. Fhye. 66. $12 E \mathrm{E}$ (1578)

Ind $\mathbf{a}$

Davlea, D. K. Ionlzaticn end ottochnert coelificients

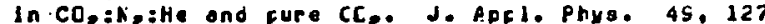

(1578)

United stotes

SInha, H. Ko: Das, S. K.: Kaelingky, H. Temperature dependence of hel iud blistering In nichel ecnccrystals. J. ApDl. Phus. 49, 170 (1578)

United stotes

Schou, Jo: Sorensen. H. The penetratien decth ch $0 . E-3-k e V$ electrons in solid hydrogen and deuteriua. J. App 1. Fhys. 49,816 (1578)

Deniark

Bottlger, Jo: Jengen, F. S.: Littoork, U. Depth profiles of jHe lcrs incloried intc aclios ot energles between 20 and 60 keV. J. Ppcl. Phys. 49, SEE (IS76) Dengark

Crese, L. A.: Cen Goxay. M. Excltotler precosas in a Pb-He discharpe. J. AfFl. Fhys. 49, $2 \in 35$ (1576) United states

Fournler, G. R.: neGeoch. H. Kinetlic wodel of the sustalned dischore excitation cl the codolum vercury excleer. J. ADDI. Fhys. 4S. $2 E 51$ (1 $1 \leq 78)$ United Kingdom
Undel
$60-200 \mathrm{heV}$

$0.5-1.1 k \in 0$

$850-2600$ ev

$15-35 k \in V$
Themas, E. W.: Incuye, H.: Faugeh, E. C. Exclted atate formed ty $B$ impact on metallic $C u$ and Po. J. ApFl. Phys. 49, 2911 (1528)

United stotes

Kaporachroer, J. Hof Fost, R. g. Use cr a Hall eccelerotor in the producticn ct negotive hydrcpen ions In cestuo vepor. J. Accl. Fhys. 49. 205S (1578) Unlted stotes

ButtertIald, K. B.: Gerotenteraer, C. C.: Shay. T.: Little. W. L.: Coliling. Ge Je Ccillstcnol quenching of xeo $2 p$; and He 2 is metostaties by colclum and otrentilum metal vopora. J. Appl. Phyo. 45, 308E (1578)

Unt ted Etotes

Allo. R. C.: Blcon. S. Seccndory-electron-escope Drobabilities. J. Appl. Fhys. 4S, 2476 (1S7E) United Etoles

Muller. H. H.: Echroder. B. Electron tackocatterling frco thin silicen cryotols. J. AcEl. Ihys. 4S. zsS5 (1578)

Weat Geraany 


\begin{tabular}{|c|c|}
\hline $\begin{array}{l}\text { REF. } \\
\text { NC. }\end{array}$ & \\
\hline 480 & $\begin{array}{l}\mathrm{E} 02 \\
e+N, \\
\mathrm{E} 03 \\
\mathrm{e}+\mathrm{N}, \\
\mathrm{E}+5 \\
\mathrm{e}+\mathrm{N}, \\
\mathrm{E} 17 \\
\mathrm{e}+\mathrm{N}, \\
\mathrm{G}+\mathrm{N}, \\
\mathrm{e}+\mathrm{N},\end{array}$ \\
\hline
\end{tabular}

481

482

DO 4

$e+\mathrm{Cl}_{3}+\mathrm{K}+\mathrm{Na}+\mathrm{Sb}$

482

$\mathrm{DO4}$

a $+\mathrm{N}$

D0 6

$e+N_{2}: e+c$

C+N,

484

$\mathrm{CO}_{\mathrm{H}}+\mathrm{C}$

$\mathrm{CO}^{+}+\mathrm{C}$

$\mathrm{H}^{3}+\mathrm{C}$

AO 4

$\mathrm{H}^{+}+\mathrm{C}$

$485 \mathrm{EO}$

e+ He*

486

EOS

e.Ar

E.27

e+Ar

487

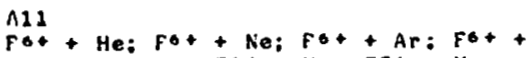

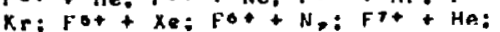
$F>+$ Ne: $F>+$ Ar: Fit $+\mathrm{Kr}: \mathrm{Fr}_{+}+$

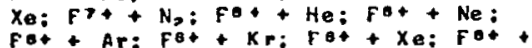
Ns: rot: lle: ros: Ho: Fot t Ars

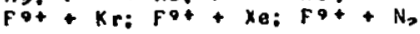

$488 \quad E 17$

- + He

$\mathrm{EO} 2$

$\mathrm{a}+\mathrm{CO}$,

$\mathrm{COS}$

$\mathrm{e}+\mathrm{CO}=$

$\mathrm{C}+\mathrm{CO}$,

490

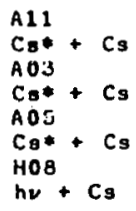

491
REACTANT S

$\begin{array}{ll}\text { EXP } & \text { ENERGY } \\ \text { OR } & \text { PANGE } \\ \text { THEOR } & \\ & \\ \text { E T } & \text { EO2: EO Z: } \\ & \text { EOS: E1 T } \\ & \text { J-7O OV } \\ & \text { GCS } \\ & S-E O \mathrm{eV}\end{array}$

E

$10-30 \mathrm{keV}$

E

E

E

I

E

$0.5-50$ ev
3-90 ev

E

A03: AOE:

A11

$300 \times$

408

$5636-8161$

40

$2-1361$ ev
E03

e $+\mathrm{H}$
Carturight, D. C. Rate coeffictents and ineiastic moentua transfer cross secticns lcr electrcnic excitation of $N$, ty electrens. J. ApFl. Phys. 4S. 3855 (157e)

UnItod States
Borgesen, P.; Bottlger. J.: Moller, H. Ranges ct 10-30-keV deutercns leflanted intc solids. J. Aprl. Phys. 49.4401 (1578) Dernark

Ghosh, C.: Varma. B. P. Secondary ealsaion frca multialkail photocathodes. J. Appl. Ftiys. 4S, $4 \leqslant \leqslant 4$ (1578) India

Sorensen, H.: Schcu, J. Interactlist tarween solid nitrogen onc 1-z-keV electrons. J. Apcl. Phys. 49. 5311 (1STE)

Denmark

Gabllard. A. J.: Gegeell, D. S.: Gcldring, E.: Levine, I.: Pletgel. W. J.: PClzat, J. C.: Fatkewski, A. J.i Reillileux. J.: Vager, 2.: 2abranaky. E.J. Experitentol deteralnation cl the etructure of $\mathrm{H}^{+}$. Fhys. Fev. A 17, 1797 (1578) United stotes

Wlllidoon. W.. Jra: Fodter, G.: Koong, R. Glauber exchange anclituder lor the scattering of electrong erco hycrogenlike lons. Fhys. Rev. A 17. 18a? (1578) United stotes

Hong. E. P.: Beaty, E. C. Neagureaenta of the triple-difforentlai crcss sectich lce low-enersy electrcn-ladact ionizotion cl argor. Fhys. Rev. A 17, 1829 ( $(1 \subseteq 7 E)$

United states

Brenn. Fo: hooklns, F,: Scrcuse, G. D. Hypertine interacticns in teb-electron livorine icns recciling in uases. Phys. Rev. A 17, 1E37 (1578)

We st Geroony

Konnerly. R. Ea: Bonhow, F. A. Eleotecn-hellug abolute tctal scotering crcss sections frca $0.5-50$ eV. Frys. Fev. A 17,1844 (1574) United states

Shyn. T. W.: Sharp. W. E.: Carignon, G. R. Angular digiributicn ce electrcis elooticoliy cottered from che. Fhys. Reve A l7. 1855 (IS7E) United States

Tax, A. C.: Yabuzak 1, 1.: Curry, S. M.: Hou, H.:

Happer. H. Inelastic cross secticrs in Cs(nzD sut j) + Caleas:, i collisions. Phyg. Feve. 17, lege (is?ó) UnIted stotes

Chan, F. 1.; Chang, C. H.: Leter, H.: KIm, Y.-K. Cooparlson of the Forn and Glouber generallized oscillator atrenoths for the $28+30$ trapsiticn cl atoalc hydeoger. fhye. fove A 17, Jaes (1STE) United stotes 
REF.

REACTANTS

No.

$492 \quad \begin{aligned} & \mathrm{E} O 3 \\ & \mathrm{C}+\mathrm{H}_{2} \\ & \mathrm{E} 04+\mathrm{H}_{2}\end{aligned}$

$493 \quad$ A.07

Under

AO 3

494

Bo?

$\because+\mathrm{H}$

H06

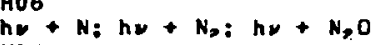

HO 2

$h_{\nu}+A r: h_{\nu}+N_{2}: h_{\nu}+N_{2} O$

$496 \quad \mathrm{H} 12$

Under

497

803

$-+\mathrm{D}$,

$\mathrm{EO} 4$

$C+D$,

Fo1

498

HOG

hr + Be sea

499

EO2

a $+\mathrm{Be}$

500

co7

$\mathrm{Kr}+\mathrm{C}_{\mathrm{Z}} \mathrm{Ge}^{+}+\mathrm{C}$

$501 \quad$ FO1

in sea

502

$\mathrm{CO}^{+}+\mathrm{C}^{\mathrm{C}} \mathrm{N}^{+}+\mathrm{C}: \mathrm{O}^{+}+\mathrm{C}$

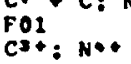

$503 \mathrm{HO1}$

Under

$504 \quad B 01$

Under

$\mathrm{FO}_{2}$

Under

505

$\mathrm{HO2}$

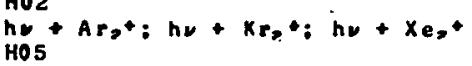

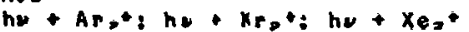

506

$\mathrm{Ho2}$

hiv + Mn

507

$\mathrm{CO} 2$

$\mathrm{H}^{*}+\mathrm{PERT}: \mathrm{He}^{+}+\mathrm{PERT}: \mathrm{LH}^{+}+\mathrm{PERT}$ :

Be* + PERT: B $^{*}+$ PERT: C* + PERT:

$\mathrm{N}^{*}+$ + PERT: $\mathrm{O}+$ + PERT; F+ + PERT
EXP ENERGY

GE GANGE

T $\quad 10-40 \mathrm{eV}$

Chung, S.: Lir. C. C. Apclication of the

clcse-ccupl ing eetrod to excitaticn of electronic

states ond disoctotion of $H_{2}$ by lecticn lapoct.

Fhys. Fev. A 17, 1874 (1578)

United Stotes

Under

Nakamura, H.: Shiral, I. Nakal. Y. Iafroved imful se approximaticn for lonizotion collitions between atces. Phys. Fev. A. 17, 1892 (1578)

France

Under

Brandl. H. S.: Kollier, B.: Lins de Barroo, H. G. P.: MI randa, L. C. M.: Castro. J. J. Theciy ol

electren-hydrogen-eton colliolicis in the oresence of o lasar tield. Phys. Rey. A 17, 1560 (1578)

Brazil 1

E 390-450 eV

Blanconl, A.: Petersen, H.: Brcun, F, C.: Bechrach, A. 2. K-stell photoobsorption spectro of $N$, and $N, D$ using synchretron radiaticn. Phws. Fev. A 17, 1907 (1978) Unlted States

Under

Barmlck, J. Clasalcal thecsy ci rodletive trangitions. Phus. Rev. A 17. 1S12 (157e)

United States

Nowak, C.: Borst, h. L.: Frlcke, J. I lifetime

deterainaticn of the O(s $S^{\circ}$ jetastoble state via

13E6-Ao using time-ot-plight technlque. Fhys. Fev. A 17. 1921 (1578)

West Germany

60-1600 eV Watson. D. K.: Dalgarnc, A.: Sterort, F. F.

Inner-stell ohotclicnizoticn of the teryllitur

isoelectronic sequence. Fhyo. Fev. A 17. 1928 (1578) Unitod stotes

Resclanc. T. N.: Rccurdy. C. Y.. dro: Crel, A. E. Extensicns of the coaplex-cooroinate sethcd to the study ct resonances in aanyelectren syoteds. Fhys. fev. A 17,1931 (1978)

United stoties

Younger, S. M.: Wlese. W. L. Theoretical bilulatien of bear-rcil decay cuives lor rescnance tronsitions ct heavy Icns. Fhys. Fev. A 17, 1544 (1578)

United stotes

FIscher. C. F.: Hensen. J. E. Thecretical cactllator strongthg $f(c)$ the rescrance tronsitiong in the $2 n I$ isceloctronic sequence. Fhys. Fev. A 17, iset (1S7E) Unted states

$\mathrm{CO} 7$

LIvingstcn. A. E.: Berry. H. G. Fine trueture of the

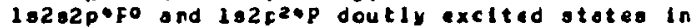
lithlusilke certon, nitropen, ond caypen. Phys. Fev. A 12. 1566 (1578)

United states

Undel

Srivastovas h. F. Trieoreticel investisotion of energy-trapping ochanism by atoalc syoteos. Physe Rev. A 17. 1576 (157E)

Canada

a'Connell, 5. F. Fydberg atates In strcna electric and magnetle Ilids. Fhys. Rev. A 17, Ised (1578) United Etates

$6200-8600$

Lee, L. C.: Solth. G. F.: Mlller, 1. M.: Cosby, P. C. Phetodisociation cross sectlons 01 Ar, $K_{r}$, . and

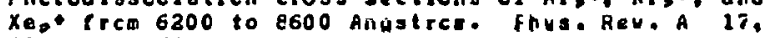
$2005(1572)$

unitod stotes

$45-60$ e

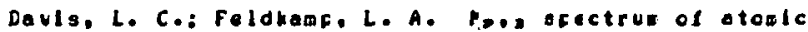
Mn. Fhys. heve A 17.2012 (157E)

United stotes

Undef
Ritchle, R. He: Brandt. H. Projectile-charge defenderce cf stopfing cowers. Fhys. Fev. A 17, 2102 ( 1578 )

Unted stotes 


\begin{tabular}{|c|c|c|c|c|}
\hline $\begin{array}{l}\text { REF. } \\
\text { NO. }\end{array}$ & REACTANTS & $\begin{array}{c}\text { EXP } \\
C R \\
\text { THEOR }\end{array}$ & $\begin{array}{l}\text { ENERGY } \\
\text { FANGE }\end{array}$ & FEFE EENCE \\
\hline 508 & $\begin{array}{l}E 02 \\
E 17+N= \\
e+N=\end{array}$ & $T$ & $0-30$ eV & 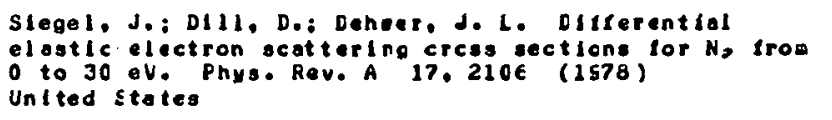 \\
\hline 509 & $E 02+A r: e+K r$ & 1 & Undef & 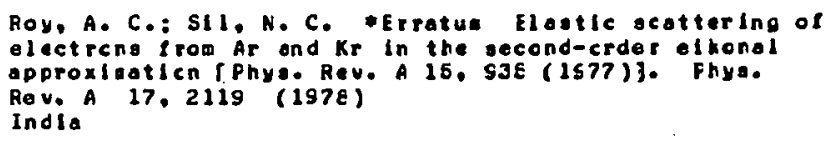 \\
\hline 510 & $\begin{array}{l}\text { Ho } 3 \\
\text { Under } \\
\text { Hos } \\
\text { Under }\end{array}$ & $T$ & Undet & 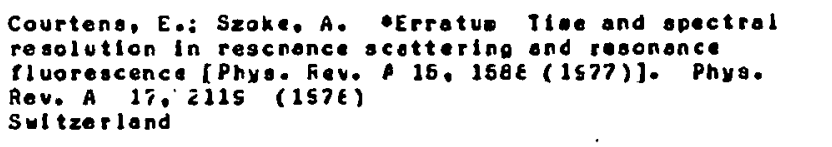 \\
\hline$\leqslant 11$ & $\begin{array}{l}\text { DIS } \\
\text { Art+ }+\mathrm{Pd}_{2} \mathrm{SI}: \mathrm{Ar}^{+}+\mathrm{NISI}, \\
\mathrm{COB}+\mathrm{Pd}, \mathrm{SI}: \mathrm{He}^{+}+\mathrm{NISI} \text {, }\end{array}$ & E & $\begin{array}{l}015 \\
100 \mathrm{keV} \\
\mathrm{COg} \\
1.5 \mathrm{mov}\end{array}$ & 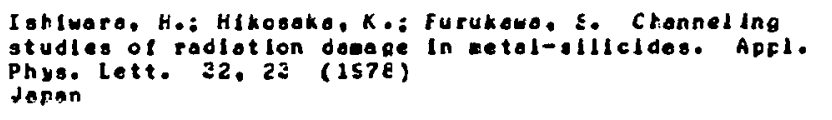 \\
\hline 512 & 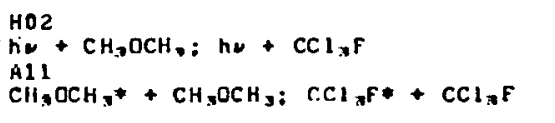 & E & $\begin{array}{l}402 \\
10.6 \\
A 11 \\
300 R\end{array}$ & 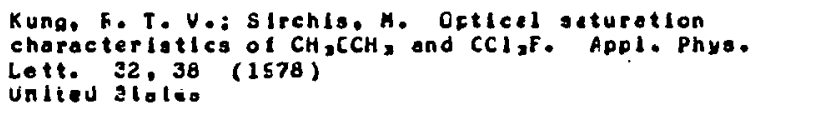 \\
\hline 513 & 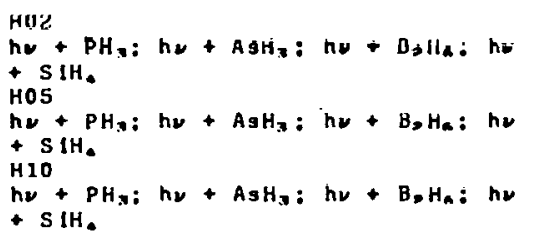 & $\boldsymbol{F}$ & $6 \mathrm{eV}$ & 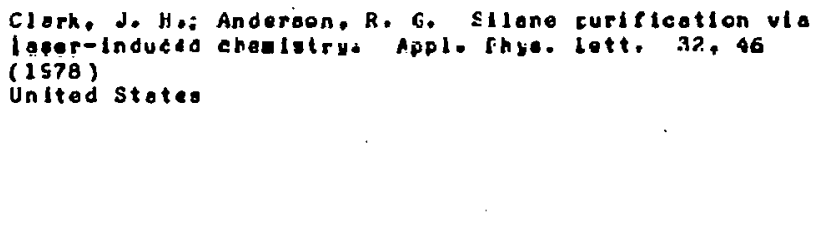 \\
\hline$\leqslant 14$ & $D 27+$ GOAs & $\mathbf{E}$ & $400 \mathrm{~K}$ & 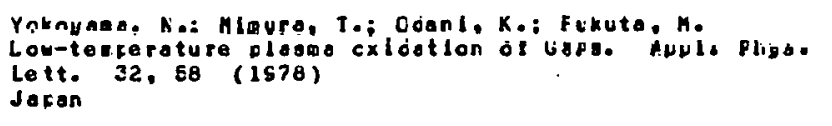 \\
\hline 515 & 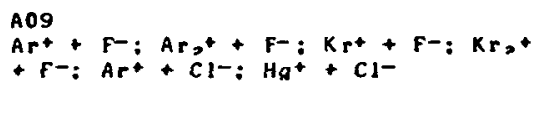 & $T$ & $300 \mathrm{~K}$ & $\begin{array}{l}\text { Yadehro. J. Mo: Bordsley. J. A. Colculoticns In } \\
\text { lon-lon reccobinoticn potes ot high pressures. Appl. } \\
\text { Phys. Lett. } 32.76 \text { (1978) } \\
\text { United States }\end{array}$ \\
\hline 516 & $\begin{array}{l}\mathrm{DO}^{+} \\
\mathrm{He}_{+}^{+}+\mathrm{SI}: \mathrm{He}^{+}+4 \\
\mathrm{CO}^{+}+\mathrm{SI}^{\mathrm{H}} \mathrm{He}_{+}^{+}+6\end{array}$ & $\mathbf{E}$ & $0.4-3 M \in V$ & 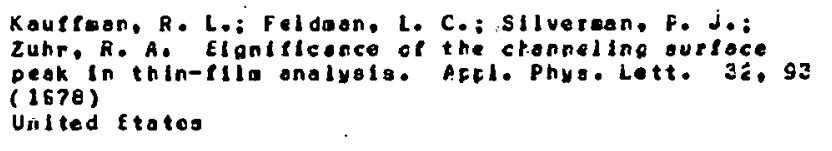 \\
\hline 517 & $\begin{array}{l}A 09 \\
K r+F- \\
A 11 \\
K r F *+F=: K r F *+K r \\
E 16 \\
e+[K r+F,]\end{array}$ & $\mathbf{E}$ & $\begin{array}{l}\text { AOS } \\
300 \mathrm{~K} \\
\text { A11 } \\
300 \mathrm{~K} \\
\text { E16 } \\
1 \leq 0 \mathrm{keV}\end{array}$ & 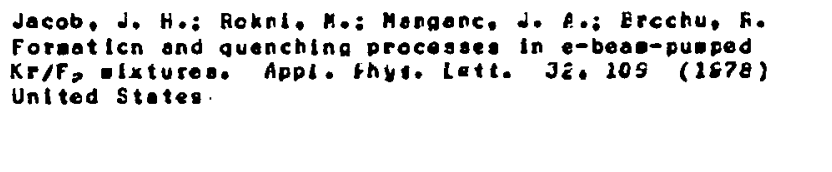 \\
\hline 518 & $\mathrm{DPC}_{\mathrm{B}}+\mathrm{SI}$ & $\mathbf{E}$ & $35 \mathrm{keV}$ & 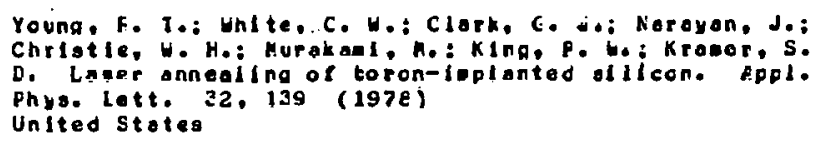 \\
\hline $51 \mathrm{~s}$ & $\begin{array}{l}\mathrm{D} 16 \\
\mathrm{BF}++\end{array}$ & $\mathbf{E}$ & $150 \mathrm{kgV}$ & 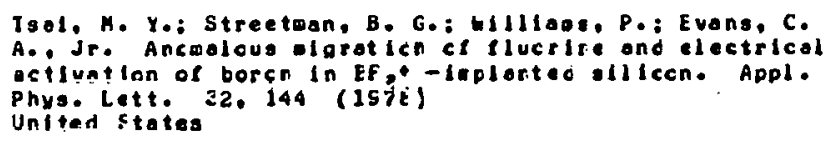 \\
\hline 520 & $\begin{array}{l}\mathrm{Dl6}^{2} \\
\mathrm{Te}^{+}+\mathrm{GaA} \mathrm{s}\end{array}$ & E & $50 \mathrm{keV}$ & 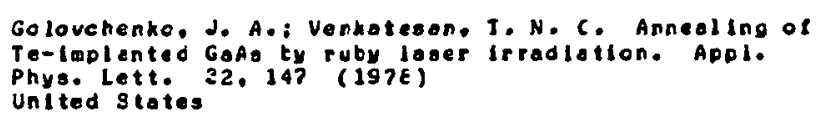 \\
\hline 521 & $\begin{array}{l}\text { HOG } \\
\text { ho }+\mathrm{U}\end{array}$ & $\mathbf{E}$ & $3.5 \mathrm{eV}$ & 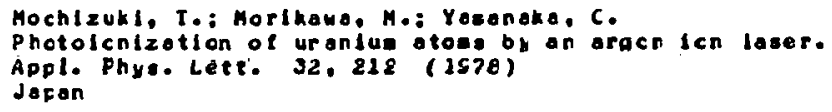 \\
\hline
\end{tabular}




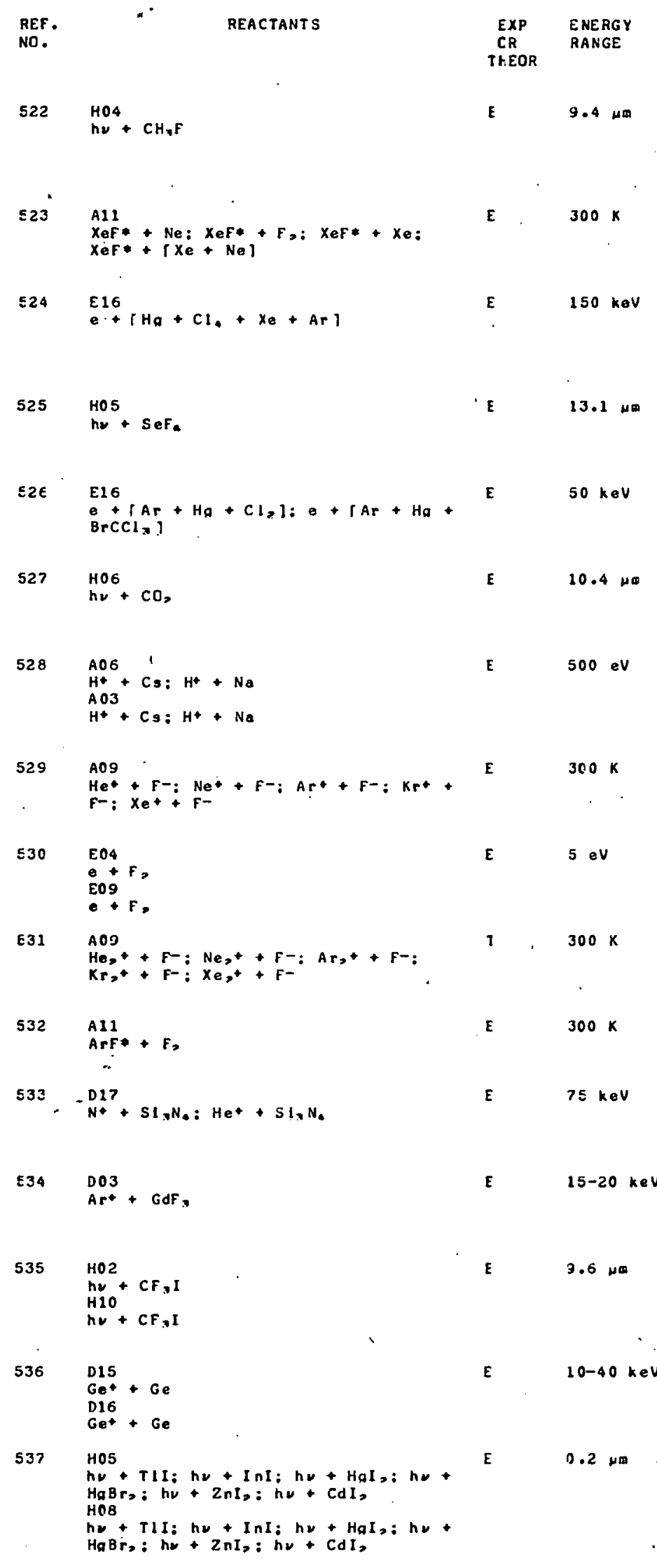

Prosn 1 tz, D.: Jacota, F. F.: B1schel, W. K.: Fhodes, C. excltotion of nethyl fluorice. Affl. Fhys. Lett. 32, 261 ( $1 \leq 78)$ United States

Roknl, H.: Jacob, J. H.: Menganc, J. A.: Ercehu, R. Formaticn ond quenching of $x_{e} F^{*}$ in $N e / x_{e} / F$, oletures. Appl. Phys. Lett, 32, 223 (1S7E)

United Stotes

Tang, K. Y.: Hunter, R. O., Jr.: Oldenettel, J.: Howton, C.: Huestis, D.; Eckstros, D.: Ferry, E. McCusker. M. Electron-teos-ccnticlleo discharpe HaCl * looer. Apdl. Phys. Lett. 32, 226 (1578) United stotes

itlee, J. J.: blttig, C. Isotoplcally selectlue Ir phctodisscciaticn ci Sefa. Accl. Fhys. Lett. 32,236 (1578)

United States

Whltney. W. T. Sustalned olscharge excltotion of $\mathrm{HgCl}$ and $\mathrm{HgB}_{\mathrm{g}} \mathrm{B}^{2} \mathrm{Y}^{+}, \mathrm{Ch}^{2} \mathrm{y}^{+}$, I lasers. Apfl. Phys. Lett. 32, 239 ' (is7a)

United states

Chin, S. L.: Faubert, D. Energy decendence of collisicnless aultiphoten icnlzeticn ci D,C molecules by TEA-CC, loser. Aorl. Fhys. Lett. 32, $30 z$ (1S7E) conodo

Hovelsen. D. C.: Mohr, H.: Cassidy, J. C.: Tang, C. L. Copeland. D. A.: Horteon. P. L. Lyoura raciotion trom

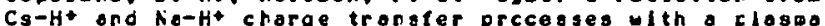
gur. AfDl. Phyo. Lett. $3 a, 30 E$. (LSTE) United Stotes

Flannery. H. F.: Yong. 1. P. Ienle reccobination ol rare-gos otcolc long $x+$ wlit $F$ - in a denge-gas $x$. Appl. Fhys. Lett. $32,327(1 \leq 7 e)$ United states

Nycoard, K. J.: Hurter, S. F.: Fletcher. J.: Fcltyn, S. $R$. Electron ot tachment in divte flucrine-hellue mixtures. ADFl. Fhys. Lett. 32. IEl (1S7E) United Stateg

Flannery, $M$. F.: Yong. $T$. P. lenic receobirotion of rare-gos aolecular long $x_{3}$. With $F-$ in a dense gas $x$. Apfl. Fhys. Lett. 32, 356 (1578) : United Stotes

Chen, C. Hig: Payne, M. G. Ar,F* rodlative llletlle meosuresent. Acol. Phya. Lett. 3á. 3Ee (1S78) United states

stein, H. J. Ien-tomtordment-induced transfer of $H$

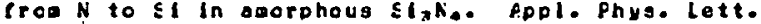
32,379 (157A)

United States

Tsong. I. S. T.: Bhalla. A. S. Hyorogen ond tluctine oroflies in GdF, Pllas ceosured by ofutter-inouceo optical ealssion. ADOl. Fhys. Lett. Zá. $3 E l$ (lSTE) United stotes

Pumer, tio: Egaleston, J.: Rlachel, W. K.: Fhodes, C. $K$. Uitraviclet absorption Cf CFa I induces ty excitotion of the vitrational made ot G.E ul. Appl. Phys. Lett. $=2,427$ (ISTE)

United States

Groczyk. J. F.: Choudherl. F. Elfects cl lon iaclantetion on the structure of acorchous gerantum. ApEl. Fhys. Lett. 32,466 (2S7E)

United Stotes

Maya, J. Flucrescence ylelds of cetal hallide vapors exclted by photoolssociaticn. Appl. Phys. Lett. 32. 484 (1578) Unlted Stotes 


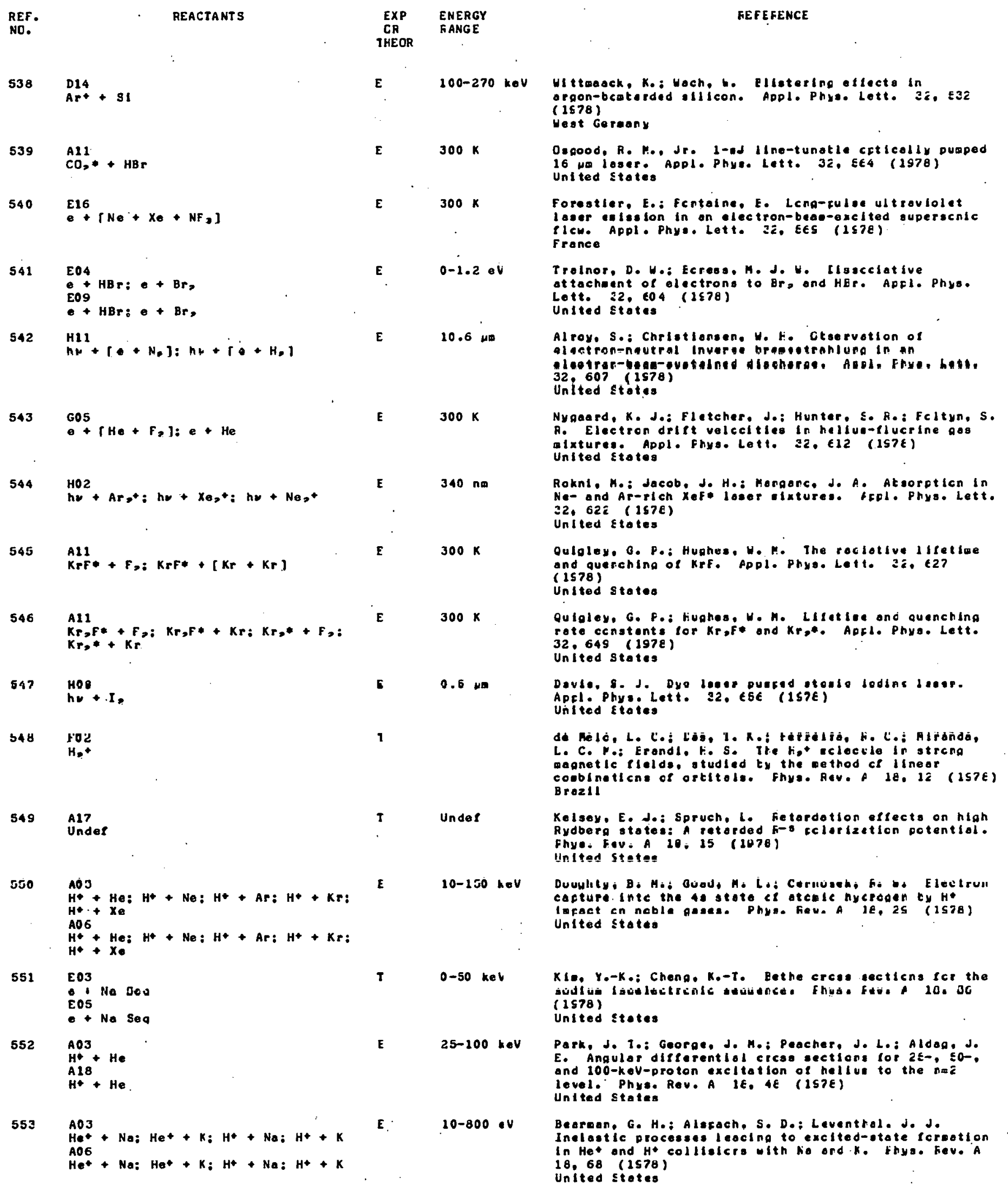


REF.

REACTANTS

$$
\begin{aligned}
& \text { REF . } \\
& \text { NO. }
\end{aligned}
$$

$\mathrm{He}^{2+}+\mathrm{He}: \mathrm{He}^{2+}+\mathrm{Ne} ; \mathrm{He}^{2+}+\mathrm{H}_{2}$ $\mathrm{He}^{2+}+\mathrm{C}$,

A 06

$\mathrm{He}^{2+}+\mathrm{He}: \mathrm{He}^{2+}+\mathrm{Ne} \mathrm{He}^{2+}+\mathrm{H}_{2}$ : $\mathrm{He}^{2+}+\mathrm{C}$,

AO 1

Under

A 04

$\mathrm{H}_{3}^{+}+\mathrm{Ar}: \mathrm{H}_{3}++\mathrm{H}_{2}: \mathrm{H}_{3}++\mathrm{Alr}$

A06 6

A O 7

$\mathrm{H}_{3}^{+}+\mathrm{Ar}: \mathrm{H}_{3}+\mathrm{H}+\mathrm{H}_{3}+\underset{\mathrm{Al}}{\mathrm{r}}$

560

HO5

$\mathrm{hv}+\mathrm{H}$,

Ho6

hV $+\mathrm{H}$,

$561 \quad \mathrm{BO2}$

Under

Und

Undef

\section{B07}

Undef

Fo1

$\mathrm{Fe}^{23+}: \mathrm{Fe}^{22+}$
$\mathrm{H}_{3}^{+}+\mathrm{Ar}: \mathrm{H}_{3}++\mathrm{H}_{2}: \mathrm{H}_{3}++\mathrm{Alr}$

$\begin{array}{ll}\text { EXP } & \text { ENERGY } \\ \text { CF } & \text { FANGE } \\ \text { IHEOR } & \end{array}$

ร

E

27-2000 eV

E

$4-600$ eV

$\boldsymbol{T}$

Under

Under

$400-800 \mathrm{keV}$

HOS

Under

Fo 1

He 11

He* + He

FO 1

H,

\section{E A11}

$300 \mathrm{~K}$
$15-200 \mathrm{keV}$
Rahman. N. K.: Gionturco. F. A.: Lewanra, U. I. Gl ouber aplitudes for rototional excltation in e-mclecule scattering vio quadrupole potentiala. Fhys. Rev. A 18,74 (1978) It aly

Feeney, R. K.: Sayle, L. E.. II: Divire. T. F. Absolute experiaental cross sections icr the electrcn-impact ionizotion of Rot Ions. Phys. Rev. A $18.82(1978)$

United Stateg

Myers, C. C.: Autrceo, J. G.: Joses, P. B.: Leventhal. J. J. Spln conarvation In electren-corture collidicnse Phys. Rev. A 18. es (1578) United stotes

Thorsen. H. R.: Delos, J. B. Theory ct near-aolatatic collisicns. I. Electrcn transloticn foctor cethod. Phys. Rev. A 1E, 117 (1S7E)

Canada

Thorson, H. R.: Delcs, J.E. Theory cl neor-ociatatic collisicns. II. Scattering coordinote wethod. Phys. Reve A 18. 135 (1978) Canado

NIr. D.: Fcsner. E.: Monn. A.: Kontcr, J. Chorge-state distribution of products frop diseclative cclilialons of $400-800-k e V H_{3}$ ions: Exferieent onc sealeafirical aodel. Fhys. Rev. A 18, 156 (157e) Is rael

Dehaer, J. L.: Dill. D. Fhetclen engulor distributions In disecclative phetolonlzation of h, at $304 \mathrm{AO}$. Phys. Rev. A 18,164 (1S7E)

United stotes

Lau, A. M. F. Laser-induced releculor frediascietion Wthout absoption or exission cl lager photona. Phys. Rev. A 18,172 (1578)

United states

Havroyonnla, C. Two-atco rescnonce tlucreacence. Phys. Rev. A 18, les (1STE)

Canade

KIE, Y. S.: Oh, S. D.: Fratt, R. H. Fnalytic

descriftion of inner-shell bound-bcund transitions. Phys. Fiv. A 1e, 194 (ISTE)

United states

Dletrlch, L, L.: Leavitt, J. A.: Eashir. So: Conwoy, J. G.: Could, H.: HacDcnald, I.: Morrute R.: Jchnson. B. M.: Fego. D. J. Oocliliotor strengtis of the

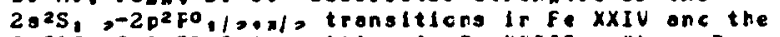
$2 a^{2} S_{0}-29203 P: 0$ transition in fe XXIII. Fhys. Rev. A 18,208 (1578)

United stotes

Kramer, P. B.: PIpkin, F. M. Net deasurement of the fine siructure in the 3 ip otote of He. Fhyo. Fev. A 18,212 (1S7E) United states

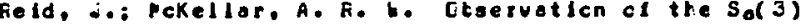
pure rotaticnal quadrupole ironsition ct $H_{2}$ with tunable diode loger. Fhy. Fev. A $1 \varepsilon_{1} 224$ (1578) canada

Shokestaft. R. Pngular distrituticn far electron cacture by protons from hydrogen atoes in the energy range 15-200 heV. Fhys. Fev. A 1E. 3G7 (IS2f) Unted siotes

Kelsey. E. J.: Sucher. J. Erratue 2 J, $11 s_{n}+$ one phcton in hellublike lons: Exact result for the lowest-crder effoct of the electrcr-elictrcn Interaction [fhyas. Rev. A 11, 1E29 (1575)]. Phys. Rev. A 18 , zos (1STE)

United Stotes 


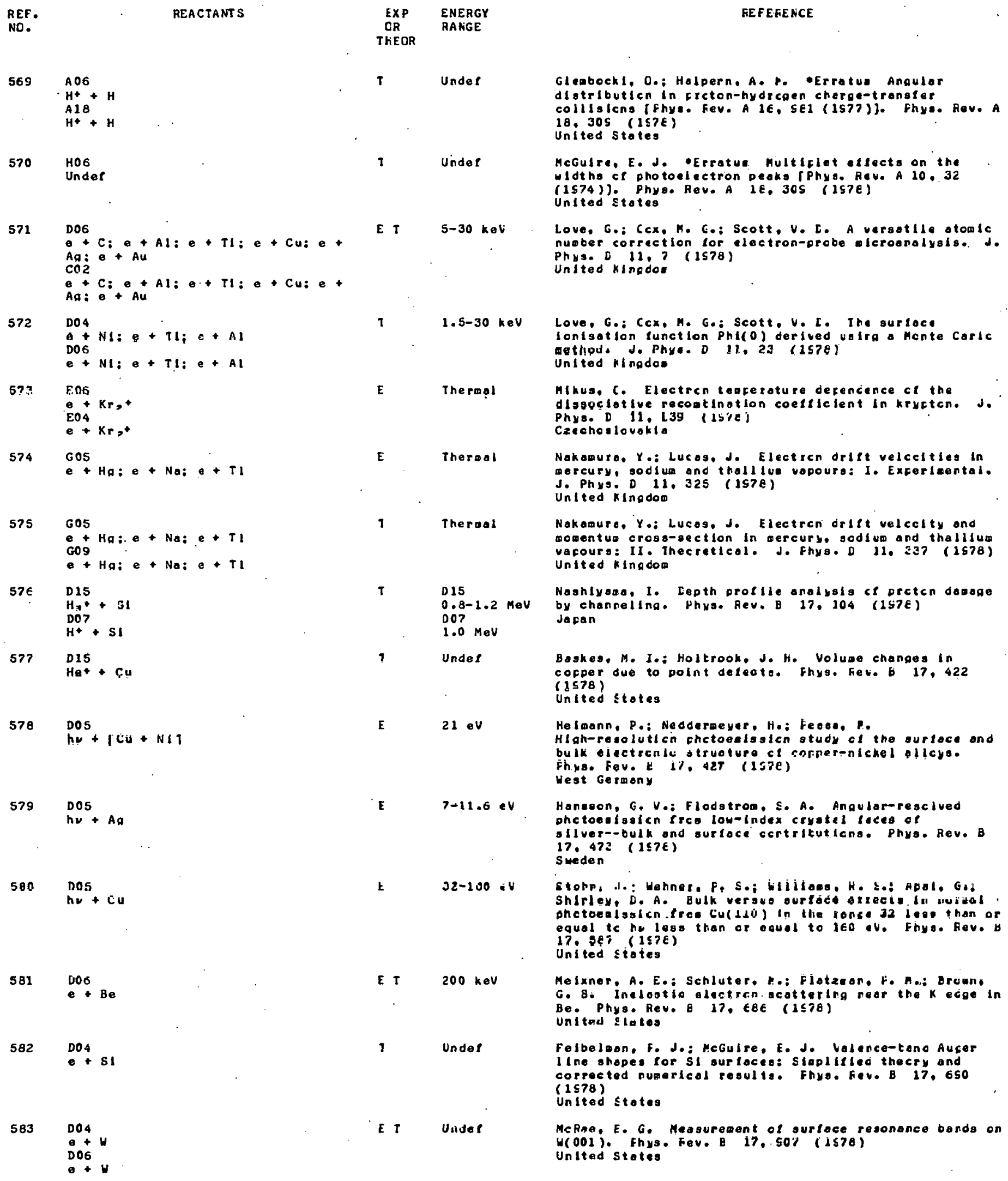




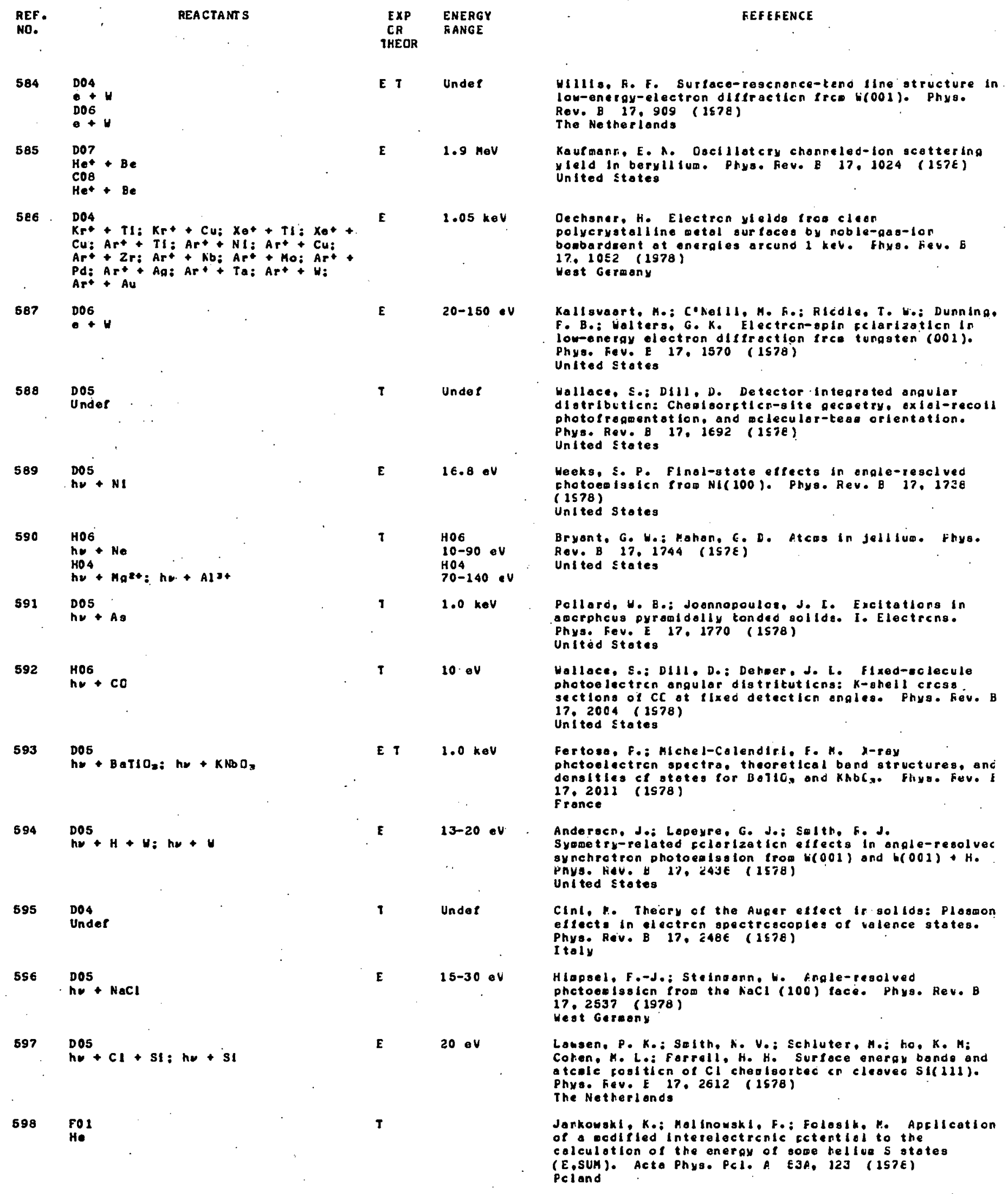




\begin{tabular}{|c|c|c|c|c|}
\hline $\begin{array}{l}\text { REF. } \\
\text { NO. }\end{array}$ & REACTANTS & $\begin{array}{c}\text { EXP } \\
\text { CR } \\
\text { THEOR }\end{array}$ & $\begin{array}{l}\text { ENERGY } \\
\text { GANGE }\end{array}$ & FEEEGENCE \\
\hline 599 & $\begin{array}{l}\mathrm{FOI} \\
\mathrm{H},\end{array}$ & 1 & . & 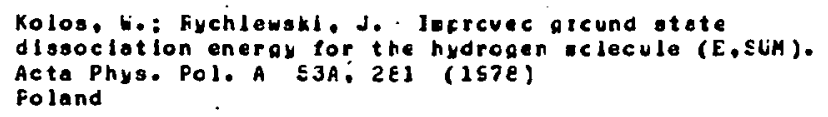 \\
\hline 602 & $\begin{array}{l}\text { Fol } \\
\text { SI+ }\end{array}$ & $\mathbf{T}$ & & 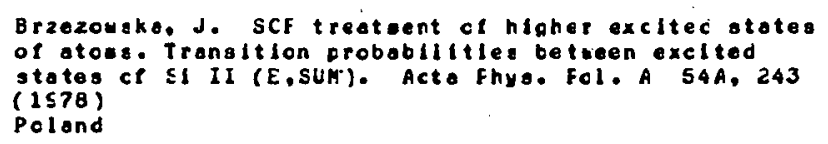 \\
\hline 603 & $\begin{array}{l}E 03 \\
e+H\end{array}$ & $\mathrm{~T}$ & $2-300 E / \Delta E$ & 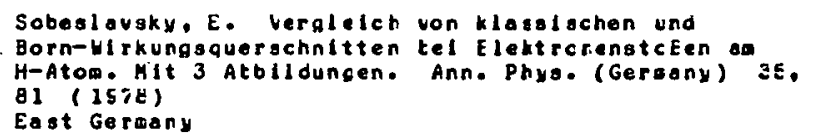 \\
\hline 604 & $\begin{array}{l}\text { Ho2 } \\
\text { Review }\end{array}$ & E & $9.2-10 . \varepsilon$ & 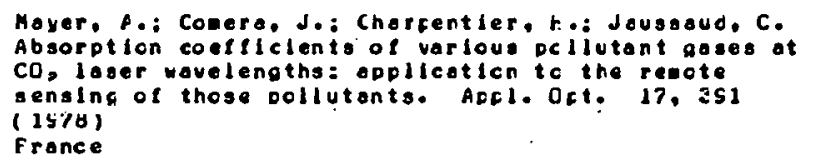 \\
\hline 605 & $\mathrm{HOS}_{\mathrm{H}}^{\mathrm{H}}+\mathrm{NU}_{2}$ & $\boldsymbol{E}$ & $\begin{array}{l}6600-4200 \\
4^{0}\end{array}$ & 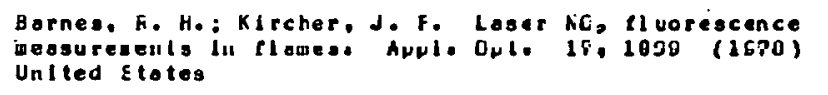 \\
\hline 606 & $\begin{array}{l}\mathrm{HO} 2 \\
\mathrm{h \nu}+\mathrm{NO}\end{array}$ & E & $22 \epsilon 2 A^{\circ}$ & 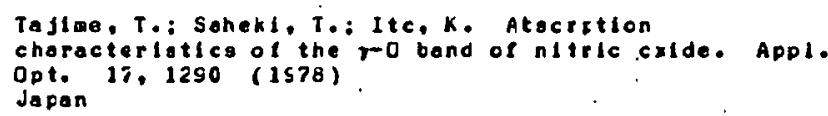 \\
\hline 607 & $\begin{array}{l}\mathrm{HO2} \\
\mathrm{hW}+\mathrm{H}>\mathrm{O}\end{array}$ & E & $1200 \mathrm{~cm}^{-1}$ & 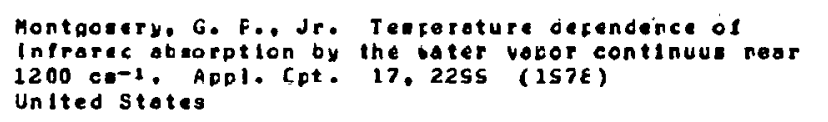 \\
\hline 608 & $\mathrm{HOZ}_{\mathrm{H}}^{\mathrm{HO}}+\mathrm{H}>\mathrm{C}$ & $\mathbf{E}$ & $4.0-3.5 \mu \mathrm{m}$ & 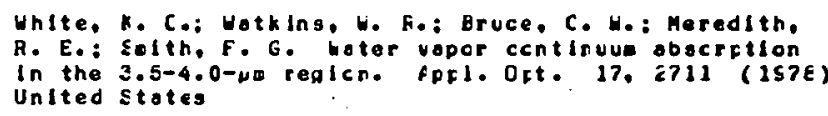 \\
\hline $60 \mathrm{~s}$ & $\begin{array}{l}\mathrm{HO}^{2} \\
\mathrm{~h}\end{array}$ & E & $\int_{\text {Nit }}^{2.10-6.5 s}$ & 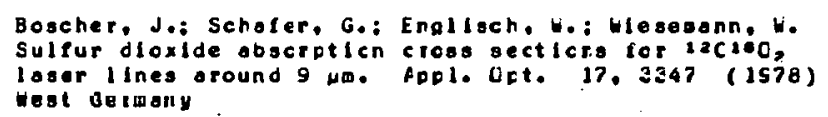 \\
\hline 610 & $\begin{array}{l}013 \\
e+(0,+W] \\
017+\left[0_{2}+W 1\right.\end{array}$ & $\mathbf{E}$ & $100 \mathrm{ev}$ & 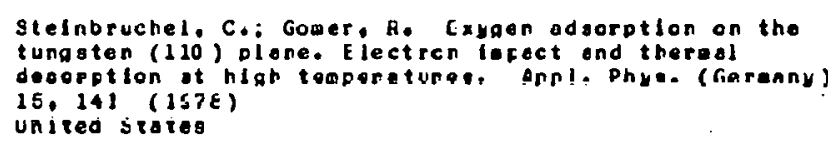 \\
\hline 611 & $\begin{array}{l}\mathrm{D} 13 \\
\mathrm{e} \\
\mathrm{CCO}+\mathrm{Ru})\end{array}$ & $\cdot E$ & $100 \mathrm{eV}$ & 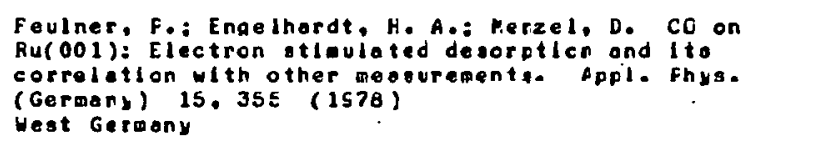 \\
\hline 612 & $\mathrm{NO}_{\mathrm{Ni}}+\mathrm{NI}$ & E & $\begin{array}{l}0.075-3.0 \\
k \in v\end{array}$ & 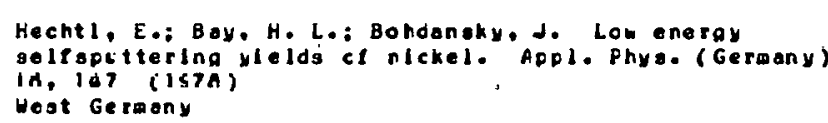 \\
\hline 613 & 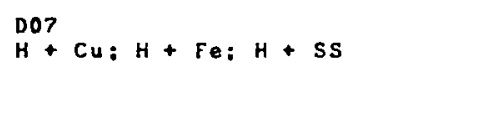 & $T$ & $0.1-100 \mathrm{kov}$ & 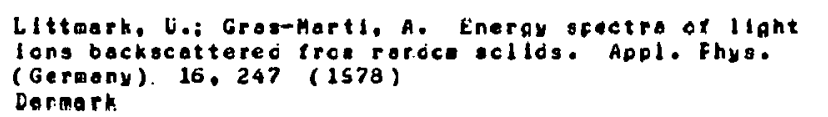 \\
\hline 614 & $\begin{array}{l}D O_{2} \\
A r^{+}+\left[O_{2}+[i]: A r^{+}+\left[O_{2}+V\right]:\right. \\
N_{2}+\left[O_{2}+T i\right]: N_{2}^{+}+\left[O_{2}+V\right\}\end{array}$ & E & $11 \mathrm{keV}$ & 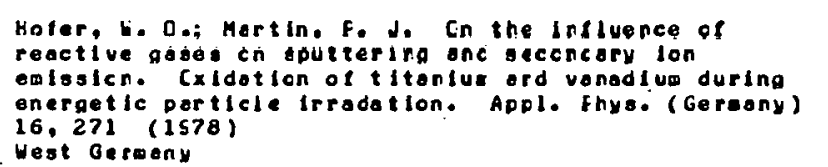 \\
\hline 615 & $\begin{array}{l}\mathrm{D} 14 \\
\mathrm{He}\end{array}+\mathrm{Rh}$ & E & $21 \mathrm{keV}$ & 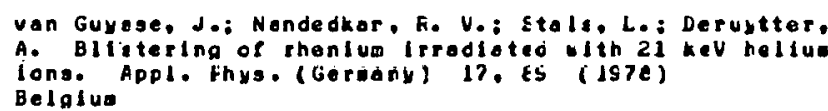 \\
\hline 616 & $\begin{array}{l}\mathrm{DOT} \\
\mathrm{H}+\mathrm{NI}\end{array}$ & $\tau$ & $0.1-5 \mathrm{keV}$ & 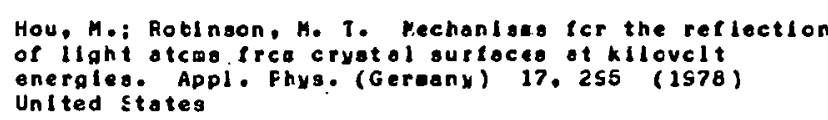 \\
\hline
\end{tabular}




\begin{tabular}{|c|c|c|c|c|c|}
\hline $\begin{array}{l}\text { REF. } \\
\text { NO. }\end{array}$ & REACTANTS & & $\begin{array}{l}\text { EXP } \\
\text { CR } \\
\text { THEOR }\end{array}$ & $\begin{array}{l}\text { ENERGY } \\
\text { RANGE }\end{array}$ & FEFE KENCE \\
\hline 617 & 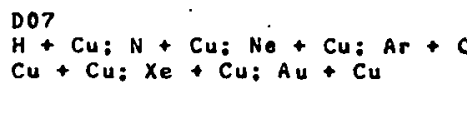 & Cu; & 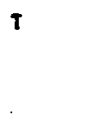 & $0.1-7.5 \mathrm{keV}$ & 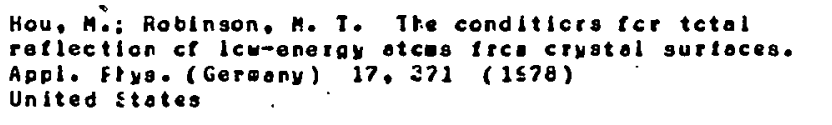 \\
\hline 618 & $\mathrm{HOS}_{\mathrm{H}}^{\mathrm{H}}+\mathrm{AsH}$ & & $\mathbf{E}$ & $2900 A^{\circ}$ & 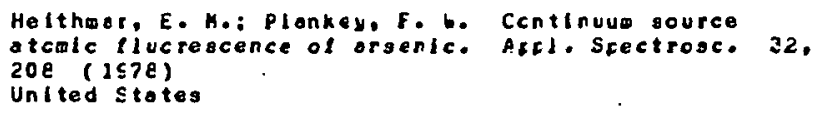 \\
\hline 619 & $\begin{array}{l}\mathrm{HO2}_{\mathrm{h}}+\mathrm{SF}_{a}\end{array}$ & & $\mathbf{E}$. & $\begin{array}{l}540-5 \leqslant 2 \\
c a-1\end{array}$ & 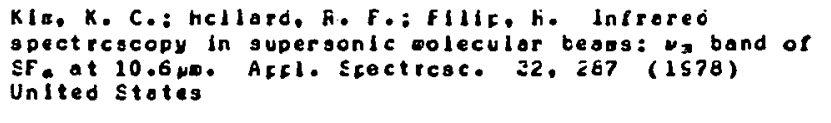 \\
\hline 620 & $\begin{array}{l}\mathrm{HO2} \\
\mathrm{h \nu}+\mathrm{Na} \\
\mathrm{HOB} \\
\mathrm{h \nu}+\mathrm{No}\end{array}$ & . & E & $5890 \mathrm{~A}^{0}$ & 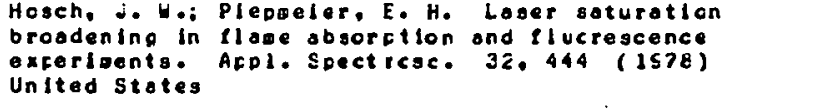 \\
\hline 621 & $\begin{array}{l}\mathrm{HO}_{2} \\
\mathrm{CH}_{4}: \mathrm{CH}_{2}+\mathrm{C}_{2} \mathrm{H}_{2}\end{array}$ & & E & $2948 \mathrm{~cm}^{-1}$ & 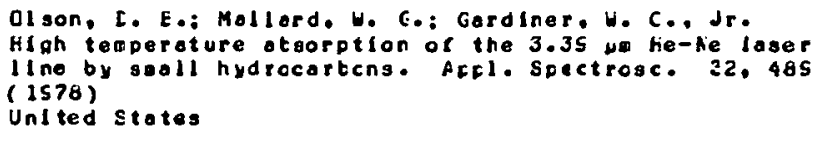 \\
\hline 622 & 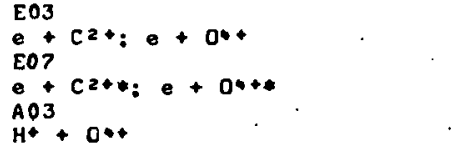 & & I & $105 \mathrm{~K}$ & 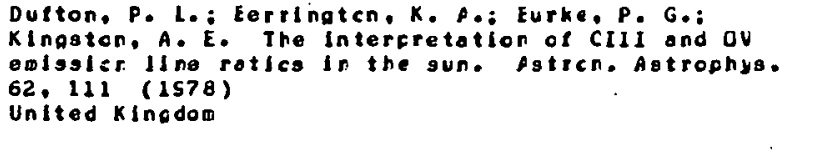 \\
\hline 623 & $\begin{array}{l}\text { Fo1 } \\
\text { sit }\end{array}$ & . & $\mathbf{E}$ & & 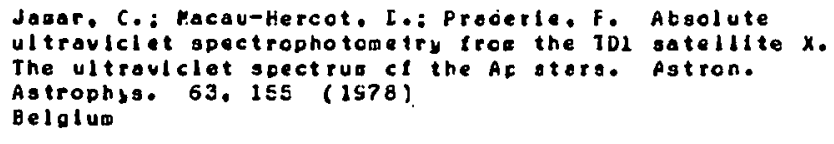 \\
\hline 624 & $\begin{array}{l}\mathrm{HO}_{\mathrm{h}} \mathrm{O} \\
\mathrm{h}\end{array}$ & & E & $2200 \mathrm{~A}^{\circ}$ & $\begin{array}{l}\text { RudkJobing, M. Intergtellar heatling by photcelectrons } \\
\text { (rom negative oxygen. Asticn. Astrophys. EZ, } 185 \\
\text { (1578) } \\
\text { Dermark }\end{array}$ \\
\hline 625 & $\begin{array}{l}\text { H11 } \\
\text { hw }+e \\
\text { h12 } \\
\text { hv }+e\end{array}$ & & I & Unde $P$ & 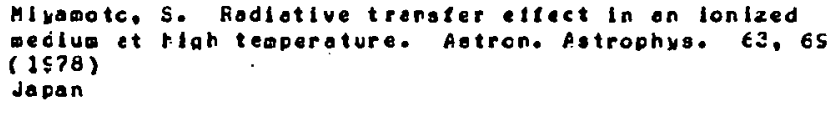 \\
\hline $62 \epsilon$ & $\begin{array}{l}\mathrm{CO}^{\mathrm{O}}+\mathrm{H}: \mathrm{Cl}^{3+}+\mathrm{H}: \mathrm{N+}+\mathrm{H}: \mathrm{N}^{2+}+ \\
\mathrm{O}^{2+}+\mathrm{H}: \mathrm{Ne}^{2+}+\mathrm{H}^{+} \mathrm{S}^{+}+\mathrm{H}^{+} \mathrm{S}^{3+}\end{array}$ & ${ }^{H} ; \mathrm{H}$ & $\tau$ & $10 \cdot k$ & 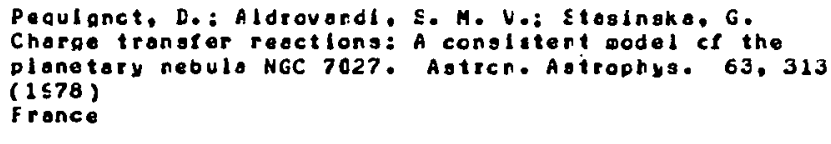 \\
\hline 627 & $\begin{array}{l}\text { FO1 } \\
\text { Ti }\end{array}$ & & E & & 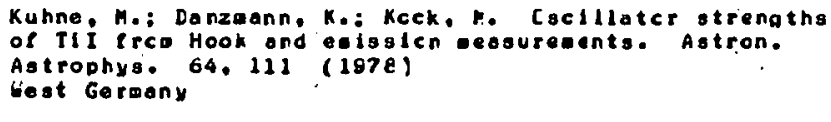 \\
\hline 628 & $\begin{array}{l}F_{01} 01 \\
C^{2}+\end{array}$ & & $\mathbf{T}$ & . & 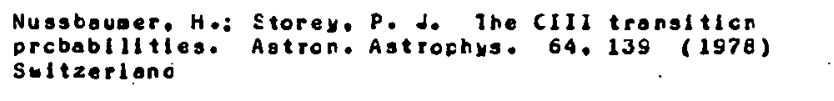 \\
\hline 625 & $\begin{array}{l}\text { FO1 } \\
\text { NI* }\end{array}$ & & $\boldsymbol{E}$ & & 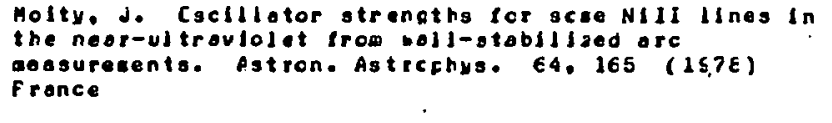 \\
\hline 630 & $\begin{array}{l}\mathrm{FOl} \\
\mathrm{TI}\end{array}$ & & E & & 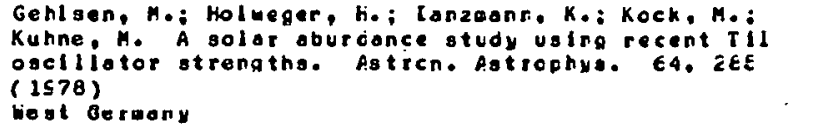 \\
\hline
\end{tabular}


REF.

REACTANT $S$

NO.

631

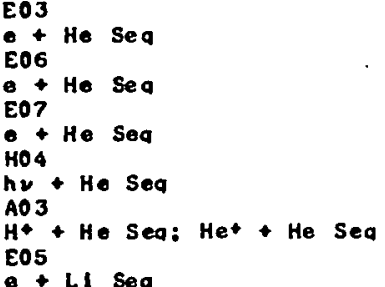

632

E03

- + He Sea

E06

t He Sea

E07

- + He Seq

EOS

+ Ll Sea

633

E03

$-02+2.04+$

63

BO 1

Under

Bo?

Under

Bo 1

Under

$\mathrm{EO}$
$\mathrm{C}+\mathrm{OH}$

AO3

$\mathrm{H}+\mathrm{OH}: \mathrm{H}+\mathrm{OH}$

637

503

$\div+4$

E05

$\because$ LI

HO6

he + LI

638

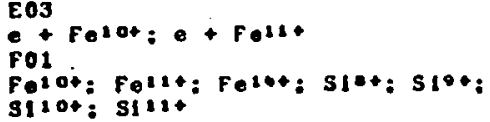

$\mathrm{EO} 2$
$\mathrm{H}+\mathrm{H} 1$

hy + .

640 A06

$\mathrm{C}+\mathrm{H}: \mathrm{C}+\mathrm{H}+$

641 HOS

he + $\mathrm{CO}$; he + $\mathrm{OH}$; hv + $\mathrm{CH}$; he

$\mathrm{H}, \mathrm{CO}: \mathrm{No}+\mathrm{NII}$

642

A06

$\mathrm{H}^{*}+\mathrm{D}$

043

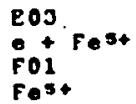

$\begin{array}{cl}\text { EXP } & \text { ENERGY } \\ \text { OR } & \text { RANGE } \\ \text { THEOR } & \\ \end{array}$

T. $10^{3}-10^{\circ} \mathrm{K}$

T

$10 \cdot K$

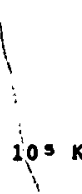

$\mathbf{T}$

1

EA3; Fos

2000-5000 K

HOE

$3500-1200$

A०

E $\mathbf{I}$

$E 03$
$100 K$

EO2

10 oV

H11

$2 E 20-8350 \mathrm{~K}$

$1 \quad 109 x$

1

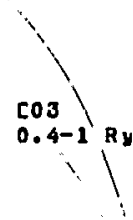

REFEFENCE

Mewe. Fo: Schrifuer, de Helluelike lon line

intengt tles. I. Staticnory closeos. Astrcn. Astrophys. 65. 99 (1978)
The Netrerlonos
Meve. Fo: Schriluer, do Heliuel the lan line infensliles. II. Nen-gtoticnary clocese. Agtren. Átrophys 65, $11 E$ (157E)

The Netreriands
Foldman, U.: Loechek, C. A. The electrcn denalty at iask In differant reatons at the oclar atocachere derived frce on Intersusten line ct 0 IV. Astrcn. Aotrophys. $E E, 216$ (157E) United stotes

Landi DegleInnocenti. E. han-LIE line formaticn in a agnetle field. The tuc-level otol uith o irequency Independent source lunction. I: Foreuloticn. Astrcn. Astrophys. 66, 115 (1S7E)

Italy

Stentlc. J.C. Rescnance-ilne cclofizotion. 111. The Hanle eifect in a compact non-LIE radiotive trangter foraulaticn. Astrch. Ast tcinya. E6, 241 (1578) Sueden

Gulbert, J.: Elitzur. M.: Nguyenm-Rieu OHexcitotion In Intersteil or clouds. Astron. Astrechys. 66, 395 (1578) Fronce

da la Feza. Ra: Querci. F. Lithlus lire sormotion in carbon atare. Astron. Astrcphys. E?, (1578) France
$5000-1000$

$A^{\circ}$

25-100 K

$\rightarrow-2$

Kastner. S. D.: Hocon. H. E. Lisb-brlahtening observaticns for the OEC-7 sotellite. III. Comcorison of EUV line intensities of Fe Xli. Fe XI. Fe Xi. EI $x$ and $S X I I$, SI $I X$ and $S X I$ ith predictions. Astrcn. Astrophyo. 67, 119 (157E)

Un lied stotes

John, T. L. Neutral Rremestrahlung teca oclecular hyorogen ond nitrogen. Aetron. Aatrochyos 67. 355 (1578)

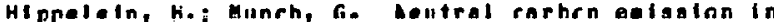
M42. Aetrcn. Aetrcphys. CE, IT (2STE) Heot Geraeny

Sandell, te. Lithtiad ot ableculed in a dark cioua model. Astron. Astrophys. 6S,ES (1578) rinland

Watson. Y. L.: Christensen. R. E.: Lelasler, F. J.

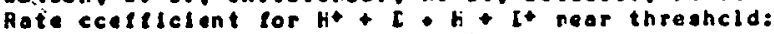
sianificance for interatellar clouda. Aatrcn. Aatrophys. 69, 1ES (1578)

United stetes

Nuesbouner, Hes Btoray, P. d. [Fo VI] ealedion under nobular cenditicne. Abtrcin. Astrcthys. 70, 32 (1978) sultariand 


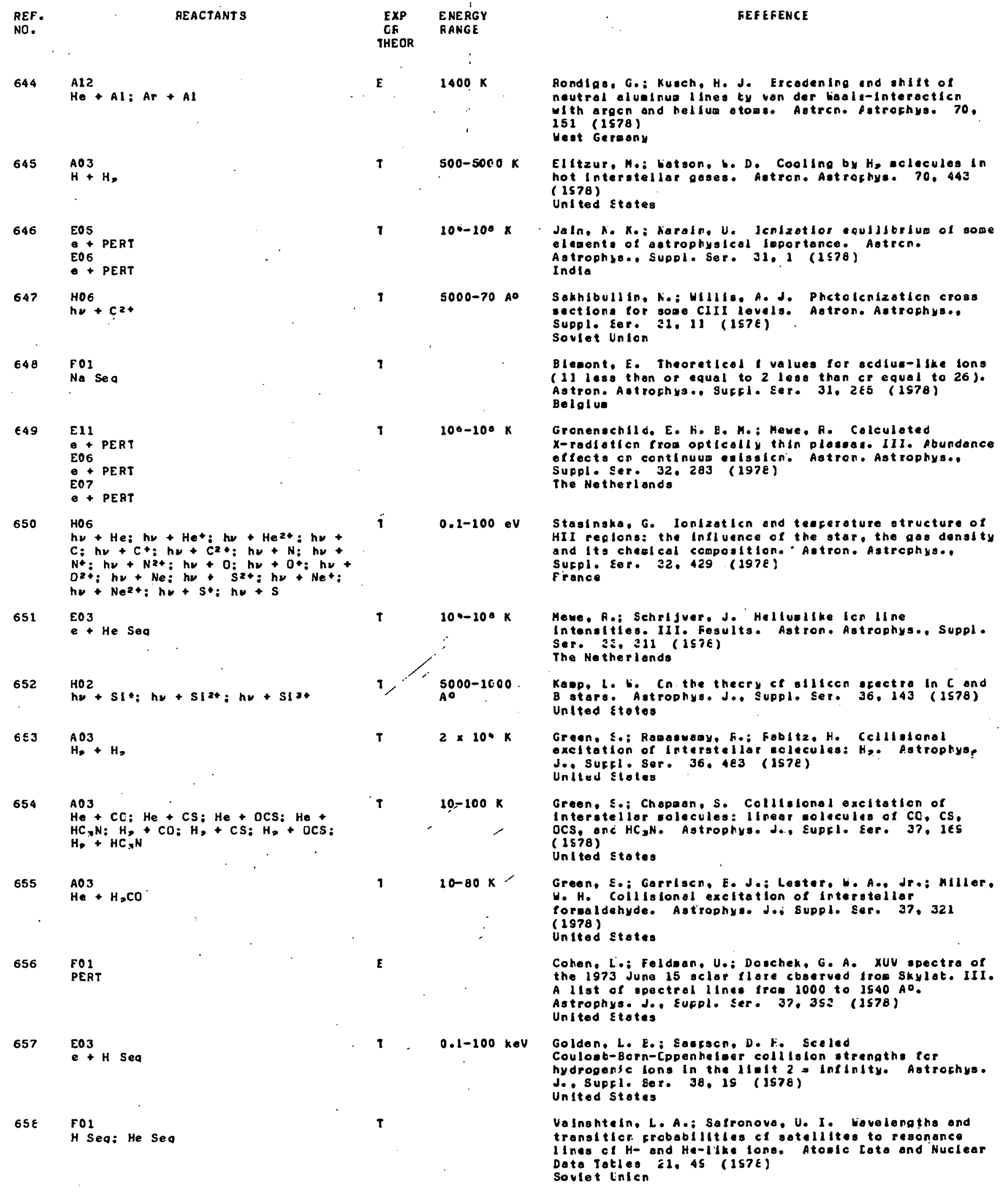




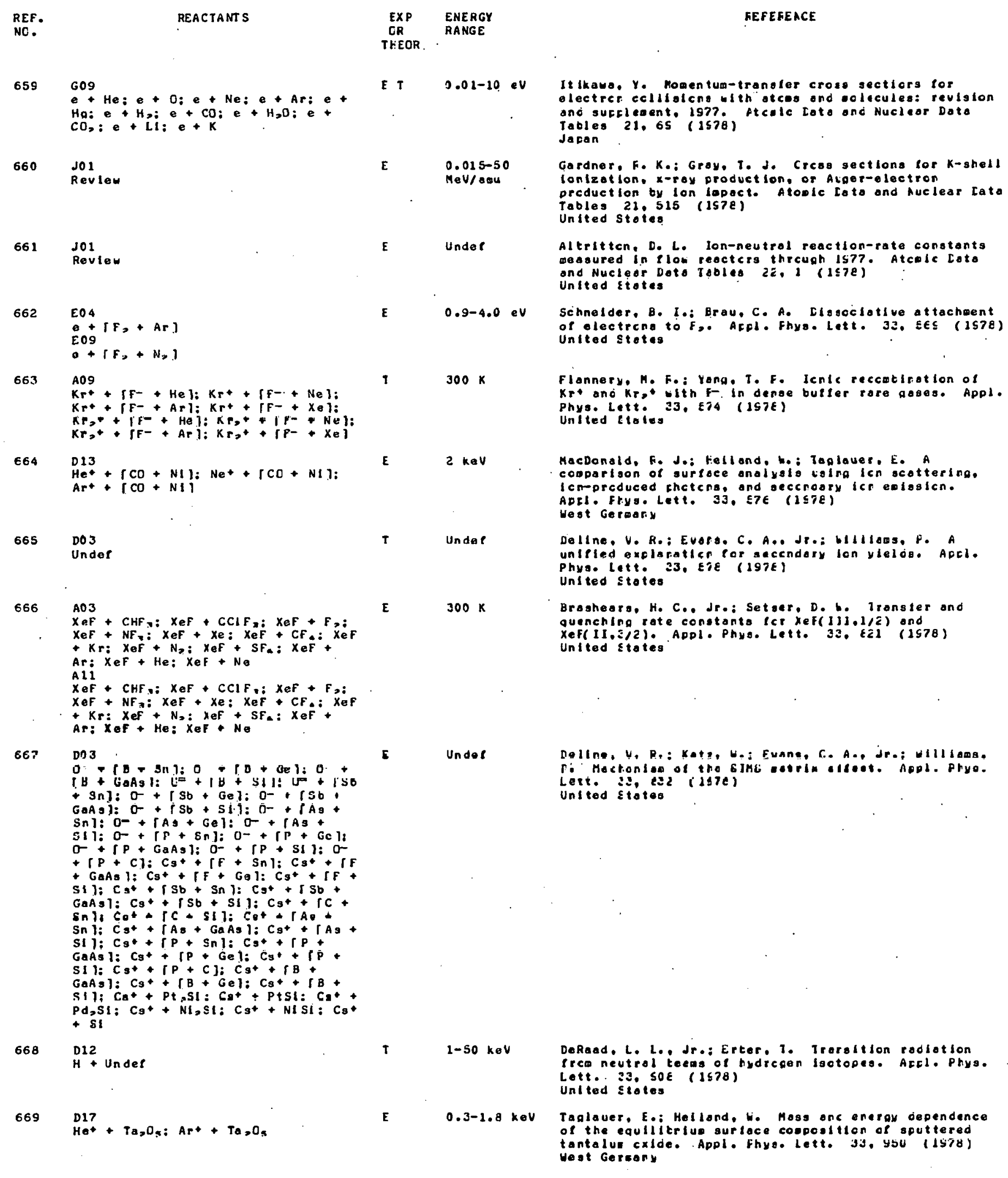




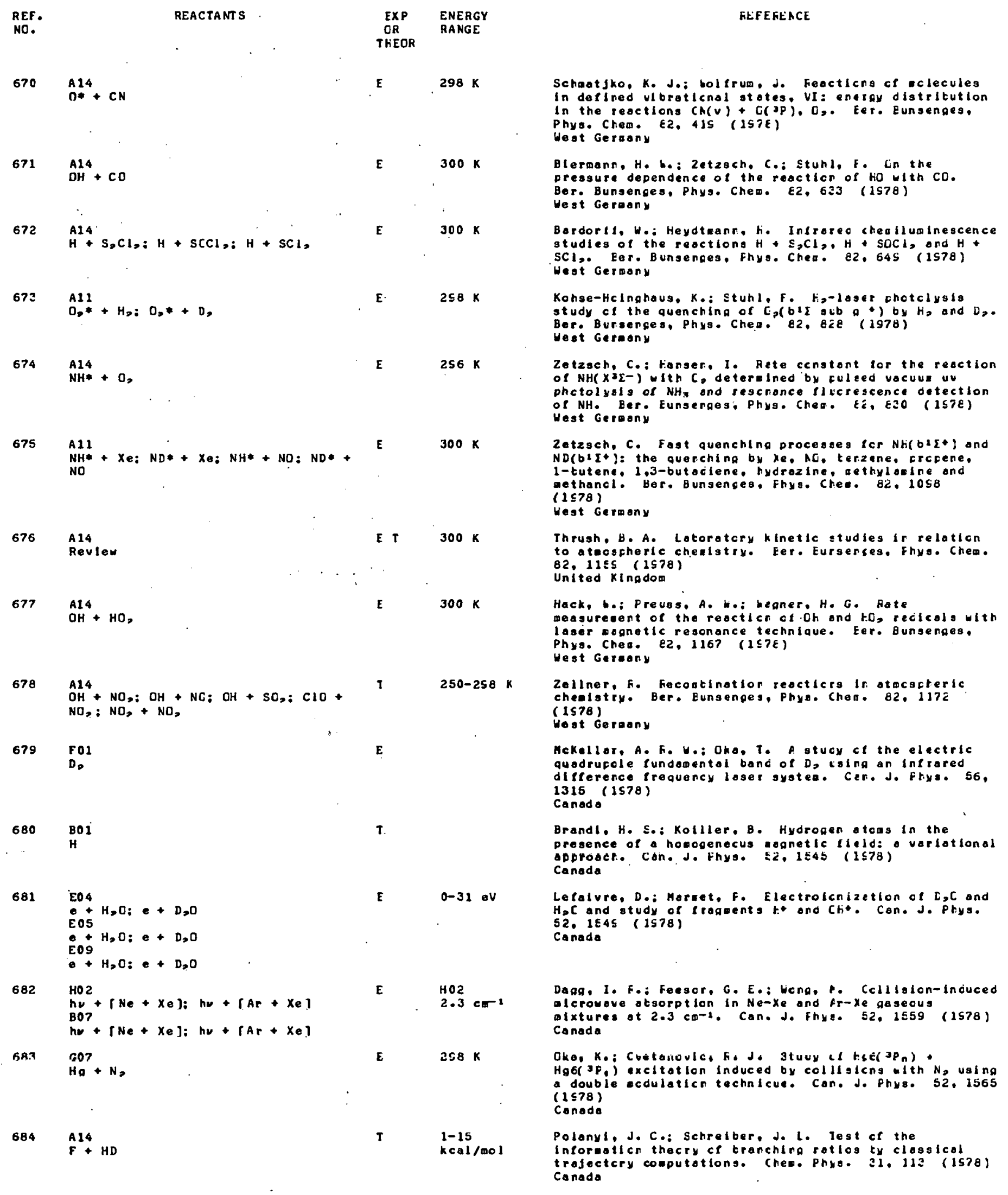




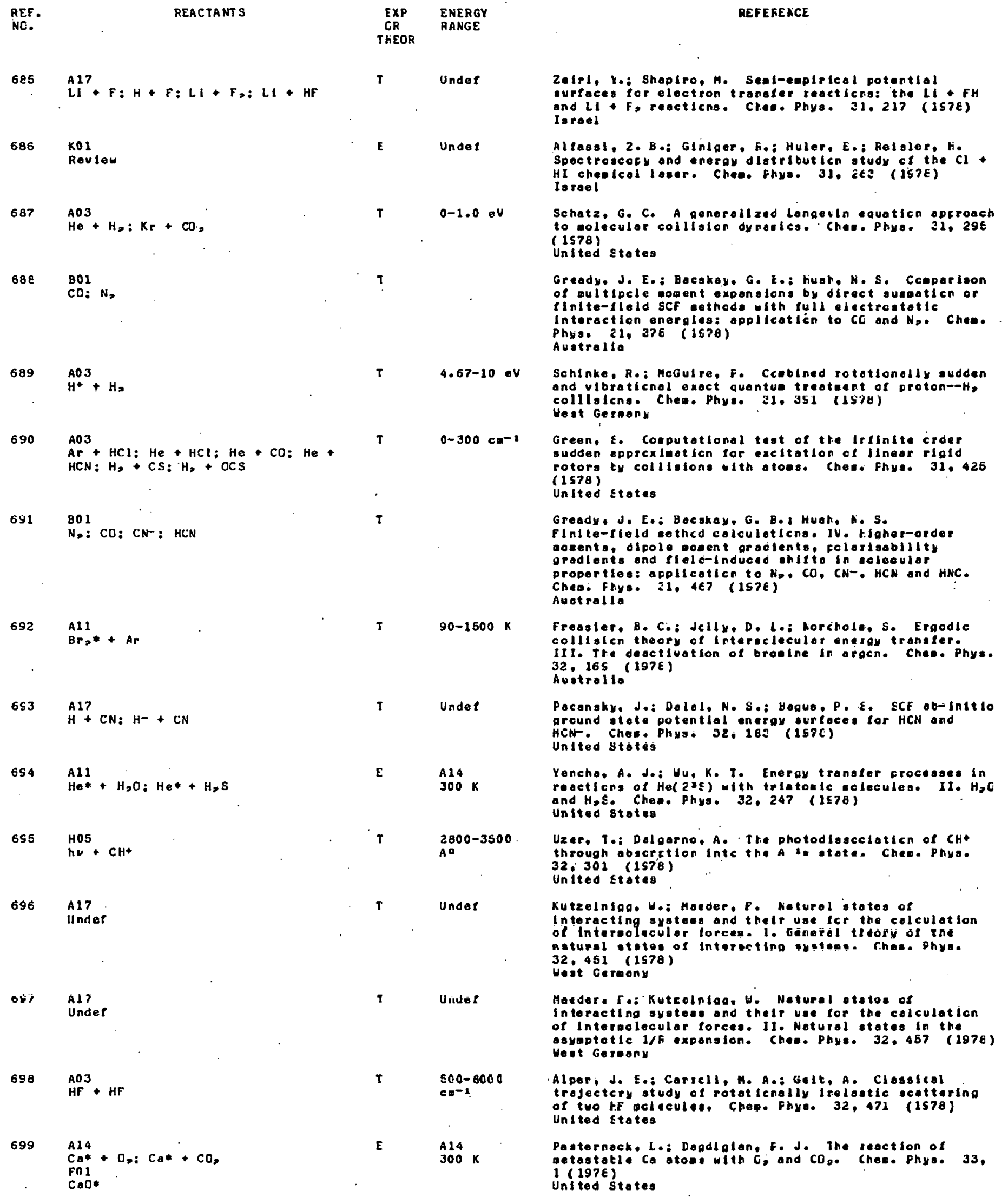




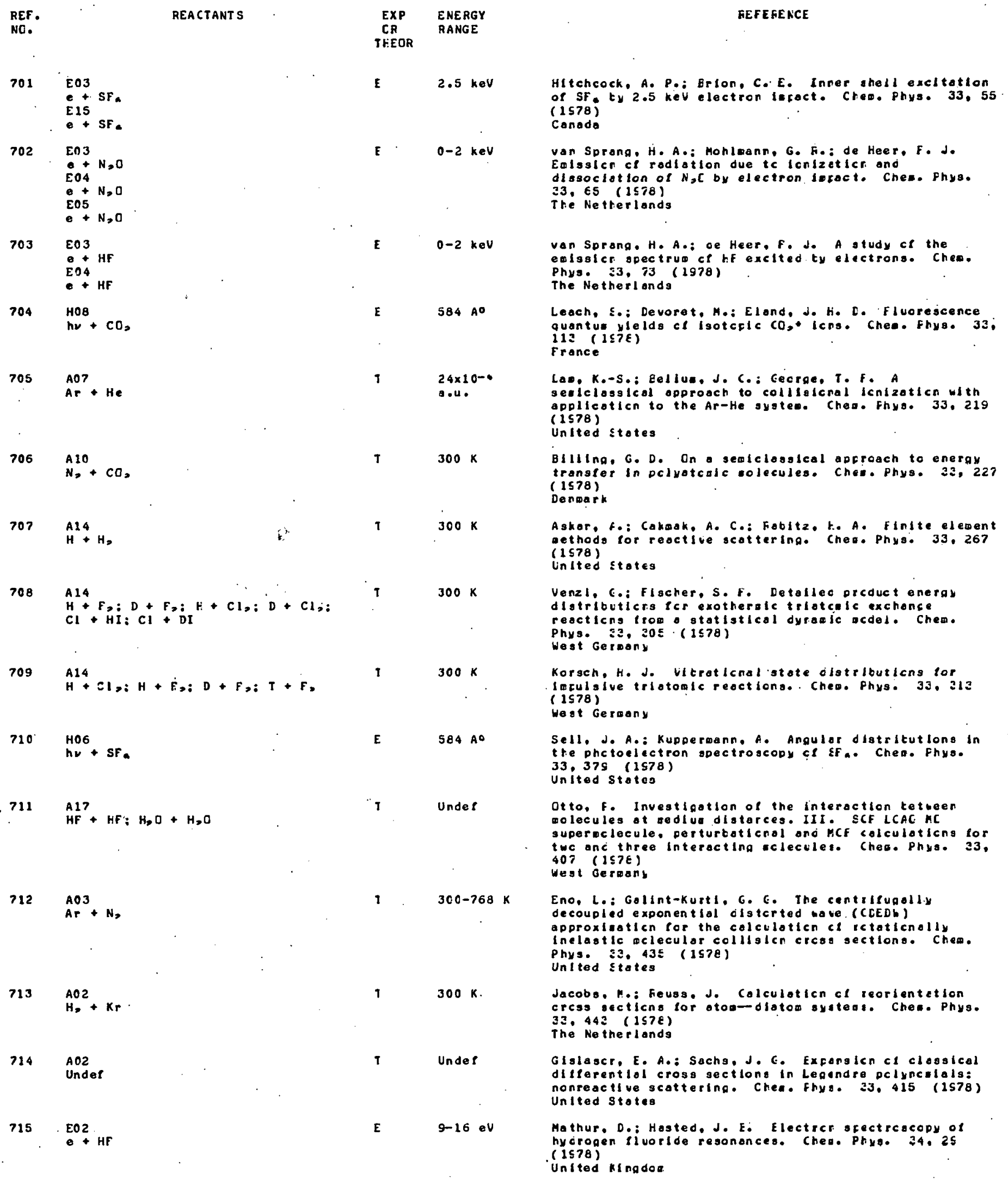




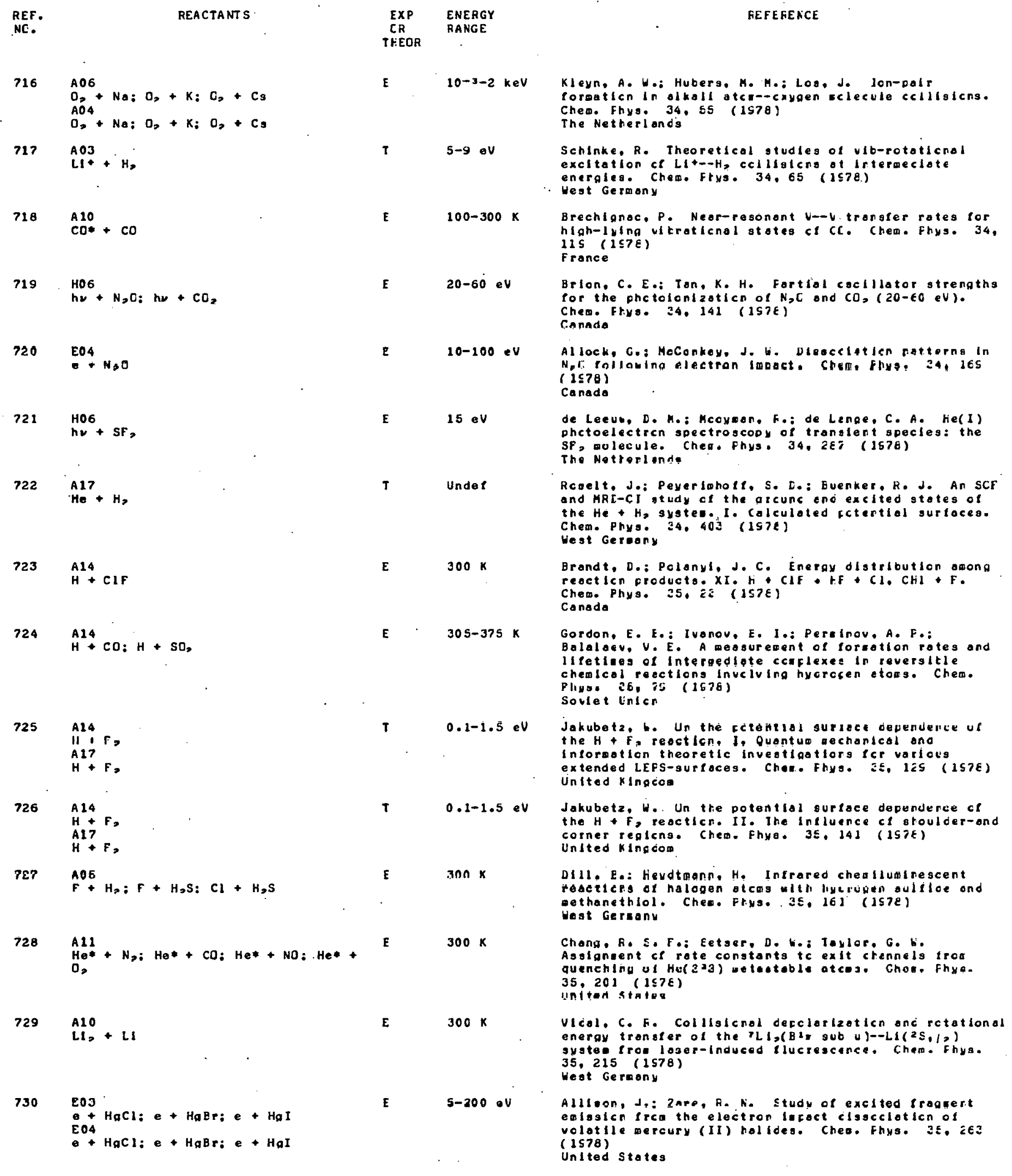




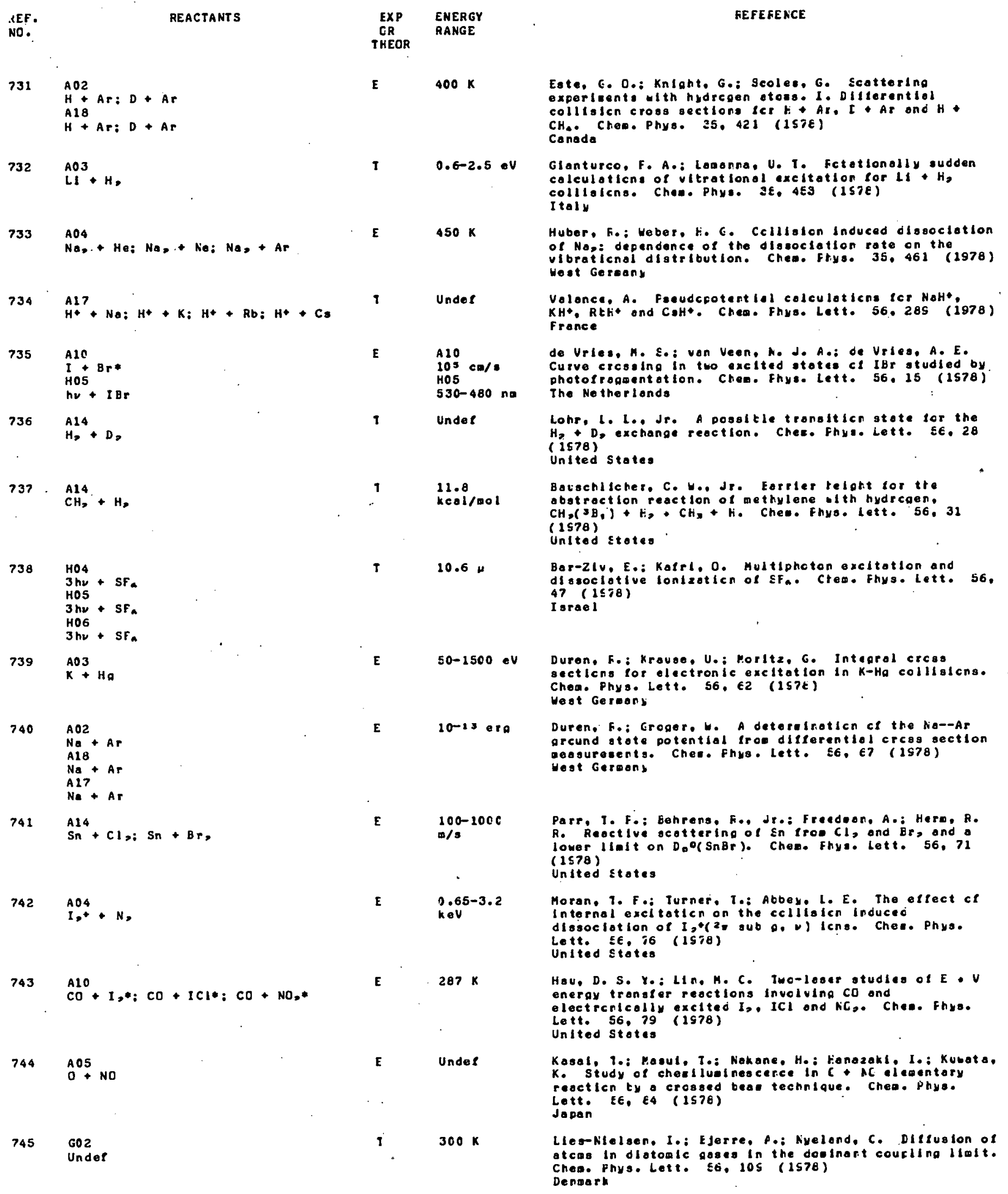




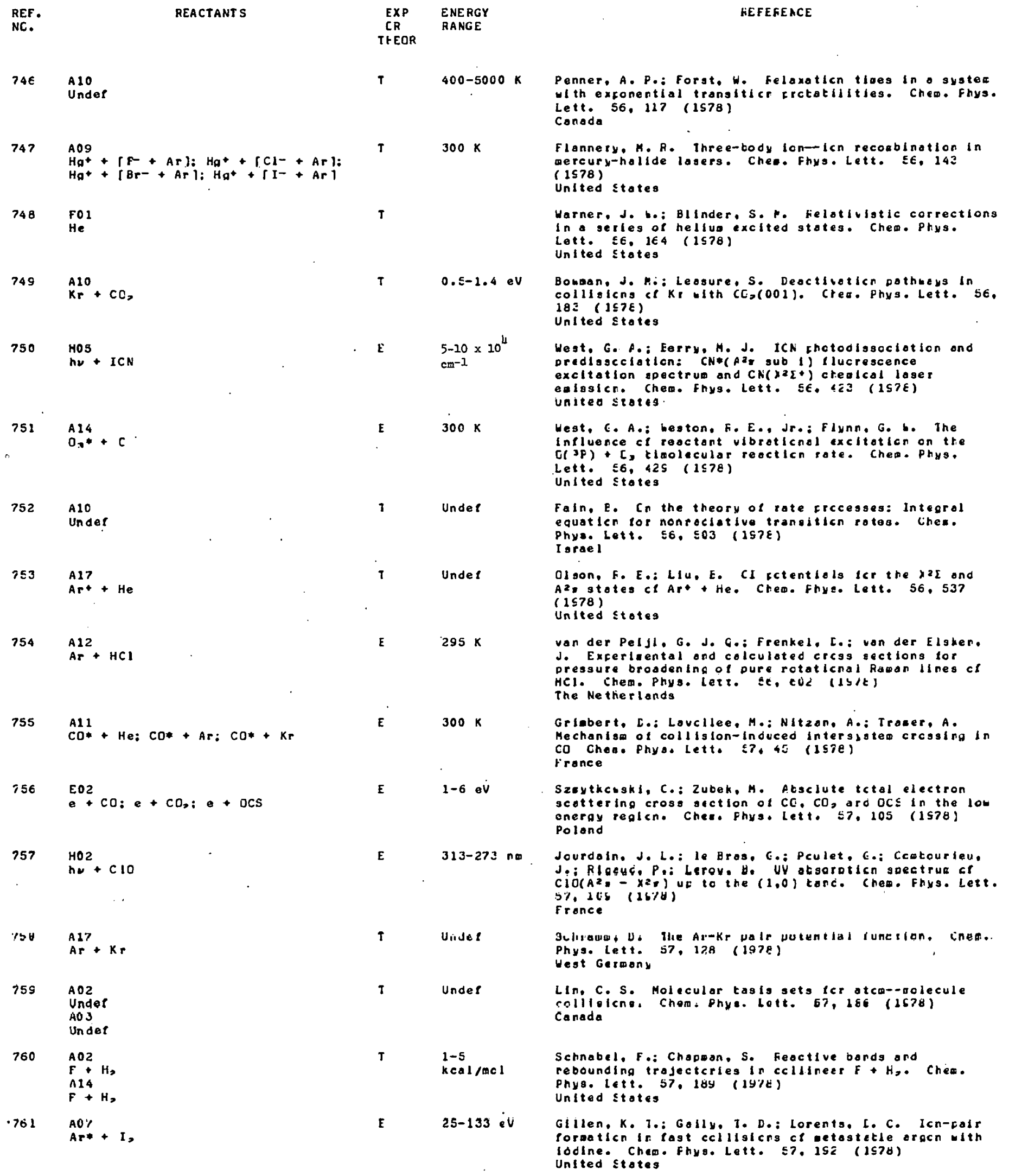




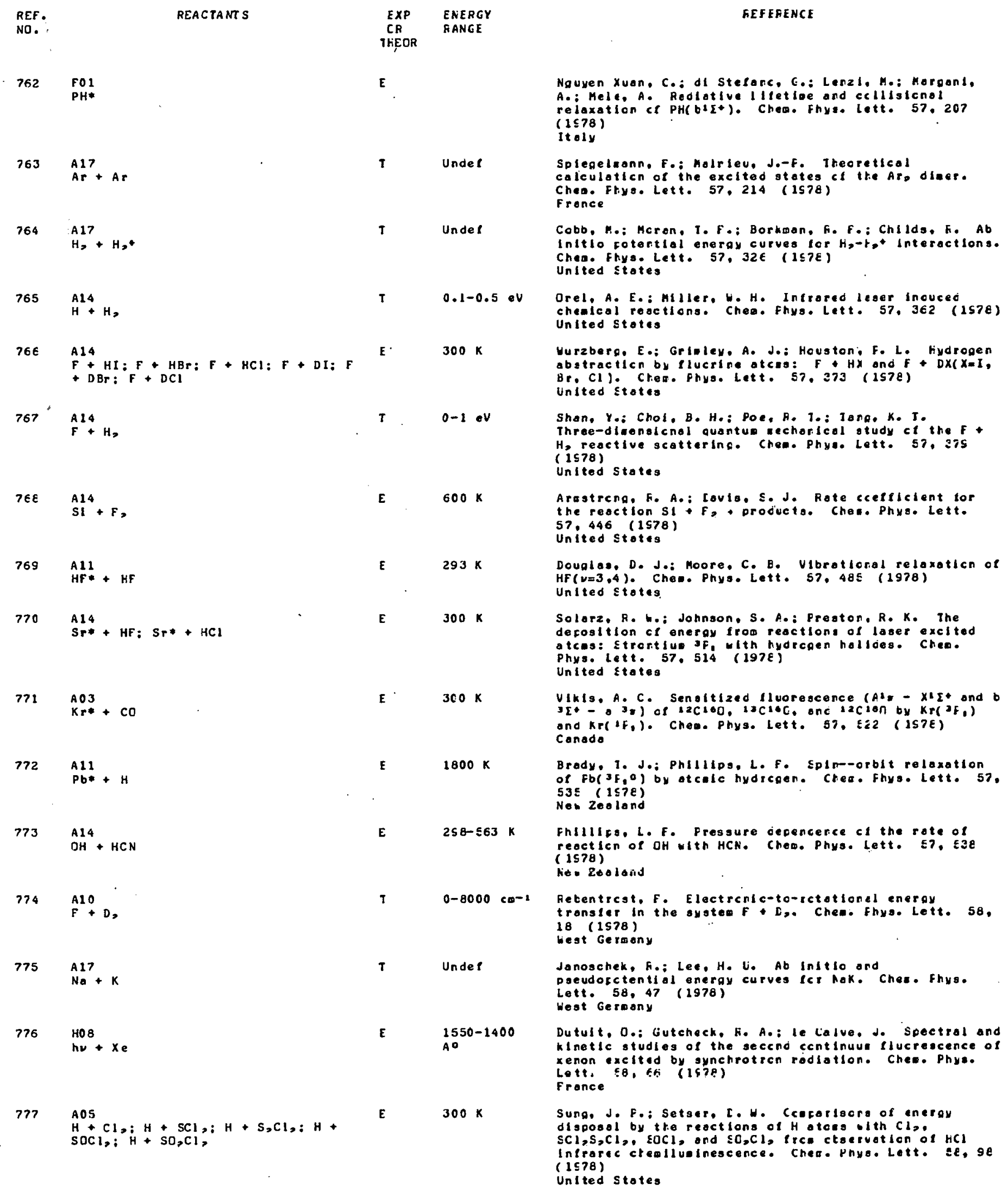




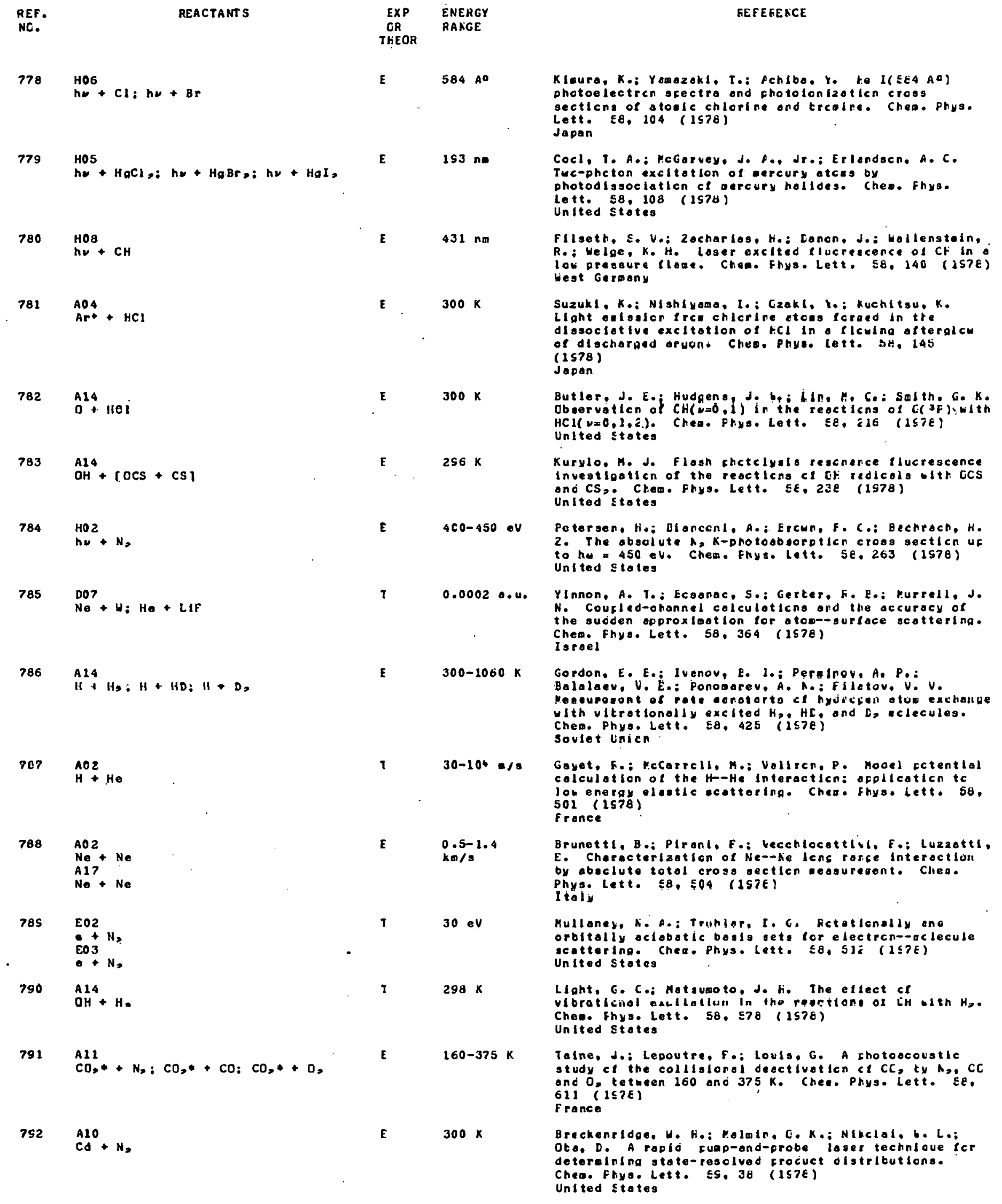


REF.

NC.

793

A14

C + PbO

REACTANTS

794

HOS

$h \nu+k x e$

A.1

795

796

A14

$\mathrm{Cl}+\mathrm{NO}$,

797

$\mathrm{AlZ}^{+}+\mathrm{H}_{2} \mathrm{O} ; \mathrm{N}++\mathrm{H}_{2} \mathrm{O} ; \cdot \mathrm{CO}++\mathrm{H}_{2} \mathrm{O}$

$\mathrm{CO}_{2+}^{+}+\mathrm{H}_{2} \mathrm{O}: \mathrm{CH}_{2}+\mathrm{H}_{3} \mathrm{O}: \mathrm{HCO}^{+}+\mathrm{H}_{2} \mathrm{O}:$

$\mathrm{H}>\mathrm{S}^{+}+\mathrm{H}>\mathrm{O}: \mathrm{HS}^{+}+\mathrm{H}>\mathrm{O}$

798

$\mathrm{OH}^{+}+\mathrm{CO}_{3} \mathrm{OH}_{+}^{+}+\mathrm{CO}_{3}: \mathrm{OH}_{+}+\mathrm{O}_{3}: \mathrm{OH}^{+}$

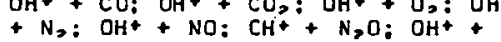

$\mathrm{H}>\mathrm{S}: \mathrm{H}_{>} \mathrm{O}^{+}+\mathrm{NO}: \mathrm{H}_{>} \mathrm{O}^{+}+\mathrm{O}_{>}: \mathrm{H}_{>} \mathrm{O}^{+}+$

$\mathrm{H}>\mathrm{S}$

799

A 14

$\mathrm{He}+\mathrm{H}_{2}+$

$800 \quad A 03$

$H_{2}+N$,

$801 \quad A 05$

$\mathrm{Xe}+\mathrm{Br}$ ?

802

1100

$2 \mathrm{hv}+\mathrm{Na}$

803

HO 8

$3 \mathrm{h \nu}+\mathrm{I}$,

804

$\mathrm{HO}_{4}$

$2 \mathrm{hv}+\mathrm{NO}$

805

E05

e $+\mathrm{H}, \mathrm{S}$

$B 00$

A17

$\mathrm{He}+\mathrm{Ar}$

807

A10

$\mathrm{O}$, $+\mathrm{H}$,
E

EXP

THEOR

E

$300 \mathrm{~K}$

ENERGY
RANGE

RANG

$540-400 \mathrm{~nm}$

$300 k$

$300 k$

$300 \mathrm{~K}$

$\varepsilon$

$300 \mathrm{~K}$

$0.5-1$ eV

$200-300 \mathrm{~K}$

$0.04-1.2 \mathrm{eV}$

589-337 nim

E

$44813-51601$
$c^{-1}$

E

$4540-3 E \leq 0$

ก

E $\quad 3.5 \mathrm{keV}$

E T Under

$167-424 K$

AEFEFEACE

Sridharan, L. C.: DIGluseppe, T. G.: HcFadden, L. L.: Davidovits, P. Cheallualrescence from the gos phase reaction of atcolic carben ith leac cxice. Chem. Fhys. Lett. E9, 43 (15i8)

United States

Weboter. C. R.: Rcstas. F. The dye laser incuced fluorescence excitotion and ealssicn orectrum of the Kxe molecule. Chem. Fhys. Lett. ES, E? (1578)

France

Fotel 1. H. T.: Hazl, A. U. Quenching ct hlgh concentrations of Se( 25$)$ produced by chotclysis. Chea. Phys. Lett. 59 . il (ISTE)

United Stotes

Nikl, H.: Moker, F. D.: Sovage, C. M.: Breltentach, Lo P. Fourier transtorm if apectrcsccolc cbservatice ci

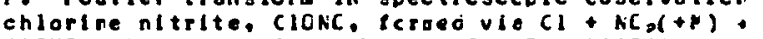
CICNO $+p)$. Ched. Fhys. Lett. SS, iE (ISTE) United stotos

Karpas, 2.: Anicich, V. G.: Huntress. L. T., Jr. An Ion cycloticn rescnance study ci reacticns cl sowe otamic and a iople cclyatoalc lcrs bith uater. Cheo. Phys. Lett. Eg, EA (ISTE)

United States

Karpas, 2 : Huntress, b. I.. Jr. Heactlons of Crt and $H, C+$ lcns bith some diotomic and gleple pclyatcolc molecules. Chem. Fhys. Lett. ES. E7 (1S7E) United states

Sathyawurtiy. N. Eflect ct potential-well in or ondotheralc sustes: reactive orc vitrolionally inelastic He t, ${ }^{\circ}$ collisicns. Chea. Fhys. Lett. ss, s5 $(1 \leqslant i \varepsilon)$

Conada

Bergercn, C.: Lefcrestler, C.: Louray. J. M. On the IIxed-nuclei approximation os applied to rctaticnal excitation cr aclecules by otcas. Chea. Fhys. Lett. 59.129 (1578) Frence

Rettner, C. T.: Simons, J. F. Fotaticral cclarization

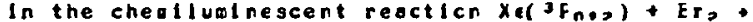
XeEr* B Br. Chea. Fhys. Lett. SS, ITE (ISTE) United KIngdom

Strand. M. P.: hiansen, J.: Chlen, F.-l.: Berry. F. S. Intluence $c$ f nucleor spln cn onguler distritution and polarlzotion of photoelectrons: resonart twc-chctcn lontzation of No. Chen. Fryselett. Es. 205 (1578) Unlted stateg

Chen, K.: Steenhcek. L. E.: Yeung. E. S. Rescnance enhancec ttree-photon atsorpticn cl acleculor lcdine. Chem. Fhys. Lett. 5S. 222 (1StE)

Unlted States

Asscher. M.: Hoos, $Y$. Twe-chctcr excltaticn of nitric oxide tc levels neor ono otove the disscciation $11 \mathrm{mit}$. Chem. Frys. Lett. SS. 231 (157e)

Is rae I

Brlon. C. E.: Ccok. J.F. I.: Ter. K. K. The valence shell tinding enersy spectrum of $H, S(E-34$ eV) $t y$ difole (e.2e) and tinary (e.2e) srecticsccsy. Cheo. Fhyo. Lett. 55. 241 (1578)

Canada

Ke 11. M.: Kucteroonn. A.: Elonkes, J. 1. Ar accurate deterwiraticn of the tie--A von der hollg cctentiol. Ches. Frys. Lett. SS, 335 (1S78) United States

Mac, C. $-R$ : Huy. J.: Gordon, F. J. Vitraticnal relaxation cl ozcne by caro orc ncrabl hydrcaen. Chem. Phya. Lett. ES. 425 (1978) United States 


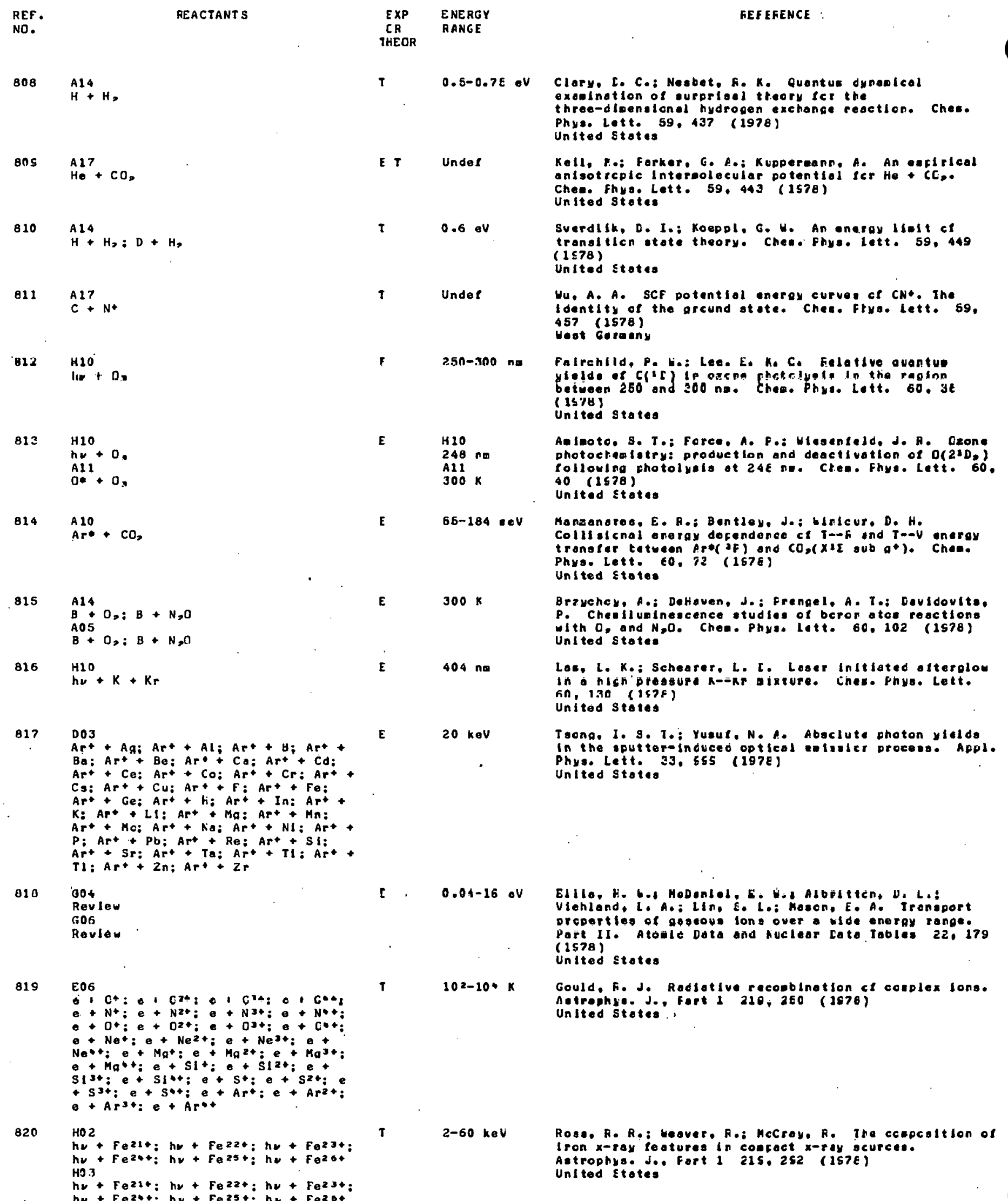




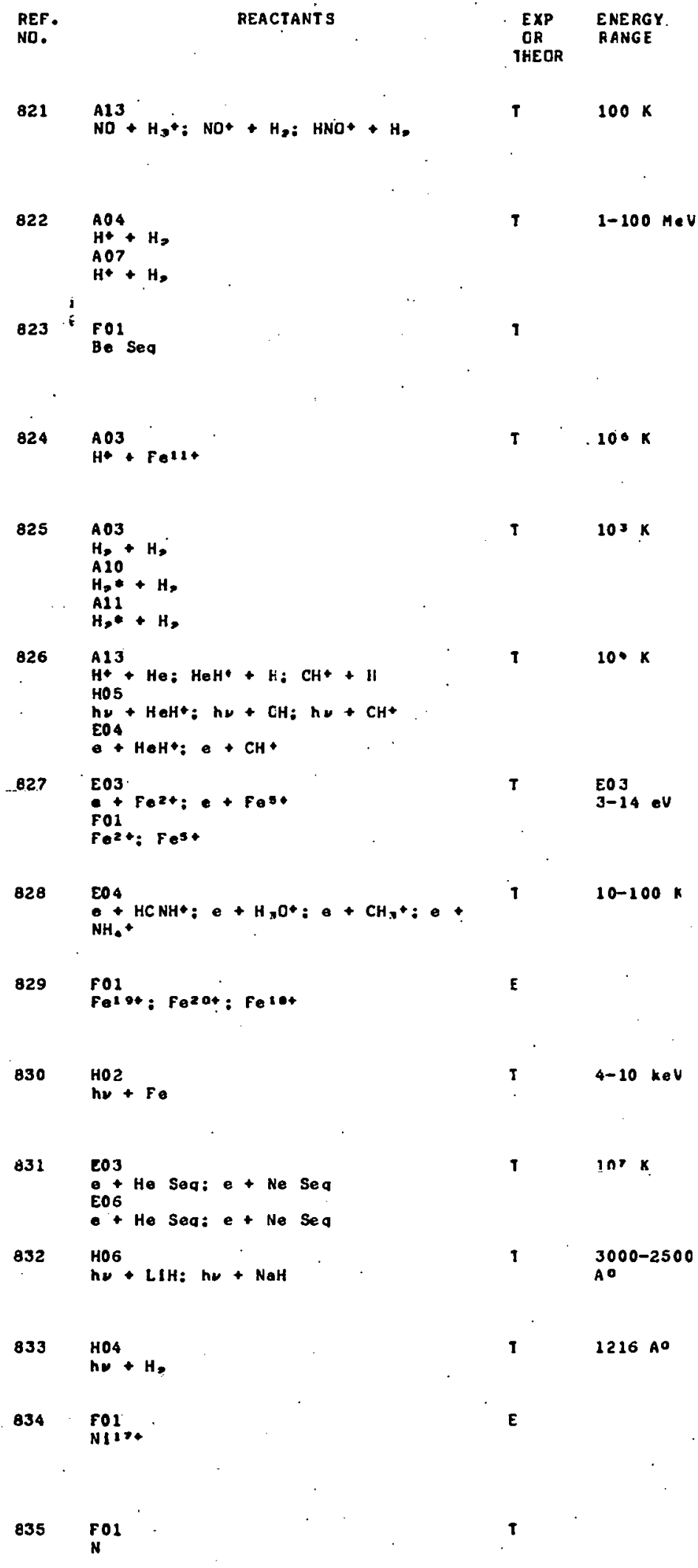

Loew, G. H.: Berkcult2, D. S.: Chang, S. Condicate Intersteliar solecules torued Irca icr-aolecule reocticns of NO. Aotrophye. J.. Fort 1 215,456 (1578)

United stotes

Crovens, 7. E.: Dalgorno, A. Ionizaticn, disscciotion. and heding efficlencles ct cesalc rays in cos ci molecular hydrosen. Aatrophys. J.. Part 1 zis, iso (1578)

United States

LIn. D. L.: Flelder. U.. Jr.: Arestrcnc, L.. Jr. Hulticcneiguration Hartree-fock calculoticn ci iagnetlo quadrupcle tronalticns ct Ee Iscelecticnic sequence. Astrophy. J.. Fort. 1 215, 10S3 (157E)

United Stotes

Londaan, D. A. Proton collislonal excitaticn in the grcund configuroticn cl Fe+13. Astrceryg: J.. Fart 1 $220,36 \epsilon$ ( IS78)

United States

Shull. J. Ho Hollenboch. D. J. H, cocling.

disaoclaticr. and Infrared ealsalcr. ir shocked

oleculor clouds. Astrcohye. J.e Fort 1 ZzO. E2E

( 1578 )

United states

Bleck, d. H. Holecules in planetary nebulae.

Aatrophys. J.. Part 1222 , 125 (1S7e)

United Etates

Garatang. R. H.: Fotb, W. D.: Rcuntree. S. F. Electron colliatcnol excitoticn cross secticrs icr Fe III and Fe $V I$ ond Ircn abundonces in gaseoug retulae. Agtrochys. J.. Part 1 z 22 , JE4 (ISTE) United ctates

Herbst. E. What ore the crcoucts ct cclyotcolic icn-electron disgoclative recentinoticr reacticns Aatrophsg. J.. PArt 1 222, SOE (JS?E)

United Stotes

WIding, $K$. $G$. Fortidden lines of fe $X I X$, fe $x x$, and Fe $X \times 1$ in color flores. Astrechys. J.. Fert 1222,735 ( 1578 )

United stotes

Langer, S. H.: Rose. F. R.: MeCray, F. Coticolly thich x-ray transfer: the shell gase. Astrcihys. J.. Fort 1 2Eź. gEs (1578)

United Stotes

Ravaond. J. C. Cn dielecticnic reccotinaticn ond resonances in excltaticn crosa gectlone. Astrcinys. J.. Part 1 222, 1114 (1 157 )

United states

Kl rby: K.: Lalgarnc, A. Nar and Lit. In diltuse

Intergteliar cloucs. Potrophys. J.. Port 1 Ė4. 444 ( 1578 )

Unt ted States

Shull. d. H. H, resonance llucrescence with Lyman-a. Astrophys. J., Fort $1224, E 41$ (1978)

Unltod stateo

Peqg, I. J.: GriffIn, F. M.: Jchnsen, E. H.: Jenes, K. W.; Kruse, T. H. A meacureoent of oaclilatcr strengths for solar XiV flare lines in AI XVIII. Aslruphys. J.: Fart 1224,1056 (1578)

United states

Lucger. P. M.: York. D. G.: Blanchord, I.: Mortcn, L. C. Nes ouclilatol strangths tcr le rescnence lines of $N$ I anc the Interatellar atundance of nitrogen. Aatroohys. J., Port 1 224, 1055 ( $157 E$ ) Unt ted ctotes 


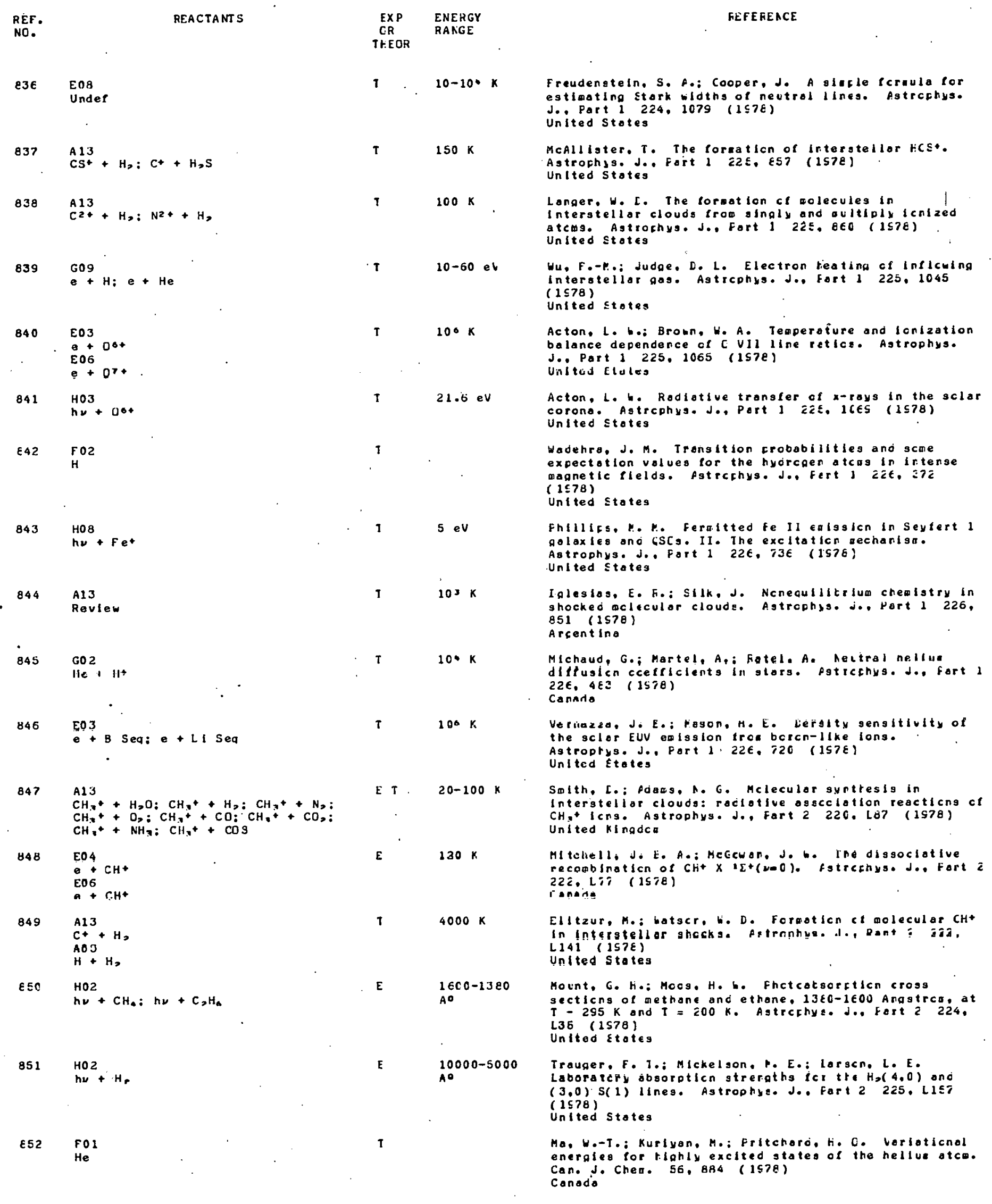




\begin{tabular}{|c|c|c|c|c|}
\hline $\begin{array}{l}\text { REF. } \\
\text { NO. }\end{array}$ & REACTANTS & $\begin{array}{l}\text { EXP } \\
\text { OR } \\
\text { THEOR }\end{array}$ & $\begin{array}{l}\text { ENERGY } \\
\text { RANGE }\end{array}$ & FEFERENCE \\
\hline 853 & $\begin{array}{l}\text { G06 } \\
\text { Under }\end{array}$ & E & Undet & $\begin{array}{l}\text { Sennhouser. E. S.: Armotrong, L. A, lcnmobliltles in } \\
\text { gaseous aumenia. Can. Jo Ches. SE, 2337 (1578) } \\
\text { Canado }\end{array}$ \\
\hline$\varepsilon 54$ & $\mathrm{O}^{\mathrm{AL}}+\mathrm{HBr}: \mathrm{O}+\mathrm{HI}$ & $\mathbf{E}$ & $258-554 K$ & 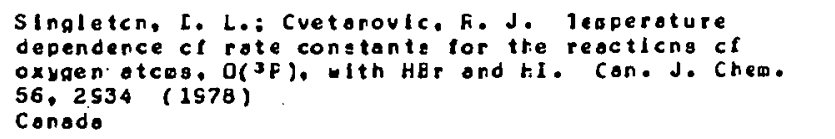 \\
\hline 855 & 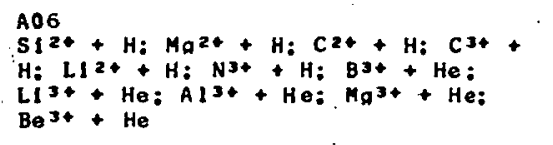 & $\mathbf{T}$ & $10 \cdot \mathrm{K}$ & 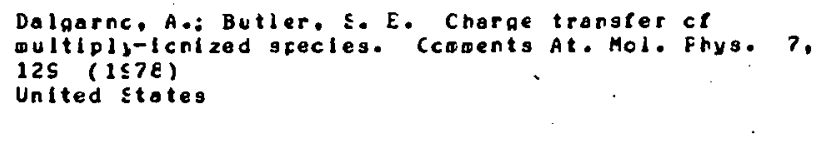 \\
\hline 856 & $E 17+\operatorname{LIF}$ & $E T$ & $20 \mathrm{eV}$ & $\begin{array}{l}\text { Schnelder. E. I. Theoreticol otcalc ord wolecular } \\
\text { physlcs at X ICFEAC. Comoents At. Mol. Phys. 7, } 157 \\
\text { (1S78). } \\
\text { Unlted stotes }\end{array}$ \\
\hline 857 & $\begin{array}{l}\text { El1 } \\
\text { + }\end{array}$ & E T & $0-21$ eV & 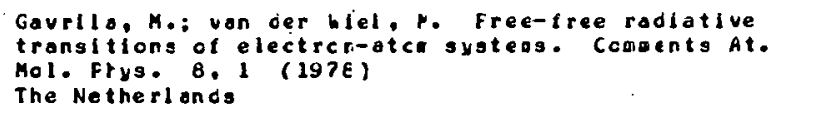 \\
\hline 858 & FOL & $\tau$ & & 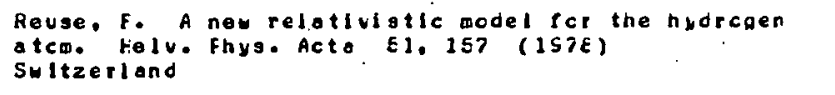 \\
\hline 859 & $\begin{array}{l}\text { Al? } \\
\text { Under }\end{array}$ & $T$ & Undet & $\begin{array}{l}\text { Dreyfus, 1. The number ol states tcunc by non-certral } \\
\text { potentidis. Helv. Phys. Acto S1, } 321 \text { (1978) } \\
\text { Sultzerland }\end{array}$ \\
\hline 860 & $\begin{array}{l}G 10 \\
e+\mathrm{CO}_{2}: e+\mathrm{Cl}_{2}: \mathrm{e}+\mathrm{Br}=\mathrm{e}+\mathrm{SF}_{a} \\
\mathrm{G11}+\mathrm{CO}_{2:} \mathrm{e}+\mathrm{Cl}_{3}: \mathrm{e}+\mathrm{Br} 2 \mathrm{e}+\mathrm{SF}_{a}\end{array}$ & E. T & $\begin{array}{l}10-1000 \\
V /(\cos \text { Torr })\end{array}$ & 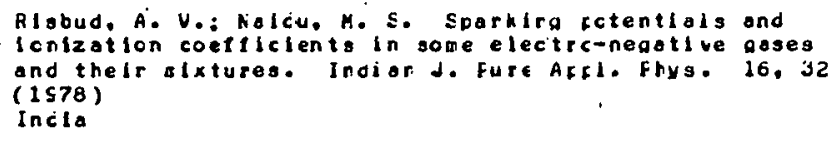 \\
\hline 861 & 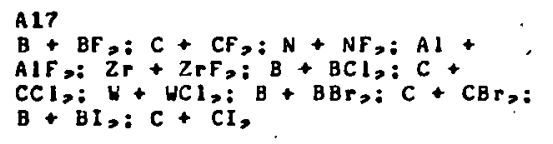 & $\mathrm{T}$ & Under & 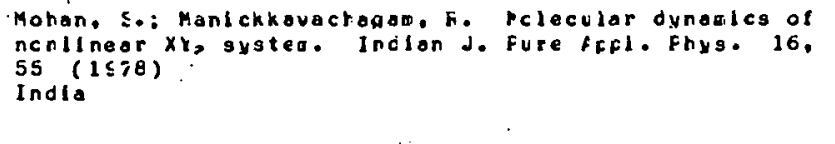 \\
\hline 862 & $\begin{array}{l}E 03 \\
e+L i ; e+N a ; e+K ; e+A b ; e+\end{array}$ & $T$ & $1-50 \mathrm{eV}$ & 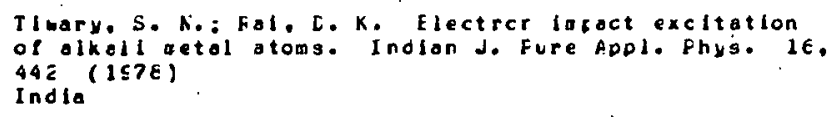 \\
\hline 862 . & $\begin{array}{l}A 14 \\
0+D C 1: 0+D B r\end{array}$ & $\mathbf{E}$ & $25 E-489 \cdot K$ & 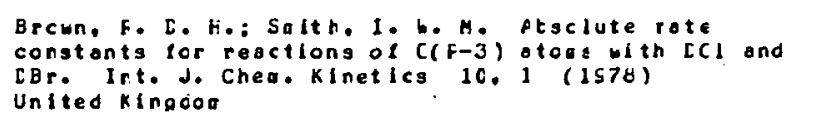 \\
\hline 664 & $\begin{array}{l}\text { A14. } \\
S+N D\end{array}$ & E & $258 \mathrm{~K}$ & 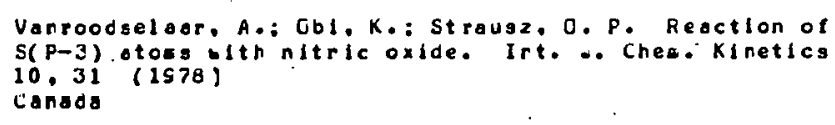 \\
\hline 865 & HO, + $\mathrm{NO}: \mathrm{HO}_{2}+\mathrm{NO}$ & $\mathbf{E}$ & $245-328 \mathrm{~K}$ & $\begin{array}{l}\text { Simonaltis, R.: Helcklen, J. Temeeratire dependerce cf } \\
\text { reacticna cP } H O \text {, Hith } A C \text { and } A C \text {, Int. J. Chea. } \\
\text { Kinetics } 10,67 \text { (1978) } \\
\text { United Stotes }\end{array}$ \\
\hline 866 & $\begin{array}{l}\mathrm{HOB} \\
\mathrm{he}+\mathrm{SO}\end{array}$ & $\mathbf{E}$ & $\begin{array}{l}3273-2975 \\
A 0\end{array}$ & 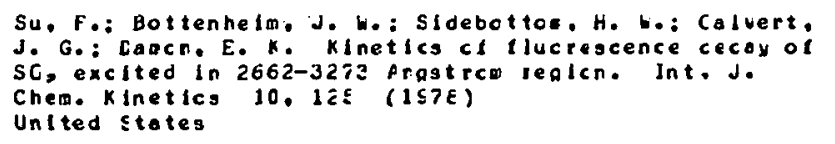 \\
\hline 867 & $\begin{array}{l}\text { A14 } \\
\text { Roviow }\end{array}$ & E & $300 \mathrm{~K}$ & 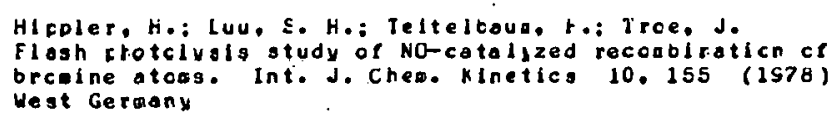 \\
\hline 268 & $\stackrel{A 14}{H+[H+M]}$ & $T$ & $77-1000 \mathrm{~K}$ & 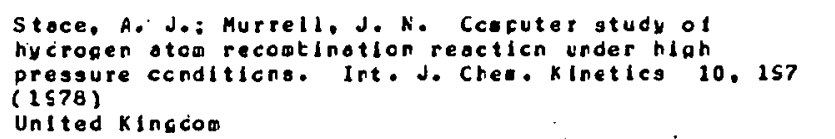 \\
\hline
\end{tabular}




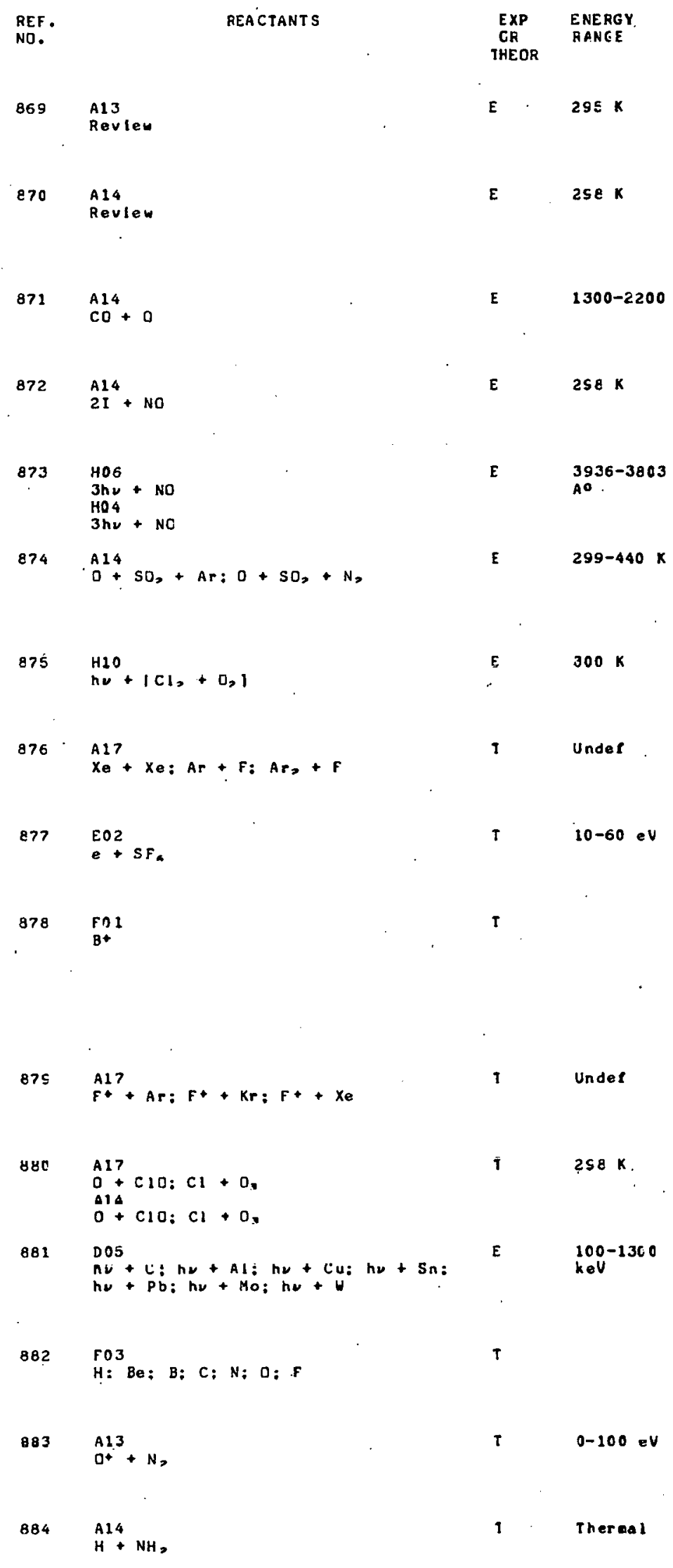

sleck, L. W. Reactlons of $(\mathrm{CO},)_{0}$. Ond ( $\left.\mathrm{CC}\right),+$ associaticn tons. Int. J. Ches. Kinetics jo, 335 (1578)

Unlted States

Clyne, M. A. A.: Nip, W. S. KInetics ct lluorine stoe reseticns using resononce obsorpticn ecectrceetry in

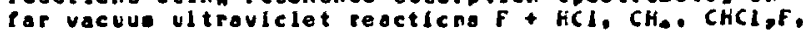
and CHCIF,. Int. J. Chem. Kinetica 10, 367 (1SiE) United Kingdos

Hardy, J. E.: Gordiner, W. C.: Burcat, A. Recombinaticn of corben ocrcxide ond oxyoen otcase. Int. J. Ches. Kinetics 10. 503 (1578) Unlied states

Baseo, A.: Hunt, J. E. Hecentinatien cl lodine otons in presence of nitrle oxide. Int. J. Ches. Kinetics 10.732 (1S7E) Canado

Jackson, Wo Ho: Lir., C.S. Ccecetiticr between aultiplicton sluorescence end ultichotcn lonlzotion in HO. IPt. J. Chee. Kincelles i0, 015 (2630) United stetes

Atkinscr. Ro: Pitts, J. N. KInetics cl reocticn $O(J P)$ $+\mathrm{SO}_{2}+M$ - SO + Hover tecterature ronge cl 299-degrees-440-degrees $K$. Int. J. Chas. Kinetics 10, 1081 (1510) United States

Asterd, F. D.: Goseo. N. I Hunt, J. E. Kinetico of ClO formaticn in liash photolyals of chlosine-oxygen alatures. Int. J. Chea. Kiretics 10, 1233 (1578) canado

Michels, H. Ho: Hotbs, Re fi.: Holaht, L. A.: Conncliy, J. H. D. Electrcilc atructure cl exclaer eclecular lasers. Int. J. Guantur Ches. 12, 1es (1578) United stotes

Benedict, H. G.: Gyemant, I. Total ceces sectlon of the SF aclecule Pcr elostic electicn scottering. Int. J. Quantue Ghen. 33, 687 (1578) Hungary

Larson. E. G. Copparisons of the FFA, SCFFA.

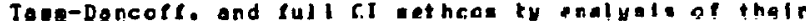
tronaltion oenglty aetrical. oscliletcs etrengthe, ond

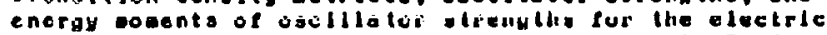
dicole tronstitions trca the grcund stete of the $8 *$ ton (Irozen K-stelit mocel). Int. J. Quantua Chea. i2, 743 $(1 \leqslant 78)$

United States

Sonnlgrahl, A. E.: [a, E. F. Selected valence-electron spltt-sheli MC coliculations on ArF*, KrF*, and XeF*. int. J. Quantum Chese. it, sís (is78)

indis

Forantos, S. C.: Murrall, J. N, closgicel oynenica of the $0+\mathrm{Cl}_{10} \cdot \mathrm{Cl}+\mathrm{O}_{\text {, }}$ and $\mathrm{Cl}+\mathrm{C}_{3}+\mathrm{Cl}_{\mathrm{C}}+\mathrm{C}_{2}$ reactions. Int. d. Qivinlum clien 1t, ceg (13To)

United Kingdo:

Nokamuro, Ko: Klkuchl, E. The eneray scoctro of secondery electrons from the netalile elements

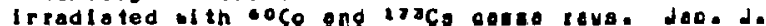
Aptl. Phys. i7, 1403 (1570)

Jafor

Dewar. H. J. S.: Kzepo, H. S. Calculotions of electron affinitios lolng the MNDO sesieafiricel SCF-MC sethod. J. Am. Chea. Soc. 100, 7E4 (1578) United stotes

Hopder, D. G. Mechanliss Ct the Pactich ol positive a tcule cxygen icne with nitrogen. J. Pa. Ches. Soc. 100, 1015 (1578)

United States

Nakatsujl, his: Kogo, 1.: Kcrde, Ko: Ycrezane. T. Force and density study on the crenical reaction frocese $N H_{\text {? }}$ + H. Mr., J. Aw. Chea. SOC. 100. 10aS (1S78) Japan 


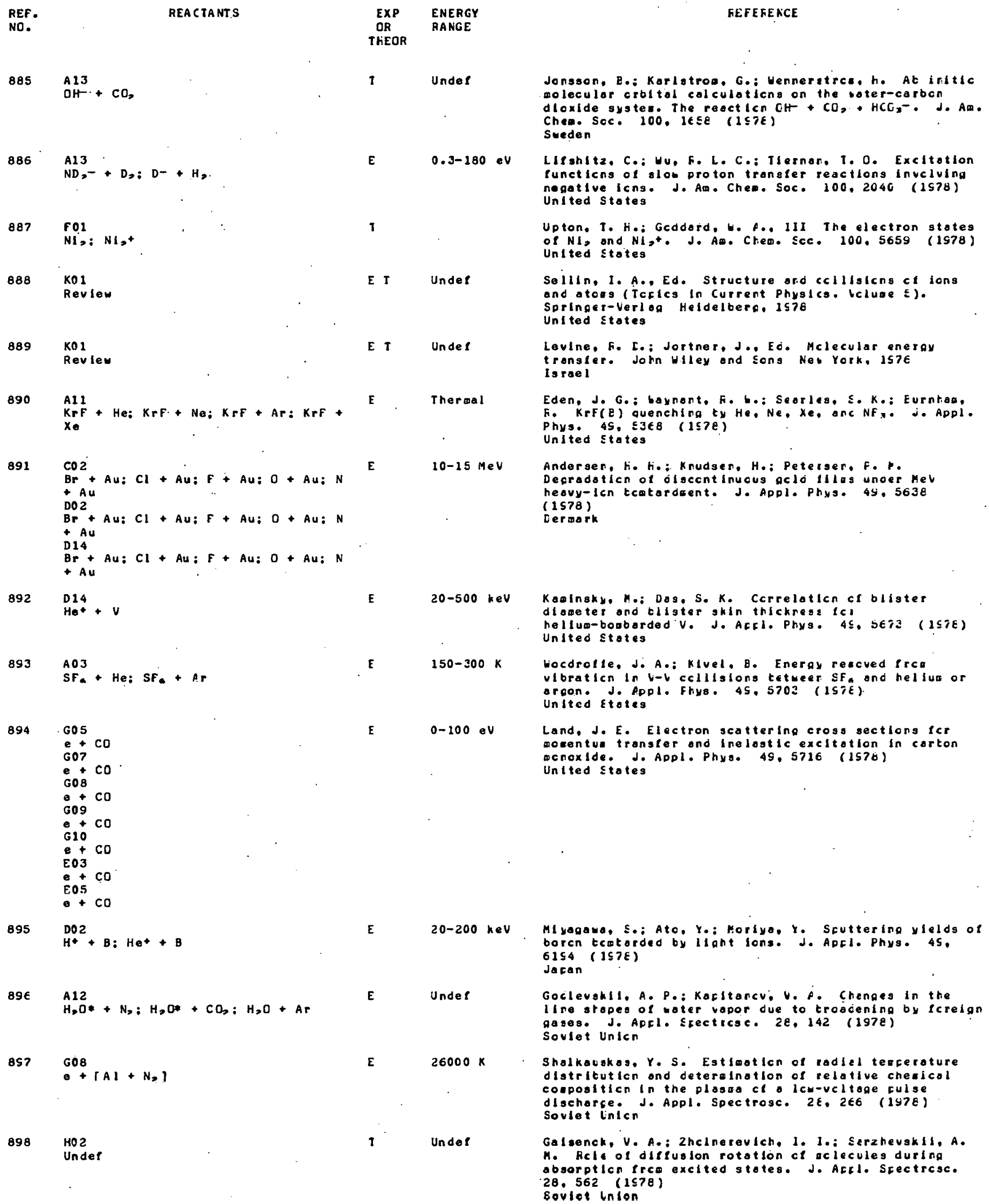




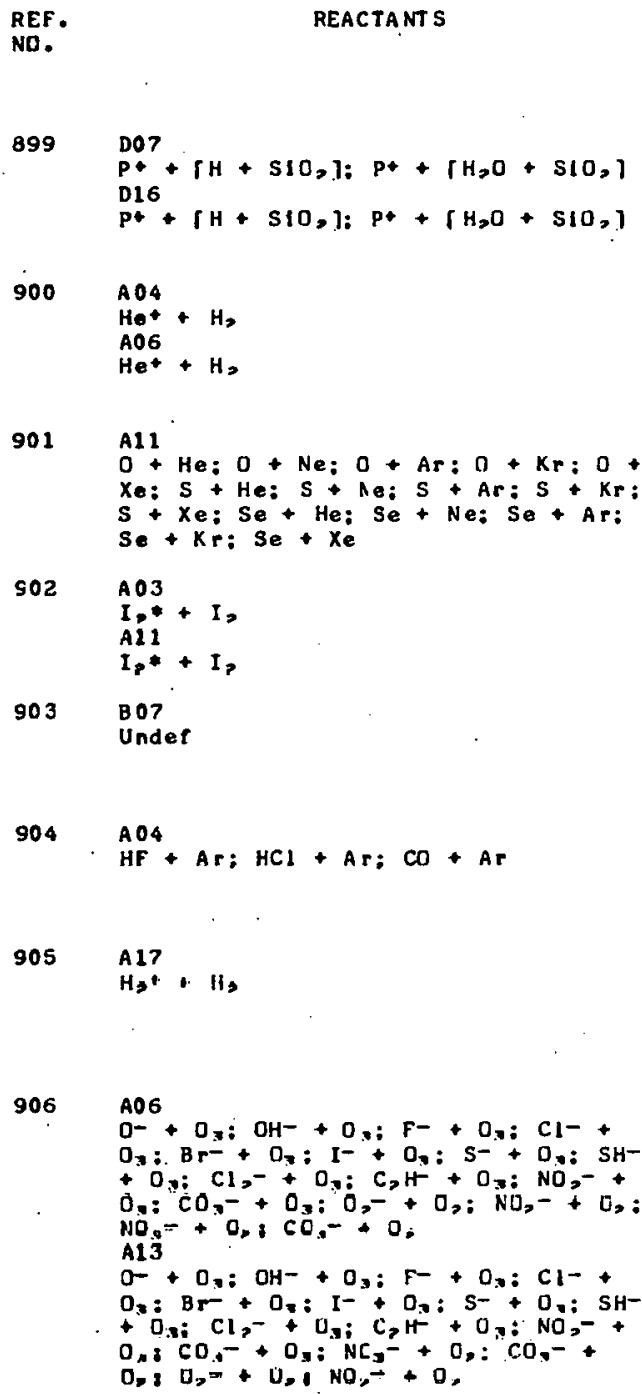

$.005-0.28$

$2500-9500 \mathrm{~K}$

Undel
$0.65-3.2$ kev

$100-400 \mathrm{~K}$

$1200 \mathrm{~K}$

$300 k$

$300 \mathrm{~K}$

Under
GEF EFENCE

Geraslzenkc, A. N.: Kovolevskave. T. 1.: Tseltlin. G. M. Investipation of hyorocen coptcre in icn-bcaborded siliccr dicxlde liles ty the atF dethoc. J. Accl. Spectrcac. 28, 600 (1S7E) Sovlet Union

Preston, F. K.: Thoepson, [. L.: McLaughlin. D. F. A theoretical orediction cl vibrotionol enhancesent for disociotive charge tronster in the het, syster. d. Chem. Frys. 68,13 (ISTE) United ftotes

Jullenne, P. S. Theory of rare gas-grcup VI is-1 I collisicn-inouced transit icrs. J. Chea. Phys. GE, 32 ( 1578 )

United states

Koto, H. Molecular collisions and decclarizaticn cf emlsalcn freal, In the oos phase exclted by clrcularly polarised light. J. Chem. Fhys, CE, E6 (157E) Jacan

Belluo, J. C.: George, T. F. Quantud recharical theory of collialcnal lenizaticn in the freserce ot intenge

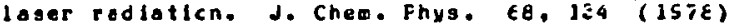
United States

Rasakriohna. H.: Bobu, S. V. Steady gtote dissociotion of shoch heoted HF. HCl. oro CC in excess Ar. J. Chem. Fhys. $68,163(1578)$

St Ine, J. A.: Muckermon, J. T. Chorge exchonge and chemleel reactlen In tha $H_{0}+$ to systec. I.

Characterlzation of the potentlal eneray surfaces and nonadiatatic rapleng. J. Chem. Fhys. 68, 185 (1578) United States

Llegitz, C.: Hu, F. L. C.: Tiernar, I, O.:

Termilliper, D. T. Necative Icn-aclecule reacticng of ozcne and their laflicotions on the thermochealstiy of O3-. J. Chem. Fhys. 68. 247 (15TE)

United Exates

Moran, 1. F.: Hilecx, J. B.: Attey, L. E

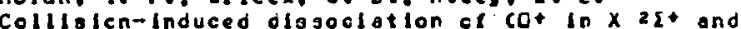
metastotle atates in CC+-CC interacticra. Jo Chea. Phys. 68, 261 (1578)

United States.

Keoper, P, F.: Nellson, P, V.; Farent, L.: Echers, M. T. Touraratura decendunes et tha pactian er kriblth $H, D$, and $H C$ : on unuaval isctece ofiect. J. Chea. Phys. C8, i2z (1578) United states

Krenos, J. Cn electrcnic eqlasicn intensity in chesilueinescant reactinn.. J. Ches. Phyg. 6E, 343 (1578)

Unitod stateo

Xystris. N.: Lahler. J.S. A reoctive collisicn mode for use in kinetic theory. J. Ches. Phys. 68, 345 (1578) United stotes

Xystris, N.: Dahler. J. S, Mass and acmentum trangcort indilite reacting gases. J. Ches. Fhyse E8, 354 (1578)

United States

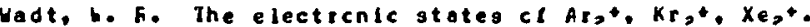
I. Potential curves ilh and ithout ocin-crbit coupling. $\mathrm{J}$. Chem. Phyo. E8, 4C2 (1578) United states 
REF.

REACTANTS

NC.

913. $\mathrm{AOS}$

$\mathrm{CO},+\mathrm{Ne}: \mathrm{CO}_{2}+\mathrm{Ar}_{2} \mathrm{CO}_{2}+\mathrm{Kr}$

914

A11

$\mathrm{HCl}+\mathrm{O}$

A14

$\mathrm{HCl}+\mathrm{O}$

915

A05

$\mathrm{He}_{2}++\mathrm{H}_{2} \mathrm{O}: \mathrm{He}_{2}++\mathrm{CH}_{3} \mathrm{CN}$

A11

$\mathrm{He}_{2}+\mathrm{H}_{>} \mathrm{O}: \mathrm{He}_{2}++\mathrm{CH}_{3} \mathrm{CN}$

$\mathrm{He}_{2}+\mathrm{H}_{2} \mathrm{O}: \mathrm{He}_{2}+\mathrm{CH}_{3} \mathrm{CN}$

916

A14

$K+\mathrm{NaCl}: K+\mathrm{NaO}$

917

HOG

$h_{\nu}+\mathrm{Ne}: h_{\nu}+\mathrm{Ar}_{\mathrm{S}} \mathrm{h}_{\nu}+\mathrm{CH}_{0}: \mathrm{h}_{\nu}+$

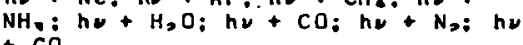

$+\mathrm{CO}$,

A0 3

$\mathrm{He}_{\mathrm{O}}+\mathrm{H}_{2} \mathrm{:He}+\mathrm{O}_{2}: \mathrm{Ar}+\mathrm{N}$,

A11

$\mathrm{He}+\mathrm{H}$,

920

$\mathrm{AO2}$

$\mathrm{He}+2 \mathrm{He}$

921

HOG

$h_{\nu}+Z_{n}: h_{\nu}+C d$

922

$H_{3}^{A 17}+A r: H_{2}+K r ; H_{2}+X e: D_{2}+N e$

923

HOS

hv + H,

924

E03

e + NO

925

$\mathrm{F}^{+}+\mathrm{H}$,

926
Al?

$\mathrm{Cs}+\mathrm{Cg}_{\mathrm{S}} \mathrm{Rb}+\mathrm{Rb}$

HO 2

$h_{\nu}+C_{3}: h_{\nu}+R b$,
1

\section{EXP ENERGY \\ THEOR}

I $0.1-10 \mathrm{eV}$

E

2s6 $\mathrm{K}$

$300 \mathrm{~K}$

$300 k$

$21-1253 \mathrm{eV}$

$0.05-9.4 \mathrm{eV}$

$3000 K$

$300 K$

412-1052 eV

Under

$1.11-14 \circ V$

$5-7.4 \mathrm{eV}$

Undef

$4230-78 \varepsilon 0$
FE F ERE NCE

Suzukswa, H. H.. Jr.i. Holtsebera. H.: Theapsen. D. L. A quaslclesslcal trojectory otudy of the energy transfer in CO,-rare gas syatess. J. Chen. Physe EE, 4EE (1578)

United states

Macdonald, F. G.: Heore, C. E. Reecticn anc deoctivetion of HCl ( $V=i, 2)$ by o ators. J. Chee. Phys. 68,512 (1S7E)

United states

Binns. W. F.: Ahl. J. L. Excltoticn ond quenchine

reoctions in $E-t e o n$ excited $\mathrm{He} / \mathrm{H}_{3} \mathrm{C}$ and he/CH, $\mathrm{CN}$

syotems. J. Chen. Phys. E8. E3E (1578)

United States

Pollak. E. Prior otatiatlcol datifibutions for the collisicn of on atom ith a diatos. J. Ches. Phya. 68.547 (157E)

United States

Allison. D. A.: Covell. R. G. Fhotoelectron

spectrcscopy ith $2 r$ M(sub aeta) (15l U V) radiotion. A atudy of the variotion of relative onctcionizotion cross cecticns of colecules ccntoiring ilrst rcw otons (C,N,C) witt exciting rodioticn Irca hel to $\mathrm{Mg}$ (sub a) linits. d. Chao. Fhys. EE, ES3 (1S7E) Canada

Tarr. S. M.: Fabltz. H. Fefio ond accurate evaluetion ct inelogtic dolecular cross oecticns. J. Chea. Phyo. $68.64 \bar{a}$ (157E)

Unlted Stotes

Torr, S. M.: Fabltz, h. High-tenferoture

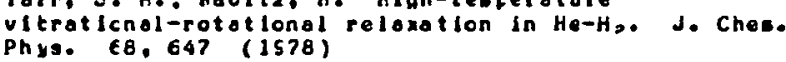

United States

Lia. T. K.: Durty. K. Faddeev-UPE thecsy and the three -He-atce gyatea: tound ot ates and zerc-eneray atoo-diotce scattering. J. Chea. Fhys. EE. 655 (1578)

Unitod stotes

Banna, M. S.: Froot, D. C.; McDowell, C. A.: lellbank, B. Free-atco core binding energies isce $x$-roy photoelectrcn spectrosccpy. I. Zinc onc codelume Je Cheq. Pryo. EB. ESE (ISTE)

Canado

Dunker. A. P.: Gordon, F. G. Ecuinc otcordiotculc molecula ccoplexes. Anlsotroplc Interacleculor potentiola lor the hydrcger-rere gas oysteos. J. Chem. Phys. EB, 200 (1578)

United states

Ritchle. B.: Tambe. B. R. Theoretical studies in phctoelectrcn scectrosccpy: extrection cl dynoelcol and structurel intoration ircs the oncular distributions for orlented cr rotaticnally resclued unoriented aclecular oaples. J. Chee. Phys. 6e.75s (1578) United states

Fruoholz, R. P.: RIando, R.: Kuperoanr. A. Coublet quortet transiticns in nitilic cxide oa detected by electrcn-iofact spectroscopy. J. Cher. Physe 68.775

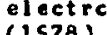
(1578) United stotes

Mahan, E. H.: Schoeler, H. F.. III: Ungeaach, S. F. Sore ceatures or the cctentlol energy aurfoces tor the

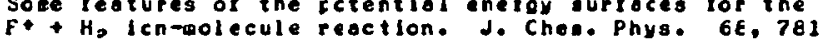
(1578) United States

Gupta. F.: hacper, Wo: Wagner, de: Herrayr. E. Absorption studles of $C s$ and $R b$, clecular bonds in the vioftle and neor visitle. J. Ches. Phys. 68.799 (1578) United stotes 


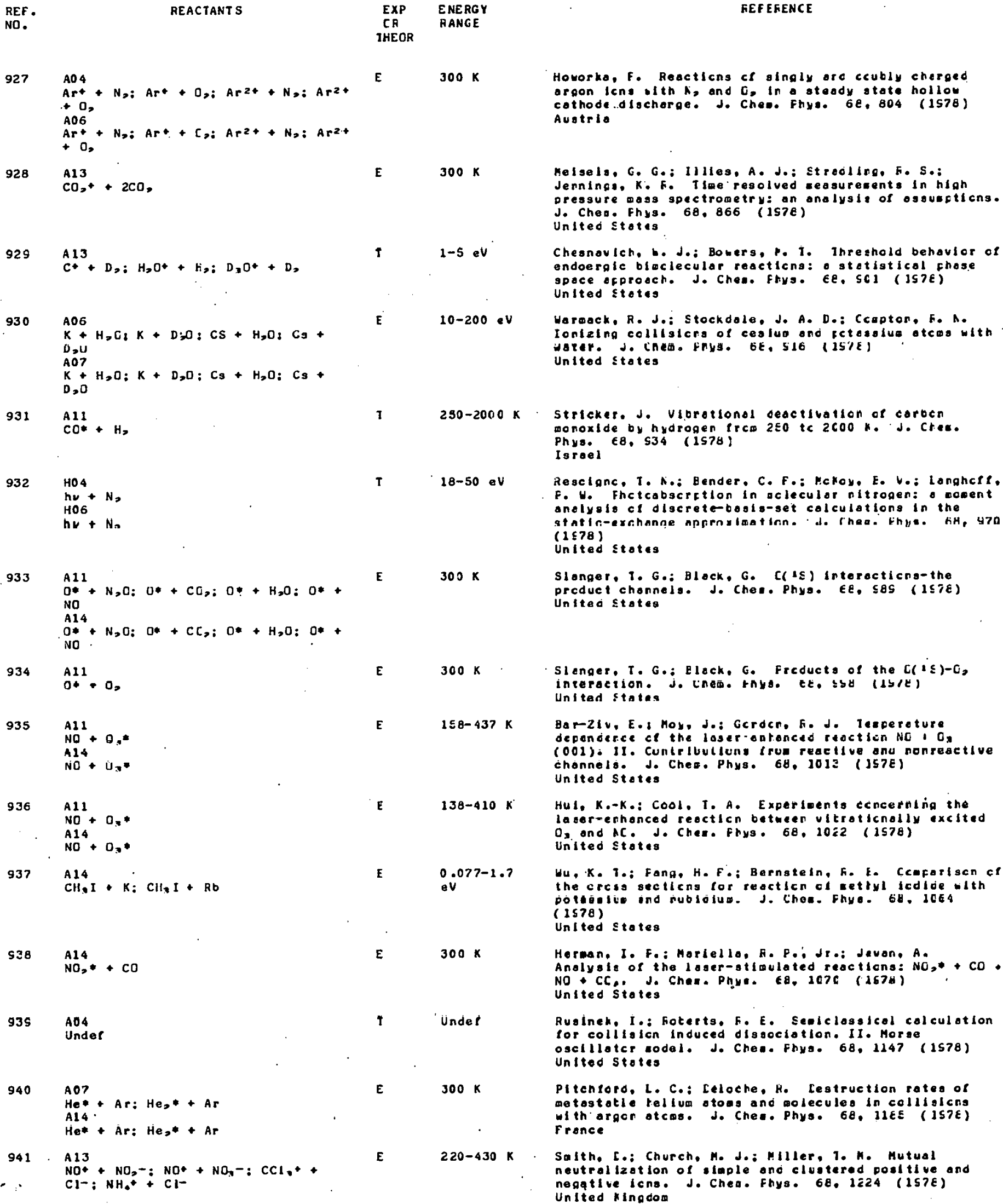

A11

$\mathrm{O}^{*}+\mathrm{N}, \mathrm{O}: \mathrm{O} *+\mathrm{CO}_{2}: \mathrm{O} *+\mathrm{H}_{2} \mathrm{O}: \mathrm{O}_{*}+$ NO

Howorka, F. Reactlicns cl gingly are ccubly cherged arcon Icns ith $A$, and $C$, In ateady atate hollou cothode.dischorge. J. Cheo. Fhyo. 6e.804 (1578) Austria

Nelsels, G. G.: lllies, A. J.: Etrooling, F. S.: Jerning:. $K$. F. Tlee resolved ceasuresents in high pressure oass spectrometry: an onalysiz of osausticns. J. Chea. Fhys. 68,866 (157e) United Etotes

Chesnavlah, b. J.: Bowers, P. I. Ihreshold behavior of endoergic blaclecular reacticna: otetistical chase space afproach. J. Chas. FHys. Ee. SCI (JSTE) United stotes

10-200 eV Haroack, R. J.: Stockdale, J. A. D.: Ccapton, F. A. Ionizing collisicris of cesluo ond fetasilus otcms with Worer. J. Cneb. Fnys. be, S16 (15)E) United states

$250-2000 \mathrm{~K}$

stricker. J. Vibrationd deactivalion of corocn wonoxlde by hydrogen trco 250 tc $2000 \mathrm{k}$. J. Cres. Phys. E8, 534 (1578)

Is rael

$18-50$ eV

Resclgnc, T. N.; Bender, C. F.: Mckay. E. V.: Longhcle, F. H. Fhctcobscrition in aclecular nitrogen: acent anelyals ci discrete-bools-set calculations in the atatic-exahanne nperneluation. A. Thea. thye. ti, y7a ( $1 \leqslant 78$ )

United states

$300 \times$

Slonger, T. G.: Block. G. C(IS) interecticng-the prcduct chonnela. J. Ches. Phya. Ee. SOS ( $1 \leq 7 \varepsilon$ ) Uniteo stateo

Slenger, T. G.: Black, G. Frcducts of the C(IS)-G,

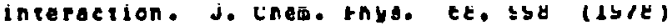
United states

E $\quad 158-437 K$

gar-Ziv. E. I Moy, J.: Gcrdch, F. J. Teeceroture dependerce ce the loser-atenced rioction NO:O,

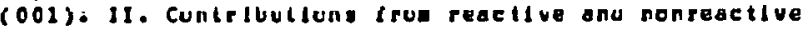
chonnels. J. Ches. Phys. 68, 1013 (J57E) Unted Stotes

$138-410 K$

Hul, K.-K.: Cool, I. A. Experiments ccncerintig the laser-enhonced reacticn between vitroticnaliy excited 0, and $A C$. J. Cher. Fhys. 68, 10z2 (1578) United States

$0.077-1.7$ ev

Hu, K. 1.: Fang, H. F.: Bernsteln, F. F. Ccararlacen ef the cross secticns cor reacticn of cetivl icdiof tith

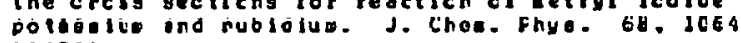
( I 578$)$

Unlted states

Herman, I. F.: Mariello, R. P.: Jre: Jevon. A. Analysis of tha laser-atioulated reacticna: $\mathrm{NO}, *+\mathrm{CO}$. NO + CC.. J. Char. Phye. ER, 107C (1674) United States

Ruolineh. I.: Foterts. F. E. Seelclosslcol calculation tor colliticn induced dissociotion. II. Morae oscillotcr model. J. Chen. Fhys. 68, 1147 (1578) Untied stotis

Pitchioro, L. C.: Celoche, H. Cestruction ratea of metostotle lel lum otoas and molecules in colliglcns w th argon atcos. J. Ches. Phys. 6a, I1EE (ISTE) Fronce

Salth, I.: Church, M. J.: Miller, 1. M. Hutual neutralization of siaple ano cluetered postive and negatlve icna. J. Chea. Fhys. 68. LE24 (ISTE) United kingdom 
REF.

NO.

942

943

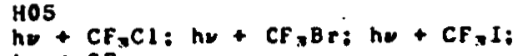

he $+S F_{\text {a }}$

944

A07

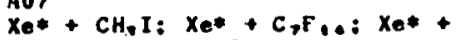

$\mathrm{C}_{a} \mathrm{~F}_{a}: \mathrm{Xe}_{0}+\mathrm{CH}_{\mathrm{a}} \mathrm{B}$

605

$+x_{0}$

A06

$\mathrm{He}_{+}+\mathrm{Kr}_{\mathrm{S}} \mathrm{He}_{2+}+\mathrm{H}_{2} \mathrm{H}_{\mathrm{O}}++\mathrm{O}_{20}$

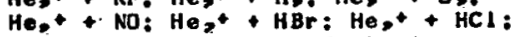

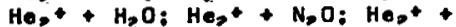

$\mathrm{CCI} \mathrm{F}_{n-\mathrm{H}} \mathrm{He}_{2}++\mathrm{NC}_{p-\mathrm{H}} \mathrm{He}++\mathrm{C}_{3} \mathrm{H}_{n}$ :

$+4 \mathrm{NH}_{3}$

A17

$\mathrm{H}+\mathrm{H} ; \mathrm{H}+\mathrm{He} ; \mathrm{H}+\mathrm{LI}: \mathrm{H}+\mathrm{N}: \mathrm{H}+$

$\mathrm{O}: \mathrm{H}+\mathrm{H}_{3}: \mathrm{H}+\mathrm{N}, \mathrm{O} \mathrm{H}+\mathrm{O}_{2}: \mathrm{H}+\mathrm{NO}$ :

$\mathrm{H}+\mathrm{N}_{0} \mathrm{O}: \mathrm{H}+\mathrm{H}_{2} \mathrm{O}: \mathrm{H}+\mathrm{NH}_{3}: \mathrm{H}+\mathrm{CH}_{4}$

$\mathrm{He}+\mathrm{il}_{2} \mathrm{He}+\mathrm{N}$ : $\mathrm{He}_{e}+\mathrm{O}: \mathrm{He}+\mathrm{H}_{2} \mathrm{O}$

$\mathrm{He}+\mathrm{N}_{2} \mathrm{O} \mathrm{He}+\mathrm{O}_{3}: \mathrm{He}+\mathrm{NO}: \mathrm{He}+$

$\mathrm{NoO}: \mathrm{He}+\mathrm{H}_{2} \mathrm{O}$ : $\mathrm{He}+\mathrm{NH}_{3}: \mathrm{He}+\mathrm{CH}_{0}:$

$\mathrm{LI}+\mathrm{N}: \mathrm{LI}+\mathrm{O}: \mathrm{LI}+\mathrm{H}_{9}: \mathrm{LI}+\mathrm{N}_{7}$

$\mathrm{LI}_{1}+\mathrm{O}_{2}: \mathrm{LI}_{1}+\mathrm{NO}: \mathrm{LI}+\mathrm{N}_{2} \mathrm{O}: \mathrm{LI}+$

$\mathrm{H}_{0} \mathrm{O}: \mathrm{Li}+\mathrm{NH}_{1}: \mathrm{LI}+\mathrm{CH}_{0}: \mathrm{LI}+\mathrm{H}$ : LI

$+\mathrm{Ho}: \mathrm{LI}+\mathrm{LI} ; \mathrm{N}+\mathrm{H}$ : N + He: N +

LI: $+N$ : $N+0 ; N+H_{2}: N+N=O N$

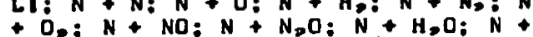

$\mathrm{NH}_{3}: \mathrm{N}+\mathrm{CH}_{0}: \mathrm{O}+\mathrm{H}_{\mathrm{O}} \mathrm{O}+\mathrm{HeO}^{-} \mathrm{O}+$

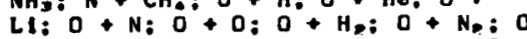
$+\mathrm{O}_{2} \mathrm{O} \mathrm{O}+\mathrm{NO}: \mathrm{O}+\mathrm{N}_{2} \mathrm{O} ; \mathrm{O}+\mathrm{H}_{2} \mathrm{O}: \mathrm{O}$ + $\mathrm{NH}_{3}: \mathrm{O}+\mathrm{CH}_{0}: \mathrm{H}_{2}+\mathrm{H}_{2} \mathrm{H}_{2}+\mathrm{HeO}_{2} \mathrm{H}_{2}+$

LI: $\mathrm{H},+\mathrm{N}: \mathrm{H}_{2}+\mathrm{O}: \mathrm{H}_{2}+\mathrm{H}_{2}: \mathrm{H}_{2}+$

$\mathrm{N}_{p}: \mathrm{H}_{2}+\mathrm{O}_{2}: \mathrm{H}_{2}+\mathrm{NO}_{2} \mathrm{H}_{2}+\mathrm{N}_{2} \mathrm{O}: \mathrm{H}_{2}$

$+\mathrm{H}_{2} \mathrm{O}: \mathrm{H}_{2}+\mathrm{NH}_{3}: \mathrm{H}_{3}+\mathrm{CH}_{4}: \mathrm{N}++\mathrm{H}$ :

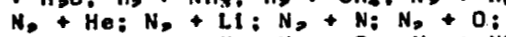

$\mathrm{N}_{0}+\mathrm{H}_{-}=\mathrm{N}_{0}+\mathrm{N}_{2}=\mathrm{N} \mathrm{N}_{2}+\mathrm{O}_{2}=\mathrm{N}_{2}+\mathrm{NO}_{3}$

$\mathrm{N}_{0}+\mathrm{N}_{2} \mathrm{O}: \mathrm{N}_{2}+\mathrm{H}_{2} \mathrm{O}: \mathrm{N},+\mathrm{NH}_{3}: \mathrm{N}+$

$\mathrm{CH}_{0}=\mathrm{O},+\mathrm{H}: \mathrm{O}_{2}+\mathrm{He}: \mathrm{O}_{2}+\mathrm{Ll}: \mathrm{O}$, $\mathrm{N}=\mathrm{O}_{3}+\mathrm{O}_{2} \mathrm{O}_{2}+\mathrm{H}_{3}: \mathrm{O}_{2}+\mathrm{N}_{2}: \mathrm{O}_{2}+$

$\mathrm{O}, \mathrm{O} \mathrm{O}_{2}+\mathrm{NO} ; \mathrm{O},+\mathrm{N}, \mathrm{O}: \mathrm{O},+\mathrm{H}>\mathrm{O} ; \mathrm{O}_{2}$

$+\mathrm{NH}_{3}: \mathrm{O} \mathrm{O}_{2}+\mathrm{CH}_{4}: \mathrm{ND}+\mathrm{H}: \mathrm{NO}+\mathrm{He}:$

$\mathrm{NO}+\mathrm{L}_{1}:$ NO $+\mathrm{N}$ : NO $+\mathrm{O}$ : NO $+\mathrm{H}_{3}$ :

$\mathrm{NO}+\mathrm{N}: \mathrm{NO}+\mathrm{O} ;$ : $\mathrm{NO}+\mathrm{NO}$; $\mathrm{HO}$ -

$\mathrm{N}, \mathrm{O}: \mathrm{NO}+\mathrm{H}_{2} \mathrm{O}: \mathrm{NO}+\mathrm{NH}_{3}: \mathrm{NO}+\mathrm{CH}_{4}:$

$\mathrm{N}=\mathrm{O}+\mathrm{H}: \mathrm{N}=\mathrm{O}+\mathrm{He}$ : $\mathrm{N}>\mathrm{O}+\mathrm{LI}_{2} \mathrm{~N}_{2} \mathrm{O}+$

$N: N_{2} O+O ; N, O+H_{2}: N_{2} O+N_{2}: N=O$ $+\mathrm{O}=\mathrm{N} \mathrm{N}_{2} \mathrm{O}+\mathrm{NO}=\mathrm{N}_{2} \mathrm{O}+\mathrm{N}>\mathrm{O}: \mathrm{N}^{\circ} \mathrm{O}+$ $\mathrm{H}_{0} \mathrm{O}: \mathrm{N}, \mathrm{O}+\mathrm{NH}_{2}: \mathrm{N}, \mathrm{O}+\mathrm{CH}_{0}: \mathrm{H}_{0} \mathrm{O}+\mathrm{H}$; $\mathrm{H}>\mathrm{O}+\mathrm{He}: \mathrm{H}>\mathrm{O}+\mathrm{LI}: \mathrm{H}>\mathrm{O}+\mathrm{N} ; \mathrm{H}, \mathrm{O}+$ $\mathrm{O}: \mathrm{H}>\mathrm{O}+\mathrm{H}>\mathrm{O} \mathrm{H}>\mathrm{O}+\mathrm{N}>\mathrm{H}, \mathrm{C}+\mathrm{O}>$ $\mathrm{H}>\mathrm{O}+\mathrm{NO}: \mathrm{H}>\mathrm{O}+\mathrm{N}>\mathrm{O}: \mathrm{H}>\mathrm{O}+\mathrm{H}_{>} \mathrm{O} ; \mathrm{H}>\mathrm{O}$ $+\mathrm{NH}_{3}: \mathrm{H}_{2} \mathrm{O}+\mathrm{CH}_{4}: \mathrm{NH}_{3}+\mathrm{H}: \mathrm{NH}_{2}+$ $\mathrm{He}: \mathrm{NH}_{2}+\mathrm{LI}_{1}=\mathrm{NH}+\mathrm{N}$ : $\mathrm{NH}_{\mathbf{3}}+\mathrm{O}_{2} \mathrm{NH}$, $+\mathrm{H}_{2}: \mathrm{NH}_{3}+\mathrm{N}_{2:} \mathrm{NH}_{3}+\mathrm{O}_{2}: \mathrm{NH}_{\mathrm{N}}+\mathrm{NO}:$ $\mathrm{NH}_{2}+\mathrm{N}_{2} \mathrm{O}: \mathrm{NH}_{2}+\mathrm{H}_{2} \mathrm{O} ; \mathrm{NH}_{3}+\mathrm{NH}_{3}:$ $\mathrm{NH}_{3}+\mathrm{CH}_{4}: \mathrm{CH}_{0}+\mathrm{H}: \mathrm{CH}_{0}+\mathrm{H}_{0}: \mathrm{CH}_{0}+$ LI: $\mathrm{CH}_{4}+\mathrm{N}: \mathrm{CH}_{+}+\mathrm{O}_{\mathrm{O}} \mathrm{CH}_{4}+\mathrm{H}_{0}: \mathrm{CH}_{*}$ - $\mathrm{N}_{2} \mathrm{C} \mathrm{CH}_{0}+\mathrm{O}_{2}, \mathrm{CH}_{0}+\mathrm{NO}_{1} \mathrm{CH}_{0}+$ $\mathrm{N}_{0} \mathrm{O}: \mathrm{CH}_{0}+\mathrm{H}_{0} \mathrm{O}: \mathrm{CH}_{0}+\mathrm{NH}_{3}: \mathrm{CH}_{0}+$ $\mathrm{CH}_{0}: \mathrm{He}+\mathrm{H}_{\mathrm{O}} \mathrm{He}+\mathrm{He}$

EXP ENERGY TEFEFEACE

OR RANGE

THEOR

Unde 1

Band. Y. E.: Freed. K. F. Lesign ct notural ccliliolon cocidinates to describe discoclaticn cl polyatcolc nolecules. J. Chen. Phys. 68, lasz (1S7E)

United st ot tes

$929-1090$

Sucbo, A. S.: Schulz, P. A.: Grant, E. F.: Shen, Y. R.: Lee, $Y$. I. Multichotcn disoociaticn icoucts froo halogenotid hydrocarbcna. J. Chea. Physe te. 1206 (1578)

UnItad Stotes

HIIdebrondt, G. F.: Kellert, F, G.: [urning, F, B.: Salth. K. A.: Stetbinas. F. F. Ionlzotion ct xench ateos in aelected high fucterg stotes ty collision with

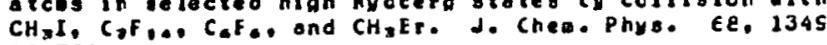
(1578)

United stotes

$0.05-5, v$

Hueng. s, 8.-S.: Freeson. G. F. Electecn acbllitieg in gaseous. critical, and liquid renor: densitx. electric fleld. ond temperature efiecta: oussilccalization. J. Ches. Fhys. E8, 13E5 (1578)

Canada

$300 \mathrm{~K}$

Collins, C, B.: Lee, F. W. Meosuresent of the rate coefricionto for the bimoleculor ord taraclecular lcn-molecule reoctions of res ith alected otcalc and wolocular scectea. J. Chec. Fhyo. 6e.1391 (1578) United states

Unde $\boldsymbol{f}^{\circ}$

Margollogh. De do: Meoth. L. J. Pseudcsoectral dipole osclilator ot rength ditrituticne enc ecee reloted two body interaction ccefficients icr H. He, Li, N, O, Ho, $\mathrm{N}_{2}, \mathrm{O}, \mathrm{NO}, \mathrm{N}_{,} \mathrm{O}, \mathrm{H}, \mathrm{C}, \mathrm{NH}_{3}$, and $\mathrm{CH}_{4}$. J. Chole. Phys. 68. 1426 (1578)

Canoda

Johnson, E, G.: Krener, L. K.: Petral, C. d.: Cross, F. J. Jy. Crcsed teas atudies of D- L, GD + D. J. Chew. Physe $E 6,1444$ (1STE)

United Etotes 


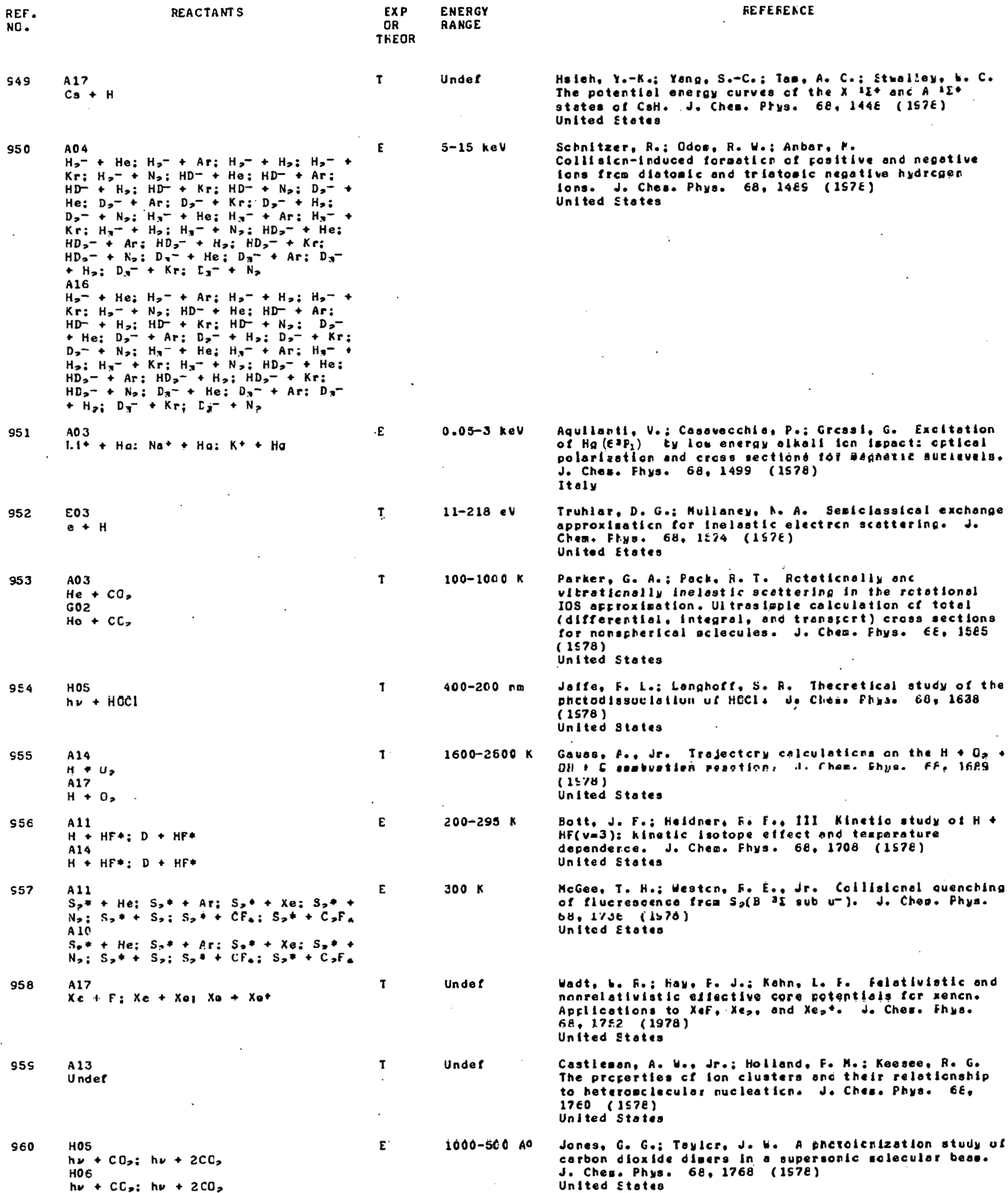

549 A17

950

$\mathrm{HO}_{2}+\mathrm{He}_{2} \mathrm{H}_{2}-\mathrm{Ar}: \mathrm{H}_{2}-+\mathrm{H}_{2}: \mathrm{H}_{2}-$ +

$\mathrm{Kr}: \mathrm{H}_{2}-+\mathrm{N}_{3}: \mathrm{HD}^{-}+\mathrm{He}: \mathrm{HD}^{-}$Ar:

$\mathrm{HD}^{-}+\mathrm{H}_{2}: \mathrm{HD}^{-}+\mathrm{Kr}_{\mathrm{r}}: \mathrm{HD}^{-}+\mathrm{N}_{2}: \mathrm{D}_{2}+$ He: $D_{2}-+A r: D_{2}-K_{H}: D_{2}-+H_{2}:$

$\mathrm{D}=-+\mathrm{N}=\mathrm{H}_{3}^{-}+\mathrm{He}: \mathrm{H}_{3}^{-}+\mathrm{Ar}: \mathrm{H}_{5}^{-}+$

$\mathrm{Kr}: \mathrm{H}_{3}-+\mathrm{H}_{2}: \mathrm{H}_{3}-+\mathrm{N}_{2}: \mathrm{HD}_{2}+\mathrm{He}^{-}$

$\mathrm{HD}_{2}-\mathrm{Ar}: \mathrm{HO}_{3}-\mathrm{H}_{3} \mathrm{O} \mathrm{HD}_{3}-\mathrm{Kr:}$

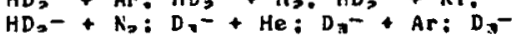

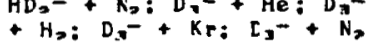

A16

$\mathrm{H}_{2}-\mathrm{He}: \mathrm{H}_{2}-+\mathrm{Ar}: \mathrm{H}_{2}-+\mathrm{H}_{2}: \mathrm{H}_{2}-+$

$\mathrm{Kr}: \mathrm{H}_{0}-+\mathrm{N}_{2}: \mathrm{HD}^{-}+\mathrm{He}^{-} \mathrm{HD}^{-}+\mathrm{Ar}:$

$H D^{-}+\mathrm{H}_{3}: \mathrm{HD}^{-}+\mathrm{Kr}: \mathrm{HD}^{-}+\mathrm{N}_{2}: \mathrm{D}_{2}-$

$+\mathrm{He}_{\mathrm{O}} \mathrm{D}_{2}+\mathrm{Ar}: \mathrm{D}_{7}-\mathrm{H}_{2}: \mathrm{D}_{2}-\mathrm{Kr}$ :

$\mathrm{D}_{2}^{-}+\mathrm{N}_{2}: \mathrm{H}_{3}^{-}+\mathrm{He}_{\mathrm{O}} \mathrm{H}_{3}^{-}+\mathrm{Ar}_{2}: \mathrm{H}_{0}^{-}$

$\mathrm{H}_{2}: \mathrm{H}_{3}+\mathrm{Kr}: \mathrm{H}_{3}-+\mathrm{N}_{3}: \mathrm{HD}_{2}-$ + He:

$H D_{2}-+A C: H D_{2}-+H_{2}: H D_{2}-+K r:$

$\mathrm{HO}^{2}-\mathrm{N}^{2}: \mathrm{D}^{3}+\mathrm{He}: \mathrm{D}^{-}-\mathrm{Ar}: \mathrm{D}^{3}-$

$+\mathrm{H}_{2 i} \mathrm{D}_{3}^{-}+\mathrm{Kr}_{\mathrm{i}} \mathrm{L}_{j}^{-}+\mathrm{N}_{3}$

951

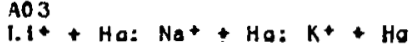

952 EO3

e $+\mathrm{H}$

953

$\mathrm{A03}$

$\mathrm{He}+\mathrm{CO}_{0}$

GO2

$\mathrm{He}+\mathrm{CC}_{7}$

$954 \quad H 05$

hv + HOCl

A14

$4 * U_{2}$

$\mathrm{H}+\mathrm{O}$,

$\$ 56$

H11 $+H F *: D * H F *$

A14

$H+H F *: D+H F *$

$\$ 57$

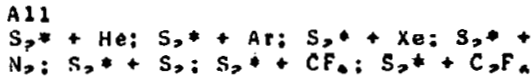
A 10

$S_{0} *+$ He: $S_{0} *+A_{1}: S_{2}+X_{e}: S_{0}+$

958

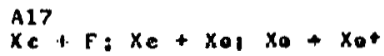

955

A 13

Under

960

E 5-15 keV

$0.05-3 k e v$

$11-218$ eV

$100-1000 \mathrm{~K}$

$400-200 \mathrm{~nm}$

$1600-2600 \mathrm{~K}$

$200-295 \mathrm{~K}$

$300 k$

Unde

Unde?

$1000-500 A^{0}$

Heleh, Y.-K,: Yong, S.-C.: Tan, A, C.: Etwolley,b. C. The potentiol energy curves of the $X$ is onc $A$ iE. states of CoH. J. Ches. Prys. 68, I44E (157E) United stotes

Schnltzer, Ro: Odoe, R. Ho: Anbar, M.

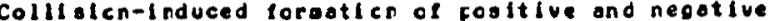
lons Irem diatonfe and triatosic negatlve hydregen lons. J. Chea. Phys. 68, l4ES (ISTE) United States

Aqulisnil, V.: Casovecchio, P.: Greasl, G. Excltation ol Ha (ET) iy low energy olkall icn ispact: cotlcal polarlation and cross gections top papheric uncievels. J. Ches. Fhys. 68,1499 (1578) Italy

Truhlar. D. G.: Mullaney. A. A. Sextclassical exchange approxliaticn for Inelastle electren scattering. Jo approxlaticn 6r, 1574 (1S7E) United Etates

Parker, G. A.: Pack. R. T. Retaticnolly onc vitraticnally inelogt ic scotering in the rctotional IOS afrroxisation. Ul trastople calculation ct totol (differential. integral, and tronsert) cross sections for nonspherical oclecules. J. Chem. Fhys. Ge. 15es ( 1578$)$

United States

Jafte, F. Le: Langhoff, S. R. Thecretfeal otudy of the ohctodissuelalluil uf HeCl, Jo Clitie Fhase 68, 1638 (1578) United states

Gauso. A. Jre. Irojectery calculaticna on the H OP.

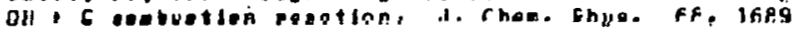
(1578) United states

Bott, J. F.: Heidner, Fi F.. 11I Kinetio atudy ot H $H F(v=3)$ : Kinetle lsotope effect ond terperature dependerce. J. Chem. Fhys.68, 1708 (1578) United statis

McGee, T. H.: Heatch, F. E.. Jr. Colllsicnal quenching of lluereacenco Pras $S_{2}(B$ as oub u-). J. Chod. Phys. bu, $1 \%$ se (is 70 )

United Etateo

Hadt, ho Foy. Fo J.: Kahn, L. F. Felat lulatlc and nonrelativiatic eliective core potentiolo tcr xencn.

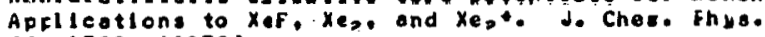
$6 A+1752$ (1978)

United stotes

Castlean, A. H., Jr.: Holland, F. H.: Kesesee, R. G. The preferties ce lon clustera ond their relotionship to heteroecleculor nucleaticn. J. Ches. Phys. GE, $17 \in 0$ (JS7e)

United states

Jones, G. G.: Taylcr. J. H. A Berolenlzatlon study uf carbon dioxide diecr in a supereonic rolecular beas. J. Chem. Phys. 68.1768 (157e) United ctotio 
REF.

REACTANTS

961

DOS + Si: $\mathrm{N}_{2}++\mathrm{SIO}: \mathrm{N}_{2}++\mathrm{S} 10$,

Di?

$\mathrm{N}_{2}+\mathrm{SI}: \mathrm{N}_{2}+\mathrm{S1O}: \mathrm{N}_{2}+\mathrm{S1O}$

562

$\mathrm{HOS}$

hv $+\mathrm{Ha}_{2}$

963

A 14

$\mathrm{La}+\mathrm{H}, \mathrm{C}: \mathrm{La}+\mathrm{D}, \mathrm{O}: \mathrm{Sc}+\mathrm{H}, \mathrm{O}: \mathrm{Sc}+$

$\mathrm{D}, \mathrm{O} ; \mathrm{Y}+\mathrm{H}, \mathrm{O} ; \mathrm{Y}+\mathrm{D}, \mathrm{O}$

964

EO9

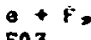

$\mathrm{EO} 3$

S65 H1O

$\mathrm{H} 10$
$\mathrm{hv}$

966

HO2

hv + o,

96

BO 3

FO

$\mathrm{N} *$

S68

$\mathrm{H}^{\mathrm{A}}+\mathrm{LI:H}+\mathrm{Na: \textrm {H } ^ { - }}+\mathrm{Cs}$

s6s

A 10

$\mathrm{Kr}+\mathrm{CC}_{\text {。 }}$

$970 \quad$ A17

Under

971

A14

$\mathrm{CHF}+\mathrm{O}, \mathrm{CHF}+\mathrm{NO}$

572

$\mathrm{Al1}$
$\mathrm{N}, *+\mathrm{SO}_{2}$

$\sin$

A11

$\mathrm{NH}_{\mathbf{3}}+\mathrm{NH}_{\mathbf{3}}$
$\mathrm{AO2}$

$\mathrm{Ar}+\mathrm{I}$

$\mathrm{AO3}_{\mathrm{Br}}^{\mathrm{Br}} \mathrm{I}$

S75

A 06

$\mathrm{Na}+\mathrm{SF}_{a}: \mathrm{Na}+\mathrm{SeF}_{a}: \mathrm{Na}+\mathrm{TeF}_{a}: \mathrm{No}$

$+\mathrm{MoF}_{a}: \mathrm{Na}+\mathrm{HF}_{a}: \mathrm{Na}+\mathrm{ReF}_{a}: \mathrm{Na}+$

IrFa: $\mathrm{N}_{a}+P \mathrm{PF}_{a}: K+\mathrm{SF}_{a}: K+\mathrm{SeF}_{a}:$

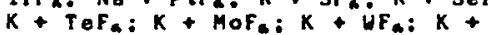

Ref a: K $K$ Irfa: K $+P t F_{a}: C_{s}+S F_{a}:$

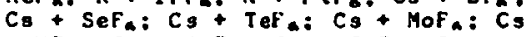

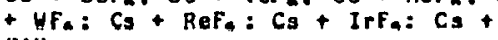

P+F,
E.

$300 K$

\section{EXP ENERGY \\ T HEOR}

$\mathbf{E}$

$30-3000$

$250 \mathrm{~nm}$

1

$0.05-5 \mathrm{eV}$

E

1470-1302

AO

E

T

Undet

.

Undef

Undel

$300 K$

$300 K$

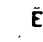

$\varepsilon$

Undef

Unde?

E

$0-40 \mathrm{eV}$
REFEFE NCE

Taylor. J. A.: Lancaster, G. M.: Ianatlev. A.:

Rabalais. J. H. Interocticrs ci lcn teass ith

surfaces. Heocticng ot nitrcgen with sillicon ond lis

oxides. J. Cheo. Ehys. EE. 177E (157e)

United states

Stock. M.: Selth, E, W.: Crullinger, R. E.; Hessel, H. M.: Pourcin, J. Analysis of the decay of aclecular fluorescence in optlcally exclted vereiry vaccr. Je Chew. Fhys. 68, 1285 (1578).

United Stotes

Liu, K.: Parson, J. M. Mechaniana of central D-atoo abotraction reacticns: oclecular teco. loser-incuced tluorescence study of prouk IIIE + HOr systemg. J. Chem. Fhys. EB, Jis4 (ISTE)

Unfted stotes

Hall. F. J. Dissoclative ettocheert ond vitrotlonol exclitation of $f, t y$ slow electrons. J. Chea. Phys. $68,1803(1578)$

Unlted stotes

slanger. T. G.: Elock, G. CO, chatolyolg revialteo. J. Cheo. Phus. $6 E: 1844$ (ISTE)

United states

Mehloon, G.: Ederer, D. L.: Solcoer, E. B. The

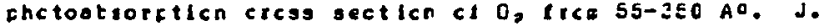
Chem. Pryse EB, IEEC (1STE)

United States

Kocher, C. P.: folrchlld, C. E. Tlae-ct-tlight deterainaticn of rodative cecay rates for hlgh Ryoberg states In otoulc ritrcgen. J. Chea. Fhys. 6e, 1884 (1578)

Untied stotes

Karo, A. M.: Garoner. M. A.; HIskes. J. F. Ab Inltio HC-SCF arcurd-state pctential enercy curves fcr liti. Natt. ond $\mathrm{CaH}^{-}$. J. Chem. Frys. EE. IS42 (ISTE) United states

Scratz, G. C.: Moser, D. D. The laportance of anharacniclty on the rotes cl energy transter in rare oas/CC, syotems. J. Crem. Fhys. Ee. is92 (1ste) United stotes

Gray. S. K.: bright, J. S. Cn the soline inteiccloticn of cotertiol eneroy data. J. Cheo. Frya. EB, zOOZ (1578) United stótes

Lin. M. C. Energy distrituticn in tif trom the resction cf CHF th 0 , ond AD. J. Chea. Phys. 6E, 2004 (1578)

United stotes

Whitson, H. E., Jr.: McNesl, F. J. Quenching of

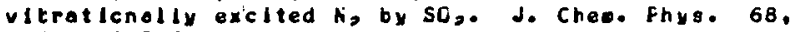
2014 (197E:

United Stotes

Kano, S.: Rerlta. N.: Hanc, I.: Stlalzu. T. Noté on Infrarecolcroweve doutle resonance gtudy cl collisicnal relaxation cf $\mathrm{AH}_{3}$. J. Chell. Phys. 6e, $2020(1578)$

Joran

Falst. M. B.: Bernatein. F. B. Erratue Ccoputational study ci elestic and electicnicaliy irelastic scottering cl Br ty ground atote I atcas: rcle cf potentlal curve crcosing (J. Chea. Fhys.64. 2571 (1576)1. J. Cheor. Fhys. GE, 2022 (1578)

United stotes

Ccapton. R. N.: Relnharot. F. W.: Coocer. C. L. Colllalcnol icnizotion tetween last olhall otoes end gelectec texallucride aclecules. J. Chea. Phya. 68, $20 \leq 3$ (ISTE)

United stotes 


\begin{tabular}{|c|c|c|c|c|}
\hline $\begin{array}{l}\text { REF. } \\
\text { NO. }\end{array}$ & REACTANT $S$ & $\begin{array}{l}\text { EXP } \\
\text { CR } \\
\text { THEOR }\end{array}$ & $\begin{array}{l}\text { ENERGY } \\
\text { RANGE }\end{array}$ & $\begin{array}{l}\text { FEFEFENCE } \\
\qquad\end{array}$ \\
\hline S7E & E05 He & E & $300-500 \mathrm{eV}$ & 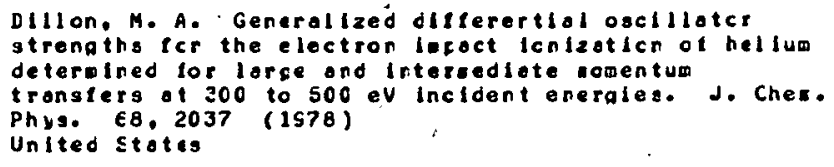 \\
\hline s77 & $\begin{array}{l}\text { HoS } \\
\text { Under }\end{array}$ & $\tau$ & Undef & 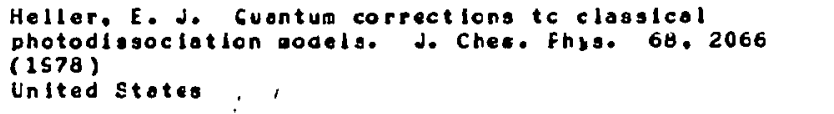 \\
\hline s78 & 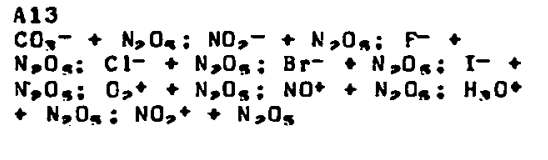 & $\mathbf{E}$ & $300 \mathrm{~K}$ & 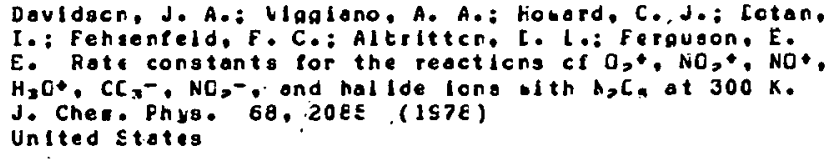 \\
\hline 970 & $\begin{array}{l}\text { A10 } \\
\text { Br: }+H>0 \\
A 11 \\
B=+H>0\end{array}$ & E & $300 \mathrm{~K}$ & 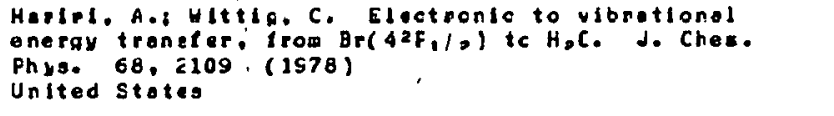 \\
\hline 980 & $\stackrel{A 17}{M g}+M a$ & $\mathbf{T}$ & Undéf & 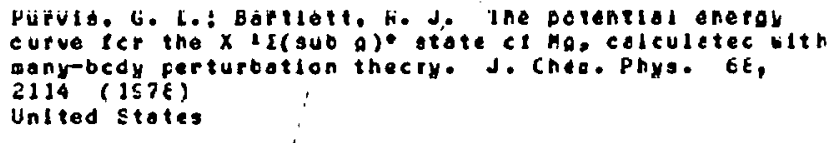 \\
\hline 981 & $\mathrm{EO2}_{\mathrm{e}}^{\mathrm{H}} \mathrm{H}$ & 1 & $100-2000 \mathrm{eV}$ & 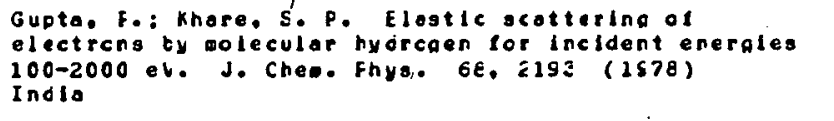 \\
\hline 982 & 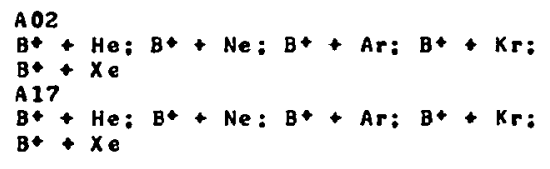 & $\mathbf{E} \mathbf{T}$ & $4-64$ ov & 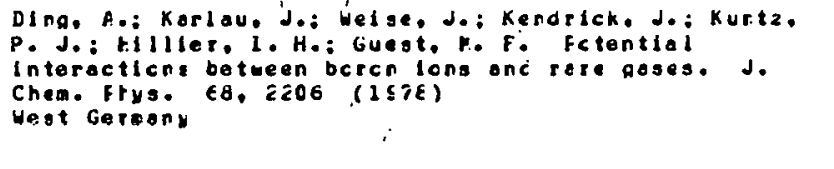 \\
\hline 983 & 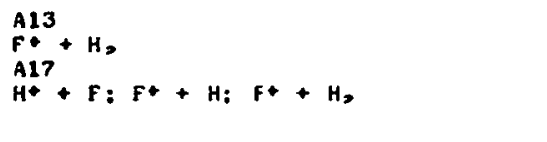 & 1 & $300 \mathrm{~K}$ & 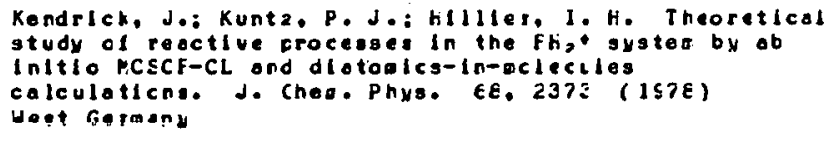 \\
\hline 584 & $\begin{array}{l}H 05 \\
h v+K r=0 \\
\text { K } 17 \text { r } \\
K r+\end{array}$ & $\mathbf{E}$ & $\begin{array}{l}7993-4680 \\
A^{0}\end{array}$ & 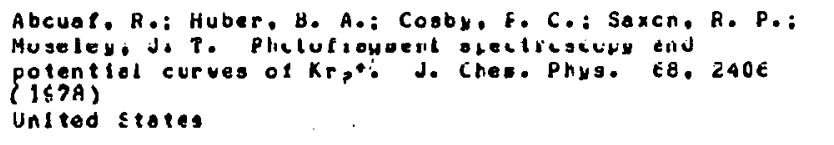 \\
\hline 985 & $\begin{array}{ll}A 14 \\
C I\end{array} D_{3}$ & 1 & $\begin{array}{l}4-15 \\
k<a 1 / 001\end{array}$ & 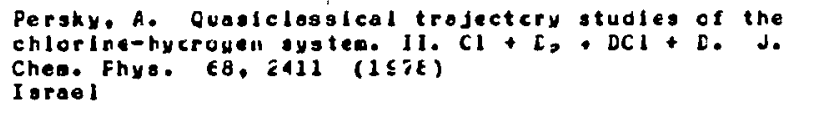 \\
\hline 986 & 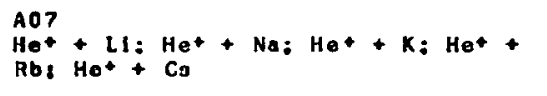 & 1 & $10-100 \mathrm{keV}$ & 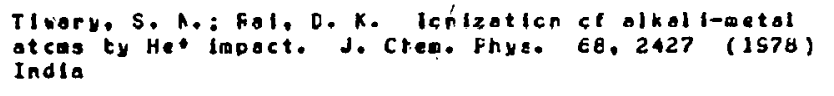 \\
\hline 987 & $\underset{U F_{a}}{D 09}+C: U F_{a}+P t_{i} U F_{a}+U$ & $\mathbf{E}$ & $300 \mathrm{~K}$ & 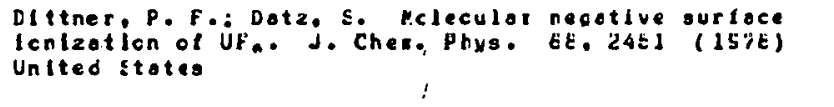 \\
\hline 800 & $\stackrel{H}{H}+\{\mathrm{IH}+\mathrm{HI}$ & $\mathbf{T}$ & Undef & 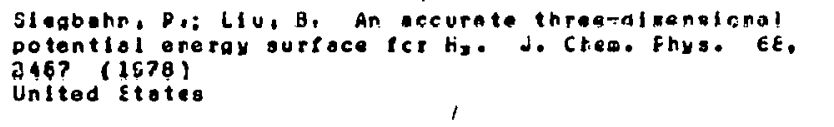 \\
\hline 989 & $\begin{array}{l}\mathrm{A} 27 \\
\mathrm{H}\end{array} \mathrm{H}$ & $T$ & Under & 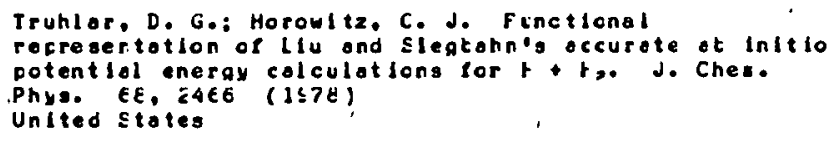 \\
\hline 950 & $\begin{array}{l}\mathrm{HOG} \\
\mathrm{hv}+\mathrm{Na}\end{array}$ & $E$ & $515-458 \mathrm{nt}$ & 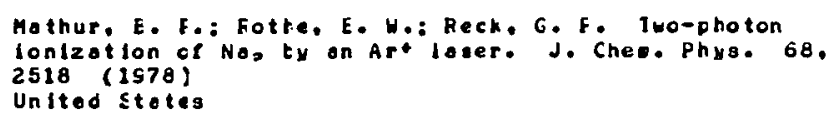 \\
\hline
\end{tabular}


REF.

REACTANT S

NO.

991

$\mathrm{HO}_{4}^{+}+\mathrm{H}_{2} \mathrm{O}: \mathrm{O}++\mathrm{H}_{2} \mathrm{O}: \mathrm{N}++\mathrm{H}_{2} \mathrm{O}: \mathrm{O}_{2}+$

$+\mathrm{H}_{2} \mathrm{O}: \mathrm{Ar}^{+}+\mathrm{H}_{2} \mathrm{O}$

\begin{tabular}{|c|c|}
\hline 992 & $\begin{array}{l}\mathrm{All}_{\mathrm{N}} \\
\mathrm{NH}_{3}+\mathrm{NH}_{3} \\
\mathrm{AOZ}_{3} \\
\mathrm{NH}_{3}+\mathrm{NH}_{3}\end{array}$ \\
\hline 993 & $\begin{array}{l}\mathrm{Al}_{17} \\
\mathrm{HO}_{2}+\mathrm{H}_{2}: \mathrm{H}_{2}+\mathrm{HCl} \\
\mathrm{H}_{2}+\mathrm{H}_{2}: \mathrm{H}_{2}+\mathrm{HCl}\end{array}$ \\
\hline 994 & $\begin{array}{l}\mathrm{HOB} \\
\mathrm{hu}+\mathrm{Na}= \\
\mathrm{A} 17 \text {. } \\
\mathrm{Na}+\mathrm{Na}\end{array}$ \\
\hline 995 & $\begin{array}{l}\mathrm{A}_{13} \\
\mathrm{~N}_{4}^{+}+\mathrm{C}_{2}: \mathrm{N}_{4}+\mathrm{CO}_{3}: \mathrm{N}_{4}+\mathrm{H}_{3}: \mathrm{N}_{4}+ \\
+\mathrm{O} O 6 \\
\mathrm{~N}_{4}++\mathrm{N}_{2}\end{array}$ \\
\hline
\end{tabular}

956 . HOS

$\mathrm{H}_{\nu}^{\mathrm{HO}}+\mathrm{HCN}: \mathrm{h \nu}_{\nu}+\mathrm{ICN} ; \mathrm{h \nu}+\mathrm{CO}$,

997

A13

Undef

SS8

A14

$\mathrm{N}_{2}+\mathrm{CO}_{2} \mathrm{~N}_{2}+\mathrm{NO}: \mathrm{N}_{2}+\mathrm{CH}_{4}: \mathrm{N}_{2}+$

$\mathrm{CO}_{3}: \mathrm{CO}+\mathrm{CO}$

AOS

$\mathrm{N}_{2}+\mathrm{CO}: \mathrm{N}_{2}+\mathrm{NO}: \mathrm{N},+\mathrm{CH}_{4}: \mathrm{N}_{2}+$

$\mathrm{CO}_{3}: \mathrm{CO}+\mathrm{CO}$

A 07

$\mathrm{N},+\mathrm{CO}: \mathrm{N}_{2}+\mathrm{NO}: \mathrm{N},+\mathrm{CH}_{4}: \mathrm{N},+$

$\mathrm{CO}_{3}: \mathrm{CO}+\mathrm{CO}$

004

$\mathrm{N}$, + $\mathrm{Au}: \mathrm{CO}+\mathrm{Au}$

$999 \quad H 06$

hv + NO

1000

E09

$+N>0$

1001

$\mathrm{Al}^{\mathrm{Al}}+\mathrm{H}: \mathrm{Al}+\mathrm{H}^{+}$

T

Unde $f$

1002

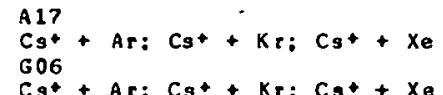

1003

HOS

$\mathrm{hr}+\mathrm{CH}$

HOG

$\mathrm{h} \nu+\mathrm{CH}$

1004
T

$\mathbf{T}$

E

E

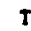

$5-30$ ev

H08

A.7

Undef

av
EXP ENERGY

CF RANGE

E

$300-1307 k$

. $200-14000 \mathrm{~cm}^{-1}$

$5145-47 \epsilon E$

$0.04-0.16$

Unde?

A 14: $A 06$ :

A 07

50-1000 eV

$\mathrm{CO} 4$

22-12Co ev

$1337-1232$

AO

$300 \mathrm{~K}$

$\mathbf{E}$

A 14

$\mathrm{O}+\mathrm{H}$,
E Under

t 5-85 eV
FE F EREN C E

Nuasbaua. G. Hi.: Cothera. A. F. Production of the excltec CH raolical in collitalcng of E0-250 ket Het, 0 t, $\mathrm{N}, \bullet, 0, *$, ord $A r^{*}$ Ions ath $H_{2} C$ nolecules in water vafor. J. Chem. Phys. 6E, 25 ál (157E) United stotes

Duquette, G.: Elllo, T. H.: Scolea, Ge: Watts, R. O.: Kleln. M. L. An interecleculor cctential lor (NiH) J. Chen. Fhys. 68, 2544 (1578) Canada

Hell. I. E.: Green, S.: Kourl, D. de The coupled stated opfrcxiaticn fer scettering ot two clatcose. J. Chem. Frys, EB, 2SE2 (1STE)

United ctotes

Kusch, P.: tiessel. M. M. Ar onelvel, ce the B loloub u) $-x$ I I (sub g)t tand syated cl Nes. J. Chem. Phys. $68,2551(1578)$

United States

LIndinger, t. : Eotan, I.: Albrittcr, L. L.: Feheenfeld, F. C. Feocticna of $\mathrm{N}_{4}+$ with $\mathrm{C}_{2}, \mathrm{CO}_{2}, \mathrm{H}_{2}$, and $\mathrm{C}_{2}$ and mobilities of $\mathrm{N}^{+}$in initropen. J. Chea. Phys. 68 , 2607 (1578)

Unlted stotes

Bond, Y. B.: Morae. M. D.: Freed, K. F. Coaporiscn of sealcloislcal treatments fcr evolueting franck-condon tronsiticn eapiltudes for molecular disocioticn.J. J Chea. Phys. EQ, z702 (1S7E)

Unted states

Verbool, G. M. L.: Melgel., G. C. Angular Eomentum distritutions in collition frocesses and the lccaticn of the trensition atote in lon-bolecule reacticns.J. Chew. Fhys. 68. 2714 (1578) United Stotes

Ut tertock, N. G.: van Zyl, E. Low energy Icnizing colllalcng tetween $N_{2}$ and CE tase nclicules onc CE. Na,

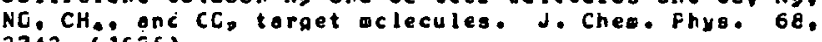
2742 (ISTE)

Unlted states
Mescher, E.: Lee, Y. T.: Curtler, P. Autolonization structure of nitric oxide (ND) at the firat Icnization lieit. J. Chea. Phys. 6e, $27 E 3$ (ISTE) Sulter land

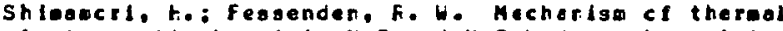
electron attacheent in $N, C$ and $N, D-h y d r o c a r b c n$ ixtures in the gas Fhase. J. Chea. Phys. 66. 2757 (1578) Unltad stateo

Sotelli, N. H.: Kortor, M.: Benedeh, R.: Glitert, 1. L. SCF potential curves for $A$ IH and $A 1 H+$ in the ottroctlve and refulal ve reglens. J. Chete. Fhyse 68, 2767 (1578)

United stotes

Thackstcn, M. G.: Fope, W. M.: Eloele, F. L.: Ellla, H. W.: Meconiel. E. H. Moblitities anc interoction potentials for $\mathrm{Cs}^{+-A r}, \mathrm{Cg}^{4}-\mathrm{Kr}$, and $\mathrm{Cs}^{*}-\mathrm{X}_{e}$. J. Chese Phys. $6 E$, 2775 (1578)

Unlted troies

Barsuhn, J.: Nesbet, F. $K$. The chctolcnlzotlon and photodissociation of $\mathrm{Ch}$ in the vicinity of the Ionlzation threshcld. J. Ches. Fhys. E8, 2783 (1978) United Stotes

Light. G. C. The etect ol vibrational excltation on the reaction of $O(O P)$ with $H$, and the ditribution of vibraticnal energy in the croouct Ch. J. Chea. Phys. $68.2831(1978)$

United stotes 


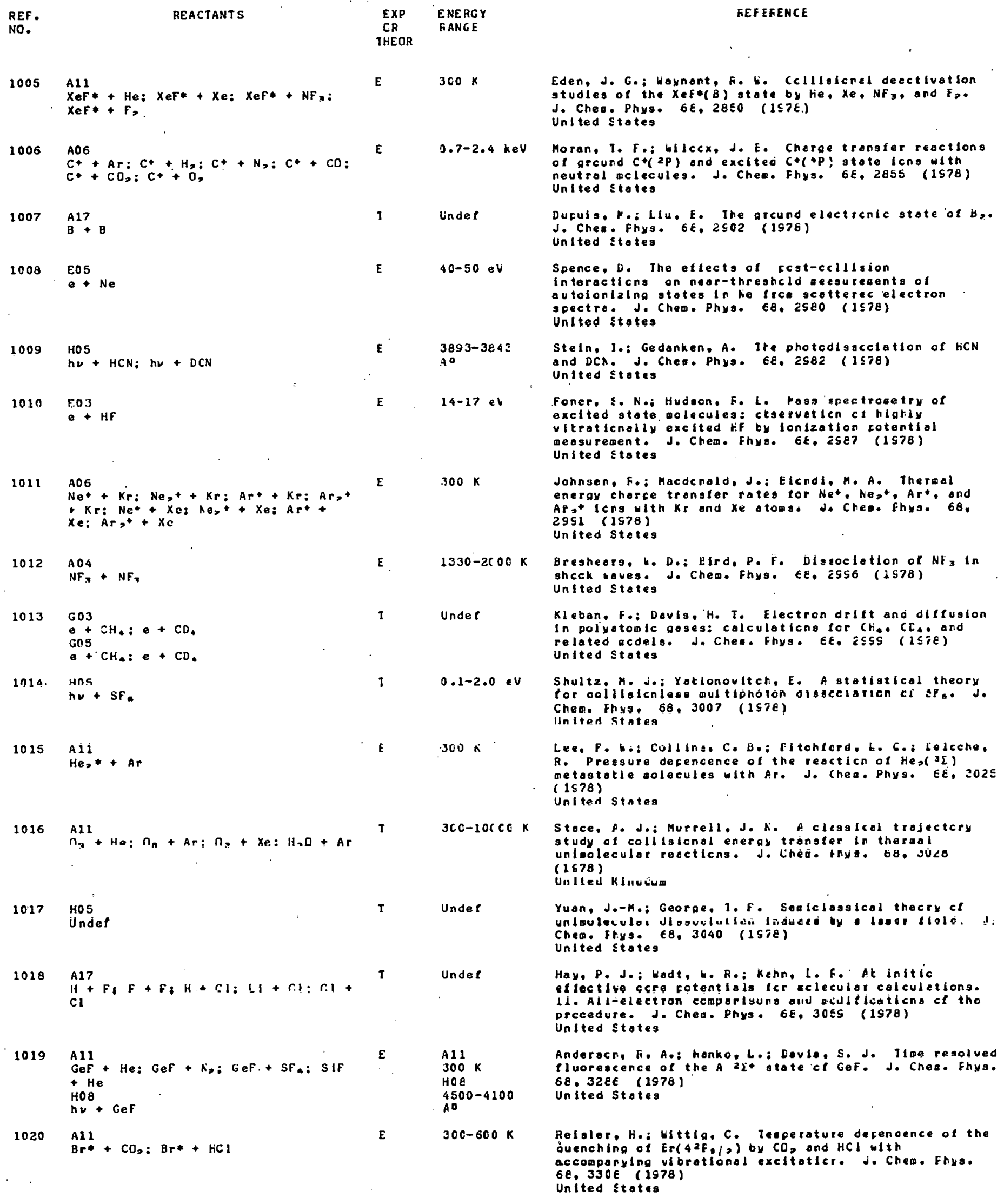




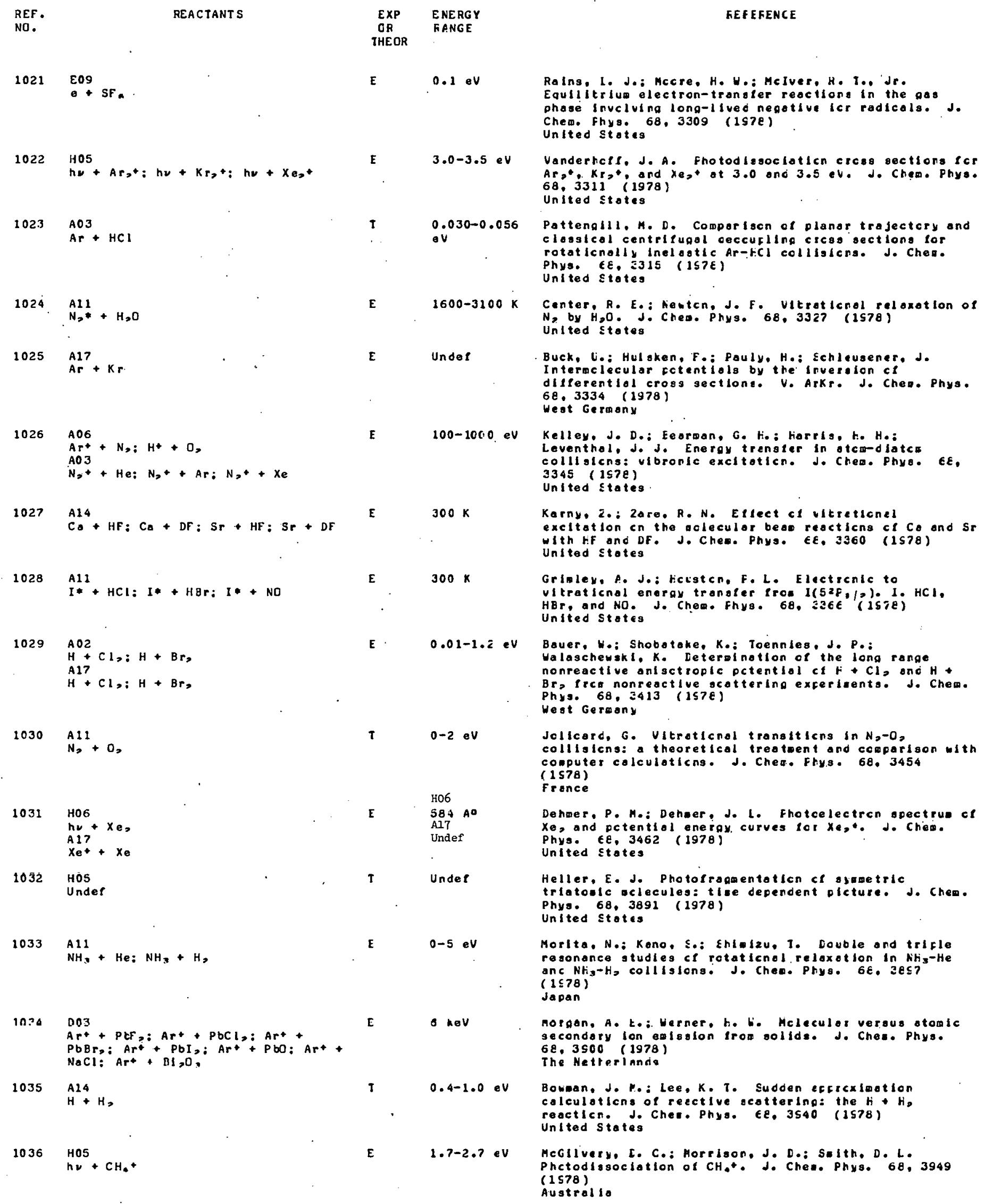


REF.
NO.

REACTANT S

1037

$\mathrm{CO}_{3}+\mathrm{Ar}: \mathrm{Cs}_{3}+\mathrm{Kr}_{\mathrm{C}} \mathrm{Cs}_{3}+\mathrm{XO}_{0}$

$1038^{\circ}$ Allo

1039 FO1

Cd: $\mathbf{H a}$

$1040 \quad$ A17

$1041 \mathrm{H} 10$

$\mathrm{HLO}_{\mathrm{HW}}+\mathrm{HN},+\mathrm{Hg} \mathrm{j}$

$\mathrm{Ha}^{\mathrm{AO}}+\mathrm{N}_{3}$

1042 A14

A14 H,

2043

A14

Br $+0_{\text {* }}$

1044

AL 7 .

HON*

hv + HCN

$1045 \quad 602$

$\mathrm{CH}+\mathrm{Ar}: \mathrm{O}+\mathrm{He}$

$2046 \quad$ A21

$\mathrm{Ha}_{0}=+\mathrm{Hg}$

1047 HOS

ho $+S F_{*}$

1040 ${ }^{\text {HOS }}$

1049 A02

Under

1050

A06

$\mathrm{He}^{2+}+\mathrm{He} \mathrm{He}^{2+}+\mathrm{Ne} \mathrm{He}^{2+}+\mathrm{Ar}$

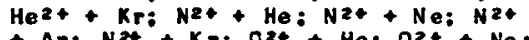

$+\mathrm{Ar}: \mathrm{N}^{24}+\mathrm{Kr}^{2+} \mathrm{O}^{24}+\mathrm{He}: \mathrm{O}^{2+}+\mathrm{Ne}$;

$\mathrm{O} 2++\mathrm{Ar}: \mathrm{O}^{24}+\mathrm{Kr}_{\mathrm{r}} \mathrm{Ne}^{24}+\mathrm{He}^{2} \mathrm{Ne}^{2+}$

$+\mathrm{Ne}: \mathrm{Ne}^{2+}+\mathrm{Ar}: \mathrm{Ne}^{2+}+\mathrm{Kr}: \mathrm{Ar}^{2+}+$

He: Ar2t + Ne: Ar2t + Ar: Ar2t+

Kr: Ar") + Hol Arde + Ma: Ar3t

Ar: Ar ${ }^{3+}+K_{r}: K_{r 2+}+H_{e}: K_{r 2+}{ }^{2+}$

Ne: $K_{r}{ }^{2+}+A_{r}: K_{r}{ }^{2+}+K_{r}$

1061 $\begin{array}{cc}\underset{1 X P}{\text { EXPERGY }} & \text { ENERG } \\ \text { CR } & \text { RANGE } \\ \text { THEOR } & \end{array}$

E : $\quad 0.02-4.7 \mathrm{eV}$

TUnde?

TUnder

T Undef

H10.

$H 10$
363.7 क
$A 07$

$300 \mathrm{~K}$

$3.300 \mathrm{~K}$

$200-360 k$

Under

$295 \dot{k}$

473-1072K

944 1/ea

$266 n$

5

$\mathbf{T}$

Undet

$\mathbf{E}$

$$
0-40 \text { eV }
$$

FEF EFENCE

Thacksten, H, G.: Elsele, F, Le: Pcpe, W. H.: Ellis, H. W.: McDaniel, E. H. Further testo of the generallzed El natoln relation: $C^{*}$ long in Ar. Kr. and Xe. J. Chen. Fhys. 68, 3950 (197e)

United stotes

Altlck, P. L.: ShIn. H. K. A model calculaticn of vibraticn-translaticn onergy transfer. J. Cheo. Fhys. $68,3973(1578)$

United stotes

Basch, H.: Neuton, M. D.: Jolrl, J.: Hcokobltz, J. h.: Toclol. S. Etfectlve ccre cotentiolo ict the codalue and aercury atoos. J. Chea. Phys. EE. 400E (ISTE) United statea

Penderaost. P.: Hoyes, E. F. All-electron ncneaplrical study of the grcund stoto CI MgF.. J. Chem. Frys. 68, 4022 (1978)

Unitod Etotes

Deant. A.; Rosenteld, Eo: Yotalive E. Vibroticnal

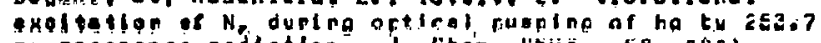
au rescnance radiotion. J. Chét Hriga. 6e.404l (1578)

Iorae 1

Garrett. B. C.: Miller, W. H. Cuontug wechonlcol reactive ecotterins vid exchange kernelo: opolicaticn to the collinear $H+H$, reaction. $J$. Ched. Fhys. 68, 4051 (1978)

United stotes

Michael, J. V.I Lee, J. H.: Payne, L, A.: Stlet, L. J. Absolute rote of the reocticn ci broblre atcms ilth ozone trom 200 to $360 \mathrm{~K}$. J. Cheo. Physe ER. 40S3 (1578)

United states

Henioul. J. P.: Gellay, C.: Lcrouet, J.C. Anomalous eflects in photoelectron spectrcocepy. The thith. electrcnic otote of HCNt. J. Cheo. Fiys. GB, 4ios (1578)

Belglue

Judelkio, H. S.: bun. H. heagureaent ce chiogine oton diffugten. J. Chea. Fhys. 6E. 41Ë (1978) United states

Stuch, Po: 2alth, E, Wel Drullingor, F, E.: Hesegl, M. H. Reloxation of the ifirst ancltec $1($ ab u) stote of Hgp. J. Ched. Fhys. 66. 4167 (IS7E) United stotes

Bado, P.; von den Bergh, H. Fresouro dependence in the multipheten dlssocloticn cl i2EF. J. Choe. Fhyo.' 6E, $41 E 8$ (1978)

Sultzerland

K.rnger, P. M.: RIlev. S. J.: Kmel, G, h. Pclyhallde phetor gaguent spectra. II. Citgoviclet ohotodlasoclation oynanios ct Uf.. J. Chea. Phys. oe, 4156 (IS7E)

United States

Budenhelzer, F. E.: Gislascr. E. A, Clesslcal differentiol croso sectlons lor anisotrople pctentiols. J. Choo. Phyo. 9A, 4222 (157E) Unlted Stotes

Maler. H. B.. II Stewart, E. Elactrce tronatio in ccllistcno ce doutly chorged atoric icrs ith rare-gas atcas ecr pileary-icnenergies telcw $100 \mathrm{eV}$. J. Chem. Phys. 68, 4228 (1978)

Unlted States

Das. G.: Wahl, A. C.: Zemke, W. T.: Stwalley, b. C. Accurate ab initio potential curves fcr the $x=$ isub

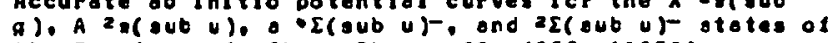
the $0_{2}-$ icn. J. Chem. Fhys. E6, 125a (157e) United Etotes 


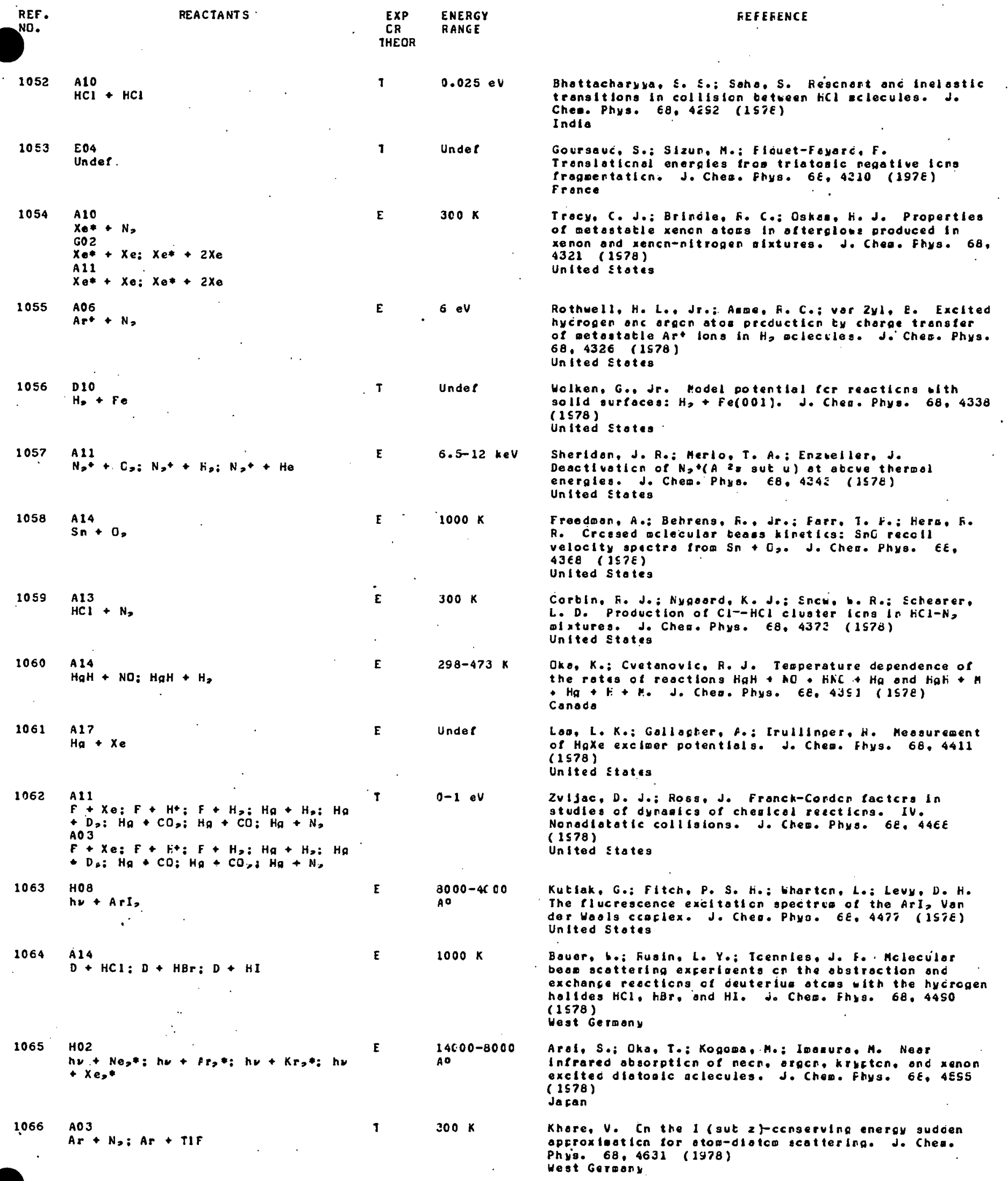




\begin{tabular}{|c|c|c|c|c|}
\hline $\begin{array}{l}\text { REF. } \\
\text { NG. }\end{array}$ & REACTANTS & $\begin{array}{l}\operatorname{EXP} \\
\text { OR } \\
\text { THEOR }\end{array}$ & $\begin{array}{l}\text { ENERGY } \\
\text { RANGE }\end{array}$ & FEFEFEACE \\
\hline 1067 & $\begin{array}{l}E 16 \\
i+\left[F_{2}+A r l: e+\left[F_{2}+K r l: e+\right.\right. \\
{[C l=A r l: e+[C l,+X e]}\end{array}$ & $\varepsilon$ & $500 \mathrm{~K}$ & 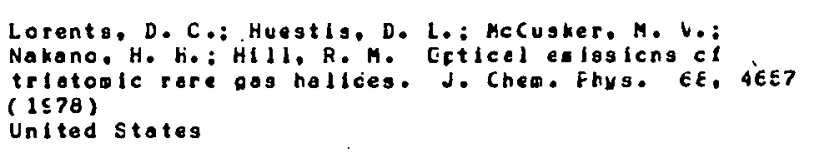 \\
\hline 1068 & $\begin{array}{c}\mathrm{Al}_{2},+\mathrm{H}: \mathrm{Ne},+\mathrm{H}^{+}: \mathrm{Ne}+\mathrm{H}: \mathrm{Ne}+\mathrm{H}^{*} \\
\text {. }\end{array}$ & 1 & Undee & 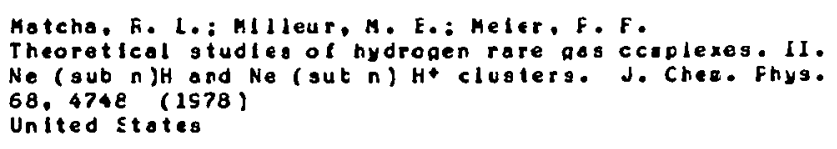 \\
\hline 1069 & $\begin{array}{l}\text { HOS } \\
\text { he }+0 .+\end{array}$ & E & $1.6-2.8 . \mathrm{eV}$ & 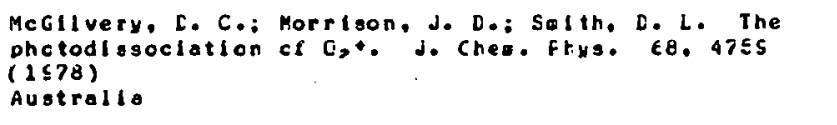 \\
\hline 1070 & $\begin{array}{l}\mathrm{CO}_{4} \\
\mathrm{Cs}_{3}+\mathrm{He}: \mathrm{Cs}^{+}+\mathrm{Ne} \\
\mathrm{COS}^{+}+\mathrm{He}: \mathrm{Cs}^{+}+\mathrm{Ne}\end{array}$ & $\varepsilon$ & $0.04-10 \mathrm{eV}$ & 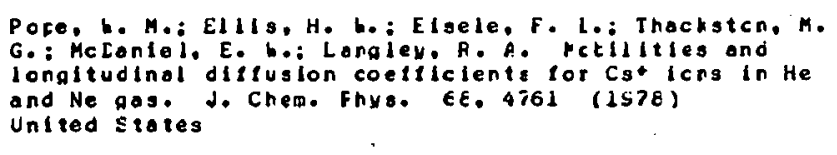 \\
\hline 1071 & $\mathrm{H}_{\mathrm{He}}+\mathrm{Ar}: \mathrm{He}+\mathrm{Kr}: \mathrm{He}+\mathrm{Xe}$ & $T$ & Under & 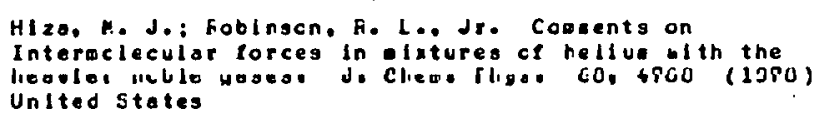 \\
\hline 1072 & $\begin{array}{l}A 17 \\
H+B r=\end{array}$ & $\mathbf{T}$ & Unde? & 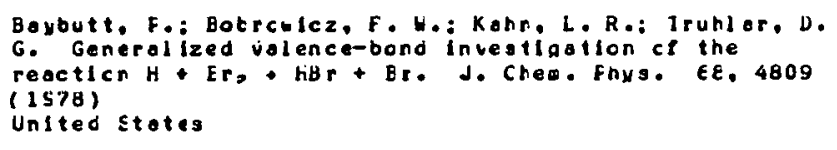 \\
\hline 1073 & 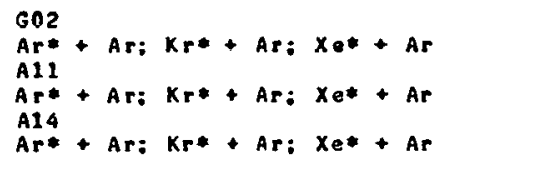 & F & $300 \mathrm{~K}$ & 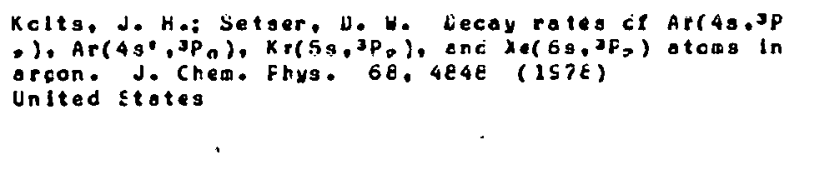 \\
\hline 1074 & $\mathrm{~A}^{\mathrm{A}+}+\mathrm{OH}-$ & $T$ & Under & 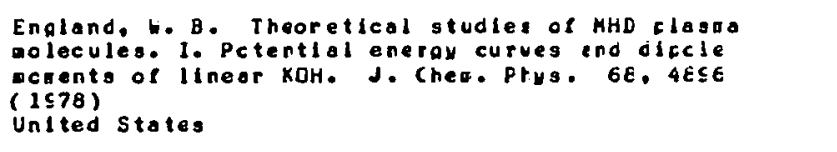 \\
\hline 1075 & $\begin{array}{l}\mathrm{AOS}^{0}+\mathrm{C}_{2} \\
\mathrm{O}_{2}++{ }_{2}\end{array}$ & $E$ & $1-40$ eV & 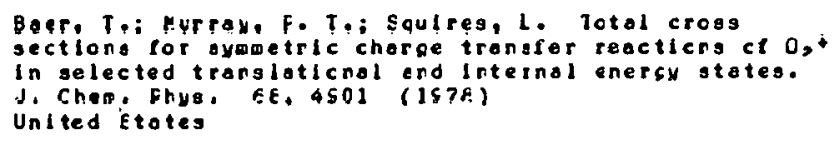 \\
\hline 1076 & 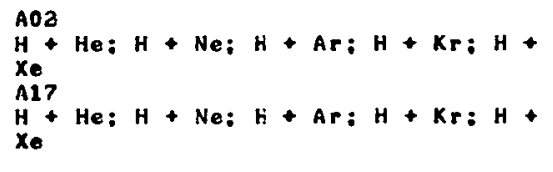 & $T$ & Under & 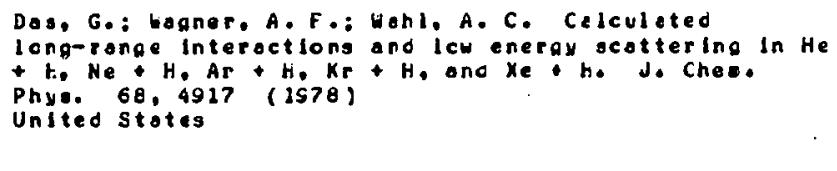 \\
\hline 1077 & 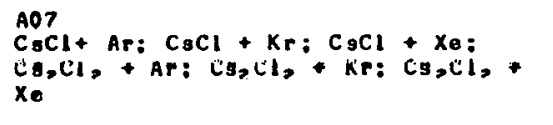 & E & $3-18$ eV & 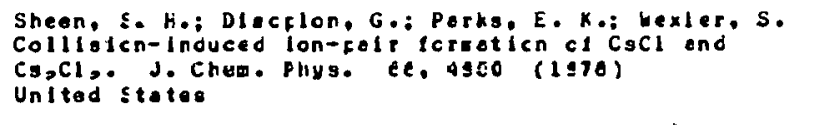 \\
\hline 1070 & $\hat{A R P}+N$ & 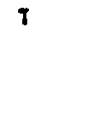 & Under & 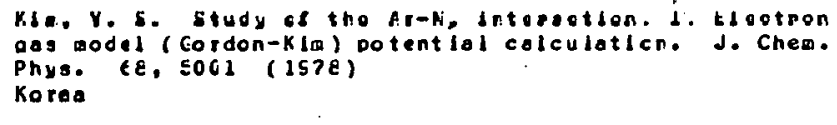 \\
\hline $1 n 70$ & $\operatorname{Hng}_{\mathrm{Hin}}^{\mathrm{H}}+\mathrm{Na}$ & F. & $1487 \mathrm{eV}$ & 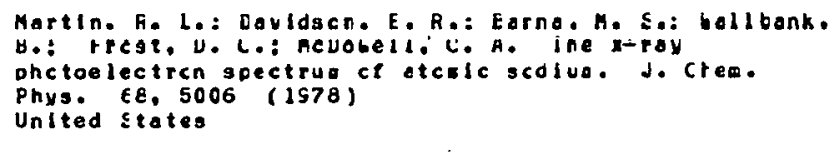 \\
\hline 1080 & $\begin{array}{l}\mathrm{Al7}_{\mathrm{H}}+\mathrm{He}: \mathrm{HCl}+\mathrm{Ar}: \mathrm{ClF}+\mathrm{Kr} \\
\mathrm{H}\end{array}$ & $\tau$ & Undef & 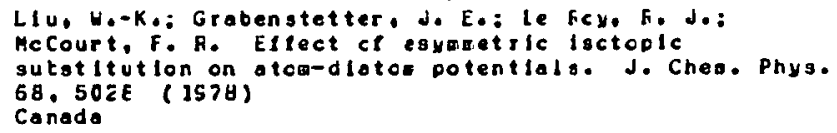 \\
\hline
\end{tabular}


REF

REACTANTS

NO

108

A 07

He* + He*

E

$0.01-10 . v$

1082

$\stackrel{A 11}{H F}+\mathrm{HF}$

T

1083

A11

$\mathrm{HF} * \mathrm{HF}$

$\tau$

1084

E16

$e+[A r+X e+F>1$

1085

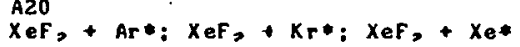

E

1086

A11

$\mathrm{HCl}+\mathrm{Cl}$

1087

he + Ar o*

1088

1089

A1I

$\mathrm{Ar}_{r *}+\mathrm{H}_{2} \mathrm{O}: \mathrm{Ar} *+\mathrm{C}>\mathrm{O}: \mathrm{Kr} *+\mathrm{H}_{2} \mathrm{O}$

A17

$\mathrm{He}^{*}+\mathrm{He}_{\mathrm{He}}+\mathrm{He}_{\mathrm{H}} \mathrm{He}+\mathrm{Ne}: \mathrm{He}^{*}+$ Ar: $\mathrm{He}^{*}+\mathrm{Kr}: \mathrm{He}^{+}+\mathrm{Xe}_{e}$

1090

A03

$\mathrm{He}+\mathrm{H}$,

$1091 \quad H 08$

ho $+\mathcal{C d}$,

1092

A13

$\mathrm{Ne}^{+}+\mathrm{Ne} \mathrm{Ar}^{+}+\mathrm{Ar}: \mathrm{Kr}_{+}^{+}+\mathrm{Kr}_{\mathrm{r}} \mathrm{Xe}^{+}+$

$x e$

1093 A11

$\mathrm{OH}^{*}+\mathrm{He}^{-} \mathrm{OH}_{*}+\mathrm{Ar}: \mathrm{OH}_{*}+\mathrm{H}_{2}: \mathrm{OH}_{*}$ $D_{D}: \mathrm{OH}_{*}+\mathrm{N}_{2}: \mathrm{OD} * \mathrm{He}: \mathrm{OD*}+\mathrm{Ar:}$ $O D *+H_{3}: O D *+C_{2}: O D * N_{2}$

$1094 \mathrm{Al}_{\mathrm{BC}}+\mathrm{H}_{2}$

$1095 \quad A 16$

$\mathrm{Cl}_{1}+\mathrm{NO}+\mathrm{N}$,

1096

A 23

$\mathrm{O}-+\mathrm{HCl}_{2} \mathrm{O}_{2}-+\mathrm{HCl}^{-} \mathrm{NO},-+\mathrm{HCl}_{2}$

$\mathrm{CO}_{3}+\mathrm{HCl}^{2}: \mathrm{CO}_{4}-+\mathrm{HCl}: \mathrm{ClO}^{-}+\mathrm{NO}:$

$\mathrm{ClO}^{+} \mathrm{NO}, \mathrm{C} \mathrm{ClO}^{-}+\mathrm{SO}_{3}: \mathrm{ClO}^{+}+\mathrm{CO}^{\circ}$

ENERGY

PANGE

$300-3500 \mathrm{~K}$

$200-1000 x$

$100-1000 \mathrm{~K}$

$650-1050$

$\mathrm{m} / \mathrm{s}$

I Undef

$300 \mathrm{~K}$

$200-400 K$
FEFEFERCE

eynaber. R. H.: Magnugon. G. D.: Tang, S. X Cheol-ienizotion in collis lens cl wetostatie hellum w th netastoble helluo. J. Chen. Fhys. 68, E112 (1578)

Unlted States

Poulsen, L. L.: Bllling, G. D.; Etelnteld, J.I. Teoperature dependence of HF vibraticnal relaxoticn. J. Ches. Phys. 68, 5121 (157e)

United stotes

Bllling, G. D.: Fculsen, L. L. Thecry ce $V-V$ ond $V-T / R$ eneray tranafer for HF $(n=1$ to 7$)+H F(0)$. J. Chea. Phys. 68, 5128 (1978)

Dernark

Tellinghulsen, J.: Tellinghulsen, F, G.: Tlsone, G. C.: Hoffran, d. H.: Hoys, A. K. Epectrosccplc tudies of diatoric noble gas halides. II. Anolyala of $x \in f$ atoo AC band syotem. J. Ches. Fhys. 6e,E177 (1578) United states

Tellinghulsen. P. C.: Tellinghulsen, de: Coxone d. A.: Velazce, J. E.: Setser. D. W. Scectrcocoplc studies os diotomic notle gos halides. IV. Vibraticnal and rotaticnal constants $f(x$ the $X$, B. And $D$ stetes ol Xef. J. Chec. Fhys. 68, 5187 (1578) United states

ShIn. H. K. Ironoler ot vibrational eneray tc

osclilatory, restricted roteticnol. onc tronslotional ootion in $\mathrm{HCl}+\mathrm{Cl}$. J. Crea. Phys. EE. S2EE (157e) Unlted Etates

Reselgno, I. N.: Hezl. A. U.: Crel. A. E. Calculation of the chotcicnizotion cross gecticn ct the iz (aubu) exclmer stote of Ar,. J. Cheo. Phys. 68.5283 (197E) United ctates

Sheldcn, J. H.: Muschlitz. E. E.. dre Guenching crcss

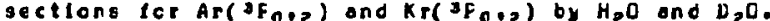
J. Cheo. Phys. 68 , $526 e$ (ISTE) Unt ted states

Prcetor. T. F.: Stwolley. b. C. The lcng-ranpe Interacticns of wetastable heliue ators (2 $2 \mathrm{~s}, 2 \mathrm{as}$ ) it th rare gos and hydrcgen tcoo. J. Ches. Fhys. 68 . 5252 (1578)

United stotes

Olgen: C. P.: Wortell, H. A. Inelogtic scattering at moderate collisicn energies. Je Chee. Fhys. EE. 5294 (1578)

United stotes

Drullinger, F. E.: Stock. H. The $C c_{0}$ excleer:

lluorescence tand shape and decay rates. J. Cheo. Phys. Ce, E2ss (157E)

Unltod stotes

Hele, H.; Varney, F. N: On the cenversion ce otoric rare aos lons $X \cdot\left(2 P_{1} / 0\right)$ into rare gas dimer lcna. $J$. Ches. Fhys. 68, 5301 (1578)

Austrio

Cenoel, F, K.: Crosiey. D. R. Energy tranofer in 2 I OH. II. Vibrational. J. Chea. Fhya.68,5309 (1978) United stotes

Kivel. E. Theory for BCl, th, laser induced chedistiy. J. Chea. Phys. 68, 637e (1978) United stotes

Lee, J. H.: Mlehael, J. U.: Poyne, W. A., Jro: Stief, l. J. The temperature dependence cl the rate conatont

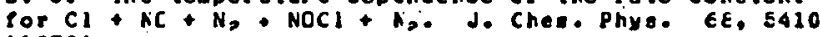
(1578)

United stotes

Dotan, 1.: Albritton, D. L.: Fehrenteld, F. C.: Streit, G. E.: Ferguacn. E. E. Rate ceratants lor the

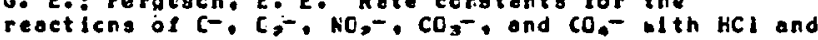

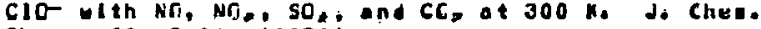

Phre. 68; 5414 (1628)

United stotes 


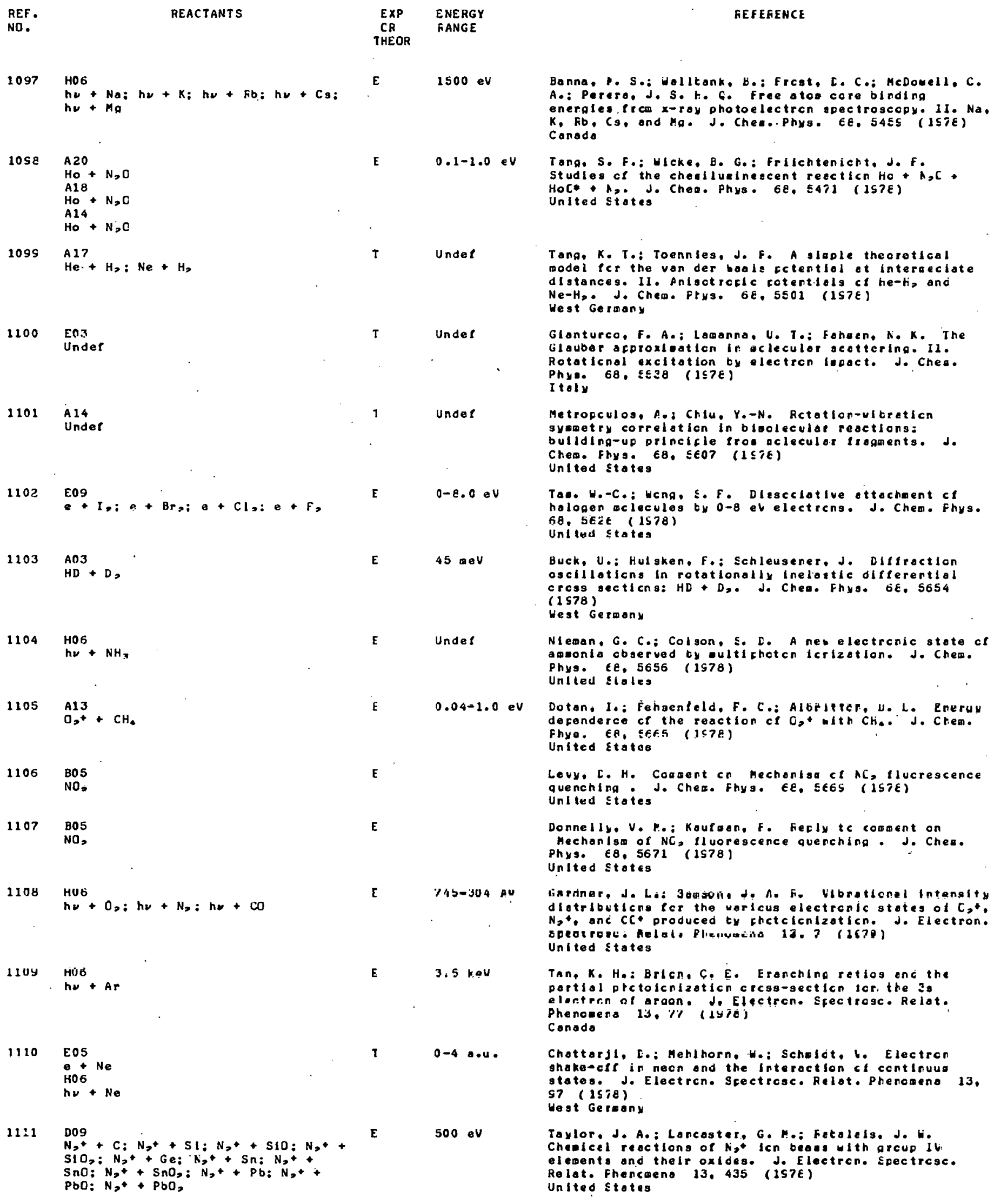




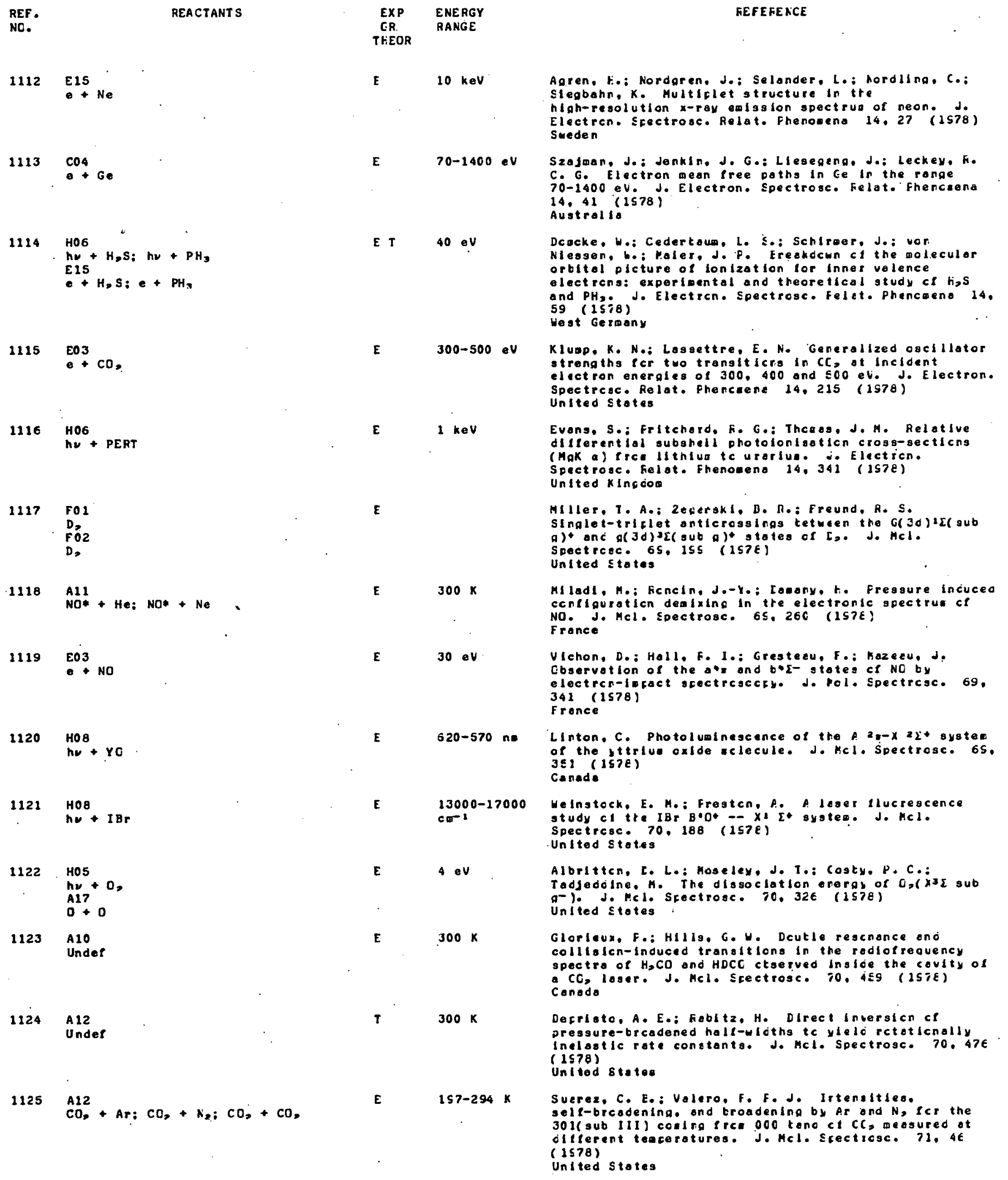




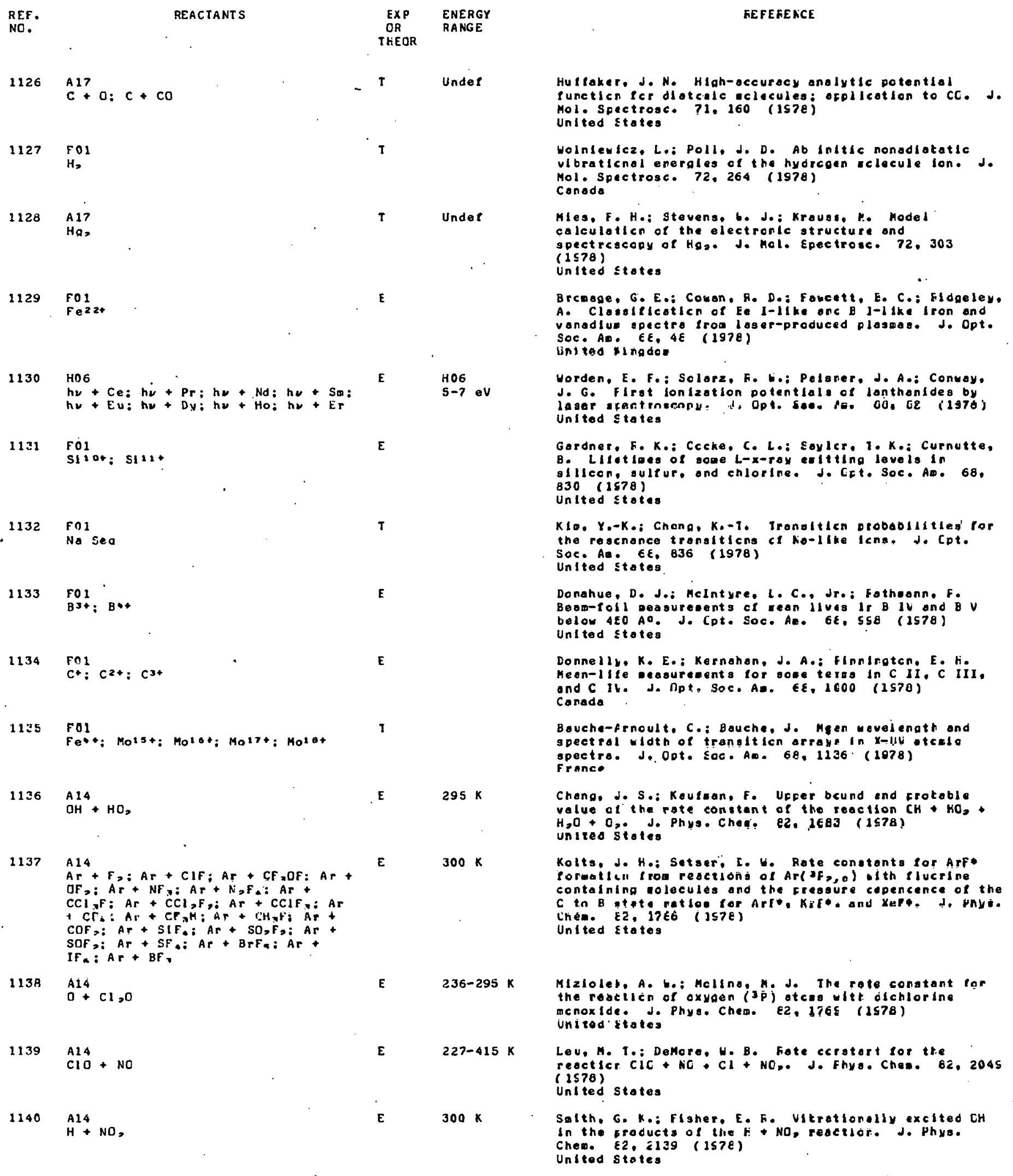




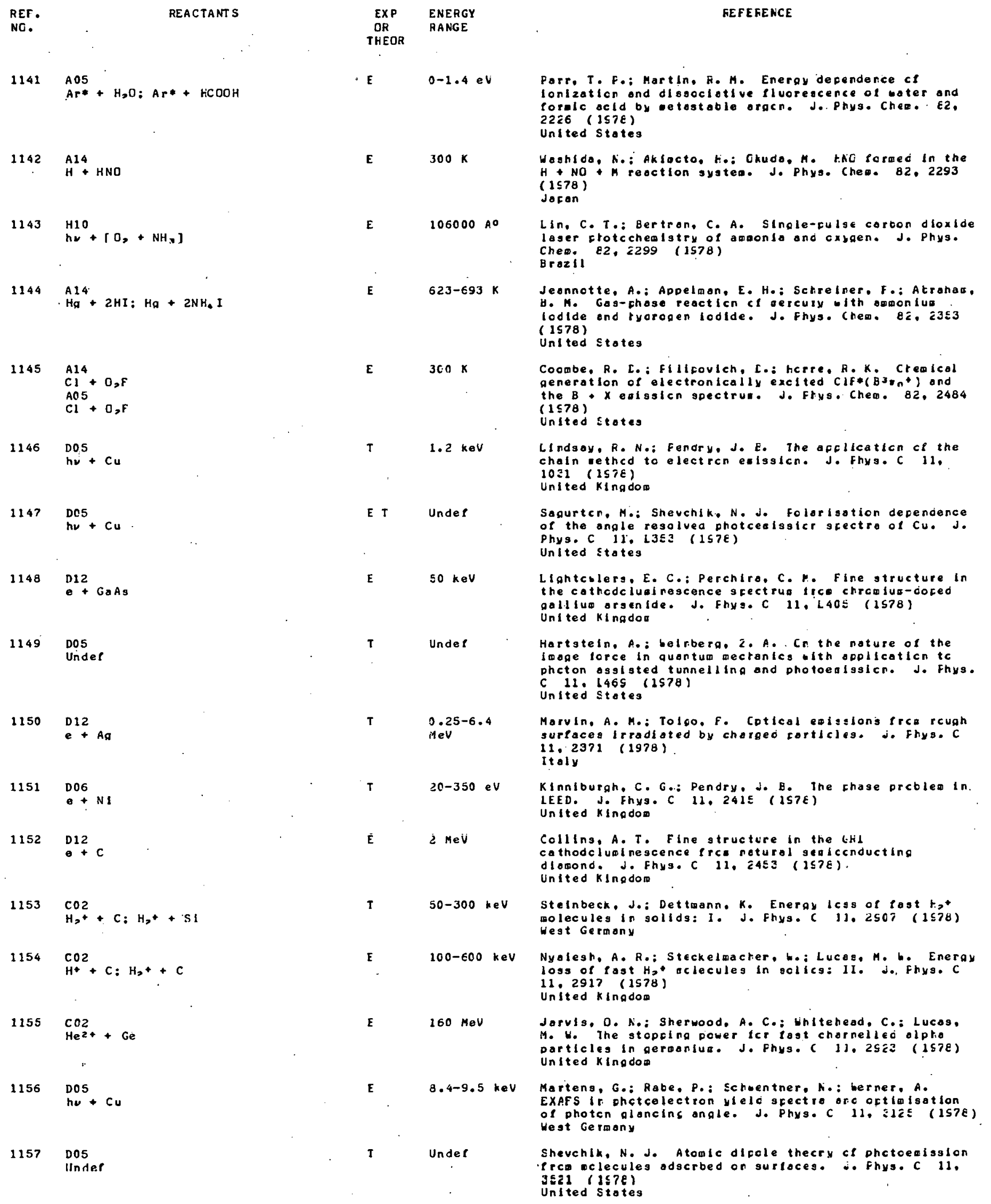




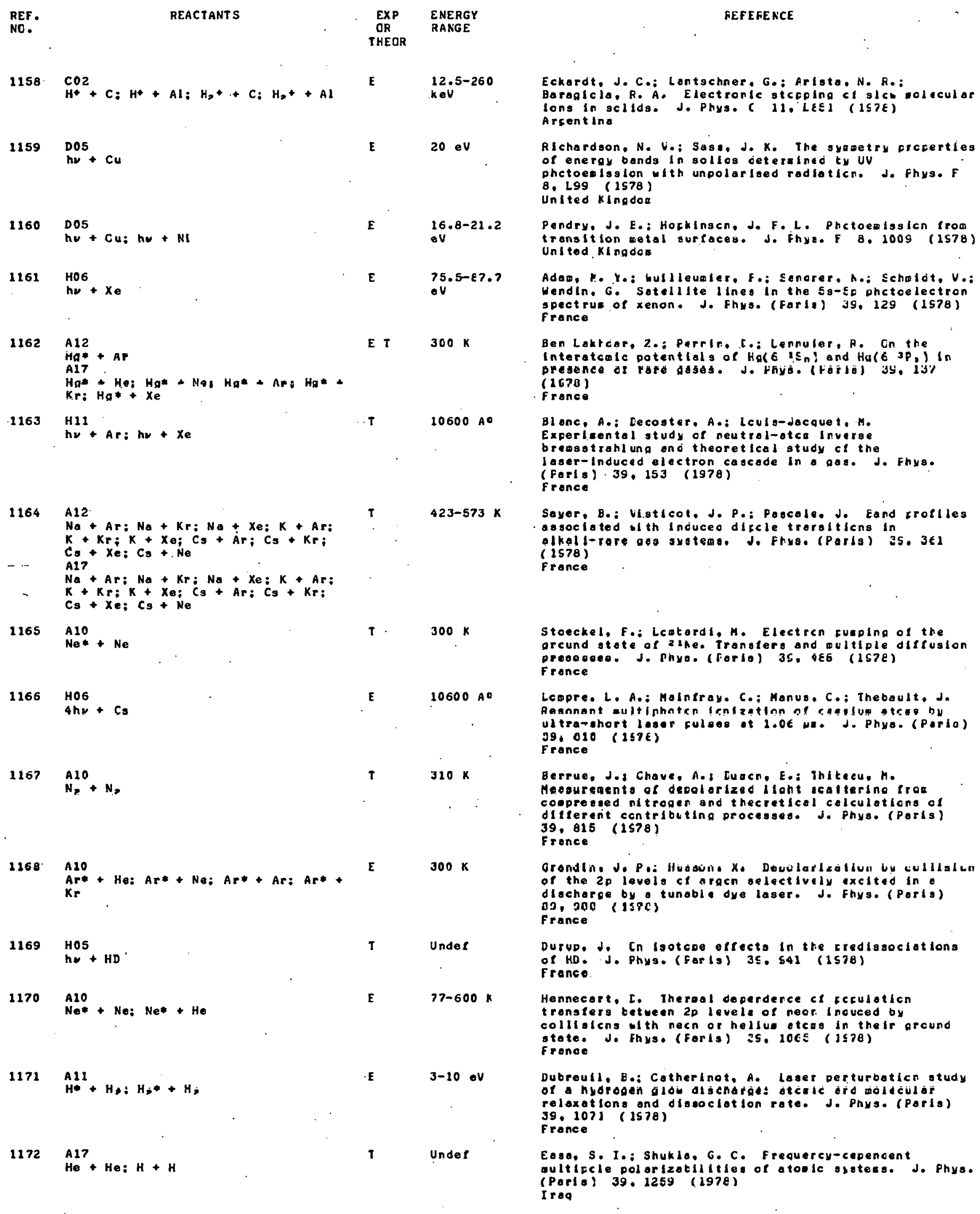




\begin{tabular}{|c|c|c|c|c|}
\hline $\begin{array}{l}\text { REF. } \\
\text { NO. }\end{array}$ & REACTANTS & $\begin{array}{l}\text { EXP } \\
\text { OR } \\
\text { THEOR }\end{array}$ & $\begin{array}{l}\text { ENERGY } \\
\text { FANGE }\end{array}$ & FEE EFENCE \\
\hline 1173 & $\begin{array}{c}603 \\
e+A r\end{array}$ & 1 & $\begin{array}{l}0.046-0.010 \\
\text { Id }\end{array}$ & 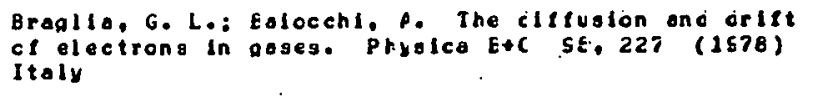 \\
\hline 1174 & $\begin{array}{l}\text { Alo } \\
\text { Undet }\end{array}$ & $\mathbf{T}$ & $300 \mathrm{~K}$ & 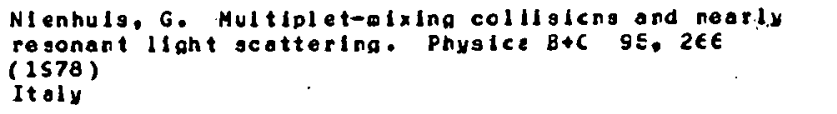 \\
\hline 1175 & $\begin{array}{l}\text { El1 } \\
\text { Under }\end{array}$ & $\mathbf{T}$ & Under & $\begin{array}{l}\text { Geltwan. S. Stimulated wultiphoton breagstrahiung In } \\
\text { electrcn-icn collisions. J. Res. Eur. Stond. Fhys. } \\
\text { Chem. E2, } 173 \text { (1S77) } \\
\text { United States }\end{array}$ \\
\hline 1176 & $\begin{array}{l}013 \\
e+r F+S S 1: e+[H+S S !\end{array}$ & $\mathbf{E}$ & $0.2-6.0 \mathrm{keV}$ & 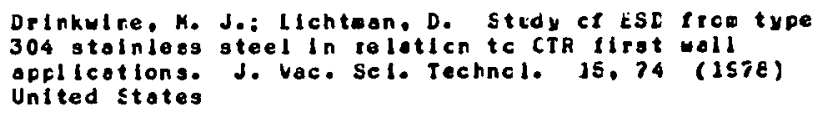 \\
\hline 1177 & 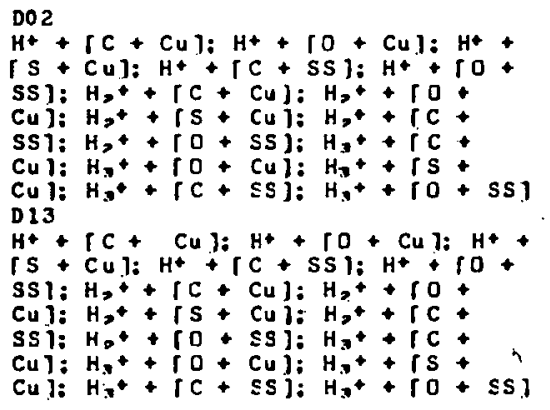 & $\mathbf{E}$ & $0.6 \mathrm{kaV}$ & 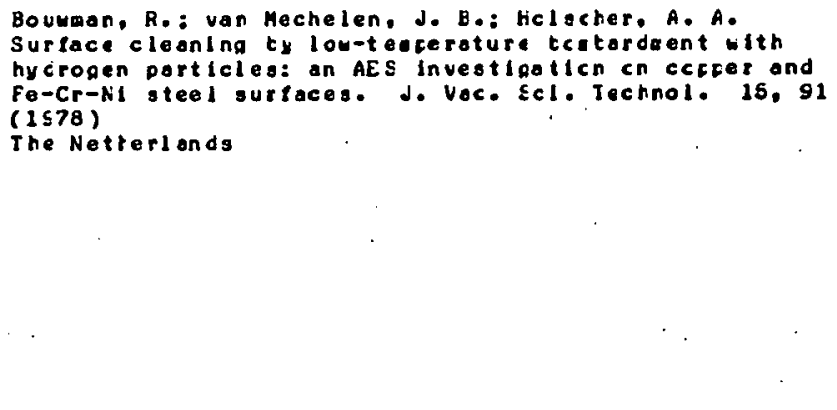 \\
\hline 1178 & $\begin{array}{l}\mathrm{De} 7 \\
\mathrm{He}+\mathrm{Pt}: \mathrm{O},+\mathrm{Pt}: \mathrm{CO}_{2}+\mathrm{Pt}_{\mathrm{t}}\end{array}$ & $E$ & $300 \mathrm{~K}$ & 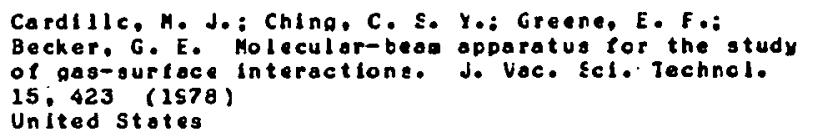 \\
\hline 1179 & $\begin{array}{l}\text { DOS } \\
h_{\nu}+[\mathrm{CO}+\mathrm{NiT} \\
\mathrm{HOG}_{\mathrm{H}}+\mathrm{CO}=\mathrm{h}_{2}+\mathrm{N}_{2}\end{array}$ & $\mathbf{T}$ & $\begin{array}{l}\text { DOS } \\
21 \text { eV } \\
\text { HO6 } \\
14-16 \text { eV }\end{array}$ & 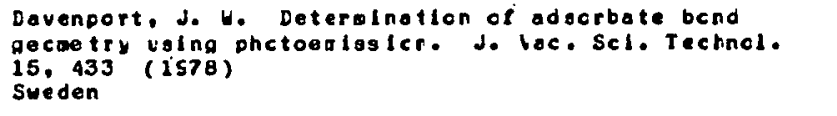 \\
\hline 1180 & $\begin{array}{l}\mathrm{DO4}+\mathrm{TIC}: \mathrm{C}+\mathrm{TI}: \mathrm{T}+\mathrm{Al}: \mathrm{e}+\mathrm{Fe} \\
\mathrm{DOG} \\
e+\mathrm{TIC}: \dot{\mathrm{C}}+\mathrm{TI}: \mathrm{e}+\mathrm{Al}: \mathrm{e}+\mathrm{Fe}\end{array}$ & $\mathbf{E}$ & $0-600 \mathrm{eV}$ & 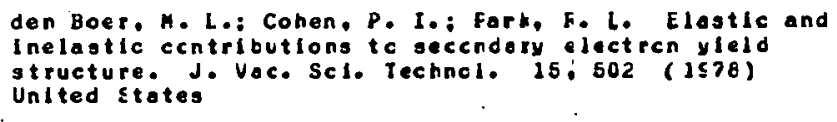 \\
\hline 1181 & $\begin{array}{l}\mathrm{DO3} \\
\mathrm{Ar}+\end{array}+\left[\mathrm{O}+\mathrm{MO}^{+}\right]: \mathrm{Ar}^{+}+\mathrm{CO}+\mathrm{Col}$ & E & J & 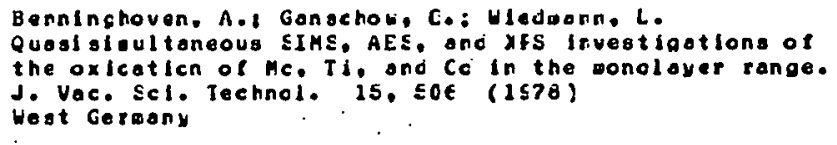 \\
\hline 1182 & $\begin{array}{l}D 15 \\
H^{+}+N 1: H+C U: C 11: H++N b: n+H 11\end{array}$ & $E$ & $10-26 \mathrm{MeV}$ & 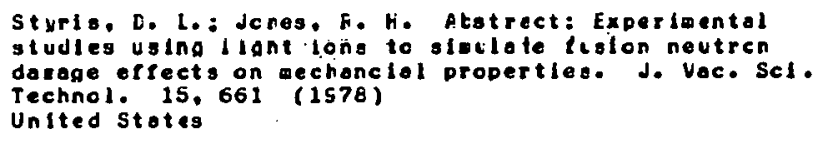 \\
\hline 1183 & $\mathrm{Ne}^{+}+\mathrm{PC}+\mathrm{HI:Ne+}+\mathrm{HO}+\mathrm{MOI}$ & E & $\begin{array}{l}0.15-2.0 \\
k \in V^{-1}\end{array}$ & 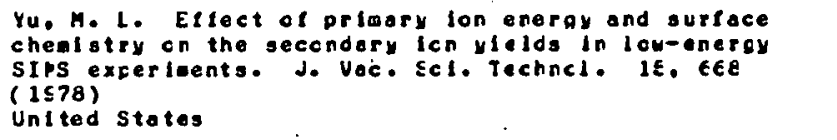 \\
\hline 1184 & $\begin{array}{l}\mathrm{Dl} \\
\mathrm{He}+\text { + } \mathrm{Mo}\end{array}$ & $E$ & $75-350 \mathrm{keV}$ & 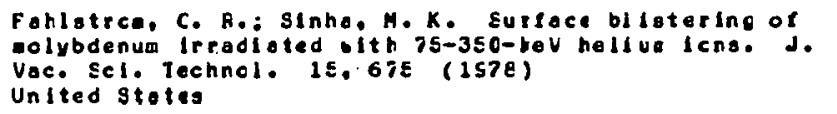 \\
\hline 1185 & $\begin{array}{l}\mathrm{DO} 2 \\
\mathrm{Ar}+\mathrm{Cu}\end{array}$ & E & $1-3 \mathrm{keV}$ & $\begin{array}{l}\text { Lundaulst, T. R. Eneray distributions ce scuttered } \\
\text { copoer neutrals and lons. d. Voc. Scl. Technol. 15, } \\
684 \text { (IS78) } \\
\text { United stotes }\end{array}$ \\
\hline 1186 & $\begin{array}{l}\mathrm{DOZ} \\
\mathrm{He}^{+}+\mathrm{Ta}_{\mathrm{O}} \mathrm{O}: \mathrm{He}^{+}+\mathrm{CuO}\end{array}$ & E T & $0.5-3.0 \mathrm{keV}$ & 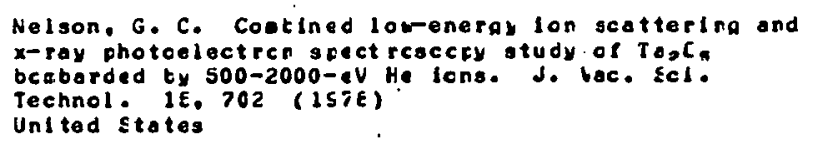 \\
\hline
\end{tabular}




\begin{tabular}{|c|c|c|c|c|}
\hline $\begin{array}{l}\text { REF. } \\
\text { NC. }\end{array}$ & REACTANTS & $\begin{array}{l}\text { EXP } \\
\text { OR } \\
\text { THEOR }\end{array}$ & $\begin{array}{l}\text { ENERGY } \\
\text { RANGE }\end{array}$ & REFERE ACE \\
\hline 1187 & $\begin{array}{l}\text { D 16 } \\
D^{*}+S S\end{array}$ & $E$ & $7 \mathrm{keV}$ & 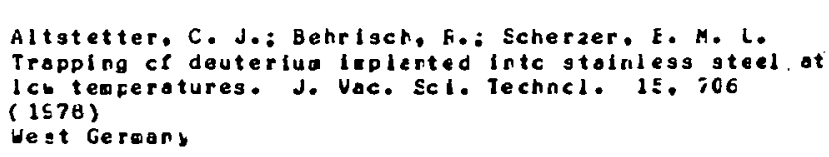 \\
\hline 1188 & $\mathrm{DL}^{\mathrm{D}}+\mathrm{MO}$ & $E$ & $40-120 \mathrm{keV}$ & $\begin{array}{l}\text { Dos, S. K.: Kamingky. M.: Lusza, P. Surfoce oonace of } \\
\text { molybdenum ond T2M alloy under Dt impoct J. lace Sci. } \\
\text { Technol. } 15,710 \text { (197e) } \\
\text { United Stotes }\end{array}$ \\
\hline 1189 & $\mathrm{Ar}^{\mathrm{O}} 2+\mathrm{Fe}_{\mathrm{O}}$ & $\mathbf{E}$ & $1-15 \mathrm{keV}$ & 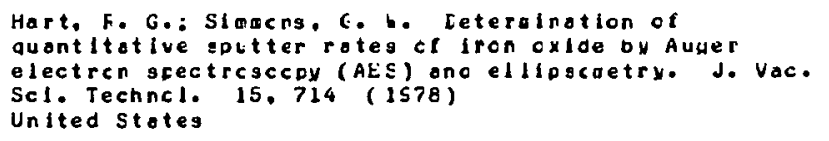 \\
\hline 1290 & $\begin{array}{l}\mathrm{D} 16 \\
\mathrm{Cr}^{+}+\mathrm{Fe}: \mathrm{Cu}^{+}+\mathrm{Fe}\end{array}$ & $\cdot E$ & $150 \mathrm{keV}$ & 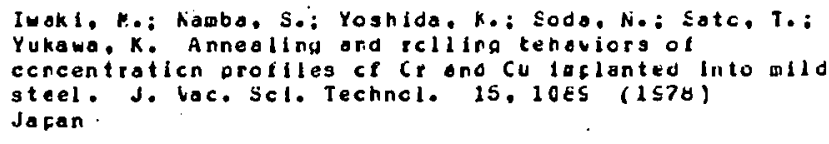 \\
\hline 2192 & 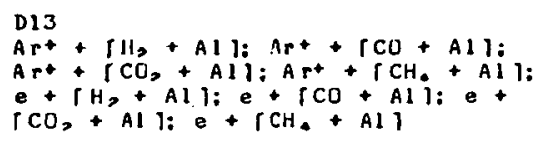 & $\varepsilon$ & $0.5-1.0 \mathrm{kèV}$ & 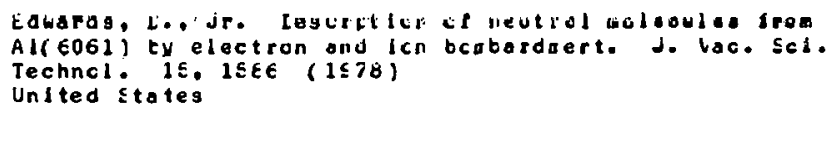 \\
\hline 1192 & $\begin{array}{l}\mathrm{D} 16 \\
\mathrm{Au}+ \\
+\mathrm{Fe}: \mathrm{Au}^{+}+\mathrm{Cu}\end{array}$ & $E$ & $150 \mathrm{keV}$ & 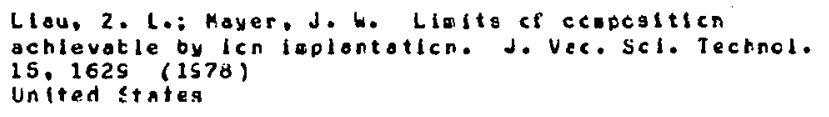 \\
\hline 1193 & $\begin{array}{l}\text { D16 } \\
\text { Review }\end{array}$ & E T & Unde ? & $\begin{array}{l}\text { Poote. J. M. Metostable alioy forgaticn. J. Vac. Sci. } \\
\text { Technol. IE. } 1636 \text { (IS78) } \\
\text { United States }\end{array}$ \\
\hline 1194 & $\begin{array}{l}\text { D16 } \\
\text { Review }\end{array}$ & E & Under & 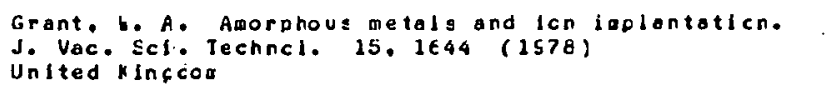 \\
\hline 1195 & $\begin{array}{l}\text { D16 } \\
\text { Revien }\end{array}$ & E & Undef & 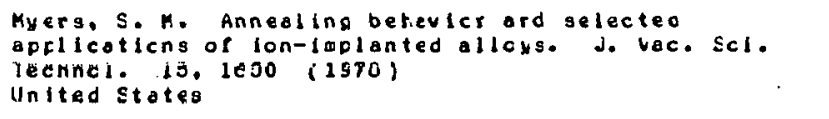 \\
\hline 2196 & 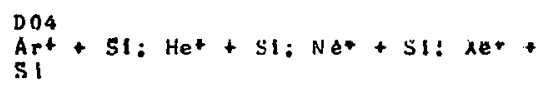 & $E$ & $1-3 \mathrm{keV}$ & 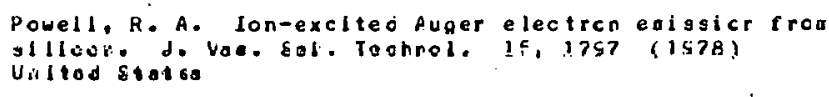 \\
\hline 1157 & $\stackrel{\mathrm{H} 17}{\mathrm{H}}+\mathrm{CN}: \mathrm{H}+\mathrm{NSI}$ & $\tau$ & Unde 1 & 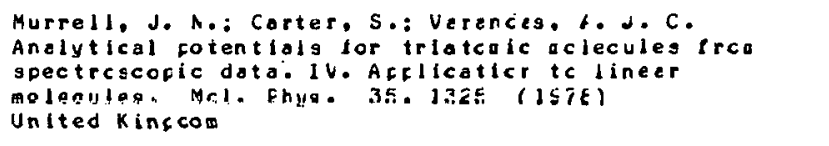 \\
\hline 1198 & $\begin{array}{l}A 17 \\
K r+X e: A r+X e: N e+X_{O}: A r+K r: \\
N e+K r: N e+A r\end{array}$ & $\mathbf{T}$ & Undef & 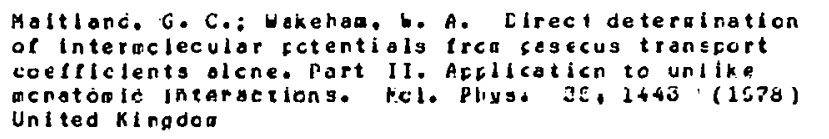 \\
\hline 1199 & $\begin{array}{l}\mathrm{A17} \\
\mathrm{NH}_{2}+\end{array}$ & 1 & Unuer & 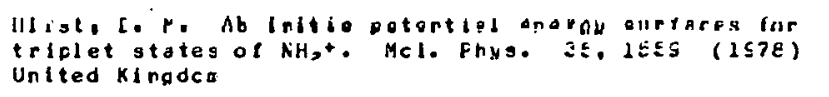 \\
\hline 1200 & $\begin{array}{l}\text { Ho6 } \\
\text { nid } \\
\text { a17 } \\
0\end{array}$ & $T$ & Undet & 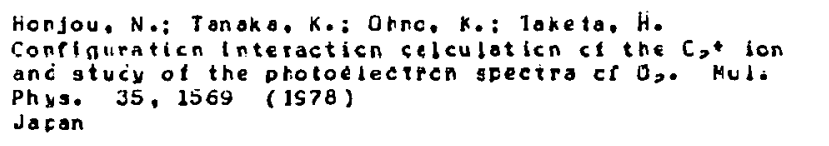 \\
\hline 1201 & $\begin{array}{l}\text { A13 } \\
\text { Art+ }+H_{2}\end{array}$ & $T$ & $0.5-0.7 \mathrm{eV}$ & 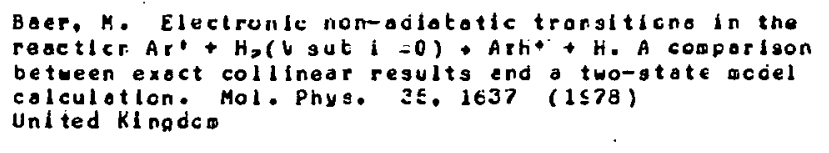 \\
\hline 1202 & $\begin{array}{l}\mathrm{HO2} \\
\mathrm{h}\end{array}$ & $E$ & $150 C 0 \mathrm{AO}$ & 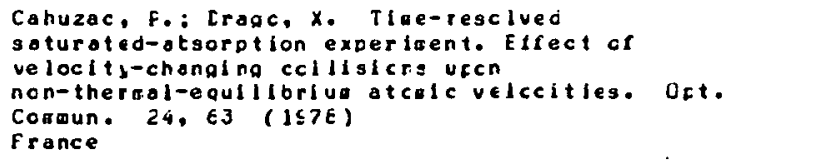 \\
\hline
\end{tabular}




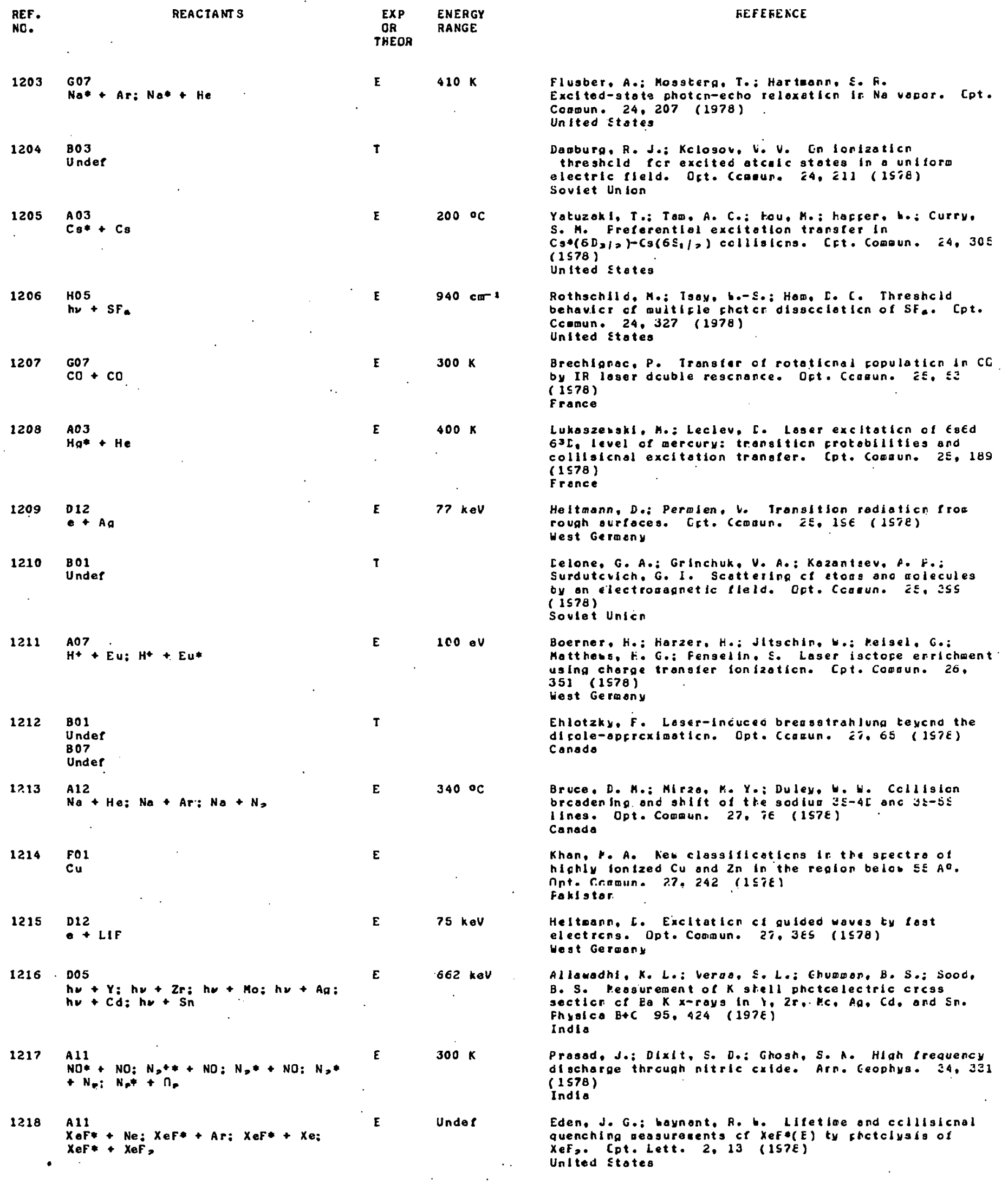




$$
\text { REF . }
$$

REACTANT.S

NO.
1219

ho2

$\mathrm{hv}+\mathrm{rSr}+\mathrm{Ar} 1$

A 12

B07

$2 h v+S r$

HO 6

$2 h v+s r$

1221

$\mathrm{HO}_{4}$

nhe $+5 F_{\text {. }}$

1222406

1223 HOG

$2 \mathrm{hv}+\mathrm{NO}$,
$\mathrm{Sr*}+\mathrm{Sr}$

$1229 \quad 404$ nhe + SF

$1225 \mathrm{HO}$

nhv + SF

$\mathrm{HO4}$

nhe + SFa

$1226 \mathrm{HO2}$

nhv + SF

$\mathrm{HOS}$

nhe + SFa

$1227 \quad A 07$

Sr*t Sr

A 14

Sr* + S

$1228 \mathrm{H} 34$

$3 h v+Y b$

Yo:

$1229 F 01$

He

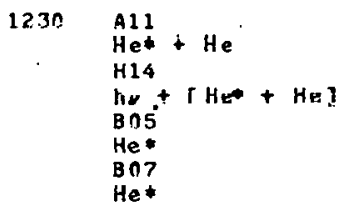

$1331 \quad 104$

$4 h v+S r$

1232

$\mathrm{HOC}$ $h_{\nu}+\mathrm{Ba}: \mathrm{hv}^{+} \mathrm{TI} \mathrm{h}_{\nu}+\mathrm{Pb}: \mathrm{hv}^{+} \mathrm{BI}$

$1232 \mathrm{HO2}$

$n h w+S F_{\text {a }}$
E

\section{$\begin{array}{ll}\text { EXP } & \text { ENERGY } \\ \text { OR } & \text { AANGE }\end{array}$ \\ IHEOR}

E

$\mathrm{HO2}$

A12

$300 \mathrm{~K}$

$2680 \mathrm{~A}^{\circ}$

$945 \mathrm{~cm}^{-1}$

1

Unde $P$

$4700-4400$

A 0

$10.6 \mu$

$T$

$10.6 \mu$

E

Undel

E

$6000 A^{\circ}$

E

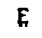

A11
$300 \mathrm{~K}$
H14: $368 \mathrm{E}$
AU

E
$4607 \mathrm{~A}^{0}$
$4250-4500$

AO

$3080 \mathrm{Aa}^{\mathrm{a}}$

10.6 i
FE F E FENCE

Sxcke, A. Cptlcal collistcrs In or Intense lager lleld. Cpt. Lett. 2, 36 (1578) United stotes

Green. W. F.: Falccne. F. b. Inversicr of the resonance line of Srt produced by cptleally puring $8 r$ atcos. Oot. Lett. 2,115 (1S7E)

United Etates

Cantre 11. C. D.: Fcx, K. Effects ct eclecular rototion ond vitraticn-rotation interaction cn ccillsicrless multiple-photon excitation of SF.. Gpt. Lett. 2, 15l ( $1 \subseteq 78)$

United states

Ben-Aryeh. X. Molecular lonizotion by abacrption of spentarecus eulosicn. Cot. Lett. 2. if4 (iste) Uilled alolies

Antonov, V. S.: Knyazev, I. N.: Letokhcu, V. S.: Matluk. V. M.: Houghev. V. G.: Fctofov. V. K. Stepwlae lajer ulutulunteol luil uf chlecules III a was

spectrcmeter: a new aethod for probing and detection of polyatcic dolecules. Cot. Lett. 3 , a? (ISTE) Soviet Union

Quigley, G. P. Collisionol effects in quitifle Infrared chcten absorption in sf.. Crt. Lett. 3, 106 (1\$78)

United stotes

Galbralth. H. Wo; Ackerhalt, d. fi. Colculaticn ot the temperoture dependence cf the rultifhoton atgorption spectrua of SF.. Cpt. Lett. a. 105 (1S78) United states

Galbralth. H. W.; Ackerhalt, J. F. Ccabarioon cf muliple-chctco excitotion iccels. Ort. Lett. 3,152 (1578)

United Stetes

Worden. E. F,: Paloner, J. A.: Ccrway, J. G. Aosociolive lonleotlon ce lasei-exeltec fydterg states in atrontiue vapor. Opt. Lett. 3, 156 (1578) United Etotes

Bekav, E. I.: Letakhow, V. S.: Matueov, O. I.I Mlehin. v. I. Singlo-aton dotection of yttorbiue by selective laser exctiotion ond lleld Ionizatich trom byobera states. Cot. Lett. 3. ISS (1578)

Soviet Union

Fal cone, R. W.: Hilliscr, J. F.: Ycung, J. Fei Horsis, S. E. Mesourenent of the He 182. isn igotofic shift uolng. tunoble VUV anti-stckes light source. Cpt. lett. ¿ i l 2 ( $1 \leq 78$ ) United Etates

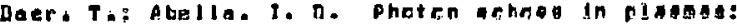
collisicnol relaxotion in hellug on 2 as -3 spopo Opt. Lett. 3, 170 (1578)

Untod statoo

Eecnorou, h. H.: treétón, f. H.: EJcrklund, G. C. Ellects of magnetic lields on four-wave gixing processes in otonlc vopora. Cpt. lett. 3, 205 (1978) United States

Burnhar, Fo: Ejou, N. Eftlcient Reos conversion of XeCl-leser radiation In metol vapora. Opt. Lett. 3. $21 E$ (1S7E) United states

Lyoun. J. L.: Anderson. R. E.: Flsher, R. A.: Fel doon, B. J. Atsorption of pulsec CO,-laser radiaticn by SF. at $140 \mathrm{~K}$ Oft. Lett. 3. $23 E$ (157E)

United stotes 
$\mathbf{T}$

$$
\begin{array}{ll}
1234 & \text { FO1 } \\
\text { He Seq }
\end{array}
$$

1235 FoI

He

$1236 \quad \begin{aligned} & E 03 \\ & \end{aligned}+E u$

1237

$\mathrm{DO3}$.
DDE

$\left.\mathrm{Art}+\mathrm{Cu}: \mathrm{Ar}+\mathrm{CH}+\mathrm{C}_{3}\right)$

$123 \mathrm{HO}^{\mathrm{HO}}$

1239

$1240 \quad F$

$1241 \quad 801$

Under

1242. E0

$6+119$

EOS

$\mathrm{e}+\mathrm{Hg}$

$1243 \quad \mathrm{FO}_{2}$

H

$1244 E 03$

e+N,

$1245 \quad F O 1$

$\mathrm{H}_{\mathbf{r}}$

1246

$\mathrm{AO} 6$
$\mathrm{Ne}+$
$\mathrm{AO}$

$\mathrm{AO} 7$

$\mathrm{Ne} *+\mathrm{Zn} ; \mathrm{Ne}^{+}+\mathrm{Zn}$

$1247 \quad 501$

MOl

1248 EOJ

$e+A r$
$\mathrm{Rl}^{\mathrm{Al}}+\mathrm{He}: \mathrm{Rb}+\mathrm{Ne}: \mathrm{Rb}+\mathrm{Ar}: \mathrm{Rb}+\mathrm{Kr}:$ $R b+X e$
$25 \mathrm{keV}$

$2000-18000$ A 0

Unde

47-400 eV

$10-1000 \mathrm{eV}$

Thermal

$0-100$ eV

$450 \mathrm{~K}$
Ivonov, L. N.: Ivanova, E. F.: Sofronowa, U. I.: Shovtrollghulil. I. A. Relativistic celculation of the level-widths of the-electrcn otcelc systems. Cpt. SDectrC8C. 44. E (ISTE)

Soulet $\ln i \mathrm{cn}$

An Islocua, G. P.: Fyzhohova, E. I.: Semenou, R. I. Fine structure of the triplet ters of the 1930 configuraticn of He I. Dft. Efectrosc. 44, 10 Soviet Unicn

(1578)

Golovetok, N. V.: Garpa. I. I.: Snleon. L. L.

Exferimentol study on electron-iapoct exclioticn cl spectrol litres of eurociue. Cet. Srectrosc. 44,13 ( 1578 )

Sculet linton

Koval. A. G.: Logochev, Y. E.: Flzqeer, B. . Lurinosity spectrua of excited forticles prciuced on bcabardeent cn copper tarcet by an Art-ion ceas. Cet. spectreac. $44,2 \equiv(1 \leq 78)$

Soviet Unicn

Ancreev. S. I.: Vorlancv. Y. V. Oetlcel preperties of a sodium plasma. Opt. Spectrosc. 44.29 (1578) Sowlet $\ln \operatorname{con}$

Makushkin. Y. S.: Llenikov, C. N. Petentisl. Tunction of diatosic moilecule. Opt. Spectrcsc. 44, 41 (1578)

Soviet Unlen

Malakhch, Y. I. Lifetiees ct Cul levels. Cipt. Spectrcoc. 44, 12E (1巨?E)

Soviet inlon

MInaev. B. F. Spin-ortitol interocticn in aolecules and mectantsm of the elfect of on external oognetlc tield cn lualnescence. Opt. Spectrose. 44.148 (1578) Soviet inion

Seaenova. I. U.: Soirnov. Y. M. Leteroination of excltaticn crcss secticns ord tronelticn crotatilities of Hg 11. CDt. Spectrosc. 44, 245 (1576)

Soviet lnicn

Shestakcu. A. F. Ceneral teraulo tor cifole polarizability of a hycrogen atod in a spectrcscoflc state. Opt. Spectrosc. 44. 3ET (1S7E) Sovlet $\ln l \mathrm{cn}$

Kosoruchkina, A, D.: Trekhcv, E. S, Electrcn-lopoct excitotion functicn of the (J lilsut u) stote of $N_{2}$. Opt. Spectrose. 44, ISS (ISTE) Soviet inicn

Lavrov, B. F.: Dtcrbaev, E. K. Ioent itication of vorlous lines of the fulcher syster of the t, aclecule. Opt. Bieotreos. 44. 360 (157P) Sovlet Unicn

Kartazoev, V. A.: Flotrouskil. Y. P.: lcluochev, Y. A. Chorge exchange ono Penning ionizollon In a culged discharged in alxture of necn anc zinc. Cot. Spectrcsc. 44, JE2 (1S7E) Soviet Unton

Driker, M. He: Ivanov, L. N. Calculaticn cf ccaflex atcalc lans using relativiatic perturtotion thecry with a radel rotentiol. Ort. Sfectrcsc. 44. 365 (1SiE) Soviet Unton

Bogdancua, I. P.: Marusin, V. D.: Yokhentovo, V. E. Some oreciol teatures cp the exciteticr of ardon otoms by slow electrons. Oct. Scectrcse. 44, JeE (1578) Soviet $\ln i o n$

Kazontsev, S. A.: Kal Iteevakil, K. I.: Flsh, C. H. Aprlicaticn of the mapnet ic scannira technicue for measuring the broacenings ond shifts of the $D$. resonance line of rubidiut caused by irert gases. Cpt. Spectresc. 44,372 (1578) Soviet Union 


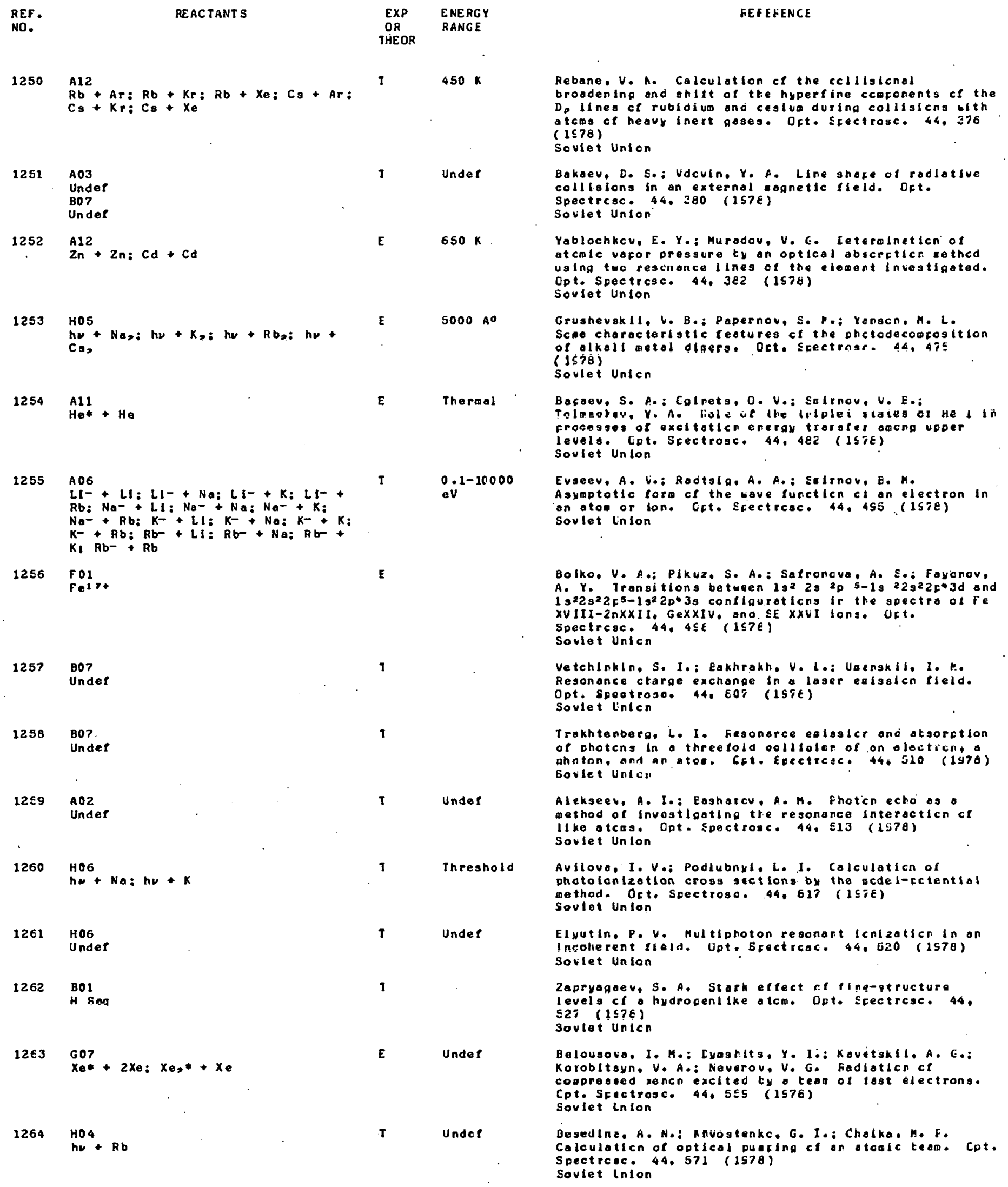




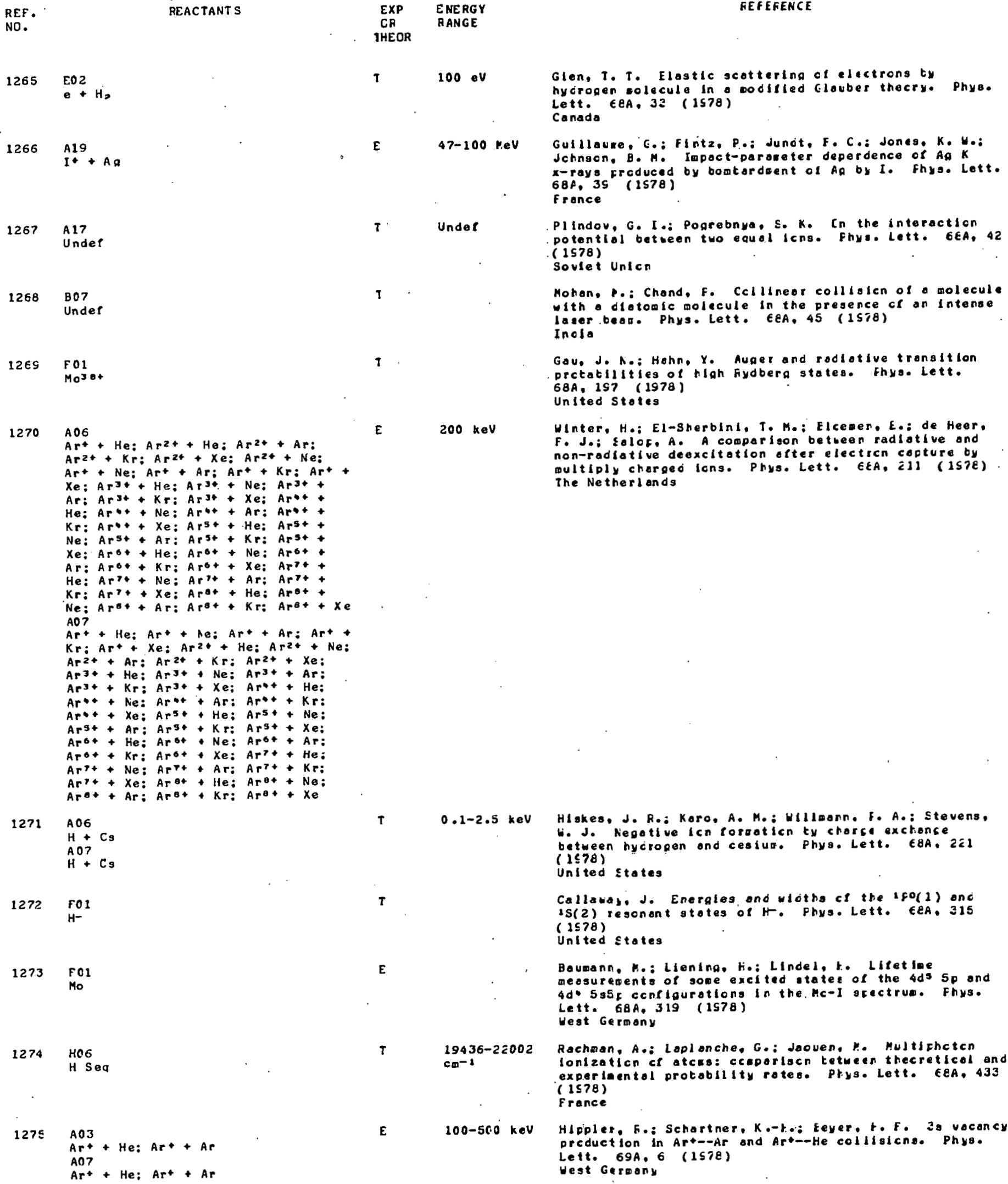

Under

Undet

1268 B07

Under

$1269 \quad F 01$

Mosor

Hiskes, J. Re: Koro, A. Mo: Hillmonn. F. A.: Stevens b. J. Negotive icn Poroaticn ty chorce exchence between hycropen ond cesiur. Phys. Lett. $E A A$, 221 ( $1 \leqslant 78$ ) hycrogen ollecule ln oodfled Glovber thecry. Phyo. Lett. $6 \mathrm{EA}, 32$ (1578)

Canada

Gulllaure, G.: Fintz, P..: Junot, F. C.: Jones, K. W.: Jchnson, B. M. Impoct-parareter deperdence of $A g k$ $x$-rays froduced by bomtordoent of Ag by I. Fhyo. Lett. $68 \mathrm{~A}, 39$ (1578)

Frence

Plindov, G. I.: Pogrebnya, S. $K$. In the interactlon potentiol between two equel icns. Fhys. Lett. GEA, 42 (1578)

Sovlet Unicn

Mohon. P.: Chond, f. Callineor collisicn of a molecule with: diotoolc molecule in the presence of an intense lacer bear. Phys. Lett. EEA, 45 (1578) Inolo

Gaug J. A.: Hohn, $x$. Auger and rodiotive transition prctatillties of hioh fiyduerg states. Fhys. Lett. 68A, 1s? (1978) United Stoles

Hinter. H.: El-Sherblni, T. Mo: Elceser. E.: de Heer. F. J.: solor. A. A comparison between radlotilue and non-radiative deexcliotion ofter electicn copture by non-radiative deexcltation ofter electrcn copture wultiply charged
The Netheriands

United states

$1272 \quad \mathrm{FO}^{-}$

Callawald J. Energles ond wioths ef the Ifo(1) ond is(2) resonont gtotes of H. Phys. Lett. GEA, 315 ( 1578 ) United States

Baumann. Mo: Liening, Ho: Lindel, t. Lifet lae measurements of some excited tates of the ads $5 p$ and $4 d^{\circ} 535$ f cenfigurations in the Hc-1 acectrua. Fhys. Lett. 68A, 319 (1978)

Vest Germony

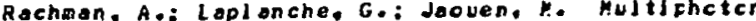
lonlzoticn of atcas: ccaporlacn tetween thecretlcol and experiacental protobility rotes. Pryge Lett. CEA, 433 experian France

Art + He: Art + Ar

HIppler, F.: Schartner, K.-k.:- Eeyer, t. F, zo vocancy preduction in Art--Ar ond Ar:--He collitsicns. Phys. Lett. 69A. 6 (1578) vest Germany 


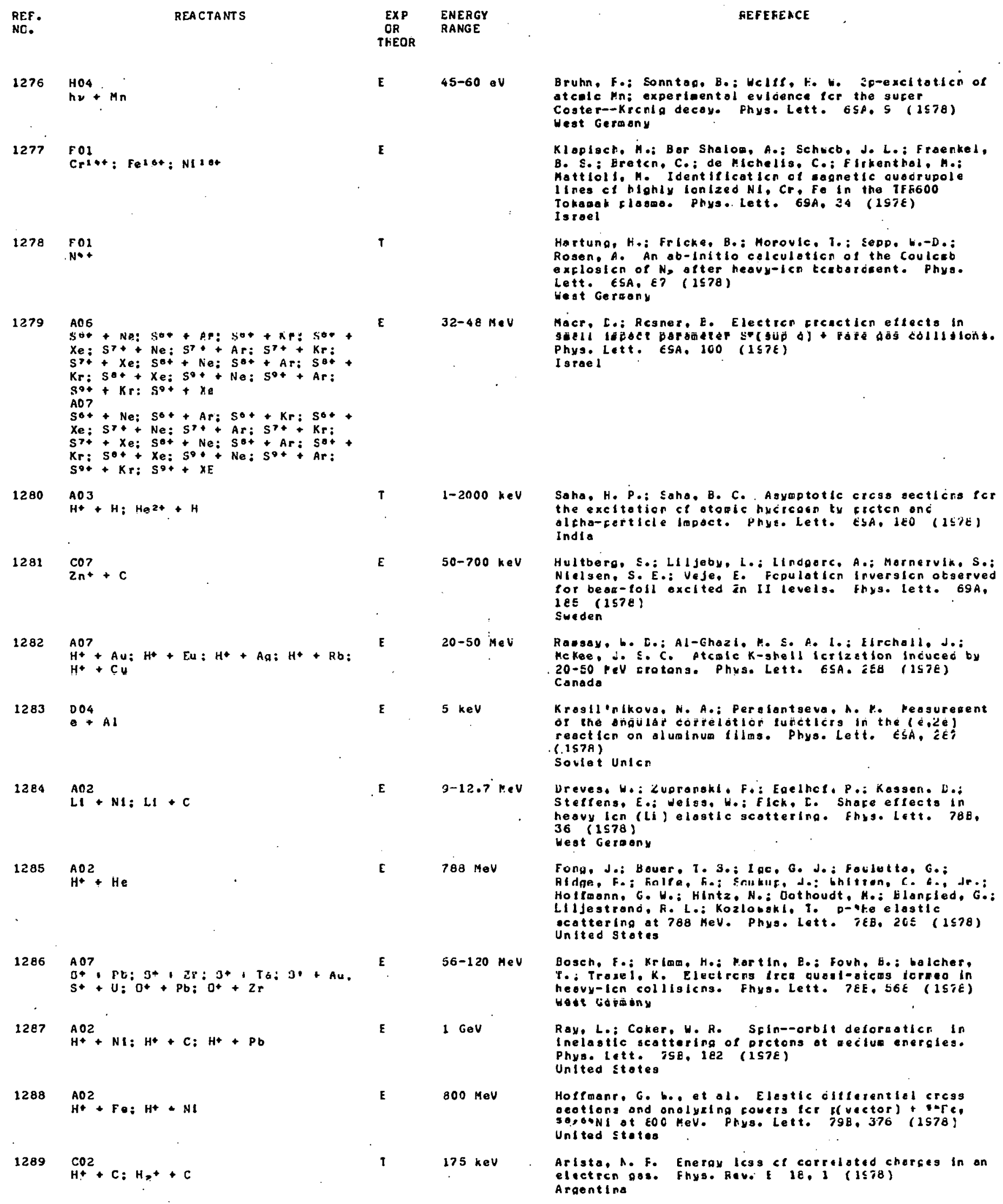




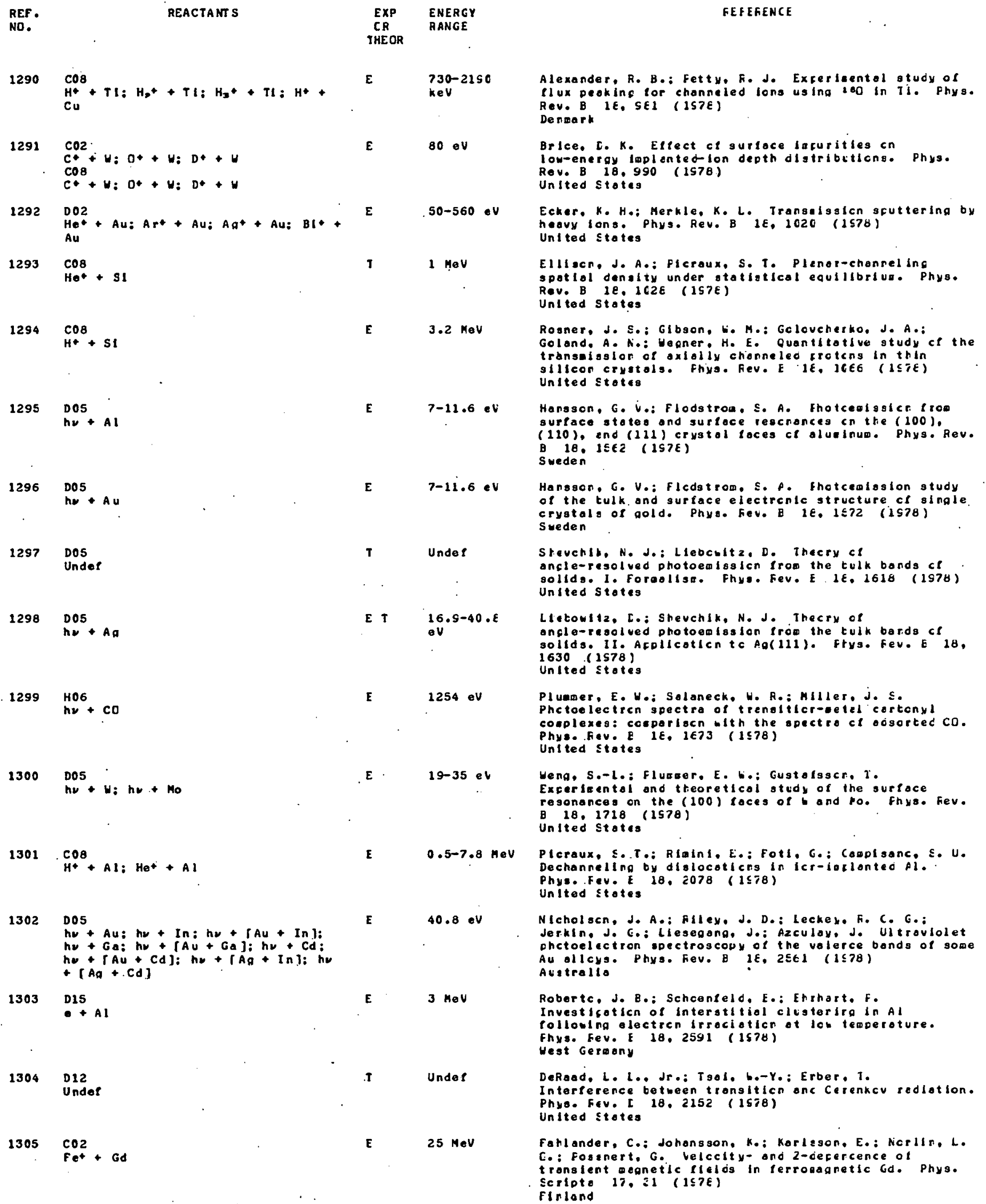




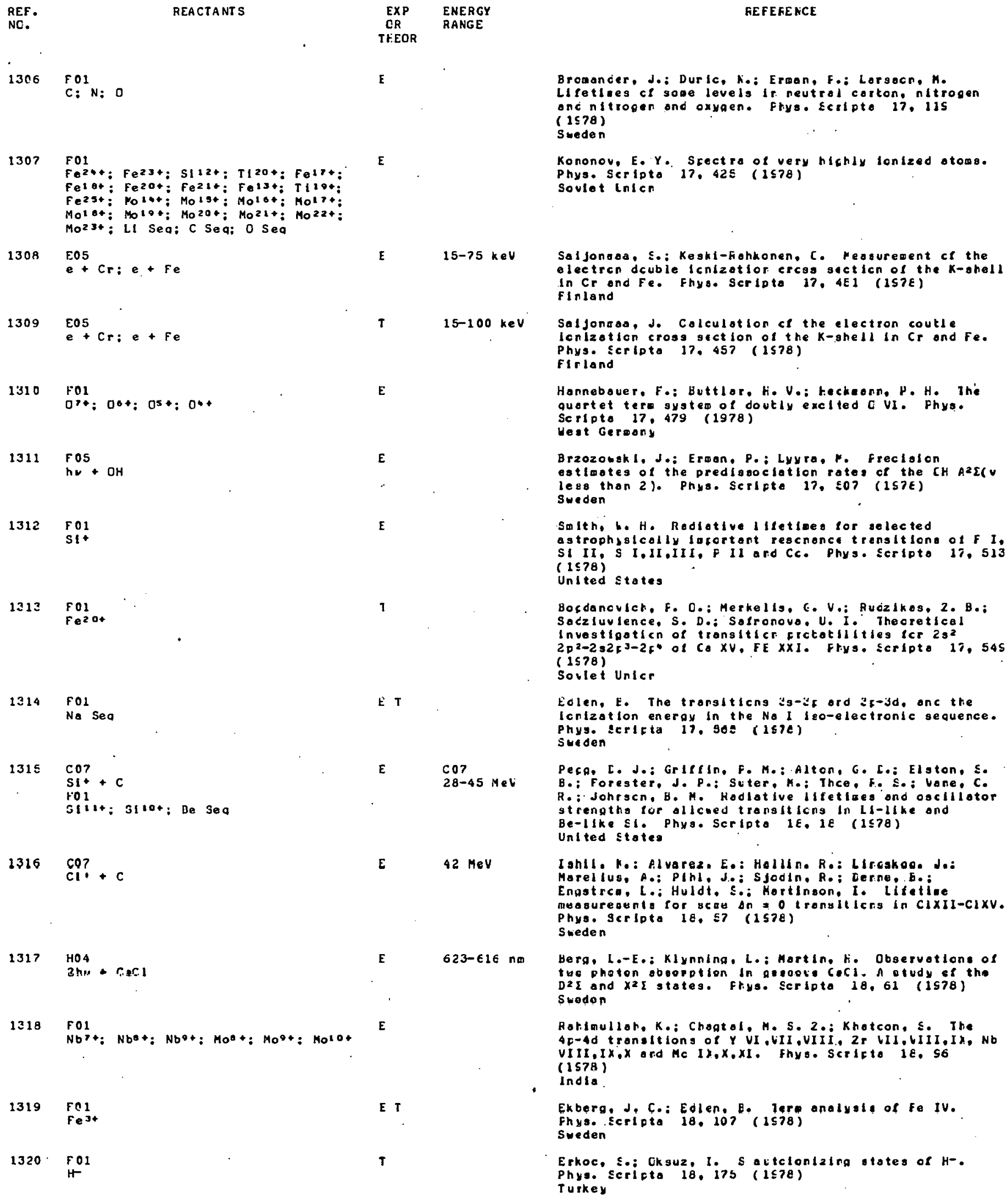

E05

1308

$e+C r: e+F e$

C 07

$28-45 \mathrm{MeV}$

$623-\epsilon 16 \mathrm{~nm}$

(1)
Lifetiles cl sode levels ir. neutral carton. nitrogen anc nitrogen ond oxyaen. Frys. Ecripte 17, 11s (1578) Sweden

Kononov, E. Y. Srectro ol very hichly lonized otoma. Phys. Seripto 17, 425 (1578) Soviet Inicr

SaI jonada, S.: Keski-Fohkonen, C. Measuredent cr the electrcn dcuble Icnizotior crcos sucticn of the K-ohell in $C r$ ond Fe. Fhyse Scripta 17. AEI (ISZE) finland

Saljonada, J. Colculotion cl the lectron coutle icnlzeticn cross section of the K-ghell in Cr and Fe. Phys. Scrlpta 17, 457 (1578)

Firland

Hannebaver. F.: Buttlar. H. V.: Heckaenn, H. H. The quartet tero system of doutiy exclied C VI. Phys. Scripto 17, 479 (1978)

Heat Geroony

Brzozostk I, J.: Erman. P.: Lyyra. F. Freclaton estimotes of the predisoociation rates of the CH AzE(V lego thon 2). Phys. Serlote 17, 507 (1S7E) Sweden

Smith, h. H. Radiotive lifetlaes for aclected astrophsifcally lofortant rescnance transitiona ot $I_{\text {. }}$ SIII, S I.II,III, F II ard CC. Phys. Scripto 17, 513 $(1 \leq 78)$

United States

Bosdanculch. F. D.: Merkells, C. V.: Rudzlkos, 2. B.: Saczluvience. S. D.: Solronovo. U. I. Theoretical Investlgaticn of tronsiticr cretatilitieg ter $2 g^{2}$ $2 p^{2}-2 s^{3}-2 f^{4}$ of Ca XV. FE XXI. FHys. Scripta 17, 54S (1578)

Sowlet Unicr

Eolen, E. The tranglticng ag-2t ord az-3d, anc the lanization energy in the Na I leo-electronic sequence. Phys. ecrita 17, 5os (1670) suiden

Pecg, [, J.: GriffIn, F. M.: Alton, G. L.: Elston, S. B.: Forester, J.P.: Suter, H.: Thce, F. S.: Vane, C. R.: Johrscn, H. M. Hadlative iffetiacs ond osclilator strengtho for allicued transiticns in Li-like and Be-like si. Phys. Scripta $1 E, 18$ ( $1 \leq 78)$ United statea

Iahil. Ro: Alvarez. E.: Hallino Ro: LIrgakog. Ja: Marelius, A.: PInl. J.: Sjodin. R.: Cerne.E.: Enastrca. L.: Huldt. E.: Hortinson, I. Litetiue

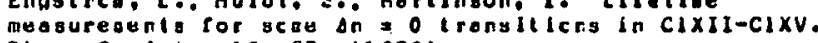
Phys. Sertota 18, 57 (1578)

Sweden

Berg, L.-E.: Klynning, L.: Marting hi, Observotions of tuo photon oboeption In pecos. CoCi. A otudy of the $D^{2} \Sigma$ and $X^{2} I$ states. Fryse Scripta 18,61 (1s78) Swodon

Rolimulloh, K.: Chogt ol H. S. 2.: Khotcon, s. The 4 - id transitions of $Y$ VI, WII,VIII. $2 r$ VII,VIII,IX. Nb VIII,IX,X ord Mc IX,X,XI. Fhyso. Scricto iE, S6 (IS78)

Ekberg. J.C.: Edlen, B. Tere analyale of Fe IV. Fhys. Ecripta 18,10? (1578) Sweden

Erkoc, S.: Gksuz, I. S attclontzing tates of $H^{-}$. Phys. Scricto 18,175 (1578) Turkey 


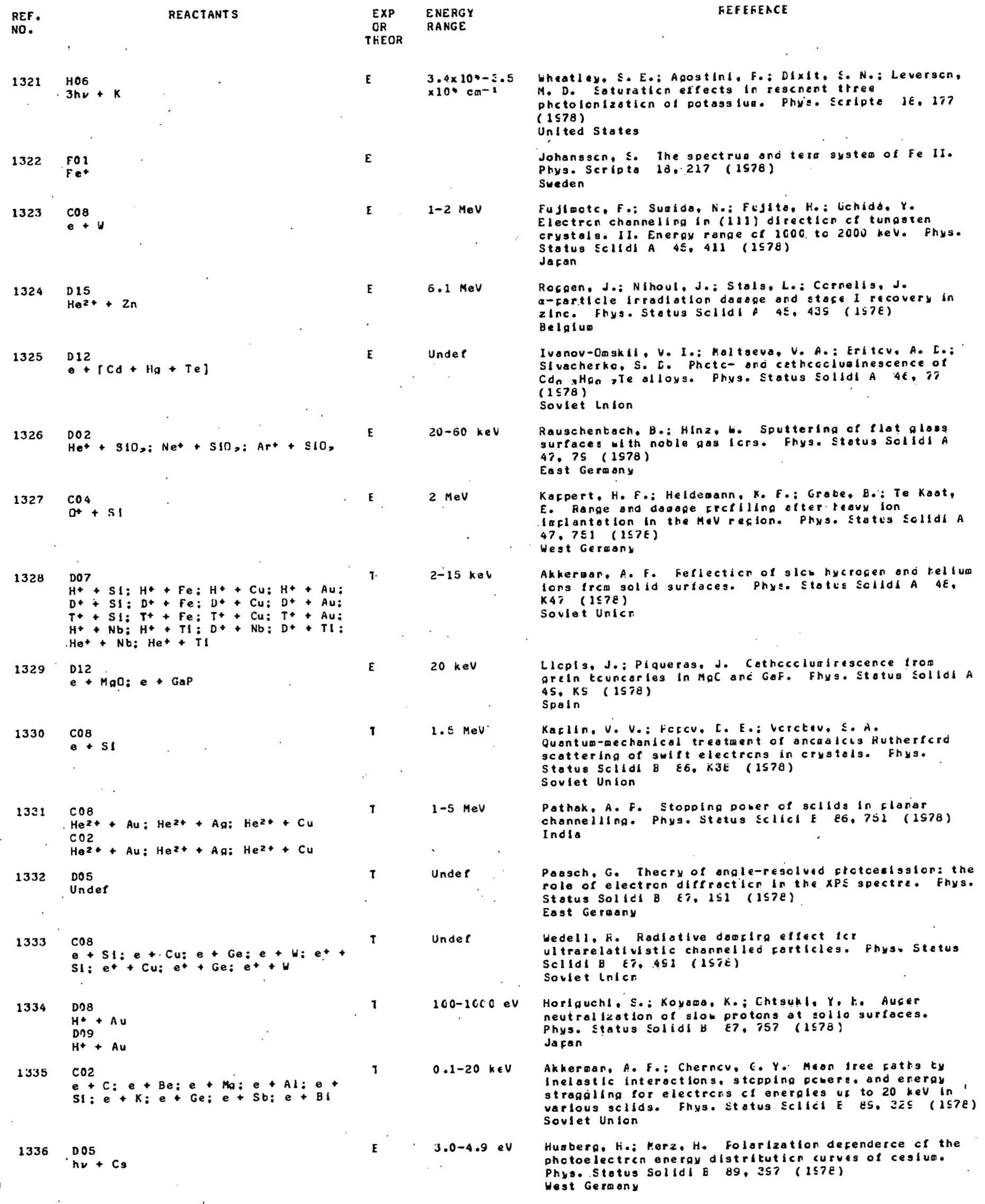


REF.

REACTANTS

1337

$\mathrm{CO} 2$
$\mathrm{NO}^{+}+\mathrm{C}: \mathrm{Ne}^{+}+\mathrm{C}$
$\mathrm{N}^{+}+\mathrm{C}: \mathrm{Ne}^{+}+\mathrm{C}$

1338

DoS + PbI, i hv + GaAs

1339 A09

Unde?

1340

$0_{0}^{A 09}+0_{0}-: 0,++\left[0_{0}-+0,1\right.$

$1341 \quad$ A 06

1342

$\mathrm{O}_{2} 13+\mathrm{O}_{2}: \mathrm{N}_{2}++\mathrm{N}_{2}$

$1343 \quad \mathrm{AlP}^{+}$
$\mathrm{H}^{*}+\mathrm{H}$

$1344 \quad E .09$

e HCl

1345

A10

$\mathrm{Na} *+\mathrm{Na}_{2}: \mathrm{Na}+\mathrm{Na}$

A11

$\mathrm{Ha}+\mathrm{Na}$ : $\mathrm{Na}$ + $\mathrm{Na}$

$1346 E \delta \hat{L}$

C+ $\mathrm{H}_{2}+$

$1347 \quad \mathrm{AO3}$

$\mathrm{H}_{2}+\mathrm{D}_{2}$

$\mathrm{AO}_{4}$

$\mathrm{H}_{2}+\mathrm{D}$,

A14

$\mathrm{H}_{2}+\mathrm{D}_{2}$

$1,348 \stackrel{\mathrm{Al}}{\mathrm{Cs}}+\mathrm{Ar}$

1349 HOG

$4 \mathrm{h \nu}+\mathrm{NO}$

1350

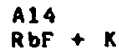

\section{$\begin{array}{ll}\text { EXP } & \text { ENERGY } \\ \text { CR } & \text { RANGE }\end{array}$ \\ THEOR}

E

$1 \mathrm{~S}-\epsilon 3 \mathrm{keV}$

E $2-6 \mathrm{eV}$

J

Theroal

1

$k \in$

$300 \mathrm{~K}$

$1-7+06$

$\mathbf{T}$

$0-50 \mathrm{eV}$

Undef

E

490-400 nm

E

$0.78-20.1$

KeV

Theraal

Undef
$1730-247 \mathrm{~K}$

Mlller, W. Jo: Gould, F. K. Electron ottnchment

kinetlc. in riases. II. Dissoclative ottachment tc HCl. J. Ches. Fhys. 68, $3 E 42$ (1578)

United stotes

Lan, L. Ko: Fujlaoto, T.: Gallogher, A. Co: Hessel. Mo H. Collisicnol excitation tronsler telween No onc Nas. J. Chea. Fhys. 68, 3553 (1578) United Etotes

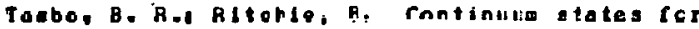
hydrogen oleculo: a study ct cenvergence in e--Hot scottering equatlong. J. Cheo. Phys. 68, zest (IS7E) United srares

Brcun, N. J.: Sllver. D. M. Feactlive ond IneldattC scattering Cl $\mathrm{H}_{2}+\mathrm{D}_{2}$ using. reculgive model cotentlal energy surface. J. Chag. Phyge 6E, 3607 (1978) United states

Dayor, E.l Forray, M.: Willent, J. F.; Lozinact, J,

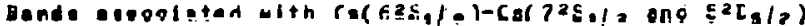
transt ticne perturted ty heliuir. A. Chew. Fhys: $C E$. $3018(1570)$

France

Zakheid, D.l Johncon, F. Iog- and three-phicter resonances in the lour-chotcn icnizeticn soectrum of nitric cxide ot lon teaperoture. J. Chew. Phys. 68. उeas (1988? United States

Zondee, L.: Bernsteln, F. E. Rctoticnel energy elfect ufcn the tronching fraction for resctlue aecay of the RbF K ccllisicn ceqplex. J. Chea. Frys. 68, 3760 (157a)

United stotes 
REF.
NO.

REACTANTS

1351

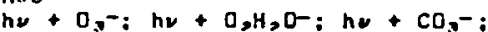
$\mathrm{h}_{2}+\mathrm{CO}_{4}-\mathrm{C}_{2}+\mathrm{h}_{2}+\mathrm{CO}_{3} \mathrm{H}_{3} \mathrm{O}-\mathrm{O}_{2} \mathrm{~h}_{2}+$

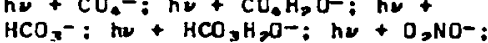
$\mathrm{h}_{2}+\mathrm{O}, \mathrm{NOH}>\mathrm{O}-: \mathrm{h}_{2}+\mathrm{NO}_{>} \mathrm{H}_{>} \mathrm{O}-\mathrm{h}_{\nu}+$ $\mathrm{CO}_{3} \mathrm{H}_{3} \mathrm{O}=$

HO 7

$h_{\nu}+\mathrm{O}_{3}-\mathrm{h} \nu+\mathrm{O}_{3} \mathrm{H}_{2} \mathrm{O}-\mathrm{h} \nu+\mathrm{O}_{3} \mathrm{H}_{3} \mathrm{O}_{-}$ $h \nu+\mathrm{CC}_{3}-h_{\nu}+\mathrm{CO}_{8}-: h_{\nu}+\mathrm{CC}_{4} \mathrm{H}_{2} \mathrm{O}_{\text {- }}$ $h_{\nu}+\mathrm{HCO}_{2}-$ : he $+\mathrm{HCO}_{x} \mathrm{H}_{3} \mathrm{O}-\mathrm{Ch}_{2}+$ $\mathrm{O}_{2} \mathrm{NO}^{-}: \mathrm{hL}^{2}+\mathrm{O}_{2} \mathrm{NOH}_{2} \mathrm{O}^{-}: \mathrm{h}_{2}+\mathrm{NO}_{2} \mathrm{H}_{2} \mathrm{O}$; $\mathrm{h} \boldsymbol{\nu}+\mathrm{CC}_{3} \mathrm{H}, \mathrm{O}-$

1352

19

Undef

E15

Under

1353 A 17

$\stackrel{A 17}{A r_{2}}+F_{i} K_{r_{2}}+\mathrm{F}$

1354

$\mathrm{DO} 2$

1355

G07

$e+N_{2}: e+H_{2}: N_{2}+\mathrm{Ne}: N_{2}+H_{2}$

1356

FO 1

$\mathrm{He:} \mathrm{Ll}^{+}: \mathrm{Be}^{2+}: \mathrm{B}^{3+}: \mathrm{C}+$

FO3

1357

$\mathrm{CO}_{2} *+\mathrm{CO}_{2}$

1358

EOg

$C+\mathrm{IO}_{2}+\mathrm{N}_{2}$

1.359

E02

$\mathrm{e}+\mathrm{H}_{2} \mathrm{O}$ : $+\mathrm{OH} ; 0+\mathrm{KOH} ; \mathrm{O}+\mathrm{O}_{2}: e$

$+\mathrm{CO}_{7} ; \mathrm{e}+\mathrm{CO} ; \mathrm{e}+\mathrm{H}_{7} ; \mathrm{e}+\mathrm{K} ; \mathrm{e}+$

$\mathrm{H}: \mathrm{e}+\mathrm{N}>\mathrm{O}$ e $+\mathrm{NO} ; \mathrm{e}^{+} \mathrm{Cs} ; \mathrm{e}+\mathrm{Cs}:$

$\mathrm{e}+\mathrm{Na}: \mathrm{e}+\mathrm{CsOH}$

$1360 \quad D 02$

$n r^{*}+W$

$1361 \quad \mathrm{EO}_{2}$

a $+N_{\text {? }}$

1362

A14

$\mathrm{Cs}+\mathrm{CO}_{7}$

$700-760 \mathrm{~K}$

$13 \in 3 E 03$

e+ N,

1364

DO2

PEHT + SI: PERT + CU: PERT + AQ:

PERT + AU: PERT + Nb
$\mathbf{E}$

$\begin{array}{cc}\text { EXP } & \text { ENERGY } \\ \text { CR } & \text { RANGE } \\ \text { IHEOR } & \end{array}$

$\mathbf{E}$

1

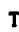

A 0

Undet

Under keV

Undef
$8400-7000$

$0.25-5 . c$

$10-18$ ev
$300 \rightarrow 1600 K$

$0.1-3.0$ ev

$0.01-4.0 \mathrm{eV}$

$0.04-3.7 \mathrm{eV}$

Seattering of lectrong with $E=0.04-3.7$ eV tW $N_{0}$

molecules. High Temp. 1E, JEI (JSTE)

Soviet inton

stotakl1, G. I.: Ivancy, F. S.: Korclev, N. M.: Malikov. M. M. Fote ol lnterectlen ol cealum end $\mathrm{CO}$, In a seove dixture in a ropidix circtiating laser. HIgh Tetr. 16,572 (1978)

sovilet Unton

Vichon. D.: Gresteou, F.: Hetz, A.: Hozeau, J Resonant exeltaticn of "new veifece state in $t_{p}$ electrcn impact. J. Mol. Efectrcsc. i3, $40 E$ (1578) France

Imada. M. Mass dependence of the couttering yleld--bouncary elfect. J. Phys. Scc.. dap. 4E, 1557 ( 1578$)$ th. C. Fo: Lee, L. C.: Ccsby, P. C.: Petersen, J. Atrospherle icns froo 7000 tc $8400 \mathrm{Ao}$. J. Chem. Fhys. $6 \varepsilon: 3 E 1 E(1578)$

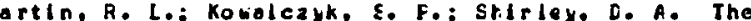
$x$-ray protoemisalcn spectra. J. Crea. Physe 68, 282S

H.: Hay, F. J. Electrenle stetes of ArgF ond F,F. J. Chem. Phys. EB, aEEO (1S7E)

Kazsokl. J.: Kothinger. L. Computer eputtering proceoges. Acta Phys. Acad.

Mlchel, P.: Peau, S.: Hinkler. R. Grellawold: Der Einfluse der Stooskenndaten aut die Elektronenklnetlk schuachicnisierten Plschilaseen. Ann. Fhyo. 5y In tho electron otcilc aysteos. Indian J. Fhyse India

- No So: Neudakh, Crlevo L. No: ulbraticnal wede ct $\mathrm{CO}$, oclecules as function of tha somperature. J. Appl. Spectrosc. 28.664 (IS7E)

A leksandrcv. N. L. Mechanla ct electron binding ct oxygen colecules in nitropen closeo. righ teop. 16. $19 \bar{a}$ (157e)

A. Zelener, E. Vo: Yakukcu, I. I. The preductions of hydrcearton fuels with olkall impurity. Hich Teap. 16, 326 (2578)

V. No: Lyodin. A. A.: Churgin. Ho H. iputering $C P$ algh-current tunosten cothoce. High reap. 10, 349 (1570) soviet linion 


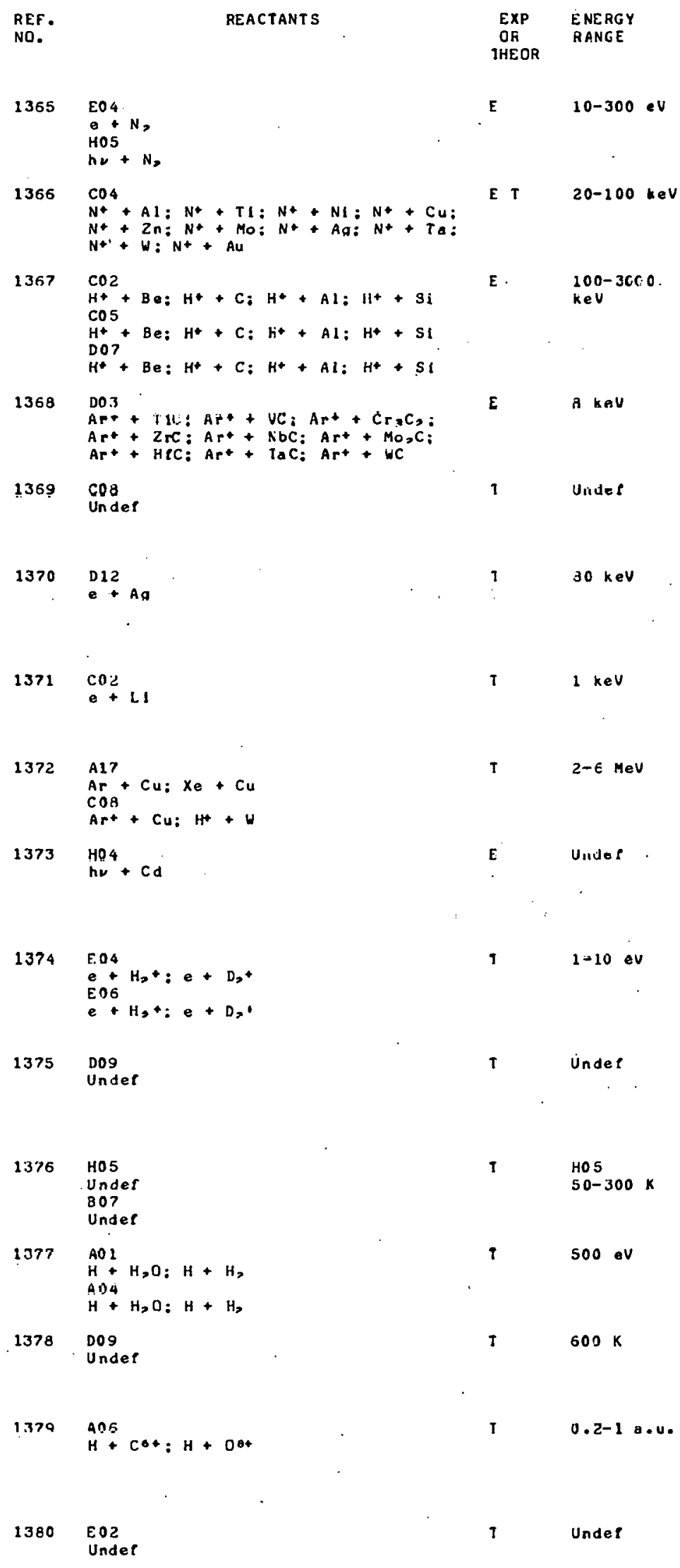

Zlof, E. C.: Mclaughlin, F. H. Cn the olsacciotion ot nifrogen by electron lacact ond by e.u.v.

phcto-absorption. Planet. Space Scl. 26, 449 (1978) Unlted States

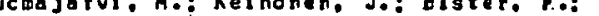
Ranges of is $N^{*}$ lons in ten wetals deasured by $(D, a y)$ resononce broodenting. Fhys. Fev. F $1 \varepsilon .4657$ (1978) Firland

Langley. F. A.: Erice, C. K. Eneroy stragfling ot prctons in Be, $C, A 1$. and Si. Fhys. Reve E le, 4673 (1578)

United Stateg

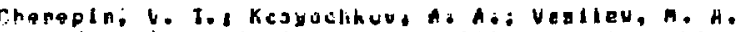
secondory ien eission of trangiticn getal carbides. Phys. Etatus solidi A so, N113 (157E)

Soviet Unicr

bafysnetskil. V. G.: Grublch, Á. Cig: Dutrovakoyo, I. Y. Diffraction of radiation frow channelled ohoraed particles. Phys. Status Sclidi B 88. 351 (1578) Soviet Unlcn

Heltuanri. C. Thecry ard exceritent of the licht emissicn of surlace plasmong induced by last electrcns on modulated surfoces. Phys. Status Sclidi $B$ e. ( 1578$)$

Heat Geroony

Zhivoplataev, F. A.: Hernandez, F, C. Fast electrcn acnttering in wotols wh the tacitaticn cl a ccre electrcn. Fhys. Stetus Sclidi E 50.441 (1578) Soviet lnler

Pathak. A. F.: Srivastava, H. F. Chanceling ono genistatistical acoel ler the interotcalc cctential. Phys. Stotus Solidi B So, 703 (1578) Indio

kinedovor, V. A.: Chigle, K. A. Investlgaticn of the

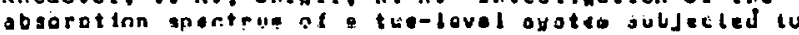
Intense two-photon excitaticn. Sov. Fhys.-JETF 47,3 (1579)

sovlet Unlúi

Zncanov. V. P.: Chiblsov. P. I. Closoclative recosbiraticn of electicns cn the oclecular lons tipt and $D_{3}$. Ith prcductich of strcnaly exclted otoms. SOV. Phys.-JETP $47,3 E$ (1578) soviet inlcn

Kagan, Y.: Kencnets, Y. V.: Dzhaoorkyzcu, A. K. Dunamic-intaraction itoeto in the decoy uf ras molecules in thin lilms. Sov. Fhys.-deIf 47. I48 ( $1 \leq 73)$

Soviot inlon

Akulin, V. M.: Allopiev. S. S.: Karlov, N. V. Mechanior ct collisicnal disscciaticn ct pclyotcolc molecules. Sov. Fhys.-JETF 47,257 (1578) Sovlet linion

Neudachln, h. G.: Leulin, V. G.: Selrncy, Y. F.

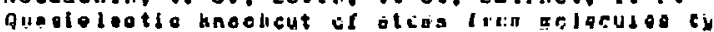
high-energy atoms. Sov. Prys.-JETF 47.267 (1978) Sowiet Inicr

Kogan, E. Y.: Mal'nev, V. M. Accosoodoticn coefflcient of diatcalc aclecular aas. Scr. Fhys.-JETf 47. $27 \mathrm{E}$ ( 1578$)$

Soviet Inion

Abramov, V. A.: Boryshnikov, F. F.: Lisitsa, V. S. Charge transfer between hycroger atcag ond the nuclel of aul tichorged lons ilth ollowance $I C r$ the degeneracy of the Inal states. Scr. Fhys.-JETf 47. 465 (1978) Soviet Union

Aous'ya. H. Y.: Kuchlev. H. Y. Analytic crccerties of tho anplituce for elastic forbard tcattering ce electrers by otcas. Scu. Fhys.-JETf $47.4 E 4$ (IS7E) Soviet unton 


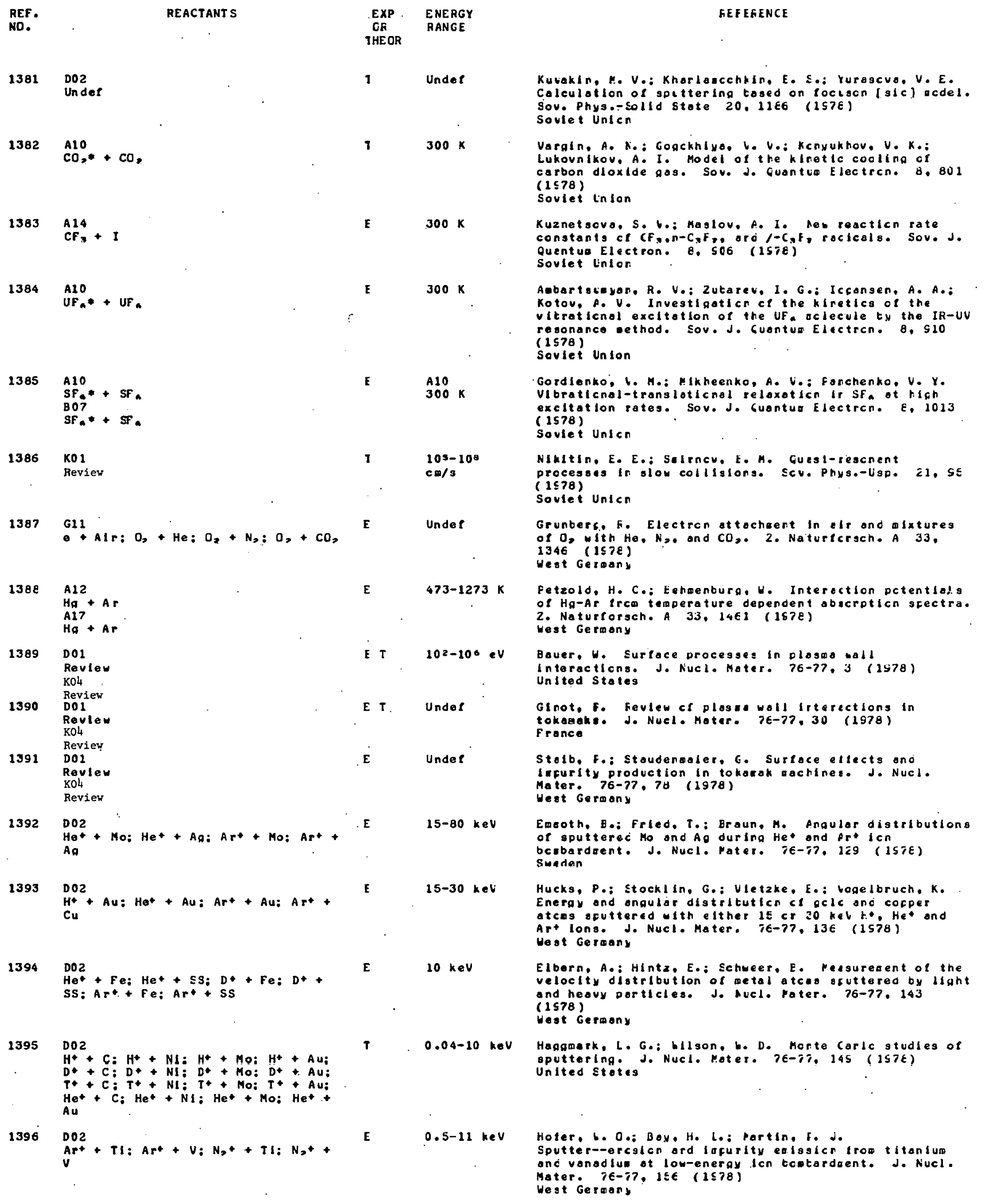




\begin{tabular}{|c|c|c|c|c|c|c|}
\hline $\begin{array}{l}\text { REF. } \\
\text { NC. }\end{array}$ & REACTANT & & & $\begin{array}{l}\text { EXP } \\
\text { OR } \\
\text { THEOR }\end{array}$ & $\begin{array}{l}\text { ENERGY } \\
\text { RANGE }\end{array}$ & FEF E FENCE \\
\hline 1397 & $\begin{array}{l}\mathrm{DO}^{+} \\
\mathrm{H}^{+}+\mathrm{B}_{4} \mathrm{C}: \mathrm{H}^{+}+\mathrm{SIC}: \mathrm{H}^{+} \\
\mathrm{C}: \mathrm{D}^{*}+\mathrm{B}_{4} \mathrm{C}: \mathrm{D}^{+}+\mathrm{SIC} \\
+\mathrm{C}: \mathrm{He}^{+}+\mathrm{B}_{*} \mathrm{C}: \mathrm{He}^{+} \\
\mathrm{TIC}: \mathrm{He}^{+}+\mathrm{C}\end{array}$ & $\begin{array}{l}\text { +TIC: } H^{+} \\
\text {DIC: TIC: } \\
\text { SIC+ }\end{array}$ & $D^{+}$ & E & $0.1-8 \mathrm{kel}$ & 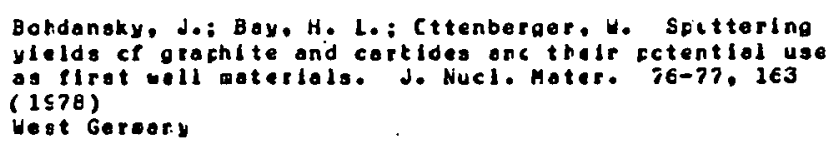 \\
\hline 1398 & $\begin{array}{l}\mathrm{DO}^{2} \\
\mathrm{H}^{+}+\mathrm{SS}: \mathrm{H}^{+}+\mathrm{C}: \mathrm{H}^{+}+\end{array}$ & BeO: D*+ & ss : & $\boldsymbol{\varepsilon}$ & $0.33-9 \quad k \in V$ & 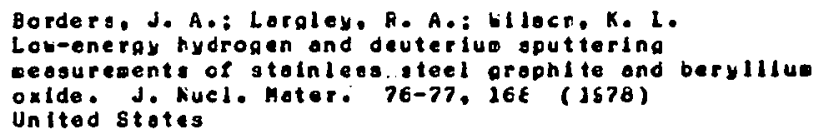 \\
\hline 1399 & $\begin{array}{l}\text { Do2 } \\
\text { Undet }\end{array}$ & & & $\mathbf{T}$ & Unde 1 & $\begin{array}{l}\text { Dzloba, S.: Kelly, R. Fecall Ieplantalion fros a thin } \\
\text { source: J. Nucl. Mater. 7E-77, I7s (1978) } \\
\text { Canada }\end{array}$ \\
\hline 1400 & $\begin{array}{l}\text { D17 } \\
H^{+}+\mathrm{SS}: \mathrm{Ar}^{+}+\mathrm{SS}\end{array}$ & & & E & $3 \mathrm{keV}$ & 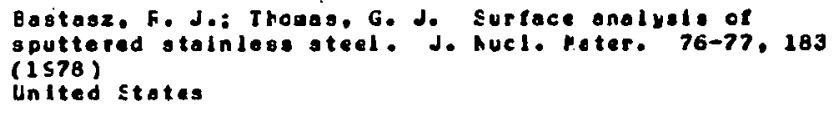 \\
\hline 1401 & $\operatorname{DQZ}^{+}+\mathrm{Mo}$ & & . & $E$ & $0.2-10 \quad n \in V$ & 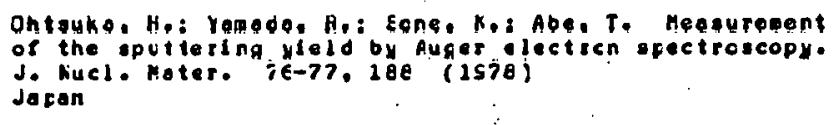 \\
\hline 1402 & $\begin{array}{l}\text { Doz } \\
\text { Undef }\end{array}$ & & ' & $T$ & Undef & $\begin{array}{l}\text { Andersen, H. H. A note on nevtron-spettering } \\
\text { measuresents. J. Hucl. Mater. 76-77, ISO (1578) } \\
\text { Denask }\end{array}$ \\
\hline 1403 & $\underset{H \rightarrow+}{\mathrm{DO} 2}+[\mathrm{C}+\mathrm{Pt}\rceil$ & & & E & s keV & 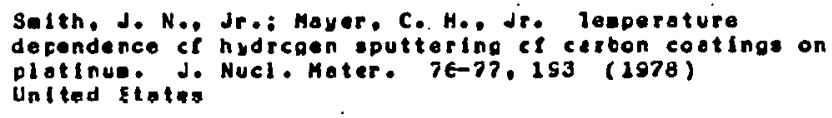 \\
\hline 1404 & 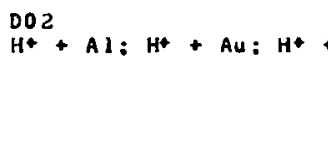 & + SS & & $\mathbf{E}$ & $100-200 \mathrm{eV}$ & 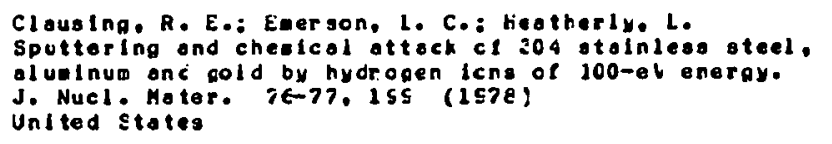 \\
\hline 1405 & $\begin{array}{l}D 17 \\
D^{+}+S 1 C\end{array}$ & & & $\mathbf{E}$ & $0.7 \mathrm{keV}$ & 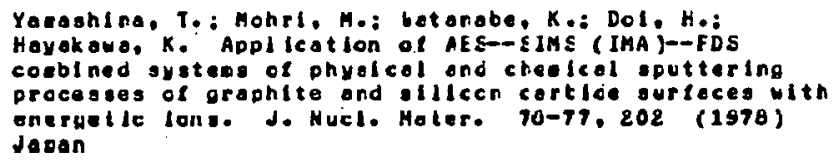 \\
\hline 1406 & 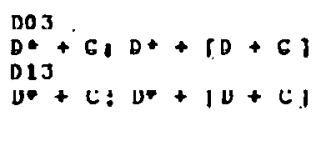 & & & E & $5-30 k \in V$ & 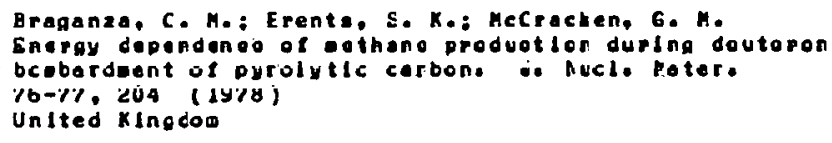 \\
\hline 1407 & 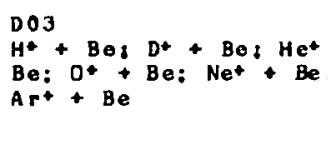 & $=\mathrm{Kr}^{+}+\mathrm{Bes}+$ & & E & $0.2-15$ hev. & 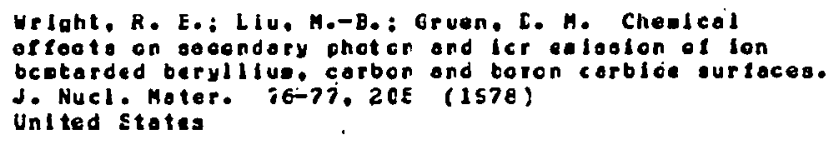 \\
\hline 1408 & $\begin{array}{l}\text { D14 } \\
\text { HAT + S4 }\end{array}$ & & & $E$ & $175 \mathrm{keV}$ & 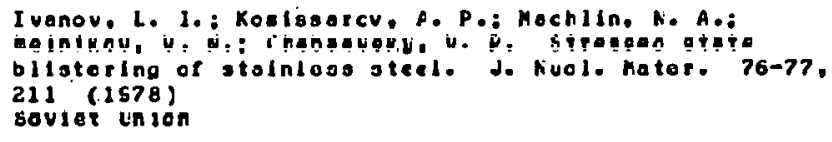 \\
\hline 1409 & $\begin{array}{l}\text { Dl4 } \\
\text { Hat + Ni: Hat + Ra }\end{array}$ & & & $\mathbf{E}$ & $20-500$ hev & 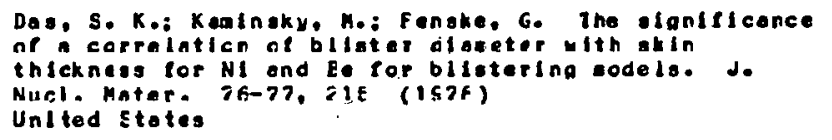 \\
\hline 1410 & $\begin{array}{l}\mathrm{Dl} 4 \\
\mathrm{He}+\mathrm{V}\end{array}$ & . & & $E$ & 2 MeV & 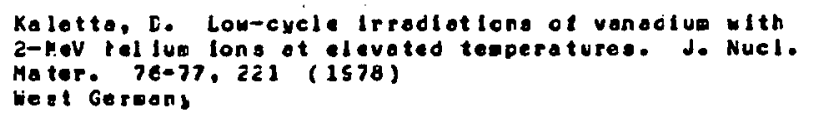 \\
\hline 1411 & $\mathrm{HL}^{\mathrm{D}}+\mathrm{V}: \mathrm{H}^{+}+\mathrm{TI}: \mathrm{He}+$ & $+\mathrm{V}: \mathrm{He}++$ & TI & $E$ & $10-40 \mathrm{k} \in \mathrm{V}$ & 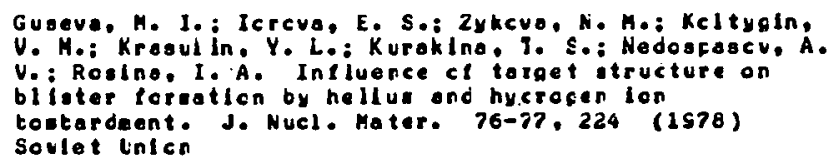 \\
\hline
\end{tabular}




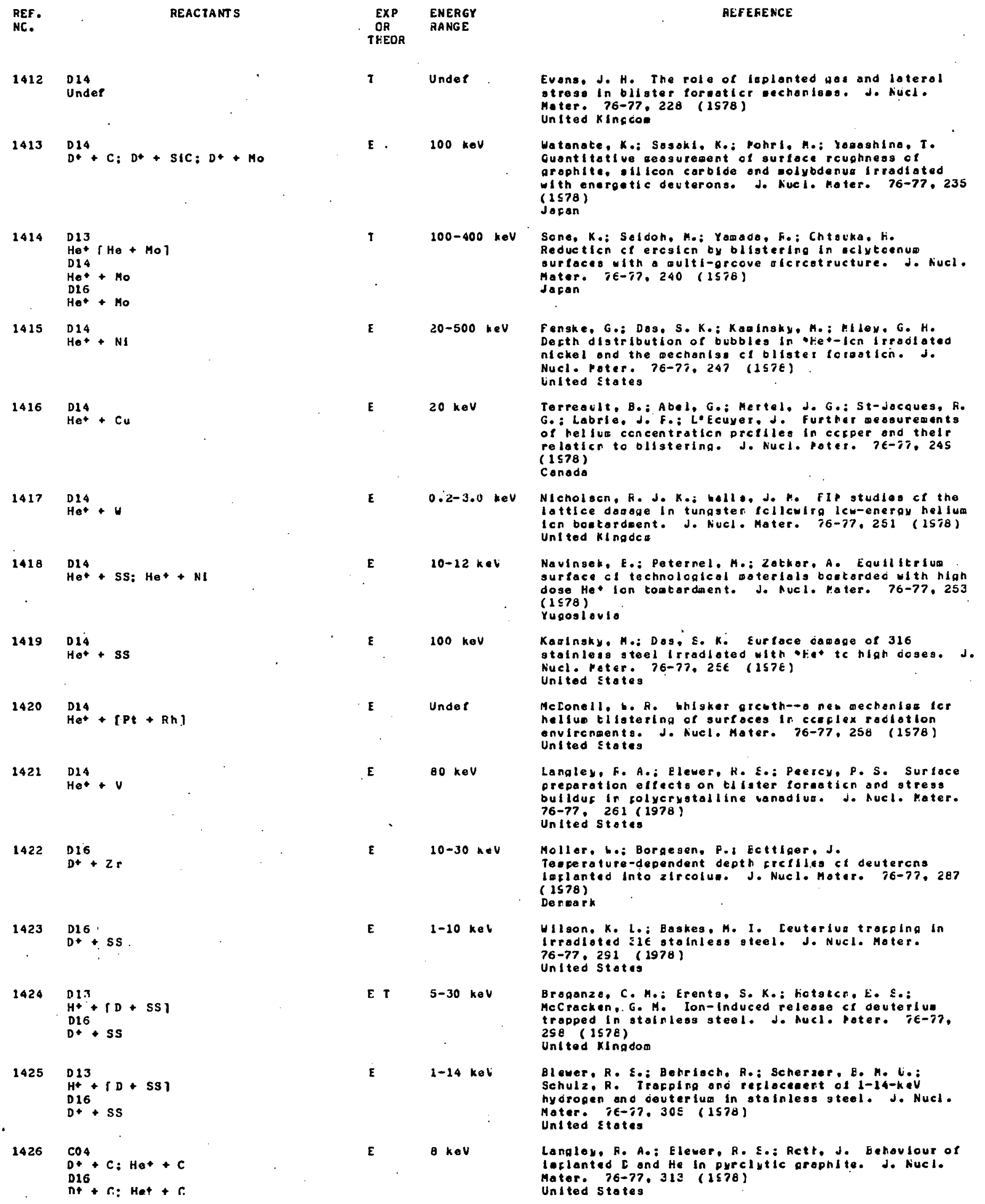


RE

1427

D13

$D+[D+S S]$

D16

D*+ SS

1428

$\mathrm{He+}+[\mathrm{H}+\mathrm{H}]: \mathrm{He}^{+}+[\mathrm{O}+\mathrm{TI}]: \mathrm{He}^{+}$

$+[\mathrm{O}+\mathrm{H}]: \mathrm{He}+[\mathrm{O}+\mathrm{Ni}]: \mathrm{He}^{+}+\mathrm{HO}$ 4. Pd

1429 D15

D*. + SS

1430

$\mathrm{DOP}$
$\mathrm{He}+$

1431

Do?

$\mathrm{He}^{+}+\mathrm{Cu} \mathrm{He}^{+}+\mathrm{Ag:} \mathrm{He}^{+}+\mathrm{Au}$

1432

D07

$\mathrm{H}^{+}+\mathrm{C}_{3} \mathrm{H}^{+}+\mathrm{TI} ; \mathrm{H}^{+}+\mathrm{MO} ; \mathrm{H}^{+}+\mathrm{H}$

$\mathrm{H}^{+}+\mathrm{AuZ}_{\mathrm{Z}} \mathrm{H}^{+}+\mathrm{TICH} \mathrm{CH}^{+}+\mathrm{TIH}_{3}: \mathrm{D}^{+}$

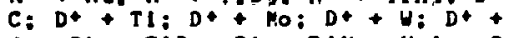
Au: $\mathrm{D}^{+}+\mathrm{TID}: \mathrm{D}+\mathrm{T}_{3}+\mathrm{TH}_{2}: \mathrm{He}^{+}+\mathrm{C}_{\text {; }}$ $\mathrm{He}^{+}+\mathrm{TI}_{3} \mathrm{He}^{+}+\mathrm{MaO}^{+} \mathrm{He}^{+}+\mathrm{H}_{0} \mathrm{He}^{+}+$

$\mathrm{Au}: \mathrm{He}^{+}+\mathrm{TID}_{>}: \mathrm{He}^{+}+\mathrm{TIH}_{2}$

1433

D 07

$T^{+}+T_{1} T_{+}+F e: T+T^{+}+H_{3}: T++$

FeH $:$ : T + FeH: T* + FeT: T+ ?

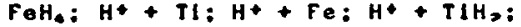

$\mathrm{H}^{+}+\mathrm{FeH}_{3}: \mathrm{H}^{+}+\mathrm{FeH}: \mathrm{H}^{+}+\mathrm{FeT}^{+} \mathrm{H}^{+}+$

$\mathrm{F} \in \mathrm{H}$.

$\mathrm{DO} 2$

$\mathrm{T}^{+}+\mathrm{TI}: \mathrm{T}_{+}+\mathrm{Fe}: \mathrm{T}^{+}+\mathrm{TIH}_{3} \mathrm{~T}_{+}+$

$\mathrm{FeH}_{\mathrm{O}} \mathrm{T}+\mathrm{TH}+\mathrm{FeH}$ : $\mathrm{T}+\mathrm{FeT}$ : Tt

$\mathrm{FeH}_{4}: \mathrm{H}^{+}+\mathrm{TI}: \mathrm{H}^{+}+\mathrm{Fe}: \mathrm{H}^{+}+\mathrm{TIH}_{3}$

$\mathrm{H}^{+}+\mathrm{FOH}_{3} \mathrm{OH}^{+}+\mathrm{FeH}^{+} \mathrm{H}^{+}+\mathrm{FOT}^{+} \mathrm{H}^{+}+$

$\mathrm{FeH}$.

D13

$\mathrm{He}+[\mathrm{O}+\mathrm{MO}]$

1435

D06

D+ $\dot{H}_{3}: e+D_{2}$

$\mathrm{DO}_{4}$

$C+H_{2}: E+D_{0}$

1436 D13

- $+\left[\mathrm{H}_{2}+\mathrm{SS}\right]:$ e + $[\mathrm{CO}+\mathrm{SS}]: e+$

$\left[\mathrm{CH}_{4}+\mathrm{SS}\right] \mathrm{O} e+\left[\mathrm{H}_{2}+\mathrm{Al}\right] \mathrm{Z}+[\mathrm{CO}$

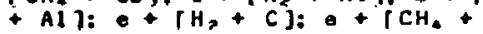

Al $\mathrm{I}_{\mathrm{e}}+[\mathrm{CO}+\mathrm{C}] \mathrm{C} e+\left[\mathrm{CH}_{+}+\mathrm{Cl}: \mathrm{e}\right.$

$+\mathrm{H}_{2}+$ Inconel 1: : + $\mathrm{rCO}+$

Inconel 1: e + [chis + Inconel

1437

$\mathrm{Cu} 4$

$\mathrm{Nb}+\mathrm{Nb}$

D15

$\mathrm{Nb}+\mathrm{Nb}$

$1438 \quad 003$

$\mathrm{DO3}^{+}+\mathrm{SS}: \mathrm{H}^{+}+$In conel: $\mathrm{H}_{3}+$ + SS:

$\mathrm{H}_{2++}^{+}$Inconel: $\mathrm{H}_{2}^{+}+\mathrm{SS}^{+} \mathrm{H}_{2}^{+}+$

Inconel

1439

D14

$\mathrm{Ta}^{3+}+\mathrm{No}_{\mathrm{O}} \mathrm{He}^{+}+\mathrm{Mo}^{2}$

1440

DI4 14 SS
EXP ENERGY

OR RANGE

E $\quad 15-750$ ㄴ

$\varepsilon$

E

5-10 kev

$0,2-20 \mathrm{keV}$

$0.1-2.0 \mathrm{heV}$

$1=1,5 \mathrm{k}, \mathrm{s}$

E $\quad 1-3 \mathrm{k} \in \mathrm{V}$

E

$100-150 \bullet V$

$45-675 k e V$

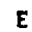

E

$5 \mathrm{keV}$

$0.3-7.2 \mathrm{KeV}$

$46 \mathrm{MeV}$
FEFEREACE

Farrell. G.: Donnelly. S. E. Theras desorticn and bobardient-l nduced release of ceuterlue ieclonteo into steinless steels ot low energy. J. Nucl. Moter. $76-77,222$ (1578)

United Kingdo:

Taglaver. E.: Hellend, W. Ien lefact cesorfticn cros sections of hydrogen ano oxygen frca detalo by llaht icn boubardment. J. Nucl. Pater. 76-77, 328 (1578) West Geraany

Ihcoas. G. J.: Hilscn. K. L. Micicatructure ct lou energy ceuterium Implonted toinless steel. J. Nucl. Mater. 76-77, 332 (1578)

United States

Foblinecn, J. E.: Jackson, I. P. Ecattering of ket hydrogen and hell un lons and neutralg tram cryaen covered surfaces. J. Nucl. Mater. TE-77, 35z (1578) Conoda

Sehou, do: Sorensen. H.: Lltteark, U. Eneray reflecticn coefflcients for $5-10 \mathrm{k}$ t $\mathrm{He}$ lons incident on AU. Ag. and CU. J. Nucl. Mater. iE-77, aES (ISTE) Dermark

Ecksteln, W.: Verteok. H. Feflection ef H. D ond lle erce C, Ti, NI. Me, W, and Au, J. Muct. Mator. 76-77. 368 (is78) Weat Geroony

Oen. D. S.: Roblinson. M. T. Cosputer liaulaticn ef the retlecticn ce hydrogen ond the ocuttering ot hyorcgen iroe eital hydrides. J. Nucl. Meter. 76-77, $=70$ (1578)

Hest Geraony

Akelohl, K.l Mlyohora, Aol Cayara, A. Lüelleayy lun scattering study ce abscred loyers cn colytcenue surface. J. Aucl. Mater. 76-77, E78 (1S7E) joran

Sorensen. H.: Schou. J. [n seccndary electron eaissicn frea sclid ti, and $c_{70}$ J. Aucl. Mater. 76-77, EZ4 (1578) Dengork

Lelegard, J.: Schram. A. Induced cesciction, cilfusion or production of gases by 100 ev electicn bcabordaent on sone woll soteilals lor tokemaks. J. nucl. pater. $76-77,637$. (1978)

France

Blersack, J. P.: Kaczerougkl, W.: NeV, J.: Rahio, B. K. H.: Riccoto. A.: Thacker. G. F.: liecker. H. Siouloticn of 14 MeV neutrins by crotcrs ci highel energies. J. Nuel. Peter. 76-77. 640 (157e) West Germony

Salth. d. N.. Jr. Secondary lon exlsolcn frco Talnies teel and lnccnel due to hyorooen ion tomardaent. d. Nucl. Mater. 78, 117 (1S7E) United Etates

Klsalnger, H. E.: Brimhal L, J. Lo: Slecnen, E. F.: Charlot, I. A. [uol-bear icn tcetordeent of molvbdenum. J. Nucl. Mater. $79, i 10$ (Iste) Untied States

Hayns, M. R. Nuierleal evaluaticn ct catired self-icn Injecticn ond vacancy loof frecucticn in heevy-ion Irradiotions. J. Nivel. Mater. 78.225 (1578) United KIngdo. 


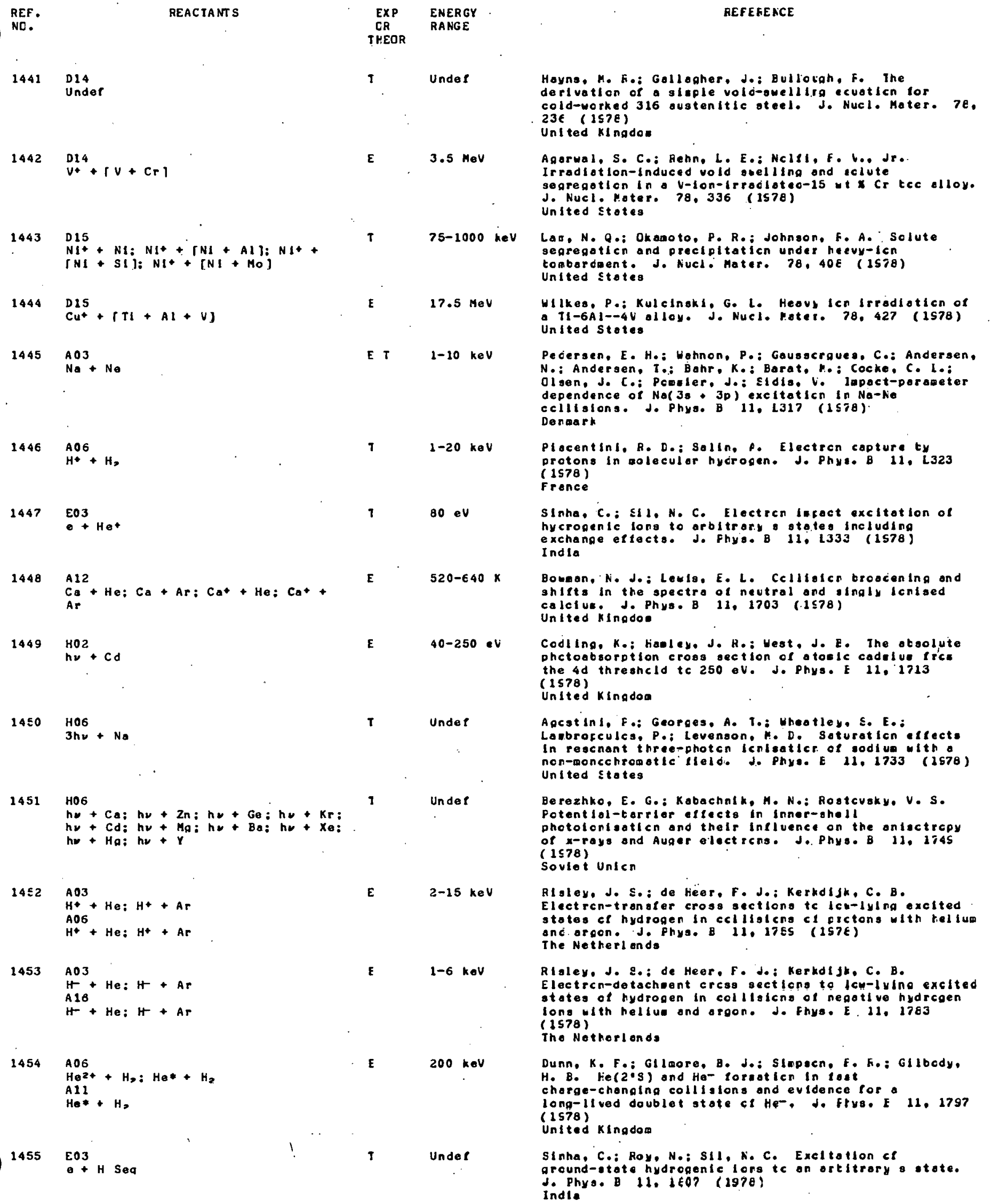




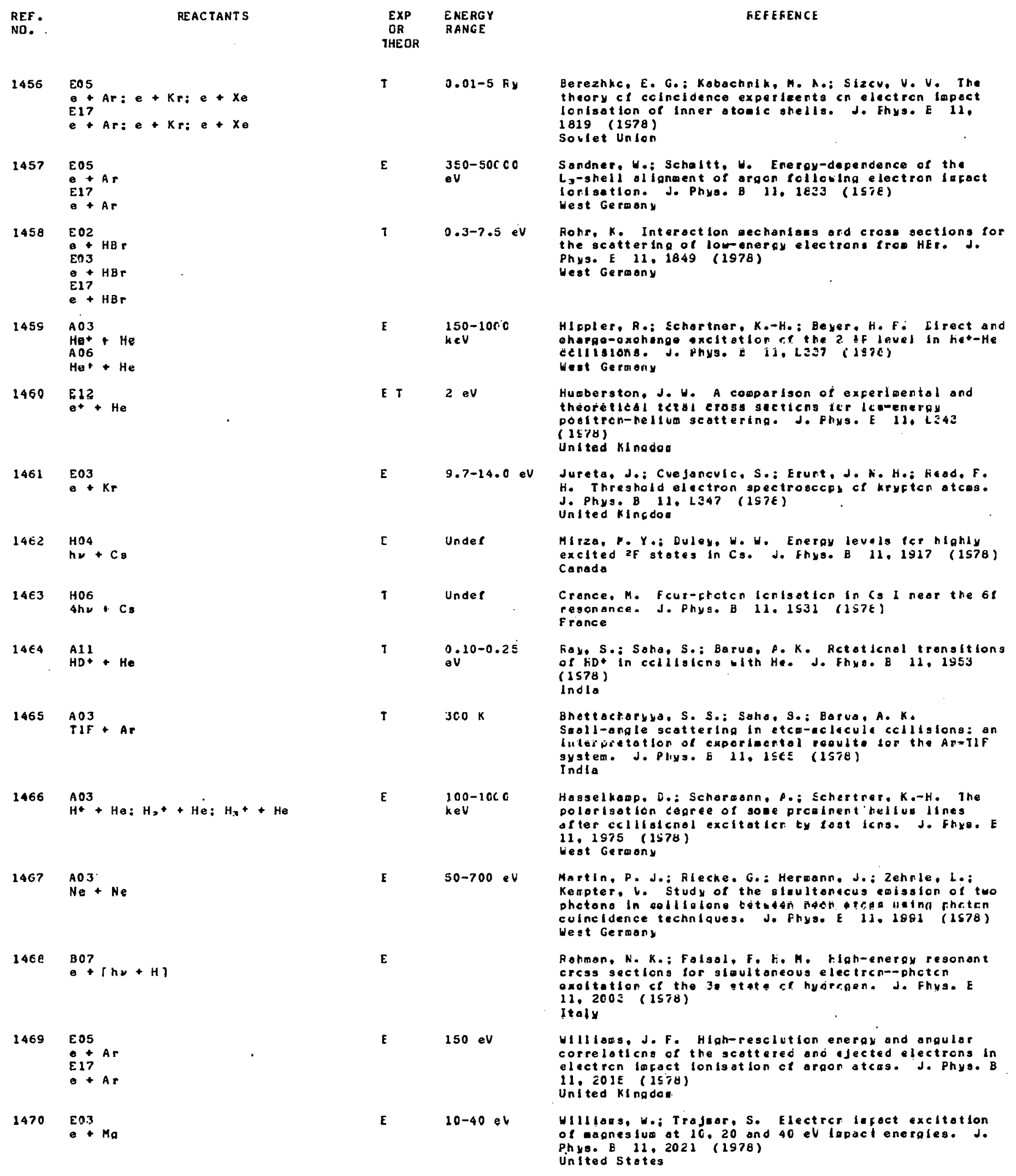




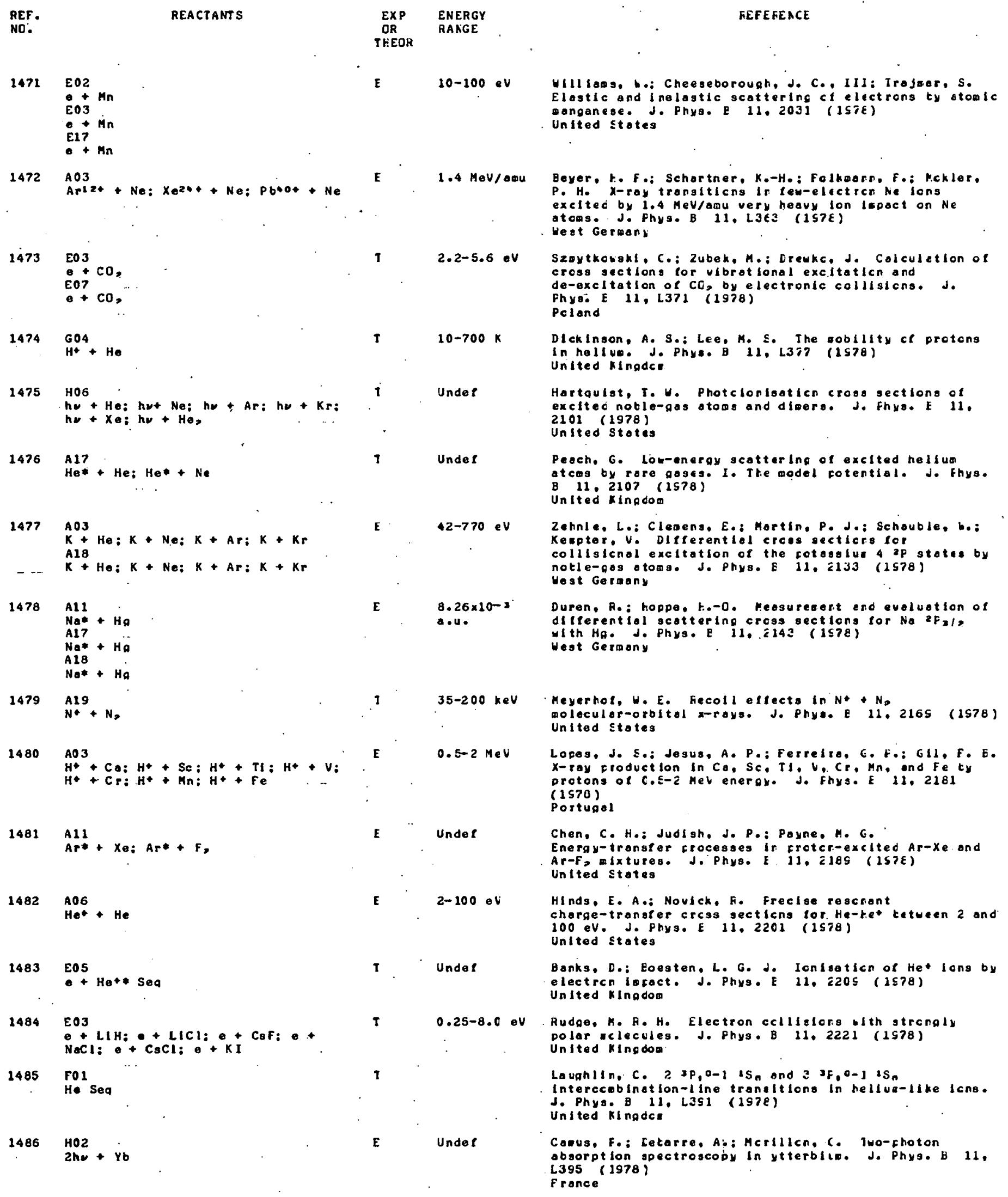




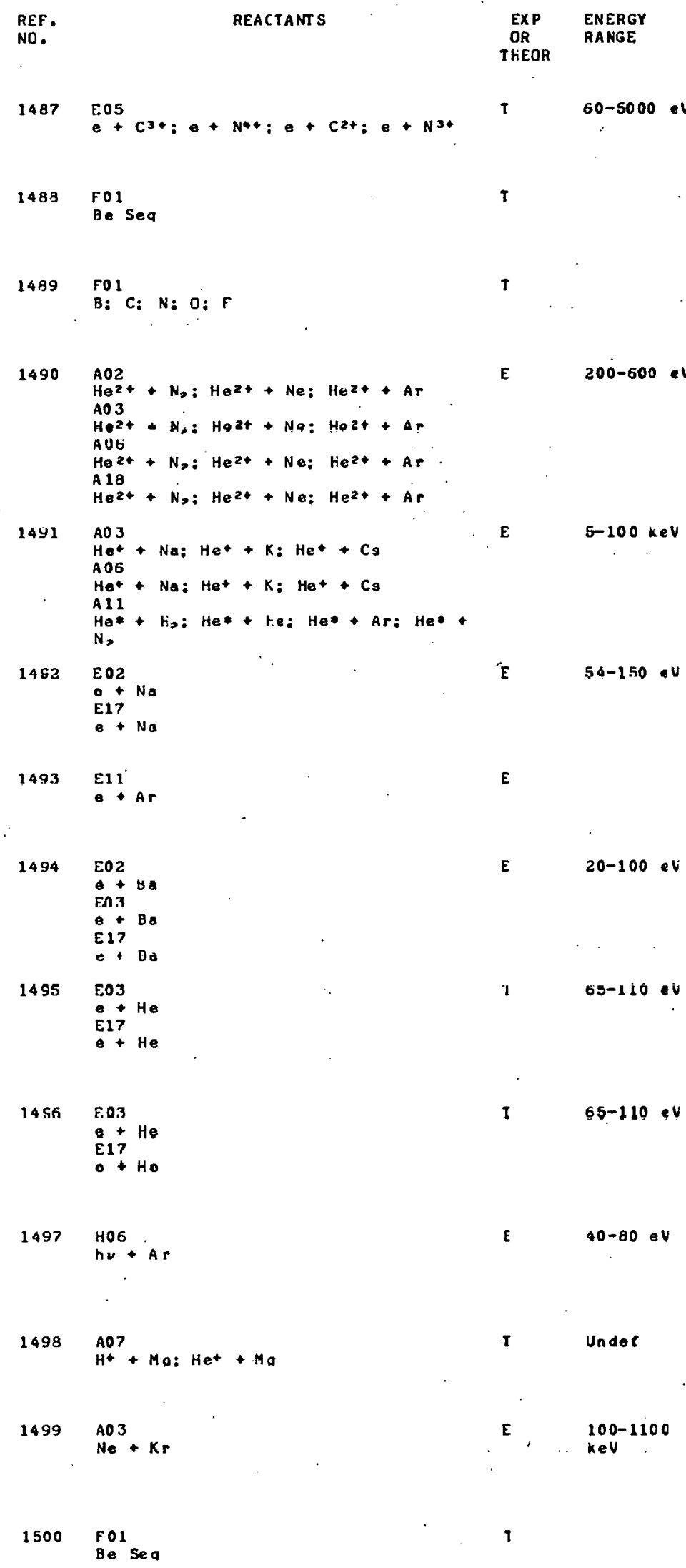

Mocres, D. L. Electron Impact Ionisaticn of LI-like and Be-like carbon and nitrogen lcra. J. Ftiya. E 11, L403 (1978)

Untted Kingoom

Laughlin, C.: Constontinldes. E. F.: Victor. G. A. Tworvalence-olectron adel-cctentiol studies of the be I Isoelectronic sequence. J. Phys.E 11, 2243 (1578) United Kingdom

Clasa, R.: HIbbert. A. The hyperline structure of the ground atates of first-row otces. J. Fhys.B 11, 2257 ( 1578 )

United KIngdo:

Rocers, W. T.: Eering, J. H.: Jchnacn, R. E.

Scattering of Hezt from $N_{2}$ : ccaparlson of Hezt $+\mathrm{N}_{2}$

w th Hezt+ Ne ond Ar. J. Ehye.E 11, 2319 (1978)

UnIted States

McCullcugh, R. H.: Colle, T. V.: Gllbcey, H. B. Foraction of last metostable hellua atcas In electron capture by Het lons in alkall-petal vacours. J. Fhys. B 11,2333 (1978)

Unitad Kingdon

Teubner. P.J.C.: Buckeen. S. J.: Ncble. C.J. Differential crosa sections for the elostlc scottering of Intermediate-eneray electrcrs lice sodium. J: Phys. B $11,2345(1578)$ Australia

Andrick. D.: Langhans. L. Mesuregent cl the tree-free cresa section of e-Ar scattering. J. Phys. B 11. $23 E 5$ (ISTE)

vest Germany

Jensen, S.: Reglater. L.: Irojoor, S. Elogtlc ono

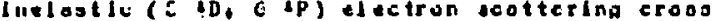
sectlong for bartume J. Fhys. E 11, 2367 (1S78) United states

Hickerson, L, L: : Hodlnow, L.: Dohler Ja St Intluence of electronic correlaticn on the electren lecact excitation of the doutly exclted JF(oub a) and ad aub u) states of hel lua. I. The Ecrn-depenhelacr apfroxiaticn. J. Phys. E, 11, 2377 (1578) United stotes

Hl ckerson, D. L.: Leung, C. N.: Kuong, b. K.: Lohler, J. S. Influence ct electrcnic ccreiletion cn the electron lepoct excltation cf the dcully excitid ofiout

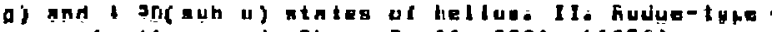
approxiratiens. J...Phyo. E 11, 2391 (1S7E) United Stotes

Adom, M. Y.: Wullieumler, F.: Krumacher, E.: Schaidt, V.: Mehihorn, W. Correlation satellites in the auter-shell photoelectron afectrua ct orgon. J. Fhuse B 11, 1413 (1578) France

Berezhko, E. G.: Kabachnik, N. Me: Slzcv, V. V, On the ol igneent of lons in inner-ghell icnlaotion by heovy particle inpact. J. Fhys. B 11. 1421 (1978) Sovlet linicn

Hoerlee, P. H.: Fortner, F, J.: Decrn, S.: Hoogkoder. T. P.: Sor Is, F. We Enhanced $K-L$ voconcy-shoring ratlos observed in lou-velocity Ne-Rr cellislofis. J. Phys. B 11. 2425 (1978) The Nethorl on ds

Gless. F.: Hitbert, A. The use of the Brelt Interaction: the $F_{\text {. }}$ is intercoublination line in beryllilua-1lke oystegs. J. Fhys. E 11, 2413 (1S7E) Untied xincoso 


\begin{tabular}{|c|c|c|c|c|}
\hline $\begin{array}{l}\text { REF. } \\
\text { ND. }\end{array}$ & REACTANTS & $\begin{array}{l}\text { EXP } \\
\text { OR } \\
\text { THEOR }\end{array}$ & $\begin{array}{l}\text { ENERGY } \\
\text { RANGE }\end{array}$ & FEF EFENCE \\
\hline 1501 & $\begin{array}{l}\text { HoG } \\
\text { hu }+\mathrm{He}\end{array}$ & $\mathbf{T}$ & Under & $\begin{array}{l}\text { Stewart, A. L. The phctolcnisoticr ct reliue using } \\
\text { perturtotion theory. J. Pliyse E 11. E44S (1578) } \\
\text { United Kingdog }\end{array}$ \\
\hline 1502 & $\begin{array}{l}\mathrm{H} 06 \\
\mathrm{hv}+\mathrm{Ne} \\
\mathrm{HO4} \\
\mathrm{h} \mathrm{H}^{-\mathrm{Ne}}\end{array}$ & $\mathbf{T}$ & Under & 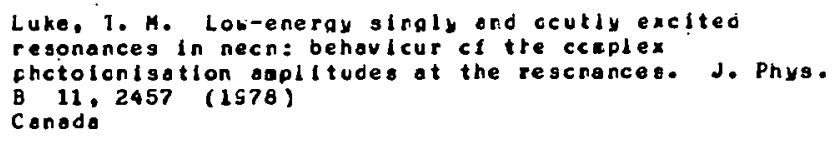 \\
\hline 1503 & $\begin{array}{l}H 06 \\
h \nu\end{array}$ & 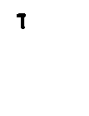 & Unde & 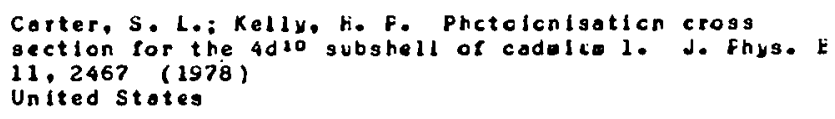 \\
\hline 1504 & $\begin{array}{l}\mathrm{All} \\
\mathrm{Ha} *+\mathrm{N},\end{array}$ & $\mathbf{E}$ & Undef & 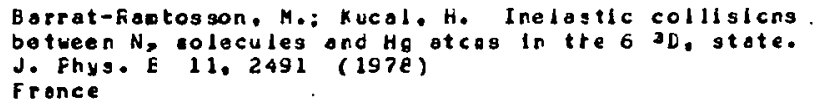 \\
\hline 1505 & $\begin{array}{l}\mathrm{AOZ}^{+}+\mathrm{Ag}: \mathrm{He}^{2+}+\mathrm{Pb} \\
\mathrm{AlO}^{+} \mathrm{AG}: \mathrm{He}^{2+}+\mathrm{Fb}\end{array}$ & $\mathbf{T}$ & $10-25 \mathrm{MeV}$ & 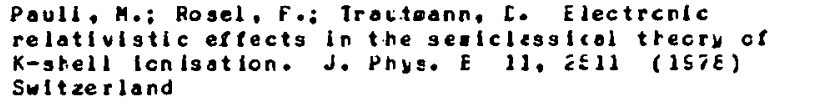 \\
\hline 1506 & $\mathrm{AOS}_{\mathrm{Xe}}^{\mathrm{AOO}}$ & $\mathbf{E}$ & $1-10 \mathrm{MeV}$ & 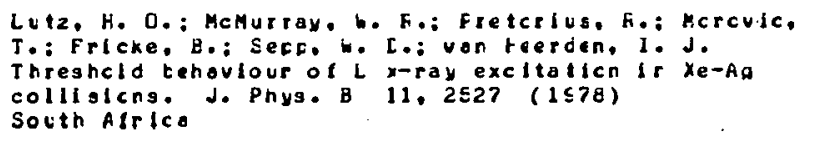 \\
\hline 1507 & $\begin{array}{l}E 02 \\
e+H \\
E 10+H \\
O+H\end{array}$ & $T$ & Unde $f$ & 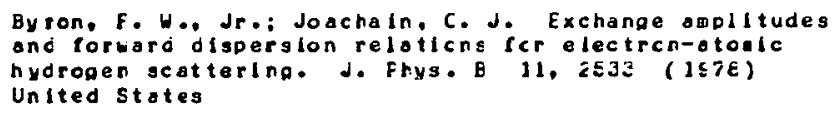 \\
\hline 1508 & $\begin{array}{l}E 13 \\
\mathrm{O}+\mathrm{H}^{-}\end{array}$ & $\mathbf{T}$ & $1.5-1000 \mathrm{eV}$ & 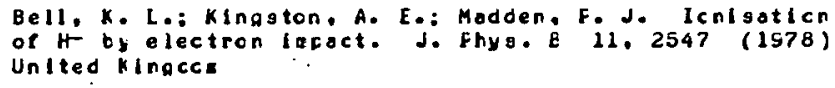 \\
\hline $150 \mathrm{~s}$ & $\begin{array}{l}E 12 \\
e^{+}+[H e+A P]\end{array}$ & $\varepsilon$ & Unde? & 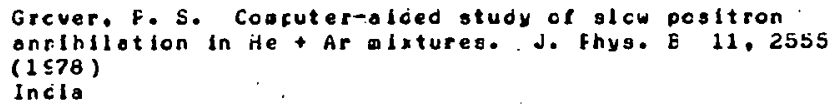 \\
\hline 1510 & $\begin{array}{l}\text { FOL } \\
\text { He* } \\
\text { HOG } \\
\text { ho }+\mathrm{He}^{*}\end{array}$ & $\mathrm{~T}$ & $\begin{array}{l}\text { Ho6 } \\
1-29 \mathrm{eV}\end{array}$ & 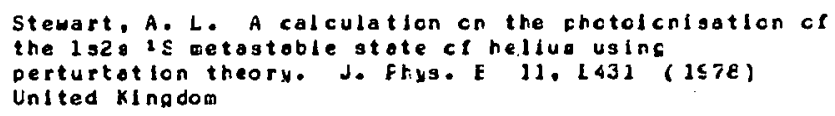 \\
\hline 1511 & ${ }_{h \nu}^{H 06}+A r: h \nu+X e$ & $\mathbf{T}$ & Unde? & 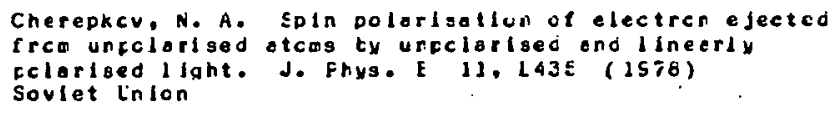 \\
\hline 1512 & $\begin{array}{l}\text { H06 } \\
3 \mathrm{h \nu}+\mathrm{Cs}_{3}: 4 \mathrm{h \nu}+\mathrm{Cs}_{3}\end{array}$ & $\mathbf{T}$ & Under & 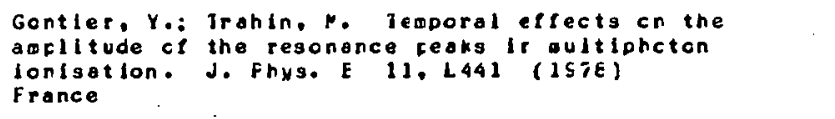 \\
\hline 1513 & $\begin{array}{l}\mathrm{A06}+\mathrm{H}: \mathrm{H}^{+}+\mathrm{He} \\
\mathrm{H}^{+}+\mathrm{H} 18+\mathrm{H}: \mathrm{H}^{+}+\mathrm{He} \\
\mathrm{H}^{+}+\mathrm{H}\end{array}$ & $\tau$ & $\begin{array}{l}10-80 \\
\text { MeV/omu }\end{array}$ & 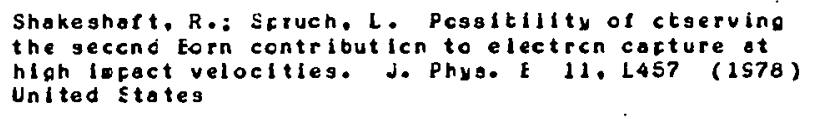 \\
\hline 1514 & 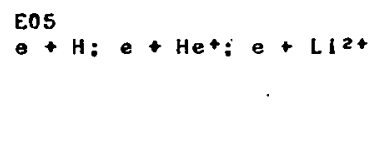 & $T$ & Unde? & 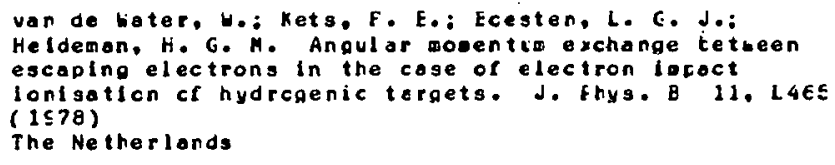 \\
\hline 1515 & $e^{+4}+H$ & 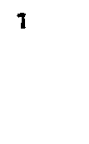 & Unde & 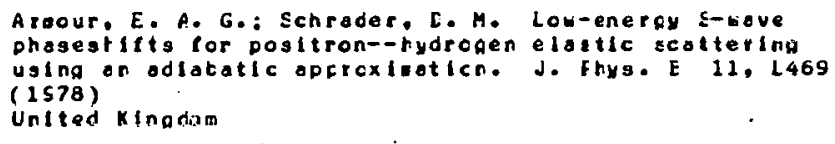 \\
\hline 1516 & 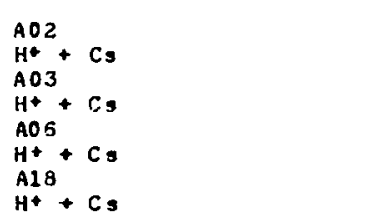 & $\dot{\mathbf{E}}$ & $\begin{array}{l}13.4-24.2 \\
\text { ev }\end{array}$ & 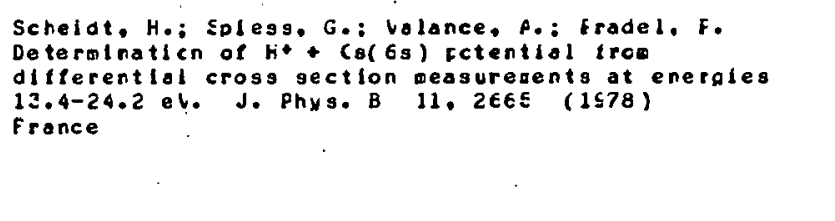 \\
\hline
\end{tabular}




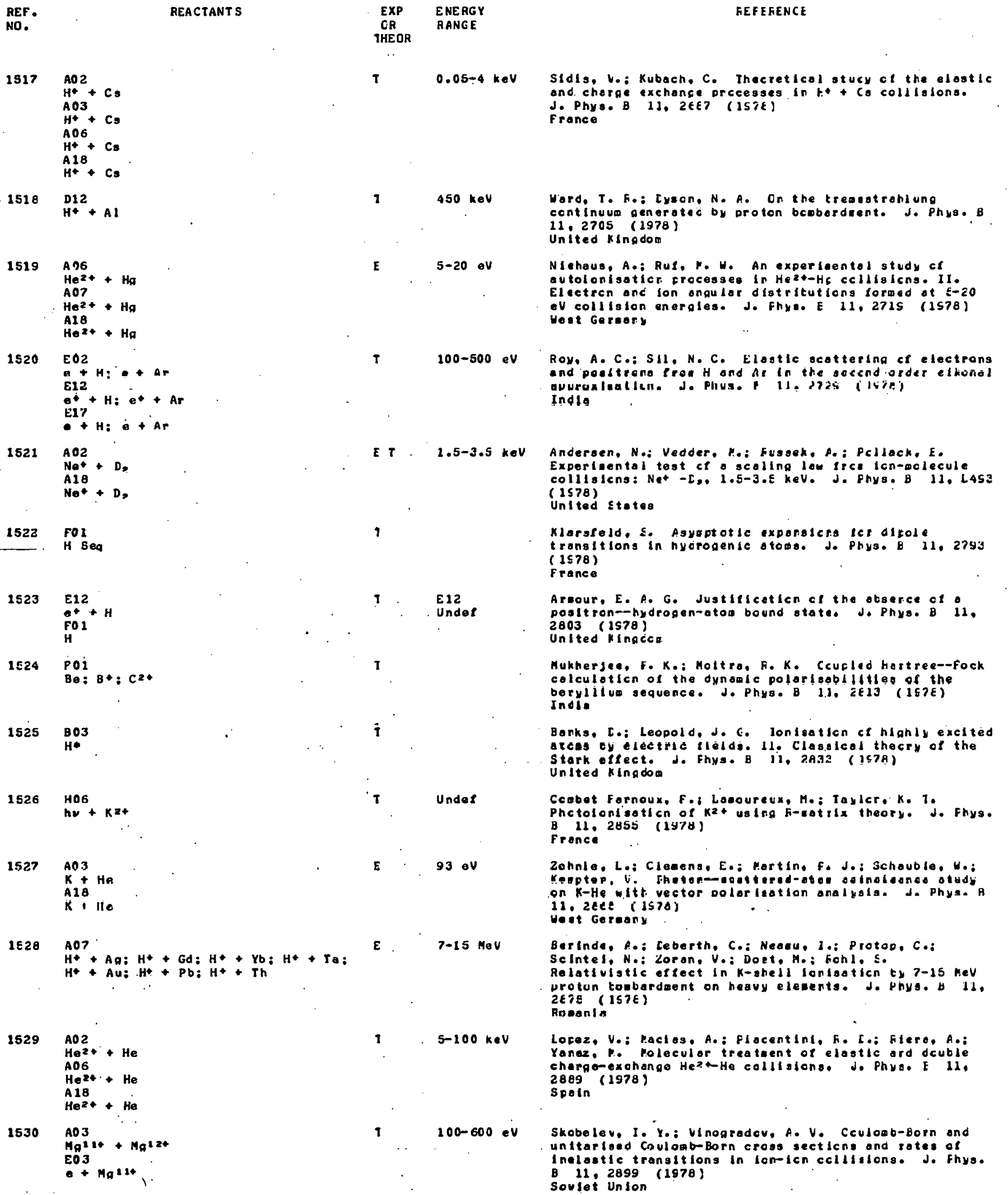


REF.

REACTANTS

NO.

\begin{tabular}{|c|c|}
\hline 1531. & $\begin{array}{l}\mathrm{EO} 2 \\
\mathrm{e}+\mathrm{H}\end{array}$ \\
\hline 1532 & $\begin{array}{l}\mathrm{E}^{0} 3 \\
+\mathrm{He}\end{array}$ \\
\hline 1533 & $\begin{array}{l}\mathrm{EO} 3 \\
0+\mathrm{N}\end{array}$ \\
\hline 1634 & $\begin{array}{l}E 04 \\
e+I \\
E 09 \\
e+I\end{array}$ \\
\hline & FO1 \\
\hline
\end{tabular}

$\mathrm{F01}$
$\mathrm{M} 033$

$1537 \quad H 06$

$1538 \quad H 05$

Under

1539 EOJ

$\mathrm{B}+\mathrm{H}$

E12

$e^{+}+\mathrm{H}$

E17 $H$

$1540 \mathrm{FO}$

$C: N: O: B$

1541 FOI

$\mathrm{Cu}: \mathrm{Au}$

$1642 \quad \begin{aligned} & \text { I10 } 1 \\ & \text { Under }\end{aligned}$

$1543 \quad \mathrm{HOA}$

$2 h \nu+$ No

$2544 \quad \begin{aligned} & \mathrm{AO3} \\ & \mathrm{He}+\mathrm{H} \\ & \mathrm{Al5} \\ & \mathrm{He}+\mathrm{H}\end{aligned}$

$\begin{array}{cl}\text { EXP } & \text { ENERGY } \\ \text { CR } & \text { RANGE } \\ \text { THEOR } & \\ & \end{array}$

T $2-30$ eV

1

E

T
Under

$290-450 \mathrm{~K}$

Undef

Undel

$54.4-200 \mathrm{eV}$

Undef

$0.001-1 \mathrm{eV}$
GEF EFENCE

Yau, A. H.: HeEochron. F. Fo: Stoutfer, A. I. Electron scotering lroo notle goses. J. Phys. B 11, 290 \% (1578)

Canada

Tully. J. A. Total crcas sectiens ler electron lapact excitation of the lis-2 is tronalition in He-like lona. J. Phys. B 11, 2923 (1S7E) France

Mileev. V. A.: Sofin. V. I.: strakhevo. S. I. The excltation of heavy atose by electrons in multiple-cellisicn diffracticn thecry. J. Fhys. E 11 . 2941 (1578) soviet $\ln i$ on

BIrtulatle, D. T.: Modinos. A. Disoclative attacheent In lodine. J. Phys. B 11, 2545. (1578) United Kingdo:

MIgdalek, J.: Baylis, t. E. Influence of atoblc core Dolarisoticr cn oscillator gtrangths ter

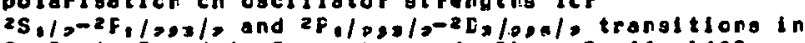
CuI. Ag I and Au I spectre. d. Five. E I1, I4ST (1578)

Canada.

Bolko, V. Ai: Plkuz, S. A.: Solroncua, A. S.: Foencu, A. $Y$. X-roy spectra of $X X X X I$ and $A C X X I V$ lons Irom laser-froduced plasmas. J. Phyae.E 11.L603 (1578) Soviet inlcr.

Chang. E. S.: Taylcr, K. I. Anguler diatritution of photolecticns ejected roi the 2 shell of corton. d. Phys. E 11. L507 (157E)

United Kingdoo

Mcclean, h. A.: Swaln, s. Thecry cl N-choton Ionisotion with short laser pulses. J. Phys. B 11. LSis (IS7E) Unlted KIngdce

Gupta, G. Fo: Mothur, K. C. Diflerentiol crose section for the excltation of the 20 . stote of otonlc hydrcgen by lectrcn and cesitrcn lucoct ot interaed ate energlee. J. Phys. B 11, LS21 (157e) India

Eggarter, E.: Eqgarter. T. P. Atcelc correletion energles III: secondorder corrections to the Hertree-Fcck ground stote ct B. C. A. C enc F. J. physe 11, 2969 (ig7e)

Phye:

Hotner, P.: Schwar2, W. H. E. Atoelc tranglticn probabllities erco the relativistlc caludopotential affroach. J. Phys. B 11. 2975 (157e) Hest Geriony

Lee, H. W.: Stehle, P. Interaction of on atow with a laserfulie. J. Fhys. E 11, 3015 (1578) United Stetes

Marx, B. R.: Allen, L. The wavelength dependence $c$ f the polerisetion selection rules ct the 3s-6s twe-photon trangition in atcalc scolua. J. ihye. B 11. $30<2$ (1578) UnIted Kingdca

Falcon. C.: Coradolce, Le: Placentinl. F. D. Unfolarlsed and apin-change collisicng between Het and $h$ ot lo enerates. J. Phye. B 11.3033 (1978) Argentina 
REF.

REACTANTS

NO.

1545

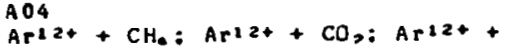

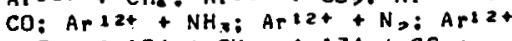

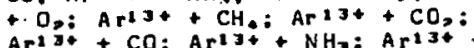

$\mathrm{N}_{32} \mathrm{Ar}^{23+}+\mathrm{C}_{7}: \mathrm{Kr}^{2 \mathrm{St}}+\mathrm{CH}_{4}: \mathrm{Kr}^{25}$

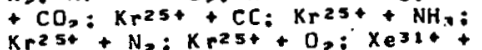

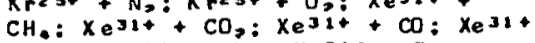

$+\mathrm{NH}_{3}: \mathrm{Xe}^{31+}+\mathrm{N}_{2}: \mathrm{Xe}^{31+}+\mathrm{O}_{2}$

$\mathrm{AO} 7$

$\mathrm{Ar}^{2+}+\mathrm{CH}_{2}: \mathrm{Ar}^{2+2+}+\mathrm{CO}_{3}: \mathrm{Ar}^{2} 2+$

$+\mathrm{O}_{2}: \mathrm{Arl}^{3+}+\mathrm{CH}_{0}: \mathrm{Ar}^{3} 3++\mathrm{CO}_{3}:$

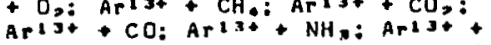

$\mathrm{N}=:$ Ar13+ $+\mathrm{D}_{2}: \mathrm{Kr}^{25+}+\mathrm{CH}_{*}: \mathrm{Kr}^{25+}$

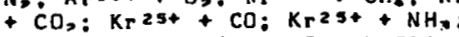

$\mathrm{Kr}_{\mathrm{r}} 25+\mathrm{N}_{2}: \mathrm{Kr}_{\mathrm{r}} 25++\mathrm{O}_{3}: \mathrm{Xe}_{31+}+$

$\mathrm{CH}_{4}: \mathrm{Xe}^{31+}+\mathrm{CO}_{2}: \mathrm{xe}^{32+}+\mathrm{CO}^{3} \mathrm{Xe}^{31+}$

$+\mathrm{NH}_{2}: \mathrm{Xe}^{31+}+\mathrm{N}_{3}: \mathrm{Xe}^{32+}+\mathrm{O}_{2}$

A 18

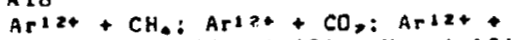

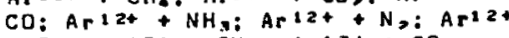

$+\mathrm{O}_{3}: \mathrm{Ar}^{13+}+\mathrm{CH}_{4}: \mathrm{Ar}^{2} 3++\mathrm{CO}_{3}:$

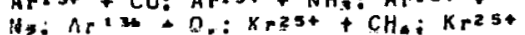

$+\mathrm{CO}_{3}: \mathrm{Kr}^{23+}+\mathrm{CC}: \mathrm{Kr}^{23}++\mathrm{NH}$ :

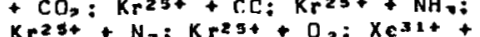

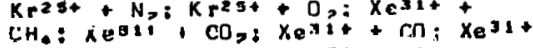

$+\mathrm{NH}_{2}: \mathrm{Xe}^{31+}+\mathrm{N}_{2} \mathrm{Xe}^{31+}+\mathrm{O}_{\text {, }}$

1546

$\mathrm{EO} 2$

O+ He+

E17

e $+\mathrm{He}^{+}$

$1547 \quad 802$

$e+\mathrm{CH}_{a} \mathrm{~S} e+\mathrm{C}_{3} \mathrm{H}_{a} \mathrm{O} e+\mathrm{C}_{3} \mathrm{H}_{a} \mathrm{O} e+$

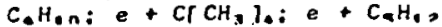

GO5

e+ $\mathrm{CH}_{a} \mathrm{O} e+\mathrm{C}_{2} \mathrm{H}_{n} \mathrm{O} \mathrm{e}+\mathrm{C}_{a} \mathrm{H}_{n} \mathrm{O} e+$

C. $\mathrm{H}_{\text {On }} \mathrm{e}+\mathrm{Cr}_{\mathrm{C}} \mathrm{CH}_{3} \mathrm{l}_{4}: \mathrm{e}+\mathrm{C}_{4} \mathrm{H}_{4}$,

1548 EO2

$\mathrm{C}+\mathrm{H}$

1549

cós

Fit He

o. He

1550

E06

$a+\mathrm{He}: e+C: e+\mathrm{O}: e+N$

$15 \bar{s}$

C.1

1552 HOG

he + o,

1553

A07

$\mathrm{He}^{+}+\mathrm{Mg:} \mathrm{H}^{+}+\mathrm{Mg}$

E05

$a+M a$.

1554

$\mathrm{EO4}$

$+\mathrm{N},:=+\mathrm{NO}$

EOg

- $N_{2}: e+N O$

EXP ENERGY

RANGE

AEFEFEACE

THEOR

Monn, R.: Folkmann, F.: Peterson, F. S.: Szobc, G.: Grceneveld, K, - C. Auger electron eulsion lrow torget icrs uncer heouy-icn locact after aclecular dissoctotion. J. Fhys. B 11.304E (1578) he at Gerang:

$100-200 \mathrm{eV}$

$257-673 K$

$\mathbf{E}$

.

$0.25-2.25$

$80-256 \mathrm{eV}$

$7-1000 \mathrm{eV}$
$20-45$ av

Undet

8-12 eV
Singh. S. N.: Kumor. S.: Srivagtavo. N. K. e--Het elastic ocottering in acoified Cculcat-Glouter opfroxiation. J. Phxs. E 11, 30El (1978) India

McCorkle, U. L.: Chrlstephcrcu, L. G.: Hoxey. L. V.: Corter. J. 6 . Foasauer--Tcunseno alniag in the electron-gcattering cross gecticns of folyatcalc gases: methane, ethane, cropane, tutore ord neopentone. J. Fhys. E 11,3067 (1578)

United stotes

Poet, R. Tre exact soluticn tor a gallifled ucdel cr electrcin scotzering by hycrcgen atcas. J. Fnys.. E 12 , 3081 ( 1578 )

United Kingcos

Bronsden, B. Hi.: Saith, J. J.: bintel=a K, h. Disrarted-acue oposonitioticra tcr the triple-cifierentiol crose ecticil tor icnisaticn ci hollua ty electror infact. J. Fhys.e 11, 30S5 ( I\$78)

Unitad kingood

Brook, E.; Harrison, M. F. A.: Solth, f. C. H. Masuredents of the electicn lacact icrisation cross sections of He, C. O and N atoos. J. Fhys. B 11,315 ( 1578$)$

Unlted kingdow

Curtls, L. J.: Ellis, I. G. A tcraula Lor concellation Ulsouvecioned of atrolis asclilator gtrengths. J. phye. F 11. L543 (1978)

United stotes

MeCoy, C. G.: Morten, J. M.: Marr. G. W. The anqular distritution of photoelectrons as e eurcticn ce chction energy for the arcund state chictolicnigetion of moleculor oxysen. J. Fhys. E 11. L547 (1578) United in hado:

Hodbro, M.: [utaris, R.l Eoheldt, H. L, $L_{1} M_{1}$, Auper electron study ot colliglonally induced allanaent for $e^{-} H^{*}$ and he impoct lonisation ir atcalic eanesilus. $e^{-} H^{+}$and he Impoct lon
J. Phys: E 11, LSEl (1978) Denark

Hozeau, J.: Gresteou, F.: Hall, R. I.: husta, A, Energy and idth of $\mathrm{N}-(3 \mathrm{P})$ froo ctserveticn of its Corgaticn disgociative attachate tc $N$, ond NO. J. Phys. E 11. LSE? (ISTE)

fronoe 
$\mathrm{GO}_{4}$ $\mathrm{O}_{3}++\mathrm{O}_{2}: \mathrm{N}_{3}++\mathrm{N}_{2}: \mathrm{N}_{0}++\mathrm{N}_{2}: \mathrm{C}_{2} \mathrm{O}_{2}+$

HO7 hy + Rb-

(1)

1566

Fo6 + SI

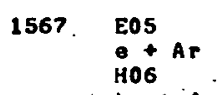

1568

hy + Ar

$\mathrm{FO}_{\mathrm{H}}$

Unde $P$

\section{$0.7-35 \mathrm{MeV}$}

$160-230 \mathrm{eV}$

Thermal

\section{$0.4-2.4$}

MeV/omu

Undef

T Undef

Steon. L.: Azrlo..F.: Ironc. M. Irterferences in the resonant vitrational excitotion of 5,0 J. Phys. B 11. LSE 1 (1S78) France

Coapeanu, R. I.: [ubau, J. Pcoltren-meon elastic sedtering. J. Phys. B il, L5e7 (157e) Rouanl:

BIshop. C. M.: Cheung.. L. P. Hceent functicns (Including stat ic dipole pclarlabillties) and raclative corrections for $H_{2}+$ J. Fhyte L 11, 3133 ( 1578 )

canada

Stre. E. O.: Kohlaeler, G. H.: Novak. E. A clasical trajectcry otudy of T-l energy trarafer of C hishly vitrationolly excited Morie oscillatcr in ccillinear collisicn with on otom. I. De-excliaticn crecesses. J. Phys, E 11. 3145 (1978)

West Geraony

Sire, E. D.; Kohlooler, G. H.: Hovak, E. A classical trajectery study of T-l energy trassler of o highy vitrationolly excited Mcrse oscillatcr in collineor collisicn ith on otom. II. Excitation ond disccioticn proceses. J. Phys. E 11. 31Ee (15TE) West Germeny

Ntelsen. S. E.: Ancersen, N.: Andersen. T.: Oloen, J. O.: Dahler. J. E. Direct excltoticn cili I (rl) levis in $k \in V$ Li-He collisicns. J.Phys. B 11. ale? (1578)

Denark

Aoundaen. F. A. On the rcle ct diccle trangltiong In the sediclosical opproximaticn for K-shell Icnisotion. J. Phys. B 11, 315? (157E) Norway

Golden. L. B.: Sorrson. D. fi.: Oolcuar. K. Ionlsotlon erca tie is sub-level of highiy charged iono.'J. Phys. B $11,3235(1978)$

Unjted stotes

Hitchcock, A. P.: Erion, C. E.: van der bilel. M. J. Ionle rrogentaticn of Sf a lcriseo in the sulphur 2p anell. J. Fhys. E 11.3245 (1S?E) Canado

Alcer, S. R.: Stefonsson. T.: Rees, J. A. Measurements of the lateral diffusicn cf $C_{2}$ icrs ir oxysen. $\Lambda_{s}+$, onc $\mathrm{N}_{*}$ lons in nltrogen, and $\mathrm{CO}^{+}$. CO icns in corton monoxide. J. Phya. B 11, 32ES (1S78) United Kingdo:

frey. F.: Erever. F.: hotch, t. H lah resolution photodetachment from the rutidiue negative icn arcund the Ro(SP./.) threshold. J. Fhys. E 11. L58S (1978) Weat Geraony

LIn, C. D. Guasi-aclecular binding ccrections to the twc-stote tomic expansion wethed for electrcn carture at low energies. 'A. Phys. B 11, 15s5 (1978) United states

McCarthy. I. E.: Uylings, F.: Ferfe, F. Ccaporison of (e.2e). photoelectron and ccnventicnal opectroscoples lot the Ar II Ion. J. Fhys. E 11.32SS (1578) The Ne the rlands

Sicola. J.: Virtagc, J.. Energy levelo ol hyorogen otcos in strong monetic lield. J. Physe. B 11. $330 \mathrm{~s}$ (1578) Finland

Geltmar. S. Icnlaticn duratica of ocoel otom In an electrostatic fleld. J. Frys. E 11. 3323 (1578) United stotes

Goldberg. A.: Shore. E. U. Modelling loger tonlsotion. J. Phys: B 11, 3339 (1S7E) United stotes 


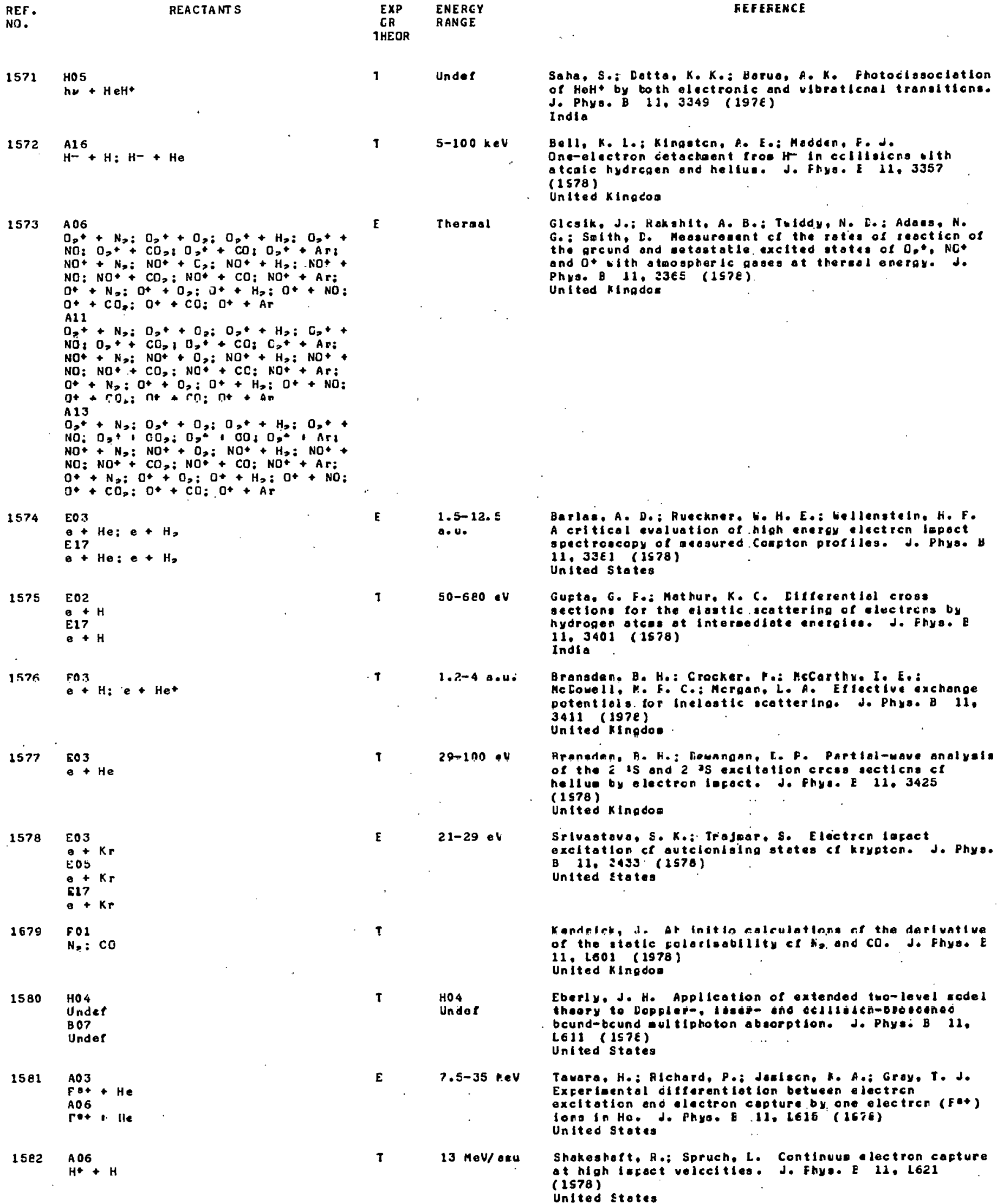

1571 HOS

hv + $\mathrm{HeH}^{*}$

1572

$\mathrm{H}^{-}+\mathrm{H}: \mathrm{H}^{-}+\mathrm{He}$

1573

$\mathrm{O}_{2}+\mathrm{N}_{3}: \mathrm{O}_{2}++\mathrm{O}_{2}: \mathrm{O}_{0}+\mathrm{H}_{2}: \mathrm{O}_{2}+$ $\mathrm{NO}: \mathrm{O}_{2}+\mathrm{CO}_{3} \mathrm{O}_{3}+\mathrm{CO}^{\circ} \mathrm{O}_{3}+\mathrm{CH}^{+}$ $\mathrm{NO}+\mathrm{N}_{-}: \mathrm{NO}_{+}+\mathrm{C}_{2}: \mathrm{NO}+\mathrm{H}_{3}: \mathrm{NO}++$ $\mathrm{NO}: \mathrm{NO}++\mathrm{CO}_{3}: \mathrm{NO}+\mathrm{CO}: \mathrm{NO}^{+}+\mathrm{Ar}$

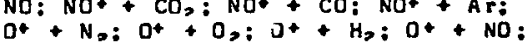
$\mathrm{O}^{+}+\mathrm{CO}_{2}: \mathrm{O}^{+}+\mathrm{CO}^{\circ} \mathrm{O}^{+}+\mathrm{Ar}$

A11

$\mathrm{O}_{3}^{+}+\mathrm{N}_{2}: \mathrm{O}_{3}^{+}+\mathrm{O}_{3}: \mathrm{O}_{3}+\mathrm{H}_{7}: \mathrm{C}^{+}+$

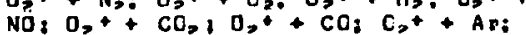
$\mathrm{NO}+\mathrm{N}, \mathrm{NO}^{+}+\mathrm{O}_{3}: \mathrm{NO}^{+}+\mathrm{H}_{2}: \mathrm{NO}+4$ $\mathrm{NO}: \mathrm{NO}++\mathrm{CO}$ : $\mathrm{NO}+\mathrm{CC}^{-} \mathrm{NO}+\mathrm{Ar}:$ $\mathrm{O}^{+}+\mathrm{N}_{3}: \mathrm{O}^{+}+\mathrm{O},: \mathrm{O}_{+}+\mathrm{H}_{3}: \mathrm{O}^{+}+\mathrm{NO}:$

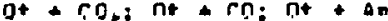

A 13

$\mathrm{O}_{3}+\mathrm{N}_{3}: \mathrm{O}_{3}+\mathrm{O}_{2}: \mathrm{O}_{3}+\mathrm{H}_{3}: \mathrm{O}_{*}+$

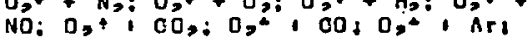
$\mathrm{NO}++\mathrm{N}_{2}: \mathrm{NO}^{+}+\mathrm{O}_{2}: \mathrm{NO}_{+}+\mathrm{H}_{3}: \mathrm{NO}_{+}^{+}$ $\mathrm{NO}: \mathrm{NO}^{+}+\mathrm{CO}_{7} \mathrm{ONO}+\mathrm{CO}^{+} \mathrm{NO}^{+}+\mathrm{Ar}$ $\mathrm{O}+\mathrm{N}_{3}: \mathrm{O}^{+}+\mathrm{O}_{3}: \mathrm{O}_{+}^{+}+\mathrm{H}_{3}: \mathrm{O}^{+}+\mathrm{NO}:$ $\mathrm{O}^{+}+\mathrm{CO}_{>}: \mathrm{O}^{+}+\mathrm{CO}: \mathrm{O}^{+}+\mathrm{Ar}^{+}$

$1574 \quad E 03$

e+He: $\mathrm{e}+\mathrm{H}_{\text {, }}$

E 17

e $+\mathrm{He}: \mathrm{e}+\mathrm{H}_{2}$

$1575 \quad E 02$

- $+\mathrm{H}$

E17

a 4

157 FO3

e+H: $\mathrm{H}+\mathrm{He}^{+}$

$1577 \quad 503$

e $+\mathrm{He}$

1578

E03

- Kr

EOS

G17 Kr

c $+\mathrm{Kr}$

$1679 \quad \mathrm{FO}$

$\mathrm{N}, \mathrm{CO}$

$\begin{array}{ll}1580 & H 04 \\ & \text { Undet }\end{array}$

B 07
Undef

$1581 \quad \mathrm{AO} 3$

$\mathrm{Fot}+\mathrm{He}$

AOG

1582

A 06

$\mathrm{H}+\mathrm{H}$

Undef

$5-100 \mathrm{keV}$

1

E

Therest

1.5-12. 5

a.u.

$50-6 e 0 \in V$

1.? -4 a.4:

29-100 14

21-29 eV

HO4

Undor

$7.5-35$ HeV

$T$

13 MeV/ oru

Saha, S.; Datta, K. K.: Herue, A. K. Fhotoclasoclation of $\mathrm{HeH}^{+}$by toth electronic and vibratlcnal transiticnse J. Phys. B 11.3349 (197E) India

Bell, K. L.: KIngetcn, A. E.: Madden, F. d. One-electron cetachaent irou H in cclllsicns ith atcalc hydrcgen ond heliud. J. Fhyo. E 11.3357 (1578)

United Kingcos

Glcsik. J.: Rakahlt, A. B.: Tulddy, N. E.: Adaes, N. G.: Swith. D. Meosuresent ct the roties of reocticn of the arcund and eetastatle. excited at ote of $0 . *$, $N C^{*}$ and 0 . With atmospberlc gaseg at thereol energy. J. Phys. B 11, 33EE (1S7E) United ringdos

Berlas. A. D.: Rueckner. H. H. E.: tiellenstein. H. F. A critical evaluation of high energy electron lapoct spectroscopy of measured.Corpton piofiles. J. Physe. $11,33 E 1$ (is78)

United States

Gupta, G. F.: Mathur, K. C. Cifferentiol cross sectlons for the elastic ecattering of electrins by hydrogen otces of intereediote enerolies d. Fhys. 11. 3401 (1578) India

Bransden. B. H.: Crocker. H.: HcCarthy. I. E. Mc Lowell. H. F. C.: Mcraan. L. A. Eflective exchange potentiols. tor Inelastic acattering. J. Phys. B 11. 3411 (197e)

United kingdoe

Aponeren, A. H.: Dewangan. C. P. Partial-wave analyals of the $z$ is and 2 is exctiotion crces eecticna cl hellue oy lection lacact. J. Fhye. 11.3425 ( 1578 )

United KIngdom

Srtuastavo, S. K.: Trajear, S. Electrcn lofact excltation ch autcioniding states ce krypton. J. Phyo. B 11. $2433(1576)$ United states

Kandefirk, d. Ar Initin ralculatione of the derivative of the statle colorleablility cl fo. ond $C O$. J. Fhyo, E 11. 1601 (1978) United Kingdoe

Eberly, J. H. Application of extended two-level codel

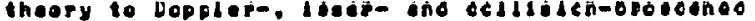
bcund-bcund aul tiphoton absorption. J. Phys: B 11. L611 (ISTE) United States

Tavara, He: Alchard, P.: deslecn. H. A.: Groy, T. J. Excerieental disterentiotion between electrcn excitotion endelectron cepture by one electrcn (Fet) long in Ho. J. Phyo. .11, teje (1676) United states

Shokeshoft, R.: Spruch, L. Continuue electron capture at high incoet velceities. J. Fhyo. E 11, L621 (1578)

Untted stotes 
NO

$1583 \quad$ E12

$\mathrm{O}^{+}+\mathrm{He}$

1584

$e^{* 12}+H e$

1585 E03

C No

1586 FO1
CP: Fe: NI

$1587 \quad A 03$

$H^{+}+H_{2}: A r+T I F$

$1588 \quad \mathrm{AOT}^{+}$
$\mathrm{H}^{+}+\mathrm{H}$

1529

$\mathrm{Al}^{\mathrm{H}} \mathrm{H}+\mathrm{He}: \mathrm{Kr}++\mathrm{Kr}$

1590

$\mathrm{AO6}$
$\mathrm{B}^{5+}+\mathrm{H} ; \mathrm{C}^{6+}+\mathrm{H} ; \mathrm{N}^{7+}+\mathrm{H} ; \mathrm{O}^{8+}+\mathrm{H} ;$
$\mathrm{Ne}^{10+}+\mathrm{H} ; \mathrm{Si}^{14+}+\mathrm{H}$

1551

$\mathrm{He}^{2+}+\mathrm{H}$

1592

H06

Unde?
B0?

e + Ne

HII

hu + No

$1594=04$

$\mathrm{E}_{\mathrm{E}}^{\mathrm{E}}+\mathrm{H}_{3}+\mathrm{O}+\mathrm{O}+\mathrm{H}_{3}+$

$\mathrm{O}+\mathrm{H}_{2}+\mathrm{O} \mathrm{O}+\mathrm{H}_{2}+$

$15 S 5 E 03$

$e+\mathrm{HCl}: e+\mathrm{HF}$

$1596 \mathrm{FO1}$

Under

1557

$\mathrm{AO}^{+}+\mathrm{H}^{+}$

1598

A06

Hez+ $+\mathrm{He}$
E $\quad 1.0-6.0 \mathrm{eV}$

$\mathbf{E}$

$\mathbf{T}$

T

$3.7 \mathrm{eV}$

$2-500$ ev

$25-200 \mathrm{keV}$

Under

Unde?

$3-1000 \mathrm{keV}$

Under

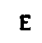

HבI

$510-480 \mathrm{~nm}$

$0.3-1.0 \mathrm{eV}$

$\tau$

$0-5$ eV

$0.1-1 \cos 0$

kov

$10-1700 e V$
Wllson, W. G. Scottering ci lew-erergy pogitions frow hellum otcms. J. Fhyg. B 11. Le2S (1578) West Gersany

Grifelth, T. C.: Heylond, G. R.: Lines, K. S.: Thcoey, $T$. F. Peopralsol of the exceriaentel crcss sections for low-energy positron-hellua scottering. J. Physe B 11. 1635 ( 1978$)$ United kingdoo

Stumpt, B.: Becker, K.: Schulz, G. Electren lafact excltation of the spectral resolved no-l lines. J. Fhys. E 11, 1639 (1978)

wegt Germony

Abtott, D. C. The structure and transiticn probabilities of icnlsed Cr. kn. Fe anckil. J. Fhys. E $11,3475(1978)$. United states

Dickinson, A. D.: Alchords, D. Cn an p-congerulng approxleaticn in time-deperdent theorics of rototional excitation in atoe-molecule ccllisions. J. Phys. B 11,3512 (1578) United kingdca

Belkle, r. A quantum thecry cl icrigation in last collisicn tetueen long and atculc gystems. J. Phys. B 11 , $3 E z \leq$ (1578)

Yugoslabla

Nikulin, V. K.: Guschino, N. A. Clatetic correlotion diagrag for the guasi-aolecular descrifticr of iontatca ccllisicns. J. Fhys. B 11. 5553 (1578) Soviet union

Greenlond, f. T. Highly strlpoed lons en nydregen atoms: the edlatatic approoch. I. Eneray aop and couplings ot fseuccercsings. J. Fhys. B 11, zEE (1 158 ) United kingdoo

Greenlond, $F$. T. Highly atripped ions cn hydrcoen atoms: the odlabatic offrcoch II. Energy gop ond coupl ingo of pseucocrassings. J. Phyg. B 11, EEja ( 1578$)$

United MIngdca

Sulrnou, Y. F.: Poulltcherikev, A, L.: Levin, U. G.: Neudatcitin, $V$. G. A gtudy of the tho-e lectron fcurler amplitudes of atoulc and oclecular mave functicns using the (l, 2e) and (e, $3 e$ ) prccesses at high energles. J. Phys. $B$ 11, zot? (1SiE)

Soviet inicr.

Longendsa, f. J. K.: von der Wiel, M. ¿. Fine structure of the neon $1 E .5-1 E .7$ eV rescnances resclued by eens cl resonant tree-free radlotibe absorfticns. J. Phys. B 11, 3603 (197E) The Netherlends

Mathur, D.: Khan, S. U.: Hogted, J. B. Disscclatlive

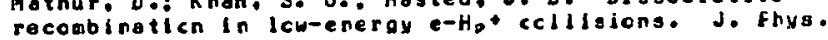
B 11,3615 (1578)

United Kincdom

Fatrikant, 1. I. The influence of leng-range interacticn on the vibraticnal excltaticn of pelar dolecules ty electrons. J. Phys. E IJ, zeal (1sat) Soviet Union

Pleart, Jo: Eomono., A, R.: Tran MInh, A. Extracclaticn to high oríclidol quentua nuaters of racial integrals in the couloet ofrexidaticn. J. Phyo, E 1\%,LES (ISTE)

france

Crethers, F. S. F.: Tcad, N. F. Regpcrse to Nitikin and Reznikov: sotal cross gecticns (Cr protcn-Be* chorge tronster. J. Phys. E 11 . 1663 (2578) United kinodom

Jognaux, A.: Eroulllard, F.: Szucs, E. Cherge exchonge in low energy Hot-He2+ collisteng. J. Physe 11 , L 669 (1578) Belalue 


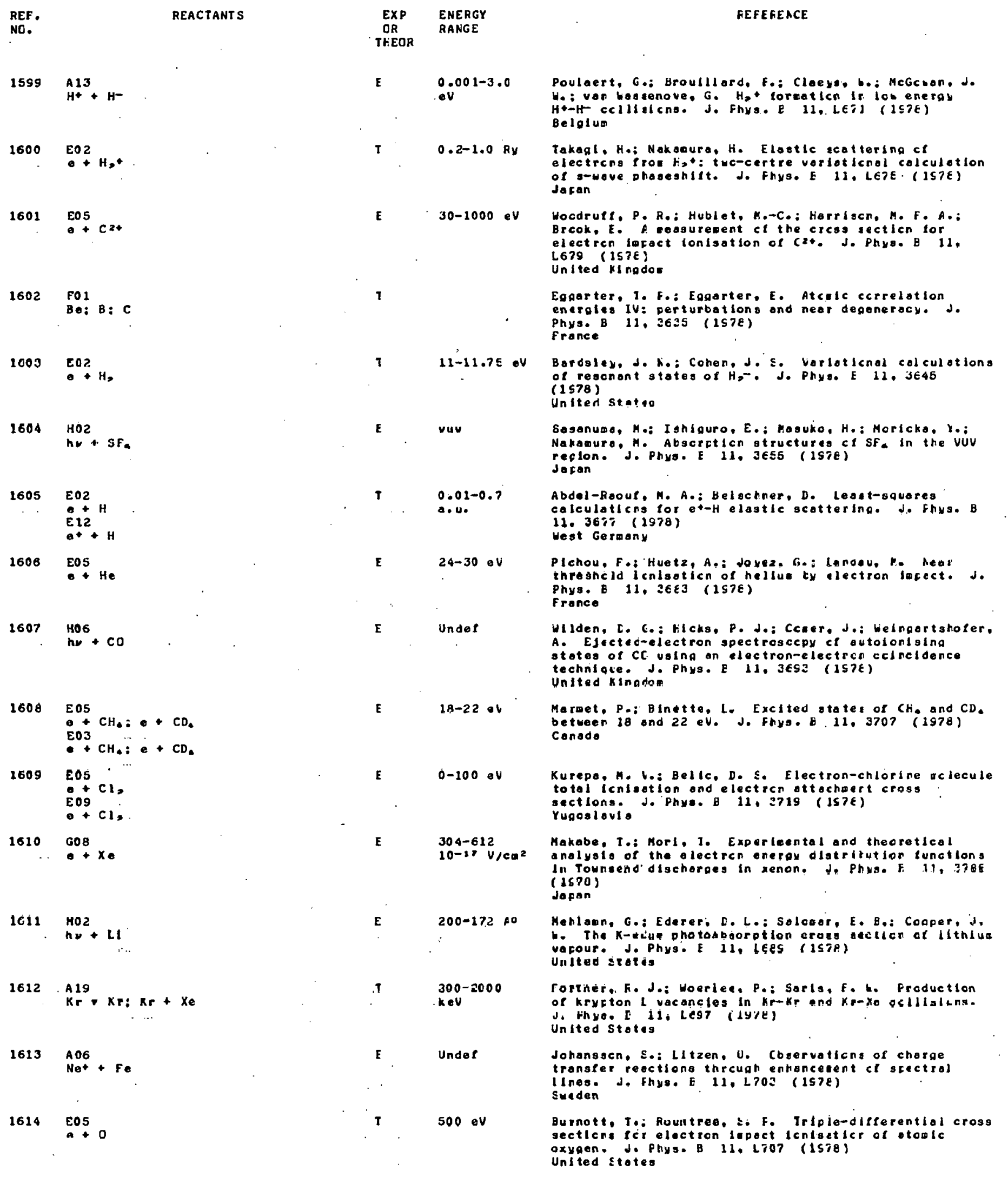


RE

REACTANT S

161

$\mathrm{EO} 2$

1616

A 12

$K+A r: K+K r: K+X e: K+H e: K+$

Ne

1617 HO7

hu $+\mathrm{H}-$

1618 A

$\mathrm{Na*}+\mathrm{He} \mathrm{Na*}+\mathrm{Ne}$

$1615 \quad \begin{aligned} & \mathrm{A06} \\ & \mathrm{He}^{2+}+\mathrm{H}^{-}\end{aligned}$

$1620 \quad 406$

$\mathrm{H}^{+}+\mathrm{He}: \mathrm{H}^{+}+\mathrm{Ar}$

$1 € 21 \varepsilon 03$

$$
\text { e+o, }
$$

$1622 E 03$

e+ D,

$1623 \mathrm{GO}$

$\mathrm{He}_{2}+\mathrm{He}: \mathrm{Ne}_{+}^{+}+\mathrm{Ne}$ : $\mathrm{Ar}_{2}+\mathrm{Ar}$ :

$\mathrm{Kr}_{\mathbf{2}}+\mathrm{Kr}$

1624 HOG

hv +0

$1625 \quad \mathrm{HO}$
$\mathrm{hl}$

$1626 \quad A 07$

$\mathrm{Xe}+\mathrm{Pb}$

1627

A07

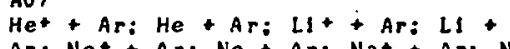
Ar: $\mathrm{Ne}^{+}+\mathrm{Ar}: \mathrm{Ne}+\mathrm{Ar}: \mathrm{Na}^{+}+\mathrm{Ar}$ : No + Ar: $\mathrm{K}^{+}+\mathrm{Ar}: \mathrm{K}+\mathrm{Ar}: \mathrm{Ar}^{+}+\mathrm{He}$

$162 \&$

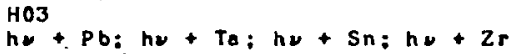

$1629 \quad \begin{aligned} & \mathrm{A} 16 \\ & \mathrm{H}^{+}+\mathrm{H}\end{aligned}$

1630

$\mathrm{H}+\mathrm{He}: \mathrm{H}+\mathrm{Ne}: \mathrm{H}+\mathrm{Ar}: \mathrm{H}+\mathrm{Kr}: \mathrm{H}+$

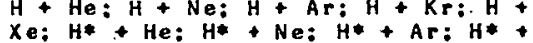
$\mathrm{Kr:H*}+\mathrm{Xe}: \mathrm{He}+\mathrm{He} \mathrm{He}+\mathrm{Ne} \mathrm{He}_{\mathrm{He}}$

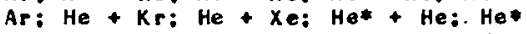
$+\mathrm{Ne}: \mathrm{He}^{*}+\mathrm{Ar}$ : He + $\mathrm{Kr}$ : He* + $\mathrm{Xe}$ : $\mathrm{LI}$ + He: LI* + Ne: LI* + Ar: Ll* + $\mathrm{Kr}: \mathrm{LI}+\mathrm{H}+\mathrm{Xe}$

A 26

$\mathrm{H}^{-}+\mathrm{He}: \mathrm{H}^{-}+\mathrm{Ne}: \mathrm{H}^{-}+\mathrm{Ar}: \mathrm{H}^{-}+\mathrm{Kr}$ :

$\mathrm{H}-+\mathrm{Xe}: \mathrm{He}^{-}+\mathrm{He}^{-} \mathrm{He}^{-}+\mathrm{Na}^{-} \mathrm{He}^{-}+$

Ar: $\mathrm{He}^{-}+\mathrm{Kr}$ : He- $\mathrm{He}^{-}$

\section{EXP ENERGY \\ THEOR}

E $5.2 \mathrm{eV}$

E

Therool

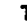

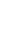

$0.001-0.7$

Ry

1 Unde?

I $\quad 100-8000$

$k \in V$ $\mathrm{keV}$

E

$14-100000$ keV/amu

$77-255 k$

$1-15 \mathrm{kev}$

$1 \cdot 5-1060$ $k \circ$
FEF ERENCE

Heddle, D. W. 0 . The 5.2 eV rescrence in electren-aercury ecottering. J. Phys. E 12, 1711 (1578)

United KIngdoo

Luin, A.: Hecortan. D. G. Ccllisicn trcadening of the potassiud resonance lines ty noble gasea. J. Phys. B 11.3841 (1978)

United Kingica

Stewart. A. L. P perturbation-varloticn study of phctodetochment from H-. J. Physe E 11. 3851 (1978) United Kingdoe

Derouard, J.: Lootordl. K. Thecretical cross sectlons fcr collialcnol oncular mosentum trangter ir otcalc Rydberg stotes. J. Phys. B 11. 3E?5 (1S78) Weat Geroony

Banyard. K. E.: Hecre. J. C. Electron copture Irod Hby fast olphe porticlea. J. Physe B 11.3895 (1978) Uni ted Kingdoe

Belkie, D.: Solin. A. Iitferential creas oections lor charge exchonge ot high enerales. J. Fhys. B 11, 3905 (1578)

Yugoslaula

Hakiya. K. Differential ond intearal cross sections for the electron lapact excitation of $G_{3}$. I. Octically al loved tronsitions frca.the ercund stete. J. Fhys. E 11. 3913 (1878)

Jofan

Hakiya, K. Differentlal and Integral cross secticns

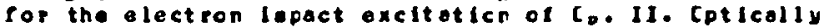
forbldden tronsiticns fros the ground stote." J. Phys. B 11. ZS:1 (1S7E)

Jaran

Helm. H.: Elford, M. T. Hoblittles onc reacticns of diatoolic rare-gos lons in thelr parent goses, hellud, neon, orgon ond krypton. Je Fhys.E 11, 3939 (1978) Australla

Prodhan. A. K. Fhetolcnlsoticn crcss oecticn of otonle oxygen. J. Phys. B 11. L725 (1578) Unlted KIngoce

Lochet. J. Fluorescence indulte for looer de 10 golecule Pop. J. Fhys. B 11. L73E (1578) fronce

Amundsen, P. A. Impact-paroreter decerience of $\mathrm{K}$-shell vocancy procuction in the heauy partner in ion-atca colliglons. J. Phys. B 11, 1737. (1578)

Norway

Jorgengen. K.: Andersen. N.: Olsen. J. C. Autolonising levels in orgon exclted.by low-energy heavy-ion lacect. J. Phys. B 11. 3SS1 (197E) Denark

Pragad. H. S.: Faju, G. K.: Navesicha Hurty, K.: Narasimto Murty. V. A.: Laksheinarayana. U. Elastic scatterino ef $145 \mathrm{keV}$ gango rays. J. Fips. B 11, 3969 (1578)

India

Ball K. La: KIngston, A. E.: Madden, Fo J. Electron detaoheent irou $H^{-}$ions by ficten lefact. JeFhys. B $11,35>7$ ( 1578 ) United KIngdo:

Dewangon. D. F.: Weltera, H. F. J. Electron lase trow

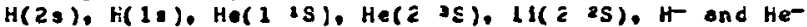
prcjectiles pasalng throuoh the inert poseg- the iree-cclilision model. J. Fhys. B 11.3983 (1978) United KIngdce 


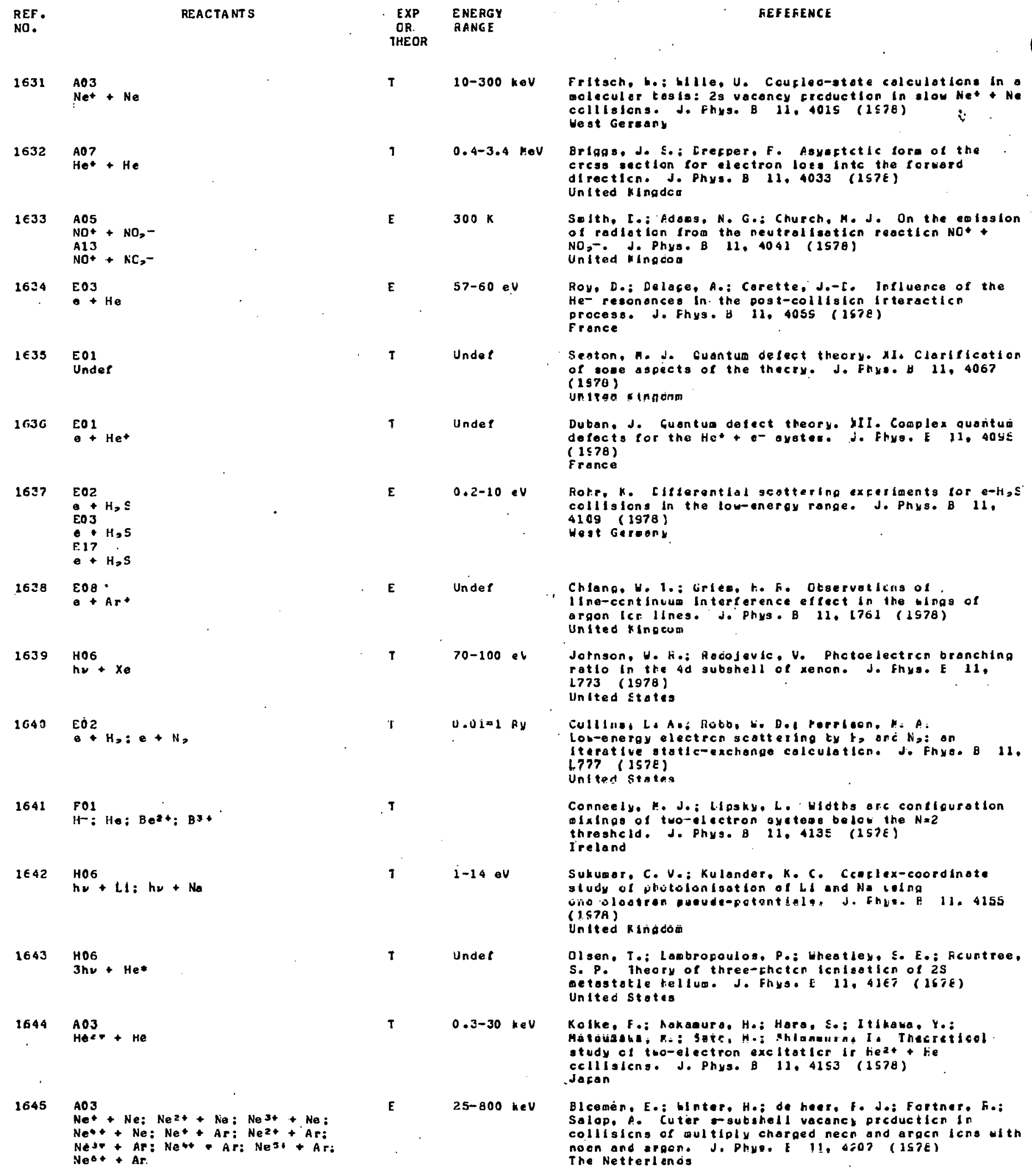


REF.

REACTANTS

NO.

1646

A0 3

$\mathrm{He}_{\mathrm{AO}}+\mathrm{AU}$

$\mathrm{He}^{2+}+\mathrm{AU}$

A 18

$\mathrm{He}^{2+}+\mathrm{Au}$

$1 \in 4 ?$

A13

$\mathrm{He}^{+}+\mathrm{N}_{2} \mathrm{O}: \mathrm{N}+\mathrm{C}_{3} \mathrm{O}^{+}+\mathrm{O}_{2} \mathrm{CC}^{+}$

$\mathrm{O}, \mathrm{O}+\mathrm{C}^{+}+\mathrm{CO} ; \mathrm{O}, \mathrm{H}^{+}+\mathrm{CO}_{\text {, }}$

1648

A06

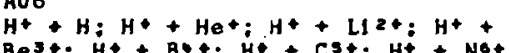

$\mathrm{H}^{4}+\mathrm{O}^{+4} \mathrm{H}^{+}+\mathrm{Fe}^{23+}$

A 18

$\mathrm{H}^{+}+\mathrm{H}^{3} \mathrm{H}^{+}+\mathrm{He}^{+}: \mathrm{H}^{+}+\mathrm{Ll}^{2+} \mathrm{H}^{+}+$ $\mathrm{Be}^{3+} \mathrm{H}^{+}+\mathrm{Bot}^{+4} \mathrm{H}^{+}+\mathrm{Cs}^{+} \mathrm{H}^{+}+\mathrm{Nat}$

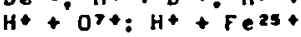

$1649 \quad \mathrm{FO}$

He

1650

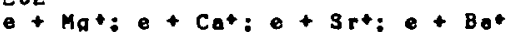
EO 3

$\mathrm{O}+\mathrm{Ma}^{+} \mathrm{Z} e+\mathrm{Ca}^{+} \mathrm{a}+\mathrm{Sr}^{+} \mathrm{Z} \mathrm{e}+\mathrm{Ba}+$

1651

E0.3

$e+\mathrm{Sm}_{\mathrm{i}} \mathrm{e}+\mathrm{Ho}$ : e + Eri e + BI

1652 FO 1

H Sea: He Sea: $L$ I Seq: Be Sea: Ne Seq: Co Seq: Felot: Fellt: Felst; $\mathrm{Fe}^{204}$ : $\mathrm{Fe}^{204}$ : $\mathrm{Fe}^{21+}$ : $\mathrm{Fe}^{2+}$ : $\mathrm{Fe}^{23+}$ : Tasse: Tasot

1653

$\mathrm{H}_{3}+\mathrm{He}$

$\mathrm{HO} 2$

$h_{2}+H_{2}: h_{2}+\left[H_{2}+H_{e}\right\}$

$16{ }_{4}$

HO2

h⿻ +0 ,

$\mathrm{Al}^{2}+\mathrm{O}$,

$1655 \quad \mathrm{~F} 01$

$1656 \quad H 06$

$h_{2}+F^{+}: h_{\nu}+F_{e}{ }^{4+}: h_{\nu}+F^{17 t}: h_{\nu}$

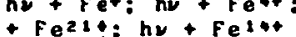

1657 E11

e+ N

$165 \theta$

503

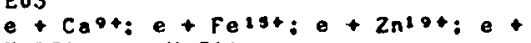

Krast: $e^{2}+M_{032}$

1659

A12

LI* + Ar: LI* + He

A17

LI* + Ar: LI* + He

\section{EXP ENERGY \\ THEOR \\ RANGE}

E $\quad 15-20 \mathrm{MeV}$

E $100-300 k$

$20-i c 0000$ keV

Under

$300-600 \mathrm{keV}$

E

E

A 12

$300 \mathrm{~K}$

$\mathrm{HO2}$

20-900 co-1

1

$\mathrm{HO2}$

9-60 $\mathrm{GHz}$

A 12

$300 \mathrm{~K}$

E

kev

$10-1.4 \mathrm{eb}$

1

$0.04-20.0$
$k \in V$
$400-1100 k$
Baver, K. G.: Fazly, Q.: Hesasen, ke: Schurkes, P. L ohell icnisotion probobility of gold by alcha carticles of 12-50 MeV. J. Phys. B 11. 4227 (1578) We et Geroany

Rakshlt, A. B.: Stock, H. H. P.: tierelng, D. P. Tulddy. N. D. Some ion-molecule reacticn rate coefficlent measusedenta ot 300 onc $100 \mathrm{k}$ in. tesperature-varlatle flculing-olterglcu epparatua. Je Phys. B 11, 4237 (157E)

United Kingdoa

Lal. M.: Irlpathl. A. N.: Srluastovo. H. K. Charge-trongler croos sectlong for prcteng colllding wth hydrogente lons I. J. Phys. E 11. 4249 (1978) Indla

Winkler, P.: Yarla, R. Electron satering resonances using dilatation transforseticna. 11l. Varlational study of the tabllity of resonance elgenvalues. J. Physe. E 11.4257 (1978)

Weat Germony

Burgess, A.: Tully, J. A. On the Eethe approximation. J. Phys. B 11, 4271 (197E)

Unitad Kingdor

RIcz, S.; 8chlenk, B.: Eerenyl, D.: Velek, A.: HCck, G.: Self el Nasr, S. A. H. I xroy prcduction crcas section $P O F$ SE, lio. Er ond $E l$ at ouveral hundred keV electron Imract. J. Prya. E 11. 4283 (2578) Hungary

Bolko, V. A.: Faencu, A. Y.: Plkux, S. A. X-roy spectrcscocy of eultiply-chorged lcng lzon laser plosmas. J. Quent. Spectrcac. Fadiat. Iranster 19, 11 ( 2578 )

Sortet Union

Blenbous. G. For-infrored abseretion in ti, eno $H,-H E$ intures. J. Quant. Spectrose. Radiat. Iransfer 19, 51 ( 1578$)$

United stotes

Mizushias, M. Frasure-incuced otoortion cl olcrowave rodloticn by the oxygen molecule. J. Cuante spectrcac. Radiat. Tronsfer 19.62 (1S7E)

United stotes

Abdalloh, H. Ho: Meraet, J. H. The tehovior ef nitrooen exclted in an inductlvely ccucled orgen ploama. J. Guant. Spectrcse. Foofot. Tronafer 15, 83 ploses France

Huebner, H. F.: Argo, H. F.: GAlsen, L. D. Photoelectric cross sectlcrg for lcns ocoleo troo their neutral atoms. J. Quant. Spectrosc. Hediat. iransfer 19. 93 (1578) United states

John, T. I.: Hillloos, R, de Eresastrohlung ty neutral atcolc nitiogen. J. Cuant. Epectrcsc. Radlat. Ironster 15. 99 (1578)

United kingoe

Bloha, H.; Lavis, J. Electron lecect exclteticn of highiy chergec sodIum-itke lons. J. Otont. Spectrcac. Radiat. Ironster 19, 227 (157e) Untted states

Etty. N.: Hentcer. S. Ercodening ond shlit of lines of the princlpal series of neutral lithlua atce crcduced by collisicn with heliue orc orgen. J. Guant. Spectrose. Fadiot. Tronster 1s, 247 (1578) Fronce 


\begin{tabular}{|c|c|c|c|c|c|}
\hline $\begin{array}{l}\text { REF. } \\
\text { NO. }\end{array}$ & & REACTANTS & $\begin{array}{l}\text { EXP } \\
\text { OR } \\
\text { THEOR }\end{array}$ & $\begin{array}{l}\text { ENERGY } \\
\text { FANGE }\end{array}$ & EEEEEENCE \\
\hline 1660 & $\begin{array}{l}\mathrm{H} \mathrm{O}_{2} \\
\mathrm{~h}\end{array}$ & & $\varepsilon$ & $4350-10600 \mathrm{~A}^{\circ}$ & 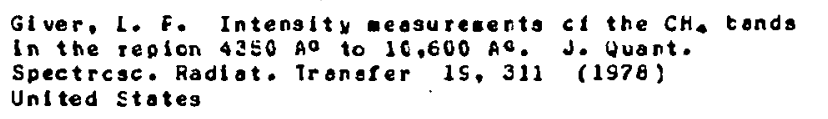 \\
\hline 1661 & 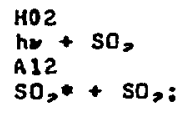 & $\mathrm{SO}, *+\mathrm{N}_{2}: \mathrm{SO}, *+\mathrm{H}, \mathrm{O}$ & $\mathbf{E}$ & $\begin{array}{l}H 02 \\
91.5-95.5 \\
6 H 2 \\
A 12 \\
295 \mathrm{~K}\end{array}$ & 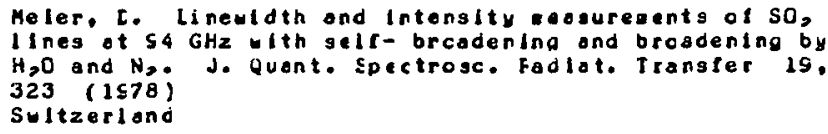 \\
\hline 1662 & 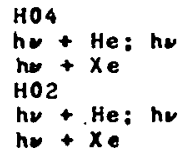 & $\begin{array}{l}+\mathrm{Ne}: \mathrm{h \nu}+\mathrm{Ar}: \mathrm{h \nu}+\mathrm{Kr} \text { : } \\
+\mathrm{Ne}: \mathrm{h \nu}+\mathrm{Ar}: \mathrm{h \nu}+\mathrm{Kr} \text { : }\end{array}$ & $\mathbf{T}$ & $\begin{array}{l}10000-300 \\
\mathrm{AO}\end{array}$ & 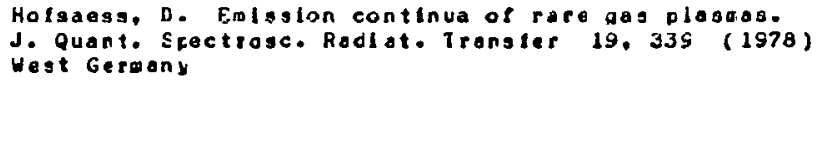 \\
\hline 1663 & $\begin{array}{l}\text { A } 02 \\
\text { Under }\end{array}$ & & $\mathbf{T}$ & $300 \mathrm{~K}$ & 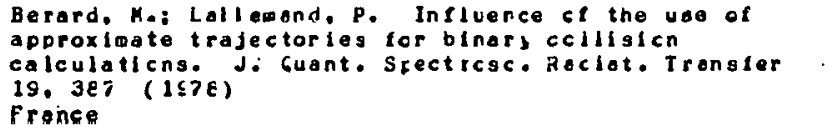 \\
\hline 1864 & $\begin{array}{l}\mathrm{BO} 1 \\
\mathrm{~N} \\
\mathrm{FO2} \\
\mathrm{N}\end{array}$ & & $\mathbf{T}$ & & 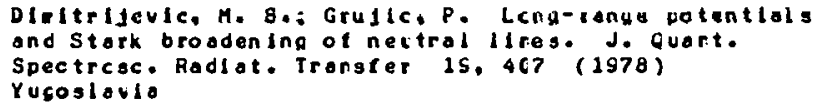 \\
\hline 1665 & $\begin{array}{l}\mathrm{A} 12 \\
\mathrm{Cs} *\end{array}$ & & $\mathbf{I}$ & $300 \mathrm{~K}$ & 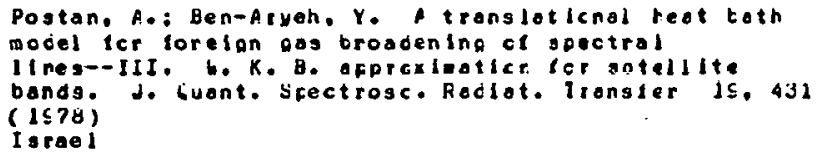 \\
\hline 1666 & $\begin{array}{l}A 17 \\
A 1\end{array} 0$ & & $\mathbf{T}$ & Undef & 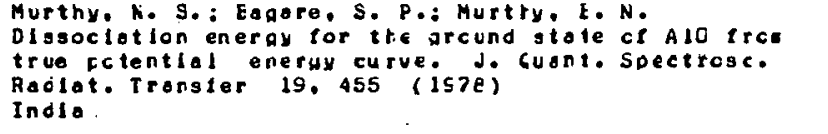 \\
\hline 1667 & $\begin{array}{l}H 02 \\
h v+N,: h v \\
N=0: h v+C: \\
F: h v+C .1\end{array}$ & 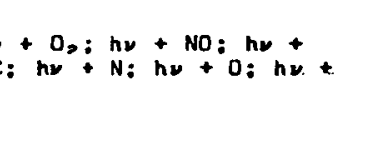 & $\mathbf{E}$ & $50-340 \mathrm{~A}^{\circ}$ & 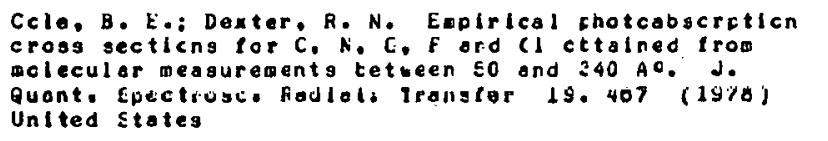 \\
\hline 1668 & $\begin{array}{l}H 13 \\
h 10+h y\end{array}$ & & 1 & Undel & 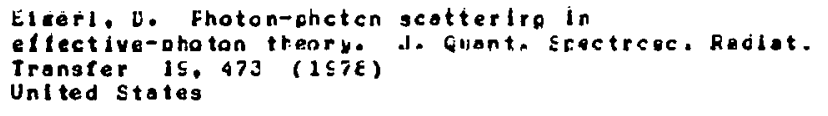 \\
\hline 1669 & $\begin{array}{l}\mathrm{FO2} \\
\mathrm{Al}\end{array}$ & & $E$ & & 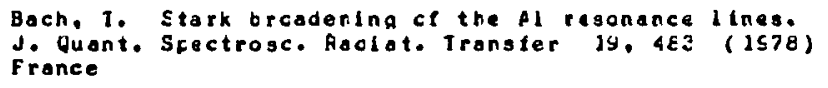 \\
\hline 1670 & $\begin{array}{l}\mathrm{Al2} \\
\mathrm{CO}_{2} *+\mathrm{Ar}:\end{array}$ & $\mathrm{CO}_{7} *+\mathrm{N}_{2} \mathrm{CO}_{2} *+\mathrm{CO}_{2}$ & $\mathbf{E}$ & $197-294 \mathrm{~K}$ & 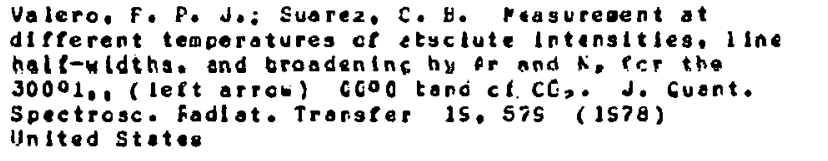 \\
\hline 1671 & $\begin{array}{l}\text { Fo2 } \\
\text { Under } \\
\text { A12 } \\
\text { Under }\end{array}$ & ' & $\mathbf{T}$ & $\begin{array}{l}\text { Al2 } \\
\text { Undef }\end{array}$ & 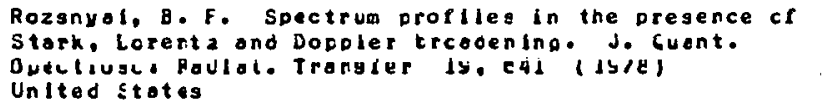 \\
\hline 1672 & $\begin{array}{l}A 12 \\
C=*+x e \\
C=*+x e\end{array}$ & & $\mathbf{E}$ & $298 \mathrm{~K}$ & 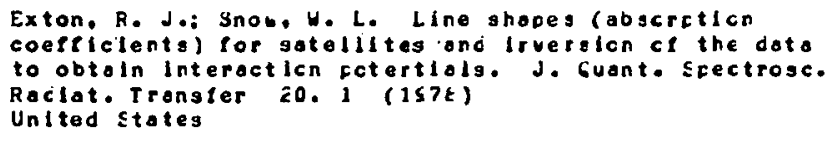 \\
\hline 1673 & $\begin{array}{l}\mathrm{Al2} \\
\mathrm{HCl}+\mathrm{HCl}: \\
\mathrm{HF}\end{array}$ & $\mathrm{HCl}+\mathrm{HF} ; \mathrm{HF}+\mathrm{HCl}: \mathrm{HF}+$ & $\mathbf{E}$ & $2 \$ 8 K$ & 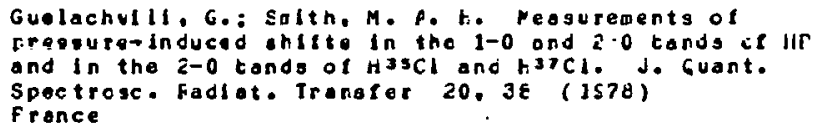 \\
\hline 1674 & $\begin{array}{l}\mathrm{HO4} \\
2 \mathrm{hv}+\mathrm{Na}\end{array}$ & & E & $1800 \mathrm{~K}$ & 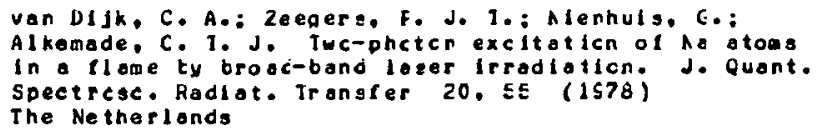 \\
\hline
\end{tabular}




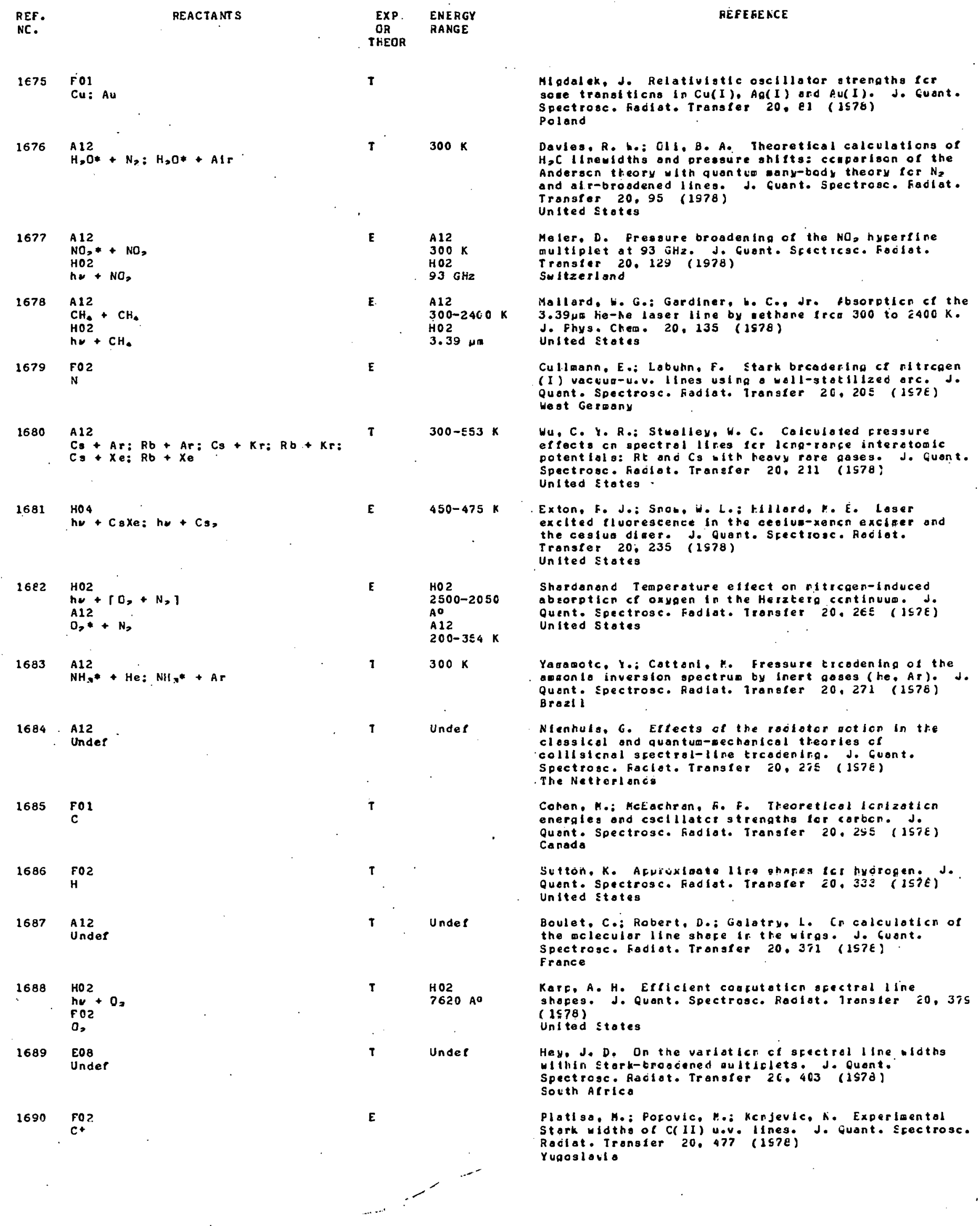




\begin{abstract}
REF.
NO.
\end{abstract}
REACTANT S

1651

EO3

E $+A r+$

507

- $+A r^{*}$

$1692^{\circ} A 12$

$\mathrm{CO}=+\mathrm{CO}$;

E

$\mathrm{FO}_{2}$

1

1694

F01

1695

Al2
Ha* No

1696

Ho 3

ho + Na

1697

E08

C A P

1698

FO1

H: $\mathrm{He}^{-}$

e+H: e+He

1699501

He

1700

$\mathrm{BO7}^{+}+\mathrm{P}: \mathrm{B}_{+}+\mathrm{K} ; \mathrm{B}^{+}+\mathrm{Ca}_{\mathrm{B}} \mathrm{B}^{+}+\mathrm{SC}$

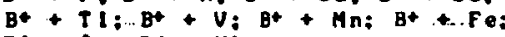

$\mathrm{B}^{*}+\mathrm{CO}^{\circ} \mathrm{B}+\mathrm{B} i$

1701

A07

He* $+\mathrm{H}$

A 17

Het $+\mathrm{H}: \mathrm{Het}+\mathrm{H}+$

$1702 \quad A 07$

KO $+x=$

1703

$\mathrm{CO2}$

$\mathrm{H}^{+}+\mathrm{Ge}^{+} \mathrm{H}^{+}+\mathrm{Se}_{\mathrm{O}} \mathrm{H}^{+}+\mathrm{Pd}^{+} \mathrm{H}^{*}+\mathrm{Al}^{\circ}$ $\mathrm{H}^{+}+\mathrm{SB}^{+} \mathrm{H}^{+}+\mathrm{BI}: \mathrm{He}^{+}+\mathrm{Ge}$ : Het Se: $\mathrm{Het}+\mathrm{Pd:Het}+\mathrm{Ag}_{\mathrm{O}} \mathrm{He}^{+}+\mathrm{Sb}_{\mathrm{B}}$. Het.t B!

$\cos$

$\mathrm{H}^{+}+\mathrm{Ge}: \mathrm{H}^{*}+\mathrm{SE}: \mathrm{H}^{+}+\mathrm{Pd}: \mathrm{H}^{+}+\mathrm{Ag}:$

$\mathrm{H}^{+}+\mathrm{Sb}^{+} \mathrm{H}^{+}+\mathrm{Bl}: \mathrm{He}^{+}+\mathrm{Ge}^{+} \mathrm{He}^{+}$

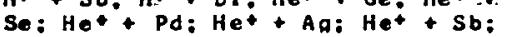

$\mathrm{He}+\mathrm{BI}$

1704

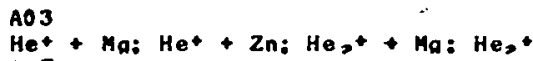

$5890 A^{0}$

$20000 \mathrm{~K}$

$\mathrm{EO} 2$

20 eV.

$\mathbf{T}$

$+2 \mathrm{Zn}$

EXP
OR
THEOR

$\mathbf{E}$

$4-38 \operatorname{MeV}$

A 07

0.05 ev

n17.

0-n. 3 ?

napt ree

7

$42 \mathrm{HeV}$

E

$2-800$ el

$2.6-3$ eV
$200-300 x$

$1860-2270 \mathrm{~K}$

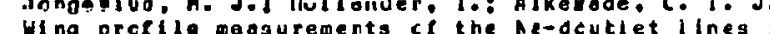

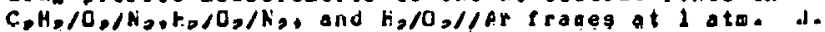
Quent. Ivectrose. hadiot. Tranoter 20.59s (1978) The Ne therlands

Jongerlug. H. J.: von der ElJ, J. L.: t.cll ander, T.: Alkenode, C. T. J. Hoylelgh ocattering by sodiur vacour in Clames. J. Guant. Spectrose. Fadiat. Transfer 20,609 (1978)

The Netherionds

Behringer, Ko: Thosa, F. Electron lafact ldths of sode Ar(II)-u.v.-gulticlets. J. Giont. Spectrosc. Radlat. Transfer 20, 615 (ISTE)

West Geraany

Junker. B. F.: Huona. C. L. Coeplex-cccrainate dethod. Structure of the wove functicn. Ftys. Fev. A 18.313 (1578)

United Etates

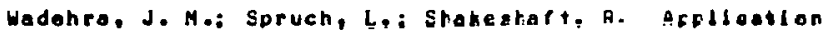
or an extrenon prinelpie te the valioticnal

determination of the generallzed oscillator gtrengths of heliuw. Phys, Fov: $\Lambda$ 18, 3t4 (1STE)

United stotes

Montgold, G.: MeDantel, F. C.: Luggan, J. La: Rice, R.: Toten. A.: Mehta. F.: Milier. F. D. p-shellichlation

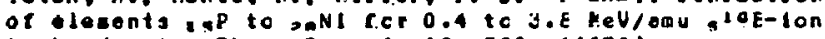
bcubarcoent. Phys. Rov. A 18,280 (1S78) United states

Blenfek, R. J. Cosplex petential end elactron acectrus In atoric cellisicns involuing fast electranir

lransirions: Penning onc assoclative lcnizaticn, Phus.

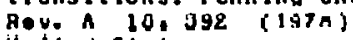

Untited states

Meverhol, E. Ionleotion or the su d nolecular ortital in heouy-ion collialone. Ihys. Fev. $A$ 18, 414 (1578)

United stotis

$20-260 \mathrm{keV}$

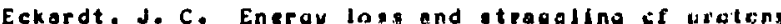
ard hellue lchs traversing scoe thin scild tollo.

Phys. Fev. A 18,426 (2S7e) A rgentina
Myers. G. D.: Leventhal. J. J. Inelastle collisions of 2-800-eV He and Fe, and Mg and $2 \mathrm{r}$ atcase Physe Fev. A 18.434 (157e)

Unlted Stotes 


\begin{tabular}{|c|c|c|c|c|}
\hline $\begin{array}{l}\text { REF. } \\
\text { NC. }\end{array}$ & REACTANTS & $\begin{array}{l}\text { EXP } \\
\text { THER }\end{array}$ & $\begin{array}{l}\text { ENERGY } \\
\text { RANGE }\end{array}$ & FEFEFENCE \\
\hline 1705 & $\begin{array}{l}E 03 \\
e+A r *: e+K r *\end{array}$ & $T$ & $3-100$ ev & 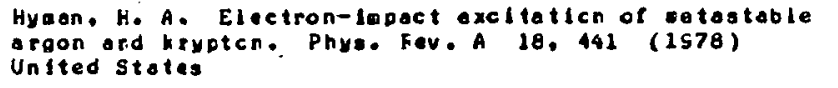 \\
\hline 1706 & 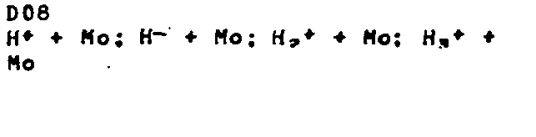 & $\mathbf{E}$ & $\begin{array}{l}0.2-10.0 \\
k \in V\end{array}$ & 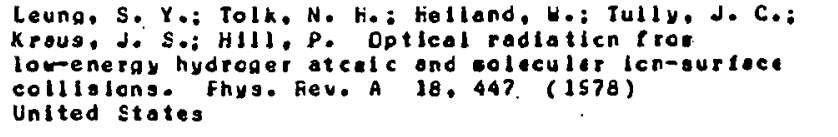 \\
\hline 1707 & Eo3 + Heq: e+He+ & $\mathbf{T}$ & $40-1000 \mathrm{eV}$ & 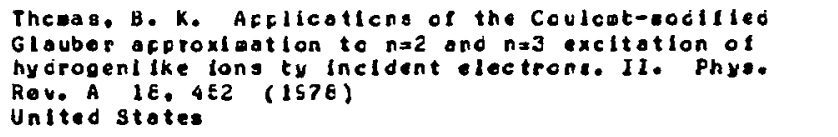 \\
\hline 1708 & 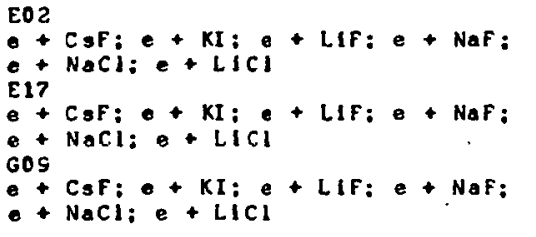 & $\mathbf{T}$ & $\begin{array}{l}\text { E02: E17 } \\
0.13-20.0 \\
\text { eV } \\
\text { GOg } \\
1.0 \text { oV }\end{array}$ & 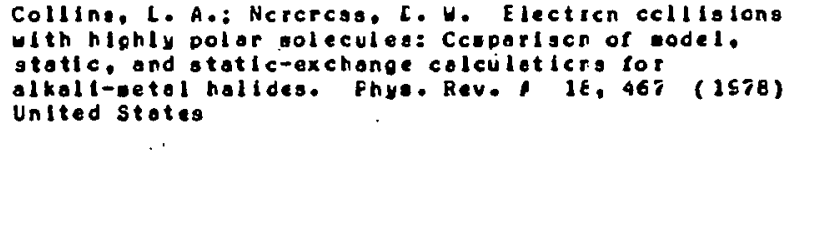 \\
\hline 1709 & 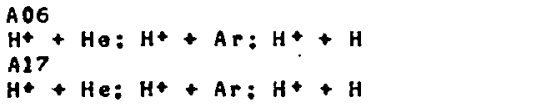 & 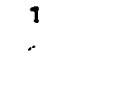 & $\begin{array}{l}120-60 \mathrm{CO} \\
\mathrm{keV}\end{array}$ & 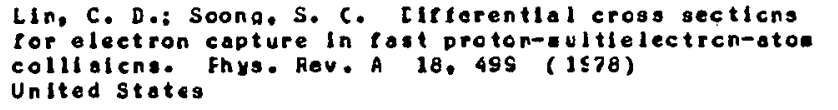 \\
\hline 1710 & $\begin{array}{l}A 07 \\
H+N_{2}: H+D_{2} \\
A 18 \\
H+N=H+0\end{array}$ & $\mathbf{E}$ & $50-3000$ eV & 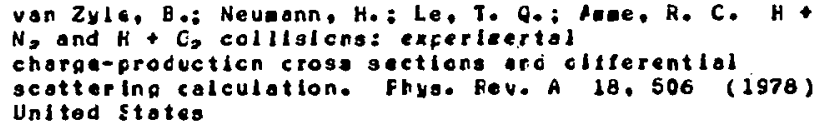 \\
\hline 1711 & $\begin{array}{l}\text { BO7 } \\
\text { he + e }\end{array}$ & $\mathbf{T}$ & & 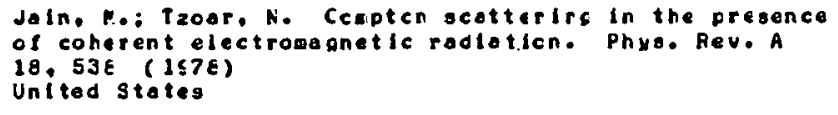 \\
\hline 1712 & $\begin{array}{l}\mathrm{HO3} \\
\mathrm{he}+\mathrm{Ne}: \mathrm{hL}_{2}+\mathrm{Ar}: \mathrm{hv}_{\mathrm{N}}+\mathrm{Kr}\end{array}$ & $\mathbf{I}$ & Under & 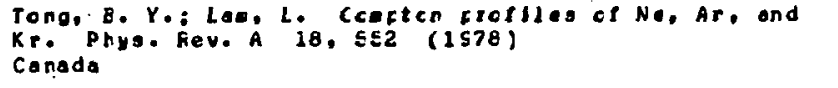 \\
\hline 1713 & $\begin{array}{l}\text { FO } 1 \\
\text { He }\end{array}$ & $\mathbf{E}$ & & 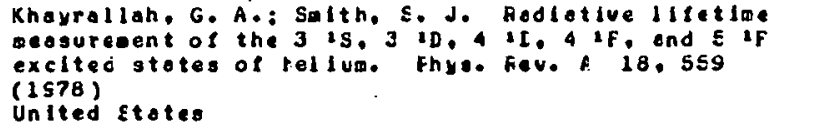 \\
\hline 1714 & $\begin{array}{l}H 06 \\
h v^{-}+\mathrm{O}: \mathrm{h} \boldsymbol{H}+\mathrm{H}\end{array}$ & $\varepsilon$ & $90-76 \mathrm{na}$ & 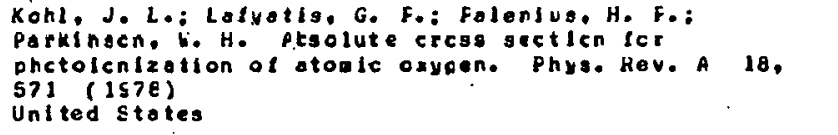 \\
\hline 1715 & $\begin{array}{l}\text { Fo1 } \\
\text { He }\end{array}$ & $E$ & & 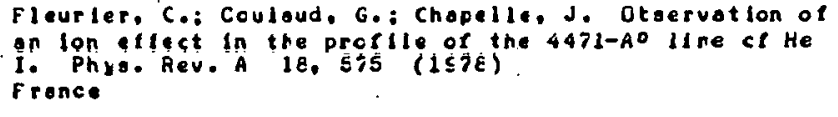 \\
\hline 1716 & 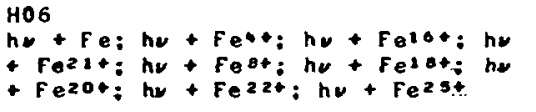 & $T$ & $0.0-11 \mathrm{k} \in \mathrm{V}$ & 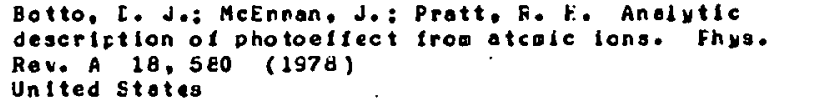 \\
\hline 1717 & $\begin{array}{l}\text { Ho6 } \\
\text { Under. }\end{array}$ & $t$ & $\begin{array}{l}\text { HOG } \\
\text { Undef }\end{array}$ & 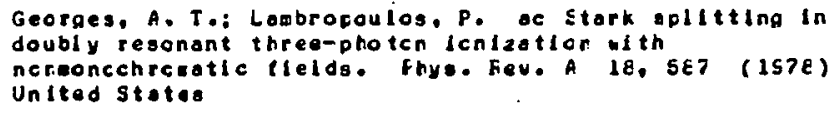 \\
\hline 1718 & 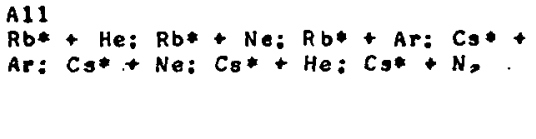 & $\mathbf{E}$ & $300 \mathrm{~K}$ & 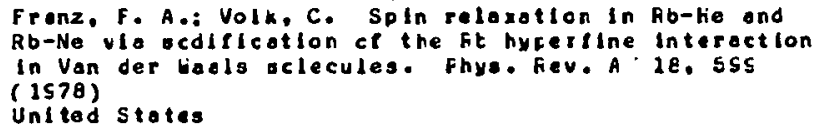 \\
\hline 1719 & $\begin{array}{l}\text { Ho8 } \\
\text { Under. }\end{array}$ & $\mathbf{T}$ & Undef & 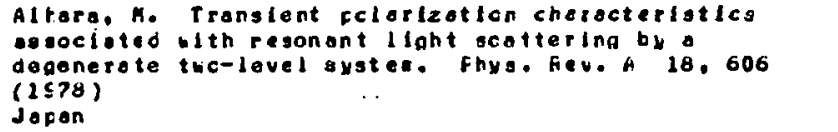 \\
\hline
\end{tabular}




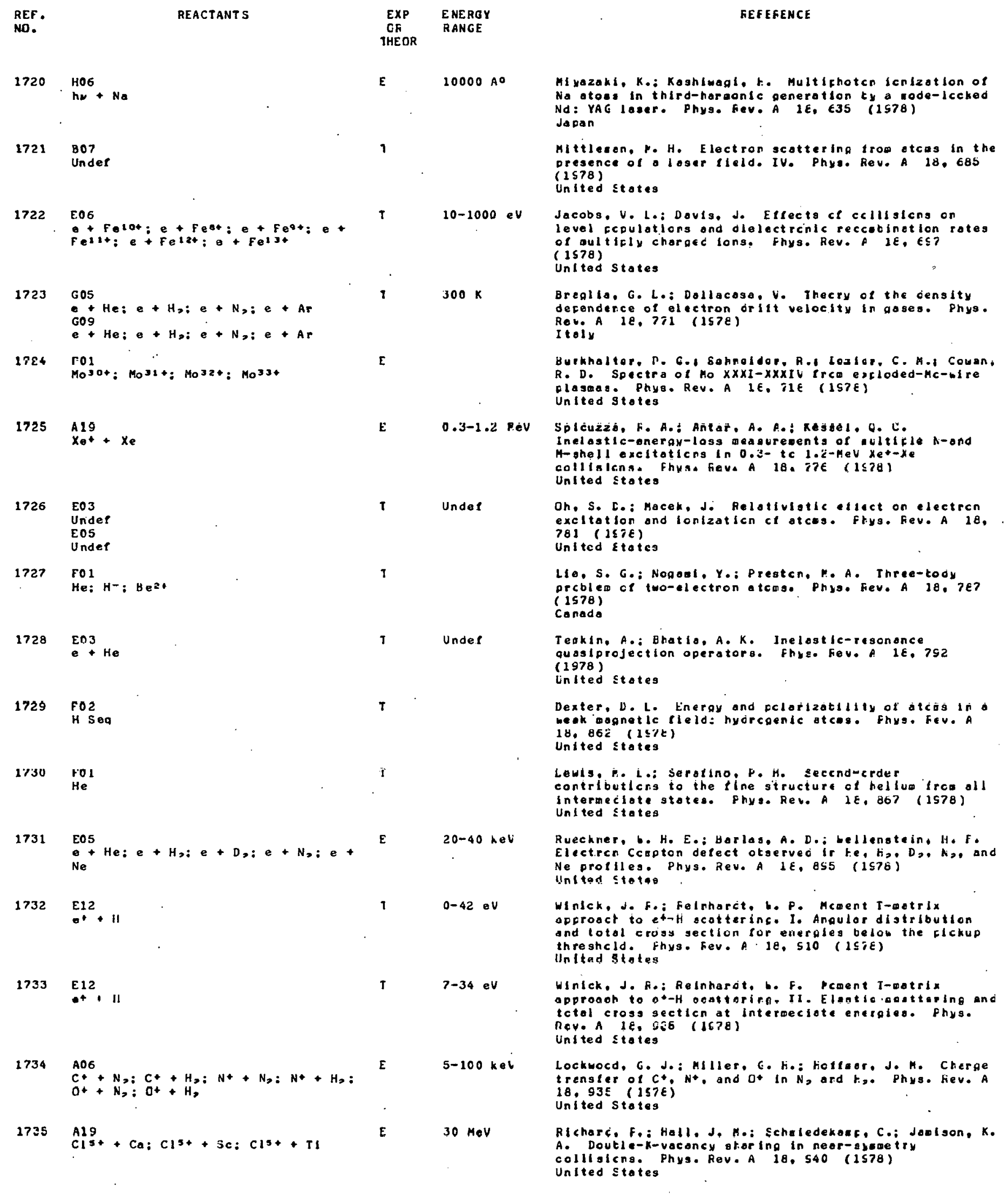




$\begin{array}{cl}\text { EXP } & \text { ENERGY } \\ \text { OR } & \text { RANGE } \\ \text { THEOR } & \\ & \\ \text { E } & 7-180 \text { eV }\end{array}$

$\mathrm{Cl}^{\mathrm{A}}+\mathrm{Ne}: \mathrm{Cl}^{-}+\mathrm{Ar}: \mathrm{Cl}^{-}+\mathrm{Kr}: \mathrm{Cl}^{-}+$

$\mathrm{Xez} B r-$ Ne; Br $-\mathrm{Ar}: \mathrm{Br}^{-}+\mathrm{Kr}$ :

$B r-x_{e}$

1737

E01

Under

E05

e+Ar: e+NI; e+Y: e+Aa: e+ $\mathrm{Ba}$ : e $+\mathrm{HO}$ : e+Au: e+BI: e+U

1739

Undef

$\mathrm{Co2}$

Under

$\mathrm{CO} 3$

Under

$1740 \quad 606$

$\mathrm{He}^{2+}+\mathrm{He} \mathrm{Ne}^{2+}+\mathrm{Ne} \mathrm{Ar}^{2+}+\mathrm{Ar}$

$\mathrm{Kr}^{2+}+\mathrm{Kr}_{\mathrm{r}} \mathrm{Xe}^{2+}+\mathrm{Xe}^{2}$

1741

A06

He2t $+\mathrm{He}: \mathrm{Ne} 2+$ He: $\mathrm{Ar}^{2+}+\mathrm{Ar}:$

$\mathrm{Kr}^{2+}+\mathrm{Kr}^{2} \mathrm{Xe}^{2+}+\mathrm{XeO}_{\mathrm{O}} \mathrm{He}^{2+}+\mathrm{CHe}^{+}$

He ]: $\mathrm{Ne}^{2+}+[\mathrm{Ne}+\mathrm{Ne}]: \mathrm{Ar}^{2+}+\mathrm{HAr}$

Ar 1: Kr2t + I Kr $+K r$ I: $X_{e}^{2+}+\left[X_{e}+\right.$

$x \in 1$

1742

$\mathrm{AO} 6$
$\mathrm{He}$
$\mathrm{A}+\mathrm{I}+\mathrm{He}$

A17

$\mathrm{He}^{+}+\mathrm{He}^{+}: \mathrm{He}^{2+}+\mathrm{He}$

FO1

17.43

$\mathrm{He}^{2+}+\mathrm{He}$

GOS

$\mathrm{He}^{2+}+\mathrm{He}$

1744

EO3

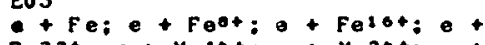

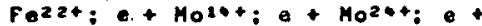

Moj2+

EOS

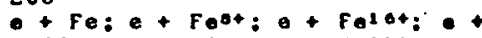

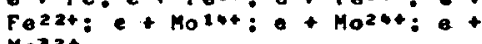

$\mathrm{MO}^{2} \mathrm{H}^{\circ}$

$1745 \quad$ A17

LIF Ile: LIF + No: LIF + Ar: LIF + Kr: LIF + Xe

$1746 \quad \mathrm{EO} 2$

$+\mathrm{H}$,

1747

Fol
Mol2t; $w * *$

1748

A22 $H: H+H e: H+N e: H+A r: H+$

Kr: H+Xe: LI + H: LI + He: LI +

Ne: LI $+A r: L I+K r: L I+X_{B}: N a+$

$\mathrm{H} ; \mathrm{Na}+\mathrm{He:} \mathrm{Na}+\mathrm{Na:} \mathrm{Na}+\mathrm{Ar}: \mathrm{Na}+$

$\mathrm{Kr:} \mathrm{Na}+\mathrm{Xe}_{\mathrm{K}} \mathrm{K}+\mathrm{H}: \mathrm{K}+\mathrm{He}: \mathrm{K}+\mathrm{Ne}$;

$\mathrm{K}+\mathrm{Ar}: \mathrm{K}+\mathrm{Kr}: \mathrm{K}+\mathrm{Xe} \mathrm{Kb}+\mathrm{H}: \mathrm{Kb}$

- He: $\mathrm{Fb}+\mathrm{Ne:Rb}+\mathrm{ArZ}_{\mathrm{Rb}}+\mathrm{Kr}: \mathrm{Rt}$

+ $\mathrm{Xe:} \mathrm{Cs}_{3}+\mathrm{H}_{1} \mathrm{CS}_{3}+\mathrm{He}: \mathrm{Cs}_{3}+\mathrm{Ne}: \mathrm{Cs}$

$+\mathrm{Ar} \mathrm{Cs}+\mathrm{Kr} \mathrm{Cs}_{\mathrm{C}}+\mathrm{Xe}$ $\mathbf{7}$

$5 \times 10 \cdot-109$
ov

Undef

A06: A17 $0.001-10 \mathrm{eV}$

$\mathbf{T}$

606

$80-2000 \times$

.609

2-80 neV

1

4-36 kel

$1300 \mathrm{~K}$

Undet

$300 K$
FEFEFEACE

Solth, E. I.; Ecuards, W. F., III: Deveraclke, L. L.: Chapicr. R. L. Total electrcn-detachaent cross sections for collitions ot nepotive halcoen lons and rare gaes tor energies around threshcld. Phys. Fev. A 18,945 (1578)

United Stotes

Nesbet. R. K. Varlational dethods for aultichannel scattering. II. New variant ct cetialzed ancmaly-iree method and restricted intercolotec oncualy-free method. Phys. Rev. A le, SE5 ( $1 \leq 7 \varepsilon$ )

United States

Scolield. J. H. K-and L-shell icrizaticn ol atoms ty relotiviotic electrons. Fhys. Rey.A 18, 563 (1578) United states

Rau, A. R. P.: Inckutl, M.: Deuthat, D. A. Verloticnol treatment of electron degrodaticn end yielda of initlal molecular specles. Phys. Fev. A 18, $\$ 71$ (1578) United States

Johnsen. R.: Blcndl. H. A. Hctllitles cl dcutly chargec rare-gas icns in thelir carent cases. Fhyso Rev. A IE, SEg (1578)

Unl ted states.

Johnsen, F.: Rlondl. M. A. Meosurementa of rociotive charge-trongfer reoctions of doubly ano singly chorged rare-gas icng with rare-gos otces ot theraol enerfies. Fhys. Fev. A $18, \$ 96$ ( $157 \varepsilon)$

united stotes

Cohen, J. S.: Bardsley, J. A. Calculaticn ct radiatlue single-charge-transfer crcss aeticng icr ccllisions of Hezt with He at lob energy. Phys. Fev. A 18, 1004 (1578) United states

Hadohra, Jo M.: Cohen, J. S.: Eardeley, J. A. Mctllity of partlcles In hellua. Phys. Rev. f IE. ICOS (1578) United States

Hahn. $Y$. cistorted-wove thecry ct electron-ion collisicns. II. Auger lonizoticn and excitaticn fluorescence. Phyo. fiev. A 1e, 1628 (1578) United States

Menottl, F. R.: Bedding. D.: Hcror, T. I. Peosureoent of the onfoctropy ct the interacticn tatween Lif ond the inert goses. Fhys. Hév. A le. 1038 (1978) United stotes

Fliflet, A. W.: Hckey, We Varloticnolly corrected discrete-tosis-set calculation fer electrcn-gclecule scatering in the statlc-exchange apprexiaticn. Fhys. Rev. A 18, 1048 (1978)

United stotes

Shcrer. F. Eflecto of 3d oubshells cr rescnance osclilator atrengthg for the zinc isoelectrcilc sequence. Fhys. Fev. A $1 E, 1060$ ( $J \leq 7 E)$ United States

Hu, C. Y. R.: Stualley,W.C. Calculated cresoure brcadening ond shift for alkoli-getol toms perturted by rare goses: twc-phcten s-s transiticns. Physe Rev. A 18, $10 E E$ (1STE)

United Stotes 


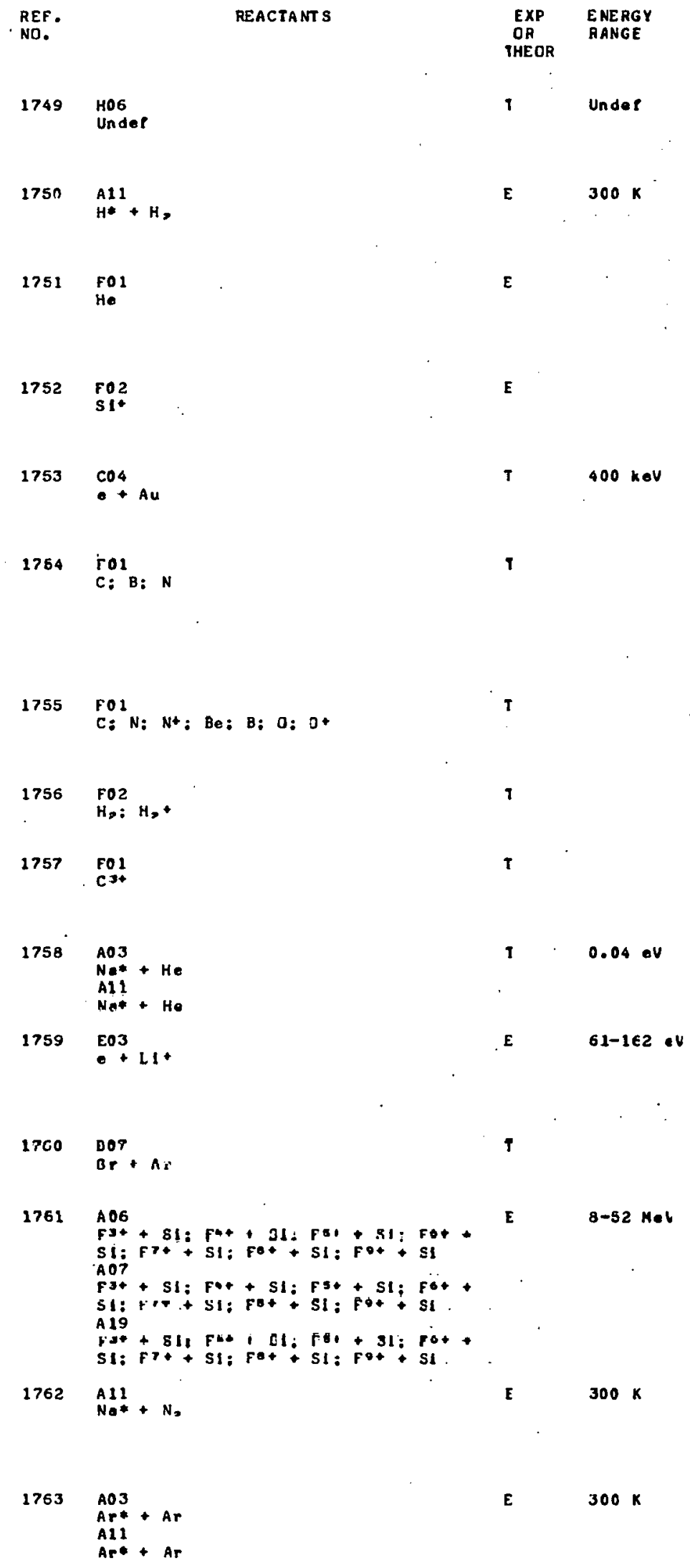

Georges, A. T.: Lenbrocoulcs, F. Quontun beots In photolonlzotion frod a colierent ouparcestiticn cl el me-gitructure levels. Phys. Fev. A 18, 1072 (1978) United States

Catherlnot. A.: Dutreuli, E.: Gend, M. Guenchlng of atcolc states in a low-preasure hydropen glew diacheree. Phys. Rev. A 18, 1057 (1s78) France

Rosenbluh, M.: Fancek, F.: Lox, B.: Mlller, T. A. Motlonol-st orh-ellect spectroncopy: 7 i\&-s ip energy socaretion end zeenan tuning coracetere for iHe. Phys. Rev. A 18,1103 (1978)

United Etotes

Chlang. H. T.: Grlea. H. F. Measurederts of the Stark braedenting of tentzed sllicon llnes lice. Ollo0000 Phyo. Rev. A 1E. 1169 (1578) inlted stoteo

Nardl. E.: 21 nasen, 2. Eneray decesition ty relotivistlc electrons in high-teaceroture torgets. Phys, Rev. A 18, 1246 (1578) Terae I

HI coleices, C. A.: Beck, E. R. Cosent on the Luken-s Inonoplu Doper Theory of atosle otructuree Including alectron correloticn. V. Exclted stotes not lowest of their aymaetry and oscliletcy otrengths in

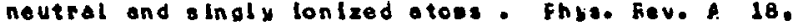
1307 (1578)

Greece

Luken. H. La: sinanoglu, C. Feply to Cowent en Theory cf atonle atructures Ineluding electron correlotion. $V$. Fhys. Rev. A 18, 1213 (157E) Unl ted Etates

zaucer, M.: Azmon. A. Holecules it strong megnetlc rields. Fiys. Fev. A 18, 1320 (1S7E) Yugosiovis

Ahlenlus, To: Lorsoon, E. Vorlotlenal soleuletien of the lowest $2 p 0$ ond opo st otes ef $L 1$ ond $C z$ ky the Hyllerase dethod. Phys. Hev. A $1 E, 1329$ (1S78) sueden

HIcksan, A. P. Theory of engular ecoentur elxing in

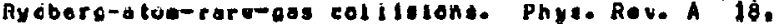
1529 (1874) Unitad states

Rogers. U. 1.: Clagn. J. O.: Cunn, G. t. Atsolute oniesten crose uection lor electron-lacoct excitetion of $\mathrm{LI}$ + to the (2 ip) 1evel. Fhy . Fev. A 18, 1363 (1578) United stotes

Ltght, de: Exoke. A. Faur=athth esdol of entleal

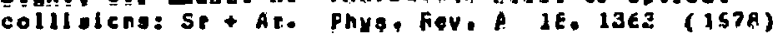
United stotes

Towara, Ho: Rlchard, P.: Geay, T. J.: Neweceb, J.I Jasl sun; K. H.: senuldokdac. C.: Hall, J. H. SI $k$-ehell lenizetion ond eleotecn trenster cross sections: sol id torgets. Fhyse Fen. A 18,1373 (1578)

United states

Huaphrey, L. M.: Gellaghar, T. F.: Cooke. ti. E.: Edelsteln, E. A. Collitalonal deoctivotion cf higher Na s and conifcid atates ty N,. Phya. Rev. A 18, 1383 (1 1588$)$ Unlted Etotes

Nguyen, T. C.: Sadegh 1, N. Rote ecellicienta tor collisicnal population tranafer betweer 3r sap argen levels at 300 oK. Phya. Fev. A 1E, 1388 (1978) France 


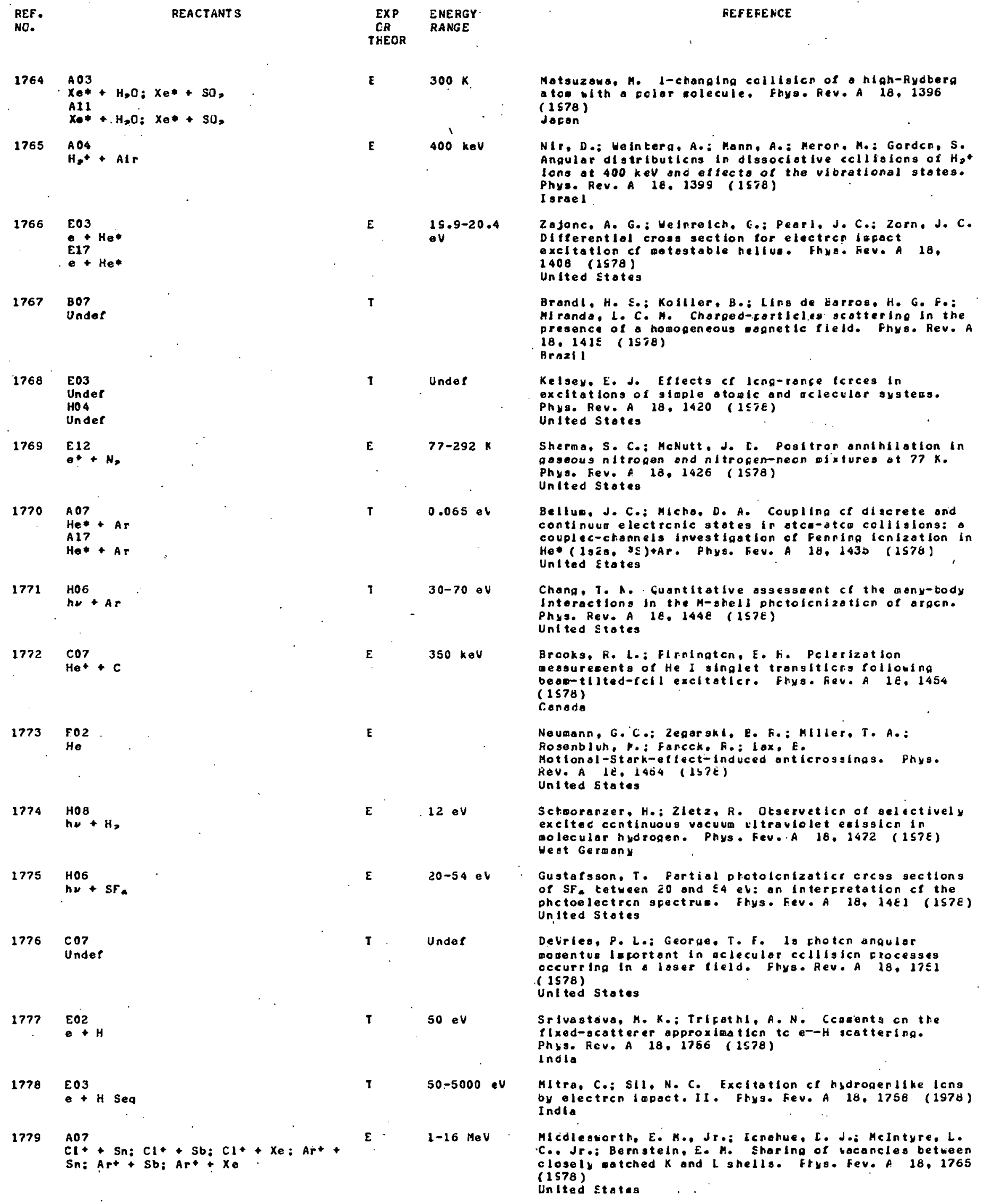




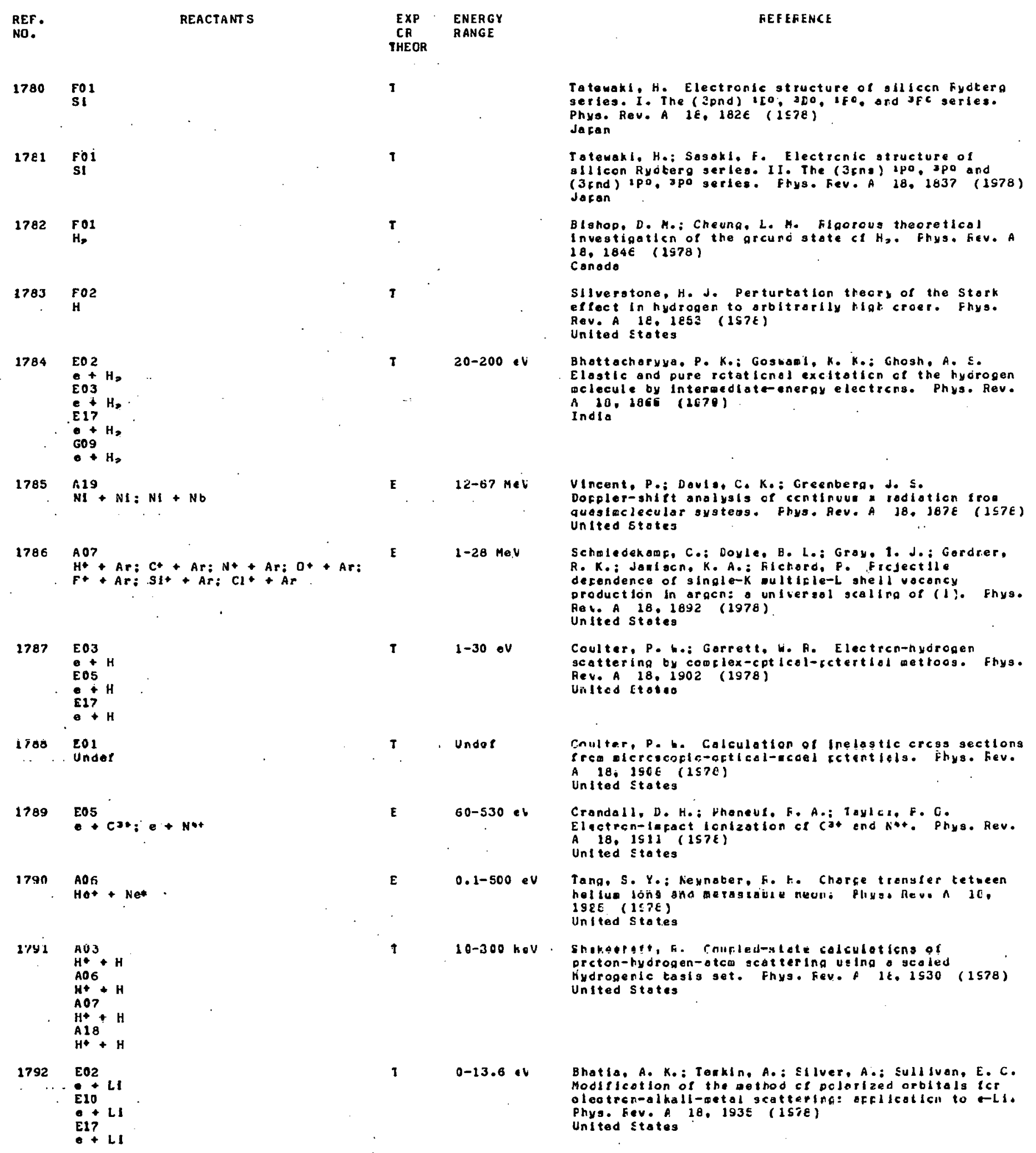


REF.
NC.

1793

A 07

$\mathrm{S}^{+}+\mathrm{Ar}_{\mathrm{C}} \mathrm{Cl}^{+}+\mathrm{Ar}: \mathrm{Art}^{+}+\mathrm{Ar}^{\mathrm{Mn}} \mathrm{Mn}^{+}$ Ar: $\mathrm{Cu}^{+}+$Ar: $\mathrm{NI}^{+}+\mathrm{Kr}_{\mathrm{r}} \mathrm{Cu}^{+}+\mathrm{Kr}_{\mathrm{r}}$ : $\mathrm{Ge}^{+}+\mathrm{Kr}_{\mathrm{r}} \mathrm{As}^{+}+\mathrm{Kr}: \mathrm{Se}^{+}+\mathrm{Kr}: \mathrm{Br}+$ $\mathrm{Kr}: \mathrm{I}^{+}+\mathrm{Kr}: \mathrm{Br}^{+}+\mathrm{Xe}_{\mathrm{C}} \mathrm{I}^{+}+\mathrm{Xe}$ A19

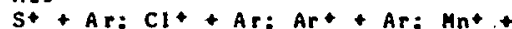
Ar: $\mathrm{Cu}^{+}+\mathrm{Ar}: \mathrm{NI}^{+}+\mathrm{Kr}_{\mathrm{r}} \mathrm{Cu}^{+}+\mathrm{Kr}_{\mathrm{r}}$;

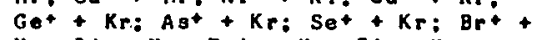
$K_{r}: I^{+}+\mathrm{Kr}_{\mathrm{B}} \mathrm{Br}^{+}+\mathrm{Xe}_{\mathrm{C}} \mathrm{I}^{+}+\mathrm{Xe}_{\mathrm{e}}$

1794

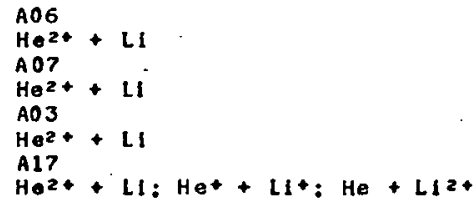

1795

A 07

$\mathrm{H} \mathrm{Seq}+\mathrm{Ne}: \mathrm{HSeq}+\mathrm{N}: \mathrm{H} \mathrm{Seq}+\mathrm{Ar}$ $\mathrm{AO3}$

H Seq + He: $\mathrm{H} \mathrm{Seq}+\mathrm{N}: \mathrm{H} \mathrm{Seq}+\mathrm{Ar}$ $\mathrm{AO} 2$

H Seq + He: H Seq + N: H Seq + Ar

A0 6

$\mathrm{O}^{\circ+}+\mathrm{H}: \mathrm{He}^{2+}+\mathrm{H}: \mathrm{H}^{+}+\mathrm{H}$

A06

Under

$\mathrm{CO}^{+}+\mathrm{LI}^{+} \mathrm{H}^{+}+\mathrm{Be}: \mathrm{H}^{+}+\mathrm{B}: \mathrm{H}^{+}+\mathrm{C}^{+}$

$\mathrm{H}^{+}+\mathrm{Ne}: \mathrm{H}^{+}+\mathrm{Al}: \mathrm{H}^{+}+\mathrm{Sl}: \mathrm{H}^{+}+\mathrm{Ar}^{+}$

$\mathrm{H}^{+}+\mathrm{Ca}: \mathrm{D}^{+}+\mathrm{LI}: \mathrm{D}^{+}+\mathrm{Be}: \mathrm{D}^{+}+\mathrm{B}:$

$D^{+}+\mathrm{C}: \mathrm{D}^{+}+\mathrm{Ne}: \mathrm{D}^{+}+\mathrm{Al}: \mathrm{D}^{+}+\mathrm{S} 1:$

$D^{+}+A r: D^{+}+C a$

$1799 \quad E 12$

$\mathrm{e}^{+}+\mathrm{Hi} \mathrm{e}^{+}+\mathrm{He}$

1800

Co6

$\mathrm{H}^{+}+\mathrm{C}: \mathrm{C}^{+}+\mathrm{C}^{+} \mathrm{N}^{+}+\mathrm{C} \mathrm{O}^{+}+\mathrm{C}: \mathrm{Na}^{+}$

$+\mathrm{C}_{\mathrm{O}} \mathrm{Mg}^{+}+\mathrm{C}: \mathrm{S}^{+}+\mathrm{C}_{i} \mathrm{Kr}^{+}+\mathrm{C}$

$\mathrm{CO} 7$

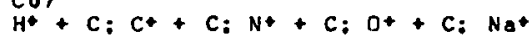
$+\mathrm{C}: \mathrm{Ma}^{+}+\mathrm{C}^{2} \mathrm{~S}^{+}+\mathrm{C}: \mathrm{Kr}^{+}+\mathrm{C}$

$\begin{array}{ll}1801 & E 17 \\ & 8+H \\ & E 02 \\ & B+H\end{array}$

1802 A 06

$\mathrm{H}^{+} 06+\mathrm{H}$

A 18

$\mathrm{H}^{+}+\mathrm{H}$

1803

A07

$\mathrm{He}+\mathrm{H}$

1804

$\mathrm{HeZ}_{\mathrm{He}}^{\mathrm{O}}+\mathrm{H}_{2}$ : He* $+\mathrm{D}_{2}$

2805

A03

He* $+H_{0}$

A11

$\mathrm{He}+\mathrm{He}$ $\begin{array}{ll}\text { EXP } & \text { ENERGY } \\ \text { OR } & \text { RANGE } \\ \text { THEOR } & \end{array}$

$0.1-1000$

$k \in V$

Under

$0.025-1600$ kev

Under

$20-10 \cdot k e V$

Under

$100-400 \mathrm{keV}$

$0.5-1.0 \mathrm{keV}$

$25 \mathrm{keV}$

E

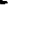

$0.015-0.10$ ev

$0.04-0.2 \mathrm{eV}$

$2.8-4.7 \cdot \mathrm{V}$
Lennard, t. N e: Mitchell, I. V.: Farater, J. S Unt verool scoling law Pcr k-voconcy ficduction in heavy ion-aters single ccllisicns. Fhys. Hev. A 18 , is $4 \mathrm{~S}$ (1578)

Canada

Shlpsey, E. J.: Redeon, L. T.: Browne, Je Co: Clsen, F. E. Electrcn-cacture ord lanlzaticr. cicss sections for colllislens of Heat with LI: production of Het(2I) at lou velecitles. Phys. Fev. A 1E.1961 (1578) United stotes

Glllesple, G. H. Excitation and ICndzotlon contributions to sum-rule Born cross sectiors tcr collisicng ct one-electron lons with otcos. phys. Rev. A $10,15 E 7$ ( $1 S 7 E$ )

Unlted stotes

Ryufuku, H.: Hatonabe, T. Charge trangler in

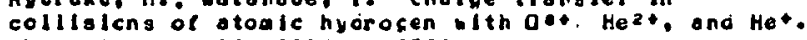
Phys. Rev. A 18, 2005 (1578)

Jecan

Spruch, L. High-lepact-velcclty: aró cherse transter tran high-fyobers itates as a clasalcol process. Phys. Rev. A IE, 2016 (ISTE) United Stotes

Gertner, I.: Heron, Ma: Rosner, E. Electronlc eneroy lass of lons in solids in the eneray range 10-10" kev/nuclecn. Phys. Rev. A 18, 20 c2 (1978) Is roel

dean, Y.-C.: Schroder, I. M. Secend-order non adistatic polarizotion potentlals for positren-hydrogen and poeftrcn-hellum elastic sceftering. Frys. Fev. A 18 , 2030 (2578)

Unlted steites

Christensen, B.: Vele, E.: Huelplund, F, Expericental study cf chorge-state olstributicns anc photon easicns trca bear-tllted-tcll interoctions. Phys. Rev. A $18,2042(1678)$

Denuark

Shakestolt. R. Electron excharpe scottering Pron. hydrogenl ike atom of high Inpact velocitles. Fhys. Fe v. A 18,2047 . (1978)

Unirea siates

Halpern. A. M. Vorlatlonally conelatert acrrexleation schewe for charge trangfer. Fhyo. Fev. A 18, 2058 (1578)

United States

Fort, J.: Loucagne. Jo Jo: feonelle, A.: Watel, G. Veloct ty derendence of the crcas sectlcng tor fenning and associotive lonization of $A$ and $D$ otcos by He( $2^{3} S$ ) motastable atcas. Phys. Fev. A $1 E, 2063$ (1S78) France

Fort, J.: Eclzlnger, T.: Ccrnc, L.: Etcing. T.: Pesnelle. A. Velocity dependence of the crcos sections Por Perning, reorrangeaent, and totol ienizotion of $\mathrm{H}_{2}$ ano $D$, colecules by He( $\left.2^{3} S\right)$ and he( $\left.z^{2} \leq\right)$ detestoble atcas. Physe Rev. A 16, 207E (1570) fronce

Burrell, C. Fo: Kurze, ho-J. Cellisienol tronster potes bitwean excited levels in hellug. phys. kev. A $18,2081 \cdot(1978)$ United Stotes 


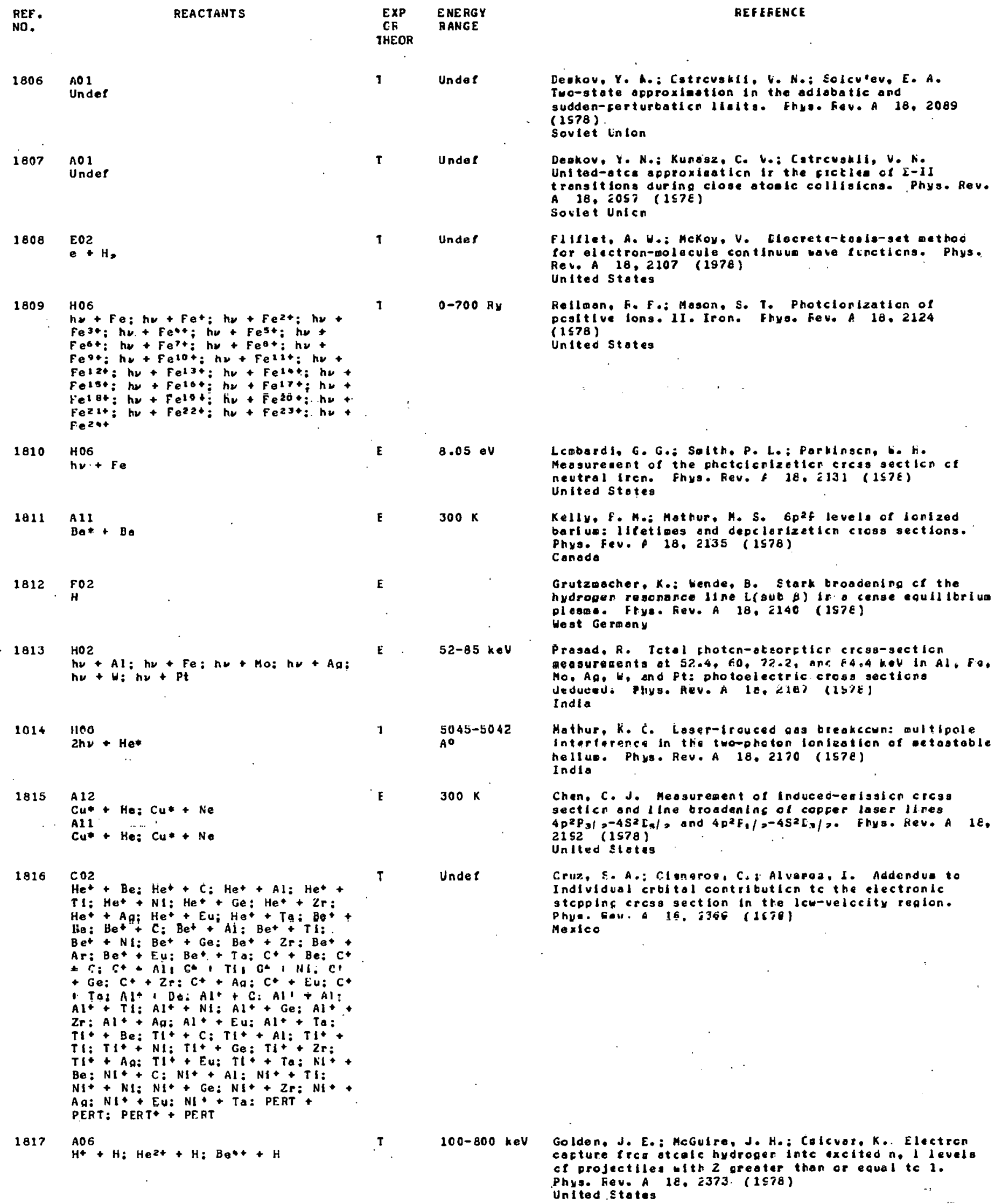

$0-700$ Ry

$8.05 \mathrm{eV}$

$300 \mathrm{~K}$

Unde?

A 0

$300 \mathrm{~K}$

Teokoy, Yo Ao: Eatreuskit Vo No: Solculev, E. A. Two-state opproxiaction in the adiobatic and sudden-certurtaticn lifitg. Fhye. Fev. A 18, 2089 (1578)

soviet inton

Deakov, Y. N.; Kundezz, C. U.: Catrcuakll, V. Nio United-atce approxianticn ir the cickles of $\sum-I I$ tronsitions during close otoelic collisicnse. Phys. Rev. a 18 , Z05 ( $1 \subseteq 7 \varepsilon$ )

Soclet Unicn

fliflet, A. W.: Mekoy, V. Clocreta-tosis-get gethoo for electron-molecule continuun wave tincticna. Phys. Rev. A 18,2107 (1978)

United States

Relloon. F. F.: Hoson, S. T. Photclonization of pcsltlue lons. II. Iron. Ehyo. Fev. A 18,2124 (1s78) Untted Stotes

Lcabardi, G. G.: Salth, P. L.: Porkingen, b. H. Measureant of the phctcicolzeticr crcss sectlon ce neutral Iren. Fhys. Rev. \& 1E, 213 ( $1 \leqslant 7 \varepsilon)$ United Stotes

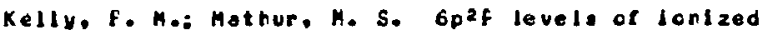
barlue: lifetloes ond depclorizeticn cross sections. Phys. Fev. A 18,2135 (1578)

Canodo

Grutzwacher, K.: bende, B. Stark broadentro cf the

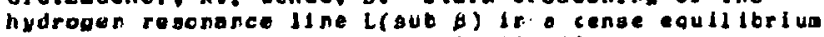
plesea. Frys. Fev. A 18 , E140 (1S7E) West Ge raony

Prasad, R. Ictel choten-otgorfticr crcsa-secticn measureants at 52.4 , fo, 72.2, anc 84.4 kol in Al, Fo, Mo. Ag, $W$, and $F t$ : photoelectric cross sections Jedued: Plivg. Aev. A lo, Lie? (IS)E) Indla

Mathur, $k$. $c$. Lager-lirouced oag breakccun: multipole interference in the two-pheton ionleation of eetosteble hellus. Phys. Rev. A 18, 2170 (157e) India

Chen, C. J. Meosurement of Induceo-ealisicn cress sectlcn ond line broadenling of copter laser li.res $4 D^{2} P_{3} /=-4 S^{2} I_{a} /$, and $4 D^{2} F_{1} /=-4 S^{2} E_{3} / 2$. Fhys. Rev. A $1 \varepsilon$, 2152 (1578) Uniled gtetes

C.ruz, S. A.: Cienqrop, C. Alvaroz, I. Addendua to Individual croltol contributicn tc the electronic stcppinf crcss section in the lcw-velccity region. Phy=. Gau. 1 16. 3365 (1679) Mexico

100-800 keV Golden, J. E.: HeGulre, J. H.: Calcror, K.. Electron corture ficg otcale hydroger intc excited $n$, I levels ef prosectlles with 2 areater than or equal te 1 . Phys. Fev. A 18, 2373. (1578) United stotes 
REF.

NO.

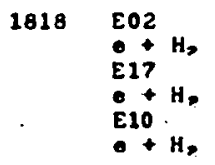

1819

A07.

$1820 \quad A 01$

Under

E01

Under

1821

$\mathrm{Al2}+\mathrm{Ar}$

$\mathrm{AO} 3$

No* + Ar

A11 AT

$\mathrm{Na}+\mathrm{Ar}$

$1822 \mathrm{EO} 3$

$0+\mathrm{H}$

E1?

$1823 E 02$

rot He

Fo1

1824

DO4

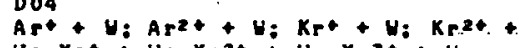

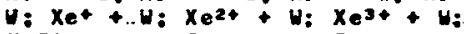

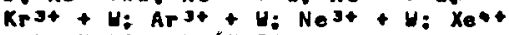

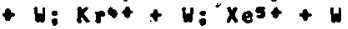

1825

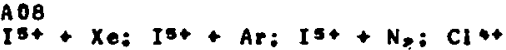

+ Xe; Clit + Ar: Cint $+N$,

1826

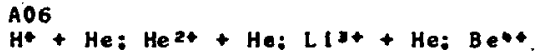
He: Bs+ $+\mathrm{He}$ : Cor $+\mathrm{He}$ : NPt+ He: $\mathrm{Oot}+\mathrm{He}$

A0?

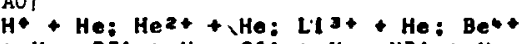
$+\mathrm{He}$ : Bst + He: Cat + He: Nrt+He: $\mathrm{Out}+\mathrm{He}$

$\cos$

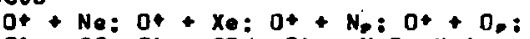
$\mathrm{O}+\mathrm{CO}_{\mathrm{O}} \mathrm{O}^{+}+\mathrm{CO}_{3}=\mathrm{O}+\mathrm{H}_{3} \mathrm{O} \mathrm{X}_{4+}+$

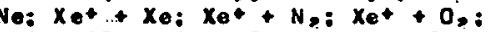

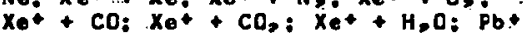

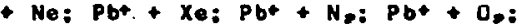
$\mathrm{Pb}+$ + $\mathrm{CO}: \mathrm{Pb}^{+}+\mathrm{CO}_{2}: \mathrm{Pb}^{+}+\mathrm{H}_{>} \mathrm{O}$

1828

$\mathrm{Cl}^{\mathrm{O}}+\mathrm{Cl}: \mathrm{Cl}_{+}+\mathrm{II}_{\mathrm{C}} \mathrm{Cl}++\mathrm{NI}$

1829

A 12

$\mathrm{Het}^{+} \mathrm{He}^{\mathrm{C}}$

$\cos$

CHA

1830

\begin{tabular}{|c|c|}
\hline T & $300 \mathrm{keV}$ \\
\hline & \\
\hline \multirow[t]{3}{*}{$\mathbf{E}$} & $300 \mathrm{~K}$ \\
\hline & $\cdot$ \\
\hline & $54-100 \cup v$ \\
\hline & \\
\hline \multirow[t]{2}{*}{$i$} & $\begin{array}{l}E 02 \\
\text {. Under }\end{array}$ \\
\hline & $!$ \\
\hline $\mathbf{E}$ & $100 \mathrm{eV}$ \\
\hline & \\
\hline $\mathbf{E}$ & $20 \cdot \mathrm{MeV}$ \\
\hline
\end{tabular}

T $\quad 100-8000$

$k \in b$

E T $50 \mathrm{keV}$

$35 \mathrm{MeV}$

$\mathbf{E}$

I Under

1

$\begin{array}{cc}\text { E } & 50 \text { keV } \\ & \\ \text { E } & \\ & \end{array}$

\section{OR PANGE \\ THEOR}

$\mathbf{T}$

$300 \mathrm{~K}$

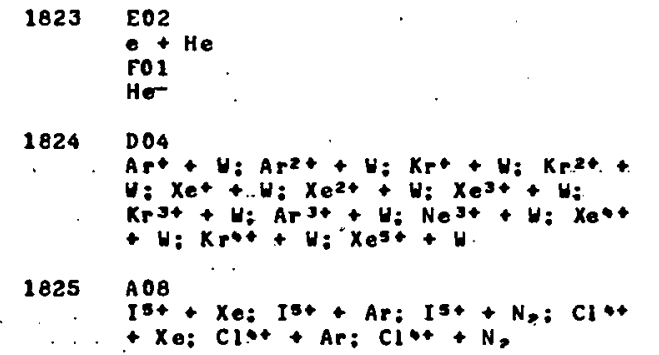

$0.003-5.0$

GEFERENCE

SrIvastovo, M. K.: Trlpathl, A. K.: Lol, M. Exchange effocts In the independent-atce eccel ct eletrcn-molecule ocattering. Phys. Rev. A JE, 2377 (1578)

Indla.

Shakeahoft, R. Coweent on the cirst-crder faddeev approxisoticn for icnizot icn cl otcos ty ion iopoct. Phys. Fev. A 18, 2381 (1978)

United stotes

Sur, S. K.: Mukherjee, S. C.: Stl. A. C. Coagent cn the Glouber scattering omflitude fcr hellug torgets. Phys. Fev. A is, 2384 (157e) India

Grunberg, G.: Blraben. F.: Kolwas. M.: Cagnoc, H. Cowbent on collisicn-prccesses inveluic in Tmo-photon near-resonance light scattering trca or otosic level. Phyo. Fev. A 18,2387 (1578)

Fronce

SI ngh, S. N.; Kuad, S.: Erivastove, H. K.

Electrcn-lacact excitation cr hycrcger. otom in the twc-potential elkonal apprcxidaticr. Fhys. Hev. A 18 , 2351 (IS7E)

Ind Ia

Junker. B. F. Coaclex-cocrdinate dethci. II. Fesonance calculotion with correlated target-giote wave Puncticns. Phys. Rev. A 18, 2427 (1578) United stotes

Varga, F.: Uinter, H. Deteralinaticn cf wetastable fractions in noble-gas-ion beasa. fhys. Fevep 18 , 2453 (1978)

Austria

Scctt, H. A.: Briduell, L. E.: Hoak, C. D.: Altch, G. E.: Jones, C. M.: Milier. F. D.: Eeyer. Fo C.: Kessel, Q. C.: Antor, A. Electron-icss crcss sections for 20-MeV Cl to and Ist long Incldent cn thin gasecus targets: exfegleantal geasureants ano fotential-model data ondyses. Fhys. Fev. f 18,2459 (1578) United States

0lson. Fi. E. Electron-capture and lococt-icnizaticn crose secticns for.ultiply chergec icro colliding with hell tum. Fhys. Fev. A 18, 2464 (1578) United states

Besentocher, F, Helneseler, J.: Huelclund, P.: Knudsen. Fi. Iniluence or patial correlaticn between target tces cn the scotering of eneicetic ions: nuitiple ocettering in coleculor geses. Phys. Hev. A $18,2470(1578)$

Denoark

Tserruyo, I.: Schaldt-Eocking, H.: Schuch, R. K-shell Ionlzation probobility in energetic neorly gyodetric

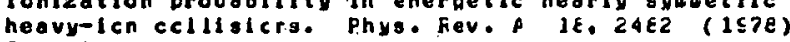
Is rael

Bassalo, J. Mo: Cottant. M. Electron-fapact withs and shifts ci neutrol heliug lines in olosma. Fhys. Hev. A. 18.2488 (1978) Brozi!

Mathur. K. C. Rotational excitaticn of $\mathrm{CH}^{+}$by electron leract. Phys. Rev. A 18, 2493 (1578) 


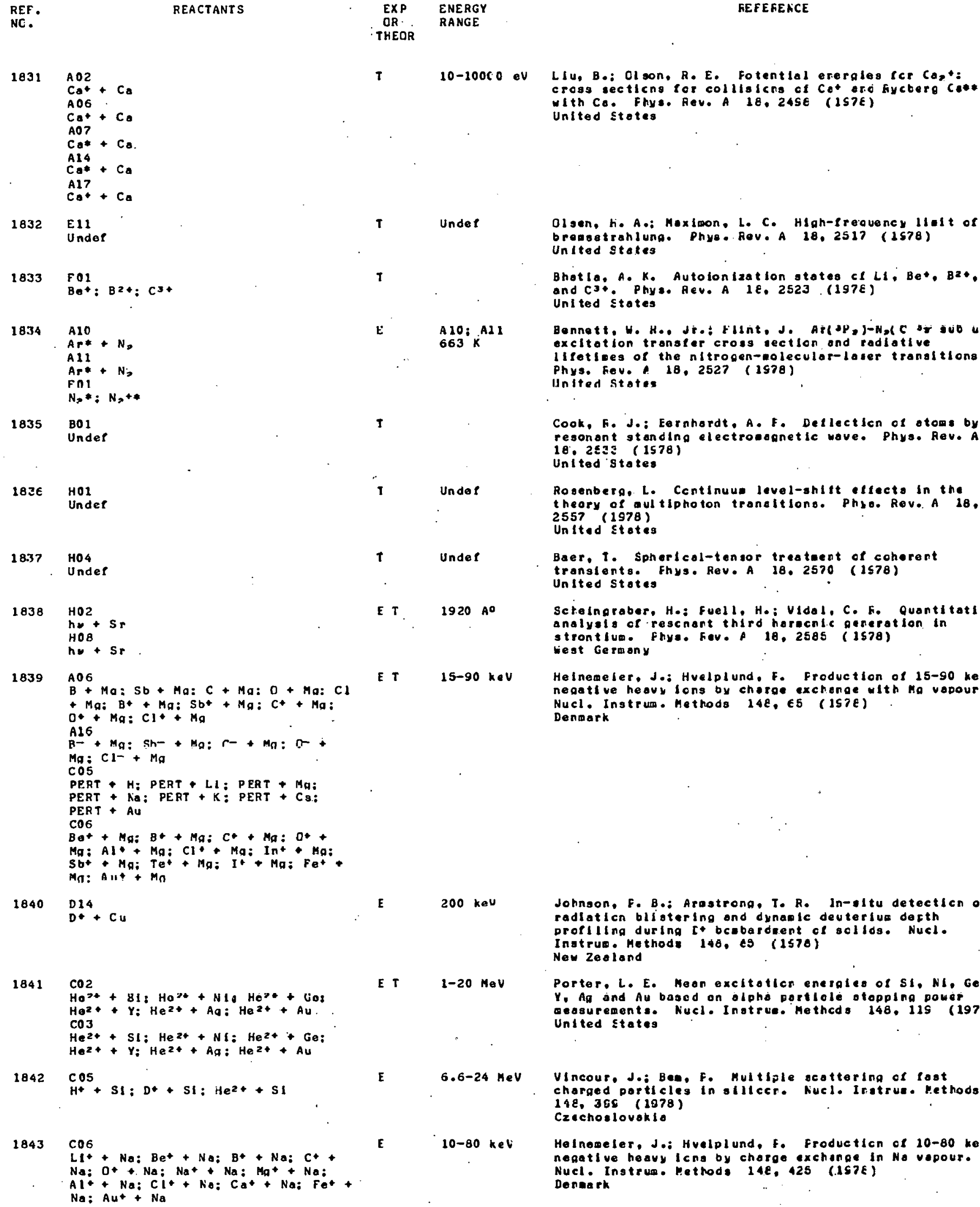

REF.
NC. THEOR

LIU, B.: Olson, R. E. Fotentlal erergles CCr Ca, : cross secticns Lor collisichs of Cot erio fiycterg Ca*k with Ca. Fhys. Rev. A 18, 2456 (1S7E) United states

$1833 \quad F 0$

$\mathrm{Be}^{+}: \mathrm{B}^{2+}: \mathrm{C}^{3+}$

A10

Al1.

Ar: $+\mathrm{N}$;

FnI

$N_{>} *: N,+*$

$1835 \quad 801$

Under

L8ze HO1

A06

$\mathrm{B}+\mathrm{Ma}: \mathrm{Sb}+\mathrm{Ma}: \mathrm{C}+\mathrm{Ma}: \mathrm{O}+\mathrm{Ma}: \mathrm{Cl}$

$+\mathrm{Maz} \mathrm{B}_{+}+\mathrm{Ma}_{+} \mathrm{Sb}^{+}+\mathrm{Mg} \mathrm{Cl}_{+}+\mathrm{Ma}$

$\mathrm{O}++\mathrm{Mg}: \mathrm{Cl}^{+}+\mathrm{Mg}$

A16.

${ }_{B-}^{A 16}+M_{g}: S h-+M_{Q}: r-+M_{a}: 0-$

$\mathrm{Mg}: \mathrm{Cl}^{-}+\mathrm{Mg}$

COS

PERT + H: PERT + Ll: PERT + Ma:

PERT + $\mathrm{NA}:$ PERT + K: PERT + CS:

PERT + Au

C06

$\mathrm{Ba}^{+}+\mathrm{Mg}_{\mathrm{O}} \mathrm{B}^{+}+\mathrm{Mg}_{\mathrm{O}} \mathrm{C}^{+}+\mathrm{Ma}: \mathrm{O}^{+}+$

$\mathrm{Mg} ; \mathrm{Al}+\mathrm{Mg} ; \mathrm{Cl}+\mathrm{Mg}^{\circ} \mathrm{In}_{+}+\mathrm{Mg}$;

$\mathrm{Sb}^{+}+\mathrm{Mg}^{+} \mathrm{Te}^{+}+\mathrm{Mg}_{\mathrm{g}} \mathrm{I}^{+}+\mathrm{Mg}^{+} \mathrm{Fe}^{+}+$ $M^{n}: M_{1}+M_{n}$

Undes

Unde P

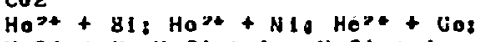

$\mathrm{Ho}^{2+}+\mathrm{Y}_{i} \mathrm{He}^{2+}+\mathrm{Aq}_{\mathrm{i}} \mathrm{He}^{2+}+\mathrm{AU}$.

$\mathrm{CO} 3$

$\mathrm{He}^{2+}+\mathrm{Sl}: \mathrm{He}^{2+}+\mathrm{NI}: \mathrm{He}^{2+}+\mathrm{Ge}$ :

$\mathrm{He}^{2+}+\mathrm{Y}_{\mathrm{i}} \mathrm{He}^{2+}+\mathrm{Aq}_{\mathrm{G}} \mathrm{He}^{2+}+\mathrm{AU}$ 


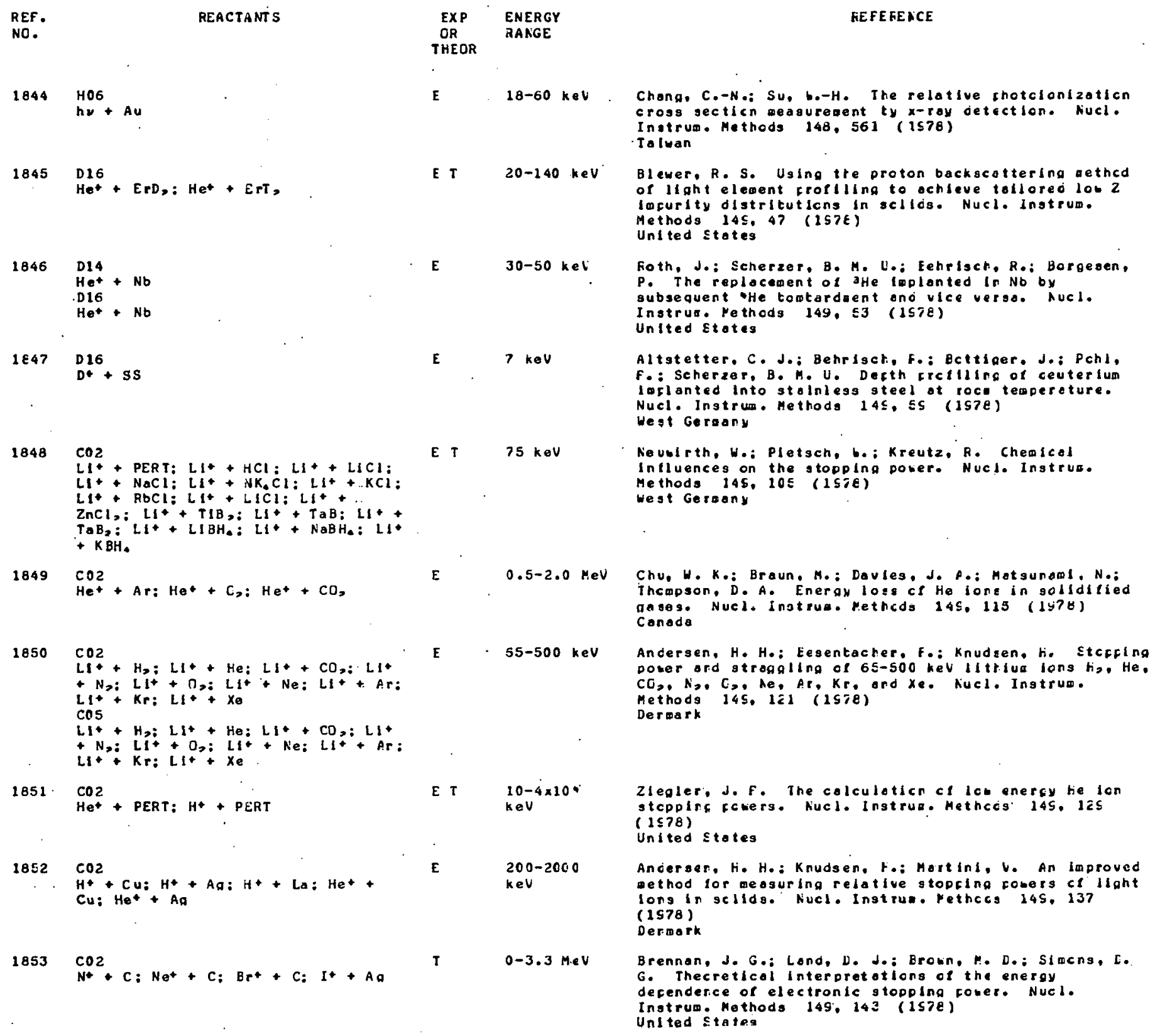




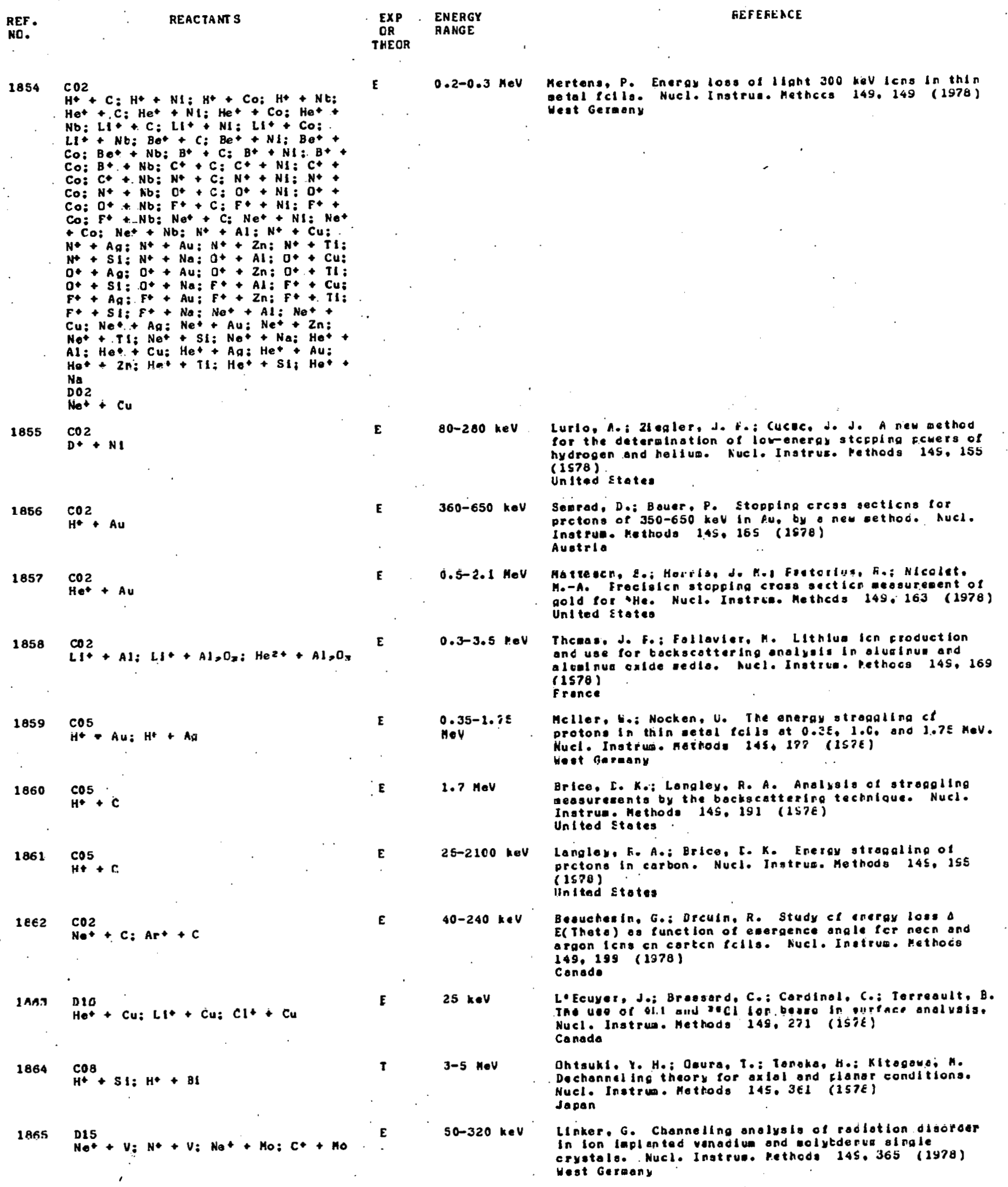




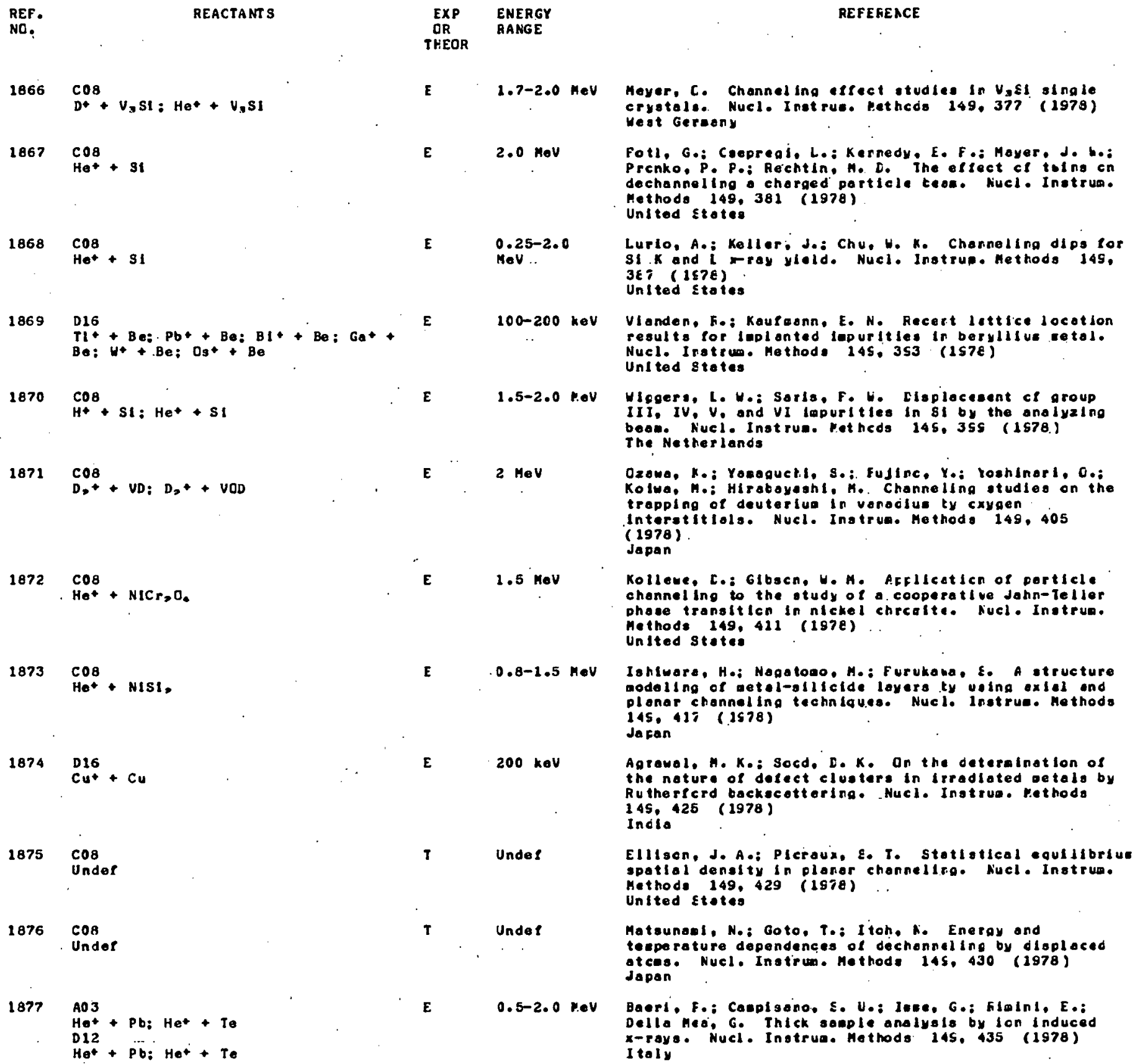

1873

$\mathrm{COB}$

Het+ HISI,

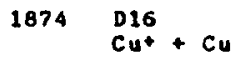

$1875 \cos$

Under

$1876 \quad \cos$

1877

REFEREACE

Fotl, G.: Caepreal, L.: Kernedy, E. F.: Meyer, J. b.: Prcnko, P. P.: Reichtin. H. D. The elfect cl tolng cn dechanneling a charged partiele teos. Hucl. Inetrua. Methodo 149.381 (1978)

United stotes

$0.25-2.0$

Lurlo, A.: Keller: J.: Chu, H. N. Channelling dips for si. K and $i$ ray yleld. Nucl. Instrup. Methods 149, उE ( $1 \leqslant 7 E$ )

United states

100-200 keV Vianden, Fo: Kaufoonn. E. N. Fecert lettice locotion results for Iaplanted Iapuritles In beryllius retal. Nucl. Iratrum. Hethode 14S, 353 (1S7e) Unlted Stetes

E $\quad 1.5-2.0 \mathrm{H.OV}$

Higgers, L. W.: Sorls. F, W. Cieplaceent ct group III. IV. $V$, and $V I$ Impurities in 81 by the analyzing beas. Nucl. Instrua. retheds 145. 35s (1578.) The Netherlands

E $2 \mathrm{MeV}$

Ozawa, Mo: Yosaguetl, S. Fujinc, Yo: Yoahlinarl, O.: Kolwa. M.: Hiraboyashl. M. Channeling atudies on the

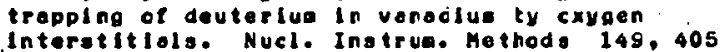
(1978).

Japan

Kollewe, c.: Gibsen. H. A. Acllcaticn or portlcle channeling to the otudy of a cooperative Jahn-ieller phase transiticn in nickel chrcalte. Nucl. Instrua. Methods 149, 411 (197e)

United state:

Iahiware, H.: Nagatono. M.: Furukana. E. A etructure modeling of metel-alliclde levero ty using exlel and plonar channeling techniques. Nucl. Inatrue. Methoda 145. 417 (1578)

Ja can

E $200 \mathrm{koV}$

Agrawal. M. K.: Socd, D. K. On the deteratination of the nature of defect cluetera in irradiated detalo by Rutherferd backecottering. Nucl. Inatrua. rethodo 145, 425 (1978)

India

Undes

Ellison, J. A.: Pleraui, E. T. Stotlstical equllibriue soatlal denalty in olanor chonnelira. Nucl. Instrue. Hethode 149, 429 (1978) Unlted stetes

T Unde?

Matsunasi. N.: Goto. T.: I toh. H. Eneray and teaperature dependences of dechenneling by disolaced atcas. Nucl. Instirun. Methods 14s. 430 (1978) Japan

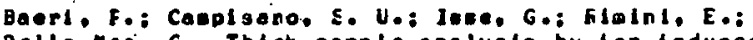
Della Hed, G. Thick somple analysis by ion Induced $x$-raya. Nucl. Instrua. Methods 14S, 435 (1978) Ita Iy 
REF

1878

AO 3

$\mathrm{H}^{+}+\mathrm{Tl}^{2} \mathrm{H}^{+}+\mathrm{Fe}: \mathrm{H}^{+}+\mathrm{Cu}: \mathrm{H}^{+}+\mathrm{Se}$

$\mathrm{H}^{+}+\mathrm{Y}_{\mathrm{O}} \mathrm{H}^{+}+\mathrm{Sn} \mathrm{H}^{+}+\mathrm{CS}^{+} \mathrm{H}^{+}+\mathrm{Ce}^{+}:$

$\mathrm{H}^{+}+\mathrm{Sm:} \mathrm{H}^{+}+\mathrm{HO}^{\circ} \mathrm{H}^{+}+\mathrm{H}: \mathrm{H}^{+}+\mathrm{AU}^{+}$

$\mathrm{He}^{2+}+\mathrm{IIO}^{\mathrm{H}} \mathrm{He}^{2+}+\mathrm{Fe}^{+} \mathrm{He}^{2+}+\mathrm{Cu}^{2+}$

$\mathrm{He}^{2+}+\mathrm{Se}: \mathrm{He}^{2+}+\mathrm{Y}: \mathrm{He}^{2+}+\mathrm{Sn}:$

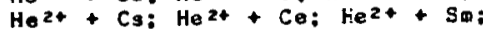

$\mathrm{He}^{2+}+\mathrm{Ho}^{\mathrm{O}} \mathrm{He}^{2+}+\mathrm{W}: \mathrm{He}^{2+}+\mathrm{Au}$

AQ7 7

$\mathrm{H}^{+}+\mathrm{II}: \mathrm{H}^{+}+\mathrm{Fe}: \mathrm{H}^{+}+\mathrm{Cu}: \mathrm{H}^{+}+\mathrm{Se}$

$\mathrm{H}^{+}+\mathrm{Y}_{\mathrm{O}} \mathrm{H}^{+}+\mathrm{Sn}_{\mathrm{O}} \mathrm{H}^{+}+\mathrm{Cg}^{+} \mathrm{H}^{+}+\mathrm{Ce}^{+}$

$\mathrm{H}^{+}+\mathrm{Sm}: \mathrm{H}^{+}+\mathrm{Ho}^{+} \mathrm{H}^{+}+\mathrm{H}^{+} \mathrm{H}^{+}+\mathrm{Au}^{+}$

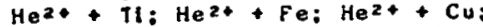

$\mathrm{He}^{2+}+\mathrm{Se}: \mathrm{He}^{2+}+\mathrm{Y}: \mathrm{He}^{2+}+\mathrm{Sn}:$

$\mathrm{He} e^{2+}+\mathrm{Cs}_{\mathrm{S}} \mathrm{He}^{2+}+\mathrm{Ce} \mathrm{He}^{2+}+\mathrm{Sa}:$

$\mathrm{Het}+\mathrm{Ho} \mathrm{He}^{2+}+\mathrm{H}: \mathrm{He}^{2+}+\mathrm{Au}$

D12

$\mathrm{H}^{+}+\mathrm{TI}^{+} \mathrm{H}^{+}+\mathrm{Fe}^{+} \mathrm{H}^{+}+\mathrm{Cu}^{+} \mathrm{H}^{+}+\mathrm{Se}:$

$\mathrm{H}^{*}+\mathrm{Y}_{\mathrm{O}} \mathrm{H}^{+}+\mathrm{Sn} \mathrm{H}^{+}+\mathrm{Cs}^{+} \mathrm{H}^{+}+\mathrm{Ce}^{+}$

$\mathrm{H}^{+}+\mathrm{5m}: \mathrm{H}^{+}+\mathrm{HO}^{\circ} \mathrm{H}^{+}+\mathrm{H}^{+} \mathrm{H}^{+}+\mathrm{AU}^{+}$

$\mathrm{Ha}^{2+}+\mathrm{Tl}: \mathrm{He}^{2+}+\mathrm{Fe}^{2} \mathrm{He}^{2+}+\mathrm{Cu}$ :

$\mathrm{He}^{2+}+\mathrm{Se}: \mathrm{He}^{2+}+\mathrm{Y}=\mathrm{He}^{2+}+\mathrm{Sn}$

$\mathrm{He}^{2+}+\mathrm{Cs}_{\mathrm{S}} \mathrm{He}^{2+}+\mathrm{Ce}^{2+} \mathrm{He}^{2+}+\mathrm{Sm}:$

$\mathrm{He}^{2+}+\mathrm{HO}: \mathrm{He}^{2+}+\mathrm{W} \mathrm{He}^{2+}+\mathrm{Au}$

$187 y$

$\mathrm{H}^{+}+\mathrm{C}: \mathrm{C}^{\circ}+\mathrm{C}: \mathrm{N}+\mathrm{C}: \mathrm{O}+\mathrm{C}$

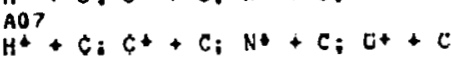

$\mathrm{HL}^{+}+\mathrm{C}_{\mathrm{O}} \mathrm{C}^{+}+\mathrm{C}^{+} \mathrm{N}+\mathrm{C}^{+}: \mathrm{O}^{+}+\mathrm{C}$

$1880 \quad$ D03

Review

1881 DO 3

$\mathrm{Ar}^{+}+\mathrm{Al}: \mathrm{Ar}^{+}+\mathrm{NI}: \mathrm{Ar}++\mathrm{Cu}: \mathrm{Ar}^{+}+$

Ar: Art + Au: Art+ SIC: Art+GaP

Art + GaAs: Art+ InAs: Art+ InSt

$1882 \mathrm{HOB}^{+}+\mathrm{MO}: \mathrm{H}^{+}+10+\mathrm{MO} 1$

1883

$\mathrm{DOB}^{+}+\mathrm{C}: \mathrm{Ne}^{+}+\mathrm{C}: \mathrm{Ar}^{+}+\mathrm{C}$

1884

$\mathrm{HU}_{4}^{+}+\mathrm{SI}_{1}: \mathrm{H}_{2}+\mathrm{SI}^{+}$

$\mathrm{HOS}^{+}+\mathrm{SI}_{1} \mathrm{H}_{2}++\mathrm{S} 1$

$\mathrm{H}^{+}+31: \mathrm{H}_{2}^{+}+\mathrm{SI}$

D 16

$\mathrm{H}^{+}+\mathrm{SI}: \mathrm{H}_{2}++\mathrm{SI}$

18 8.

$\mathrm{Al}^{+}+\mathrm{K}_{\mathrm{i}} \mathrm{Ar}^{+}+\mathrm{Al}_{\mathrm{i}} \mathrm{Ar}^{+}+\mathrm{II}: \mathrm{O}_{2}^{+}+$

1886 DOL
Revien
D17

D17

1887

$\mathrm{n} n 3$

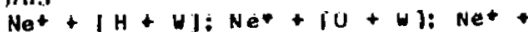
$[D+4]$

1888

DO3

$\mathrm{Kr}^{+}+B e: \mathrm{Kr}^{+}+\mathrm{BeU}: \mathrm{Kr}^{+}+\mathrm{Al}: \mathrm{Kr}^{+}$

$+A l_{>} O_{3}: K_{r}+\mathrm{SC}^{+} \mathrm{Kr}^{+}+\mathrm{GaAs}^{+} \mathrm{Kr}^{+}$

$+Y: K r++I n: K r++T I$

1889

$\mathrm{HOB}^{+}+\mathrm{AU}: \mathrm{H}^{+}+\mathrm{Si}: \mathrm{H}^{+}+\mathrm{Al}: \mathrm{H}^{+}+\mathrm{BI}:$

$\mathrm{He}^{+}+\mathrm{Au}: \mathrm{He}^{+}+\mathrm{Sl}: \mathrm{He}^{+}+\mathrm{Al}^{\mathrm{S}} \mathrm{He}^{+}+$

$\mathrm{BI}: \mathrm{Ne}^{+}+\mathrm{Zr}: \mathrm{Ne}^{+}+\mathrm{Cu}: \mathrm{Ne}^{+}+\mathrm{NI}$ $\begin{array}{ll}\text { EXP } & \text { ENERGY } \\ \text { CF } & \text { FANGE }\end{array}$

THEOR

E $4-30 \mathrm{HeV}$

$0.001 \cdot 32$ i $\varepsilon \mathrm{V}$

$1-100 \mathrm{keV}$

Unide
$0.05-3.0$

30- 1 co biev
$12 \mathrm{keV}$
Poncet. Engeleann. C. Study ct the $x$-roy eoission incuced by 4 to $30 \mathrm{MaV}$ protons or alpha particles. With a view tc andiytical application. nucl. Inatrue. Me thods 149,461 (1978)

Fronce

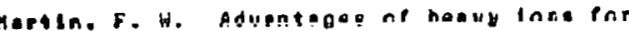

high-resolution afcroscopy. Aucl. Instrue. netheds 1 AS, 475 (1978)

United stales

Kelly. F.: Goed-zanin, C. Je: Shehato, M. T.j Squires, D. B. in otteart to understand seconcory photon ealsicn. Nucl. Ingtruo. Hethods 14S.562 (ISZE) Canada

Brcngersad, H. H.: Buck, T. H. Low-energy ton ceatering (LEIS) lor ccarcsiticn ond atructure analysis of the octer surface. Nucl. Instrua. Methods 145. 565 ( 1578 )

The Natherlands 
REF.

REACTANT $S$

1890

$\mathrm{COB}^{\circ}+\mathrm{Si}$

DOB

$\mathrm{H}^{+}+\mathrm{SI}$

1892

DO8

$\mathrm{He}^{+}+\mathrm{Pb}: \mathrm{He}^{+}+\mathrm{BL}: \mathrm{He}^{+}+\mathrm{BL}_{2}, \mathrm{O}$ :

$\left.\mathrm{He}^{+}+\mathrm{POCl}_{3} \mathrm{:He}^{+}+\mathrm{Pb}^{\circ} \mathrm{NO}_{3}\right]=$

1892

D08

$\mathrm{Net}+\mathrm{Au}_{\mathrm{O}} \mathrm{Ne}+\mathrm{SI}^{+}$

1893

D08

$\mathrm{H}^{+}+\mathrm{SI}: \mathrm{He}^{+}+\mathrm{SI}_{1} \mathrm{~N}^{+}+\mathrm{SI}^{+} \mathrm{H}^{+}+$

$\left[A_{q}+\mathrm{SI}\right]: \mathrm{He}^{*}+\left[\mathrm{Aq}_{\mathrm{q}}+\mathrm{SI}\right]: \mathrm{N}+$

$[A \mathfrak{A}+\mathrm{SI}]$

1894

DO 8

$\mathrm{He}++\mathrm{NI}: \mathrm{Ne}+* \mathrm{NI}$

1895

Het +[CO+NI]: Hat+[H, $+W]$ :

$\mathrm{Ne}+\left[\mathrm{CO}+\mathrm{N}[]_{i} \mathrm{Ne}+[\mathrm{S}+\mathrm{NI}]\right.$

1896

$\cos$

$H^{*}+$ LIF

1897

$\mathrm{CO} 2$

$\mathrm{He}+\mathrm{Fe}: \mathrm{He}^{+}+\mathrm{NI}$

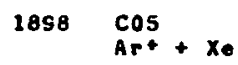

1899

$\mathrm{H}^{*} \mathrm{C}^{\circ}+\mathrm{Cr}: \mathrm{H}^{*}+\mathrm{Cu}$

$\mathrm{H}^{*}+\mathrm{Se} ; \mathrm{H}^{*}+\mathrm{NI} ; \mathrm{H}^{*}+\mathrm{Cu}_{2} \mathrm{H}^{*}+\mathrm{Ge}$

1900

A05

$\mathrm{Het}+\mathrm{Ar}$

1901

$I^{C 06}+N_{*}: I++A r: I+* K r: I *+X=$

1902

$\mathrm{NO}^{+}+\mathrm{C}_{2} \mathrm{~N}^{*}+\mathrm{Al}: \mathrm{N}^{*}+\mathrm{Cu}: \mathrm{N}^{*}+\mathrm{Ge}:$

$N^{+}+A_{9:} N^{+}+S n: N^{+}+B I$

1903

$\mathrm{CO} 2$

$\mathrm{He}^{2+}+\mathrm{H}_{2}: \mathrm{He}_{2+}+\mathrm{He}_{\mathrm{H}} \mathrm{He}_{2+}+\mathrm{N}_{2}$ :

$\mathrm{He}^{2+}+\mathrm{O}_{2} \mathrm{H}^{2+}+\mathrm{He}^{2+} \mathrm{He}^{2+}+\mathrm{Kr}_{\mathrm{r}}$

$\mathrm{He}^{2+}+\mathrm{Xe}_{\mathrm{e}}$.

1904

012

$0+\mathrm{NI}: \mathrm{H}^{+}+\mathrm{NI}$
E

$\mathbf{E}$

$20 \mathrm{MeV}$

$\begin{array}{ll}\text { EXP } & \text { ENERGY } \\ \text { OR } & \text { PANGE }\end{array}$

THEOR

E T 20-100 keV

165-2500 eV

Under

E $5-20 \mathrm{keV}$

$1.5-15 k \in V$

$0.2-2.0 \mathrm{keV}$

$100 \mathrm{keV}$

$0.3-2.6 \mathrm{HeV}$

A19

$80-140 \leqslant 4$

$\mathrm{CO} 2$

100 keV

Unde $f$

\section{E}

T
E $6.5 \mathrm{MeV}$

E $\quad 1-8.5 \mathrm{M} \in \mathrm{V}$

$0.013-1000$ GeV
REFEFEACE

IchInokewo, T.; Kasamura, T.: Nakadura, H.: Fujlectc, F.: Korakl. K. Angul or vorlationg of tackscattering vield with $20 \mathrm{keV}$ frotcn tcabardeent. Nucl. Instrum. Methods 145. 572 (15TE)

Jacan

Chrlgtersen. L. L.: Mossottl. V. E.: Fusch, T. W.: Erlckson. F. L. Caclllatory scattered lon yields from ncnconductlve ooterials. Aucl. Instrus. Methcos 149, SE7 (1578)

United Etotes

Buck, T. M.: theotley, G. H.: Mller, C. L.: Ficbinacn, D. A. H.: Chen, Y.-S. Concerisen cl otime-of-lilght syatew ith on lectrostatic analyzer in low-energy ion scattering. Nucl. Instrum. Methode 145, 591. (1978) United Etates

Agaey. E. A : Roblrson. J. E. Surtoce onalygla uoling mediug eneray ion end neutral scatterirg. Nucl.

Inatrue. Methods 145. Eg5 (1578)

Canada

Eckatelr. H.: Mclchanov. V. A.: Verteek. H. The charge state of He and Ne backscottering lros Ni in the energy range cf 1.5-15 kel. Aucl. Ingtrua. Hethods 149, 599 (1578)

Weat Germany

Taglauer, E.: Beltat, L.: Helland, b. Inveatlgatlen of ion lopoct desorpticn ci atcos and aclecules by low energy, Ion scattering (ISS). Nucl. Inotrus. Methcds 145. $\cos (1578)$

Weat Geroany

Goleckl, I.: Jaceard, $C$. fn offaretus for chonneling excerlaents ot Torr pressures. Nucl. Inatrue. Methcds 149, 66S (1978)

sultzerland

Baglin. J. E. E.: Chu, H. K. StcFing cower of $0.3-2.6$ MeV He ions in fe and NI. Nucl. Insirum. Methcds 14S. ESE (15>8)

Unlted states

Slguund. F.: Helneater, J.: Eesenkocher, F.: Huelplund, P.: Knucisen. H. Seall-angle eulticle scottering of leng In the screenes-coulcob reglon. Ill. Cobbinec angular and laterol spread. Nucl. Ingtrum. Hethods 150, zél (1STE)

Denuarh

Marsholl. F. E, El Flcl, A. R.: Klimer, J.K.

Heasuresent of topoling powergi ualra Icn-induced $x$-ray enlasicr. Nucl. Irstrue. Pethcds 150, 241 (1978) United stotes

Kenno, S.: Takohoshl. T. Fecogtinotion lualnescence in the scintillation of high fregare aracn gog Induced by alfha farticles. Nucl. Ingtrua. Hethcis 150,517 (1578)

Jafan

Hoak. C. D.: Briduell, L. B.: Scott, H. A.: Alten, G. D.: Jonee, C. Mo: Miller, F. L.: Seyer, F. Co: Keasel, Q. C.: Antor. A. Absolute charge tate ylelds cf 20 MoV izil lons coeralng fros o ga atriffer. Aucl. Inetrua. Hethods 150.529 (1578) United states

Rud, N.: Bottlger, J.: denaen, F, s. Heasuretents $c$ r energy-losedistrituticns tor $E .5$ ReV $15 N$ lcns In sollds. Nucl. Instrua. Matheds 1E1, 447 (1578) Denmark

Hanke, C.: Laursen, J. Stcpping crose sectlicns lcr a

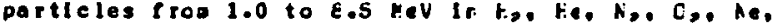
$K r$. and $X e$. Nucl. Instrum. Methods $1 E 1$, eEz (ISTE) Denmark

2relov. V. P.: Ruzlcka. J. Scae pecullioritleg of the optlcal transiticn radiaticn erce bloh-energy forticlea at Inclined incidence (III). Nucl. Instrus. Methods 151. 355 (1578)

Soviet Unicn 


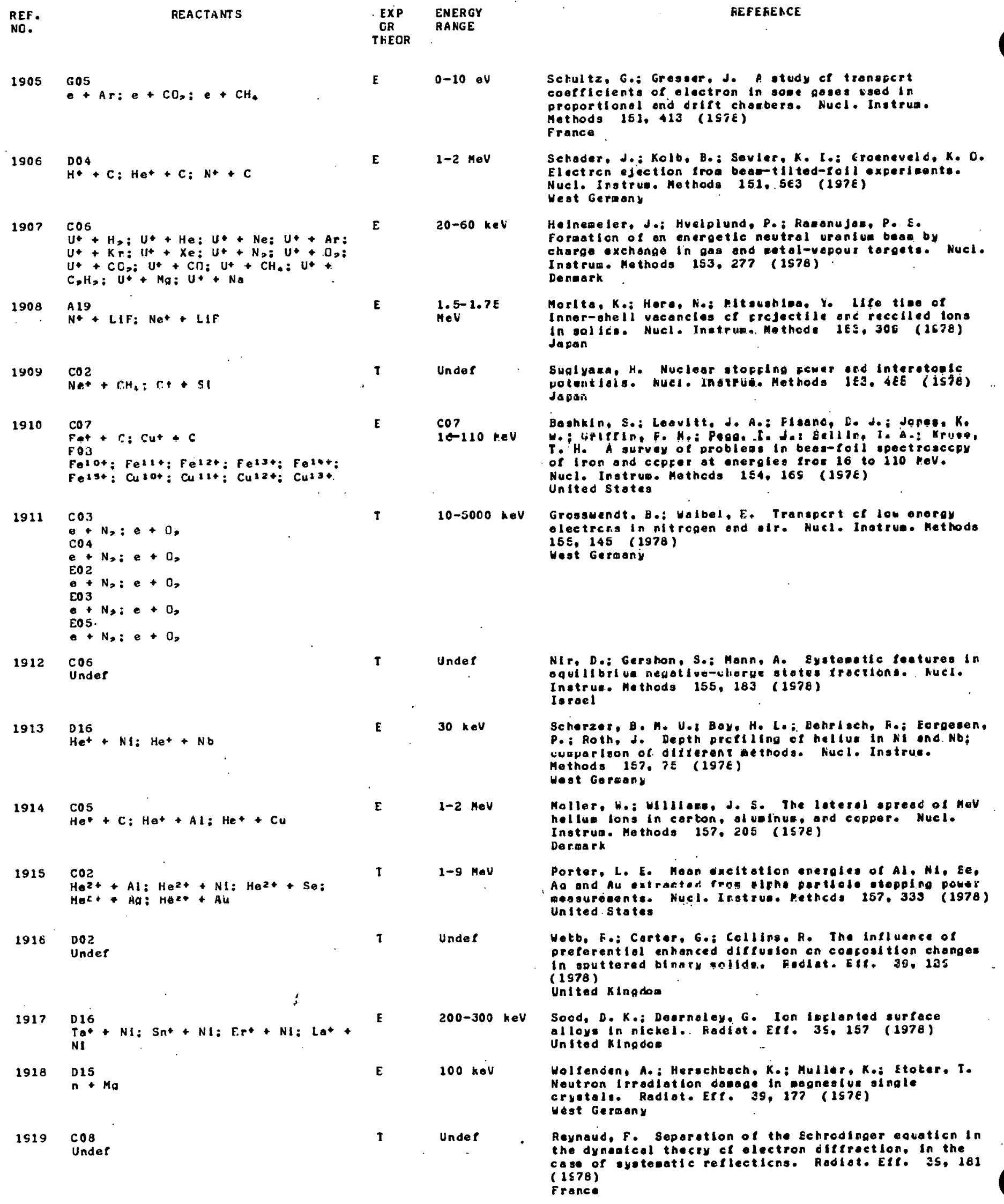




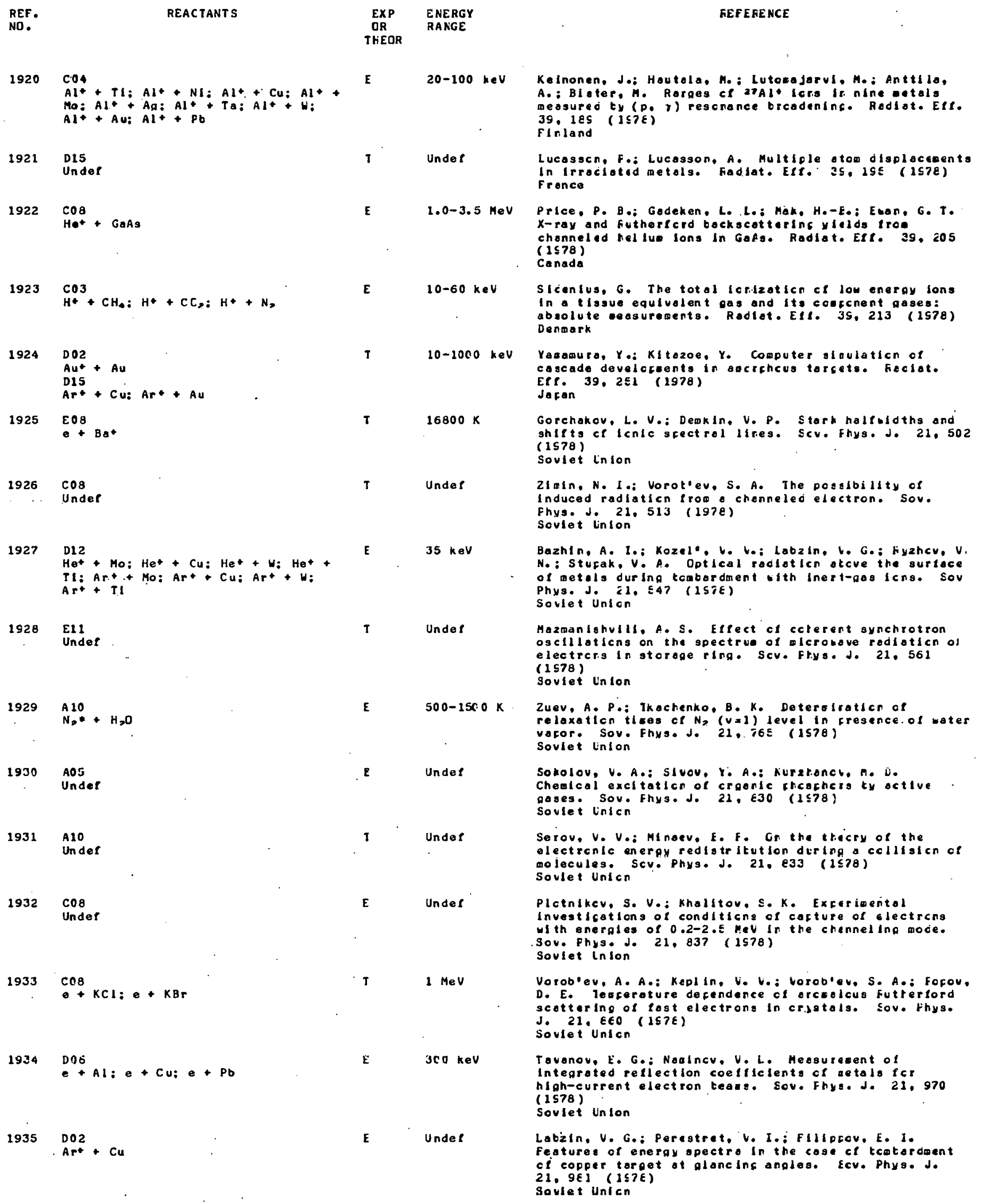


REF.
NO.

REACTANTS

$1936 \begin{aligned} & E 05 \\ & C_{0}^{+} x_{0} \\ & \text { FO }_{0}\end{aligned}$

1937

$\mathrm{CO} 2$

$++\mathrm{KCl}: \bullet+\mathrm{NaCl}$

1938

E16

$-+x_{e C l}$

1939

D16
BF $-1+31=81+31$

1940

D 17

Apt + VO,

1941

H1O

$h w+[B r+A r]$

1942

$H 04$

$2 h \mathrm{C}+\mathrm{Rb}$

hV + Rb

1943

CO4

- $+\mathrm{NaCl}$

1944

H06

$2 h \nu+L i$

1945

D09

CO* + Pyrex

$1946 \quad \mathrm{Al1}$
$\mathrm{CO}$

$1947 \mathrm{HQG}_{\mathrm{hL}}^{\mathrm{HO}}+\mathrm{He}$

I9AA nox,

e+cu

$1949 \quad$ DOS
ho + St

1950
$\mathrm{HO} 4$

$h v+T I: h v+V: h v+S I: h w+G e$
EXP
CR
THEOR

THEOR

E

E

E

$\mathbf{E}$

$\mathbf{T}$

$\mathbf{E}$

E

$20-50 k \in V$

$6000 \cdot A^{0}$

$1 \bullet v$

$53 \cdot 4-52 \cdot 2$

$3.4-5.2 \cdot v$
Ogurtsov, G. N.: Mikuahkin, V. M.: Flabs, I. P. Coster-Kronig trongiticns in the A shell of xenon. Sov. Tech. Fhys. Lett. 4, E (1S7E) Soviet inion

Vorobiov, S. A.: FlotnIkove S. V.: Fazua, E. I. Diotrloutlon cf the eneray otscrtec trca on electron teoo In crystal. Sove Tech. Phyo. Lett. 4.7 (1578)

Soviet Unien

Bychkov, Y. I.: Korlov, N. V.: Losev, h. F.: Kesyotes, G. A.: Prokhorov, A. M.: Torasenko, U. F, XeCl laser w th electicn-bean-exclted dischorge. Sov. Tech. Fhys. Lett. 4, 34 (1978)

Soviet inton

Tyogal, V. A.: Lyoenko, V. S.: Kraslkc. A. A.: Codtlinedi, A. M.: Lukahir, M. M.

Antsotroplc-electro-retlectance deleralinaticn os surface lottlce delects in lon loplantotion. Scv. Tech. Fhys. Lett. 4. 3s (1578) Soviet Union

Lanskayo, 1. G.: Suvorov, A. U.: Terukcu. E. I. Ellect of Ion tcobardment on the cetol--olelcteric phese trangition in VCg. Sov. Tech. Phys. Lett. 4.45 (1578)

Soviet Unicn

Antipenko, E. H.: Legtyorev, Y. L.: Nikclaev, L. E.: Tarasenko, V. V. Fhotoinduced reccmbination ci atcalc brcaine. Scv. 1ech. Fhys. Lett. 4, 65 (1578) Soviet Unicn

Arkhipkin, V. G.: Fopov, A. K.: Tleoseev, V. F. Cenversion of $A=1.06 *$ light lntc the 420-na rance in rublalua varer. Scu. Tech. Fhys. lett. 4, is (1578) soviet unton

Grochev, B. D.: Gudouskikh, V. A.: Korcbechko, Y. S.: MIneev. V. I.: Petrochenko, A. F.: kozlovakil. S. E. Frecise eeourewents ct the overoge electron ronge in a diefectrle. Sov. Teeh. Phys. Lett. 4, 83 (1578) Sovlet inicn

Delone, N. B.: Zon, E. A.: Fedorcv, H. V. Folerlzotion of nuciet in resonent otoelc lonizoticr. Scv. Iech. phys. Lerr. 4, i4 (15it)

Sovlet Unicn

Ionlkh, Y, Z,: Kuranov, A. L.: Penkin. N, P.: Sharkov, V. F. Reloxation of vibritionally excited CO t o glass ourfoce in discharge. Sov. Tech. Fhys. Lett. 4.101 (1978) soviet linion

IonIkh, Y. 2.t Kuranov, A. L.: FenhIn, A. F.; Sharkav,

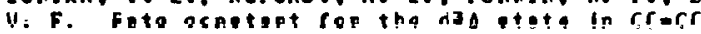
colligicns, Sov. Tech, physe Lete. 4, 10E (1ste) goviet linlun

Bellk, Y, P,i Boboshey, S, V, Shmoenck, L. A. Hasonance absorption of the ealselich trom. loeer-producod ploean. Sev. Toeh. Phye. Lett. 4, 108 (1578)

Suviot Unien

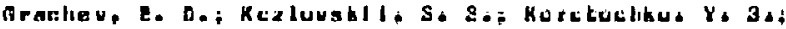
Hineev: V. I.: Fetrochenko, A. F. Angclar distributicn of rast electrong sattered Inelagtically ir ccFper. Sov. Tech., Fhys. Lett. 4, 110 (1578) Soviet Unton

Gritsenko, V. A.: Hogll'nikov. K. F. Featureg in the phctoealsicn of electicns cue to the tend otructure of illicon. Sou. Tech. Phys, Lat?, 4. 15? (1578) Sovitet Unton

Loskutcy, H. F.: Ulyakcw. F. I. Scectral-ilne absorption in the loser evorcraticr ct materials. Sov. Tech. Fhys. Lett. 4, 136. (1978)

Soviet inlon 
REF.

REACTANT S

1951

$\operatorname{Co} 4$

e+ Gas

1952

$\mathrm{N}^{+}+\mathrm{VO}_{2}: \mathrm{Ar}^{+}+\mathrm{VO}_{2}$

$1953 \quad D^{*}+S 1$

1954

A0?

$\mathrm{Ho*}+\mathrm{H}$,

$1955 \mathrm{CO}$

- Plasma

1956

A17

$x_{e}+x_{e}: x_{e}+x_{e}+x_{e}+x_{e}$

1957

A17

$\mathrm{Au}+\mathrm{H}: \mathrm{Au}+\mathrm{Cl}: \mathrm{Hg}+\mathrm{H} ; \mathrm{Hg}+\mathrm{Cl}$,

$1558 \begin{aligned} & E 03 \\ & \text { Get } \\ & \text { G02 } \\ & 0, * 0, \\ & \text { Al1 } \\ & 0, * 0,\end{aligned}$

$1559 \quad B 07$

$\mathrm{H}_{2}{ }^{+}+\mathrm{He}$

$1960 \quad \mathrm{Sn}^{\mathrm{Al}}+\mathrm{N}, \mathrm{O}$

$1 s \in 1$

$\mathrm{Hl}^{\mathrm{He}}+\mathrm{He}: \mathrm{He}+\mathrm{Ne}: \mathrm{He}+\mathrm{Ar}: \mathrm{He}+\mathrm{Kr}:$

$\mathrm{Kr}+\mathrm{Kr}_{\mathrm{r}} \mathrm{Ne}+\mathrm{Ne}: \mathrm{Ne}+\mathrm{Ar}_{\mathrm{r}} \mathrm{Ne}+\mathrm{Kr}$ :

$A r+A r: A r+K r$

1962

$\mathrm{SF}_{a}+\mathrm{CsCl}_{3} \mathrm{SF}_{4}+\mathrm{Cs}_{2} \mathrm{Cl}_{2}$

1963 HOS

nhe $+\mathrm{SF}_{\mathrm{a}} \mathrm{Cl}$

1964 HO4

$H 04$
nhv $+S F_{n}$
$30 \mathrm{keV}$

Unde?

$100 \mathrm{keV}$

77-300 K

2 HeV

Under

$300-550 k$

Undef

$0-11$ eV

0.1 eV

E
FEF EFENCE

Didenko. A. H.: Foh, V. S.: Usov, Y. P.: Tevethov, U. I.: Shetanov, A. A.: Shuloev. N. E. Irangport of high-curront alcrosecond electron bas. in a gas. Sov. Tech. Fhys. Lett. 4. 167. (1578)

Soviet Union

Abroyan, 1. A.: Vellehko, V. Y.: Govrllyuk, A. I.: Zakharchenya, B. Fo: Chudncuskil, F. A. Eltect ot fon torbardeent on the optical characteristlcs of the phase tronsition in VC, liles. Sov. Tech. Fhys. Lett. 4. $22 E(1578)$

Soviet unton

Gerosteenko. N. N.: Pon'kin, V. G.: Svitashev. K. K.: Iseltlln, G. M. Fcroation ci c vovegulde leyer in the bootarisent of allicon dicxide by torcr ions. sove bootardeent ol allicon dicxide by torct lone. sove
tech.iphys. Lett. 4, 232 (ISTE) Sovlet inien

Zhitnikcu, f. A.: Kartcahkir, V. A.: Klementev, G. V. Ioctople ellects in the Interaction of rolecular hydrogen uth atceic hellue in the $2{ }^{3}$, state. Scu. rech. Fhys. Lett. 4, 272 (1578)

Soviet Unton

BerezIn. A. Ko: Grlohaev. I, Ao: Zeldlits, W. P.:

KItelev, V. A.: : Sotroncv, V. G.: Fintera. Y. E.:

Fursov. G. L. Interaction ct a highly relotivistic monoenirgetlc electron beas bith dense plosed sciv. Toch. Fhyo. Lett. 4. 255 (1578)

soviet Union

Ereler, H. C.: Lee, Y. S.: FItzer, K. So: Hinter, N. H. Ab Int tio efrective core fctentiolo including

relativiatic ellects. II. Pctentiol energy curves for $x$ en: $x_{e}+$ and $x_{0} *$. J. Chea. Fhyse ES, s76 (1978) United states

Hay, F. J.: Wodt, H. R.: Kahn, L. F.: Ecbrculcz, F, H. At initlo tudies of AuH, AuCl, tat ond HoCl, using rolativistic effective core potentials. J. Ches. Fhys. 69. $984(2578)$ United states

Lawton, S. A.: Phelps, A. V. Exclaticn ct the 0 is (oub g) otote cl Co by lctenergy electrong. J. Chem. Fhys. 69. 1065 (1578)

United Etoteo

Brand1, H. S.: Kolller, B.: LIna de barros, H. G. P.: MIranda, L. C. H. Inert ges otco-aclecule ccllisions in. loser lield: vibraticnal excltoticns.in hosopolar noleculor Iong. J. Chen. Fhyse.65, 1096 (1578) Brazti

Felder, H.: Fentijn. A. HTFFF hinetica studies of $\mathrm{Sn} / \mathrm{N}, \mathrm{O}$, a highly effictent chelluolinescent reaction. J. Ches. Phys. 6S. 1112 .(1.57E) United states

Brual. G.. Jr.: Rothgteín, S. M. Fore gos interectiong using on limproved statlotical eethcd. J. Ches. Phys. 69.1177 (1978)

Conada

Parks, E. K.: Sheen, S. H.: Wexler, E. Thresheld behavicr $t$ cr colliaion-inducad dissociation of Cacl and

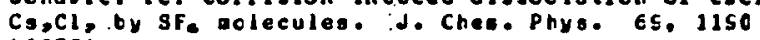
(1578)

United States

Karl, F. A.. Jr.: Lyaon, d. L. Investigaticn of the wulticle-photon dissocistion of SF. Cl ith real tive chlorine-stca diegnestic.. J. Chese. Fhyse 69.1196 (IS78)

United states

Ackerhalt, J. R.: Galbroith, H. H. Celllsicriless wultiple phctco excitation.ce sfo: a cepposieon of anharmonic oscillators ith and ithout octahedral splitting in the presence of rotationol offects. J. Chei. Fhyo. 69, 1200 (157E)

United stotes 


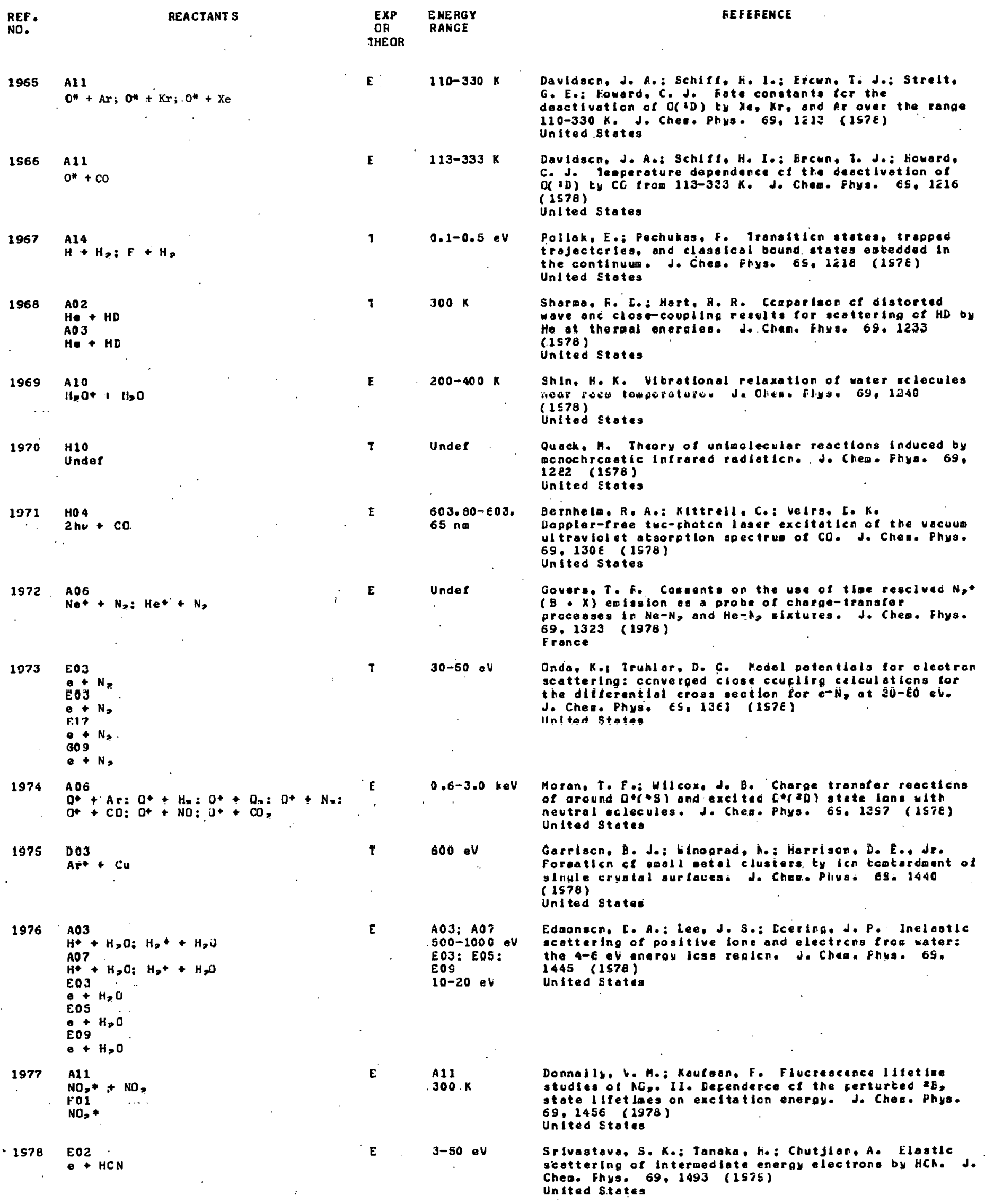


REF
NO.
$1979 \begin{aligned} & H 06 \\ & \text { nhu }\end{aligned}$

$2580 \quad$ A14

1981 AOJ

Ar* $+N_{\text {. }}$

1982

1983

1984

$\mathrm{E} 09$
$\mathrm{E} 05$

$0+\mathrm{H}_{2} \mathrm{O}$

1985

LI + 2 LI

19.86

$A 05$
SP*
$A 14$

Sr* + $\mathrm{N}_{\mathbf{0}}$

2987 AO?

He * Ar

Be? + Ar

He* Ar

1988

A24 + .

1989

A11

CIF* A Ar: CIF* + CIF

1990

A11

I* + Br;: I* + $\mathrm{H}_{>} ; \mathrm{I} * \mathrm{CH}_{3} \mathrm{I} \ldots$

1991

A 14

I* B

1992

HO7

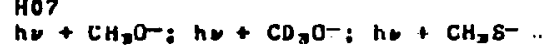

1993

H10

ho + so, $\begin{array}{ll}\text { EXP } & \text { ENERGY } \\ \text { CR } & \text { RANGE } \\ \text { THEOR } & \end{array}$

$\boldsymbol{\varepsilon}$

$600-360 \mathrm{~nm}$

E $90-180 k$

E $\quad 0-0.8 \mathrm{eV}$

E

A05

2 HeV

300

E $\quad 0-6 \bullet V$

\section{E05}

$0-20$ eV

EO9

$0-14$ eV

1 Under
E $300 K$

E $\quad$ A07

$300 \mathrm{~K}$

E. $300 \mathrm{~K}$

E

$488 \mathrm{~nm}$ 


\begin{tabular}{|c|c|c|c|c|c|c|}
\hline $\begin{array}{l}\text { REF. } \\
\text { NO. }\end{array}$ & REACTANTS & & $\begin{array}{l}\text { EXP } \\
\text { GR } \\
\text { THEOR }\end{array}$ & $\begin{array}{l}\text { ENERGY } \\
\text { RANGE }\end{array}$ & & REFEFENCE \\
\hline 1994 & $\begin{array}{l}A 11 \\
\operatorname{co*}+0\end{array}$ & & $\mathbf{E}$ & $265-389 K$ & k & 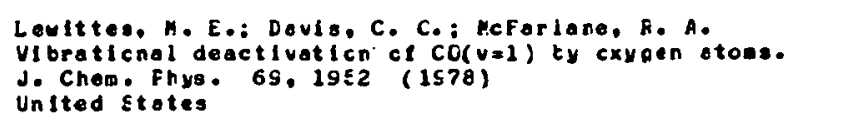 \\
\hline 1995 & 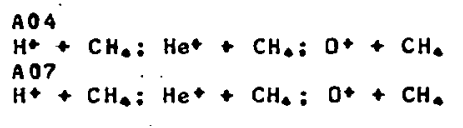 & & $\mathbf{E}$ & $1 \mathrm{MeV}$ & & 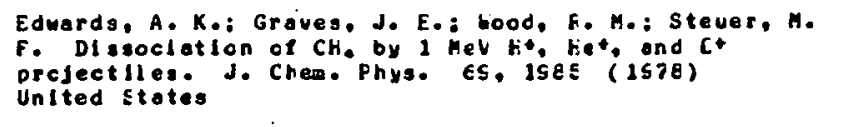 \\
\hline 1596 & $\begin{array}{l}A 11 \\
X \in F *+H e: X e F *+N e: X e F *+X \\
X \in F *+F_{>}: X e F *+N F,\end{array}$ & & $\mathbf{E}$ & $300 \mathrm{~K}$ & & 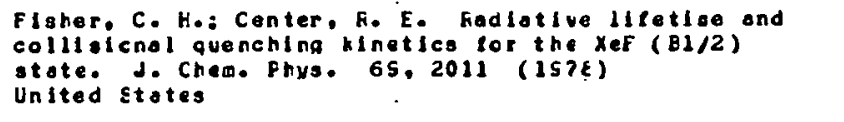 \\
\hline 1997 & $\begin{array}{l}\mathrm{H} 14 \\
2 \mathrm{hN}\end{array}+\mathrm{H}>*$ & . & $\mathbf{E}$ & $0-3 \cdot e V$ & & 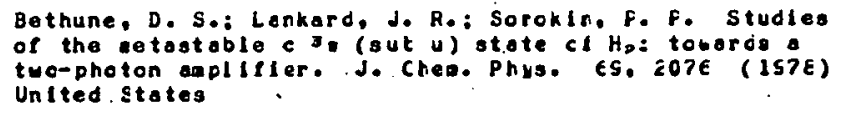 \\
\hline 1998 & $\begin{array}{l}\text { Ho6 } \\
h=+N,\end{array}$ & & $\varepsilon$ & $5-42$ ov & & 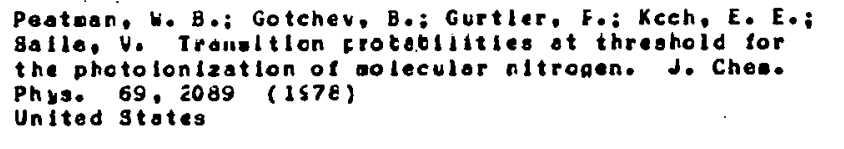 \\
\hline 1993 & hoo $+\mathrm{H}$, & & 1 & $15-70$ ev & & 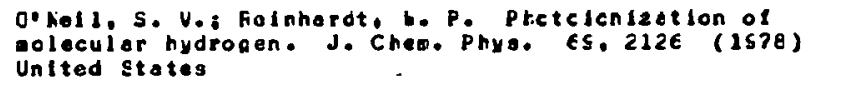 \\
\hline 2000 & 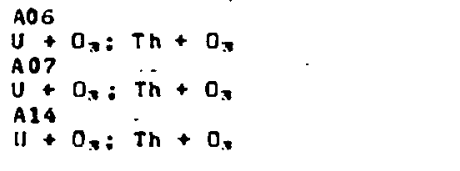 & & $\mathbf{E}$ & $\begin{array}{l}7.2-10 * \\
\mathrm{cas} / \mathrm{s}\end{array}$ & & 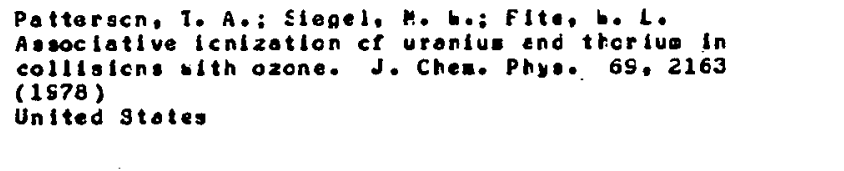 \\
\hline 2001 & $\begin{array}{l}\text { Hog } \\
h_{2}+U F_{n} \\
H 10 \\
h+U F_{a}\end{array}$ & . & E & $480-3,40 \mathrm{n}$ & ne & 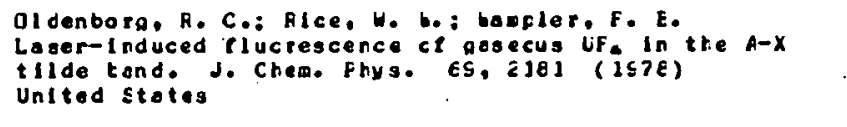 \\
\hline 2002 & 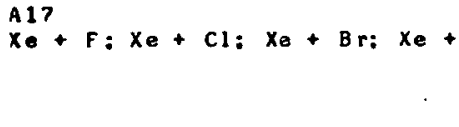 & +1 & $T$ & Unde $P$ & & 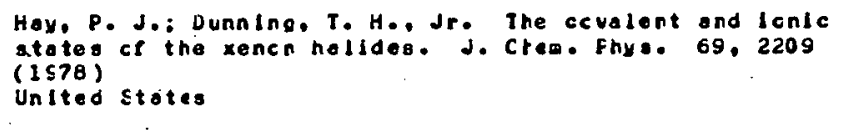 \\
\hline $\begin{array}{r}2003 \\
.\end{array}$ & $\begin{array}{l}\text { HOS } \\
\text { ho }+T 1 B r\end{array}$ & . & E & $2660 A^{0}$ & & 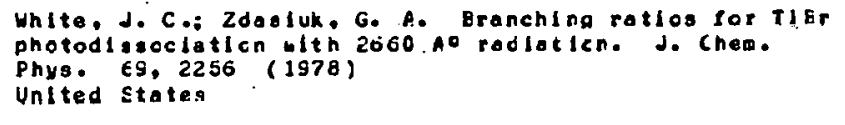 \\
\hline 3004 & 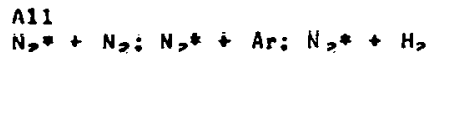 & & E & $300 \mathrm{~K}$ & & 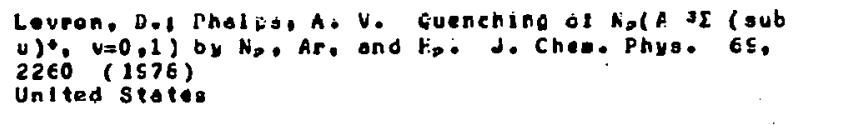 \\
\hline 2005 & $\begin{array}{l}\mathrm{Al} 7 \\
\mathrm{Ne}\end{array}$ & .. & $\mathbf{T}$ & Undef & & 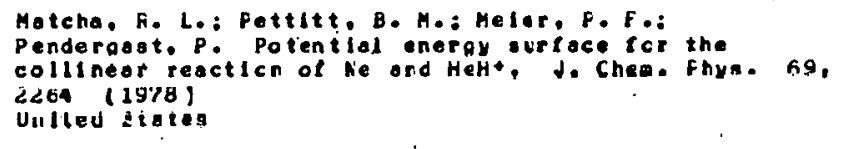 \\
\hline 2000 & 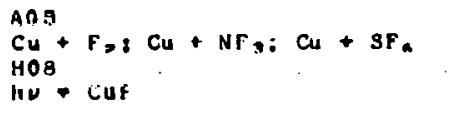 & & $\mathbf{E}$ & $1000-400$ & $\mathrm{~nm}$ & 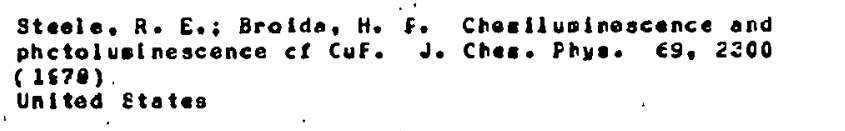 \\
\hline 2007 & $\begin{array}{l}A 10 \\
I * H>O ; I *+H D O=I *+D, O= \\
+H>0: D>0 *+D>0\end{array}$ & $\mathrm{H}, \mathrm{O}$ & $\mathbf{E}$ & $300 \mathrm{~K}$ & & 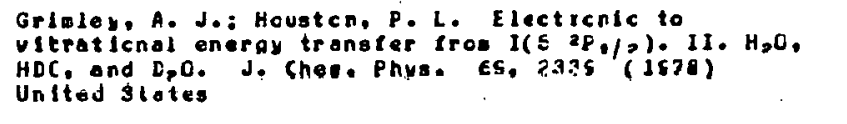 \\
\hline 2008 & $\begin{array}{l}\mathrm{AO}_{\mathrm{He}}+\mathrm{NH}_{3} \\
\text {. }\end{array}$ & & 1 & $\begin{array}{l}50-1000 \\
1 / \mathrm{cm}\end{array}$ & & 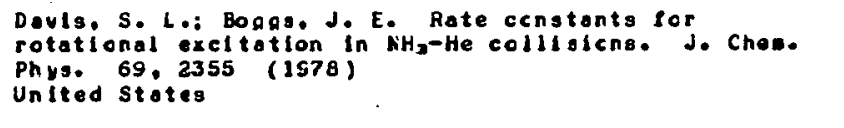 \\
\hline 2005 & $\begin{array}{l}A 02 \\
F+x e \\
F 1 ? \\
F+x e\end{array}$ & & E & $\begin{array}{l}2.1-13 . s \\
k e a t / \infty o l\end{array}$ & & 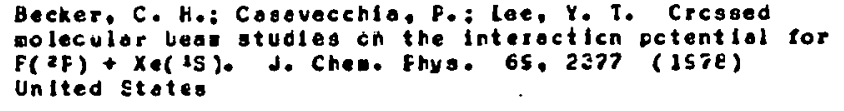 \\
\hline
\end{tabular}




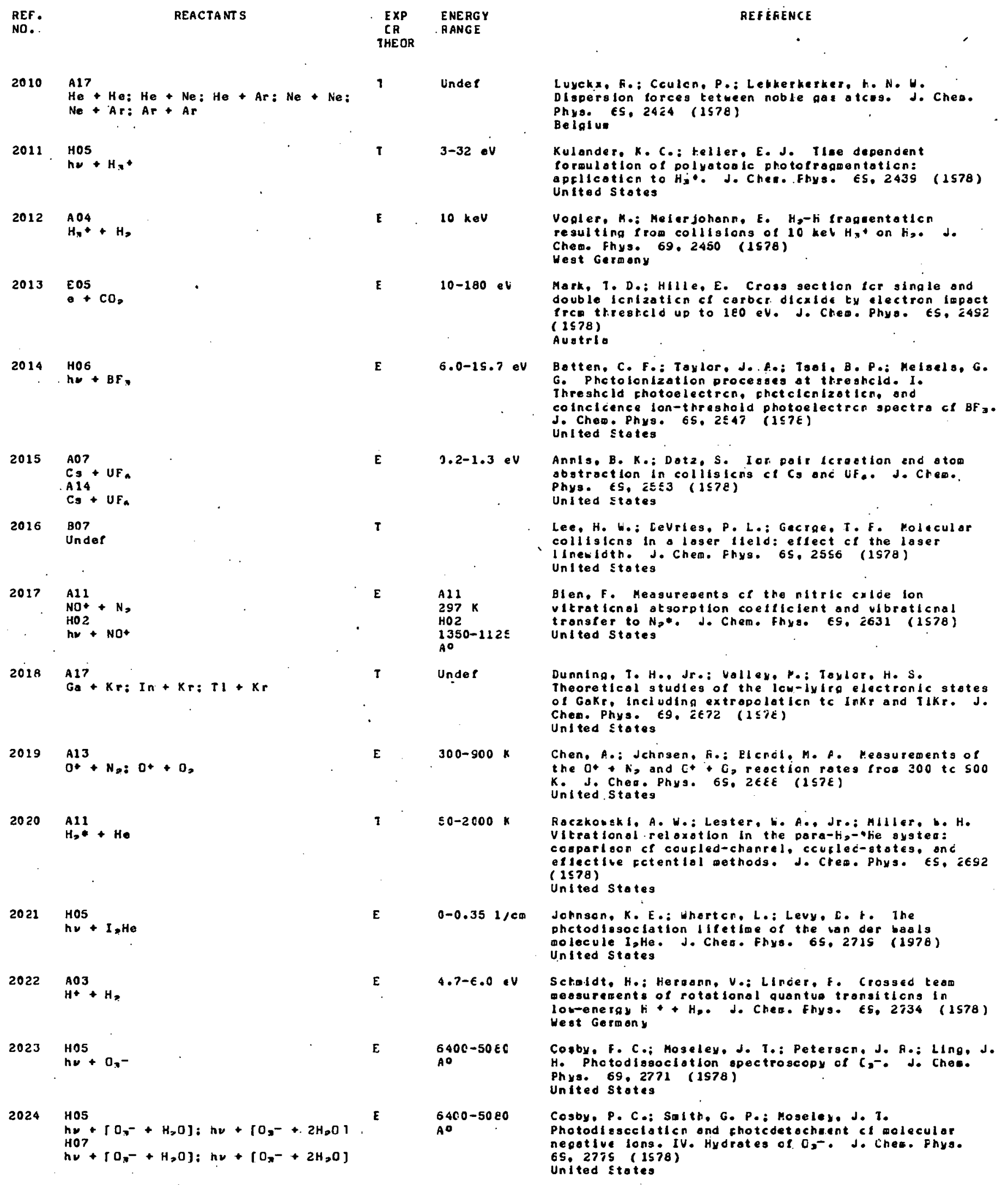




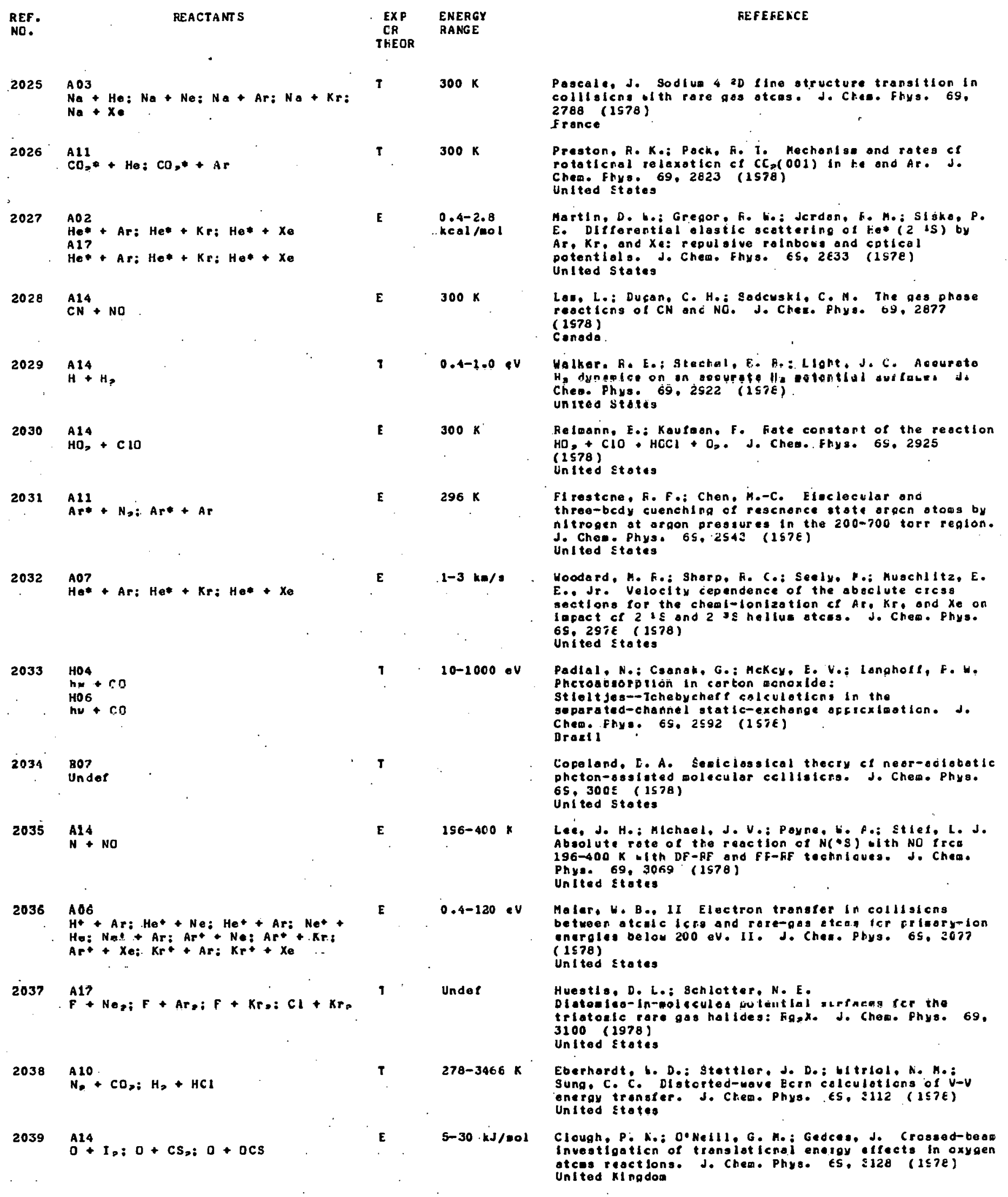




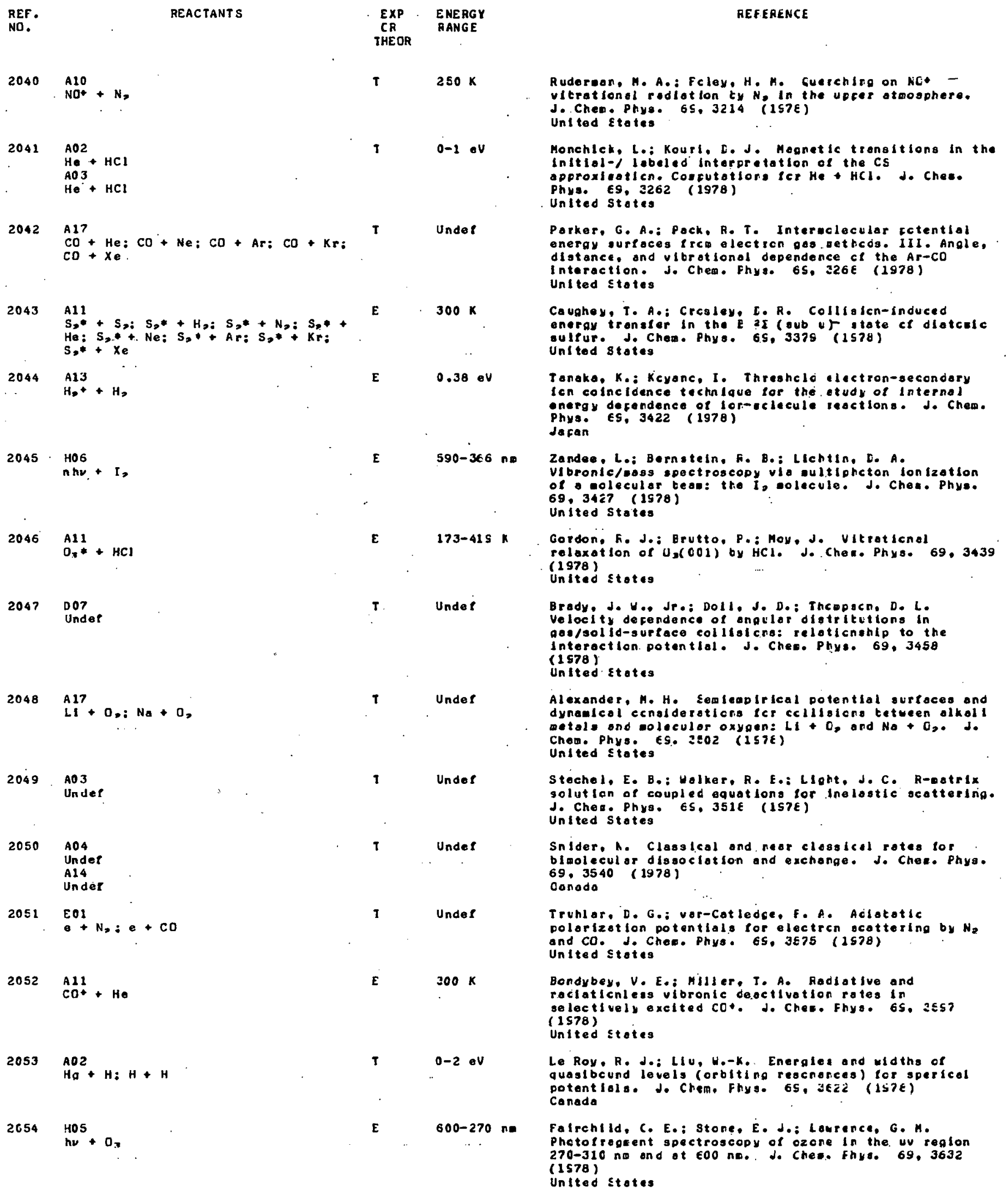




\begin{tabular}{|c|c|c|c|c|}
\hline $\begin{array}{l}\text { REF. } \\
\text { NO. }\end{array}$ & REACTANTS & $\begin{array}{l}\text { EXP } \\
\text { CF } \\
\text { THEOR }\end{array}$ & $\begin{array}{l}\text { ENERGY } \\
\text { GANGE }\end{array}$ & FEFEFENCE \\
\hline 2055 & $\begin{array}{l}\mathrm{AlO} \\
\mathrm{Br} * \mathrm{CO}\end{array}$ & E & $296-600 \mathrm{~K}$ & 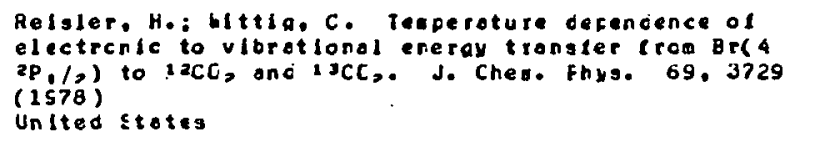 \\
\hline 2056 & $\begin{array}{l}H 04 \\
\mathrm{hW}_{\mathrm{N}}+\mathrm{H}_{2} \mathrm{O} \\
\mathrm{HOS} \\
\mathrm{h \nu}+\mathrm{H}>\mathrm{O}\end{array}$ & $\mathbf{E}$ & $813-500$ P0 & 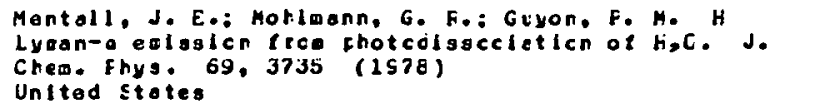 \\
\hline 2057 & $\stackrel{A}{F}+\mathrm{H}=$ & $\tau$ & $0-0.5 \mathrm{eV}$ & 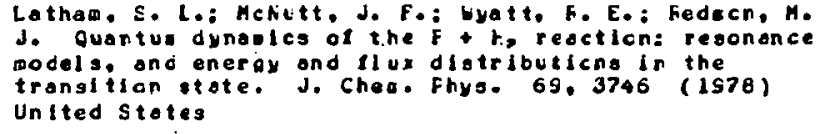 \\
\hline 2058 & 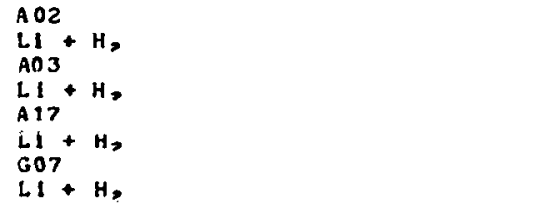 & T & $\begin{array}{l}0-40 \\
k<a 1 / m o l\end{array}$ & 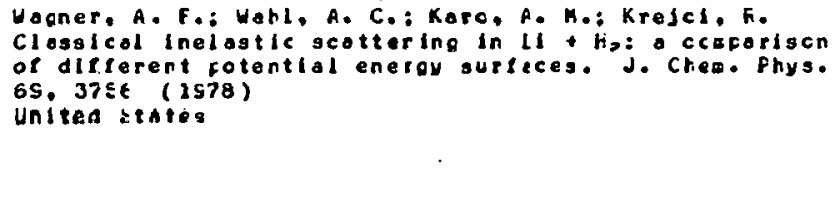 \\
\hline 2059 & $\begin{array}{l}\mathrm{Ald} \\
\mathrm{Be}+\mathrm{HF} \\
\mathrm{Al7} \\
\mathrm{Be}+\mathrm{HF}\end{array}$ & 4 & $\begin{array}{l}15-40 \\
x \in 01 / \infty<1\end{array}$ & 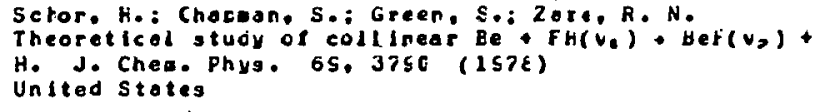 \\
\hline $20 \in 0$ & $\begin{array}{l}A 2 I \\
I *+H g I \\
H 05 \\
h \nu+H a I_{2}\end{array}$ & E & $320-265 \mathrm{rm}$ & 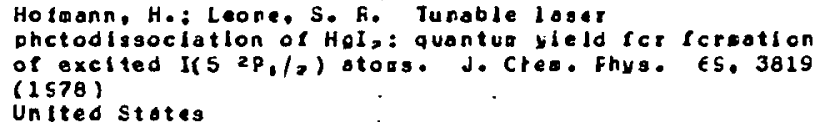 \\
\hline $20 \in 1$ & 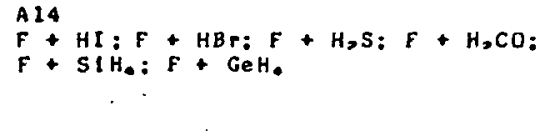 & $\mathbf{E}$ & $300 \mathrm{~K}$ & 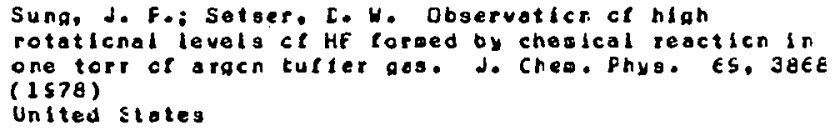 \\
\hline 2062 & $\begin{array}{l}\text { Go4 } \\
R_{6 e}+K_{r}: R b++X_{e}: K++K r: K++\end{array}$ & $\varepsilon$ & $0-6 \mathrm{eV}$ & 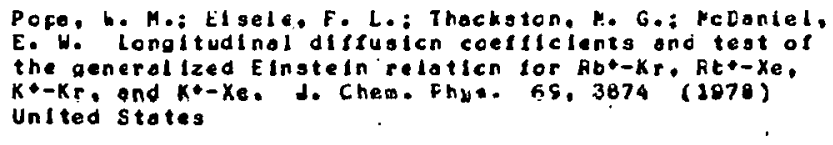 \\
\hline $20 \epsilon \overline{3}$ & All + Ar & E & $300 k$ & 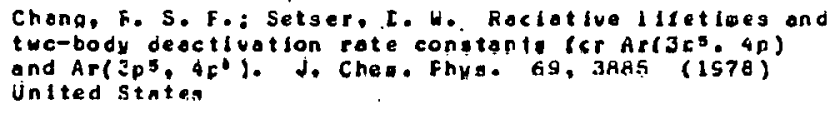 \\
\hline 2064 & ${ }_{e}^{\mathrm{E} 03}+\mathrm{Cs}_{3}:-+\mathrm{ocs}:-+\mathrm{sO}_{2}$ & E & $25-70$ eV & 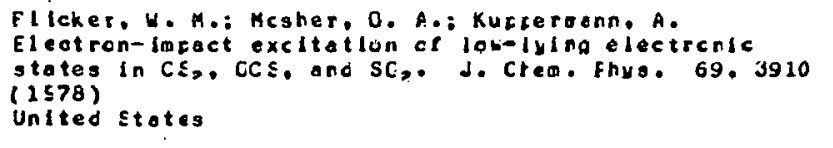 \\
\hline 2065 & $\mathrm{AlP}_{\mathrm{He}}^{\mathrm{APr}}$ & $\mathbf{T}$ & Unde? & 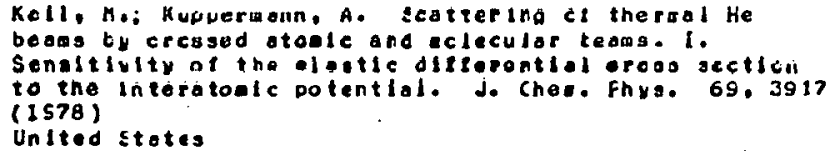 \\
\hline 2066 & $\mathrm{Hog}+\mathrm{N}=$ & E & $27-31$ ov & 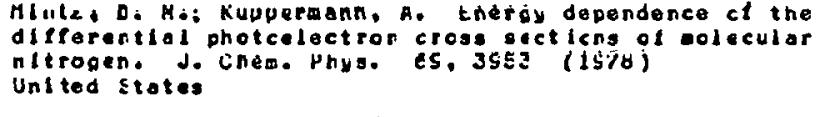 \\
\hline $206 \%$ & $\stackrel{A 11}{0 *}+0,: 0,0 *+0$ & E & $300 \mathrm{~K}$ & 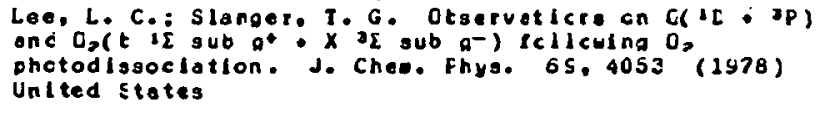 \\
\hline $2068^{\circ}$ & 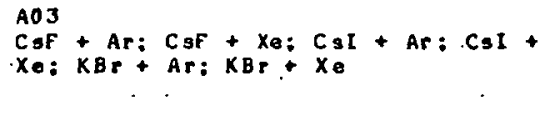 & $\mathbf{E}$ & $0-4 \mathrm{eV}$ & 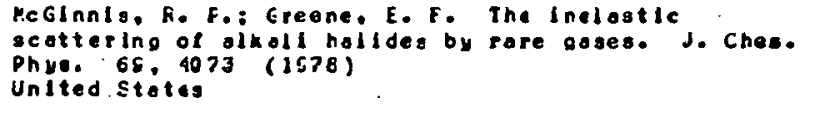 \\
\hline
\end{tabular}


REF.

REACTANT S

NC.

2069

Al1

aCs + He: OCS + Ap

2070

A14 $+H$

T

$300 \mathrm{~K}$

$2071 \quad$ A14

Under.

2072

A17

$\mathrm{H}+\mathrm{H}: \mathrm{D}+\mathrm{D}$

2073

A17

$\mathrm{TI}+\mathrm{H}: \mathrm{Tl}+\mathrm{He}: \mathrm{Tl}+\mathrm{Ne}$ : Tl + Ar:

$\mathrm{TI}+\mathrm{K}: \mathrm{r} ; \mathrm{T} .1+\mathrm{Xe}$

2074

D11

$\mathrm{CO}+\mathrm{Ir}$

$\mathrm{CO}^{2}+\mathrm{Ir}$

2075

A14

$H+B r_{p}: D+B r$,

2076

$\mathrm{AO4}$
$\mathrm{Kr} * \mathrm{H}, \mathrm{O}$
$\mathrm{HOS}$
$\mathrm{hy}+\mathrm{H}, \mathrm{O}$

2077

$\mathrm{HO}$
$\mathrm{hm}$
$\mathrm{HOG}$

no + NO

2078 AL1

$\mathrm{Ar}^{*}+\mathrm{Xe}_{\mathrm{O}} \mathrm{Ar}^{*}+\mathrm{Kr}: \mathrm{Ar} *+\mathrm{Hg}$ : $\mathrm{Ar} *$

$\mathrm{H}_{3}$ : Art $+\mathrm{D}_{9}$ : Ar* $+\mathrm{CO}$ : Ar* + $\mathrm{N}_{2}$ :

Ar* + NO: Ar* + N,0: Ar* + O, : Ar*

+ SO $_{3}:$ Art + CO, : Ar* + COS: Ars . +

HCl : Art $+H B_{r:}$ : Ar* + HI: Art...Fo:

Art + Cl pi Ar* + Brp; Ar* + ICl;

$A r *+I B r: A r *+C l F: A r *+O F$ :

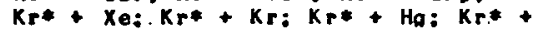

$\mathrm{H}_{2}: \mathrm{Kr} *+\mathrm{D}_{8}: \mathrm{Kr} *+\mathrm{CO}: \mathrm{Kr} *+\mathrm{N}_{2}:$

$\mathrm{Kr} *+\mathrm{NO} ; \mathrm{K}^{*}+\mathrm{N}_{>} \mathrm{O}: \mathrm{Kr}_{*}+\mathrm{O}_{2}: . \mathrm{Kr}^{*}$

$+\mathrm{SO}_{3}: \mathrm{Kr}^{*}+\mathrm{CO}_{2}: \mathrm{Kr}^{*}+\operatorname{Cos} \mathrm{Kr}^{*}+$

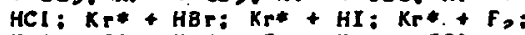

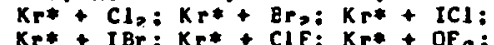

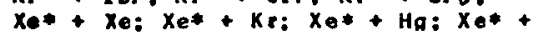

$H_{0:} x_{e}+D_{9}: x_{0}+\mathrm{CO}_{0} x_{0 *}+N_{2}:$

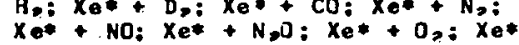

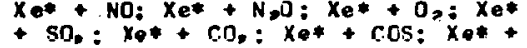

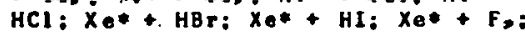

$X_{0 *}+C_{13}: X_{e *}+B_{2}: X_{e *}+I C 1:$

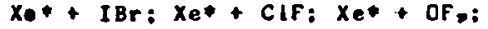

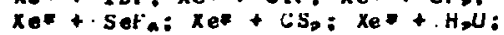

$\mathrm{Xe}^{*}+\mathrm{H}_{3} \mathrm{~S}^{*} \mathrm{Xe}^{*}+\mathrm{NH}_{3}: \mathrm{Xe}^{*}+\mathrm{HCH}^{\circ}$

$\mathrm{X}_{0} *$ - BrCN: $\mathrm{Kr}_{*}+\mathrm{SeF}_{0}: \mathrm{Kr}_{*}+\mathrm{CS}_{2}:$

$\mathrm{Kr}+\mathrm{H}_{2} \mathrm{O} ; \mathrm{Kr} *+\mathrm{H}_{2} \mathrm{~S}: \mathrm{Kr}_{r} *+\mathrm{NH}_{\mathrm{N}}:$

$\mathrm{Kr}_{r}+\mathrm{HCN}: \mathrm{Kr}^{*}+\mathrm{BrCN}_{\mathrm{C}} \mathrm{Ar}^{*}+\mathrm{SeF}_{a}$

$A r *+C S_{0}: A r *+H_{0} O=A r *+H_{D} S:$

Ar* + $\mathrm{NH}_{3}$ : Ar* + HCN: Ar* + BrCN
Unde $f$

$\cdot$

Under

Under

.

E

$90-1300 \mathrm{~K}$

E

$0-10$

keal/mol

E $\quad \mathrm{AO}^{4}$

$300 \mathrm{~K}$

300
$H 05$

$.124 \mathrm{no}$

T

$0-10 \mathrm{eV}$

E

joo $K$
REFE RE ACE

Green, s. On the onount of Infcraticn in rototicnal relaxation experisents with offliceticn to elcrowave transient $T$, ond $T$, rates. J. Chea. Ptys. 6S. 407E (1578)

United States

Folst, H. E.: Muckermon, J. T.: Echukert, F. E. Incortonce sampling áne hietograculc representaticns ce resctlvity functicns ord creduct olstitutions in Monte Carlo quastclosafcol trajectory calculoticna. J. Chea. Phys. 69, 4087 (1978)

United stotes

Augustin. S. D.: Fabltz. H. The classical fath approxicaticn in time-dependent quantur collisicn theory. J. Ches. Fhys. 6S, 41S5 (1STE)

United stotes

Sllvera, I. F.: Goldman, V. V. The Iactroplc interoolecular rotent lois $i c r H_{0}$ ord $E$, in the solio and gas phases. J. Chem. Phys. ES, 4 LOS (157E) The Netherlonds

Hu, C. Y. R.: Stwalley, H. C.: Frcctor, T. F.

Long-rarge interactions of the thelliue $72 S_{0} /$, state and broadening and ahlt ct the thelliub viclet and green lines by rare aoses. J. Chen. Fhys. ES, 4 aze (1578)

United States

Toylor, J. L.: Ibtctscr. C. E.: Weinterg, W. H. The chealsorpticn of $C C$ on clean and oxldized Ir(120). J. Ches. Physe 69, 4258 (1STE)

United Stotes

Hepburn, J. W.: Klleek, D.: Lilu, K.: FClanyl, J. C.: Halloce, $S$. C. Reoctive crogo section os a function ef collisticn eneray. I. H(D) * Br, HEr(IEr) + Er. J. Cheo. Fhys. 69.43il (ISTE)

Canodo

Vikis, A. C. The CH (A $2 \Sigma * 1$ interral eneray

distributicr froduced ty the $\operatorname{Kr}\left(3 F_{1}\right)$ garsitizeo

decompositicn of HoO. J. Cheos. Phis. ES. 4314 (ISTE) Canada

Creasshl, P.: Jchnson, P. M.: hhllten. J. L. Multiphcten icnlzation spectrcscocy: theoretical onolysis of the NC spectrua. J. Chea. Phys. 69,4341 (1578)

United states

Velazcc, J. É: Kclts, J.H.: Setser..L. b. Hote constants. and quenching mechanleog for the eletestoble stotes ct. orgcn, kryoten, ard xencr. $\therefore$ Chem. Fhys. 69. 4357 (1978)

United Stotes 


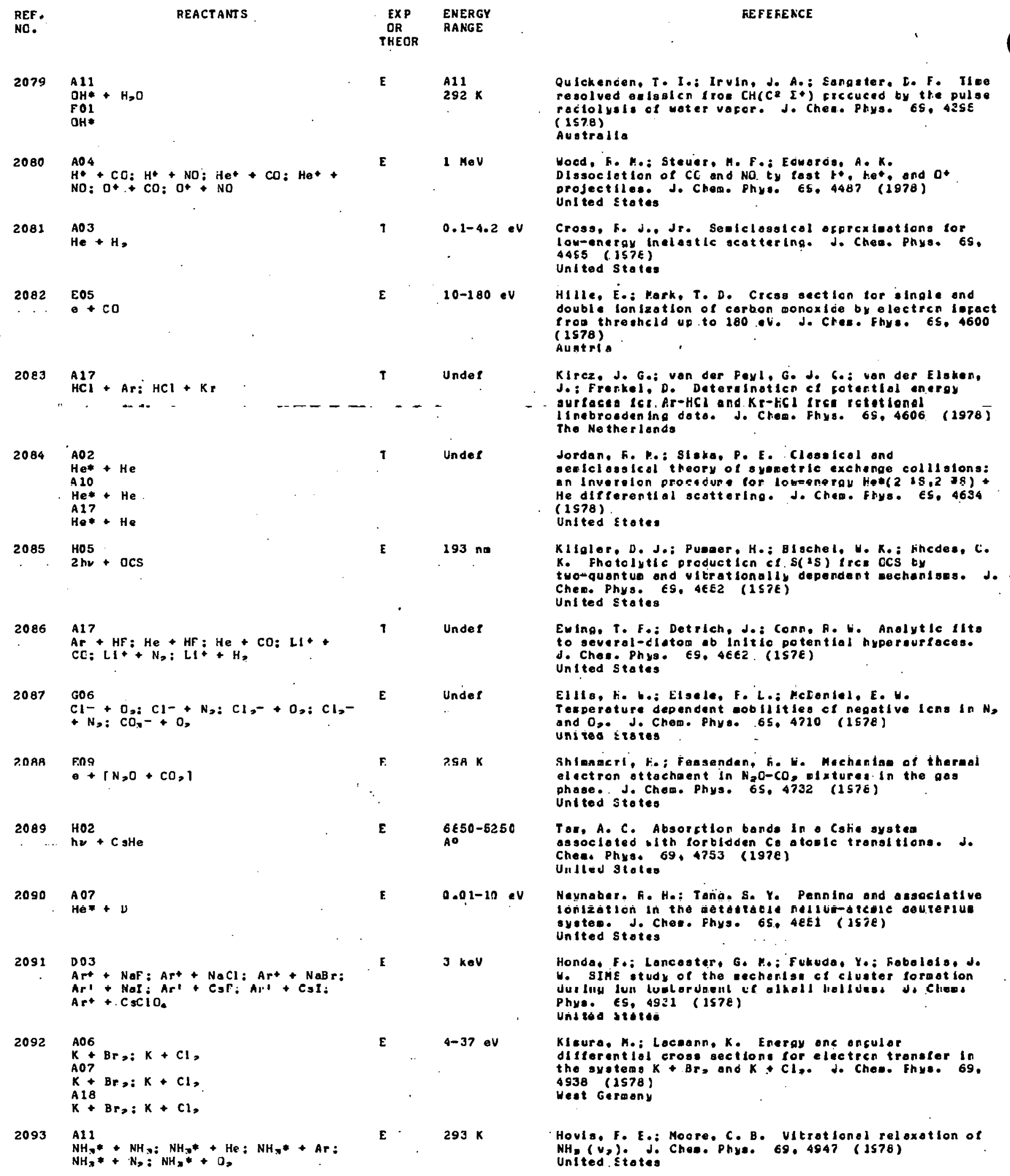


REF.
NO.

REACTANTS

2094

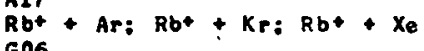

$\mathrm{Rb}^{\mathrm{G}+}+\mathrm{Ar}_{\mathrm{r}} \mathrm{Rb}^{+}+\mathrm{Kr}: \mathrm{Ab}++\mathrm{Xe}$

2095

$\mathrm{H}+\mathrm{H}$

AO4

$\mathrm{H}+\mathrm{H}$,

A14

2096

A1 7

Kr* + Ar: Xe* + Ar: Ar* + He: Ar*+

Ar: Ar* $+K r: A r *+X_{e}: H e *+A r$

2097

A10

$\mathrm{Ne} *+\left[\mathrm{Ne}+\mathrm{X}_{0}+\mathrm{NF}_{2}\right] \mathrm{F} *+[\mathrm{Ne}+$ $\left.X_{e}+N F_{0}\right] ; F_{p}+\left[N_{0}+X_{0}+N F_{n}\right]:$

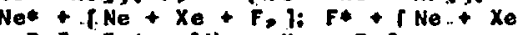

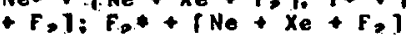

2098

A 10

$\mathrm{NF} *$ + Bt

2099

$\mathrm{HO2}$

$h y+\mathrm{SeO}_{\mathrm{i}} \mathrm{hy}+\mathrm{YO}$; hy $+\mathrm{LaO}$

2100

$\mathrm{Ne}^{+}+\mathrm{Ne}: \mathrm{Ar}++\mathrm{Ar}: \mathrm{Kr}++\mathrm{Kr}: \mathrm{Xe}++$

2101 A11

$\mathrm{H}_{2} \mathrm{O}^{+}+\mathrm{H}_{2} \mathrm{O}: \mathrm{H}_{2} \mathrm{O}^{+}+\mathrm{He}^{+} \mathrm{H}_{2} \mathrm{O}^{+}+\mathrm{H}_{7} \mathrm{O}$

$\therefore \ldots \mathrm{H}_{2} \mathrm{O}^{+}+\mathrm{H}_{2} \mathrm{OH} \mathrm{H} \mathrm{O}^{+}+\mathrm{CO}$

$2102 \quad A 14$

$\ldots \ldots$ SF + HF

2103 E03

$2104 \quad \mathrm{AO2}$

$\mathrm{CH}+\mathrm{H}_{2}: \mathrm{H}+\mathrm{H}_{2}$

$\mathrm{AOS}_{+}+\mathrm{H}_{2} \mathrm{O} \mathrm{H}^{+}+\mathrm{H}_{3}$

$\mathrm{LI}+\mathrm{H}^{-} \mathrm{H}+\mathrm{H}^{\circ}$

A18

$\mathrm{Li}+\mathrm{H}_{*}: \mathrm{H}^{+}+\mathrm{H}$,

$2105 \quad A 17$

$\mathrm{Ma}+\mathrm{H}$

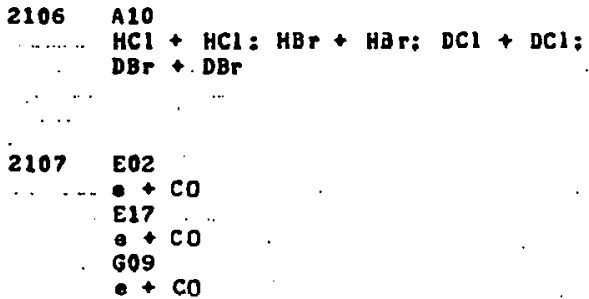

\section{EXP ENERGY \\ C.R TANGE}

THEDR

E

$300 \mathrm{~K}$

$300 \mathrm{~K}$

$300 K$

$630-360$ ad

I Under

E

$300 \mathrm{~K}$

$800 K$

e

$0-40$ oV

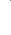

$0.6-3.7 \mathrm{eV}$

Under

$300 \mathrm{~K}$

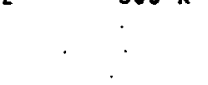

E. 3-100 el
Gatland, I. R.: Lane, De Fio: Thocketon, M. G.: Fope, W. M.: Elocle, F. L.: Eilis, F. Ma: Meloniel. E. h. Mobilities ond Interaction.cotentiols lcr Fb+-Ar,

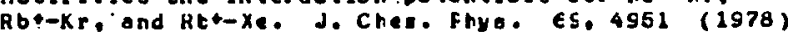
United stotes

Kulander, K. C. Collialon induced diacclation in collinear $H+H_{2}$ : quantum mechanlcal crcbablilites ualng the time-dependent wavefocket effrcact. $\mathrm{J}$. Chem. Phye. Es, EOE4 (1578)

United Kingdom

Vollee, C.: Glasser, J.: Forsen, P.: Chepelle, J. The interactions tetween exclted and grcund atate rare gas atoms. J. Chew. Phys. 6S, Sosi (is7e)

France

Huest IA, D, L.: HIll, K. Ho: Nokanc, H. Ho: Lorents, D. C. Quenching of Ne*, F*, and $F *$ in $\mathrm{Ne} / \mathrm{Xe} / \mathrm{NF}$, and No/xe/fo intures. J. Chee. Fhys. 6S. 5133 (1978) United Stotes.

Cacelle; G. A.: Sttton. D. G.: steinfeld, d. i. Near-resonant electronic energy trangler trco Nf(o is) to B1. J. Cheis. Fhys. 69, 5140 (1STE) United stotes

Sehenck, P. Ko: Hallard, W. G.: Iravie, d. C.: Suyth, K. C. Atacipticn spectra cf letol culces uaing oftogalvanic apectrosccoy. J. Cher. Plys. 65, E147 (1578)

Unlted stotes

Michelo. H. H.: Hobbs, R. H.: Wright, L. A. Electronic structure of the noble pa divar leno. I. Pctential onergy curveg and scectrosccile cersterts. J. Chem. Phys..69.5151 (1978)

United stotes

Kagugo. T.: KuzQ. Ha: Shlolzu, 1. Deterainaticns cf rolaxation rate ccratorts cr the za Gtiz rototional transition of $\mathrm{H}, \mathrm{O}$ ty ccherent transient spectrcsccoy. J. Choo. Phys. 6S, 51SE ( $1 \subseteq 7 E)$

Japan

Karny, 2.; Estler, F. C.: Zore, R. N. Elfect ol reapent orlentation and rotation urch croduct otate distribution in the reocticr $E r+h F(v=1 . J)$. Srf(v'.d) + H. J. Chea. Fhys. 6S. SlsS (1S7E) United States

Dehmer. J. L.: Slegel, Jo: DIII, D. Shope rescrances in e-SF scottering. d. Chee. Fhys. ES, $520 E$ (1S28) United stotes

MeCann, K. J.: Flonnery. M. F. Elagtlc scattering and rotatlcral excltation in lcn-aclecule ccllialong. II. $L^{*-H}$, and $H^{+-H}$, ccllisions. J. Chew. Physe ES, 5azs (1578)

United stotes

Sazon, F. F.: Kirby, K.: Liu, E. At InItio conflguraticn interaction study of the low-lying electrente totes ef Hati. J. Cheo. Fhyse ES, 5301 (1578)

- United stotes

Horultz, A. B.: Leone, E. F. Laser-axcited rescnant lactople $Y$. Venaray trangter: $h$ oCl-talCl.

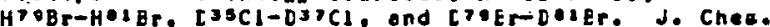
Phys. ES, E319 (ISTE)

United Stater

Tanaka, H.: Srluastava, S. K.: Chutjion, A. Absolute ilestlc dilierantiol alectron ecatterira crcsa ections In the Interwedlate anergy. reglen. IV. CC. J. Chem. Phys. 6S, 5329 (1978)

United stites 


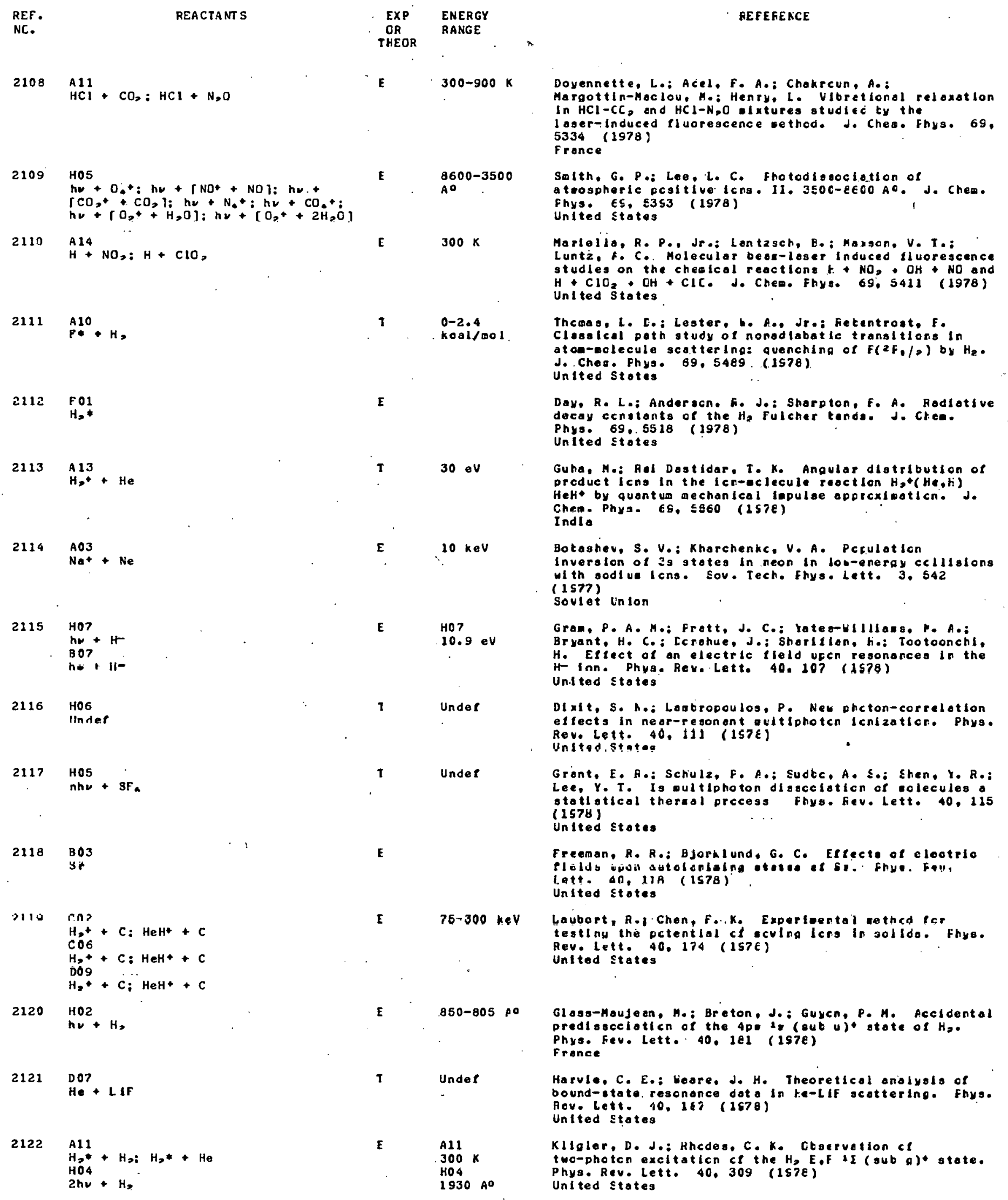




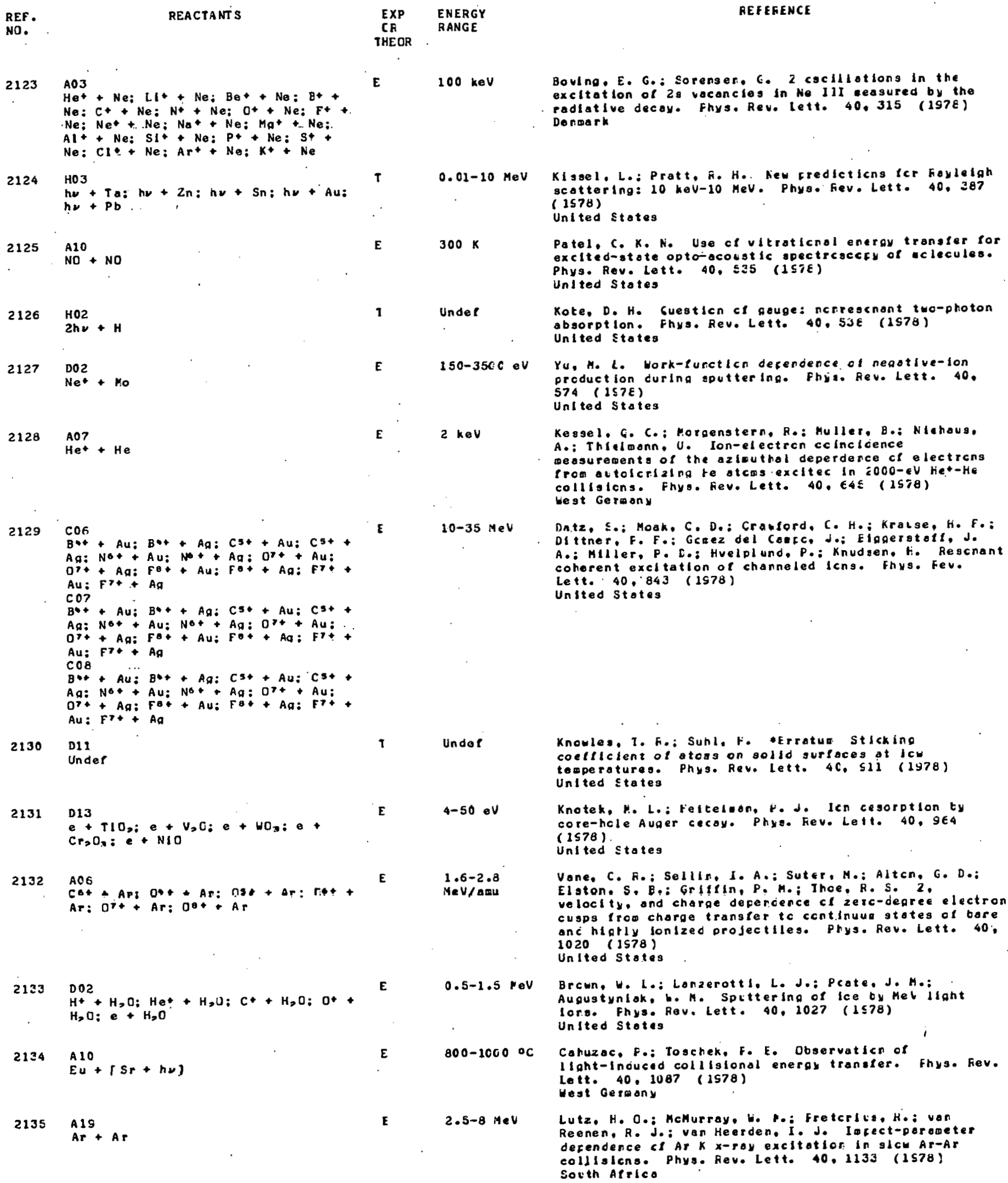

$100 \mathrm{keV}$

$0.01-10 \mathrm{MeV}$

$300 \mathrm{~K}$

Undef

$150-350 \mathrm{C}$ eV

$2 k \in V$

E

10-35 MeV

Undo?

$4-50$ eV

$1.6-2.8$

MeV/amu

E

$0.5-1.5 \mathrm{MeV}$

REFEFENCE

Boulng. E. G.: Sorenser. C. 2 cacillotions in the excltation of 2 s vaconcios in Ne Ill ceasured by the radiative decay. Fhys. Reve lett. 40.315 (197E) Denmerk

Kisgel. L.: Pratt. F. H.. New credicticns tcr Hayleigh gcotering: 10 kev-10 Mev. Phys. Fev. Lett. 40. 387 (1578)

United States

Patel. C. $K$. N. Uge ch vitraticnol energy trangler for exclted-stote opto-acoustlc spectrcsecfy of aclecules. Phys. Rov. Lett. 40, 5as (1STE) United States

Kote, D. H. Guesticn cl gauge: ncriescrant two-photon absorption. Fhys. Rev. Lett. 40, S3E (1578) United states

Yu, h. L. Work-lunctlcn defendence. of negotive-ion production curing sputtering. Fhis. Rev. Lett. 40. 574 (157E)

Unl ted States

Kegsel. G. C.: Morgenstern, R.: Muller, B.: Nlehaug. A.: Thieloamn. U. Ion-electrcn celnclience weasurementa of the azieuthal deperderce cf electrons fron actolcriaing te atcusexcitec in $0000-6 \mathrm{~V} H \mathrm{H}^{+-H E}$ colligiens. Fhys. Fev. Lett. 40. C45 (1578) We st Gerouny

Datz, S.: Moak, C. D.: Cranford, C. H. K Kralse, H. F.

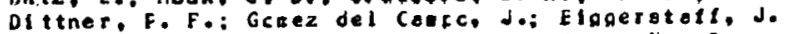
A.: Milier, P. E.: Huelolund, P.: Knudsen. H. Rescnant coherent excitation of channeled icns. Fhys. Fev. Lett. 40,843 (1978)

United Stotes

Knowles. I. F.: Sunl. F. efrratur Stickino coeflicient of atoas on salid surfoces ot $\mathrm{ICw}$ tomperatures. Phys. Reve Lett. 4C, S1i (1978) United states

Knotek. H. L.: Fetteison, H. J. Iten cegorption ty core-hcle Auger cecoy. Phys. Fev. Lett. 40,964 (1578).

United States

Vane, C. Fo: Sellin, I. A.: Suter, M.: Altcn, G. D.: Elston. S, B.: Gritiin, P, M.: Ihoe, R. S. 2 . velocity. and charae decercence cl zeac-degree electron cusps troo charge transfer tc centinuug atotes of bare anchigtiy lonlzed projectiles. Phys. Reve Lett. 40 , 1020 (1578)

Unlted stotes

Brcun, W. L.: Lonzerott1. L. J.: Pcate. J. H.: Aupustyntok. W. M. Spcttering of les oy Hel isght lors. Fhys. Aev. Lett. 40, 1027 (1578) Unted Stotis

$800-1000$ oc

Cahuzac. F.: Toschek, F. E. Observaticn of liont-inouced colligional energy transer. Fhys. Fev. lett. 40.1087 (1578)

Hest Germony

$2 \cdot 5-8 \mathrm{HeV}$

Lutz, H. O.: McHurray, b. Me: Fretcrlte, Ho: van Reenen. R. J.: van Heerden. I. J. Iofect-parameter decendence cl AT $K x-$ ray excltatior In slcu Ar-Ar collislons. Phys. Fev. Lett. 40.1133 (1s78) South Africo 


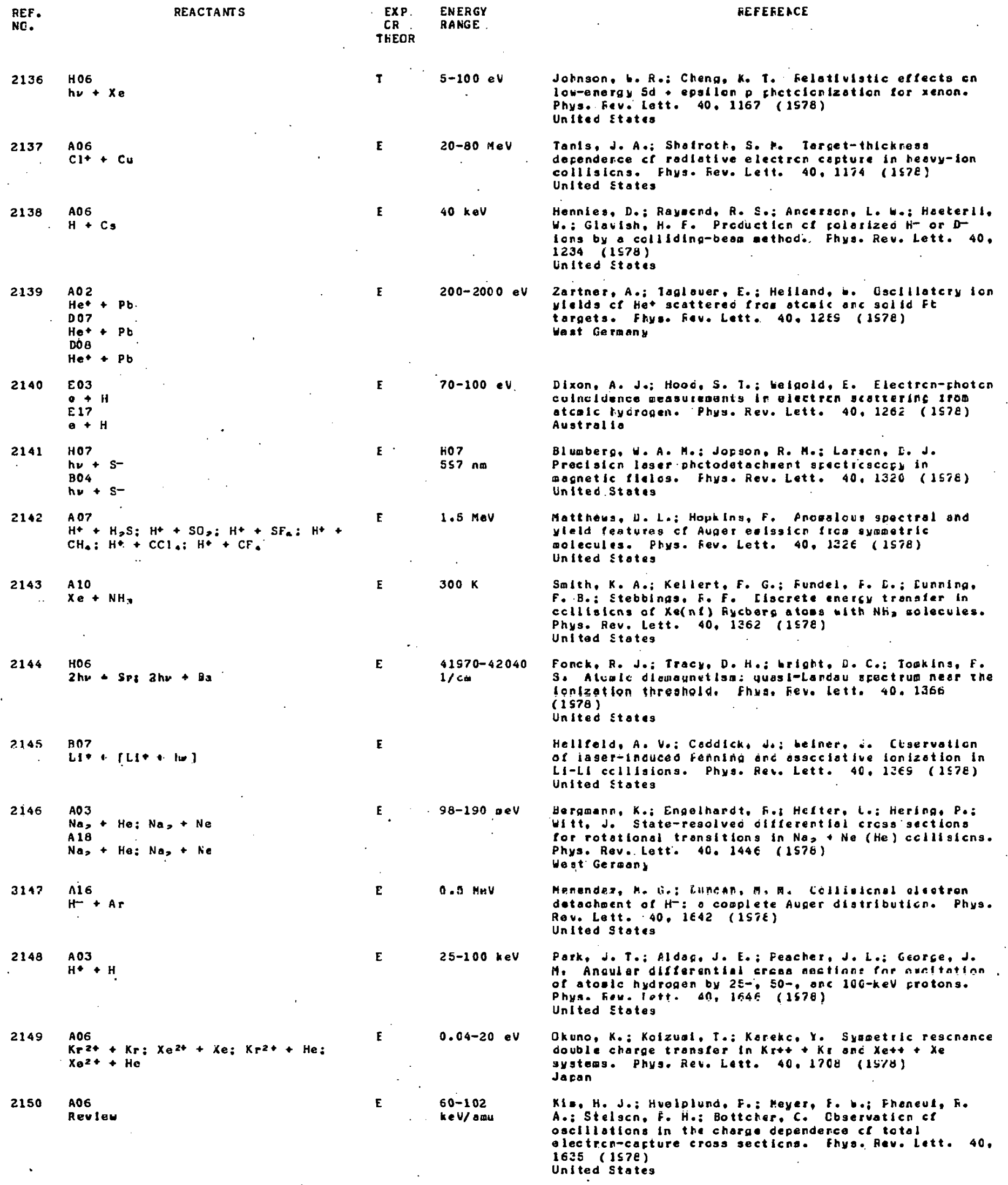


REF.
NO.

REACTANT S

2151

D 07

He + Lif

$2152 \quad B 01$

H.

$2153 \quad 803$

2154

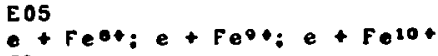

E06

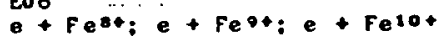

2155

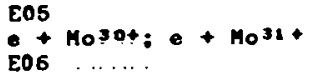

e+Mojot: $e+M_{03}{ }^{+}$

$2156 \quad 106$

${ }_{\text {hy }}^{406}+\mathrm{Kr}: \mathrm{h \nu}+\mathrm{Xe}$

2167

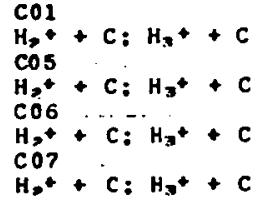

2158

AO8
H + PERT

$2159 \quad 406$

3he + Ne

$2160 \quad 103$

$\mathrm{He}+\mathrm{Ne}$

A17

$\mathrm{He}+\mathrm{Ne}$

A18

$\mathrm{He}+\mathrm{Ne}$

$2161 \quad 605$

$0+\mathrm{He}$

2162

$\mathrm{HO4}$

hy $+[\mathrm{Co}+\mathrm{Ar}]$

hv $+\mathrm{ICs}+\mathrm{Ar}$ I

$2163 \quad \mathrm{Al}_{-}+\mathrm{Ne}$

$\mathrm{EO} 3$

- $+\mathrm{NaNe}$

2164

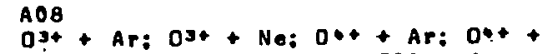

Ne: Ost Ar: $\mathrm{O}^{+}+\mathrm{Ne}$ : $\mathrm{O}^{+}+\mathrm{Ar}$

os: Ne: Sliot + Ar: Sllot t. Ne:

S1114 t.Ar: SI11t+Ne: SI12t. Ar:

Sil2t + Ne: Slist + Ar: S1134. Ne:

Silst $+\mathrm{Ar}$ : Slist $\mathrm{Ne}$
E T

E $\mathbf{T}$

\section{EXP ENERGY \\ RAMGE \\ THEOR}

E

Undef

E

$65-200$

$1.6-2.2 \mathrm{keV}$

$64-118 \bullet V$

1 keV/anu

E

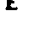

E

E

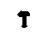

$0.064 \mathrm{gV}$

Under

Under

$4550 A^{\circ}$

A17

Unde?

$\mathrm{EO} 2$

$0.13 .6 \mathrm{eV}$

$1.6-3.9$

MeV/amu
REFEFEACE

Fronk 1, D. L.: Leoner, D.: Krlohnasuasy. S. V.: Derry, G.: 0.Gerear. T. Selective-odacrfticn-induced intensity maxias in He/L if gcatteing. Phys. Rev. Lett. 41, to (157e)

United States

Koch. F. F. Fesonont states In the ncnferturtotive reglos: the hydrogen atog in on intense alectric lieldo Phys. Rev. Lett. 41, $\$ S$ (1578)

United States

LIttaan. M. G.: Kash, M. M.: KlefFrer, C.

Fiald-ionizetion processe. in exclted otoos. Physe Rev. Lett. 41. $10 z$ (1S7E)

United states

Brooks, F. L.: Dat la, R. U.: Grier, H. R. Meosuremen of dielectronic recombinetion rete $f(C$ the Ircn icna Fe $I x-x I$. Phys. Rev. Lett. 41. 107 (1978) United stotes

Breton, C.: de Michelis, C.: Finderthel, Ma: Hattioli, H. Iontzotion and recoublation rete ccefficients ct highly Ionlaed olybdenue icns frce ofectrooccicy ci tokarak clasoas. Fhys. Rev. Lett. 41. 110 (IS7E) fronce

Eberhordt, k.: Kalkolten. G.: Kunz, C. Measurement of the Auger decay after rescrance excltotion $c t x e 4 d$ and Kr 3d resonance ilines. Phys. Rev. Lett. 41, IEt (1578)

West Gereany

Golllarc, H. J.: Fctzat, J.C.: Reallileux, J.

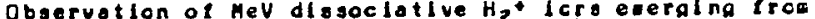
very thin fcils. Fhys. Rev. Lett. 4l. 159 (1978) France

lson. F. E : Berkner, K, t.: Grahea, h. G.: Pyle. R. V.: Schlachter. A. S.: stearng. J. H. Charge-otate decenderce of electron loso fros H by ccllisicns ith heavy. highly strlcped lona. Phya. Heve Lett. 41. 163 (1578)

United stotes

Hocon, F. B.: Solth, S. J.: Cecrges, A. I.:

Labrocoulos, P. ac Stark eplitiling In rescnont wultiphctcricnizotion with orcocteno loserg. Fhys. Rev. Lett. 41. 229 (1978).

United stotes

Gerber. R. B.: Shopiro, K.: Buck, L.; Schlecsener, J. Quantus-aechanlcol inversicn ce the dilierentiol cross cection: deteraination of the fe-Ne fotential. Phys. Rev. Lett. 41. 236 (IS7E)

Is roe I

Scluarz. $K$. H. Anomalous electron mobilitieg In dense helluw gas: Phys. Rev. Lett. 41.235 (1978) Unted'states

Nayleh. H. H.: Hurst, C. S.: Payne. M. G.: Young. J. F. observation of new satelilites in the Ca-Ar gyster using resonance icnizoticn scecticsccry. Phya. Feve Lett. 41. 302 ( $1 \leqslant 7 E)$

United stotes

Hagnou-seeurs. F.: Phllippe, M.: Valirch, F. Model-cetentlal calculaticns cn the eclecular system Node. Fhys. Fev. Lett. 41. 3SE (1578) France

Suter. M.: Vane, C. R.: Sellin, I. A.: Elstcn, S. E.: Alton, G. D.: Thoe. R. S.: Loutert, F. Cbaervetion of Ascilotory (Interierence) structure in the tcrword peak frco east-projectile.electrcn losa. Fhyo. Feve Lett. 41. 399 (1978)

United stotes 


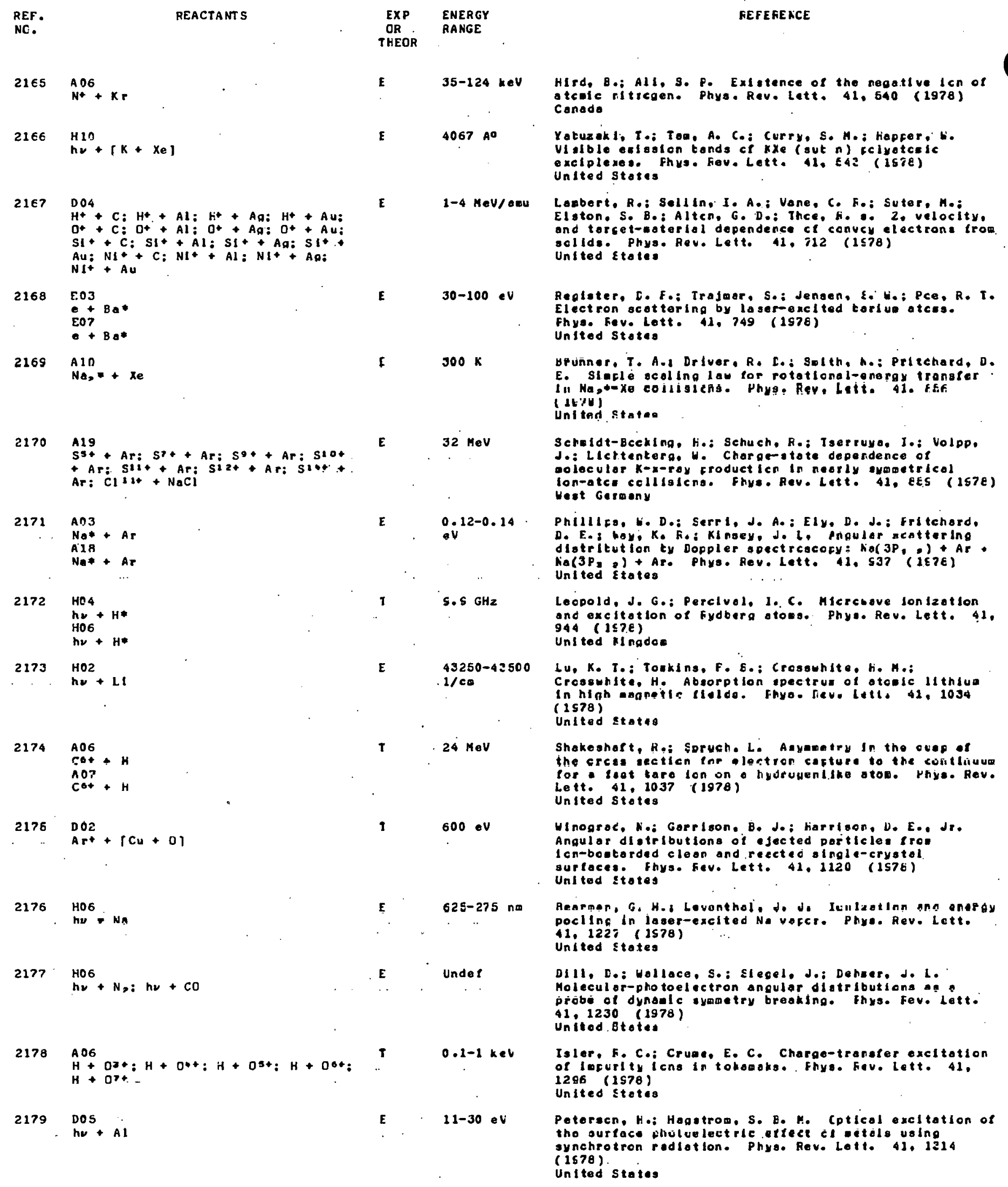




\begin{tabular}{|c|c|}
\hline $\begin{array}{l}\text { EXP } \\
\text { OR } \\
\text { THEOR }\end{array}$ & $\begin{array}{l}\text { ENERGY } \\
\text { RANGE }\end{array}$ \\
\hline $\mathbf{E}$ & $\begin{array}{l}\text { Ho6 } \\
3005-25 \in 0 \\
A^{0}\end{array}$ \\
\hline$E$ & $\begin{array}{l}1.2-1000 \\
k e V\end{array}$ \\
\hline
\end{tabular}

$2181 \quad \mathrm{AOG}^{\mathrm{MO}}+\mathrm{MQ:}^{+} \mathrm{MQ}^{2+}+\mathrm{Mg}_{9}$

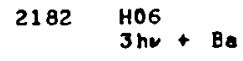
$\underset{\mathrm{Pb}}{\mathrm{DOO}}+\mathrm{Pb}: \mathrm{H}^{+}+\mathrm{Pb}: \mathrm{H}_{+}+\mathrm{Pb}^{+} \mathrm{H}_{+}+$

$2184 \stackrel{A 11}{B a *}+B a *$

$2185 \quad A 03$

$\mathrm{H}+\mathrm{NI}$

$2126 \quad \begin{gathered}E 09 \\ e+H_{7} ; \mathrm{e}+\mathrm{D}_{2}\end{gathered}$

$2187 \quad E 09$

C $+\mathrm{H}_{7}$.

$2128 \quad 602$

$X_{0 *}+A r$

Al1 $x_{0 *}+\ldots$

$2189 \quad \mathrm{AOS}$

$\mathrm{Na}+\mathrm{No}$

A10

$\mathrm{Na*}+\mathrm{No}$

2 hv + No

$2190 \mathrm{AO2}$

$\mathrm{Kr} *+\mathrm{He}$

2191

A0?

$\mathrm{He}^{2+}+\mathrm{Alr}$

$2192 \quad \mathrm{HO2}$

hv $+T h: h_{\nu}+U$

2193
E $4555 \mathrm{AO}^{\circ}$

$0.6-10$

keV/nucleon

E $\quad 1 / 25 \mathrm{eV}$

E

$3.15 \mathrm{MeV}$

E

$1-5$ eV

...

$2.1-4$ ev

$300 \mathrm{~K}$

E

$270 \circ \mathrm{C}$

Thapmel

E. $4 \mathrm{MeV}$
GE FE FE NCE

freemen, R. R.: Economou. A. F.: Bjorklund, G. C.: Lu, K. T. Coservoticn of electric-ilelic-lroucec resonance above tre icnizotien lialt in a cne-electron otom. Phys. Rev. Lett. 41, 1463 (1578)

United $\leqslant$ tates

Pedergen, E. H.: Hikkelsen, Je Ve: Vaoken, Jo:

Taulbjero. Interterence eftect in rescnont double-charce trangfer. Fhya. Feve Lett. 41,1541 (1578)

Denpark

Cocke, b. E.: Gollogher, T. F. Cboeruoticn of falr splltiligs In the autolonlzoticn sfectius of Es. Fhys. Rev. Lett. 41. 1648 (197e) Unlted states

Tolk. N. H.: Tully, d. C.: Kraua, j. S.: Helland, W.: Neff, S. H. Elliftic folorization cf kolder radiotion Prea lon-energy grezing-incidence ccllliglcng of hydoger. Fhyo. Fev. Lett. 41.643 ( 1578 )

United stotes

White. J. Co: 2dogluk. G. A.: Young, J.F.: harris, S. E. Oboervaticn of radiot Ive ccllialcnel flucreacence. Phys. Fev. Lett. $41,170 \mathrm{~s}$ (1578) United states

Blalr, J. E.: Dyer, P.: Snover, K. A.: Trainor, T. A. Nuclear tie delay ond $x$-roy-cicter celincioences near nuclear scottering resonance. Phyo. Feve Lett. 41, 1712 (ISTE)

United stotes

Allan. M.: Hara. S. F. Éflect of vitrationel ond rotational excitation on dissoclative attachaert in hydroger. Fhys. Fev. Lett. 41, 1751 (1578)

United stotes

Wocehro, J. M.: Eardsley, J. N. Vibrotional-ond rotaticnal-otote cependence of dissociotive attacheent in e-H, cclilsicns. Phys. Fev. Lett. 41. IISE (IS7E) United stoteg

Chenevler, H.: Sodeght. N.: Febay-Feyrcula, J. C.

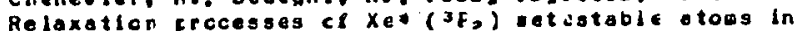
arpon-nenon wixtures. d. Fhya. Lett. (Parls) as, Lios ( 1578 )

France

Biraben. F.: Rercli, K.: Glocetinc, E.: Grynterg, G. Relaxation of the $4 D_{3} /$ and $4 D_{s} /$, lavels if scdilu otca perturbed by noble gases. J. Fhys. Lett. (foris) 39, perturbed to

France

Hrechianace C.: vetter, R.; Eerson. P. F. Fersistence of velceity rellobing elastic collisicna. J. inys. Lett. (Faris) as. Lizi (1S7E)

France

Fointu, A. M.: Fitalre. M. Methco tcr the weacurement ct the cean lonlzing crosg-section of last farticles and of the correscending cherge crcouced. je Ftys. and of the correscending cherge France

Cukler. M.: Dhez, F.; Gauthe. H.: joegle. F.: hehenkel, C. L.: Corbet Farncux, F. Fhctcobscrition of Th ond by direct aesureaent and last electrco energy loss apecte near the Ed thresholdse J. Fing. Lett. (Faris) spectro neor the
39, Lis (1978) Fronce

VIalle, J.-L. Sensitive detection of auticle threshclds for lectric lleld icnizaticn of soclum Rycberg stotes. J. Phys. Lett. (Parig) 39. L365 Rycbers
(' $1 \leq 78)$ France 


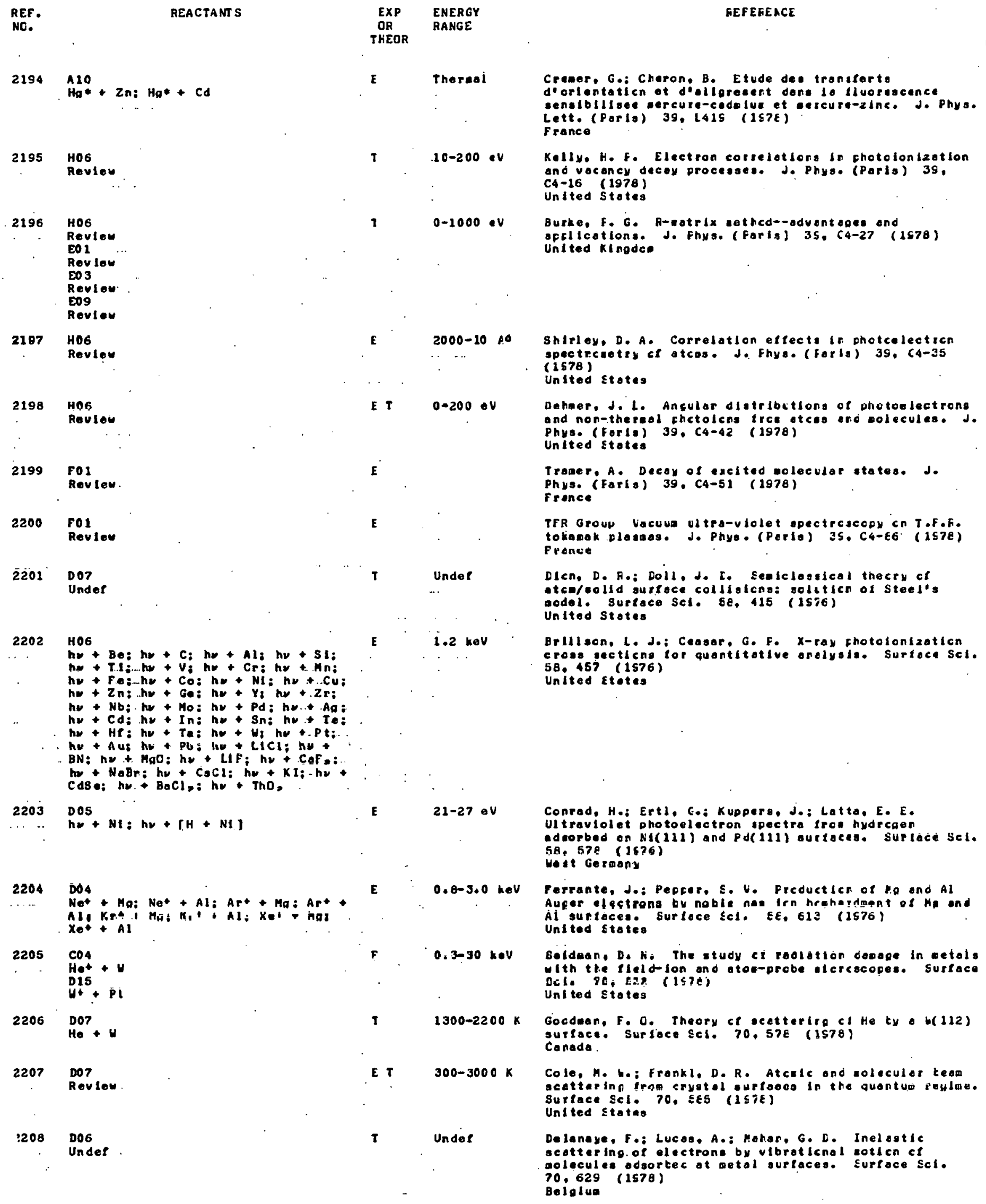




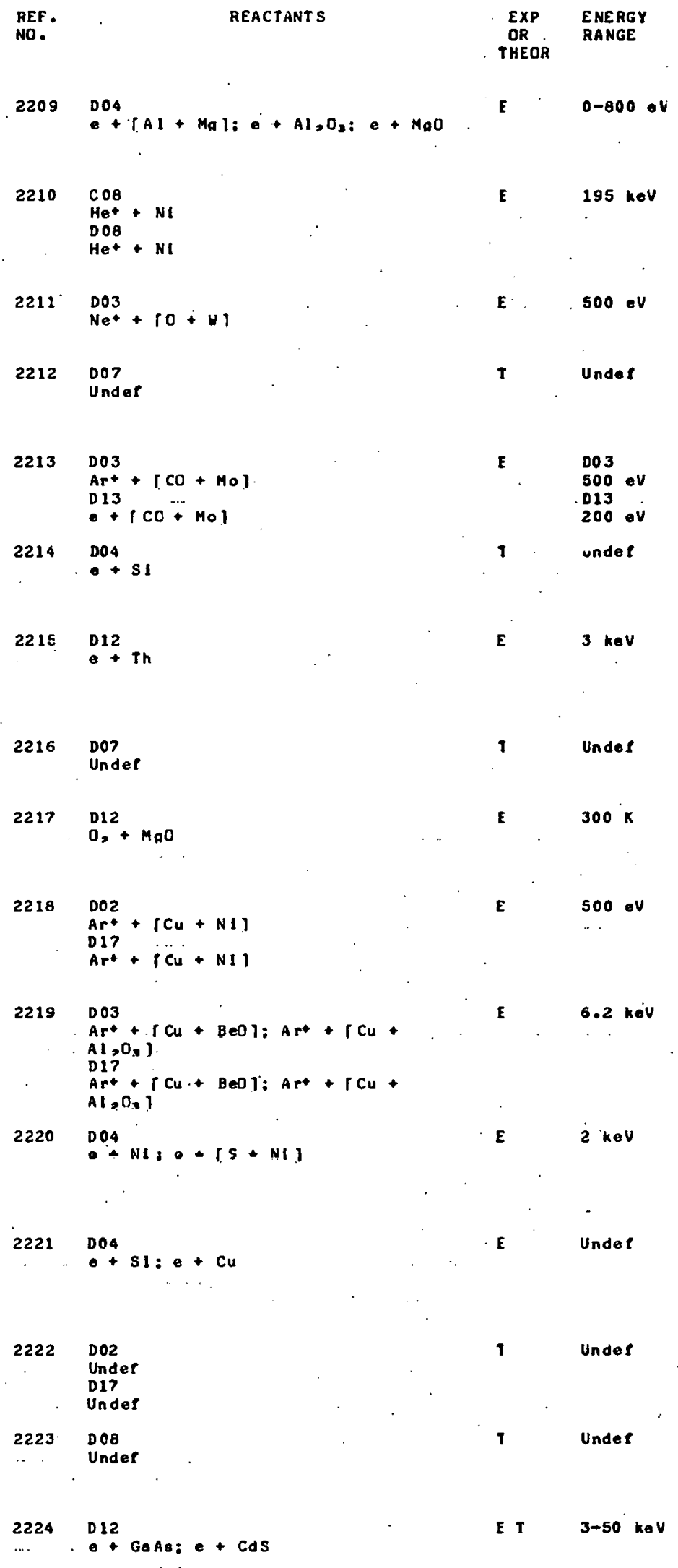

Goldstein. E.: Cresner, J. Grouth of MaO Pllse with hloh secendery electron ealasion on Al- $\mathrm{Hg}$ alloys. Surface Sel. 71 , 15 (197E)

United stoties

Varelas, C.: Slzasnn, F. Light ealestcn by oulft tiet lons after socual no and chonneling interactlons on acnocryotelline nickel surfoce. Surtece Scl. il. El acnocryot

(1578)

United stotes

Yu, M. L. The SIKE secet rue of the $0--4(100)$

cheofisorption systew. Surfoce Scl, 71.12) (1578) Unltod Etetes

Garcla, N. Threshold and lennard-jones rescnances and olastic lifetises in the scattering cl otom frow cryotalline surfoces. Surface Scl. 71, 220 (1578) Spaln

Dawson. P. H. The adsorpticn of CC on solybdenue otudled by low enerai SIML ond EIV. Surfoce Sci. 71. 247 (157e).

Canade

De Andreo, A.; Del scle, R. Thecry cl $(e, 2 e)$ reaction near solid ourfoces: opplication tc sl. Surfoce Ecl. $71,30 \epsilon \quad(1 \leq 7 \varepsilon)$

Italy

Batasz, Fi, Colennores, C. A.: Sogorjel, G. A. Electron Induced luminescence os. technique $R C S$ studying the odsoritice of exyeen end the oxidetion of therlum. Sur tace Ecl. 71,357 (1578) United Etotes

Logos, *. Scottering of lewenergy otces by watallic surfaces. Surfoce Sci. 71, 414 (157E) Chile

Roose, R. F.: Offergeld, G. lloht ealeston during adsorption end deacrpticn ct cxyoen on eagneglus oxide. Sustace SCI. 71, $4 \in 2$ (ISTE).

be lol ue

Saexi, N.: Shiolzu, R. Thickress ond in-depth ccapositicn prollle of oltered layer coused on CU-NI alloy surfoce due to preferentiol iputlering. Eurfoce ScI. 71, 479 (1978)

Jaran

Blolse, G.: Lyon, C.: fioques-Carwes, C, Sputterirg and secondary icn enisglon of twc-chese aystes cooposed of small cxide precipltotes discersed in copper matilx. Surfoce sci. $71, \epsilon 20 \quad(1 \leq 78)$

Fronce

Matsudelre, T.; Onctil, M. Angular distributlena of Auser electron eatosion; clean ond pas-odscrbed polycryetalline $\mathrm{Ni}$ surtoces. Surfice sct. IĆ, $\leq$ (1578)

Jazan

Unite, S. J.: WoodrufP, D. Fo: Medennell, L. Angulor decendence of Auger elect ren ealsolen trom si ong cu( 100$)$ surloces in the presence of overlayers. Surface Eel. 72, 7 ? (197, $)$ Unsted Mingdo:

Ho, P. S. Elfocts of enhancec clllusicn cn preferred sputtering of homogeneous olloy susfaces. surfoce Scl. 72,253 (1578) United stotes

Blcos, W.: Hone, D. Theory el choige exchinge scottering trom surfaces. Surface sel. 72,277 ocottion

Unlted Stotes

Dalt ruk, N. Le: Litovetienko, U. G.: Talat, G. H. The eflect of the surface siace charge reglen cn the cathodol um Inescence of seniconductcrs. Surface Scl. $72,321$ ( $157 E)$

Soviet unicn 


\begin{tabular}{|c|c|c|c|c|}
\hline $\begin{array}{l}\text { REF. } \\
\text { NC. }\end{array}$ & REACTANTS & $\begin{array}{l}\text { EXP } \\
\text { OR } \\
\text { THEOR }\end{array}$ & $\begin{array}{l}\text { ENERGY } \\
\text { RANGE }\end{array}$ & FEFEFEACE \\
\hline 2225 & 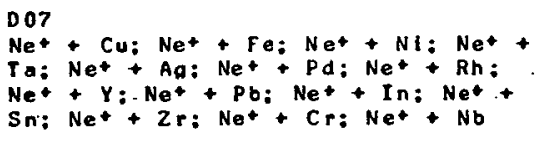 & $\mathbf{E}$ & $0.1-2.5 \mathrm{keV}$ & $\begin{array}{l}\text { Boun, b. L. Multiple ocattering featuras ir necn lon } \\
\text { scattering spectro (ISS).trce fclycrystelline } \\
\text { moterlels. Surtace Scl. } 72 \text {. } 56 \text { (ISTE) } \\
\text { Unlted Stotes }\end{array}$ \\
\hline 2226 & $e^{D 13}+\operatorname{CO}+\operatorname{Ir} 1$ & $\mathbf{E}$ & $\begin{array}{l}0.09-2.5 \\
\mathrm{keV}\end{array}$ & 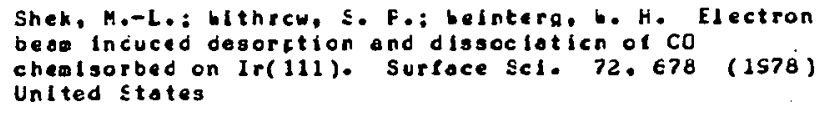 \\
\hline 2227 & $\begin{array}{l}\text { Do3 } \\
A r_{+}^{+} \mathrm{LIF}: \mathrm{Ar}+\mathrm{NaF}: \mathrm{Ar}^{+}+\mathrm{KF}: \mathrm{AF}^{+} \\
+\mathrm{NaBF}: \mathrm{Ar}^{+}+\mathrm{KF}\end{array}$ & $E$ & $0-3.0 \mathrm{k} \in \mathrm{V}$ & 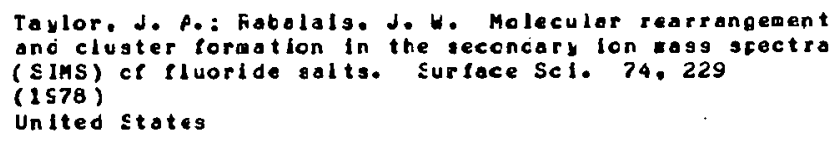 \\
\hline 2228 & $\begin{array}{l}\text { Do7 } \\
\text { Undef }\end{array}$ & $\mathbf{T}$ & Unde $f$ & 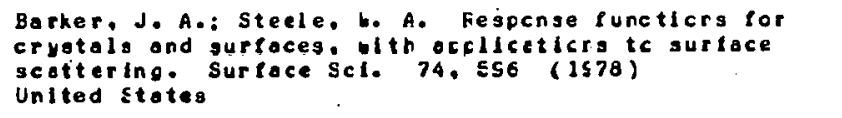 \\
\hline 2229 & 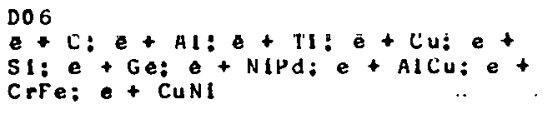 & $\mathbf{T}$ & Unde & 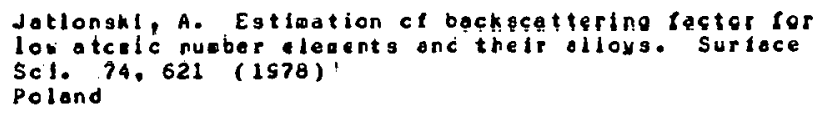 \\
\hline 2230 & $\mathrm{~N}_{2}++\mathrm{SI}_{4}: \mathrm{O}_{2}++\mathrm{SI}: \mathrm{He}^{+}+\mathrm{SI}$ & $\mathbf{E}$ & $6 \mathrm{keV}$ & 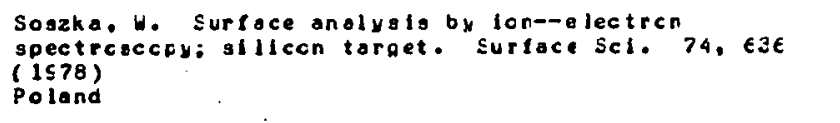 \\
\hline 2231 & $\begin{array}{l}\text { D10 } \\
\mathrm{HeF}+\mathrm{LIF} ; \mathrm{He}+\mathrm{HaF}: \mathrm{LIF}: \mathrm{D}+\mathrm{NIF}: \mathrm{H}+ \\
+\end{array}$ & 7 & $\begin{array}{l}0-2.2 \times 10-3 \\
0 \mathrm{~V}\end{array}$ & 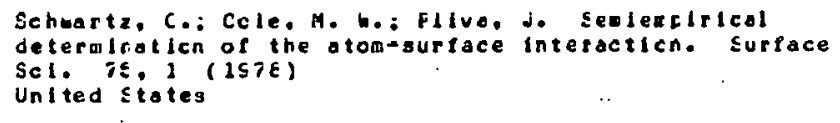 \\
\hline 2232 & $\begin{array}{l}\mathrm{DO3} \\
\mathrm{Ar}+\mathrm{GAA}=\end{array}$ & $E$ & $8 \mathrm{keV}$ & 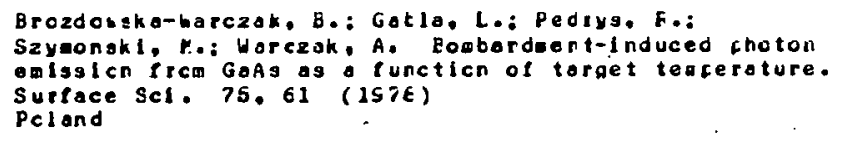 \\
\hline 2233 & 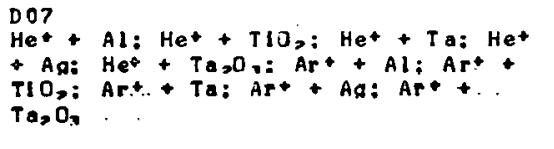 & $\mathbf{E}$ & $0.1-2.5 \mathrm{keV}$ & 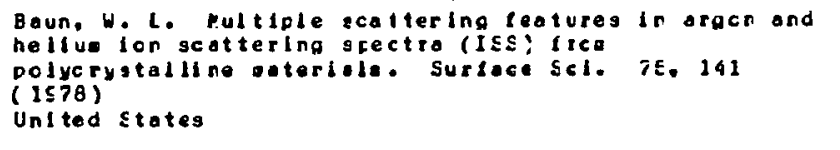 \\
\hline 3234 & $\begin{array}{l}003 \\
\mathrm{Ar}^{+}+\mathrm{SS} ; \mathrm{Ar}+ \\
+ \text { Invar }\end{array}$ & $\mathbf{r}$ & $94 k \in V$ & 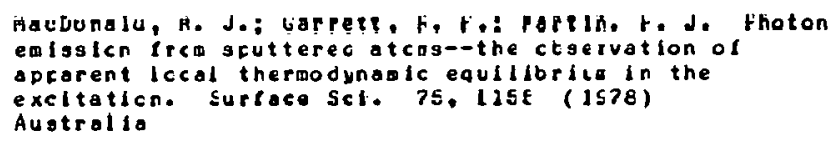 \\
\hline 2235 & DO3 $\mathbf{r}^{+}+\mathrm{SS:} A \mathbf{r}^{+}+\operatorname{Invar}$ & $\mathbf{E}$ & $54 \mathrm{keV}$ & 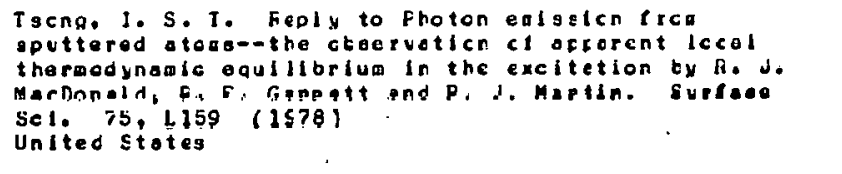 \\
\hline 2236 & $e+A u: e+A g: e+M g$ & E & $0-160 \mathrm{ev}$ & 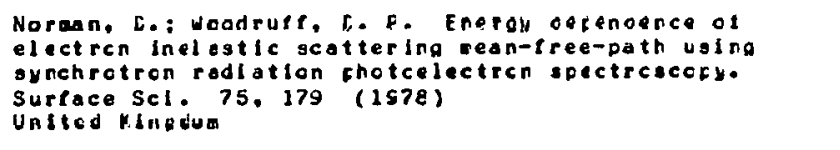 \\
\hline 2.377 & $\begin{array}{l}\mathrm{ng}_{\mathrm{S}}+\mathrm{Cu} \\
\mathrm{D} 1 \mathrm{O} \\
\mathrm{H}\end{array}$ & $\mathbf{T}$ & $\begin{array}{l}6-11 \\
k c a l / 001\end{array}$ & 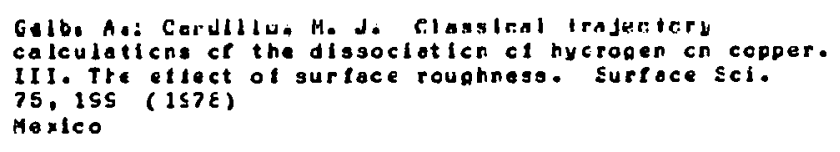 \\
\hline 2238 & $\begin{array}{l}\mathrm{DO4} \\
e+B .0\end{array}$ & E & $3-40$ eV & 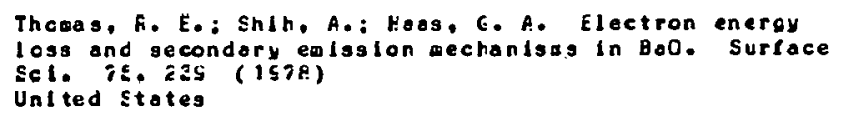 \\
\hline $\begin{array}{l}2239 \\
.\end{array}$ & $\begin{array}{l}\text { Co5 } \\
\text { Undef }\end{array}$ & $T$ & Unde $\boldsymbol{l}$ & 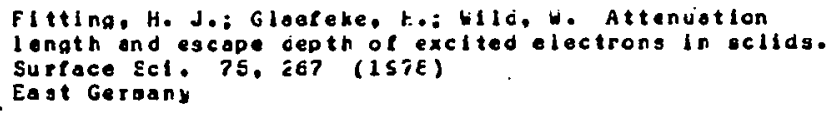 \\
\hline
\end{tabular}




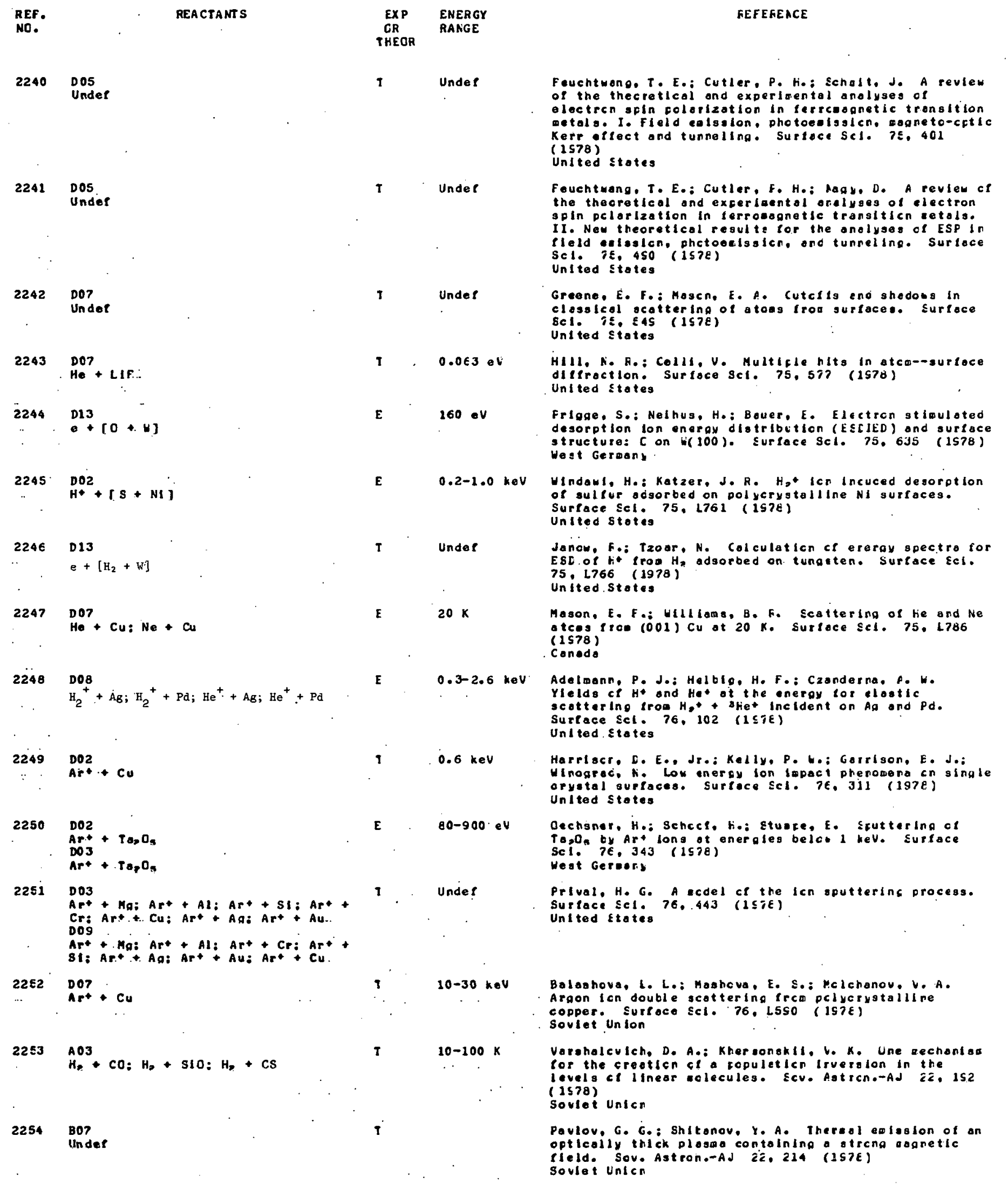




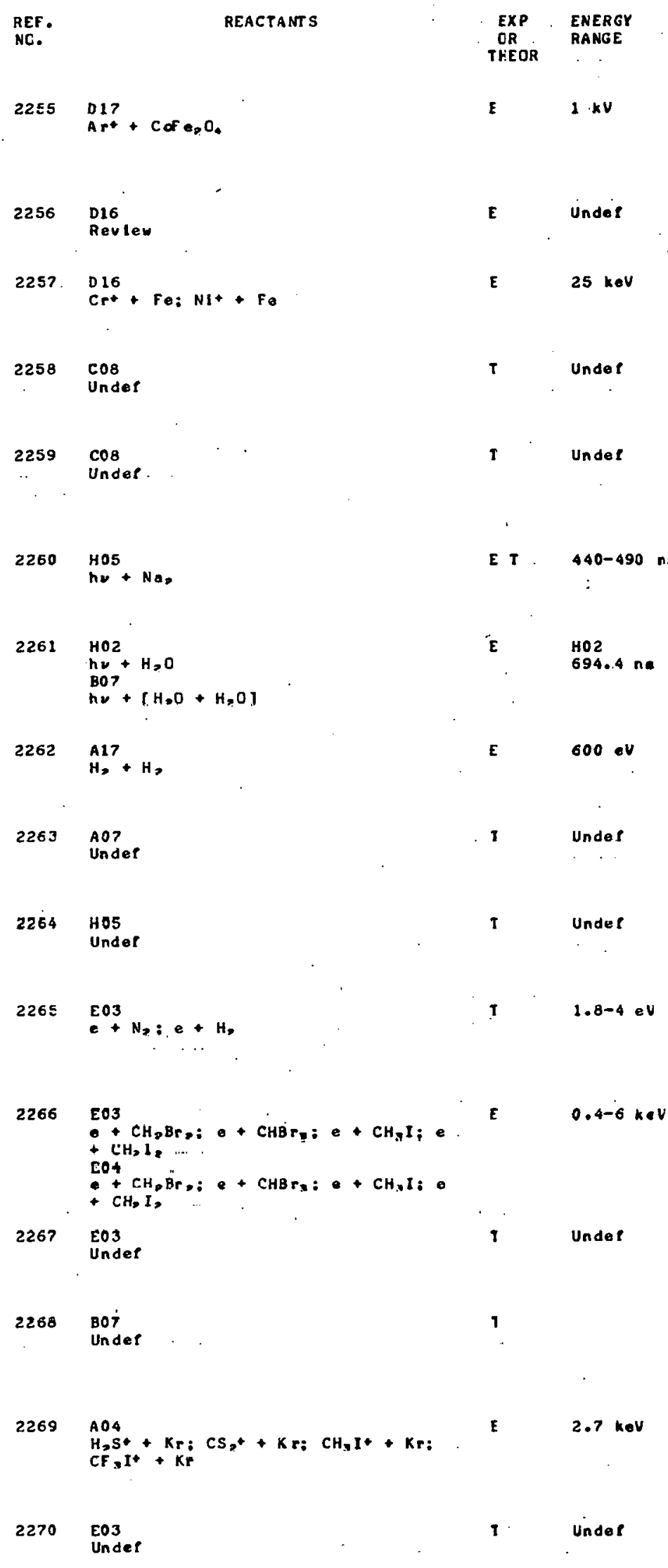

Chuang. T. J.: Erundle, C. R.: Mandelt, K. An x-ray phetoelection spectroscopy otudy of the cherlcal changes In calde and hydrcxide surtoces incucec by Art lon bowtardeent. Thin Solid Filus.63, 19 (1978) Unl ted Etates

Dearnaley. G.: Hartley. N. E. b. Ien Ieflantotion intc cetal ond carbidea. Thín Eclid fllea 54.-2ie (ISTE) Untted Xingdoe

Sartweil. B. D. Foradion of corrcsion-resiatant surface alicys by cetel icr.leclantoticn. Thin Solid flins 54,233 (1578)

United stotes

Akhiezer, A. I.: Boldyshev, U. F.: Shul'ga. N. F. Theory of the radiaticn ol relativistic channelod porticles. Sov. Physe-Loklady 22,565 (1977) Soviat Unien

Beloahttakil. V. V.: Kudahhci: H. A. Elfect ol oniliscoticn of Induced enlasicn cn teod of channeled relotivistic particles. Sov. Phys-Dchlady 22,645 . (1977)

soviet union

Pacernov, S. H.: Shlyafnlkev, G. U.: Yenson, M, L. Photodiasoclation of vibrationally oxclted colecules. Sov. Phys.-tokledy 23, 56 (1678) Soviet Union

Zuev, V. E.:- Lopasov, V. F.: Ponoarev, Y. N. Nazrouling of the collitolon shape of the eclecular aboortion lino of atecopheric weter vecor in eirong light Ifeld. Sov. Physo-Doklady 23, 261 (1978) Sovtet tinl ca

Leonas, V. E.: Khrceov, V. N. Experientol inveaticaticn of ahort-range Interucleculor forces in hydrogen. Eou. Phys--Dokleoy 23, 330 (1578) Soviet Unicr.

Devdartenl. A, Z,: Datrcuakl1, V. N.: Sebyokin, X. N. Electrcn epectro irow the autoionizaticn ol quasieclecules. Ecv. Fhws.-JETf 46.215 (1977) soviet union

Kuz"uIn, M. V.: Sozonov, V. N. Theory ef excltation of a ouantud nenilinear osclilotor by a horsonic icrce. Dove Phyo.-detp to, 220 (1977) soviet Union

Golubkcv, G. V.: Dellochlk, F. I.: Ivanov, G. K. Vibraticnal oxcitation ce oclecules during resonence scottering of electrong. Ecv. Phya.-JEIP 4E. zao (1577)

Soviet Unten

Dentlewak If, h, F, : Kocpe, V. I, Kovel, A. G.I Khevanaktl. N. A. Farantirn at arelted tregnente If the distoctation ci.holcgenetec athene coevounds ty

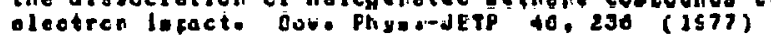
olectren lisact

Smirnov, E. M.: Firaov, O. B. Crous dectien of excltation of molecular vitrational level by electron iefaet. sou. Phyo.-JEIf 46, 200 (1677) Soviet tintan

Alekseer, A. L.: Evseev, I. V.: Ermochenko, V. M. Fesslbility of investlagting atosic cclllolens by the photon-echc technique. Sov. Fhys--JETf 46, 246 (1577)

soviet inton

Manvelyan, A. V.: Kuprlyanov, S. E.: Ferov, A. A.: Potapov, V. K. Isoact disacciation of mieculer ions prcduced ty photolonizotion of vericus colecules. Sove Phys.-JE1P 46,442 (1577) Soviet Inton

Deakov. Y. A.: Detrovekil. V. N. Exchenge excttetion of on ator ty electron with high ongular doenta. Sov. Phys,-JETP 4C, 44S (1977) Soviet Unl cr 


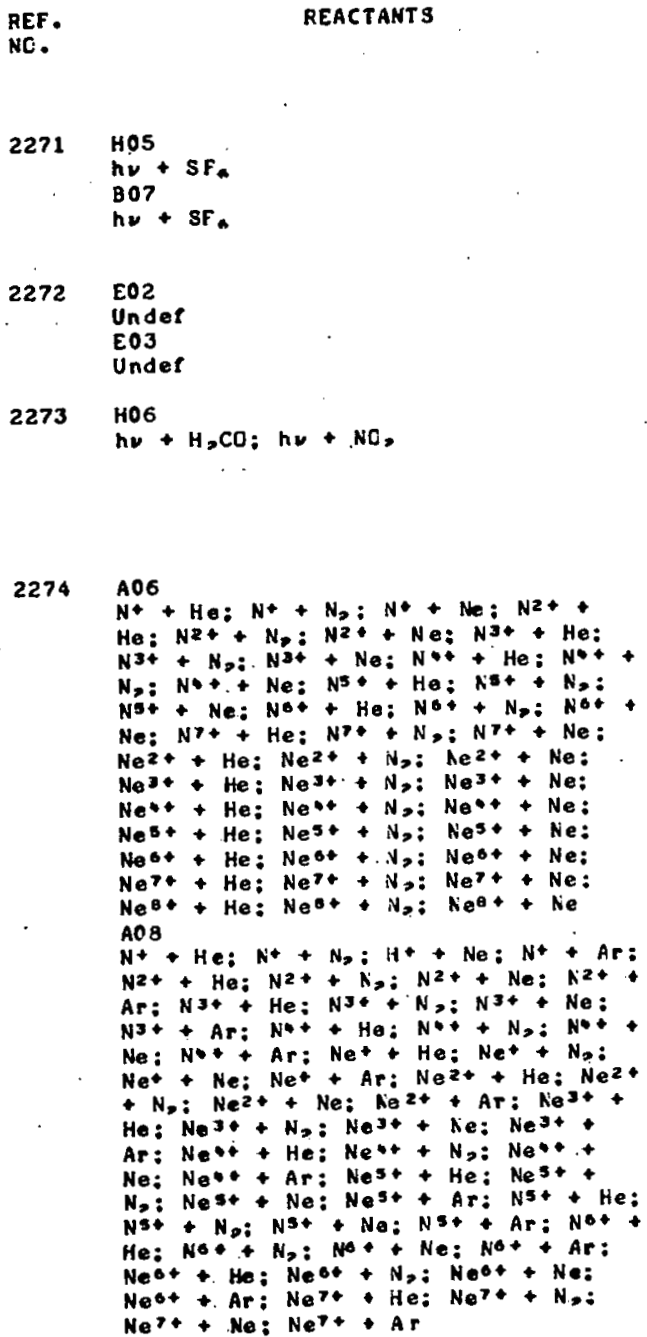

$\mathrm{NO}^{+}+\mathrm{He}: \mathrm{N}^{+}+\mathrm{N}_{2}: \mathrm{N}^{+}+\mathrm{N} \mathrm{N}^{-\mathrm{N}^{2}+}+$

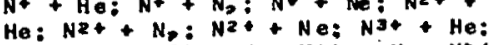

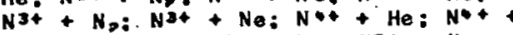

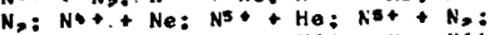
Not + Ne: NOt+ He: NOt + No: Not $\mathrm{Ne}: \mathrm{N}++\mathrm{He}: \mathrm{NH}+\mathrm{N}, \mathrm{N} \mathrm{N}^{+}+\mathrm{Ne}$ : $\mathrm{Ne}+\mathrm{He}^{+} \mathrm{Ne}^{2+}+\mathrm{N}_{2} \mathrm{Ne}^{2+}+\mathrm{Ne}:$ $\mathrm{Ne}^{2+}+\mathrm{He}: \mathrm{Ne}^{3+}+\mathrm{N}: \mathrm{Ne}^{3+}+\mathrm{Ne}$ $\mathrm{Ne}^{3+}+\mathrm{He}: \mathrm{Ne}^{3+}+\mathrm{N}: \mathrm{Ne}^{3+}+\mathrm{Ne}$

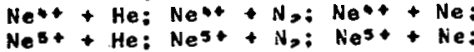
$\mathrm{Ne} 6+$ He: $\mathrm{Ne}^{\circ+}+\mathrm{N}: \mathrm{Ne}_{+}+\mathrm{Ne}$ $\mathrm{Ne}{ }^{7 t}+\mathrm{He}: \mathrm{Ne}^{7+}+\mathrm{N}$ : $\mathrm{Ne}^{7+}+\mathrm{Ne}$ $\mathrm{NeB}++\mathrm{He}^{-} \mathrm{Ne}^{\circ+}+\mathrm{N}: \mathrm{Ne}^{+}+\mathrm{Ne}$ AOB $\mathrm{NO}^{+}+\mathrm{He}: \mathrm{N}^{+}+\mathrm{N}_{2} ; \mathrm{H}^{+}+\mathrm{Ne} ; \mathrm{N}^{+}+\mathrm{Ar}:$ $\mathrm{N}^{2+}+\mathrm{HaZ} \mathrm{N}^{2+}+\mathrm{N}=\mathrm{N} \mathrm{N}^{+}+\mathrm{Ne}: \mathrm{N}^{2+}+$ Ar: $\mathrm{N}^{3+}+\mathrm{He}: \mathrm{N}^{3}+\mathrm{N}_{3} \mathrm{~N}^{3+}+\mathrm{Ne}$ $\mathrm{N}^{+}+$Ar: $\mathrm{N}^{4+}+\mathrm{He}^{+} \mathrm{N}^{+}+\mathrm{N}_{2}: \mathrm{N}^{*}+$ $\mathrm{Ne}: \mathrm{N}+{ }_{+}+\mathrm{Ar}: \mathrm{Ne}^{+}+\mathrm{He}: \mathrm{Ne}^{+}+\mathrm{N}_{2}$ : $\mathrm{Net}+\mathrm{Ne} \mathrm{Ne}_{+}^{+} \mathrm{Ar:} \mathrm{Ne}^{2+}+\mathrm{He}: \mathrm{Ne}^{2}$ $\mathrm{Ne}^{+} \mathrm{Ne:} \mathrm{Ne}^{+} \mathrm{Ar:} \mathrm{Ne}_{+}^{+} \mathrm{He}^{+} \mathrm{Ne}^{2}$ He: $\mathrm{No}^{3+}+\mathrm{N}=: \mathrm{Ne}{ }^{+}+\mathrm{Ne}$ : $\mathrm{Ne}^{3+}$ AF: Neot + He: $\mathrm{Ne}+t+\mathrm{N}>\mathrm{S} \mathrm{Ne}^{+*}+$ $\mathrm{Ne}: \mathrm{No}{ }^{+}+\mathrm{Ar}: \mathrm{Ne}^{5+}+\mathrm{He}$ : $\mathrm{Ne}^{3+}+$ $\mathrm{N}=\mathrm{Nest}+\mathrm{Ne}$ : Nest + Ar: NSt + he:

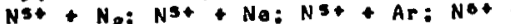

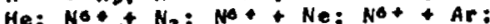
$\mathrm{NeOt}+\mathrm{He}$ NeOt $+\mathrm{N}, \mathrm{N}_{004}+\mathrm{Ne}$ :

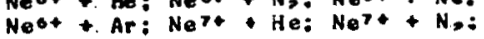
$\mathrm{Ne}^{\mathrm{T}+}+\mathrm{Ne}: \mathrm{Ne}^{\mathrm{T}+} \mathrm{Ar}^{\mathrm{s}}$

$2275 \quad A 03$

Unde?

$2277 \quad 405$

$X_{0}+F_{0}$

807

$h v+\left[X_{e}+F, J\right.$

$2278 \quad A 12$

Under

2279

A10

$\mathrm{CH}_{3} \mathrm{~F} * \mathrm{CH}_{3} \mathrm{~F}$

2280

$$
\begin{aligned}
& \mathrm{EO3} \\
& \mathrm{EO}+\mathrm{H}_{2}: \mathrm{C}+\mathrm{D}_{2} \\
& \mathrm{E}+\mathrm{H}_{3}: \mathrm{O}+\mathrm{D}_{2}
\end{aligned}
$$

\section{HEFEREACE}

Sazonov, V. N.: Finkel'shtein, $V_{\text {. }}$. fnalysia ct model ce radiative dissocioticn ct celyatcolc molecules in the lield ef laser radiation. Sov. Phys.-JETP $46.6 E 7$ (1577)

Soviet inicn

Fabrikant, 1. I. Threahold behovicr cl the crcas actiona for ucettering of electrcrs ty polor molecules. Sov. Phys.-JETF 4E, E\$3 (1977). soviet linicn

Antonov. V. S.: Knyozev. I. N.: Letokhcv, V. S.: Movshev. V. G. Photolontzotion of eolicules frcs the ground end galectively-excited to tes by radiaticn Proc a vacuugultraulalat h, loser. ScV. Ffyse-jETf $4 E$, $697(1 \leqslant 77)$

Soviet Unicn

Doltriev, I. S.; Tashaev, Y. A.: Nikoloev, W. S.: Teclove, $Y$. A.: Popov, B. M. Experloental atudy ce the loos and cafture cl electecrs ty lest aulticly chorged itroger anc neen ions in voricus casea. Scr. Phys.-JETP AE. EE4 (1577) soviet linica
Belgaon. 1. L. Cross actions ce transitions between highlyexcited levels os o result ct ccllistonewith chorged porticles. Sov. Prye-JETF 4 . SOE (1S77) Soviet inton

2hltalkcy. R. A.: Kor toshkin, V. A.: Mlesent'ev, G. V. Coherence transter in aetogtatility exchange in the inture of the Soutet 10 isn

Guczenkc, L. I.: Gurvlch, L. V.: Lutov, V. S.: Yakovienkc. S. I. Chealcat radiative cclilisicnse Sov. Physe-JETP 46. 1002 (1977)

Soviet Unicn

Detrouskll. V. N. Interacticn cl on excltoc hydrogenlike otom with chorged porticie in the dipole aprodiatien. Eou. Fhys.-JETf $4 E, 10 E 8$ (1577) Soviet Unton

Chesnokcu, E. N.: Ponfllov, V. A. Vibrotional energy trangfer. tolueen the orthe-ond cara-accilleaticns ol $12 \mathrm{CH} F$ and $1 \mathrm{CH}, \mathrm{CH}$ olecules. Scu. Phys.-JETP 46, 1112 (1577)

Soviet Unlen

Polyakcua, G. N.: Eanyuk, A. I.: Erkc, W. F. Kinetic-eneray diatribution ol exclted atcos prcauced when $H$, and $D$, eolecules ore olescelotid by electron Iapact. Sov. Phys.-JETF tE, 1117 (1577) Soviet Union 


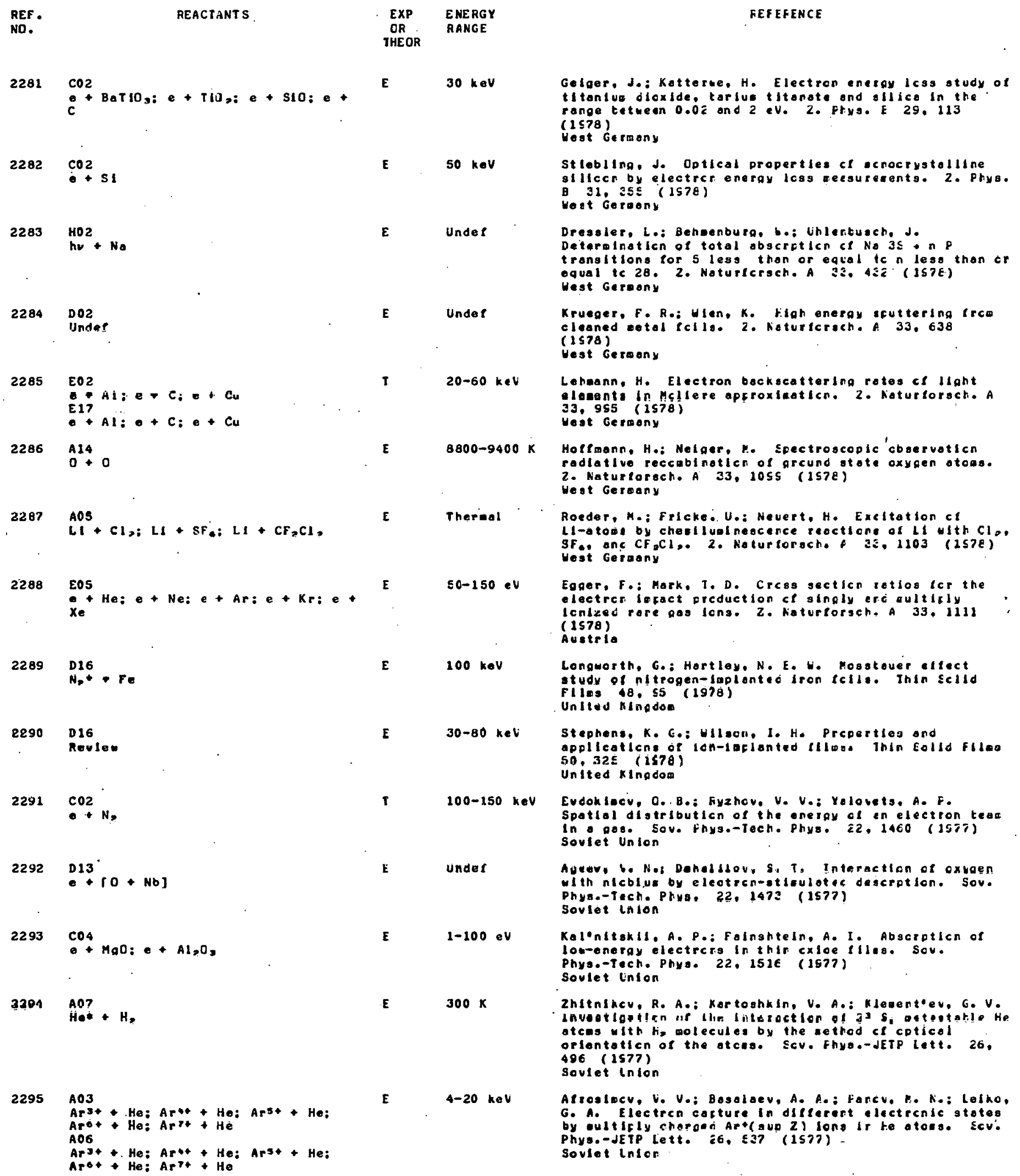


REF.
NO.

REACTANT S

2296

A 06

ON+ $+h$

2257

B07

Under

$2298 \mathrm{JOL}$

Review

2299 FOI

He Seq

2300

D17

$\mathrm{He}^{3+}+\mathrm{PbF}_{9}: \mathrm{He}^{2} \mathrm{I}+\mathrm{CaF}$,

2301 D12

$e+[G a N+Z n]$

2302

D07

$\mathrm{He}^{*}+\left[\mathrm{MO}_{\mathrm{O}}+\mathrm{O},\right]$

2303

$\mathrm{CO}^{+}+\mathrm{H}>\mathrm{O}: \mathrm{He}^{+}+\mathrm{H}_{2} \mathrm{O}: \mathrm{O}++\mathrm{H}, \mathrm{O}: \mathrm{Ar}^{+}$

$+\mathrm{H}>\mathrm{O}: \mathrm{Fe}^{+}+\mathrm{H}_{>} \mathrm{O}$

$\mathrm{CO} 3$

$\mathrm{H}^{+}+\mathrm{H}>\mathrm{O}: \mathrm{He}^{+}+\mathrm{H}>\mathrm{O}: \mathrm{O}+\mathrm{H}_{2} \mathrm{O}: \mathrm{Ar}^{+}$

$+\mathrm{H}_{2} \mathrm{O}: \mathrm{Fe}^{+}+\mathrm{H}_{2} \mathrm{O}$

E0S

$\mathrm{C}+\mathrm{H}>\mathrm{C}$

2304

$\mathrm{He}^{+}+\mathrm{SI}_{\mathrm{O}} \mathrm{He}^{+}+\mathrm{GOP}: \mathrm{He}^{+}+\mathrm{GaAs}$

D15

$\mathrm{H}^{+}+\mathrm{Sl}^{\mathrm{O}} \mathrm{H}^{+}+\mathrm{GaF}: \mathrm{H}^{+}+$GaAS: $\mathrm{N}^{+}+$

S1: $\mathrm{N}^{+}+\mathrm{GaP}: \mathrm{N}^{+}: \mathrm{GQAS}^{+}$

2305

$\mathrm{He}+\mathrm{Cu}$

2306

DO3

Art + SC: Art+ II: Art \& V:Ar+ +

Cr: Art + Fe: Art+ Nl: $\mathrm{Ar}^{+}+\mathrm{Cu}$ :

$\mathrm{Ar}^{+}+2 \mathrm{Zn}: \mathrm{Ar}^{+}+2 \mathrm{r}: \mathrm{Ar}^{+}+\mathrm{Al}: \mathrm{Ar}^{+}+$

Si: Art+ SiO,: Art + Al,0,: Art. $\mathrm{NaCl}$ : $\mathrm{Ar}^{+}+\mathrm{KCl}$

2307

$\mathrm{He}^{+}+\mathrm{C}$

2308 D15

o + Mo

2309

$\operatorname{COB}$

$\mathrm{H}^{+}+\mathrm{SI}$

$\begin{array}{ll}\text { EXP } & \text { ENERGY } \\ \text { OR } & \text { RANGE } \\ \text { THEOR } & \end{array}$

Under

$\mathbf{T}$

E

E

Undef

E $2.5 \mathrm{MeV}$

$2-30 k \in V$

$0.5 \cup V$

$\mathbf{I}$

CO2: $\cos$

MeV

EOS

10-10s eV

$T$

$\mathrm{COB}$

$1 \mathrm{MeV}$

D 15

$40-60$ keV

E

$2-10 \mathrm{kel}$

E

$43 \mathrm{keV}$

$2 \mathrm{MeV}$

$0.5-3.0 \mathrm{MeV}$

$\tau$

$1 \mathrm{MeV}$
REFEFENCE

Abramov, V. A.: Baryshnikov, F, F.: Lleltso, V. S. Change cr intenalty of spectrol licea cl ouiticly charged Icna os a result of charpe exchonge with atoalc hydroger. Sov. Phys.-JETf lett. E?, 464 (1S7E) Sovlet inion

2hukovo, N. I.: Kazanteev. A. Fo Cooling ard heatling of atora cclliding in on crtical tielo. Sci. Phys.-dEtf lett. 27, 601 (1578) Soviet inton

Johnstcn, hi. 6.: Podolike, J. Interpretaticns of stratoscheric photceheilstiy. Fev. Gecchys. Space Fhys. 16, 491 (1978)

United states

Bolko, V. A.: Pikuz, S. A.: Salroncva., U. L.: Foenovo A. $Y$. Sotelilter to the He-ilke Icn $10^{2} 2 S_{0-i a D_{0}} \mathrm{IF}_{0}$ ines ith $2=12-15$ in laser plasese. Acn. Not. RCy. Astron. Soc. 186.789 ( 1578$)$ soviet inici

PIstre, J. I.: Canto, Y.: Eoloroenze, J.; Setoyo, B.; Chemin, d. F. Comparative oboervalion cr lenle algraticn $c t$ PbF, $B$ and Cof, thin Illas by Futherford ocattering. Fov. Fhys. Afri. (Peris) 13. c12 (157e) fronce

Boulou, M.: Jocot, G.: Bols. D. Cothcdolueinasence study ct 2 n doped GaN. Rev. Fhys. Aopl. (Ferls) 13. SEE ( $1 \leq 7 \varepsilon)$

France

Eolzilau, C.: Nuvolone. F.: Fovosel, d. Reflecticn of netastatle hellud atosos on oclybcenualilo/ surface: eflect ct cxygen adsorption. Fiev. Physe Aprl. (Parls) 13. 571 (157E) France

Poretzke, H. G. Cn prifary coeege ond oeccncery electrch dacace in heavy icn tracks if plastlca. Radiot. Eft. 34, 3 (1977)

Weet Goraons.

Wolker, R. S.: Thcepacr. I. A.: Fcehleen, 8. h. A seateerirical vethcd ot applying the dechanreling correction in the ixtraction of dieorder distribution. Radiat. EfI. 34, 157 (1577) Conada

Verhelf. L. Ko: Foelaede, E.: Eoere, A. L. Neutrallizaticn and lonlzaticn cl lev energy hellua lons scattering from copper aurface. Rodiote Ell. 34 , $162(1577)$

the Netherlands

Boyly. A. Fo: HocDcnald, F. J. The energy epectro of seccndory icno entted during Ion teaberdment. Radiat. EPt. 24. leS (1577)

Australta

Veprek, S.: Portmonn, A.: hebt, A. F.: Stuessl, h. Sustace worchclcay charpes cf arochite irrocleted with energetic nel lus ions. Rodlat. El1. 34. 183 (1577) sultzerland

Nishida, T.: Izul, K.: Furuno, S. Locallzotion ot clectrcn current censity and lta effect on dasage preduction in a eclybdenua cryotol. Feclat. El1. 34. 217 (2577)

Jarnon

Beerl. Fo: Carnera. A.: Desalvo, A.: Ficea, F. Feeding-lin and blocking phenowen excleined In teros ol a elople diffuston podel. Fadiat. Elf. 34, $26 z$ (1577)

Itoly 
REF

REACTANTS

NO.

2310

D10

PERT + PERT

2311

Bo 1

Under

2312

HO4

Undef

HOS

Under

2313 HO1

Under
$\mathrm{BO} 7$

Unde?

$2314 \quad \mathrm{EO2}$

Under

$2315 \quad \begin{aligned} & \text { Hol } \\ & \text { Under } \\ & \text { B07 }\end{aligned}$

Under

2316 .

$\mathrm{AO3}$

$\mathrm{Ca}^{+}+\mathrm{Ne} \mathrm{Ca}^{+}+\mathrm{Ar}: \mathrm{Ca}^{+}+\mathrm{Kr}: \mathrm{Ba}_{+}+$

$\mathrm{Ne}$; Ba+ + Ar: Srt: Ar: Srt? Kr

A06

$\mathrm{Sr}^{+}+\mathrm{Kr}_{\mathrm{r}} \mathrm{Sr}^{+}+\mathrm{Ar}: \mathrm{Ca}^{+}+\mathrm{Kr}: \mathrm{Ba}^{+}+$

Ar

$2312 \mathrm{HOL}$

Under

2318

E04

$\mathrm{O}+\mathrm{H}_{3}+$

EOS

$\mathrm{C}+\mathrm{H}_{x}+$

2319

$\mathrm{AO}^{3}$

$\mathrm{ClI}_{3} \mathrm{~F}+\mathrm{CH}_{4}$

$\stackrel{\mathrm{A} 10}{\mathrm{CH}_{3} \mathrm{~F}}+\mathrm{CH}$.

$2320 \quad H 13$

Undet

2321

$\mathrm{COS}_{\mathrm{CO}}^{\mathrm{OOS}} \mathrm{CO}=$

B07

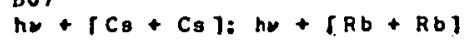

$2322 \quad H 04$

Unde?

$2323 \quad$ HO4

Undef

2324
EXp

THEOR

ENERGY

RANGE

T Under

$\tau$

Undef

HOI

Unde?

I Unde?

Under

$200-500 k$

$300 \mathrm{~K}$

Undef

$A O B$

Innta?

Unde?

$\tau$

Undet 


\begin{tabular}{|c|c|c|c|c|c|c|}
\hline $\begin{array}{l}\text { REF. } \\
\text { HO. }\end{array}$ & . & REACTANTS & & $\begin{array}{l}\text { EXP } \\
\text { CR } \\
\text { THEOR }\end{array}$ & $\begin{array}{l}\text { ENEAGY } \\
\text { RANGE }\end{array}$ & FEFEFENCE \\
\hline 2325 & $\begin{array}{l}\text { E11 } \\
\text { Under } \\
\text { E12 } \\
\text { Under }\end{array}$ & & & $\mathbf{T}$ & Under & 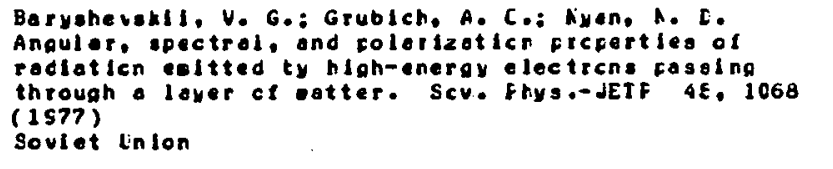 \\
\hline 2326 & $\begin{array}{l}\text { E13 } \\
0+H\end{array}$ & . & & $T$ & $4-20 \bullet V$ & 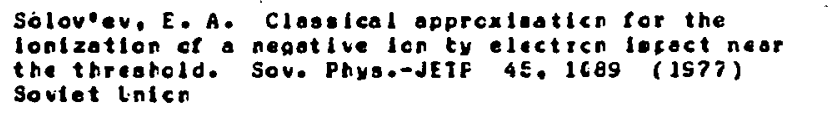 \\
\hline 2327 & $\begin{array}{l}\text { Eo3 } \\
\text { Under }\end{array}$ & . & $\cdot$ & $\mathbf{T}$ & Undef & 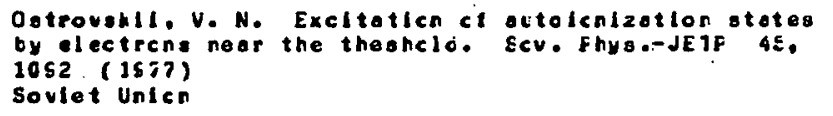 \\
\hline 2328 & $\begin{array}{l}\text { A10 } \\
\text { Under }\end{array}$ & & & $\mathbf{1}$ & Under & 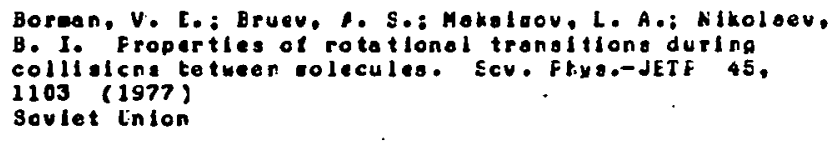 \\
\hline 2329 & 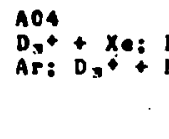 & 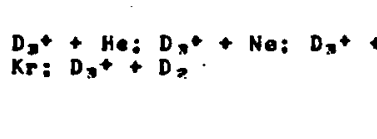 & 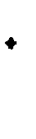 & $\mathbf{E}$ & $1-40 \cdot v$ & 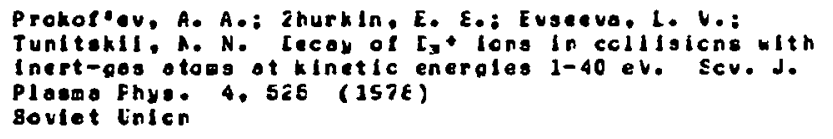 \\
\hline 2330 & $\mathrm{EO2}^{\mathrm{E}} \mathrm{CO}$ & & & $E$ & $\begin{array}{l}0.06-6.0 \text { ev } \\
\text {. }\end{array}$ & 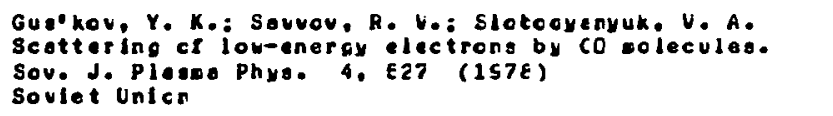 \\
\hline 2331 & $\begin{array}{l}\text { A06 } \\
H^{*}+\text { PERT }\end{array}$ & - & & $\mathbf{T}$ & $103-10 s e v$ & 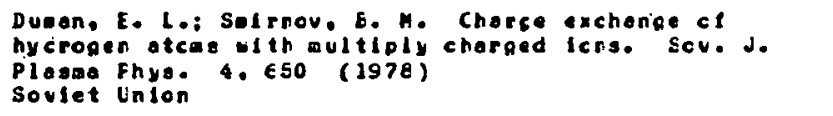 \\
\hline 2332 & $E_{-H *}^{E 03}$ & & & $\mathbf{I}$ & Under & 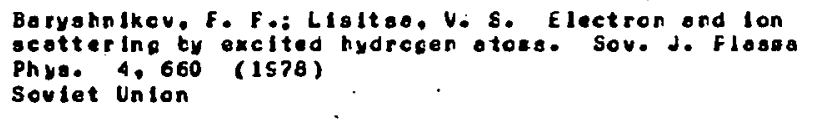 \\
\hline 2333 & $\begin{array}{l}\text { AL? } \\
\mathrm{LI+}+\mathrm{He:}\end{array}$ & $K \bullet A r$ & & $T$ & Under & 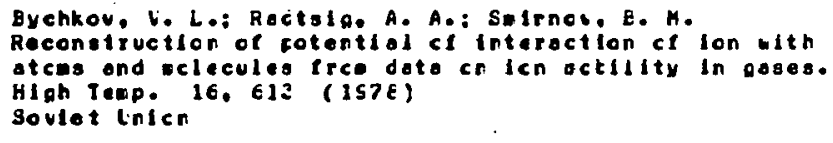 \\
\hline 2334 & 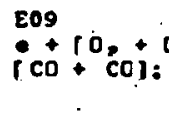 & $0,1: 0+\left[\mathrm{CO}_{2}+\mathrm{CO}_{2}\right]: \cdots$ & $\bullet$ & E & $77-300 \times$ & 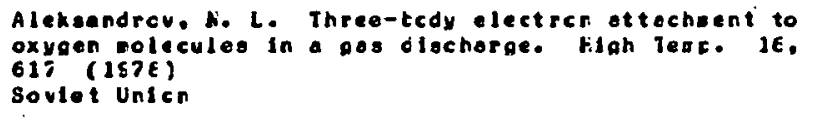 \\
\hline $2335^{\circ}$ & $\begin{array}{l}\text { ALG } \\
\text { NO- Ho: }\end{array}$ & $\mathrm{NO}-+\mathrm{CO}$ & & $\mathbf{T}$ & $200-500 k$ & 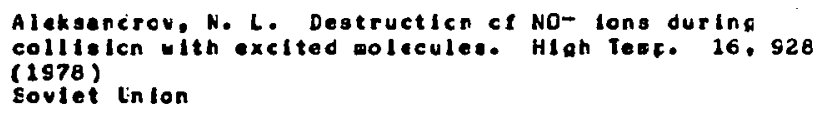 \\
\hline 2336 & $\begin{array}{l}A 10 \\
B r+*+A r\end{array}$ & & & $\mathbf{T}$ & $300-900 \times$ & 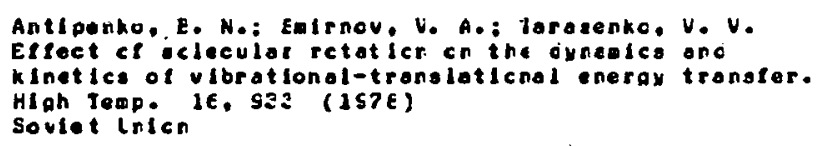 \\
\hline 2337 & $\begin{array}{l}A 17 \\
L I+F: L I \\
N a+F: N a \\
+F: K+C \\
R b+C l: R \\
C a+C 1: C\end{array}$ & 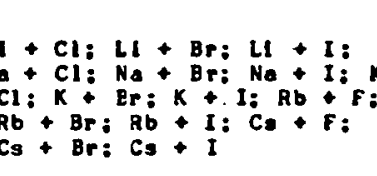 & & $\mathbf{T}$ & $\begin{array}{l}0.68-203 \\
c a-1\end{array}$ & 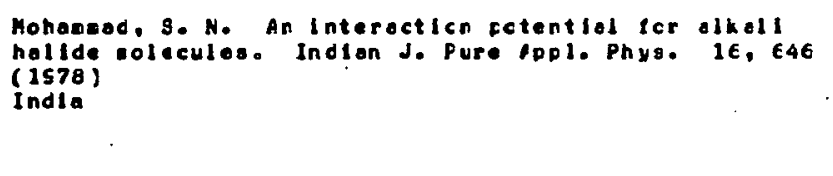 \\
\hline 2338 & $\begin{array}{l}\operatorname{EO3} H, \mathrm{H}, \mathrm{O} \\
\mathrm{EOg}+\mathrm{H}, \mathrm{O}\end{array}$ & & & $\mathbf{t}$ & $0.25 \bullet v$ & 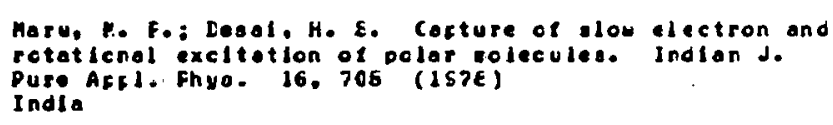 \\
\hline 2339 & $\begin{array}{l}\text { A12 } \\
\text { OC } 3 * \text { Ho: }\end{array}$ & ocs* +Ar & & $\mathbf{T}$ & $60-220 \mathrm{~K}$ & 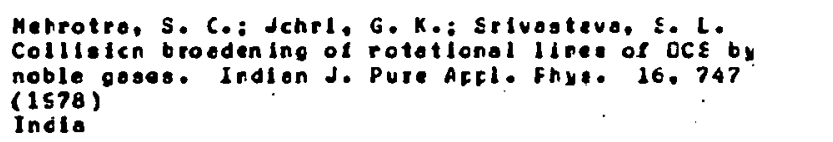 \\
\hline
\end{tabular}




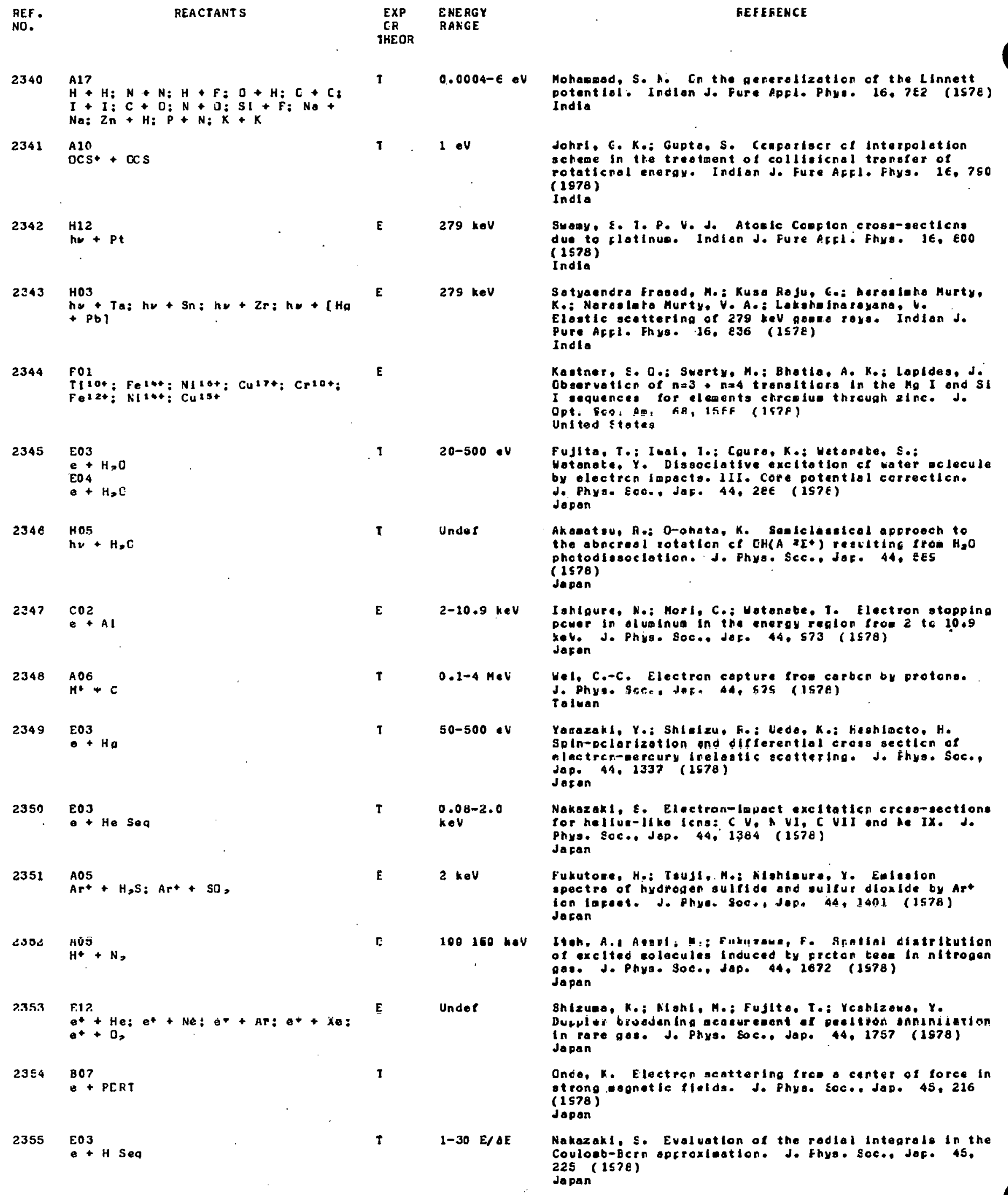




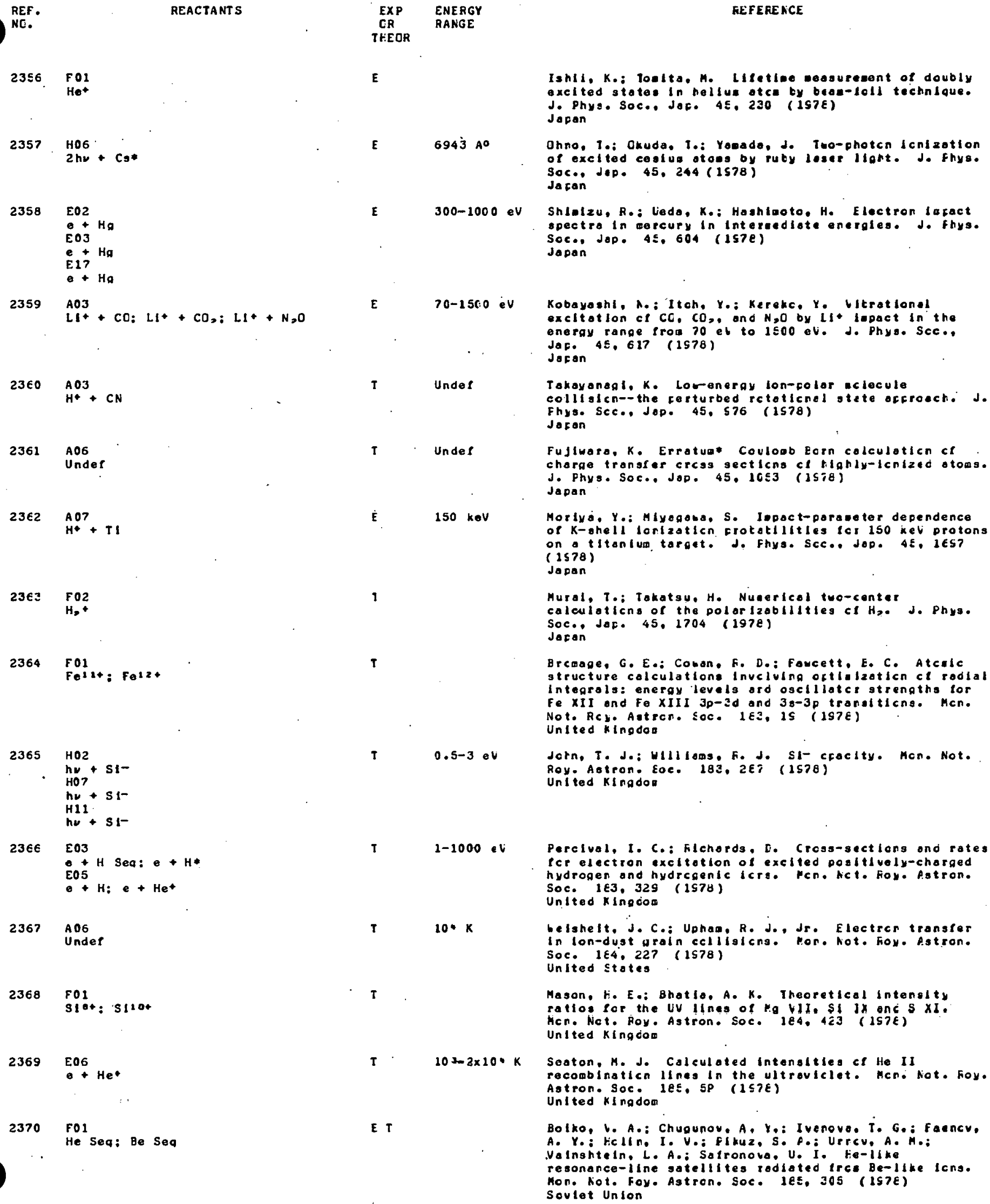

Under

H11.$$
\text { he + S1- }
$$

$\varepsilon 03$

- + H Sea: $\mathrm{e}+\mathrm{H}^{*}$

EOS

e $+\mathrm{H} ; \mathrm{e}+\mathrm{He}^{+}$

Ishll, K.: loolta. M. Lifetloe meosuresent of doubly excited stotes in nelluk otce by beos-ioll technlque. J. Phys. SOC.. JOF. AE. 230 (ISTE) Jopon

Ohno, 1.: Ckuda, 1.: Yoeado, d. Two-photcn Icnlaotion of exclted cogiva otons by ruby loser light. J. Fhys. Soc., Jip. 45, $244(1578)$ Jafon

Shlolzu, R.: ledo, K.: Hashicoto, H. Electron lafact spectro in warcury in Interadiote energles. J. Fhys. Soc., Jap. 4S. 604 (157E) Japan

Kobayashi, A.: Itoh, Yo: Kerekc, Y. Witrat lonel excltotion CP $\mathrm{CC}, \mathrm{CO}$, , and $N, 0$ by $L_{1}$ t lupact in the energy range frou 70 et to 1500 ev. J. Physe. Scc., JaF. $4 E, 617$ (1578)

Jakan

Takayanagl, K. Lomenergy lon-color iclecule collisicn-- the certurbed retelicnel otete offroech: J. Fhys. SCC.. JOD. 45, S76 (1578)

Jogon

Fujluara, K. Erratua* Couloob Eorn colculaticn cr charge trangfer crcos secticns cl tighly-icnized otoms. J. Phys. Soc.. Jop. 45, $10 \leq 3$ (15i8) Japan

Morlyd. Y.: Miyogona. S. Iopoct-poraceter dependence of K-ohell lonizaticn. crotatillties $f(i) 150$ ket protons On A tltanlum target. J. Fhys. ScC.. JOD. 4E, LES? (1578)

Jopan

Mural, T.: Takatsu. H. Nucerlcol two-cantar calculaticns of the polarlzabilitieg cl $H_{p}$ J. Phys. Soc., Jaf. 45, 1704 (1972)

Jofan

Brcmage, G. Eo: Cowan. F. D.: Faucett, E. C. Atcalc structure colculations invelulng oftlizeticn cl rodial Integrols: energy levels ord osclliatcr strengtho tor fe $X I I$ and $F e X I I I$ Jp-3d and $38-3 p$ troneiticns. Men. Not. RCy. Astren. Soc. lE3, is (1S7E) Unlted kingdoo

Jorn, T. J.: Hill ilams, F. J. SI- CFacity. Hen. Not. Roy. Astron. Eoc. 183, $2 E 7$ (1578) United kingdor

Perelval, I. C.: Flehords, D. Cross-sectlons ond rates fer electron excitotion of exclted positluely-charged hydrogen and hydrcgenlc icra. Pcn. Hict. Foy. Patron. Soc. $1 \in 3,329$ (1578)

United Kingcod

helshelt, J. C.: UDhaa, R. J., Jr. Electrcr trangter in ton-dugt groin cclligicns. Mon. Not. Foy. Astron. Soc. $1 E 4,227$ ( 1578 )

United States

Mason, H. E.: Bhatlo. A. K. Theoretical Intenglty ratiog for the UV lines of Kg VII, SI IX enc $S X I$. Hen. Net. Poy. Astron. Soc. 1E4, 423 (ISTE) Unlted Kingoom

Seaton, M. J. Caleulated intensitles of He II racombinaticn lines in the ultroviclet. Mcn. Not. Foy. Astron. Soc. 1EE. 5P (1STE) Unlted kingdoa

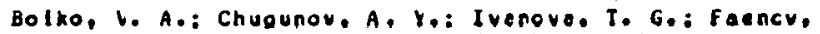
A. Y.: ticll $n_{0}$ I. V.: Flkuz, S. P.: Uerev, A. M.:

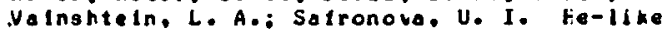
resonance-iline gatellites rodiated tras Be-like lans. Hon. Not. Foy. Agtron. Soe. leE, 305 (ISTE) Soviet Union 


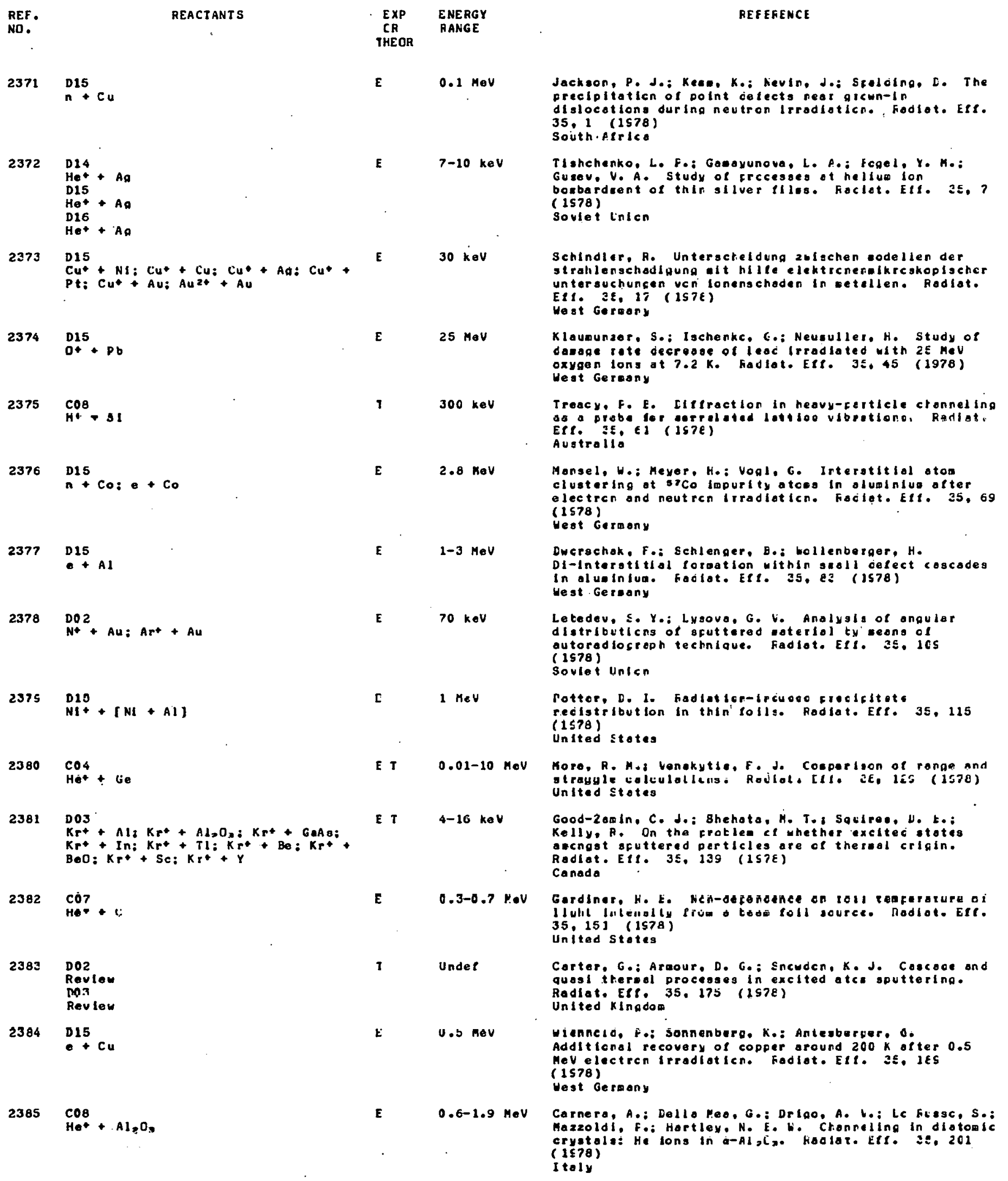




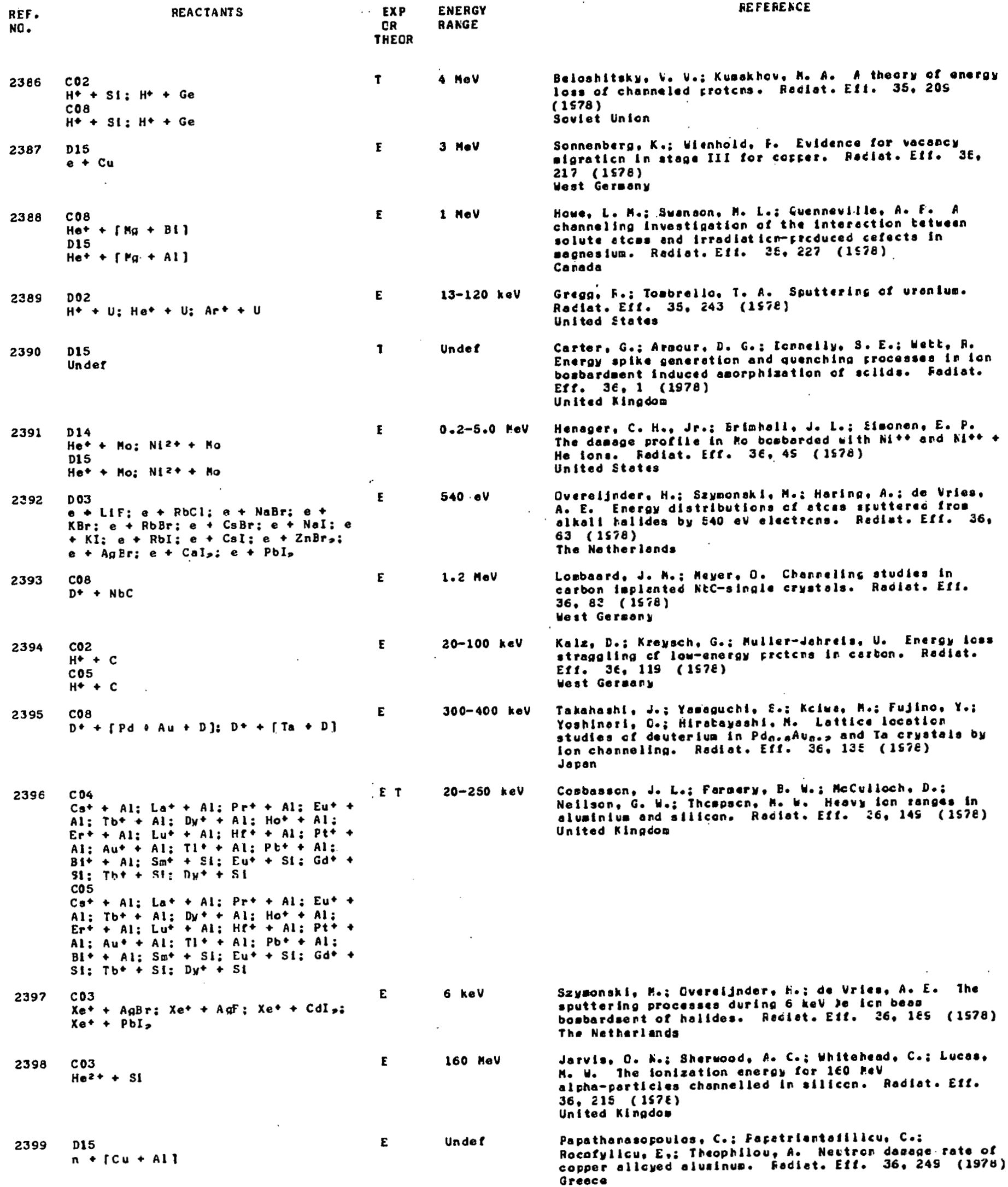

\begin{tabular}{|c|c|}
\hline $\begin{array}{l}\text { EXP } \\
\text { CR } \\
\text { THEOR }\end{array}$ & $\begin{array}{l}\text { ENERGY } \\
\text { RANGE }\end{array}$ \\
\hline$T$ & $4 \mathrm{MeV}$ \\
\hline E & $3 \mathrm{H} \bullet \mathrm{V}$ \\
\hline $\mathbf{E}$ & $1 \mathrm{M} \in \mathrm{V}$ \\
\hline $\mathbf{E}$ & $13-120$ \\
\hline 1 & Under \\
\hline
\end{tabular}

E $540 \cdot 1$

E

E

$20-250 \mathrm{keV}$

Beloahlteky, V. V.: Kusakhov, M. A. A theory of energy logs of channeled crotcns. Radlat. Elt. 35, 209 (1578)

soviet Unton

Sonnenberg, K.; Hienhold. F. Evidence tor vacancy ligratien in ítag III lor cokcer. Rediat. Elt. 3E. 217 ( 1578 )

West Geroany

Howe, L. H.: Susnson, H. L.: Cuennevill le, A. F, A channeling Investigation of the Interection tetreen colute atces and irradiat icm-crcaced celects in eogneslum. Rodiot. Ell. $3 \varepsilon, 227$ (1578) Canada

Grega; F.: Toebrello, T. A. Sputtering of urentua. Radlat. Ell. 35. 243 (157e) United Statos

Carter, G.: A raour. D. G.: Icanelly, S. E.: Wett, R. Energy sike generotion and quenching crocesses in ion bosbardeent Induced acorphisation of eclldse Fadiat. EfP. $3 E .1$ (1978)

United Kingdow

Henoger, C. H.. Jr.: Ertmhall, J. L.: Eleonen,E. P.

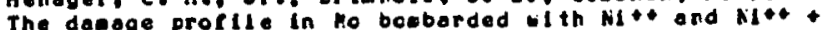
He lona. Fadlat. Eft. 3E, AS (1578) United stotis

Overelfinder. Ho: Szymonok I. Mo: Harlng, Aa: de Vries, A. E. Eneroy distributions of etces auttereo fron

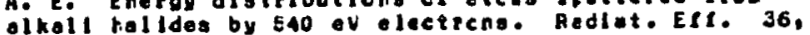
$63(1578)$

The Netherlonds

Lombard, d. M.: Heyer, 0 . Channelling atudies in carbon Ieplanted Nic-single cryatols. Radlot. Efl. 36. 82 ( 1578 ) Hest Gereony

Kalz, D.: Kreysch, G.: Muller-dehrele, U. Energy loes etragoling cr lom-eneigy crctcrs in cerbon. Rodict. EY1. 36, 119 (157E)

West Germany

Takahaghl, J.: Yagoguchl, E.: Kelue, H.: FuJino, Y.: Yoantnorl. O.: Hitrotayashi. M. Lattica locotion studies of devterium in Pdn.aAnos and Ta cryatalo by lon chonnoling. Rodiat. ElP. BG. 13E (1STE) Jovon

Cosbaseon, J. Le: Foraery, B. H.: HeCulloch, D.: Nellson, G. H.: Thespocn, M. Heavy Ion ranges in alueintive and ilicen. Rootat. Elf. 36,145 (is78) United kingdom

Szywonsk I. He: Overalijnder, ti.: de Vrles, A.E. The suttering processes during 6 hev he icn bead boubardeent of hallides. Haciot. Elt. 36, les (1578) The Netherlands

Jarvis. O. Ni: Sherwood. A. C.: Whitehead, C.: Lucas, H. $U$. The lontzation eneroy tor $1 \in 0 \mathrm{MeV}$ al oha-partleles channelled in ilicen. Hadlat. ElR. 36. 215 (157E)

United Kingdoe

Papathanasogoulos, C.: Fafatrlantofllicu, C.: Rocofyllcu, E,: Theophllou, A. Nectron danage rate of copper alloved alualnuo. Fodiet. Ell. 36.249 (1978) oreece 


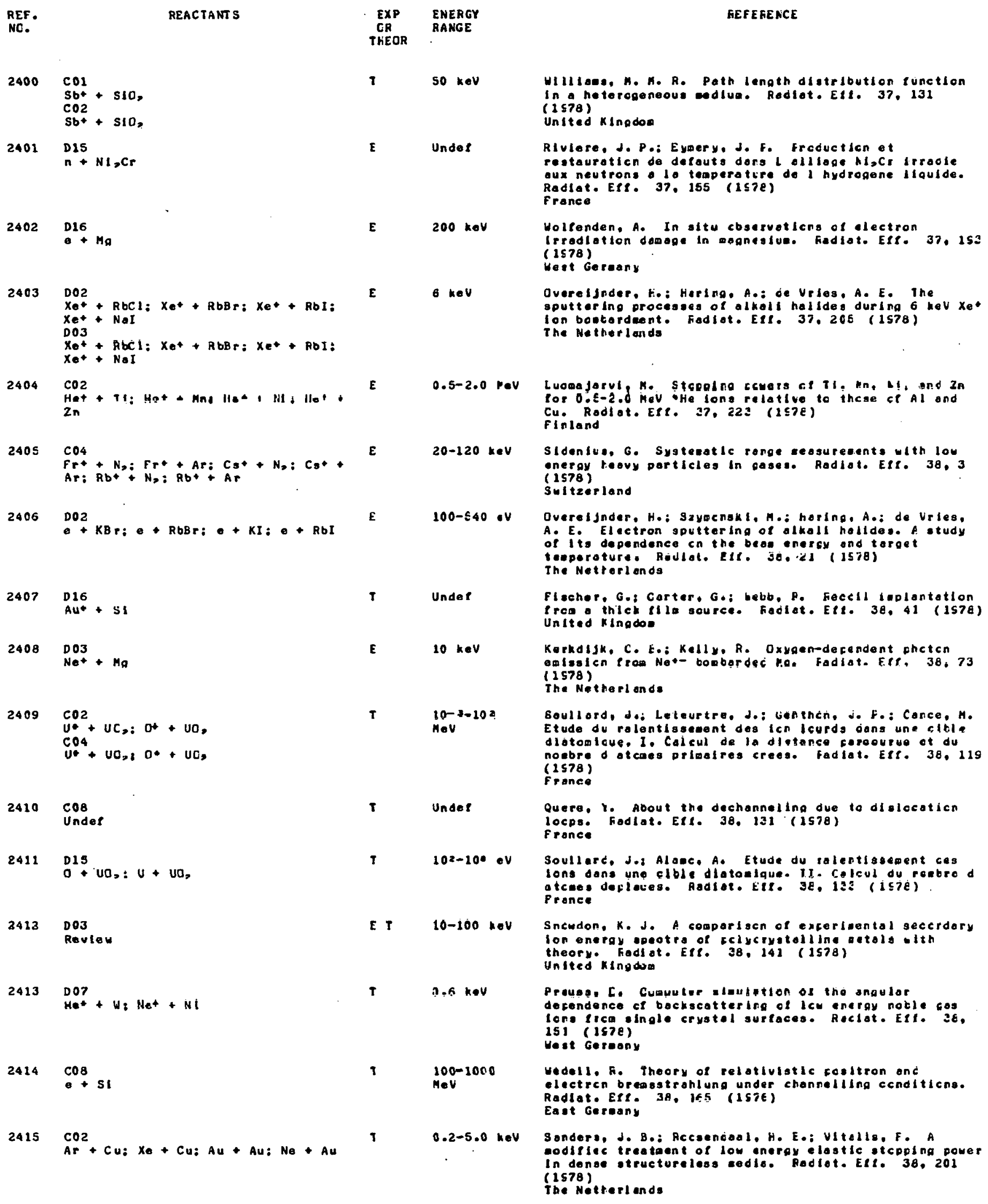


REF.
NO.

REACTANTS

2416 D15

n $+N b$

$2417 \quad \underset{002}{\mathrm{Cut}+\mathrm{Cu}}$

$2418 \mathrm{CO}$

$\mathrm{CO}^{*}+\mathrm{SI}: \mathrm{Ar}+\mathrm{Cu}$

$2419 \quad 016$

Under

2420

Dos
Art $+C u$

2421016

Undier

$2422 D 16$

Under

$2423 \quad D 15$

- + BI

2424

D15

- ss: n+ss

2425

D07
$H^{*}+A L: H^{+}+A U: H^{+}+S S$

2426

$\mathrm{CO}$
$\mathrm{B} \bullet+\mathrm{si}$

2427

D07

$\mathrm{H}^{+}+\mathrm{NI}: \mathrm{D}^{+}+\mathrm{NI}: \mathrm{He}^{+}+\mathrm{NI}: \mathrm{N}^{+}+$ NI

$2428 \quad D 15$

$+\mathrm{Cu}$

$2429 \quad \begin{aligned} & C 02 \\ & \text { Under }\end{aligned}$

$2430 \quad 1906$

Under

$2431 \quad c 08$

$+51$

D10

$\rightarrow \$ 1$

$\begin{array}{ll}\text { EXP } & \text { ENERGY } \\ \text { CR } & \text { FANGE } \\ \text { THEOR } & \end{array}$

$0.1 \mathrm{MeV}$

$40 \mathrm{keV}$$$
\text { E }
$$

Unde?

10-35 kev

Unde P

Undef

$0.6-1.2 \mathrm{MeV}$

$1 \mathrm{MaV}$

\section{T}

\section{E}

T

$\mathbf{T}$

T

Under

1 1-5 MeV
GEF EFENCE

Iransalasion electron wicrogccpy cbservatlcn cl

interatitial clusters in neutren-irracioted nlotium.

Raclat. Etr. 38. all (1S78)

United stotieg

van Wyk, G. N.: Solth, H. d. Ion tcatordmert Inouced pretarentiol crientoticn ir fclycrystelline Cu targets. foclat. Elt. 38, 245 (1578)

South Alrlco

Yacamura, Y.: Inuog. H. Sonine expanefcn of range and daese distributicns. Fadot. Ell. IE. $2 E$ (ISTE) Jacon

Doeura, J.: Coussement, R.: Pottyn, H. Model tcr the sI te pofulaticns ct heovy icns lafiantec in metols in the case where oll detects are lactile Rodiot. Eff. 39.11 (157e)

Belgiue

Ba lashcua, L. La: Borlscu, A. H.: Maghucua, E. S .: Molchoncv, $v$. A. Energy digtributicns cr last lonized recolls. Radiat. EfI. 35. 1S (1578: Soviet inicn

Winterbcn. K. B. Icn-ieplantation diatrituticns in non-unllorm torgets: crejected ronge. Radiot. Elt. 39. 31 (IS7E)

Canada

Wintertcn. K. B.: Sandera, J. E. Fnalyticol calculaticna of some lon-iaplantatjon decth ditributicna. Radiat. Etf. 35. is (1578) Conodo

Quelard, G.: Dural, J.: Ardonceau, J.: Lesueur, D. Energle seuil de deflocenent dons le tismuti. Radiat. Ef1. 39. 45 (1978)

Fronce

Ghonles. N. H.: Kulcinskl. G. L. P rate theory approach to the tie decendent icicstivctural develocment during irrociation. Radiate tff. 3s, 47 (1578)

Unitod States

Barrogon, A.: Garcia Sant Itonez, F, Surtoce roughness eflecto on the energy dispersion of bockscattered prctons. Radiat. Ert. 39, 61 (1578) Hexico

Horcovlch, A.: Eahlr, G.: Eernatein, T.: Kallsh, F. A nethod for ceasuring stopping powers cl channeled lons: boron in SI. Hadiat. Etf. 3S, EE (1578) boron

Hou, H.: Eckstein, H.; Verbeek, H. Sall ongle backscattering cf hydrogen. detterluo, reltio ond neon frow single and pclycrystalline nickel. Reolot. Ell. 39. 107 ( $1 \leqslant 7 \varepsilon)$ He ot Gereany

$0.5-3.0 \mathrm{meV}$ Tenenboum. A. Forooticn cf Frenkel calis in electron Irrodiatid copper. Rodiat. Etf. 29. 119 (ISTE) It aly

Edvabnyl, I. W.: Yalovets, A. F. Icnizoticn lcsses of past chorged particles in on onisctrocle wectue. Sov. Phys. J. 21.7 (1578)

Soviet linion

Andryushin. A. I.: Fedorov, H. W. Fescnant interaction between intense jectrcagnetic waves curing ionization cl an otom. Sov. Fhys.J. 21,45 (1578) sovitet linicin

Vorobect, A. A.: Fcrov, D. E.: Vcrcterev, S. A.: Kaplin, V. V. Eand structure of transverge-energy eigenvalues and strcng electron scattering by otouic plones ir angle cryotals. Sov. Fhyo.J. il S4 (ISTE) soviet Union 


\begin{tabular}{|c|c|c|c|}
\hline $\begin{array}{l}\text { REF. } \\
\text { NO. }\end{array}$ & REACTANTS & $\begin{array}{l}\text { EXP } \\
\text { CR } \\
\text { THEOR }\end{array}$ & $\begin{array}{l}\text { ENERGY } \\
\text { FANGE }\end{array}$ \\
\hline 2432 & $\begin{array}{l}C O 2 \\
e+C u: e+A 1 \\
E 11 \\
e+C u: e+A 1 \\
D 12 \\
e+C u: e+A 1\end{array}$ & $\mathbf{E}$ & $4-30 \mathrm{MeV}$ \\
\hline 2433 & $\begin{array}{l}\text { Al2 } \\
\text { Unde? }\end{array}$ & 9 & Under \\
\hline 2434 & $\begin{array}{l}\mathrm{Al}_{2} \\
\mathrm{HH}_{x} *+\mathrm{NH}_{3}\end{array}$ & $\mathbf{T}$ & Under. \\
\hline 2435 & $\begin{array}{l}\text { BO1 } \\
\text { Under } \\
\text { HO1 } \\
\text { Under }\end{array}$ & $\mathrm{T}$ & $\begin{array}{l}\text { HOI } \\
\text { Under }\end{array}$ \\
\hline 3436 & $\begin{array}{l}101 \\
818\end{array} \leq 1+$ & $t$ & \\
\hline 2437 & $\begin{array}{l}\text { Fol } \\
\text { SI+: }\end{array}$ & $\mathbf{T}$ & \\
\hline & & & \\
\hline 2438 & $\begin{array}{l}\text { Ho } 2 \\
\text { Under }\end{array}$ & I & Under \\
\hline 2439 & $\begin{array}{l}\text { BOI } \\
\text { Under } \\
\text { HO1 } \\
\text { Under }\end{array}$ & 1 & $\begin{array}{l}\text { Ho1 } \\
\text { Under }\end{array}$ \\
\hline $20 \Delta 0$ & $\begin{array}{l}\text { HOS } \\
\text { Under }\end{array}$ & 1 & Undof \\
\hline 2441 & 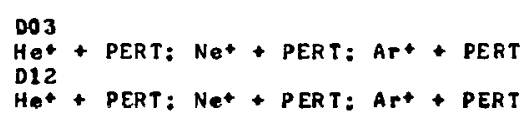 & E & $30 \mathrm{kov}$ \\
\hline 2442 & $\begin{array}{l}\text { Bo6 } \\
\text { Under } \\
\text { HO4 } \\
\text { Under }\end{array}$ & $\boldsymbol{T}$ & $\begin{array}{l}\mathrm{HO}_{4} \\
\text { Under }\end{array}$ \\
\hline 2443 & $\begin{array}{l}\text { 80? } \\
\text { Undef }\end{array}$ & $T$ & \\
\hline 2444 & $\mathrm{AeG}_{\mathrm{Ha}} \mathrm{O}+\mathrm{He}$ & $E$ & $2-60 \mathrm{keV}$ \\
\hline 2445 & $\begin{array}{l}E 04 \\
e+H_{-}: e+H D: e+D_{0} \\
E O S+H_{2}: e+H D: C+D\end{array}$ & $\boldsymbol{T}$ & $3-5 \mathrm{eV}$ \\
\hline 2446 & 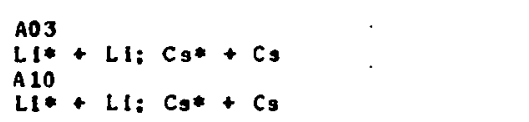 & $\mathbf{T}$ & Under \\
\hline
\end{tabular}

Lisin, V. A.: Yundo. N. T. An. investigotion ol electrcn-photon Pluxes ofter two-layer barrlers irradiated by a bea of lost wonoenergetic electrons. Sov. Fhys. J. 21,240 (157e)

Soviet Union

Cherkascv, H. R. Ispact thecry cl broodening of spectrol ilines. I. Method of calculating reloxiticn parametera. Sov. Fhys. J. 21.264 (1578) Soviet Unicn

Cherkascu. R. R. Impact thecry cl broadenting ct spectral ilinea. II. Selt-brcadening of inversicn lines of the $\mathrm{NH}_{3}$ colecule and rototional Iines of the Chis dolecule. SOv. Fhys. J. El, 2EE (15TE) Soviet Union

Ancsov. H. D. Interaction of on aton olth etreng classlcal radiaticn. Sev. Fhys. J. 2i. 35i (1978) Soviet Union

Paviov, R. N.: Veapisnali, F. V.: Mrivitakll, V. V. aicells Iunctluil aelliul fur lie calculation of the prepertles of atoms ith tro inner heles. I. Absclute ionizetion energies. Sov. Fhya.J. al. 354 (1578) soviet Union

Pavlov, A. N.: Vedringkll, F. E.: Krivitgkll, V. V. Greens tunctlcn eethod lor the colculation ct the - rcpertles cl atous wh twe inner holes. II. Cheolcal shifts of $x-r$ ay I ines. Sov. Fhye. J. 21. 358 (1978) soviet Unicn

Averbukh, I. S.: Kevarakit, V. A.: Parelean, N. F. Etfecta of the Landau-zener type in the noties erestre of Doleculas. Sov. Phys.-JETf 47, 64E (1578) Soviet Unicn

Letokhov, V.S.: Hinogin. V. E. Quontue notion of atces in the resonont lleld of a standing light wove Sov. Phis,-JEIP 47, ESO (ISTE) Soviet Unicn

Fadorov, H. U. Polarleatlin ef electreng procuced as a result of the regonance lonizetion of atoes by

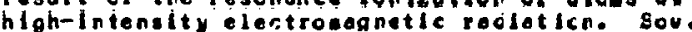
Phys--JETF 4\%, 202 (157E)

Soviet inton

Klyan, T. S.: Gritsuns, V. V.: fooki, J. H. En the continuous spectra enitted by particies knocked out by

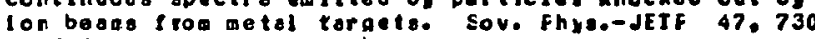
( 1578 )

Soviet Union

Nevessk I1. N. E. Two-level ayated in al nuscidal electric lield. Sev. Phys.-JETf 47, e19 (1578) Soviet Unton

Uarthnyen, T. A.: Frzhlbel'shli, S. G. Optleol excltotion ce colliding atcas te stote of quesibound motion. Sov. Phys.-JETf 47, E24 (1578) Soviet inten

Afroslacv. V. V.: Easalaev, A. A. i Lelthe, G. A.i Fanạu, H. N. Foradtion of Het long In varlous electrcilc stotes in Hoz+ + He collialona. Scv. Fhyg.-dFTF $\triangle 7$, ajt (j5is) Soviet inton

Deukarev, G. F.: Pozdneev. E. A. Application ef the Foddee equations to celculoticna ci dissoclative at taohwent eross sectlons. Sov. Phys.-JETP 47. 1045 $(1 \subseteq 78)$

soviet linicn

Chlbisov. H. I. Centributicn cithe treory of excitation transfer in tloo ccillalona of llke otose SOv. Phys.-JEJP IE. $2 E$ (IETE)

Soviet Union 
REF

REACTANT S

NO.

2447

Unde

HOG

Undet

2448

A13

$\mathrm{COD}++\mathrm{D}_{2}: \mathrm{COD}+\mathrm{Ne}_{\mathrm{O}} \mathrm{D}_{3}++\mathrm{CO}$

2449

$\underset{\mathrm{H}}{\mathrm{H}_{3}+\mathrm{Ne}^{+}}+\mathrm{H}_{3}++\mathrm{Kr}: \mathrm{D}_{3}++\mathrm{Ne}: \mathrm{C}^{+}+$

2450

HOC

hy $+N$,

2451

$E 03$

$e+H_{2} \mathrm{O}: e+D_{2} \mathrm{O}$

$e+H, O ; e+D>D$

2452

A14

$\mathrm{N} \rightarrow *+\mathrm{O}_{2}$

$e+\mathrm{N}_{2}+\mathrm{O}_{2}$

2453

A14

$\mathrm{H}+[\mathrm{H}+\mathrm{H}]$

2454

A13

Art $+\mathrm{H}=\mathrm{O}$

2455

Al3

$\mathrm{Ar}^{+}+\mathrm{H}_{2} \mathrm{O}$ : $\mathrm{Xe}^{+}+\mathrm{H}_{2} \mathrm{O}$

2456

A13

$A r^{*}+\mathrm{H}, \mathrm{O}$

2457

A11

$S F_{a} *+S f_{a}$

$2458 \quad \mathrm{HO} \mathrm{I}$

Under

2459 BO7

Under

2460

$\stackrel{A I I}{I>*}+I_{>} I_{>} *+X_{\theta}$

2461

rol

He Seq
ENERGY

RANGE
E

H06

Under

$1-12 \mathrm{eV}$

Theraol

E $\quad 2-10$ eV

o keV

Unde?

Undef

$625-802 K$

Under

\section{I}

T 293-413k
90-1000 eV

Delone. N. B.: 20n, B. A.: Kralnov, V. F. Cilfusion wechanise ce icnizaticn of highly excited ofces in on alternoting electrcagnet ic lield. Scu. Phyg.-JETf 48. 223 (IS7E).

Soviet Unicn

Zhurkin, E. S.: Sargayon, G. N.: Helkhesyon, R. I.: Tunitskil. N. N. Study of the forwari and back seccndory lcn-mclecule reocticns CCD*+ D, soling to or coulng fres $\mathrm{D}_{3}+$ + $\mathrm{CO}$. High Energy Chea lá. C2 $(1 \leq 78)$

Sorlet Unlc

Sargsyon, G. K.: 2hurkin, E. S.: Melkhesyan, F. T. Crcss sections of secondary icn-oclecule reacticns cf $\mathrm{D}_{3}{ }^{\circ}\left(\mathrm{H}_{3}{ }^{+}\right)$lans with Inert ooses. High Energy Ches. $12.62(1578)$

Soviet Unton

Draevsk 11, A. N.; Suchkov, A. F.: Shateko. Y. N. Possible creparaticn of chealcaliy octive nitrogen in $v-v$ exchange processes in a ncreoulilibilum secium of vitrationally excited aolecules. High Energy Cher. 12.135 (1578)

Soviet Union

Polyakova, G. N.: Fonvuk, A. I.: Erko, V. F.:

Pavilchenko. 0 . S. Forgaticn ci het excltec hycregen atcas by electron impact cn water aclecules. Hiah

Energy Chea. 1द, 167 (157E)

Sorlet unicn

Basov, K. E.: Lonllychev, V. A.: Penteleev, V. I.: Pofoulctev, V. I.: Gagul'skil, V. V.: Suchkcu, A. F.: Falzullcu, F. S.: Shebeko, Y. A.: Gorozhankin. E. V.: Kurankcy, V. V. Electrcicnlzotlcn syrthesis ol

nitrogen oxides. high Energy Chea. 12,224 (1578) Soviat linicn

Levitokil. A. A.: Folak, L. S. Study ct the

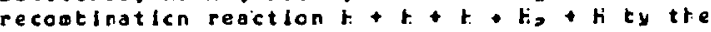
classical trajectorles wethco. High Erergn Ches. 12, $245(1578)$

Soulet Union

Nikolaev. E. N.: Tortsyrev. G. [. Forooticn ci thermodynaticaliy unlovoratle $(t, 0)$ sut $n^{+}$clusters Ith $n=1-30$. High Energy Chex. 12, 253 (1578) Soviet Unicn

Arlfov, U. A.: Pozharov, S. L. Mechanism of the coraticn of $\mathrm{H}^{+}(\mathrm{H}, \mathrm{C})$ sut $n-O H$ clusters. High Energy Chew. 12, 227 ( 1578$)$

Soviet unicn

Arlfov, U. A.: Fozharav, S. L. Inftiol stageg of clustering reocticns in water vopor. High Eneray Ches. 12,331 (1578)

Soviet Union

Vasilenko, L. S.: Fopova. T. Y.: Fubtgcva, N. N.: Skvortscy, M. N. Investigoticn of dolecular relaxation In SF ky tre amolitude-mcdulated vave sethod. Scu. J. Quentud Electron. 8, E? (ISTE)

Soviet Unicn

Baklanov, E. V.: Lubetskil. E. Y. Iwc-cheten absorpticn resonance in the presence cl transit effects. Scr. J. Guantus Electrcn. e, 51 (1S78) Sovlet Unicn

Yakovlerke, S. I. Laser-ircuceo rodietive cclligions (review). Sou. J. Quantud Electrcr. E. 151 (1978) Soviet linicn

Stcllov, Y. Y. Invest igaticn of the lluorescence of Boleculor icdine in the 340 ni band. Scu. J. Quantum Electrcn. $E, 2 z 3$ (2S7e) Soviet Unicn

Bolko, V. A.: Plkuz, S. A.: Foenov, A. Y. Intenslties of rescnance line satellites of hellug-like lons with $Z=12-23$ in laser closed $x$-ray eElselon. Sov.J. Quantua Electron. 8,226 (1S7E) Soviet Union 


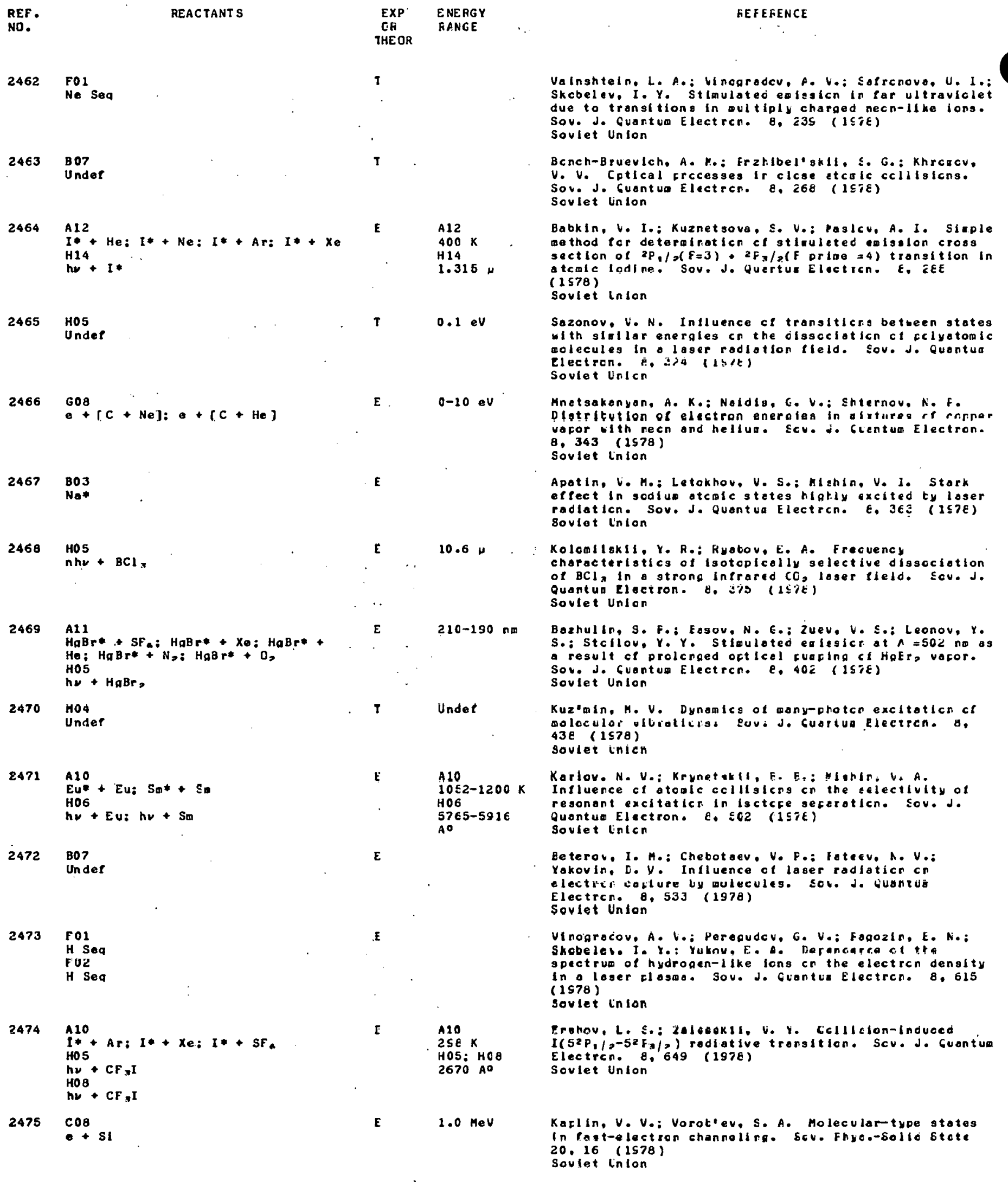


REF.

REACTANT S

NC.

$2476 \mathrm{CO} 2$

e+Au

E18

e $+\mathrm{Au}$

2477 D15

$\mathrm{H}^{*}+\mathrm{Al}$

2478006

e+[Al + Ho $]$

2479 DOZ

$\mathrm{Ar}^{+}+\mathrm{SI}$

$2480 \quad 004$

$\mathrm{Het}+\mathrm{SI}$

$2481 \quad 004$

- GaP

$2482 \quad 006$

$+4$

2483 D06

C + Mo

2484

$\mathrm{H}_{\mathrm{H}}^{\mathrm{A}} \mathrm{O}+\mathrm{He}: \mathrm{Kr}+\mathrm{Kr}$

A17

$\mathrm{He}^{2+}+\mathrm{He}: \mathrm{Kr}++\mathrm{Kr}$

$2485 \quad A 07$

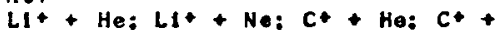

$\mathrm{Ne:} \mathrm{N}^{+}+\mathrm{He} \mathrm{N}^{+}+\mathrm{Ne} \mathrm{Ne}^{+}+\mathrm{He}: \mathrm{Ne}^{+}$

$+\mathrm{Ne}: \mathrm{Na}^{+}+\mathrm{He}$ : $\mathrm{N}_{+}^{+}+\mathrm{Ne}: \mathrm{Ha}^{+}+\mathrm{He}$ $\mathrm{Ha}^{+}+\mathrm{Ne}$

2486

A07

$\mathrm{Na}++\mathrm{Ne}: \mathrm{Mg}^{+}+\mathrm{Ne}$

2487

$\mathrm{EO} 2$

e+He: e+Ne; e+Ars e+Kr; e+

A0 3

$\mathrm{Ba}_{\mathrm{Xe}}+\mathrm{Kr}: \mathrm{Ca}++\mathrm{Ar}: \mathrm{Ca}^{+}+\mathrm{Xe}: \mathrm{Sr}^{+}+$

$2489 \quad E 02$

e+Aq: $\mathrm{A}+\mathrm{Ne}$

E17

e+Ag: $e+N e$

$2490 \quad$ D 13

$\mathrm{H}+\mathrm{ZrC}$
EXP ENERGY

CR RANGE

THEOR

E

5-30 keV

T

$0.2 \mathrm{HeV}$

$\mathbf{E}$

$0-50$ ev

E

$150-500 \in V$

E

1-10 keV

E.

$0 \cdot 2-3.0 k \in V$

2-50 eV

$0.5-1.5 \mathrm{keV}$

Undet

$100-2000$

$100-2000$ eV

$0.025-1 \mathrm{eV}$

$0.1-2 k \in v$

$0.6-8 \mathrm{kev}$

T

$300-1600 \mathrm{~K}$
REFEFENCE

Vyotsk In, A. Y०: Selrnov, B. N. Electron-beos eneray losse in a buik gcld target. Sov. Phys,-solic stote 20,143 (1578)

Sovlet Union

Taratin. A. M.: Vorob'ev. E. A. Characteriotles cl the energy and opatial diatributicns ol pileary knceked-out atose in olngle cryotals. Sov. Phya--Eolld state 20. $56 E(1 \leqslant 78)$

Soviet Unicn

Erohova, T. P.: Korablev, V. V.: Herezcu, X. A.

Choracterlotics of elootle reflection ct olcu electrons fres ofle-single-crystal systea. Scu. Phya--Solid

Stote 20,710 (1978)

soviet inion

Labunov, V. A.: Borlsenko, V. E, Temperature

dependence of the eputtering ccelslctert ot allicon.

Sov. Phys.-Solid state 20,712 (1978)

Sovlet tinion

Dorozhkin, A. A.: Petrov. A. A.: Fetrev. N. N. Fele of the Auger processes In electrcn eslasicn due to boberdaent with light lons. Sov. Physo-solid Etate 20. 734 (1S7E)

Soviet Unien

Atonlna, L. F.: Stuchlnekil, G. D. Eneroy apectro of secondsiy electrons amitted froe gallius phcaphide with a reduced work function. Sov. Fhyse-Sclid state 20 . 874 (1978)

Soviet Unlon

Ershovo, T. P.: Korablev, V. V.: Herozov, Y. A. Elastle rellection of low electrons fros tungaten Ingle crystal. Sov. Phys.-Solld Etate 20,905 ( 1578$)$

Sovlet Unton

Gotoyunova, M. V.: Zaslovskli, S. L.: Fronin, I. I. Anleotropy of the elatic refiecticn of electrcno fros - Dolybdenue single crystal. Sov. Phys.-Solid stoto 20. 919 (2978)

Soviet Union

Nikulin, V. Ko: Guahchina, N. A. Shielded-orbital model for quasi oolecule analysis of icr-aton collisicns. Sov. Fhys.-Tech. Phys. 23.7 (1578) soviet Union

Letypov. 2. 2.: Shoporenko. A. A. Ionizaticn cl otoalc

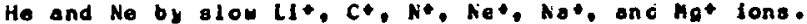
Sov. Phys,-Tech. Fhys. 2J, 12 (157E) Soviet Union

La typov, 2. 2.: Shaporenke. A. A. Use of effective lonization cross sections to exsalne ncnadiabatic transition. In the Nat-Ne and hat-he systesa. Sov. Phys.-Tech. Phya. $23: 114$ (1578) Soviet Unicn

Gus'kov, Y, K.: Sovwov, R. V.: Slobodyonyuk, V. A.

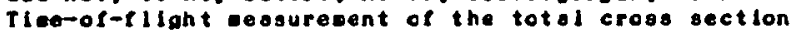
for elostlc scattering of low-eneray electrons $(E=0.02 E-1.0$ U) ty He. Ne, $A r, K r$, and $X e$. Scu. Phys.-Jech. Phys. 23, 167 (1S78) Soviet Union

Borlsov, V. B.: Egcrov, V. 8.: Zetserkcunyuk. H. M.: Poetor, A. A.: Forts, H. E. Ion ccaposition of a pulsed elcrowove diechorge in on lrert pas. Scu. Phys.-Tech. Phys. 23. 171 (1978) Boviet Union.

Akkermonn, A. F.; Chernov, G. $Y$. Elastle scattering of - lectrcns by atose in tho keV ronge. Scu. Fhyso-Tech. Phys. 23, 247 (1578) Sovlet Union

Matskevich, T. Lo: Krachlno, T. V. Hycrogen adsorption on zircenlus corbide and tis rhentur olloya. Bov. Phys.-Tech. Phya, 23, 314 (1578)

Soviet Union 


\begin{tabular}{|c|c|c|c|c|}
\hline $\begin{array}{l}\text { REF. } \\
\text { NO. }\end{array}$ & REACTANT S & $\begin{array}{c}\text { EXP } \\
\text { CR } \\
\text { IHEOR }\end{array}$ & $\begin{array}{l}\text { ENERGY } \\
\text { RANGE }\end{array}$ & REFEFENCE \\
\hline 2491 & $\mathrm{CO}^{\mathrm{C}}+\mathrm{Al}$ & $\tau$ & $0.5-500 \mathrm{keV}$ & 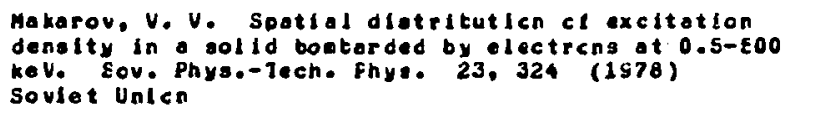 \\
\hline 2492 & $\begin{array}{l}\text { Do9 } \\
\text { Under }\end{array}$ & 1 & $1-100 e V$ & $\begin{array}{l}\text { Kishinesskil, M. E. Secondory negative-ion exisalon. } \\
\text { Sov. Physe-Tech. Physe 23, } 456 \text { (1978) } \\
\text { Soviet. Union }\end{array}$ \\
\hline 2493 & $\mathrm{H}^{\mathrm{AOS}}+\mathrm{H}^{+}: \mathrm{SI}^{2+}+\mathrm{Ca}$ & $\mathrm{T}$ & $\begin{array}{l}100-100 \\
c a / s\end{array}$ & 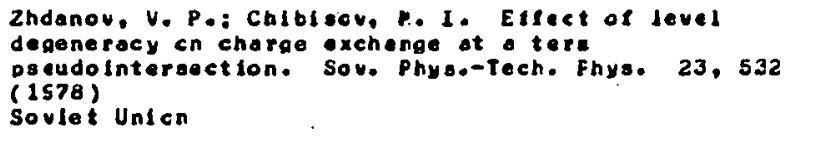 \\
\hline 2494 & $\mathrm{e}^{\mathrm{GO}}+\mathrm{Ne}$ & E & $470-1600 \mathrm{~K}$ & $\begin{array}{l}\text { Dealdou, V. I.: Kolovolev. N. E. Callisions of the } \\
\text { second kind between ol cetrong and exclted neon atcess. } \\
\text { Sov. Physo-tech. Phys. 23, } 610 \text { (1S78) } \\
\text { Soviot Union }\end{array}$ \\
\hline 2496 & $\begin{array}{l}\text { Do6 } \\
\text { Unde? }\end{array}$ & $\mathbf{T}$ & Undet & 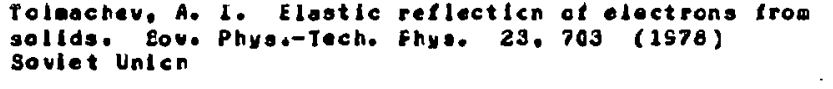 \\
\hline 2496 & $\begin{array}{l}\mathrm{Al}_{2} \mathrm{~N} \\
\mathrm{CH}_{0} ; \mathrm{C}_{2} \mathrm{H}_{a} ; \mathrm{CO},\end{array}$ & E & $00-800 \mathrm{~K}$ & 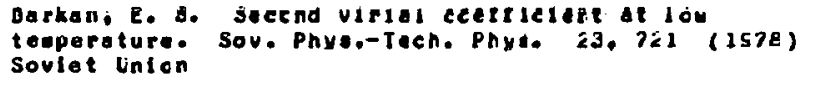 \\
\hline 2497 & 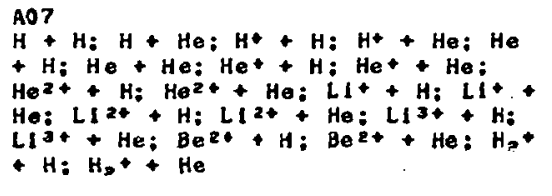 & $\mathbf{T}$ & $\begin{array}{l}100-6 \times 104 \\
\mathrm{~cm} / \mathrm{s}\end{array}$ & 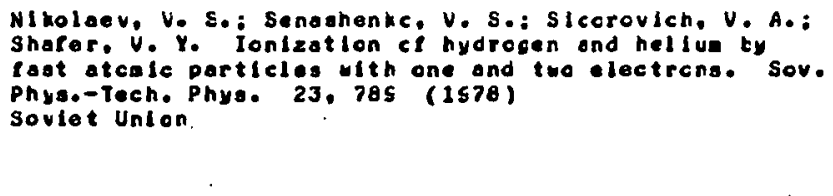 \\
\hline 2498 & 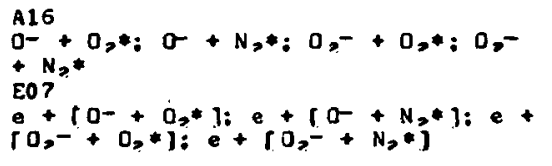 & $\mathbf{T}$ & $0.1-10 \bullet V$ & 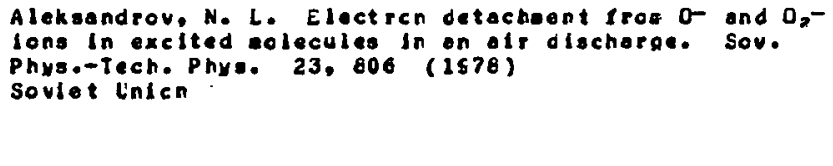 \\
\hline 2499 & $\mathrm{H}^{+4}+\mathrm{Ar}: \mathrm{H}^{+}+\mathrm{Sb}$ & $\mathbf{T}$ & $10-1000 \mathrm{keV}$ & 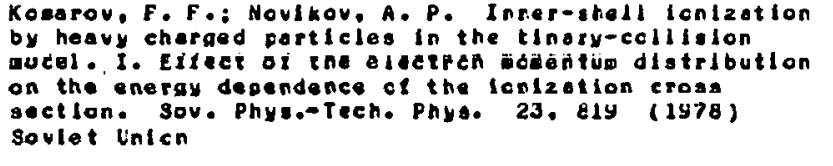 \\
\hline 2500 & $A_{17}^{A 17} \cdot K_{1}$ & $\boldsymbol{T}$ & Undẹf & 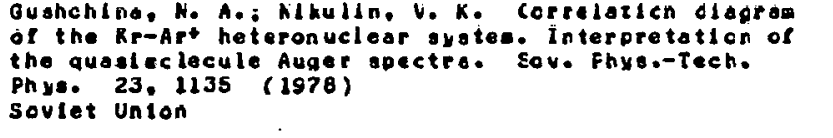 \\
\hline 2501 & $\mathrm{H}_{\mathrm{Ag}}^{\mathrm{AOP}}+\mathrm{Cu}: \mathrm{H}^{+}+\mathrm{Ag}: \mathrm{He}^{+}+\mathrm{Cu}: \mathrm{He}^{+}+$ & E & $150-800 \mathrm{keV}$ & 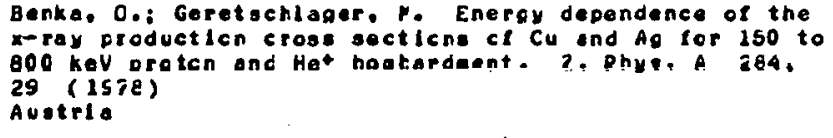 \\
\hline 2502 & 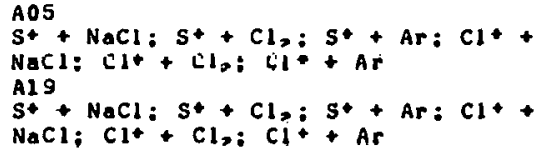 & $\mathbf{E}$ & $3.82-48 \mathrm{MeV}$ & 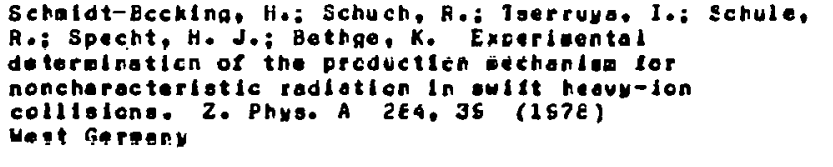 \\
\hline 2503 & $\begin{array}{l}A 03 \\
P b+A G: P b+A U \\
A 19 \\
P b+A g: P b+A U\end{array}$ & E & S.A KaV/Amu & 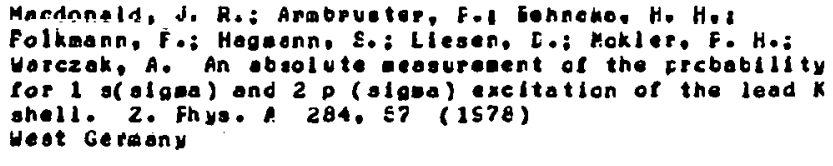 \\
\hline 2504 & $\mathrm{NOB}_{\mathrm{Ne}}+\mathrm{Cu}: \mathrm{Ar}+\mathrm{Cu}: \mathrm{Kr}+\mathrm{Cu}$ & $\mathbf{T}$ & Under & 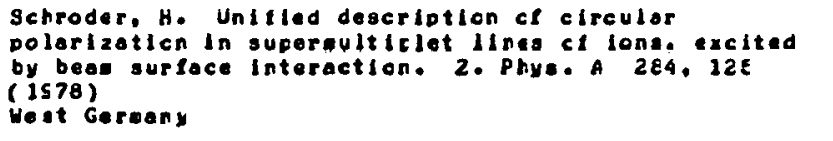 \\
\hline 2505 & 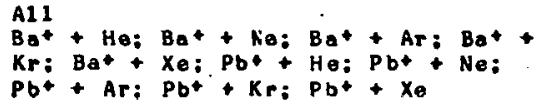 & E & Therael & 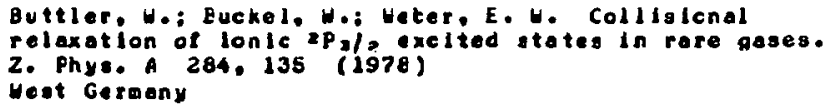 \\
\hline
\end{tabular}




\begin{tabular}{|c|c|c|c|c|c|c|}
\hline $\begin{array}{l}\text { REF. } \\
\text { No. }\end{array}$ & & REACTANTS & & $\begin{array}{l}\text { EXP } \\
\text { OR } \\
\text { THEOR }\end{array}$ & $\begin{array}{l}\text { ENERGY } \\
\text { RANGE }\end{array}$ & GEFEFEACE \\
\hline 2506 & $\mathrm{Cs}_{\mathrm{C}}^{\mathrm{A} 15}+\mathrm{Ca:} \mathrm{Na}$ & $+\mathrm{N} \bullet$ & & $\mathbf{E}$ & Theraal & $\begin{array}{l}\text { Heter. H. G. Studies of the atod-dider polarlzation } \\
\text { trongter. Port II. Z. Phys. a } 284,145 \text { (1978) } \\
\text { Hest.geraony... }\end{array}$ \\
\hline 2607 & 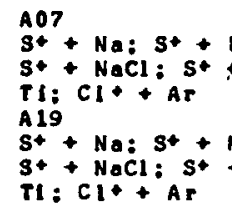 & 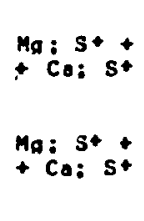 & 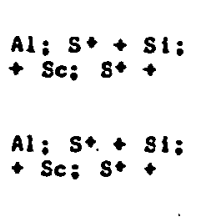 & E & $3.9-48 \mathrm{M} \cdot \mathrm{V}$ & 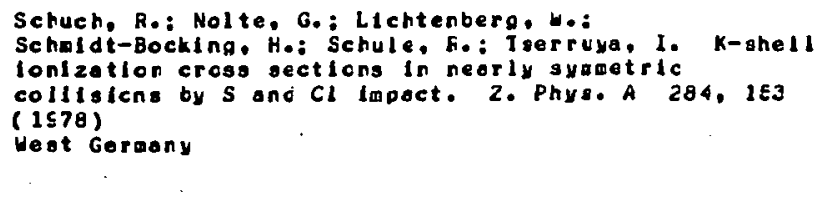 \\
\hline 2508 & $\begin{array}{l}H 06 \\
h v+N\end{array}$ & & & $\mathbf{T}$ & $0.1-1 \mathrm{RY}$ & $\begin{array}{l}\text { Hempe, K. Phctolicnizatlon crose secticn of atcolic } \\
\text { nitrogen colculated by the gany-chonnel quantus defect } \\
\text { aethod. 2. Phys. A } 284.247 \text { (1S78) } \\
\text { West Geraany }\end{array}$ \\
\hline 2509 & 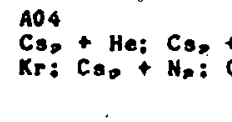 & $\begin{array}{l}+\mathrm{Na}: \mathrm{Cs}_{*} \\
\mathrm{Cs}=+\mathrm{CH}_{0}\end{array}$ & $+\operatorname{Ar}: \mathrm{Cs}_{+}+$ & E & Thermal & 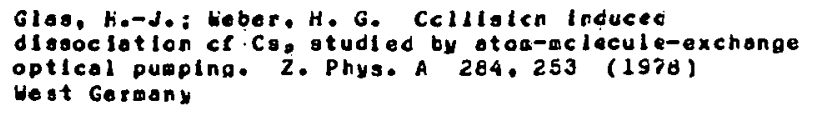 \\
\hline 2510 & $\begin{array}{l}\mathrm{HOA} \\
\mathrm{hN} \\
\mathrm{HOB}+\mathrm{BO} \\
\mathrm{he}+\mathrm{Ba}\end{array}$ & & . & $\mathbf{E}$ & ses na & 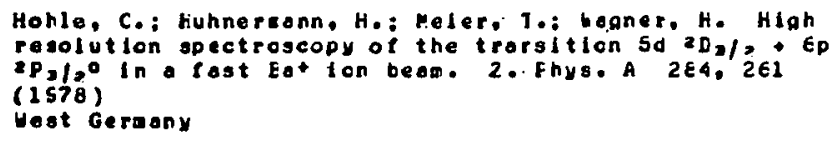 \\
\hline 2511 & $\begin{array}{l}\mathrm{AOS}^{05} \\
\mathrm{H}^{+}+\mathrm{Al}: \mathrm{H}^{+}+\end{array}$ & S1: $H^{*}+$ & $T I: N^{+}+A l:$ & $E$ & $0.5-14 \mathrm{KeV}$ & 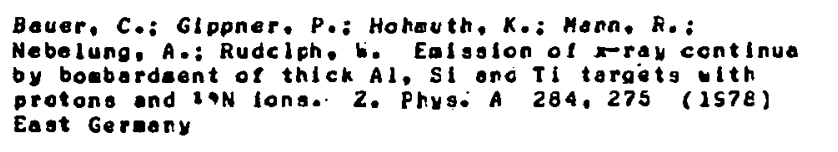 \\
\hline 2512 & $\begin{array}{l}\text { E1s } \\
\text { Under }\end{array}$ & & & E. & .. Under & 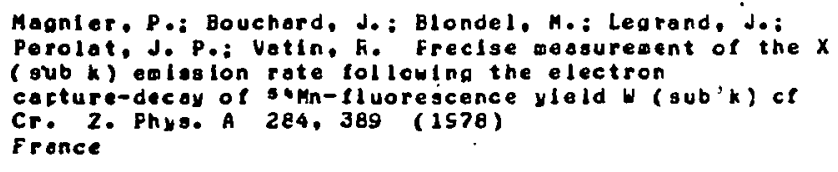 \\
\hline $2 \varepsilon 13$ & $\begin{array}{l}\mathrm{H} 08 \\
\mathrm{Hu}_{0}+\mathrm{Cs}\end{array}$ & . & & E & $6231 A^{\circ}$ & 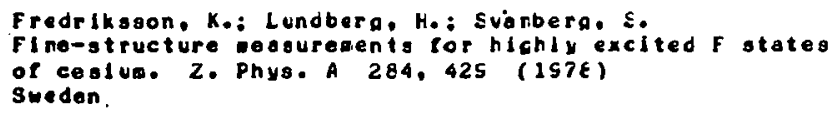 \\
\hline 2614 & $\begin{array}{l}\text { A07 } \\
\text { Under }\end{array}$ & & . & $\mathbf{T}$ & $\begin{array}{l}550-1400 \\
\mathrm{HeV}\end{array}$ & 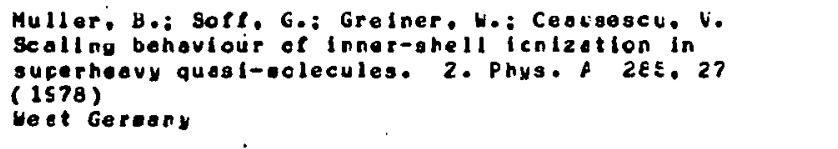 \\
\hline 2515 & $\begin{array}{l}\mathrm{CO} 2 \\
\mathrm{He}^{2+}+\mathrm{SI}\end{array}$ & - & $\therefore$ & $\mathbf{E}$ & $\begin{array}{l}\text { G.05-8.7E } \\
\mathrm{MeV}\end{array}$ & 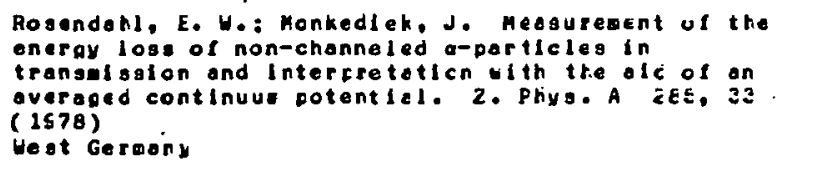 \\
\hline 2616 & $\mathrm{U}_{\mathrm{A}}^{\mathrm{A} 7}+\mathrm{U}: \mathrm{U}+\mathrm{Pb}:$ & $\mathrm{Pb}+\mathrm{U}:$ & $\mathbf{P b}+\mathbf{P b}$ & $\mathbf{T}$ & 4.7 Movjesu & 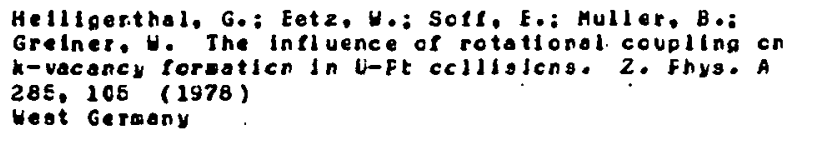 \\
\hline 2517 & $\mathrm{Cs}_{\mathrm{C}}^{15}+\mathrm{RO}$ & . & . & E & Theraal & 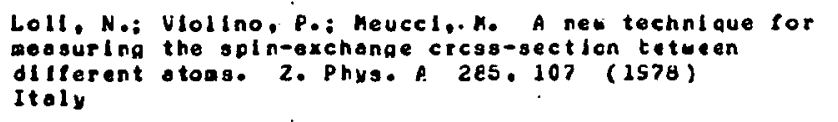 \\
\hline 2518 & $\begin{array}{l}H 08 \\
\text { ho }+\mathrm{Ba}\end{array}$ & & & $\mathbf{E}$ & $\begin{array}{l}1000-5000 \\
A O\end{array}$ & 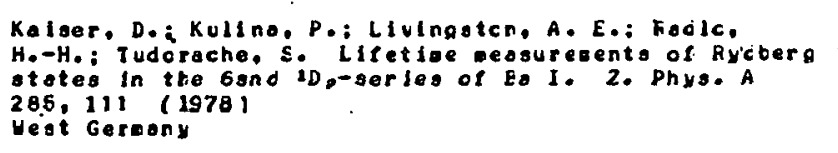 \\
\hline 2519 & $\begin{array}{l}\text { Bo7 } \\
\text { Under }\end{array}$ & & & $\mathbf{T}$ & & 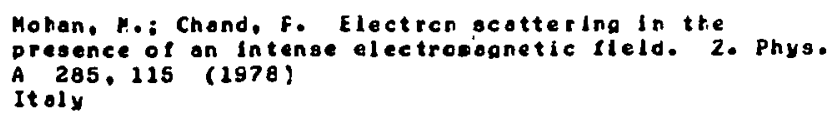 \\
\hline
\end{tabular}




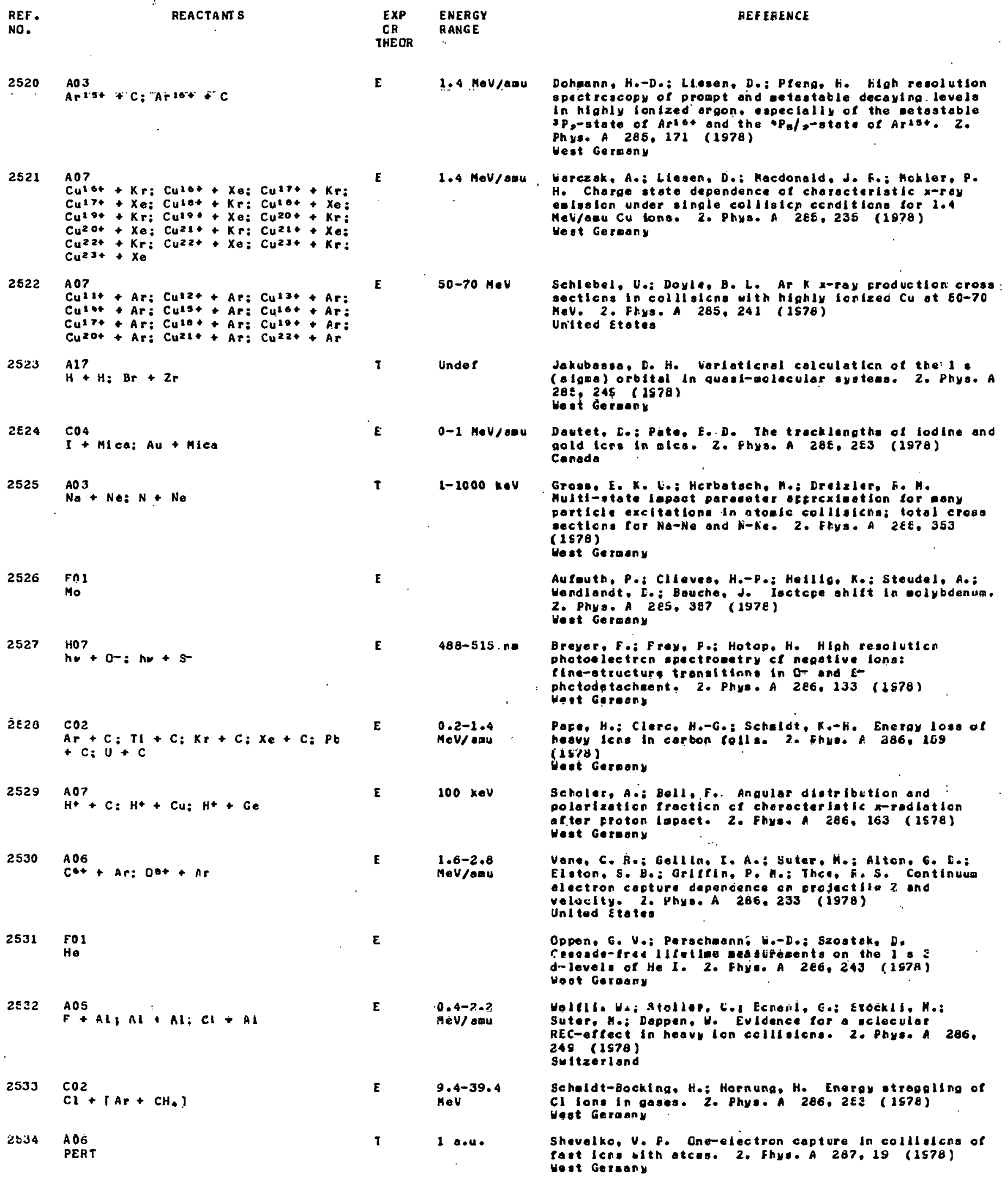


REF •

NC.

2535

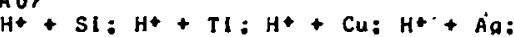
$\mathrm{He}^{+}+\mathrm{Si}$ : $\mathrm{He}^{+}+\mathrm{Tl}_{1} \mathrm{He}^{+}+\mathrm{Cu}^{-} \mathrm{He}^{+}$ $\mathrm{Ag}: \mathrm{N}^{+}+\mathrm{SI}^{+} \mathrm{N}^{+}+\mathrm{TI}^{\circ} \mathrm{N}^{+}+\mathrm{Cu}^{+} \mathrm{N}^{+}+$ Ag.

2536

A05

$\mathrm{Ag}+\mathrm{Ag}: \mathrm{Nb}+\mathrm{Nb}: \mathrm{Br}+\mathrm{Br}: \mathrm{Ge}+\mathrm{Ge}:$ $\mathrm{Fe}+\mathrm{Fe}$.

$\begin{array}{ll}2537 & \begin{array}{l}\mathrm{E03} \\ \mathrm{e}+\mathrm{H}\end{array} \\ 2538 & \mathrm{AO7} \\ & \mathrm{H}+\mathrm{H} \\ 2539 & \mathrm{AO3} \\ & \mathrm{Nb}+\mathrm{Kr}: \mathrm{Kr}+\mathrm{Nb} \\ \mathrm{AO5} \\ \mathrm{Nb}+\mathrm{Kr}: \mathrm{Kr}+\mathrm{Nb}\end{array}$

$2540 \quad H 08$

$2 h y+L I$

$2541 \quad A 03$

$\mathrm{Ne}^{3+}+\mathrm{N}_{2}: \mathrm{Ne}^{3+*}+\mathrm{N}_{2}$

$2542 \quad \mathrm{CO}^{+}+\mathrm{C}$

FO 1

$\mathrm{N+}: \mathrm{N} 2+$

2543 AO6

$\mathrm{H}_{3}+\mathrm{H}_{3}: \mathrm{H}_{2}+\mathrm{He}_{\mathrm{O}} \mathrm{H}_{2}+\mathrm{Ar}^{+}$

2544

$\operatorname{Co7}$

Arist +C: Arsot $+C$

2545

A 06

$\mathrm{Cs}^{+}+\mathrm{Cs}_{\mathbf{s}}$

2ะ40

$\mathrm{COZ}+\mathrm{C}: \mathrm{Ar}+\mathrm{Al}: \mathrm{Ar}+\mathrm{AQZ}: \mathrm{Ar}+\mathrm{AU}:$

$A r+C: A r+A l: A r+A G: A r+A U:$
$A r+B I: K r+C: K r+A l: K r+A g:$

$K_{r}+A u: K r+B I: X_{e}+C: X_{e}+A l:$

$X_{e}+A g: X_{e}+A U: X_{e}+B i: U+C: U$

$+A l: U+A g: U+A u: U+B I$

$\cos$

$A r+C: A r+A l: A r+A g: A r+A u ;$

$\mathrm{Ar}+\mathrm{Bl}: \mathrm{Kr}+\mathrm{C}: \mathrm{Kr}+\mathrm{Al}: \mathrm{Kr}+\mathrm{Ag}_{\mathrm{g}}$

$\mathrm{Kr}+\mathrm{Au}: \mathrm{Kr}+\mathrm{Bi}: \mathrm{Xe}+\mathrm{C}: \mathrm{Xe}+\mathrm{Al} ;$

$X_{e}+A_{a}: X e+A u: X e+B i: U+C: U$

$+A l: U+A g: U$ + $A U: U+B I$

$2547 \quad 0.5$

$\mathrm{H}^{+}+\mathrm{In}: \mathrm{H}^{+}+\mathrm{Sn}: \mathrm{H}^{+}+\mathrm{Nd}: \mathrm{H}^{+}+\mathrm{DY}:$

$\mathrm{H}^{*}+\mathrm{Pt}^{\circ} \mathrm{H}^{+}+\mathrm{All}$

$2548 \quad A 07$

$\mathrm{NI}+\mathrm{NI}$

\section{$\mathrm{AO3}$}

$\mathrm{He}^{+}+\mathrm{Ne}$

A 06

$\mathrm{He}+\mathrm{Ne}$ $\begin{array}{cl}\text { EXP } & \text { ENERGY } \\ \text { OR } & \text { RANGE } \\ \text { THEOR } & \end{array}$

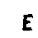

$\mathbf{E}$

$0.17-2$
MeV/anu

$18 \mathrm{MeV}$

E 007

1-10 MeV

E $\quad 10 \mathrm{keV}$

E 1-3 MeV

T $12-90 \mathrm{MeV}$

E 10-355 keV
FEFEFENCE

Baver. C.: tonn. E.: Fudoloh. W. K-shell ienization of Si. II. $C u$ and $A g$ for incident retone. He and $1+N$ lons in the energy range of 0.17-2.0 Mev/amu. 2. Phys. A $287,27(1578)$

East Geraany

Stcller, C.: toltli, H.: Bcrani, G.: Mcrenzonl, E.: Stcckl1. H. The onisotrofy of the MO-EO (slama)-radiaticn obgerved in syodetric heavy icn collisicns. 2. Phys. A 2E7, 33 (1S7E) Switzerland

Blum, K.: Fitchard, E. E.: Kleinpospen, H. Ccherent excltation of the $n=3$ level cl hyorogen in ollelc tree replon. 2. Hhys. A 287,127 (1sie) Sectianc

Ittel, H.: Bell, F, N-stell ionizaticn of tungsten by prcton Irfoct. 2. Fhys. A 287, 142 (1978) West Germony

Frank. Ho: Koun, K.-H.: Monfrass. P. The excitaticn cr the ls (sigaa) and $2 p$ ( 9 lgou) quagleolecular rodlation in Nb+Kr collisicns. 2. Fhys. A $2 E 7$, IAS (ISTE) Soviet Union

Koyal gki, J.: Neumonn, F.: Suhr, H.: binkler, K.: zu Putlitz. G. Iwo-phctćn intracavity dye laser

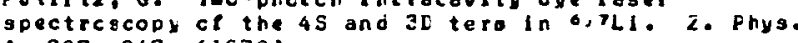
A 287,247 (1578)

West Germon

Schule, fi.: Steintauser. f.: Stafast, t..: Bethoe, Ko Collisicnal conflguration-rearrangewent in fast neon projectiles. $Z$. Phys. A zE?, $34 Z$ ( 1578$)$ west Geriony

Brink, J. A.: Coetzer, F. J.: Cliver, J. F. I.: van der Westhulzen, P.: Pretorlag, F.: Hchurrob, W. F. Level lifatias in $A I$ and $N$ III: opforotus ano results. Z Phys. A $26 E, 1$ (1S7e)

South Africa

Vogle, M. Lissociative charge excrange in collisions c $10 \mathrm{keV} \mathrm{H},+$ long on t., He, ond fr torgets. 2. Phys. A 288,7 (1S7E)

Weat German

Dohmann. H. D.: Ffeng, he Measureaent $c$ f the porulaticn cf the "Fs $p^{-3}$ tate in Arist by cascadirig processes. 2. Phys. A 28E, 2S (1S7E)

West Germony

Nienstoct, H.: Ganlik, C.: zu Pullitz, G.: beber, H. Go Cress secticn for the rescrent chorge exchange Cs+ $+\mathrm{Cs}^{+}$ at theral energieg. Z. Phys. A 286 . jos (1STE) Wegt Germony

Nlckel, f.: Marx, L.: Guttner, K.: holaann, S.: Munzenberg. G. Multiple gcatiering arc ellesiny luss of fost heevy lons in thir sclid targets. 2. Fhys. A $28 E, 12 E(1578)$

Hest Germany

Khon, H. F.: Hopking, B. G.: Cruooton. D. Protcn induced $L x-r a y$ crcss-secticns for In, Sn, No, Ly, ft. and $A u$. 2. Phys. 288.133 (1SiE) United ingdor

Antolt, R. Theory of the angular distributicn cr molecular ctital $K$ z-ray geen in heavy-ion-otor

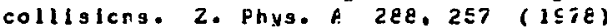
west Gerrany

Wittmann, Wo: Andro. H. J. Coservaticr of the hel $33 p$ polarizaticn ofter dedium eneroy fiet-Ae colliglons with incoct paroweter selection. 2. Phys. A ZeE, 335 (1578)

Hopt reran 


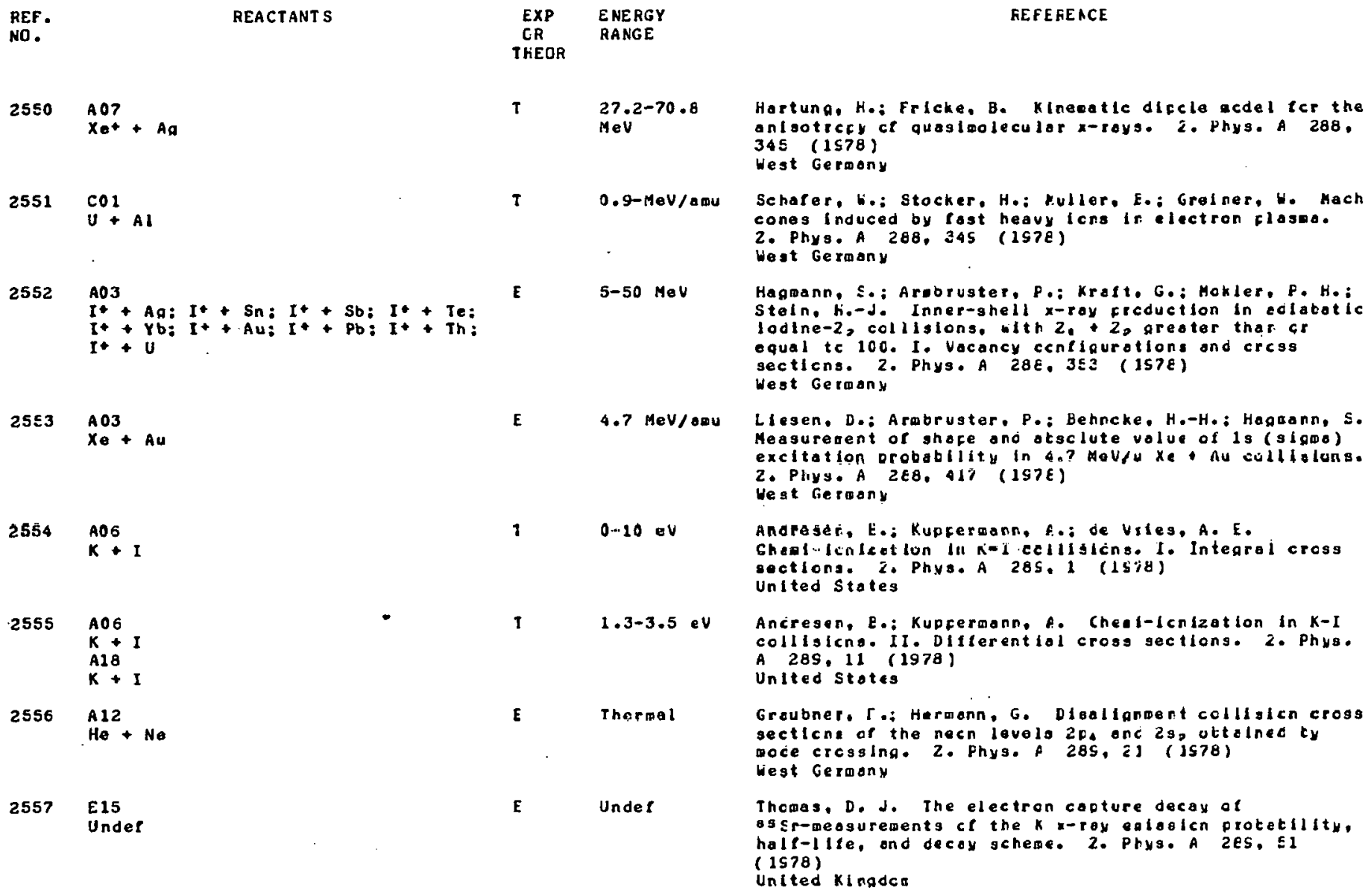

$27.2-70 \cdot 8$
$\mathrm{MeV}$

T

$u+A I$

2552

$\mathrm{A03}$

$I^{*}+\mathrm{Ag}^{\circ} \mathrm{I}^{*} \mathrm{Sn}: \mathrm{I}^{+}+\mathrm{Sb}: \mathrm{I}^{*}+\mathrm{Te}:$

$\mathrm{I}_{+}^{+} \mathrm{Yb}: \mathrm{I}^{+}+\mathrm{Au}: \mathrm{I}^{+}+\mathrm{Pb}: \mathrm{I}^{+}+\mathrm{Th}:$

$\mathrm{I}+\mathrm{U}$

$2553 \quad \begin{aligned} & \mathrm{AOS}_{\mathrm{X}} \\ & \mathrm{Xe}_{\mathrm{O}}+\mathrm{Au}\end{aligned}$

2554

A06
$K+I$

2555

$A 06$
$K+I$
A18

A18
$K+I$

$2556 \quad$ A 12

He + Ne

2557 E15

Under

E $4.7 \mathrm{MeV} / \mathrm{amu}$

1

$0 \cdots 10 \mathrm{eV}$

$1.3-3.5$ eV

Tharmal

E

$0.9-\mathrm{HeV} / \mathrm{amu}$

5-50 $\mathrm{MeV}$

Under

KEFE FEACE

Hartung. H.: Frlcke, B. Klneatle difele acdel lcr the an isoticey ct quasimolecular x-roys. 2. phys. A 288. $345(1 \leqslant 78)$

west Geroony

Sehafer, H.: Stacker. H.: Muller, E.; Gralner, H. Hach cones linduced by fast heauy lcns ir electron flasea. 2. Phys. A 288 , 345 (1978)

Weat Germany

Hagmann, S.: Arobrugter, P.: Kratt, G.: Hokler. F, H.: Stelin. fi.-d. Inner-ghell $x$-roy creduction in colatotic lodine-2, collisions, with 2 . 2 , greoter thar 4 r

equal te 100. I. Vacancy cencigurotions and crcss secticns. 2. Phys. A $28 E, 3 E 3$ (157E)

West Germany

Llesen. D.: Armbruster. P.: Behncke. H.-H.: Haguann, S. Measurerent or shafe ano otsclute volue of ls (slgwo)

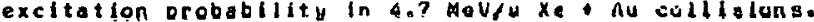
2. Pliys. A $2 E 8,417$ (197E) Hest Gerany

Anareser. L.: Kupcermann, f.: de Viles. A. E. chealulenlection III $N=1$ ceilisicns. I. Integrai cross sectiong. Z. Phys. A 285. 1 (15it) United States

Ancresen, E.: Kupceraann, A. Cheat-lenization in K-I collisicns. II. Diflerentiol cross gectlons. 2. Phys. A 289.11 (1978)

Untied stotes

Graubner. T.; Hermonn, G. Dibalignwent collialicn cross secticne of the necn lovels $2 p$. onc 23 , ottalnec ty moce crcsing. 2. Phyg. A 28S, E) (js78) liest Germony

Thomas, D. J. The electron capture decay of Bs sr-meosurewents of the $k$-roy eglosicn protetility, half-lite, ond decoy scheme. 2. Prys. A 2es, El (1528)

(1578)

United Kingdca 
REACTANT INDEX 
General

$\mathrm{H}+\mathrm{H}_{2}$

A02

HEAVY PART ICLE - HEAVY PARTICLE I NTERACT IONS

Elastlc Scattering Collisions

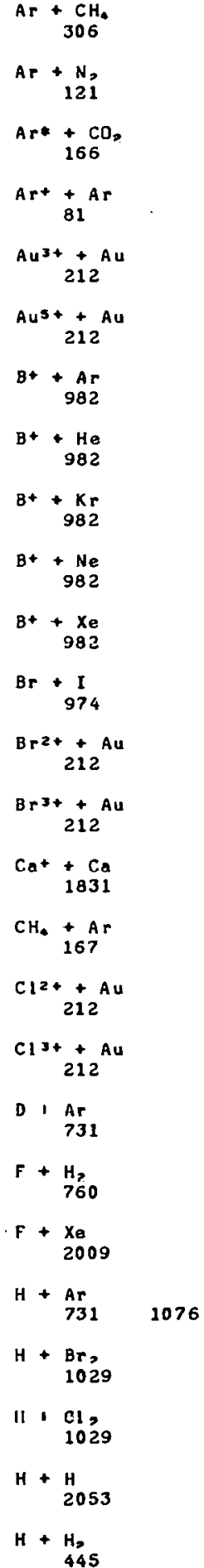

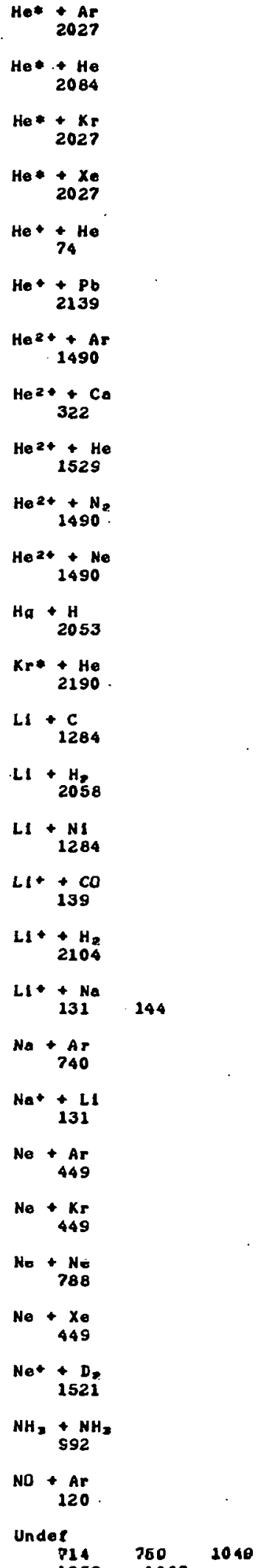


$n 03$ HEAVY PARTICLE - HEAVY PARTICLE
IUTERACTIONS

Excltation

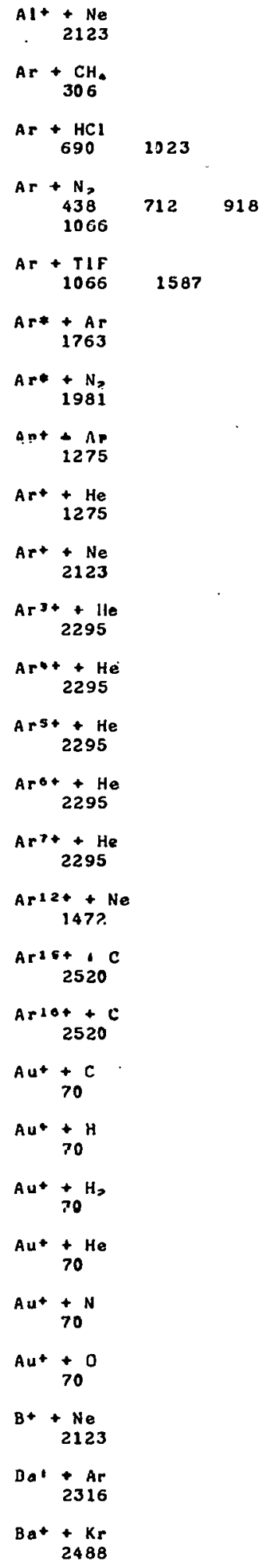

$\mathrm{Ba}+\mathrm{Ne}$
2316

$\mathrm{Be}+\mathrm{Ne}$

2123

$B \mathbf{B}+\frac{I}{974}$

$c+c$

1879

$\mathrm{C}+\mathrm{Ne}$

2123

$\mathrm{Ca}+\mathrm{Ar}$

23162488

$\mathrm{Ca}+\mathrm{Kr}$

2316

$\mathrm{Ca}+\mathrm{Ne}$

2316

$\mathrm{Ca}^{+}+\mathrm{Xe}$

2488

$\mathrm{CH}_{3} \mathrm{~F}+\mathrm{CH}_{4}$

2319

$\mathrm{Cl}+\mathrm{He}$

19

$01 * \frac{H i}{2123}$

$\mathrm{CO}_{2}+\mathrm{Ar}$

913

$\mathrm{CO}_{2}+\mathrm{Kr}_{\mathrm{r}}$ 913

$\mathrm{CO},+\mathrm{Ne}$

913

$\mathrm{Cs}_{\mathrm{s}} * \underset{490}{+\mathrm{Cs}_{3}}$

1205

2446

Cs. $+\mathrm{C}$

$\mathrm{C}_{9}+{ }_{70} \mathrm{H}$.

$\mathrm{Ca}_{70}+\mathrm{H}_{2}$

$\mathrm{Cs}_{70}^{+} \mathrm{He}$

$\mathrm{Cs}+\mathrm{N}$

$\mathrm{Cs}_{3}+\mathrm{O}$

70

$\mathrm{CsF}+\mathrm{Ar}$

2068

$\mathrm{CsF}+\mathrm{Xe}_{\mathrm{e}}$

$20 \mathrm{AA}$

CgI $*$ Ar

$C_{s I}+x_{e}$

2068

$\mathrm{D}_{2}+\mathrm{He}_{151}$

F $+\mathrm{H}^{4}$

1062

$F+\mathrm{H}_{2}$
1062

$r+x e$

1062

$F++\mathrm{Ne}$

2123

Fot $+\mathrm{He}$
$H+\underset{5}{C O}$

$\begin{array}{rlll}H+ & H_{7} & & \\ 107 & 645 & 849 \\ 2095 & & \end{array}$

$H+\underset{5}{N}$

H $+\mathrm{O}_{5}$

H $+\mathbf{C H}$

. 636

H* + Ar

$550 \quad 1452$

$H *+\underset{56}{A 4}$

1878

$\mathrm{H}^{+}+\mathrm{C}$

1879

$\mathrm{H}^{+}+$

$56 \quad 1480$

$\mathrm{H}^{*}+\mathrm{Ce}$

1878

H+ + CN

$2 J 00$

$H^{+}+\mathrm{Cr}$

1480

$\mathrm{H}+\underset{\mathbf{5} \dot{2} 8}{\mathrm{C}}$

$\begin{array}{lll}528 & 1516 & 1517\end{array}$

$\mathrm{H}+\mathrm{Cu}$

1878

$\mathrm{H}^{+}+\mathrm{Fe}$

$1480 \quad 1878$

$\mathrm{H}+\mathrm{Fe}^{\mathrm{It}}$

824

$H *+H$

$\begin{array}{lll}196 & 213 & 1280 \\ 1791 & 2140 & \end{array}$

$\mathrm{H}+\mathrm{H}_{3}$ $214 \quad 689 \quad 1587$ $2022 \quad 2104$

$11++\begin{array}{r}H>0 \\ 1976\end{array}$

H* He $550 \quad 552 \quad 1452$

$H++H e$ Seq 631

H* + Ho 1878

$\mathrm{H}++\underset{5}{\mathrm{~K}}$

553

$\mathrm{H}^{+}+\mathrm{Kr}_{3} \mathrm{S50}$

$H+\div \operatorname{Mn}_{1480}$

$\mathrm{H}^{+}+\mathrm{Na}$

$528 \quad 553$

$\mathrm{H}+\underset{56}{\mathrm{No}}$

$\mathrm{H}^{+}+\mathrm{Ne}$

550

H* $+\mathrm{NI}$

2185

H* $+\mathrm{OH}^{+}$

622 


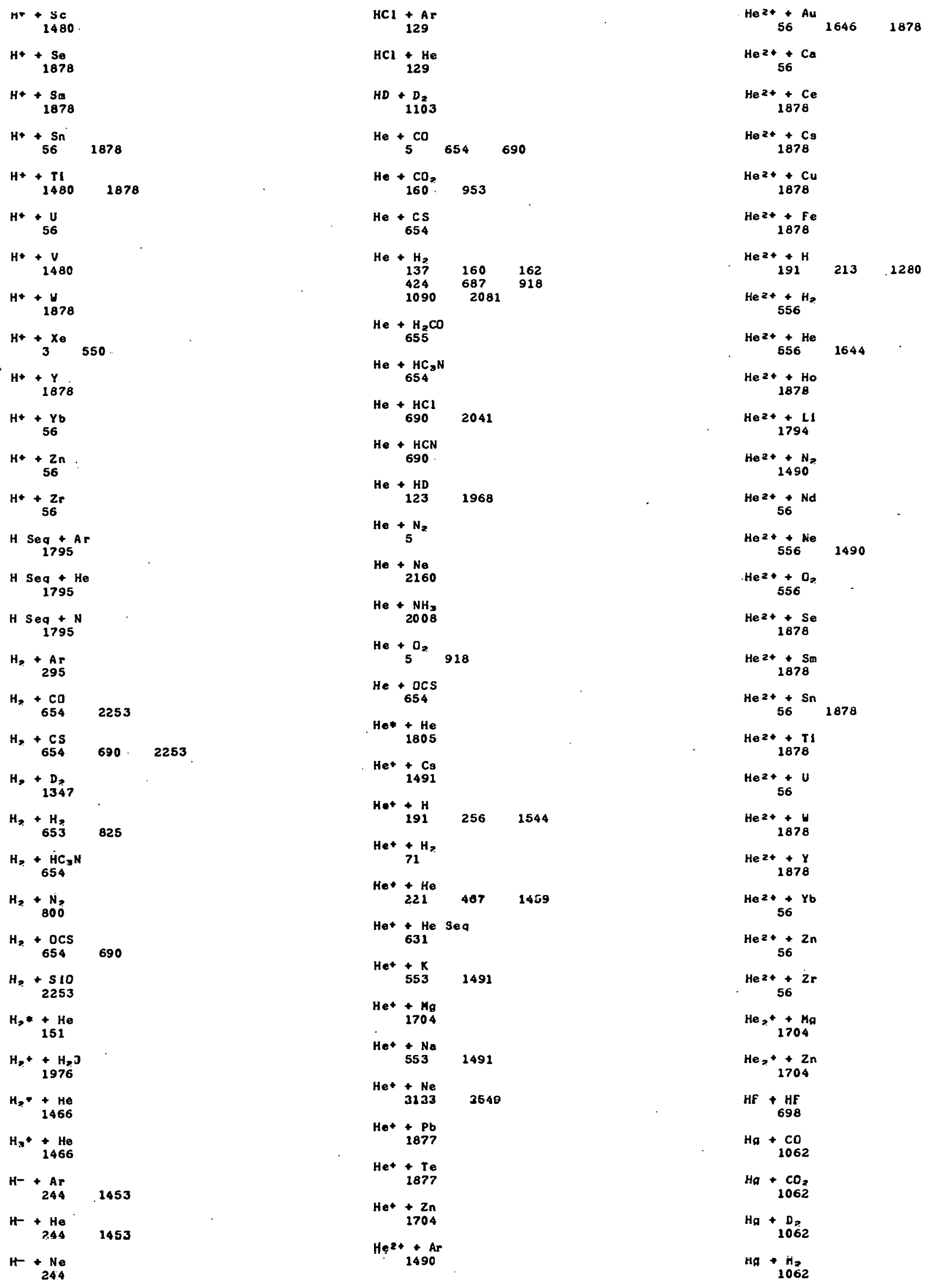




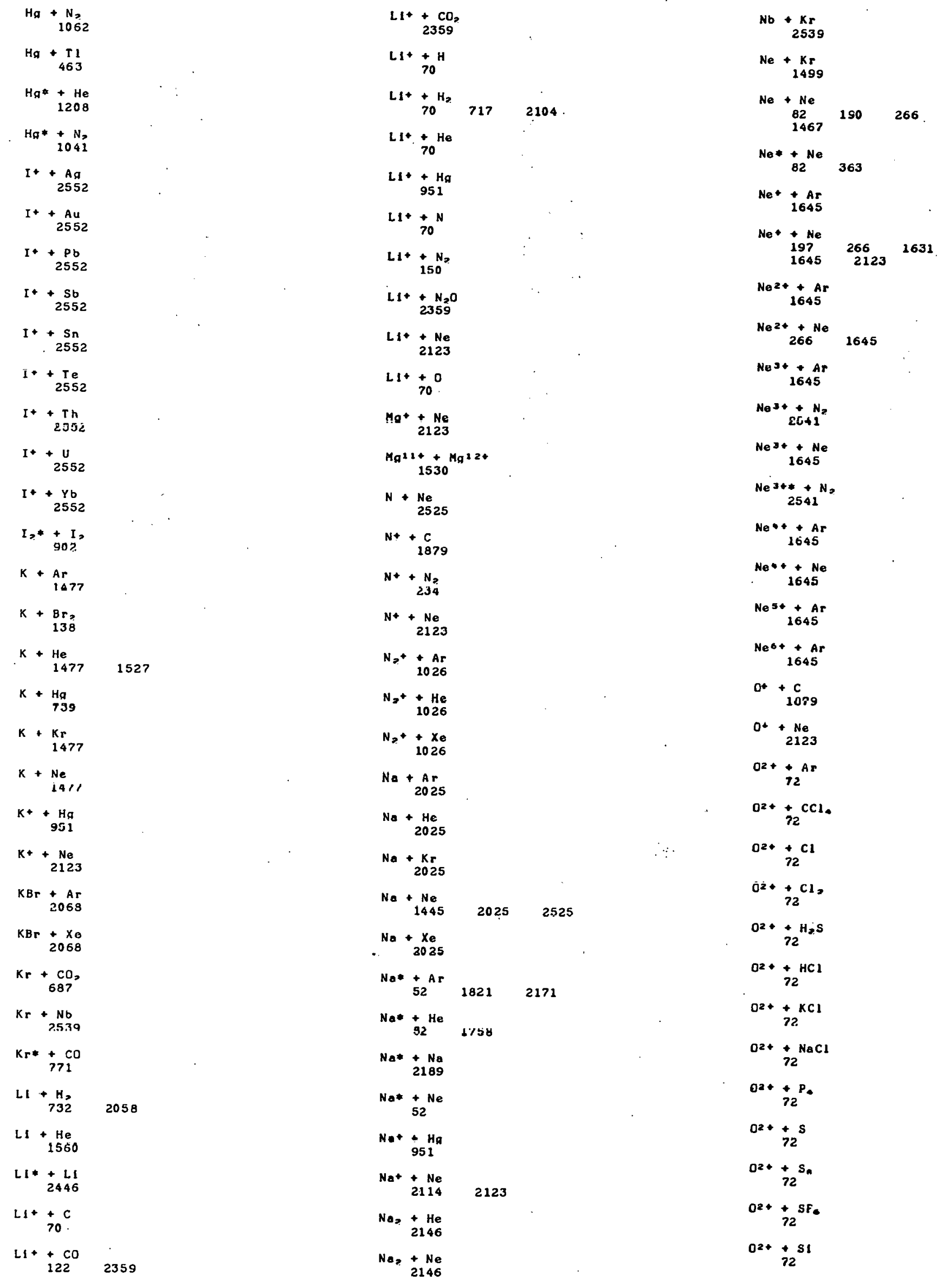




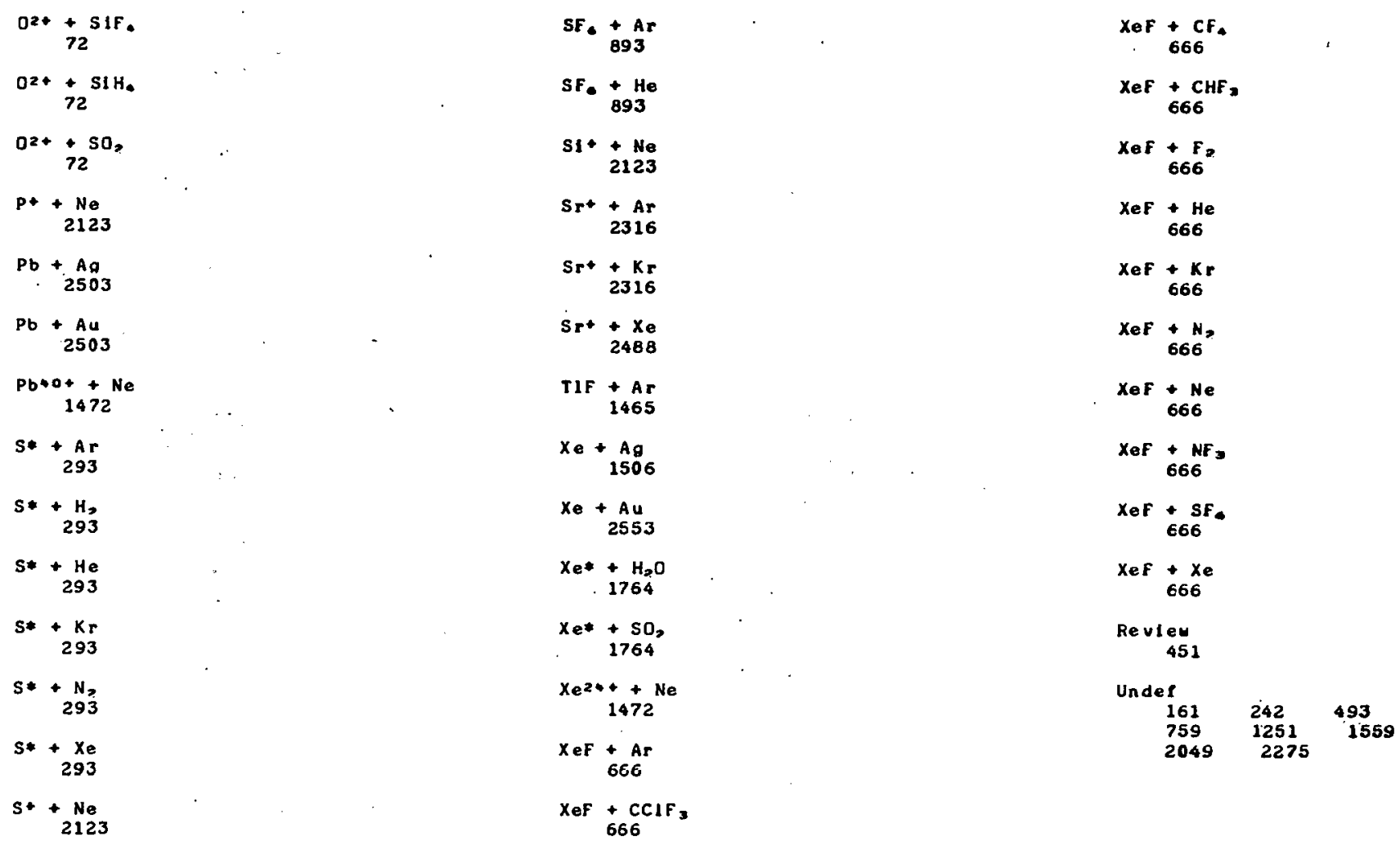


A04

HEAUY PART ICLE - HEAUY PARTICLE INTERACTIONS

Dissociation

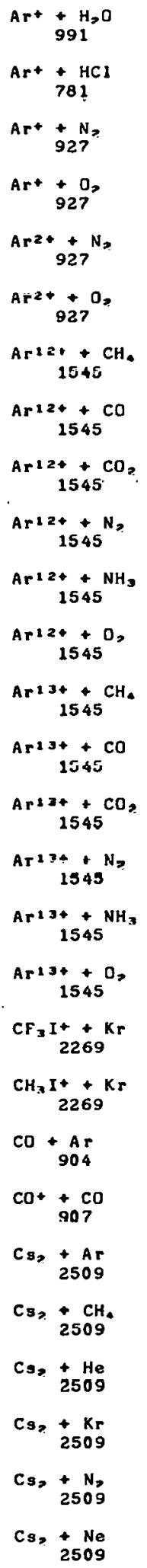

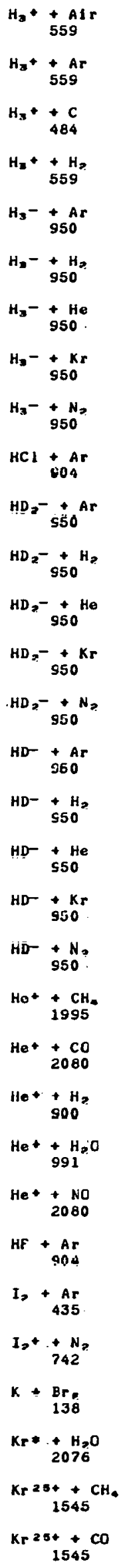


$\mathrm{Kr}^{25+}+\mathrm{CO}_{2}$

1545

$\mathrm{Kr}_{1545}^{2 \mathrm{St}}+\mathrm{N}$,

$\mathrm{Kr}^{25+}+\mathrm{NH}_{\text {t }}$

1545

$\mathrm{Kr}^{25+}+\mathrm{O}$,

1545

$\mathrm{N}_{2}++\mathrm{H}_{2} \mathrm{O}$

991

$\mathrm{Na}, \underset{733}{+A \mathrm{r}}$

$\mathrm{Na}, \underset{733}{+\mathrm{He}}$

$\mathrm{Na},+\mathrm{Ne}$

733

$N F_{x}+\mathrm{NF}_{10}$

1012

$N O *+N_{\text {? }}$ 311
$\mathrm{O}+\underset{1995}{\mathrm{CH}_{4}}$

$\mathrm{O}+\mathrm{CO}$

2080

$\mathrm{O}+\mathrm{H} \mathrm{O}$

991

$\mathrm{O}+\underset{2080}{\mathrm{NO}}$

$a_{2}+C_{3}$

$0,+\underset{716}{K}$

$0,+\begin{array}{r}\mathrm{Na} \\ 716\end{array}$

$\mathrm{O}_{2}+\underset{991}{+\mathrm{H}_{2} \mathrm{O}}$

$\mathrm{SF}_{-}+\mathrm{Cs}_{2} \mathrm{Cl}_{2}$

1962

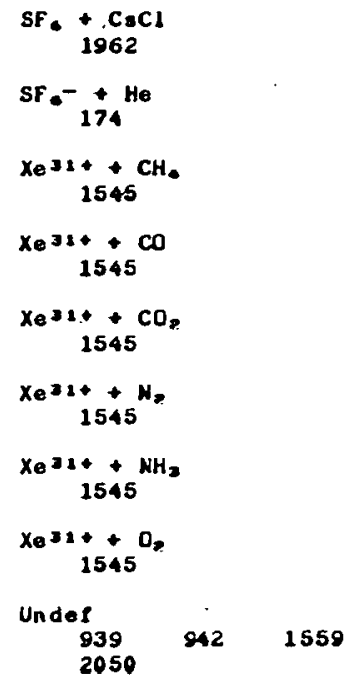

1545

$x \in 314$

$+\mathrm{CO}^{\circ}$

$+N_{2}$

$++\mathrm{NH}$

t. 0

of

2050 
A05

HEAUY PARTICLE - HEAUY PARTICLE INTERACTIONS

Themil un Inescence, Fl uorescence, and Lubinescence (photon ealsaion by unspecifled procesies)
$A g+\underset{2536}{A g}$
$A 1+A 1$
2532
$A \mathbf{r}+\mathrm{H}_{2} \mathrm{O}$
1141
Ar* + HCOOH
1141
$\mathrm{Ar}^{+}+\mathrm{H}_{2} \mathrm{~S}$
2351
$\mathrm{Ar}++\mathrm{SO}_{2}$
$B+\begin{array}{r}N>0 \\ 815\end{array}$
$B+0_{3}$
$\mathrm{Br}+\mathrm{Br}$ 2536
$\mathrm{Cl}+\mathrm{Al}$
$\mathrm{Cl}+\underset{727}{\mathrm{H}_{2} \mathrm{~S}}$
$\mathrm{Cl}+\mathrm{K}$,
909
$C 1+0, F$
1115
$\mathrm{Cl}+\underset{2502}{\mathrm{Ar}}$
$\mathrm{Cl}^{+}+\mathrm{Cl}_{-}$
2602
$\mathrm{Cl}++\mathrm{NaCl}$
2502
$\mathrm{ClO}+\mathrm{C}$
$\mathrm{Cl}=\underset{420}{+} \mathrm{Ha*}$
$\mathrm{Cl}_{2}+\mathrm{Sr}^{\circ}$
$\mathrm{Cs}+\mathrm{Cg} *$
2321
$\mathrm{Cs}_{490}^{+\mathrm{Cs}_{9}}$
$\mathrm{Cu}+\underset{2006}{F}$

$\mathrm{Cu}+\mathrm{NF}_{3}$

2006

$\mathrm{Cu}+\underset{2006}{\mathrm{SF}_{6}}$

F + Al 2532

$\mathrm{F}+\mathrm{H}_{2}$
$\mathrm{72}$

$\mathrm{F}+\mathrm{H}_{2} \mathrm{~S}$

$\mathrm{Fe}+\mathrm{Fe}_{2536}$

Ge + Ge 2536

$\mathrm{H}+\mathrm{Cl}_{2}$

$H+1.1$

108

$H+\mathrm{Lt}_{2}$
108

$\mathrm{H}+\mathrm{S}_{2} \mathrm{Cl}_{\text {? }}$

777

$H+\mathrm{SCl}_{2}$
777

$\mathrm{H}+\mathrm{SO}_{2} \mathrm{Cl}_{2}$

777

$H+\operatorname{socl}_{727}$

$\left.H++\underset{1982}{[A r}+F_{2}\right]$

$\mathrm{H}+\mathrm{Al}$

2511

$\mathrm{H}^{+}+\mathrm{Au}$

$\mathrm{H}^{+}+\underset{2547}{\mathrm{DV}}$

$H^{+}+$In

$\mathrm{H}^{+}+\mathrm{N}_{\mathrm{P}}$ 2352

$\mathrm{H}^{+}+\mathrm{Nd}$

$\mathrm{H}+\mathrm{Pt}$

2547

H+ $+\begin{gathered}5 \mathrm{i} \\ 2511\end{gathered}$

$\mathrm{H}^{+}+\mathrm{Sh}$

2547

H+ + T !

2511

$\mathrm{Het}+\mathrm{Ar}$

1900
$\mathrm{He}_{2}++\mathrm{CH}_{\mathrm{a}} \mathrm{CA}^{\prime}$

915

$\mathrm{He}_{2}+\mathrm{H}_{2} \mathrm{O}$

$\mathrm{Hg}+\mathrm{Tl}$

$\mathrm{Kr}+\mathrm{Nb}$

2539

$\mathrm{La}+\mathrm{O}_{2}$
307

$\mathrm{LI}+\underset{2287}{\mathrm{CF}_{7} \mathrm{Cl} l_{2}}$

$\mathrm{LI}+\mathrm{Cl}^{2}$
$228 ?$

$1+S F_{6}$

N+ Al

2511

$N++51$

N+ $+\mathrm{TI}$

- 2512

$\mathrm{Nb}+\mathrm{Kr}$ 2539

$\mathrm{Nb}+\mathrm{Nb}$

2536

$\mathrm{NO}+\mathrm{O}_{3}$

$\mathrm{NO}+\mathrm{NO}_{2}-$

1633

D+NO

744

$S^{+}+\underset{2502}{A r}$

$s+c 1$

2502

$31-N a c l$

Sc $+\underset{30 ?}{0,}$

$S r++N_{2} O$

Xo + Dr,

801

Xe $\begin{array}{r}\Gamma_{227} \\ \text { X }\end{array}$

$Y+0_{3}$
307

Undet 1930 
A06

HEAUY PARTICLE - HEAUY PARTICLE INTERACTIONS

Electron Capture

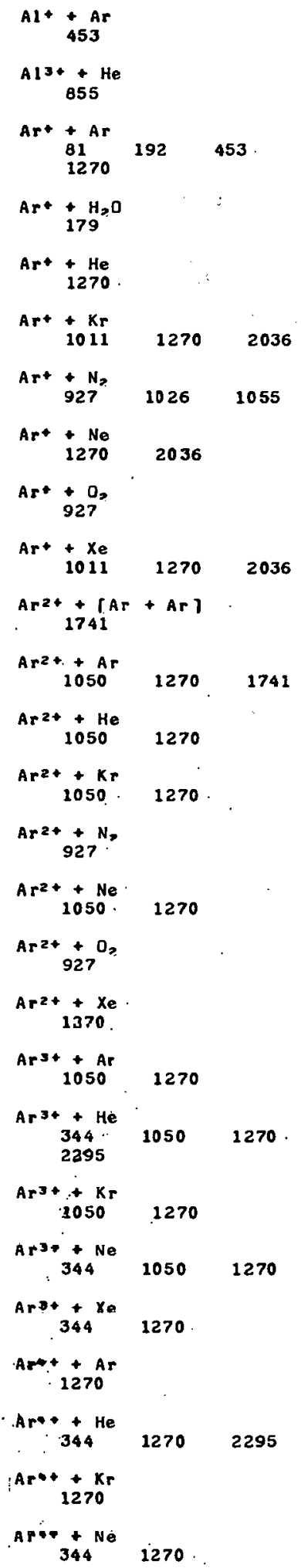

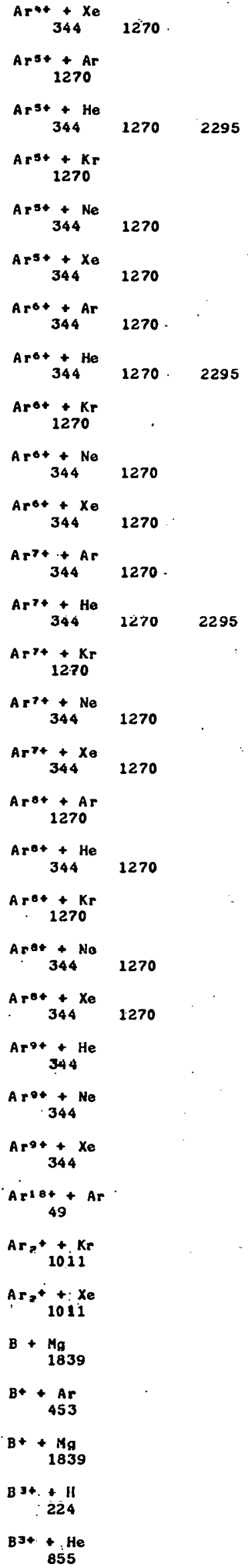

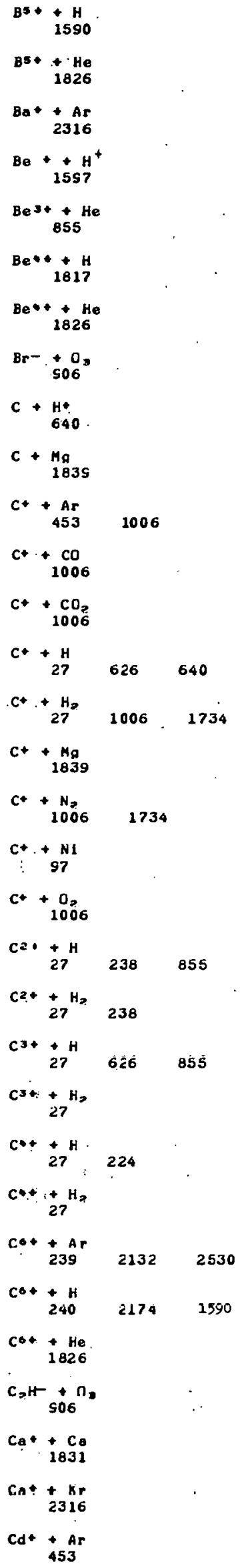




$$
\begin{aligned}
& \mathrm{Cl}+\underset{1839}{\mathrm{Ag}} \\
& \mathrm{Cl}+\underset{453}{+} \\
& \mathrm{Cl}++\mathrm{Cu} \\
& 2137 \\
& \mathrm{Cl}+\stackrel{\mathrm{Mg}}{\mathrm{H}} \\
& \mathrm{Cl}+\mathrm{H}+\mathrm{Kr} \\
& 239 \\
& \mathrm{Cl}_{2}-\underset{906}{+}+\mathrm{O} \\
& \mathrm{Cl}+\underset{906}{+} \mathrm{O}_{\mathrm{x}} \\
& \begin{array}{r}
\mathrm{CO}+\mathrm{Co} \\
998
\end{array} \\
& \mathrm{Hint}_{310} \mathrm{Th}^{\mathrm{T}} \\
& \mathrm{CO}_{3}+\mathrm{Cu}_{310}^{\mathrm{U}} \\
& \mathrm{CO}_{906}+{ }_{90}^{+} \\
& \mathrm{CO}_{306}^{+}+\mathrm{O}_{3} \\
& \mathrm{Cr}+\mathrm{Ar} \\
& 453 \\
& \mathrm{Cs}+\underset{930}{\mathrm{D}>0} \\
& \mathrm{CS}+\underset{930}{\mathrm{H}} \mathrm{O} \\
& \mathrm{Cs}_{9}+\operatorname{IrFa}_{975} \\
& \mathrm{Cs}+\mathrm{MoF} \text {. } \\
& 975 \\
& C_{s}+\underset{975}{11 P_{2}} \\
& \mathrm{Cs}+\mathrm{ReF} \text {. } \\
& \mathrm{C}_{3}+\mathrm{SeF} \text {. } \\
& \mathrm{Cs}_{s}+\underset{975}{S F_{\alpha}} \\
& C_{3}+\operatorname{Ter} \\
& \text { Cs + } \\
& \mathrm{Cs}^{+}+\mathrm{Ar} \\
& 453 \\
& \begin{array}{r}
\mathrm{Cs}+\mathrm{Cs} \\
2545
\end{array} \\
& \mathrm{Cs}_{3}+\mathrm{H} \\
& 215 \\
& \mathrm{C}_{3}+\underset{215}{+} \\
& \mathrm{Cu}++\mathrm{Ar} \\
& \begin{array}{c}
\text { D+ } \\
97
\end{array} \\
& \mathrm{D}+\mathrm{NI} \\
& F^{+}+\underset{97}{\mathrm{NI}} \\
& \text { F3+ + 51 }
\end{aligned}
$$

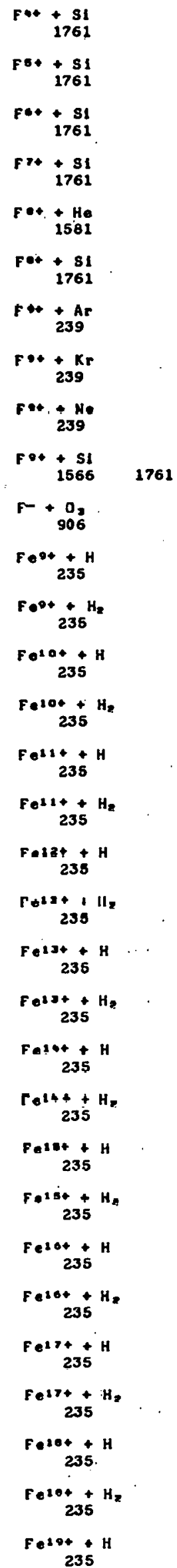



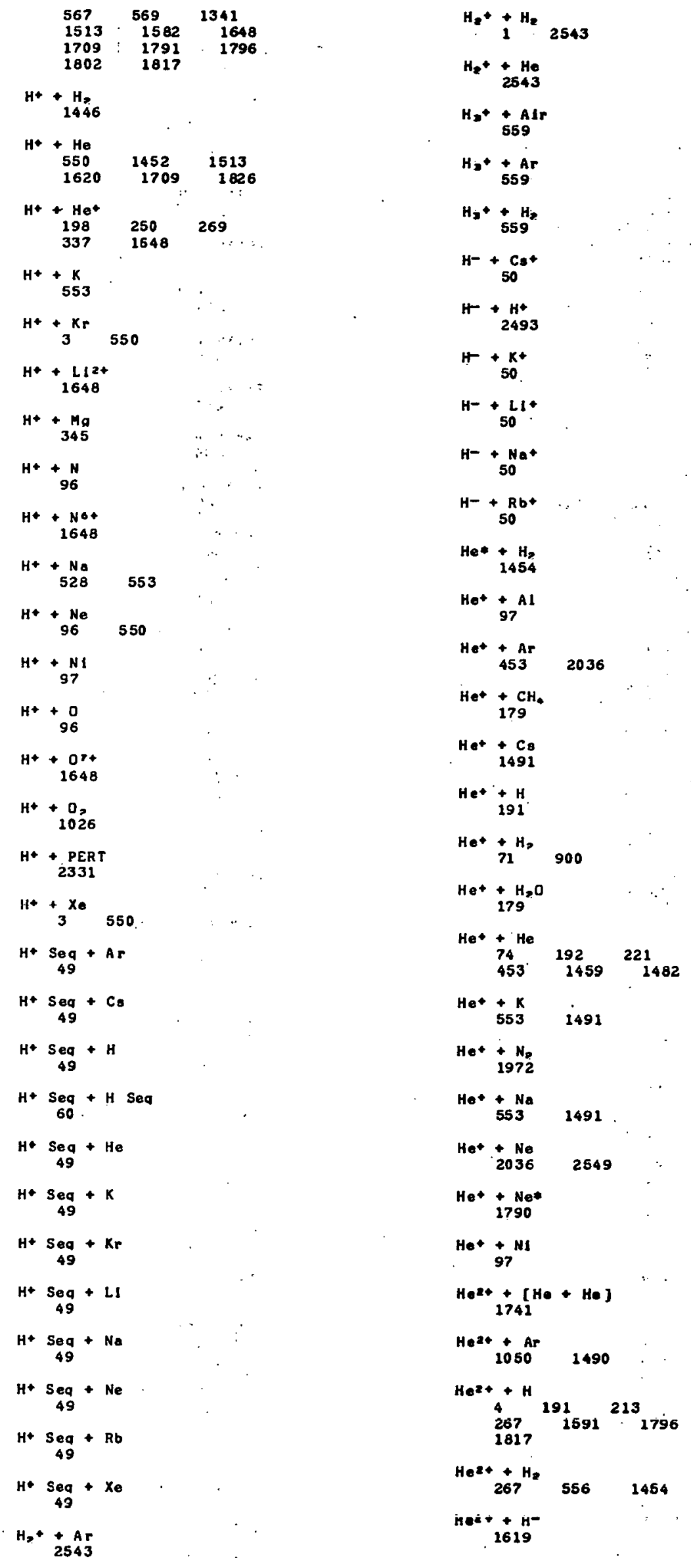


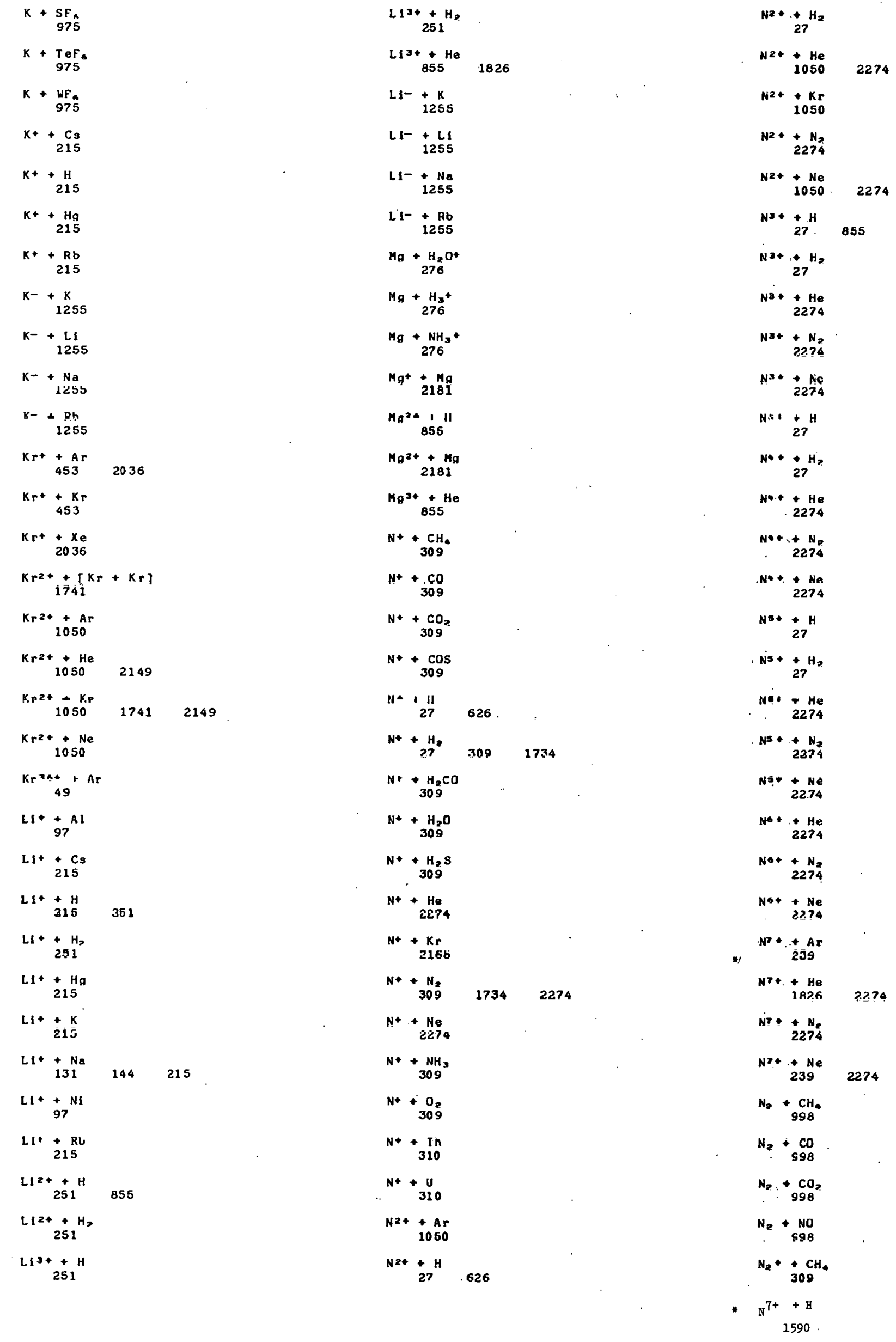




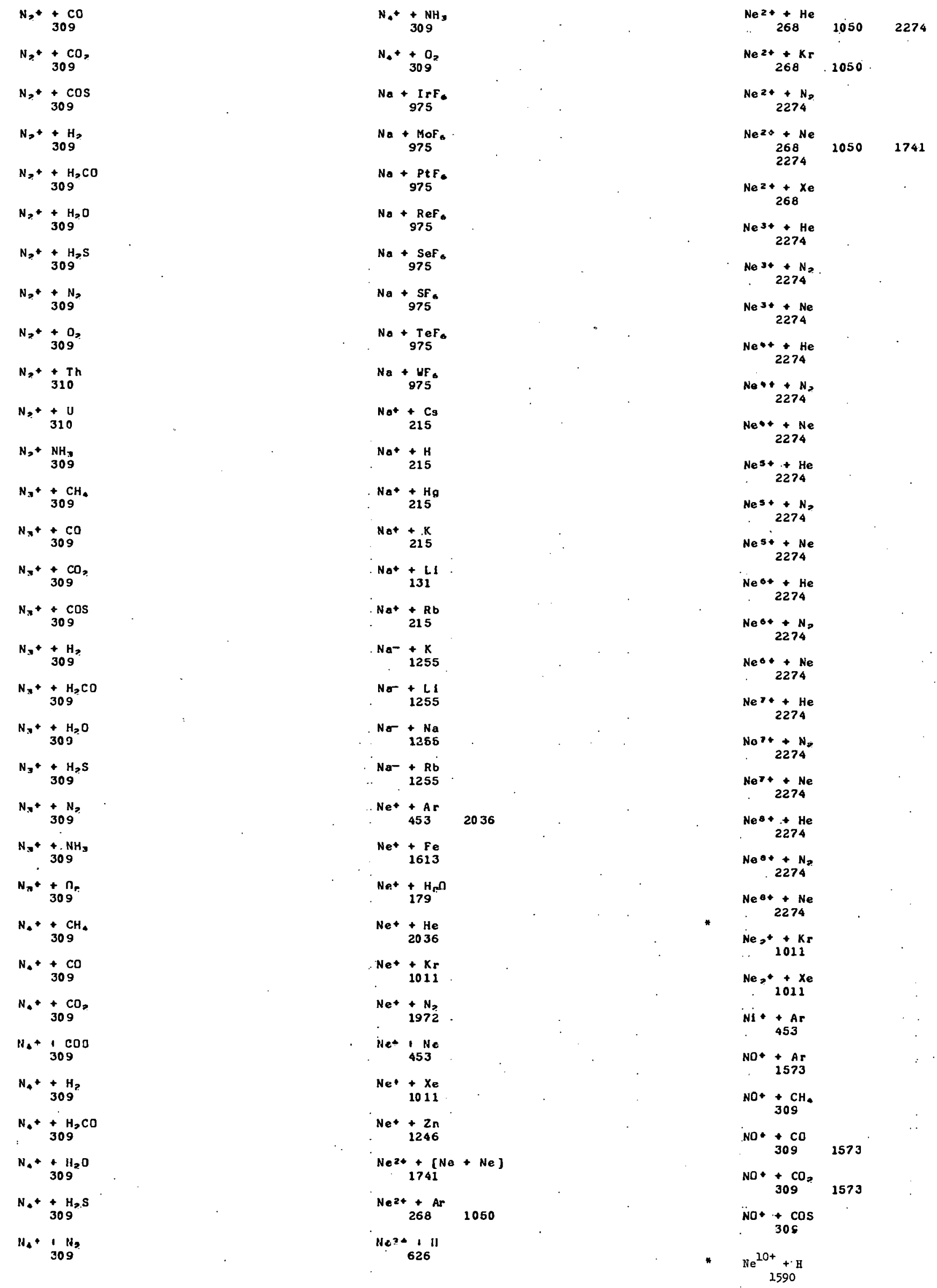



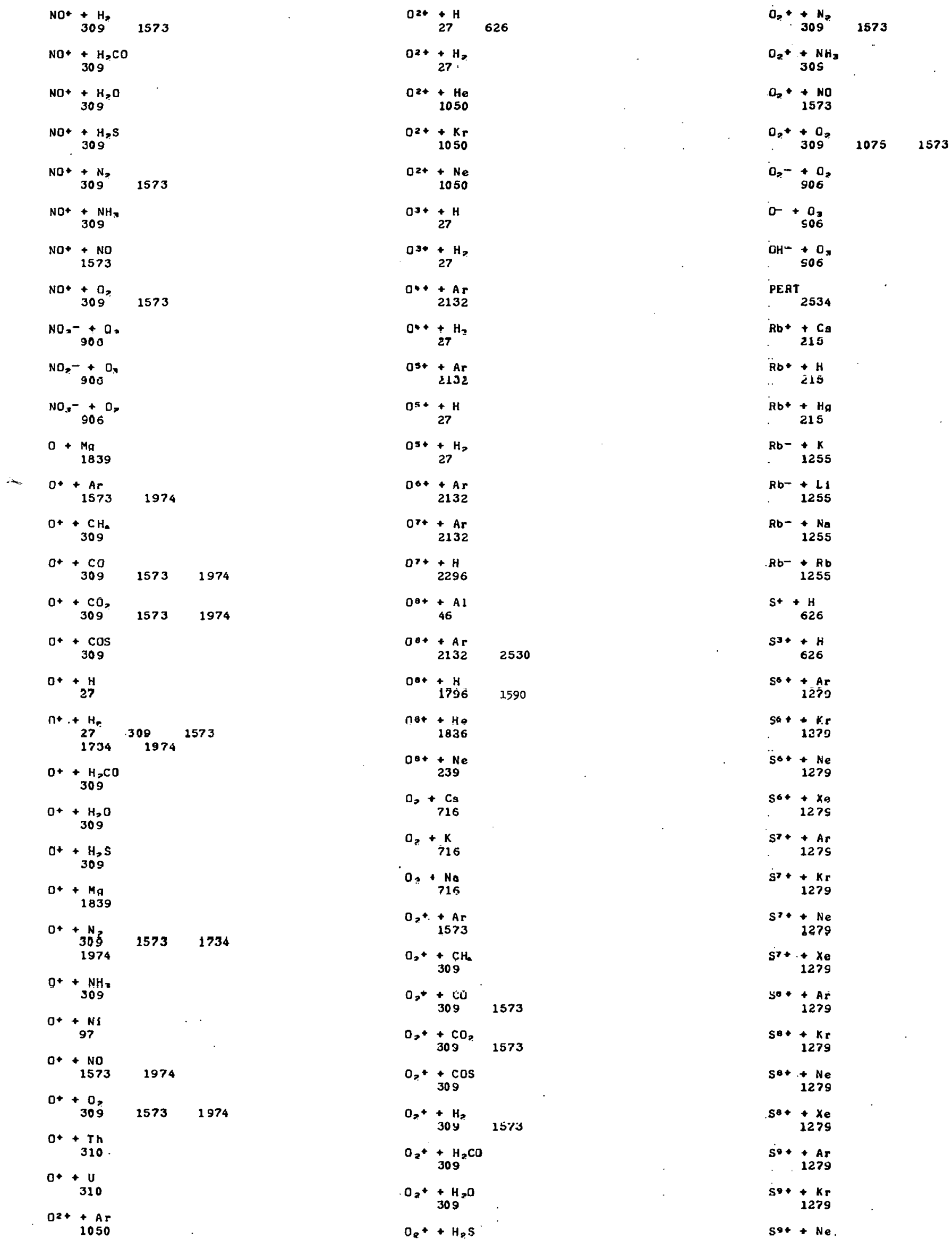


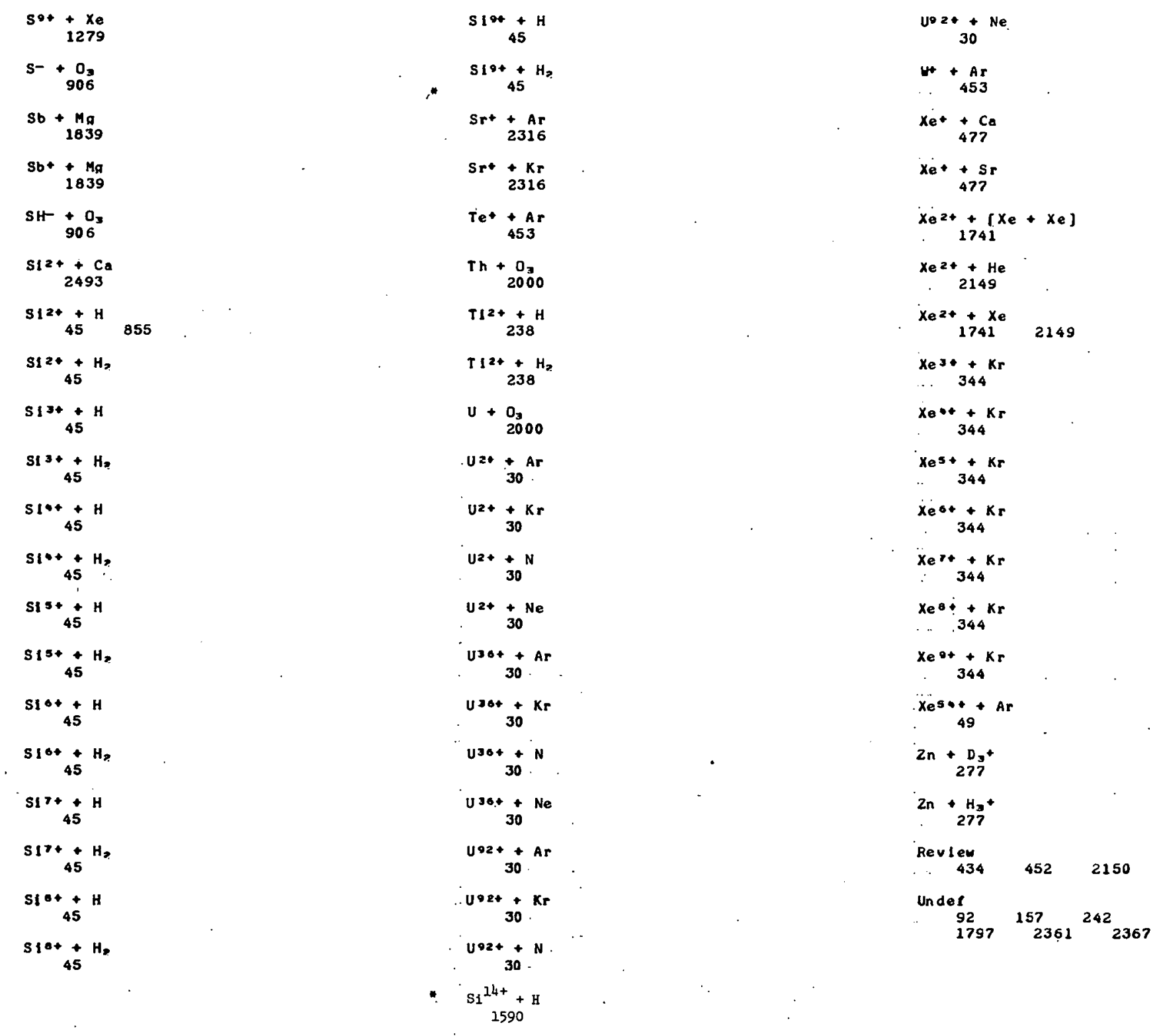


HEAUY PARTICLE - hEAUY PARTICLE INTERACT IONS

Ionl zation

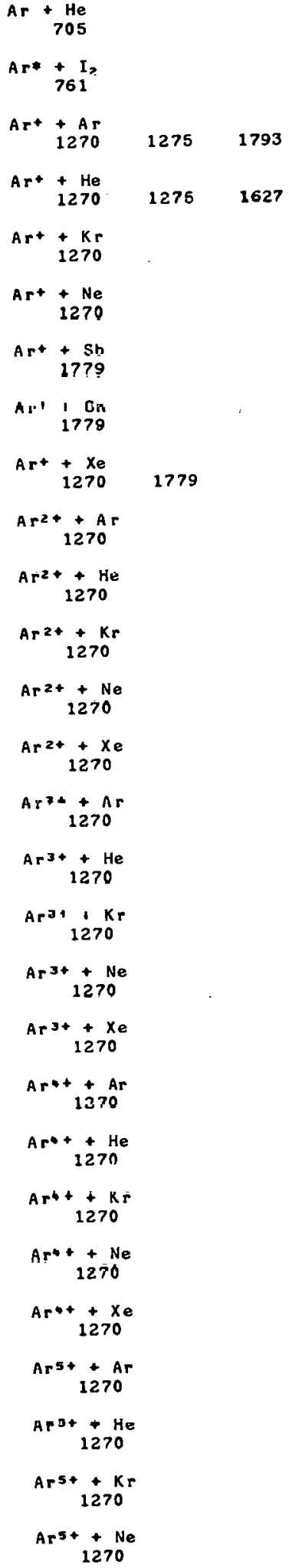

A r $6+$ Ar 1270

Arst $+\mathrm{He}$

$\mathrm{Ar}^{6+}+\mathrm{Kr}$ 1270

Arot $+\mathrm{Ne}$

$\mathrm{Ar}+\mathrm{Xe}$ 1270

$A r^{2+}+A r$ 1270

Arot + He . 1270

$\mathrm{Arr+}+\mathrm{Kr}$ 1270 .

$\mathrm{Ar}^{7+}+\mathrm{Ne}$ 1270

$. \mathbf{r}^{2+}+\mathrm{Xe}$ 1270

Arel Ar 1270

Arst $+\mathrm{He}$ 1270

Arot + Kr 1270

A rot $+\mathrm{Ne}$

A rot $+X e$ 1270

$\mathrm{Ar}^{3} 2+\mathrm{CH}$. 1545

Ar12+. + CO

Ai $2.34+60$. .1545

$\mathrm{Ar} 12++\mathrm{N}_{2}$ 1545

A $100 . .+\mathrm{NII}_{3}$. 1545

$A r^{2+}+\mathrm{O}_{2}$ $1545^{2}$.

$\mathrm{Ar}^{3+}+\mathrm{CH}_{4}$ 1545

$\mathrm{Ar}^{13+}+\mathrm{CO}$
1646

$\mathrm{Ar}^{23}+\mathrm{CO}_{2}$

1545

Ari3t+ 1545

$\mathrm{Ar}^{3}+\mathrm{NH}_{3}$ 1545

$\mathrm{Ar}^{13+}+\mathrm{O}_{2}$ $1545^{\circ}$

$\mathrm{As}+\mathrm{Kr}$

1793

$A$ u + C $C$

$\mathrm{Au}+\mathrm{H}$

$A u+\underset{70}{+}$
$\mathrm{Au}++\mathrm{N}$

Aü +0

$B++\mathrm{Ca}$ 1700 .

$\mathrm{B}+4 \mathrm{CO}$ 1700

Bt+Fe

B $+K$ 1700

$B *+\mathrm{Mn}$ 1700

Bt $+\mathrm{NI}$

. 1700

B十 $+\frac{P}{1700}$

$5++5 \mathrm{C}$ 1700

B* $+\frac{T 1}{1700}$

B $* V$

1700

$\mathrm{Be}^{2+}+\mathrm{H}$ 2497

Be $2+$ He

*

Brt $+K r$ 1793

$\mathrm{Br}+\mathrm{Xe}$

. 1793

$c+t \underset{1786}{\operatorname{Ar}}$

$c+c$

. 1879

"ct. He 2485

C $+\mathrm{He}$

c. .

$\ddot{C^{6}+t H}+\frac{H}{2174}$

Ca $+\mathrm{Ca}$

1831

Cl + Ar

1793 2507

$c l+t+C l$

$\mathrm{Cl}+4 \mathrm{NI}$ 1828

$\mathrm{Cl}+$ Sb

$\mathrm{Cl}+\mathrm{sn}$ 1770 .

$\mathrm{Cl}++\mathrm{TI}$

cl $+x e$

$\mathrm{CO}+\mathrm{co}$

- $\mathrm{B}^{5+}+\mathrm{He}$

wh $. c^{6+}+\mathrm{He}$ 


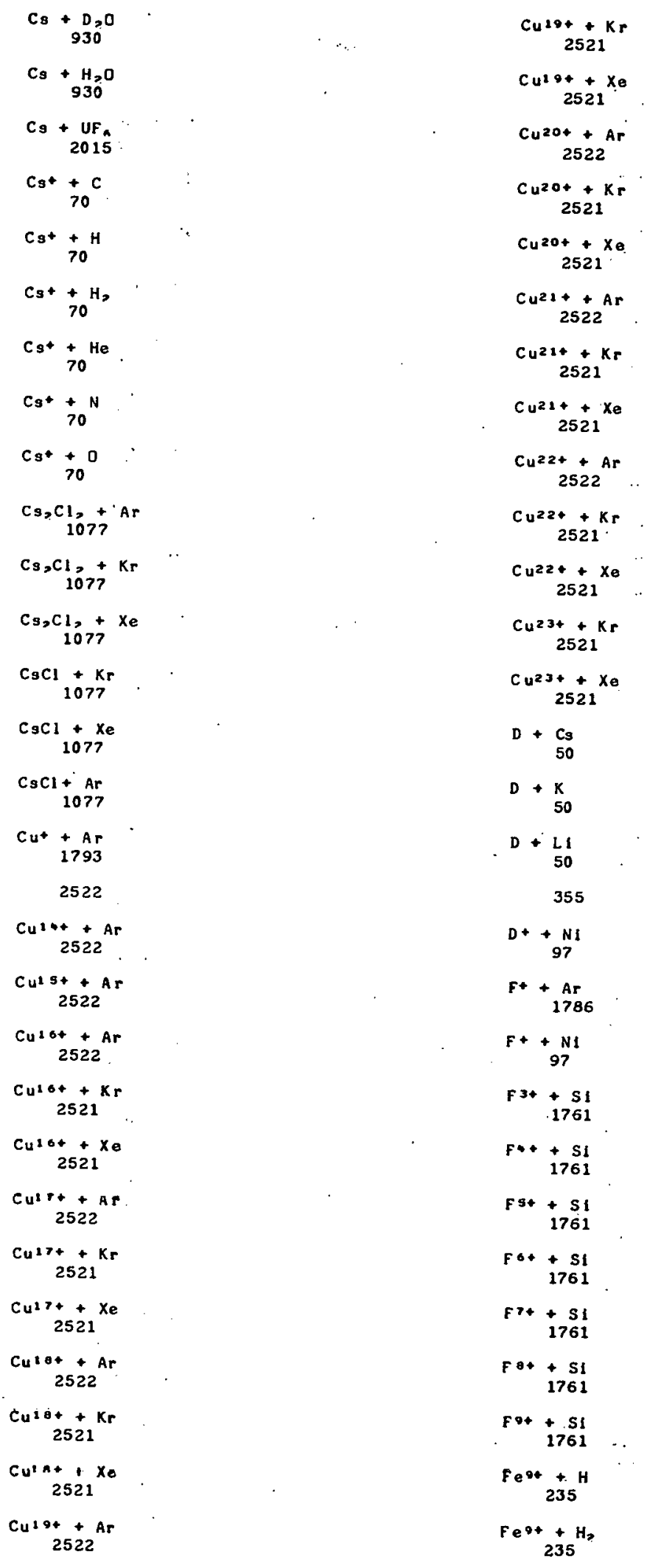




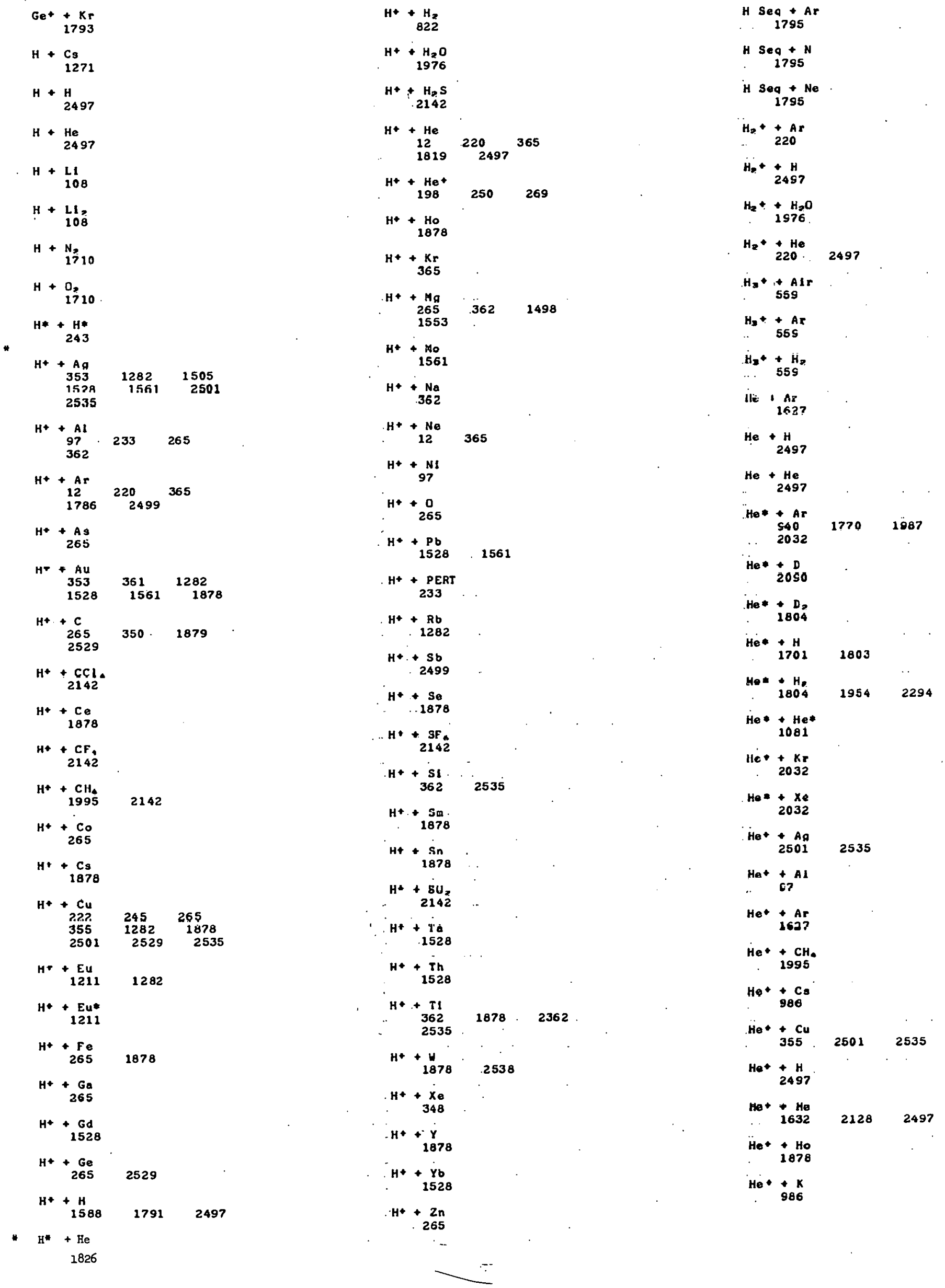




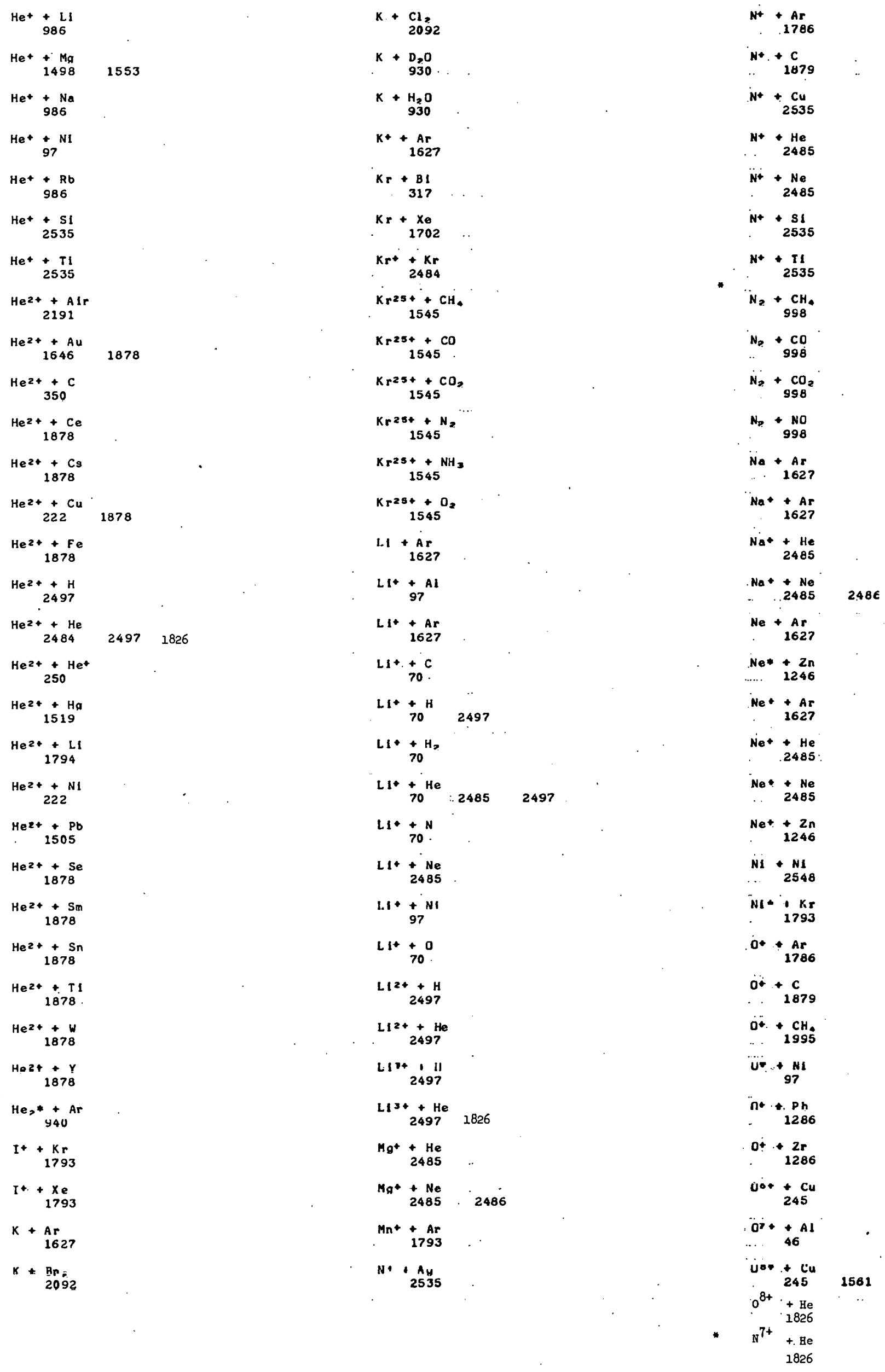




$$
\begin{aligned}
& \mathrm{Pb}+\underset{330}{\mathrm{~Pb}} 2516 \\
& \text { Pb. }+\underset{2516}{U} \\
& \text { S* * Al } 2507 \\
& S++\underset{1793}{A r} \\
& S++\underset{12 u}{1286} \\
& S++\underset{2507}{C a} \\
& S++\underset{19 g}{2507} \\
& \mathrm{~S}+\underset{2507}{\mathrm{Na}} \\
& \text { s+ }+\mathrm{NaCl} \\
& \mathrm{St+PB} \\
& \mathrm{S}+\mathrm{Sc} \\
& s++\frac{51}{2507} \\
& S++T a \\
& S++T 1 \\
& S++\underset{1286}{U} \\
& S++\underset{1286}{Z r} \\
& \text { Sot }+\begin{array}{r}
\text { Ar } \\
1279
\end{array} \\
& \text { Sot }+K r \\
& 56++\mathrm{Ne} \\
& \mathrm{S}^{6+}+\mathrm{Xe}
\end{aligned}
$$

$S^{7+}+A r$
1279

$S>++K r$

$\mathrm{S}+\mathrm{Ne}$

$s>+\quad X e$

So+ + Ar

S*+ $+\mathbf{K r}$

$\mathrm{S} \cdot+\mathrm{Ne}$

So+ $+\mathrm{Xe}$

$\mathrm{C}^{\circ+}+\mathbf{A r}$

S $0+\div \mathrm{Kr}$

$\mathrm{Sg}+\mathrm{Ne}$

So* $+X E$

$\mathrm{Se}+\mathrm{Kr}$

$\mathrm{SI}^{+}+\mathrm{Ar}$

$S r *+S r$

$T h+0_{3}$

$\mathrm{U}+\mathrm{D}_{2}$

$u+0_{3}$

U. PU . 2516 $u+u$

$X e+A 4$
326

$\mathrm{Xe}+\mathrm{BI}$

326

$\mathrm{Xe}+\mathrm{Pb}$

1626

$x e+$ In

$X_{e} *+C_{6} F_{*}$

Xe* $+C_{7} F_{1}$

$\mathrm{Xe} * \underset{\mathrm{S4}}{+\mathrm{CH}_{3} \mathrm{Br}}$

$\mathrm{Xe}+\underset{94.4}{\mathrm{CH}_{7} \mathrm{I}}$

$X e+\begin{array}{r}A g \\ 2550\end{array}$

$+\mathrm{XeS}_{2+}+\mathrm{CH}_{4}$

$\mathrm{Xe}_{1545}^{32+\mathrm{CO}}$

$\mathrm{Xe}^{31+t \mathrm{CO}_{2}}$

$\mathrm{Xe}^{31+}+\mathrm{N}_{\text {, }}$

$\underset{1545}{\mathrm{Xe}^{3+}+\mathrm{NH}_{3}}$

$\mathrm{Xe}_{1545}^{31+t \mathrm{H}_{2}}$

Review

Under $P$

$\begin{array}{lcc}264 & 327 & 493 \\ 2263 & 2514 & \end{array}$ 
HEAUY PART ICLE - HEAUY PARTICLE

INTERACTIONS

stripplng

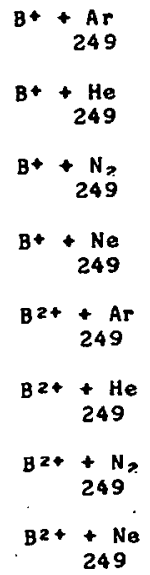

$\mathrm{H}^{*}+\mathrm{Ne}$

. 2274

$\mathrm{He}+\underset{1630}{\mathrm{Ar}}$

$\mathrm{He}+\mathrm{He}$

$\mathrm{He}+\mathrm{Kr}$ 1630

$\mathrm{He}+\mathrm{Ne}$ 1630

$\mathrm{He}+\mathrm{Xe}$ 1630

$\mathrm{He}+\mathrm{Ar}$ 1630

$\mathrm{He} *$ + He 1630

$\mathrm{He} *+\mathrm{Kr}$ 1630

$\mathrm{He}+\mathrm{Ne}$ 1630

$\mathrm{He}+\mathrm{xe}$ 1630

$\mathrm{He}^{+}+\mathrm{Ar}$

249

$\mathrm{He}^{+}+\mathrm{H}$

$\mathrm{He}^{+}+\mathrm{He}$

249

$\mathrm{He}^{+}+\mathrm{N}_{3}$
249

$\mathrm{He}^{+}+\mathrm{Ne}$

249

Ist+Ar

1825

$I^{5+}+N_{1825}$

$I^{5+}+X e$

$L I *+A r$ .1630

$L L *+$ He

$\mathrm{L}+\mathrm{Kr}$ 1630

$L I *+\mathrm{Ne}$

$11 *+x_{6}$

1630

$. \mathrm{LI}+\begin{array}{r}+H \\ 361\end{array}$

$\begin{aligned} \mathrm{LI}+\mathrm{H} & = \\ 251 & =\end{aligned}$

$\mathrm{LL}_{249} \mathrm{Ar}$

$\mathrm{LI}^{2+}+\mathrm{H}$

251

$\mathrm{Cl}_{2+}+\mathrm{H}_{2}$
$\mathrm{Li}_{249}^{+} \mathrm{N}_{2}$

LI $2 *+\mathrm{Ne}$

249

$\mathrm{LI}^{3+}+\mathrm{H}$ 251

Lis + H ?

$$
251
$$

$N+\underset{249}{A r}$

2274

$\mathrm{Nt} \cdot+\underset{24 \mathrm{He}}{\mathrm{He}}$ 22.74

$N++N$ $249 \quad 2274$

$\mathrm{N}+\mathrm{Ne}$

$\mathrm{N}^{2}+$ Ar $249 \quad 2274$

$\mathrm{N}^{2}+\mathrm{H}$ 2492274

$\mathrm{N}^{2}+\mathrm{N}$ $249 \quad 2274$

$\mathrm{N}_{2}+\mathrm{Ne}$ 249 2214

$\mathrm{N}^{3+}+\mathrm{Ar}$ $249 \quad 2274$

$\mathrm{N}^{3+}+$. He 249 2274

$\mathrm{N}^{3+}+\mathrm{N}_{2}$ $249 \quad 2274$

$\mathrm{N3}+\mathrm{Ne}$ 249 2274

$N *++A$ $249 \quad 2274$

$\mathrm{N} *+\mathrm{He}$ 2492274

$N *++N_{2}$ $249 \quad 2274$

$\mathrm{N} *+\mathrm{Ne}$ $249 \quad 227$

N5 + Ar 2274

$\mathrm{NS}+\mathrm{He}$ 2274

Ns * + No 2274

N5 + Ne 2274

No + + A r 2274

$\mathrm{No}+\mathrm{He}$ 3371

No + N? 2274

$\mathrm{No}+ \pm \mathrm{Ne}$

$\mathrm{Na}_{\mathrm{a}}+\mathrm{Ar}$ 


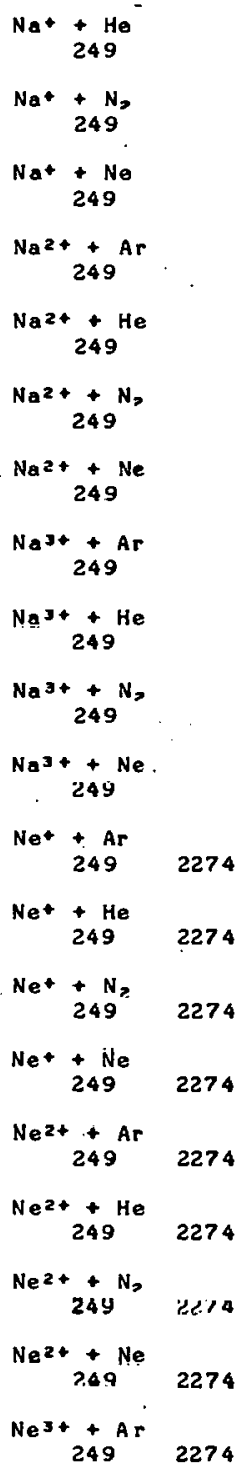

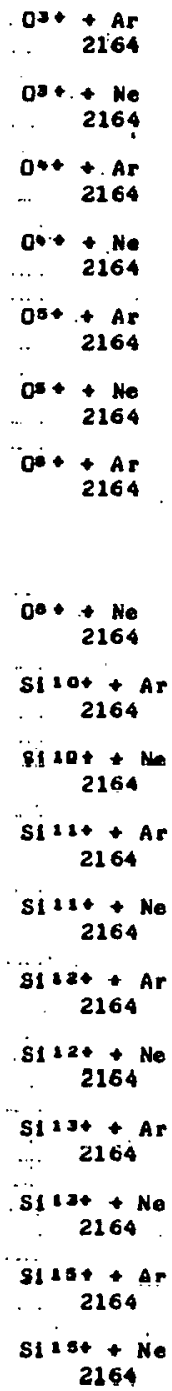


A09

HEAVY PART ICLE - HEAUY PARTICLE INTERACT IONS

Reconbination or Mutual Meut rel ization Leadina to Neutral iProdueta (Ion-Ion)

\begin{tabular}{|c|c|c|}
\hline Art & $\begin{array}{l}+\mathrm{Cl}^{-} \\
515\end{array}$ & \\
\hline$A r^{+}$ & $\begin{array}{l}+F^{-} \\
515\end{array}$ & 529 \\
\hline Ar, & $+\underset{515}{+F-}$ & 531 \\
\hline $\mathrm{H}^{+}+$ & $+\underset{272}{H-}$ & \\
\hline$H+$ & $+\underset{50}{\mathrm{C}_{3}}$ & \\
\hline $\mathrm{H}$ & $+\underset{50}{K+}$ & \\
\hline $\mathrm{H}^{-}$ & $+\mathrm{LI}_{50}$ & r \\
\hline$H^{-}$ & $+\mathrm{Na}_{50}^{\mathrm{Nat}}$ & \\
\hline $\mathrm{H}-$ & $+\underset{50}{R b+}$ & \\
\hline $\mathrm{He}^{+}$ & $+F$ & \\
\hline
\end{tabular}

$$
\mathrm{He}_{2}+\underset{531}{+}
$$$$
\mathrm{Hg}^{*}+[\mathrm{Br}-\cdots+\mathrm{Ar}]
$$$$
747
$$

$\mathrm{Hg}^{\circ}+[\mathrm{Cl}-+\mathrm{Ar}]$

747

$\mathrm{Hg}^{+}+\underset{747}{+[\mathrm{F}-\mathrm{Ar}]}$

$\mathrm{Hg}^{+}+[\mathrm{I}-+\mathrm{Ar}]$

747 .

$\mathrm{Hg}^{+}+\underset{515}{\mathrm{Cl}-}$

$\mathrm{Kr}+\underset{663}{+\left[F^{-}+A r\right]}$

$\mathrm{Kr}+\left[\mathrm{F}^{-}+\mathrm{He}\right]$ 663

$\mathrm{Kr}^{+}+\left[\mathrm{F}^{-}+\mathrm{Ne}\right]$

$\mathrm{Kr}+[\dot{\mathrm{F}}+\mathrm{Xe}]$ 663

$\mathrm{K} *+F$

517529

$\mathrm{Kr}_{2}++\left[\mathrm{F}^{-}+\mathrm{Ar}\right]$ 663

$\mathrm{Kr}_{\mathrm{s}}+\underset{663}{+}\left[\mathrm{F}^{-}+\mathrm{He}\right]$
$\mathrm{Kr} \geqslant \underset{663}{+}\left[\mathrm{F}^{-}+\mathrm{Ne}\right)$

$\mathrm{Kr}_{2}+\underset{663}{+}[\mathrm{F}-+\mathrm{Xe}]$

$\mathrm{Kr}_{2}+\mathrm{F}_{515}$

$\mathrm{Ne}+\mathrm{F}^{-}$ 529

$\mathrm{Ne}_{2}+\mathrm{F}$

$\mathrm{NO}+\mathrm{Br}^{-}$ 110

$\mathrm{NO}+\mathrm{Cl}^{-}$ 110 .

NO * + I110

$0_{4}+t\left[0_{1340}-+0_{2}\right]$

$0 .+0$. 1340

$\mathrm{Xe}+\mathrm{F}-$

. 529

$\mathrm{Xe}_{2}+\mathrm{F}$

531

Under

1339 


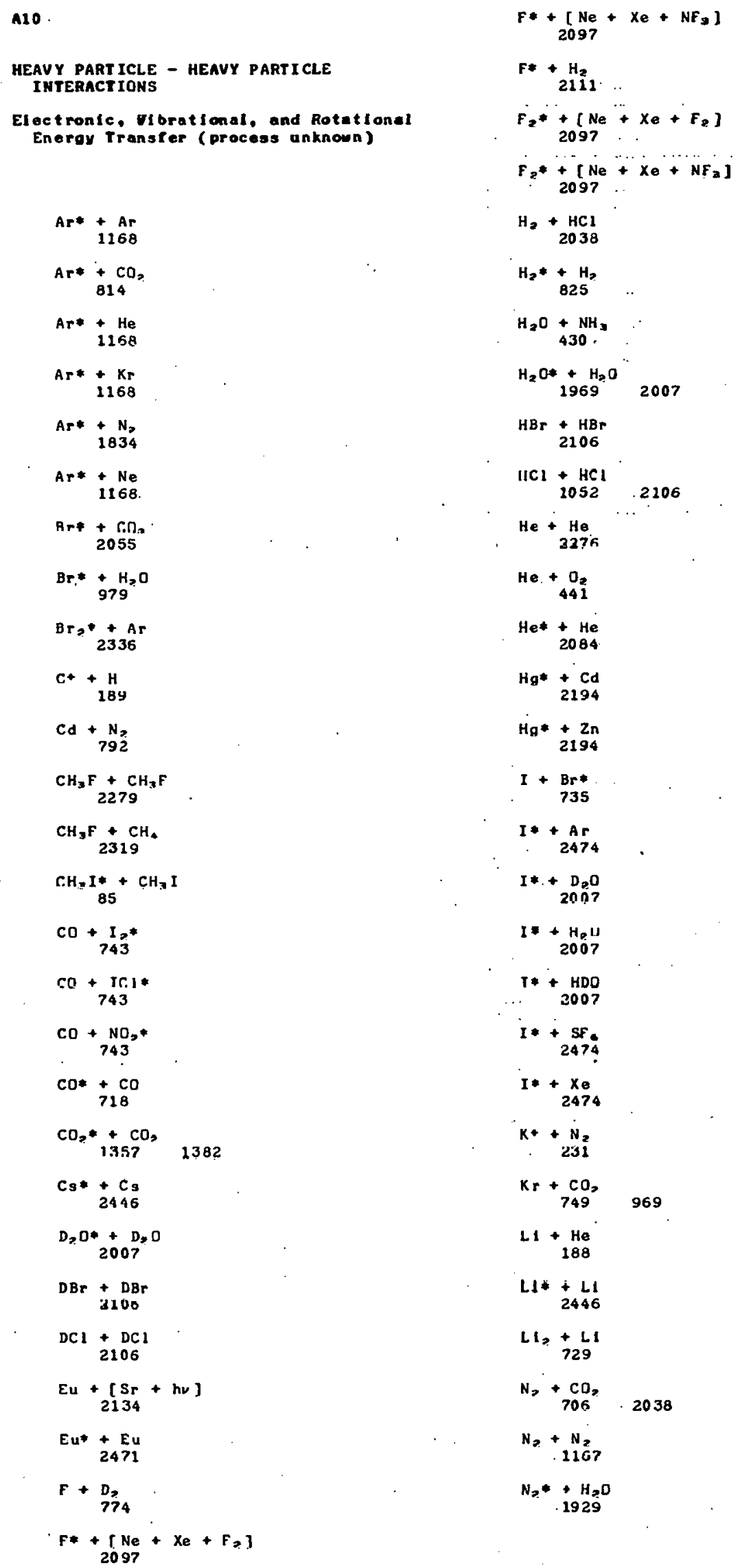

$\mathrm{Na}+\mathrm{N}_{2}$

255

$\mathrm{Na} * \mathrm{.52}_{\mathrm{t}}^{\mathrm{Ar}}$

$\mathrm{Na}+\mathrm{He}$

52

$\mathrm{Na} *+\mathrm{Na}$ 1345

2189

$\mathrm{Na}+\mathrm{Na}_{3}$

1345

$\mathrm{Na} * \mathrm{Ne}$

52

$\mathrm{Na}_{2} * \mathrm{Xe}$

2169

$\mathrm{Ne} *+\mathrm{He}+\mathrm{Xe}+\mathrm{F}, \mathrm{I}$ 2057

$\mathrm{Ne} *+\left[\mathrm{Ne}+\mathrm{Xe}_{\mathrm{e}}+\mathrm{NF}_{3}\right]$ 2057

$\mathrm{Ne} *+\mathrm{He}$

1170

$\mathrm{Ne*}+\mathrm{Ne}$

1165

1170

$N F *+E I$

ND

NO + NO

.. 2125

$\mathrm{NO}+\mathrm{N}_{2}$

$\mathrm{O}+\mathrm{H}_{2}$

ocs + ocs

2341

$S_{2} *+A r$

S57

$\ddot{S},+\underset{957}{+C_{p} F_{6}}$

$S_{p} *+C F$.

S57

$s_{+} \neq \underset{857}{+H e}$

$\mathrm{S}_{2} * \mathrm{~N}_{2}$

S57?

$S * S_{557}$

$\dot{s}_{p} *+x e$

g5?

$\mathrm{SF}_{*}+\mathrm{Cr}$

- 1385

$\sin =5 \mathrm{~m}$

- 2471

UF: $= \pm \mathrm{UF}_{1384}$

xe $\mathrm{NH}_{3}$

2143

$\mathrm{Xe} *+\mathrm{N}_{2}$

2054

Under

$145 \quad 746,752$

$1038 \quad 1123 \quad 1174$ 
A11

HEAUY PART ICLE - HEAVY PARTICLE INTERACTIONS

Collisional De-Excltation (total)

Ar* + A 1073
2063 $1763 \quad 2031$

$A r *+B r$ 2078

$\mathrm{Ar} *+\mathrm{BrCN}$

2078

$\mathrm{Ar} *+\mathrm{Cl}$

$\mathrm{Ar} *+\mathrm{CLF}$

2078

$A r *+C 0$

$A r *+C O$

$166=2078$

$\begin{aligned} & A r *+\cos \\ & 2078\end{aligned}$

$A r *+C S$

2078

Ar* $+D_{2}$

$A+* \mathrm{D}_{2} \mathrm{O}$

$\mathrm{Ar} * \mathrm{~F}$,

14812078

$A r *+H_{3}$

$\mathrm{Ar} *+\mathrm{H}, \mathrm{O}$

$1088 \quad 2078$

$\mathrm{Ar} *+\mathrm{H}_{>} \mathrm{S}$

$\mathrm{Ar} *+\mathrm{HBr}$

2078

Ar* $+\mathrm{HCl}$

$A r^{*}+\mathrm{HCN}$

2078

$A r * \begin{array}{r}H g \\ 2078\end{array}$

Ar* $+\mathrm{HI}$

2078

$A r *+I B r$

2078

$A r *+I C$

207A

$A r *+K r$

2078

Ar* $+N$,

$1834 \quad 2031 \quad 2078$

Ar* $\underset{2078}{+N_{2} \mathrm{O}}$

$A r *+\mathrm{NH}_{3}$

2078
$A r *+N O$

2078

$A r * 0$,
+2078

Ar* + OF

$A r *+5 e F$ 。

2078

$\mathrm{Ar} *+\mathrm{SO}_{2}$

2078

$A r *+X e$

14812078

$A r, F * F$,

1982

$A r F *+F_{2}$

$532^{? .1982}$

$A r F *+2 A r$

1982

$B a *+B a$

1811

Ba* $\underset{2184}{+\mathrm{Ba*}}$

$\mathrm{Ba}+\mathrm{Ar}$

2505

$\mathrm{Ba}+\mathrm{He}$

2505

$\mathrm{Ba}+\mathrm{Kr}$

2505

$\mathrm{Ba}+\mathrm{Ne}$

2505

$\mathrm{Ba}+\mathrm{Xe}$

$B r *+B r$

423

$\mathrm{Br} * \mathrm{BrCl}$

423

$\mathrm{Br} *+\mathrm{Cl}$.

423

$\mathrm{Br}+\mathrm{Cd}_{2}$

$1020^{\circ}$

$\mathrm{Br} *+\mathrm{H}>\mathrm{O}$

979

$\mathrm{Br} * \underset{\mathrm{HCl}}{1020}$.

$B \mathbf{r} * \mathbf{I}$,

423

$\mathrm{Br}+\mathrm{IBr}$

423

Br* + ICl

423

$B r_{2} *+A r$

692

$\mathrm{CCl}_{3} \mathrm{FH}+\mathrm{CCl}_{3} \mathrm{~F}$

512

$C d *+D_{283}$

$\mathrm{Cd} * \mathrm{H}_{3}$

283

$C d *+H D$

283

$\underset{512}{\mathrm{CH}_{3} \mathrm{OCH}_{3}}+\mathrm{CH}_{3} \mathrm{OCH}$

$\mathrm{Cl}_{2} * \mathrm{Cl}_{2}$

152
CIF* $+A r$

$\mathrm{ClF}+\mathrm{ClF}$ 1989

$\mathrm{CO} *+\mathrm{A}$

288755

$\mathrm{CO} * \mathrm{CO}$

$\mathrm{CO} *+\mathrm{CO}_{2}$

$\mathrm{CO}+\mathrm{D}_{28}$

$\mathrm{CO}+\mathrm{H}$ 288 (93)

$\mathrm{CO}+\mathrm{He}$

$\mathrm{CO} * \mathrm{Kr}_{\mathrm{r}}$

755

$\omega=N_{28}$

$\mathrm{CO}+\mathrm{O}$

1994

$\mathrm{co} *+\mathrm{o}_{0}$

288

co* $+x e$

288

$\mathrm{CO}+\mathrm{He}$ 2052

$\mathrm{CD}_{*} * \mathrm{Ar}$

2026

$\mathrm{CO}_{2}+\mathrm{CO}$

. $7 \$ 1$

$\mathrm{CO}_{2} * \mathrm{HBr}$

$\mathrm{CO}_{2} * 4 \mathrm{He}$

.... 2026

$\mathrm{Cu}_{2} \underset{791}{+} \mathrm{N}_{2}$

$\ddot{\mathrm{CO}} \mathrm{O}_{2} * \mathrm{O}_{2}$

$$
791
$$

Ca + A

.. 1726

$\mathrm{Cs}+\mathrm{Cs}$

.. 490 .

$\mathrm{Cs} * \mathrm{He}$

1718

cis* $\mathrm{N}_{2}$

$\ddot{C s}+N$

2718

Cu* +.He

1815

$\mathrm{Cu}+\mathrm{Ne}$

1815

D. + HF*

- 956

$\ddot{D}_{2}+\mathrm{H}$

251

F $+\mathrm{H}$

1062

$\dot{F}+\mathrm{H}_{2}$ 


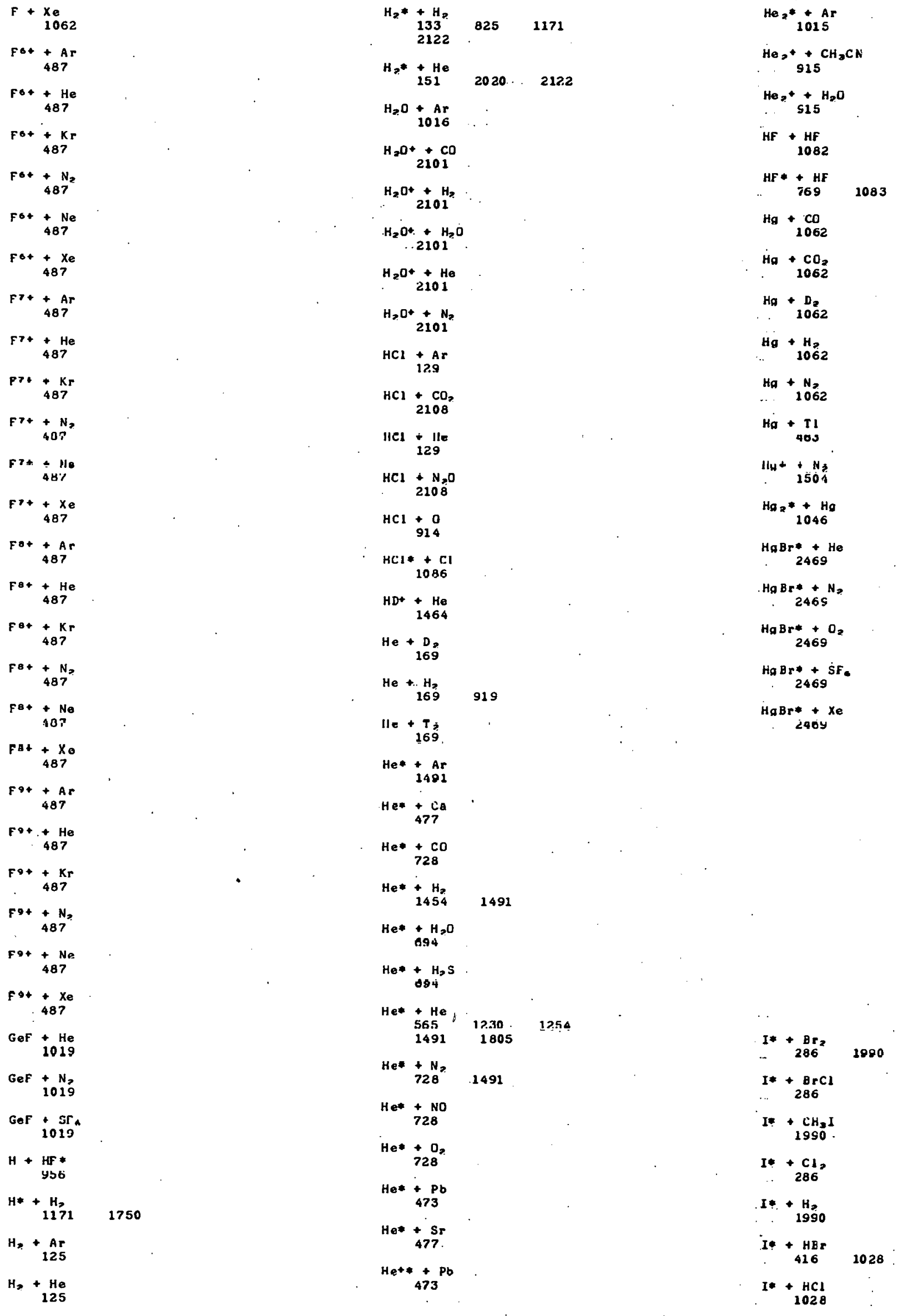




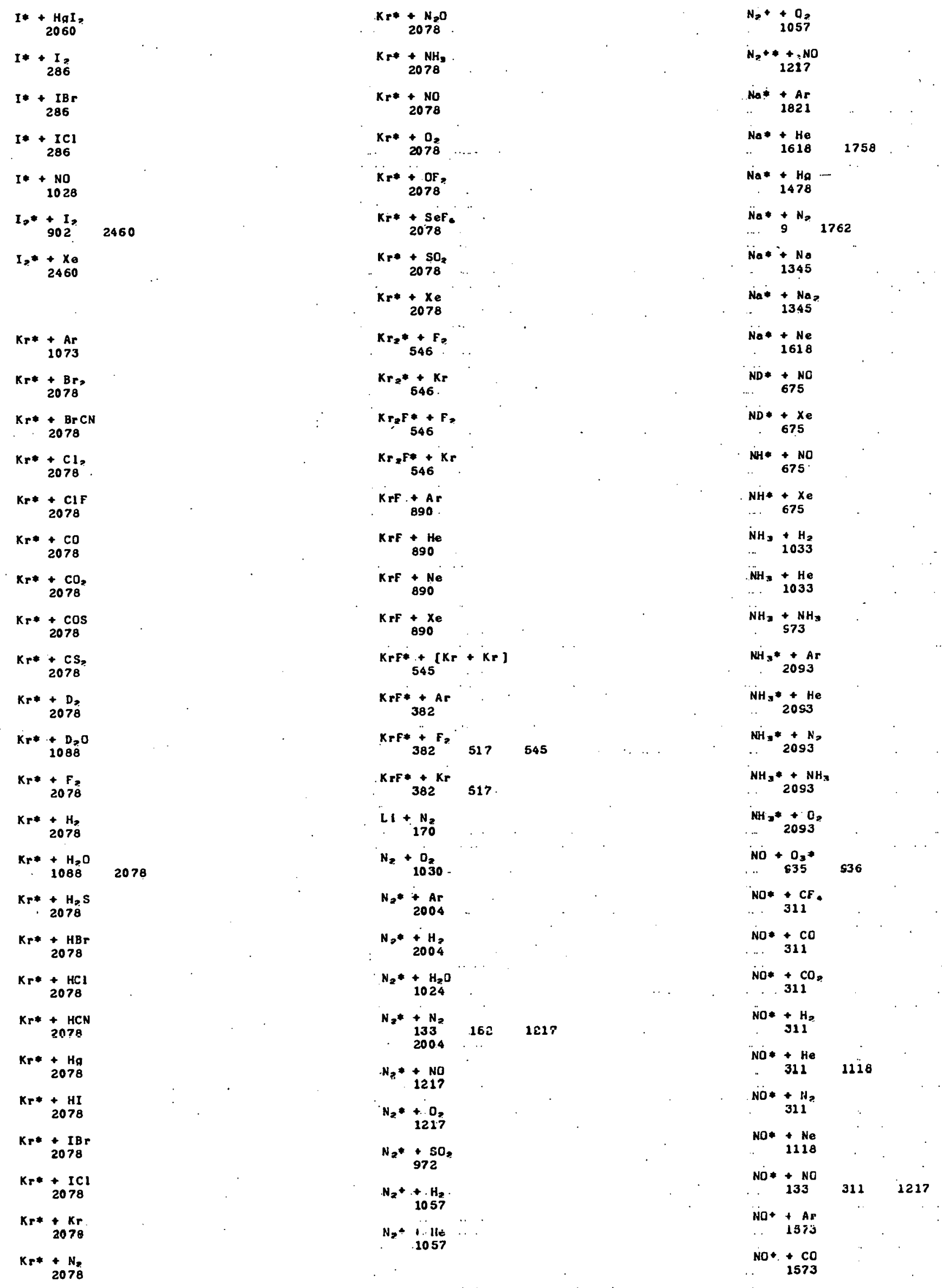




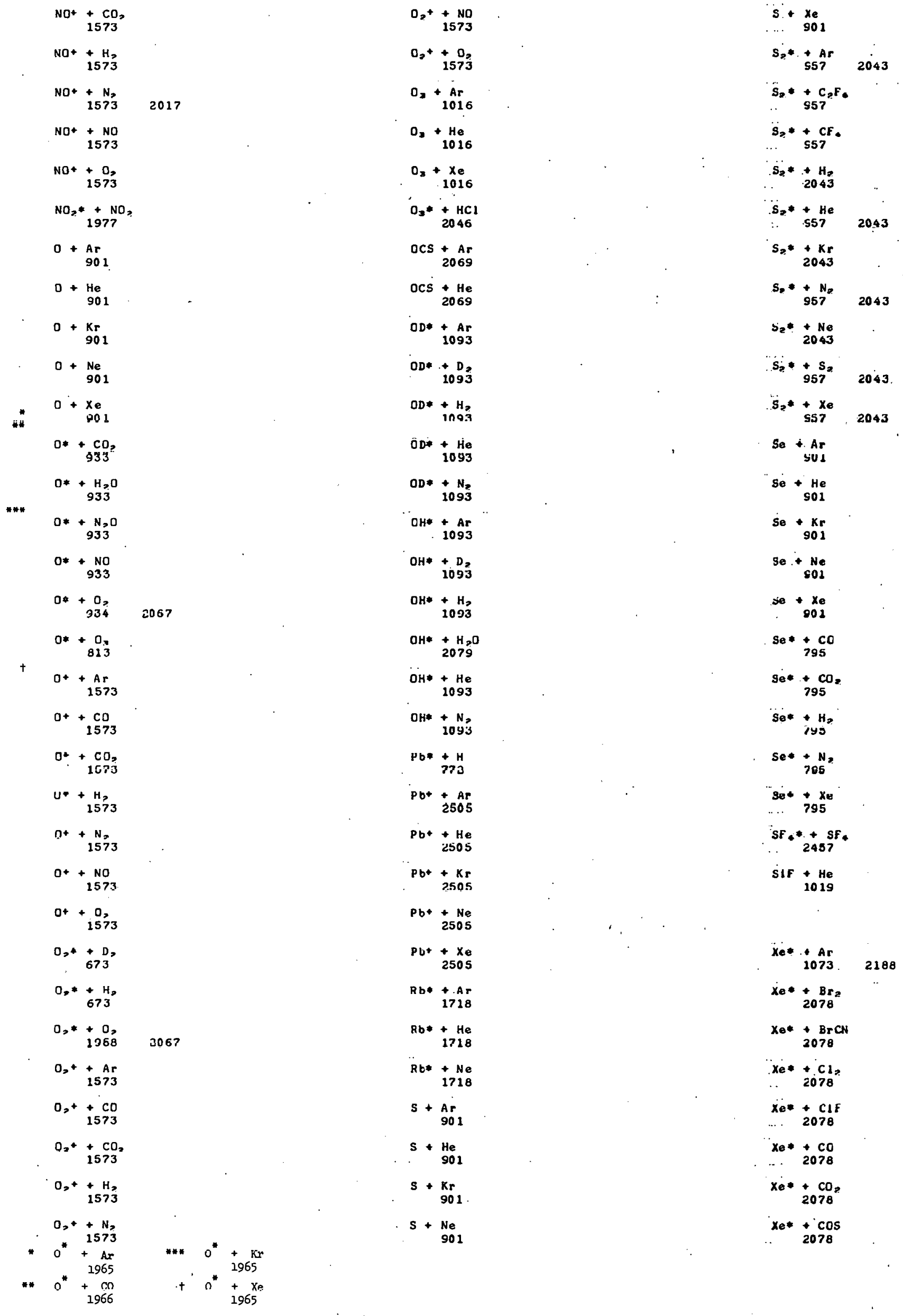




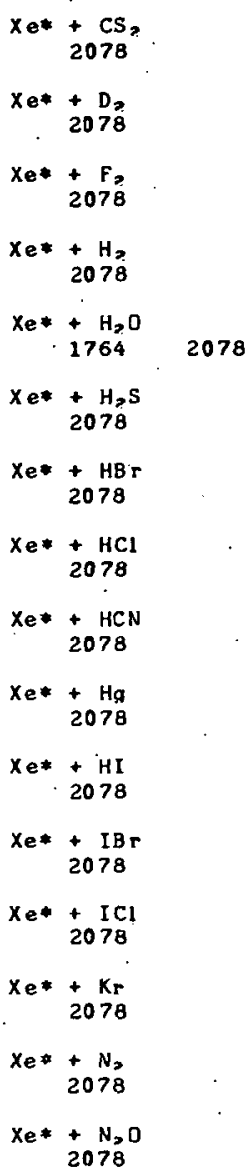

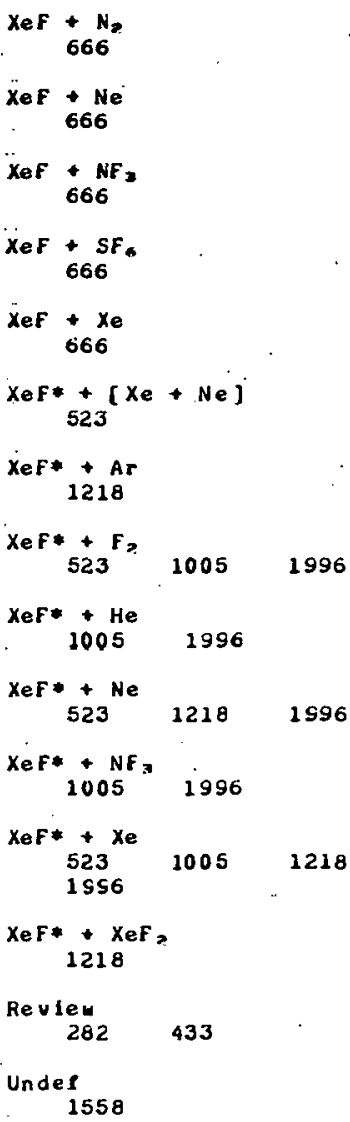


A12

HEAVY PART ICLE - HEAUY PARTICLE INTERACTIONS

Collisional Line BroadenIng

$$
\begin{aligned}
& \text { Ar }+\begin{array}{r}
\text { Al } \\
644
\end{array} \\
& \mathrm{Ar}+\underset{754}{\mathrm{HCl}} \\
& A r+X e \\
& \mathrm{Ca}+\mathrm{Ar} \\
& 1448 \\
& \mathrm{Ca}+\mathrm{He} \\
& 1448 \\
& \mathrm{Ca}+\underset{144 \mathrm{Ar}}{\mathrm{Ar}} \\
& \mathrm{Cot}+\mathrm{Ho} \\
& 1448 \\
& c d+c d \text { d } \\
& \text { CF. }+\mathrm{CF}_{102}
\end{aligned}
$$$$
\text { CH. } \underset{1678}{\mathrm{C}}
$$$$
\mathrm{CO}_{2}+\mathrm{Ar}
$$$$
1125
$$$$
\mathrm{CO}=\underset{465}{+} \mathrm{CO}
$$$$
465 \text { 1125 }
$$$$
\mathrm{CO}_{2}+\mathrm{N}_{2}
$$$$
1125
$$$$
\mathrm{CO}_{*} * \underset{1670}{+\mathrm{Ar}}
$$$$
\mathrm{CO}_{2} * \mathrm{CO}_{3}
$$$$
1670^{+\mathrm{CO}_{2}} \quad 1692
$$$$
\mathrm{Cu}={ }_{1670}^{+N}
$$$$
\mathrm{Cs}+\text { Ar }
$$$$
\begin{array}{lll}
1164 \quad 1250 \quad .1680
\end{array}
$$$$
\text { Cs } * H
$$$$
1748
$$$$
\mathrm{Cs}+\mathrm{He}
$$$$
1740
$$$$
\mathrm{Cs}+\mathrm{Kr}
$$$$
1164
$$$$
1250 \quad 1680
$$$$
\begin{aligned}
& \text { Ne } \\
& 1164
\end{aligned}
$$$$
1748
$$$$
C s+x e
$$$$
1164 \quad 1250 \quad 1680
$$$$
C_{s} * A r
$$$$
\begin{array}{r}
A r \\
1665
\end{array}
$$$$
C_{s} *+x \theta
$$$$
1672
$$$$
\mathrm{Cu} * \mathrm{He}
$$$$
1815
$$$$
\begin{array}{r}
\mathrm{Cu} * \mathrm{Ne} \\
1815
\end{array}
$$$$
H+\text { Ar } 1748
$$

$$
\text { H }+ \text { H }
$$$$
1748
$$$$
\text { H }+\mathrm{He}
$$$$
1748
$$$$
\text { H }+K_{r}
$$$$
2748
$$$$
\mathrm{H}+\mathrm{Ne}
$$$$
1748
$$$$
H+\mathrm{Xe}
$$$$
1748
$$

$\mathrm{H}_{2}+\mathrm{He}$

$\because \mathrm{H}_{2} \mathrm{O}+\mathrm{Ar}$

$$
896
$$
$\mathrm{H}_{2} \mathrm{O} * \underset{1676}{+\mathrm{AIr}}$

$\mathrm{H}_{2} \mathrm{O} *+\mathrm{CO}_{2}$ 896

$\mathrm{H}_{2} \mathrm{nt}+\mathrm{Ne}^{\mathrm{C}}$ คP6 $16 ? 6$

HCL + HCI 1673

$\mathrm{HCl}+\mathrm{HF}$

He $+\mathrm{Al}$

. 644

$\mathrm{He}+\mathrm{Ne}$

$\mathrm{He}+\mathrm{Ne}$

35

Het ${ }_{1829}$

$\mathrm{HF}+\mathrm{HCl}$

1673

$H r+\underset{1673}{H}$

$\mathrm{Hg}+\mathrm{Ar}$

1300

$1 g+A r$

1162

I* Ar

2464

$I * \ldots \mathrm{He}$

246A

$\mathrm{I} *+\mathrm{Ne}$

. 3464

$I * \frac{X 6}{2464}$

$K+A r$

1164 "1616 1748

$\mathbf{K}+\mathbf{H}$ 1748 .

$\mathrm{K}+\mathrm{He}$

$1616 \quad 1748$

i $+\mathrm{Kr}$

$1164 \quad 1616$

1748

$\mathrm{K}+\mathrm{Ne}$

K. N616 - 1748

$x+x_{0}$

$1164 \quad 1616$

$\mathrm{Kr}+\mathrm{Na}$

75

$\mathrm{Kr} * \dot{91}^{\mathrm{Ar}}$
$K r *+1$

Li $+\underset{1748}{A R}$

$1 i+\underset{1748}{H}$

$\ddot{L i}+H e$ 1748

$L \mathbf{L}+\mathbf{K r}$ 1748

ii $+\mathrm{Ne}$

il $+x e$

$\ldots 1+1748$

$21 * \underset{1659}{A r}$

$\mathrm{LI}+\mathrm{He}$

.1659

$N=N_{\Delta \rightarrow 5}$

मi

$1164 \quad 1213 \quad 1748$

No $+H$

$\mathrm{Na}+\mathrm{He}$

1213

1748

$\mathrm{Na}+\mathrm{Kr}$

1164

1748

$\ddot{\mathrm{Na}}+\mathrm{N}_{3}$

1213

Na $+\mathrm{Ne}$

. 1748

$\mathrm{Na}+\mathrm{Xe}$

1164

1740

NA - AP

$\mathrm{Na} * \mathrm{No}$

. 1696

$\mathrm{Ne}+\mathrm{Ne}$

... 35

$\mathrm{Ne}+\mathrm{Xe}_{\mathrm{e}}$

No 466

$\mathrm{NH}_{3}=\underset{1683}{+}$

$\mathrm{NH}_{\mathbf{2}}+$ He

... 2683

$\mathrm{NH}_{8} *+\mathrm{NH}_{3}$

... 2434

$\mathrm{NO}_{2} * \mathrm{NO}^{2}$

1672

$0 .+0$

$\mathrm{D}_{2}+\mathrm{N}_{2}$

1682

ócse + Ar

. 2339

ocs* + He

2339

$R \dot{b}+A r$

Ar
1249
1748

Rb $+\mathrm{H}$

1748 


\begin{tabular}{|c|c|c|c|}
\hline & 1249 & 1748 & \\
\hline Rb & $\begin{array}{c}K_{r} \\
1249 \\
1748\end{array}$ & 1250 & 1680 \\
\hline $\mathbf{R ~ b}$ & $\begin{array}{l}+\mathrm{Ne} \\
1249\end{array}$ & 1748 & \\
\hline Rb & $\begin{array}{r}x \text { Xe } \\
1249 \\
1748\end{array}$ & 1250 & 1680 \\
\hline
\end{tabular}

$$
\begin{gathered}
\mathrm{SO}_{2} *+\mathrm{H}_{60} \\
1661 \\
\mathrm{SO}_{2} *+\mathrm{N}_{2} \\
1661 \\
\mathrm{SO}_{2} *+\mathrm{SO}_{2} \\
1661 \\
\mathrm{Sr}+\mathrm{Sr}
\end{gathered}
$$

$\mathrm{Xe}+\mathrm{Na*}$
.75

$z_{n}+z_{n}$ 1252

Under $1124 \quad 1671 \quad 1684$ 1687 
$\mathrm{ClO}+\mathrm{NO}$

HEAVY PAAT ICLE - HEAVY PARTICLE INTERACT IONS

Heavy Particle Interchange,

Rearrangement, and Assoclation (one or more Ionic reactants)

$A r^{+}+A r$

1092

$\mathrm{Ar}+\mathrm{H}_{2}$

$120 \mathrm{i}$

$A r^{+} \underset{2454}{+H_{20}} \quad 2455 \quad 2456 \quad 797$

$\mathrm{Br}+\mathrm{N}^{2} \mathrm{O}_{5}$

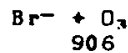

$\mathrm{C}++\mathrm{CO}_{2}$

1647

$C+D ?$
929

$\mathrm{rt}+\underset{440}{\mathrm{H}_{n}} \quad 849$

$\mathrm{C}+\underset{837}{\mathrm{H}_{2} \mathrm{~S}}$

$\mathrm{C}+\mathrm{O}$ ?

1647

$\mathrm{C}^{2+}+\underset{838}{\mathrm{H}}$

$\mathrm{C}_{>} \mathrm{H}^{-}+\mathrm{O}_{\text {. }}$

$\mathrm{CCl}_{3}+\ldots+\mathrm{Cl}^{-}$

941

$\mathrm{CH}++\mathrm{H}$

826

$\mathrm{CH}_{2}+\underset{847}{+} \mathrm{CO}$

$\mathrm{CH}_{3}+\underset{847}{\mathrm{CO}}$ ?

$\mathrm{Cs}^{3}+\operatorname{CnS}$

847

$\mathrm{CH}_{3}+\underset{847}{+\mathrm{H}_{3}}$

$\mathrm{CH}_{3}+\mathrm{H}_{>} \mathrm{O}$

847 .

OII: ${ }_{847}^{1 \mathrm{H},}$

$\mathrm{CH}_{3}+\underset{B 17}{+} \mathrm{NH}_{3}$

$\mathrm{CH}_{347}+\underset{8}{ }$

$\mathrm{Cl}_{2}+\mathrm{O}^{-}$

vys

$\mathrm{Cl}-\mathrm{N}_{2} \mathrm{O}_{5}$
$\$ 78$

$\mathrm{Cl}+\mathrm{O}=$

$610^{\circ}+60$

1096

$\mathrm{ClO}+\mathrm{NO}$

- $\mathrm{CH}_{2}{ }^{+}+\mathrm{H}_{2} \mathrm{O}$

797

- $\mathrm{CO}_{797}^{+}+\mathrm{H}_{2} \mathrm{O}$

** $\mathrm{CO}_{2}^{+}+\mathrm{H}_{2} \mathrm{O}$

$+\mathrm{HCO}_{797}^{+}+\mathrm{H}_{2} \mathrm{O}$
$\mathrm{ClO}^{-}+\mathrm{SO}_{2}$

. 1096

$\mathrm{CO}_{2}+2+2 \mathrm{CO}_{2}$

$\mathrm{CO}_{3}-+\mathrm{HCl}^{-}$

1096

$\mathrm{CO}_{3}-\underset{978}{+\mathrm{N}_{2} \mathrm{O}_{3}}$

$\mathrm{CC}_{3}-\underset{906}{+} \mathrm{O}_{2}$

$\mathrm{CO}_{3}-\underset{906}{+} \mathrm{D}_{3}$

$\mathrm{CO}_{4}^{-}+\mathrm{HCl}^{-1}$

1096

$\mathrm{COD}+\mathrm{D}_{2}$

2448

$\mathrm{COD}+\mathrm{Ne}$

2448

$\mathrm{CS}+\mathrm{H}_{2}$ $83 \%$

$\mathrm{D}_{3}+\mathrm{CO}$

$244 B$

$\mathrm{D}_{\mathbf{3}}+\underset{2449}{\mathrm{Kr}}$

$\mathrm{D}_{3}+\mathrm{Ne}$

$\mathrm{D}_{3} \mathrm{O}+\mathrm{D}_{2}$

929

$\mathrm{D}-+\mathrm{H}_{\mathrm{B}}$

$\mathrm{F}+\mathrm{H}_{2}$ 983

$\mathrm{F}-\underset{978}{\mathrm{~N}_{2} \mathrm{O}_{5}}$

$\mathrm{F}+\mathrm{O}_{3}$

906

$\mathrm{H}+\mathrm{H}^{-}$

$\mathrm{H}+\mathrm{Ho}$

826

$\mathrm{H}_{2}+\underset{2044}{+\mathrm{H}_{2}}$

$\mathrm{H}_{2}^{+}+\mathrm{He}^{+}$

2113

$\mathrm{H}>\mathrm{O}+\underset{176}{+} \mathrm{H}>929$

$\mathrm{H}_{3}+\mathrm{Kr}$

2449

$\mathrm{II}_{3}+1 \mathrm{HO}_{2449}$

$\mathrm{H}_{-} \mathrm{nt} \underset{\mathrm{s78}}{+} \mathrm{N}_{\mathrm{a}} \mathrm{ng}$

$\mathrm{HCl}+\mathrm{N}_{2}$

1059

$\mathrm{He}^{+}+\mathrm{N}_{2} \mathrm{O}$

1647

$\mathrm{He}+\underset{378}{2 \mathrm{He}}$

$\mathrm{He}_{2}^{+}+\mathrm{CH}_{3} \mathrm{CN}$

$\mathrm{He}_{2}+\mathrm{H}_{2} \mathrm{O}$

*** $\mathrm{H}_{2} \mathrm{O}^{+}+\mathrm{NO}$

$\mathrm{H}_{2} \mathrm{O}^{+}+\mathrm{C}_{2}$

$\mathrm{H}_{2} \mathrm{O}+\stackrel{798}{+}+\mathrm{H}_{2} \mathrm{~S}$

*. $\mathrm{HgCl} *+\mathrm{Ar}$

$38{ }_{1}$
$\mathrm{HgCl}+\cdot \mathrm{CCl}$
384
$\mathrm{HeH}+\mathrm{H}$

826

$\mathrm{HNO}+\mathrm{H}_{2}$

821

$\mathrm{I}+\underset{454}{\mathrm{CH}}$

$\ddot{I}=\underbrace{N_{2} \mathrm{O}_{3}}_{S 78}$

$I-+\begin{array}{r}03 \\ S 06\end{array}$

$\overline{\mathrm{K}}++\mathrm{D}_{2}$

508

$\mathrm{Kr}+\underset{\mathrm{SO}}{+\mathrm{H}_{2}}$

$\mathrm{Kr}++H \mathrm{D}$

-. 908

$K \boldsymbol{r}++K r$

1052

$\mathrm{Mg}+\mathrm{H}_{3} \mathrm{O}^{+}$

.. 276

$\mathrm{Hg}+\underset{? .76}{\mathrm{H}_{3} \mathrm{~S}}$

$\mathrm{N}+\mathrm{CH}_{*}$

309

$\mathrm{N}^{+}+\mathrm{CO}$

309

$N++\mathrm{CO}_{30}$

$\mathrm{N}+\mathrm{COS}$

309

$\mathrm{N}+\mathrm{H}_{2}$

$\mathrm{N}+\cdots \mathrm{H}_{2} \mathrm{CO}$

305

N* $+\mathrm{H}_{2} \mathrm{O}$

$\ddot{N}+\mathrm{H}_{2} \mathrm{~S}$

309

$\mathrm{N}+\mathrm{N}^{3}$

$\therefore$ Jog

$\mathrm{N*}+\mathrm{NH}_{3}$

$30 \mathrm{~s}$

$\mathrm{N}+\mathrm{O}_{2}$

$309 \quad 1647$

$\mathrm{N} 2++\mathrm{H}_{2}$

838

$\mathrm{N}_{2}++\mathrm{CH}_{4}$

305

$\mathrm{Nr}++\mathrm{CO}$

309

$\mathrm{N}^{2}+\underset{30 \mathrm{C}}{\mathrm{CO}}$

$\mathrm{N}^{+}+\operatorname{Cos}$

suy

$N+\underset{309}{+}$

$\mathrm{N}_{2}+\mathrm{H}_{2} \mathrm{CO}$

309

$\mathrm{N}_{2}+\mathrm{H}_{2} \mathrm{O}$

$=309^{2} .797$

$\mathrm{N}_{2}+\mathrm{H}_{2} \mathrm{~S}$.

$30 \mathrm{~s}$

$\mathrm{N}_{2}+\underset{309}{+N_{2}}$ $309 \quad 1342$

$\mathrm{HgCl}_{8}+\mathrm{Cl}_{2}$

$\mathrm{HgCl} *+\mathrm{HCl}$

$\mathrm{HgCl} *+\mathrm{He}$ $\mathrm{HgCl}^{3}+\mathrm{N}_{2}$ $\mathrm{HgCl}^{3}+\mathrm{Xe}$

$\mathrm{H}_{2} \mathrm{~s}^{+}+\mathrm{H}_{2} \mathrm{O}$

384 


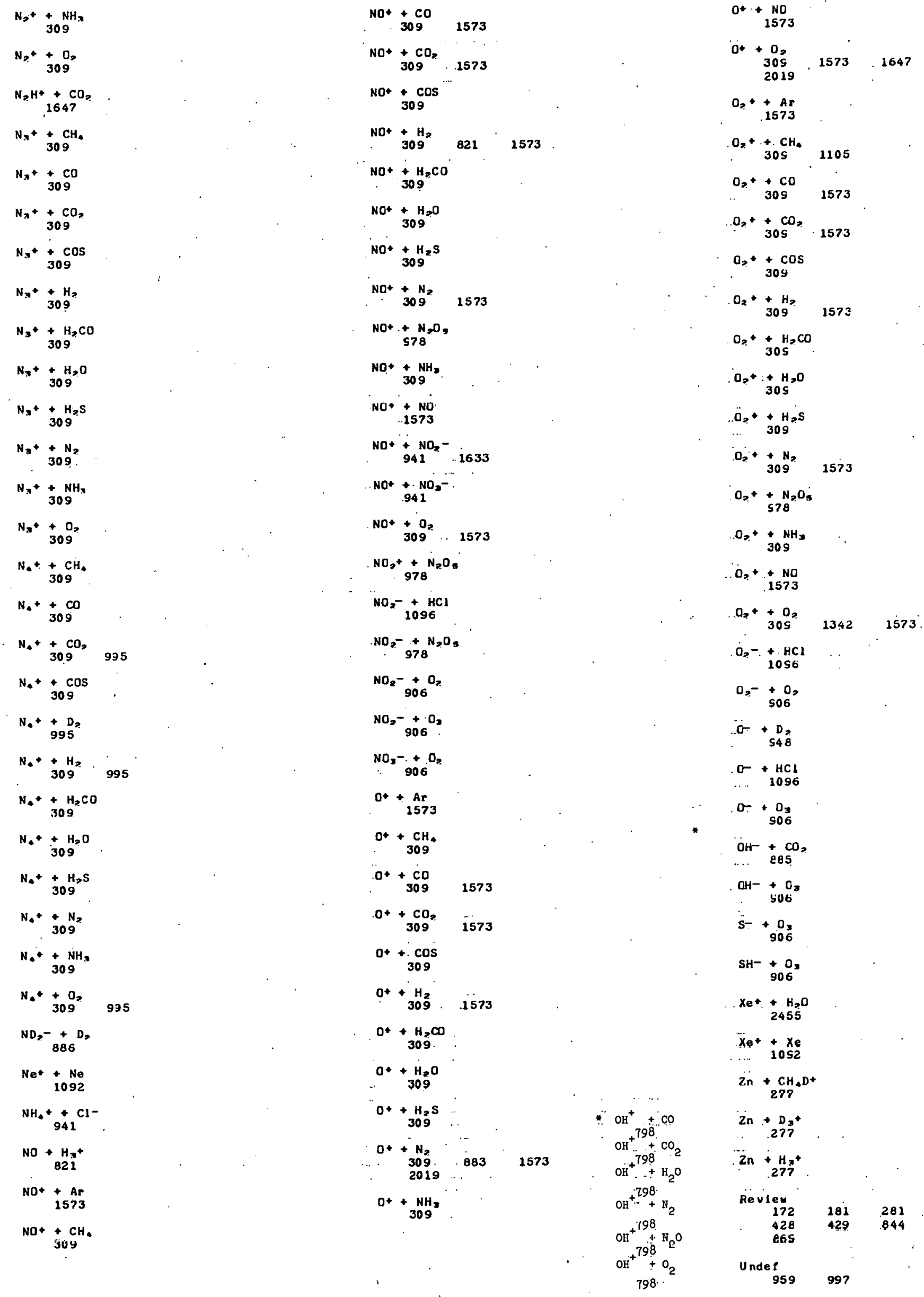


A14

\section{HEAUY PART ICLE - HEAVY PARTICLE INTERACT IONS}

Heavy Particle Interchange. Rearrangenent, and Association (only neutral reactant $s$ )

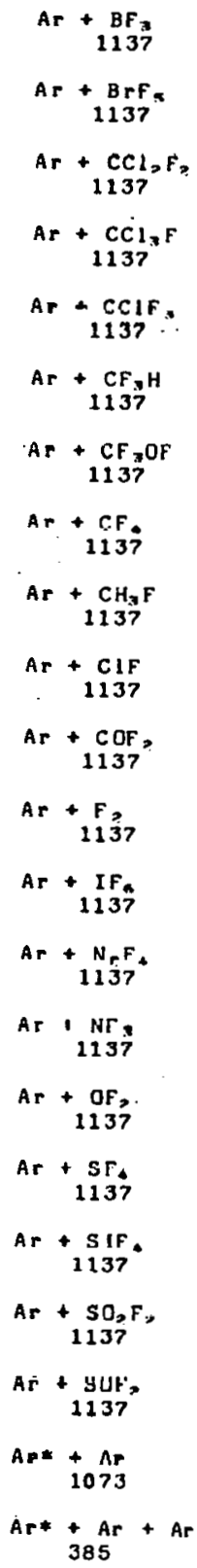

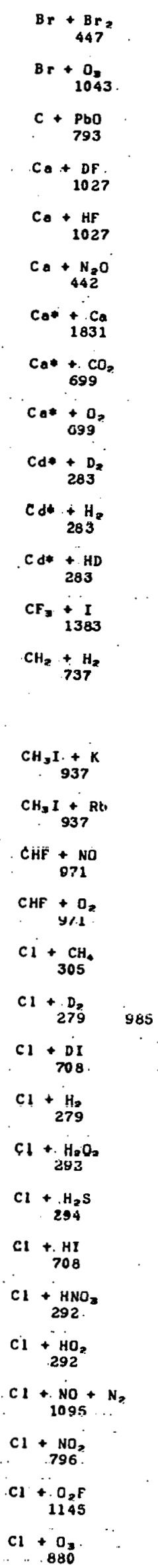




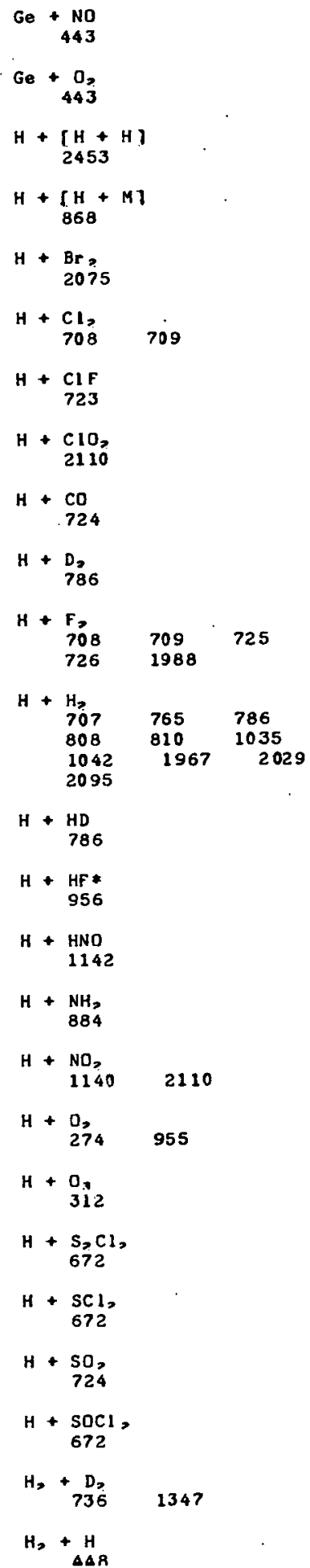




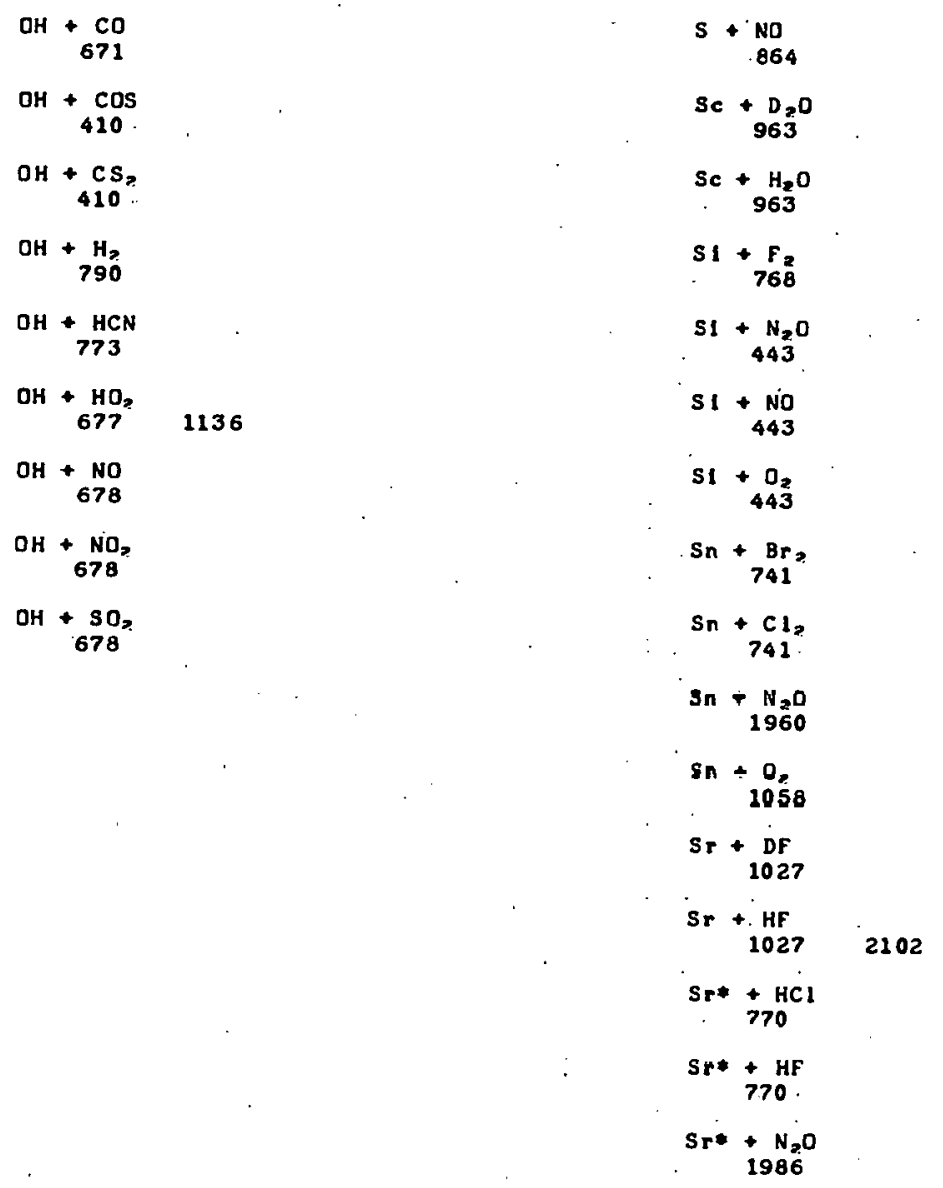

$\mathrm{Sr}+\mathrm{sr}$

1227

$T+F_{7}$

$T+\mathrm{HT}$ 290

Th +03 2000

$\mathrm{U}+\mathrm{O}_{2}$ $4 i s$

$\dot{u}+\mathrm{C}_{3}$

2000

$\dot{x e}+2 x e$

Xe* + Ar

.. 1073

$X_{\theta}+\theta r_{2}$

418

Y..,+ 0
563

$Y+\mathrm{H}_{5} \mathrm{O}$

Q.5.

$2 I+N D$

Review

$111 \quad 124 \quad 145$

$\begin{array}{lll}275 & 280 & 070\end{array}$

Unde?

161278.303

421426.910

$11012050 \% 2071$

$R$ RbF $+K$ 
A15

HEAUY PART IClE - heavy PARTICle INTERACTIONS

Spin Exchange

$$
\begin{gathered}
\mathrm{Cs}_{3}+\underset{\mathrm{Rb}}{2517} \\
\mathrm{Cs}_{2}+\mathrm{Cs}_{25} \\
2506 \\
\mathrm{H}^{+}+\underset{\mathrm{Au}}{56} \\
\mathrm{H}^{+}+\underset{56}{\mathrm{Ca}} \\
\mathrm{H}^{+}+\underset{56}{\mathrm{Nd}} \\
+56
\end{gathered}
$$

$$
\begin{aligned}
& \mathrm{H}+\underset{56}{\mathrm{Sn}} \\
& \mathrm{H}+\underset{56}{\mathrm{U}} \\
& \mathrm{H}+\underset{56}{\mathrm{Yb}} \\
& \mathrm{H}^{+}+\underset{56}{\mathrm{Zn}} \\
& \mathrm{H}+\underset{6 r}{\mathrm{Zr}} \\
& \mathrm{He}^{+}+\mathrm{H} \\
& 1544 \\
& \mathrm{He}^{2+}+\mathrm{Au} \\
& 56 \\
& \mathrm{He}^{2+}+\mathrm{Ca} \\
& 56
\end{aligned}
$$

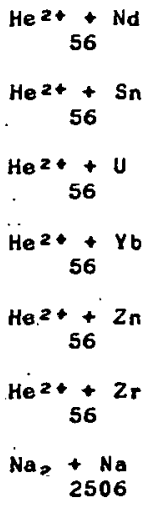


A17

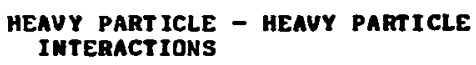

Interaction Potentlal a

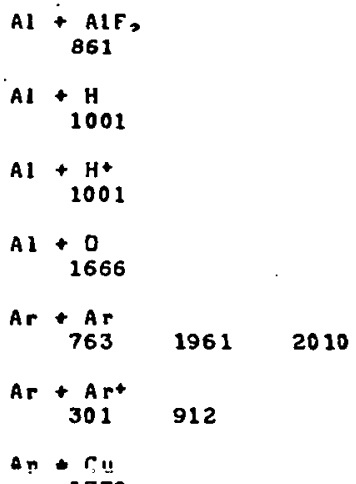

B* + A r

B+ $+\mathrm{He}$

982

$B^{+}+\underset{982}{K_{r}}$

$\mathrm{B}++\mathrm{Ne}$

982

$\theta^{\circ}+\times 6$
982

$\mathrm{Ba}+\mathrm{Xe}$

185

$\mathrm{Be}+\mathrm{HF}$

2059

$B r+28$

$c+\mathrm{CBr}$ 861

$c+\mathrm{CCl}_{2}$.

$c+\mathrm{CF}$,

861

$\mathrm{C}+\mathrm{CI}_{\mathrm{B}}$

$$
c+\mathrm{Co}
$$$$
1126
$$$$
C+N
$$$$
439.811
$$

$c+0$ $\begin{array}{lll}304 & 1226 & 1239\end{array}$

$C=\mathrm{H}_{2}$

$\mathrm{Ca}+\mathrm{Ca}$ 1031

CH, -

143

CH.

$\therefore 2496$

$\mathrm{CH}_{4}+\mathrm{CH}_{4}$

947

$\mathrm{CH}_{4}^{+}+\mathrm{H}$

CH. $+H_{947}^{+}$

$\mathrm{CH}_{4}+\mathrm{H}_{7} \mathrm{O}$ 947

$\mathrm{CH}_{4}+\mathrm{He}$ 947

CH. $\underset{947}{+\mathrm{LI}}$

CH. $+\underset{947}{+N}$

CH. $+\mathrm{N}_{94}$

$\mathrm{CH}_{4} \underset{947}{+\mathrm{N}_{2} \mathrm{O}}$

$\mathrm{CH}_{4}+\mathrm{NH}=$ 947

CH.
CH. + D

CH. $\underset{947}{+O_{2}}$

$\ddot{c l}+c_{1}$

... 1018

$\mathrm{Cl}+\mathrm{HBr}$

. 298

$\ddot{c l}+\underset{2037}{K r=}$

ci $+\mathrm{O}_{3}$

CIF $+K r$

. 1080

Co + Ar

2042

$\ddot{\mathrm{CO}}+\mathrm{H}_{\mathrm{s}}$

. 127

$\mathrm{CO}+\mathrm{He}$

2042

co + Kr 2042

CO $+\mathrm{Ne}$

co $+x e$ $x e$
2042

$C 0=2496$

Cs + Ar 1164

1348

$\mathrm{Cs}+\mathrm{Br}$ 2337

$\mathrm{Cs}^{\prime}+\mathrm{Cl}$ 2337

$\mathrm{Cs}+\underset{926}{\mathrm{Cs}}$

Co $+F$, coor

$\mathrm{Cs}+\mathrm{H}$ $\stackrel{+1}{\$ 49}$

$\ddot{6 s}+1$

$\dot{C}_{3}+K r$ 1164

$19+N 4$ 1164.

$c_{3}+x_{e}$ $x e$
1164

$89 * \begin{array}{r}\times 9 \\ 1672\end{array}$

$\ddot{C}++A r$ 1002

$\mathrm{Cs}+\mathrm{Cs}_{\mathrm{s}}$

. 313

Ca $+k$

C 313

$\mathrm{Cg}^{+}+\mathrm{KT}_{\mathrm{T}}$ 1002

$\mathrm{Cs}+\mathrm{Na}$

… 313

Cat+Rt

. 313

$\dot{C}_{8}++X_{0}$ 


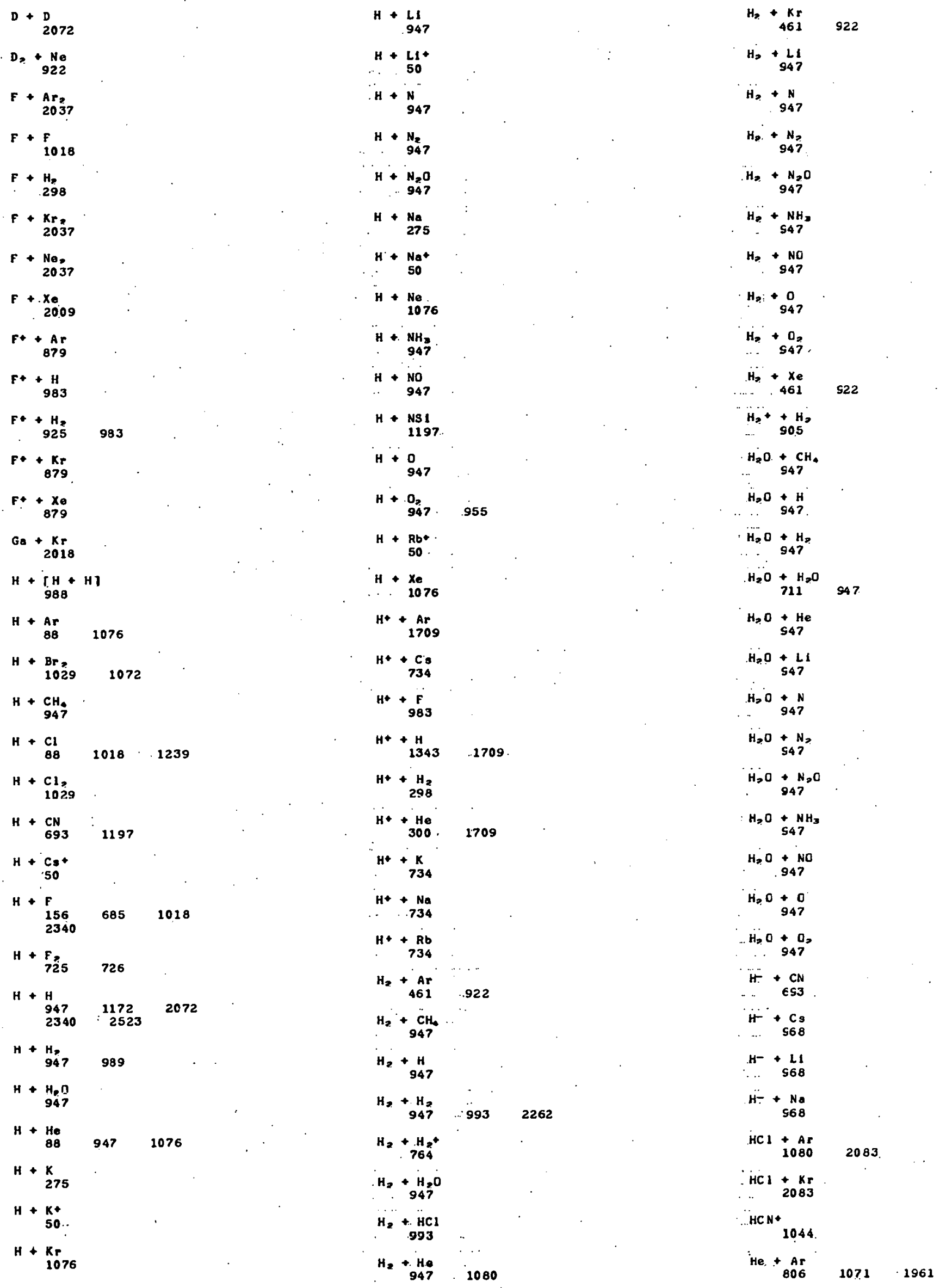




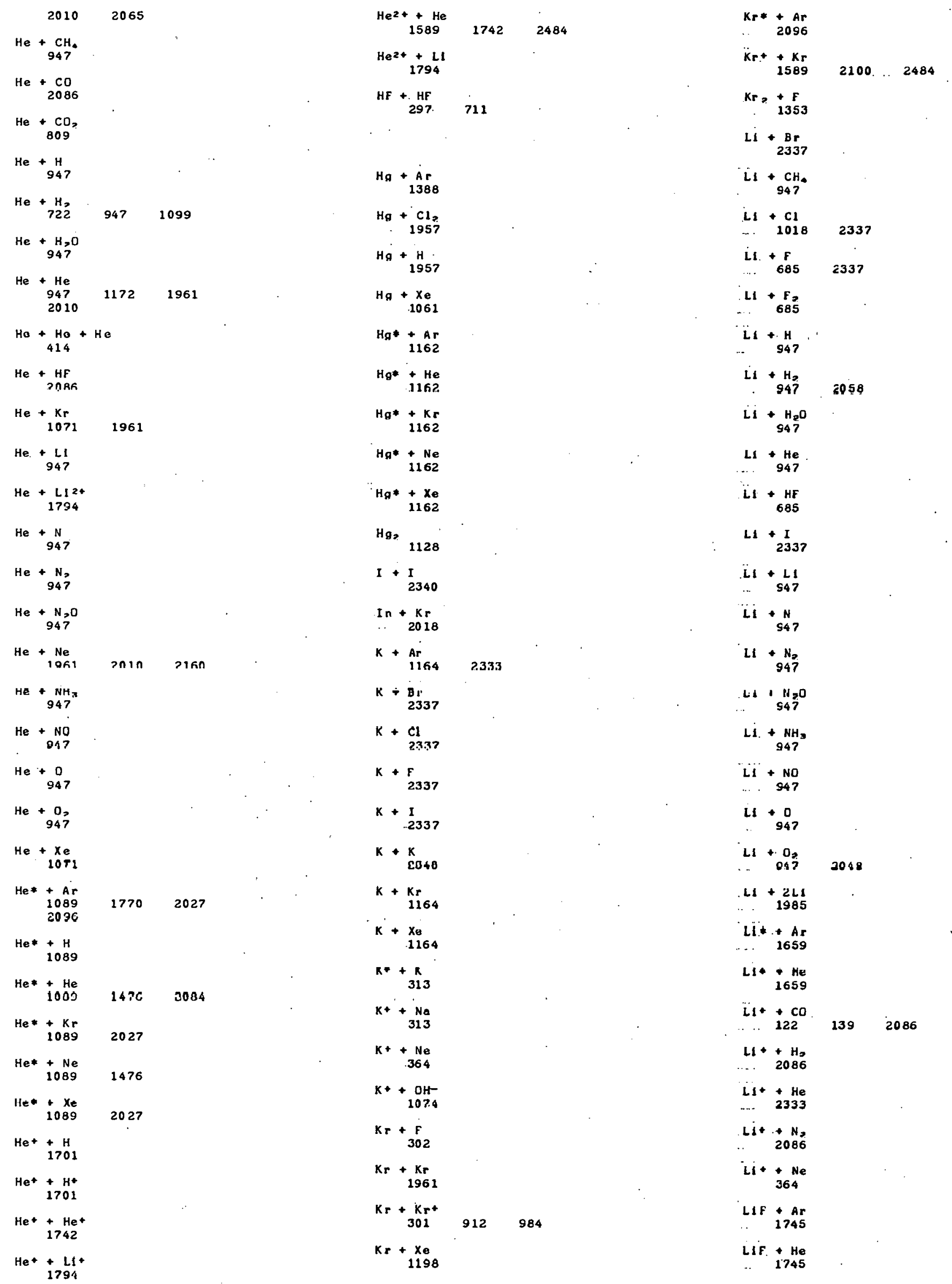




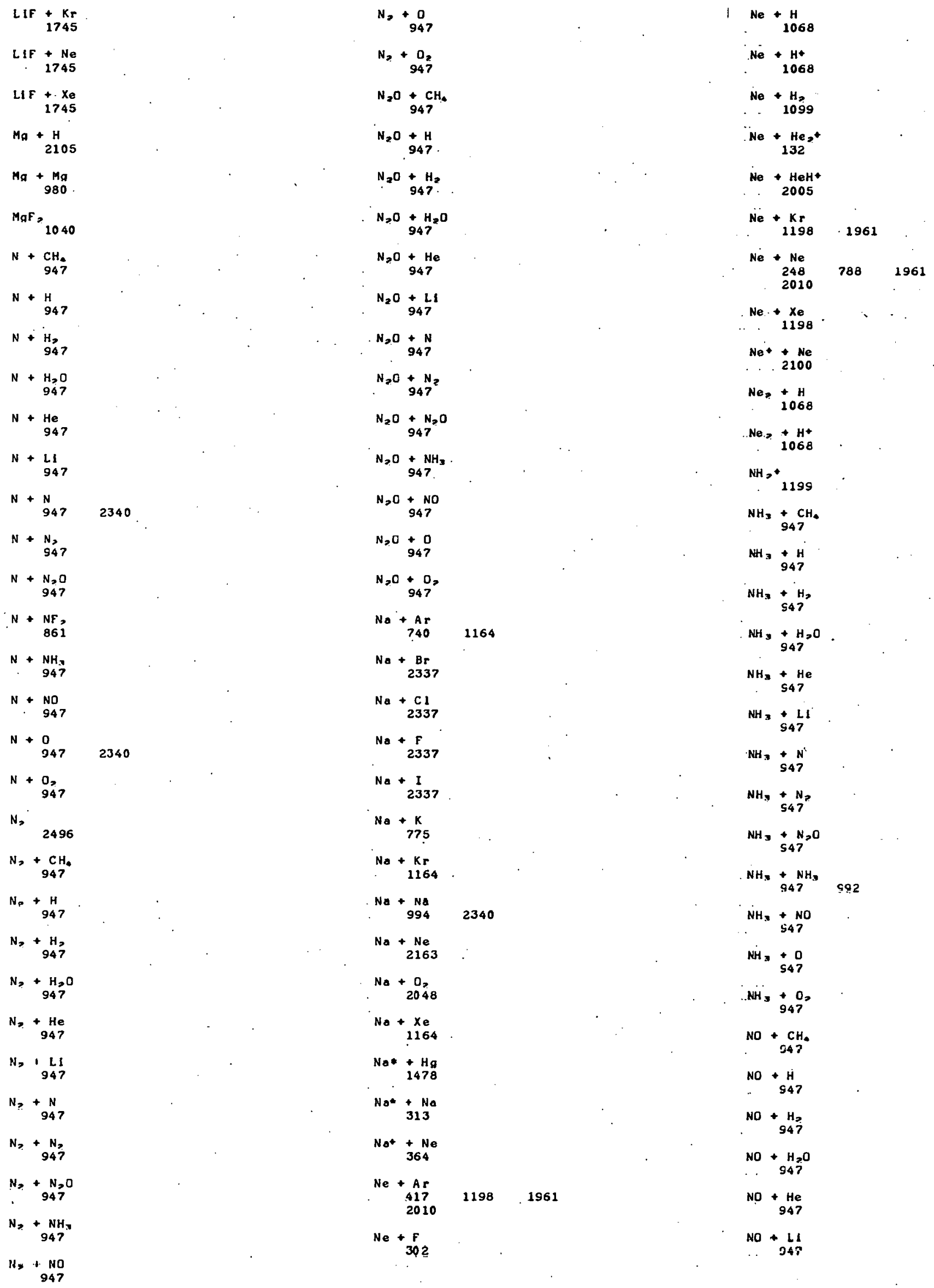




\begin{tabular}{|c|c|c|}
\hline NO $+\underset{947}{N}$ & & \\
\hline$N O+N_{947}$ & & \\
\hline $\mathrm{NO}+\underset{947}{N_{8} \mathrm{O}}$ & & \\
\hline $\mathrm{NO}+\underset{947}{\mathrm{NH}}$ & & \\
\hline $\begin{array}{r}\mathrm{NO}+\mathrm{NO} \\
947\end{array}$ & $\therefore$ & \\
\hline NO $+\underset{947}{O}$ & . & \\
\hline $\mathrm{NO}+\underset{947}{\mathrm{O}_{3}}$ & & \\
\hline $\mathrm{O}+\underset{947}{\mathrm{CH}_{4}}$ & & \\
\hline $0+\begin{array}{c}c 10 \\
880\end{array}$ & & \\
\hline$n: I_{947}$ & 2310 & \\
\hline $\mathrm{O}+\underset{947}{\mathrm{H}_{7}}$ & & \\
\hline $\begin{aligned} \mathrm{O}+ & \mathrm{H}>\mathrm{O} \\
& 947\end{aligned}$ & . & \\
\hline $\begin{array}{l}0+\mathrm{He} \\
947\end{array}$ & & \\
\hline $0+\operatorname{Lif}_{947}$ & & \\
\hline$O+\underbrace{N}_{947}$ & & \\
\hline $\begin{aligned} & 0+ N_{2} \\
& 947\end{aligned}$ & . & \\
\hline $\begin{aligned} O+N_{2} O \\
947\end{aligned}$ & & $\cdot$ \\
\hline $\mathrm{D}+\underset{947}{\mathrm{NH}_{3}}$ & & \\
\hline$U+\underset{94 \%}{\text { Nu }}$ & & \\
\hline $\begin{aligned} & 0+ 0 \\
& 947\end{aligned}$ & 1132 & 2340 \\
\hline $\begin{array}{r}0.03 \\
947 .\end{array}$ & & \\
\hline $\mathrm{O}^{2}+\underset{347}{\mathrm{H}_{3}}$ & & \\
\hline $0_{3} \quad 1200$ & ' & 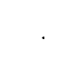 \\
\hline $\mathrm{O}_{2}+\underset{947}{\mathrm{CH}_{0}}$ & & \\
\hline
\end{tabular}

$\mathrm{O}_{2}+\underset{947}{\mathrm{H}}$

$\mathrm{O},+\begin{array}{r}\mathrm{H}_{2} \\ 947\end{array}$

$\mathrm{O} 2+\underset{947}{\mathrm{H}_{2} \mathrm{O}}$

$\mathrm{O},+\begin{array}{r}\mathrm{He} \\ 947\end{array}$

$0 .+24$

$\mathrm{O}_{2}+\underset{947}{\mathrm{~N}}$

$\mathrm{O}_{2}+\begin{array}{r}\mathrm{N}_{2} \\ 947\end{array}$

$\mathrm{O}_{2}+\underset{947}{\mathrm{~N}_{2} \mathrm{O}}$

$\mathrm{O}_{2}+\mathrm{NH}_{2}$

$0 .+\underset{34 T}{N 0}$

$\mathrm{O}_{2}+\mathrm{O}_{947}$

$\mathrm{O}_{2}+\mathrm{O}_{92}$

$0,-$

1051

$\mathrm{P}+\mathrm{N}$

2340

$\mathbf{R b}+\mathbf{B r}$

$\mathrm{Rb}+\mathrm{Cl}_{1}$

2337

$R b+\underset{2337}{F}$

Rb + I

2337

$R 6+\frac{R b}{026}$

$\mathrm{Rb}+\mathrm{Ar}$

2094

$R^{R+}+K$

Rbt + Kr

2094

Rn! + Ná

$\mathrm{Rb}+\mathrm{Rb}$
$R b++X e$

2094

$s i+\underset{2340}{F}$

Ti $+A=$

2073

$I 1+\underset{2073}{H}$

$\mathrm{Tl}+\mathrm{He}$

2073

$\mathrm{I}+\mathrm{Kr}$ 2028 2073

$\mathrm{Ti}+\mathrm{Ne}$

2073

T1 $+\mathrm{Xe}$

.. 2073

$\cdot \omega+4 C 1$.

xe. $\mathrm{Br}$ snos

$\mathrm{Xe}+\mathrm{Cl}$

$\dot{x e}+c u$

$\mathrm{Xe}+\mathrm{Cu}_{1372}$
$-\quad{ }^{2}$

$X e+\underset{302}{F}$

$\cdot \$ 58$

2002

$X e+\frac{I}{2002}$

$x e+x e$

$876 \quad 558$

58

1956

$x e+x e^{*}$

Xe $+\mathrm{Xe}^{+}$ $912 \quad 558 \quad 1956$

$X e *+A r$

2096

$X e++\frac{X e}{1031}$

$\mathrm{Zn}+\mathrm{H}$

2340

$2 \dot{r}+2 r F=$

Review

15+ 401

IInder

$54 y$ oyo ost 


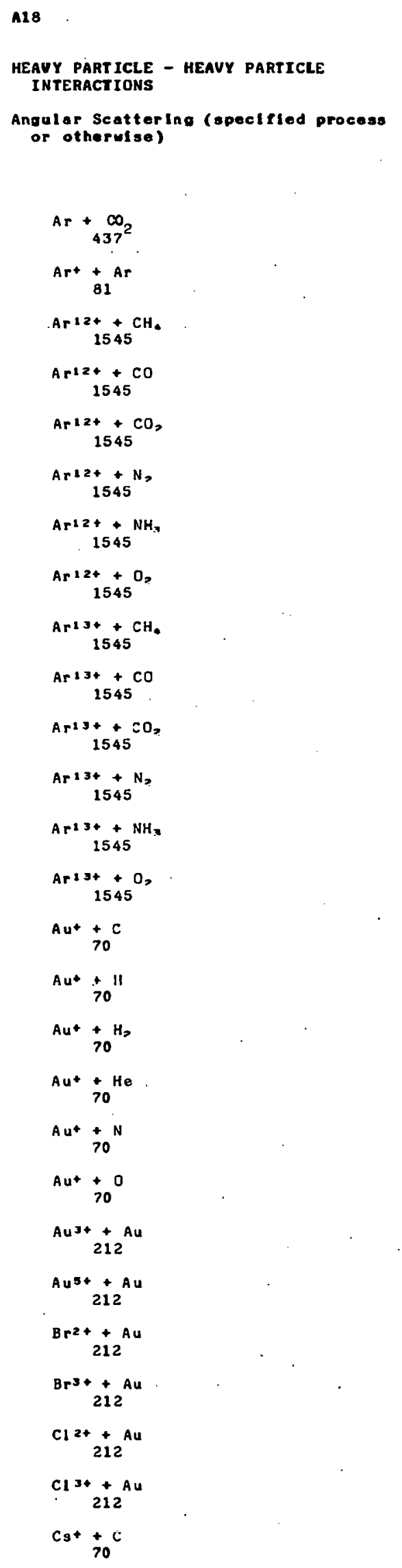

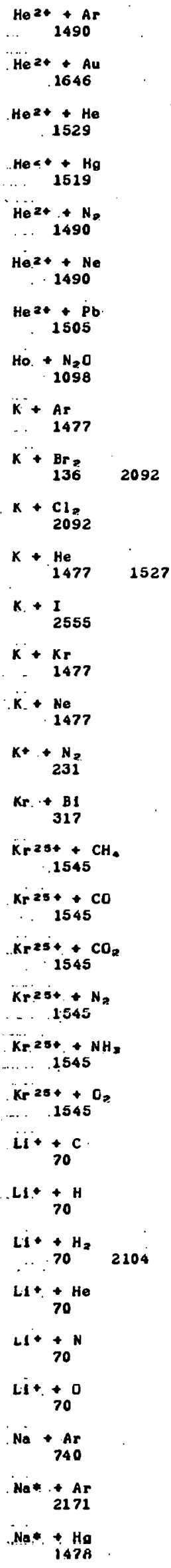


$\mathrm{Na},+\mathrm{He}$

2146

$\mathrm{Na}_{2}+\mathrm{Ne}$

$\mathrm{Ne}+\mathrm{D}$,

1521

$\mathrm{Xe}+\mathrm{BI}$
$\mathrm{Xe}^{32}+\mathrm{CH}_{3}$

1545

$\mathrm{Xe}^{31+}+\mathrm{CO}$

1545

$\mathrm{Xe}^{31+}+\mathrm{CO}_{2}$

1545

$$
\begin{gathered}
\mathrm{Xe}^{31+}+\mathrm{N}_{2} \\
1545 \\
\mathrm{Xe}^{31++\mathrm{NH}_{3}} \\
1545 \\
\mathrm{Xe}^{31+}+\mathrm{O}_{2} \\
1545
\end{gathered}
$$


A19

\section{HEAVY PART ICLE - HEAVY PARTICLE INTERACT IONS}

Inner-shell Interactlons (not covered under other specified categories)

$\mathrm{Al}+\mathrm{Al}$

$A 1++A r$

11

$\mathrm{Al}+\mathrm{Cu}$

11

$A 1++5$

11

Ar + Ar

$\mathrm{Ar}+\mathrm{Al}$

11

$A r+t$ Ar 11793

$\mathrm{Ar}^{+}+\mathrm{Cu}_{4}$

11

$\mathrm{Ar}^{+}+\mathrm{S}$

$\mathrm{As}+\mathrm{Kr}$

1793

$\mathrm{Br}^{+}+\mathrm{Kr}$
1793

$\mathrm{Br}^{+}+$PERT

$\mathrm{Br}^{+}+\mathrm{Xe}$

1793

$\mathrm{Cl}+\underset{57}{+\mathrm{Ag}_{\mathrm{G}}}$

$\mathrm{Cl}+\mathrm{Al}$

$\mathrm{Cl}+\mathrm{Ar}$

25n? $1793 \quad 2502$

$\mathrm{Cl}_{57}^{+} \mathrm{Au}$

$\mathrm{Cl}_{1}+\underset{57}{+\mathrm{BI}}$

$\mathrm{Cl}+\mathrm{Cl}$

$\mathrm{Cl}++\mathrm{Cu}$

11

$\mathrm{Cl}_{57}^{+} \mathrm{HF}$

$\mathrm{Cl}+\underset{57}{+} \mathrm{Ho}$

$\mathrm{Cl}_{57}^{+\mathrm{Mo}}$

$\mathrm{Cl}+\ldots \mathrm{NaCl}$

2502

$\mathrm{Cl}+\underset{57}{+\mathrm{Pb}}$

$\mathrm{Cl}+{ }_{57}^{\mathrm{Pr}}$

$\mathrm{Cl}^{+}+\mathrm{Pt}_{57}$
$\mathrm{Cl}_{57}^{+\mathrm{Re}}$

$\mathrm{Cl}+\mathrm{S}$

. 11

$\mathrm{Cl}+5 \mathrm{Sm}$

$\mathrm{Cl}+\underset{57}{\mathrm{Sn}}$

$\mathrm{Cl}+\mathrm{Ta}_{57}$

$\mathrm{Cl}+\mathrm{Th}$

$\mathrm{Cl}+\mathrm{U}$

$\mathrm{Cl}+\mathrm{Y}$

$\mathrm{Cl}^{5+}+\underset{1735}{\mathrm{Ca}}$

$\mathrm{Cl}^{3+}+\mathrm{Sc}$

1735

$\mathrm{Clst}_{+} \mathrm{TI}$

1735

$\mathrm{Cl11}_{+}+\mathrm{NaCi}$

2170

$\mathrm{Cu}+$ Ar

1793

$\mathrm{Cu}^{+}+\mathrm{Kr}$

1793

$F^{+}+\underset{57}{A 9}$

$+F^{+} \mathrm{Au}$
57 .

$F++57$

$F+\underset{57}{+\mathrm{He}}$

Ft+Ho

$5 \%$

$F++\underset{57}{M 0}$

$\mathrm{F}+\underset{57}{\mathrm{~Pb}}$

$\mathrm{F}^{+}+\underset{57}{\mathrm{Pr}}$

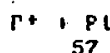

$F++\underset{57}{R e}$

$f^{+}+5 \mathrm{Sm}$

$\mathrm{Ft}^{+}+\mathrm{sn}$

$F++\underset{57}{\mathbf{T a}^{\circ}}$

Ft th

$F^{+}+\underset{57}{U}$

$\mathrm{F}^{+}+\mathbf{5 7}$

$F^{3+}+51$ 1761

$F++51$
$\mathrm{F}^{\mathrm{F}+}+\mathrm{S1}$

$F^{6}+$ + S1

1761

$\ddot{F} \ddot{?}++s 1$

1761

F6* + 51

. 1761

$\mathrm{Fot}^{+} \mathrm{si}$

1761

$\mathrm{Ge}^{+}+\mathrm{Kr}$

1793

$\stackrel{H^{\circ}+A_{59}^{A g}}{57}$

$H+{ }_{6}^{A l}$

$\dddot{H+}+\underset{\text { S6 }}{A x}$

H* + A U

57

$H+{ }_{6}$

$\mathrm{H}^{+}+\mathrm{BI}$

.... 57

$\mathrm{H}+\underset{96}{\mathrm{C}}$

$\mathrm{H}+\mathrm{CO}_{6}$

$\ddot{H}^{+}+\mathrm{Cr}$

-. 1899

$H+\mathrm{Cu}$

. 1899

$\mathrm{H}+\mathrm{H}$

H+ $+\mathrm{HP}$

... 57

$\mathrm{H}+\mathrm{HO}$

... 57

$\mathrm{H}^{+}+\mathrm{MO}_{57}$

H+ $+N$

$\mathrm{Ht}+\mathrm{Ne}$

S6

H. + 0

$\mathrm{H}^{*}+\mathrm{Pb}$

- 57

Ht $+\mathrm{Pr}_{57}$

$\ddot{H}+. P$

$\dddot{H}+\underset{5 ?}{\mathrm{Re}}$

$\mathrm{H+}+\mathrm{SI}$

$\mathrm{H+}+\mathrm{SP}_{57}$

$\dot{H} *+s_{n}$

. 57

$\mathrm{H}+\mathrm{Ta}^{\mathrm{T}}$
+57

Hit $+\mathrm{Th}$ 


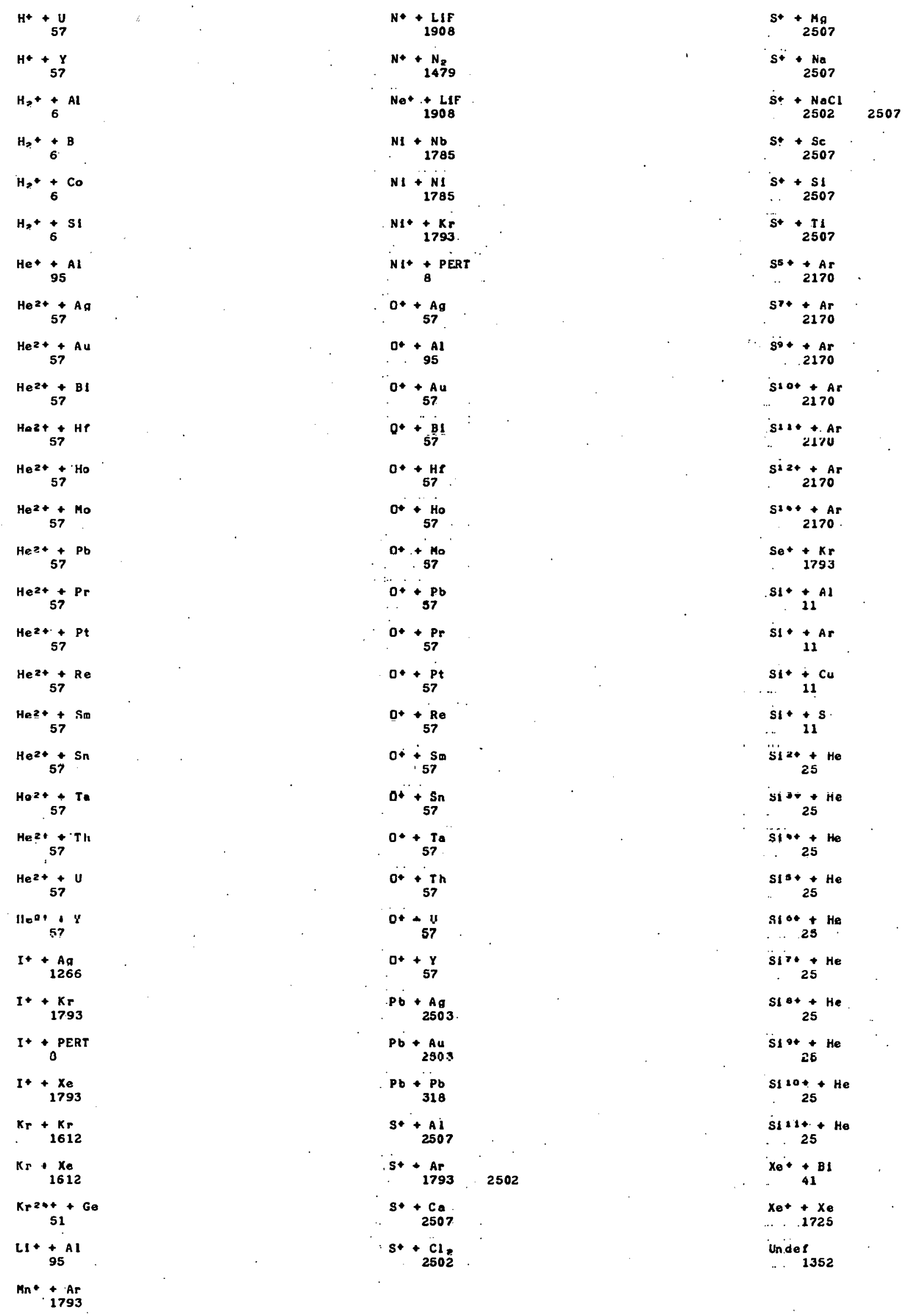


120.

HEAUY PART ICLE - HEAUY PARTICLE INTERACT IONS

Attenuation (unspecifled process)

$H+\mathrm{Cs}_{\mathbf{4}}$

B01.

INTERACTIONS HITH STATIC OR TIME-VARYING ELECTRIC AHD MAGAETIC FI ELDS

General

CN- 691

co

688691

B02

INTERACT IONS UITH STATIC OR

TIME-VARYING ELECTRIC AND MAGNETIC FIELDS

Dissoctation

Under

561

B03
INTERACTIONS UITH STATIC OR
TI HE-UARYING ELECTRIC AUD MAGNETIC FI ELDS

Iontzation

H

1525

B04

INTERACTIONS WITH. STATIC OR

TIME-VARYING ELECTRIC AND MAGUETIC

FIELDS

ptacheent

hข $\div$ S-

$$
\begin{aligned}
& \mathrm{H}+\mathrm{Cs}_{476} \\
& \mathrm{Ho}+\mathrm{N}_{2} \mathrm{O} \\
& 2098 \\
& \mathrm{Kr}+\mathrm{BI} \\
& +317 \\
& \mathrm{Xe}+\mathrm{BI} \\
& 317
\end{aligned}
$$$$
X e F= \pm A r *
$$

$\mathrm{XeF}_{2}+\mathrm{Kr}_{\mathrm{r}}$

XeF 2

$\ddot{x e f}_{2}+\mathrm{xe}_{\mathrm{e}}$

1085

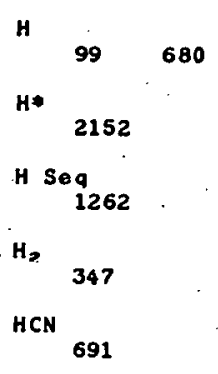

H*

$$
\text { H Seg }
$$$$
1262
$$$$
347
$$

691

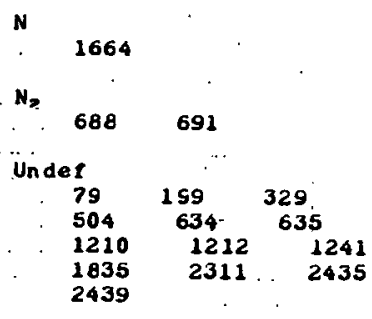

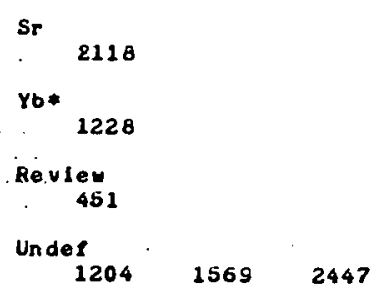

$4 \operatorname{seq}$

$182 \quad 186$

$L I * .2153$

$N *$

Na*

$65 \quad 2193 \quad 2467$
Review

451 
$\mathrm{CO}_{2}$

PART ICLE PENETRATTYN IN MACROSCOP IC

ÄATtË (IONS, NEUTRALS, AND ELECTRONS)

Energy Loss

$A 1+\underset{66}{A r}$

$A 1+A G$

1816

$A 1++A 1$

1816

$\mathrm{Al}+\mathrm{Be}$

1816

$A 1 *+C$

1816

$\mathrm{Al}+\mathrm{Eu}$

1816

$\mathrm{Al}+\mathrm{Ge}$
1816

$\mathrm{Al}+\mathrm{Ni}$

1816

$\mathrm{Al}+\mathrm{Ta}$
1816

$\mathrm{Al}++\mathrm{TI}$

1816

$\mathrm{Al}+\mathrm{Zr}$

1816

$A r+A g$

2546

$A r+A l$

2546

$\mathrm{Ar}+\mathrm{Ar}$

Ar + Au

2546

$A r+B I$

$A \mathbf{r}+\mathrm{C}$

$+\underset{2528}{C} 2546$

$\mathrm{Ar}+\mathrm{Cu}$

2415

$\mathrm{Ar}^{+}+\underset{1862}{\mathrm{C}}$

$\mathrm{Ar}+\underset{\mathrm{H}_{2} \mathrm{O}}{+}$

$\mathrm{Au}+\mathrm{Au}$

2415

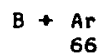

$\begin{array}{rl}B & B \\ 10\end{array}$

$B+8$ B
10

$B+C$

$B+F$

$B+\underset{10}{H}$

$\mathrm{B}+\mathrm{He}$
10
$B+\frac{1}{10}$

B $+\underset{10}{\mathbf{N}}$

$\mathrm{B}+\mathrm{Ne}$

$B+0$

10

B+ + B

$B++\frac{B e}{10}$

B + + C

1854

$\mathrm{Bt}+\mathrm{Co}$

B + $+F$

$B++H$

10.

B + $+\mathrm{He}$

10

B+ $+\underset{10}{\mathrm{LI}}$

$B++N$

B* + N.b

.1854

B+ $+\mathrm{Ne}$

$\mathrm{B}++\mathrm{Ni}$

2854

B ++0

B + + PERT

507

$\mathrm{B}^{+}+\mathrm{SI}$

$\mathrm{Be}+\underset{66}{\mathrm{Ar}}$

Be +8

$\mathrm{Be}+\mathrm{Be}$

$\mathrm{Be}+\mathrm{C}$

$\mathrm{Be}+\mathrm{F}$

$B e+\underset{10}{H}$

$\mathrm{Be}+\mathrm{He}$

10

$B e+\underset{10}{i}$

$\mathrm{Be}+\mathrm{N}$

$\mathrm{Be}+\mathrm{Ne}$

10

$\mathrm{Be}+0$

$\mathrm{Be}^{+}+\mathrm{Al}$

1816

$\mathrm{Be}^{+} \mathrm{Ar}$
$B e+.8$

$\mathrm{Be}+\mathrm{B}$

$10^{1816}$

Be* *

$10 \quad 1816 \quad 2854$

$\mathrm{Be}+* \mathrm{Co}$

1854

$\mathrm{Be} *+\mathrm{Eu}$

1816

Bet $+\mathrm{F}_{10}$

$\mathrm{Be}+$ + Ge

1816

$\mathrm{Be}+{ }_{10}^{\mathrm{H}}$

Be. $+\mathrm{He}$

Bet + LI

10

Bet+ $+N$

.10

$\mathrm{Ba}++\mathrm{Nb}$

1854

$\mathrm{Be}+\mathrm{Ne}$

Be* + N1

1816

1854

$\mathrm{Be}++0$

Be * + PERT

607

Be + Ta

1816

Bet + TI

1816

$\mathrm{Be}+.+2 \mathbf{Z}$

Br $+\mathrm{Au}$

891

$\mathrm{Br}+\mathrm{C}$

C. Ar

C. +10

$C .+B \dot{e}$

c. $+\mathrm{C}$

10 .

$\begin{aligned} & c . F \\ & 10\end{aligned}$

C $+\mathrm{H}$

c. $+\mathrm{He}$

$\dddot{C}+\begin{aligned} & 1 \\ & 10\end{aligned}$

c..* N

C. $+\mathrm{Ne}$

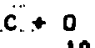




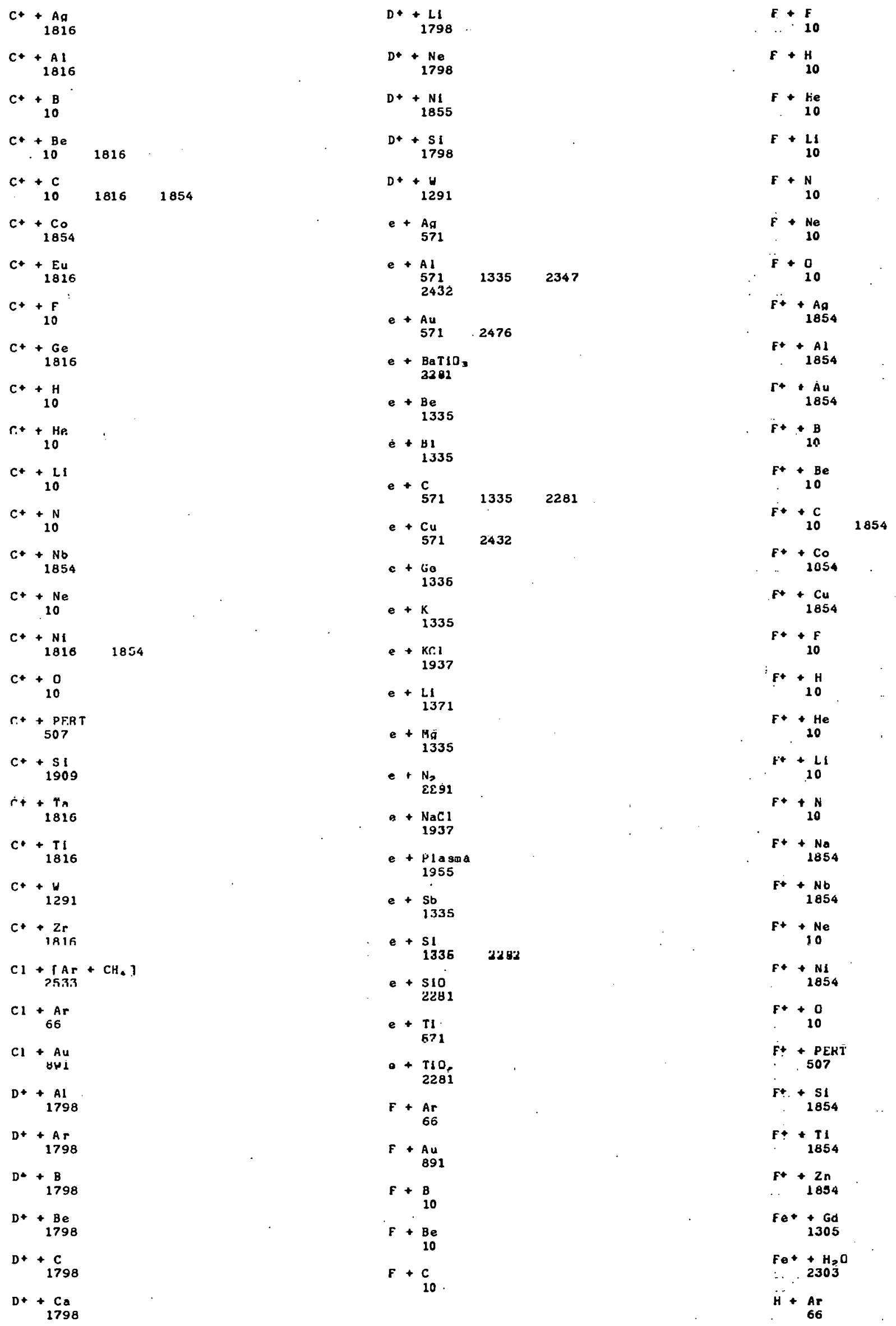




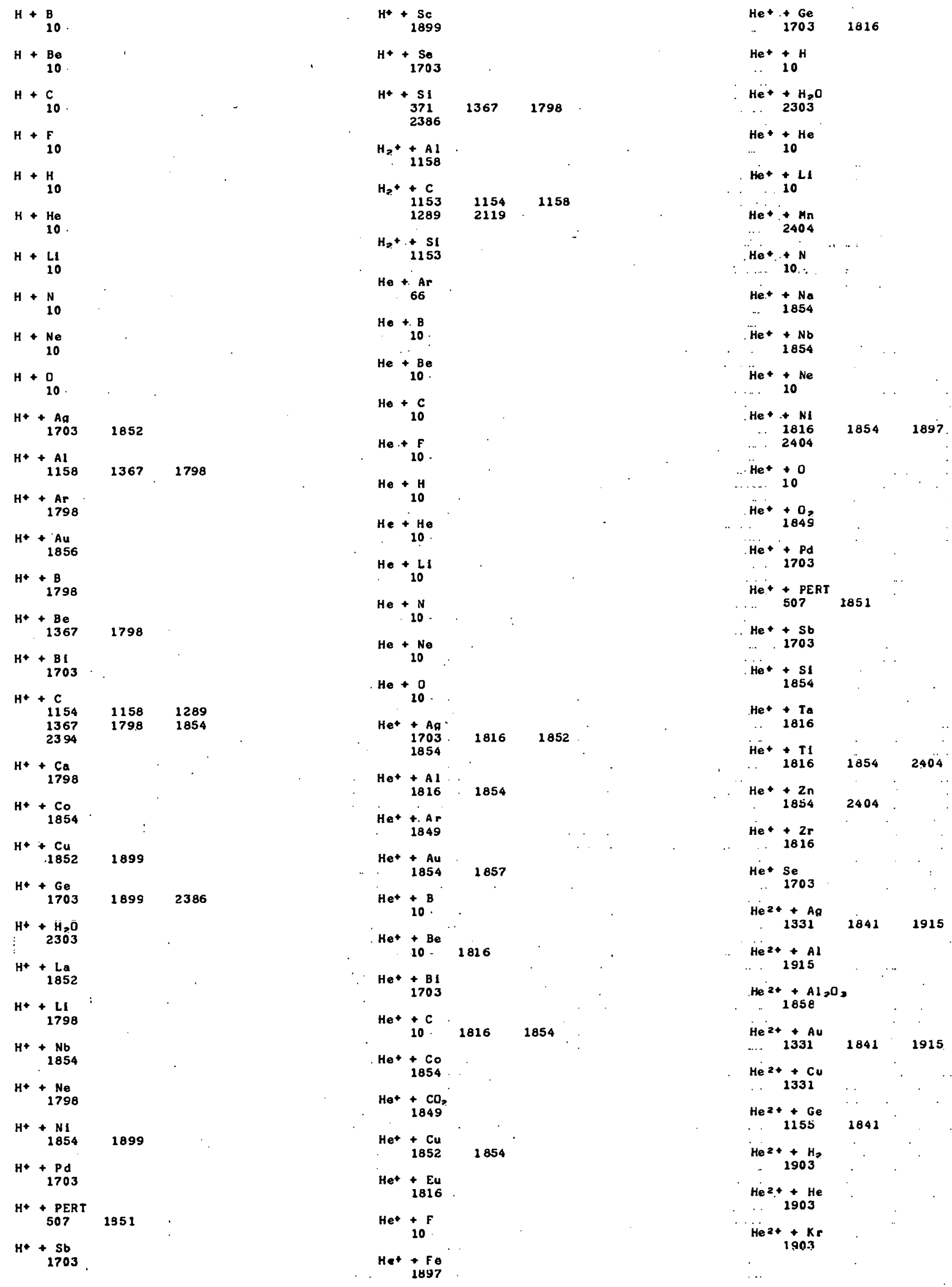




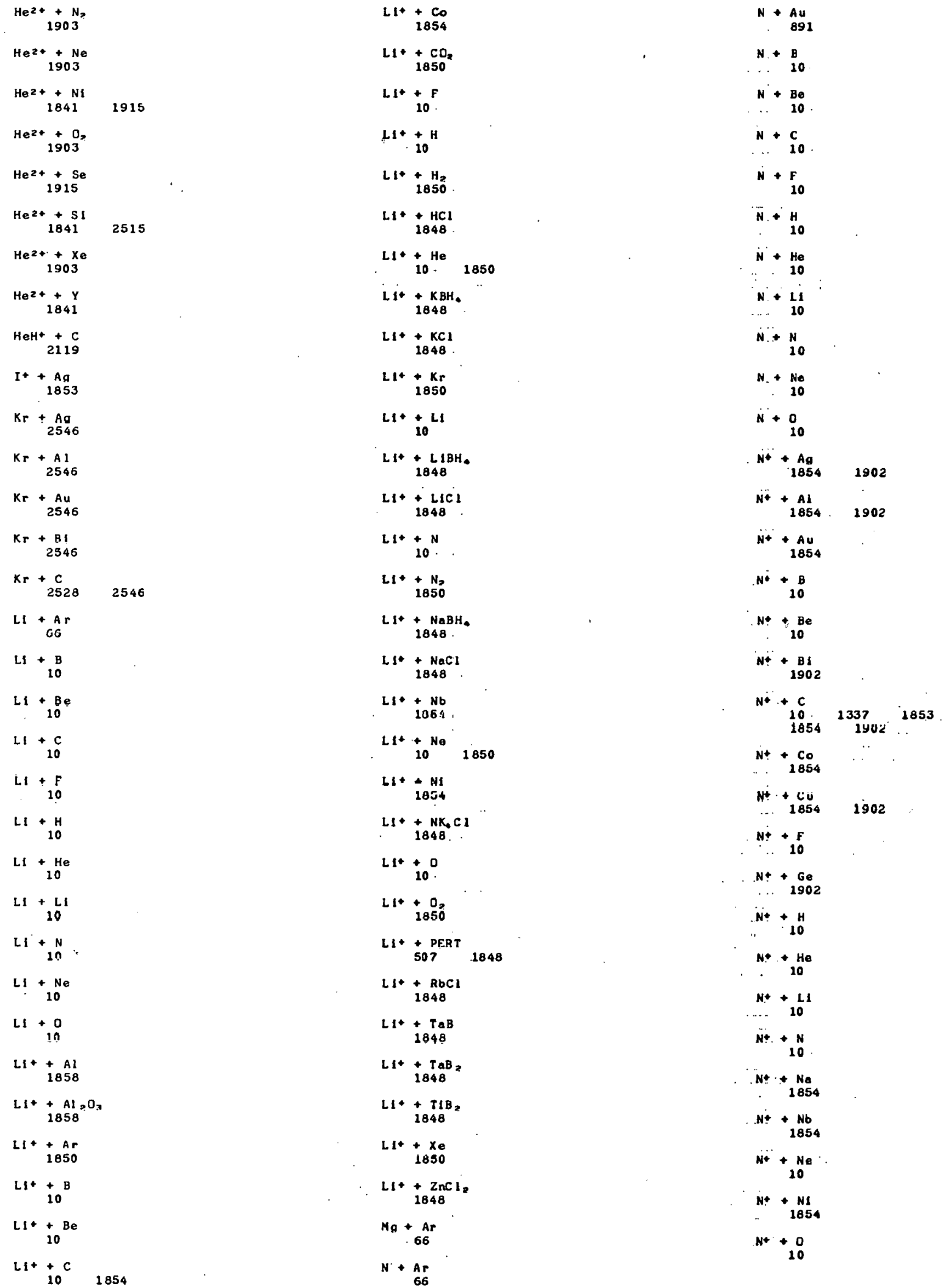




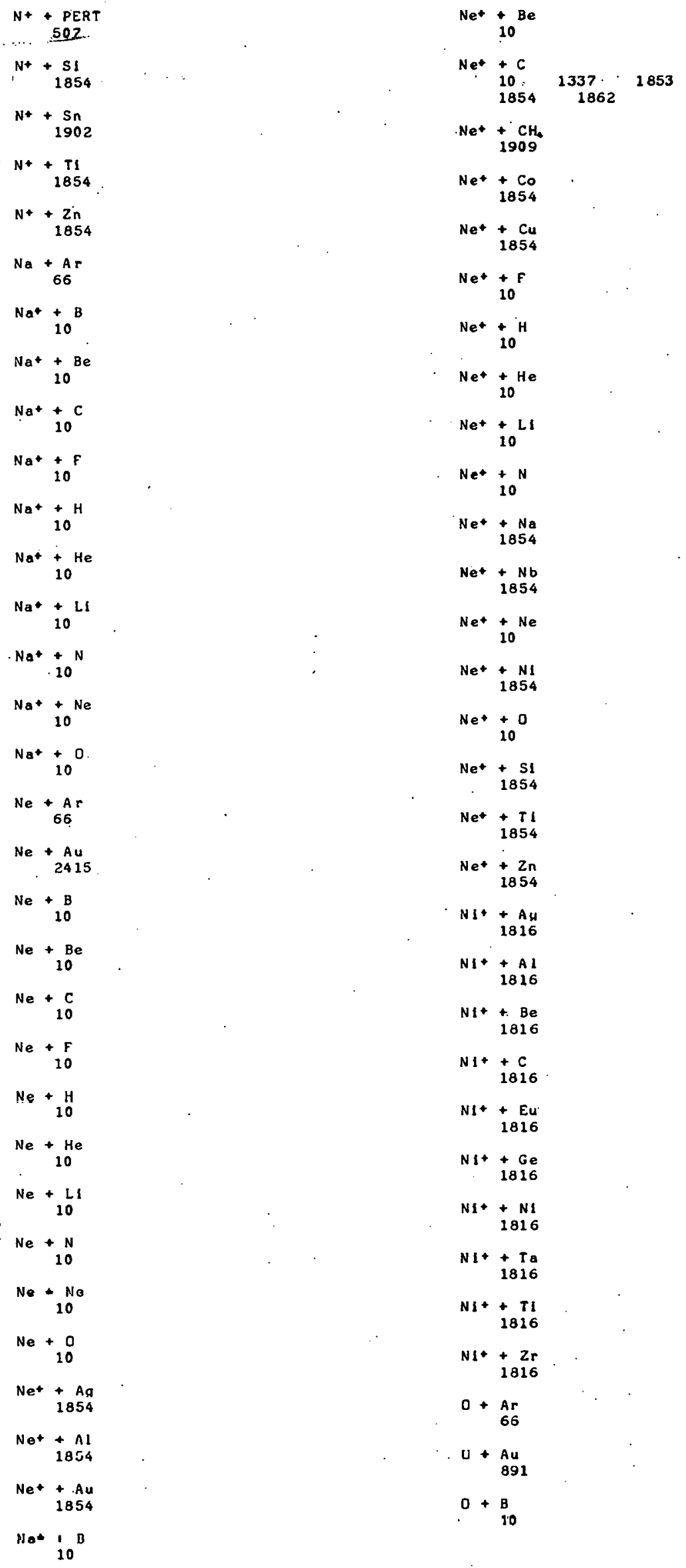

$0 . \mathrm{Be}$
10

$0+\frac{c}{10}$

$0+5$

$0+H$

$0+\mathrm{He}$
10

$0+11$

$0+\frac{N}{10}$

$\mathrm{O}+\mathrm{Ne}$

10

$0 .+0$
10

$0 *+A g$

$D^{+}+\underset{46}{A 1} 1854$

$0++A 4$

O+ + B

O+ $+\mathrm{Be}$

$0++\mathrm{C}$

$10 \quad 1854$

$0++C_{0}$

$\mathrm{O}+\mathrm{Cu}$

$0++\underset{10}{f}$

$\mathrm{O}+\underset{10}{\mathrm{H}}$

O+ $+\begin{array}{r}\mathrm{H}_{2} \mathrm{O} \\ 2303\end{array}$

$\mathrm{O}+\mathrm{He}$

$0++\frac{\mathrm{LI}}{10}$

0..$N$

O+ $+\mathrm{Na}$ 1854

$\mathrm{O}^{+}+\mathrm{Nb}$

1854

$\mathrm{O}+\ldots \mathrm{Ne}$

10

$\mathrm{O}+\mathrm{NI}$

1854

$0 \div+0$

o+ + PERT

507

$0++51$

: 1854

$0++T 1$

1854

$\overline{0}^{+}+\mathbf{u O}_{2409}$ 


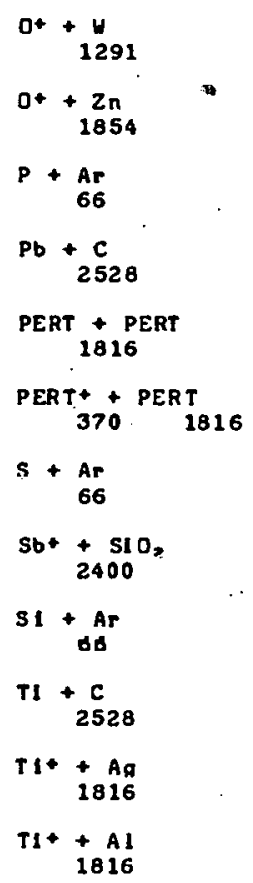

$\cos$

PART ICLE PENETRATION IN MACROSCOPIC

MATTER (IONS, NEUTRALS, AND ELECTRONS)

Energy to Creote an Ion Pair

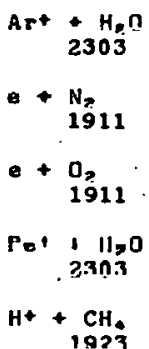

U + BI 2546

$u+c$

2528 2546

U+ + UD,

$240 S$

$X e+\begin{gathered}A g \\ 2546\end{gathered}$

$\mathrm{Xe}+\mathrm{Al}$

2546

$x e+2546$

$X_{0}+B 1$

2546

$\mathrm{Xe}+\mathrm{C}$

$2528 \quad 2546$

$\mathrm{Xe}+\mathrm{Cu}$

2415

Review
$\therefore \quad 68$

Uñdor

$328 \quad 1739 \quad 2429$.

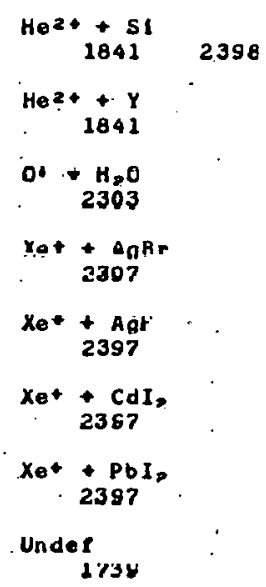


cos

PART ICLE PENETRATION IN MACROSCOPIC

MATTER (IONS, NEUTRALS, AND ELECTROUS)

Particle Range

\begin{tabular}{|c|c|c|}
\hline$A I^{*}$ & $\begin{array}{r}+A g \\
1920\end{array}$ & \\
\hline$A I^{+}$ & $+\begin{array}{c}A U \\
1920\end{array}$ & \\
\hline Al+ & $+\underset{1920}{C u}$ & \\
\hline$A 1+$ & $+M_{1920}$ & \\
\hline$A 1^{+}$ & $\begin{array}{l}+\mathrm{NI} \\
1920\end{array}$ & \\
\hline$A 1+$ & $\begin{array}{c}+P_{0} \\
1920\end{array}$ & 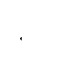 \\
\hline$A 1^{*}$ & $\begin{array}{r}+\mathrm{Ta} \\
1920\end{array}$ & \\
\hline$A I^{*}$ & $+\frac{T 1}{1920}$ & \\
\hline$A 1+$ & $+\underset{1920}{y}$ & \\
\hline$A r^{+}$ & $\begin{array}{r}+c u \\
2418\end{array}$ & \\
\hline Art & $+L_{394}^{L 1,0}$ & \\
\hline Au. & $+\underset{2524}{M 1 c a}$ & \\
\hline $\mathrm{Au}^{*}$ & $\begin{array}{r}+A 1 \\
2396\end{array}$ & \\
\hline$B+$ & $+\begin{array}{c}S 1 \\
2418\end{array}$ & \\
\hline$B t^{+}$ & $\begin{array}{l}+A 1 \\
2396\end{array}$ & . \\
\hline & $\begin{array}{l}+A 1 \\
2396\end{array}$ & \\
\hline $\mathrm{Cs}^{\circ}$ & $\begin{array}{c}+A P \\
2405\end{array}$ & \\
\hline $\mathrm{Cs}_{9}+$ & $\begin{array}{l}+N_{2} \\
2405\end{array}$ & \\
\hline$D+1$ & $+\underset{4 B I}{A I}$ & \\
\hline D+ & $+\frac{C}{481}$ & 1426 \\
\hline$D+$ & $\begin{array}{r}N I \\
457\end{array}$ & 481 \\
\hline$D^{*}+$ & $\begin{array}{r}2 r \\
481\end{array}$ & \\
\hline$n_{n+}+$ & $\begin{array}{l}+A 1 \\
2396\end{array}$ & \\
\hline $\mathrm{D}_{\mathrm{H}}+$ & $\begin{array}{l}+51 \\
2396\end{array}$ & \\
\hline
\end{tabular}

e+Al

2491....

Ho* + AI

e+ $\mathrm{Al}_{2} \mathrm{O}_{\mathbf{1}}$ $2293 \ldots$

e + Au

1753

$+\mathrm{D}_{2}$

e Gas 1951

e $+\mathrm{Ge}$ 1113

e+ $\mathrm{H}_{2}$

e MgO 2293

$e+N_{2}$

1911

e+ NaCl

1943

$c+0_{2}$

1911

Ert $+A$ 2396

Eut $+A 1$ 2396

$\mathrm{Eu}^{+}+\mathrm{SI}$ 2396

$\mathbf{F r}^{+}+\mathrm{Ar}$

$\mathrm{Fr}^{+}+\mathrm{N}_{\mathbf{H}}$ 2405

$\mathrm{Gd}^{+}+\mathrm{SI}$ 2396

$\mathrm{H}^{+}+\mathrm{SI}$ 1884

$\mathrm{H}_{2}+\mathrm{SI}$ 1884

$\mathrm{He}+\mathrm{Al}$

472

$\mathrm{He}+\mathrm{C}$
472

1426

$\mathrm{He}+\mathrm{Ge}$

2380

$\mathrm{He}+\mathrm{NI}$
457 472

$\mathrm{He}+\mathrm{SI}$

472

Het $+v$

$\mathrm{He}+4$

$\mathrm{He}+\mathrm{Zr}$ 472

$H++\underset{2396}{H+}$
$\mathrm{I}+\mathrm{HICa}$ 2524

in: + $A 1$ 2396

Lut + AI

... 2396

N*. Ag

.. 1366

N+. $+A 1$

.. 1366

$\mathrm{Ni}$. $\mathrm{AL}$

. 1366

$\mathrm{N}+\mathrm{Cu}$ 1366

N* + Mo

... 1366

N*". + NI 1366

N* $+\mathrm{Ta}$

1366

$N+\ldots+T I$ 1366.

$N++H$ 1366

$N+\ldots \mathbf{2 n}$ 1366

$\mathrm{Nb}++\mathrm{Nb}$

$0 \div+\$ 1$ 1327

0* + UD?

Pb $+A 1$ 2396

Pr* +21

$P t++A 1$

... 2356

R.b * A

… 2405

$\mathrm{Rb}+ \pm \mathrm{N}_{\mathrm{D}}$

2405

Sm + $+\mathbf{S}$ 2396

..TÖ+ $+A 1$

2396

$\mathrm{Tb}+\mathrm{st}$ 2396

. 2396

U.. $+40_{2}$
2409
$\mathrm{I}++A 1$ 
PARTICLE PENETRATION IN MACROSCOPIC MATTER (IONS, NEUTRALS, AND ELECTRONS)

$\mathrm{H}+\mathrm{Au}$

1859

Mult I ple Scattering

$$
\begin{gathered}
A 1+\underset{6 r}{66} \\
A r+\underset{A g}{2546} \\
A r+A l \\
2546 \\
A r+A r \\
66 \\
A r+A 4 \\
2546 \\
A r+B 1 \\
2546 \\
A r+C \\
2546
\end{gathered}
$$$$
\mathrm{Ar}^{+}+\mathrm{Xe}_{\mathrm{e}}
$$$$
\mathrm{Au}+\mathrm{Al}
$$$$
2396
$$$$
B+\begin{array}{r}
\text { Ar } \\
66
\end{array}
$$$$
\mathrm{Be}+\mathrm{Ar}
$$$$
\mathrm{Bl}+\mathrm{Al}
$$$$
2396
$$$$
C+\begin{array}{r}
\text { Ar } \\
66
\end{array}
$$$$
\text { C) }+\underset{66}{\mathrm{Ar}}
$$$$
\mathrm{Cs}^{+}+\mathrm{Al}
$$$$
2396
$$$$
\text { D* + SI }
$$$$
\text { Dy + }+A 1
$$$$
2396
$$$$
\text { Dy+ }+\underset{2396}{\text { SI }}
$$$$
\text { e+ Ag }
$$$$
\text { - I Au }
$$$$
2236
$$$$
\text { - I } \mathrm{Ma}
$$$$
2236
$$$$
\begin{aligned}
& E++A 1 \\
& 2396
\end{aligned}
$$$$
\text { Eut }+\underset{2396}{A l}
$$$$
\mathrm{Eu}_{+}^{+} \mathrm{SI}
$$$$
2346
$$$$
\begin{array}{r}
f+A r \\
66
\end{array}
$$$$
\text { Gd + }+ \text { SI }
$$$$
+51
$$$$
H+\begin{array}{r}
A r \\
66
\end{array}
$$$$
\mathrm{H}+\hat{\mathrm{Ag}}
$$

$H++\frac{B e}{1367}$

$\mathrm{H}+$ + B 1

1703

H+ + C

1367

2394

1860

1861

$H+\mathrm{Ge}$
1703

$\mathrm{H}^{+}+\underset{1703}{\mathrm{Pd}}$

$\mathrm{H}+\mathrm{Sb}$

1703

$H++\mathrm{Se}^{\mathrm{He}}$

$\mathrm{H}^{+}+\mathrm{SI}$

1367

1842

1884

$\mathrm{H}_{\mathrm{x}}+\underset{2157}{+\mathrm{r}}$

$\mathrm{H}^{+}+\mathrm{SI}$

1884

$\mathrm{H}_{3}+\underset{484}{\mathrm{C}}$

2157

$\mathrm{He}+\mathrm{Ar}$

$\mathrm{He}^{+}+\mathrm{Ag}$
1703

$\mathrm{He}^{+}+\mathrm{Al}_{\mathrm{I}}$

1914

$\mathrm{He}+\mathrm{BI}$
1703

Het $+\mathrm{r}$.

1914

$\mathrm{He}^{+}+\mathrm{Cu}$

1914

Hot +59

1703

Hel $+\mathrm{Pd}_{1703}$

$\mathrm{He}^{+}+\mathrm{Sb}$

1703

llel $\begin{array}{r}\text { Je } \\ \text { DOM.3 }\end{array}$

$\mathrm{He}^{+}+\mathrm{ss}$

$\mathrm{He}^{2+}+\mathrm{s}$

1842

Het+ Al

$\mathrm{HC}^{+}+\mathrm{Al}$

2396

$K r+\underset{2546}{A 9}$

Kr A Al

2546

$\mathrm{Kr}+\mathrm{Au}$

$\mathrm{Kr}+\mathrm{BI}$
. 2546

$\mathrm{La}^{+}+\mathrm{Al}$

2396

$L_{1}+\underset{66}{A r}$

L $1+$ Ar 1850

$\mathrm{LI}++\mathrm{CO}_{2}$

$\mathrm{Li}+\mathrm{H}_{3}$ 1850

LIt + He

$\mathrm{Lt}+\mathrm{Kr}$ 1850

$\mathrm{LI}+\mathrm{N}_{\text {? }}$ 1850

i,i- $+\mathrm{Ne}$ 1850

$\mathrm{LI}^{+} \mathrm{C}_{2}$ 1850

$\mathrm{LI}+\mathrm{Xe}$

1850

Lu+ + Al 2396

$\mathrm{Mg}+\mathrm{Ar}$

66

$N+\begin{gathered}\text { Ar } \\ 66\end{gathered}$

$\mathrm{N}^{+}+\mathrm{C}$ 1337

$\mathrm{Na}+\mathrm{Ar}$ 66

$\mathrm{Ne}+\mathrm{Ar}$

66

$\mathrm{Ne}+\mathrm{C}$ 1337

$0+A r$

$0++\frac{A 1}{46}$

$\mathrm{O}+\mathrm{CO}$

1827

$\mathrm{O}+\mathrm{CO}_{183}$

$0++\begin{array}{r}H>0 \\ 1827\end{array}$

$0++N_{2}$

0. - Ne

$\mathrm{O}+\mathrm{O}_{2}$

$0++58$

D+ $+\mathrm{Xe}$ 1827

$P+4 r$

$\mathrm{Pb}++\mathrm{Al}$ 2356

$\mathrm{Hb}++20$ 
$\mathrm{Pb}++\mathrm{CO}$,
1827

$\mathrm{Pb}+\mathrm{H}=\mathrm{O}$ 1827

$\mathrm{Pb}+\mathrm{N}$,
1827

$\mathrm{Pb}++\mathrm{Ne}$ 1827

$\mathrm{Pb}+\mathrm{O}$,

$\mathrm{Pb}^{+}+\mathrm{Xe}_{\mathrm{e}}$

1827

PERT + Au
1839

PERT + Cs

1839

PERT + $H$

1839

PERT + K

1839

PERT + LI

1839

PERT + $\mathrm{Mg}$

1839

PERT + Na

1839

$\mathrm{Pr}^{4}+\mathrm{Al}$
$\mathrm{Pt}_{+2396}$

$S+A r$

66

$S 1+\underset{66}{A r}$

$\mathrm{S}^{+}+\mathrm{SI}^{+}$

2396

$T$ to + Al

$T b++51$

2396

+ + Al
2396

$\mathbf{U}+\mathbf{A g}$

2546

$U+A I$

2546

$U+A u$

2546

i + BI

2546

$u+c$

2546

$\mathrm{Xe}+\mathrm{Ag}$

2546
$X e+\underset{2546}{A 1}$

$\mathrm{Xe}+\mathrm{Au}$

2546

$\mathrm{Xe}+\mathrm{BI}$

2546

$\mathrm{Xe}+\mathrm{c}$

2546

$\dot{x e}^{+}+\mathrm{co}$

1827

$\mathrm{Xe}+\mathrm{CO}_{2}$

$\mathrm{Xe}+\mathrm{H}_{\mathrm{P}} \mathrm{O}$
1827

$\mathrm{Xe}^{+}+\mathrm{N}$,

$\mathrm{Xe}+\mathrm{Ne}$

1827

$\mathrm{Xe}^{+}+\mathrm{O}_{2}$

$\dot{x}_{e}++x_{e}$

1827

Under

2239 
PART ICLE PENETRATION IN MACROSCOPIC MATTER ( IONS, HEUTRALS, AND ELECTROUS)

Charge State Populations

$A 1+\underset{25}{C}$

$\mathrm{Al}+\underset{1839}{\mathrm{Mg}}$

$\mathrm{Al}+\mathrm{Na}$

1843

$\mathrm{Ar}+\mathrm{C}$

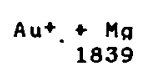

$\mathrm{All}^{+}+\mathrm{Nn}$

1843

$B+\frac{C}{258}$

B+ + Ma

1839

$\mathrm{B}++\mathrm{Na}$

1843

$B+++A g$

2129

$\mathrm{B}++$ A

2129

$\mathrm{Be}^{+}+\mathrm{Ma}$

$\mathrm{Be}++\mathrm{Na}$

1843

$\mathrm{C}+\mathrm{C}$

1800

$\mathrm{C}++\mathrm{Mg}_{1039}$

$\mathrm{C}++\underset{1843}{\mathrm{Na}}$

$\mathrm{Cs}^{5+}+\mathrm{As}$

2129

$\mathrm{Cs}_{+\mathrm{Au}}$

$\mathrm{Ca}+\mathrm{Na}$

1843

C. $+\mathrm{Hn}$

1838

$\mathrm{Cl}+\mathrm{Na}$

1843

$f+c \cdot$

$\mathrm{F}^{++}+\mathrm{Ag}$
Fet + Ag 2129

Fo +Au

2129

$\mathrm{Fe}+\mathrm{C}$

258

$F e^{+}+M a$

$\mathrm{Fe}+\mathrm{Na}$

1843

$\mathrm{H}+\mathrm{C}$

1800

$\mathrm{H}_{2}+\mathrm{C}$

2119

2157

$\mathrm{H}_{3}+\mathrm{C}$

$\mathrm{HeH}++\mathrm{C}$

2119

$I++A r$

IYU 1

$I++K r$

1901

$\mathrm{I}+\underset{1839}{\mathrm{Mg}}$

$\mathrm{I}+\mathrm{N}_{2}$

1901

$I++X_{0}$

1901

$\mathrm{In}+\mathrm{Mg}$

1839

$K r+c$ C

$\mathrm{LI}+\mathrm{Na}$

1845

$\mathrm{Mg}+\mathrm{C}$

258

$\mathrm{NG}^{4}+\mathrm{C}$

1800

$\mathrm{Mg}+\mathrm{Na}$
1843

$N+\mathbf{C}$

258

$\mathrm{N}^{*}+\mathrm{C}$

1800

$\mathrm{Not}+\mathrm{Ag}$

2129

$\mathrm{N}+4 \mathrm{AU}$

2129

$\mathrm{Na}+\mathrm{C}$

258

$\mathrm{Na}+\mathrm{C}$
Na. Na

$\mathrm{Ne}+\mathrm{C}$

258

$\ddot{0}+c$

‥ 258

$\ddot{0}+c$

1800

$0++\mathrm{Hg}^{\mathrm{Hg}}$

$\mathrm{O*}+\mathrm{Na}$

1843

D) * Ag

. 2129

$\ddot{0}++A \omega$

.. 2129

$s+c$

1800

$\ddot{s b}++M g$ 1839

$\mathrm{Te}+\mathrm{Ho}$

1839

U*. + Ar

$\mathrm{Ut}+\mathrm{C}_{2} \mathrm{H}_{2}$

U? + $\mathrm{CH}_{4}$

2907

U* : + co

vit $+\mathrm{CO}^{2}$

1907

$\mathrm{U}++\mathrm{H}_{2}$

U. + He

U. +
He
I9O

Ut $+K \mathbf{r}$

1907

U+ + Hq

. 1907

$u+* N_{2}$

U.. + No

1907

u+ . Ne

... 1907

$U++\mathbf{O}_{8}$

$0+0_{87}$
$\ldots \quad 1907$

$u++x e$

. 1907

Undef

1912 
$\cos$

PARTICLE PEMETRATION IN MACROSCOPIC

MATTER (IONS, NEUTRALS, AND ELECTRONS)

Exelted state Populations

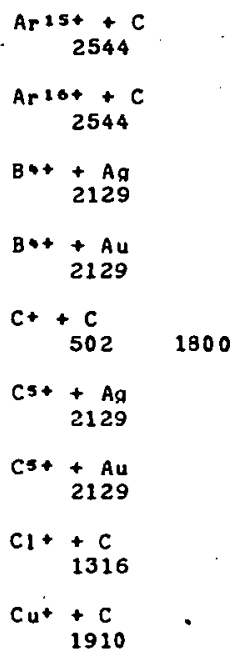

\begin{tabular}{|c|c|c|}
\hline$F^{2+}$ & $\begin{array}{l}+\quad A g \\
2129\end{array}$ & \\
\hline$F^{7+}$ & $\begin{array}{r}+A C \\
2129\end{array}$ & \\
\hline Fo* & $\begin{array}{r}+A g \\
2129\end{array}$ & \\
\hline for & $+\begin{array}{c}\text { Au } \\
2129\end{array}$ & \\
\hline $\mathrm{Fe}^{+}$ & $\begin{array}{l}+c \\
1910\end{array}$ & \\
\hline $\mathrm{Ge}^{+}$ & $+{ }_{500}^{c}$ & \\
\hline $\mathrm{H}^{+}$ & $+\underset{1800}{c}$ & \\
\hline $\mathrm{H}_{2}+$ & $\begin{array}{l}+c \\
2157\end{array}$ & \\
\hline $\mathrm{H}_{3}+$ & $\begin{array}{l}+c \\
2157\end{array}$ & \\
\hline $\mathrm{He}^{+}$ & $\begin{array}{l}+c \\
1772\end{array}$ & 2382 \\
\hline $\mathrm{Kr}^{+}$ & $\begin{array}{l}+c c \\
500\end{array}$ & 1800 \\
\hline $\mathrm{Mg}_{\mathbf{g}^{+}}$ & $\begin{array}{l}+c \\
1800\end{array}$ & \\
\hline
\end{tabular}




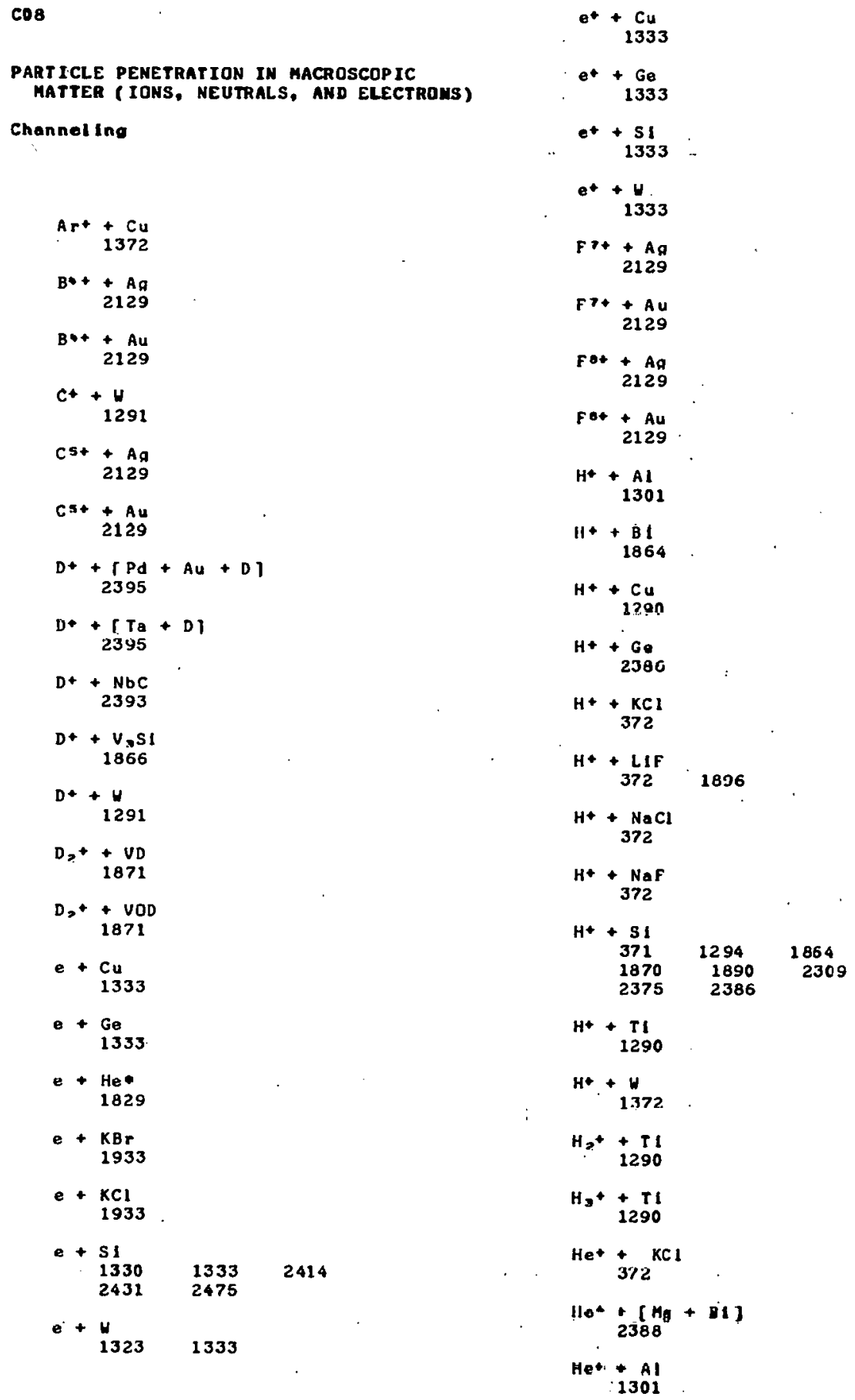

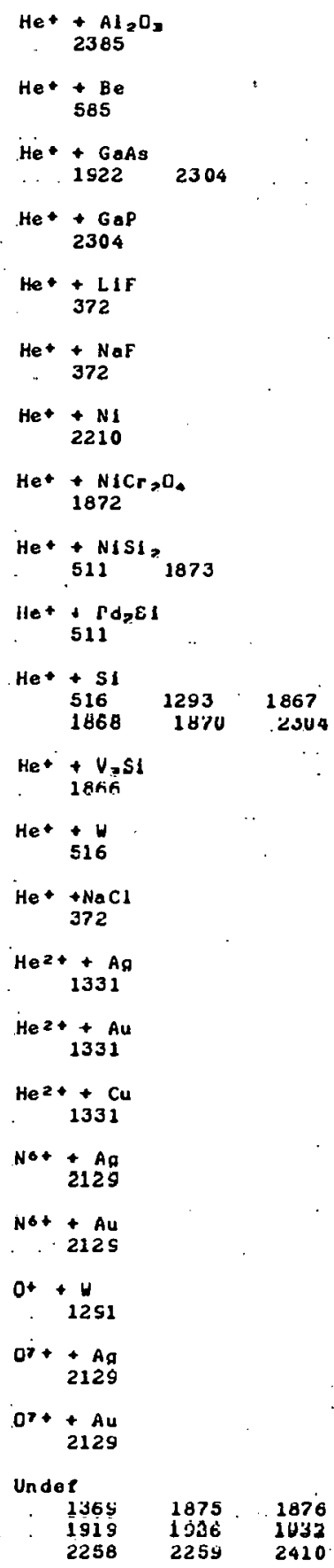

Do1

PART ICLE INTERACTIONS UITH SOLIDS

General

Review 
002

PARTICLE INTERACTIONS UITH SOLIDS

Spattering by Electrons, Neutrons, and Heavy Particles (total renoval

coefficlent s)

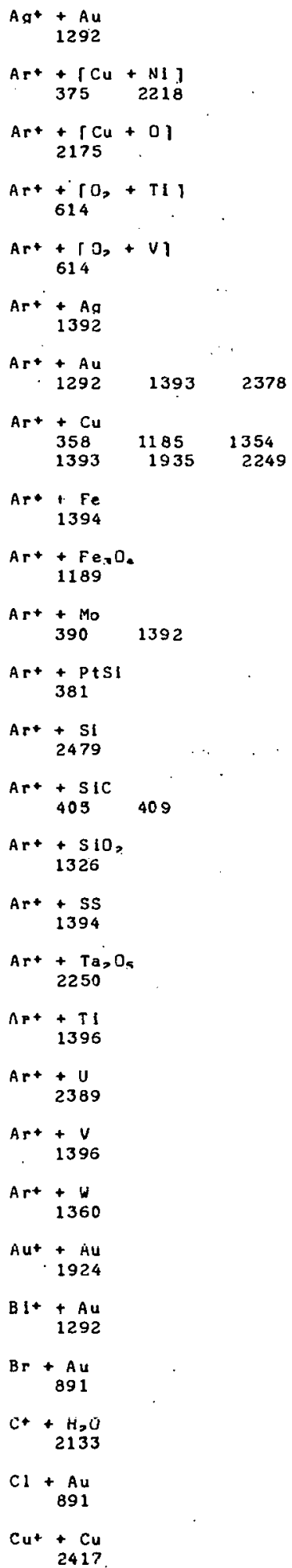

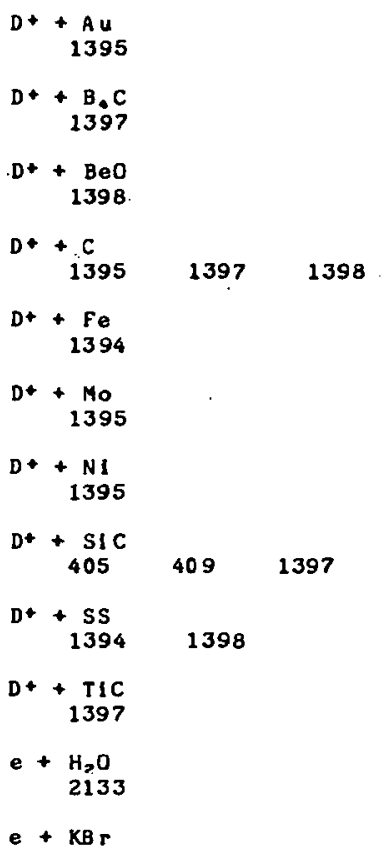

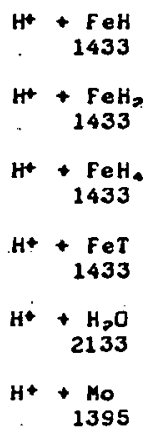




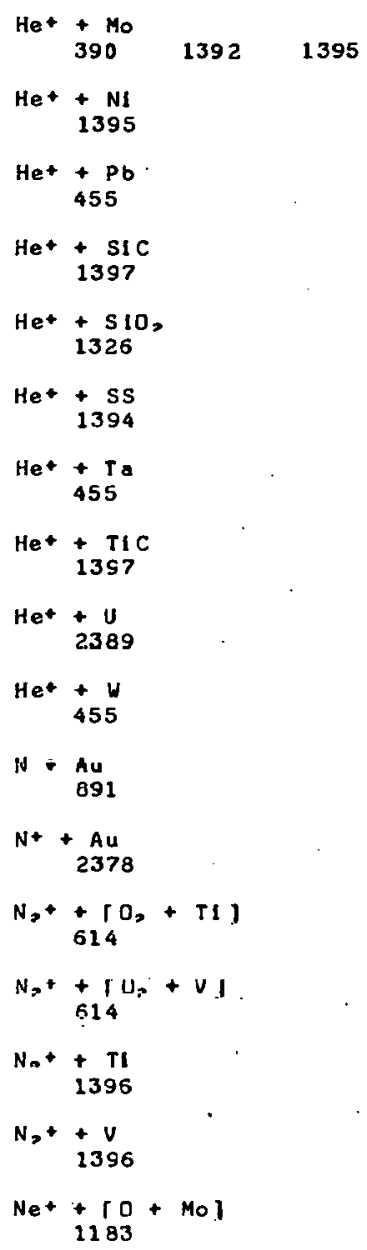

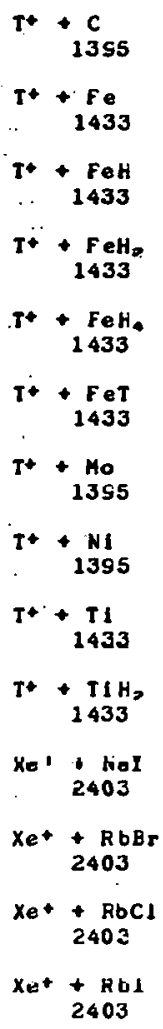


$\mathbf{0 0 3}$

PARTICLE INTERACTIONS UITH SOLIDS

sputtered Particle Charge and Quntun (Exelted) state Distribution

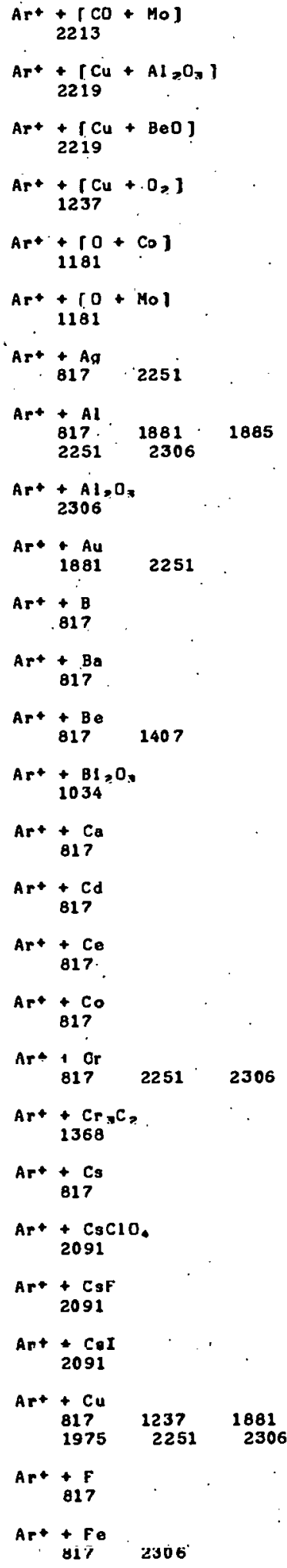

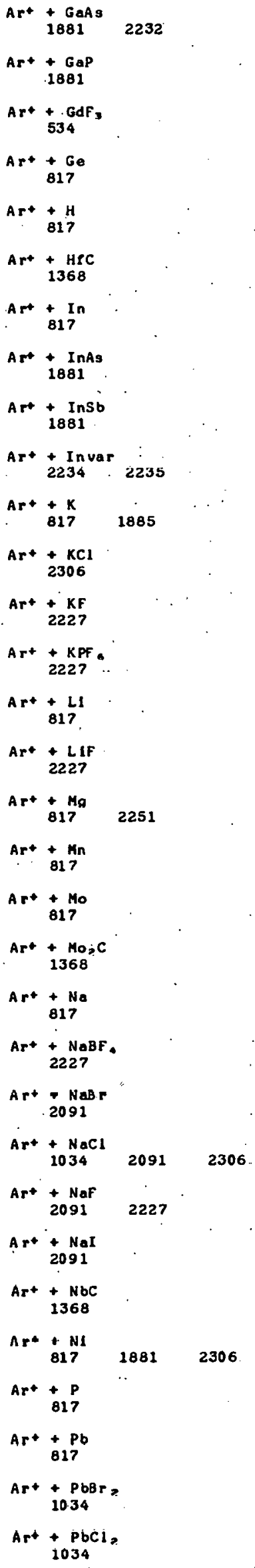




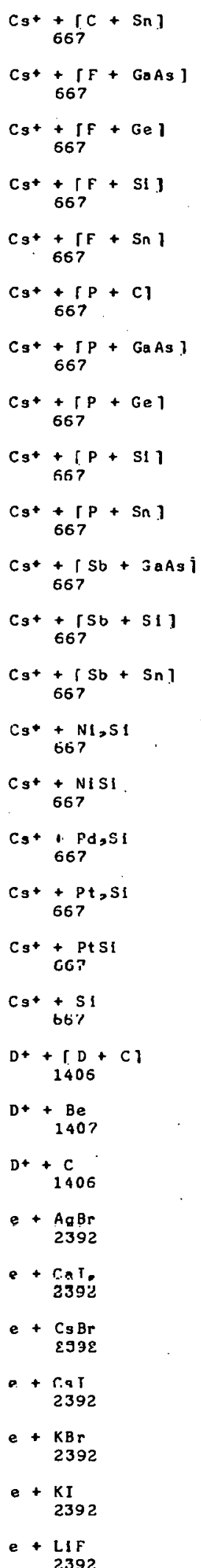

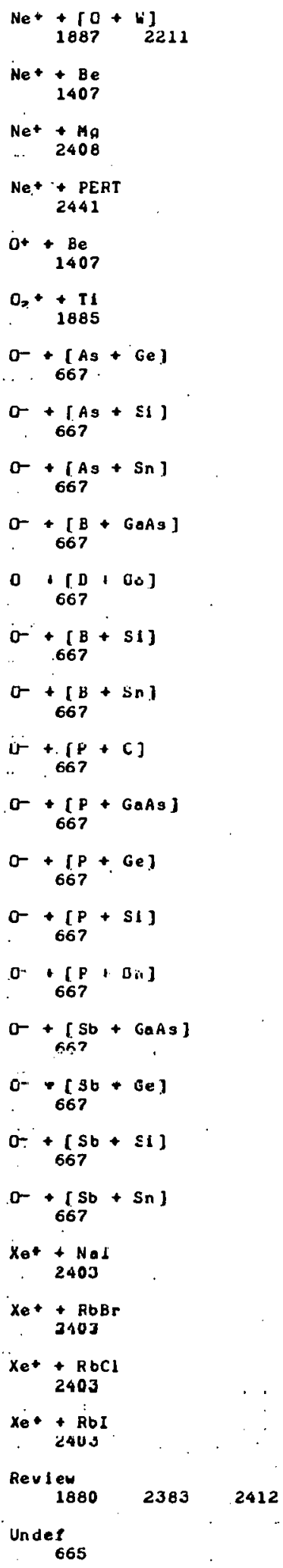


Do4

PARTICLE INTERACTIONS UITH SOLIDS

Secondary Electron Ejection by Heavy Particles and Electrons

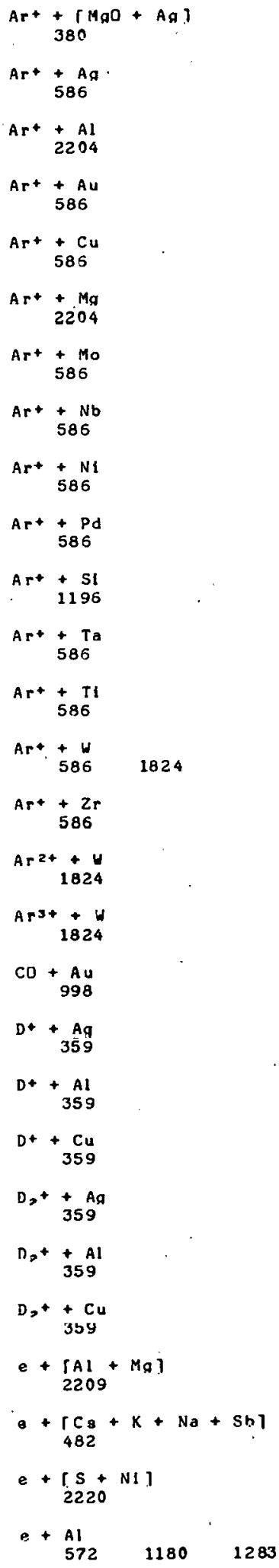

$\mathrm{e}+\mathrm{Al}_{2} \mathrm{O}_{3}$
2209

e $+\mathrm{BaO}$ 2238

e $+\mathrm{Cu}$ 2221

e $+D_{47}$

1435

$\mathrm{e}+\mathrm{Fe}$

e + GaP

2481

e+ $\mathrm{H}_{2}$

471.1435

e + Mg0

2209

$e+N_{48}$

e+N1

$572 \quad 2220$

$e+{ }_{582} 2214$

2221

$\mathrm{e}+\mathrm{T1}$
$572 \quad 1180$

e + IIC 1180

$e+w$ 583 、 584

$\mathrm{H}^{+}+\begin{array}{r}\mathrm{Ag} \\ \mathbf{3 5 9}\end{array}$ 2167

$H *+A 1$

2167

$H^{+}+A U$
2167

$\mathrm{H}+\mathrm{C}$ $1906 \quad 2167$

$\mathrm{H}^{*}+\underset{359}{\mathrm{Cu}}$

$H_{0}++A q$

$\mathrm{H},+\mathrm{Al}$ 359

$\mathrm{H}_{5}+\underset{359}{+\mathrm{Cu}}$

$\mathrm{He}^{+}+\mathrm{C}$ 1906

$\mathrm{He}+\mathrm{Si}$ $2230 \quad 2480$

$\mathrm{Kr}+\mathrm{Al}$

2204

$\mathrm{Kr}^{+}+\underset{\mathbf{5 8 6}}{\mathrm{Cu}}$

$\mathrm{Kr}+\mathrm{Mq}$ 2204

$K r^{\prime}+\mathrm{Tl}$ 586

$\mathrm{Kr}^{+}+\mathrm{H}$

$\mathrm{Kr}^{2+}+\underset{1824}{W}$

$\mathrm{Kr}^{3+}+\mathrm{W}$ 1824

Krot $+W$
$N++C$ 1906

$N_{?}+A u$

$\mathrm{N}_{2}++\mathrm{SI}$ 2230

$\mathrm{Ne}+\mathrm{Al}$

iet +4 $H 9$
2204

$\mathrm{Ne}+\mathrm{SI}$

1156

$\mathrm{Ne}^{3+}+$ 1824

$\mathrm{Ni}+\mathrm{Ag}^{-}$

$\mathrm{Ni}++\mathrm{Al}$ 2167

$\mathrm{Ni}+\mathrm{Au}^{\mathrm{u}}$ $216 \%$

$\mathrm{NI}++\mathrm{C}$ 2167

$\left.\mathrm{O} *+\int_{42} \mathrm{Cu}+\mathrm{Ee}+0\right\}$

$0++A g$ 2167

O+ + Al 2167

$\mathrm{D}^{+}+\mathrm{Au}$ 2167

$\mathrm{O}^{\circ}+\mathrm{C}$ 2167

$\mathrm{O}_{0}^{+}+\mathrm{Si}$ 2230

$\mathrm{SI}+\mathrm{Ag}$ 2167

$S I++A I$ 2167

$\mathrm{Si}+\mathrm{Au}$ 2167

$\mathrm{Si}+\mathrm{C}$

2167

$\mathrm{Xe}^{+}+\mathrm{Al}$ 2204

$\mathrm{Xe}_{\mathrm{C}}+\mathrm{Cu}$

$\mathrm{Xe}+\mathrm{Hg}$ 2204

$\mathrm{Xe}^{*}+\mathrm{SI}$ 1196

$\mathrm{Xe}^{+}+\mathrm{Ti}$ 586

$X e+t W$

$\mathrm{Xe}^{2+}+\mathrm{H}$ 1824 $\mathrm{Xe}^{3+\underset{W}{1824}}$

$\mathrm{Xe} *+$ 1824

$X e^{5+}+W$ 1824 Under $478 \quad 595$ 
D05

PART ICLE INTERACTIONS UITH SOLIDS

Photoelectric Ejection of Electrons

(coefricients)

$$
\begin{aligned}
& h v+\underset{1302}{[A g}+\mathrm{Cd}] \\
& \left.h v+\underset{1302}{\Gamma_{13 g} \mathrm{Ag}}+\mathrm{In}\right] \\
& h v+\underset{1302}{[\mathrm{Au}}+\mathrm{Cd} 1 \\
& h v+\underset{1302}{[\mathrm{Au}}+\mathrm{Gal}
\end{aligned}
$$

hv $+[\mathrm{Au}+\operatorname{In} 1$

hv $+[\mathrm{CO}+\mathrm{NI}]$

1179

$\left.h w+r_{578} r_{n} n+N i\right]$

he $+[11+N 1]$

2203

hv $+\begin{array}{r}A g \\ 579\end{array}$

$1216 \quad 1298$

hv $+A l$

$1295 \quad 2179$

he + As

591

$h \nu+A u$

$383 \quad 1296 \quad 1302$ hv + BaT10

$\mathrm{hv}+\mathrm{C}$

881

$h v+c d$

$1216 \quad 1302$

$h v+\underset{597}{C l}+S I$

$\mathrm{h}_{2}+\mathrm{Co}_{\mathrm{O}}+\mathrm{N}$

374

$\mathrm{he}+\mathrm{Cs}_{\mathrm{s}}$

1336

he $+\mathrm{Cu}$

$580 \quad 881 \quad 1146$

$\begin{array}{llr}1147 & 1156 & 1159 \\ 1160 & \end{array}$

$h v+G a$

1302

hy
$377^{-G a s}$

A. $+\mathrm{H}+\mathrm{H}$

594

nv $*$ In

1302.

$\mathrm{hu}^{+} \mathrm{KNbO}$.

593

he + Mo

$881 \quad 12161300^{\circ}$

he $+\mathrm{NaCl}$

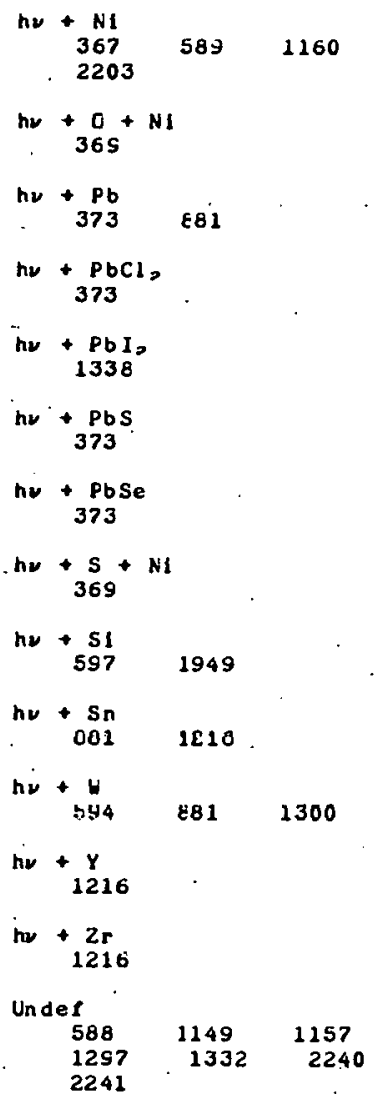

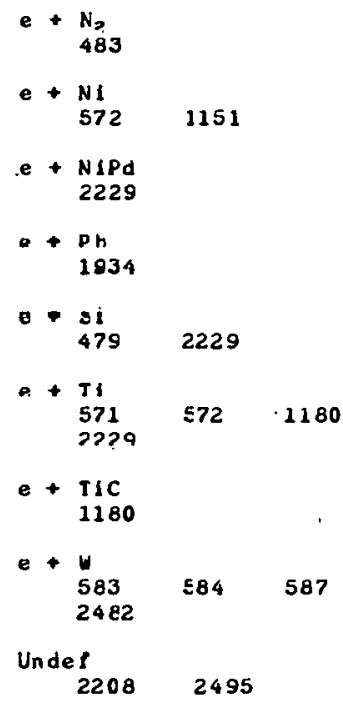


Do7

PART ICLE INTERACTIONS UITH SOLIDS

Reflection of Heavy Particles fro: surfaces (total reflection coefricienta)

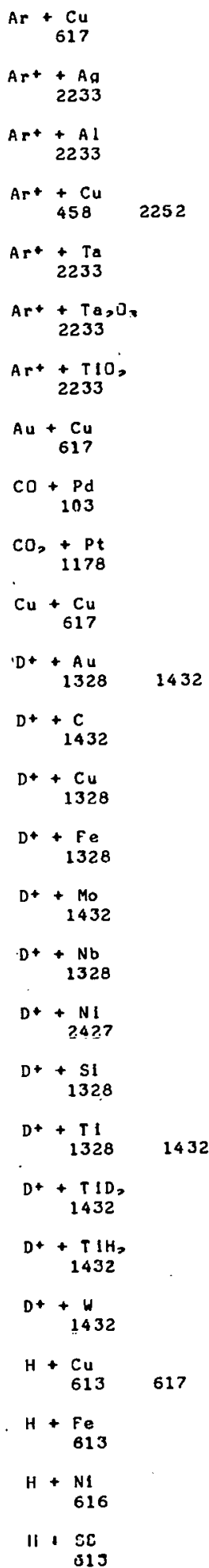

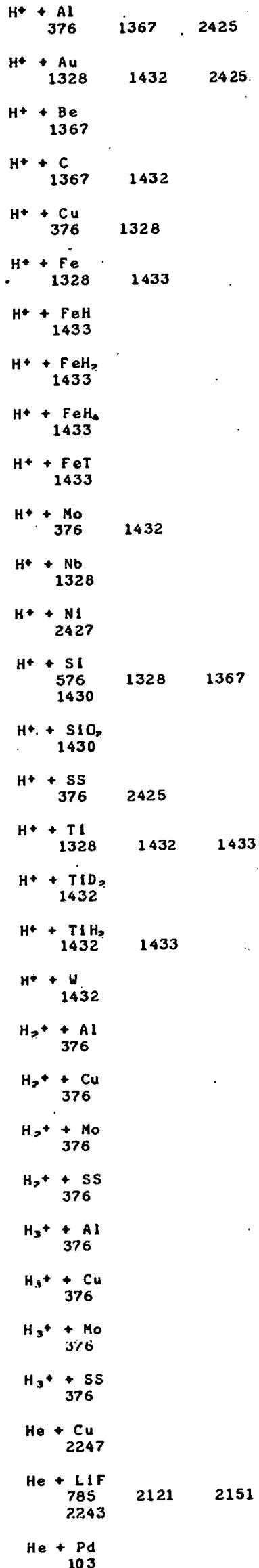

He $+\mathrm{Pt}_{1178}$

He $+w$

.. 2206

$\mathrm{He} *+\left[\mathrm{Ho}_{\mathrm{O}}+\mathrm{O}_{2}\right]$ 2302

$\mathrm{He}+\mathrm{Ag}_{44} 1431 \cdots 2233$

He+ $+\mathrm{Al}_{2233}$

$\mathrm{He}++\mathrm{Au}$ 1431

1432

$\mathrm{He}+\mathrm{Be}$

$\mathrm{He}+\mathrm{C}$ 1432

$\mathrm{He}+\mathrm{C}_{4}$ 1431

$\mathrm{He}^{+}+\mathrm{CuO}$ 1186

$\mathrm{He}^{+}+\mathrm{Ho}$ 1432

$\mathrm{He}++\mathrm{Nb}$ 1328

$\mathrm{He}++\mathrm{Nb}_{2} \mathrm{O}_{5}$ 44

He + NI $455 \cdot 2427$

Het $+\mathrm{Pb}$

$\mathrm{He}+\mathrm{si}$ $516 \quad 1430$

$\mathrm{He}_{+}^{+} \mathrm{S}_{3} \mathrm{O}_{2}$ 1430

$\mathrm{He}++\mathrm{Ta}$ 2233

$\mathrm{He}++\mathrm{Ta}_{2} \mathrm{O}_{3}$ 2233

$\mathrm{He}+\mathrm{T}_{2} \mathrm{O}_{5}$

$\mathrm{He}++\mathrm{TI}$ $1328 \quad 1432$

$H \mathrm{He}^{4: .+I 1 D_{2}}$

$\mathrm{He}+\mathrm{TIH}_{2}$ 1432

$\mathrm{He}+\mathrm{T} 10$,

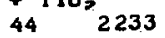

Het $+416 \quad 1432,2413$

$\mathrm{He}^{+}+\mathrm{WO}_{\mathrm{a}}$

, 44

$\mathrm{He}^{+}+2 \mathrm{rO}_{2}$

44

N. + Cu

$\mathrm{Ne}+\mathrm{Cu}$ 617 2247

$\mathrm{Ne}+\underset{785}{4}$

$\mathrm{Ne}+\underset{455}{+\mathrm{Ag}}$

$\mathrm{Ne}+\underset{2225}{\mathrm{Cr}}$ 


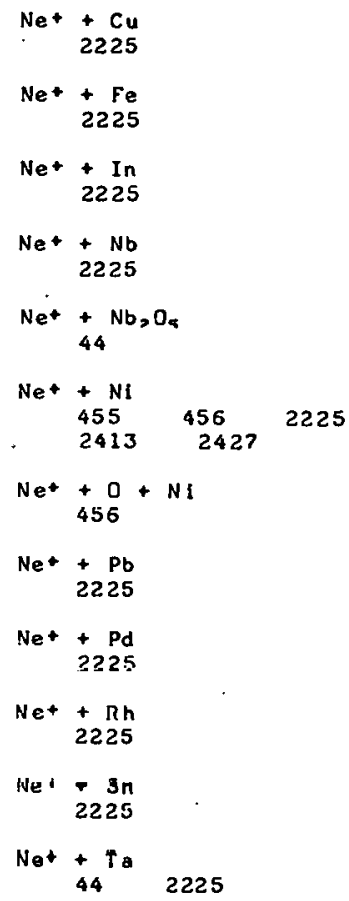
$\mathrm{Ne}_{44}^{+} \mathrm{Ta}_{2} \mathrm{O}_{5}$

$\mathrm{Ne}+\mathrm{T}_{10}$

44

$\mathrm{Ne}+$ MO 44

$\mathrm{Ne}+\mathrm{Y}$

2225

$\mathrm{Ne}^{+}+\mathrm{Zr}$

2225

$\mathrm{Ne}^{+}+2 \mathrm{rO}$,

44

$0>+\frac{P d}{103}$

$\mathrm{O},+\mathrm{Pt}$

1178

$\mathrm{P}++[\mathrm{H}+\mathrm{SIO}>]$

899

$\mathrm{P}+[\mathrm{H}, \mathrm{O}+\mathrm{S} 1 \mathrm{O}$,

899

$\mathrm{T}+\mathrm{Au}$ iseo

$\mathrm{I}+\mathrm{Cu}$ 1328
$\mathrm{T}+\ldots \mathrm{Fe}$

1328

1433

$\mathrm{T+}+\mathrm{FeH}$

T* + $\mathrm{FeH}_{2}$ 1433

T+ $+\mathrm{FeH}_{\text {. }}$ 1433

$\mathrm{T}+\mathrm{FeT}$ 1433

$\mathrm{Tt}+\mathrm{Si}$ 1328

$T++\begin{gathered}T 1 \\ 1433\end{gathered}$

$T++T H$,

1433

$\mathrm{Xe}+\mathrm{Cu}_{617}$

Review

2207

Under

$\begin{array}{lll}2042 & 2201 & 2212 \\ 2210 & 2228 & 2242\end{array}$ 
DDB

PARTICLE INTERACTIONS WITH SOLIDS

Charge and quantiu state Distributions of Reflected Heavy Particles at Hacroscople Distances fros Surfaces

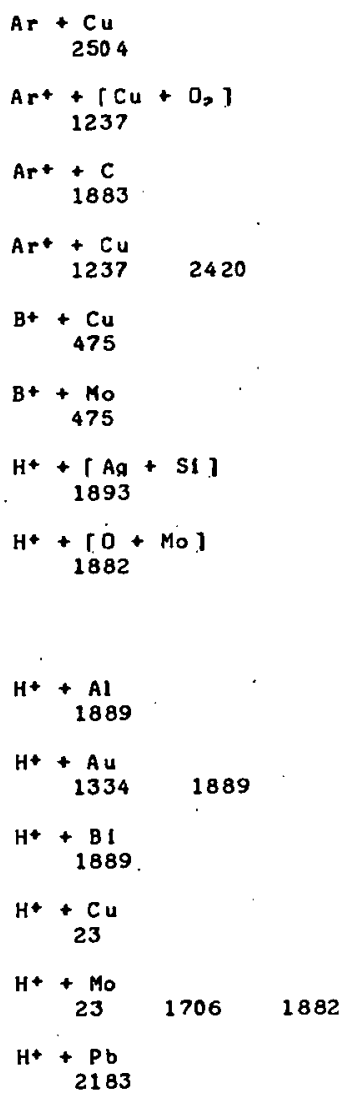

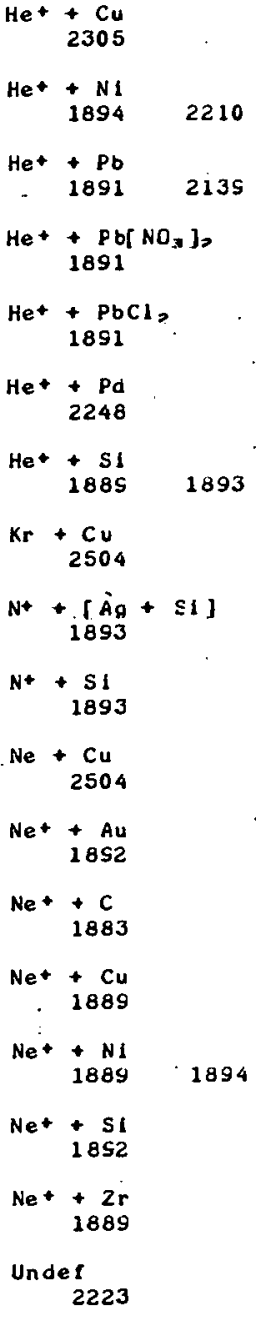




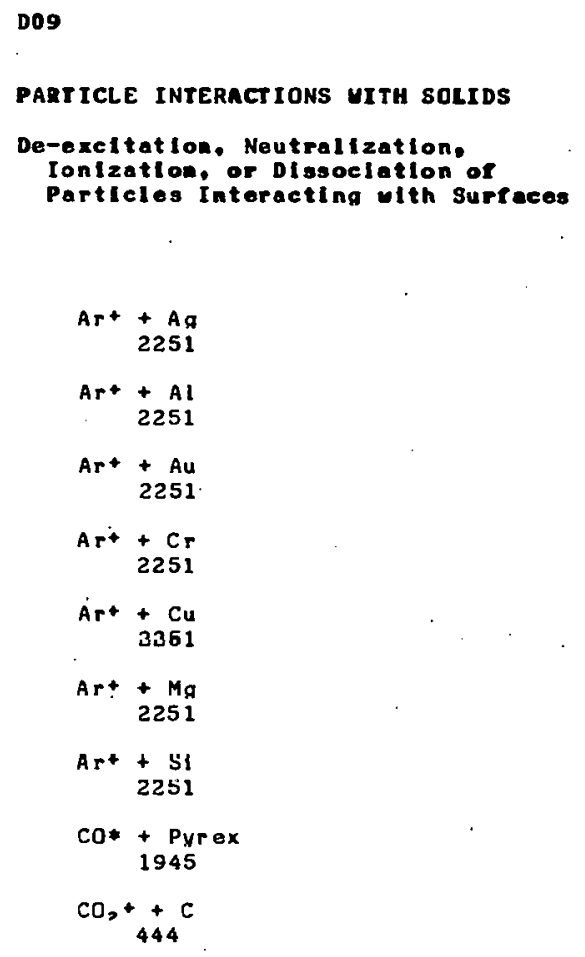

$C u+C u$

$\mathrm{H}+\mathrm{Cu}$ 340

$\mathrm{H}^{+}+\mathrm{Au}$ 1334

$\mathrm{H}_{2}+\mathrm{Cu}$ 2237

$\mathrm{H}_{2}++\mathrm{C}$

2119

HE + Y

413

$\mathrm{He}+\mathrm{Cu}_{340}$

$\mathrm{HeH}+\mathrm{C}$

2119

$\mathrm{N}_{2}++\mathrm{C}$

1111

$\mathrm{N}_{2}++\mathrm{Ge}$

1111

$\mathrm{N}=+\mathrm{Pb}$

1111

$\mathrm{N}_{2}++\mathrm{PbO}$

1111
$\mathrm{N}_{2}++\mathrm{PbO}_{2}$

1111

$\mathrm{N}_{2}++\mathrm{SI}$

961

1111

$\mathrm{N}_{2}+: \mathrm{S10}$

961

1111

$\mathrm{No}++\mathrm{S1O}$

961

1111

$\mathrm{N}_{2}++\mathrm{Sn}$

1111

$\mathrm{N}_{0}++\operatorname{snO}$

1111

$\mathrm{N}^{2}++\mathrm{SnO}^{2}$

1111

$\mathrm{N}_{2} \mathrm{O}++\mathrm{C}$

444

if $+c$

$\mathrm{UF}_{4}+\mathrm{Pt}_{\mathrm{t}}$

- 587

iv. +4

Unde? 1375

1378

2492
D10

PARTICLE INTERACTIONS UITH SOLIDS

Interaction Potent 1 al s Between Surfaces and Free Particles Located External to the Surface (olectrons and heary particles)

$$
D+L i F
$$
$D+\mathrm{NaF}$
2231
e + si 2431
H + Lif 2231
$H+$ NaF 2231
$\mathrm{H}_{2}+\mathrm{Cu}$

D11

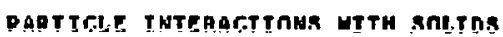

Sticking Coefficlents (thermal energles)
$\mathrm{CO}+\mathrm{Pd}$

$\mathrm{Ha}+\mathrm{PA}$

103
H. + Fe

He + Lif

$\mathrm{He}+\mathrm{NaF}$

... 2231

PERT + PERT

. 2310 .

CO + Ir

2074 
PARTICLE INTERACTIONS UITH SOLIDS

- + Th 2215

Electromagnetic Radiation Induced by Electron or Heavy Partlele Inpact on Surtaces

$\mathrm{Ar}++\mathrm{Cu}$

1927

Art + Mo 1927

Ar + + PERT 2441

$A r^{+}+\mathrm{TI}$ 1927

Art +4

$C++C$ 1879

$\mathrm{Cl}^{6+}+\mathrm{Cu}$

$e+\left[\mathrm{Cd}+\mathrm{Hg}_{q}+\mathrm{Te}\right]$

1325

$a+[\mathrm{GaN}+\mathrm{Zn}\}$ 230.1

e+Ag $1209 \quad 1370$

e+Al 2432

$e+c$ 1152

$e+$ CdS 2224

e $+\mathrm{Cu}$ 2432

+ GaAs 1148 2224

e+GaP

1329

$e+L 1 F$ 1215

e + MgO

1329
$H$ * Under 668

$\mathrm{H*}+\mathrm{Al}$ 1518

$\mathrm{H}++\mathrm{AU}$

1878

$\mathrm{H}++\mathrm{C}$ 1879

$\mathrm{H}+\mathrm{Ce}$

$\mathrm{H}+\mathrm{Cs}$ 1878

$H *+\mathrm{Cu}_{1878}$

$\mathrm{H}+\mathrm{Fe}$

1878

$\mathrm{H}+\mathrm{Ho}$

$\mathrm{H}+\mathrm{NI}$

1904

$\mathrm{H}+\mathrm{Se}$

$\mathrm{H}^{+}+\underset{\mathrm{Sm}}{1878}$

$\mathrm{H}^{+}+\underset{1878}{\mathrm{Sn}}$

$\mathrm{H}+\mathrm{Ti}$

$\mathrm{H}+\mathrm{H}$ 1878

$\mathrm{H}+\mathrm{Y}$

1878

$\mathrm{He}^{+}+\mathrm{Cu}$

$\mathrm{He}^{+}+\mathrm{Mo}_{\mathrm{O}}$

1927

$\mathrm{He}^{+}+\mathrm{Pb}$

1877
$\mathrm{He}+\mathrm{Te}$ 1877

$\mathrm{He}^{+}+\mathrm{TI}$

$\mathrm{He}+\mathrm{H}$ 1927.

$\mathrm{He}^{2+}+\mathrm{Au}$

$\mathrm{He}^{2+}+\mathrm{Ce}$ 1878

$\mathrm{He}^{2+}+\mathrm{Cs}$

$\mathrm{He}^{2+}+\mathrm{Cu}$ 1878

$\mathrm{He}^{2+}+\mathrm{Fe}$ 1878

$\mathrm{He}^{2+}+\mathrm{HO}$ 1878

$\mathrm{He}^{2+}+\mathrm{Se}_{1878}$

$\mathrm{He}^{2+}+\mathrm{Sm}$ 1878

$\mathrm{He}^{2+}+\mathrm{Sn}_{1878}$

$\mathrm{He}^{2+}+\mathrm{II}$

1878

$\mathrm{He}^{2+}+\mathrm{H}$

$\mathrm{He}^{2+}+\mathrm{Y}$

1878

$\mathrm{N}+\mathrm{C}$

1879

$\mathrm{Ne}+$ PERT

2441

$0 *+\mathrm{C}$

1879

$\mathrm{O}+\mathrm{MgO}$

.. 2217

Undet 1304 
D13

PART ICLE INTERACTIONS UITH SOLIDS

Desorption of Gases fron Surfaces

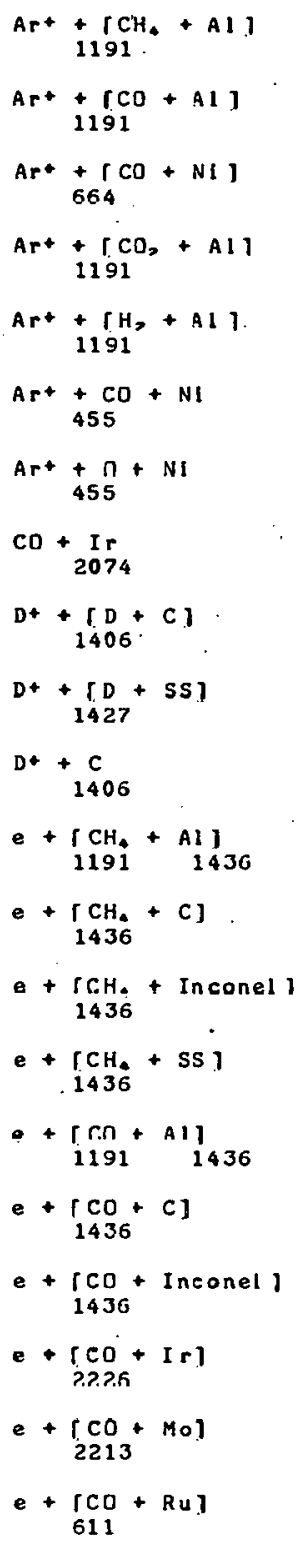

$e+\underset{1436}{100+S S T}$ $\left.e+\underset{\left[\mathrm{CO}_{2}\right.}{1191}+\mathrm{Al}\right]$

$e+\underset{1176}{f F}+5 s 1$

e+ $\underset{1126}{[H+S S]}$

$e+\underset{2246}{\left[\mathrm{H}_{2}+W\right]}$

$e+\left[\mathrm{H}_{2}+A 1\right]_{1436}$ $\left.e+\underset{1436}{\left[H_{2}\right.}+C\right]$

$e+\underset{1436}{\left[H_{2}\right.}+$ Incone 1$\}$

e $+\left[\mathrm{H}_{7}+\$ \mathrm{~S}\right]$ 1436

$e+\underset{22}{\mathrm{O}}+\mathrm{Nb} 1$

$e+[0+W]$ 2244

$e+\underset{61.0}{[0, W]}$

e $+\mathrm{Cr}_{\mathrm{r}} \geqslant \mathrm{O}$,

2131

$e+N 10$ 2131

e+ T10?

2131

$e+v_{20} 0$

e $+\mathrm{WO}_{3}$

2131

II 1 2rC 2490

$\mathrm{H}+\mathrm{I} \mathrm{CH}+\mathrm{Lui}$ 1177

$\mathrm{H}^{+}+[\mathrm{C}+\mathrm{SS}]$ 1177

ii) T [ + 33$\}$ $1424 \quad 1425$

$\mathrm{H}+[\mathrm{O}+\mathrm{Cu}]$ 1177

$\mathrm{H}^{*}+[\mathrm{O}+\mathrm{SS}]$ 1177

$\mathrm{H}++[\mathrm{S}+\mathrm{Cu}]$ 1177 
D16

PART ICLE INTERACTIONS UITH SOLIDS

Blistering. Volds, and Surface straln In Metals

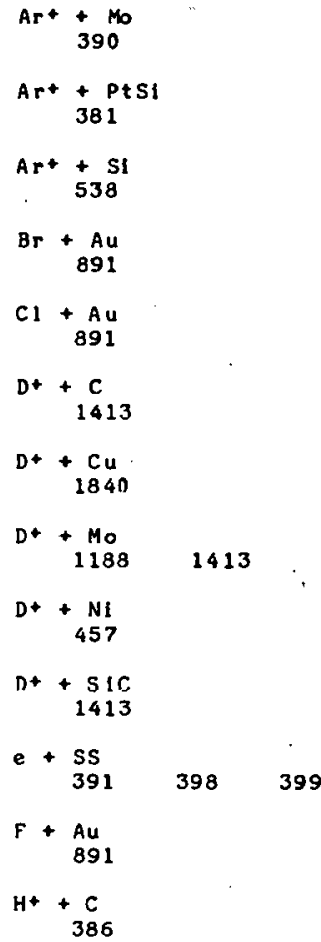

$\mathrm{H}^{+}+\begin{array}{r}\text { Mo } \\ 389\end{array}$

$\mathrm{H}+\mathrm{TI}$

1411

$\mathrm{H}+*$

1411

$\mathrm{He}+\mathrm{Rh}$

615

$\mathrm{He}+[\mathrm{Nb}+\mathrm{Zr}]$

396

$\mathrm{He}^{+}+[\mathrm{Pt}+\mathrm{Rh}]$

1420

$\mathrm{He}+\mathrm{Ag}$
2372

$\mathrm{Het}+\mathrm{Be}$

1409

$\begin{array}{rl}+\mathrm{He} & \mathrm{C} \\ \mathbf{3 8 6} & \end{array}$

$\mathrm{He}+\mathrm{Cu}$

1416

Het + Ho

1184.141

1439

$\mathrm{He}+\mathrm{Nb}$

$396 \quad 1846$

He* + NI

$\begin{array}{lll}457 & 470 & 1409\end{array}$

1415

1418

$\mathrm{He}^{*}+\mathrm{SS}$

$400 \quad 404 \quad 1408$ 1418

404
1419

$\mathrm{He}+\mathrm{TI}$

1411

$\mathrm{He}^{+}+\mathrm{V}$

1410
1421

$\mathrm{He}+t$

.1417

n. $+\mathrm{Al}_{393} \mathrm{O}_{3}$

N"+ Au

891

$N_{2}+[N !+A l]$

407

$\mathrm{Ne}+\mathrm{C}$

$\mathrm{Ne}++\mathrm{Nb}$

402

$\mathrm{Ni}++\mathrm{NB}$

392

N1 * SS

1440

$\mathrm{Ni}+\mathrm{Ti}+\mathrm{Nb}$

392

$\mathrm{Ni} 2+$ + $\mathrm{Ho}$ 2391

$0+A \mathrm{Au}$

891

$0++5 S$

SI $6+500$

$\mathrm{Ta} 3++\mathrm{Ho}$

1439

$\left.v+[]_{1442}^{v}+C r\right]$

Unde $f$

$403 \quad 1412-1441$ 
D15

PARTICLE INTERACTIONS UTTU SOLIDS

Radiation Danage in Metals

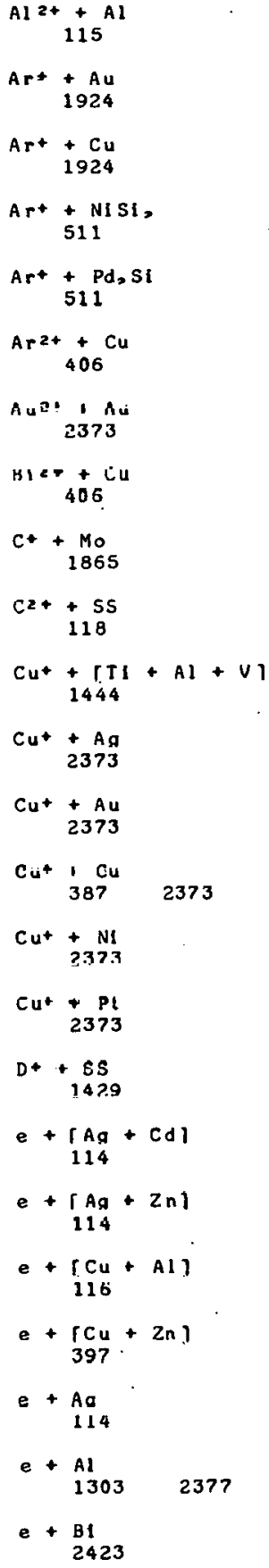

e + co

2376

$\mathrm{e}+\mathrm{Cu}$

$117.119 \quad 406$

$\begin{array}{lll}2384 & 2387 & 2428\end{array}$

e + Mo

2308

e + Nb

112

e + SS

$118 \quad 2424$

$+2 \mathbf{r}_{2} \mathrm{Al}$

395

$\mathrm{Ge}+\underset{536}{+\mathrm{Ge}}$

H+ Al

$\mathrm{H}+\mathrm{Cu}$

$406 \quad 1182$

$\mathrm{H}+\mathrm{GinAs}$

2304

11. Gar

2304

$\mathrm{H}^{+}+\mathrm{Nb}$

1182

$\mathrm{H}++\mathrm{NI}$

1182

$\mathrm{H}+\underset{2304}{\mathrm{SI}}$

$\mathrm{H}_{\mathbf{3}}+\mathrm{SI}$

576

$\mathrm{He}+[\mathrm{Mg}+\mathrm{Al}]$

2388

Het $+A$ An
2372

$\mathrm{He}^{+}+\underset{2307}{\mathrm{C}}$

$\mathrm{Hot}+\underset{387}{6 u} 577$

Het+ Mo

$\mathrm{He}+$ + SS

404

$\mathrm{He}^{2 *}+\mathrm{Zn}$

1334

$n+\left[\begin{array}{l}B 1 \\ 113\end{array}\right.$

$n+\underset{2399}{[\mathrm{Cu}}+\mathrm{Al}]$

$n+B !$

113

$n+C_{0}$
$\mathrm{n}+\mathrm{Cu}$

1182

2371

$n+M g$

. 1928

n. + Nb

$1182 \quad 2416$

n. $+N$

1182

$\mathrm{n}+\mathrm{NI}_{2} \mathrm{Cr}$

$n+s s$

n 2424

$\mathrm{Nt}+$ GaAs

2304

$N+$ GaP

2304

N* + 31

$N++$ V

.. 1865

$\mathrm{Nb}++\mathrm{Nb}$

1437

$\mathrm{Ne}^{+}+\mathrm{Mo}$ 1865

$\mathrm{Ne}+\mathrm{V}$

1865

$N 1+\underset{1443}{[N I+A 1]}+2375$

$\mathrm{NI}+\left[\mathrm{NI}+\mathrm{MO}_{0}\right]$

1443

$\mathrm{Ni}+\left[\mathrm{Ni}+\mathrm{Si}_{1}\right]$

1443

$\mathrm{NI}+\stackrel{\mathrm{Cu}}{ }$

387

$\mathrm{NI}+\mathrm{NI}$ 1443

Ní? + no

2391

$0+U C_{0}$ 2421

$0++P b$

$0 *+s S$

404

$U+\mathrm{NO}_{2}$

$\omega *+p t$

2205

Uitde? 19212390 
D26

PART ICLE INTERACTIONS UITH SOLIDS

Particle Implantation in Motals

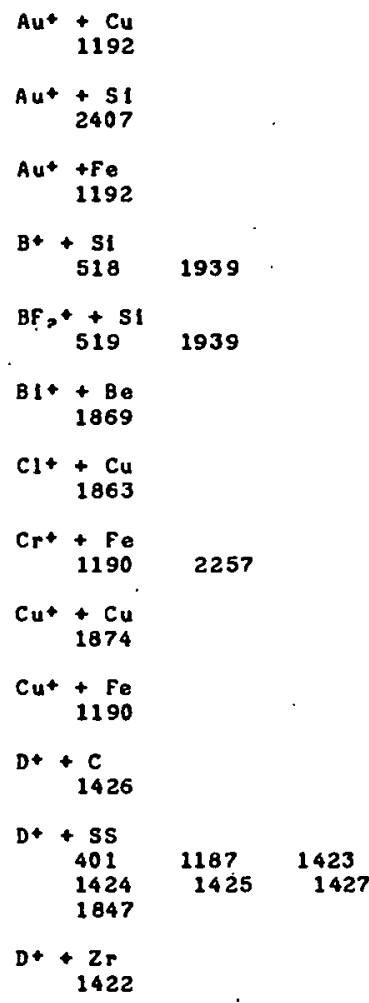

$$
\mathrm{D}_{2}+\underset{401}{+\mathrm{SS}}
$$$$
\text { e }+\mathrm{Mg}
$$
2402

Er+ + N1

$6 a^{+}+B e$ 1869

$6 e++6 e$

$\mathrm{H}+\mathrm{SI}$ 1884

$\mathrm{H}_{2}++\mathrm{SI}$ 1884

$\mathrm{He}^{+}+\mathrm{Ag}$ 2372

$H e++C$

$\mathrm{He}^{+}+\mathrm{Cu}$

1863

$\mathrm{He}^{+}+\mathrm{ErD}_{2}$ 1845

$\mathrm{He}^{+}+\mathrm{ErT}$,

1845

$\mathrm{He}^{+}+\mathrm{Mo}$

1414

$\mathrm{Het}+\mathrm{Nb}$

1846

1913

$\mathrm{He}+\mathrm{NI}$
1913

$\mathrm{He}^{+}+\mathrm{SS}$

404
Lat + NiI

$\mathrm{CI}_{3}+\mathrm{Cu}$

1863

$\mathrm{N}++\mathrm{Fe}$

$\mathrm{Ni}+\mathrm{Fe}$ 2257

$0+$ ss

404

$\mathrm{Os}^{+}+\mathrm{Be}$

1865

$\left.P++\underset{89}{[H}+S_{10}\right]$

$\mathrm{PH}+\left[\mathrm{H}_{2} \mathrm{O}+\mathrm{SIO}_{2}\right]$ 899

$\mathrm{Pb}+\mathrm{Be}$ 1869

$\mathrm{Sn}+\mathrm{NI}$

1917

$\mathrm{Ta}+\mathrm{NI}$ 1917

Te+ + GaAs

520

$\mathrm{Ti}+\mathrm{Be}$ 1869

$w+B e$

1869

Revleu

$1193 \quad 1194^{\circ} \quad 1195$

Under

$2419 \quad 2421 \quad 2422$ 
DI7

PARTICLE INTERACTIONS UITH SOLIDS

Electron-, Ion-, and Photon-Induced Cheatcal Changes to Surfaces

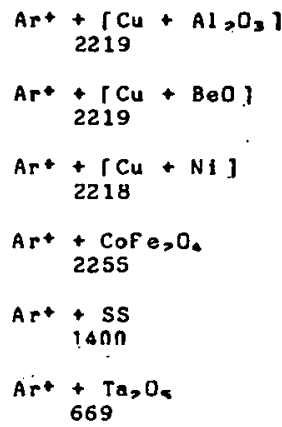

EO1

ELECTRON-PARTICLE INTERACTIONS General

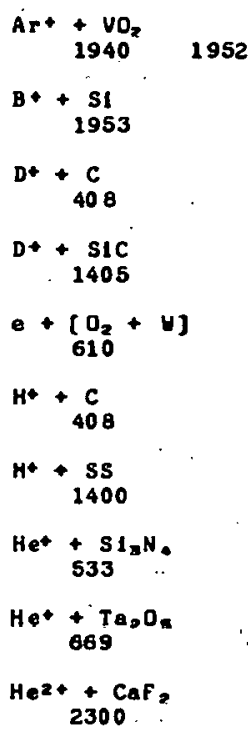

Review 2196

Under 1635 1820 
$\mathrm{EO} 2$

ELECTRON-PARTICLE INTERACTIOAS

Elastic Collislons

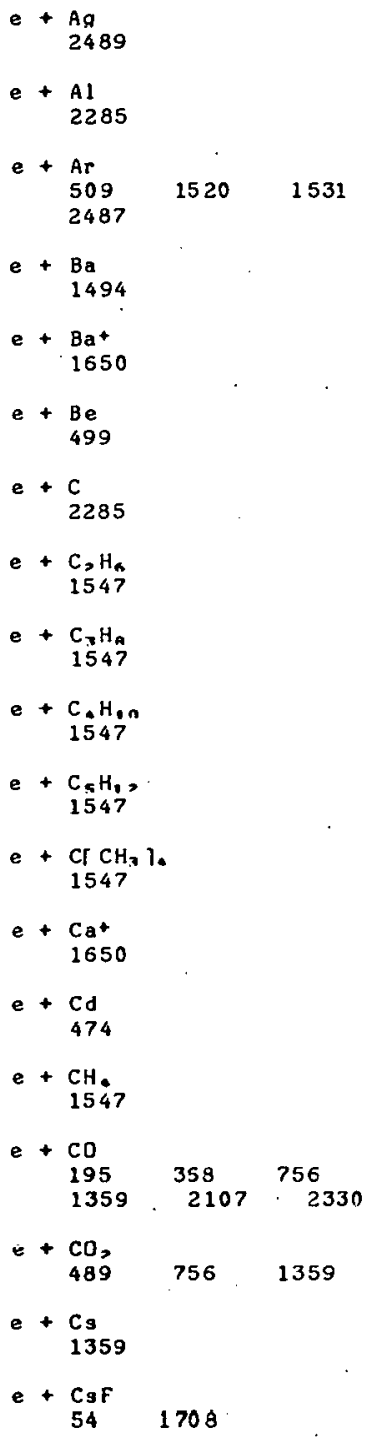

e $+\operatorname{Cs} \mathrm{OH}$ 1359

$\mathrm{e}+\mathrm{Cu}$ 2285

$\mathrm{e}+\mathrm{H}$ $\begin{array}{llr}59 & 77 & 200 \\ 201 & 216 & 253 \\ 1359 & 1507 & 1520 \\ 1548 & 1575 & 1605 \\ 1698 & 1777 & 1801\end{array}$

$\mathrm{e}+\mathrm{H*}$
26 260

$\mathrm{e}+\mathrm{H}$ $\begin{array}{lll}\mathrm{H} & & \\ 53 & 273 & 368\end{array}$ $\begin{array}{llr}639 & 981 & 1265 \\ 1359 & 1603 & 1640\end{array}$ $\begin{array}{lll}1359 & 1603 & 1640 \\ 1746 & 1794 & 1808\end{array}$ 1818

$\mathrm{e}+\mathrm{H}_{3}+$ 1346.1600

$\mathrm{e}+\mathrm{H}_{2} \mathrm{O}$ $-1359$

$\mathrm{e}+\mathrm{H}_{2} \mathrm{~S}$ 1637

$e+H B r$

1458

e+ HCN 1978

$\mathrm{e}+\mathrm{He}$ $\begin{array}{lll}14 & 90 & 183 \\ 200 & 354 & 1531\end{array}$ $\begin{array}{llr}1698 . & 1823 & 2487\end{array}$

e+ $+\mathrm{He}^{+}$ 1546

$e+H F$

715

$\mathrm{e}+\mathrm{Hg}$ $1615 \quad 2358$

e $+k$

1359

e+KI

54.1708 .

$\mathrm{e}+\mathrm{KOH}$

1359

$e+k r$ $509 \quad 2487$

$e+L i$
e1C1
1708

e LIF

1208

e+ $\mathrm{Ma}^{+}$

1650

e. Mn

1471

$\mathrm{e}+\mathrm{N}_{2}$
13 13
195 508 1359 1911 $\begin{array}{ll}53 & 67 \\ 368 & 480\end{array}$ $639 \quad 789$

e + Na 1359

e $+\mathrm{NaCl}$ 1708

e $+\mathrm{NaF}$ 1708

e+Nolio 2163

$\mathrm{e}+\mathrm{Ne}$ $\begin{array}{lll}\text { So } & 236 & .1531 \\ 2487 & 2489 & \end{array}$

e $+\mathrm{NO}$ 1359

$e^{i}+0$, $1359 \quad 1911$

e GCS
756

e+CH 1359

e+SFa 2103

$e+5 r^{+}$
1650

e $+\mathrm{Xe}$

2487

Review
54

Undet

$284 \quad 1380 \quad 2272$ 1361 1973

1492 1640 
E03

ELECTRON-PARTICLE INTERACTIONS

\section{Excitation}

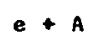

$1248 \quad 1533$

e + Ar* .

1705

e + Art

e B Seq

846

e Ba
1494

$++8 a 4$

2168

$0 .+1650$
.1650

$\mathrm{e}+\mathrm{Be}+$

$39 \div 259$

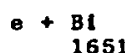

e $+\mathrm{c}^{2+}$

622633

$\mathrm{Ca}+$
$259 \quad 1650$

e+Ca+

1658

$+C d$
474

$e+C D$.

$0+\mathrm{CH}^{+}$

1830

e $+\mathrm{CH}^{2} \mathrm{Br}$

2266

$++\underset{2266}{\mathrm{CH}_{2} \mathrm{I}}$,

e+CHII

2266

$a+\mathrm{GH}_{4}$

e + CHBr

2266

$\begin{array}{rll}0 & 60 \\ 83 & 195 \quad 368 \\ 894 & & \end{array}$

$\mathrm{CO}_{2}$
$11 \mathrm{ij} \quad 1473$

Cs
$29 \quad 184 \quad 862$

$\mathrm{e}+\mathrm{Cs}_{2}$
2064

e cor:

1484

C $\begin{array}{ll}\text { CsF } \\ 54\end{array} 55 \quad 1484$

$\begin{array}{rl} & \text { D? } \\ 206 & 210 \quad 271 \\ 2280 & \end{array}$
$\therefore+D_{2} 0$

2451

$e+$ Er

e Eu

1236

$e+F_{2}$

$\mathrm{e}+\mathrm{Fe}$

1744

$\mathrm{Fe} 2+$
827

e Fest

643827

$\mathrm{Fe}^{64}$
1744

e $+\mathrm{Fe}^{10}$

639

$+\underset{638}{F Q^{11}}$

e Fete

1658

e Fe16*

1744

e Fe 2 .

1744

$+4$

193
324
$6 n 3$

$\begin{array}{lll}1576 & 1787 & 1822\end{array}$

e+1*

2332

2366

- H Seq

208
1707

$657 \quad 1455$

2366

- $+\mathrm{H}_{2}$

$$
396
$$

273
1574

2280

$.200 \quad 210$

$368 \quad 403$

$1764 \quad 2265$

- $+\mathrm{H}_{2} \mathrm{O}$

1976

$2338 \quad 2345$

$H_{2} 5$
1637

- + HBF

2468

c + HCI

1595

- $+\mathrm{H}^{\circ}$

$\begin{array}{lcc}7 & 183 & 202 \\ 203 & 237 & 2.5 ? \\ 1495 & 1496 & 1574 \\ 1577 & 1634 & 1728\end{array}$

$\mathrm{He}$
24

e $+\mathrm{He}^{+}$

$\begin{array}{lll}48 & 225 & 485 \\ 1447 & 1576 & 1707\end{array}$

- He Seq

$\begin{array}{lcc}93 & 631 & 632 \\ 651 & 831 & 1532\end{array}$

2350

- $\leftrightarrow \mathrm{HF}$

703 1010. 1595
$\mathrm{e}+\mathrm{Hg}$

$1242 \quad 2349 \quad 2358$

c+ $\begin{gathered}\mathrm{HgBr} \\ \mathbf{7 3 0}\end{gathered}$

e. $+\mathrm{H}_{\mathfrak{q} \mathrm{Cl}}$

730

$\mathrm{HgI}$
$\mathbf{7 3 0}$

e.. Ho

1652

e $k$

$\begin{array}{lll}k 9 & 184 & 325 \\ 862 & & \end{array}$

e $+K I$

$54 \quad 1484$

$a+\frac{K r}{1461} 1578$.

- $+K \mathbf{r} *$

17̄0์

e... Krase

- +11

$325 \quad 637 \quad 862$

. $+11+$

1759

- Li Seq

846

e $+1 C$

.. 1484

e +1 Lf

- LIH

. 2484

a + Mg

1470

e $+\mathrm{Mg}^{\circ}$

$25 y$

1650

e+ $\mathrm{Ha}_{12}$

$15.3 n$

e. + mn

1471

e $\mathrm{Mol}^{\mathrm{H}+}$

1744

e+ $\mathrm{Ho}^{20}$.

1744

- 1 Moti.

1658

- I No 20

1744

e+N

$$
\begin{aligned}
& 13 \text { 31 } 67 \\
& 195 \quad 368 \quad 480 \\
& 95470 y \quad 184 t \\
& \begin{array}{llr}
1363 & 1911 & 1973
\end{array} \\
& 325 \quad 662 \quad 2585 \\
& 551 \\
& 1484
\end{aligned}
$$$$
\text { e. }+\mathrm{N}_{20}
$$$$
\text { e }+\mathrm{Na}
$$$$
\text { - Na Seq }
$$$$
\text { e }+\mathrm{NaCl}
$$$$
\text { e }+\mathrm{Ne}
$$ 


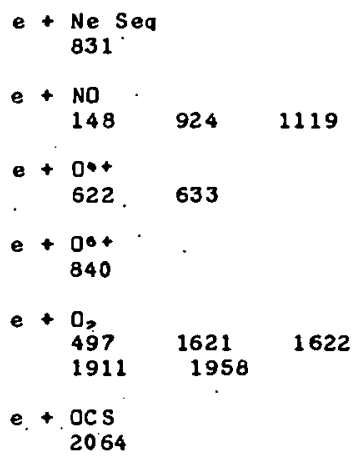

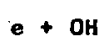

OH
636

- +86

$29 \quad 184 \quad 862$

e+ $5 F_{0}$

e + Sm 1651

$\mathrm{SO}_{2}$
$1555 \quad .2064$ e
Sr+
1650

$2 n 19 \circ$
1658

Review

$6455: 2196$

Under

.. 1100

2267

2327

\section{$1726 \quad 1768$ \\ $\begin{array}{ll}1726 & 1768 \\ 2270 . & 2272\end{array}$}

e $\mathrm{HgBr}$

730

- $\mathrm{HgCl}$

. 730

e $\mathrm{HgI}$

730

e+ $I_{2}$

$+\underset{573}{K r_{p}}$

- $+\mathrm{N}^{2}$

$1951365 \quad 1554$

- $+\mathrm{N}_{2} \mathrm{O}$

$702 \quad 720$

$e+\mathrm{NH}_{82 \mathrm{~B}}+$

- + NO

1664

$\mathrm{O}_{2}$
497

$+S_{1563}$

Revi ew

Under 
EOS

ELECTRON-PARTICLE INTERACTIONS

Iontzation

e+ $\mathrm{TN}_{24}+0,1$

2452

e + Ag

1738

e Ar

\begin{tabular}{llc} 
Ar & \multicolumn{2}{c}{468} \\
486 & 1456 & 1457 \\
1469 & 1567 & 1738
\end{tabular}

e
$27 r^{+}$
270

e $+\mathrm{Au}$

1730

$\mathrm{e}+\mathrm{B}^{3+}$

218

e $+\mathrm{Ba}$

1738

$0+B 1$

1738

$e+C$
1550

$2+\mathrm{C} *$

$204 \quad 351$

$e+\mathrm{C}^{2}+$

$204 \quad 1487 \quad 1601$

e+C $\mathrm{C}^{3+}$

$1487 \quad 1789$

$e+C+$
218

e+Cs+

318

$e+\mathrm{Ca}_{\mathrm{Cl}}$

$e+C d$

$e+\frac{C D}{1000}$

$\mathrm{e}+\mathrm{CH}_{\text {. }}$

1608

$++4 ?$

$e+\begin{array}{lll}\mathrm{co} & & \\ 178 & 195 & 894\end{array}$

2082

$0+\mathrm{co}$,

2013

$\mathrm{e}+\mathrm{Cr}$

$1308 \quad 1309$

$++\mathrm{Cs}_{184}$

$e+D_{3}$

1731

$e+\begin{array}{r}D, 0 \\ 681\end{array}$

$\mathrm{e}+\mathrm{Fe}$

$1308 \quad 1309 \quad 1744$

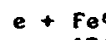

$1744 \quad 2154$

$+N_{1550}^{N}$

$+\mathrm{Fe}^{94}$

2154

e $+\mathrm{Fe}^{10+}$

2154

et Fe16+

1744

e $+\mathrm{Fe}^{22+}$

1744

e $+\mathrm{H}$

$\begin{array}{lll}230 & 1514 & 1787\end{array}$

$\begin{array}{ccc}e+\mathrm{H}_{2} & & \\ 43 & 158 & 178 \\ 205 & 2731 & \end{array}$

$\mathrm{e}+\mathrm{H}_{7}+$
158

$\mathrm{e}+\mathrm{H}>\mathrm{O}$

$1970 \quad 1981$

e $+\mathrm{H}_{2} \mathrm{~S}$

ROS

e+He

$34 \quad 40$ AS

$\begin{array}{lll}94 & 219 & 237\end{array}$

$\begin{array}{lcc}323 & 976 & 1549 \\ 1550 & 1606 & 1731\end{array}$

2288

e $+\mathrm{He}^{+}$

$230 \quad 1619 \quad 2365$

e+He+* Seq

e + He Seq

93

$\mathrm{e}+\mathrm{Hg}$

a $+\mathrm{He}$

1738

e $+K$

$184 \quad 261$

- $+K r$

$\begin{array}{lll}468 & 1456 \quad 1578\end{array}$

2288

$e+\underset{b J}{L i}$

$e+\operatorname{Li}_{631} \operatorname{Sea} 632$

e+112+

1314

$e+\mathrm{Mg}$

$194 \quad 1553$

e + Molt+

1744

o + Mo24

1744

e+ $\mathrm{Ho}^{30+}$

2155

e + Mo 314

e+ Mo $32+$

1744 e $+N^{+}$

351

e+N2+

351

e+N3+

1487

e $+\mathrm{N} \bullet+$

$\begin{array}{lll}218 & 1487 & 1789\end{array}$

$e+N$

$\begin{array}{llc}43 & 178 & 195 \\ 480 & 1731 & 1911\end{array}$

$\mathrm{N}, 0$
702

e Na Seq

551

e+Ne

$\begin{array}{llll}33 & 34 & 43 & \\ 468 & 1008 & 1110\end{array}$

1731 ट2К

e $+\mathrm{Ne}^{6+}$

218

e $+\mathrm{Ne}^{\mathrm{T}}+$

218

e $+N 1$

1738

e + NO

e. +4

$1550 \quad 1614$

$\mathrm{e}+\mathrm{C}+$

$204 \quad 351$

$+42+$
204
351

e+C+

e+05+

ith

e +92

191

$e+\underset{339}{\text { PERT }} 646$

$\mathrm{Rb}$
184

$e+F b$ *

555

$+\quad \mathrm{CF}_{153}$

e $+\mathrm{U}$

1738

e $+x_{e}$

$\begin{array}{lcc}X e & & \\ 33 & 400 & 1+56 \\ 1936 & 2288\end{array}$

e $+\mathrm{Y}$

1738

Roviet
280

Unde $f$ 1テCE 1736 
$E 06$

ELECTAON-PARTICLE INTERACTIONS

Recombination (alectron-ion)

e Art

819

e $+\mathbf{r r}^{2+}$

819

e Ar3+
819

C $+A r+t$

819

e+Ar10+

58

e Ar12.

58

$e+\operatorname{Ar}_{58}^{124}$

e $+\mathrm{Ar}^{134}$

58

e+ $\underset{58}{\operatorname{Ar} 104}$

e Arist

58

e Ariot
58

C+Ar2t

58

$A+\mathrm{Ar}^{+}$
47

e $+\mathrm{C}^{+}$

e+C2+

$\mathrm{e}+\mathrm{C}^{3}+$

e $+\mathrm{C}+$

819

$\mathrm{CH}^{+}$
848

- + $\begin{array}{r}D_{2}+ \\ 1374\end{array}$

$++\underset{366}{+t}$

e+F2+

$a+\mathrm{Fe}^{\circ+}$
1722

$1722 \quad 2154$

e $+\mathrm{Fe}^{\circ+}$

$1722 \quad 2154$

e Fe104
1722

$1722 \quad 2154$

$9+$ Fol t:

1722

$e+F a 12+$
1722

e Felat

1722

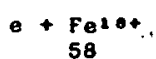

e $+\mathrm{Fe}^{194}$

58

$+\mathrm{Fe}^{20+}$
58

58

e+Fe21+

58

$-F e^{22+}$
58

e+Fe23*

58

$\begin{aligned} &+ \mathrm{Fe}^{2+4} \\ & 58\end{aligned}$

e $+\mathrm{Fe}^{25}$

58

$\mathrm{H}+\mathrm{H}_{2}^{+}$
$\mathrm{I374}$

e+ $\mathrm{H}_{3}+$

1594

2318

e+ $+\mathrm{H}_{54}^{+}$

e $+\mathrm{He}^{+}$

2369

e+He Sog

$631^{632} 831$

$+K_{r_{2}}$
573

$++M_{819}$

e $+\mathrm{Mg}^{2+}$

819

$\mathrm{C}$
$\mathrm{Bg}$
$\mathrm{Mg}^{3+}$

e $+\mathrm{Mg}^{\circ}$ t

819

e $+H^{30+}$

2155

e+ $\mathrm{Ho}^{3} 1$ +

2155

e+Mo3nt

58

e + Hosst

58

e $+M_{0}^{36+}$

58

- + Mopro $^{\circ}$

58

e $+\mathrm{Mo}^{3} \mathrm{C4}$

58

C $+\mathrm{Mo}^{30}$

58

- + Mo $*$ ot

58

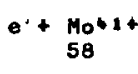

a + N+

819

- $+\underset{32+}{\mathrm{N} 2+}$

819

e+N3+

819

e+ $\mathrm{N*}$
- $+\mathrm{Na}^{2+}$

$366^{\circ}$

e+ $\mathrm{Na}^{3}+$

366

e+ Ne*

819

- Ne Seo

831

$+\mathrm{Ne}^{2+}$
366

819

e $+\mathrm{Ne}^{3+}$

$366 \quad 819$

- Ne*t

819

e $+\mathrm{NH}_{+}+$

e $+\mathrm{NO}$

109

c +0 .

819

e $+02+$

366

819

e+ $0^{3}+$

$366 \quad 819$

$0+0$
819

$++\frac{07+}{840}$

e + PERT

646

649

e +5

e+s2t

819

e 30
819

e $+9 *$

819

e+ $51+$

a $+512+$

819

e $513+$
819

e $+\mathrm{St} *$

$61 \mathrm{~S}$

4004
58

e $+467+$

58

e+1460+ 58

e +460 .

58

e $470+$

58

a + Ho 1 .

e+4724 58

et w13. 
E07

ELECTRON-PARTICLE INTERACTIONS

Coll Istonal, De-Excitation

$$
\begin{aligned}
e+ & \underset{2498}{2498}+N=1 \\
e+ & \underset{20,-}{[0,08}+0_{2} * 1
\end{aligned}
$$

EOB

ELECTRON-PART ICLE INTERACTIONS

Collisional LIne Broadening

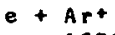

$1638 \quad 1697$

E09

ELECTRON-PARTICLE I NTERACTIONS

Negat I ve I on fornation

$$
\begin{aligned}
& e+\mathrm{COO}_{+} \mathrm{CO} \\
& 2.3 .34 \\
& e^{\cdot}+\mathrm{CCO}_{2}+\mathrm{CO}, 1 \\
& 2334 \\
& e+\underset{662}{[F}+N=1 \\
& e+\underset{2334}{\mathrm{I}_{2}}+\mathrm{N}, 1 \\
& e+[N, D+C O,\} \\
& 2088 \\
& e+\Gamma_{13 \overline{3} 8}^{0}+N_{2} ! \\
& \text { - } \prod_{2334}^{n_{j}}+n_{e} 1
\end{aligned}
$$

E10

ELECTEON-PARTICLE INTERACTIONS

Spln Exchange

$$
\begin{aligned}
& \mathrm{e}+\mathrm{H} \\
& 1507
\end{aligned}
$$

$$
\begin{aligned}
& \text { e }+\left[\mathrm{O}^{-}+\mathrm{N}_{2} *\right] \\
& \left.e+\underset{2498}{[0-}+\mathrm{O}_{2} *\right] \\
& \text { e+ Art } \\
& \text { e }+\mathrm{Ba} \\
& 2168 \\
& \text { e+C } \mathrm{C}^{+*} \\
& 622
\end{aligned}
$$

- $+\mathrm{Ba}+$

1925

$$
\begin{aligned}
& ++\mathrm{CO}_{1473} \\
& \begin{array}{r}
\text { e He* } \\
168
\end{array} \\
& \text { e t He Seq } \\
& 631 \quad 632 \\
& \text { e+ 0*+ } \\
& 622 \\
& \text { e + PERT } \\
& 649
\end{aligned}
$$

Undet

$$
\begin{aligned}
& \begin{array}{l}
\mathrm{Br}= \\
541
\end{array} \quad 1102 \\
& \begin{array}{r}
e+C_{1} \\
1102
\end{array} \\
& 1609 \\
& \mathrm{e}+\mathrm{CO}_{2} \\
& \text { e+ } \mathrm{D}_{3} \\
& 2186 \\
& 2445 \\
& e+D_{2} u \\
& \text { e+ F⿻. } \\
& 964 \quad 1102 \\
& ++\mathrm{H}_{2186} \\
& 2187 \\
& 2445 \\
& \text { e }+\mathrm{H}_{3} \mathrm{O} \\
& 681 \quad 1976 \quad 1984 \\
& \text { e+ } \begin{array}{r}
\mathrm{HBr} \\
\mathrm{E} 41
\end{array}
\end{aligned}
$$$$
\text { e }+\mathrm{HCl}
$$$$
1344
$$$$
\text { e + HD }
$$$$
2445
$$$$
\begin{array}{r}
\text { e I, } \\
1102
\end{array}
$$$$
1534
$$$$
\begin{array}{r}
e+N_{2} \\
1554
\end{array}
$$$$
+\mathrm{N}_{2} \mathrm{U}
$$$$
1000
$$$$
1983
$$$$
\text { e + NO }
$$$$
1664
$$$$
\text { e+sfa }
$$$$
1021
$$$$
\begin{array}{r}
\mathrm{e}+\mathrm{SO}_{2} \\
1555
\end{array}
$$$$
\text { Review }
$$$$
2196
$$

$$
\begin{array}{r}
\mathrm{e}+\mathrm{H}_{2} \\
1828
\end{array}
$$

e $+\frac{11}{1792}$ 
E11

ELECTRON-PARTICLE INTERACTIONS

Free-free Transitions (Breasstrahlung)

$$
\text { e+Al }
$$
2432

E12

ELECTROH-PARTICLE INTERACTIONS

Positron Collistons

$$
\begin{aligned}
& e^{+}+\underset{1509}{\underset{H e}{H e}+A r l} \\
& e^{+}+\underset{1520}{A r} 2353
\end{aligned}
$$

E13

\section{ELECTRON-PARTICLE INTERACTIONS}

Electron Detacheent from Negatlve Ions

$$
e+15
$$

E15

ELECTRON-PARTICLE INTERACTIONS

Inner-Shell Interactions

$$
\text { e+ } \mathrm{H}_{2 \mathrm{~S}}
$$

$$
\begin{array}{rl}
e+ & \text { Ar } \\
857 & 1493 \\
e+ & C \\
98 \\
e+ \\
+ \\
\\
2432 \\
e+\frac{N}{1657}
\end{array}
$$

$$
\begin{aligned}
& \hline+ \text { Ne } \\
& e 57 \\
& \text { P PERT } \\
& 649 \\
& \text { UndeI } \\
& 1175 \\
& 2325
\end{aligned}
$$

$$
\begin{aligned}
& e^{+}+\underset{460}{H} \quad 1515 \quad 1520 \\
& \begin{array}{lll}
460 & 1515 & 1520 \\
1523 & 1539 & 1605
\end{array} \\
& \begin{array}{lll}
1732 & 1733 & 1799
\end{array}
\end{aligned}
$$

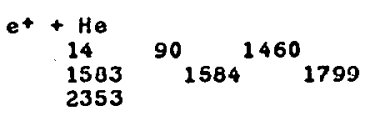

$$
\begin{aligned}
& e++\frac{N_{2}}{1769}
\end{aligned}
$$

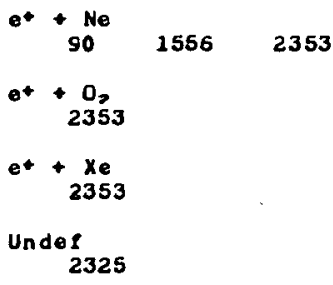


E16

ELECTRON-PARTICLE INTERACTIONS

Fluorescence and Lual nescence

$e+[\mathrm{Ar}+\mathrm{Hg}+\mathrm{BrCCl}$, 526

$\mathrm{e}+\left[\mathrm{Ar}+\mathrm{Hg}+\mathrm{Cl}_{7}\right]$ 526
C $+\left[\mathrm{Ar}+\mathrm{Xe}_{0}+\mathrm{F}_{2}\right]$ 1084

o+ $\mathrm{Cl}_{1067}+\mathrm{ArI}$

$+\underset{1067}{[C l}=\left(X_{e}\right]$

$e+\left[F_{2}+A r\right]$

$+\left[F_{2}+K \mathbf{r}\right]$

1067

$-\leftarrow \underset{524}{\mathrm{CHg}_{4}}+\mathrm{Cl}+\mathrm{Xe}_{\bullet}+\mathrm{Arl}$
e+[Kr+F, $]$

517

$e+\left[\mathrm{Ne}+\mathrm{Xe}+\mathrm{NF}_{3}\right]$ 540

e 4 Ar 385

e+ Hz,

e $+\mathrm{XeCl}$ 1938
E17

ELECTAON-PARTICLE INTERACTIONS

Angular scattering (speciried process)

$\therefore$

$+\underset{2489}{+ \text { Ag }}$

e $+A \mathfrak{i}$

2285

- Ar

$\begin{array}{lll}486 & 1456 \quad 1457\end{array}$

$1469 \quad 1520$

e+Ba $1494 \quad \therefore$

e $+\mathrm{C}$

2285

$e+\mathrm{CO}$

$\therefore \div \quad 388 \quad 2107$

$\mathrm{e}+\mathrm{CO}_{48}$
489

$e+C=F$

1708

e $+\mathrm{Cu}$

2285
- $+\mathrm{H}$ $\begin{array}{llc}384 & 353 & 1520 \\ 1539 & 1575 & 1787\end{array}$ $1801 \quad 1822 \cdot 2140$

$e+\frac{4 *}{260}$

e+ $\mathrm{H}_{2}$ 368
1818

1574.1784

- $+\mathrm{H}_{2} \mathrm{~S}$ 1637

e $+\mathrm{HBr}$ 1456

$\therefore+$ He

$\begin{array}{lll}69 & 219 & 237\end{array}$

$323 \quad 488 \quad 1495$

14961549.1574

- + He: 1766

- Hẹt. 1546

- $+\mathrm{Hg}_{2358}$ 2358

$+K I$

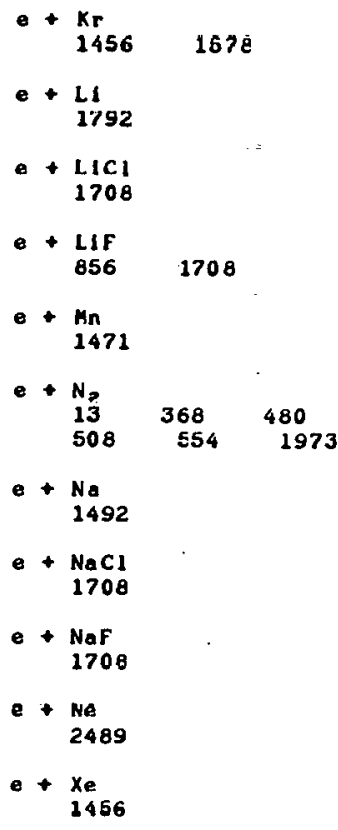

E18

ELECTRON-PARTICLE INTERACTIONS

Attenuation (unspeclified process)

e $+\because 4 u$ 
F01

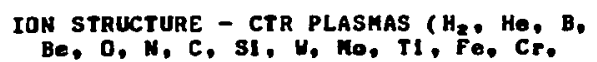
Nb, Au, Cu, Ni, H, HeH*)

Transition Probabilities, Daclliator Strengths, Eneray Levels, Lifet ines

AIO 135

A rCl

ArH 88

Au

$\begin{array}{lll}563 & 1535 & 1541 \\ 1675 & & \end{array}$

B

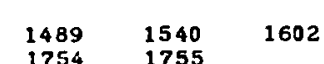

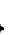

$878 \quad 1524$

B 2+ 1833

B3 +

$\begin{array}{lll}1133 & 1356 & 1641\end{array}$

Bu+

1133

Be

$1524 \quad 1602 \quad 1755$

Be+

1833

Be Sea

$823 \quad 1315 \quad 1488$

$1500 \quad 1652 \quad 2370$

$\mathrm{Be}^{2+} 1356 \quad 1641 \quad 1727$

c

$\begin{array}{lll}1306 & 1489 & 1540\end{array}$

$1602 \quad 1685 \quad 1754$

c*

1134

c seq

1307

c2*

$\begin{array}{lll}628 & 1134 & 1524\end{array}$

c3+

$\begin{array}{lll}502 & 1134 & 1757\end{array}$

$c *+1356$

$\mathrm{C} \times 0 *$

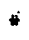

$\begin{array}{lll}C D & 304 & 1579\end{array}$

Co Seq

1652

Cr 1586

Cr10+

2344

Crt+

1277

- Cd 1039 cu

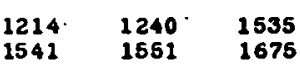

Cuss.

2344

$\mathrm{Cu}^{2}>+$

2344

D* $679 \quad 1117$

F 1489

Fe 1586

F * 1322

$\mathrm{Fe}^{2+}$

$\mathrm{Fe}^{3}$

1319

Fet.

$\mathrm{Fe}^{54+} 643 \quad 827$

ferot.

638

$\mathrm{Fe}^{12+} 638 \quad 2364$

$\mathrm{FOO}_{2344} 2364$

$\mathrm{Fe}^{\mathrm{i3}+}$

1307

$\begin{array}{rr}638 & 2344\end{array}$

Fe10!

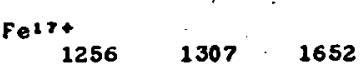

Feto+ $829 \quad 1307 \quad 1652$

Fe $19 *$

$829 \quad 1662$

$\mathrm{Fe} 204$

$829 \quad 1307 \quad 1313$

16521307

ferat

$1307 \quad 1652$

$\mathrm{Fe}^{22+}$

$564 \quad 1129 \quad 1652$

$\mathrm{Fe}^{234} \mathrm{564} \quad 1307 \quad 1652$

$F e^{20 *}$

1307

$\mathrm{Fe}^{25+}$

1307

H

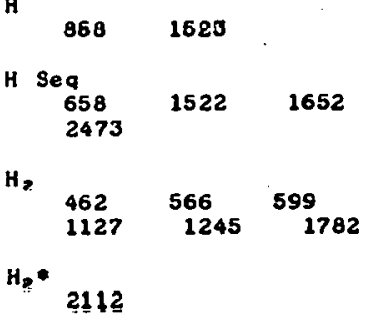

$H_{2} * 1657$

H. 291

tr

$1272 \quad 1320.1642$

$\begin{array}{ll}1272 & 1320 \\ 1698 & 1727\end{array}$

He.

\begin{tabular}{lll}
336 & 346 & \multicolumn{1}{l}{666} \\
698 & 748 & 862 \\
1229 & 1236 & 1356 \\
1641 & 1649 & 1699 \\
1713 & 1716 & 1727 \\
1730 & 1751 & .2631
\end{tabular}

He*

1610

He* 2366

He sea

$\begin{array}{lll}568 & 668 & 1234\end{array}$

$\begin{array}{lll}1486 & 1652 & 2299\end{array}$

$\mathrm{He}_{2}=$

1742

He- $1698 \quad 1823$

HeH 88

$\mathrm{Ha}+$

300

$11 * 1356$

L) 8eq $\begin{array}{lll}356 & 1307 & 1662 \\ 1694 & \end{array}$

LIH

$104 \quad 105$

Ho $\quad 332 \quad 1273 \quad 2626$

Ho**

1316

Mo9+

1318

Mo 10.

1328

Hol2*

1747

Not2*

338

not $2+$

$1247 \quad 1307$

Molst

$1136 \quad 1307$

Hol 1

$1136 \quad 1307$

Mol 7 -

11351307

rio to

11351307

no 20 .

1307

Holos

1307

Hoz:

1307

Ho 2 a

1307 


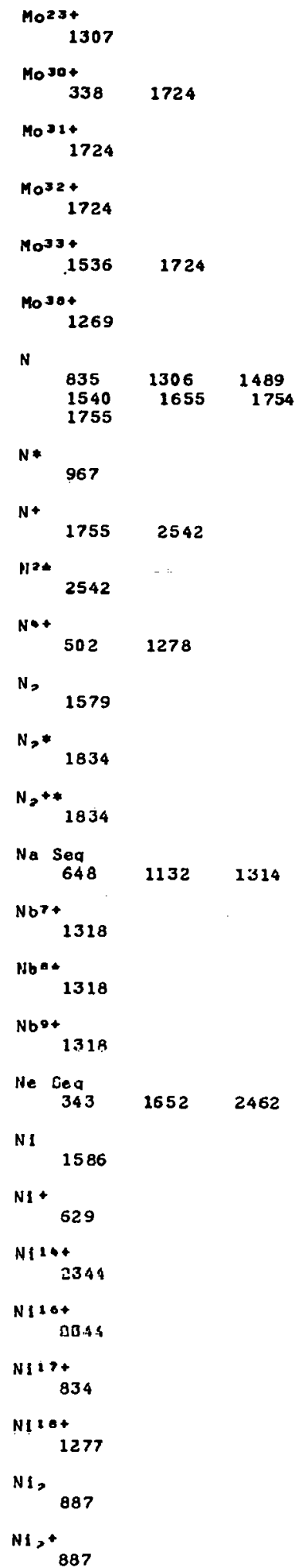

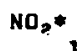

T

$$
\text { T1: }
$$


FO2

ION STRUCTURE - CTR PLASHAS ( $\mathrm{H}_{2}, \mathrm{He}, \mathrm{B}_{\mathrm{O}}$ Be, $0, N, C$, si, $U$, Ho, Ti, Fe, Cr. Nb, Au, Cu, Ni, Hะ*, HeHt)

shirts and Line Broadenting by FIelds

A 1

1669

c*

1690

Cu2 $0^{+}$

1910

$\mathrm{Cu} 1 \mathrm{t+}$

1910

Cu22+

1910 .

$\mathrm{Cu}+\mathrm{J+}$

1910
$\mathrm{D}_{2}$

1117

Ferot 1910

Fein :

1910

$\mathrm{Fe}^{12+}$

1910

Fetse

1910

Fet*

1910

$\mathrm{Fe} \mathrm{st}^{\mathrm{s}}$

1910

$\begin{array}{lll}352 & 842 & 1243\end{array}$

$\begin{array}{lll}1568 & 1686 & 1783 \\ 1812 & 2324 & \end{array}$

H Seq

$1729 \quad 2473$

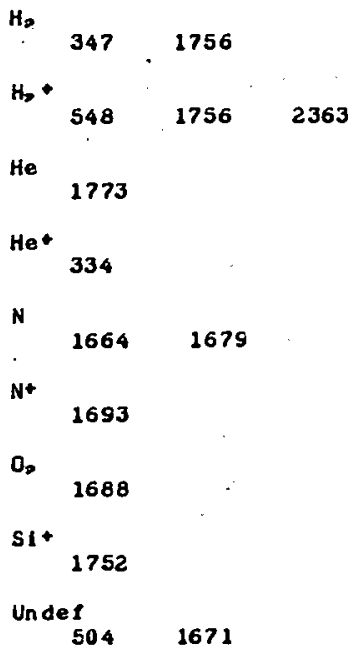

Ho

63

He* 334

1688

Unde 1

$504 \quad 1671$
Fo3

ION STRucture - CTR plasmas ( $\mathrm{H}_{\mathrm{a}}$, He. B. Be, D. N, E, si. W, Ho, TI. Fe, Cr. Nb, Au, Cu, Ni, H*t, Hett)

Electron Afrintey

882

F05

ron structure - ctr plashas ( $\mathrm{H}_{\mathbf{2}}$. He. B,
Be, O, N, C, si, H, Ho, II, fe, Cr.

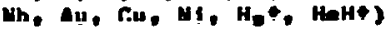

hv + OH
H

1356

C

882

882

882
882

0 
602

TRAH8PORT PHENONEHA AND AVERAOE phopentIEs IN GAsEs

Diffusion of Neutrels

$$
\begin{aligned}
& A r+K r \\
& 417 \\
& \text { Ar }+\frac{x}{314} \\
& \text { Are Ar } \\
& \text { CI }+A r
\end{aligned}
$$$$
\operatorname{AO}_{1045}
$$

603

TRAMSPORT PHENOCE MA AND AVERAOE PAOPERTIES IH GASES

Di reuaton of Elcetrans

$$
\text { - Ar }
$$

604

\section{TRAMSPORT PHENORENA AHD AVERAGE} PROPER IEs In oAses

Diffueton of lone

$$
\begin{aligned}
& C_{2} 0,+1664 . C O \\
& C=+\underset{1037}{A r} \\
& C=+H_{1070}
\end{aligned}
$$

$\cos$

TRAMSPORT PHENONENA AHB AUERAeE PROPERTIES IN GASES

Drift Veloetty of Electrone

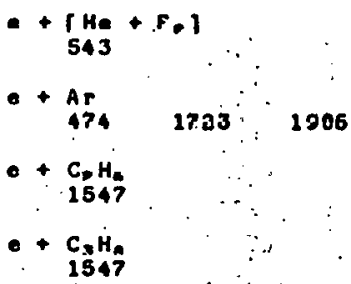

$H \cdot \circ \mathrm{CO}^{\circ}$

983
He + H०
845

He $+x_{0}$

$K r+K r$

326

$\mathrm{kr}: \mathrm{x}_{0}$

324

Kro his

1073

Me + Ar

47

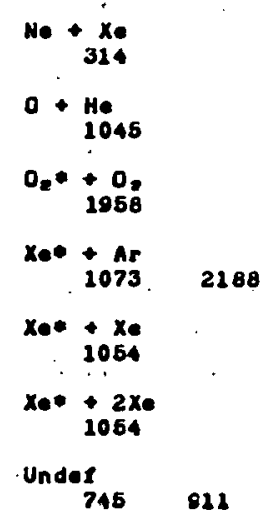

$-+\cos _{10}$

- $+\mathrm{CH}_{0}$

1013
$H_{-}^{*}+N_{*}$

$\mathrm{N}_{*} \bullet+\mathrm{N}_{*}$

$0_{2}+0_{*}$

1664

$R b+* 4$

$R b+* X e$

2062

Revien $\because \mathrm{C}_{46} \mathrm{H}_{90}$

- $+\mathrm{CiH}_{\mathrm{i}}$

1547

$++\frac{\mathrm{Cr}_{\mathbf{1}} \mathrm{CH}_{4}}{1647}$

$-+\mathrm{CD}_{10}$

- $\mathrm{CH}_{\mathrm{i}}$

1013 1647. 1008

- 60

894

$-+60$.
- $+\mathrm{H}_{2723}$

$-+\mathrm{He}$

$543 \quad 1723 \quad 2161$

-

EFE

- $+\mathrm{Ne}_{2}$

1723

- $\begin{aligned} & \text { Ne } \\ & \text { 574 }\end{aligned}$

676

- +1

$674 \quad 675$

$x_{e}$
.946 
606

TRANSPORT PHENOMENA AND AVERAGE PROPERTIES IN GASES

Drift Veloclty of Ions

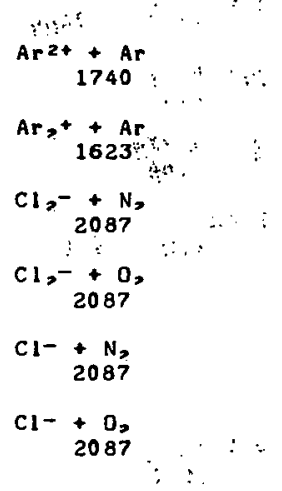

G07

TRANSPORT PHENOMENA AND AVERAGE PROPERTIES IN GASES

Seattering and Eneray Los: Paraneters of Electrons; Neutrals and Ions in Gases

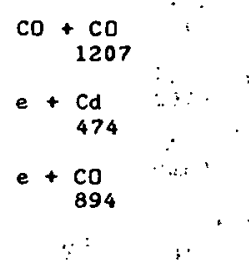

GOB

TRANSPORT PHENOMENA AND AVERAGE PROPERTIES IN GASES

\section{Energy Distribution (energy}

distribution of lons and electrons with applied electric and nagnetic (ields)

$$
e+\underset{897}{\{A l}+N>?
$$
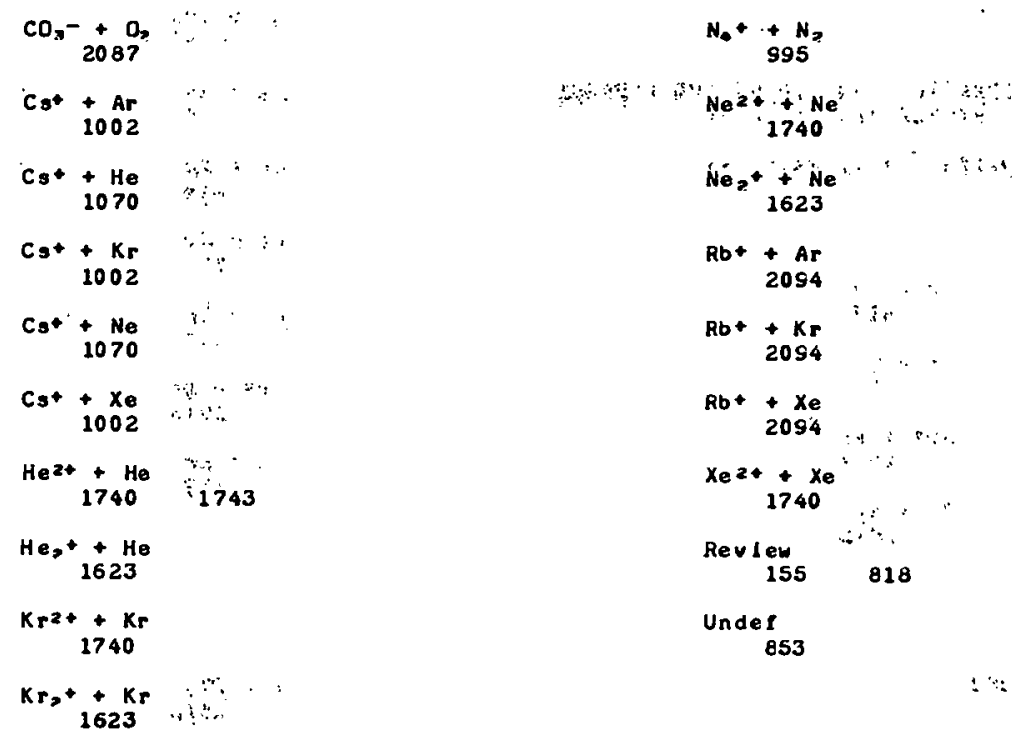

$$
\begin{aligned}
& \text { e }+\mathrm{H}_{2355} \\
& \text { e+N, } \\
& 1355 \\
& \text { e+Ne* } \because \therefore: \cdots \\
& 2494 \\
& \mathbf{H g}+\mathbf{N}_{\mathbf{2}} \quad \because \quad \cdots
\end{aligned}
$$

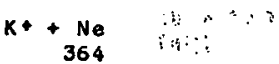

$$
\begin{aligned}
& \mathrm{LI}+\underset{2058}{\mathrm{H}_{3}} \therefore \\
& \mathrm{LI+}+\mathrm{Ne} \\
& N_{2}+\underset{1355}{H_{2}}
\end{aligned}
$$

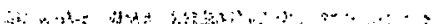

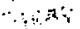

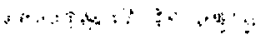

$\mathrm{N}_{2}+\mathrm{Ne}$ 1355

$\mathrm{Na} *+\mathrm{Ar}^{+}:$ 1203

$\mathrm{Na} *+\mathrm{He}$ 1203

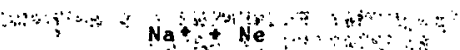
364

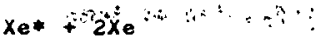
1263

$\mathrm{Xe}_{2} *+\mathrm{Xe}$ 1263

Undef

$$
\begin{aligned}
& 316 \because \because-4 y \\
& \therefore ; \quad:
\end{aligned}
$$

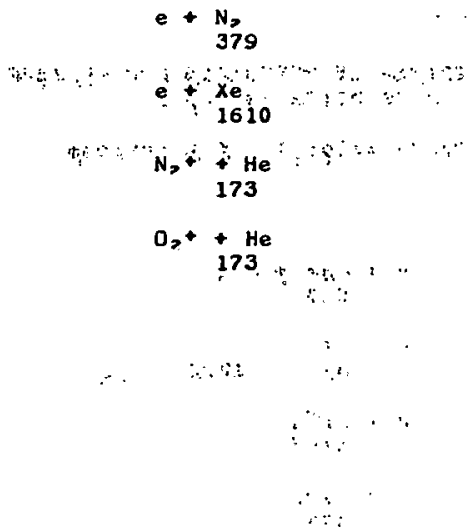


009

TRANSPORT PHENOMENA AND AUERAGE PROPERTIES IN GASES

Nomentue Transfer

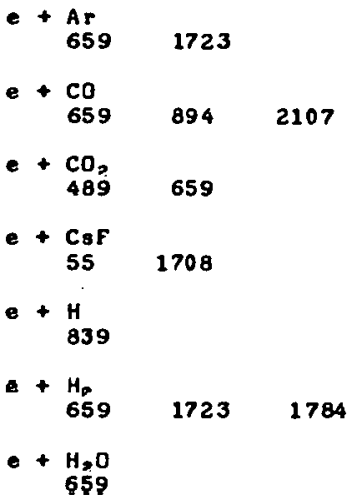

610

TRANSPORT PHENORENA AND AVERAGE PROPERTIES IN GASES

First and Second Townsend Coefficlents $-\div\left[\mathrm{CO}_{2}+\mathrm{N}_{2}+\mathrm{He}\right]$ $-469$

G11

TRANSPOAT PHENOMENA AND AVERAGE PROPERTIES IN GASES

Filertmn Attachmont roperfig fiente

$$
\begin{array}{r}
\text { e A1r } \\
1387
\end{array}
$$

HO1

PHOTON COLLISIONS UITH HEAVY PARTICLES, ELECTRONS AND PHOTONS IN GASES (ho $<$ $100 \cdot \mathrm{keV}$ )

General

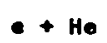

- $+K I$ 1708

- 4 LI

659

- LICI 1708

$-+\operatorname{LIF}$ $65 \quad 1708$

- $+\mathrm{N}_{\mathrm{2}}$ $\begin{array}{ll}N_{2} & 53 \\ 1723 & 2973^{480}\end{array}$

- $\mathrm{Na}$ 575

- $\mathrm{NaCl}$ 1708
- NaF

1708

$+N e$
659

$-+0$

- +1

675

$H_{D}+A r$

125

$H_{2}+\begin{array}{r}\mathrm{He} \\ 125\end{array}$

$\mathrm{He}^{2+}+\mathrm{He}^{\circ}$ 1743

Revilew 55

Undes S11

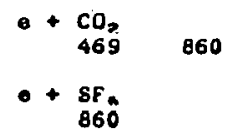

D. $\quad \mathrm{CC}_{1387}$

$\mathrm{O}$, + He

1387

0, + Ny $-\begin{aligned} & \mathrm{Rr} \\ & 860\end{aligned}$

$-\mathrm{Cl}_{2}$
860

$-+\frac{60}{660}$

- $+3 F_{80}$ 
PHOTON COLLISIONS UITH HEAVY PARTICLES, ELECTROHS AND PHOTONS IN GA8ES the 100 keV)

Total Absorptlon

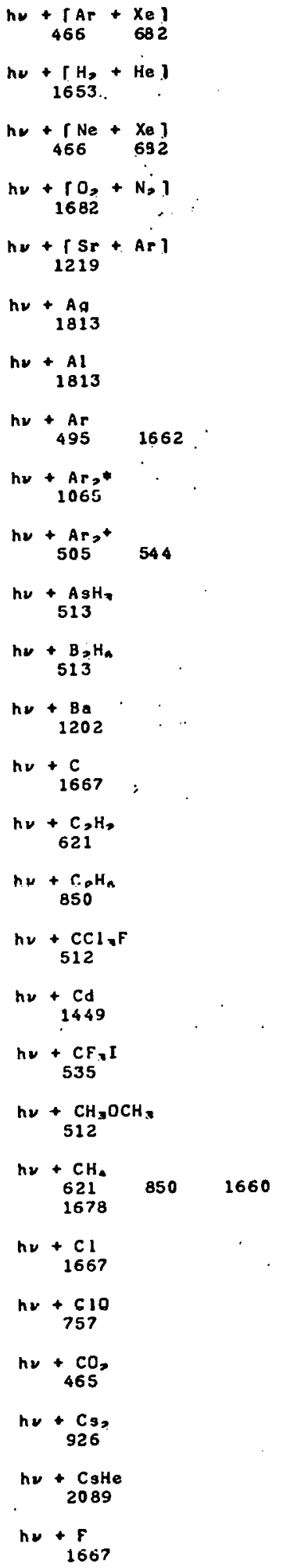

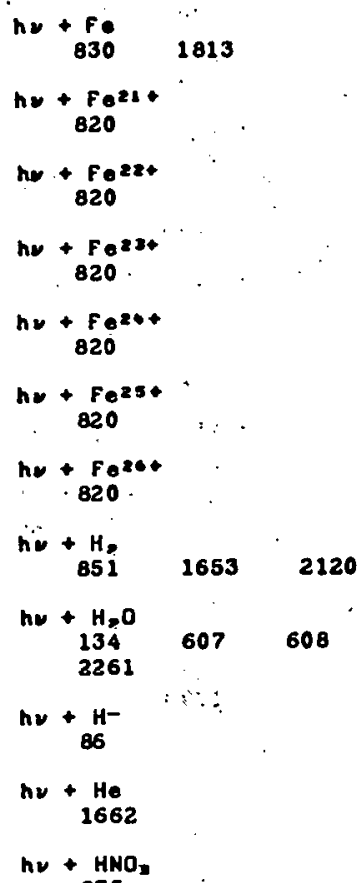

$h=+F$ $B 20$

ne $+\mathrm{Fe}^{22+}$ 820

he $+F^{2 x}$ 820

he $+\mathrm{Fezt}^{2+}$ 820

$\mathrm{hl}+\mathrm{Fe}^{25 *}$ 820

he $+\mathrm{Fe}^{20+}$ 820

$\ddot{n i v}+\mathrm{H}_{8}$ $85 i \quad 1653 \quad 2120$

hv $+\mathrm{H}=\mathrm{O}$ $\begin{array}{lll}134 & 607 & 608\end{array}$

2261

$h v+\underset{86}{H}$

nv + He

1662

hv + HNO 275

hv $+\mathrm{Kr}$

$h v+K r *+A r$

91

hv + Krt + He

hv + Krsto 1065

$h v+\underset{505}{K r} \rightarrow$

hy + LaO

2099

he + L!

2173

hv $+M n$

So 6

hv + Mo

1813

$\mathrm{he}+\mathrm{N}$

2667

hv $+\mathrm{N}$

$241 \quad 465 \quad 495$

$784 \quad 1667$

$h v+\underset{241}{N}>0$

$h y+{ }_{275}, O_{5}$

he $+\mathrm{Na}$

$620 \quad 1238 \quad 2283$

he + Ne

1662

ne + Neg:

1065

hv + Nes*

544

ho + NO

$241606 \quad 2667$ ho $+\mathrm{NO}_{2}$

275

$167 ?$

hv $+\underset{275}{\mathrm{NO}_{3}}$

he +0

.1667

he $+\underset{24}{0}$

241

566

1654

hv $+\underset{613}{\mathrm{PH}_{2}}$

hw + Pt

1813

he + $\mathrm{Ab} ;$

hv $+\mathrm{seO}$

2099

$h v+S F_{0}$

1604

hy $+51+$

he $+51^{2}$

652

ho $+513+$ 652

hv $\frac{51^{-}}{2365}$

hv + SiH 513

hv $+\mathrm{SO}_{\text {}}$

609

1661

$h \nu+S_{1838}^{S r}$

he + Th

2192

nu $+u$

2192

hu +4

1813

hy $+x e$

1662

$h_{1}+\mathrm{Xe}_{2} *$ 1065

he $+X_{e}$ ? $505 \quad 544$

hv + YO

nhe + Sf . 1224 1233

$1225 \quad 1226$

$2 h v+H$

Lni +80 1486

Reviow

Unde? $159 \quad 898 \quad 2438$ 
H03

PHOTON COLLISIONS UITH hEAUY PARTICLES, ELECTRONS AND PHOTONS IH GABES (he < 100 keV)

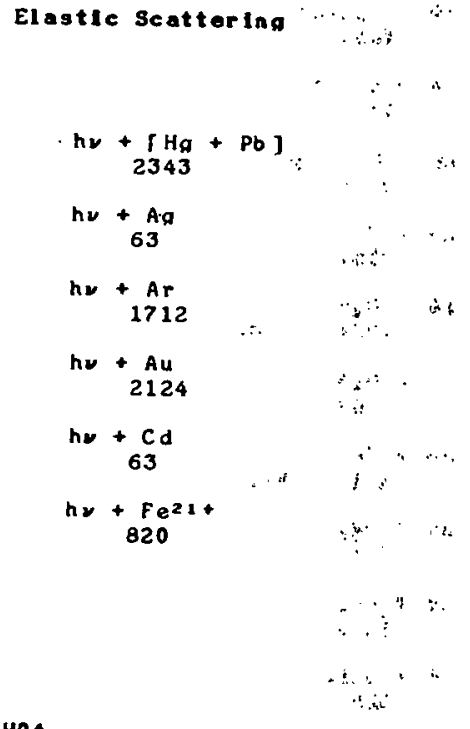

HO4

PHOTON COLLISINANS UITH HEAVY PARTICLES, ELECTRONS AND PHOTUNS IN GASES (ho < $100 \mathrm{keV}$ )

Encitation
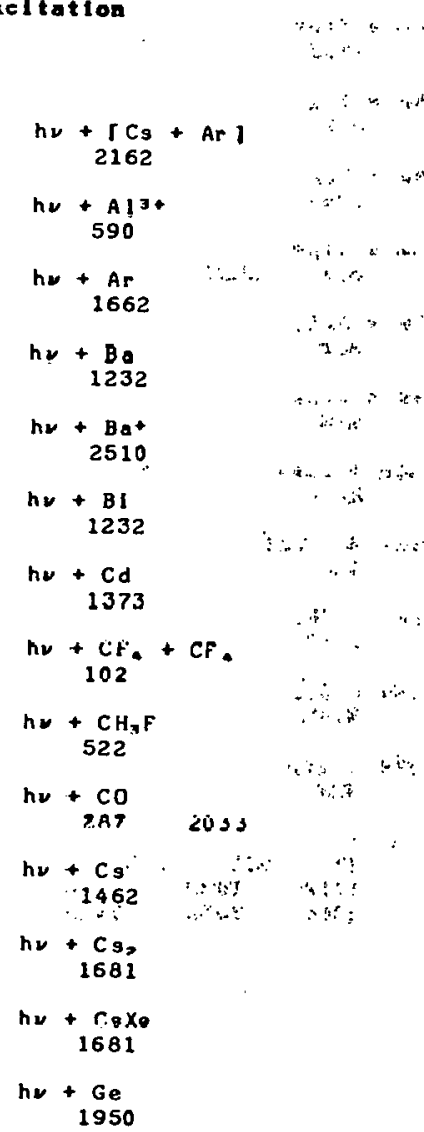

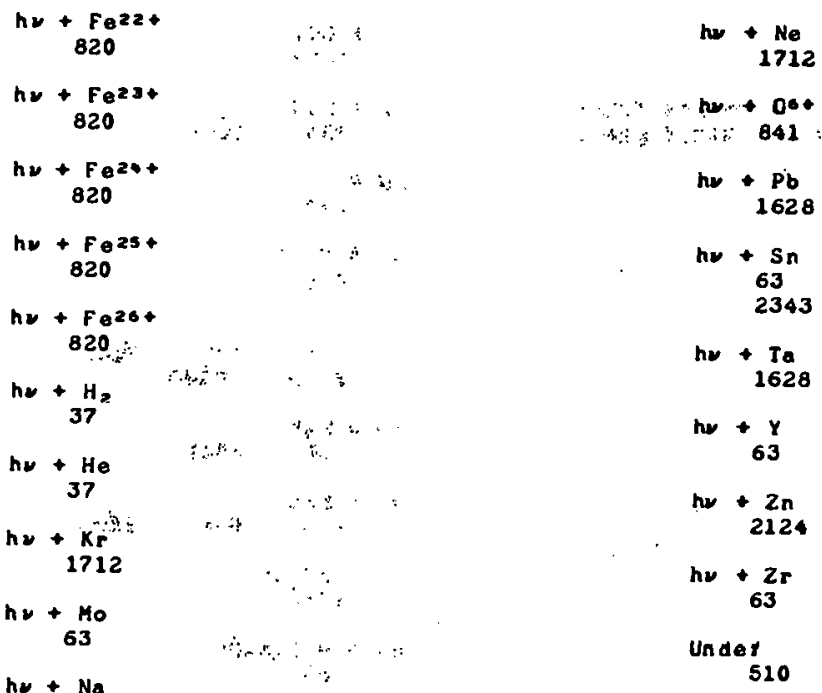


ho $+\dot{c} \dot{c}_{\mathbf{s}}$

1283

PhOtON COLLI8IONB UITH hEAV pam ICLES, ELECTRONS AND PHOTONE IN GASEs the < 100 keV)

Diseoclation

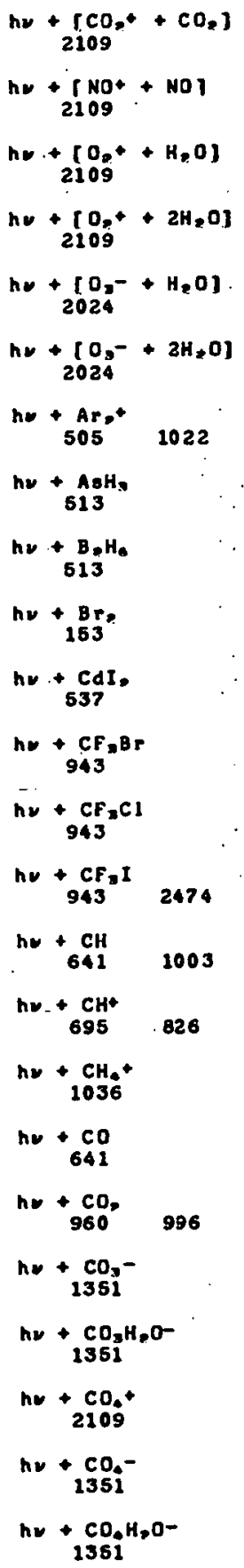

hv + DC

$436 \quad 1009$

ne $+\dot{H}_{60}$

ne $\mathrm{H}_{\mathrm{o}} \mathrm{CO}$

. . 641

$h \in+\underset{134}{\mathrm{H}_{2} \mathrm{O}} \cdot{ }_{2076}^{187} 2346: 2086$

ho $+\underset{130}{H} 0^{\circ} 2011$

hy Hen

KCN
436

he + $\mathrm{HCO}_{-}-$ 1351

hy $+\mathrm{MCO}_{2} \mathrm{H}_{2} \mathrm{O}$ 1351

Hu + KD 1169

ho + HeH* $826 \quad 1671$

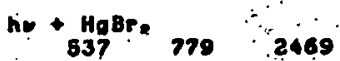

hy $\therefore \mathrm{HoCl}$. 770

hy $+\mathrm{Hal}^{\mathrm{S}}$ 779.2060

he $\leftarrow \mathrm{HN}_{3}$ 141

he + Höcl 954

ho + Iake

hv + I Br .163736

he $+\mathrm{ICN}$

$+760^{\circ} .996$

hv $\operatorname{InI}_{857}$

he $\mathrm{K}_{\mathbf{a}}$

1253

he $+\underset{606}{K r} * 084 \quad .1002$

nv $+\underset{1365}{N_{8}}$

ne $: \mathrm{N}_{2169}$

ho ¿ No.

1253

2280

hv $\div$ NHs

$\mathrm{hv}+\mathrm{NO}_{\mathrm{g}} \mathrm{H}_{2} \mathrm{O}$

1351

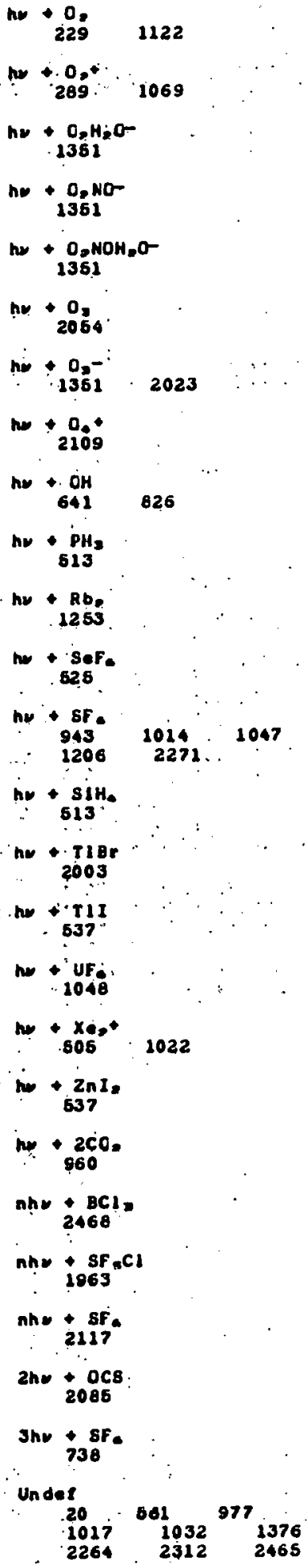




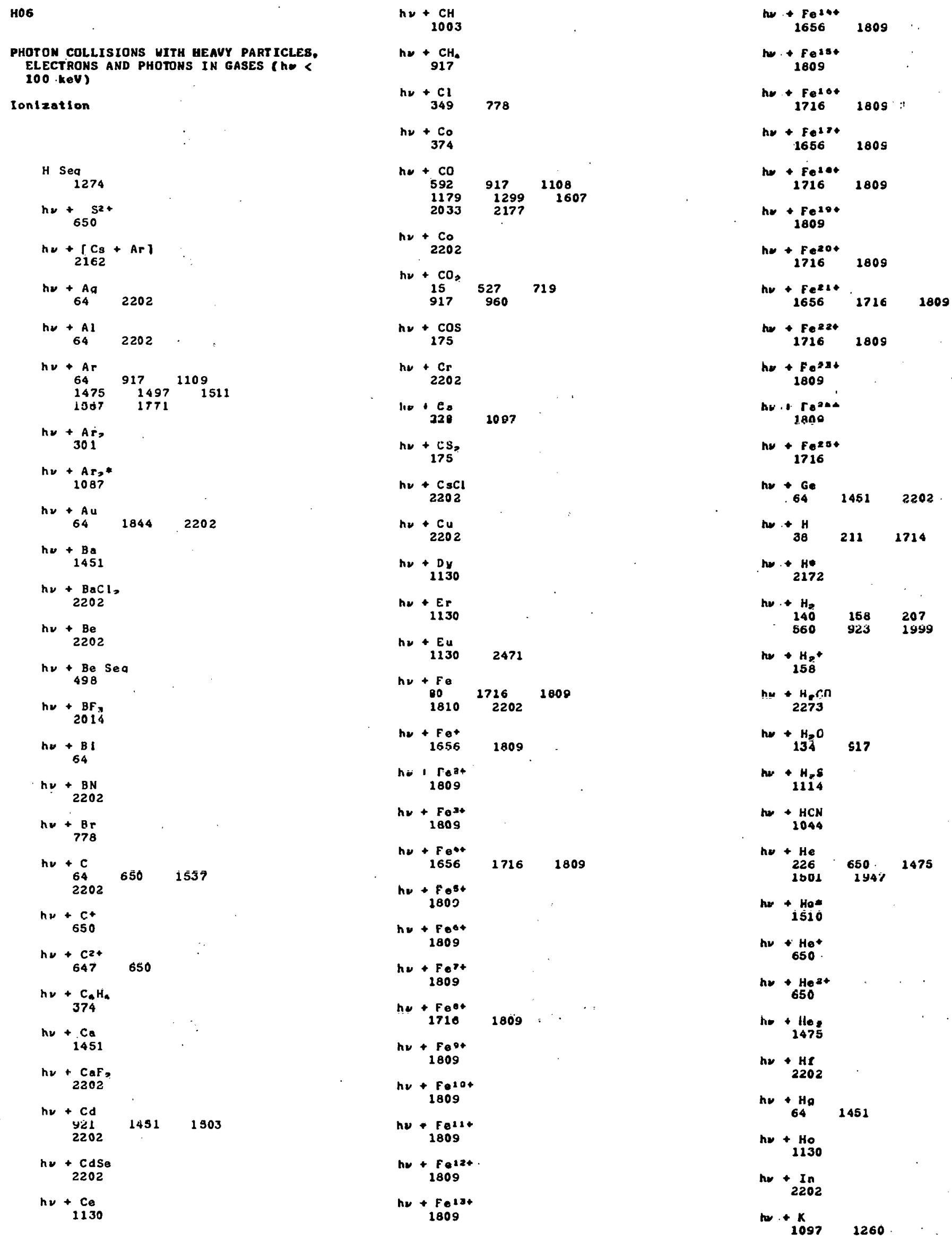




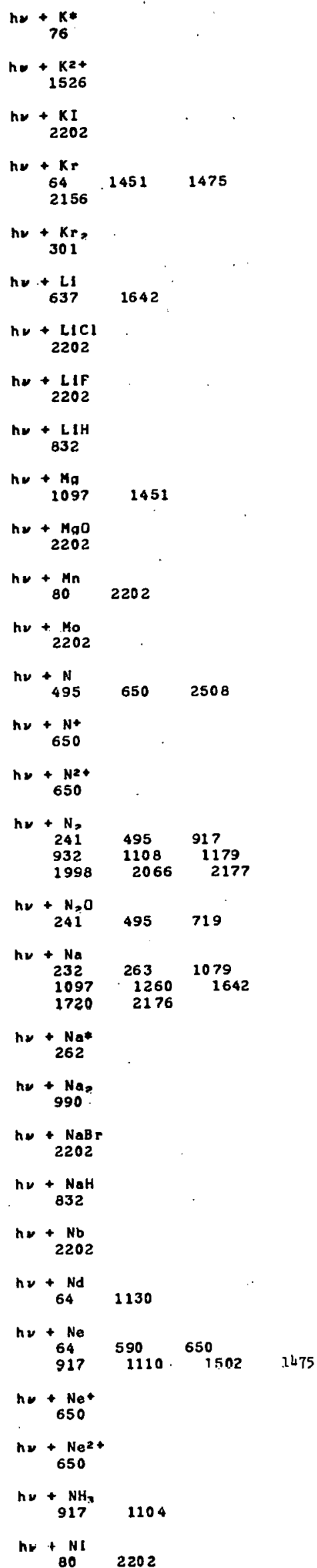

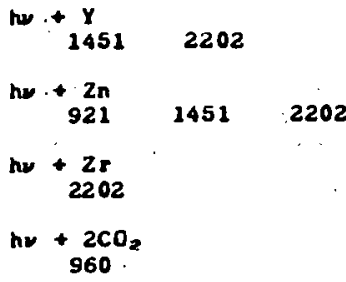


407

PHDTON COLLISIONS UITH HEAVY PARTICLES, ELECTRONS AND PHOTONS IN GASES ( ho . $100-k e v)$

Photodetachnent

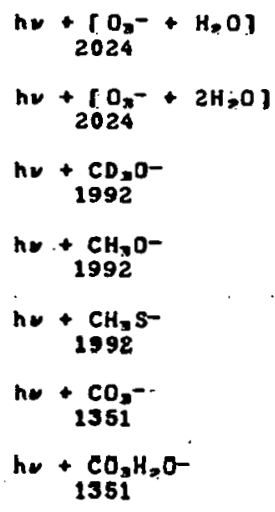

$\mathrm{HOB}$

PHOTON COLLISIONS UITU GEAUY PARTICLEs. ELECTRONS AND PHOTONS IN GAgEs (ho < 100 (nev)

Pluoresence and Lupinescence Ylelde

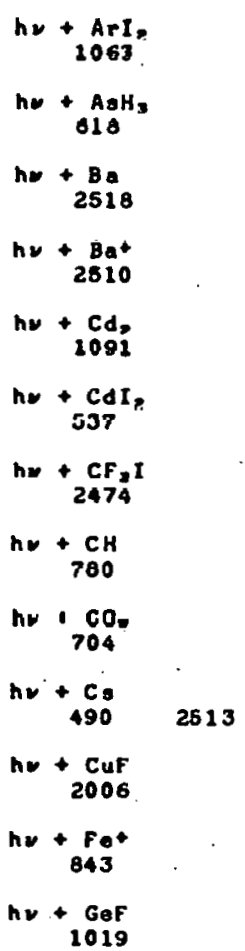

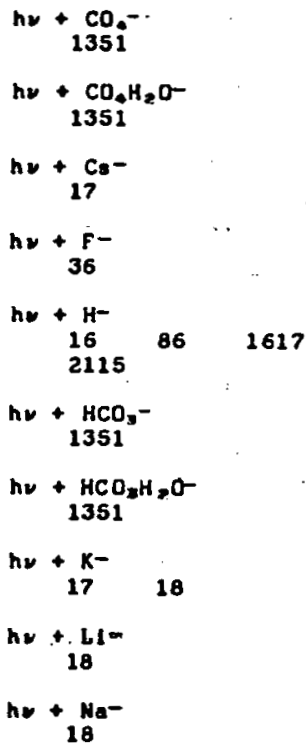


H10

\section{PHOTON COLLISIONS UITH HEAVY PARTICLES ELECTRONS AND PHOTONS IN GASES (he < $200 \cdot k e V$ )}

Photocheel stry

$$
\begin{aligned}
& h v+[B r+A r ? \\
& 1941 \\
& \mathrm{hu}+\underset{875}{\mathrm{C} \mathrm{Cl}_{2}}+\mathrm{O}_{2} \mathrm{I} \\
& h \nu+\left\{K+X_{e}\right\} \\
& 2166 \\
& \text { hv + } \mathrm{rN}_{2}+\mathrm{Hal} \\
& 1041
\end{aligned}
$$

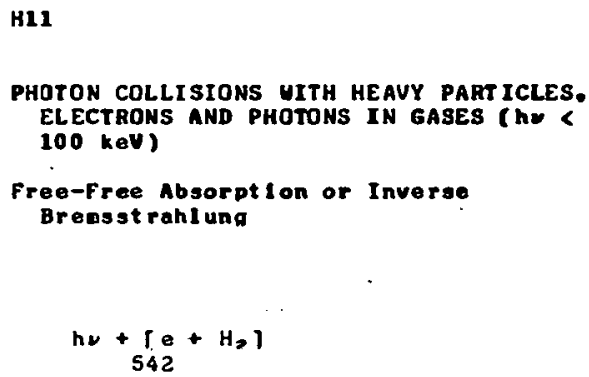

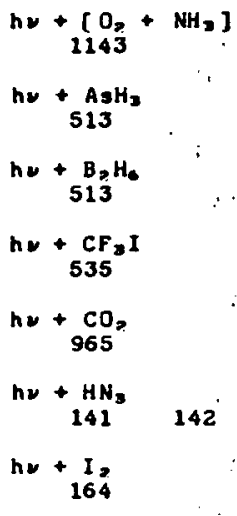

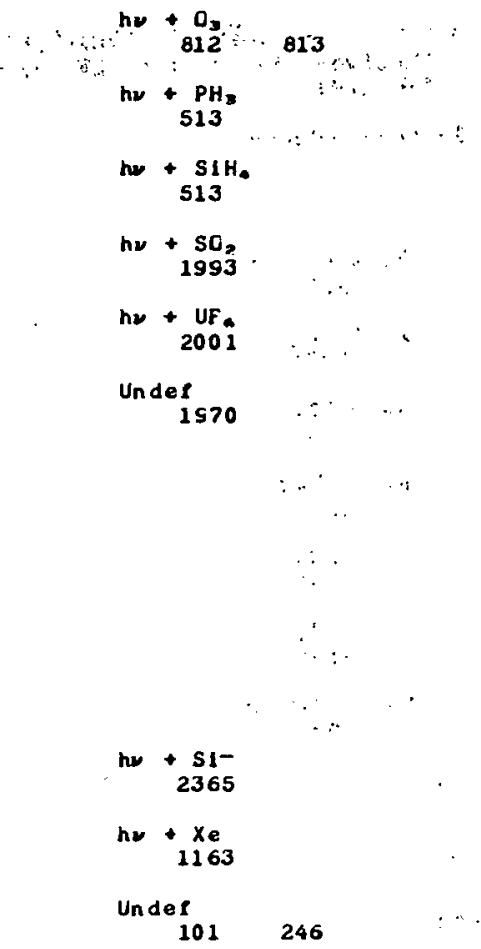

$h \boldsymbol{L}+K+K r$

816

$+0_{3}$

$+\mathrm{PH}$

513

200

def

$x$

def

$\mathrm{h} \nu+\mathrm{Ne}$ 
PHOTON COLLISIONS UITH HEAVY PARTICLES. ELECTRONS AND PHOTONS IN GASES (hV < $100 \mathrm{keV}$ )

Photon-Electron Seattering

$\mathbf{4 2 3}$

PHOTON COLLISIONS UITH hEAVY PARTICLES, ELECTRONS AND PHOTONS IN GASES ( ho $<$ $100 \cdot(\mathrm{keV})$

Photon-Photon Colll bbons
Under

2320

hv $+h_{1668}$

114

$$
\begin{gathered}
h v+e \\
21 \\
h v+I *
\end{gathered}
$$

2hv $\underset{1997}{\mathrm{H}_{2} *}$

PHOTON COLLISIONS UITH HEAVY PARTICLES ELECTRONS AND PHOTONS IN GASES ( hy < $100 \mathrm{keV})$

Photo-De-Excltation, Quenchlng, and st Inulated Eission (cross sections)

$h v+\underset{1230}{[\mathrm{He}}+\mathrm{He}]$ 
101

DATA COMPILATION

Heavy Particle

$\begin{array}{rrr}\text { Rev lew } & & \\ 660 & 661 & 2298\end{array}$

ross

888

889

REVIEWS AND BOOKS

Heavy Particle

Review

450 .

451

686

1386

$\mathrm{KO4}$

REVIEWS AND BOOKS

Particles on surfaces and Solids

$\begin{array}{rrrr}\text { Review } & 1389 & 1390 & 1391\end{array}$ 
AUTHOR INDEX 
A01

HEAVY PART ICLE - HEAUY PARTICLE INTERACT IONS

General

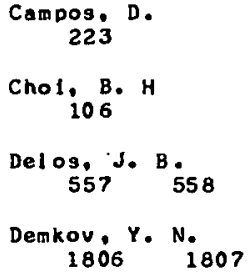

Eu. B. c.

126

Kruger, H.

223

Kunasz, C. V. 1807

Levin, V. G .

1377

Mukher jee, S. C 1820

Neudachin, v. G. 1377

Oatrovsk11, V.N. 1806 1807
Poe, $\underset{106}{R . T}$.

sil. N. C.

1820

Solirnov, Y. F 1377

Solov'ev, E. A. 1806

Sur. S. K. 1820

Tang. K. T. 106

Thorson, H. R. $557 \quad 558$ 
A02

HEAVY PART ICLE - HEAVY PARTICLE INTERACT IONS

Elastic Seattering. Collisions

Alekseev. A. I.

$$
1259
$$

Alexander. M. H.

121

Andersen, N 1521

Arnold, L. G. 319

Basharov, A. M. 1259

Baver. T. S. 1285

$-==$ Bauer. W

Becker, C. H.

Berard. $M$. 1563

Berman, P. R. 2190

Berngteln, R. B. y/4

Blakley, C. R.

Blanpled. $G$. 1285

Borlng J. W.

Brechl gnac. $c$. 2190

Arunetti. B. 449,788

Budenholzer, $F$. E. 1049

Burg I, H. R.

$$
321
$$

Casavecchla, P. 2009

Champl on, $R$. L. 131

Chapman, s.

Clark, B. C. İ

Coker. W. R. 1287

Connor, J. N, L. 167

Dee, 6. 1076

Dav Is, J. P. 464

de Vreugd, $C$. 131

Dlercksen, G. H. F. 139
D In 9: $A$

Dol egchall, P. 321

Dondl, M. $G$. 445

Dreves, 1284

Durfy. $K$.

Duquette, $G$. 992

Duren. R. 740

Egelhof, P. 1284

El119. T. H. 992

Este, G. 0 . 731

Falst, H. B.

F lck, D . 1284

Flannery, M. R. 2104

Fong, J.

Pralles; U. L 166

Futrel 1, J. ${ }_{74}$.

Gayet, R. $\rightarrow 8 ?$

Georglev, B. 2.
3a0

GIlesple, G. H. 1795

Gislason. E. A. 714 1049

Goldelan. R. 285

Green, $S$. 285993

Gregor, H. W. 2027

reroner, 740

Grcsier. J. 144

Gruebler: 321

Gubler. H. P. 322

Guest, M. F. 982

Hart, $R$. R.

Heidenrel ch, G. 321

HeIl, T. G.

Hell and. $w$. 2139
HI111er, I. H. S82

Hintz, N. 1285

Holfeann, G.H. 1285

Holfeann, G. H... ot al. 1288

IaO, G. J. 1285

Jacobs, $H$. 713

Jakubetz, 167

Jenny. $B$. 321

Johnson, R. E. 1400

Sordan; R. $M$ $2027 \cdot 2084$

Kaplau, j. Y४2

Karo, A. H. 2058

Kassen, 5 . 1284

Kendrick, $d$.

K.lobolo, U. 322

Klacssen, $D$. 120

Klein. M. L. 992

RnIght, G. : 231

Konlg. v. 321

Kourl, D. J. $285 \$ 593$ 2041

Kozlowsk1, T. 1285

Kraemer . W. P. 1.35

Kr ejc i R 2068

kubach, $c$. 1517

Kuntz, P. J. c82

lallomend, P. 1663

Lee, $Y . T$. 2009

Le fioy, k. J. 2053

Lil jestrand, R. L. 1285

LIO, T. K. s20.

LIn, C. S. 769

LI U, B. 1831 


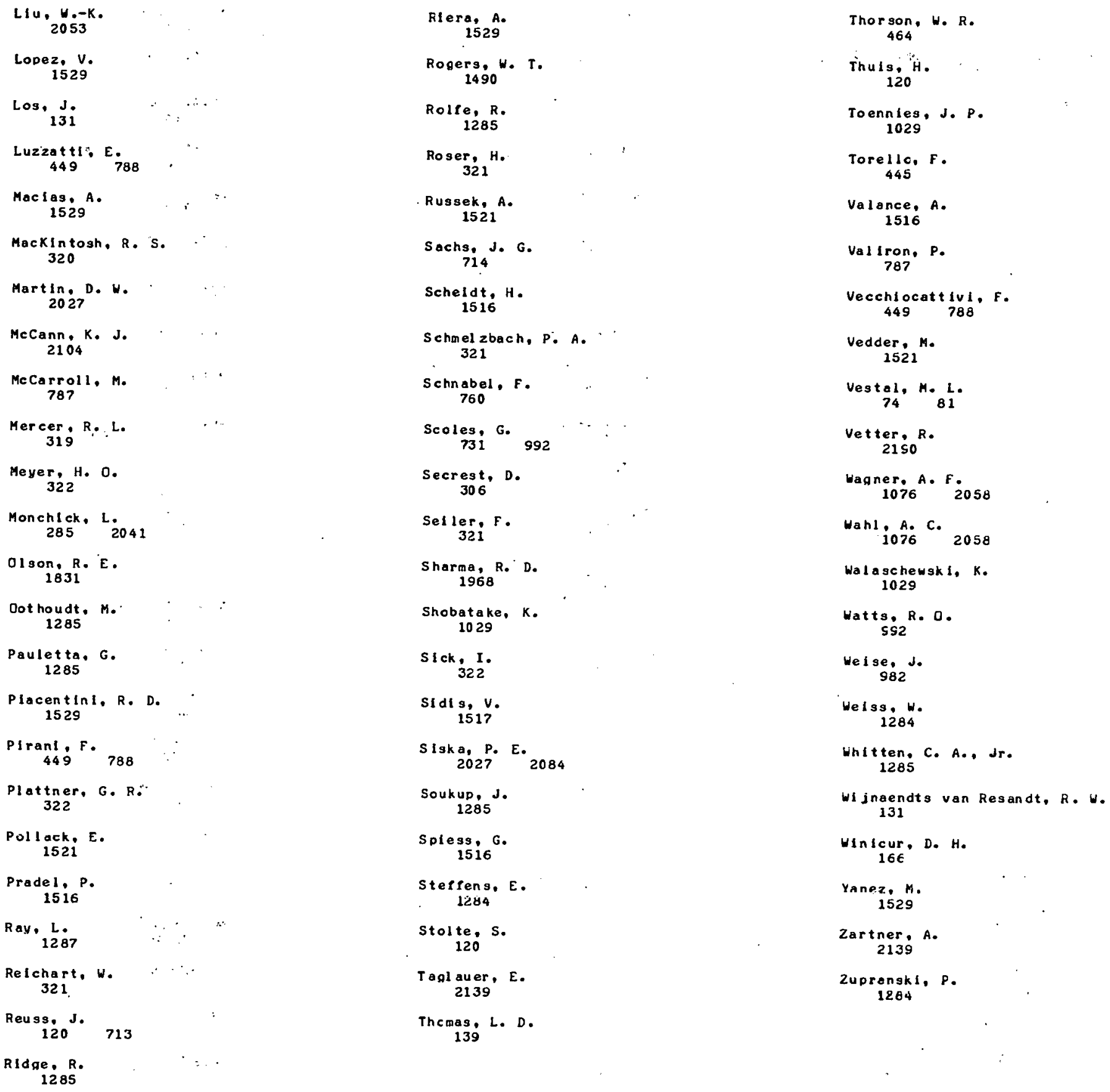


Berrington, K. A. 622

HEAVY PART ICLE - HEAUY PARTICLE INTERACT IONS

Exe I tation

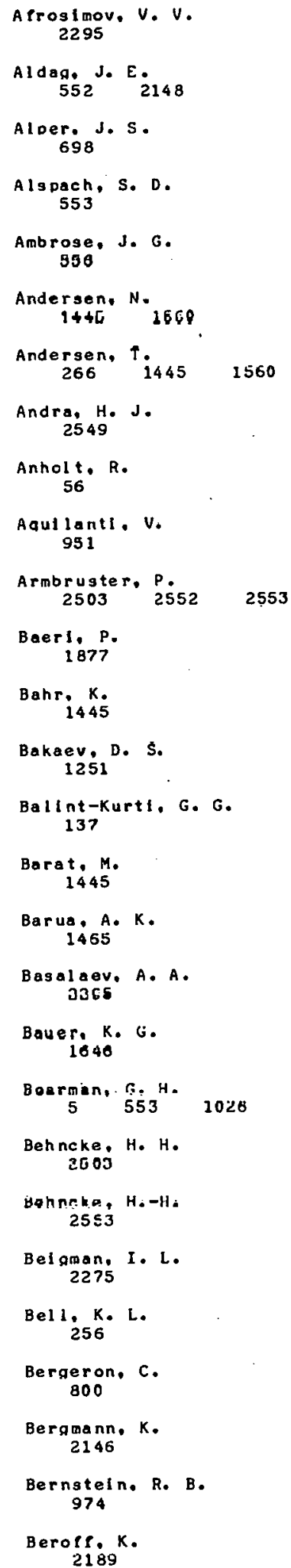

Bethge, $K$.

2541

Bever, H. F.

1275 . $1459 \quad 1472$

Bhat tachar yya, S. S. 1465

Billing. 6. D. 151

Bl raben, $F$. $1821 \cdot 2189$

Black, 0 . 293

Bloirs. S. S.

B1 a 1s. N. C. 295

Bloemeng E.

Bobaghev, S. V. 2114

Bogas, J. E. 2008

Boring, J. $u$. 1490

Borisov. V. B. 2488

Bottner. R. 150

Boving, E. 266

BovIng, E. $G$. 2123

Brandon. F. L. 3

Broshears, H. C., Jr. 666

Bray, $c$.

Brown, N.J. 1347

Browne, J. C. 1794

Buck: $U_{0}$ 11032160

Burko, p. E. 622

Burrel I, C.F. 1805

Cagnac, $B$. 1821

Camplsano, S. U. 1877

Carrol 1. A. A. 698

Casavecchía,P. 951

Case, D. A. 161

Cassidy, J. C.

Cernogek, R. $H$.
Chepman, S.

654

Cheng: $K$.

Chesnokov, E. N. 2319

Chiblsov, H. I. 2446

Chol, 8. H. 107

$\mathrm{Cl}$ eneng. E. $1477^{1527}$.

Cocke, C. L. 1445

Cohen. J. S. 82

Collina, L. A. B2

cooke, H. E. 62

Coolen, f. L. A. 363

Copeland, D. A. 528

Croge. R. J., Jr. 2081

Crothers. D. S. F. 242

Curry. S. M. $490 \quad 1205$

Caajkowakl. M. 463 Dahler, J. S.

Davla, S. L. 2008

Degani. J.

de Heer, Fo J. $1452 \quad 1453 \quad 1645$

Deil a Mea, $G$. 1877

Demareat, J.A. 72

Dẹvengan, D. P. 190

Dhuifeq. D. 244

Dickinaon, A. D. 1507

nieribatn, G, H. F. 122

Doering. d. P. 1976

Do hmann, H.-D. 2520

Doorn. S 1455

Doughty. B. H. 3550

Drelzler. R. $n$. 2525

Dutton, P. L. $\epsilon 22$ 


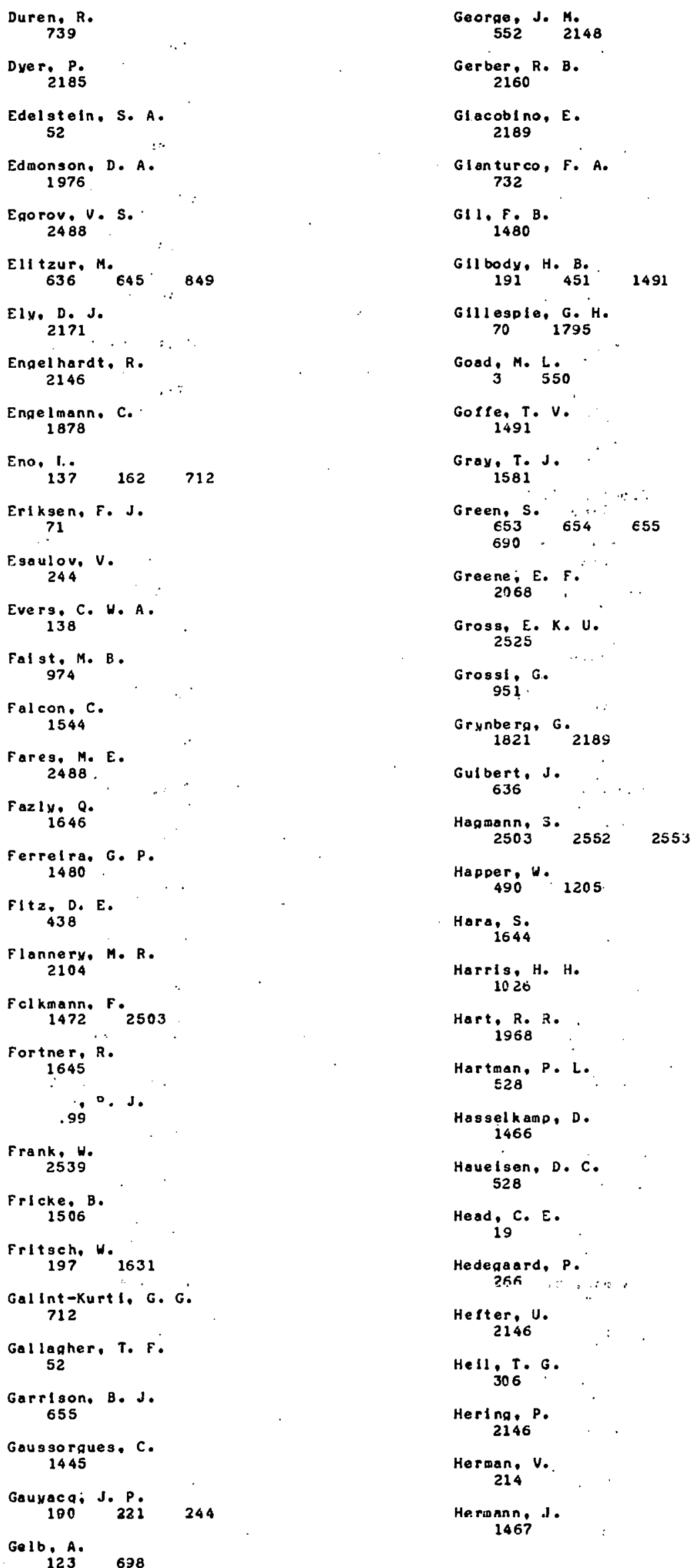

Hermonn, v.

2022

Herschbach, D. F. 161

Hickian. A. P.

$$
1758
$$

Hi poler, $R$ $1275 \quad 1459$

Hollenbach, D. J. $\varepsilon 25$

Hoogkamer, T. P. 2341499

Horbatsch, H. 2525

Hou, M. $450 \quad 1205$

Hulsken, F. 1103

Imme, $G$. 1877

It lkata, $Y$. 1644

It oh, $Y$. 235s

Jaecks, D. H. 71

James, P. B. 55

Jasiscn. K. A 1581

Jesus, A. P. 1480

Johnsen, R. E. 1450

Kanekc, $Y$. $235 \mathrm{~S}$

Karo, A. M. 2058

Katc, H. S02

Kaun, K, $-H_{0}$ $253 \mathrm{~S}$

Kellev, J. D. 1026

Kempter, $v$ $1467 \quad 1477$ 1527

Kerkoljk, C. B. 1452.1453

Kharchenko, V. A. $2114 \quad 2316$

Khare, v. $10 \in 6$

Khersonskil, V. K. $22 \leqslant 3$

KI T. $Y .-k$.

King, E. B.

Kingston, A. E. $256 \quad 622$

Kinsey, J. L. 2171

Kivel, $B$. 893 


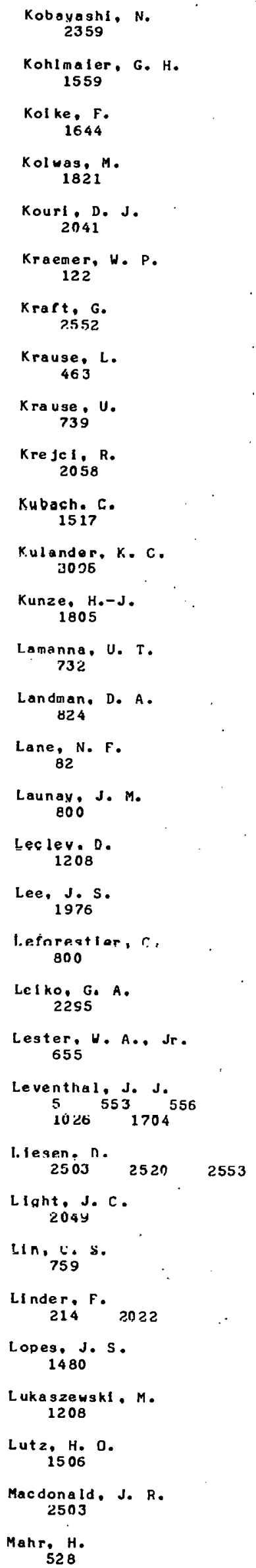

Opradclce, $L$.

1544

Ovehinnikov, v. L. 2316

Pack, F. T \$53

Panfilov, V. N. Z319

Panov. M. N. 2295

Park, J. T $552 \quad 2148$

Parker, G. A. SE3

Parr, T. P 1581

Pascale, J. 2025

Pastcr, A. A. 2488

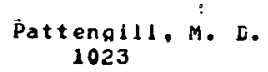

Posohor. J. L.

Pedersen. E. H. 1445

Pfeng, $H$. 2520

PhI111\%s, w. v. 2171

Placentini, R. D. 1544

Poe, F. T. 107

Polanyi, J. C. 123

Pontiler, J 1445

Poncet, $M$ 1878

Popre, D. 150

Pradel, F. 1610

Pretorlus, $R$, 1500

Prins. $M$

Pritchard, $T$. E 2171

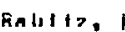
$\in \leq 3$ S1

Ramaswamy. F. $\epsilon \leqslant 3$

Redacn, L. I. 1754

RI chard. P. 1581

Aichards, D. 1587

Rlecke, G. $146 \%$

Rioini, E. 


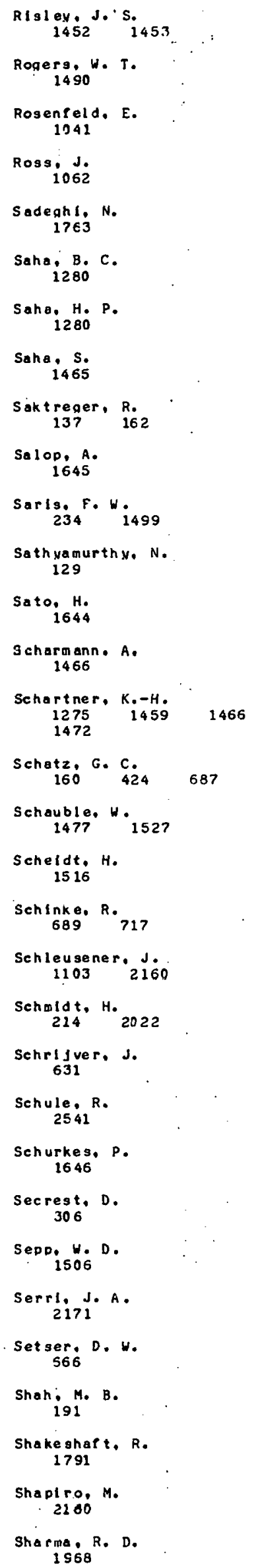

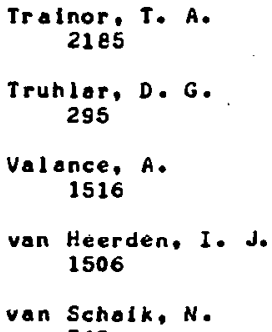

Zat serkounyuk, N. M. $24 e 8$ $\begin{array}{lll}1467 & 1477 & 1527\end{array}$ 
Kleyn, A. W.

$$
716
$$

HEAVY PART ICLE - HEAVY PARTICLE INTERACT IONS

Dissociation

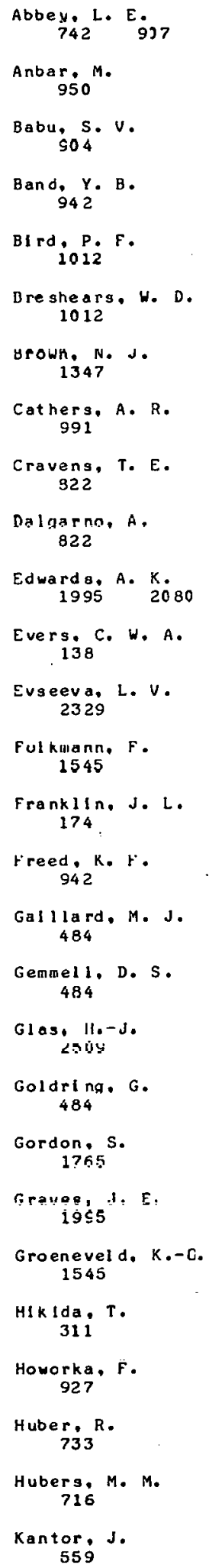

Kohlmaler, G. H. 1559

Kuchitsu, K. 781

Kul ander, $K$. $C$. 2095

Kupriyanov, S. E. 2269

Levin, v. G. 1377

Levine, I. 484

Los, J.

Mann. A . buy 176u

Mann. R. is 85

Manuelyan, R. $V$. 2269

McLaughlin, D. R . 900

Melerjohann. B. $1 \quad 2012$

Meron, M. 1765

Moran, T. F. $742 \quad 9.07$

Morl, Y.

311

Neudachin, V. G 1377

NIT. D. $559 \quad 1765$

NI ghivana, I TO1

Novak, E.

1559

Nus sbaum, G: H. 991

fidcm. R. W. 950

LESK1, Y. 781

Parks, C. K. 1962

Perov. A. A. 2269

Peterson, R. S. 1545

Pletsch, id. J 484

Polzat, J. C. 484

Potapov, V. K. 2269

Preston, R. K. 900

Prokof'ev, A. A. 2329
Rawakrishna, H. 904

Ratkcuski, A. J. 484

Refaey, K. M. A. 174

Reaillieux,J。 484

Roberts. R. E. S3S

Rosner, E. 555

Rusinek, I. s39

Schnitzer. F。 s50

Sneer, S. H. 1962

Silver, D. M. 1347

Sire, E. C. 1559

Smirnov, Y. F 1377

Snlder, N. 2050

Stace, A. J. 435

Stever, M. F. $1595 \quad 2080$

Suzukl. K. $\rightarrow \varepsilon i$

Szabo, G. 1545

Thomoson. D. L. 900

Tunitskil. N. N. 2329

Turner, $T$. 742

Vager. $Z$. 484

Vikia. A. C. 2076

Voglón, A. 1: 2012

We ber. 11 . G. 2509

Heinterg. A. 1765

Wexler, s.

wl cox, J. B. yo?

Wood, R. M i995 2080

Zabronsky. E.J. 484

Zhurkin. E. S. $232 \$$ 


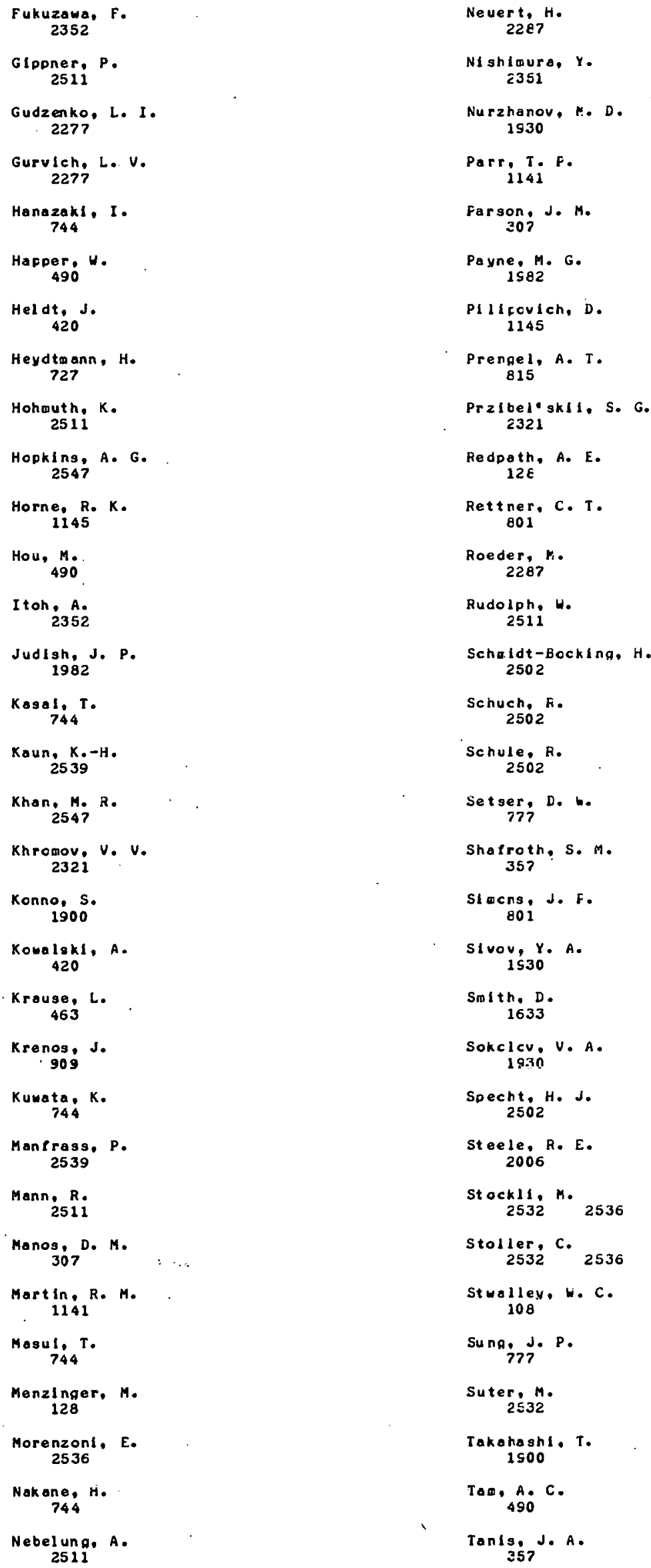

Nevert.
2287

Nishimura, $Y$. 2351

Nurzhanov. H. D. 1530

Parr, T.F 1141

$\underset{307}{\underset{30}{*} \text { J. M. }}$

Payne, M. G. 1582

PI I fovich, D. 1145

Prenge 1, A. T. 815

Przloel"skil. S. G. 2321

Redpoth. A. E.$$
12 \varepsilon
$$

Rettner, C. T. 801

Roeder, $M$. 2287

Rudolph, W. 2511

Schaldt-Bccking, $H$. 2502

Schuch, F. 2502

Schule, R. 2502

Setser, D. W. 777

Shafroth, S. M. 357

Simens, d. F. 801

SIvor, Y. A.

SmIth, D. 1633

Sokclev, V. A.

Specht. H. J. 2502

St ee le, R. E. 2006

St ock11, $2532 \quad 2536$

$\underset{2532}{\operatorname{stol1er}}{ }_{2536}$

Stwalley, w. C. 108

Sung. J. P.

Suter, $M$. 2532

Takahashi. T. 1500

Tom, A. C. 490

$\operatorname{TanIs,~J.~A.~}$ 
Tserruya, I.
2502

Tsujl. M.

2351

Hade. M. K.

463

Hax: K. R
UI Icomb, B. E.

$$
1986
$$

nol $111, y$

$2532 \quad 2536$

Wu, C. - Y. R. 108
Yebuzakl. T. 450

Yakovlenko, S.I. 2277

Yana, $s_{0}-c$. 


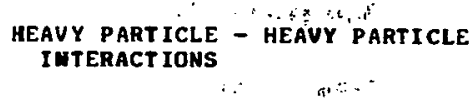

Electron Capture $\cdots$

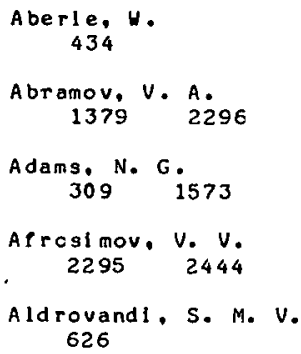

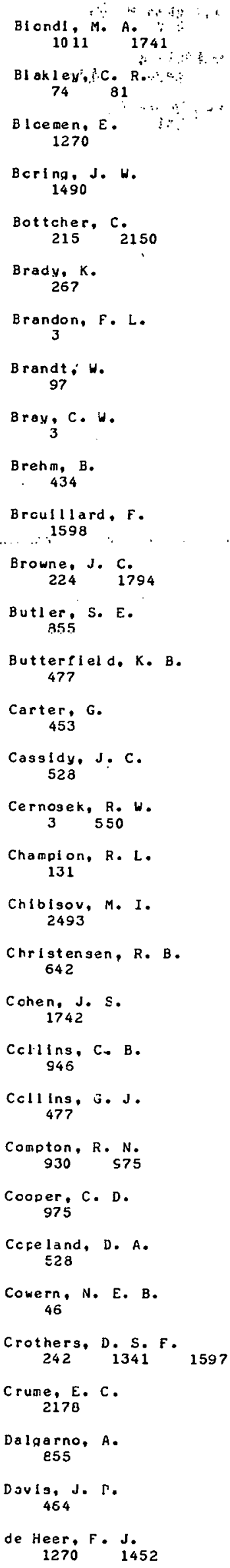

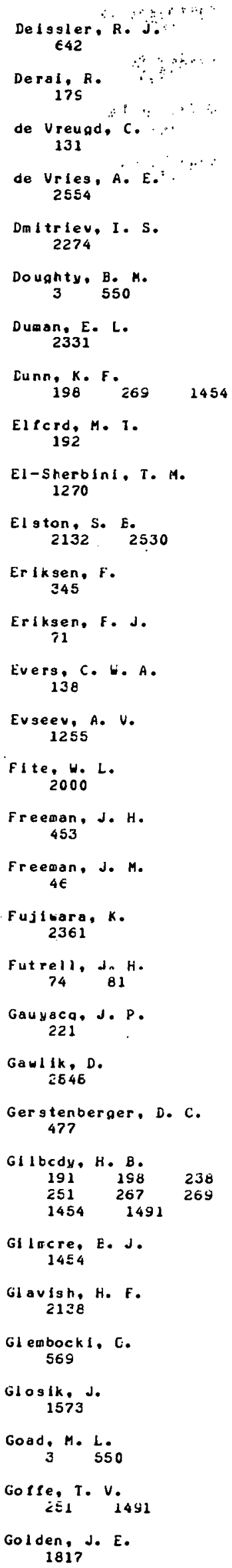




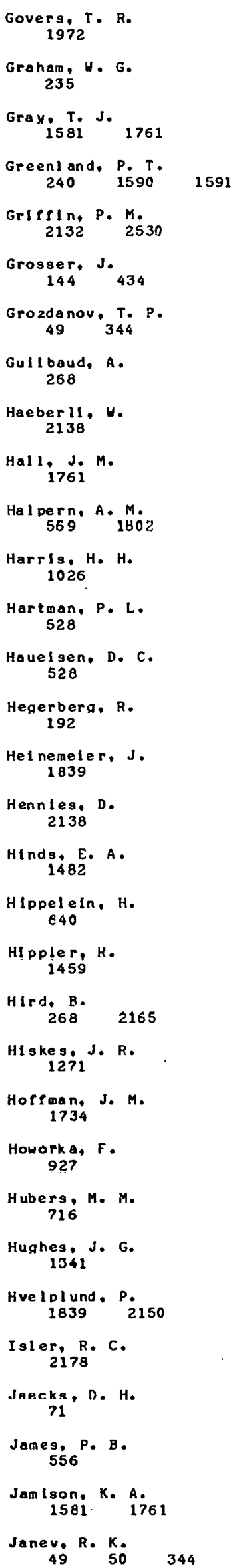

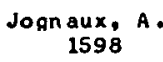
Little, W. L.

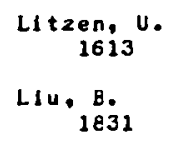

Hyerg, G. D. 


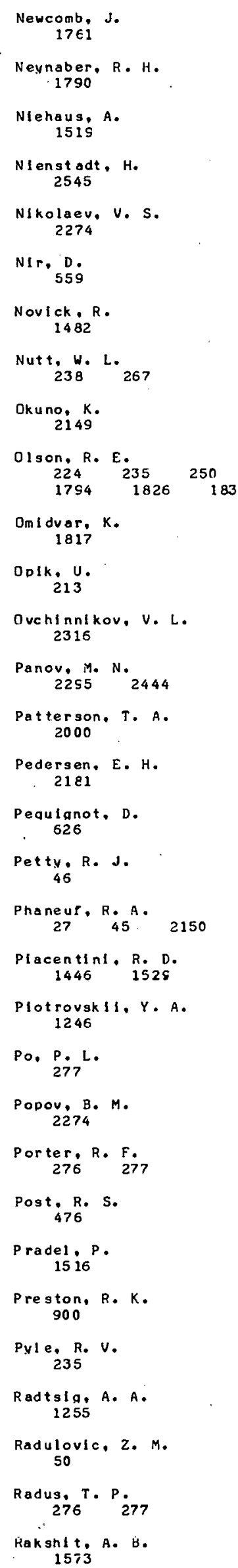

Roo, B. K.

Raymond, R. S. 2138

Redmon, L. T. 1794

Reinhardt; P. $H$. 975

RIchard, P. $1581 \cdot 1761$

Riera, A. 1529

Risley, J. S.

Rogers, W. T. 1490

Rcsner: $B$. 1279

Rethuell, H. L., Jr. 1055

RuP, M. $H$. 15 is

Rutherford, J. A. 310

Ryuruku, H . 1796

Sol1n, A. $1446 \quad 1620$

Salop, A. $1270^{\circ}$

Salzborn, E. 452

Echartner, K.-H. 1459

Scheldt, $H$. 1516

Schlachter, A. S. 235

Schmledeka mp, c. 1761

sellin, I. A $2132^{2530}$ $\underset{198}{\text { Sewell, E. C. }} 269$

Shatroth. S. M. 2137

Shah, M. Bं. $191^{251} 267$

Shakeshaft, $R$. $\begin{array}{llr}60 & 567 & 1513 \\ 1582 & 1791 & 2174\end{array}$

Shav, T.

Shevelko, V. P. 2534

Shipsey, E. J. $157,224 \quad 1794$

Shpenik, 0.8 . 2316

sidis, $v$ $221 \quad 1517$

siegel, M. W. 2000
Si 1, N. C.

SImpson, F. R. 1454

Sulrnov, B. M. $1255 \quad 2331$

Silth, s. $30 \mathrm{~S} \quad 1573$

Solield, C.J. 46

Soong, S. C. S6 1709

Spless, G. 1516

Spruch, L. 1513. $1582 \quad 1797$ 2174

Squires, L. 1075

Srivastava, M. K. 1648

stasinska, $\in$. $\epsilon 2 \epsilon$

Stearng, J. W. 235

stelangson, $T$. 192

Stelson, P. H . $45 \quad 2150$ Stern, ${ }_{221}^{B}$.

Stevens, W. J. 1271

Sterart, B. 1050

Stockdale, J. A. D. 930

Suk, H. C. 268

Suter, $A$ $3132 \quad 2530$

Szucs, S. $15 S 8$

Tang. C. L. $\leqslant 2 \varepsilon$

Tang, S. $Y$. 1750

Tanis, J. A. 2137

Tashoev, $Y$. A. 2274

Taulbjerg, $K$. 2181

Tawara, H. $1581 \quad 1761$

Teflcua, Y. A. 2274

Terw1111ger, D. T. 506

Thoe, R. S. 2132 S 2530

Thompson, D. L. 500 
$\underset{464}{\text { Thorson, W. R. }}$

Tlernan, $T \cdot 0$. 906

Todd. N. R. 1557

Tolmachev, $Y$. A. 1246

Tridathi, A. N. 1648

Tripathy. D. N. 32

Tunne 1 1, L. N. 96

Turner, J. E. 92

TwIddy, N. D. 1673

Upham, R. J.. Jr. $230 \%$

Itterback, N. G 998

vaaben, J. 2181

Val ance, $A$. 1516
Vane, C. R. $2132 R$

van $2 y 1, B$.

$998 \quad 1055$

Vegtal, M.

Vogle, M.

2543

Voaler. $M$.

Volovich, P. N. 2316

Vroom, D. A.

Warmack. R. J.

930

batanabe, $T$.

1736

Watson, W. D.

642

Weber, H. G.

2545

Wè 1. L. . L.

2348
Weisheit , J. C
2367

Wijnaendts van Resandt, $R$. W. 131

Hilccx, J. B. 1006 i974

WI l lann, P. A. 1271

winter, $H$. 1270

Winter, T. G. 4

witmann, $t$ 2549

Hu. F. L. C. 506

Yanez. 1 .

Zhdencv, V. F. 2493

zu Putlitz, 6 . 2545 
A07

HEAVY PART ICLE - HEAVY PART ICLE IN TERACT IONS

Ionlzation

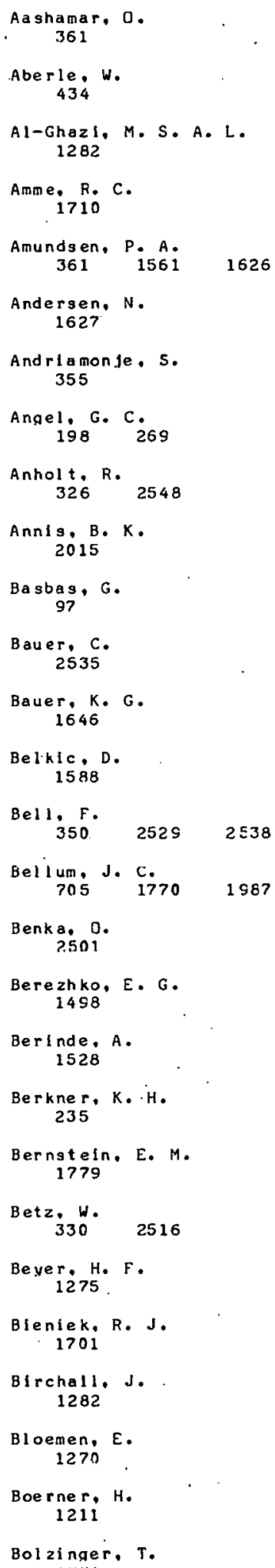

Bosch, $F$.
1286

Brondt, wo

$$
97
$$

Brehm, B.

434

Briggs, J. S

$$
1632
$$

Browne, J. C.

1794

Ceausescu, v. 2514

Chatterjee, P. N. 365

Chemin, J. F. 355

Cheng, $K$.

$$
70
$$

Chlu. K. C.R. 220

Compton, R. N. 930

Conway, J. G. 1227 Corno, D.
1804

Cowern, N. E. B. 46

Cravens, T. E. 822

$\underset{108}{\operatorname{Crccks}, \text { J. B. }}$

Dal garno. A.

Datz, S.

2015

Davidovic, D. M. 233

De, J. N.

317

Deberth, $C$. 1528

dé Heer, F. J. 1270

Deloche. R. 940

Devdarlani, A. 2 . 2263

Dirocion, G. 1077

Doer ing, $J$. P. 1976

Donahue, D. J. 1779

Dost, $M$.

Doyle, B. L. 1786.2522

Drepper, $F$. 1632

DuBols, R. 1553

Duggan, J. L.
Dunn, K. F 269

DunnIng, F. B. $\$ 44$ Ebding, $T$.

Edmonson, D. A. 1976

Edwords, A. K $1 \$ 952080$

El-Sherbini, T.M. 1270

En gelmann, $C$. 1878

Fazly, Q. 1646

Fitalre, M. 2191

Fite, $W$. L $415 \quad 2000$

Folkmann, F. 1545

Forgter, J. S 1753

Fort: J. $1803 \quad 1804$

Freenon, J. M. 46

Fricke, E. 2550

GaIly, T. D.

Gardner, $R$. K. 1786

Gayet, $R$.

George, T. F. $705 \quad 1987$

Geretschloger, M. 2501

Cil body. H. U. $198 \quad 269$

Gillen, K. I. 761

Gillegple, G. H. 10 1750

Grahom, $G$. 235

Graves, J. E. 1955

Gray, T. J. 1761 . 1786

GreIner, $330 \quad 2514 \quad 2516$

Groeneveld, $K .-O$. 1545

Grosser, J. 434

Gushchina. A. A. 2484

Hall. J.M. 1761

Hartung. $H$. ¿550 


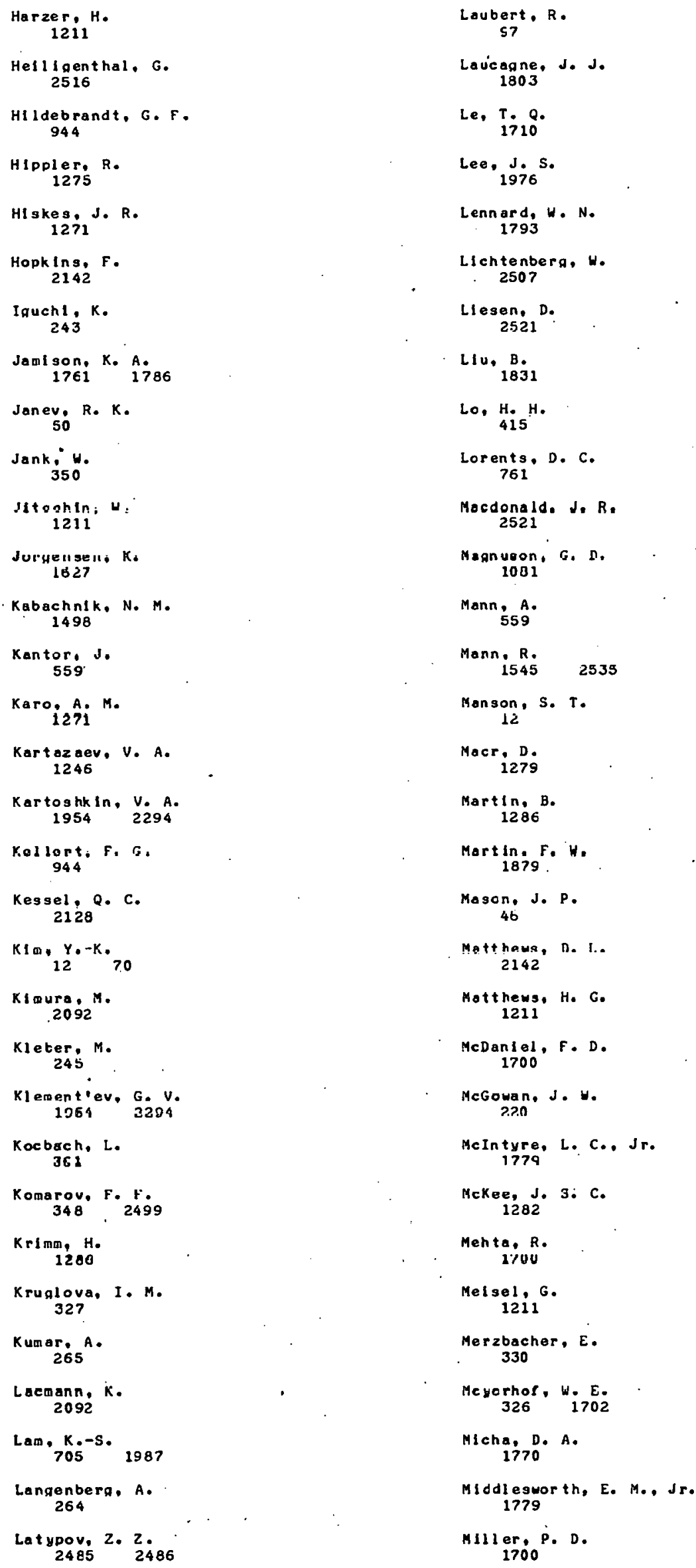

Harzer, H. 1211

Helligenthal, G. 2516

HIldebrandt, G. F. 944

Hippler, R. 1275

HI skes, J. R. 1271

Hopkins, F. 2142

Iquehl, $K$. 243

Jamison, K. A. $1761 \quad 1786$

Janev, R. $K$. so

Jank:

Ittoghin: U: 1211

Juryensen; K. 1627

Kabachnlk, N. M. 1498

Kantor. J. 559

Karo, A. M. 1271

Kartazaev, V. A. 1246

Kartos hk In, V. A. $1954 \quad 2294$

Kollort, $F, G$. 944

Kessel, Q. C. 2128

$K 10 . Y_{12}{ }^{-K K}, 70$

Kimura, M. 2092

Kleter, M. 245

Klement'ev. G. v. 10542204

Kocbach, L. 361

Komarov, F. F. $348 \quad 2499$

$\mathrm{KrImm}, \mathrm{H}$. 1280

Kruglova, I. M. 327

Kumar. $A$. 265

Laemann, K. 2092

Lam ${ }_{705}^{K .-S .} 1987$

Langenbera, $A$. 264

Latypov, Z. Z. 24852486

Laubert, R. 57

Laveagne, J. J. 1803

Le, T, Q. 1710

Lee, J. S. 1976

Lennard, $W . N$. 1793

Lichtenberg, W. 2507

Llesen, D. 2521

LIU, B. 1831

Lo, H. H. 415

Lorents. D. C. 761

Hocdonald. J, R. 2521

Magnueon, G. D, 1001

Mann, A.

Mann, R. $1545 \quad 2535$

Manson, S. T. 12

Macr, D 1279

Martin, B. 1286

Martin. F. H. 1879 .

Magon, J.P. 46

Matt haus, D. I. 2142

Hatthews, H. G. 1211

McDaniel, F. D. 1700

McGowan, J.U. 2.2.0

MeIntyre, L. C.. Jr. 1779

Mckee, J. 3: C. 1282

Mehta. R. 1700

Melsel, G. 1211

Merzbacher, $E$. 330

Meyorhor, W. E.

Micha, D. A. 1770

Middlesworth, E. M., Jr. 1779

Mlller, P. D. 1700

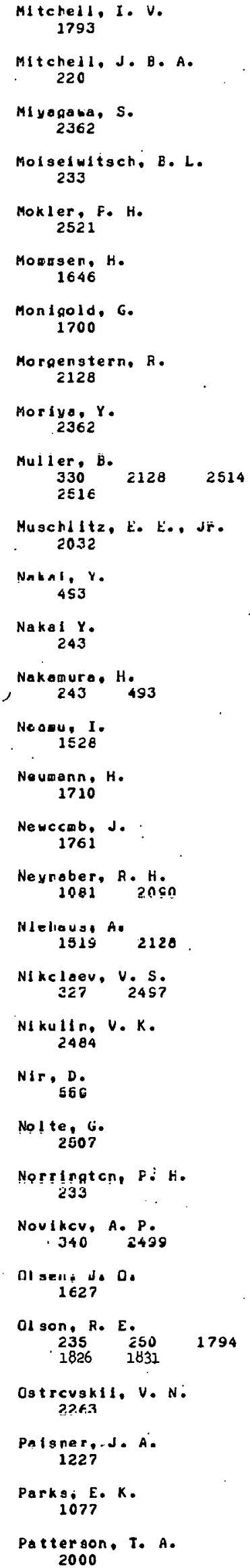




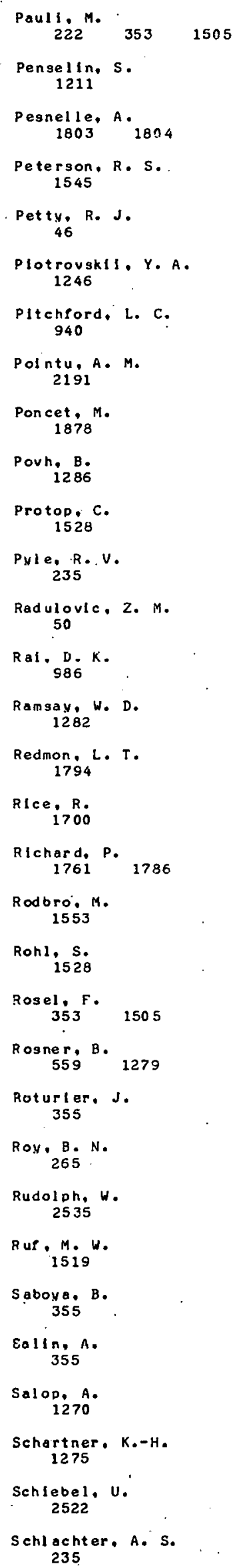

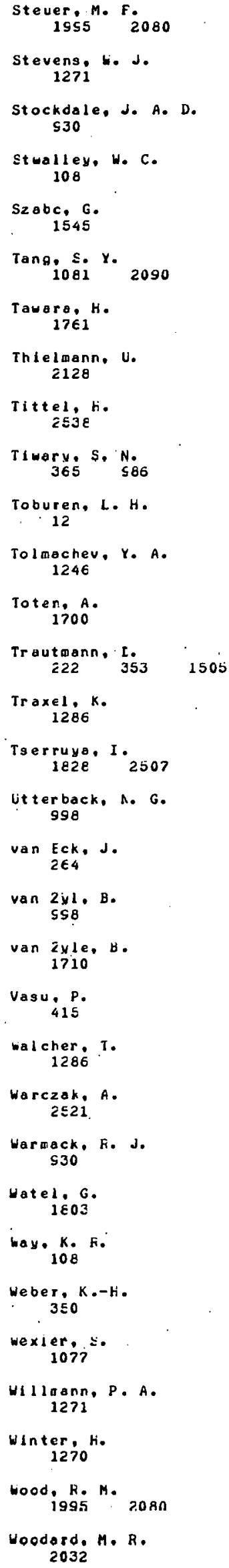


Vorden. E. F. 1227

Uu, ${ }_{108}-\mathrm{Y} \cdot \mathrm{R}$.

AOB

HEAUY PARTICLE - HEAUY PARTICLE I NTERACT IONS

stripoing

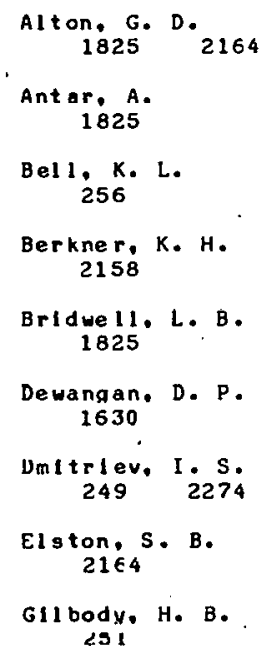

Recogbination or hutual Neutrol ization Leading to Neutral Products (ion-Ion)

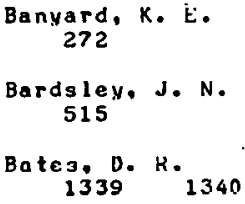

$Y$ an $, . S,-\hat{L}$.

108

Zhithikov, R. A. $1954 \quad 2294$

Gorfe, T. V.

Graham, i. G. 2158

Jenes, $C$. M.

1825

Kessel. 0. C. 1825

KI ragton, A. $E$. 256

Laubert. $R$. 2164

Miller. P. D. J.8.25

Moak, C. D. 1825

Nikolaev, V. S. $249 \quad 2274$

Olson, R. E 2158

Popov, B. M. 2274

Pyle, R. V. ?158 zoran, v. 1528
Saver, f. 0 . 1825

Schlachter, A. S 2158

Scott, H. A.

1825

Sellin, I. A. 2164

Shah, M. B. 251

Stearns. J. W 2158

suter. Mo 2164

Tashaev, Y. A. $245 \quad 2274$

Télcua, Y.A. $245 \quad 2274$

Thce, R. S. $21 \in 4$

Vane, C. F. $21 \epsilon 4$

Wailters, H. R. J. 1630

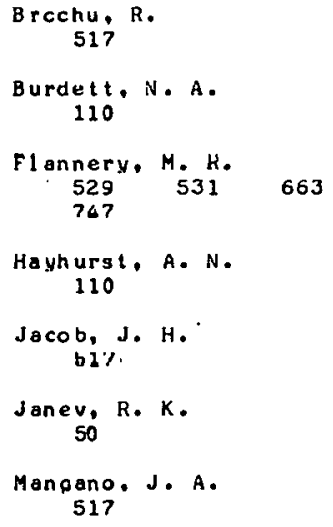

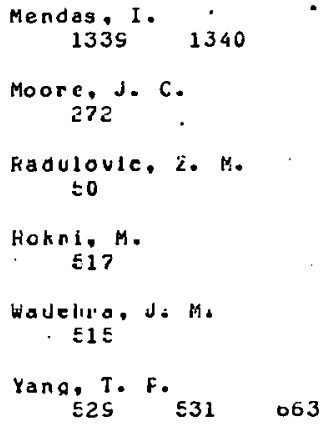


A10

HEAUY PART ICLE - HEAVY PARTICLE
INTERACTIONS

Electronic, Uibrational, and Rotationel Energy Transfer (process unknown)

Altick, P. L. 1038

Amaee, B.

$$
255
$$

A mbart sum yan, R. V. 1384

Ant ipenko, B. N.

$$
2336
$$

Arimondo, E.

$$
85
$$

Bennet t, $W$. R. Jr. 1834

Bentley. J.

$$
814
$$

Berote $k$.

2189

Berrue, J. 1167

Bhattacharjee, R. C. 145

Bhat tacharyya, S. S. $10 \leqslant 2$

BI 1 IIng, G. D. 706

Biraben, F. 2189

Borman, V. D.

Bottcher. C. 255

Bowman, J. M.

Brechl an ac, $P$. 718

Breckenridge, W. H. 792

Brindle, R. C. 1054

Bruev, A. S. 2328

Brunner, $T$. A . 2169

Cahuzac, P. 2134

Capelle, G. $n$. 2098

Chave, A. 1167

Che ron. 8.

2194

Chesnokov, E. N. $2279 \quad 2319$

GHilisur. M. I. 2446
Churakov, v. v. 1357

Ccoke. W. E. 52

Cremer, $G$. 2194

de Vries, A. E. 735

de Vrles, M. S. 735

DrIver. R. D. 2169

Dumon, B. 1167

Dunning, $F$. B. 2143

Eberhardt, W. D. 2038

Edelstein. S. A.

Ershov, L. S. 2474

Faln, B.

$F I$ in t, $J$. 1834

Foley, H. M. 2040

Forst w $145 \quad 746$

Fujimoto, T. 1345

Gallagher, A. C. 1345

Gall agher, T. F.

Gi accbino. E. 2189

Glorleux, P $85 \quad 1123$

Gogokhlya, v. V. 1382

Gordienko, V. M. 1.385

Gcroon, R. J.

Grandin, J.P. 1168

GrImley. A. J. 2007

Grunberg, G. 2189

Gupta, 5 .

Harel, C. 189

Horiri, A. 979

Hennecart, D. 1170

Herl cmont. F. 430

Hessel. M. M. 1345
HII , R. M: 2057

H1119, G. 4. 1123

Hollentach, D.J. 825

Horwitz, A. B. 2106

Houston, P. L. 2007

Hs U. D. S. Y. 242

Huestis, D. L. 2097

Husscn, $x$. 1168

Io gansen, A. A. 1384

Johrl. G. K. 2341

Jordan. F. M. 2084

Karlcu, N. V.

Kartoshkin, V. A. 2276

Kellert, F. G. 2143

Klement ev, G. $V$. 2276

Konyukhov, v. K. 1382

Kotcu, A. V. 1384

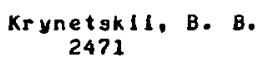

Lam, L. K, 1345

Leasure. $s$. 749

Lemolre. $J$. 430

Leone, S, R. 2106

Leshenyuk. N. S. 1357

Legter, $W_{*} A_{\text {. . Jr. }}$ 2111

LIn. M. C.

Lomterd1, M. $11 \in 6$

Lopez, $v$. 189

Lorents, D. C. 2057

Lukounlkov, A. I. 1382

Lyszyk, H. 430

Makslmov, L. A. 2328

Maloin, O. K. 792 


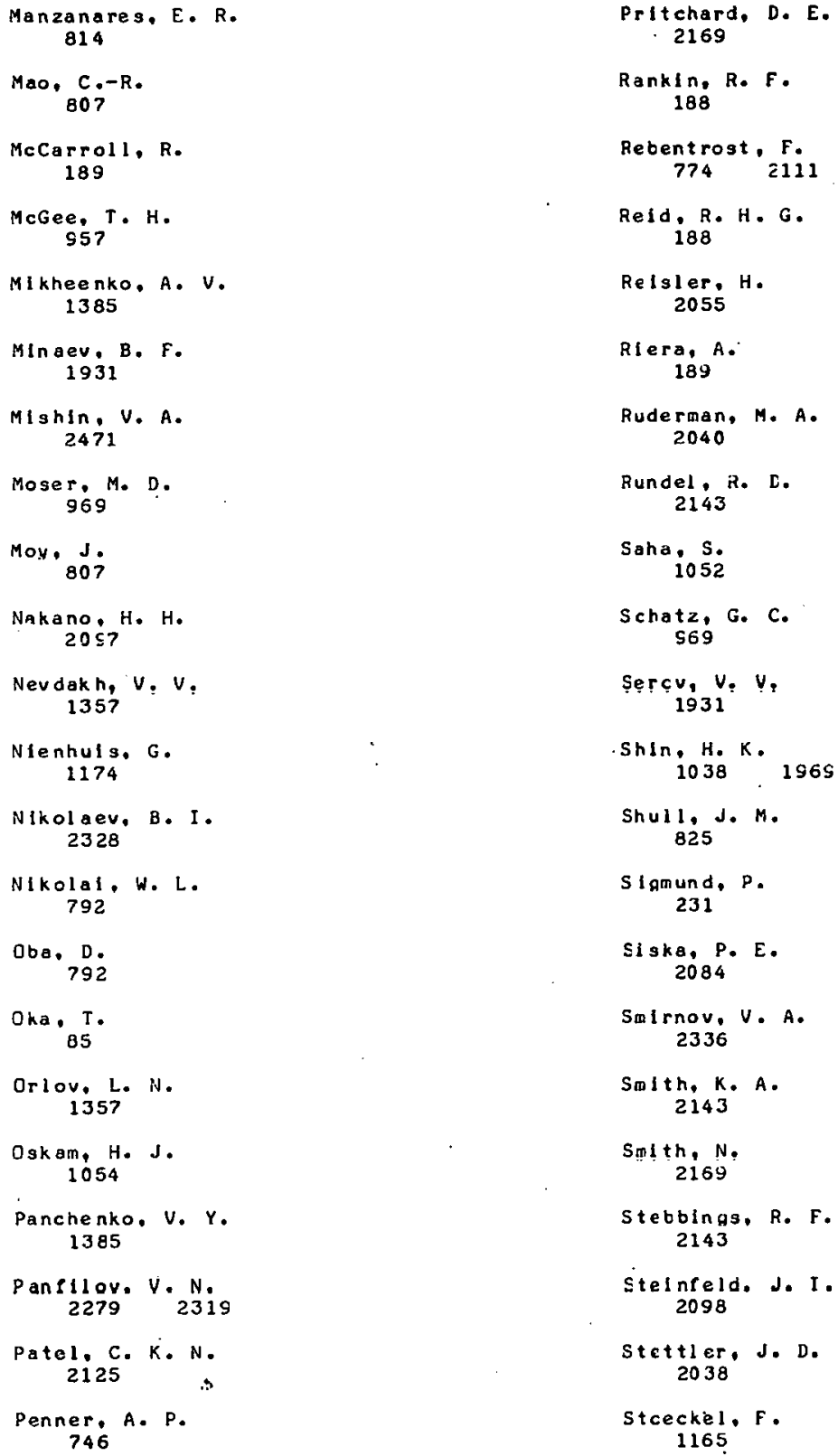

Manzanares, E. R. 814

Mao, $\mathrm{C} \mathrm{.}_{807^{-R}}$.

MeCarroll, R. 189

$\underset{\text { Ms? }}{\operatorname{McGee}}$ T. H.

Mikheenko, A. V. 1385

Minaev. B. F. 1931

Mishin, v. A.

2471

Moser.M. D.

Mov, J

Nakano, H. H. $20 \leq 7$

Nevdak h, $v, v$, 1357

Nienhuis, G. 1174

Nikolaev, B. I. 2328

Nikolai. W. L 792

Oba, D.

Oka. T.

Orlov, L. N. 1357

Oskam, H, J 1054

Panchenko, V. Y. 1385

Pansilor. V. N. $2279 \quad 2319$

Patel, C. K. N. 2125

Penner. A. P. 746

Pritchard, D. E. 2169

Rankln, R. F. 188

Rebentrost, $F$ $774 \quad 2111$

Reid, R. H. G. 188

Reisler, H.

2055

Riera, $A$. 189

Ruderman, $M$. $A$. 2040

Rundel, R. L. 2143

Saha, 5 .

Schatz, G. C. $\$ 69$

Serçu, ve v, 1931

ShIn, H. K. $1038 \quad 1969$

Shull, J. M. 825

$S$ igmund, $P$. 231

siska, P. E. 2084

SmIrnov, V. A. 2336

Smith, K. A. 2143

Smith, N. 2169

Stebbings, R. F. 2143

stelnfeld. J. I. 2098

Stettler. J. D. 2038

Stceckel, F.

1165

Sung, C. C.

2038

Sutten, D. C. 2058

Tarasenko, V. $V$. 2336

Thiteau, M. 1267

Thomas, L. D. 2111.

Tkachenkc, $F . K$. 1529

Toschek, P. E. 2134

Tracy, C.J. 1054

Trushin, S. A. 1357

van Veen, N. J.A. 735

Varoin. $A, N$. $13 \varepsilon 2$

vidal, C. R. 729

wanncn, P. 185

weston, F. F., dr. 557

Winicur, D. H. E14

ditrlol, N. M. 2038

wittia, C 579 2055

zalesskil, $Y, Y$. 2474

2hlinikcv, F: A. 2276

\%ubarev. I. G. 1384

Zuev, A. F。 1929 . 
A11

\section{HEAVY PART ICLE - HEAUY PARTICLE INTERACT IONS}

Collisional De-Excitation (total)

Abella, I. D 1230

Ade is . N. G.

1573

Ade 1, F. A. 2108

Ahl, J. L. 915

Amano, $T$

$$
973
$$

Am Imoto. S. T.$$
813
$$

Anderson, R. A.

$$
1019
$$

Baer, T.

$$
1230
$$

Bagaev, S. A. 1254

Barrat-Rambosson. M. 1504

Barua, A. K. 1464

Bar-Z1 v, E.

Basov, N. G. 2469

Bazhul in. S. P. 2469

Bennet t, $W . R ., J r$. 1834

Bien, F.

BIIIIng, G. D. $151 \quad 152 \quad 1082$ 1083

Binns, $H$. R.

Blraben, $F$.

$$
1821
$$

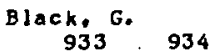

Bon dy bey, V. E. $20 \leq 2$

Bott. J.F.

Brady, T. J .

$$
7 \% 2
$$

Brashears, H. C., Jr. 666

Breckenridge, w. H.

$$
282 \quad 283
$$

Brenn. $R$.

$$
487
$$

Brindle, R. r. 1054

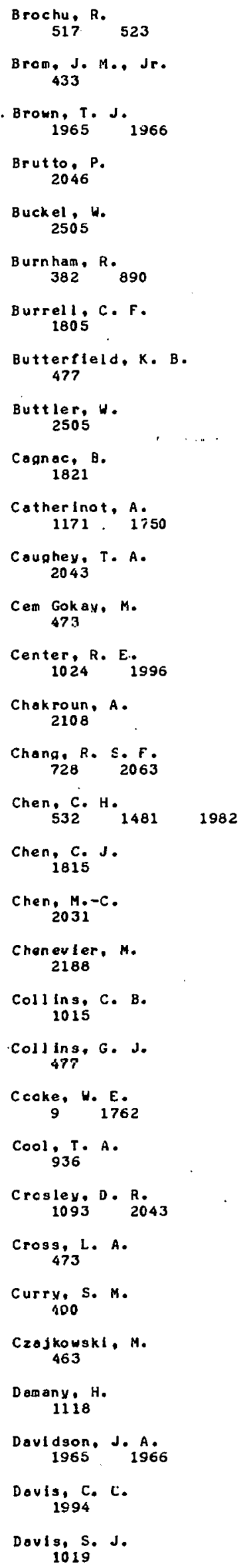

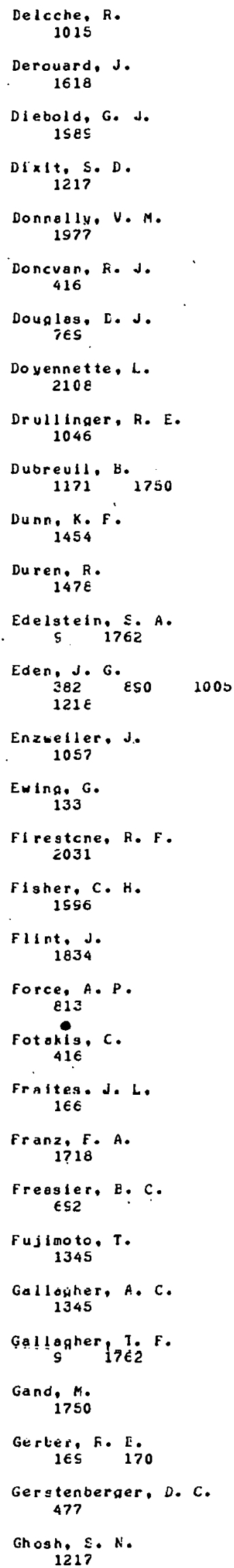




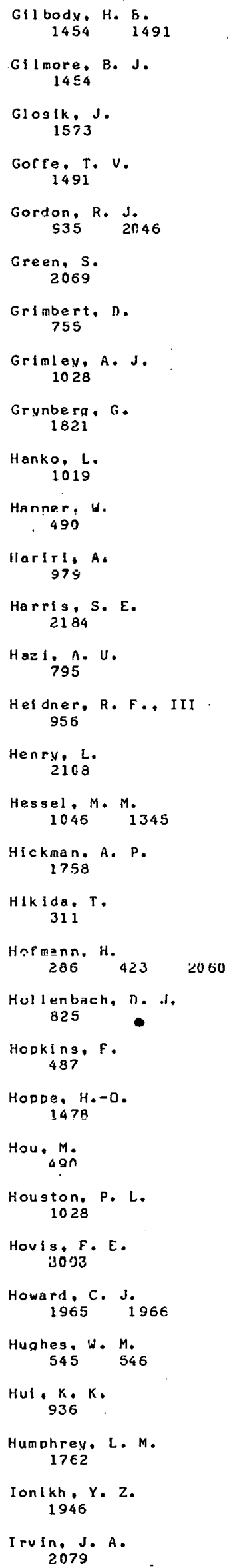

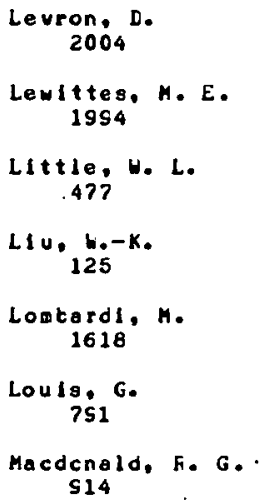




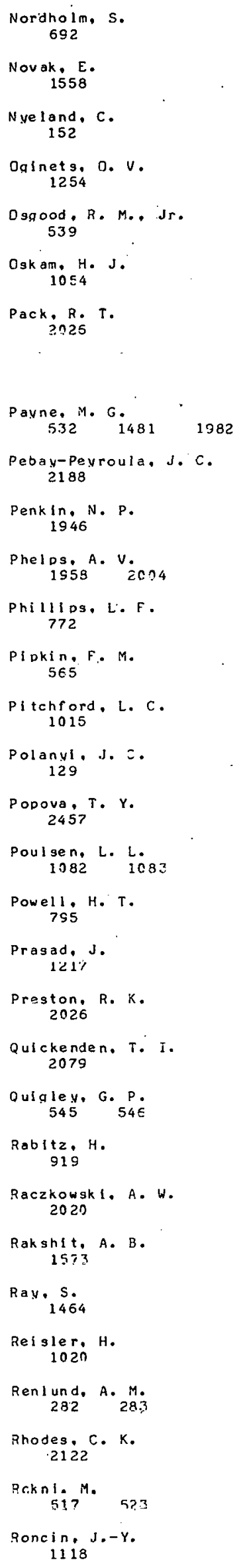

Stricker, J

G31

Stuhl, $F$

Taine, $d$

791

Tan. A. C. $4 \subseteq 0$

Tarr, s. M. 919

Taylcr, G. H. 728

To lmochev, $y$. A. 1254

Tracy, C.J. 1054

Tramer, A. 755

Twidoy, N. D. 1573 vasilenkc, L. S.
2457

Velozcc, J.E. $207 \varepsilon$

VIKIs, A. C.

Vo $1 k, C$. 1718

wade, M. K. 463

baynant. R. W. $382 \quad$ \&9 $\quad 1005$ 1218

Weber, $E$. W $2 \leq 05$

weston, R. E., Jr. ऽ57

White, J. C. ¿ 184

whitgnn, M. F... Jr. S72

Liegenfeld, J. F. e13 1990

binlcur. L. H. $1 C C$

wittig, $C$. ST 1020

Wolk, G. L. 1 SSO

Wu, K. T. 694

Yabuzaki, T. 490

Yencha, A. J. $f \$ 4$

Young. J. F. 2184

Zaritsky. N. C $165 \quad 1 \% 0$

Zdagiuk, G. A. z $1 \& 4$

Zeizgch, C. t')

Zuev, v. $s$. 2469

Evt jac. D.J. 
A12 HEAVY PART ICLE - HEAUY PARTICLE
INTERACTIONS

Collislonal Line Broadening

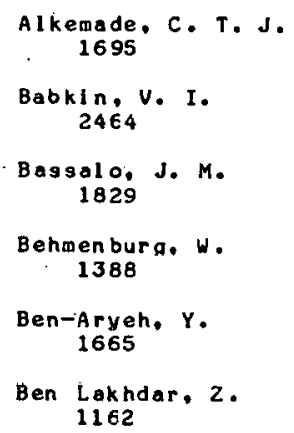

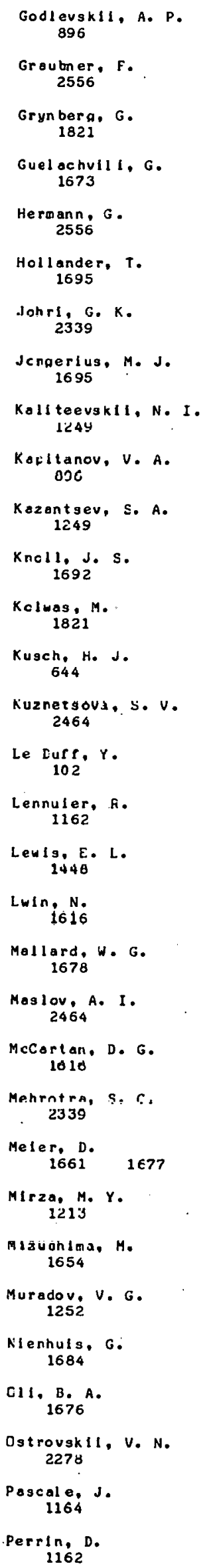


A13

HEAUY PARTICLE - HEAUY PARTICLE
INTERACTIONS

Heavy Particle Interchange.

Rearrangement, and Association (one

or more lonic reactants)

Adams. N. G.

$\begin{array}{lll}181 & 309 & 428 \\ 429 & 847 & 1573\end{array}$

1633

Ah1, J. L.

$$
915
$$

Albritton, D. L.

978 995 1096

1105

-

Arifov, U. A.

$2455, \quad 2456$

Baer. M.

1201

Bates, D. R.

1342

Becker, M.

Berkowltz, D. S. 821

Binns, W. R.
gis

Biondt, M. A.

$$
2019
$$

Black. J. H.
826.

Bowers, M. T.

$908 \cdot 929$

Broulllard, $F$. 1599

Castleman, A. W.. Jr. 959

Chang, 5 .

Chen, A.

2019

Chesnoulich, W. J. Y $2 \dot{y}$

Chureh, M. J.

Claeys, w. 1599

Corbin. R. J. 1059

$\underset{948}{\operatorname{Crosg}, R^{\circ} \mathrm{J} ., \mathrm{Jr}}$ $\underset{\substack{\text { Davidson, J. A. } \\ 078}}{\text {. }}$

Dotan, I. $978 \quad 995 \quad 1096$ 1105

Filitzur, M. $849^{\circ}$

Fehsenfeld, F. C. $978995 \quad 1096$ 1105

- Anicich, v. G. 797
Ferguson. E. E.

$978 \quad 1096$

Fukuda; K.

Glosik, J.

1573

Grlet, D.

Guha, M.

2113

Hasegawa, K. 281

Hell gelst, M.

$$
454
$$

Helm, H. 1092

Herbst, E.

440

Herman, $z$.

Hillier. I. H.

983

Holland, P. M. 959

Hepper, D. $G$.

883

Howard, C. J. 978

Iglesias, E. R. 844

Illles, A. J.

$\$ 28$

Jennings, K. R. 928 .

Johnsen, R. 2019

Johnson. S. G.

Jensson, B.

$$
885
$$

Karlat rom,
885

Keesee, R. G. 959

$\underset{908}{\text { Kemper, }}$ P. R.

Kendrick, J.

$$
983
$$

Kcyano, I. 20.44 .

Kremer, L. N.

$$
948
$$

Kuntz, P. J. 983

Longer, W. D. 838

Lifshitz, C. 886906

Lindinger. $w$. S95

Loew, G. H. e21

Malkhasyan, $K$. T.

Mandl, A..
Matauura, Y. 378

McAl1 ister, T. E37

McGowan. J. H. 1599

HeIsels, G. G. $928 \quad 597$

Metral, C. J. SAE

Mlller, T. M. 305.541

Munson, B.

172

Nel lson, P. V. 508 .

Néta, P.

Nikolaev, E. N. 2454

Nygaard, K.J. 1059

Pacak. V. 126

Parent, $c$. SO8

Po. F. $\mathrm{L}$

Polley, C. b. 172

Porter, R. F. 276.277

Poulaer $t$, $G$. 1555

Pozharcu, S. L. $2455 \quad 2456$

Radus I I P P
.277

Ral Lastidar, $T$. K. 2113

Rakghit, A. B. $1573 \quad 1647$

Sarggyan, G. N. $2448 \quad 2449$

Schearer, L. D. 1059

sleck, L. H. E69

SIIK. J. $\varepsilon 44$

SmIth, D. $\begin{array}{lll}181 & 309 & 428 \\ 429 & 847 & 941\end{array}$ $1573 \quad 1633$

Snow, W. R. 1059

stock, H. M. P. 1647

Straditng, R. S. $52 \mathrm{E}$

Strelt, G. E. 1056

Sull Ivan, J. P. 440

Parks, J. H. 
Tanaka. K. 2044 Tantsyrev, G. D.
2454

Terwllifiger. D. T 906

$\underset{886}{\operatorname{Tlernan}}$ T. $\underset{906}{0 .}$

Tunitgkii, N. N. 2448

Twi ddv. N. D. $1573^{N} i 647$ van Hassenove, $G$. 1599

Varney, R. N.

1092

Verboom, G. M. L 997

Vigglano, A. A. 578

Wareing. D . P. 1647 watson, W. D. wennerstrom, $H$. 885

Holf, G. K. 454

Hu, F. L. C. Eee $\$ 06$ Yencha, A.j. 176

Znurkin, E. S. 2448 244S 
A14
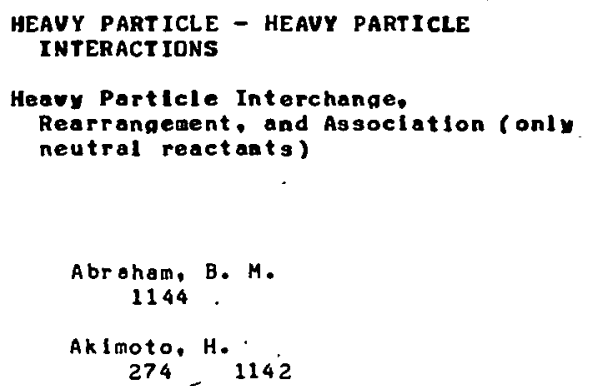

Breckenrldge, W. $H$. 283

$\underset{796}{\text { Brel tenbach. L. P. }}$

Brown. Na J. 1347

Brcwn, R. D. H. 863

Erunet, $H$

$$
299
$$

Brzychey, A. 815

Burcat A.

Butler, J.E. 782

Butler, ili R.

Cakmak, A. C. 707

Caledonia, G. E. 1980

Caupbe11, I. M. 111

Cage, D. A. 303

Chanil, .I. $S$. 1136

Chapman, F. M.. Jr. 308

Chapman. S. 760 2059

Chiu, $\mathrm{Y}_{\mathbf{L}}-\mathrm{N}$. $110 i$

Chol. B. H. 767

Clarvo D. C. 女ó 8

Clewens, L. 308

Clough, P. N. 2039

Clyne. M. A. A. 870

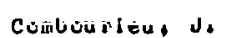
292

Connor, J. N. L. 171

Conway, J. G. 1227

Cool ${ }_{936}$ T. A.

Cccmbe, R. D. 1145

rugtanoula, P: e54 1060

Dogdiglan, P. J. 442 6\$9 1986

Dahler. J. S.
910 .

Danl lychev, V. A. 2452

Datz, 5 . 2015

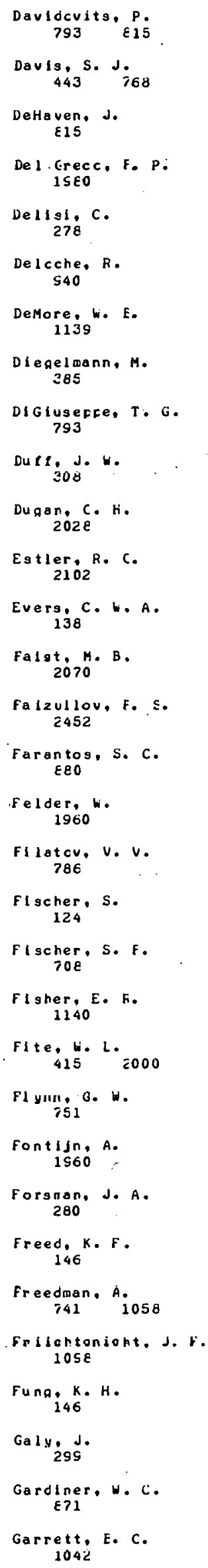




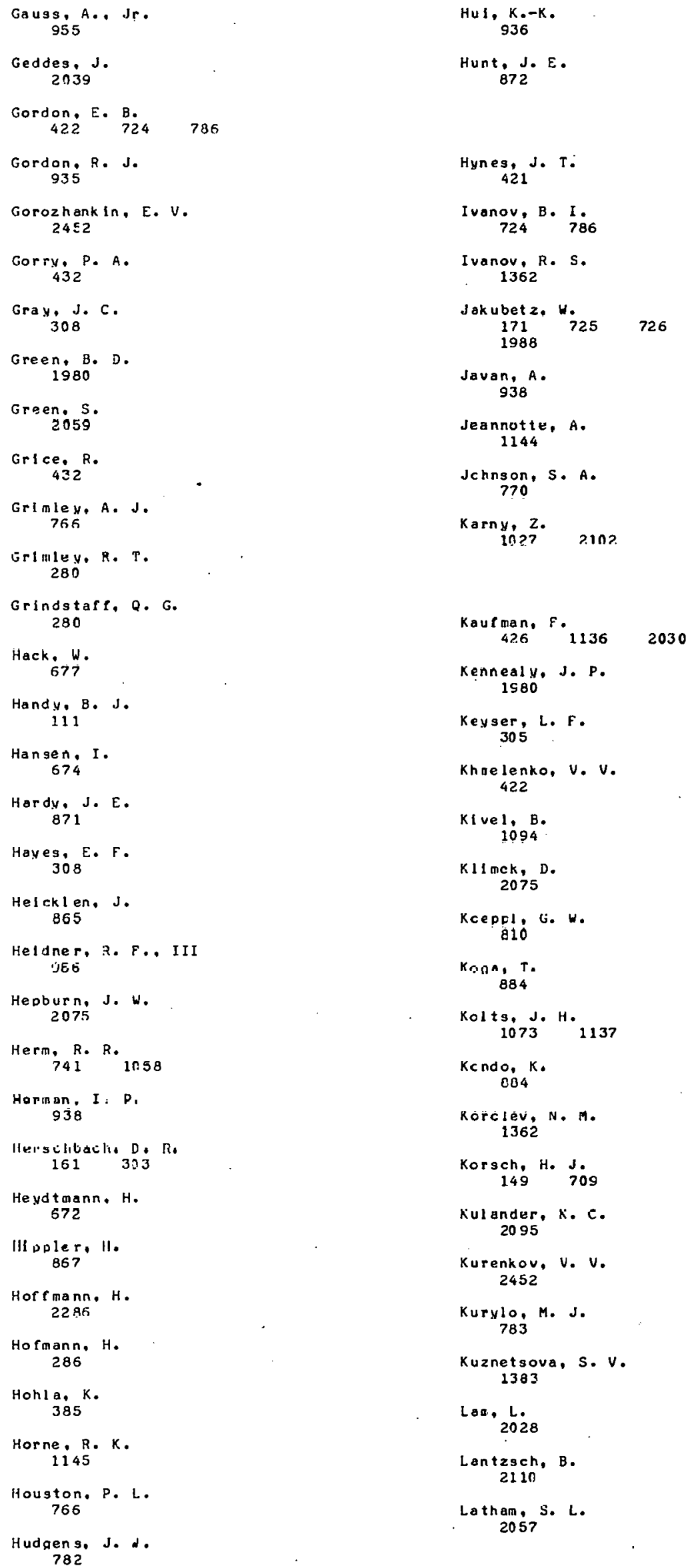

Gauss, A. . Jr. 955

Geddes, $J$. 2039

Gordon, E. B. $422 \quad 724 \quad 786$

Gordon. R. J.

935

Gorozhank in. E. V. $24 \leqslant 2$

Gorrs. P. A.

Gray, J. C.

308

Green. B. D. 1980

Green. $S$. 2059

Grice, ${ }_{432}^{R .}$

Grimle $x$. A. J. 766

Gr1m1Ey.R. T. 280

Grindstafe, Q. G. 280

Hack, W

Handy, B. J. 111

Hansen, I. 674

Hardy, J. E.

$$
871
$$

Haves, E. F. 308

Helckl en, J. 865

Heldner, R. F., III JEs

Hepburn, J. W. 2075

Herm, $R$. $R$. $741 \quad 1058$

Horinan. I; P,

$$
938
$$

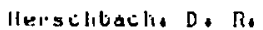
161303

Heydtmann, H. 672

III ppler, H. 867

Hof mann, H. 22 ค6

Hofmann, H. 286

Hohl a, K.

Horne, R. K. 1145

Houston. P. L.
766

Hudgen s, J. d.

782

Karny, Z 1ก?? $2.10 \%$

Kaufman, $F$ $426 \quad 1136 \quad 2030$

Kenneal $y, J$. P. 1980

Keyser, L. F. 305

Khaelenko, V. V. 422

Kivel, $B$ 1094

KIImck, D. 2075

Kcepol, G. W. à

Kona, T. 884

Kolts, J. H. $1073 \quad 1137$

Kc nd O, K. 004

Rórclév, N. M. 1362

Korsch, H. J. 149709

Kul ander, K. C. 2095

Kurenkov, v. $v$. 2452

Kurvio, M. J. 783

Kuznetsova, S. V. 1383

Las, L.

2028

Lantzach, B 2110

Latham, S. L. 2057

LeBras, $G$ 292

Lee, J.: H. 312 1043 1095 2035

Lee, K. I 1035

Leore, S. R. $286 \quad 294$

LeU, M. T 1135

Levine, fi. D. $149 \quad 426$

Levitskil, A. A. 2453

Levy. M. R. 418

Light, G. C. $750 \quad 1004$

LIght, J. C. 2029

Lin. M. C . 782 sid

LIU, E. 1831

LI U, K. $563 \quad 2075$

LO, H. H. 415

Lohr, L. L., Jr. 736

Looakin, L. A. 448

Luntz, A. C. 2110

LuU, S. H. 867

Macuoneld, Fo G. 914

Maker, P, r. 796

Malikcv, M. M. $13 \in 2$

Manog, D. M. $2 \subseteq \epsilon$

$\operatorname{man} x, j$ 171

Nariello, R. P., Jr. $938 \quad 2110$

Maslev, A. I. 1383

Matsumoto, J. H. 790

Maxscn, V. I. 2110

Mcclelland, G. M. 161

Mef adden, D. L. 793

McNutt. J. F 2057

Met repculos. A. 
Mezhov-Deglin, L. P. 422

Michael, J. V.

$312 \quad 1043 \quad 1095$ 2035

ller, W. H. $765 \quad 1042$

MIllet, P.

299

Miziolek, A. W. 1138

Mol.ina, M. J. 1139

Moore, C. B. 914 Morrell, G. 0.

Mov, J.

Muckerman, J. T. 2070

Murrel1. J. N. 868 880

Nakatsuji.
884

Nel ger, $M$. 2286

Nesbet, R. K. 808

Niemezyk. T. M. 443

Niki, $\underset{796}{H .}$

NIP. W. $S$.

Northrup, S. H. 42.1

Nowlkow, C. V. 432

Numrich, R. W. 275

abi, $\frac{K}{864}$

กв., K.

1060

$\underset{274}{\text { Okuda, } M .} 1142$

Olson, R. E.

1831

$\Pi \cdot N e I 11, G . M$.
2039

Diel, A, L.

765

Dsherov, V. I. 448

Pal sner, J. A. 1227

Pang. H. F. 937

Pantel eev. V. I. 2452

Parr, T.P. 1058

Parson: J. M.
Pasternack, L. 699

Potterson, T. A. 2000

Payne, A. A. $312 \quad 1043$

Payne, W. A.. Jr. 1095

Payne, H. A. 2035

Pechukas. P. 1967

Perminov. A. P. $724 \quad 786$

Ferry, F. A. 410

Persky, A. 985

PhIllips, L. F. 773

Pill covich, D. 1145

Pitchford, L. C. $\$ 40$

Pitts, J. N., Jr. 410

Fitts, J. N. 874

Foe, R. T.

Pol ak, L. S. 2453

Pol any 1, J. C. $\begin{array}{lll}147 & 296 & 684 \\ 723 & 2075 & \end{array}$

Fellak, E. \$16 1967

Feromarev, A. N.

Puirs-Germain. 8 . 299

Popovicher, V. I. 2452

Poul et, G. $2 \ddot{2} 2$

Prenge 1, A. T . 815

Preston, R, K.

770

Preuss, A. W. 67

Puoachev, O. F. 422

Fatitz, H. 2071

Ratitz, H. A.

Fagul'skli, V. V. 2452

Hatner, M. A. 124

Fedmon, M. J. 2057

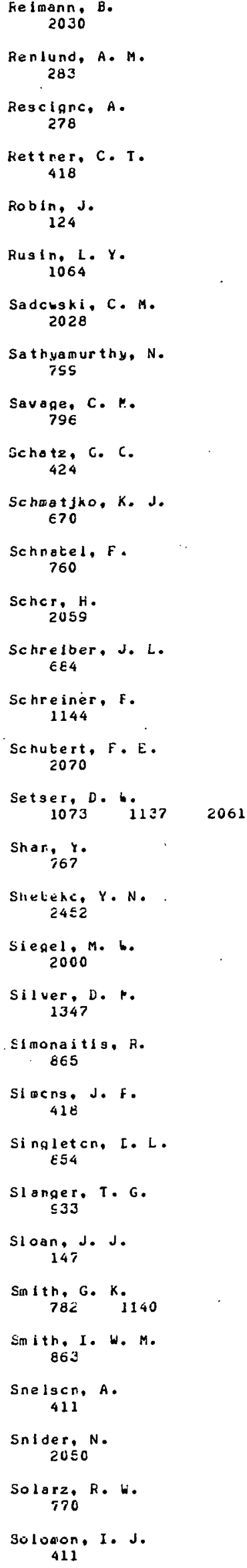




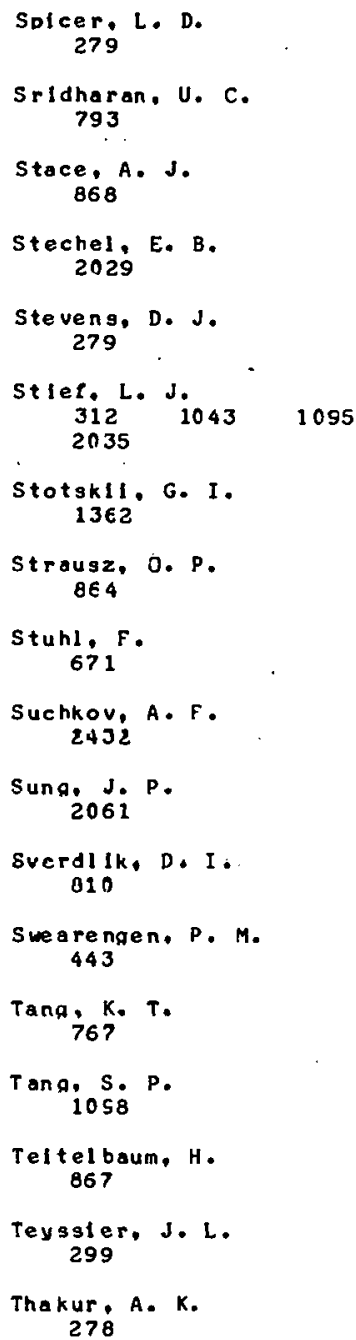

Soicer. L. D.

Srldharan. U. C. 793

Stace, A. J. 868

Stechel, E. B. 2029

Steveng, D. J. 279

Stlet. L. J.

$312 \quad 1043 \quad 1095$ 2035

stotskil. G. I. $13 \in 2$

Strausz, O. P. 864

Stuhl , F.

Suchkov, A. F . $24 J 2$

Sung, J.P.

sverdl lk, D. I .

010

Swearengen. P. M. 443

Tang, K. T. $76 ?$

$T$ ang. S. P. 1058

Teltel baum, $H$. 867

Teyssier, J.L. 299

Thakur. A. K.

Thompson, D. L. 447

Thrush, B. A. 676

ToennIes, J. P. 1064

Troe, $J$ 867

Truhlar, D. 6.

Ushakov, V. G. 448

Utterback, N. G. S98

Vanroodsel aar, A 864

von $2 \times 1, B$. 998

vosu, P.

415

Vaughn, C. 424

Ven $21, G$. 12470 ค

Wagner, H. G. 677

Walker, R. B. 2029

Wallace, S. C. 2075

Washlda, N. $274 \quad 1142$

West, G. A. 751

Weston, R. E., Jr.
Wi cke, B. G. 1058

wesenfeld, J. R. 1951

bill comb, B. E. ISEG

Wolerus.J $E>0$

Wo $1 k, G .1$. 1991

worden, E, F. 1227

Wright, J. S.

Wratel, W. G. 385

wu, K. T.

turzberg, E 7 a

wratt. F. E. 2057

xyotrisen. 910

Yonezama. $T$. e\&4

Zandec, $L$. 1350

Zare, fi. $1027 \quad 2055$ 2102

zellner, $F$. 678

Zetzsch. C. $671 \quad E 74$ 
A15

HEAVY PART ICLE - HEAUY PARTICLE INTERACT IONS

Soin Exchange

$$
\text { Anholt, R. }
$$

A1 6

\section{HEAVY PART ICLE - HEAVY PARTICLE INTERACT IONS}

Electron Detacheent froe Negative Ions into Continuum

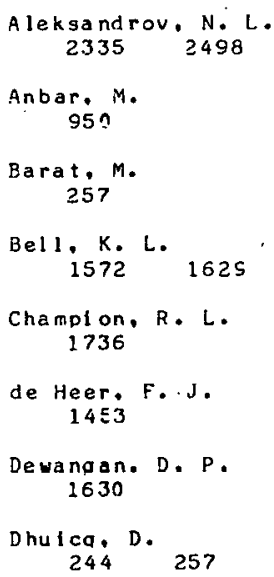

Falcon, $C$.

1544

Lol1, N.

Meucel, M. 2517

Opradolce. L. 1544
Placentini, R. D. $1 \subseteq 44$

Vicll no, $P$. 2517

Weber, h. G. 2506
Doversp Ike, L. D. 1736

Duncan, $M$. $M$ : 2147

Edwards,,.$R_{0}$, III 1736

Esaulov. V. 244

Fayeton, J 257

Gauyaca, J.P. 244

Helnemeler, J. 1839

Huelpl und, $P$. 1839

Kerkdijk, C. B. 1453

Kingston, A. E. 1572 1629

LIU. 8.
Madden, P. J. 1572 i625.

Menendez, M. $G$.

2147

Moruzzi, J. L. $180^{\circ}$

Ddca, R. H. S5O

ULsen, F. E. $8 B$

Fayoent, S. W. 180

Risley, J. S. 1453

Schnitzer, R. s50

SnIth, B. I 1736

Walters, H. R. J. 
A17

HEAVY PART ICLE - HEAUY PARTICLE INTERACT IONS

Interaction Potent Ials

Abouar. $R$
.984

Albritton, D. 1 . 1122

Alexander. M. $H$. 2048

Arora, P. S 417

Bagare. S. P. 1666

Bagus, P. S. 593

Dárusi ay. 'J. N. $1+4 c$

Borkan. E. S. 2496

Bartlett, R. J. sao

Bauer. W. 1029

Bavbut $t, P$. 1072

Becker. C. H. 2009.

Bedding, D.

1746

Behmen bura. 1388

Bollum. J. C. $17>0$

Benedek, $R$. 1001

Benaurla, $R$.

Ben Lokhdar. 2. 1162

Blenlek, R. J. 1701

Bobrowlez, F. W. 1072,1957

Borkman, R. F 764

Browne, J. C. 3no 2794

Brual, G. Jr 1361

Brunetti, B. 188

Buck, U 10252160

Buenker, R. J.

722

Bychkov, U. L. 2333

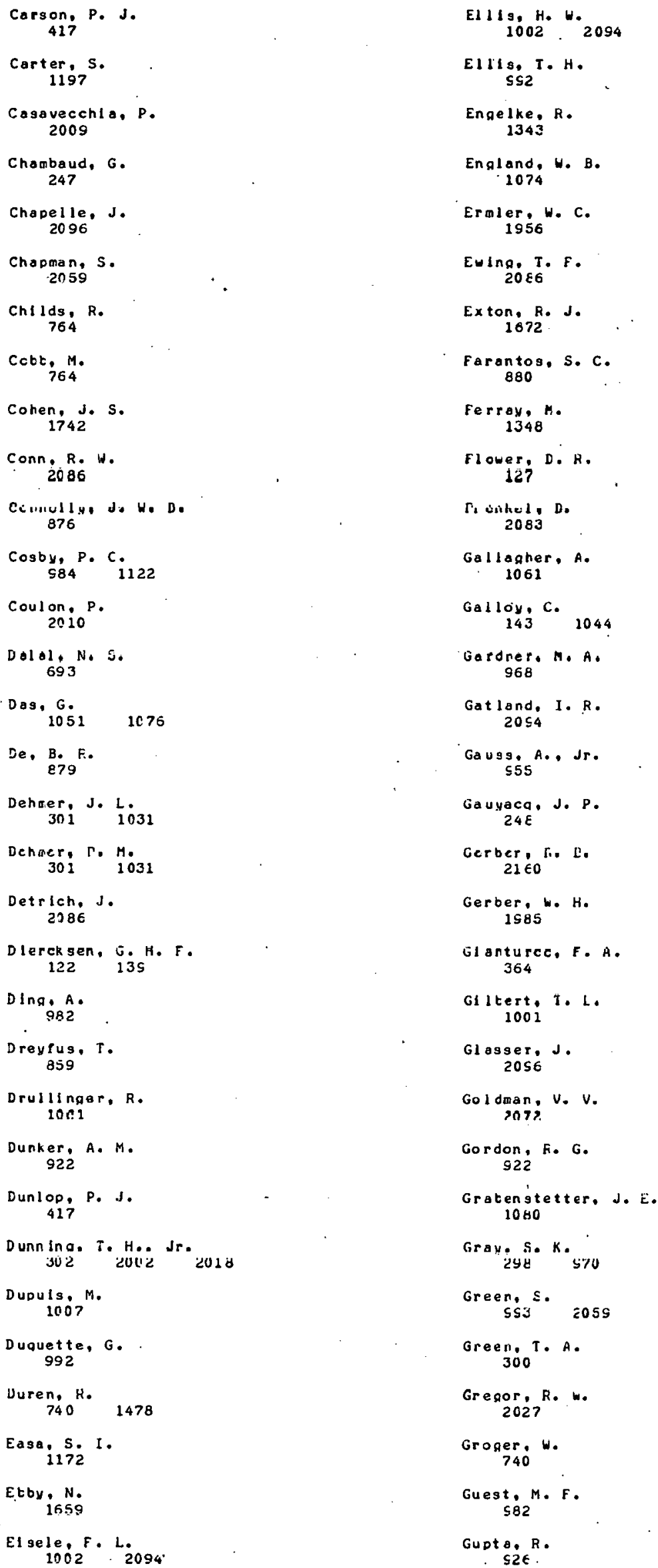

El $11 \mathrm{~s}, \mathrm{H}, \mathrm{H}$

1002.2094

EIIIs, T. H SS2

Engelke, R. 1343

Engl and, $W$. 1074 Ermler. W. C.

EuIng. T. F. $20 \& 6$

Exton, R. J 1672

farantos, S. C. 880

Ferray,

Flower, D. R, 127 $r$ intiels $D$.

Gallagher, A. 1061

Galloy, C. $143^{1044}$

Geronet. W. A. 968

Gatland, I. R. $20 \leqq 4$

Gauss, A., Jr. $\$ 55$

Gauyaca, J. P. $24 E$

Gerber, fi. E. $21 \in 0$

Gerber, W. $H$. 1585

Glanturec, F. A. 364

G1 Itert, $T$. L. 1002

Glasser. J. 2056

Goldman, v. $v$. xas?

Gordon, F. G. 922

Grateng'tetter, J. Ë. 1060

Gray. S. K. 2yt $\$$

Green, $s$. SS3 $\quad 205 s$

Green. T. A. 300

Gregor, R. w. 2027

Groger. W. $740^{\circ}$

Guest. M. F. 582

Gudto, R . 


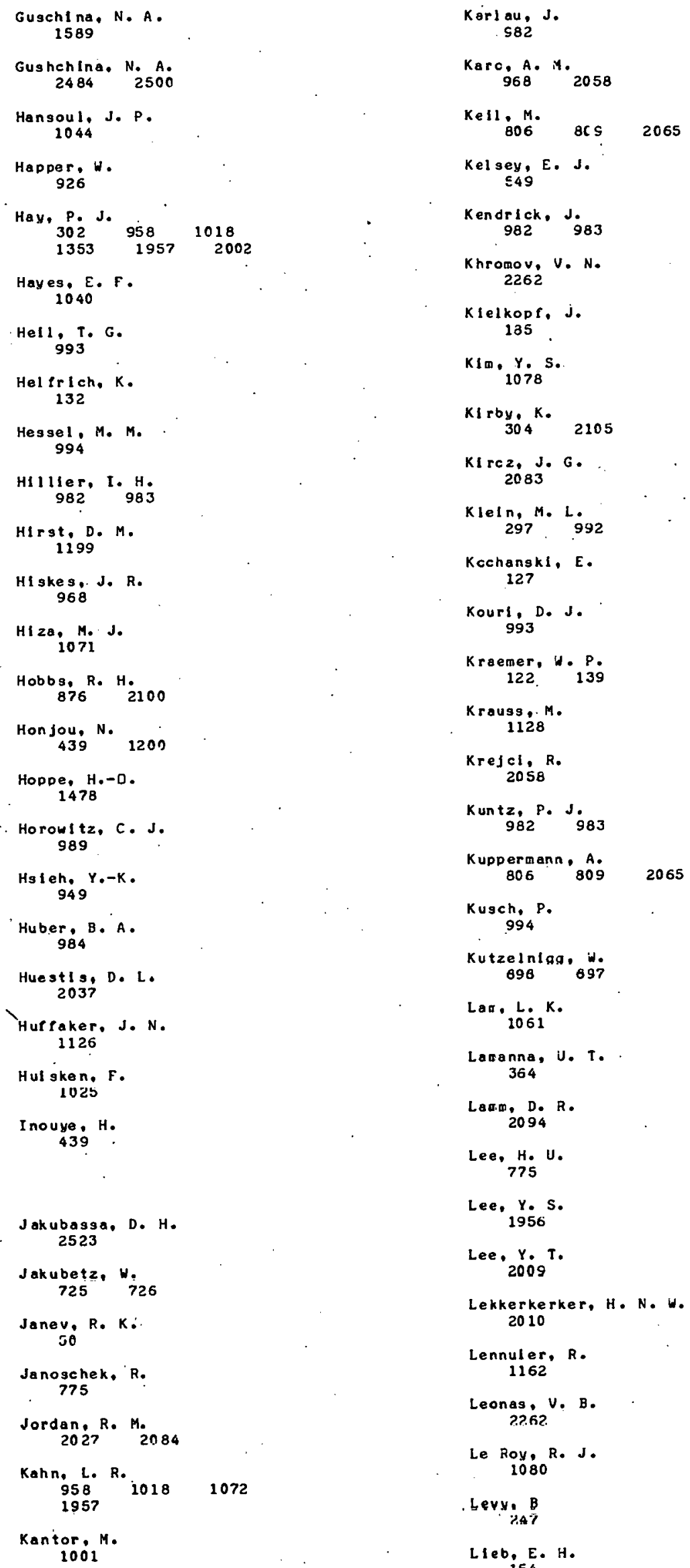

Kerlau, J

Karo, A. M. $968 \quad 2058$

Kell, M.

Kel sey, E. J. $£ 49$

Kendr I ck, J.

Khromov, V. N. 2262

Kielkopf, j. 135

KIm. Y. S. 1078

KI rby, K. $304^{k .} 2105$

Kircz, J. G. 2083

KleIn, M. L. $297 \quad 992$

Kcehanskl, E. 127

Kourl, D. J. 993

Kraemer, H. P. $122 \quad 139$

Krauss, M. 1128

Krejci, R.

$K$ untz, P. J. 982 S83

Kuppermann, A. $806 \quad 809 \quad 2065$

Kugch, P. 994

Kutzelnigg, H. 698 697

Lar. L. K. 1061

Laranna, U. T. 364

Laum, D. R. 2094

Lee, H. U. 775

Lee, Y. S. 1956

Lee, Y. T. 2009

Lekkerkerker, H. N. W. 2010

Lennuler, $R$. 1162

Leonas, V. B. 2262

Le Roy, R. J. 1080

Leyr, B

Lleb, E. H.

Lin, C. D.

1709

Liu: $\begin{array}{llc}\varepsilon 8 & 304 & 753 \\ 588 & 1007 & 1831\end{array}$ 2105

Liu, W. $-K$. 1080

Lloyd, J. 414

Lorquet. J. C. $143 \quad 1044$

Lozingot, J. 1348

Uyckx, F. 2010

Luzzatti, E. 788

$\underset{696}{\operatorname{maeder}} F$, ES?

Mahan, E. H. 925

Malt land, G. C. $11 \subseteq \varepsilon$

Makushkin, Y. S. 1235

Malrieu, J. $-P$. $7 \in 3$

Manickkavachagam, $R$. $\varepsilon \in 1$

Margollash, D. J. $\$ 47$

Martin, D. W. 2027

Masnou-Seeurs. F. 2163

Matcha. Fi. L. 1068 2005

MeCcurt, F. F. 1080

McDaniel. E. W. $1002 \quad 2094$

McDenold, I. R. 257

McGuire, $P$. 12

Meath, J. J. 547

Meler. P. F. 10682005

Menotti, F. F. 1745

Micha, D. A 1770

Michels, H. H. 300 E76 2100

Mies, F. H 1128

MI I lcur, M. B. $106 \varepsilon$

Millie, P. 247

Mo hammad, S. $N$. 2337.2340 


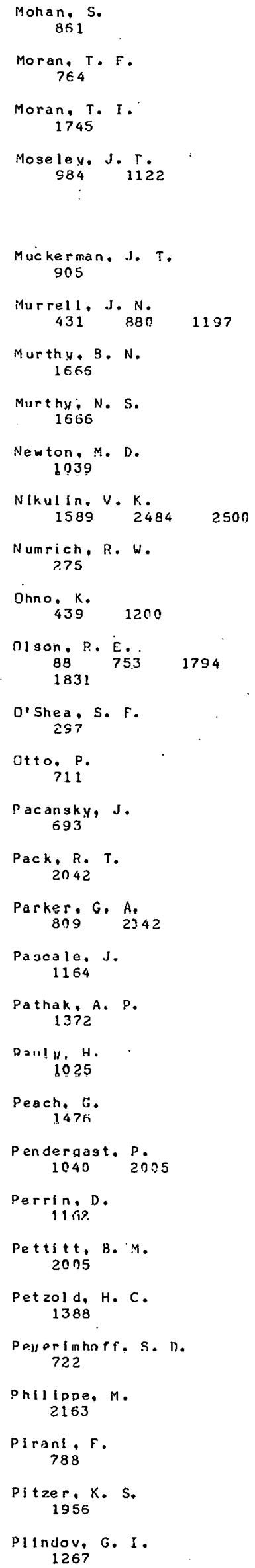

Pogrebnva, S. K. 1267

Pope, W. M. 10022094

Prissett, J. 127

Proctor, T. R. 10892073

Pugh, D. 414

Purvis, G. D. 930

Radtsig, A. A. 2333

Radulovic, 2. M. 50

Ranson, $\mathrm{P}$. 2096

Redmon, L. T. 1794

Rebingon, R. I.., Jr. 1071

Romelt, J. 722

Rcthsteln, S. M. 1961

Rulis, A. M. 461

Sabelll, N. H. 1001

Sagara, M. 439

Sannigrahi, A. B. 879

Sexsen, $P, D$. $984^{2} 2105$

Sayer,

$1164 \quad 1348$

Schaefer, H. F., III 925

Schleusener, $J$. $1025 \quad 2160$

Schlotter, N. E. Pn 37

Sehmidt, H. M.

132

Senór, $H$. 2059

Jitiratring, $D$. 758

Schumacher, E. 1985

$\underset{461}{\text { Scoles, G. } 992}$

Shapiro, it. $695 \quad 2160$

Shimakura, N. 439

Shipsey, E. J. 1794

3liobatake, $k$. 1029

shukla, G. C.
Siegbahn, F. 588

Silvera, I. F. 2072

Siska, P. E. 20272084

Slankas, J. T 806

Sm Irnov. E. M. 2333

Sn Ith, K. M. 461

Snow, H. L. 1672

Soong, S. C. 1709

Splegelmann. $F$. 763

Spruch, L. 549

SHIvastava. M. P. 1372

Stevens, h.: J. 1128

St Ine, J. R. sos

Stwolley, H. C. S4S $\quad 105 i \quad 1089$ 2073

Tadjedine, M. 1122

Taketa, h. 1200

Tam, A. C. S4S

Tanaka, $K$. 1200

Tang, K. T. 1099

Taylcr, H. S. 2018

Thackston, N. G $1002 \quad 2094$

Thcads: L. L

Tueninies, J.P. 1025 10s

Truhlar, D. G. $275 \quad 689 \quad 1072$

Ulenikcv, O. N. 1239

Ungea ach, S. H. 525

Valance, $A$. $313 \quad 734$

Vall Iron, P.

$$
21 \in 3
$$

Vallee, 0 2096

Valley, H. 2018 
van der Elaken, J. 2083

van der Peyl, G. J. Q. 2083

Varandas, A. J. C 1197

Vecchiocattivi, F. 788

Visticot. J.P. $1164 \quad 1348$

von Hirschhausen, $H_{0}$ 132

Wadt, W. R. $912958 \quad 1018$ 13531957

Wagner, A. F. $1076 \quad 2058$ Wagner, J.
Hahl. A. C.

$105121076 \quad 2058$

Wakeham. W. A. 1198

Wol aschewski, K. 1029

Watts, R. 0 992

WeIse, J

Wentger, $s$. 1659

$\underset{\text { S2 } 6}{\text { Wennmyr. }}$.

Wilson, s. 156

WInter, N. W.
Wright, J. S.
298

Wright, L. A. E76 2100

Wu, A. A. 811

Wu, C. Y.R. 2073

Yang, s. $-c$.

Zare, R. N. $20 \leq 5$

ZeIrI, Y $\epsilon \varepsilon 5$

Zerke, w. T $10 \leqslant 1$ 
Á1 8

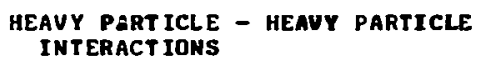

Angul ar Scattering (specified process

or otherwise)

$\checkmark$

Aldag, J. E. 552

Amme, R. C. 1710

Andersen, $\mathbf{N}$.

1521

And resen, B. 2555

Andritamon jo. S.

355

Baver. K. G. 1646

Bergmann, $K$. 2146

Blakley, C. R. 81

Boring, J. W. 1490

Buck . U. 2160 Chemin, J. F.

Cheng, K. 70

Clemens. E. 1477 1527

Davis, J. P. 464

De. IO if 317

de Vris, A. E. 136

Duren, $R$. $740^{R} \quad 1478$

EIy, D. J. 2171

Engelhardt. $n$. 2146

Este. O. 0 . 731

Evara, G. W... 136

Fazly. Q. 1646

Flannery. M. R. 2104

Folkmann, $F$.

1545

Frlichtenlcht, J. F. 1098

Futrel1, J. H.

Gayet. R. 355

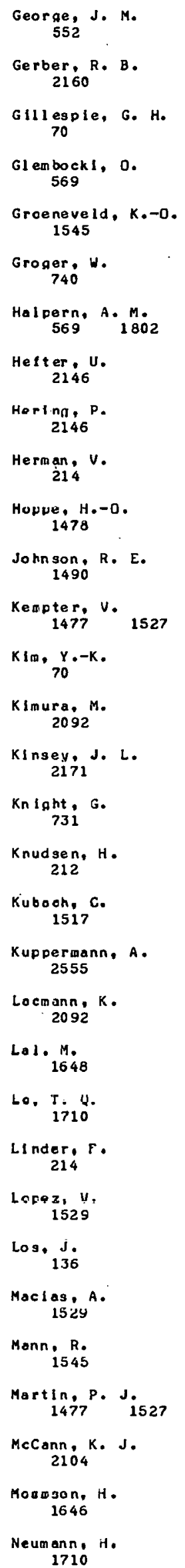

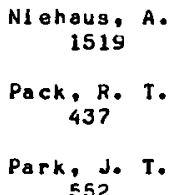


Sidis, $v$.

1517

Sigmund, $P$. 231

SmIth, K. M.

461

Sperber. D. 317

Spiess, G.

1516

Sprueh, L. 1513

Srivastava, M. K. 1648

Szato, G. 1545
Tang. S. P.

1098

Thorson, $W$. R. 464

Trautmann, D. 1505

TripathI, A. N. 1648

Volance, A.

van Zyle, B. 1710

Vedder, M. $\underset{\text { Vestal. M. L. }}{\text { Q }}$

Hay, K. F. 2171

Wlcke, E. G. lose

wit t J.

Yanez, $M$.

Zehnle, L.
1477 
A29

\section{HEAUY PART ICLE - HEAUY PARTICLE INTERACT IONS}

Inner-Shell Interactlons (not covered under other specifled categories)

Andersen. H. H.

$$
6
$$

Anholt, R.

$$
\text { oft. R. } 4157
$$

Antar, A. A. 1725

Armbruster, P. 2503

Behncke. H. H.

Bernstein, E. M. 11

Delline, í, 2502

Betz, $W$

318

Davis, C. K. 1785

Uonahue, b. I 11

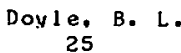

Duggan, J. L.

$$
1700
$$

Elchler, J.

C1 FIYI, A. R. 1899

Ellsworth L. D. 25

Fel dịnañ. L. C.

Fintz. P. 1266

Folkaann, $F$. 2503

Forster, J. S 1795

Fortner. R. J. 1612

Grav. T. J. i7fi Greenhern, t. S:
l7e5

Greiner, $W$. 318

Guil llaume, G. 1266

Hagmann, $\$$. 2503

Hal 1.J.M. 1735 M. 1761

Hara, N. 1908
$\underset{95}{H o p k \ln s,} F$

Jaglson, K. A. 95 $1735 \quad 176$

Johnson, B. M. 1266

Joneg, K. $W$. 1266

Jundt, F, C. 1266

Kessel, Q. C.

Kirsch, J. 328

KI lwer, J. K.

Kcwalezyk, S, P. 1352

Lennar d. $W$. N. 1793

Lichtenbery. W. $2170 \quad 2507$

11000 , D. 51.2503

LIn, C. D.

Lur lo, A.

$$
6
$$

Lutz, H. O.$$
2135
$$

Mocdonold, J.R. $251^{2} 2503$

Marshall, R. E. 1899

Martin, R. L. 1352

Matthews, D. L. S5

MeDaniel, F. D. 1700

McIntyre, L. C.. Jr. 11

MeMurray, W. M. 2135

Merta. R.

Meyerhof, W. E. $8 \quad 1479$

Middlesworth, E. M.. Jr. 11

MIIIer, P, H. 1700

MItche11. I. V. 1793

Mitsushina, Y. 1908

Mckler. $P$.
51

Mokler, P. H. 2503

Mcnigold, $G$. 1700

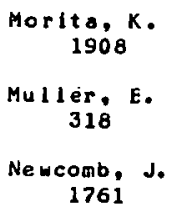


A20

HEAVY PARTICLE - HEAVY PARTICLE INTERACT IONS

Attenuation (unspeclifled process)

Coxon, J. A.

1085

De, J. N.
Frilichtenlcht, J. F. 1098

Kamperschroer, J. H.

Post, R. S. 476

Setser, D. W. 1085

Sperber, D. 317
Tang, S. P

1058

Tellinghuisen, J. 1085

Tellinghuisen, $P$ : $C$. 1085

Velazco, J.E. 1085

Wicke, B. G. lose
BO1

INTERACTIONS UITH STATIC OR TIME-VARYING ELECTRIC AND MASNETIC FIELDS

General

And ree $v, S . P$. ¿311

Anosov, M. D. 2435

Bac skay, G. B. $688 \quad 691$

Bernhardt, A. F. 1835

Brand I, H. S. 680

Cook, R. J. 1 R.35

Delone, G. A. 1210

vimitrijevic. M. S. 1664

Ehlotzky. F. 1212 $\underset{99}{\text { Ehrich, H. }}$

Gready, J. E..

$688 \quad 691$

Grinchuk, V. A. 1210

Grujic, P.

Hugh, N. S. $688 \quad 691$

Kazontsev, A. P. 1210

Kelleher, $[$. $E$. 99

Klyuchnik, A. V. 347

Koch, P. M. 2152

Kollier. $B$.

Landi Degl'Innocenti, E. 634

Letokhov, V. S. 2439

LIsitsa, V. S. 2311

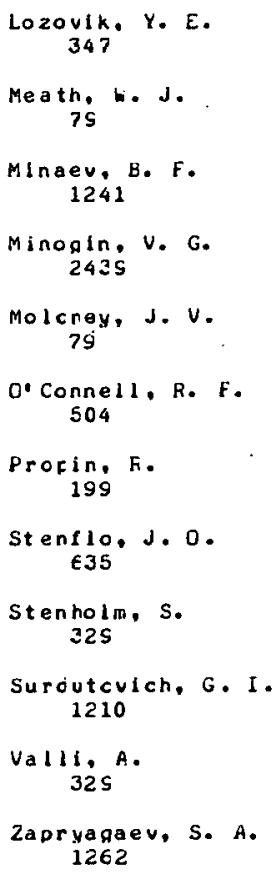


$\mathrm{BO3}$

$$
<
$$

INTERACTIONS UITH STATIC OR TIME-VARYING ELECTRIC AND MAGHETIC FIELDS

Ionlzation

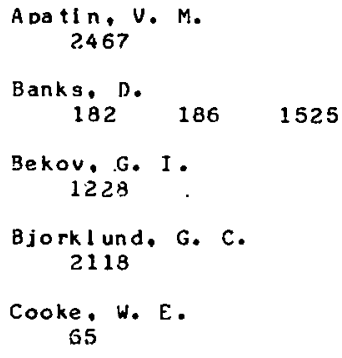

HO4

INTERACTIONS UITH STATIC OR TIME-VARYING ELECTRIC AND MAGNETIC FIELDS

Detachaent

Blumberg, W. A. M. 2141

B05

INTERACTIONS UITH STATIC OR

TIME-VARYING ELECTRIC AND MAGNETIC FIELDS

Quenrhl nu!

Abella, I. D.

1230

806

INTERACTIONS UITH STATIC OR IIME-VARYING ELECTRIC AND MAGNETIC
FIELDS

Excitation
Delone, N. B.

$$
2447
$$

Falrehild, C. E.

967

Freeman, $R$. $R$. 2118

Gallagher, T. F.

65

Geltman, S.

1569

GIlbody, H. B.

451

Kosh, M. M. 2153

Kleppner, $D$. 2153

Kocher, C. A. $96 ?$
Kologov, V. V. 1204

Kralinov, V. P. 2447

Leopold, J. G. $182 \quad 186 \quad 1525$

Letokhov, V. S. $1228 \quad 2467$

LIt toan. H. G. 2153

Matveev, 0 . I. 1228

Mishin, v. I. $1228 \quad 2467$

VIalle, J.-L. $21 \mathrm{s3}$

$20 \mathrm{n}$. B. A 24.47
GII body, H. B. 451

Jorson. R. M. 2141
Larscn, $D . d$. 2141

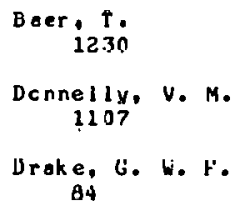

Kaufsan, F.

1107

Levy, L. H. 1106

Vă wi Jngadroen, A. O4
Economou, N. P. 2180

Freeman, R. R. 2180

Gil body. H. B. 451
Lu, K. T.

2180

Nevesakli, A. É.

2442 
Folsal, F. H. M. 1468

INTERACTIONS UITH STATIC OR TIME-VARYING ELECTRIC AND WAGHETIC FIELDS

Colllsions in Presence of Intense Electrosagnetle Fleids

Abella, I. D. 1230

Akulin, V. M. 1376

Alekseev, A. L. 2268

All mplev, S. S. 1376

Baer. $T$.

Baká̃v, D. $S$. 1251

Bakhrakh, V. L. 1257

Bellum, J. C. 903 1987

Beterov, I. M. 2472

Bonch-Bruevich, A. M. 23212463

Brand1, H. S. $494: 1767 \quad 1959$

Bryant . $H$. C. 2115

Burshtein, A. I. 2313

Caddlck, J. 2145

Cagtro, J. J. 494

Chand, $P$. $341 \quad 1268 \quad 2519$

Chebotaer, V. P. 2472

Copel and. D. A. 2034

Dagg I. R. 682

Devries, P. L. 28,2016

Doktorov, A. B. 2313

Donahue. $J$. 2115

Dubov, v. S. 2277

Eberly. J. H.

Ehlotzky, F. 1212

Ermachenko, $V$. $M$. 2268

Evseev. I. V. 2260
Fateev, N. V. 2472

Flnkel'shteln, v. Y. 2271

Fog $11 a, C$.

George, T, F。 $28 \quad 503 \quad 1587$ 2016

Gecrges, A. T. 1717

Gordienko, V. M. 1385 .

Grom, P. A. M. 2115

Gudzenko, $L$. I. 2277

Gurvich, L. v. 2277

Hellfeld. A. V. 2145

Jeln, M.

1711

Jung, $C$.

101

Karlou, N. V.

1376

Kazantsev, A. P. 2297

khromov, v. $v$. $2321 \cdot 2463$

Kolller, B. $494^{\circ} 1767 \quad 1559$

Kruger, H. 101

Lam, K. $-S$. 1987

Lambropoulos, P. 3717

Landi Degl'Innocenti. E. 634

Langendam, P. J. K. 1593

Lee, H. W. 2016

Letokhov, V. S. 2315

Liant. J. 1760

Lins de Barros, H. G. P. $494 \quad 1767 \quad 1959$

Lcpasov, V. P. 2261

Mahlab. M. 's 28

Mouroyaninis, $\mathrm{C}$. 562

Mikheenko, A. $V$ 1385

Minogin, v. G. 2315

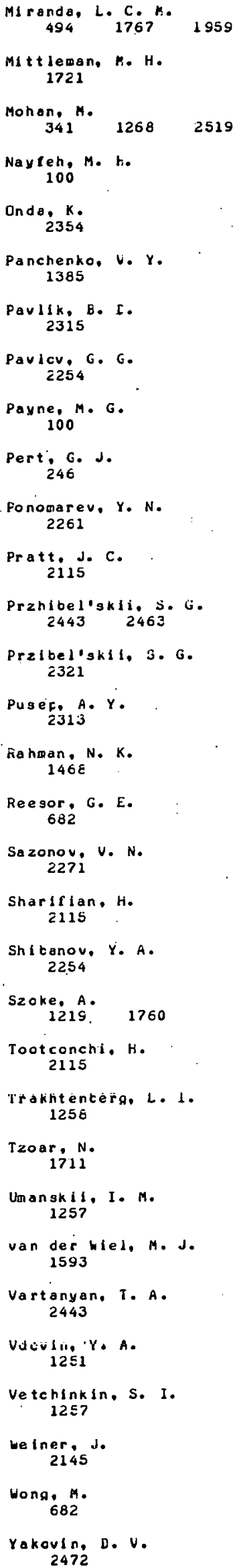


Yakovlenko, S. I.

$2277 \quad 2459$

Yates-WIII I am s, M. A. 2115

$\operatorname{col} 1$

PARTICle PENETRAT ION IN MACROSCOPIC

MATTER ( IONS, NEUTRALS, AND ELECTRONS)

General

Douthat, D. A.

1739

$\underset{484}{\operatorname{Gal} 11 \text { ard, M. J. }}$

Ge mme 11, D. S. 484

Goldring, $G$. 484

GreInor, $W$.

2551
Zhukova, N. I. 2297

Zuev, V. E. 2261
Inokut I, $M$

1739

Jengon, $f$. $E$.

360

Lauine, I .

484

Muller, $B$.

2551 .

Pletgch, W. J. 484

Polzat, J. C.

484.2157

Ratkowsk I, A.J. 484

Rau, $A, R$, P. 1739
Remlllieux, J.

$484 \quad 2157$

Schafer, $W$.

2551

St ccker. $H$. 2551

Vager, $Z$. 48.4

Watson, fi, L. 360

White, J. R.

wi lllams, M. M. R. 2400

7.abransky. E.J.

484 
$\mathrm{CO} 2$

PARTICLE PENETRATION IN MACROSCOPIC MAtTER (IONS, NEUTRALS, AND ELECTRONS)

Eneray Loss

$\underset{58}{\operatorname{Ahlen}}$ $\underset{1335}{\text { Akkerman }}$, A. F.

Alvarez, I 10 1816

Andersen, $H$. H. 891 1850 1852

Arista, N. R. $1158 \quad 1289$

Baer, $T$.

Baglin, J. E. E. I 897

Bah!

Baraglola, R. A. 1158

Bauer, $P$.

Beauchemi $n, G$ 1862

Beloghltgky, V. V. 2386

Bentin 1, G. G. 371

Berez In, A. K 1955

Bernstein. $T$.

$$
2426
$$

Besenbacher. $F$. 185 ?

Bottlger, J.

1802

Brandt, W. $370^{\circ} \quad 507$

Braun, $M$.

1849

Brennan, J. G. 1853

Brice. D. K. $1291 \quad 1367$

B rown. M. D. 1853

Cance, $M$. 2409

Carnera, A. 371

Chen, F. K.

$$
2119
$$

Chernov, G. Y. 1335

Chu. W. K. $1849 \quad 1897$

Cisneros, $C$. is 1816
Clerc, ${ }_{2528}^{H . G}$.

Cowern, N. E. B. 46

$\operatorname{Cox}$. M. G.

Cruz, S. A. io 1816

Cuomo, J. J.

Davies, J. A. 1949

$\underset{66}{\text { Dehmer, J. L. }}$

Della Mea, G. 371

Dettmann; $K$. 1153

Douthat, D. A. 1739

DrIgo, A. V.

Drcuin, $R$. 1862

Eckardt, J. C. $1158 \quad 1703$

Edvabny i, I. V 2429

El FIqI, A. R. 1899

Ellmer, $K$. 1337

Evdoki mov, $0 . B$. 2291

Fahlander, $C$. 1305

Fainberg, $Y$. B. 1955

Follavier. M. 1858

Freeman, J.M. 46

Fursov, G. L. 1955

Gel nar. J. 2281

Genthon, J. P. 2409

Gertner, I . 1798

Grishaev, I. A. 1955.

Oullner, i。 2546

Honke. C. 1903

Harris, J. M 1857

Hér inandez, F . C. 1371

Hofmann, S. 2546

Hornung. $H$. 2533
Inckut $1, M$.

$66 \quad 1739$

Is higure, $N$. 2347

Jarvis, C. N.

1155

Jensen, P. $S$. 1902

Johonssen, $K$.

1305

Kalish, R. 2426

Ka I2: 5

Karlsson, E. 1305

Katterwe. H. 2281

Ki selev, V. A. 1SSS

KII wer, J. K. 1899

Knudsen. H. 891 $1850 \quad 1852$

Kreutz. F.

1848

Kreysch, $G$. 2394

Kuaokhov, M. A. 2386

Land. $\mathrm{c}$. $J$. 1.853

Langley, R. A.

Lantsehner, $G$. 1158

Laubert, $R$. 2119

Lauraen, J. 1903

Leteurtre, J. 2409

Lisin, V. A 2432

Lo kussc. $s$. 371

Love, G. 571

Lueas, M. $H$ 1154 1155

Luomajarvi, M. 2404

Lur 10. A. 1855

harcovich, A. 2426

Marshall, R. E. $185 S$

Martinl, $v$. 1852

$\operatorname{Marx}$. D. 2546

Mason, J. P. 


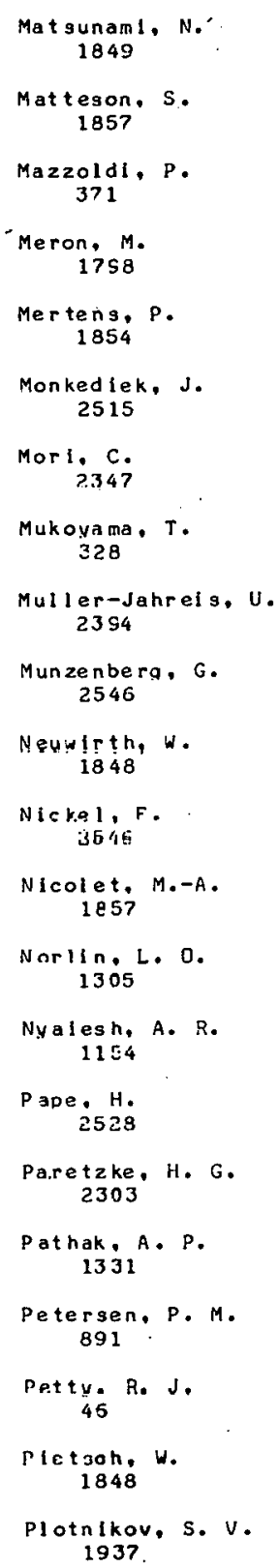

$\cos$

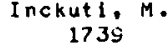

Porter, L. E.

$1341 \quad 1915$

Pcssnert, G. 1305

Pretorlus, R. 1857

Rau, A. R. P. 1739

Ritchle, R. H.

Robinson. J.E. 370

Rcciendaal, H. E. 2415

Rosendahl, E. W. 2515

Rcsner, $B$. 1798

Rozum, E. I. 1937

KUO, N. 1902

Ryzliov, V. V. 22:1

Safronov, V. G. 1955

$S$ anders. J. B. 2415

Schmidt, K. $-H$. 2528

Scholdt-Bocking, $H$. 2533

$S \cot t, v . \quad$ L.

Semrad, D. 1856

3herwood, A. C. 1155

Siuons, D. G. 1853

Smirnov, b. N. 2476 $\underset{46}{\operatorname{Set} i \text { eld }, C . J . ~}$
Soullard, J.

Steckelmacher. W. 1154

Stelinbeck, J. $11 \leq 3$

Stietling,J. 2282

St urm, J.

Sugiyama, H. 1905

Thomas, J. P. 1858

Therfson, D. A. 1849

vitalis. Fo 2415

vorcíev. S. A. 1937

Vyatatin. A. Y. 2476

Wa tanabe, T. 2347

Wat anate, $Y$. 326

whitehead, $c$. 1155

Wi lllaws, H. M. H. 2400

Yalovets, A. P. 2251 2429

Yarlogadda, B. S. 370

Yunda, N. T. 2432

Zeidlits, V. P. 1955

Zhivcpistsev, F. A. 1371

Zlegler. J. F. $186 i$ ids 
PARTICLE PENETRATION IN MACROSCOPIC MATTER ( IONS, NEUTRALS, AND ELECTRONS )

Partlele Range

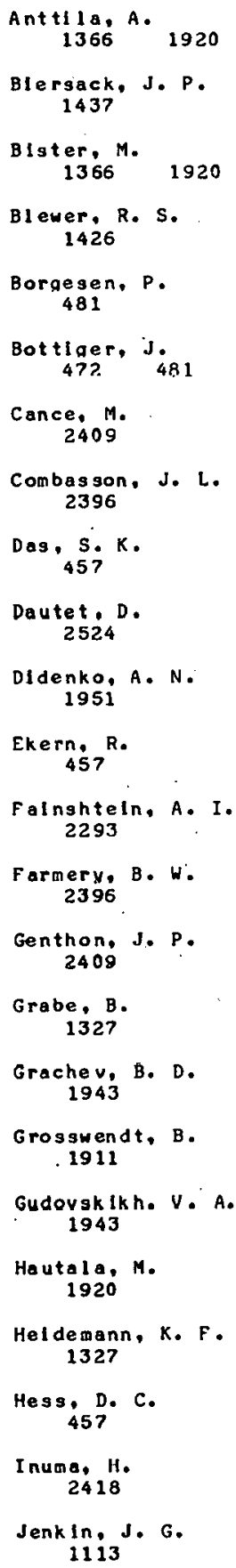

Pate, B. D.

Pet rochenko, A. F. 1943

Rahla, B. K. H 1437

Riccato, A. 1437

Roth, J. 1426

Sidenius, $G$. 2405

Sorengen, $H$. $471 \quad 483$

Soullard, J. 2409

Szajoan, Jo 1113

Tanifuji, $T$. 394

Te Kaat, E. 1327

Thacker, G. R. 1437 Thompson, H. W
2356

Isvetkev, V.I. 1951

Uchida, $k$. 394

Uecker, t. 1437

Usov, Y. P. 1951

Vengkytis, F. J. 2380

Haitel, E. 1911

Wu, C.P. 1884

Yamonura, Y. 2418

Zinamon. 2 . 1753 
$\cos$

PARTICLE PEHETRATION IN MACROSCOPIC

MATTER ( IONS, NEUTRALS, AND ELECTRONS)

Multiple Scattering

Andersen, $H$. H. 1850

Baer: $T$.

Den, P. 1842

Besenbacher, $F$. $1827 \quad 1850 \quad 1898$.

Aríce, D. K. $1367 \quad$ i $860 \quad 1861$

Choyke, W. J.

404

Combasson, J. L, 2356

Cowern, N. E. B. 46

Dehmer, J. L. 66

Eckardt, J. C. 1703

Ellmer. $k$. 1337

Farmery. B. W. $23 \mathrm{~s}$

Fit ting, H.J. 2239

Freeman, J. M. 46

Gail lard. M. J. $484 \quad 2157$

Gemme 11, D. S. 484

Glaefeke. $H$. 2239
-

Goldring, G

484

Guttner, $K$.

2546

Helinemeler, J. $\begin{array}{lll}1827 & 1839 & 1898\end{array}$

Ho tau ann, S. 2546

Huel plund, $P$ 1827 i839 1898

Inckut 1, M. E6

$K a 12, D$. 2394

Knudsen, $H$. $1827 \quad 1850 \quad 1898$

Arevaen, $t$. 2394

Lally, J. S.

404

Longley. $R$. A. $1367 \quad 1860$

1861

Levine, I.

484

Magee, C. W. 1884

Marx. D.

2546

Mason, J.P. 46

MeCulloch, D. 2396

McGruer, J. N. 404

Moller. W. $1859 \quad 1914$

Muller-Jahrels, U. 2394

Munzen berg, $G$. 2546

Nellson, G.W. 2396
Nickel, F. 2546

Nocken. U. 1855

Noraan, $D$. 2236

Petty, R. J. 46

Pletsch, W. J. 484

Polzat, J. C $484 \quad 2157$

Ratkowskl, A. J. 484

Remillieux, J. 484 2157

El gaund, $P$. 1858

Solleld, c.j. 46

SpItznagel, d. A. 404

Sturm, J.

1337

Thompson, M. H. 2396

vager. 2 . 484

Vinccur, J. 1842

Wld 1.

Will lams, J. S. 1914

WoodrufP, D. F. 2236

Wu, C. P. 1884

Zabrensky, E. J. 484 


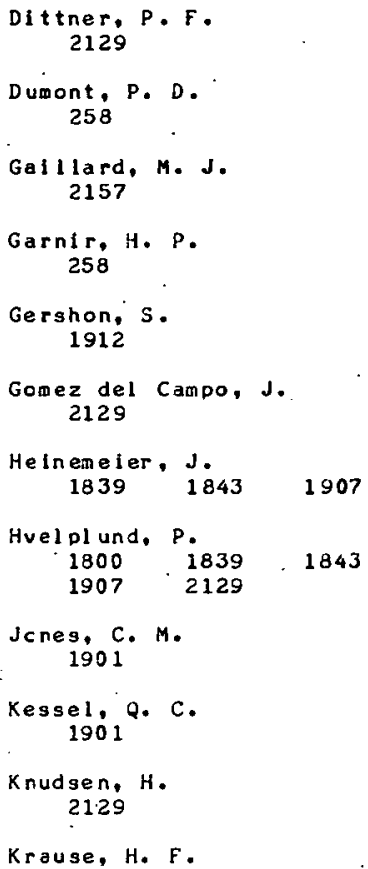

Dumont, P. D.

258

Gai 1 lard, M. J 2157

Garnir, H. P. 258

Gershon; $s$. 1912

Gomez del Campo, J. 2129

Heinemeier, $J$. $1839 \quad 1843 \quad 1907$

Hvelol und. $P$. $1800 \quad 1839$ $1907 \cdot 2129$

1843

Jcnes, C.M 1901

Kesse1, Q. C. 1901

Knudsen, $H$. 2129

Krause, H, F. 2129
Laubert, $R$. 2119

Mann. A. 1912

Aller, P. ᄃ。 $1901 \quad 2125$

Moak, C. D. 212

Nit, D. 1912

Polzat, J. C. 2157

Ramanujeo, F. S 1907

Realllieux.J. $21 \leq 7$

Sayer, $F .0$. isol

Scott, H. A. 1501

Veje, $E$. 1800 
PaRt ICle PENETRATION IN MACROSCOP IC MATTER (IONS, NEUTRALS, AND ELECTRONS)

Exclted state Populations

Alton, G. D

$$
1315
$$

Alvarez, E.

$$
1316
$$

Bashkln, S.

$$
1910
$$

Berry, H. G.

BIggerstaff, J.A.

$$
2129
$$

BrInk, J. A.

$$
2542
$$

Bunotkg : D, L:

$$
1772
$$

Chrlstensen, B.

$$
1800
$$

Cue lze 1.; F, J

2542

Crenford, 0. II. 2129

Datz. $s$. 2129

Denne, B. 1316

Devrles, P. L. 1776

nit tner, p, F, 2129

Dohmann. H. D. 2544

Clston, E. B. 1315

Engstrom. L. 1316

Forester, J. P. 1315

Gall lard. M. J 2167 Gardiner, R. B :
2382

George, T. F. 1776

Glloody, H. B. 451

Gomez del Campo, J. 2129

Grifrin, P. M $1315 \quad 1910$

Hall in, R. 1316

liuldt, s.

Hult bera, $S$. 1281

Huelplund, $P$. $1800^{\circ} 3130$

Ishi 1, K. 1316

Johnson, B. M. 1315

Jones, K. W. 1910

Knudsen, $H$. 2129

Krause, H. F. 2129

Kruse, $\mathrm{t}$. H. 1910

Leavitt. J. A. 1910

L11jeby, L. 1281

Lindgard, A. I'C 1

Lidskog. J. 1316

LIvingst on, A. E. 502

Mannervik, S. 1281

Marel Ius, A. 1316

Martinaon, I. 1316
$\underset{2542}{\operatorname{McMurray}}$. W.

Ml ller, P. F. 2129

Moak. C. D. 2125

Nielgen, S. E. $12 e 1$

Ul Iver, J. H. I. 2542

Pegg. D. J. 1315. 1910

Preng. $H$. 2544

PIhl, J. 1316

Pinnington, E. H. 1773

PI gano, D. J. 1910

Polzat, J. C. 2157

Pretcrias, fi. 2542

Reailli eux,J. 2157

Sellin. I. A. 1910

sjodin, $F$. 1316

Suter, $M$. 1315

Thoe, R. S . 1315

van der westhulzen. P. 2542

Vane. C: Fe i.315

Veje. $E$. $1281 \quad 1800$

wese. W. Li 500

Younger. S. H. 500 
608

PARTICLE PENETRATION IN MACROSCOPIC MATTER (IONS, NEUTRALS, AND ELECTROUS)

Channeling

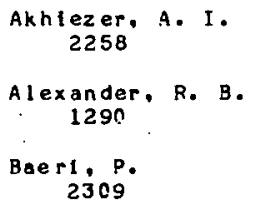

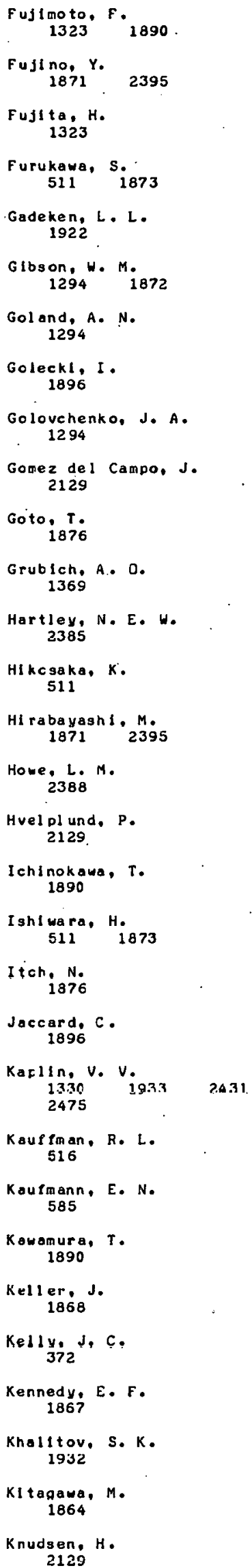

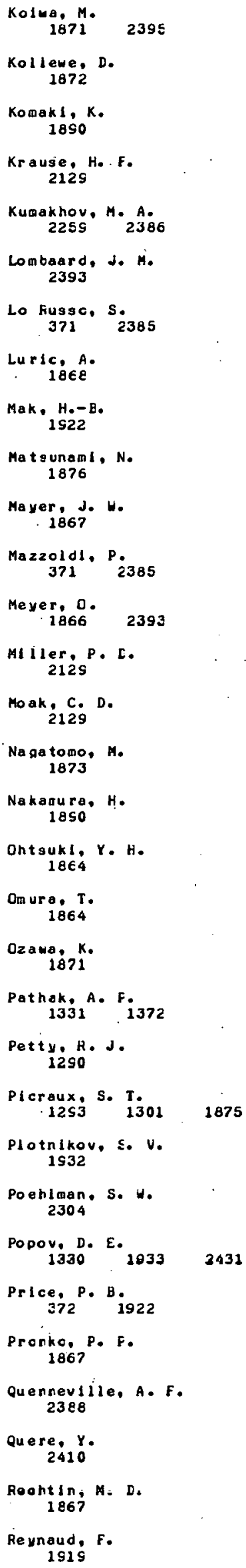

Reynaud, $F$. 1919 


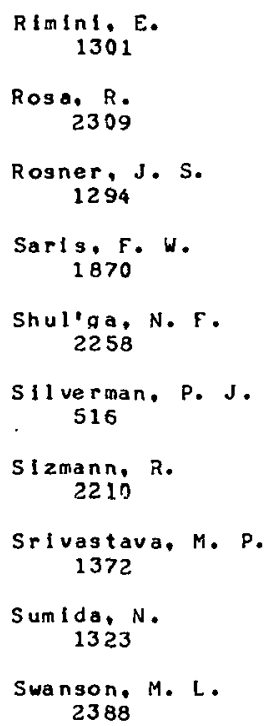

Baver.

$$
1389
$$

Staib, P.
Takahashi, J. 2395

Tanaka, $H$. 1864

Thcompson, D. A. 2304

Treacy, P. B.

Uchida, Y. 1323

Varelas, C. 2210

Vorob'ev, A. A. 1933 2431

vorobev, S. A. 1330

Vercblev, S. A. 1926 1933 2431

Walker, $f, S$.

2304

Wede 11, $R$. $1333 \quad 2414$

Wegner, H. E. 1254

wiggers, L. W. 1870

Yamaguchi, s. 18712395

Yoshinar $1 . C$. 1871 2395

Zimin, N. I.

1926

Zuhr, R. A. 516 
$\mathrm{DO2}$

PARTICLE IMTERACTIONS UITH SOLIDS

Sputtering by Electrons, Neutrons, and Heavy Particles (total renoval coetflicients)

A be. ${ }_{386}^{T}$

Andersen, $H$. H. $891 \quad 1402$

A rmour, D. G. 2383

A to, Y.

Aug ust yni ak, W. M. 2133

Bay, H. L. $612 \quad 1396 \quad 1397$

Bohdansky. J. 612 1397

Borders, J. A. 1398

Borl senko, V. E. 2479

Bouwn an, $R$. 1177

Braganza, C. M. 408

Broun 1392

Brown, W. L 2133

Carter. G. $191^{\circ} 2383$

Chursin. M. M. 1360

Clausing, R. E. 1404

Colling, $R$. 1916

de Velos, A. E. $2403 \quad 2406$

Dol . ${ }_{409}^{H}$

Dzloba, S 1399

Ecker, K. H. 1292

Elbern. A. 1394

Emerson, L. 6 . 1404

Emmoth, B. 1392

Erents, S. K. 408

Fal cone, G. 358

FI 11 ppov, E. 1 . 1935

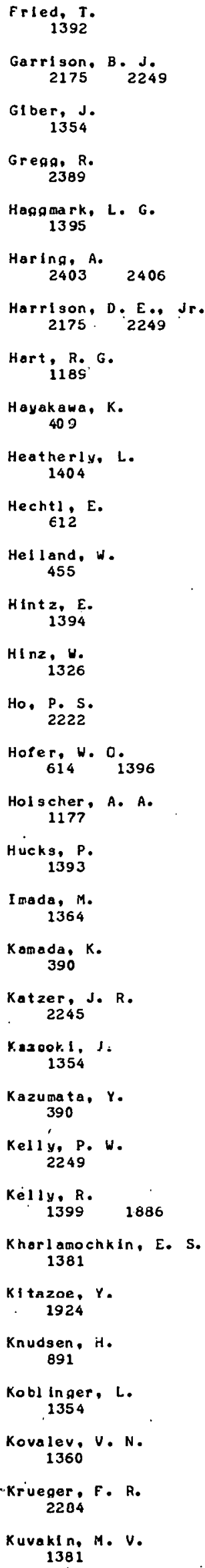

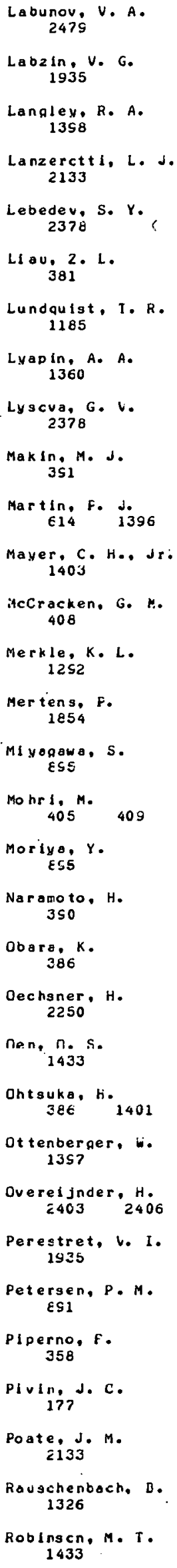
1433 
Roques-Carmes, $c$.$$
177
$$

Saek 1. N. 2218

Schoof, $\mathrm{H}$

2250

Schweer. $B$. 1354

Sheng, T. T.

381

ShImizu, R. 375 2218

Simmons. G. W. 1189

Slodzl an, G. 177

Smith, H. J.

Sinfth, J. N., Jr. 1403

Snowdon, K. J. 2383

Sono, F.. $386 \quad 1401$
Stockl in, G.

1393

Stumpe, E.

$S z$ ymonsk I, M. 2406

Taglauer, E. 455

Tombrello, T. A. 2389

van Mechelen, J. B. 1177

von Wyk, G. N. 2417

Vletzke, E 1393

Vogel bruch, $K$. 1393

Watanabe. $K$. $408 \quad 409$

Webb, $R$. wien, K. 2284

Hiscn, K. L. 1358

HIlson, W. D. 1395

HI ndowi. H.

Winograd, N. $2175 \quad 2245$

Yamado, R. $386 \quad 1401$

Yamamura, $Y$. 1924

Yaoshina. $T$. $405 \quad 409$

Yu, H. L.

Yurasova, V.E. 1381 
D03

PARTICLE INTERACTIONS WITH SOLIDS

Sputtered Partlcle Charge and Quantum (Exclted) state Distribution

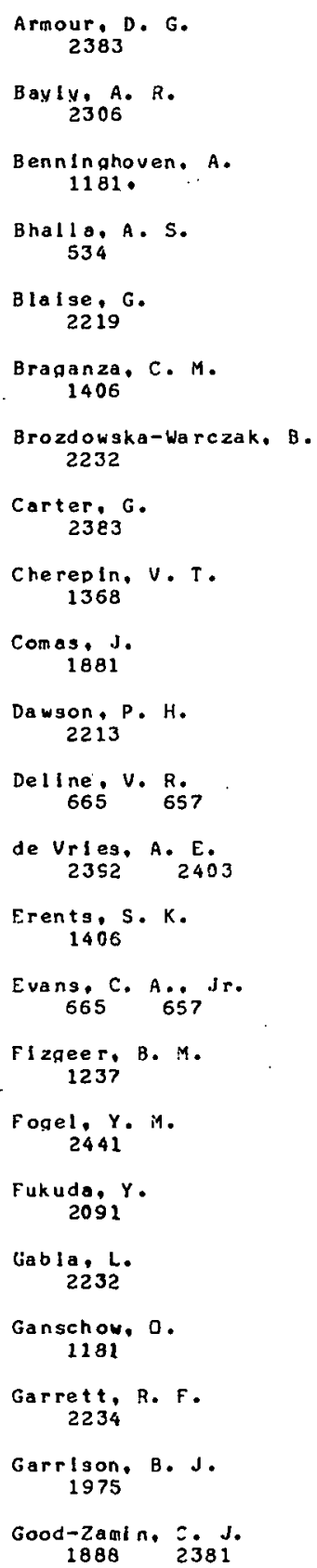

${ }_{2232}^{R}$

Prival, H. G. 2251

Rabalols. J. W. $2051 \quad 2227$

Pausch, E. 0 . 475

Roques-Carmes, $C$. 2215

Schoof, H. 2250

Shehata, M. T. $1888 \quad 2381$

Smith. J. N., Jr. 1438

Snowden, $K$. J $2383 \quad 2412$

Squíres, D. B. 1688 238?

St unfe, E.

Szymanskl, M. 2232 2392

Taylor, J. A.

Thcaas, E. W. 475

Tscng, I. S. $T$. 2235

Vasilev, M. A.

1368

warczak. A c232

werner, H. 1034

Whlte, C. W. IEEO

wiedaann, L. 1161

Will lams, P. $\epsilon \in 5 \quad 667$

winograd, N. 1975

Wright, R. E. 1407

$\mathrm{Yu}, \mathrm{M} . \mathrm{L}$ 18e? 2211

Yusuf, N. A. Ei7 
DO4

PARTICLE INTERACTIONS UITH SOLIDS

Secondary Electron Ejection by Heavy Particles and Electrons

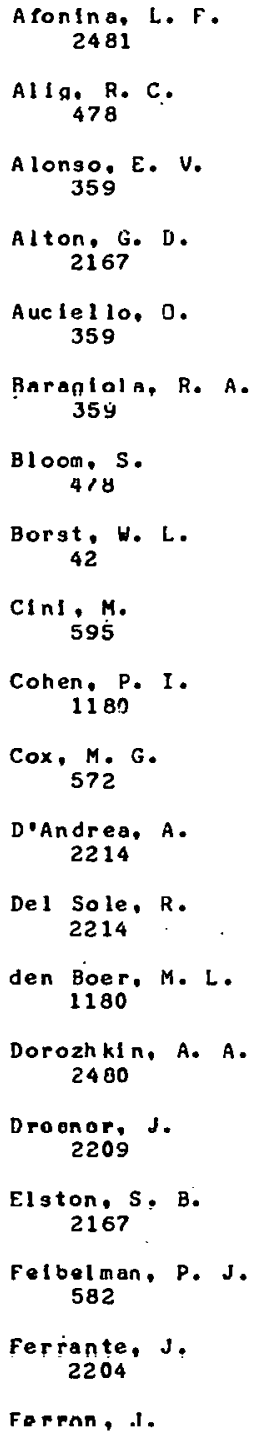

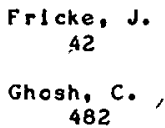
2480

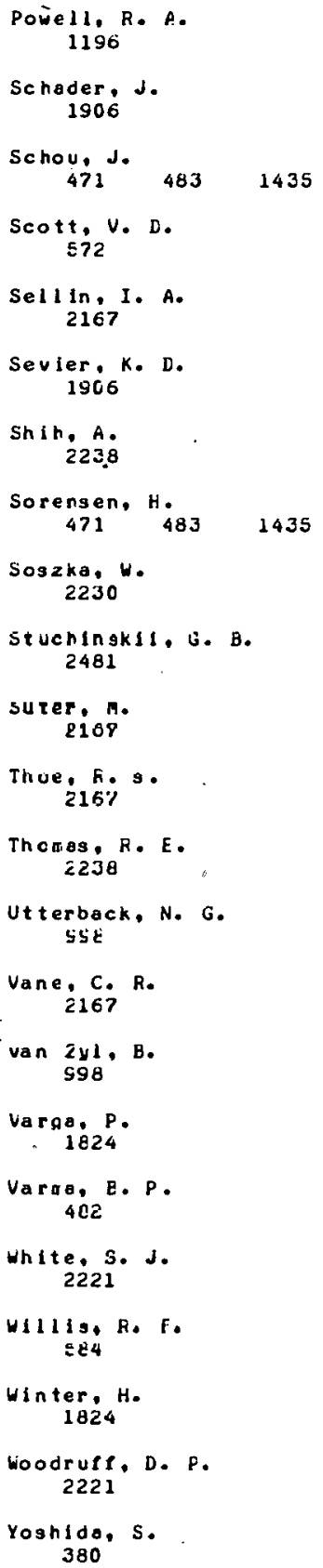




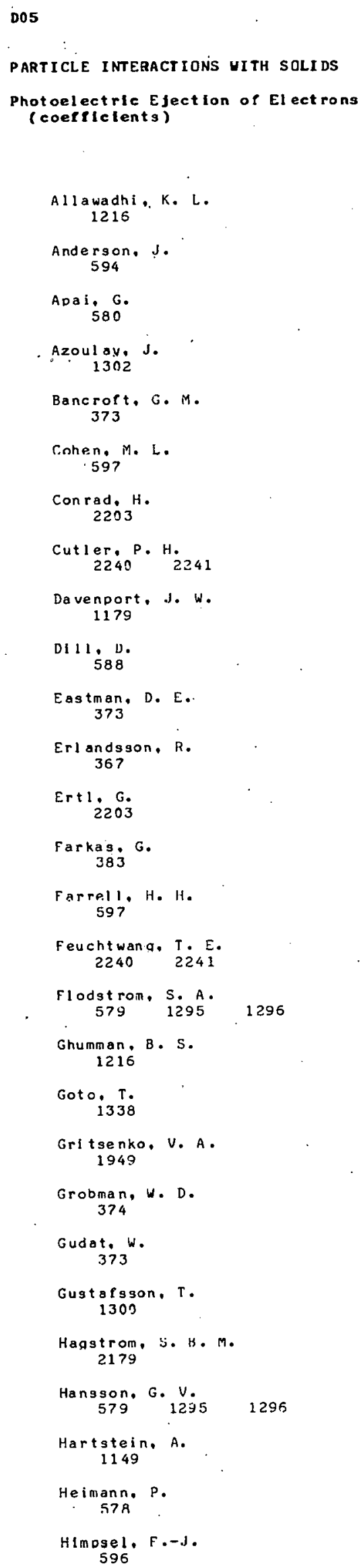

Horvath, $z$.

383

Humberg, H. 1336

Jenk in, J. G. 1302

Joannopoulog, J. D. 591

Kamada, $\mathrm{H}$. 377

Kasuya, A. 1338

Kikuchl, $B$. 881

Kudo, M. 377

Nithel, Y.

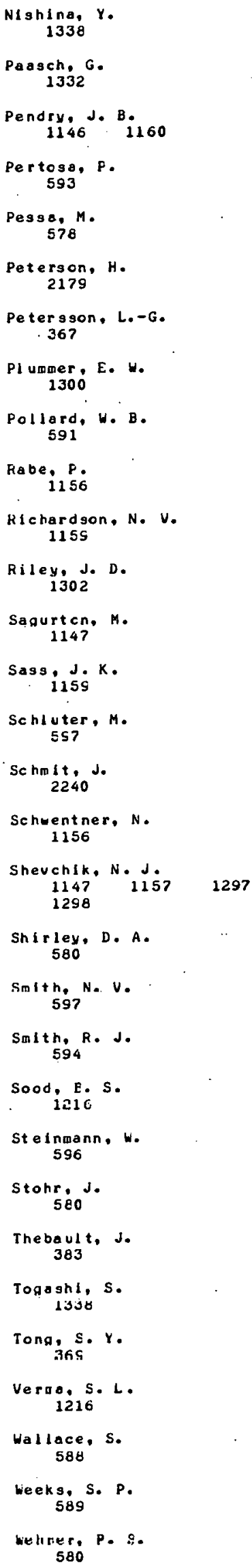


Weinberg, $Z$. A. 1149

Weng, S. $-\mathrm{L}$. 1300
D06

PARTICLE INTERACTIONS UITH SOLIDS

Herlection or Eloctrons from Surfacos

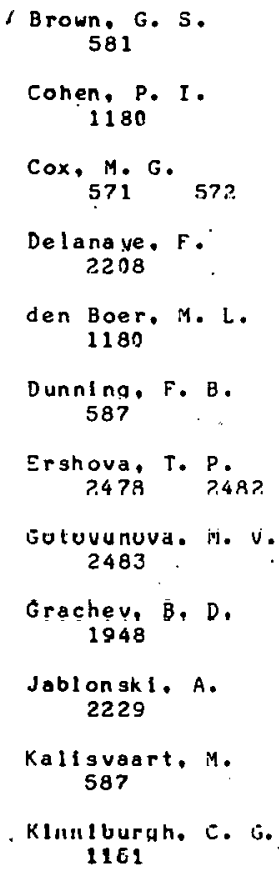
(coepticlents)
Wi 11 I ams, R. S. 580

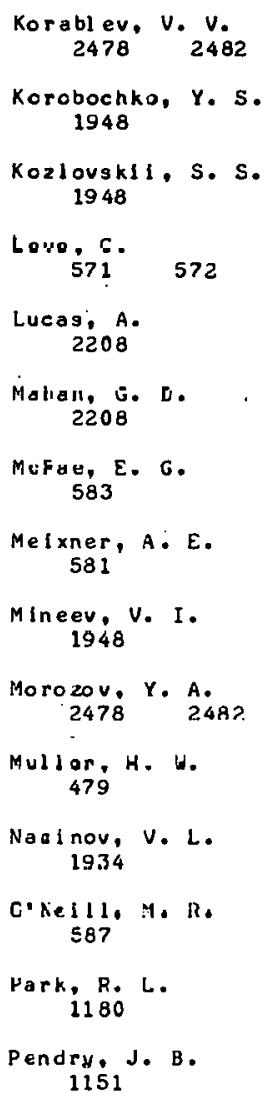


Do7

PARTICLE INTERACTIONS UITH SOLIDS

Reflection of Heavy Particles froo surfaces (total reflectlon coeff(clents)

Akkerman, A. F .

1328

A rmour. D. G. 456

Balaghova, L. L. 2252

Barker, J. A. 2228

Barragan. A.

2425

Baun. W. L . 44 2225 2233

Becker, G. E. 1178

Bolz lou, C. 2302

Bosanac, $S$. 785

Brady. J. W.. 'Jr. 2047

Brice, D. K. 1367

Cardil lo, M. J: 1178

Cel $11 . v$. 2243

Chl ng, C. S. Y. 1178

Cole, M. W. 2207 Derry, $G$.

DIon, D. R. 2201

Dol 1, s. D. 2201

EcksteIn, W. $1432 \quad 2427$

Engel $T$.

Feldman, L. C. 516

Frank1, D. L. 2151

Frank 1, D. R. 2207

Garcla, N. 2212
Garcla Sant Ibonez, F. 2425

Gerosimenko, N. N. 899

Gerber. $R . B$. 785

Goodman, F. 0 . 2206

Gras-Mart I, A.

$$
613
$$

Greene, $E$. $F$. 1178 2242

Horvie, $C$. E. 2121

Hel land, w $455 \quad 2139$

HIII, N. H. 2243

Hou, M. $616 \quad 617 \quad 2427$

Jockson, D. $P$. 1430

Kauteman, R. L. 516

Kaufmann,E. N. 585

Knudsen, H. 212

Kovalevskaya, T. I.
899

Krishnasuamy, s. v. 2151

Lagcs, M. 2216

Langley, R. A. 1367

Littmark, U. 613 i431

Mashkova, E. S. 458

Mashova, E. S. 2252

Mason, B. F. 2247

Mason, E. A. 2242

Molchanov, V. A. 458 2252

Murakam l, Y. 376

Murrel1. J.N.

Nashiyana, I. 576
Nel son, G. C. 1186

Nuvclone, $R$. 2302

Oen, 0. s. 1433

o' Gorman, T. 2151

Petersen, P. M. 212

Preuss, E. 2413

Robinscn, J.E. 1430

Roblnscn, M. T $\in 16,617^{\circ} \quad 1433$

Roussel, J. 2302

Schcu, $J$ 1491

Shibata. T. 376

Sl lveran, P. J. EI€

Sorengen $H$. 1431

Steele, t. A. 2228

Taglauer, E 455 2139

Taneka, S. 376

Thicarscn, D. L. 2047

Tseitlin, G. M. 899

van den Berg, J. A. 456

Verbeek. $\mathrm{H}$. 1432 2427

verhelj. L. K. 456

Heare, J. $H$. 2121

Hesner, $D$. 2151

WIIl lams. B. R. 2247

Yinncn, A. T. 785

Zartner, A. 2135

Zuhr, R. A. 
DOB

PARTICLE INTERACTIONS UITH SOLIDS

Charge and quantus State Distributions

of Replected Heavy Particles at

Macroscoplc Distances froo Surfaces

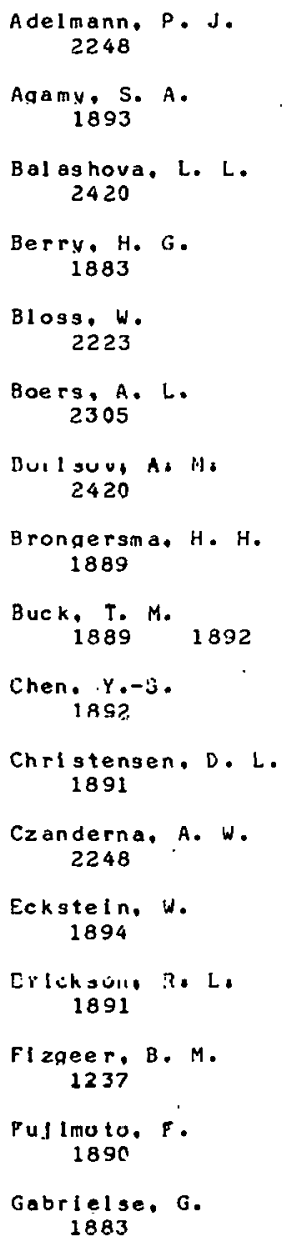


D09

PART ICLE INTERACTIONS UITH SOLIDS

De-excltation, Neutralization. Ionfzation, or Dissoclation of

Particles Interacting with. Surfaces

Cardillo. M. J. 2237

Chen, F. K. 2:119

Datz, S.

$$
987
$$

Dit tner, P.F. 987

Dzhamankyzov, N. K. 1375

Gelb. A. 2237

Gemmel 1, D. S.

Grozdanov, T. P. 340

D10

PART ICLE INTERACTIONS UITH SOLIDS

Interaction Potentials Between Surfaces and Free Particles Located External to the Surface (electrons and heavy particles)

Cardil 10, 14. J. 2237
Horl guchl, S.

$$
1334
$$

Ignat lev, A. 961

Ion ik h, Y. Z.

1945

Janev, R. K. 340

Kagan, $Y$.

,

Kanter, $E$. P 444

Kishinevsk II, M. E. 2492

Kogan, E. Y. 1378

Kcnonets, $Y, V$. 1375

Koyama, K. 1334

Kuranov, A. L. $194 S$

Lancaster, G. M. \$61 1121

Ccle, M.

2231

Gelb, $A$ 2237

Kapl in. v. $v$. 2431

Maclenald, R. J. 2310

O.Connor, D. J. 2310

P) [va, J. 2231
La uber $t, R$. 2115

Mal"nev. V. N. 1378

McCreery. J.H. 413

Ontsukt, Y. H. 1334

Penkin. N. F. 1945

Pietsch, W. J. 444

Prival, H. G. 2251

Rabalais, J. W. $s \in 1 \quad 2111$

Sharkov, V. F. 1945

Tayler, J. A. 961 i11

wolken, G., Jr. 413
PoDOY, E. E.

2431

Schwartz, $c$. 2231

Vorcbiev, A. A. 2431

Voroblev, S. A. 2431

wolken, G.: Jr. 1056 
D1 1

PARTICLE INTERACTIONS MITH SOLIDS

stleking Coefricients (thermal energies)

Engel ${ }_{10}{ }^{\mathrm{T}}$

D12

P̈́ñTICle INTERACT ION3 UTtU JOLEDS

Electronagnet Ic Radiation Induced by Electron or Heavy Particle Iapact on Surraces

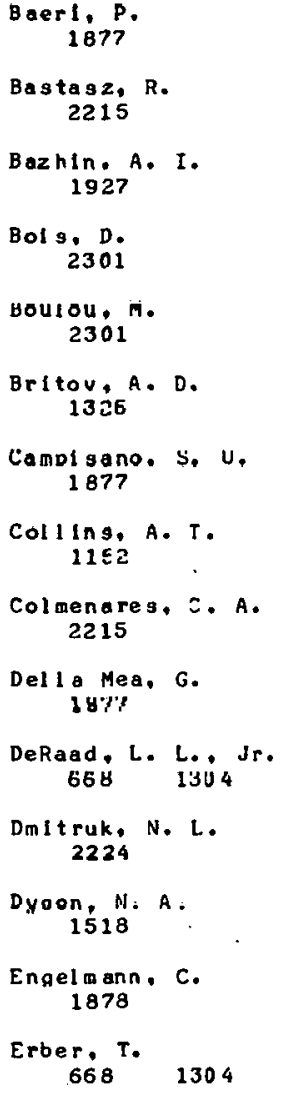


D13

PARTIClE IMTERACT IONS UITH SOLIDS

Desorption of Gases froe Surfaces

Ageev, V. N.

2292

Akaishi, K.

1434

Baver, $E$. 2244

Behrlsch, R. 1425

Beitat, $U$. 1855

Blewer, R. S. 1425

Bouwman, R. 1177

Braganza. C. M. $1406 \quad 1424$

Dawson, P. H. 2213

Donnelly. S. E.

Drinkulne, M. J. 1176.

Dzhalliov. S. T. 2292

Edwards, D.. Jr. 1191

Engelhardt, H. A. 611

Erents, S. K. $1406 \quad 1424$

Farre 1 1, G. 1427

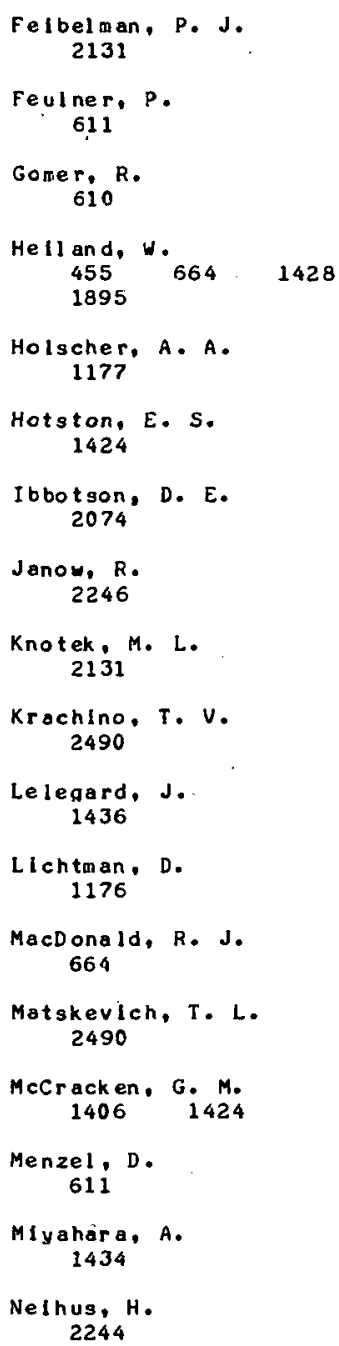

Ontsuka, H.

Pr lage, S 2244

Sagara, $A$.

Saldoh, M. 1414

Scherzer, B. H. U. 1425

Schrom, A. 1436

Schulz, R. 1425

Shek, Mo-L. 2226

Sone, $K$. 1414

ste inbruchel. C. 610

Taglauer. E. $455 \quad 664$ 1855

1428

Taylcr, J. L. 2074

Tzoor, $N$ 2246

van Yechelen, J. B. $117 ?$

Weinterg. W. H. $2074 \quad 2226$

W throw, S.,P. 2226

Yamada. $F$. 1414 
Foge 1. Y. M.

Gallagher, J. 1441

Blistering, Volds, and Surface Strain in Wetals

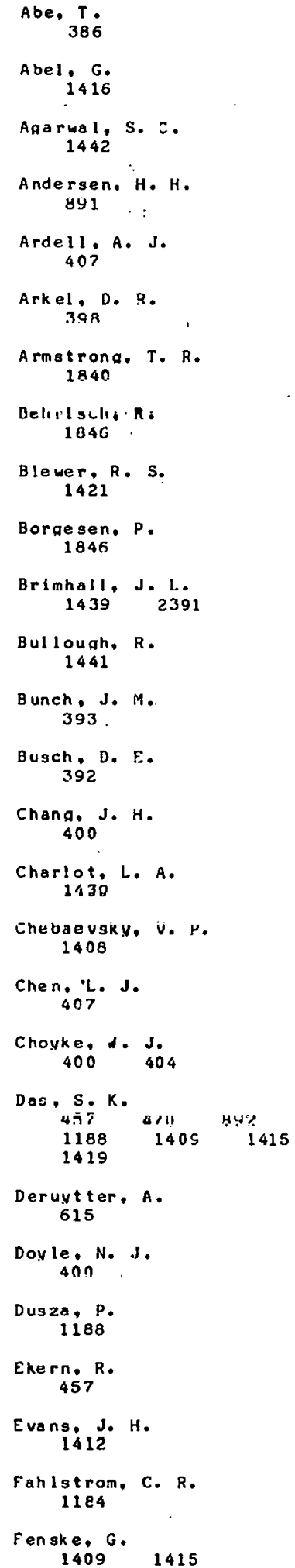

Gocdhew, P. J.
396
Gama yunova, L. A. 2372

Gerber, S. B. 392

Gusev, V. A. 2372

Guseva. M. I.

Hayns, $M . R$. $399 \quad 1440 \quad 1441$

Henager, C. H., Jr. 2391

Hoso, D. C. 457

Hoffman. I. G 393

Ionova, E. S.

$$
1411
$$

Ivanov, L. I. 1408

Johnson, P. B. 1840

Kaletta, D. 1410

Kadada, K. $390 \quad 402$

KamInsky, M. $457 \quad 470 \quad 892$ $\begin{array}{llr}1188 & 1409 & 1415\end{array}$ 1419

Kazumata, $Y$. उ88 390

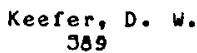

KIss Inger, H. E. 1439

Knudsen, $H$. 891

Koltygin, V.. M. 1411

Konl ssarov, A.P. 1450

Krasulin, Y. L. 1411

Kurokina, T. S. 1111

Lobrlo, J. C. 1416

Lollv. J. S.

$$
404
$$

Longley, R. A. 1421

L'Ecuyer, J 1416

Llau, Z. L.

Lccmis, B. A. 392
Machlin. N. A. 1408

Makin, H.J. 391

Mansur, L. K.

Martel, J. C. 1416

McDonell, W. R. 1420

McGrver, J. N. $400 \quad 404$

MeInikov, V. N. 1408

Miley, G. H. 1415

Mohrt: M. 1413

Nandedkar, h. v. 615

Niapometo, $\mathrm{H}$. $390 \quad 402$

Navinsek, B. 1418

Nedcspascr, A. V. 1411

Nichclecn, F. J.K. 1417

Nolf I, F, V., Jr. 1442

Obara, $K$ 386

Oht suke. H. उ86 1414

Pard. A. G. 385

Pecrcy, P. E. 1421

Heternel, M. 1418

Petersen, P. M. 891

Kehn. L. E. 1442

foosina, I. A. 1411

koth, J.

Saidch, 1414

Sasok 1 . K. 1413

Scherzer, B. M. U. $1846^{\circ}$

Sheng. T. T . 381

$S I$ monen, $E$. P $2430^{\circ} 2381$

Sinha, M. K. $470 \quad 1284$

Sone, $K$. $386 \quad 1414$

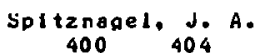


Stals, L.

St-Jac que $3, R, G$ 。 1416

Terreault, B. 1416

II shchenko, L. P, 2372

Townsend, J. R. 400

Trier, S. K.

396

van Guysse, J. 615
Venskytis, F. J. 400

Wach,

Walls, J. M. 1427

Wotanabe, $K$.

1413

HIIlams, T. H. $398 \quad 399$

WItmaack; $K$.

538

Yamada, $R$

$386 \quad 1414$
Yamashina, I.

1413

Yegso, J. D.

YoO, M. H. 403

Zabker. A. 1418

Zeltaann, A. H. 353

Zykova, N. N. 1421 
D15

PARTICLE INTERACTIONS UITH SOLIDS

Radiation Danage In Metals

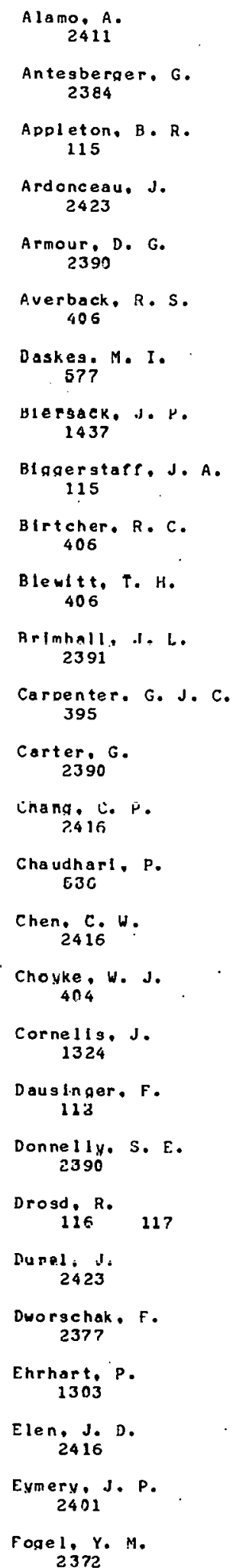

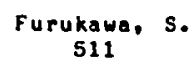

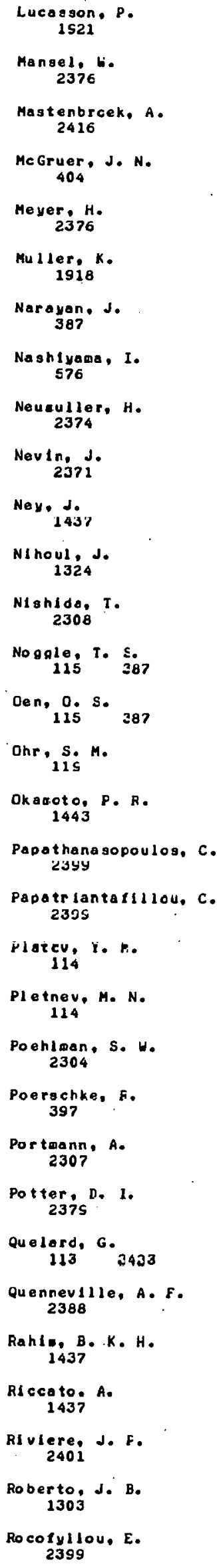


Roggen, J. 1324

sadykhov, S. I : 0 . 114

Schilnd ler, R. 2373

Schl enger, B. 2377

Schoenfeld. B. 1303

Schulson, E. M. 395

Schultz, H. 112

Schwel khardt, J. 112

SeIdman. D. N. 2205

Slmonen, E. P. 2391

Sonnenber $\mathrm{d}$. $K$. $2384 \quad 2387$

Soullard, J.

2411

Spalding: D. 2371

Spitznage 1, J. A 404

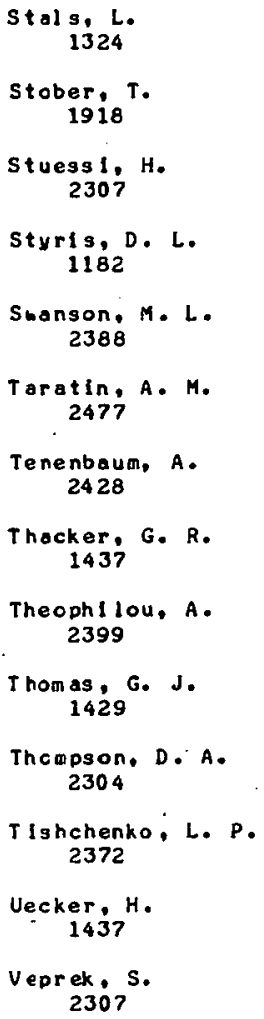

Vog 1, G

Vorob'ev, S. A. 2477

Halker, F. s. 2304

Washburn, J. $116 \quad 117$

Webb. A. P. 2307

liebb, $F$. 2390

Wienhold, P. 23842387

Wikes, P. 1444

Hill loos, J. M. 115

wilson. K. L. 1429

Woltenden. A. 1518

wo 1 lenberger, H.
397

Yaøorura, Y. 1924 
PARTICLE I MTERACTIONS UITH SOLIDS

Particle Implantation in Metals

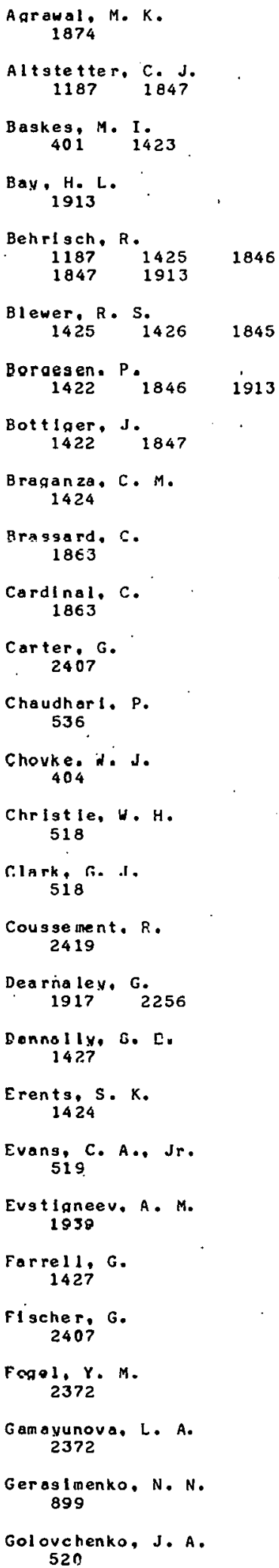

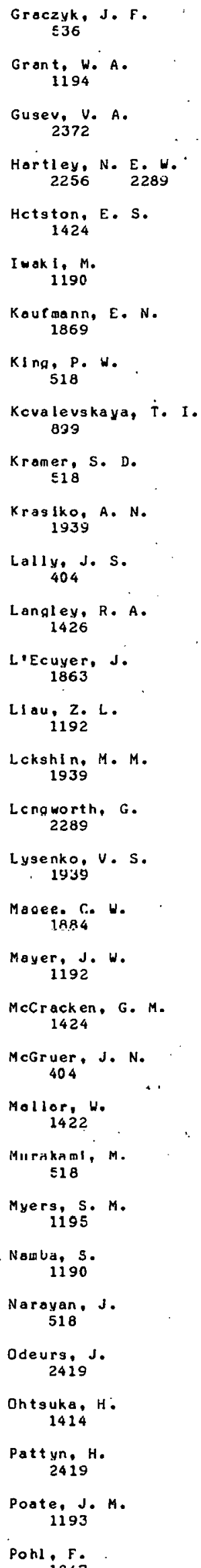

Graczyk, J,F. $\leqslant 36$

Grant, W. A 1194

Gusev, V. A. 2372

Hartley, N. E. W.

22562289

Hctston, E. S 1424

I wak i. M. 1190

Koutmann, E. N. 1869

KIng, P. W. 518

Kovaleuskaya, $\dot{T}, 1$. 839

Kramer, S. D.

Kraslko, A. N 1939

Lally, J. S.

Langley, R. A. 1426

L.Ecuyer, J. 1863

LI au, Z, L. 1192

Lckshin, M. M. 1939

Lcngworth, G. 2289

Lysenko, V. S. 1939

Maoee. C.. W. 1884

Mayer, J. W. 1192

McCracken, G. M. 1424

McGruer, J. N. 404

Mollor, He 1422

MIIrakami, M. 518

Myers, S. M. 1195

Namba, $s$. 1190

Naroyan, $J$. 518

Ddeurs, J. 2419

Ohtsuka, $H$ : 1414

Pattyn, H. 2419

Poate, J. M. 1193

Pohl. F.

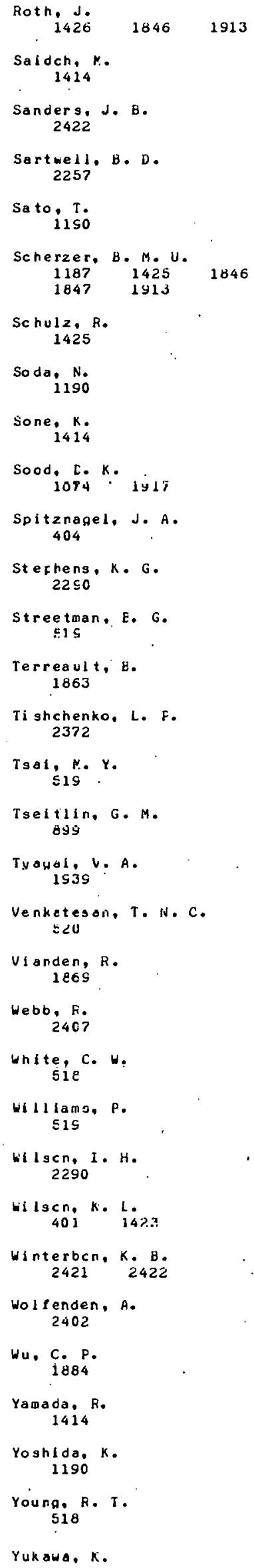


D17

PARTICLE INTERACTIONS YITH SOLIDS

Electron-, Ion-, and Photon-Induced

Chemlcal Changes to Surfaces

Abroyan, I. A. 1952

Bastasz, R. J 1400

Blalse, G. 2219

Braganza, C. M. 408

Brundle, C.R. 2255

Chemin. J. F 2300

Chuang, T. J. 2255

Chudnougkil. F. A. 1952

Danto, $Y$.

Do1. $H$.

1405

Erents, S. K. 408

Fukuta, M.

Gavril yuk. A. I. 1952

Gerast menko, N. N. 1953

Goner, R. 610

$\varepsilon 01$

ELEECTRUN-PART ICLE゙ INTERACTIONS

General

Burke. P. G. $21 \subseteq 6$
Hayakava, K.

1405

Hell and, $W$. 669

Ho, P. S.

Ign a t I ev, A $\$ 61$

Kelly, R.

Lancaster, G. M. 961

Lanskaya, T. G. 1940

Lyon, 0 . 2219

MeCracken, G. H. 408

Mimura, T. 514 Mohrl. M.

Odanl $K{ }_{14}^{K}$

Pan'kin, V. G. 1953

Plstre, J. D. 2300

Rabalals, J.W. 961

Roques-Carmes, C. 2219

Soboya. B. 2300

Saek I, N. 2218
Salardenne, $J$. 2300

Shialzu, R. 2218

Stein, H. J. 533

steintruchel, $c$. 610

Suverou, A. $V$. 1540

suitoshev, $K$. $K$. 1553

Taglaver. E. 665

Taylor, J. A。 961

Terukov, E. I. 1940

Thcous. G. J. 1400 Tseit 1 in, G. M.

Vel ichko, V. Y. 1552

Wandel $t, k$. 2255

Ya tanabe, $K$. 1405

Yawashiná, T. 1405

Yokcyams, N. 514

Zakhorchenyo, B. P. 1952

Si 1. N. C .

$1820^{\circ}$

Sur. S. K.

1820

Truhlar, D. G. 2051

van-catledge, F. A. 2051
Nesbet, R. K. 1737

Seaton, M. J. 
ELECTRON-PARTICLE INTERACTIONS

Elastic Collisions

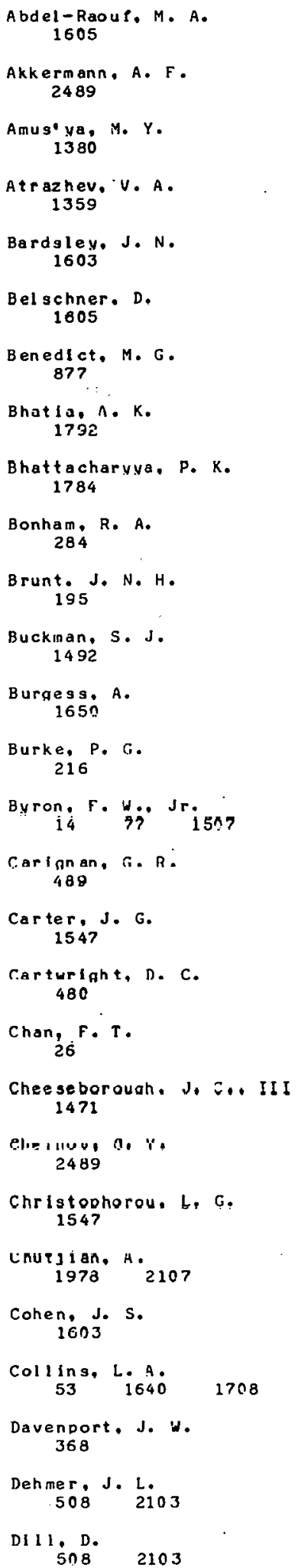

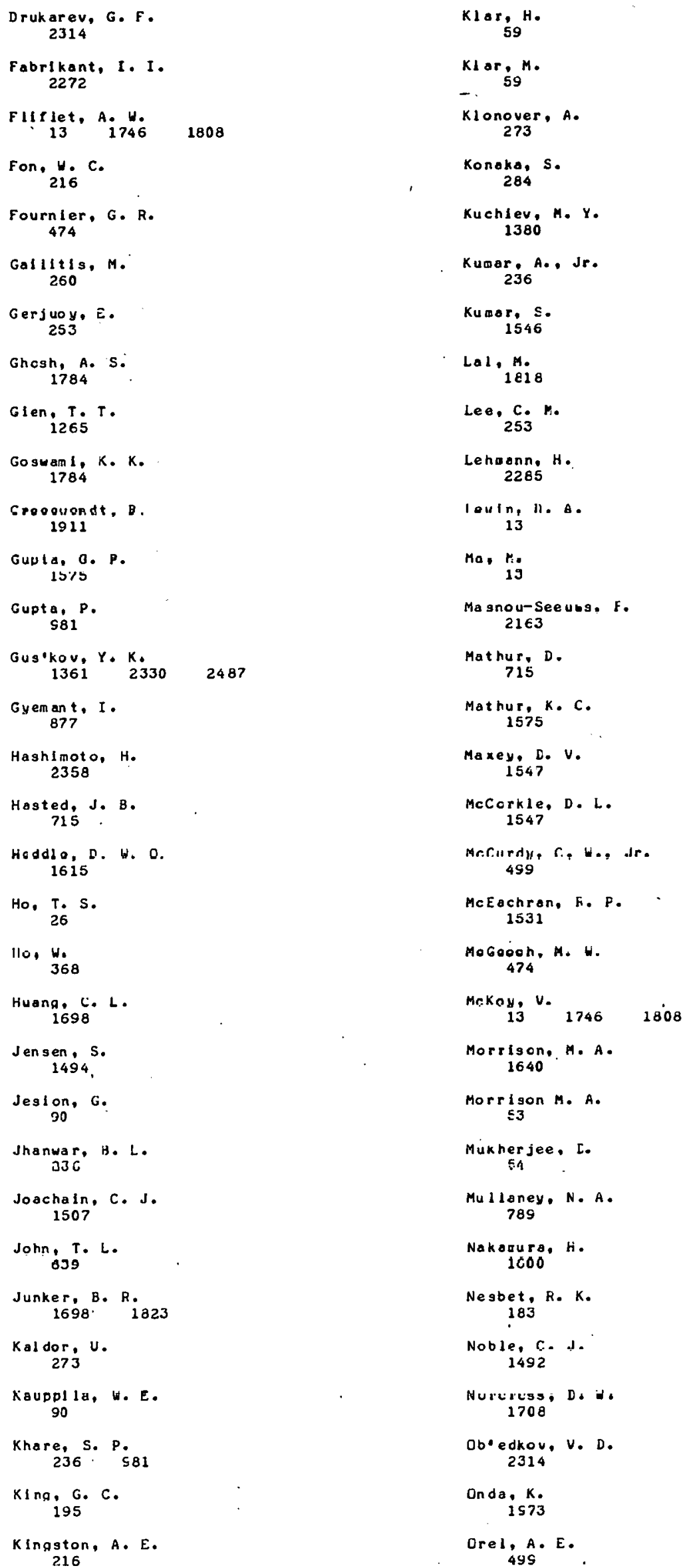


Phillppe, M.

2163

Poet, R.

1548

Pol, $V$.

Read, $F$. $H$. 195

Reglster. D.

1454

Resclano, $T$. N 499

Ritchle, B.

$$
1346
$$

Robb. W. D. 1640

Rohr. K. $1458 \quad 1637$

Rov, A.C. $509 \quad 1520$

Savuov, R. V. $1361 \quad 2330 \quad 2487$

Schrieffer, J. R 368

Seaton. M. J. 201

Shake shaf $t, R$. 1801

Sharp, W. E. 489

ShI mi zu, R . 2358

Shyn, T. W.

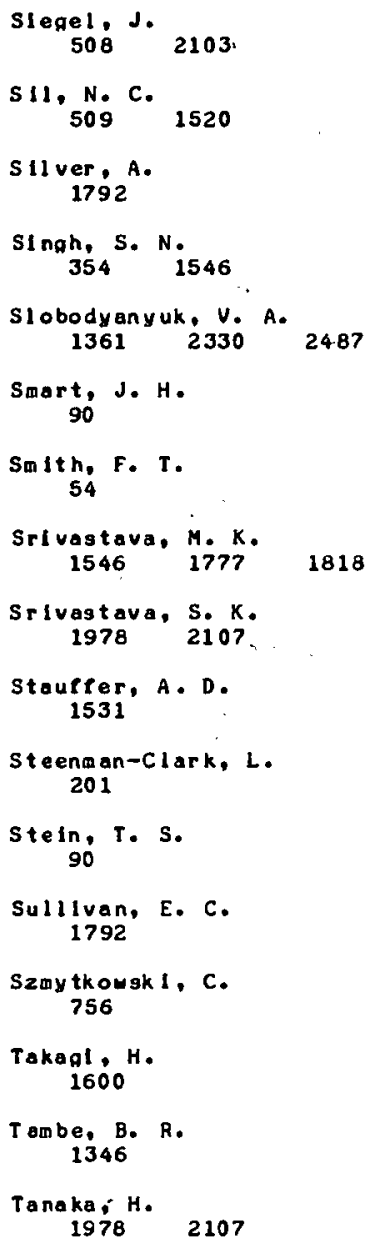

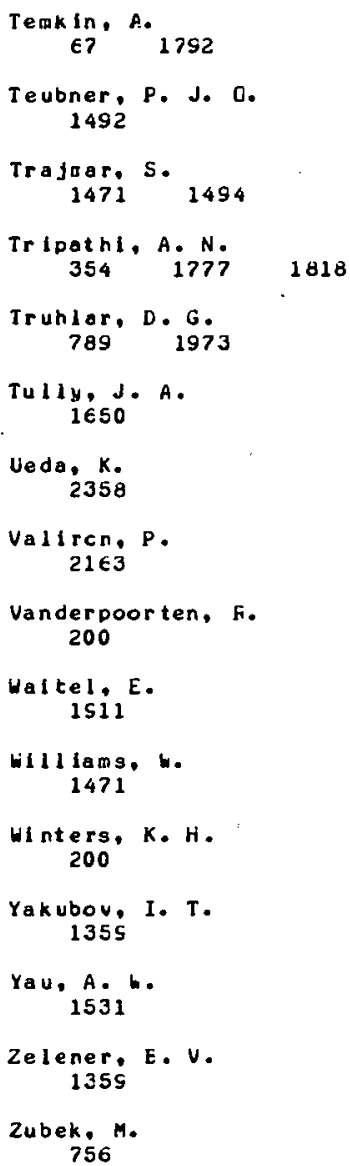


$\mathrm{EO3}$

\section{ELECTRON-PARTICLE INTERACTIONS}

Excltation

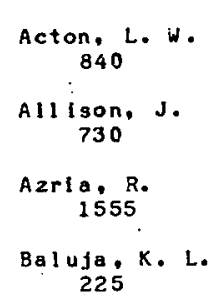

Cheeseborough, J. C.. III 1471

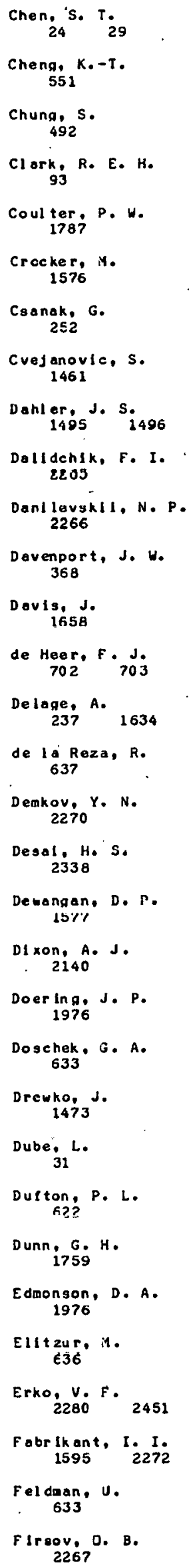


$\underset{2349}{H a s h i m o t o} H_{2358}$

Hayes. M. A

208

$\underset{39}{\operatorname{Hen} r y}$ R. J. $W$.

Herrick, D. R. 168

Hickerson, D. L. $1455 \quad 1496$

Hickman, A. P. 55

HItcheock. A. P. 701

Ho, w.

$$
368
$$

Hock , G.

Hood, 3. T . 2140

Hudson, R. L. 1010

Huetz, A. 1363

Hyan, H. A. 1705

I vanov, G. K. 2265

I wal, T . 2345

Jensen, $s$. 1494

Jensen, S. h. 2lEE

Jo 11 y, J. 1691

Jurela, J 1461

Ka 1 dor, U. 273

Karclis, C. $20 \epsilon$

Kastner, s. 0 . 638

Ke lsey. E 48

Ke l se $y$, E. J. 1768

Kennedy. J. V. 259

Khayrallah, G. A. 24

Khovanskil, N. A. 2266

$\mathrm{KIm}, \underset{491}{Y-K} \cdot 551$

KIng, G. C. 195

Kingston, A. E .
Kle inpoppen, H. 2537

Klonover, $A$. 273

KI UmP, K. N . 1115

Koppe : $V$. $T$. 2266

Kosoruchk in a, A. D. 1244

Koval, A. G. 2266

Kumar, $S$. 1822

$\underset{148}{\text { Kuppermann. }} 924$ 2064

Kwong, R. 485

K wong H. K 1496

Lamanna, J. T. $554 \quad 1100$

Land, J. E. 894

Lassettre, E. N. 1115

Lawton, S. A. 1958

Lee. J. S. 1976

Leung, $C$. N. 1456

Levin, D. A. 13

Lieber. M. 491

Llepinsh, A.

Lin. C. C. 492

Lisitsa, V. S. 2332

$\mathrm{Ma}, \mathrm{M}$.

Macek. J. $48 \quad 1726$

Marmet, $P$. 1608

Maru, M. P. 2338

Marusin, $v$ : $D$. 1248

Mason, H. E. $638 \quad 846$

Matese, J. J. 39

Mathur, K. C. 1539 i 830

Mazeau, J. $1119 \quad 1363$

McCarthy, I, E. 1576

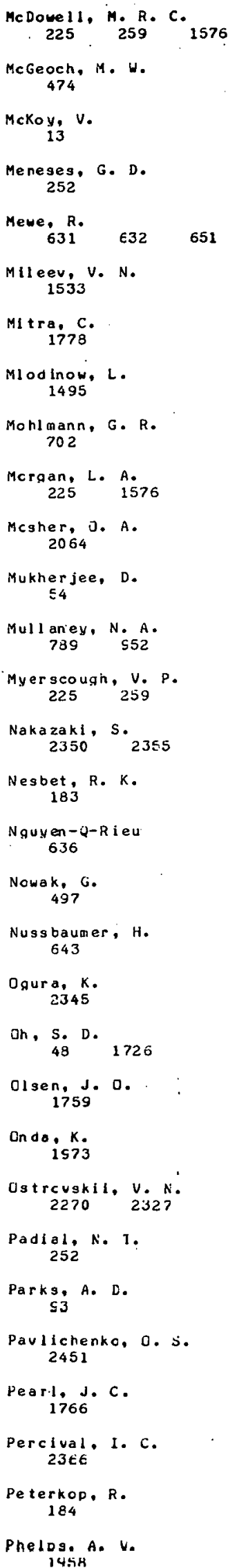




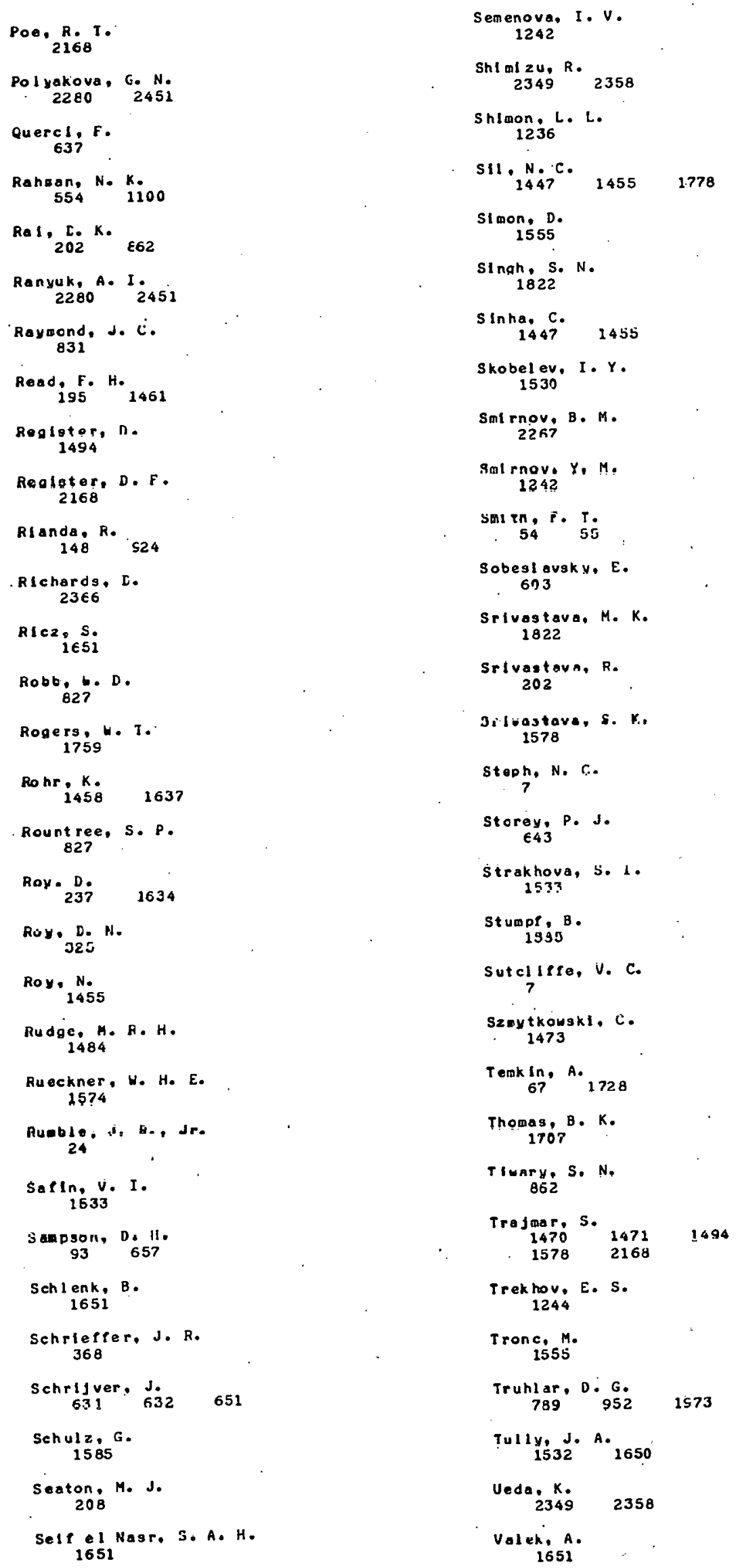

ShI mI zu, R. $2349 \quad 2358$ 
E04

ELECTRON-PARTICLE INTERACTIONS

\section{Dissoctation}

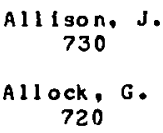

Allison, J.

$$
730
$$

Allock, G.

720

Blondt. M. A.

$$
47
$$

Birtulstle. D. T. 1534

Black, J. H.

$$
826
$$

Boness, M. J. W.

541

$\underset{83}{\text { Borst, }} \underset{497}{\text { L. }}$

Bose N.

$$
271
$$

Brau, C. A.

$$
662
$$

Brion, C. E.

Brunt. J. N. H.$$
195
$$

Chiblsov. M. I. 1374

Chung, S.

492

DanllevskI I N. P.
2266

de Heer, F. J.

702703

Drukarev, G. F. 2445

Erko, V. F. $2280 \quad 2451$

Flque $t-F$ avard. $F$. 1053

Fletcher. $\mathrm{J}$. 530

Foltyn. S. R.

$$
\begin{array}{r}
\text { tyn } \\
530
\end{array}
$$

Forsman, J. A.

280

Fricke, J.

$$
497
$$

Fujlta, T.

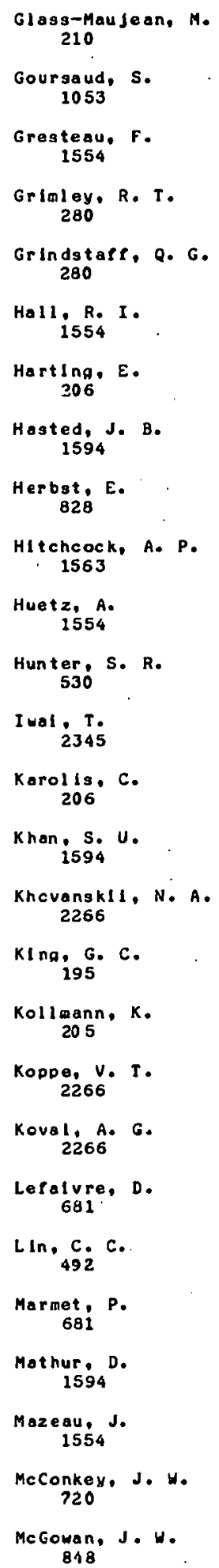


ELECTRON-PART ICLE INTERACTIONS

\section{Ionlzation}

Banks, D
1483

Barlas, A. D 1731

Basov, N. G. $24 \subseteq 2$

Beaty. E. C. $89 \quad 485$

Bellc, D. S. 1609

Berezhko, E. G. 1456

Binette. $L$. 1608

Dirkinshaw, K. 204

Boesten, L. G. J.

Bransden, B. $\mathrm{H}$. 1549

Arehm, R. 178

Breton, C 2155

Brion, C. E. 805 is63

Brook. E $1550^{\circ} 1601$

Brooks, R. L. 2154

Brunt, I. N H 106

Burnet $t$, $T$. 1614

Cantlion I. R. $94 \quad 323$

Carette, J.-D. 237

Carlwnlult. D. C. 480

Eliatland: B 1110

Cheng, K. $-T$.

Ciark, H. L. H. 93

Compton, R. N. 1984

Cook. J. P. D. 805

Coulter, P. d. 1787

Cranda 11, D. H. 1789

Dan Ilvchev. $\checkmark$. A.
Datla, R. U. 2154

de Frenes, G. 178

de Heer, F.J. 202

Delage, $A$ 237

de la Reza, R 637

de Michel is, C. 2155

D 1110 , M. A. 976

Divine, T. F. 555

DIxon, A. J . 33

Doer Ing. J.P. 1976 BuDulst ${ }^{\mathrm{A}}$ Butolg, K. D.

Eduonson, D. A 1976

Eoger.

Folzullov, f. S. 2452

Feeney, R. K. 555

Finden thal. M. 2155

rl ok s, I, P. 1936

Forsman. J.A. 280

Fournier, i. R. 474

Fuss, I . 34

Garrett. $R$ 1787

Genz, $H$.

Giardini Guidoni, A. 523

Gclden, L. B. $218 \quad 1562$

Gorozhankin. F. V. . $\angle 452$

Grien, H. R. 2154

Grioley, R. T. 280

Grindstar, U. $G$. 280

Grosswendt, B. 1911

Guldon I, A. G. 94.

Hahn, Y.
Hawdan, M. 204

Harriscn, M. F. A $270 \quad 1550 \quad 1601$

Hasted. J. E. 204

Heldeman, H. G. H. 1514

Hessel bacher, K. H. es

H $111 \mathrm{e}, \mathrm{E}$ $2013 \quad 2082$

Hi is, L. 261

Hitcheock, A. P: 1563

Hof fmann, D. H. H. 335

Hong, S. P. e9 486

llublel, H. C. 270

Hublet, M. - I. 1601

Huetz, A. 1606

Jain, N. K. 646

Joyez, G. 1606

Kabochnik, M. N. 1456

Karstensen, $F$ 194

Neshi-Folikuntels it 1308

Kets, F, E 1514

Kiti, H. 551

KIng, G. C. 195

Kl e inpoppen. $H$. $2 \in 1$

Klots, C. E. $15{ }^{2}$

Kolke, F. $40 \quad 418$

Kollmann, K. 205

Kumar. A IS 1 468

Kurenkov, $v, v$. 2452

Kurepo, M. V. $1 \in O S$

Land, J.E. 894

Landau, M. 1606

Lee. J. S.

Lefolure, D. $\epsilon 81$ 


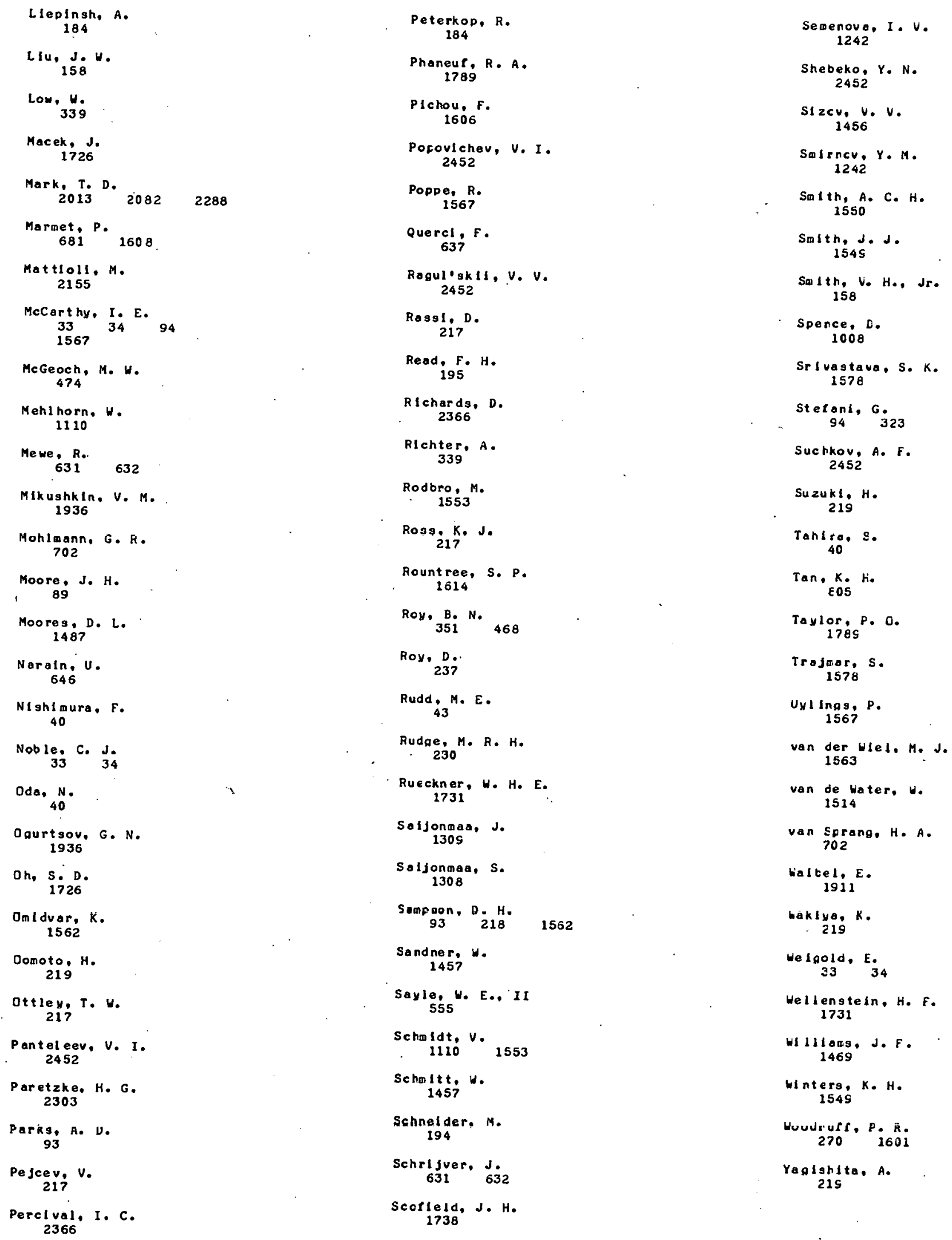


Eं06

ELECTRON-PART ICLE INTERACTIONS

Recomblnation (electron-ion)

Acton, L. W. 840

Blond I. M. A.

$$
47
$$

Breton, $C$ 2155

Brooks, R. L. 2154

Brown, W. A. 840

Burdett, N. A.

109

Chandra, $S$. 366

Chibisov. M. I. 1374

Dat la, R. U. 2154 Davis, J.

de Michel is. C. 2155

DuBol s, R. D. 73
E07

ELECTRON-PARTICLE INTERACTIONS

Collistonal Do-Exeltatios

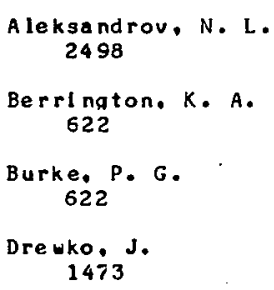

${ }_{73}^{\text {Dunn, G. H. }}$

Findenthal, $M$. 2155

Gau, J. N.

$\underset{\text { Gig }}{\text { Gould, J. }}$

Griem, H. R.

2154

Gronenschlld, E. H, B. M. 649

Gupta, v.

Hahn, Y.

Hasted, J. B. 1594

Hayhurgt, A. N. 109

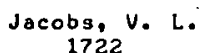

Jaln, N. K.

$\underset{73}{\text { Jeffries, J. B.: }}$

Khan, S. U. 1594

Mathur, D. 1594

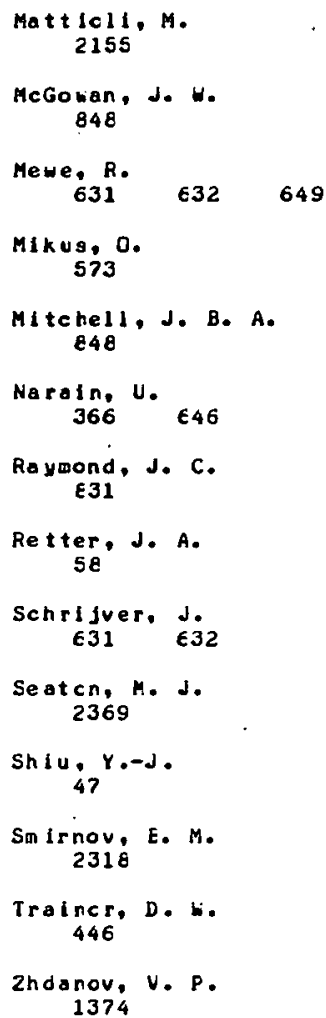

Poe. Fi. T.

Register, D. $f$. $216 \varepsilon$

Schrijuer, d. 631 G32

Szmytkowski , C. 1473

Trajmar, $S$. 2168

Zubek, $N$.
Kingston, A. E. 622

Mewe, $R$. $631 \quad 632 \quad 649$ 
EOS

ELECTRON-PARTICLE INTERACTIONS

Collisional Line Broadening

Behrlnger, $K$. 1697
Chiang, W. T.

1638

Cooper, J. 836

Denk In, v. P.

1925

Freudenstein, S. A. 836
Gorchakov. L. V. 1525

Grier, H. R. 1638

Hey, J. D. 1689

Thoma. P. 1657
809

\section{ELECTRON-PART ICLE INTERACTIONS}

Negative Ion Foreation

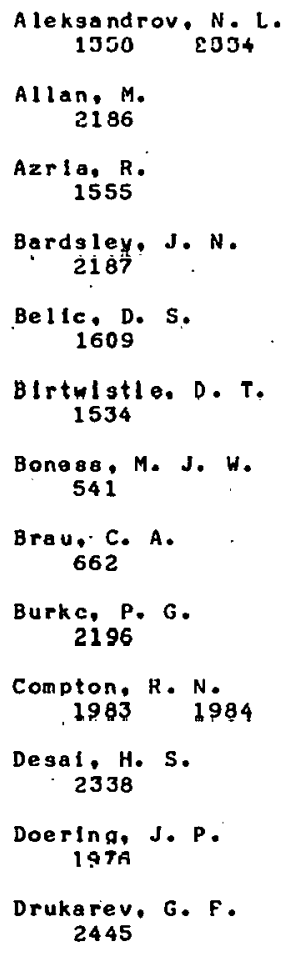

Eduonsont D. A.

$$
1976
$$

Fessenden, $R$ 1000 2088

Fletcher, J. 530

Foltyn. S. R. 530

Gculd, R. K. 1344

Gresteau, F 1554

Hall, R. I. 1554

Hall, R, I. 564

Huetz, A.

Hunter, S. R. 530

Klots, C. E. 1983 1584

Kurepa, M. V. 1609

Lee, J. 3. 1976

Lefaivre, D. 681

Mormat, $P$.

Maru, M. P. 2338
Mazeau, J.

1554

McI ver, $F$. T .. Jr. 1021

miller, h. J.

1344

Modinos, A. 1534

Moore, H. W.

1021

Nygaerd. K.J. 530

Pozdneev, S. A. 2445

Rains, L. J. 1021

Schneider, E. I . 662

ShImamor 1. F. $1000 \quad 2088$

S I mun, D.

Tam. b. -6 . 1102

Frolnar, D. H. 541

Tronc, H. 1555

Hadehro: Ja M. 2187

Wong,-S. F. 2186 
$\mathbf{E 1 3}$

ELECTRON-PARTICLE INTERACTIONS

Electron Detachment Iron Negatlve Ions

Bell, K. $_{1508} \mathrm{~L}$.

E15

ELECTRON-PARTICLE INTERACTIONS

Inner-Shel I Interactions

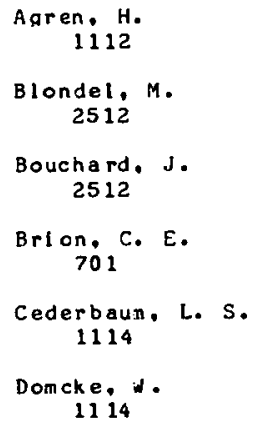

E1 6

ELECTROW-PARTICLE INTERACTIONS

Fluorescence and Lual nescence

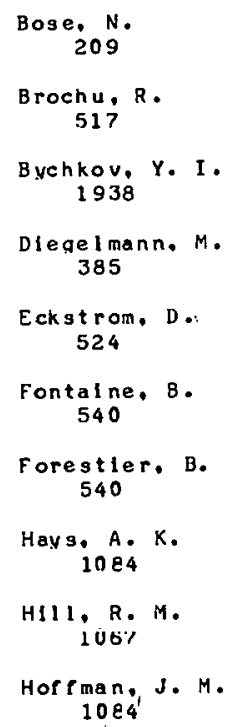

Hitchcock. A. P. 201

Kowalczyk, S. P. 1352

Lavi 11 a, R. E. 61

Legrand, $J$. 2512

Magnier, P 2512

Maler, J.P.

1114

Martin, H. L. 1352

Nordgren, J. 1112

Nordling, $c$. 1112
Solcuiev, E. A. 2326 dden, P. J. 508 
E17

ELECTEON-PARTICLE INTERACTIONS

Angular Scattering (specifled process)

Akkermann. A. $F$

$$
2489
$$

Barlas. A. D.

1574

Beaty. E. C.

89485

Berezhko, E. G.

1456

Bhat Ia, A. K. 1792

Bhallacliaryga, F. K. 1784

Bonhan, R, A. 488

Bransden . B H. 1549

Buckman, S. J . 1492

Camilloni. R. 323

Carelte, Ja-D. 237

Carlgnan. G. R. 489

Cartwright, D. C. 480

Cheeseborough, J. O., III 1471

chornoy, r. $Y$. 2489

Chuejlan, $A$. 2107

Collins, L. A. 1708

Coulter. P. 1787

Dahlor, J. S. $1495 \quad 1496$

Davenport, J. U. 368

Dehmer...J. L. 503

Delage, A. 237

D 111,5 .

Dixon, A. J 2140

Fifflet. A. W. 1.3

Gal 11 tIs. M. 260

Garrett, W. R. 1787

Ghosh, A. 3 . 1784 $\underset{554}{\text { Glanturco, F. A. }}$

Giardini Guldonl. A. 323

Gien: T. T. 333

Goswam 1, K. K. 1784

Gupta, G. P 1539 1575

Hashimoto, H. 2358

Hesselbacher, K. H. 89

H l ckerson, D. L 1495 1496

Ho, $W$. 368

Hong. S. P.

Hecu, D. T. 2140

Jensen, 3 . 1494

Kabachnik. M. N. 1456

Kennerly. R. E. 488

Kolke. F. 219

Kum ar, $\mathrm{S}$ $1546^{\circ} \quad 1822$

Kwon g, w. K. 1496

Lal, M. 1818

Lamanna, U. T. 554

Lehwann, $H$. 2285

Leung, C. N.

Levin; D. A.

Ma, M.

13

Mathur, K. C.

MeKoy,-

Mindinaw. L. 1495

Moore, J. H. 89

Noble, C. J. 1492

Norcross, D. W. 1708

Onda, $K$. 1973

o omoto, $\mathrm{H}$. 219

Pearl, J. C. 1766
Rahoon, K. K.

554

Reglster, D. 1494

Rohr, $K$. $1458 \quad 1637$

Roy, A. C 1520

Roy, D.:

Rueckner, H. H. E 1574

Sandner, $y$. 1457

Schoitt, W.

1457

Schneider, B. I. 856

Schrieffer, J. F. 368

Shateghaft . R. 1801

Sharp. E. E.

485

ShImIzU, R。 2358 Shyn, T. W.

siegel, J. SOE

S1 1. N. C.

Silver, A. 1792

Singh. S. N. $1546^{\circ} 1822$

sizov, v. v......... 1456

SmIth, J. d. $154 \mathrm{~S}$

Sr Ivastava. H. K.

Srivagtava, S. K. 1578 , 210.7

Stefani, 0 . 2.2.3.

Sullivan, E. C. 1752

Suzukl, Ho 215

Tanaka, H. $210 \%$

Tewkin. A. 1752

Teubner, P.J. U 1452

Trajmar, $s$. $1471^{\circ} 1494 \quad 1578$

Trlfothl. A. N.' 1818

Truhlor, D. G 1573

Ueda, $K$. 2358 
Waki ya, $K$.

219

Wei gol d. E. 2140

Wei nre ich, $G$.

1766

Wellensteln. H. F. 1574

E18

ELECTEOH-PARTICLE INTERACTIOHS

Attenuation (unspecifiad process)

Sal rnov, B. N.

2476
Uill lans, J. F.

1469

wi 11 ams, W.

1471

Uinters, K. H.

1549
YagIshita, A. $21 s$

Zajenc, $A . G$.

Zorn, J. C.

1766
Vyat sk In, A. Y. 2476 
FO1

ION STRUCTURE - CTR PLASKAS ( $\mathrm{H}_{2}$. He, B, $B e, 0, N, C, S i, U, H o, T I, F e, C r$.

$\mathrm{Hb}, \mathrm{Au}, \mathrm{Cu}, \mathrm{NI}, \mathrm{H}_{ \pm}^{*}, \mathrm{HeH}^{*}$ )

Transition Probabilities, Oscillator Strengths, Energy Leveis, Lifet 1 mes

Abbott $D . C$.
1586

Abdallsh. M. H. 1655 A bouaf, $R$.

Ahlenius. T. 1757

AlI. M. A.

1694

Alton, G. D. 1315

Anderson, R. J . 2112

Ani gimova. G. P. 1235

Armour, E. A. G. 1523

Armstrong, L., Jr. 823

Aufmuth, $p$. 2526

Bardslev, J.N. 1742

Bar Shalom, 4. 1277

* Bashkin, $s$. 564

Rauche.J. 2526

Bauche-Arnoul $t, c$. 1135

Baumann, $M$. 1273

Baylis. W. E. 1535

Bcok, D. R. 1754

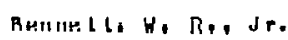
1834

Berry, $H$, G. 502

Bliail a. E. ए.

Bhatia, A. K. $1833 \quad 2344 \quad 2368$

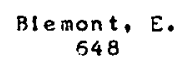

B Is hop, D. M. $1557 \quad 1782$

Bl anch ard. $T$. 835

Blinder, S. M. 748
Bogdanovich, P. 0 . 1313

Bolko, V. A. $\begin{array}{lll}1256 & 1536 & 1652 \\ 2299 & +2370 & 2461\end{array}$

Borst, $W . L$. 497

Breton, C.
1277

Brink, J. A 2542

Bromage, G. E $1129 \quad 2364$

Bromander, J . 1306

Browne, J. C. 300

Brzezowska, J 602

Burk hal ter, P. G. 1724

Buttlar, H. V. 1310

Callaway, J $346 \quad 1272$

Chagtal, M. S. Z. 1318

Chapel l c, J. 1715

Cheng, K.-T. 1132

Cheung, 1. M. 1557 i782

Chien, C. W. T.

Childs, W. J.

3.3?

Chugunov, A. Y. 2370 .

Clleves, $H .-P$. 2526

Cocke, C. L. 1131

Coetzer, F.J.

Conen, J. $\mathrm{S}$.

1712

Cohen. $L$.

Cohen, M. 1685

Connooly, M: I, 1641

Ccnstantinides, E. R. 1488

Conway, J. G.

Cosby, P. C. 289

Coulaud, G. 1715

Cowan, R. D. $1129 \quad 1724 \quad 2364$
Curnutte, B.

1131

Curtis, L. J. 1551

Dagdiglan. F. J. 699

Dal by, F. W. 462

Danzaann, $K$. $627 \quad 630$

Day, R. L. 2112

de Michelis, C. 1277

Dletrlch, D, D $5 \in 4$

di Stefano, G. 762

Donahue, D. J. 1233

Dcinolly, U. M.

Donnelly, K. E $459 \quad 1134$

Doschek, G. A. 656

Dozier, C. M. 1724

Dr Iker, M. N.

Dubke. M. 332

Duric, $N$ 1306

Edlen. B. $1314 \quad 1319$

Édởoñ s, H. H. 1596

Fagarter, E. $1640^{\circ} 1602$

Eggorter, T . P. 1540 1602

Ekberg, J. 0 . 13 ig

EIIIS. D. G. 1551

El ston, S. B. 1315

Erkec, S. 1320

Eratan. 1306

Faencu, A. $Y$. $1536 \quad 1652$ $2370 \quad 2461$

2299

Fatrchild, $C$. E. 967

ralcone, $\mathrm{n}$. W. $122 \mathrm{~s}$

Fawcett. E. C. 1125 2364

Fancnov, A. $Y$. 1256

* Basch, H. 


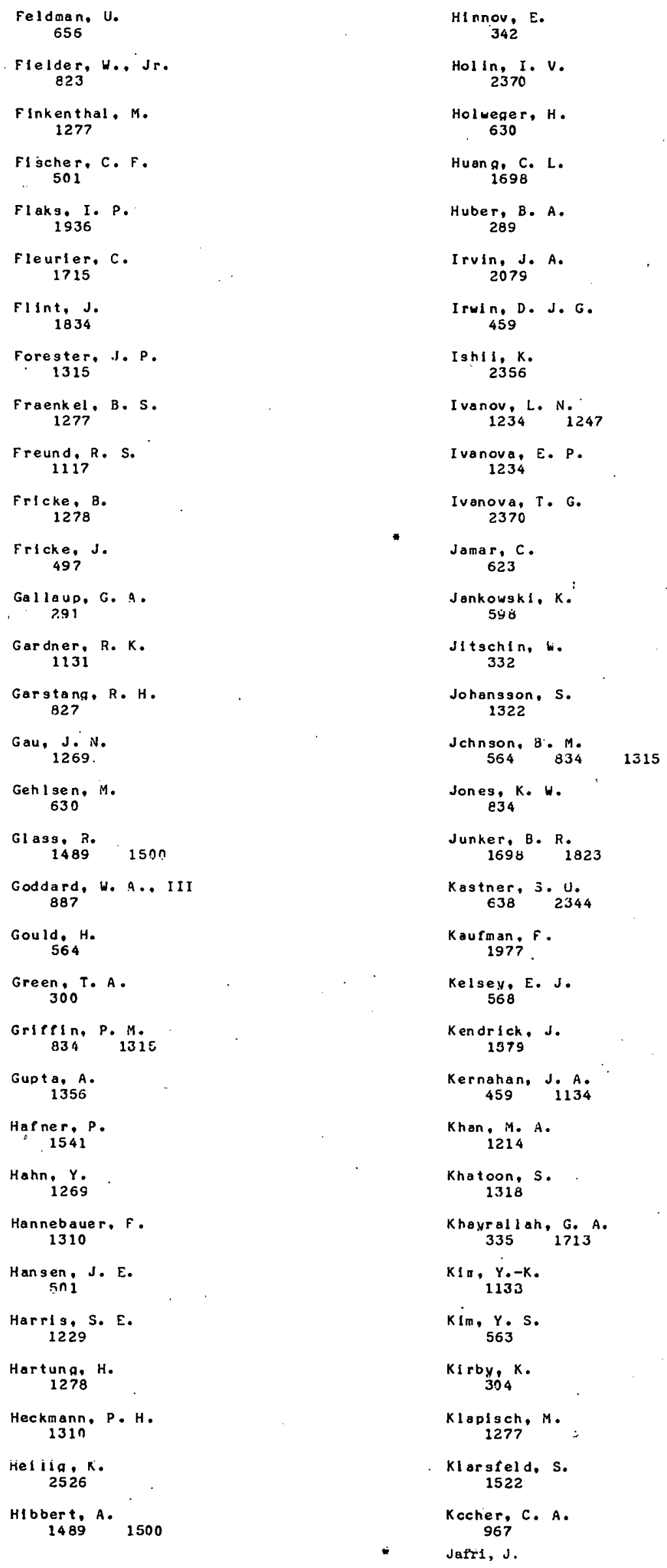

HInnov, E.

342

Hol in, I. V. 2370

Holveger, H. 630

Huang, C. L. 1698

Huber, B. A.

Irvin, J. A. 2079

I rwin, D. J. G. 459

Ishil, $k$. 2356

I vanov, L. N. $1234 \quad 1247$

Ivanova, E. P. 1234

Ivonova, T. G. 2370

Jama r, C. 623

Jonkowsk I, $K$ : $59{ }^{\circ}$

Jitsehin, w. 332

Johangson, S. 1322

Jchnson, $8 . M$. $564 \quad 834 \quad 1315$

Jones, K. W. 234

Junker, B. R. $1698 \quad 1823$

Kastner, 3. U. $638 \quad 2344$

Kaufman, $F$. 1977.

Kelsey, E. J. 568

Kendrick, J. 1379

Kernahan, J. A. $459 \quad 1134$

Khan, M. A. 1214

Khatoon, 5 . 1318

Khoyrallah, G. A. $335{ }_{1713}$

$K 1 \pi, Y_{j}-K$. 1133

Kim, Y. S. 563

Kirby, $K$.

Klapisch, M. 1277

Kl orsfeld, s. 1522

Kecher, C. A. 967

Jafri, J. 


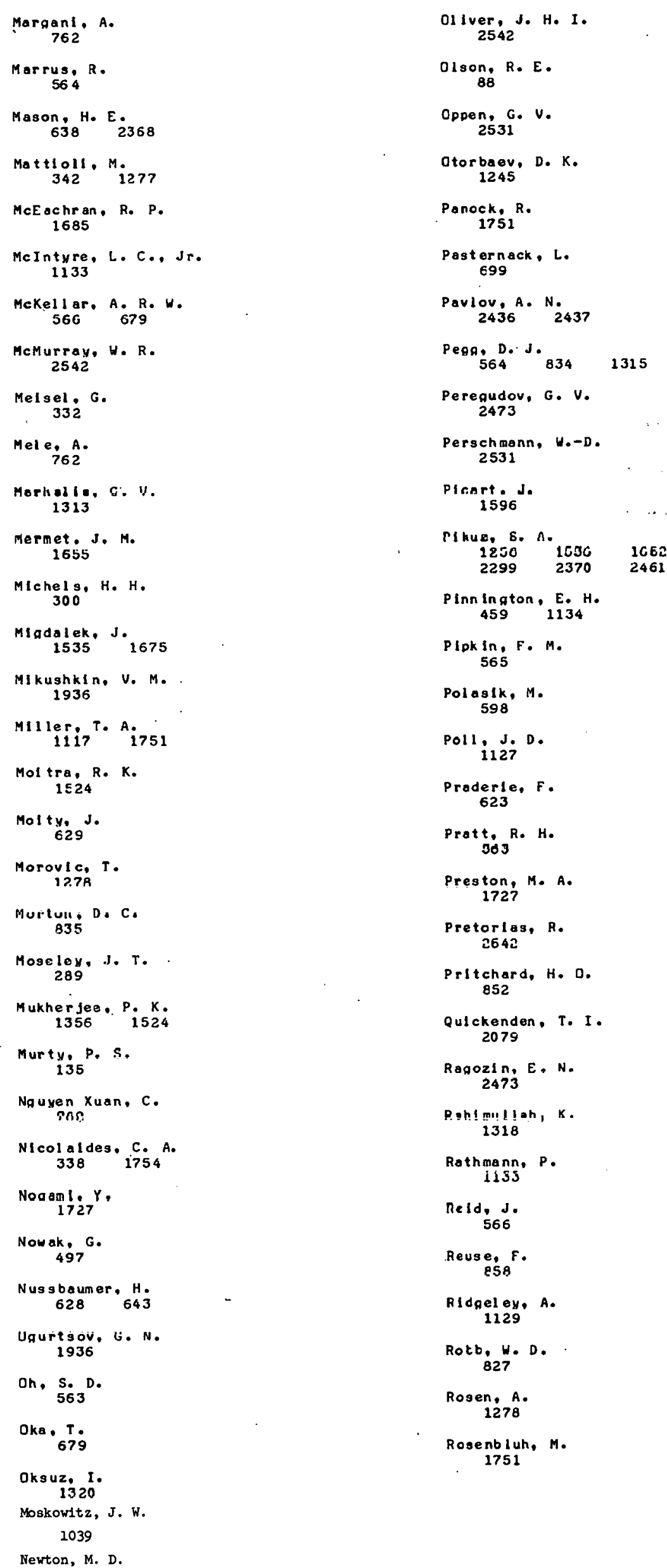

Oliver, J. H. I. 2542

Olson, R. E. 88

Oppen, G. V. 2531

Otorbaev, D. K. 1245

Panock, R. 1751

Pasternack, L. 699

Pavlov, A. N. $2436 \quad 2437$

Peg9, D. J. ${ }_{564} \quad 1315$

Peregudov, G. V. 2473

Perschmann, W.-D. 2531

PIonert. Je 1596

rikue, 8. $n$. $\begin{array}{lll}1250 & 1036 & 1652 \\ 2299 & 2370 & 2461\end{array}$

Pinnington, E. H. $459 \quad 1134$

Plok in, F. M. 565

Polaslk, M. 598

POIl, J. D. 1127

Praderle, $F$. 623

Pratt, R. H. 303

Preston, M. A. 1727

Pretorlas, R. 2642

Pritchard, H. O. 852

Qulckenden, T. I. 2079

Ragozin, E, N. 2473

P.hf mu! ! oh, K. 1318

Rothmann, P. if $55^{\circ}$

Reld, J.

Reuse, ${ }^{8}$.

Ridgel ey, A. 1129 Rotb, W. D.

Rosen, A. 1278

Rosenbluh, M. 1751

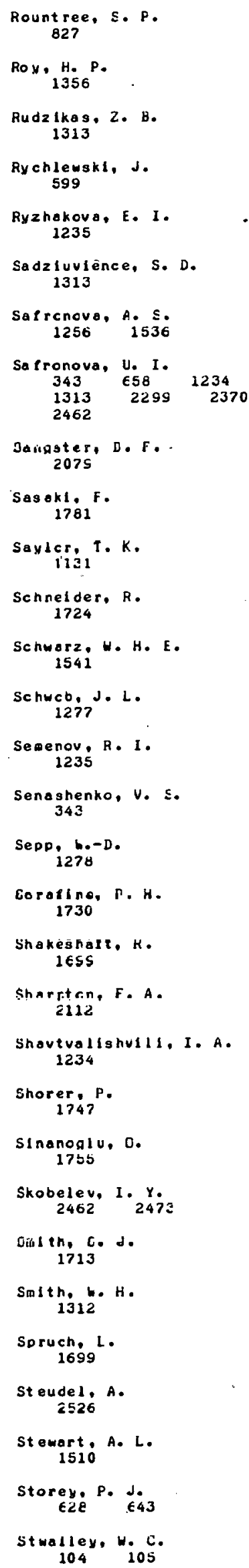




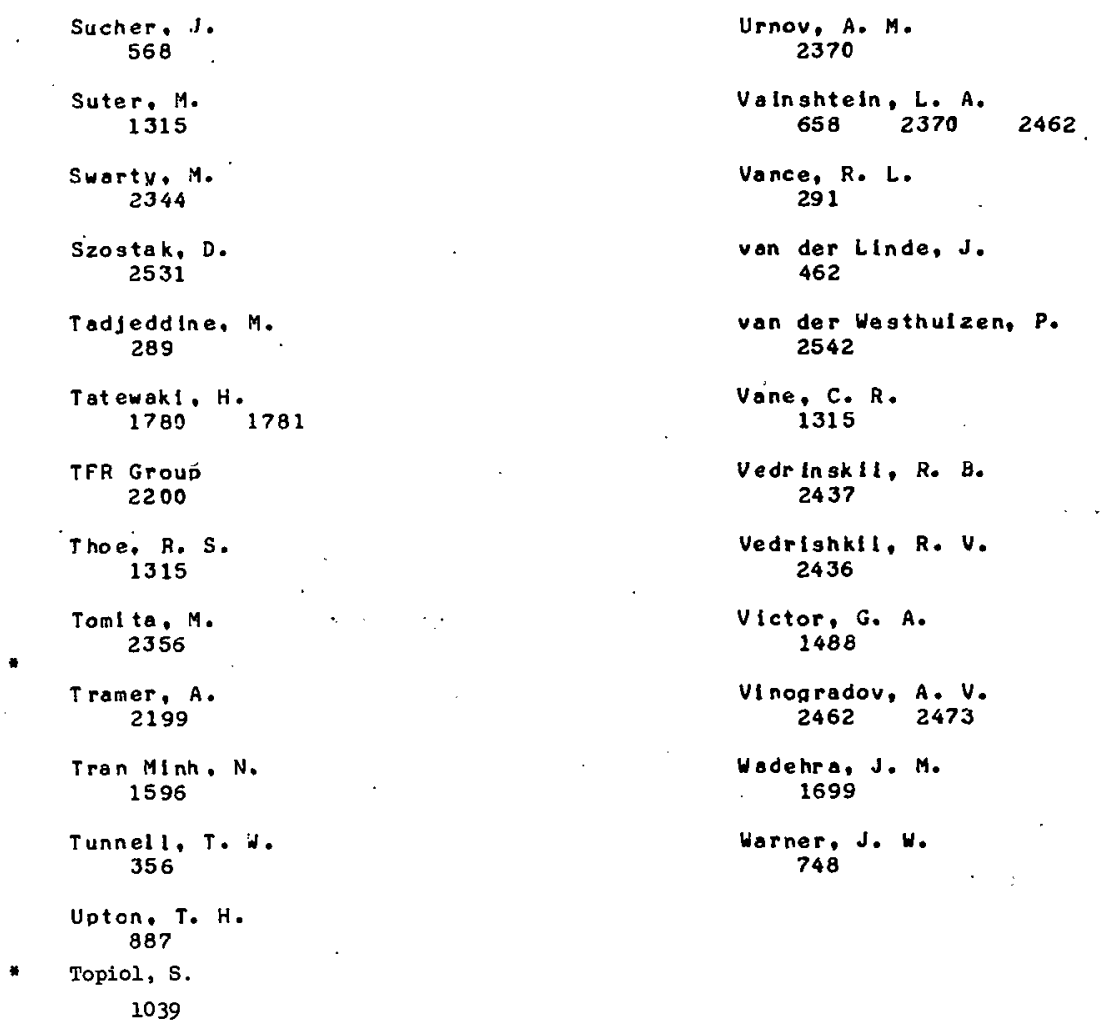


Grujtc. $P$ Grutzmacher, $K$.
1812

IOS STRUCTURE - CTR PLASMAS (Hz. He. $B_{*}$ Be, O. N, C, SI, U, Mo, TI, Fe, Cr. $\left.\mathrm{Nb}, \mathrm{Au}, \mathrm{Cu}, \mathrm{NI}, \mathrm{H}_{3}^{*}, \mathrm{HeH}^{*}\right)$

Shirts and Line Broadening by Flelds

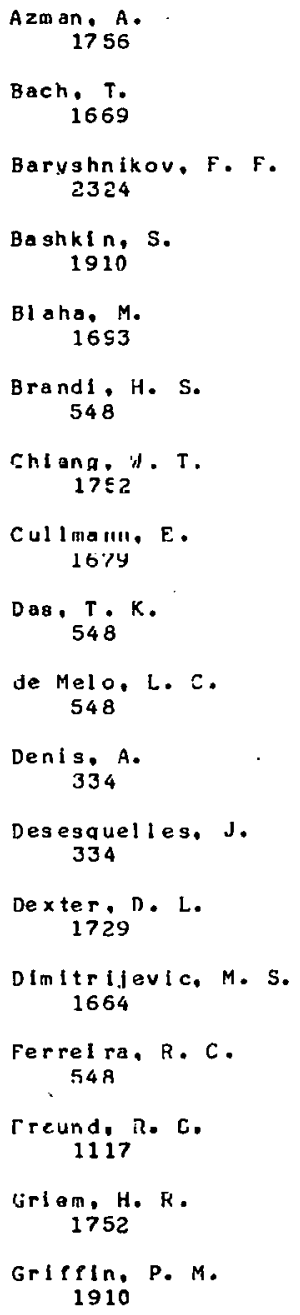

Electron Affinity

Dewal. $M$. J . 3 .

882

ION STRUCTURE - CTR PLASMAS ( $\mathrm{H}_{2}$, He, $\mathrm{B}$. Be. O. N, C. Si. u, Mo, TI. fe, Cr.

Kruse, T. H.
Hex, J. D. 1693

Jones, $K$. W.

Karp, A. i 1688

Kl yuchnik, A. V. 347

Kcnjevic, N. 1690 191

Labuhn, $F$. 1679

Lax, B. 1773

Leavttt, I. $n$.

1910

Lisilsa, v. 3i 2324

Lczovik, Y. E.

347

Miller, T. A. $1117 \quad 1773$

Miranda, L. C. H. 548

Murai, T. 2363

Neumann, G. C. 1773

O'Connell, R. F. 504

Panock. R. 177.3

TENA, D. J. 1910

Feregudov, G. $v$. 2473

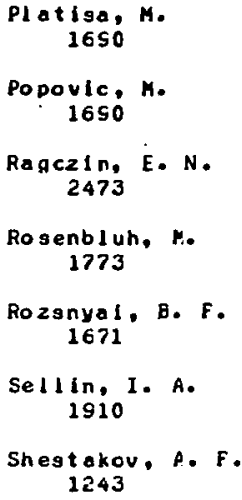

Nb, Au, Cu, NI, $\left.\mathrm{H}_{3} * \mathrm{HeH}^{*}\right)$

Brzozowskl, J.

$$
1311
$$

Eraan, P. 
602

TRANSPORT PHENOMENA ANB AVERAGE PROPERTIES IN GASES

Diffusion of Neutrals

Arora, P. S.

417

Bjerre, $A$

745

Brindl e, R. C.

1054

Carson. P. J. 417

Chenevier. $M$. 2188

Codasterano, P. 315

Dahler, J. S.

911

Dunlop, P. J. 417

603

TRANSPORT PHENOMENA AND AVER AGE PROPERT IES IN GASES

Diffusion of Electrons

Batoechl, A. 1173

304

TRANSPORT PHENOMENA AND AVER AGE PRDPERTIES IN GASES

Diffusion of Ions

Albritton. D. L.

818

Alger, S. R. 1564

Dick inson, A. S.

$$
1474
$$

Judelk Is, H. S.

1045

Kestin, J. 314

Khal ifa, H. E. 314

Kol ts, J. H. 1073

Lawton, S. A.

$$
1958
$$

Lles-Nielsen, I. 745

$\underset{845}{\operatorname{Martel}} A$.

MIchaud, jo 845 .

Nyel and, $\approx$. 745

Oskam, H. J. 1054 Pack, $R$. T

Porker, G. A. . 953

Eraglia, 3. L.

1173

Kleban, P.

1013
Pebay-Peyroula, J. C. 2188

Phelps, A. V.

1958

Fatel, $A$.

Ricei, M. A

Sadeghi, N.

2188

Setser. D. W.

1073

Tracy, c. J.

liakeham, W. A. 314

Wu $n, M$.

1045

Xystris. N. \$11

Zanza, v.
1013

Davis, H. T.

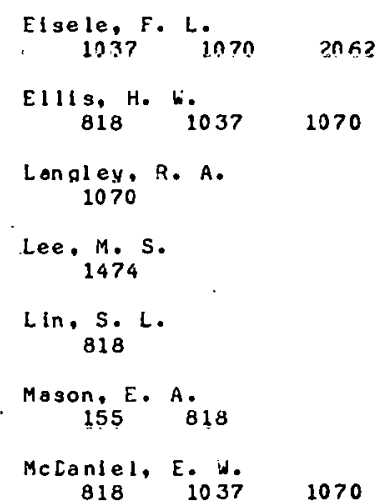

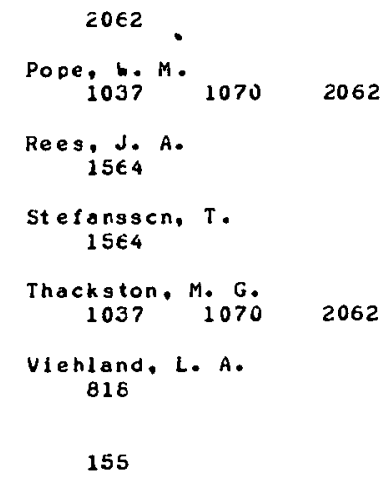


605

TRANSPORT PHENOKENA AND AUERAGE PROPERTIES IN GASES

Defft Velocity of Electrons

Bragl I a, G. L.

1723

$\underset{1547}{\text { Carter,J. G. }}$

Chrigtophorou, L. G. 1547

Dallacasa, v. 1723

Davis, H. T. 1013 rng

TRANSPORT PHENOMENA AND aVERAGE PROPERTIES IN GASES

Drift Velocity or Ions

Albritton, D. L.
818

A remstrona. D. A.

1 853

Bardsl ey, J. N.

$$
1743
$$

Blondl. M. A.

$$
1740
$$

Cohen. J. S.

$$
1743
$$

notan, $T$.

Eisele, f. 1
Fletcher. J.

$$
543
$$

Fol tyn, S. R.

543

Fournier, G. R. 474

Freeman, G. $R$. 945

Gresser, J. 1905

Huang, S. S.-S. 945

Hunter, S. R. 543

Kleban, P. 1013

Land, J. E. 894

2094

Elford, M. T. 1623

EIIIs, H. H. $\begin{array}{ll}818 & 1002 \\ 2087 & 2054\end{array}$

1070

Fehsenfeld. $f \cdot c$. 995

Gatland. I. R. 2094

He Im. H. 1623

Jchnsen, $R$.
1740

Lomm, D. R. 2094

Langley, R. A.
$10>0$

LIn. S. L. 818 $-\cdots$ $\underset{574}{\text { Lucog, J. }} 575$

Maxey. D. V. 2547

MeCorkle, D. L.
154 ?

McGeoch, H. $H$. 474

Nakenura, $x$. 574,575

Nygaard, K. J. 543

Schultz, $G$. 1905

Schworz, K. W. $216 i$

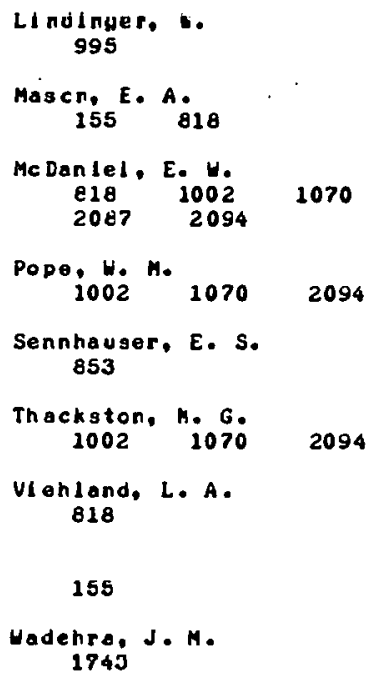

TRANSPORT PHENOMENA AND AVERAGE PROPERTIES IN GASES

Seattering and Energy Loss Parameter if Ellectiums. Neulrals and Tons in Gases

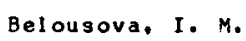


G08

TRANSPORT PHENOMENA AND AVERAGE

PROPERTIES IN GASES

Energy DIstribution (energy

distribution of lons and electron

uth applled electric and aognetic

rleids)

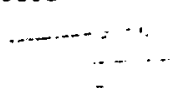

Fournler, G. R.

474

Govinda Raju, G. R.

379
Gurumurthy, G, R。 379

Hogted, J. B. 173

Land, J.E. 89

Makabe, $T$. 1610

Mathur, D. 173

McGeoch, 4. W. 474
Mn at sakanyan, A. K. 2466

Mori, $\mathrm{T}$ 1610

Naidis, G. V.

Naveed-Ullah, K. 173

Shalkauskas, Y. S. ES7

Shternov, N.P. 2466
G09

TRANSPORT PHENORENA AND AVERAGE PROPERTIES IN GASES

Momentue Trangfer

Bardsl ey. J.N. 1743

Bhat techaryya, P. K. 1784

Braglla, G. L. 1723

Carlanen. G. R. 489

Cartwriaht, D. C.

$\ldots=$

480

C Chutj!an. A

2107

Cohen, J. S.

colllng; L. 4 .

53 i 1708

Dahlor, J. S.

$91 i$

Dal l acasa. $V$. 1723
Fliflet, $\dot{A}: W$ 13

Ghosh, A. S. 1784

Gcswan I, K. K. 1784

Hickman, A. P. 55

It I kawa, $Y$. 659

Juoge, D. L. 839

Lond, J. E. 894

Levin, D. A. 13

Liu, W. $-K$.

125

Lucas, J

Ma, M.

McCourt, $F$, : 125

McKoy, $\dot{v}$. 13
Morrlson A. A.

53

Nakouura, Y. 575

Norcross. D. W 1708

Onda, $k$. 1573

Sharp. W. E. $48 S$

Shyn, T. H. 489

SmIth, F. I . 55

Sr luastava, S. K. 2107

Tanoka, H. 2107

Truhlar, D. G. 1973

wadehra, J.M. 1743

$F \cdot-M$.

e3s

Xystris, N.

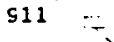

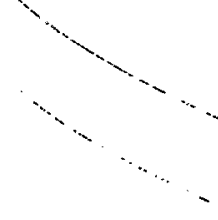
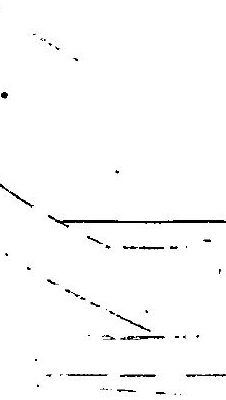
510

TRANSPORT PHENORENA AND AUERAGE

PROPERTIES IN GASES

First and Second Tounsend Coefflelents

Davies, D. K.

469

611

TRANSPORT PHENOMENA AND AUERAGE

PROPERTIES IN GASES

Electron Attachaent Coefelcients

Davies, u. $x$.

469

H01

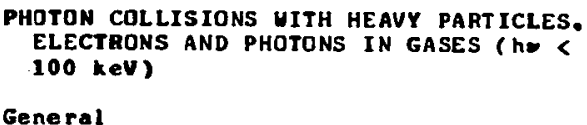

General

Anosov. M. D. 2.35

Baklanov. E, V. 2458

Berman. P. R. 2

Burghteln, A, I. 2313
Land, J.E.

$$
894
$$

Risbud, A. W. 860

Naldu. M. S. 860
Eísbud. A. V.

Grunberg, $R$.

1387

NaIdu, M. S.

860

860
Dok torov, A. B.

2313

Dubetsk I I, B. Y. 2458

Kochanov, V. P. 2317

Lee, H. W.

1642

Le Gouet; J.-L.

$L \in$ tokhov. v. $S$. 2315.2439

Minogin, V. G. $2315 \quad 2439$
Pavilk, B. D. 2315

Puser, A. Y. 2313

Rautian, S, G. 2317

Rosenberg, $L$. 1836

Shalagin, A. M. 2317

Sr ivastava, R. F. 503

Stehle, F. 1542 
PHOTON COLLISIONS UITH HEAVY PARTICLES ELECTRONS AND PHOTONS IN GASES (ho < $100 \mathrm{keV}$ )

Total Absorption

Ackerhalt J. R. $1225 \quad 1226$

Anderson, $R, G$. $513 \quad 1233$

And reev, S. I. 1236

Aral, $S$. 1065

A ve rbukh, I. $S$ : 2438

Bachrach, R. 2 495784

Behmenbura, $W$. 2283

Behringer, K.

Berman. P. R. 91 Blanconf. A.
495

Blen, F.

BI rnbaum. G. 1653 .

Blschel. W. K. 535

Boscher, J. 609

Brechlgnac, $C$. 91

Breton, J. 2120

Brickmann.J. 159

Brion, C. E. 134

Brown, F. C.

Bruce, $C$. $W$. 608

Cahuzac, P. 1202

Camus, P. 1486

Charpentler. $H$. 604

Clark, J. H.

$$
513
$$

Codling, $k$. 1449

Cole. 8. E. $241 \quad 1667$

Combet Farnoux. $F$. 2192

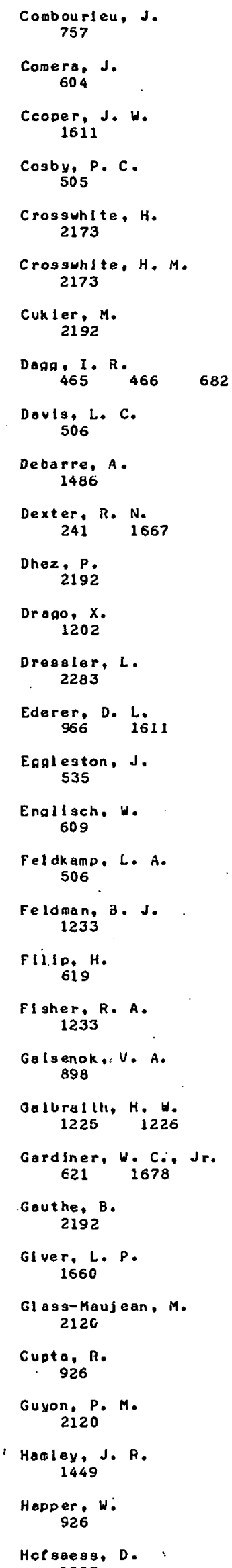

Hollard, R. F. E1s

Hosch, J. H. 620

Imasura, Mo 1065

Ishiguro, E. 1604

I to, $K$,

606

Jaccb, J. H. $\$ 44$

Jaegle, $F$ 2192

Jaussoud, $C$. 604

Jo hn. T. J. 2365

Jourdain, J.L. 757

Kamp, L. H. 652

Karp, A. H. 1688

KI田, K. C. E 19

Kobe, D. H. 2126

Kogoma. $M$. 1065

Kovargkil. V. A. 2438

Kung, F. T. V. 512

Lanqer, $S . H$. 830

Largen. L. E. $E S 1$

le Biras, G. 757

Lee, $\underset{S 05}{L .} C$.

Leroy. B. 757

Lupasur, V. P. 2261

Lu, K. T 2173

Lyman, J. L. 1233

Mailard, U. G. $\begin{array}{lll}621 & 1678 \quad 2099\end{array}$

Manganc, J. A. 544

Hoouko, H. 1604

Mayer, $A$. 604

McCray, $\mathrm{R}$ $820 \quad 830$

Mehlamn, $G$. 1611

Mehluan, $G$. 


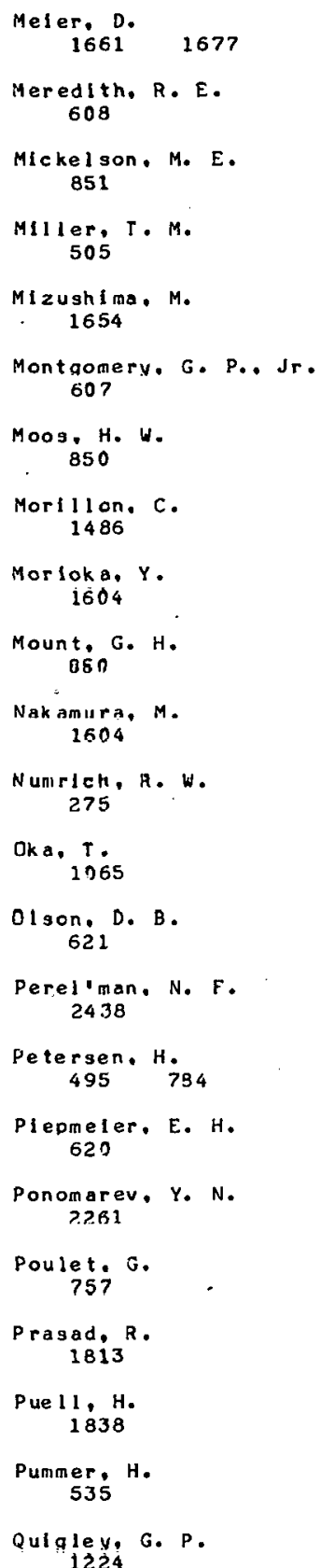

Quigley, G. P.

Reesor, G. E.

$465 \quad 466 \quad 682$

Rhodes, C. K.

535

Rigaud, $P$

757

Rokn1, M.

544

Ross, R. R. $820^{\circ} 830$

Saheki. T.

Soloman, E. B. $966 \quad 1611$

Sarzhevskil, A. Mo 898

Sasanuma, M. 1604

Schater, G. 600

Schelnaraber, H. 1838

Schenek, P. k. 2099

Shardanand 1682

Sirchis. M. 512

Smith, F. G. 608

$\sin l t h$, G. $P$. 50

sayth, K. C. 2099

Szoke, A. $12.1 \mathrm{~s}$

Tajime. T. 606

Tam. A. C. 2085

$T$ an, $K$. $H$. 134

Theria, $P$. 86

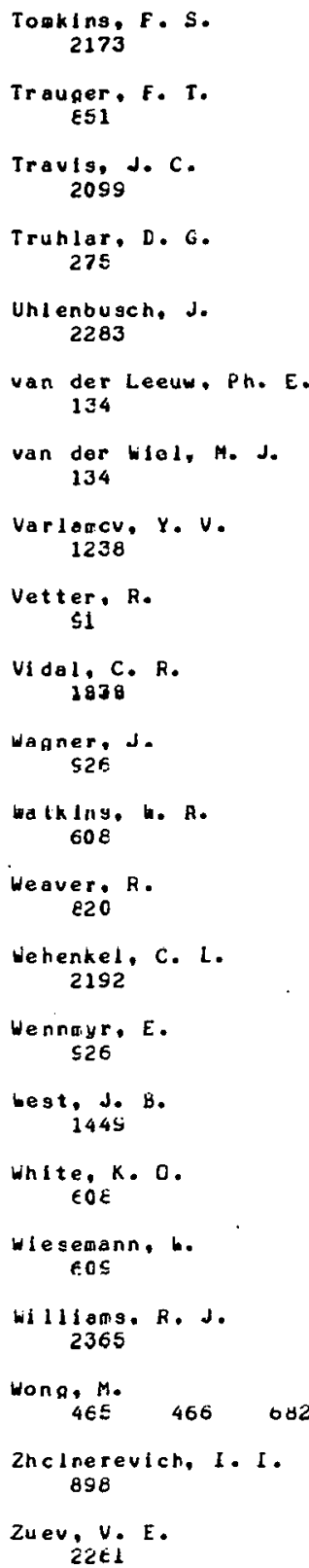


H03

PHOTON COLLISIONS UITH HEAVY PARTICLES, ELECTRONS AND PHOTONS IN GASES ( he < $100 \mathrm{keV})$

Elastic Scattering

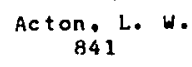

All awadhi, K. L. 63

Chen. M. H. 37

Courtens. E. 510

Crasemann, B.

$$
37
$$

Ghumgan, B. S. 53

Hol lander, T. 1696
Ice, $\underset{37}{\text { G. }}$ E.

Pratt, R. H. 2124

Jongerius, M. J: 1696

Klssel, L. 2124

Kusa Raju, G. 2343

Lok shm inarayana, $V$. $1628 \quad 2343$

Lam . L. 1712

McCray, R. 820

Narasigha Murty, K. 2343

Narasi mha Murty, V. A. $1628 \quad 2343$

Navasimha Murty, $K$. 1628

Raju, G . K

$162 \varepsilon$

Ross, R. R.

Satyoendra Prasad, M. 2343

Sood, B. S.

63

Szoke, A.

510

Tong. B. Y.

1712

van der Bli, J. J. 1696

Verma, S. L.

63

tieaver, 5 . 820
Prasad, M. S.

1628 


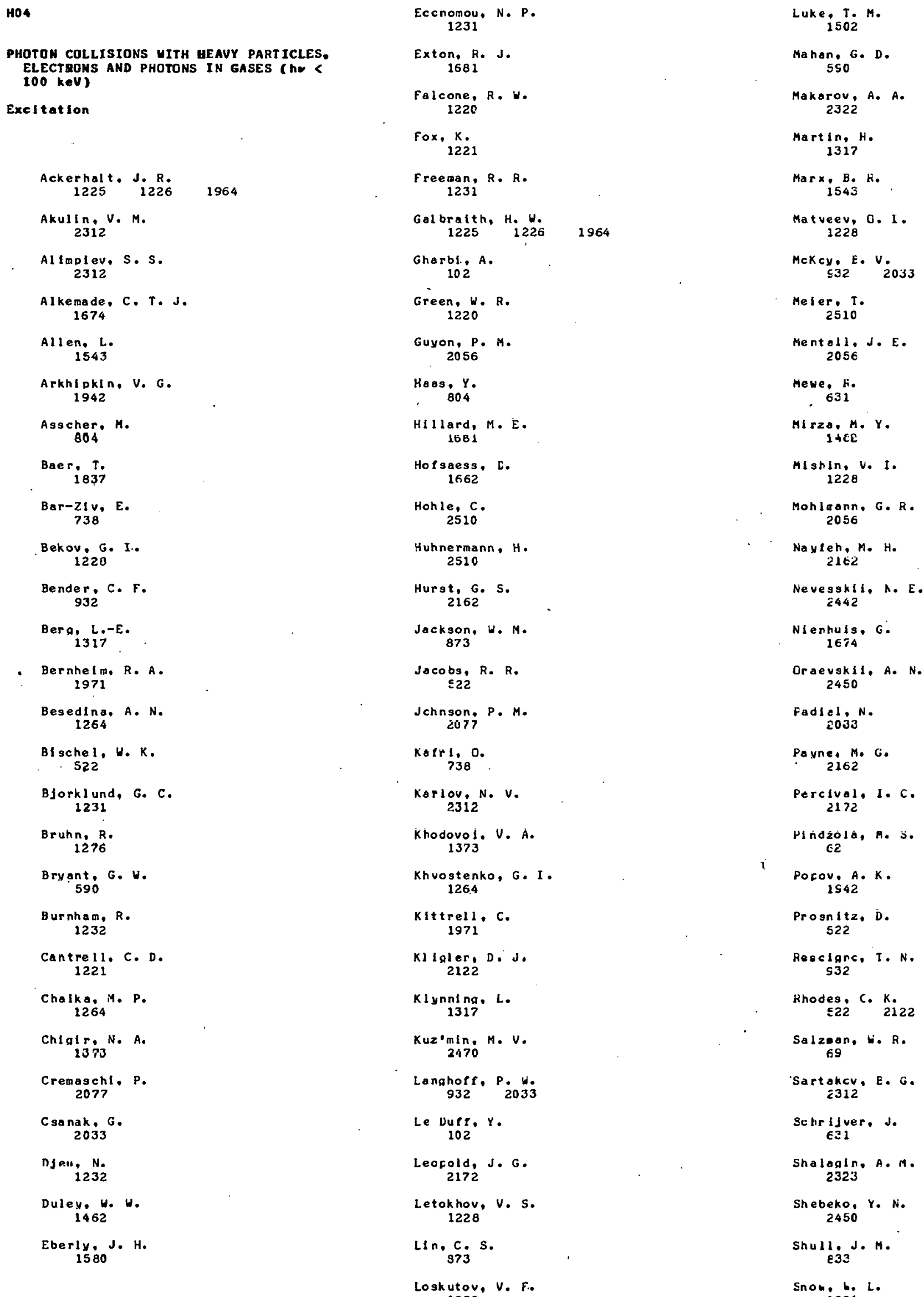

HO4

PHOTON COLLISTONS UITH hEAUY PARTICLES, ELECTRONS AND PHOTONS IN GASES ( ho < $100 \mathrm{keV})$

Excitation

Ackerhalt. J. R. 1225 i226 1964 Akulin. V. M.

Al Implev, S. S. 2312

Alkemade. C. T. J. 1674

Allen. L. 1543

Arkhipkin. v. $G$. 1942

Asscher, $M$. 804

Baer, $T$. 1837

Bar-ZIv, E. 738

Bekov. G. I.. 1220

Bender, C. F。 932

Berq, L. E. 1317

Bernhelm. R. A.$$
1971
$$

Besedina, A. N. 1264

Bi schel, W. K. 522

Bjorkl und, G. C. 1231

Brutho $R$.

Bryant. G. W. 590

Burnham, R. 1232

Cantrell. C. D. 1221

Chalka, M. P. 1264

Chlgir, N. A. 1373

Cremaschl, P. 2077

Csanak, $G$. 2033

njal. N. 1232

Dulev. W. W. 1462

Eberly, J. H. 1580

Eccnomou. N. P. 1231

Exton, R. J. 1681

Falcone, R. W. 1220

Fox, $K$.

1221

freeman, $R$. $R$. $123 i$

Gal bratth, H. W. 1225 1226 1964

Gharbi., A. 102

Green, W. R. 1220

Guyon, P. M. 2056

Heas. $Y$.

. 804

Hillard, M. E. $\mid 5 B 1$

Hofsaess, c. 1662

Hohle, c. 2510

Huhnermann, H. 2510

Hurst, G. 5 2162

Jackson, W. M. 873

Jocobs, R. R.

$$
\leqslant 22
$$

Jchnson. P. M. 2077

xotr $1,0$.

Karlov, N. V. 2312

Khodovol. V. A. 1373

Khvostenko, G. I. 126.4

KIttrell, C 1971

Kl Igler, D.J. 2122

Klynnt ng. L. 1317

Kuz'min. M. V. 2470

$\underset{932}{\underset{9 a n g h o f f}{~}} P_{2033}$

Le Vuff, $Y$. 102

Leofold, J. G. 2172

Letokhov, V. S. 1228

LIn. $\underset{873}{C .}$.

Loskutov, V. F.

Luke, T. M. 1502

Mahan, G. D. 590

Makarov, A. A. 2322

Martin, H. 1317

$\operatorname{Mar} x, B .1$. 1543

Matreev, 0. I. 1228

HeKCY, E. V. $532 \quad 2033$

Meler. T. 2510

Mentall. J.E. 2056

Mewe, b.

. 631

Mi rza, M. Y. $1+C \mathrm{C}$

Hishin, V. I. 1228

Mohlaann, G. R. 2056

Naxteh, M. H. 2162

Nevegskil, A. E. 2442

Nienhuls, $G$ 1674

Oraevakil. A. N. 2450

Fadial. N. 2033

Panne, M. G. 162

Percival. I. C. 2172

Hindzóla, R. S. 62

Pocov, A. K. 1542

Prosnttz, D. 522

Reselgrs, T. N. s32

$\underset{E 22}{\text { Khodes. }}$ K.

Salzean, H. R 69

Sartakcv, E. G. 2312

Schrijuer.J. Eal

Shalogin, A. M. 2323

Shebeko, Y. N. 2450

Shull. J.M.

Snow, b. $L$. 
Sont $89,8$.
1276.

Suchkov, A. F.

2450

Timofeev. V. P. 1942

Ulyakov, P. I .

1950

van Dijk. C. A. 1674
Veirs, $D, K$. 1971

$\underset{287}{V I k \text { is, } A . C .}$

Wagner, $H$. 2510

Whitten, J. L. 2077
Holff, H. H.

$127 \epsilon$

Young, J. P.

2162

Zeegers, P.J.T.

1674 
PHOTON COLLISIONS UITH HEAUY PARTICLES ELECTRONS AND PHOTONS IN GASES ( he < $100 \mathrm{keV}$ )

Dissoctation

Aboual, $R$ $289^{R} 984$

Ackerhalt . J. R. 20

Akamatsu, $R$. 2346

Akul in, $V$. M. $1376 \quad 2312 \cdots$

Albritton. D. L. 1122

Allmolev. 5.5 . $1376 \quad 2312$

Anaerson, $A, G$. 513

Ashiold. M. N. F, 436

Bado: $\overline{\mathrm{P}}$.

Baer. $r$. 229

Band, Y. B.

Baronavgkl . A. P. 141

Barsuhn, J. 1003

Barua, A. K. 1571

Bar-zi v. $-E \div$

Basov, N. $G$. 2469

Bazhul in. S. P. 2469

Berry, M. J.

- 750 .

Bi sche 1. W. K. 2045

… Black, J. H. 026 .

Bottcher. $C$. 130

Bot tor. R, 229

Brion, C. E. 134

Clark. J. H.

Cool. T. A

Cosby, P. C

$289 \quad 505 \quad 984$

$1122 \quad 1351-2023$

2024

Dalgarno, A. 695
Detta, K. K.

1571

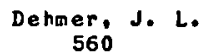

de Vries. A. E. 735

de $\underset{735}{\text { Vries, M. S. }}$

DIII. D.

Durup, J. $116 \mathrm{~S}$

Erl andson. A. C. 779

Ershov, L. S. 2474

Falrehild...C. E.2.054

Ferreira, L. F. A 229

Flukel 'slitelul' $v$. $r_{0}$ 2271

Freed, K. F. 996

Gedanken, A. 1009

Georqe, T. $F$. 1017

Govers, T. $\bar{R}$. 229

Gront. E. R. $943 \quad 2117$

Grushevski i. V. B. 1253

Guyon, P. M. 229 2056:-

$H$ an, D. 0 . 1206

Heil er, E. J. $977 \quad 1032 \quad 2011$

Hotrann. $H$. 3060

Huter, B. A. 289.584

Jaffe, R. L.

Johngon, $K: E$ :

$>2021$

_Jcnes;G. G.

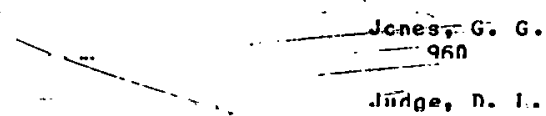

167

$K \in \operatorname{tr} 1,0$ 738

Korl, R, R, Jr 1963

Karlov, N. V.

K. $1376 \quad 2312$

KI I gler. D.J. 2085

Kolöitskil . Y. R. 2468

Kroger. P. M. 1048
Kul onde $r, K, C$. $130 \quad 2011$

Kuz'olin, M. V.

Kwei, G. H 1048

Langhoff, S. R. 954

Lau, A. M. F. 563

Lawrence, G. M. $20 \leqslant 4$

Lee, L. C. $187 \quad 505$ 2105

Lee. $Y_{\text {S43 }}$ T. 2117

Leone. S. R. 2060

Leoncv, Y. E.

Levy. D. H. 2021

LIng. J. H.

Lyman. J. L. ISEJ

Macpherson, $M_{0}$ T. 436

Mayo, J

MeDenald, J.R. 141

McGarvey, J. A., Jr 775

McGIlvery, D. C. $1036 \quad 1069$

Meloughlin, R, L. 1366

Mental1, J. E. 2056

Miller, F. G. 141

Miller, T. 505

- Mnhiminnn, G. R. 2056

Morrioon, J. D. 103e 1069

Morse. H. D.

Moseley, J. I. $289 \quad 584 \quad 1122$ $\begin{array}{lll}1351 & 2023 & 2024\end{array}$

Nenner. 1 . 225

Nesbet. F. $k$ 1003

D-ohate, $K$. 2346

Oren, L. 187

Fapernov, S. M. $1253 \quad 2260$ 


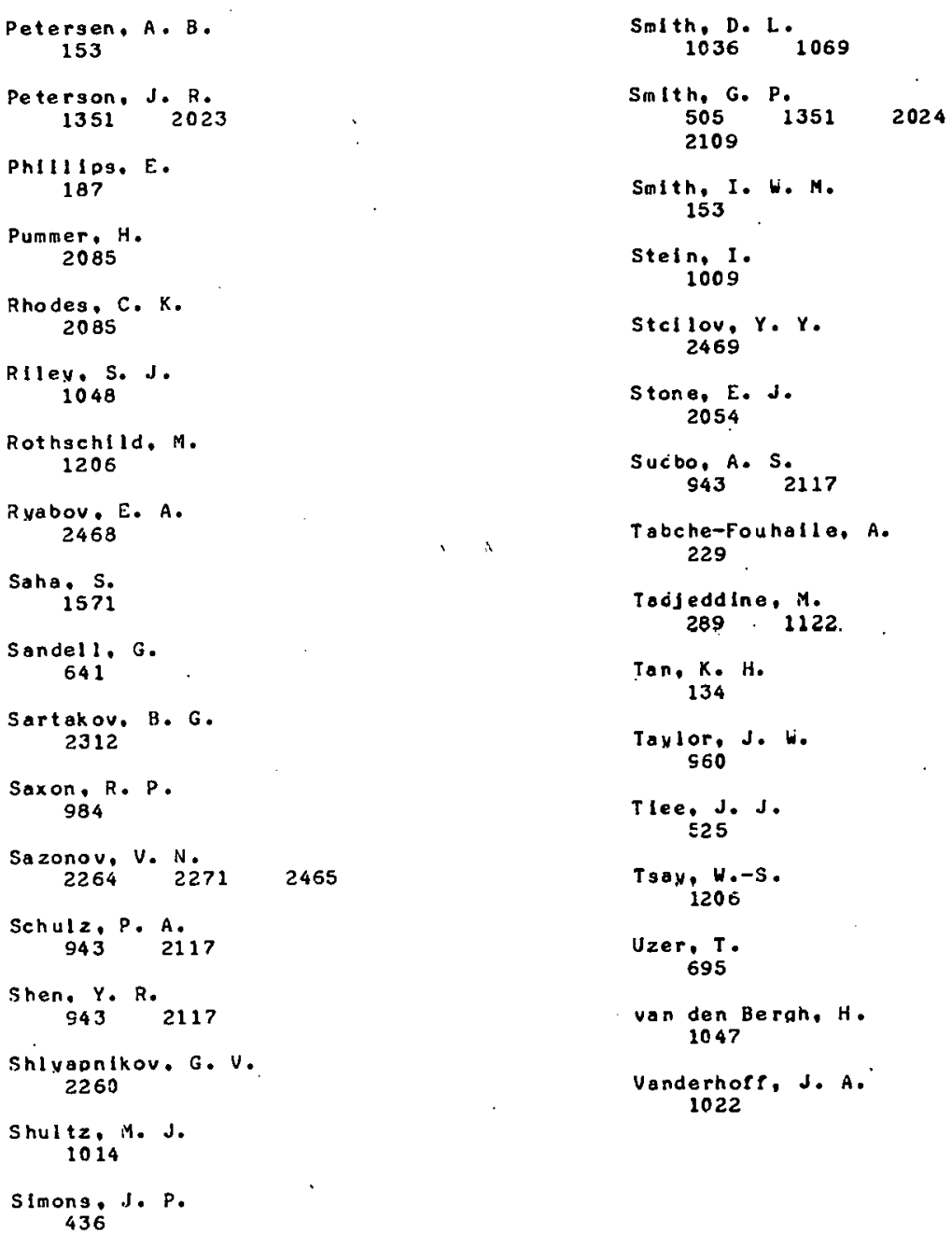

Petergen, A. B. 153

Peterson. J. R $1351 \quad 2023$

PhIll IDg. E. 187

Pummer. $H$. 2085

Rhodes, $C . K$. 2085

Rlley, S. J. 1048

Rothschild. M. 1206

Ruabov. E. A. 2468

Saha is?

Sandell, G. 641

Sartakov. B. G. 2312

Saxon, R. P 984

Sazonou, V. N. $2264^{2} 2712465$

Schulz, P. A. $943 \quad 2117$

Shen, $Y$. R. $943 \quad 2117$

Shlvapnlkov. G. V. 2260

Shultz, M. J. 1014

Simong. J. P. 436

Smith, D. L. $1036 \quad 1069$

Sinth, G. P. 2109 2024

Snith, I. W. M. 153

Stein, I . 1009

Stcllov, Y. Y. 2469

Stone, E. J. 2054

Sucbo, A. S. $943 \quad 2117$

Tabche-Fouhalle, A. 229

Todjeddine, $M$. 289.1222

Tan, K. H. 134

Tavlor, J. W.

T Iee. J.J. 525

Tsan, w.

Uzer. T . 695

van den Bergh, H. 1047

Vanderhofs, J. A. 1022

van der Leeuw, Ph. E्.. 134

van der Wlel, M. J: :

134

van Veen, N.J.AB, ? 735

Vikis, A. C.

. 2076

West, G. A 750

Wharton, L.

2021

white. J. C. 2003

$w 1+t ! a, c$. 525

Yablonoultch, E. 1014

Yanson, M. L. $1253 \quad 2260$

Yuan, J.-M.

$$
1017
$$

Zalesskil, v. $x$. 2474

2dasluk, G. A. 2003

2 ID $, E, C$. 1365

zuev, v. S. 2469 
HO6

PHOTON COLLISIONS HITH HEAUY PARTICLES. ELECTRONS AND PHOTONS IN GASES ( he < $100 \mathrm{keV})$

Ionization

Achiba, Y.

778

Adam, M. Y.

$11 \in 1 \quad 1497$

Adler, I.

80

Agostini. P.

$1321 \quad 1450$

A 11 son, ì. A. 917

Amusia, H. Y.

336

Andrushin. A, I. 2430

Antonov, $V$. S.

$1223 \quad 2273$

Argo, M. F.

1656

Avi lova, I. V. 1260

Aymar. M. 263

Bachrach, ?. 2 . 495

Baer. T.

Danitoft. G. M.

Banna. M. S 97. $1079 \quad 109$ ?

Barsuhn, $J$. 1003

Bar-ZIv, E. 738

Datton, o. r. 2014

Bearman, G. $H$. $21 \% 6$

Belik, V. P. 154 ?

Btn-Aryeh, $Y$. 1222

Bender, $C, F$. 932

Berezhko, E. G. 1451

Bernstein, R. B. 2045

Berry. R. S. 802

Blanconl. A. 495

Bjorkl und. G. C. 2180

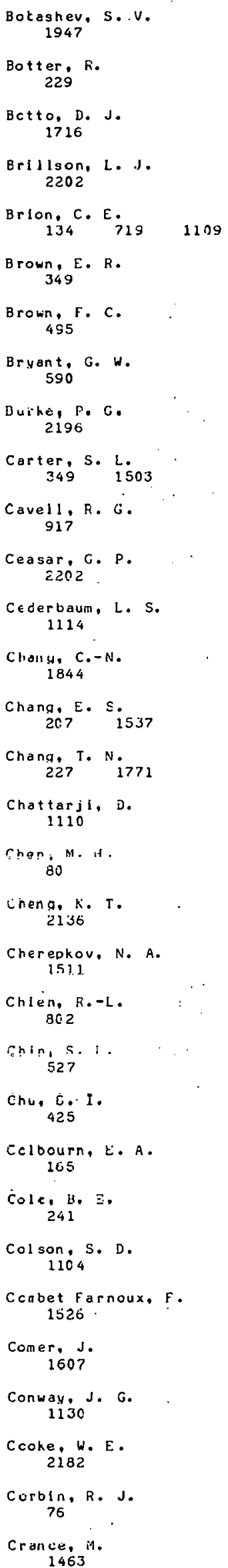

Botashev, S..V. 1947

Botter, $R$. 229

Bctto, D. J. 1716

Erilison, L.J. 2202

Brion, C. E 134 i19 1109

B rown, E. R. 349

Brown, F. C 495

Bryant, G. W. 590

Buithè, P. C 2196

Carter, S. L.

Cavell, R. G. 917

Ceasar, G. P. E202

Cederbaum, L. S. 1114

Clioll g, C. - N.

Chang, E. S. 207 1537

Chang, T. N 227 i771

Chat tarji, D. 1110

chan: M. A.

80

inena, $k . T$. 2136

Cherepkov, N. A. isi.1.

Chien, R. -L. $8 \mathrm{a} 2$

chin, s. i. 527

Chu, E. I . 425

Cclbourn, Ė. A. 165

Colc, B, $\equiv$. 241

Colson, S. D. 1104

Conbet Farnoux, F. 1526

Comer, J. 1607

Conway, J. G.

1130

Ccoke, W. E. 2182

corbin, R. J. 76

Crance, in.

Crasemann, B. $\varepsilon 0$

Creaschi, F. 2077

Csanak, G. 2033

Dalgarno, A. $4 \subseteq \varepsilon \quad \varepsilon 32$

Davenfort, J. H. 1179

Davidscn, E. R. 1075

Dehmer, J. L. $301 \quad 560 \quad 592$ $\begin{array}{llr}1031 & 2177 & 2198\end{array}$

Dehaer, P. M. $301 \quad 1031$

de Lange, C. A. $419 \quad 721$

de la fieza, R. ESI

de Leeun. D. M. $41 \mathrm{~S} 721$

Delone, $A$. $E$. $1944 \quad 2447$

de Meíjere, J.L.F. 87

Dexter, K. N. 241

$D \mathbf{i l}, \mathrm{D}$. $\leq \in 0 \quad 592 \quad \dot{1} 77$

Di $\times 1$ t, S. N. 1321 2116

Lomcke. W. 11 is

Duong, H. T. 2.32

[yke, J. N. 165

Eastan, D. E.

$15 \quad 373$

Eoerhardt, b. ż156

Eberly, J. K.

Eccncricu, N.P. $21 \varepsilon 0$

Elvutin, P. V. 1261

Evans, s. 1216

Falcone, $R$. W. 1220

Faubert. D. 527

Fedcrcu, M. $1944 \quad 2430 \quad 2440$

Ferreira, L. F.A. 225

Fonck, F. J. 2144

Freman, K. Fi. 2180 


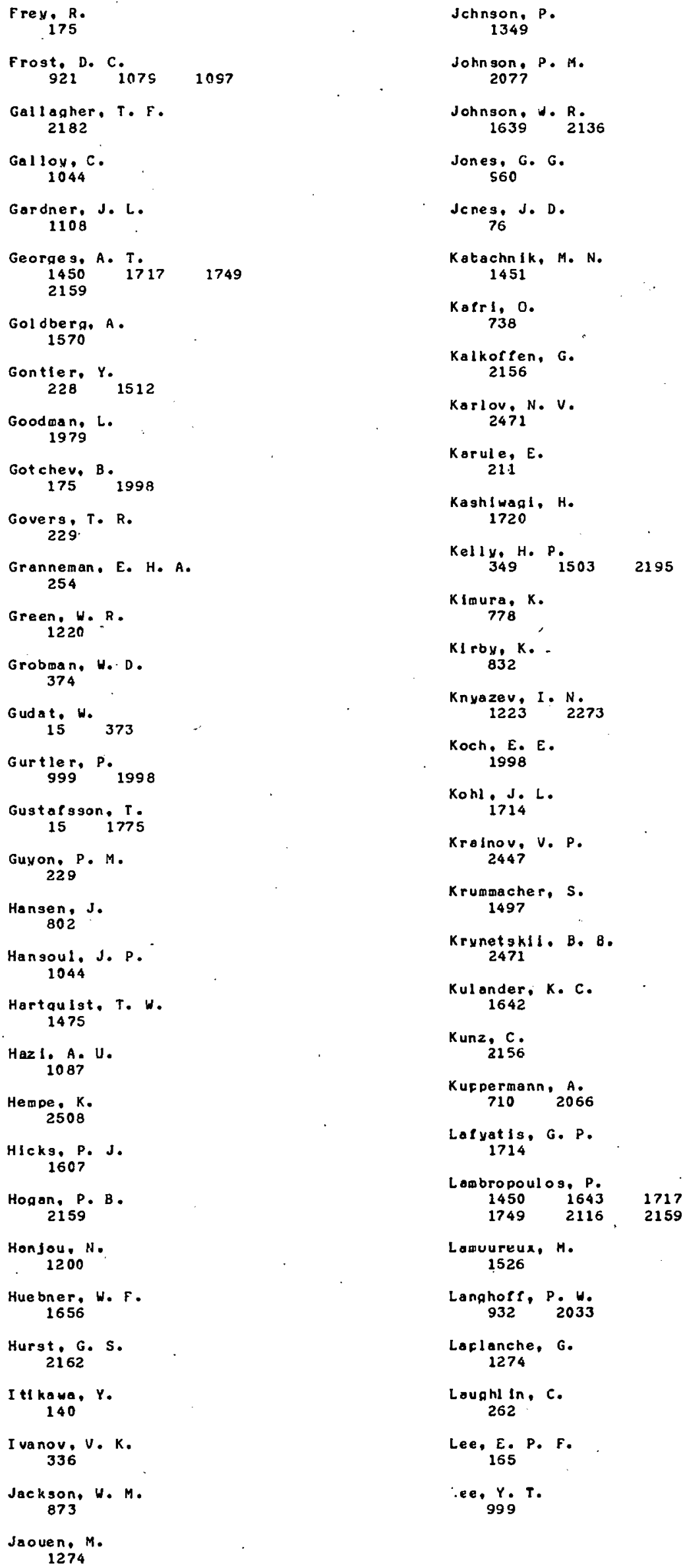

Jchnson, $P$. 1349

Frost, D. C. 921 ins 1097

Gallagher, T. F. 2182

Gallox, C. 1044

Gardner, J. L. 1108

George s, A. T $\begin{array}{lll}1450 & 1717 & 1749\end{array}$ 2159

Goldberg. A. 1570

Gontter. Y. $228 \quad 1512$

Goodman, L. 1979

Gotchev, B. $175 \quad 1998$

Govers, T. R. 229

Granneman. E. H. A. 254

Green. W. R. 1220 .

Grobman, H. D. 374

Gudat. W. 373

Gurtler. P.

Gustafsson, $T$. $15 \quad 1775$

$\underset{229}{\text { Guyan, P. M. }}$

Hangen, J. 802

Hansoul, J. P. 1044

Hartquist, T. W. 1475

Haz 1. A. U. 1087

Hempe, K.

Hicks, P. J. 1607

Hogan, P. B . 2159

Honjou. N. 1200

Hue bner. W. $F$. 1656

Hurst, G. $S$ 2162

Itlkawa, Y. 140

I vanov, V. K. 336

Jackson, W. M. 873

Jaoven. M. 1274

Johnson, P. M.

2077

Johnson. R $1639 \quad 2136$

Jones, G. $G$. $\$ 60$

Jenes, J. D. 76

Katachn ik, M. N. 1451

Kafrl, 0 . 738

Kalkoffen, G. 2156

Karlov, N. V. 2471

Korule, E.

Kashlwagi, H. 1720

Kelly, H. P. $349^{H \cdot P 03} 2195$

Kimura, $x$. 778

KI rby, $K$. -

Knyazev, I. N. $1223 \quad 2273$

Koch, E. E. 1998

Kohl, J. L. 1714

Krainov, V. P. 2447

Krummacher, $S$. 1497

Krunetgk11, B, $B$. 2471

Kul ander, $K$. C. 1642

Kunz, $c$. 3156

Kufpermann, A. $710 \quad 2066$

Lafyat is, G. P. 1714

Lombropoul os, p. $\begin{array}{lll}1450 & 1643 & 1717\end{array}$

Lamuureux, M. 1526

Langhofr, P. W.

Loflanche, $G$. 1274

Loughl In, C. 262

Lee, E. P. F. 165

.ee, Y. T. 999

Lehmann, K. K. 1575

Leofcld, J. G 2172

Letokhov, V. S. $1225 \quad 2273$

Levenson, M. D. 1321 . 1450

Leventhal. J. J. $217 \epsilon$

Levin, v. G. 1592

Lichtin, D. A . 2045

Lin, C. S. e73

LIU. J. W $15 \varepsilon$

Loabardl, G. G. 1810

Lompre, L. A. $11 \in 6$

Lorquet, J. C. 1044

Lu, K. T . $21 \mathrm{HO}$

Luke, T. M. 1502

Mahan, G. D. 550

Maler. J. P. 1114

Mainfray, C. 1166

Manus, $C$. 1166 ,

Marr, G. V. $22 \epsilon \quad 1552$

Martin, $R, L$. 1075

Mascn, S. T . 180s

Mathur, B. F. 5ว0

Mathur, $K, C$. 1814

Matiuk, V. M. 1223

Mccorthy. I. E. 1567

Mcclean, H. A. 15,4

MCCeM, I. G. 1552

Melcwell, C. A. $521 \quad 1075$

1097

McEnnan, J. 1716

McGuire, E.J. 570

HeKov. B. V. $\$ 32 \quad 2033$

Mehlhorn. W. $1110^{\circ} 1497$ 


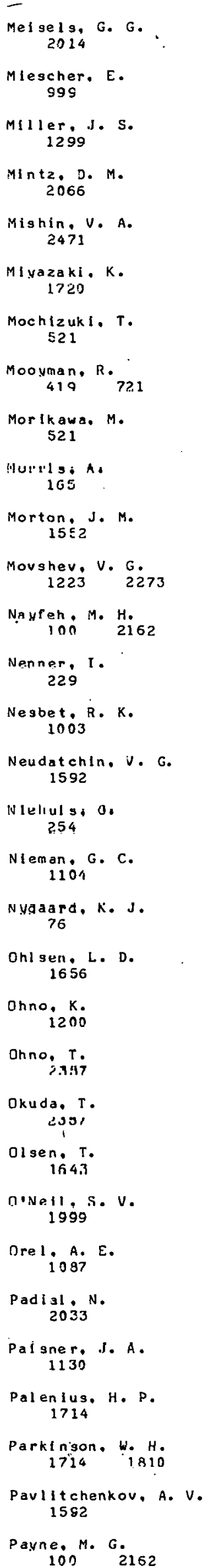




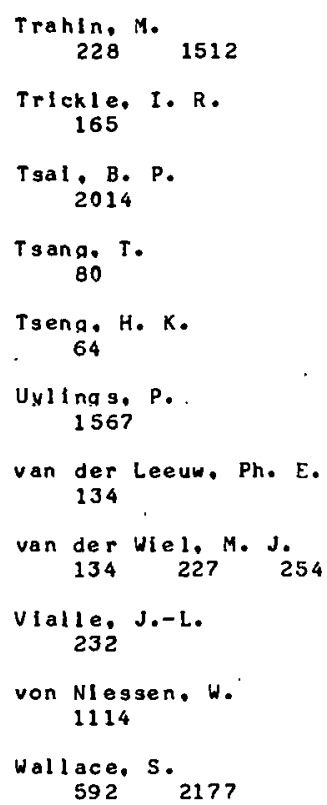

Photodetachment

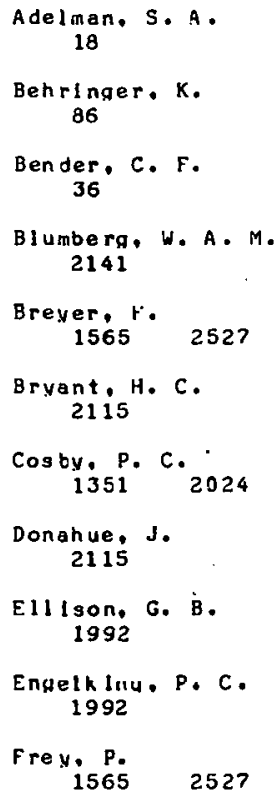

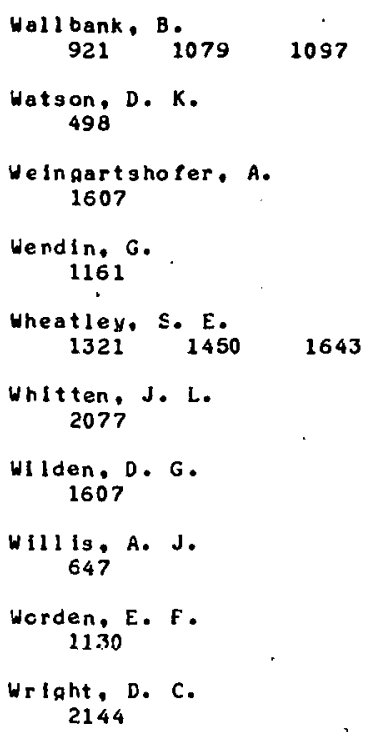

Grom, P. A. M.

2115

Hctop, H. $1565 \quad 2527$

John. T. J 2365

Jopson. R. M. 2141

Lamm, G.

$$
18
$$

Larson, D. J. 2141

Lee, L. C. 1351

Lineberyer , $w . C$ $17 \quad$ is92

Moknu, n. $v$.

Moseley, J: T: $1351 \quad 2024$

Nculck, S. E. 17 .

Peterson, J. $R$. 1351

Proll, J. C. 2115

Feod, $F: H$

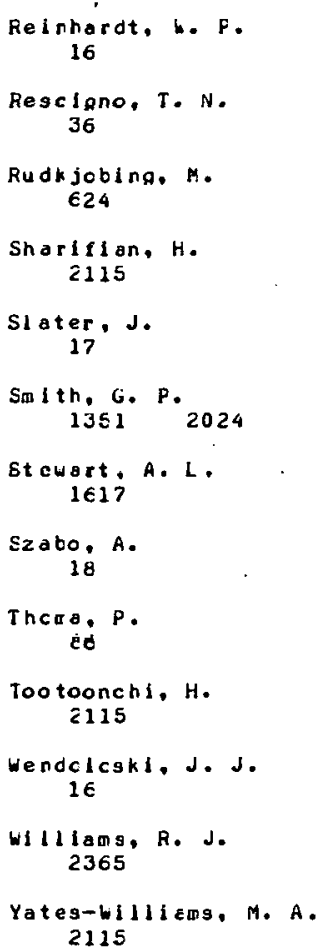


Ho 8

PHOTON COLLISIONS UITH HEAVY PARTICLES. ELECTEONS AND PHOTONS IN GASES ( he < $100 \mathrm{keV}$ )

Fluorescence and Luefinescence Ylelds

Ackerhalt, J. R.

$$
22
$$

Alharo. Me

$$
1719
$$

Anderson. $R, A$.

$$
1019
$$

A rkhipkin, V. G. 1942

Barneg R R.

$$
60
$$

Baronausk 1 A. A. 142

Berofe. K. 2189

Di raben, F. 2189

Bottenhelm. J.w. 866

Brolda, H. P. 2006

Calvert, J. G.

Chen, $K$. 803

Clark. R.

Courtens. E. 510

Curry.

Damon, E. K.

Danon, J.

Davis, S. J. nat 1U10

Devoret, $M$. Iu 4

$\underset{\text { Dค? }}{\text { Drull inger, }} \underset{1091}{R . E .}$

$n+11+0.0$
776

Eland, J. H. D.

$$
704
$$

Ershov, L. S. 2474

Filseth, s. v. 780

Fiteh, P..S. H. 1063

Frankel, D. S., Jr. 427

Fredriksgon . $K$. 2613
Ga11 75

Giacobino, E. 2189

Grynberg, G. 2189

Gutcheck, R. A 776

Hanko, $L$.

1019

Hepper, H. 490

Helthmar, E. M. 618

Hessel, M. M. $\exists 62$.

Hohl e, c.

Hosch, J. W. 620

Hou. M.

Huhnermann, H. 2510

$\underset{187}{\text { Judge , D . L. }}$

Kofser, D. 2518

Kircher. J. F. 605

KnIght, P. L. 78

Kowalski, J.
2640 Kut I ak, G.

Kulina, P. 2518

Kusch, P. 994

Leach, S $\mathrm{PO}_{4}$

le Calve. d. 116

Lee. L. C. 187

Lee, S. $-T$. 412

Lavy. i. it. 1063

Linton, C. 1120

LIvingston, A. E. 2518

Lochet, J. 1625

Lundber , $H$. 2513

Hand!. A. 384

Manuccía, I.J. 427

Motthias. E. 412
Mayo, J.

McCaffery, A. J. 163

MeDonald, J. R. 142

Meier, T 2510

Miller, F. G. 142

Molander, W. A. 78

Neumann, F. 2540

oldenbora. F. C. 2001

Oren, L.

Parks, J. H. 384

PhIllips, E. 187

PhILII Fs, M. M. 843

Plepmeler. E. H. E20

Plankex. F. W. $\epsilon 18$

Pollakcff. E. E. 412

Popov. A. K. 1542

Pourcin, J. $\$ 62$

Preaton, A. 1121

Puell. hi. $183 e$

Hadle, H. H. 2518

RIce, b. W.

fiv seliberua h. A. 412

Ruglos: P: 794

Schelnaraber. H. $1 e 3 \varepsilon$

Senacionzer, $H$. 1774

Shirley, D. A. 412

Sidebot tom, H. W. 866

Smith, E. W. $S \in 2$

Steele, F. E. 2006

Steenhoek, L. E. 803

Stoek, M. SE2 1091

strcud, C. Fi., Jr. 78 


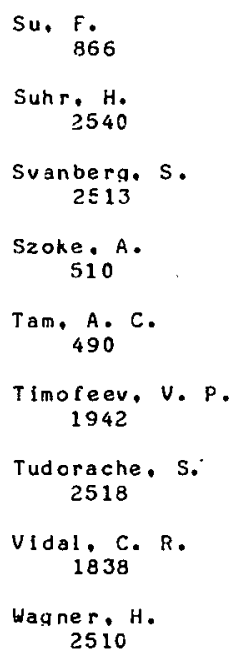

H10

PHOTON CULLISIUNS WITH HEAVY PARTICIF.s. ELECTRONS AND PHOTONS IN GASES ( he < $100 \mathrm{keV}$ )

Photocheml stry

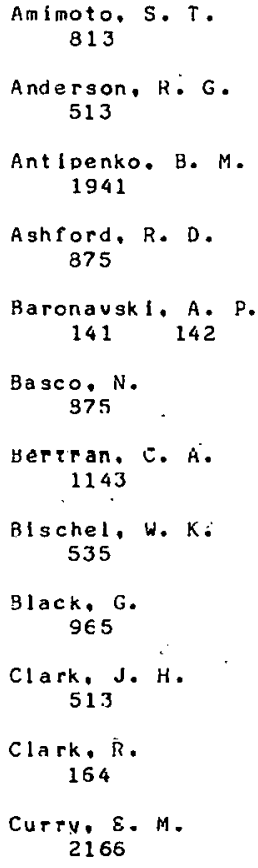

Vallenstein, $R$.

$$
780
$$

Wampler, $F$. B. 2001

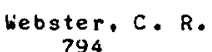

Welnstock, E. M. 1121

Wel ge, $K$. $H$. 780

West, W. P.

Wharton, L. 1063

bilte, M. G. 412

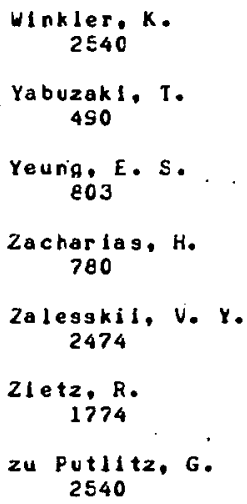

Degan I, J. 1041

Deotyarev, Y. L. 1941

Egoleston. J. 535

Fairchlld, P. $W$. 812

Fcrce, A. P. 813

Happer, W.

Hunt, J. $E$. 875

Lam. L. K. 816

Lee, E. K. C: 812

Lin, C. T . 1143

Manuin, n. C. 1993

Mccaffery, A. J: 164

McDonald, J. R $141 \quad 142$.

Miller, R. G. $14 i \quad 142$

Nikulaev, $V$. D. 1941
Odenbers. F. $C$. 2001

Pumaer, 5 .

Quack. H. 1970

Reis 9, H. 1 S\$ 3

Rhodes, C. K. 535

Rice, bi:

Rosenfeld, E. 1041

Schearer, L. D. 816

Slanger, T. G. $S \in S$

Tam, A. C. 2166

Tarosenkc, V. V. is 41

Hampler. F. B. 2001

wiegenfeld, J. F. 813

Yabuzakl, I 2166

Yatsives. 1041 
H1 1

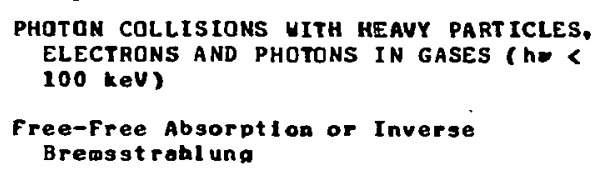

Al rov. $S$. 542

Bl anc, $A$. 1163

H12.

PHOTUN COLLISIONS UTTH HFAYY PARTICLEE. ELECTRTNS ANA PHOTONS IN GACED (Hi: : $100 \mathrm{keV}$ )

Photon-Electron scattering

Barwick,J. 486

H1 3

PHOTCN COLLISIONS UITH HEAVY PARTICIFS, ELECTRUNS AND PHOTONS IN GASES (ho $<$ $100 \mathrm{keV}$ )

phuton-Photon Collislons

Anikin, U. I,

2320
Christiansen, H. H.

$$
542
$$

Decoster, A.

1163

John. T. J.

2365

John, T. L.

639

J un a, C.

101

Kruger, $H$.

$10 i$

K.rell, N. N.

21

Hepulitn, H. A.

21

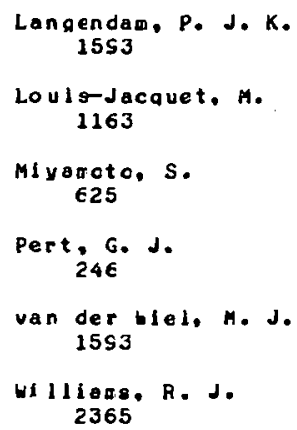

Langendam, P. J. K. $15 \mathrm{s3}$

Loulo-Jacquet. $M$.

1163

Miyarato, $S$.

625

Pert, G. J.

$24 \epsilon$

van der blel, M. J.

1593

H 11 iens, R. J.

2365

inivarotc. $s$.

625

Swany, E. T, P. V.J. J. 2342
Drobovich, K. N. 2320

Dubuilh; A. N.
Ei aerl.

1668 
H14

PKOTON COLlisIons HITH hEAUY PARTICLES. ELECTRONS AND PHOTONS IN GASES ( ho < $100 \mathrm{keV}$ )

Photo-De-Excltation. QuenchIng, ond Stinulated Eolssion (crass sect lons)

Abells, I. D. 1230

101

DATA COMP ILATION

Heavy Particle

Albritton, D. L. 661

KO1

REVIEUS AWD BOOKS

Heavy Particl

Al fass 1. 2. B.
686

GI I bod y, H. B. 451-

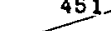

REVIEUS AND BOOKS

Partlcles on surfaces and Sollds

Bauer, $W$

1389

Ginot, $P$.

1390

Moruzz1, J. L.

450
Babkin, V. I.

2464

Boer, $T$.

1230

Bethune, D . S.

1997

Krcl1, N. H.

21

Kuznetsova. s.v. 2464
Lankard, J. R. 1S57

Maslov, A. I.

2464

MCMulin, H. A. 21

Sorokin, P. P. 1 SST?
Gardner. R. K. 660

Grov. T. J. 660
Johnston, H. S. 22ऽE

Podclske, J 2258
ReIsler, H. $\epsilon 86$

Sellin, I. A.. Ed. $\varepsilon 88$

Sol rncu, B. M. 1386

Stechens, $k$. $G$ 450

Wilson, I. H. 450

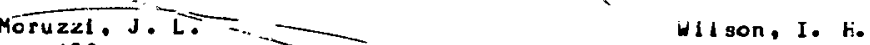

$\underset{889}{\text { Levine, R. D. }}$ 450

Nikitin, E. E. 1386

$G$ In I ger
686

Hul er, E.

Jortner, J... Ed.

- $\cdots \cdots+\ldots$
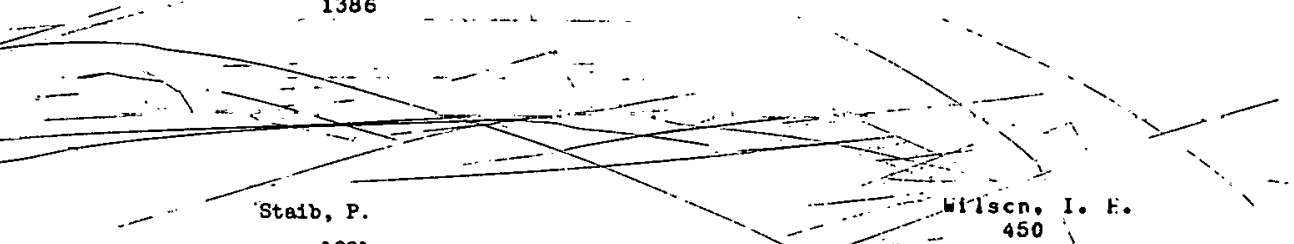

Staib, P.

1391

Staudenmaier, G.

1391

Stephens, $1, G, \cdots$ - 450, 